W

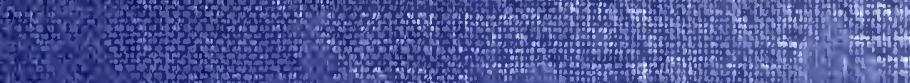
H.

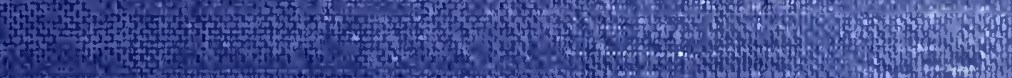

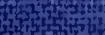

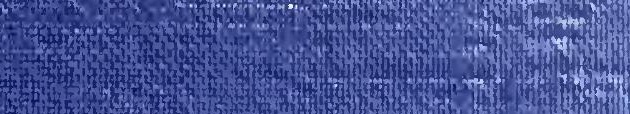

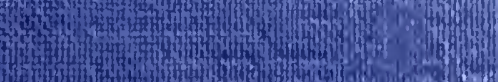

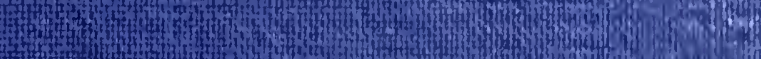
a Hith 
(3) 
. 



\section{Meyrick Types of Microlepidoptera in the British Museum (Natural History) \\ London}




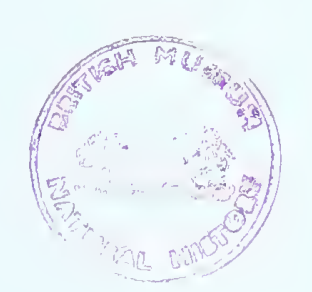




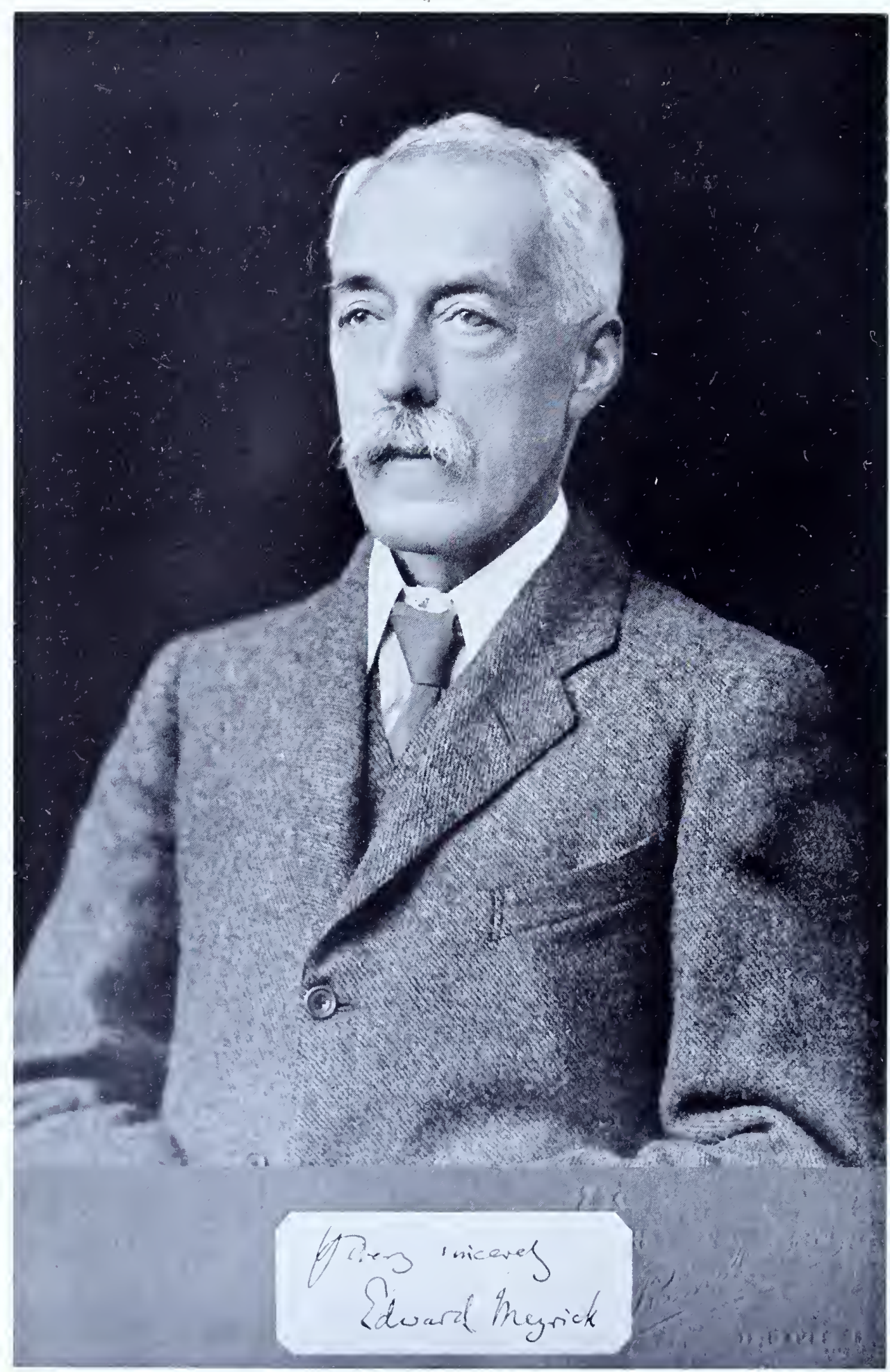

EDWARD MEYRICK 


\title{
Catalogue of the
}

Type Specimens of Microlepidoptera in the British Museum (Natural History) described by Edward Meyrick

\author{
$B Y$ \\ J. F. Gates Clarke \\ Bureau of Entomology and Plant Quarantine \\ U.S. Department of Agriculture
}

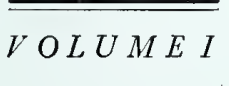

$\underline{V O L U M E I}$

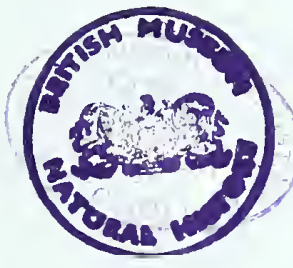

LONDON

PRINTED BY ORDER OF THE TRUSTEES

OF THE BRITISH MUSEUM 
Sold at The Britsh Museum (Natural History), Cromwell Road, S.W.7 and by

B. QuaRITCH, LitD.

and H. M. Stationery Office

MADE AND PRINTED IN GREAT BRITAIN

BY JARROLD AND SONS LTD, NORWICH 


\section{PREFACE}

THIs work owes its inception to a chance remark made to the author when, whilst serving with the United States armed forces in Europe, he paid brief visits to the Museum in July and December, 1945, for the purpose of examining type specimens in the Meyrick collection. Edward Meyrick had died in 1938, bequeathing his collection to the British Museum, where it arrived in May of the same year. It was hardly ready for critical examination when war broke out and it had to be sent out of London again for safety, for it was estimated to contain the type specimens of at least ten thousand species.

The richness of Meyrick's collections reflects the fact that for almost half a century the great bulk of all critical material, and much other as well, was referred to Meyrick almost as a matter of routine by Commonwealth microlepidopterists and by many others; a specimen identified by Meyrick was no longer a subject of inquiry in so far as its name was concerned, and for almost half a century Meyrick dominated this field. However, the use of morphological characters that Meyrick steadfastly refused to recognise began eventually to cast doubts on some of his conclusions, and it became a matter of importance that Meyrick's type specimens should be re-examined with the aid of the latest techniques. In I945 there was in the Department nobody available to undertake this work. The advent of Mr. Gates Clarke, who was already a microlepidopterist of wide experience, was therefore most opportune, for it led to his being seconded by the United States Department of Agriculture for duty in the British Museum (Natural History) for a period of two and a half years. The scope of his work he has himself defined in the Introduction to this first volume, which, it is anticipated, will be followed by at least four others. The Trustees believe that these will provide the solutions of many taxonomic puzzles and they take this opportunity of expressing their deep sense of gratitude to the author, and to the United States authorities who so generously made his services available to them.

Ist May, I954

N. D. RILEY,

KeEPER OF ENTOMOLOGY 
". . and what is the use of a book without pictures?"

ALICE IN WONDERLAND 


\section{TABLE OF CONTENTS}

$\begin{array}{lr}\text { EDWARD MEYRICK } & \begin{array}{r}\text { Frontispiece } \\ \text { Page }\end{array} \\ \text { INTRODUCTION } & \text { I } \\ \text { CATALOGUES OF THE MEYRICK COLLECTION } & 4 \\ \text { LABELS AND LABELLING } & 7 \\ \text { ARRANGEMENT AND SCOPE OF THE WORK } & \text { II } \\ \text { CLASSIFICATION } & \text { I3 } \\ \text { ACKNOWLEDGMENTS } & 28 \\ \text { ILLUSTRATIONS } & 30 \\ \text { CATALOGUE OF SPECIES } & 3 \mathrm{I}\end{array}$





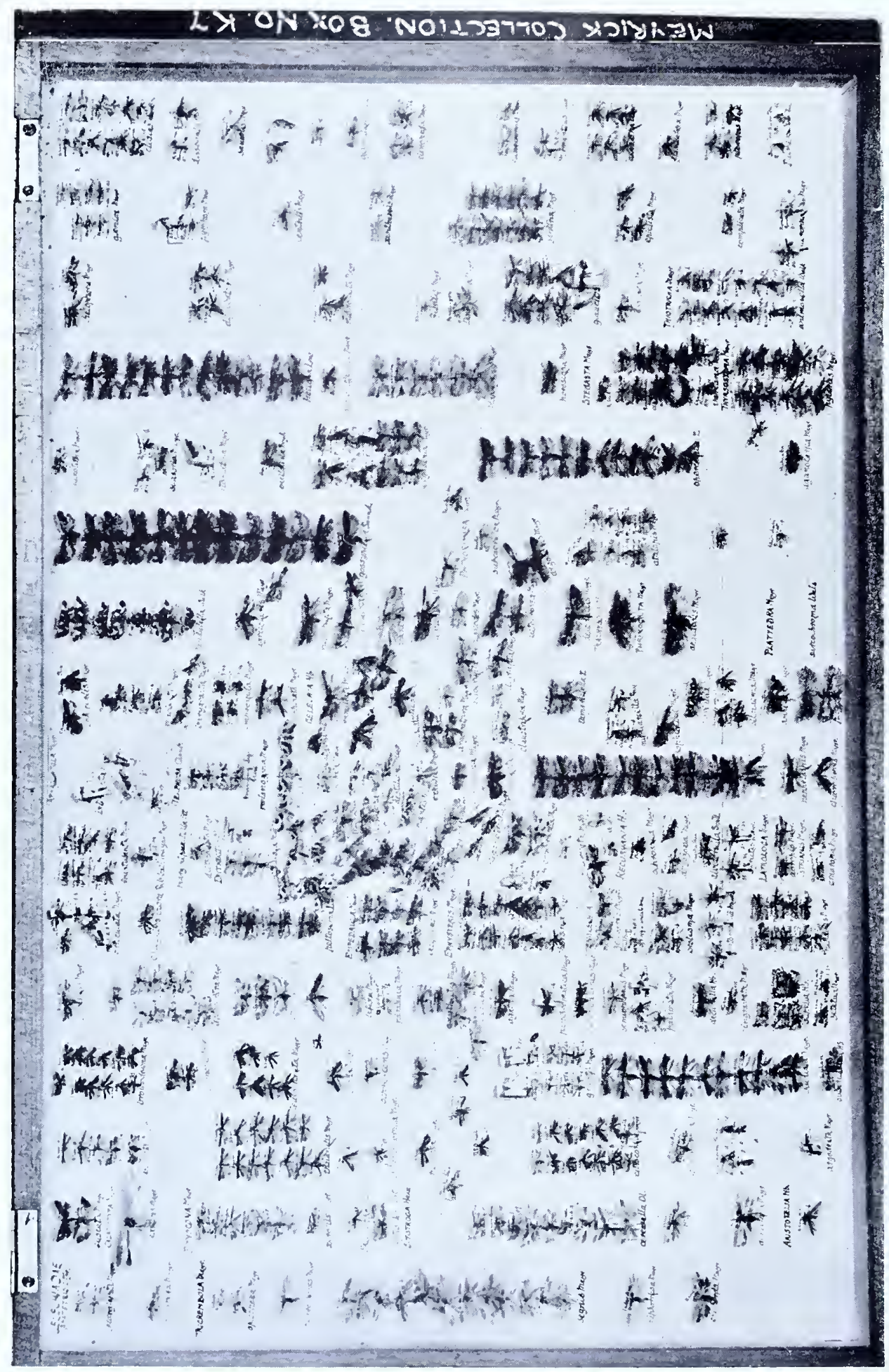

ONE SIIE OL A STORE BOX OF GELECHIIDAE FROM THE MEYRICK COLLECTION (see page I) 


\section{INTRODUCTION}

THE object of this work is to select, and designate where necessary, lectotypes of species of Microlepidoptera described by Edward Meyrick, and to establish the types when the species were described from uniques; to illustrate the wings and genitalia of the lectotypes and types and thus stabilise the nomenclature of the species examined at the British Museum (Natural History). As a secondary object a complete alphabetical list of all the species of Microlepidoptera described by Meyrick, with a citation to the original description of each, has been compiled and is published in this first volume.

This study has been undertaken as a joint project of the British Museum (Natural History), the Bureau of Entomology and Plant Quarantine, U.S. Department of Agriculture, and the Smithsonian Institution, Washington, D.C.

The Meyrick material in the British Museum consists of two parts; that placed in the Museum by Meyrick when he described species from Museum material, or specimens that found their way to the Museum in acquisitions, such as the Paravicini Collection; and that which was acquired when Meyrick's personal collection was given to the British Museum after his death.

No one appears to know when Meyrick decided to deposit his collection in the British Museum, but certainly it was not always his intention to do so. The following is a quotation from a letter addressed to Lord Walsingham. " ". . I should like, in the interests of science generally, to supply you with types of as many species as possible, which might serve to illustrate my descriptions. It would afford me much more satisfaction to endeavour to form such a collection to be placed in your hands, than to allow my specimens to be placed in the British Museum, where they would be lost in the general confusion." Later, ${ }^{2}$ however, Meyrick leaves no doubt about the ultimate disposition of his valuable collection.

Meyrick's collection consists of about Ioo,0oo specimens, containing by far the great bulk of his types, and was housed in well over two hundred double-sided store-boxes measuring II by I6 inches. On Plate 2 one side of a box of GELECHIDAE is illustrated to show how Meyrick arranged his collection. The series begin in the upper left-hand corner of the lid of each box.

All the specimens are mounted on small pins, a large proportion of which have developed verdigris, causing damage to many specimens, some to the extent that they have fallen off the pins, while others have been split.

This vast collection was obtained from numerous sources all over the world, from institutions and students who sought Meyrick's help, from collectors, presumably paid from Meyrick's personal funds, and through his own efforts.

Meyrick spent the years I877-I886 as a schoolmaster in New Zealand and Australia and obtained there great quantities of new species and genera. These specimens form the largest part of his collection from these regions, are exquisitely prepared and form the finest section of the collection. Most of the descriptions of the species from New

1 Meyrick to Walsingham in litt., Ramsbury, Hungerford, Wiltshire, 6.2.84.

2 Deuts. Ent. Zeit., Iris, 52: 73, 1938. 
Zealand occur in the Transactions and Proceedings of the New Zealand Institute, those from Australia being published in the Proceedings of the Linnean Society of New South Wales.

The chief contributors in the Asiatic region were Mr. E. E. Green, Government Entomologist of Ceylon, Mr. J. Pole, Mr. G. C. Alston, and Mr. G. B. de Mowbray of Maskeliya, Ceylon, Mr. W. Vaughan of Madulsima, and Mr. L. C. H. Young of Bombay. Between them they are responsible for almost the entire knowledge of the microlepidopterous fauna of Ceylon. Chief among the contributors from India was the late Fleet Paymaster T. Bainbrigge Fletcher, who spent more than a quarter of a century in that country. Much of his material was reared, his studies in this connection being supplemented by Meyrick's work on the taxonomy of the many species. A large quantity of material was described from the Khasi Hills, Assam, where it was collected by native collectors. The specimens from the Nilgiri Hills, North Coorg, were obtained by L. Newcome. Many others contributed to the knowledge of the fauna of the Indian region but their material was not so extensive. The great majority of the species from Ceylon and India were described in volumes I6 to 23 of the Journal of the Bombay Natural History Society.

The Chinese fauna, yet very imperfectly known, is represented mostly by material collected by Prince Aristide Caradja of Rumania and Dr. H. Höne of Bonn, Germany. Many of the types are in the Meyrick collection although some (according to information compiled by the late G. A. Bissett probably no less than 257 types) remained in the Caradja Collection now the property of the Muzeul de Istorie Naturală, Bucharest, Rumania. A considerable quantity of specimens was obtained by C. F. Baker and submitted to Meyrick by the late August Busck of the U.S. National Museum of Washington, D.C. The types of the species described by Meyrick from this last material were retained by him and all bear " $\mathrm{AB}$ " (August Busck) in the lower left corner of the pin-label.

Japanese and Formosan species were supplied by Professor S. Issiki and Professor T. Esaki, of Tokyo, and were nearly all reared.

The Chinese species supplied by Prince Caradja and by Dr. Höne were described in a separately published volume of the Deutsches Entomologische Zeitschrift, Iris, entitled "Materialien zu einer Microlepidopteren Fauna der Chinesischen Provinzen Kiangsu, Chekiang und Hunan, I935"; and in the Bulletin de la Section Scientifique of the Académie Roumaine.

The descriptions of the Japanese and Formosan species will be found in Exotic Microlepidoptera. Much niaterial was described from other Pacific areas, principally from Papua (Miss Cheesman), Pacific Islands (St. George Expedition I924-5) and the Hawaiian Islands. The Papuan and Pacific Islands species, exclusive of those from the Hawaiian Islands, were described in the Transactions of the Entomological Society of London. The Hawaiian species were described in Pacific Entomological Survey, Pub. 7, Art. 28, 1934 and in Exotic Microlepidoptera, and most of the types are deposited in Hawaii.

For a knowledge of the African fauna we are indebted primarily to Dr. A. J. T. Janse of Pretoria and to C. J. Swierstra, whose early collections provided the initial impetus for the study of African Microlepidoptera. The types of the species from these early collections are distributed in the Transvaal Museum, Pretoria, the South African Museum at Cape Town and the Meyrick Collection. Most of the species were described 
in the Annals of the Transvaal Museum and the Annals of the South African Museum. The descriptions of species described in later years, however, are to be found scattered throughout the several volumes of Exotic Microlepidoptera. Types of these last species are in the British Museum and the Belgian Congo Museum at Tervueren, Belgium.

The principal source of material from Madagascar was that collected by Dr. R. Jeannel and described in Voyage de Ch. Alluaud et $R$. Jeannel en Afrique Oriental, I9II-I2. Résultats scientifiques. Lepidoptera, II, pp. 33-I20 (Paris, A. Lhomme, I5 août I920). The types from this expedition are in the Muséum National d'Histoire Naturelle in Paris. The descriptions of other species from Madagascar, from various sources, are to be found in Exotic Microlepidoptera and most of the types of these were retained by Meyrick.

The American fauna, as treated by Meyrick, consists chiefly of descriptions of species and genera from South America, although a considerable number of North American species were also described by him. The vast preponderance of South American, and some North American, material was supplied by H. S. Parish, formerly of Toronto, Ontario, Canada. The most important contribution by Parish concerns the great number of species submitted from Bartica and Mallali, British Guiana. Actually these, and the species contributed by Eugène Le Moult from French Guiana, constitute a large percentage of the known South American forms. Brazilian species and a few hundred from other South American localities comprise the known fauna from that region.

Seventy-six species from Southern Argentina and Chile were described in the Anales del Musec Nacional de Historia Natural de Buenos Aires, vol. I, I93I, the remainder in Exotic Microlepidoptera and one or two other papers. All of the types, except some two hundred or more in the Deutschen Entomologischen Institut's collection, Berlin, Germany, about the same number in the Naturhistorisches Museum, Vienna, and a few scattered examples, were retained by Meyrick.

I have outlined briefly above the most important sources of Meyrick's material and have alluded to the disposition of the bulk of his types. It must be borne in mind, however, that the sources of supply were many and varied and types are scattered in numerous places, some of them unknown. We know also that certain types have disappeared and others have been destroyed, such as those of the Hamburgisches Zoologisches Museum und Institut, Hamburg, which were lost through bombing during World War II. Therefore, I have treated only the sources of Meyrick's material that provided large sections of his collection and have indicated the disposition of only the known types, not including the known repositories of small numbers of types; the latter are dealt with specifically in the catalogue of species.

Meyrick's descriptions of species and genera are contained in 420 books and papers of which the first was published in I875 and the last, posthumously, in I939. ${ }^{1}$ It is not known how many species of Lepidoptera Meyrick described but I list in this volume I4,I99 names of Microlepidoptera, exclusive of Pyralidoids (except the included PTEROPHORIDAE). Approximately 8000 of these were described in the first four and part of the fifth volumes of Exotic Microlepidoptera.

${ }^{1}$ For a complete bibliography, see Janse, A. J. T. and Fletcher, T. B., The Moths of South Africa, 4, part 1, xi-xxiv, 1942. 


\section{CATALOGUES OF THE MEYRICK COLLECTION}

An integral and important part of the Meyrick Collection is the set of hand-written catalogues, compiled by Meyrick for the most part in ledgers measuring about eight inches by thirteen. In order to work with the insects of this collection, it is necessary to consult frequently many of these catalogues, others less frequently. In the following pages I deal with a few of the more important catalogues in detail, and all are listed below:

No. I. Index to genera and species, cross references to Nos. 6, 7 and Io. $302 \mathrm{pp.}$ No. 2. Index to species of Pyrales, cross references to No. 13. (The pages are unnumbered.)

No. 3. A list of South African Microlepidoptera numbered serially, with index. This consists of a large ledger with 687 species listed on the first 63 pages, with references to some, and localities for all. The index, appended at the back of the volume, consists of 4 pages.

No. 4. A list of Asiatic Microlepidoptera with index. There are I235 species listed on II3 pages. The index consists of 4 pages inserted at the end of the volume. References are given for most species and localities for all.

No. 5. A list of Australian Pyrales and Microlepidoptera numbered serially. Indexed. This list consists of 3660 species on 333 pages (a few numbers are left open for additional species). The index of $\mathrm{r}_{5}$ pages is inserted at the end of the volume. References are given for nearly all species.

No. 6. A catalogue of Tineina, Hepialina (genera and species). Pages numbered IOOI-I754. Indexed in No. I.

No. 7. A catalogue of Tineina (genera and species). Pages numbered I-698. This is indexed in No. I by page No. (Plate 4.)

No. 8. A list of genera of PYRALIDAE (with descriptions). Indexed. Of the 225 double pages in this volume many are left blank for the addition of new genera. Under each genus, except synonyms, is a brief description of the characters. In the index, consisting of 6 pages, synonyms are indicated by a " + " to the left of the name.

No. 9. An early list of Microlepidoptera taken by Meyrick and arranged after Staudinger and Wocke (circa I875). $554 \mathrm{pp}$.

No. ro. A catalogue of Tortricina (PHALONIIDAE) (genera and species). Pages numbered 200I-2478. Indexed in No. I.

No. II. An alphabetical list of Pyralid Genera with type species and original references. I29 double pages. Type citations do not always conform to the International Rules of Zoological Nomenclature.

No. I2. Food plants of Microlepidoptera arranged under genera with generic index. In this catalogue $3 \mathrm{I} 6$ of the 434 pages were in use, and 4 pages of index were inserted at the end of the ledger.

No. 13. An index to the species of Pyrales arranged under genera with reference and distribution (incl. PTEROPHORIDAE). Index to genera. This catalogue 
consists of 698 pages (arranged as are Nos. 6, 7 and Io), plus Io pages of index to the genera, at the end of the volume.

No. I4. Descriptions of Tineid genera of Microlepidoptera with index. There are 683 pages of brief characterisations of the genera and I9 pages of index (about 4250 names) at the end of the volume.

No. I5. An alphabetical list of the genera of Microlepidoptera with type species and original references. $265 \mathrm{pp}$.

No. I6. Descriptions of the genera of Tortricina, with index. Only a few of the I32 double pages are used. An index of 4 pages is inserted at the end of the ledger.

No. I7. Notes on larvae and pupae. Tort. Tin. There are I59 pages, more or less filled with larval and pupal descriptions. A few drawings of wing venation will be found just past the middle of the ledger.

No. I8. Copied descriptions of CHLIDANOTIDAE, CRAMBIDAE, PHYCIDAE [PHYCITIDAE], GALLERIIDAE, TORTRICIDAE, and PSYCHIDAE. Over 300 pages in minute hand-writing in German, French, Latin, etc., done with meticulous care. Several descriptions are augmented by sketches and coloured figures.

No. I9 (I897-I9I6). No. 20 (missing). No. 2 I (I924-30).

No. 22 (I930-2). No. 23 (I933-6). No. 24 (I937-8).

These catalogues contain the lists of the thousands of determinations made by Meyrick. A card (inserted in No. 2I) from Meyrick's son, dated Io-V-40, alludes to the missing ledger: ". . I I am sorry to say that I can find no trace of the missing ledger. . . It might be worth enquiring of Messrs. Quaritch, who took most of the entomological library. ..."

No. $24(b)$. Extracts from various works. Copied descriptions of new species. Of the 332 pages only I4I were used.

No. 25. Meyrick's Diary, I872-90 (Captures). The captures are listed on I45 of the 470 pages, four columns to the page.

No. 26. Copies of descriptions of GEOMETRIDAE and PYRALIDAE with Lederer's Key to Pyrales and his figures, Plates III-IX. The plates are copied in black and white.

No. 27. Copied descriptions of genera and species of Microlepidoptera and PYRALIDAE, with structural drawings and coloured figures. $470 \mathrm{pp}$.

No. 28. A list of Australian Microlepidoptera, including HEPIALIDAE, numbered serially and indexed. There are Ioo3 species listed, with a little over 4 pages devoted to the index.

No. 29. A list of Australian Microlepidoptera (including PYRALIDAE), serially numbered from I to 2926. This list is not indexed. References to the original description, and localities, are given.

No. 3o. Descriptions of world genera, with keys and index. Consisting of 368 pages, Io $\times I 5 \frac{1}{2}$ inches, of which 346 were used. The index is missing. A few drawings of heads and palpi accompany the descriptions.

No. 3I. Species of Tineina, European. Contains copies of original descriptions, with a few coloured drawings by Meyrick. 270 pp., including "Index Methodicus" at the end of the volume.

Unnumbered. Sketches of Tineid venation. 
(I) Small notebook containing Meyrick's notes on "A Revised Handbook of British Lepidoptera, I928". 69 pp.

Two small notebooks containing pencilled notes and drawings of species in the British Museum and elsewhere on which Meyrick required further knowledge.

(2) Pp. $59^{-158 .}$

(3) Pp. I59-202.

The contents of most of the catalogues are indicated by the title, but several need elucidation. Numbers I and 2 hold the key to the other catalogues. No. I contains the references to Nos. 6, 7 and Io; and No. 2 refers to No. I3. Since the system applied is the same in No. I and No. 2, and the arrangement of Nos. 6, 7 and 10 and $I_{3}$ are identical, the explanations of two catalogues will suffice.

In No. I the pages up to p. 218 are devoted to an index of species; from p. 2 I9 on, to the generic index. Plate 3 represents most of p. 63 of Catalogue No. I. In this case, as with the genera (lower part of plate), the number following the name indicates the page on which the specific name will be found in No. 6, 7 or ro. Preceding some of the specific names will be seen "+", which denotes a synonym.

Plate 4 represents part of a page from Meyrick's genus "Gelechia" in No. 7. The vertical lines $(a)$ in the extreme left-hand column show species actually represented by specimens in the Meyrick collection. The vertical lines $(b)$ to the left of the locality represent species known to and recognised by Meyrick. Following each name is a reference to the original description but in many cases this reference is followed by others. Synonyms are entered after the name considered valid by Meyrick.

Names followed by "L46", "L9o", etc. (c), will be found also in ledger No. I2 "Foodplants of Microlepidoptera arranged under genera ..." where a notation of the host is recorded. The number following the " $L$ " is the page number in ledger No. I2.

Names followed by "Sp.67", etc. (d), are recorded in ledger No. 27 "Copied Descriptions of Genera and Species of Microlepidoptera ..." the number again referring to the page.

When a name is followed by "Nr55", etc. (e), this refers to a species in one of the small unnumbered notebooks indicated at the end of the above list of catalogues.

The names of New Zealand species are frequently followed by "Huds.I28", etc., which refers to Hudson, G. V., The Butterfies and Moths of New Zealand, I928, and the page in that work on which the particular species is mentioned.

In citing synonyms in ledgers Nos. 6, 7, ro and r3, Meyrick usually deleted the name of the synonym and added it after the valid name. Unfortunately a considerable number of names, marked as synonyms in the specific indices, were not scratched out, resulting in some uncertainty as to what Meyrick did or did not consider a synonym.

Catalogues Nos. II and 15 are alphabetical lists of world genera of Microlepidoptera and Pyralidoids. The type species of each genus, according to Meyrick's concept, is cited. These type citations are not always in accordance with the International Rules of Zoological Nomenclature and are, therefore, incorrect in many cases. 


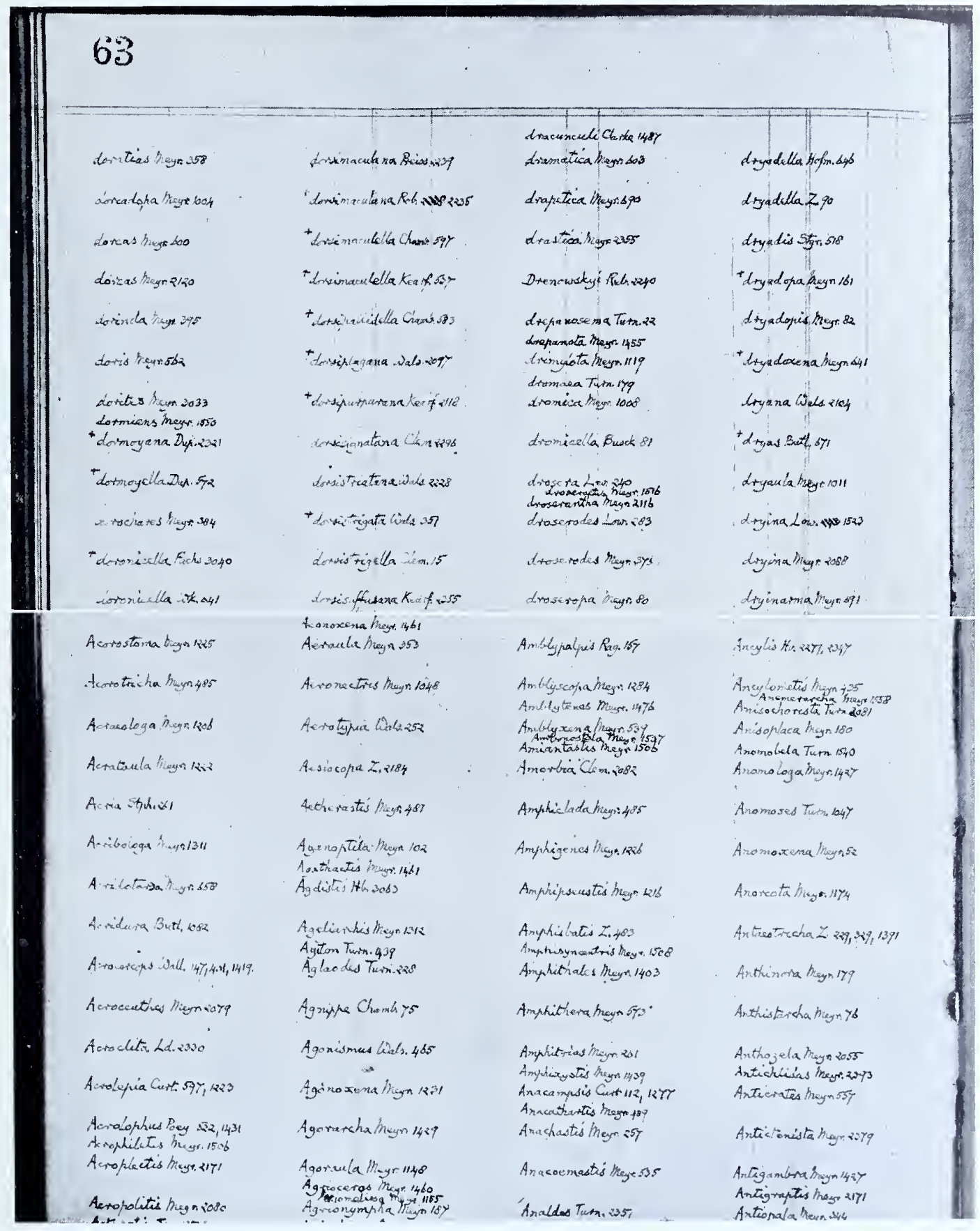

PORTIONS OF MEYRICK'S INDEX TO GENERA AND SPECIES (see page 6) 


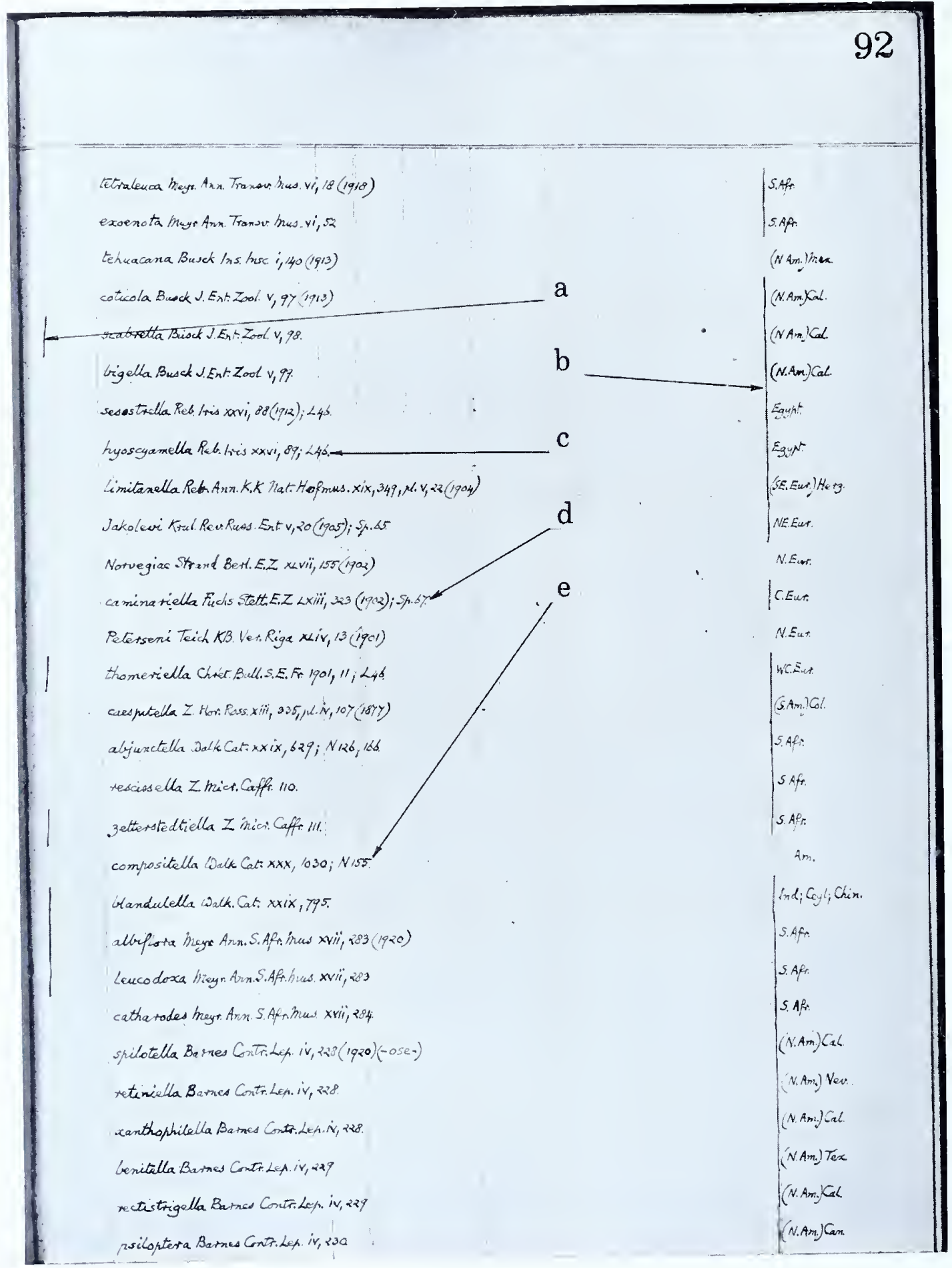

A PAGE FROM MEY'RICK'S CATALOGUE OF TINEINA (see page 6) 


\section{LABELS AND LABELLING}

TEXT figure I on p. 8 illustrates some of Meyrick's labels and also some of those that I have used in my work on the specimens in his collection.

Meyrick's labels are of two types: (I) printed and (2), handwritten. The first type was used for localities in Ceylon, Assam, North and South America, Africa, etc., from which so many specimens were acquired as to justify the printing of large quantities of labels. The second type was used for localities from which material was less frequently obtained and generally consisted of few specimens. All labels were simple and small with only the bare essentials, and frequently not those, on them. It was customary for Meyrick, upon receipt of labelled specimens which he intended to retain, to remove the original labels and write new ones in his own cramped style, to conserve space. These labels were designed to convey information to Meyrick but were made without reference to future understanding and, in many cases, it is possible to understand what was meant only by the most careful analysis of his personal records. It is, therefore, necessary to discuss certain labels to elucidate their meanings.

Almost without exception labels bearing rearing information were removed and destroyed. Such information was entered in Meyrick's personal catalogues when he considered such data of sufficient importance.

The labels to which I refer in text figure I are indicated by letters:

(a) “St. Jean, R. Maroni, Fr. Guiana. Le M. 7.I5." This label is used for a vast quantity of material supplied by the dealer, Eugène Le Moult of Paris. Le Moult's name is mentioned only twice ${ }^{1}$ in connection with the hundreds of descriptions of his material. Usually Meyrick labelled only one or two specimens "St. Jean" and the remainder of the series without that specific locality.

(b) "Bartica, Brit. Guiana. Parish. I.I3." This is the type of label used for specimens from South America supplied by H. S. Parish. Although most of the specimens bearing Parish's name were collected by him it appears that much of the material from Pará, Brazil, was actually obtained by the Rev. Miles Moss but was sent to Meyrick through Parish.

(c) "Brenster Co. Texas" was used for all material collected at "Alpine, Brewster County, Texas." As far as I know no correction of "Brenster" was ever made by Meyrick although Alpine, Texas, is a well known collecting locality, there being much material from this locality in the U.S. National Museum as well as other collections. Meyrick, without exception, published "Alpine, Texas" for his specimens.

(d) "Khasi Hills, Assam. Io.I906." Specimens bearing this label were almost invariably recorded by Meyrick from "Khasis". Just why this variation of "Khasi Hills" was used in Meyrick's publications is not clear but appears to be a personal peculiarity of the author. There is no indication from whom the specimens were obtained.

(e) "Dibidi, N. Coorg. LN. 3.4.Io." and $(f)$ "Dibidi, N. Coorg, Newcome. I2.5.07" are variations of the same thing. With few exceptions moths from this locality were recorded from "N. Coorg" or "Coorg" with rarely a reference to the specific locality.

${ }^{1}$ Exotic Microlepidoptera, 1: 45, 1915; 1.c. p. 435, 1915. 
(g) “Nilgiri Hills, S. India. HLA. 600o'. 5.07." There are several variations in the labels for this locality. Almost always the published locality reads "Nilgiris".

(h) "Pusa, Bengal. TBF. bred. I5.7.I5" and (i) "Pusa, Bihar, TBF. bred. 6.2I" represent the same locality. A third variation reads, "Pusa, India." "TBF." are the initials of the eminent authority on Indian Microlepidoptera, the late T. Bainbrigge Fletcher of Stroud, Gloucester, England. Specimens supplied by Fletcher were, with few exceptions, reared. The only indication of this given on the labels is "bred". In every case the entire rearing data were removed and such notes as Meyrick considered necessary were entered in his catalogue of reared species. (Cat. No. I2.)

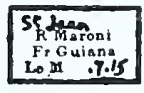

a

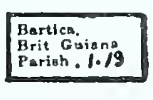

b

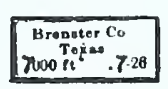

C

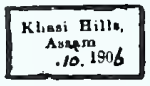

d

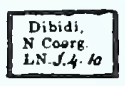

e

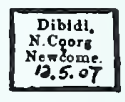

f

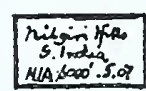

g

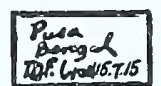

h

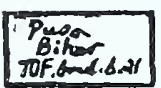

i

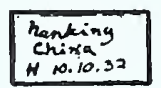

1

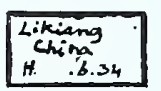

m

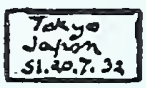

n

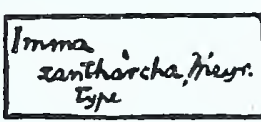

0

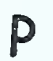

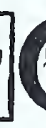
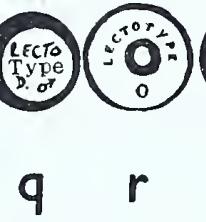

FIG. I
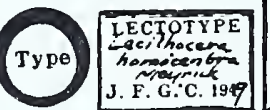

J. F. G.:C. 1949

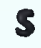

t

$\mathbf{U}$

(l) "Nanking, China. H. Io. Io.32." All specimens collected by Dr. H. Höne at Nanking but recorded from "Lungtan" bear this label.

$(m)$ The species described by Meyrick in "Materialen zu einer Microlepidopteren Fauna der Chinesischen Provinzen Kiangsu, Chekiang und Hunan", recorded from "Yul. . . ." all bear the label "Likiang, China".

(n) "Tokyo, Japan SI. 20.7.32." This label is representative of the kind used by Meyrick for moths collected in Japan and Formosa and submitted by Professor S. Issiki.

Another label, not illustrated, should be mentioned here. A substantial quantity of moths collected at "Toowong, Queensland, Australia" by Dodd, described by Meyrick from Thomas de Grey, Lord Walsingham's collection, were recorded from "Brisbane”. In fact every type I have seen recorded from Brisbane is labelled "Toowong".

(o) This is an example of a rare Meyrick "type" label. Such labels are found on certain species of Imma, Acrolophus, some species of Stenoma in the U.S. National 
Museum, and a comparatively few others. Meyrick's almost fanatical opposition to the labelling of types accounts for the scarcity of such specimens.

$(p)$ "Genotype Crocogma Meyrick." I have attached this type of red label to all specimens that typify the type species of genera.

(q) This is the kind of label used by Diakonoff for certain types of Indo-Malayan TORTRICIDAE and CARPOSINIDAE. Reference to the lectotypes selected by him is made in the appropriate place in my work.

$(r)$ The label shown here is the kind used by me in conjunction with that shown in figure " $t$ ". This is the "recognition label" and consists of a white disc with a central red ring with "LECTOTYPE" printed in black. " $t$ " shows an example of the "name label", the name being written in my hand with the dates from I947 to I949. My initials appear at the bottom of the label.

(s) This tag is used in cases in which species were described from unique specimens, and consists of a red-ringed white disc with "TYPE" printed in black in the centre.

(u) To all specimens from which I have made a preparation of the genitalia I have attached such a label. In each case the sex is indicated together with the date of the preparation and the slide number. On each slide will be found a corresponding number and date, sex and a duplication of the pin-label data so that no confusion can possibly arise.

One other label, not illustrated, should be mentioned. Specimens from Nanking, China, described in "Materialien zu einer Microlepidopteren Fauna . . . etc." are recorded from "Lungtan".

When labelling a series of specimens, Meyrick had the peculiarity of frequently dating only one specimen in the series. This leads to some confusion but, generally, it is possible to distinguish original specimens from those, if any, added to the series subsequently.

Throughout the sixty-four years of the publication of this enormous number of species and genera, Meyrick seldom marked or designated a type. His contention, borne out in correspondence, was that a type specimen was of no value but that the description was the important factor. He had absolute confidence in the accuracy and completeness of his descriptions and resented any suggestion to the contrary. In this connection I quote from a letter to Busck, " ". . . I submit that your two specimens (sexes) suggested to be =urosema and absoluta Meyr. are not in the least like my descriptions of either of those species, which are carefully described, and have abundance of points for recognition. In particular, my urosema (which is a o as stated) really has a 'strong black mark' as described on anal segment, ..."2 In another letter ${ }^{3}$ concerning the identity of two Oecophorids, in which case Meyrick was correct, he wrote. "I am much obliged for the specimens of Machimia huachucella Busck, which is a true Machimia, but in supposing it identical with my Cryptolechia diligenda you are paying small compliment to my accuracy of description; they have hardly a character in common. Structurally the pointed forewings, sinuate termen, and terminal ending of vein 7 , and in colour-characters the absence of markings on palpi, and whitish hindwings (all correctly set forth in my description, which is complete), as

${ }^{1}$ Meyrick to Busck: Thornhanger, Marlborough, 21.9.32.

2 Busck stated that Meyrick's urosema was a female, which it proved to be, when I dissected the type.

3 Meyrick to Busck: Thornhanger, Marlborough, 16.2.29. 
well as the indication of the stigmata, show clearly that it is entirely distinct. If I had not been certain (from the neuration, without the o) that it was a Cryptolechia, I should not have described it."

A short excerpt, from another amusing letter, is worth quoting. This resulted from an exchange of opinions emerging from observations on a species with venational variations which Meyrick never admitted. I quote: ${ }^{1}$ "I am simply amazed that you should venture to state positively that the wing of agyrtodes Meyr. which I sent you for study belonged to some other insect! Why did you not further display your knowledge by stating what insect it was? It was as I stated it to be, and was taken at random from a number of unset examples of the species, of which I could not examine the neuration until I had removed the wing. I will add that I believe the hindwing sent could not be attributed to any other described North American species. Also, it agrees with my description, which you alleged to be wrong. Do you suppose that I first wrote a wrong description, and afterwards manufactured a hindwing to fit it?"

${ }^{1}$ Meyrick to Busck: Thornhanger, Marlborough, 21.1.28. 


\section{ARRANGEMENT AND SCOPE OF THE WORK}

My arrangement of families, genera and species is not systematic. The families dealt with in each volume are grouped to make the volumes approximately the same size. In Volume II I have placed together the STENOMIDAE, XYLORYCTIDAE and COPROMORPHIDAE. This combines nearly related families, but this is purely a matter of convenience. Volume III will contain the TORTRICIDAE, PHALONIIDAE and OLETHREUTIDAE and probably the CARPOSINIDAE and CHLIDANOTIDAE; Volumes IV and V will deal with the GELECHIIDAE, OECOPHORIDAE, BLASTOBASIDAE and COSMOPTERYGIDAE and perhaps one or two small groups; Volume VI will contain such families as the HYPONOMEUTIDAE, GLYPHIPTERYGIDAE, TINEIDAE and ACROLOPHIDAE. This arrangement is at present tentative but is essentially that which will be followed. The genera and species included in each family are, with few exceptions, in accordance with Meyrick's concepts. But the species grouped under the genera are in line with relationships based on genitalic studies, combined with other characters, in so far as it has been possible to make proper generic assignments without proposing new generic names.

Under each family the genera are arranged alphabetically, whether synonyms or not, and the included species are also listed alphabetically under the generic headings. Synonyms are listed in their proper alphabetic positions and cross-indexed to the valid names. Generic synonyms, although appearing independently, are cited.

Each species is headed by its valid name. This is followed by a reference to the pertinent illustrations giving plate and figure numbers. Next is a reference to the original description citing the combination of generic and trivial names as they appeared when the species was described. Following this I present a brief extract from the original description to show sex, size and source of the material and, occasionally, Meyrick's remark indicating his belief as to the species relationships. This is done to establish beyond doubt that the original specimen or series was examined. I then denote the specimen selected as the "LECTOTYPE" or "TYPE". All such references are to be considered type and lectotype designations unless otherwise specified. ${ }^{1}$ Following the above designations is the number (in parentheses) of the genitalic preparation which, in the case of the types of the genera, includes the right wings. The numbers run from 4400 to approximately 9600 and all slides are in the British Museum (Natural History). Under "Labels and labelling" I have discussed the pertinent labels associated with the type and lectotype designations and those associated with the slides of the genitalia. After the above designations an explanation of figures is given.

Where synonyms have been cited and figured I have given the synonymy under the references and then have indicated by the inclusion of the trivial names in brackets (example: [accurata] ... [perjura $]$ ) that part of the text referable to the particular species.

1 Wherever I have indicated that the type or lectotype has been selected by Diakonoff the reference is to, Diakonoff, A., "The type specimens of certain Oriental leucosmidaE and carposinIDAE", Bulletin of the British Museum (Natural History) Entomology, 1, No. 4: 275-300, 1950. (illus.) 
In the second and succeeding volumes it will be noted that there are hundreds of generic transfers. Even so there is a considerable residue of species which have been left in the genera in which Meyrick placed them. Obviously a complete revision of the Microlepidoptera will require study of the types of other authors, and that is far beyond the scope of this work, so transfers have been made only in cases in which clear-cut genera already exist and the changes were mandatory.

Following is a list of the families studied and the geographical areas from which the material came. It will be noted that the entire New Zealand and almost the entire Australian faunas are omitted.

STENOMIDAE

XYLORYCTIDAE

GELECHIIDAE

BLASTOBASIDAE

OECOPHORIDAE

METACHANDIDAE

ETHMIIDAE

HYPONOMEUTIDAE

TINEIDAE

STREPSIMANIDAE

CHLIDANOTIDAE

TORTRICIDAE

OLETHREUTIDAE

CARPOSINIDAE

COPROMORPHIDAE

GLYPHIPTERYGIDAE

ACROLOPHIDAE

PHALONIIDAE

SCYTHRIDIDAE

SCAEOSOPHIDAE

COSMOPTERYGIDAE

PHYSOPTILIDAE
American family with one representative from Africa (acc. Meyrick).

Asiatic and African with two Australian species.

American, Asiatic and African with a few Australian species. American and a few African species.

American, Asiatic, some African and a few Australian species. Asiatic and African.

American, Asiatic, African.

American, Asiatic and a few African species.

American, African (few).

Asiatic.

Asiatic.

American, Asiatic, African; also a few species from Australia described from Walsingham material.

American, Asiatic, African.

American, Asiatic, African.

American, Asiatic, African.

American, Asiatic, African.

American family.

American, Asiatic, African.

American.

African family.

Those species which I have transferred from other families.

Asiatic family.

Time did not permit the study of several families from the geographical areas listed, but an attempt was made to include all the species of a given family from the entire world exclusive of New Zealand and Australia. 


\section{CLASSIFICATION}

MEYRICK's classification ${ }^{\mathbf{1}}$ is based primarily on the venation of the wings of Lepidoptera supplemented by such other characters ${ }^{2}$ as he considered important. He even went so far as to lay down principles ${ }^{3}$. of classification based solely on venational characters and ridiculed the value of other structural features, particularly those of the genitalia.

At the outset Meyrick began with a false premise and as late as $1928^{4}$ he made the following statement: "In my classification the standard aimed at is an average of about Io species to the genus and 50 genera to the family in the world fauna; which corresponds pretty nearly to the numbers found in the genera and natural orders adopted by botanists for flowering plants, whose history is bound up with that of the Lepidoptera. Some monotypic genera are of course necessary; the largest existing genus is Stenoma, which contains at present over 600 species. . .."(!)

This was an attempt by Meyrick to employ a purely artificial hypothesis, based on a mathematical formula, to provide a convenient system to which he could fit his classification and one not based on natural phenomena and evolutionary development of the insects he proposed to study.

As will be pointed out in the following pages his "genus" Stenoma is one of the largest conglomerations of unrelated species ever brought under one name in the Lepidoptera.

Let us go a step further regarding his attitude toward the distinction of genera: ${ }^{5}$ "(2) As to distinction of Platyedra and Pectinophora from one another, as genera, I should be glad if I could make it clear to you, not because of any different value set on genitalic structure, but on broad principles of the formation of genera. You wish to separate them on account of differences; I wish to unite them on account of resemblances. You will find on examination of my work that I always do so; I cannot give a better instance than that of Depressaria, where you and Walsingham and Durrant make two genera, whereas I make one, the difference of course being in neuration there. Similarly you make three genera of Cerostoma, while I make one. Look at Walsingham's and Durrant's splitting of Acrolophus, where I have consistently maintained one genus. You have plenty of supporters; every beginner and every specialist (i.e., everyone of narrow views) is on your side. That ought to show you that you are wrong. My views are wider, and I know I am right. Your views are founded on opinion, and you are anxious others should adopt them, because then you would feel you were right; mine are founded on reason, and I like others to think differently because it is more creditable to me; there is no credit in seeing only what everyone else sees."

His almost fanatical reliance on venation as a means of classification, although admitting considerable variation, coupled with an equally determined refusal to consider the genitalic organs as a means of classification not only of species but also of

\footnotetext{
1 Meyrick, E., Proc. Linnean Soc. N.S. Wales, 5: 205, 1880.

2 Meyrick, E., Trans. Ent. Soc. London, 1883, pp. 119-31.

3 Meyrick, E., Ent. Mon. Mag., 25: 175-8, 1889.

4 Meyrick, E., A Revised Handbook of British Lepidoptera, p. 13, 1928.

5 Meyrick. E., in litt., to August Busck, Marlborough, Wilts., England, 14.12.27.
} 
higher categories, led Meyrick astray in many cases. In this connection he stated, ${ }^{1}$ "Thus colour and outline, the hairs of the larvae, and the genital organs of the imagos are likely to be of slight importance in the definition of groups; whilst neuration occupies a high position, except when directly influenced by an alteration in form of wing, which is seldom the case."

Although Meyrick disdained the use of genitalia in classification he condescended to mention them on several occasions ${ }^{2}$ but then he gave only a superficial treatment of a few features which could be seen by denuding the uncus and ends of the harpes, and he never gave an illustration of one.

Regarding genera he says further, " "Genera which (according to Durrant) would be identical 'under a lens' but distinct 'under a microscope', are too fine for me. As one cannot convert all one's specimens into microscope slides. . . ."

This persistent refusal to examine the genitalia, in conjunction with other structures, not only led Meyrick into error in delimiting families and genera but accounted in large part for his misdetermination of the sexes of many of his species.

It will be noted throughout this work that I have pointed out numerous errors in the determination of the sex or sexes of the specimens before Meyrick when he described certain of his species. These errors are attributable to several causes: (I), Meyrick's inability to discover the sex of his specimens because of his use of nothing stronger than a small hand lens and his reliance on characters of the antenna, frenulum, etc., rather than genitalia; (2), probable lapsi in particular cases and, (3), misprints on the part of the compositor.

It is impossible to determine the causes of most of these errors but there are a few cases in which we can draw certain conclusions. In the description of Gelechia sarcochlora ${ }^{4}$ the specimens were recorded as "ot" and all of them are "op". It seems likely, in this case at least, since the ovipositors are clearly visible on all specimens, that this is a printer's error but we may suspect that Meyrick ignored the ovipositor and relied on the frenulum or antenna. Dissection would have removed any doubt.

To cite another example: Ethmia acontias ${ }^{5}$ was described from eight specimens and the sex was recorded as " $\overrightarrow{0}$ ". Not only dissection, but an examination of the ovipositors. of the specimens disclosed their sex to be ㅇ. Meyrick later discovered his error and corrected it $^{6}$ attributing the sexual designation to a misprint. These are only two of many examples which serve to illustrate the errors, from various causes, in the recording of sexes in Meyrick's work. A third is cited on an earlier page (urosema) and many others are disclosed in their proper places. I use the above examples to support my contention that closer examination and dissection could have eliminated many mistakes, and that the specimens I have examined are the actual types and not replacements.

But let us return to the matter of venation and examine specific cases. Meyrick ${ }^{7}$ proposed the generic name Baeonoma (Stenomidae) for mastodes, which he cited as type, and four other species. He characterised the venation of the genus as follows: ". . . Forewings with Ib furcate, $2-5$ approximated, 7 to termen, seldom 7 and 8 (leucodelta) or 8 and 9 (holarga) stalked, II from middle. Hindwings I or over I,

1 Meyrick, E., A Handbook of British Lepidoptera, p. 11, 1895.

${ }^{2}$ Exotic Microlepidoptera, 3: 164, 1925; Trans. Ent. Soc. London, 1913, pp. 192-9, 1913; Trans. Ent. Soc. London, 76:521, 1929.

Exotic Microlepidoptera, 3: 491, 1929.

${ }^{6}$ Exotic Microlepidoptera, 4: 603, 1935.

${ }^{3}$ Meyrick, E., in litt., to August Busck: Thornhanger, 18.1.15.

5 Journ. Bombay Nat. Hist. Soc., 17: 409, 1906.

7 Exotic Microlepidoptera, 1: 507, 1916. 
trapezoidal-ovate, cilia $\frac{2}{5}$ to $\frac{3}{4} ; 3$ and 4 connate, 5 somewhat approximated, 6 and 7 stalked."

With the above characterisation in mind, and also Meyrick's remarks on venation under Stenoma neastra, let us examine the characters of the other species placed in Baeonoma.

$B$. euphanes: This species has veins 2 and 3 stalked in the forewing, all others separate. The venation of the hindwing is essentially the same as that of mastodes.

$B$. helotypa: The same venation as that found in mastodes.

$B$. holarga: The forewing has 2 and 3 stalked, 8 and 9 stalked, and Io approximate to 9 at base. The hindwing is essentially the same as that of mastodes.

$B$. infamis: Veins 2, 3 and 4 are approximate and 7 and 8 are connate in the forewing. In the hindwing 3 and 4 are connate and 5 is approximate to 4 .

B. leucodelta: Veins 2 and 3 are stalked; 7 and 8 are stalked in the forewing. The hindwing is that of mastodes.

$B$. orthozona: Forewing with veins 2 and 5 equidistant from 3 and 4 respectively; 3 and 4 approximate; 7,8 and 9 approximate and equidistant. Hindwing with 3 and 4 short-stalked.

B. suavis: Forewing with 2 and 3 approximate toward base; 4 closely approximate to $3 ; 7$ and 8 short-stalked and closely approximate to 9 . In the hindwing 3 and 4 are short-stalked with 5 approximate.

By making a comparison of the details of venation enumerated above, with the figure of the venation of mastodes (Vol. II, pl. 7I, fig. Ia), the differences are immediately apparent. $B$. heloty $p a$ and $B$. mastodes are congeneric as borne out not only by venational characters but also by the genitalia. With these two species I associate favillata which I have transferred from Stenoma. Meyrick was correct in associating leucodelta with euphanes but wrong in placing them with mastodes and its congeners. They (lencodelta and euphanes) appear more closely related to some of the Old World XYLORYCTIDAE. $B$. orthozona differs from all the rest and its position is not clear. The two remaining species, holarga and suavis, are generically distinct from mastodes, helotypa and favillata and will require new genera unless they can be properly assigned to genera in the Old World XYLORYCTIDAE. I wish to point out here that the proper interpretation of the venational characters could easily have been reached with a closer observation of pattern, habitus and genitalic features.

Above I have pointed out the inadequacies of Meyrick's observations and the shortcomings of his too heavy reliance on venational characters. Meyrick himself would have admitted the close relationship of mastodes, favillata and helotypa had the characters of the genitalia, in combination with other features, been brought to his attention. The association of the other species, with the possible exception of infamis, is unnatural as demonstrated by pattern, venation and genitalia and their eventual reassignment is mandatory.

Meyrick's genera Aphanoxena (synonym of Antaeotricha), Cerconota, Chlamydastis (=Ptilogenes), Eumiturga, Mysaromima, Orphnolechia, Paraspastis, Prasolithites (synonym of Antaeotricha), Psephomeres form a related group within the family STENOMIDAE characterised by forked scales in the male genitalia (except perhaps in Mysaromima the male genitalia of which are not known) and a variously shaped, dentate, sclerotised plate for a signum in the females. 
I have already discussed Baeonoma which, in the strict sense (mastodes, helotypa and favillata) is more closely allied to the Old World XyLORYCTIDAE than it is to the true Stenomids. Dinotropa is referable to the oEcophoridae; Hyalopseustis, Lychnocrates, Petasanthes, Rhodanasa, Thioscelis, and Timocratica have Xyloryctid affinities and are peculiar to the New World Tropics.

The two closely related genera, Neophylarcha and Rhopalosetia, assigned to the COPROMORPHIDAE, certainly do not belong there and stand alone in the unique development of the frenulum which in the male terminates in a lamella; in the female at least one bristle terminates in a lamella. (See: Vol. II, pl. 259, fig. Ia and pl. 263, fig. Ia.) These two probably represent members of a distinct family. Cathelotis appears to be a higher development of Phycomorpha and the latter closely related to Ordrupia. Cathelotis, then, if a true Copromorphid, which I doubt, should be related to the Old World genera Copromorpha, Chersomorpha, Osidryas and Aegidomorpha through Phycomorpha and Ordrupia or common ancestor. For the time being I am treating all of these genera under the family heading COPROMORPHIDAE.

The genus Petalothyrsa, assigned by Meyrick to the EPERMEniIDae, belongs to the STENOMIDAE to which family I now assign it.

The genus Stenoma, as conceived by Meyrick, was a huge, unwieldy mass of more or less related species containing many forms with nearer relatives in other genera than those with which Meyrick associated them.

Of the genus Stenoma Meyrick wrote, ${ }^{1}$ under the description of Stenoma neastra, "This would be referable to Orphnolechia Meyr. [which is correct!], but I am not able to maintain that genus, since insistence on the structural distinction separates closely allied species, and groups together others which are not allied. In the aggregate of species grouped under Stenoma the stalking of veins 2 and 3, or 2-4, or the anastomosing or confluence of 2 and Ic (a frequent character which has been generally overlooked) in the forewings, or the stalking of veins 6 and 7 , or 8 and 9 , or Io and II of the forewings, or the stalking of veins 3 and 4 of hindwings, are all specific characters only, and not always constant even as such, recurring in unrelated groups of the genus, and disguising natural affinity instead of expressing it. This will be seen by inspection of the following descriptions of species, which are grouped according to approximate natural affinity, and these points of structure noted. Hence I consider Walsingham's genera Aedemoses, Catarata, Athleta, Zetesima, Menestomorpha and Mothonica as all untenable and to be suppressed."

Had Meyrick examined the genitalic structures of his species it would have been clear to him that, although his observations on the venational characters were in part true, they were not entirely so and the proper interpretation of these characters could have been established by the genitalia.

The genus Stenoma is based on a single female, Stenoma litura Zeller, now in the British Museum (Natural History), which is unlike any of the 600 or so species that have been placed as congeners with it. I have transferred I 82 species from Stenoma of Meyrick to other well established genera. Of these I have assigned 2I to Anadasmus, Io3 to Antaeotricha, I to Baeonoma, 35 to Cerconota, I6 to Gonioterma, I to Mothonica, I to Timocratica and 4 to Orphnolechia. It will be noted that the species, with few exceptions, not only agree in type of genitalia but also in general habitus, as we should expect.

${ }^{1}$ Exotic Microlepidoptera, 1: 412, 1915. 
I do not claim finality for all these new assignments but a casual glance will assure the observer that the associations are more natural than those previously made except in the residual "Stenoma". This great mass of species is still a heterogeny which will require close study, many new genera and much refinement, but it is not the purpose of this work to propose new generic names.

The genus Dinotropa is clearly Oecophorid on all characters and is accordingly transferred to the OECOPHORIDAE where it will be treated in its proper place.

When Meyrick described Peleopoda semocrossa ${ }^{\mathbf{1}}$ he placed it in the Xyloryctidae. Later $^{2}$ he assigned the species to the OECOPHORIDAE but Busck ${ }^{3}$ placed it in the STENOMIDAE because veins 6 and 7 of the hindwing are stalked. Certainly the stalking of veins 6 and 7 is rare in the OECOPHORIDAE but the genitalia unquestionably place the species there. I therefore transfer this species back to the OECOPHORIDAE.

Although Meyrick eventually recognised the family STENOMIDAE as distinct from the XYLORYCTIDAE he wrote as late as $1925^{4}$ as follows: "(3) Your evidence as to Setiostoma being a Stenomid seems to me worthless; and I have no reason to suppose that your grounds for separating the XYLORYCTIDAE and STENOMIDAE would be any more convincing."

The family XYLORYCTIDAE was proposed by Meyrick in I $890^{5}$ for a group of Australian genera and species characterised by a short cross-vein between the cell and vein 8 of the hindwing which the author, at that time, claimed to be ". . . invariably present." As time went on, Meyrick broadened his concept of the family and included in it genera and species from many tropical and temperate regions of the world and not only assigned many of his own genera to this family but included those of other authors. The thirty Meyrick genera treated in this work, assigned by him to the XYLORYCTIDAE, comprise a large assortment of types and representatives of several families. Of these genera only four, Amorbaea, Epichostis, Linoclostis and Nephantis, possess the crossvein between the cell and vein 8 on which Meyrick laid so much stress. Two other genera, Neospastis and Synchalara, have vein 8 almost anastomosing with the cell, indicating a tendency to the formation of a cross-vein, and in the genus Phthonerodes vein 8 fuses along the cell for more than one-third its length.

Amorbaea, Epichostis, Linoclostis and Nephantis form a group of closely allied genera in which there is no blind-sac in the aedeagus and the abdomen is spined. Epichostis stelota, however, is misplaced and is hereby transferred to the OECOPHORIDAE. In the hindwing of Nephantis veins 6 and 7 are stalked but in the other three genera veins 6 and 7 are well separated and divergent. In Phthonerodes the blind-sac of the aedeagus is absent but it is present in Synchalara and Neospastis.

The following genera have veins 6 and 7 of the hindwing stalked and have no crossvein between vein 8 and the cell: Aeolanthes, Amphitrias (synonym of Odites), Antithyra, Antolaea, Epimactis, Myriopleura (synonym of Odites), Procometis, Prothamnodes, Rhizosthenes, Thymiatris, Trichernis (synonym of Odites) and Trypherantis.

Aside from the two features mentioned above, there is little to recommend the close association of these genera and much less to support their relationship to xyLORYCTIDAE (s. str.). In the genus Antolaea the gnathos is divided into two spined

1 Exotic Microlepidoptera, 4: 13, 1930.

${ }^{2}$ Genera Insectorum, fasc. 180: 188, 1925.

${ }^{3}$ Lepidopterum Catalogus, Part 67, p. 8, 1935.

4 Meyrick to Busck: Thornhanger, Marlborough, 27.4.25.

5 Trans. R. Soc. S. Australia, 13:23, 1890.

M.T.M. I-3 
knobs and the aedeagus has a blind-sac. The character of the divided spined gnathos is shared by the Meyrick species placed in the genus Acria Stephens, by the two species Durrantia montivola and D. faccescens, and Odites navigatrix, but none of them has the blind-sac of the aedeagus. The spined gnathos is a common character in the OECOPHORIDAE and these species appear to be a development of that family. We cannot place them in the OECOPHORIDAE at present because of the stalking of veins 6 and 7 of the hindwing, and because we have no supporting evidence from larvae for such an association.

The genus Aeolanthes presents a peculiar but highly characteristic type of male genitalia in which the aedeagus is disproportionately large and lacks the blind-sac, the harpes are variously shaped and ornamented and always reduced, and the uncus assumes various shapes, but it is always present. The females generally have double signa of dentate plates but at least one species (sagulata) possesses no signum at all.

The species referred to the genus Odites or its synonyms conform very much to type in form of genitalia: the transtilla usually exhibits strong development of the lateral lobes. The females are diverse and, at present, do not give much help in delimiting the genus. The true species of Odites possess a peculiar type of sharply beaked gnathos, which is directed anterad, and a long narrow tegumen. In this genus Meyrick has placed such atypical species as brachyclista, concreta, continua, hermetica, mistharma, sphenodontis and spoliatrix which must ultimately be transferred elsewhere. Rhizosthenes possesses the same type of gnathos as Odites.

The genus Epimactis is extremely close to Odites, and indeed may be a synonym but, as veins 3 and 4 of the hindwing are united, I am maintaining its identity for the time being.

Thymiatris and Trypherantis are very closely related, having symmetrical male genitalia with a semi-tubular anellus, broadly attached harpe with clasper, aedeagus without blind-sac and gnathos and uncus present.

Another group of genera, Deloryctis, Metathrinca and Ptochoryctis lack the crossvein between vein 8 and the cell in the hindwing, veins 6 and 7 are well separated and divergent and the abdomens are spined. These genera are probably nearer the OECOPHORIDAE than the XYLORYCTIDAE.

I am illustrating species assigned by Meyrick to Agriophara, Gemorodes and Letogenes but, since I have not examined the types of those genera, I cannot determine whether or not the species are correctly placed.

Several other genera and species have been associated with the XYLORYCTIDAE by Meyrick and require special consideration at this point. Amniodes (synonym of Terthreutis: new synonymy) is hereby transferred to the TORTRICIDAE. Rhapsodica is also referable to the TORTRICIDAE and is hereby transferred. Thyrsomnestis has already been transferred to the STENOMIDAE, where it probably belongs, though it is atypical of that family. Plocamosaris (synonym of Noeza: new synonymy) was described in the XYLORYCTIDAE and later transferred to the OECOPHORIDAE by Meyrick. The genus is referable to the GELECHIIDAE, as clearly indicated by the genitalia and its obvious close relationship to the types of both Noeza and Neochrista, and is hereby transferred to that family. Leistogenes also belongs in the GELECHIIDAE, as revealed by genitalic examination, and is hereby transferred to that family.

Meyrick ${ }^{1}$ described Asapharca nephelomicta from New Mexico, but it does not seem

${ }^{1}$ Exotic Microlepidoptera, 3: 555, 1930. 
likely that the American nephelomicta is truly congeneric with the African species of Asapharca and, in fact, the female genitalia exhibit Gelechioid features rather than Xyloryctoid characters. Since only the type female is known the species will be left in Meyrick's XYLORYCTIDAE, with reservations.

To this family we must add the genus Lasiodictis which was described in the GLYPHIPTERYGIDAE. I hereby transfer Lasiodictis to the XYLORYCTIDAE.

The family GELECHIDAE, as at present constituted, is one of the largest families of Microlepidoptera and is world-wide in distribution. In this work I am considering only 226 genera, assigned by Meyrick to this family, although the family includes far more than that number. The proper restriction of the family will require many years of careful study.

One of the principal characters used to separate a Gelechiid from other species with similar venation is the absence of vein Ic of the forewing. This character still holds in the vast preponderance of genera but Ic is present in Anastreblotis, Apethistis, Autosticha, Chlorolychnis, Hierangela, Homaloxestis, Steremniodes and Stochastica. Some of these, however, are questionably Gelechiids and the presence of this vein may be abnormal in the other cases. All too frequently only a single specimen was available for examination so the extent to which the presence of this vein is exhibited in a given species could not be ascertained.

The above genera are included in two large, distinguishable groups of genera within the family. The separation of these two groups of genera is based on the presence or absence of a cross-vein between vein 8 and the cell of the hindwing. This cross-vein is present in Acribologa, Agathactis, Ageliarchis, Agriastis, Alsodryas, Anastreblotis, Anathyrsotis, Anomoxena, Anthistarcha, Arotromima, Atasthalistis, Axyrostola, Battaristis, Brochometis, Bucolarcha, Calamotypa, Calyptrotis, Carbatina, Catoptristis, Cerycangela, Chalcomima, Charistica, Chelophoba, Chthonogenes, Clepsimacha (=Cratinitis), Coconympha, Coleostoma, Compsolechia, Cotyloscia, Crasimorpha, Crossobela (? too badly damaged), Cymotricha, Deimnestra, Desmophylax, Dissoptila, Eripneura, Erythriastis (? no hindwing), Ethmiopsis, Ethirostoma, Eunebristis, Eupolis, Eustalodes, Glycerophthora, Hapalonoma, Haplochela, Holaxyra, Homalochelas, Hypelictis, Illingiotis, Leistogenes, Locharcha, Lophaeola, Metabolaea, Meteoristis, Molopostola, Musurga, Myrophila, Mythographa, Noeza (=Neochrista, =Plocamosaris, new synonymy), Orsodytis, Oxycryptis, Pachnistis, Pachygenia, Pachysaris, Paralectra, Pessograptis, Phrixocrita, Phylopatris, Pilocrates, Platyedra, Plexippica, Porpodryas, Promolopica, Prophoraula, Psamathoscopa, Rhynchotona, Schemataspis, Semiomeris, Semophylax, Sitotroga (=Nesolechia, new synonymy, =Syngenomictis), Sorotacta, Sterrhostoma, Stiphrostola, Symphanactis, Syncratomorpha, Taphrosaris, Telephila, Teuchophanes, Thyrsostoma, Tornodoxa, Trachyedra, Trichembola, Xenorrhythma, Zalithia and Zomeutis.

In the following group of genera the cross-vein between vein 8 and the cell is absent: Anthinora, Apethistis, Aphanostola, Apocritica, Asarista, Asmenistis, Aulidiotis, Autosticha (=Epicoenia), Beltheca (=Anterethista), Calliprora, Chlorolychnis, Cnaphostola, Colonanthes, Commatica, Compsosaris, Crambodoxa, Deroxena, Elasiprora, Empedaula, Encolapta, Encolpotis, Ephysteris, Eristhenodes, Euzonomacha, Evippe (=Tholerostola, new synonymy), Hapalosaris, Harpagandra, Heliangara, Hierangela, Homaloxestis, Inotica, Ischnodoris, Isembola, Isophrictis, Istrianis, Latrologa, Meridorma, 
Mnesistega, Narthecoceros, Oxylechia, Palintropha, Paradoris, Parallactis, Pavolechia (=Desmancha), Pelocnistis, Pharangitis, Phatnotis, Pitycona, Proselotis, Protolychnis, Semnostoma, Stagmaturgis, Steremniodes, Stochastica, Synactias, Syncathedra, Thiotricha, Thripsigenes (=Lioclepta, new synonymy), Toxotacma and Trypherogenes.

There appears to be a distinct correlation between the absence of Ic of the forewing and the presence of a cross-vein between vein 8 and the cell of the hindwing although Anastreblotis has Ic present in the forewing and a cross-vein between vein 8 and the cell of the hindwing. The type species of this genus, A. calycopa Meyrick, a female, has the pattern and facies of a Blastobasid and may well belong in that family. It will be noted also that the venation of the forewing is that of the BLASTOBASIDAE.

In the second group, those without the cross-vein between vein 8 and the cell of the hindwing, vein Ic is absent in the great majority of genera but it is present in Apethistis, Autosticha, Chlorolychnis, Hierangela, Homaloxestis, Steremniodes and Stochastica. Autosticha is doubtfully Gelechiid, not only on venational characters but also because of its atypical genitalia and the refuse-feeding habit of its larva.

The GELECHIIDAE characteristically have smooth abdomens but the abdomens of at least two genera, undoubtedly referable to this family, are clothed with spines. These are Mnesistega and Pavolechia Busck (=Desmancha).

The condition of veins 6 and 7 of the hindwing, i.e. stalked, approximate, etc., has been used frequently to determine the family position in GELECHIIDAE but there is no relation between the condition of these two veins and the assignment of any given genus to this family; nor is there any relation between the condition of these two veins and the assignment of genera to either of the two groups I have discussed above. Veins 6 and 7 may be united as in Agathactis, Calyptrotis, Pachygenia, Elasiprora, Hapalosaris, and Pitycona; stalked as in Anastreblotis, Homalochelas, Metabolaea, Pachnistis, Pessograptis, Aphanostola, Phatnotis and many others; approximate at base as in Acribolga, Eupolis, Phrixocrita, Sorotacta, Trachyedra and others; connate as in Arotromima, Cotyloscia, Symphanactis and Calliprora, or widely separate as in Atasthalistis, Glycerophthora, Trichembola, Crambodoxa, Empedaula, Pelocnistis and some other genera. In addition there are varying degrees of proximity or divergence of these two veins.

Occasionally anomalous conditions appeared which may or may not be normal for the genera but, because of the paucity of material available for study, it was impossible to determine whether or not the specimens examined were freaks. In the hindwing of Symphanactis there is a very distinct, well developed "vein 9" from the base of the wing to costa. Vein 7 and vein 8 of the hindwing of Isembola are both stalked, thus producing ten veins. These conditions may not be significant but may well indicate hereditary traits peculiar to the species.

The following genera possess characters which preclude their retention in the Gelechildae. They are: Alciphanes, Anaxyrina, Athymoris, Aulidiotis, Brachyerga, Carodista, Cophomantis, Crocogma, Cynicocrates, Cynicostola, Deltoplastis, Demiophila, Dinochares, Dolichotorna, Doxogenes, Encrasima, Enthetica, Epharmonia, Eridachtha, Gasmara Walker (=Antiochtha), Habrogenes, Heteralcis, Heterodeltis, Heteroderces, Hygroplasta, Hyperochtha, Hypochasmia, Hyptiastis, Irenidora, Lecithocera, Lysipatha, Macrernis, Merocrates, Mnesteria, Monerista, Neocorodes, Nosphistica, Olbothrepta, Oxygnostis, Parelliptis, Periphorectis, Phanoschista, Philarachnis, Philoptila, Placanthes, 
Protobathra, Psammoris, Pseudocrates, Sarisophora (?), Scaeostrepta, Semnolocha, Sphaerolbia, Sphagiocrates, Stelechoris, Stryphnocopa, Technographa, Teucrodoxa, Thymbristis, Torodora and Trichoboscis.

For these genera I propose the new family TIMYRIDAE based on Timyra Walker, List of the specimens of Lepidopterous insects in the collections of the British Museum, vol. 29, p. 782, 1864. (Typus generis.-Timyra phycisella Walker, 1.c. p. 783 [by monotypy].)

In addition to the genera listed above the following are also hereby transferred from the GELECHIIDAE to the TIMYRIDAE: Crocanthes Meyrick (I886), Frisilia Walker (I864), Thubana Walker (I864) and Tisis Walker (I864). No doubt others that I have been unable to study are referable here also.

This family is peculiar in that the venation of the genera varies widely, from complete, as in Brachyerga and Carodista, to that in which there is a great reduction as in Oxygnostis and Phanoschista. In genera in which the venation is greatly reduced the cell is open and the upper surface of the hindwing is often modified by sex-scaling or hair-pencils in the male. The family is characterised also by considerable modification of the labial palpus of the male. The male genitalia are symmetrical, harpe broadly attached at base, and are characterised by a beak-like gnathos turned anterad. The aedeagus is stout and the vesica is armed with strong cornuti. The vesica enters the aedeagus ventrally or through the proximal end. The female genitalia are various but if the signum is present it is generally a dentate plate.

The affinities of this family appear to be with Odites, and related genera of the XYLORYCTIDAE, by reason of the similar gnathos. The signum of the female suggests derivation from the OECOPHORIDAE.

The following genera: Colpomorpha, Cyrictodes and Pedioxestis, are hereby transferred from the GELECHIIDAE to the OECOPHORIDAE. The genus Harpograptis is hereby transferred from the GELECHIIDAE to the COSMOPTERYGIDAE and the Chinese genus Irenidora, a borderline genus, is assigned to the BLASTOBASIDAE with its near relative, the North American Gerdana Busck.

Orygocera amphichelota is hereby transferred from the OECOPHORIDAE to the GELECHIDAE and Athrinacia cosmophragma is removed from the GELECHIDAE and assigned to the OECOPHORIDAE. In the foregoing pages I have already disposed of Leistogenes and Plocamosaris, assigned to the XYLORYCTIDAE and OECOPHORIDAE respectively by Meyrick, and I hereby transfer Ischenophenax from the OECOPHORIDAE and Plexippica from the HyPONOMEuTIDAE to the GELECHIIDAE.

The structure of the genitalia of the male of Strepsimanes scieropis Meyrick supports Meyrick's contention that the family STREPSIMANIDAE is a derivative of the XYLORYCTIDAE.

The family PHYSOPTILIDAE appears to be very near the GELECHIIDAE, which view is supported by genitalic structures.

In $193^{1}$ Meyrick proposed the family name SCAEOSOPHIDAE for a group of genera, Scaeosopha, Allotalanta, Cyphothyris, etc., which he had formerly placed in the family OECOPHORIDAE. This family cannot be maintained because the genera and species must be assigned to other well established and recognised families. Allotalanta, Bathraula, Cyphothyris, Helicacma, Protogrypa, Scaeosopha and Scalideutis are hereby

${ }^{1}$ Exotic Microlepidoptera, 4: 332. 
transferred to the COSMOPTERYGIDAE on the basis of the genitalic structures which are characteristically those of that family. Allotalanta xanthacma is clearly Oecophorid and is hereby transferred back to the family OECOPHORIDAE.

Meyrick's OECOPHORIDAE was a very broad concept, and I have shown in the preceding pages that it is necessary to remove certain genera from the family and add certain others which obviously belong here. It is likewise necessary to transfer a large number of species to genera in other families and reallocate others in this family.

The OECOPHORIDAE constitute a homogenous family usually consistent in venational characters, and in type of genitalia the exceptions are few. Vein Ic of the forewing is usually preserved at the margin of the wing and this is correlated with separate and slightly divergent veins 6 and 7 of the hindwing. The family may be divided into two more or less natural groups based on the gnathos. The gnathos of one group is spined and usually terminates in a round or oval knob, but sometimes is divided into two similar elements; the gnathos of the other group is slender or stout but generally smooth and never terminates in a spined knob. The female genitalia vary but the signum, if present, is usually in the form of a sclerotised, dentate plate. The abdomen may be spined or smooth.

Meyrick recognised the following genera in the OECOPHORIDAE (he recognised others not covered in the scope of this work): Aeolarcha, Aeolernis, Agriotorna, Alomenarcha, Anacathartis, Anacoemastis, Anchonoma, Anorcota, Archisopha, Arctoscelis, Astiarcha, Atelosticha, Atopophrictis, Auxotricha, Calliphractis, Chariphylla, Chrysonoma, Comotechna, Critoxena, Delosaphes, Doliotechna, Elaeonoma, Elaphrerga, Eomichla, Eonympha, Epimecyntis, Epimoryctis, Epiphractis, Erithyma, Erotis, Eupselia, Exosphrantis, Halimarmara, Haploscopa, Heterodmeta, Heterozyga, Himotica, Homosaces, Horomeristis, Ischnophenax, Idiocrates, Lactistica, Langastis, Lasiochira, Lepidechidna, Lysigrapha, Macarocosma, Macrocosma, Macrosaces, Meleonoma, Melochrysis, Mesothyrsa, Mylothra, Octasphales, Orsimacha, Orsotricha, Oxycharis, Pelochares, Pelomimas, Periacma, Perilachna, Petalostoma, Phalarotarsa, Phanerodoxa, Philarga, Philomusaea, Philtronoma, Picrotechna, Porthmologa, Promalactis, Protonostoma, Psaltica, Psendocentris, Psittacastis, Scoliographa, Siderograptis, Sphaerelictis, Stereodytis, Struthoscelis, Scyllochitis, Syntomaula, Tachystola, Terthrotica, Trachypepla, Triptologa, Tyrolimnas, Xenomicta, Xystoceros, Zatrichodes, Zemiocrita and Zygolopha.

Of the above genera Anorcota and Syntomaula are immediately referable to the COSMOPTERYGIDAE and are hereby transferred to that family. Meyrick never recognised the assignment of Triclonella Busck ${ }^{1}$ to the COSMOPTERYGIDAE and all but one of his species described in Triclonella must, therefore, be transferred to the COSMOPTERYGIDAE. These are: Triclonella calyptrodes, T. elliptica, T. etearcha, T. iphicleia, T. philantha, $T$. pictoria, T. trachyxyla, T. triargyra and T. turbinalis. One species, T. anachasta, must be removed from the genus Triclonella and assigned to the BLASTOBASIDAE. The genera Critoxena and Syndroma are also hereby transferred to the BLASTOBASIDAE.

Meyrick's genus Borkhausenia is also composite. Although most of the species are properly referred to the OECOPHORIDAE a few belong elsewhere. B. longa and B. leucoritis have been removed previously ${ }^{2}$ and transferred to the COSMOPTERYGIDAE. B. exstimulata is likewise referable to the COSMOPTERYGIDAE and is hereby transferred to that family.

1 Busck, A., Proc. Ent. Soc. Washington, 34: 17, 1932.

${ }^{2}$ Clarke, J. F. Gates, Can. Ent., 74: 17, 1942. 
The genitalia of the species transferred to the COSMOPTERYGIDAE are so characteristic there can be no doubt about their position.

Other species referable and hereby transferred to the COSMOPTERYGIDAE are: Promalactis synclina, Cryptolechia chordostoma, C. synclera, Meleonoma implexa, Alloclita delozona, A. reflua, Pseudodoxia crocomitra and Mompha pentapyrga.

The genus Promalactis is composite but $P$. isothea and its type species, $P$. holozona, present a unique case. In the males of both of these species the harpe is equipped with a movable, articulated appendage, a structure which, to my knowledge, is not found elsewhere in the Lepidoptera. This genus (s. str.) will probably require a new family. Certain other species, at present known only from females, will undoubtedly have to be associated with these two species, but further study will be required to establish the limits of this unique and interesting group.

The following African and Indian species, all very closely related, cannot be retained in the OECOPHORIDAE and are hereby transferred to the GELECHIIDAE: Cryptolechia arvalis, C. nyctiphronas, Orygocera amphichelota, O. liliacea, O. propycnota and $O$. sphacterias. The genus Ischnophenax is also referable to the GELECHIDAE and is hereby transferred.

The genera Auxotricha, Comotechna, Halimarmara and Picrotechna have vein 5 of the hindwing nearer to 6 than to 4, usually a positive Ethmiid character, but the genitalia, with the possible exception of Comotechna, favour retention of these genera in the OECOPHORIDAE.

Aeolernis, Alomenarcha, Archisopha, Himotica, Meleonoma, Mesothyrsa, Orsotricha, Oxycharis, Periacma, Petalostoma, Philarga, Psaltica, Struthoscelis, Triptologa and Zygolopha, have vein Ic absent in the forewing though otherwise exhibiting chiefly Oecophorid characters. Of these genera Orsotricha and Petalostoma are hereby transferred to the GELECHIIDAE and the others are retained, with reservations, in the OECOPHORIDAE.

Epiphractis and Struthoscelis exhibit venational characters which are unique while retaining other features which are clearly Oecophorid. The former possesses a crossvein between 7 and 8 of the hindwing beyond the cell, and both have an accessory cell in the forewing. The accessory cell of Epiphractis is less well defined than that of Struthoscelis and the discal cell of the latter is greatly shortened.

Three genera, Mylothra, Xystoceros and Zemiocrita have veins 6 and 7 of the hindwing stalked. This is not an Oecophorid character, but in spite of it Zemiocrita is retained in the OECOPHORIDAE since it exhibits other Oecophorid features. The Indian Mylothra and Xystoceros are borderline cases and both are nearly related to the North American Gerdana Busck. These two genera I now assign to the BLASTOBASIDAE since they are better accommodated there than elsewhere.

The species assigned by Meyrick to the COSMOpterygidae and BLASTOBASIDAE appear, with a few possible exceptions, to have been properly placed and require no special comment. As Meyrick did not distinguish between the COSMOPTERYGIDAE and MOMPHIDAE, and since the treatment of these families in this work is largely incidental, no further comment will be made.

The small family metachandidae, confined to the African and Oriental Regions, is characterised by the loss of one or more veins from at least one wing but usually one from each. Although two types of genitalia are found in the family as it now exists, 
one type, in which the cucullus is divided and the signum is three-pointed, predominates. This type suggests a close affinity to the Gelechiid-Oecophorid complex; the second type suggests a closer relationship to the Blastobasids.

Meyrick always considered the ETHMIIDAE and HYPONOMEUTIDAE synonymous, despite the fact that the former are Gelechioids, and maintained a distinction between the HYPONOMEUTIDAE and PLUTELlidae. These last two families are inseparable, as indicated by structures of both immature and adult stages.

The family ETHMIIDAE is already well established and all the species described in the genus Ethmia by Meyrick are referable to this family. The genera Agrioceros and Probolacma are also hereby transferred to the ETHMiIDAE. Idioptila, described as a Gelechiid, has previously ${ }^{1}$ been referred to this family as a synonym of Pyramidobela Braun. One of the strongest characters of this family is the segmented harpe of the male.

The genera of the HYPONOMEUTIDAE fall into two large groups on the basis of the male genitalia. The first group consists of genera in which the harpe is ample, the aedeagus is slender and the uncus is divided into two elements. Unfortunately the males of several genera are not known to me and I am unable to place them, but the following fall in the first group with Hyponomenta: Amiantastis, Calliathla, Chalconympha, Diaphragmistis, Epichthonodes (?), Litaneutis, Lycophantis, Metanomenta, Orthosaris, Perisceptis, Philaustera and Tarphyscelis.

In the second group the uncus is a single element and this and the harpe suggest a relationship with the XYLORYCTIDAE rather than with the other HYPONOMEUTIDAE. Genera in this group are: Anticrates (=Pyrozela), Comocritis, Eriopyrrha, Lactura Walker, Spilogenes, Trichocirca (=Telosphrantis) and Thalassonympha.

Certain of the genera retained in the HYPONOMEUTIDAE require further comment. The monobasic genus Pronomeuta exhibits extraordinary features in both wing venation and genitalic type. In the forewing of the type species, sarcopis, the medial vein is strongly developed thus dividing the discal cell into two almost equal parts, and there is a well developed accessory cell lying between veins 6 and Io. The genitalia strongly suggest a Tineid affinity by the form of the harpes and the unusually great development of the vinculum.

The genus Lamyristis is noteworthy because of the presence of seven bristles in the frenulum of the female, the degeneration of the radial vein of the forewing and the tendency to form accessory cells.

Several genera, now placed in the HYPONOMEUTIDAE, require reallocation and I hereby transfer Loxozyga to the oECopHoridae, Orinympha to the SCYTHRIDIDAE and Platybathra to the cosmopterygidae. The last genus is very closely related to Syntetrernis, already placed in the COSMOPTERYGIDAE by Meyrick, and possesses the same type of genitalia.

Metharmostis will probably have to be assigned to the COSMOPTERYGIDAE on the basis of the female genitalia of $M$. asaphaula which show a striking similarity to those of Stilbosis devoluta, already placed in that family.

Stichotactis is very closely related to, if not congeneric with, Homadaula, and is hereby transferred to the GLYPHIPTERYGIDAE.

Nosymna ochrochorda appears to be incorrectly placed in the HYPONOMEUTIDAE

${ }^{1}$ Meyrick, Exotic Microlepidoptera, 3: 414, 1928. 
but, not having seen Walker's type of the genus, I cannot state whether or not ochrochorda is properly assigned generically.

The GLYPHIPTERYGIDAE present no great problems except in the genus Imma in which appear many different forms of palpi, pattern and coloration, and in which we find species that seem to be transitional between the Microlepidoptera and the Macrolepidoptera. Indeed, it is here that we may look for the origin of the PHALAENIDAE. Required study of the genus Imma is far beyond the scope of this work and can only be alluded to here.

The following families: tORTRICIDAe, Olethreutidae (=Eucosmidae, Auct.), PHALONIIDAE, CARPOSINIDAE and CHLIDANOTIDAE, comprise a natural world-wide group which, though composed of closely related families, form definable entities, each component being rather easily separable from any other. There are a few cases, however, which present difficulties but this may be due more to our lack of understanding than to any particular peculiarity of the insects themselves.

In the family TORTRICIDAE only fifty Meyrick genera are being considered and, since nearly all of these are already properly placed and require no special comment, I shall discuss only those which require attention at this point.

First it should be pointed out that the family TORTRICIDAE characteristically possesses vein Ic of the forewing, no cubital pecten on the hindwing, symmetrical male genitalia with broadly attached harpe and moderate to slender aedeagus and a subtriangular, somewhat folded, anellus. The signum, when present, consists of a single stellate plate, a sclerotised band or a single thorn with a large bulbous base. The above, of course, are not all the characters involved in the definition of the family, but will be adequate here.

Of the fifty genera considered in this work Acroplectis, Deltobathra, Diactenis, Mnesipyrga, Peteliacma and Proselena lack vein Ic of the forewing. Mnesipyrga is referable to the PHALAENIDAE and is hereby transferred to that family. It belongs in the Acidaliodes complex, having the characteristic spiculate bursa copulatrix of that group, a simple $\mathrm{Ib}$ of forewing and no vein 5 of hindwing. The other genera, except Diactenis which has aberrant venation, have little to recommend their assignment to other families.

Except for synonymy, which will be treated in Volume III, there are no other changes of genera required at this point.

Meyrick's concept of Eulia was broad and loose and he has placed a great assortment of unrelated species in it. Many of the species he included in Eulia will be reassigned, elsewhere in this work, and still others will require the erection of new genera to contain them and provide a proper classification. What is true of Eulia is, to a lesser extent, true of other genera and a reshuffling of many species will be unavoidable.

A unique group in the TORTRICIDAE, consisting of the four closely related genera Ardentica, Atteria, Polyortha and Psendatteria is peculiar in that the harpe of the male is split longitudinally along the ventral edge, forming a longitudinal pocket for the reception of an abdominal hair-pencil. The four genera are South American.

Tortrix noctivola is referable to the OLETHREUTIDAE and is hereby transferred to that family. Arotrophora balsamodes is hereby transferred to the PHALONIDAE.

Tortrix crocomis lacks vein Ic of forewing but is retained in the family TORTRICIDAE with doubt because the abdomen of the unique type is missing. 
Among the five Tortricoid families the olethreutidae are the most closely related to the TORTRICIDAE. The genitalia of this family, however, serve to distinguish any member from any species of Tortricid. In fact, there are no genitalia in the Lepidoptera that can be easily confused with those of the olethreutidae. The peculiar anellus with the aedeagus strongly fused to it is unique and is diagnostic of the family. The female of any Olethreutid may be distinguished from any Tortricid, or from species of other families of this complex, by the signum, which consists of a single or double thorn without large bulbous base, or a pair of small dentate cones. Exceptions to this are Polychrosis inficta, Proschistis lucifera, Argyroploce mniopyrrha, A. petrumacha, A. sideroxyla, $A$. streblopa and $A$. torquata, but other characters leave no doubt as to their family position.

In this family most species are assigned to other than Meyrick genera and particularly to Argyroploce without regard to subfamily distinctions. Meyrick's Argyroploce is a synonym of Olethreutes Hübner and all species placed under that name by him will be transferred to the genus Olethreutes. In this work, because of its arrangement and method of presentation in subsequent volumes, subfamily position will be ignored but the fact remains that in a correct systematic arrangement the subfamilies must be recognised. The genera, as conceived by Meyrick, like Eulia and Stenoma contain large assemblages of unrelated forms.

It is interesting to note, however, that in the assignment of hundreds of species to the olethreutidae Meyrick referred only two Tortricids to this family. A splendid record! These two species are Argyroploce ornithotypa and Eucosma torrens which I hereby transfer to the TORTRICIDAE.

The Tortricoid family PHALONIIDAE provides a multitude of genitalic types but a great constancy in structure and facies and, as far as known, vein Ic of the forewing is never present in the PHALONIIDAE. I do not know of a single exception. The absence of Ic immediately excludes TORTRICIDAE (except five genera) and all OLETHREUTIDAE.

The great bulk of PHALONIIDAE have been placed in the genus Phalonia, but this genus, as it now stands, is another "wastebasket" containing several distinct genera. Reallocations in this family will necessarily be many. Hysterosia zophocosma and Phtheochroa psychrodora are both referable to the OLETHREUTIDAE and are hereby transferred to that family.

The fourth family of this complex, the chlidanotidae, is confined to the Indian Region, Borneo and Fiji. In this family Meyrick placed Archimaga, Branchophantis, Chlidanota, Electracma, Metrernis and Trymalitis. The CHLIDANOTIDAE are characterised by the absence of Ic and one other vein ( 7 or 9) in the forewing plus the absence of cubital pecten from the hindwing. The male genitalia are simple, Xyloryctoid in character with ample, simple harpe, simple uncus, broadly triangular anellus and moderate aedeagus. The ovipositor lobes are distinctly Tortricoid but the signum consists of a central plate with a heavy pecten of long sclerotised spines.

Although Electracma undoubtedly belongs here the forewing shows twelve veins by the freakish terminal forking of vein 7 . It might be argued that 7 and 8 are longstalked and that vein 9 is present but I think Meyrick's interpretation of the veins is correct and that vein 9 is absent. It appears that the apical section in the type specimen of hemichroa is deformed, as is clearly the case in the type of Metrernis (ochrolina), in which degeneration of the apical veins has taken place. It may be inferred from these 
two examples that malformation of this sort is not unusual. This family appears to be intermediate between the PHALONIIDAE and the OLETHREUTIDAE. In my initial statement concerning this family, I limited its distribution to a small area of the Old World Tropics but it seems very likely that certain tropical American species will be referable to the CHLiDANotidaE.

In the last of the Tortricoid farnilies dealt with in this work, the CARPOSINIDAE, we encounter a group which cannot be easily confused with the other four. In this family wing characters alone will serve for distinction. The forewing is tufted and the hindwing lacks veins 4 and 6 , the latter often represented by a fold from about the middle of the discocellulars. The cell of both wings is usually closed but in Picrorrhyncha both are open. The female genitalia of this genus are also aberrant for the family and the genus may be misplaced here.

In the genitalia we find characters which generally establish the family position although a few species, apparently correctly placed here, deviate from the general pattern. Characteristically the aedeagus is very slender proximally but is greatly enlarged and heavily armed distally. In the female the signum usually consists of two forked elements but in a few species, Commatarcha palaeosema, Meridarchis eremitis, $M$. heptaspila, Paramorpha aulata, P. laxeuta and one or two others, this condition does not exist.

Meyrick did not distinguish between the TINEIDAE and ACROLOPHIDAE and is supported in this view by some contemporary workers. The larvae and adult characters, in my opinion, justify such a separation and, in this work, I regard them as distinct families. The scope of this work is limited to a consideration of the American fauna in these two families. In the TINEIDAE vein 7 runs to apex or costa, generally the latter, and in the ACROLOPHIDAE it runs to termen. The basal whorl of scales on each segment of the antenna is reduced to a whorl of bristles in the TINEIDAE, but in the ACROLOPHIDAE the two whorls are equally developed.

Since Meyrick made no distinction between these two families no criticism of his assignment of genera is warranted. Brachysymbola appears to be nearly related to Acrolophus and is hereby transferred to the ACROLOPHIDAE. Meyrick's genus Acrolophus contains several species of several genera and these will be properly placed in a subsequent volume. 


\section{ACKNOWLEDGMENTS}

Since the inception of this task I have become indebted to a large number of individuals to whom I wish to express my sincere appreciation for the help received.

To Mr. N. D. Riley, Keeper of Entomology, who suggested the project, and the Trustees of the British Museum (Natural History) who placed every facility at my disposal and without whose support the plan could not have been carried to conclusion, and to Mr. W. H. T. Tams, in charge of Lepidoptera, a constant source of inspiration and encouragement, an especial debt is due.

My sincere thanks are due my own superiors, Mr. C. F. W. Muesebeck, Leader, Division of Insect Identification and the late Dr. P. N. Annand, Chief, Bureau of Entomology and Plant Quarantine for their enthusiastic support of this undertaking and for the release from other duties which enabled me to carry out my investigations abroad. To Dr. Alexander Wetmore, Secretary, Smithsonian Institution, I owe my thanks for necessary expenses and for providing complete photographic equipment. I wish to express my appreciation also to Mr. Paul O. Nyhus, Agricultural Attaché, United States Embassy, London, and to his staff who aided me on many occasions and who supplied much needed items such as typing paper and index cards, unobtainable in London at that time, and helped me in many other respects.

To the following individuals I am much indebted for information concerning the many scattered types in institutions and private collections: To Dr. A. J. T. Janse, Transvaal Museum, Pretoria, South Africa; to Dr. A. J. Hesse, South African Museum, Cape Town, South Africa; to M. J. Bourgogne and M. P. Viette, Muséum National d'Histoire Naturelle, Paris, France; to Professor Bruno Pittioni, The Naturhistorisches Museum, Vienna, Austria; to Dr. Ramon Agenjo, Instituto Español de Entomologia, Madrid, Spain; to Mr. Elwood C. Zimmerman, The Bernice P. Bishop Museum, Honolulu, Hawaii for information concerning the types in that institution and in the collection of the Hawaiian Sugar Planters' Experiment Station, Honolulu, Hawaii; to Dr. H. Schouteden, Musée du Congo Belge, Tervueren, Belgium; to Dr. W. T. M. Forbes, Department of Entomology, Cornell University, Ithaca, New York; to Dr. M. L. Roonwal, Zoological Survey of India, Kaiser Castle, Banaras Cantt.; to Professor Dr. Ferdinand Pax, Museum für Natur- Völker- und Handelskunde, Bremen, Germany; to Professor Dr. Hans Sachtleben, Deutsches Entomologisches Institut, Berlin, Germany; to the late Professor G. D. Hale Carpenter, Hope Department, Oxford University Museum, Oxford, England; to Dr. R. T. M. Pescott, National Museum of Victoria, Melbourne, Australia; to Dr. A. Diakonoff, late of the Zoölogisch Museum en Laboratorim, Bogor, Java, and Dr. C. O. van Regteren-Altena for information on the types in the Rijks Museum van Natuurlijke Historie, Leiden, Holland; to Dr. A. B. Walkom and Mr. Anthony Musgrave, The Australian Museum, Sydney, Australia; to M. Le Marchand, Paris; to Victoria H. Raica, Muzeul de Istorie Naturală and Prince Aristide Caradja, Bucharest, Rumania; to Professor Dr. B. Klatt, Hamburgisches Zoologisches Museum und Institut, Hamburg, Germany; to Dr. J. N. Mukherjee, Indian Agricultural Research Institute, New Delhi, India; to Mr. E. A. Ellis, The 
Castle Museum, Norwich, Norfolk, England; to Dr. W. R. B. Oliver, Canterbury Museum, Christchurch, C.I., New Zealand; to Tom Harrison, Curator, Sarawak Museum, Kuching, Sarawak; to Dr. H. Höne, Museum Alexander Koenig, Bonn, Germany; to Dr. H. G. Amsel, Buchenburg bei Peterzell, Baden, Germany; and to Mr. E. P. Wiltshire, British Consulate, Shiraz, Persia.

The following enthusiasts, who worked under my direction, are due special thanks for their interest in the project and their tireless efforts to bring it to a successful conclusion: Mr. John D. Bradley, Microlepidopterist, British Museum (Natural History), my fulltime assistant, who worked with me throughout the course of my stay and relieved me of many time-consuming tasks; Mr. Harold Malies, microscopist, of Southport, Lancs., England, Fellow of the Royal Microscopical Society, who made practically all the photomicrographs; Mr. Charles Owen Harman, medical student, from Brighton, England, who photographed very many of the wings and heads of the moths; the late Miss Hazel Rough, sculpturing student from Wellington, New Zealand, who made so carefully the more than 600 drawings of the venation of the types of the genera and by her ability, enthusiasm and charm won the admiration of her many friends in the Museum; Miss Margaret Chaborel of London and Mrs. Jacqueline Beatty of Paris who aided so extensively in bringing together the catalogue of species and handled the considerable correspondence with the many individuals who supplied information.

There are others who have helped in smaller, but nonetheless important ways, and to them I give my sincere thanks also. 


\section{ILLUSTRATIONS}

THE illustrations for volume I were made by Mr. M. G. Sawyers, staff photographer of the British Museum (Natural History) who made some of the "exploratory" photomicrographs in volume II also.

The remaining photomicrographs were made by Harold Malies on a Watson "Holophot". This apparatus, however, had to be adapted, by various devices, to photograph successfully all of the various types and sizes of genitalia. It is recognised that photomicrographs of genitalia are not always entirely satisfactory as a means of illustration but the immensity of the task made the use of this method mandatory. In some cases it has been necessary to use composite photomicrographs, exposures at different levels and part of one superimposed over the other, in order to bring out diagnostic details. In some cases I have added, also, sketches in lateral aspect to show essential details.

Most of the photographs of the wings and heads have been taken by Mr. Harman with a Leica camera equipped with a Leitz Elmar $\mathrm{f}=5 \mathrm{~cm}$., $\mathrm{I}: 3.5$ lens. Extension tubes were used where necessary to enlarge the image on the film and all the prints were brought up to 3 by 4 inches. In a few instances the left wings were not in condition to be photographed and in such cases the right wings were photographed and the image reversed on the plate. 


\section{CATALOGUE OF SPECIES}

IN the pages immediately following is a catalogue of the species of Microlepidoptera described or named by Meyrick.

The purpose of this catalogue is not only to list the names and give references to the original descriptions, but also to indicate the disposition of the type when known. When a species was described a proposed repository for the type was sometimes given, but the type is not always to be found in the place indicated. Usually, however, no indication of the disposition of the type was given and it is assumed that these types were retained by Meyrick. Some have been lost and some have been destroyed and others are to be found in places other than indicated. As previously pointed out, when Meyrick synonymised one of his species with another, the two series were merged without reference to the type specimens. As a result of this practice at least one type [Stenoma niphacma labelled S. chalastis by Meyrick] was sent to August Busck of the U.S. National Museum, as an example of the valid species, yet this specimen should have been found in the Meyrick Collection.

In order to ascertain the locations of types I have written to many institutions and individuals and have been accorded a most hearty and helpful response. As a result of this generous co-operation it has been possible to acquire authentic information regarding the actual locations of several thousands of types in addition to those which I have seen, examined and authenticated myself. There are many more types in the British Museum that have not yet been studied or authenticated.

Following each reference is a symbol which represents the institution or collection in which the type will be found providing the type has been verified. If the location of the type is unknown no symbol will be found. If the type is lost or destroyed the words "Lost" or "Destroyed" are inserted. Meyrick proposed many substitute names for those which he found to be preoccupied. These have been marked "Sub".

The types of genera have been marked "*" following the generic name of which the species is the type.

Below is a list of institutions and collections, with their appropriate symbols, in which the types will be found:

The British Museum (Natural History), London

The United States National Museum, Washington, D.C.

The Transvaal Museum, Pretoria

The South African Museum, Cape Town

The Muséum National d'Histoire Naturelle, Paris

The Naturhistorisches Museum, Vienna

The Instituto Español de Entomologia, Madrid

The Bernice P. Bishop Museum, Honolulu

The Hawaiian Sugar Planters' Experiment Station, Honolulu 
The Musée du Congo Belge, Tervueren, Belgium

The Department of Entomology, Cornell University, Ithaca, N.Y.

The Zoological Survey of India, Kaiser Castle, Banaras Cantt.

The Museum für Natur- Völker- und Handelskunde, Bremen

The National Museum of Victoria, Melbourne

The Deutsches Entomologisches Institut, Berlin

The Hope Department, University Museum, Oxford, England

The University Museum, Cambridge, England

The Rijks Museum van Natuurlijke Historie, Leiden, Netherlands

The Australian Museum, Sydney

M. Lhomme's Collection, Doutelle, Lôt, France

M. Le Marchand's Collection, Paris

Mr. Fernando Bourquin's Collection, Buenos Aires, Argentina

In addition to the information received from the institutions and individuals listed above much other information, though largely negative in character, has been acquired from several other sources. Through Victoria H. Raica, Keeper, Muzeul de Istorie Naturală, Bucharest, Rumania, I have received word that the collection of Prince Aristide Caradja is in that Institution. The Caradja collection contains between 200 and 300 types but without confirmation and verification I cannot list the species. In a letter to me Prince Caradja stated that the types received by him from Meyrick are those listed in the several papers dealing with the Chinese material.

According to the Curator, Sarawak Museum, there are no Meyrick types in that collection. I failed to find the types of the species described in the Sarawak Museum Journal in the Meyrick collection and cannot give any information regarding their location.

Dr. W. R. B. Oliver, Director, Canterbury Museum, Christchurch, C.I., New Zealand, has advised me that there are no types in that Institution.

World War II has taken its toll also, as I was informed by Professor Dr. B. Klatt, Director, Hamburgisches Zoologisches Museum und Institut, Hamburg: "Zu Ihrer Anfrage vom ro Febr. muss ich Ihnen leider mitteilen, dass unsere Lepidopterensammlung bei einem der Bombenangriffe im Jahre I943 vernichtet wurde, so dass wir Ihnen von Seiten des Museums aus, zu unserem Bedauern nicht behilflich sein können." Certainly there were some Meyrick types there but the number, or the species are unknown.

In the Castle Museum, Norwich, England, there is at least one holotype (Scoparia coprista) but since the Pyralidoids are beyond the scope of this work further reference to this species will be omitted. I am indebted to E. A. Ellis, Esq., for the above information.

Dr. J. N. Mukherjee, Director, Indian Agricultural Research Institute, has advised me that there are no types either at New Delhi or in the "Indian Pusa Collection" although some species may be represented by paratypes or cotypes. 


\title{
CATALOGUE OF MICROLEPIDOPTERA DESCRIBED BY EDWARD MEYRICK
}

\begin{tabular}{|c|c|}
\hline $\begin{array}{l}\text { Specific } \\
\text { name }\end{array}$ & $\begin{array}{c}\text { Generic } \\
\text { name }\end{array}$ \\
\hline abacodes & MELASINA \\
\hline abathra & TINEA \\
\hline abathrodes & GYPSONOMA \\
\hline abductella & ERIODYTA \\
\hline abiasta & GLYPHIDOCERA \\
\hline absoluta & PHTHORIMAEA \\
\hline absolutrix & TINEA \\
\hline abstemia & EUCOSMA \\
\hline abstrusa & CRYPSITHYRIS \\
\hline academica & AUTOSTICHA \\
\hline acalyptra & ORNEODES \\
\hline acanthidias & ACROCERCOPS \\
\hline acanthis & POLYCHROSIS \\
\hline acanthodes & STAGMATOPHORA \\
\hline acanthogona & ACROLOPHUS \\
\hline acanthopa & IDIOPHANTIS \\
\hline acanthopis & GELECHIA \\
\hline acarpa & CALYCOBATHRA* \\
\hline acarta & BLASTOBASIS \\
\hline acatharta & PARADORIS \\
\hline accensa & TELPHUSA \\
\hline accincta & NEALYDA \\
\hline accipitrina & EUCosma \\
\hline accumulata & SCYTHRIS \\
\hline accurata & MELASINA \\
\hline accurata & STENOMA \\
\hline accusatrix & TINEA \\
\hline aceraea & EULECHRIA \\
\hline acerata & ANARSIA \\
\hline acerba & ASYMPHORODES \\
\hline acervalis & OPOGONA \\
\hline acervata & EULECHRIA \\
\hline acetosa & THYROCOPA \\
\hline achalinella & EULECHRIA \\
\hline acharacta & AUTOSTICHA \\
\hline acharis & NARYCIA \\
\hline acharista & LASIOMACTRA* \\
\hline acharma & $\begin{array}{l}\text { PROCOMETIS } \\
\text { [HYOSTOLA*] }\end{array}$ \\
\hline acharnaea & GELECHIA \\
\hline
\end{tabular}

\author{
Original reference and location of type specimen
}

I908, Proc. Zool. Soc. London, p. 749.

I920, Exotic Microlepidoptera, 2: 355.

I937, in Caradja and Meyrick, Deuts. Ent. Zeit. Iris, 5I: 177 . (BM)

I884, Proc.LinneanSoc. N.S.Wales, 8: 5I7. (Misidentified

by Meyrick as abductella Walker.)

1936, Veröff. Deutschen Kol.-Mus. I: 332, pl. 13, fig. 25. (н)

I9I7, Trans. Ent. Soc. London, I9I7: 44. (BM)

1930, Exotic Microlepidoptera, 3: 547. (вм)

I932, Exotic Microlepidoptera, 4: 224. (Sub.)

I917, Exotic Microlepidoptera, 2: 74 .

I922, Zool. Meded. 7: 87. (L)

1913, Ann. Transvaal Mus. 3: 269. (T)

1934, Exotic Microlepidoptera, 4: 524.

1920, Exotic Microlepidoptera, 2: 348. (BM)

I933, Exotic Microlepidoptera, 4: 428.

I9I9, Exotic Microlepidoptera, 2: 279. (вм)

I93I, Exotic Microlepidoptera, 4: 64. (BM)

1932, Exotic Microlepidoptera, 4: I95. (BM)

I891, Ent. Mon. Mag. 27: 60.

I9II, Trans. Linnean Soc. London, I4: 286.

I9I I, Journ. Bombay Nat. Hist. Soc. 20: 736. (вм)

I92I, Ann. Transvaal Mus. 8: 68. (т)

1923, Exotic Microlepidoptera, 3: 5. (BM)

I913, Ann. Transvaal Mus. 3: 274. (T)

I9I4, Ann. Transvaal Mus. 4: I97. (T)

1922, Exotic Microlepidoptera, 2: 606.

I9I6, Exotic Microlepidoptera, I: 526 (вм)

1916, Trans. Proc. New Zealand Inst. 48: 4 I9.

I883, Proc. Linnean Soc. N.S. Wales, 8: 324 .

1913, Journ. Bombay Nat. Hist. Soc. 22: 169. (BM)

I929, Trans. Ent. Soc. London, 76: 501.

I9I4, Exotic Microlepidoptera, I: 209.

I9I4, Exotic Microlepidoptera, I: I6I. (мА)

I9I5, Exotic Microlepidoptera, I: 37I.

1883, Proc. Linnean Soc. N.S. Wales, 7: 523.

1918, Exotic Microlepidoptera, 2: 153.

I920, Voyage de Ch. Alluaud et R. Jeannel en Afrique

Oriental, II, Microlepidoptera, p. Iog. (P)

I92I, Ann. Transvaal Mus. 8: Io2. (T)

1908, Proc. Zool. Soc. London, p. 73I.

1927, Exotic Microlepidoptera, 3: 348. (Bм) 


\begin{tabular}{|c|c|c|}
\hline acharnias & LEPIDECHIDNA* & I934, Exotic Microlepidoptera, 4: 522. (BM) \\
\hline acherusia & AXYROSTOLA* & I923, Exotic Microlepidoptera, 3: 29. (BM) \\
\hline achlyodes & YPSOLOPHUS & I904, Proc. Linnean Soc. N.S. Wales, 29: 432. \\
\hline chlyoessa & PHRYGANOSTOLA & I881, Proc. Linnean Soc. N.S. Wales, 5: $25^{2}$. \\
\hline achlyota & TINEA & I926, Ann. South African Mus. 23: 343. (SA) \\
\hline hlyphanes & CRYPTOLECHIA & I934, Exotic Microlepidoptera, 4: 478. (BM) \\
\hline chnastis & ANTIOCHTHA & I9o6, Journ. Bombay Nat. Hist. Soc. I7: I5o. (BM) \\
\hline chnias & EPIPHTHORA & I904, Proc. Linnean Soc. N.S. Wales, 29: 266. \\
\hline hnodes & ACROCERCOPS & I9I5, Trans. Ent. Soc. London, I9I5: 23I. \\
\hline chranta & DICHELOPA & I9Io, Proc. Linnean Soc. N.S. Wales, 35: 283. \\
\hline chroa & BORKHAUSENIA & I9I4, Exotic Microlepidoptera, I: 232. \\
\hline hroana & HETEROCROSSA & I883, Ent. Mon. Mag. 20:3I. \\
\hline hrochalca & PROTOMACHA & I889, Proc. Linnean Soc. N.S. Wales, (Ser. 2) 3: I646. \\
\hline hrosta & HARMOLOGA & I902, Trans. Ent. Soc. London, I902: 572. \\
\hline chyritis & EULIA & I926, Exotic Microlepidoptera, 3:257. (BM) \\
\hline hyrobathra & ARISTOTELIA & I933, Exotic Microlepidoptera, 4: 447. (c) \\
\hline hyrodes & SIMAETHIS & I9I2, Exotic Microlepidoptera, I: 46. (BM) \\
\hline hyropa & SCYTHRIS & I9I6, Exotic Microlepidoptera, 2: Io. \\
\hline yrota & GELECHIA & I886, Trans. Proc. New Zealand Inst. I8: I7o. \\
\hline icularis & UNTOMIA & I9I8, Exotic Microlepidoptera, 2: I46. \\
\hline iculata & BORKHAUSENIA & I928, Exotic Microlepidoptera, 3: 468. (Bм) \\
\hline ida & BORKHAUSENIA & I9II, Ann. Transvaal Mus. 3: 72. \\
\hline idata & OPOSTEGA & I9I5, Trans. Ent. Soc. London, I9I5: 240. \\
\hline :idula & ACROCERCOPS & I9II, Ent. Mon. Mag. 47: 2I3. \\
\hline inacella & GLYPHIPTERYX & I883, Proc. Linnean Soc. N.S. Wales, 7: I93. \\
\hline lea & EOCHROA & I883, Proc. Linnean Soc. N.S. Wales, $7: 45^{6}$. \\
\hline lera & PROTOLECHIA & I904, Proc. Linnean Soc. N.S. Wales, 29: 348. \\
\hline naea & HELIOCAUSTA & I888, Proc. Linnean Soc. N.S. Wales, (Ser. 2) 2: 936. \\
\hline manthes & PERONEA & I931, Ann.Mus.Nac. Hist. Nat. Buenos Aires, 36: 382. (BM) \\
\hline cmastis & MELASINA & I9I7, Exotic Microlepidoptera, 2: 94. \\
\hline mias & PHYLLOCNISTIS & I906, Trans. R. Soc. S. Australia, 30: 62. \\
\hline nodeta & HYPERECTA & I93I, Exotic Microlepidoptera, 4: 64. (BM) \\
\hline mogramma & BRENTHIA & I915, Trans. Ent. Soc. London, I9I5: 217. (BM) \\
\hline monias & PRAYS & I9I4, Journ. Bombay Nat. Hist. Soc. 23: I25. (BM) \\
\hline mophanes & ACROCERCOPS & I928, Exotic Microlepidoptera, 3: 409. (T) \\
\hline mostacta & LABDIA & I932, Exotic Microlepidoptera, 4: 213. \\
\hline motypa & THECTOPHILA* & I927, Trans. Proc. New Zealand Inst. 57: 701. \\
\hline nura & COLEOPHORA & I9I4, Exotic Microlepidoptera, I: 206. \\
\hline lasta & LECITHOCERA & I9I9, Exotic Microlepidoptera, 2: 236. (BM) \\
\hline nitis & STENOMA & I9I5, Exotic Microlepidoptera, I: 4I4. (Bм) \\
\hline ontarcha & NEPTICULA & I926, Exotic Microlepidoptera, 3: 295. \\
\hline ontias & ETHMIA & I906, Journ. Bombay Nat. Hist. Soc. I7: 409. (BM) \\
\hline ontias & STATHMOPODA & I897, Proc. Linnean Soc. N.S. Wales, 22: 318. \\
\hline ntistes & ERECHTHIAS & 1881, Proc. Linnean Soc. N.S. Wales, 5: 266. \\
\hline ontophora & LIMNOECIA & I922, Exotic Microlepidoptera, 2: 573. \\
\hline rantha & THIOTRICHA & I908, Journ. Bombay Nat. Hist. Soc. I8: 440. \\
\hline raspis & TRISSOCHYTA* & I921, Exotic Microlepidoptera, 2: 474 . \\
\hline ates & SCIEROPEPLA & I89o, Trans. R. Soc. S. Australia, I3: 68. \\
\hline atodes & STENOMA & I9I6, Exotic Microlepidoptera, I: 536. (BM) \\
\hline ratopa & PRASODRYAS & I926, Exotic Microlepidoptera, 3: 288. \\
\hline
\end{tabular}




acratopis
acratynta
acrena
acrias
acribota
acricula
acridomima
acridula
acris
acritodes
acritodes
acritopa

acrobaphes acrobapta acrobatica acrocarpa acrocausta acrocentra acrochalca acrocharis acrochlora acrocosma acrocosma

acrodactyla acrodelta acrodeta acrodina acrodisca acrodora acroglypta acrognampta acrogona acrogramma acrograpta acrogypsa acroleuca acrolitha acrolophoides acrolychna acronephela acronoma acropeda acropelta acropercna acrophanes acrophantis acrophrictis
MYLOCRITA* LITHOCOLLETIS ARISTOTELIA PTEROPHORUS STENOMA PROTOLECHIA THYLACOSCELES* STENOMA STAGMATOPHORA CAPUA PSEUDODOXIA DICHOMERIS

OCYSTOLA ANTAEOTRICHA STRUTHOSCELIS* EPICEPHALA CACOECIA DICANICA* METAPHRASTIS* CTENOPSEUSTIS HYPELICTIS* COPTOTELIA ECCOPSIS

[COSMORrhyncha*] Igo8, Proc. Zool. Soc. London, p. 7 I 7 . GELECHIA PLUTELLA BATRACHEDRA EREUNETIS CHOROPLECA LYONETIA BATTARISTIS IMMA

HAPSIFERA BUCCULATRIX APHANOXENA THYESTARCHA PROTOLECHIA CYPHOSTICHA ACHARNEODES DICHOMERIS ANTAEOTRICHA GLYPHIPTERYX PHALONIA COMMATICA THECOBATHRA* ONEBALA THIOTRICHA ASYMPHORODES
I922, Exotic Microlepidoptera, 2: 507. I9I6, Exotic Microlepidoptera, I: 622. Igo8, Proc. Zool. Soc. London, p. 724. I908, Trans. Ent. Soc. London, I907: 500. I930, Ann. Naturhist. Mus. Wien, 44:247. (v) I9I4, Exotic Microlepidoptera, I: I98. (MA) I889, Trans. Proc. New Zealand Inst. 2I: I7I. I9I8, Exotic Microlepidoptera, 2: 20I. (BM) I9II, Trans. Linnean Soc. London, I4: 283. I9I0, Proc. Linnean Soc. N.S. Wales, 35: I9o. I9I4, Exotic Microlepidoptera, I: 263. (BM) I935, in Caradja and Meyrick, Materialien zu einer Microlepidopteren Fauna der Chinesischen Provinzen Kiangsu, Chekiang und Hunan, p. 72. (BM) I885, Proc. Linnean Soc. N.S. Wales, 9: I074. (MA) I933, Exotic Microlepidoptera, 4: 434. (BM) I9I3, Trans. Ent. Soc. London, I9I3: I77. (BM) I927, Insects of Samoa, 3, Lepidoptera, fasc. 2, p. Io6. I907, Trans. Proc. New Zealand Inst. 39: II5. I9I3, Ann. Transvaal Mus. 3: 33I. I907, Proc. Linnean Soc. N.S. Wales, 32: I34. I932, Exotic Microlepidoptera, 4: 26I. (BM) I905, Journ. Bombay Nat. Hist. Soc. I6: 600. (Bм) I9I2, Trans. Ent. Soc. London, IgII: 702. (BM) I907, Trans. Proc. New Zealand Inst. 39: II7. I93I, Ann. Mus. Nac. Hist. Nat. Buenos Aires, 36: 408. I927, Insects of Samoa, 3, Lepidoptera, fasc. 2, p. 94 . I9I2, Trans. Proc. Nere Zealand Inst. 44: I22. I9I7, Exotic Microlepidoptera, 2: 79. I9I5, Trans. Ent. Soc. London, I9I5: 243. I929, Exotic Microlepidoptera, 3:505. (BM) I930, Exotic Microlepidoptera, 4: 4. (BM) I92I, Zool. Meded. 6: 200. (L) I9I9, Exotic Microlepidoptera, 2: 287. (BM) I9I5, Exotic Microlepidoptera, I: 387. (вм) I9I7, Ann. South African Mus. I7: 8. (SA) I904, Proc. Linnean Soc. N.S. Wales, 29:340. I908, Journ. Bombay Nat. Hist. Soc. I8: 825 . I93I, Exotic Microlepidoptera, 4: I28. (v) I922, Trans. Ent. Soc. London, I922: II2. (BM) I9I5, Exotic Microlepidoptera, I: 392. (BM) I888, Trans. Proc. New Zealand Inst. 20: 86. I9I2, Ent. Mon. Mag. 48: 35. (Sub.) I9I4, Trans. Ent. Soc. London, I9I4: 238. I922, Exotic Microlepidoptera, 2: 553. (BM) I9Io, Journ. Bombay Nat. Hist. Soc. 20: 457. (BM) I936, Exotic Microlepidoptera, 5: 45. (BM) I934, Pacific Ent. Surv. Publ. 7, art. 28, p. 35I. (вB) 
acrophylla

acroplecta

acroplecta

acropola

acropta

acroptila

acrosema

acrosphales

acrosticta

acrostigma

acrostropha

acrothecta

acrotherma

acrotoma

acrotropa

acroxantha

acrozona

acrozyga

actaea

actias

actinia

actinias

actinobola

actinopa

actinota

actista

actuosa

actuosa

aculeata

acuminatus

acuta

adaequata

adamantea

adamantina

adamasta

adecasta

adelina

adelocentra

adelopa

adelopis

adelphodes

adjutrix

adminiculata

administra

admiranda

adoratrix

adornata

adoxella

adulans
TELPHUSA

[SYNCOPACMA*]

PYRODERCES

ARGYROPLOCE

PHILOBOTA

LEUCOPTERA

IMMA

ARGYROPLOCE

LECITHOCERA

PTOCHORYCTIS

PERSICOPTILA

STATHMOPODA

GLYPHIPTERYX

GRACILARIA

ANARSIA

COESYRA

OCYSTOLA

HOPLOPHANES

TINEA

PROSCHISTIS

PALTODORA

TRACHYDORA

BORKHAUSENIA

GLYPHIPTERYX

ISOPHRICTIS

PROTOLECHIA

STENOMA

EUCOSMA

ODITES

COSMOPTERYX

PTEROPHORUS

DICHOMERIS

HELCYSTOGRAMMA

OMIOSTOLA

CNAPHOSTOLA*

TINEA

PACHYPSALTIS

GRACILARIA

DICHOMERIS

SCYTHRIS

XYSMATODOMA

TRICHOPTILUS

HOLCOCERA

STENOMA

AUXIMOBASIS

ASYMPHORODES

STENOMA

STENOMA

EULECHRIA

STENOMA
I9I2, Ann. Transvaal Mus. 3:65. (T)

I9I5, Exotic Microlepidoptera, I: 3I3.

I921, Ann. Transvaal Mus. 8: 6o. (T)

I884, Proc. Linnean Soc. N.S. Wales, 8: 485.

I9I4, Exotic Microlepidoptera, I: 208.

I906, Trans. Ent. Soc. London, I906: I82. (BM)

I909, Journ. Bombay Nat. Hist. Soc. I9: 6oI. (BM)

I9I8, Exotic Microlepidoptera, 2: 108.

Igo6, Journ. Bombay Nat. Hist. Soc. I 7: 403. (BM)

I9I5, Exotic Microlepidoptera, I: 320.

I938, Trans. R. Ent. Soc. London, 87: 521.

I88I, Proc. Linnean Soc. N.S. Wales, 5: 244.

I908, Journ. Bombay Nat. Hist. Soc. I8: 830.

I9I3, Journ. Bombay Nat. Hist. Soc. 22: I69. (BM)

I884, Proc. Linnean Soc. N.S. Wales, 9: 779.

I885, Proc. Linnean Soc. N.S. Wales, 9: Io66.

1897, Proc. Linnean Soc. N.S. Wales, 22: 412.

1893, Proc. Linnean Soc. N.S. Wales, 7: 539.

I9I I, Proc. Linnean Soc. N.S. Wales, 36: 260.

I904, Proc. Linnean Soc. N.S. Wales, 29: 272.

I 897, Proc. Linnean Soc. N.S. Wales, 22: 393.

I902, Trans. Ent. Soc. London, I902: 574.

I88I, Proc. Linnean Soc. N.S. Wales, 5:24I.

I929, Exotic Microlepidoptera, 3: 48I. (BM)

I904, Proc. Linnean Soc. N.S. Wales, 29: 352.

I9I3, Trans. Ent. Soc. London, I9I3: I86. (BM)

I9I3, Ann. Transvaal Mus. 3:274. (T)

I9I4, Journ. Bombay Nat. Hist. Soc. 22: 780. (BM)

I9o9, Journ. Bombay Nat. Hist. Soc. I9: 4I9.

I920, Ann. South African Mus. I7: 274. (SA)

I927, Exotic Microlepidoptera, 3: 354. (T)

IgI4, Trans. Ent. Soc. London, I9I4: 27I. (BM)

I922, Exotic Microlepidoptera, 2: 520. (BM)

I9I8, Exotic Microlepidoptera, 2: I32. (BM)

I9o9, Ann. Transvaal Mus. 2: 27, pl. 8, fig. 7. (T)

I934, Exotic Microlepidoptera, 4:5I6. (c)

I88I, Proc. Linnean Soc. N.S. Wales, 5: I42.

1920, Exotic Microlepidoptera, 2: 305. (BM)

I897, Proc. Linnean Soc. N.S. Wales, 22: 424.

I893, Proc. Linnean Soc. N.S. Wales, 7: 496.

I887, Trans. Ent. Soc. London, I887: 266.

I9I8, Exotic Microlepidoptera, 2: I6I. (BM)

I9I5, Exotic Microlepidoptera, I: 472. (BM)

I922, Exotic Microlepidoptera, 2: 54I. (ВM)

I934, Pacific Ent. Surv. Publ. 7, art. 28, p. 349. (вB)

I925, Exotic Microlepidopiera, 3: 200. (BM)

I9I5, Exotic Microlepidoptera, I: 443. (BM)

I883, Proc. Linnean Soc. N.S. Wales, 7:540. (MA)

I925, Exotic Microlepidoptera, 3: 220. (BM) 


$\begin{array}{ll}\text { adulatrix } & \text { STATHMOPODA } \\ \text { adversa } & \text { CTENOCOMPA } \\ \text { advocata } & \text { STENOMA } \\ \text { adytodes } & \text { STENOMA } \\ \text { aechmobola } & \text { LECITHOCERA }\end{array}$

$\begin{array}{ll}\text { aedifica } & \text { MELASINA } \\ \text { aedificata } & \text { ANACAMPSIS } \\ \text { aedilis } & \text { CNEPHASIA } \\ \text { aedilis } & \text { PROCHOLA } \\ \text { aegidia } & \text { ARGYROPLOCE } \\ \text { aegidopis } & \text { PHRIXOCRITA* } \\ \text { aegotricha } & \text { STATHMOPODA } \\ \text { aegra } & \text { COLEOPHORA } \\ \text { aegrella } & \text { MACHAERITIS } \\ \text { aellomacha } & \text { GRACILARIA } \\ \text { aellophora } & \text { ERECHTHIA } \\ \text { aellotricha } & \text { PROTEROCOSMA } \\ \text { aelurodes } & \text { TINEA } \\ \text { aeluropa } & \text { CAPUA } \\ \text { aeluropis } & \text { IMMA } \\ \text { aemula } & \text { ACROCERCOPS } \\ \text { aemula } & \text { OPOGONA } \\ \text { aenictopa } & \text { SYNGENOMICTIS } \\ \text { aenigmatica } & \text { CNEPHASIA } \\ \text { aeolantha } & \text { ARGYROPLOCE } \\ \text { aeolastis } & \text { GRACILARIA } \\ \text { aeolellum } & \text { CORISCIUM } \\ \text { aeolias } & \text { PHILOBOTA } \\ \text { aeolocentra } & \text { GRACILARIA } \\ \text { aeolochlora } & \text { ARGYROPLOCE } \\ \text { aeolochorda } & \text { LABDIA } \\ \text { aeolocoma } & \text { TIMYRA } \\ \text { aeolodes } & \text { PLATYPTILIA } \\ \text { aeolodoxa } & \text { TORTYRA } \\ \text { aeologlypta } & \text { LASPEYRESIA } \\ \text { aeologramma } & \text { LASPEYRESIA } \\ \text { aeolopa } & \text { LOBESIA } \\ \text { aeolopis } & \text { MESOTHYRSA } \\ \text { aeolopis } & \text { PROTOLECHIA } \\ \text { aeolornis } & \text { LASPEYRESIA } \\ \text { aeolospila } & \text { GRACILARIA } \\ \text { aeolozona } & \text { EULIA } \\ \text { aequabilis } & \text { STENOMA } \\ \text { aequata } & \text { HOMILOSTOLA } \\ \text { aequilibris } & \text { DICHOMERIS } \\ \text { COMPOEOLACHIA }\end{array}$

I9I 7, Exotic Microlepidoptera, 2: 6I.

I9I9, Exotic Microlepidoptera, 2: 264.

I9I6, Exotic Microlepidoptera, I: 525. (Bм)

1925, Exotic Microlepidoptera, 3: I96. (BM)

I935, in Caradja and Meyrick, Materialien $z u$ einer

Microlepidopteren Fauna der Chinesischen Provinzen

Kiangsu, Chekiang and Hunan, p. 75. (вм)

Ig08, Proc. Zool. Soc. London, p. 744.

I929, Exotic Microlepidoptera, 3: 507. (BM)

IgIo, Proc. Linnean Soc. N.S. Wales, 35:276. (ма)

I915, Exotic Microlepidoptera, I : 33г. (вм)

I932, Trans. Ent. Soc. London, 80: I I2. (BM)

1935, Exotic Microlepidoptera, 4: 562. (вм)

I92I, Ann. Transvaal Mus. 8: I09. (T)

I9I7, Exotic Microlepidoptera, 2: 7I.

I886, Proc. Linnean Soc. N.S. Wales, го: 772.

I88I, Proc. Linnean Soc. N.S. Wales, 5: I58.

I88 I, Proc. Linnean Soc. N.S. Wales, 5: 270.

I889, Trans. Proc. New Zealand Inst. 2I: I75.

I893, Proc. Linnean Soc. N.S. Wales, 7: 545.

I926, Sarawak Museum Journ. 3: I47.

I906, Trans. Ent. Soc. London, p. I98. (вм)

I916, Exotic Microlepidoptera, I: 628.

I9I5, Exotic Microlepidoptera, I: 358 .

I927, Insects of Samoa, 3, Lepidoptera, fasc. 2, p. 78. (BM)

I9I2, Trans. Ent. Soc. London, I9I I: 682. (BM)

I9I4, Journ. Bombay Nat. Hist. Soc. 22: 77I. (BM)

I920, Exotic Microlepidoptera, 2: 298.

I88I, Proc. Linnean Soc. N.S. Wales, 5: 167.

I889, Proc. Linnean Soc. N.S. Wales, (Ser. 2) 3: I6r2.

1922, Exotic Microlepidoptera, 2: 565.

I9I6, Exotic Microlepidoptera, I: 562. (вм)

I927, Insects of Samoa, 3, Lepidoptera, fasc. 2, p. 85.

I939, Trans. R. Ent. Soc. London, 89: 56.

I902, Trans. Ent. Soc. London, I902: 278.

I928, Exotic Microlepidoptera, 3: 423. (вм)

1936, Exotic Microlepidoptera, 4: 6I5.

I9I6, Exotic Microlepidoptera, 2: 2I. (BM)

1907, Journ. Bombay Nat. Hist. Soc. I7: 976. (вм)

I9I0, Journ. Bombay Nat. Hist. Soc. 20: I62. (BM)

I904, Proc. Linnean Soc. N.S. Wales, 29: 369.

I930, Exotic Microlepidoptera, 3: 605. (BM)

I938, in Caradja and Meyrick, Deuts. Ent. Zeit. Iris;

$52: 2 \mathrm{I}$.

1926, Exotic Microlepidoptera, 3: 252. (вм)

I9I6, Exotic Microlepidoptera, I: 5I3. (вМ)

I917, Exotic Microlepidoptera, 2: 93. (BM)

I9I4, Trans. Ent. Soc. London, I9I4: 282. (BM)

I93I, Exotic Microlepidoptera, 4: I49. (v)

I93I, Exotic Microlepidoptera, 4:65. 
aequivoca

aequorea

aequorea

aeraria

aeraria

aeria

aericincta

aerifera

aerifica

aeriventris

aerobatica

aerobatis

aerodana

aerodes

aeruginea

aeruginosa

aesthetica

aestuans

aestuaria

aestuosa

aestuosa

aestuosa

aethalea

aethalea

aethaliana

aethalopa

aethalopis

aethalota

aetheria

aetherias

aethiopica

aethocoma

aethopa

aethopis

aethoptera

aethostola

aetodes

aetopis

affabilis

affirmata

affluens

affusa

agaclita

agalmatopa

agana

aganactes
BLASTOBASIS

ENARMONIA

RECURVARIA

ARGYROPLOCE

CRYPTOLECHIA

NOTODRYAS*

AEGERIA

GLYPHIPTERYX

NEPTICULA

NEPTICULA

CACOECIA

GELECHIA

TORTRIX

EULECHRIA

COPROMORPHA

AGRIOTHERA

PERSICOPTILA

PLATYSCEPTRA*

HOMALOPSYCHA*

HOMALOXESTIS

[CYMATOPLEX*]

PLATYPTILIA

SPILONOTA

MELASINA

NEPHOGENES

HENDECASTICHA*

PHILAMETRIS*

OINOPHILA

GRACILARIA

AEOLOSCELIS

ORINYMPHA*

TELOSPHRANTIS

EPICHORISTA

PROTONOSTOMA*

OCYSTOLA

CRYPTOPHASA

MACHIMIA

CROSSOPHORA

PHILOBOTA

HAPSIFERA

PARECTOPA

ARGYROPLOCE

LECITHOCERA

EPIPYRGA*

CHOREUTIS

CLERARCHA*

CHELOPHOBA*
I922, Exotic Microlepidoptera, 2: 540. (BM)

I9I2, Ent. Mon. Mag. 48: 34. (Sub.)

I9I7, Trans. Ent. Soc. London, I9I7: 39. (BM)

I9o9, Journ. Bombay Nat. Hist. Soc. I9: 605. (Вм)

I9I0, Journ. Bombay Nat. Hist. Soc. 20: I63. (BM)

I 897, Proc. Linnean Soc. N.S. Wales, 22: 427.

I928, Exotic Microlepidoptera, 3: 466. (BM)

I9I2, Exotic Microlepidoptera, I: 57.

I9I5, Trans. Ent. Soc. London, I9I5: 255.

I932, Exotic Microlepidoptera, 4:3I2.

I9I7, Trans. Ent. Soc. London, I9I7: 7. (BM)

I924, Trans. Proc. New Zealand Inst. 55: 204.

I88I, Proc. Linnean Soc. N.S. Wales, 6: 520.

I884, Proc. Linnean Soc. N.S. Wales, 8: 321.

I9I7, Ann. South African Mus. I7: 9. (SA)

I9I3, Exotic Microlepidoptera, I: I55.

I927, Insects of Samoa, 3, Lepidoptera, fasc. 2, p. 9I.

I9I6, Exotic Microlepidoptera, I: 605.

1920, Ann. South African Mus. I7: 304. (SA)

I9I3, Ann. Transvaal Mus. 3: 295. (T)

I9I6, Exotic Microlepidoptera, I: 558.

I9I2, Journ. Bombay Nat. Hist. Soc. 2I : 854. (BM)

I907, Journ. Bombay Nat. Hist. Soc. I8: I59.

I883, Proc. Linnean Soc. N.S. Wales, 8:380.

I88I, Proc. Linnean Soc. N.S. Wales, 6: 692.

I924, Exotic Microlepidoptera, 3: I02. (T)

I930, Trans. Ent. Soc. London, 78: 32I. (P)

I881, Proc. Linnean Soc. N.S. Wales, 5: I43.

I897, Proc. Linnean Soc. N.S. Wales, 22: 327.

I927, Exotic Microlepidoptera, 3: 361. (BM)

I932, Trans. Ent. Soc. London, 80: I66. (BM)

I923, Bull. Mus. Nat. Hist. Nat. Paris, 29:563.

I9Io, Journ. Bombay Nat. Hist. Soc. 20: I67. (BM)

I902, Trans. R. Soc. S. Australia, 26: I36.

I938, Trans. R. Ent. Soc. London, 87: 5I6.

I93I, Exotic Microlepidoptera, 4: I I9. (v)

I889, Proc. Linnean Soc. N.S. Wales, 3: I673.

I889, Proc. Linnean Soc. N.S. Wales, 3: 1627.

I93I, in Joannis, Ann. Soc. Ent. France, 98 (Sup.): 745

[507]. (P)

I9I8, Exotic Microlepidoptera, 2: I78.

I921, Anu. Transvaal Mus. 8: 56. (T)

I923, Exotic Microlepidoptera, 3: 40. (BM)

I884, Proc. Linnean Soc. N.S. Wales, 9: 79 I.

I926, Exotic Microlepidoptera, 3: 305. (BM)

I89o, Trans. R. Soc. S. Australia, I3: 54.

I935, in Caradja and Meyrick, Materialien zu einer Microlepidopteren Fauna der Chinesischen Provinzen Kiangsu, Chekiang und Hunan, p. 72. (BM) 


\begin{tabular}{|c|c|c|}
\hline aganocarpa & PHTHORIMAEA & I935, Exotic Microlepidoptera, 4: 585. (BM) \\
\hline aganodes & EUCosma & I922, Exotic Microlepidoptera, 2: 5I5. (P) \\
\hline aganopa & MENDESIA & I9I I, Ann. Transvaal Mus. 2: 234. (T) \\
\hline aganophthalma & PHTHORIMAEA & I93I, Exotic Microlepidoptera, 4:6I. (BM) \\
\hline anopis & $\begin{array}{l}\text { PHAEOSACES } \\
\text { [PROSAROTRA*] }\end{array}$ & I905, Journ. Bombay Nat. Hist. Soc. I6: 605. (Bм) \\
\hline gatha & ARISTOTELIA & I9I8, Exotic Microlepidoptera, 2: II9. (BM) \\
\hline agatharcha & EULIA & I926, Exotic Microlepidoptera, 3: 253. (BM) \\
\hline agathelpis & STENOMA & I932, Exotic Microlepidoptera, 4: 303. (v) \\
\hline agathoclea & HOMOPLASTIS* & I926, Sarawak Mus. Journ. 3: I62. \\
\hline agathopa & DICHOMERIS & I92I, Ann. Transvaal Mus. 8: 85. (T) \\
\hline agathopis & ARGYROPLOCE & I927, Insects of Samoa, 3 , Lepidoptera, fasc. 2, p. 76. (вм) \\
\hline aura & BATRACHEDRA & I902, Trans. Ent. Soc. London, I902: 579. \\
\hline relaea & OCYSTOLA & I885, Proc. Linnean Soc. N.S. Wales, 9: ro7o. \\
\hline ggerata & MERIDARCHIS & I9Io, Trans. Ent. Soc. London, p. 430. \\
\hline aggerata & STENOMA & I9I5, Exotic Microlepidoptera, I: 428. (BM) \\
\hline aggesta & CRYPTOPHASA & I925, Exotic Microlepidoptera, 3: I47. \\
\hline agglomerata & MACHIMIA & I920, Exotic Microlepidoptera, 2: 375. \\
\hline glutinata & HOMALOPSYCHA & I93I, Exotic Microlepidoptera, 4: 96. \\
\hline aggravata & STENOMA & I9I5, Exotic Microlepidoptera, I: 5I4. (BM) \\
\hline aggregata & STENOMA & I9I6, Exotic Microlepidoptera, I: 527. (Bм) \\
\hline agilis & BUCCULATRIX & I920, Ann. South African Mus. I7: 301. (SA) \\
\hline agitata & PROSCHISTIS & I909, Journ. Bombay Nat. Hist. Soc. I9: 589. (Bм) \\
\hline agitata & STRUTHISCA & I9I3, Ann. Transvaal Mus. 3: 333. (T) \\
\hline aglaocarpa & ZACORISCA & I924, Exotic Microlepidoptera, 3: II3. \\
\hline aglaocrossa & PTILOTHYRIS & I935, Exotic Microlepidoptera, 4: 564. (c) \\
\hline aglaodesma & COSMOCLOSTIS* & I886, Trans. Ent. Soc. London, I886: I2. \\
\hline aglaodora & PROPHYLACTIS & I897, Proc. Linnean Soc. N.S. Wales, 22: 408. \\
\hline aglaogramma & TRICLONELLA & I93I, Exotic Microlepidoptera, 4: II7. (v) \\
\hline aglaograpta & IRINYMPHA & I932, Exotic Microlepidoptera, 4: 275. \\
\hline aglaopa & STAGMATOPHORA & I928, Exotic Microlepidoptera, 3: 389. \\
\hline aglaophanes & RHABDOCOSMA & I935, Exotic Microlepidoptera, 4: 604 . \\
\hline aglaospila & NEMOTOIS & I928, Exotic Microlepidoptera, 3: 463. (Р) \\
\hline aglaoxantha & SPATALISTIS & I924, Exotic Microlepidoptera, 3: II6. (BM) \\
\hline aglaozona & LITHOCOLLETIS & I883, Proc. Linnean Soc. N.S. Wales, 7: I99. \\
\hline aglypta & ANTAEOTRICHA & I925, Exotic Microlepidoptera, 3: I74. (Вм) \\
\hline agoraea & PSEUDODOXIA & I9Io, Journ. Bombay Nat. Hist. Soc. 20: I52. (вм) \\
\hline rastis & SARISOPHORA & I93I, Exotic Microlepidoptera, 4:78. (BM) \\
\hline agraphopis & CRITOXENA & I930, Exotic Microlepidoptera, 3: 624. (BM) \\
\hline agraria & STENOMA & I925, Exotic Microlepidoptera, 3:210. (BM) \\
\hline agraula & ODITES & I908, Journ. Bombay Nat. Hist. Soc. I8: 632. (Bм) \\
\hline estis & AUXIMOBASIS & I922, Exotic Microlepidoptera, 2: 540. (BM) \\
\hline agria & MELASINA & I9o9, Ann. Transvaal Mus. 2: 27, pl. 8, fig. 8. (T) \\
\hline agricolaris & GELECHIA & I933, Exotic Microlepidoptera, 4: 448. (BM) \\
\hline agriochlora & EUCOSMA & I929, Trans. Ent. Soc. London, $76: 495$. (BM) \\
\hline agriodes & ELINOSTOLA & I921, Exotic Microlepidoptera, 2: 472. \\
\hline iodes & GELECHIA & I927, Exotic Microlepidoptera, 3: 350. (BM) \\
\hline ogramma & CHELARIA & I926, Sarawak Mus. Journ. 3: I53. \\
\hline oma & PERONEA & I920, Exotic Microlepidoptera, 2: 342. (BM) \\
\hline iopa & ENDOPHTHORA & I888, Trans. Proc. Nere Zealand Inst. 20: 95. \\
\hline
\end{tabular}


agrioschista

agrippina

agroeca

agronoma

agrypha

agypsota

agyrtodes

alacris

alaopis

alaudana

albata

albescens

albicaudis

albicellata

albicincta

albidorsis

albiflora

albiflua

albifrenis

albifusa

albinea

albipalpis

albipennis

albiramis

albiscripta

albisignis

albitergis

albitincta

alcaea

alcestis

alcimacha

alcmaeonis

alcyonis

alcyonopa

aleatoria

aleatrix

alectryonura

aletis

aletodes

aletreuta

aleuritis

aleurodes

aleuropis

aleurota

alexandra

alexandrina

algosa

algosa

alicia

aliena
STENOMA

ANTICRATES

TORTRIX

CASMARA

TORTRIX

PROCHOLA

IDIOPTILA*

SYMMOCA

STOMOPTERYX

EPITYMBIA*

STATHMOPODA

TORTRIX .

COSMOPTERYX

SCAEOSOPHA

BRACHMIA

STATHMOPODA

GELECHIA

SCYTHRIS

TROCHASTICA*

LAMPRONIA

PSEUDODOXIA

ARGY ROPLOCE

ACROLOPHUS

APATETRIS

HYPELICTIS

LECITHOCERA

ASCALENIA

STENOMA

EPACTRIS

LECITHOCERA

POLYHYMNO

CACOECIA

STILBOSIS

IRIANASSA

POLYDRACHMA*

ATTEVA

ELEGIA

HYPERCALLIA

PSEUDODOXIA

PARECTOPA

ACROLEPIA

ANARSIA

SETIARCHA*

DISSELIA*

GONIONOTA

PLEUROTA

SPILONOTA

STENOMA

NEPTICULA

TRIPTOLOGA
I927, Exotic Microlepidoptera, 3:365. (BM)

I93o, Exotic Microlepidoptera, 3: 594. (BM)

I908, Proc. Zool. Soc. London, p. 723. (BM)

I93I, in Caradja, Bull. Sect. sci. Acad. roum. I4: 7 I (вм)

I9Io, Proc. Linnean Soc. N.S. Wales, 35:247.

I922, Exotic Microlepidoptera, 2: 580. (BM)

I927, Exotic Microlepidoptera, 3: 344. (вм)

I9I8, Exotic Microlepidoptera, 2: I56. (BM)

I921, Ann. Transvaal Mus. 8: 76. (T)

I88I, Proc. Linnean Soc. N.S. Wales, 6: 658.

I9I3, Exotic Microlepidoptera, I: 94.

I9I2, Exotic Microlepidoptera, I: 6. (T)

I932, Exotic Microlepidoptera, 4: 3I4. (v)

I93I, Exotic Microlepidoptera, 4: II8. (v)

I92I, Ann. Transvaal Mus. 8: 90. (v)

I93I, in Caradja, Bull. Sect. sci. Acad. roum. I4: 75 .

I920, Ann. South African Mus. I7: 283 (SA)

I928, Bull. Hill Mus. 2: 237. (P)

I9I3, Ann. Transvaal Mus. 3: 32I. (T)

I926, Ann. South African Mus. 23: 349. (SA)

I9I4, Exotic Microlepidoptera, I: 266. (BM)

I93I, Exotic Microlepidoptera, 4: I4O. (BM)

I93I, Exotic Microlepidoptera, 4: I03. (v)

I923, Exotic Microlepidoptera, 3: 5I.

I9I4, Journ. Bombay Nat. Hist. Soc. 22: 773. (Bм)

I9I4, Supplementa Entomologica, No. 3, p. 50. (D)

I926, Ann. South African Mus. 23: 335. (SA)

I930, Exotic Microlepidoptera, 4: 28. (v)

I924, Exotic Microlepidoptera, 3: 72.

I923, Exotic Microlepidoptera, 3: 40. (BM)

I9I8, Exotic Microlepidoptera, 2: I29. (BM)

I928, Exotic Microlepidoptera, 3: 455. (BM)

I9I7, Exotic Microlepidoptera, 2: 49. (BM)

I926, Exotic Microlepidoptera, 3: 30I.

I928, Exotic Microlepidoptera, 3: 46I.

I922, Exotic Microlepidoptera, 2: 55I. (BM)

I932, Exotic Microlepidoptera, 4: 238.

I905, Trans. Ent. Soc. London, I905: 235.

I9I5, Exotic Microlepidoptera, I: 308. (BM)

I936, Exotic Microlepidoptera, 5: 38.

I9I3, Exotic Microlepidoptera, I: I50.

I922, Exotic Microlepidoptera, 2: 502. (BM)

I932, Exotic Microlepidoptera, 4: 328. (v)

I886, Proc. Linnean Soc. N.S. Wales, Io: 799 .

I909, Trans. Ent. Soc. London, I9o9: 26.

I936, Exotic Microlepidoptera, 5: 50.

I9I2, Journ. Bombay Nat. Hist. Soc. 2I: 854. (BM)

I9r6, Exotic Microlepidoptera, I: 537. (вM)

I928, Exotic Microlepidoptera, 3: 46I. (BM)

I922, Exotic Microlepidoptera, 2: 545. (BM) 
allactica

allactis

allactopa

allantopa

allevata

alliciens

allocoma

allogama

allomima

allomorpha

alloptila

allotria

allotriopa

alludens

alluvialis

alma

alma

alogista

alopecana

alopecistis

alopecodes

alopecopa

alphesta

alphestis

alphitella

alphitodes

alphitopa

alseis

alsocoma

altercata

alticola

altilis

altisona

altivaga

altivaga

altivagans

altivola

aluminias

alvearis

alveata

alysidota

amabilis

amalleuta

amalleuta

amalodes
STROPHALINGIAS*

STEGASTA

ACROCERCOPS

DICHOMERIS

HYALOCHNA*

CHOREUTIS

MICROBELA*

HARMOLOGA

GELECHIA

PANAPHELIX

TIMYRA

GELECHIA

LABDIA

DEMOBROTIS

STENOMA

AMORBAEA

MACHIMIA

DICHOMERIS

CACOECIA

EULECHRIA

DEUTEROCOPUS

HOMALOXESTIS

MYSTACERNIS*

ARGYROPLOCE

LEPIDOTARSA

CALYPTROTIS*

OMIOSTOLA*

HYPERCALLIA

STILBOSIS

ANARSIA

HYPOSMOCOMA

TINEA

PHTHORIMAEA

ACROCLITA

CARPOSINA

MONOPIS

EPITHECTIS

CAPUA

DEMOBROTIS

NEELYSIA

GRACILARIA

EUXANTHIS

ANARSIA

OLIGOPHLEBIA

CARPOSINA
I93I, Exotic Microlepidoptera, 4: 53.

I904, Proc. Linnean Soc. N.S. Wales, 29: 3 I4.

I916, Exotic Microlepidoptera, I: 627.

I934, Exotic Microlepidoptera, 4: 5I2. (BM)

I9I8, Ann. Transvaal Mus. 6: 3o. (T)

I926, Exotic Microlepidoptera, 3: 305. (BM)

1885, Proc. Linnean Soc. N.S. Wales, 9: 1047.

I9I4, Trans. Proc. New Zealand Inst. 46: I05.

1938, Institut des Parcs Nationaux du Congo Belge,

fasc. I4, p. I2. (c)

I922, Exotic Microlepidoptera, 2: 495. (P)

I9I6, Exotic Microlepidoptera, I: 570. (BM)

I925, in Wytsman, Genera Insectorum, fasc. I84, p. 8I. (Sub.)

1923, Exotic Microlepidoptera, 3: 58. (BM)

I9I9, Exotic Microlepidoptera, 2: 253.

I925, Exotic Microlepidoptera, 3: 204. (BM)

rgo8, Journ. Bombay Nat. Hist. Soc. I8: 627. (Bм)

I9I4, Exotic Microlepidoptera, I : I74. (MA)

I935, in Caradja and Meyrick, Materialien zu einer

Microlepidopteren Fauna der Chinesischen Provinzen

Kiangsu, Chekiang und Hunan, p. 72.

1885, Trans. Proc. New Zealand Inst. I7: I47.

I889, Proc. Linnean Soc. N.S. Wales, (Ser. 2) 3: I565.

I9I I, Journ. Bombay Nat. Hist. Soc. 21 : 105.

I929, Exotic Microlepidoptera, 3: 5I8. (BM)

I9I5, Exotic Microlepidoptera, I: 370.

I92I, Exotic Microlepidoptera, 2: 449.

I883, Proc. Linnean Soc. N.S. Wales, $7: 447$.

I89I, Ent. Mon. Mag. 27: 56. (BM)

I922, Exotic Microlepidoptera, 2: 5I9. (BM)

I906, Journ. Bombay Nat. Hist. Soc. I7: 406. (BM)

I9I7, Exotic Microlepidoptera, 2: 46. (BM)

I9I8, Exotic Microlepidoptera, 2: I48. (BM)

I9I5, Exotic Microlepidoptera, I: 343.

I893, Proc. Linnean Soc. N.S. Wales, $7: 533$.

I9I7, Trans. Ent. Soc. London, I9I7: 46. (BM)

I926, Sarawak Mus. Journ. 3: I5I.

1925, Exotic Microlepidoptera, 3: I37.

I938, Institut des Parcs Nationaux du Congo Belge,

fasc. I 4, p. 26. (c)

I929, Exotic Microlepidoptera, 3: 486. (BM)

I9I2, Trans. Ent. Soc. London, I9I I: 674. (BM)

I9I9, Exotic Microlepidoptera, 2: 254.

I9I5, Exotic Microlepidoptera, I : 339.

I88I, Proc. Linnean Soc. N.S. Wales, 5: r6I.

I93I, in Caradja, Bull. Sect. sci. Acad. roum. I4: 62. (BM)

I9I3, Ann. Transvaal Mus. 3: 298. (T)

I9I0, Rec. Indian Mus. 5: 219. (K)

I9I I, Trans. New Zealand Inst. 43: 6r. 


\begin{tabular}{|c|c|c|}
\hline amalodes & PHILOBOTA & I889, Proc. Linnean Soc. N.S. Wales, (Ser. 2) 3: г626. \\
\hline amalopa & MACAROSTOLA & I907, Proc. Linnean Soc. N.S. Wales, $32: 63$. \\
\hline amalopis & ORNEODES & I927, Insects of Samoa, 3, Lepidoptera, fasc. 2, p. 96. \\
\hline amalthea & HALOGENES* & I937, Exotic Microlepidoptera, 5: I 12. (Т) \\
\hline amanda & EUCOSMA & I9I2, Ent. Mon. Mag. 48: 34. (Sub.) \\
\hline amara & BUCCULATRIX & I9I3, Ann. Transvaal Mus. 3: 329. (T) \\
\hline amara & EUCOSMA & I9I3, Ann. Transvaal Mus. 3: 273. (T) \\
\hline amata & MACHIMIA & I9I4, Exotic Microlepidoptera, I: I75. (MA) \\
\hline amaura & EULECHRIA & I883, Proc. Linnean Soc. N.S. Wales, 7: 538. \\
\hline amaurodes & TINEA & I893, Proc. Linnean Soc. N.S. Wales, (Ser. 2) 7: 536. \\
\hline maurodoxa & PLEUROTA & I935, Exotic Microlepidoptera, 4: 593. (M) \\
\hline amauropa & ISONOMEUTIS* & I888, Trans. Proc. New Zealand Inst. 20: 75 . \\
\hline amauropis & CYMOTRICHA & I923, Exotic Microlepidoptera, 3: I. (BM) \\
\hline amaurota & ANACAMPSIS & I9I4, Trans. Ent. Soc. London, I9I4: 262. (BM) \\
\hline amazonica & COMPSOLECHIA & I9I8, Exotic Microlepidoptera, 2: I39. (BM). (Sub.) \\
\hline ambiens & STENOMA & I923, Exotic Microlepidoptera, 2: 6I5. (P) \\
\hline ambitiosa & ANARSIA & I9I3, Ann. Transvaal Mus. 3: 299. (T) \\
\hline nbitiosus & PTEROPHORUS & I9II, Ann. Transvaal Mus. 2: 220. (T) \\
\hline amblopa & LIMNOECIA & I9I5, Exotic Microlepidoptera, I: 3I9. \\
\hline amblopis & EBODA & I9I I, Trans. Linnean Soc. London, I4: 267 . (BM) \\
\hline amblopis & PROTOLECHIA & I904, Proc. Linnean Soc. N.S. Wales, 29: 368. \\
\hline nblycerella & GLYPHIPTERYX & I883, Proc. Linnean Soc. N.S. Wales, 7: I89. \\
\hline ablycryptis & SYMMOCA & I929, Exotic Microlepidoptera, 3: 5I6. (BM) \\
\hline nblydectis & PLATYPTILIA & I932, Trans. Ent. Soc. London, 80: 108. \\
\hline blyopa & DEPRESSARIA & I921, Exotic Microlepidoptera, 2: 392. (BM) \\
\hline amblyptera & HIEROMANTIS & I927, Insects of Samoa, 3, Lepidoptera, fasc. 2, p. 99. \\
\hline ablystoma & PTILOGENES & I936, Arb. morph. taxon. Ent. Berl. 3: I05. (D) \\
\hline iblyteles & BRACHYNEMATA & I889, Proc. Linnean Soc. N.S. Wales, (Ser. 2) 3: I662. \\
\hline ablyxena & OPOGONA & I920, Exotic Microlepidoptera, 2: 36o. (BM) \\
\hline ambrosia & EPICROESA* & I9o7, Proc. Linnean Soc. N.S. Wales, 32: 96. \\
\hline hbrosias & EPICURICA & I922, Arkiv för Zool. vol. I4, No. I5, p. 9. \\
\hline egarta & ANARSIA & I933, Exotic Microlepidoptera, 4: 36о. (BM) \\
\hline nenena & PELTOPHORA & I888, Trans. Proc. New Zealand Inst. 20: 78 . \\
\hline nentata & OPSODOCA & I9I9, Exotic Microlepidoptera, 2: 27I. (BM) \\
\hline ethystas & PERONEA & I9I2, Exotic Microlepidoptera, I: I6. (BM) \\
\hline nethystias & ZALITHIA & Igo6, Journ. Bombay Nat. Hist. Soc. I7: I40. (BM) \\
\hline methystina & DECTOBATHRA & I904, Proc. Linnean Soc. N.S. Wales, 29: 300. \\
\hline nethystodes & SIMAETHIS & I9I4, Supplementa Entomologica, No. 3, p. 57. (D) \\
\hline amethystopa & ACROCERCOPS & I9I6, Exotic Microlepidoptera, 2: 4 . \\
\hline metris & GELECHIA & I92I, Ann. Transvaal Mus. 8: 72. (T) \\
\hline mianta & PACHYRHABDA & I927, Insects of Samoa, 3, Lepidoptera, fasc. 2, p. IoI. \\
\hline ca & MELASINA & Igo8, Proc. Zool. Soc. London, p. 748. \\
\hline amina & TRICHOPHAGA & I925, Bull. Soc. R. Ent. Egypte, 9: 216. \\
\hline & DEPRESSARIA & $\begin{array}{l}\text { I93I, Ann. Mus. Nac. Hist. Nat. Buenos Aires, 36: } 393 . \\
\text { (BM) }\end{array}$ \\
\hline amodes & AROTROPHORA & IgIo, Proc. Linnean Soc. N.S. Wales, 35: 265. \\
\hline honias & PTEROPHORUS & I9o9, Ann. Transvaal Mus. 2: 3, pl. I, fig. 6. (T) \\
\hline lonura & CHELARIA & I921, Exotic Microlepidoptera, 2: 430. (BM) \\
\hline nopleura & CRYPTOLECHIA & I920, Ann. South African Mus. I7: 29o. (SA) \\
\hline noxanthus & YPSOLOPHUS & , Proc. Linnean Soc. N.S. Wales, 29: 430. \\
\hline
\end{tabular}




\begin{tabular}{|c|c|c|}
\hline amnesta & NEOCORODES* & I923, Exotic Microlepidoptera, 3: 36. (BM) \\
\hline amnopis & BORKHAUSENIA & I9Io, Trans. New Zealand Inst. 42: 65. \\
\hline amnopis & BORKHAUSENIA & I9I I, Trans. Nere Zealand Inst. 43: 65 (homonym). \\
\hline amoebaea & PHILOBOTA & I889, Proc. Linnean Soc. N.S. Wales, (Ser. 2) 3: I623. \\
\hline amorbas & TRACHYPEPLA & I9I I, Trans. New Zealand Inst. 43: 66. \\
\hline amorbas & ZIROSARIS* & I9Io, Trans. Nere Zealand Inst. 42: 66. \\
\hline amorpha & EREBOSCAEAS* & I937, Exotic Microlepidoptera, 5:93. (T) \\
\hline amorphopa & PHAULOGENES* & $\begin{array}{l}\text { I938, in Caradja and Meyrick, Deuts. Ent. Zeit. Iris, } \\
52: 27 \text {. }\end{array}$ \\
\hline ampelurga & AEOLANTHES & I925, Exotic Microlepidoptera, 3: I46. (BM) \\
\hline ampherista & ANTAEOTRICHA & I925, Exotic Microlepidoptera, 3: I63. (BM) \\
\hline amphiactis & CRIOCHARACTA* & I939, Trans. R. Ent. Soc. London, 89:62. (T) \\
\hline iarma & DALACA & I926, Ann. South African Mus. 23: 350. (BM) \\
\hline amphibaphes & DECADARCHIS & I939, Trans. R. Ent. Soc. London, 89:6I. (c) \\
\hline nphicalyx & PARADORIS & IgII, Journ. Bombay Nat. Hist. Soc. 20:735. (BM) \\
\hline icarpa & CANTHONISTIS* & I922, Zool. Meded. $7: 82$. (L) \\
\hline nphicausta & OPOGONA & Igo7, Journ. Bombay Nat. Hist. Soc. I7: $75^{\circ}$. \\
\hline centra & EPISTOMOTIS & I9I3, Ann. Transvaal Mus. 3: 326. (T) \\
\hline chelota & ORYGOCERA & I930, Exotic Microlepidoptera, 3: 622. (BM) \\
\hline ichlora & TRICHOTAPHE & I923, Exotic Microlepidoptera, 3:4. (BM) \\
\hline mphichorda & OPOGONA & I921, Ann. Transvaal Mus. 8: I25. (т) \\
\hline chroa & CEROMITIA & I9o8, Proc. Zool. Soc. London, p. 755. \\
\hline chroma & TELPHUSA & I9I3, Ann. Transvaal Mus. 3:286. (T) \\
\hline nphichrysa & TRICHOMOERIS* & I9I3, Exotic Microlepidoptera, I: I56. \\
\hline icoma & DICHOMERIS & I912, Trans. Ent. Soc. London, I9II: 695. (BM) \\
\hline copa & PROMALACTIS & I908, Journ. Bombay Nat. Hist. Soc. I 8: 808. (BM) \\
\hline hicosma & CYMOTRICHA & I930, Ann. Naturhist. Mus. Wien, 44: 228. (v) \\
\hline phicrena & COPTOTELIA & I9I2, Trans. Ent. Soc. London, I9II: 703. (BM) \\
\hline icroca & CRYPTOPHASA & I925, Exotic Microlepidoptera, 3: I5I. \\
\hline crossa & OINOPHILA & I92I, Ann. Transvaal Mus. 8: I24. (T) \\
\hline hidelta & GRACILARIA & I918, Exotic Microlepidoptera, 2: I79. \\
\hline lidoxa & HAPLODYTA & I889, Proc. Linnean Soc. N.S. Wales, (Ser. 2) 3: I667. \\
\hline hidyas & EULECHRIA & 1888, Proc. Linnean Soc. N.S. Wales, (Ser. 2) 2: 950. \\
\hline phidyma & STATHMOPODA & I913, Exotic Microlepidoptera, I: 88. \\
\hline lignosta & PHANEROCHERSA* & I926, Exotic Microlepidoptera, 3: 244. \\
\hline igona & HOMONA & 1936, Exotic Microlepidoptera, 5: 6o. (v) \\
\hline phigramma & CRYPTOLECHIA & I9I5, Exotic Microlepidoptera, I: 305. \\
\hline grapta & LECITHOCERA & I926, Sarawak Mus. Journ. 3: I59. \\
\hline ilecta & LASPEYRESIA & I925, Exotic Microlepidoptera, 3: I45. (BM) \\
\hline hileuca & TRACHYPEPLA & I9I4, Trans. Proc. New Zealand Inst. 46: I07. \\
\hline ileucota & TRIADOGONA* & I937, Exotic Microlepidoptera, 5: I53. \\
\hline ilocha & URODUS & I924, Exotic Microlepidoptera, 3: I28. (BM) \\
\hline hiloga & PLATYPTILIA & I909, Ann. South African Mus. 5: 365 . (SA) \\
\hline hiloxa & TRACHYCENTRA & I907, Proc. Linnean Soc. N.S. Wales, 32: I44. \\
\hline hilyca & COESYRA & I884, Proc. Linnean Soc. N.S. Wales, 9: 787 . \\
\hline hilychna & SEMATOPTIS* & I931, Ann. Mus. Nac. Hist. Nat. Buenos Aires, 36: 386. \\
\hline hilyta & ANT AEOTRICHA & I9I5, Exotic Microlepidoptera, I: 503. (BM) \\
\hline himetalla & NEMOTOIS & I9I2, in Wytsman, Genera Insectorum, fasc. I33, p. 7. \\
\hline imitra & OPOSTEGA & I9I3, Ann. Transvaal Mus. 3:328. (T) \\
\hline uphimnesta & EUXANTHIS & I928, Exotic Microlepidoptera, 3: 436. (BM) \\
\hline
\end{tabular}


amphipeda amphiphracta amphiplaca amphiplecta amphiptera amphipterna amphiptila amphisaris amphiscia amphiscolia amphisticta amphiterma amphitoxa amphitricha amphitrite amphitrocta amphixantha amphixysta amphizela amphizeucta amphizeuctis amphizyga amphoritis amphorodes amplexa ampliata amplificata ampycota amydraula amydropa amydropis amydrota

amylodes amynias anacampta anacampta anavantha anachasta anachastopa anachorda anachoreta anaclastis anaclastis anaclina anaclina anaclintris anadesma anadoxa anaema
GLYPHIPTERYX

ENTRICHIRIA*

PROTOLECHIA

DOLEROTHERA*

STENOMA

PYRODERCES

PROSELOTIS

PYRODERCES

LEPIDOSCIA

BATTARISTIS

BRACHMIA

MACROSACES

PELTOPHORA

EPIPHRACTIS

TINEA

ANTIGAMBRA*

IMMA

THIOTRICHA

STRUTHISCA

LACHNOSTOLA*

STATHMOPODA

ANTAEOTRICHA

STATHMOPODA

ACRIA

GELECHIA

DICHOMERIS

PARALECTA

HOLAXYRA*

BATRACHEDRA

PHYLLOCNISTIS

SAROPLA

CTENOCOMPA

COESYRA

SCIOMYSTIS*

COESYRA

MACROBATHRA

EPERMENIA

TRICLONELLA

ACROCLITA

PHILOBOTA

HIEROXESTIS

GLYPHIPTERYX

PYRODERCES

ARGYROPLOCE

OPOGONA

ANTAEOTRICHA

LINOSTICHA

COSMOPTERYX

OECOPHORA
I920, Ann. South African Mus. I7: 295. (SA)

I921, Zool. Meded. 6: I87. (L)

I932, Exotic Microlepidoptera, 4: 352. (cU)

I9I8, Exotic Microlepidoptera, 2 : I86.

I9I3, Trans. Ent. Soc. London, I9I3: I85. (BM)

I9I7, Exotic Microlepidoptera, 2: 36 .

I92I, Exotic Microlepidoptera, 2: 425. (BM)

I922, Exotic Microlepidoptera, 2: 572.

I893, Proc. Linnean Soc. N.S. Wales, (Ser. 2) 7: 5 I3.

I9I4, Trans. Ent. Soc. London, I9I4: 248. (BM)

I9I4, Exotic Microlepidoptera, I : 279. (BM)

I9Io, Journ. Bombay Nat. Hist. Soc. 20: I46. (BM)

I889, Proc. Linnean Soc. N.S. Wales, 3: I640.

I9Io, Trans. Ent. Soc. London, I9Io: 373.

I932, Exotic Microlepidoptera, 4: 209.

I927, Exotic Microlepidoptera, 3:322. (T)

I9o6, Trans. Ent. Soc. London, I906: I93. (BM)

I929, Exotic Microlepidoptera, 3: 500. (BM)

I9I6, Exotic Microlepidoptera, I: 608.

I9I8, Ann. Transvaal Mus. 6:22. (T)

I934, Exotic Microlepidoptera, 4: $52 \mathrm{I}$.

I930, Ann. Naturhist. Mus. Wien, 44: 234. (v)

I9I3, Exotic Microlepidoptera, I: 9I.

I923, Exotic Microlepidoptera, 2: 6I2. (BM)

I925, in Caradja, Mem. Sect. Sti. A cad. Românā, 3: 380.(Bu)

I913, Journ. Bombay Nat. Hist. Soc. 22: I75. (BM)

I925, Exotic Microlepidoptera, 3: I53.

I913, Journ. Bombay Nat. Hist. Soc. 22: I76. (Bм)

I9I6, Exotic Microlepidoptera, 2: 30. (BM)

I934, Exotic Microlepidoptera, 4: 469.

I889, Proc. Linnean Soc. N.S. Wales, 3: I648.

I920, Voyage de Ch. Alluaud et R. Jeannel en Afrique

Oriental, II, Microlepidoptera, p. IIO. (P)

I884, Proc. Linnean Soc. N.S. Wales, 9: 784 .

I9I9, Exotic Microlepidoptera, 2: 243.

I9I3, Exotic Microlepidoptera, I: II8.

I9I4, Exotic Microlepidoptera, I : 217.

I9I7, Exotic Microlepidoptera, 2: 69.

I928, Exotic Microlepidoptera, 3:467. (BM)

I934, Exotic Microlepidoptera, 4: 483. (BM)

I884, Proc. Linnean Soc. N.S. Wales, 8: 499.

I921, Ann. Transvaal Mus. 8: I25. (T)

I907, Proc. Limnean Soc. N.S. Wales, 32: 130.

I897, Proc. Linnean Soc. N.S. Wales, 22: 348.

I92I, Ann. Transvaal Mus. 8: 58 . (T)

I9I5, Exot:c Microlepidoptera, I: $35^{8}$.

I9I6, Exotic Microlepidoptera, I: 499. (BM)

I889, Proc. Linnean Soc. N.S. Wales, (Ser. 2) 3: 1579.

I9o9, Journ. Bombay Nat. Hist. Soc. I9: 4I2.

I883, New Zealand Journ. Sci. I: 524. 


\begin{tabular}{|c|c|c|}
\hline anaemopa & ILYCHYTIS* & I937, Exotic Microlepidoptera, 5: I43. \\
\hline anaglypta & DEMOBROTIS* & I893, Proc. Linnean Soc. N.S. Wales, (Ser. 2) 7: 556. \\
\hline anagramma & GELECHIA & I92I, Ann. Transvaal Mus. 8: 72. (T) \\
\hline anagrapta & SYNTOMACTIS & I897, Proc. Linnean Soc. N.S. Wales, 22: 382. \\
\hline analoga & SIMAETHIS & I9I2, Trans. Proc. New Zealand Inst. 44: I22. \\
\hline analogica & ODITES & I9I7, Exotic Microlepidoptera, 2: 57. (вм) \\
\hline analoxa & SCHEMATISTIS* & I9I2, Ann. Transvaal Mus. 3: 68. (T) \\
\hline analytica & TINEA & I9I9, Exotic Microlepidoptera, 2: 248. (BM) \\
\hline anamnestis & LASPEYRESIA & I928, Exotic Microlepidoptera, 3:450. \\
\hline anamochla & PTILOGENES & I929, Trans. Ent. Soc. London, 76: 5I7. (Bм) \\
\hline ananeura & PROTOLECHIA & I904, Proc. Linnean Soc. N.S. Wales, 29: 374 . \\
\hline nantia & HELIOZELA & I897, Proc. Linnean Soc. N.S. Wales, 22: 405. \\
\hline anaphanta & STENOMA & I925, Exotic Microlepidoptera, 3: 180. (вм) \\
\hline anaphracta & PARADORIS* & I907, Journ. Bombay Nat. Hist. Soc. I7: 740. (BM) \\
\hline anaphrictis & HOMOSETIA & I9I9, Exotic Microlepidoptera, 2: 25I. \\
\hline lecta & ARGYROPLOCE & I9o9, Journ. Bombay Nat. Hist. Soc. I9: 598. (вм) \\
\hline anaptis & AROTROPHORA & I9Io, Proc. Linnean Soc. N.S. Wales, 35:269. \\
\hline anarcha & LINOSTICHA & I889, Proc. Linnean Soc. N.S. Wales, (Ser. 2) 3: I584. \\
\hline anarithma & PROTEROCOSMA & I889, Trans. Proc. New Zealand Inst. 2I : I75. \\
\hline astis & THIOTRICHA & I927, Insects of Samoa, 3, Lepidoptera, fasc. 2, p. 8I. (BM) \\
\hline anarrecta & PHILOBOTA & I889, Proc. Linnean Soc. N.S. Wales, (Ser. 2) 3: г6zo. \\
\hline anasactis & MELASINA & I907, Journ. Bombay Nat. Hist. Soc. I8: I 58. \\
\hline sa & PILOPREPES & I889, Proc. Linnean Soc. N.S. Wales, (Ser. 2) 3: I 597. \\
\hline anasticta & ODITES & I930, Exotic Microlepidoptera, 4: I8. (P) \\
\hline anastrella & TRACHYPEPLA & I883, New Zealand Journ. Sci. I : 523. \\
\hline trepta & CRUSIMETRA & I927, Insects of Samoa, 3, Lepidoptera, fasc. 2, p. 7 I. \\
\hline rota & PICROSPORA & I9I2, Ann. South African Mus. Iо: 70. (SA) \\
\hline nathyrsa & ACROLOPHUS & I9I9, Exotic Microlepidoptera, 2: 278. (BM) \\
\hline resta & STENOMA & I9I5, Exotic Microlepidoptera, I: 452. (BM) \\
\hline nazancla & PHILOBOTA & I889, Proc. Linnean Soc. N.S. Wales, (Ser. 2) 3: I6r7. \\
\hline nazona & NEPTICULA & I9o6, Trans. R. Soc. S. Australia, 30: 58 . \\
\hline ncalodes & COSMOPTERYX & I9I9, Exotic Microlepidoptera, $2: 283$. \\
\hline lopa & ORNEODES & I92I, Exotic Microlepidoptera, 2: 409. \\
\hline ncalota & AGRIOPHARA & I9I6, Exotic Microlepidoptera, I: 486. (BM) \\
\hline ncalota & ENARMONIA & I907, Journ. Bombay Nat. Hist. Soc. I8: I40. (BM) \\
\hline ps & POLYCHROSIS & I9o9, Journ. Bombay Nat. Hist. Soc. I9: 588 . (BM) \\
\hline anchiala & GEPHYRISTIS* & I9o9, Ann. Transvaal Mus. 2: 27, pl. 8, fig. 6. (T) \\
\hline cilla & LIMNOECIA & I934, Exotic Microlepidoptera, 4:508. \\
\hline ancillaris & STENOMA & I9I6, Exotic Microlepidoptera, I: 530. (вм) \\
\hline istraea & COSMOPTERYX & I9I3, Ann. Transvaal Mus. 3: 307. (T) \\
\hline ancistraula & PARECLECTIS* & I937, Exotic Microlepidoptera, 5: I44. (T) \\
\hline & $\begin{array}{l}\text { PTOCHORYCTIS } \\
\text { [METATHRINCA*] }\end{array}$ & I9o6, Journ. Bombay Nat. Hist. Soc. I7: 403. (BM) \\
\hline ancistropis & EPICEPHALA & I935, Exotic Microlepidoptera, 4: 597 . \\
\hline istrota & IMMA & I9I2, Exotic Microlepidoptera, I: 39. (BM) \\
\hline ancistrotis & SAROPLA & I889, Proc. Linnean Soc. N.S. Wales, (Ser. 2) 3: I 647. \\
\hline ancobathra & USSARA & I932, Exotic Microlepidoptera, 4: 275. (v) \\
\hline cogramma & BORKHAUSENIA & I9I9, Trans. Proc. New Zealand Inst. 51: 352. \\
\hline ancoloba & LASPEYRESIA & I922, Exotic Microlepidoptera, 2: 533. (BM) \\
\hline anconia & POLYCHROSIS & igri, Proc. Linnean Soc. N.S. Wales, 36: 257. \\
\hline
\end{tabular}




\begin{tabular}{|c|c|}
\hline anc & STATHMOPODA \\
\hline anconitis & STENOMA \\
\hline ancorata & ANCYLIS \\
\hline cosema & ARGYROPLOCE \\
\hline costyla & DECADARCHIS \\
\hline ancylacma & STENOMA \\
\hline cylogramma & HIEROMANTIS \\
\hline ncylopa & EPICEPHALA \\
\hline ancylota & TORODORA \\
\hline ancylotoxa & PHILOBOTA \\
\hline ncyristis & USSARA \\
\hline ancyropis & HOMOSETIA \\
\hline acyrota & EPIBLEMA \\
\hline cyrota & LOCHEUTIS \\
\hline adina & NEPTICULA \\
\hline nelaea & STENOMA \\
\hline nelicta & HYPERCALLIA \\
\hline nemarcha & MACROBATHRA \\
\hline lemodes & ARGYROPLOCE \\
\hline lemodes & MACROBATHRA \\
\hline anemois & OTONOMA* \\
\hline anemolia & ORNEODES \\
\hline hemonantha & PSEUDATTERIA \\
\hline anetodes & STENOMA \\
\hline aneuropa & IDIOMICTIS \\
\hline angareuta & THYLACOSCELES \\
\hline angarodes & SESIA \\
\hline angelica & ACROCERCOPS \\
\hline anguicula & PILOPREPES \\
\hline anguillana & PALAEOBIA \\
\hline anguillaris & PTOCHORYCTIS \\
\hline anguinea & CHELARIA \\
\hline angusta & AUXIMOBASIS \\
\hline animosa & MELASINA \\
\hline animosus & TRICHOPTILUS \\
\hline animula & CALICOTIS \\
\hline anisacta & OPOGONA \\
\hline anisastra & TRAPEZORITIS* \\
\hline anisaula & APATETRIS \\
\hline anisectis & GELECHIA \\
\hline anisocarpa & ODITES \\
\hline anisocentra & HOMADAULA \\
\hline anisochrysa & ATTEVA \\
\hline sodelta & EUCOSMA \\
\hline sodes & HYPERCALLIA \\
\hline sodora & MACROBATHRA \\
\hline sogramma & COMPSOLECHIA \\
\hline opa & ANACAMPSIS \\
\hline oplecta & HYPOSMOCOMA \\
\hline
\end{tabular}

I9Io, Rec. Indian Mus. 5: 223. (K)

I9I5, Exotic Microlepidoptera, I: 4I4. (BM)

I9I2, Journ. Bombay Nat. Hist. Soc. 21: 862. (BM)

I932, Exotic Microlepidoptera, 4: 3Іо. (вм)

I927, Insects of Samoa, 3, Lepidoptera, fasc. 2, p. I I4.

1925, Exotic Microlepidoptera, 3: 205. (BM)

1933, Exotic Microlepidoptera, 4: 430. (BM)

19I8, Exotic Microlepidoptera, 2: I73.

I894, Trans. Ent. Soc. London, I894: I7. (BM)

I884, Proc. Linnean Soc. N.S. Wales, 8: 475.

1920, Exotic Microlepidoptera, 2: 334. (BM)

I9I9, Exotic Microlepidoptera, 2: 25I. (BM)

I907, Journ. Bombay Nat. Hist. Soc. I7: 733. (вм)

I883, Proc. Linnean Soc. N.S. Wales, 8: 343.

I9I5, Trans. Ent. Soc. London, I9I 5: 255 .

I932, Exotic Microlepidoptera, 4: 305. (Us)

I926, Exotic Microlepidoptera, 3: 3I2. (BM)

I886, Proc. Linnean Soc. N.S. Wales, Io: 805.

I9I2, Journ. Bombay Nat. Hist. Soc. 21 : 873. (BM)

I886, Proc, Linnean Soc. N.S. Wales, Io: 8ig.

I897, Proc. Linnean Soc. N.S. Wales, 22: 358.

I929, Exotic Microlepidoptera, 3: 533. (BM)

I932, Exotic Microlepidoptera, 4: 254. (D)

I9I5, Exotic Microlepidoptera, I: 438. (BM)

I935, Exotic Microlepidoptera, 4: 572. (BM)

I922, Exotic Microlepidoptera, 2: 584 .

I92I, Exotic Microlepidoptera, $2: 443$.

I9I9, Exotic Microlepidoptera, 2: 230. (BM)

I9I3, Exotic Microlepidoptera, I: I33. (MA)

I88I, Proc. Linnean Soc. N.S. Wales, 6: 662.

I9I4, Journ. Bombay Nat. Hist. Soc. 22: 778. (BM)

I913, Journ. Bombay Nat. Hist. Soc. 22: I6I. (BM)

I922, Exotic Microlepidoptera, 2: 54I. (BM)

I9I3, Ann. Transvaal Mus. 3: 334.

I92I, Ann. Transvaal Mus. 8: 49. (T)

I9I I, Trans. Linnean Soc. London, I4: 285.

I920, Voyage de Ch. Alluaud et R. Jeannel en Afrique

Oriental, II, Microlepidoptera, p. 93. (P)

I932, Exotic Microlepidoptera, 4: 212.

I92I, Exotic Microlepidoptera, 2: 424.

I923, Exotic Microlepidoptera, 3: I9. (Sub.)

I930, Exotic Microlepidoptera, 4: I7. (BM)

I922, Ent. Mitteil. II : 47. (D)

I928, Exotic Microlepidoptera, 3: 4I5.

I923, Bull. Mus. Nat. Hist. Nat. Paris, 29:563.

I9I6, Exotic Microlepidoptera, I: 552. (BM)

I924, Exotic Microlepidoptera, 3: 98. (T)

I927, Exotic Microlepidoptera, 3: 353.

I9I8, Exotic Microlepidoptera, 2: I40. (вм)

I935, Proc. Hawaiian Ent. Soc. 9:67. (SP) 





anthracobathra SCHOENOTENES anthracodelta SCYTHRIS anthracographa HOLCOCERA anthraconesa TYROLIMNAS* anthracopa GELECHIA anthracopis PSECADIA anthracoptila EPERMENIA anthracorma DRYADAULA anthracosema SYMMOCA anthracosperma GRACILARIA anthracospora MACHIMIA anthracotis anthracura anthracuris antiarcha antiastis antibathra antibathra antibatis antibola anticentra anticentra anticeros anticharis antichlora antichorda antichroa antichroa antichroma

antichroma anticipans anticlina anticolona anticoma anticosma anticrates anticrossa anticyma anticyphas antidectis antidelta antidesma antidora antidoxa antidoxa antidroma antigama antigama antigona
LASPEYRESIA

ANACAMPSIS

ACROCERCOPS OPOGONA CHELARIA HOMALOXESTIS MOMPHA NARYCIA CTENOCOMPA LEPTOSACES THIOTRICHA IMMA STENOMA SIMAETHIS COSMOPTERYX BRACHMIA TORTRIX COERANICA

[TRACHYXYSTA*] EUCOSMA LASPEYRESIA PYROZELA BUCCULATRIX ORNEODES HYBROMA TRICHOTAPHE CRYPTOLECHIA STATHMOPODA EUXANTHIS EPICALLIMA STATHMOPODA LIMNOECIA EUCOSMA GLYPHIPTERYX PILOPREPES TINEA HERMOGENES TRICHOCEROTA TORTRIX
I938, Trans. R. Ent. Soc. London, 87:508. (BM)

I9II, Ann. Transvaal Mus. 3: 72.

1937, Exotic Microlepidoptera, 5: I45.

I934, Exotic Microlepidoptera, 4: 477. (BM)

I922, Exotic Microlepidoptera, 2: 50I. (P)

I902, Trans. R. Soc. S. Australia, 26: I65.

I93I, Exotic Microlepidoptera, 4: I6I.

I9I5, Exotic Microlepidoptera, I: 369. (MA)

I933, Exotic Microlepidoptera, 4: 359. (BM)

I93I, Exotic Microlepidoptera, 4: I7I.

I934, Exotic Microlepidoptera, 4: 476. (v)

I9I3, Ann. Transvaal Mus. 3: 279. (T)

I9I4, Trans. Ent. Soc. London, I9I4: 263. (BM)

I926, Exotic Microlepidoptera, 3: 295.

I934, Ann. Mag. Nat. Hist. I4: 4II.

I929, Exotic Microlepidoptera, 3: 5I4. (BM)

I9I6, Exotic Microlepidoptera, I: 575. (BM)

I9I4, Ann. Transvaal Mus. 4: I95. (T)

I926, Ann. South African Mus. 23: 348. (SA)

I9I9, Exotic Microlepidoptera, 2: 265.

I9I0, Journ. Bombay Nat. Hist. Soc. 20: I55. (BM)

I904, Proc. Linnean Soc. N.S. Wales, 29: 296.

I928, Exotic Microlepidoptera, 3: 422. (BM)

I9I6, Exotic Microlepidoptera, I: 5I2. (BM)

I9I2, Exotic Microlepidoptera, I: 44. (BM)

I909, Ann. South African Mus. 5: 357. (SA)

I9I8, Exotic Microlepidoptera, 2: I56. (BM)

I9I9, Trans. Proc. New Zealand Inst. 5I: 35I.

I902, Trans. R. Soc. S. Australia, 26: I37.

I912, Ent. Mon. Mag. 48: 35. (Sub.)

I927, Exotic Microlepidoptera, 3: 342. (BM)

I907, Journ. Bombay Nat. Hist. Soc. I7: 747. (BM)

I9I3, Ann. Transvaal Mus. 3: 329. (T)

I929, Exotic Microlepidoptera, 3: 536. (ВM)

I9I9, Exotic Microlepidoptera, 2: 24I. (BM)

I93I, Exotic Microlepidoptera, 4: 69. (v)

I9I5, Exotic Microlepidoptera, I: 304.

I927, Insects of Samoa, 3, Lepidoptera, fasc. 2, p. Ioo.

1936, Exotic Microlepidoptera, 5: 23.

I9I4, Exotic Microlepidoptera, I : 2I8. (вм)

I907, Journ. Bombay Nat. Hist. Soc. I7: 982 .

I9I3, Ann. Transwaal Mus. 3: 308. (T)

I92I, Ann. Transvaal Mus. 8: 53. (T)

I9o9, Journ. Bombay Nat. Hist. Soc. I9: 435. (вм)

I889, Proc. Linnean Soc. N.S. Wales, (Ser. 2) 3: I599.

I93I, Exotic Microlepidoptera, 4: 95.

I908, Journ. Bombay Nat. Hist. Soc. I8: 630. (BM)

I926, Exotic Microlepidoptera, 3: 270.

I93I, Exotic Microlepidoptera, 4: I50. 


\begin{tabular}{|c|c|}
\hline antigrapha & SIMAETHIS \\
\hline antileuca & THYMOSOPHA* \\
\hline antiloga & ULOCORYS* \\
\hline antiloxa & CYMOTRICHA \\
\hline antilyca & NEMOTOIS \\
\hline antilyra & STENOMA \\
\hline antimacha & CONOPOMORPHA \\
\hline antimeloda & MACROBATHRA \\
\hline antimetra & ISORRHOA* \\
\hline antimicras & DECADARCHIS \\
\hline antinoma & PACHYRHABDA \\
\hline antipala & ARISTOTELIA \\
\hline antipetra & ELACHISTA \\
\hline antiphanes & THISIZIMA \\
\hline antiphanopa & TYRICTACA* \\
\hline antiphila & CNEPHASIA \\
\hline antiphona & PLUTELLA \\
\hline antiphracta & TINEA \\
\hline antiphractis & LECITHOCERA \\
\hline antiplaca & COMPSOLECHIA \\
\hline antiptila & CHOREUTIS \\
\hline antipyla & PROTAPHREUTIS \\
\hline antipyramis & GELECHIA \\
\hline antiqua & ASCALENIA \\
\hline antiquata & IMMA \\
\hline antirrhoa & EUCOSMA \\
\hline antisaris & CHELARIA \\
\hline tischema & STATHMOPODA \\
\hline antisema & LECITHOCERA \\
\hline antisphena & PSEUDOCRATES * \\
\hline antistacta & OPOGONA \\
\hline antistatica & HYPONOMEUTA \\
\hline antisticha & DICHOMERIS \\
\hline antisticta & CYMOTRICHA \\
\hline antistola & PARALECTA \\
\hline antitacta & STENOMA \\
\hline ntitheta & LASPEYRESIA \\
\hline tithetis & SYNTOMACTIS \\
\hline titona & RHAPSODICA* \\
\hline antitoxa & LITHOCOLLETIS \\
\hline antitypa & FALCULINA \\
\hline antitypa & HARMOLOGA \\
\hline antizyga & DICHOMERIS \\
\hline ntricola & TINEA \\
\hline ntrifera & EUCOSMA \\
\hline
\end{tabular}

antygota EULECHRIA
I9I I, Trans. New Zealand Inst. 43: 76 .

I9I4, Ann. South African Mus. IO: 245. (SA)

I9I5, Exotic Microlepidoptera, I: 357.

I93I, in Caradja, Bull. Sect. sci. Acad. roum. I4: 68. (Bм)

I936, Exotic Microlepidoptera, 5: 42.

1925, Exotic Microlepidoptera, 3:2 I5. (BM)

I907, Proc. Linnean Soc. N.S. Wales, 32: 58.

I930, Exotic Microlepidoptera, 3: 6I4. (BM)

I9I3, Exotic Microlepidoptera, I : 79.

I907, Journ. Bombay Nat. Hist. Soc. I7: 75I.

I9Io, Trans. New Zealand Inst. 42: 72.

I904, Proc. Linnean Soc. N.S. Wales, 29: 290.

I922, Exotic Microlepidoptera, 2: 508.

I894, Trans. Ent. Soc. London, I894: 27.

1927, Exotic Microlepidoptera, 3: 372. (BM)

I9I3, Ent. Mitteil. 2: 298. (D)

I902, Trans. Ent. Soc. London, I902: 576.

I909, Ann. South African Mus. 5:362. (SA)

I92I, Exotic Microlepidoptera, 2: 435. (BM)

I922, Trans. Ent. Soc. London, I922: 92. (BM)

I9I2, Exotic Microlepidoptera, I: 56. (BM)

I930, Trans. Ent. Soc. London, 78: 322. (P)

I925, Bull. Soc. R. Ent. Egypte, 9: 209.

I925, Bull. Soc. R. Ent. Egypte, 9: 213.

I9I3, Exotic Microlepidoptera, I: 98. (BM)

I920, Voyage de Ch. Alluaud et R. Jeannel en Afrique

Oriental, II, Microlepidoptera, p. 6I. (P)

I9I3, Ann. Transvaal Mus. 3: 297. (T)

I922, Exotic Microlepidoptera, 2: 586.

I938, in Caradja and Meyrick, Deuts. Ent. Zeit. Iris, $52: 5 \cdot(\mathrm{BM})$

I9I8, Exotic Microlepidoptera, 2: 99. (BM)

I937, Exotic Microlepidoptera, 5: 87.

I93I, Exotic Microlepidoptera, 4: 87. (BM)

I926, Exotic Microlepidoptera, 3: 285. (BM)

I929, Exotic Microlepidoptera, 3: 5II. (BM)

I930, Exotic Microlepidoptera, 4: II.

I925, Exotic Microlepidoptera, 3: 2I9. (BM)

I9I I, Proc. Linnean Soc. N.S. Wales, 36: 289.

I897, Proc. Linnean Soc. N.S. Wales, 22: 388.

I927, Exotic Microlepidoptera, 3: 364. (BM)

I9I5, Trans. Ent. Soc. London, I9I5: 222.

I9I7, Exotic Microlepidoptera, 2: 58. (BM)

I9I4, Trans. Proc. New Zealand Inst. 46: I05.

I9I3, Ann. Transvaal Mus. 3: 303. (T)

I924, Rec. Indian Mus. 2: II4. (K)

I935, in Caradja and Meyrick, Materialien zu einer

Microlepidopteren Fauna der Chinesischen Provinzen

Kiangsu, Chekiang und Hunan, p. 56. (BM)

I9I3, Exotic Microlepidoptera, I: I6I. 


$\begin{array}{ll}\text { anxia } & \text { PARAPSECTRIS } \\ \text { anympha } & \text { LECITHOCERA } \\ \text { aoristana } & \text { CAPUA } \\ \text { aoropis } & \text { LATROLOGA } \\ \text { apanthes } & \text { OECOPHORA } \\ \text { aparthena } & \text { PHILONYMPHA } \\ \text { apathetica } & \text { PHTHINOSTOMA } \\ \text { apathodes } & \text { HAPLODYTA } \\ \text { apellodora } & \text { CERANTHES } \\ \text { aphanatma } & \text { SCYTHRIS } \\ \text { aphanes } & \text { COESYRA } \\ \text { aphanodes } & \text { CTENOCOMPA } \\ \text { aphanodesma } & \text { STENOMA } \\ \text { aphanogramma } & \text { LABDIA } \\ \text { aphanombra } & \text { COLEOPHORA } \\ \text { aphanopa } & \text { DICHOMERIS } \\ \text { aphanoptis } & \text { URODUS }\end{array}$

aphanozona

aphaura

aphileta

aphilodes

aphiltra

aphorista

aphoristis

aphranassa

aphrias

aphridias

aphristis

aphrobapta

aphrobola

aphrochoa

aphrocoma

aphrocrossa

aphrocyma

aphrocymba

aphrodora

aphrogama

aphrogenes

aphrogramma

aphromorpha

aphronesa

aphroneura

aphrontis

aphrophanes

aphrosema

aphrospila

aphrospora

aphrostcha
COPHOMANTIS

EULECHRIA

MEGACRASPEDUS

BLASTOBASIS

ARISTOTELIA

PERONEA

ARGYRESTHIA

COSMOPTERYX

EPIBLEMA

EPIMESOPHLEPS

MACROBATHRA

EULIA

MACHETIS*

PLEMYRISTIS*

TRACHYDORA

COLEOPHORA

ACROCERCOPS

SPILONOTA

IMMA

URODUS

AGRIOPHARA

STENOMA

ARISTOTELIA

EPERMENIA

CEROMITIA

CREMNOGENES

STENOMA

PERSICOPTILA

LASPEYRESIA

ZELLERIA

[sic!]
I9I7, Ann. South African Mus. I7: 4. (SA)

I9I6, Exotic Microlepidoptera, I: 593.

I88I, Proc. Linnean Soc. N.S. Wales, 6: 446.

I9I8, Exotic Microlepidoptera, 2: I32. (BM)

I883, New Zealand Journ. Sci. I : 524.

I884, Proc. Linnean Soc. N.S. Wales, 9: 723.

I92I, Ann. Transvaal Mus. 8: I I4. (T)

I9I4, Exotic Microlepidoptera, I: 240.

1926, Ann. South African Mus. 23:336. (SA)

1933, Exotic Microlepidoptera, 4: 369.

1884, Proc. Linnean Soc. N.S. Wales, 9: 782.

I9I9, Exotic Microlepidoptera, 2: 264.

I9I5, Exotic Microlepidoptera, I: 478. (BM)

I93I, Exotic Microlepidoptera, 4: 5I.

I9I3, Ann. Transvaal Mus. 3: 323. (T)

I92I, Ann. Transvaal Mus. 8: 83. (T)

I930, Ann. Naturhist. Mus. Wien, 44: 263, pl. I, fig. 39.

(v)

I926, Exotic Microlepidoptera, 3: 290. (BM)

I888, Proc. Linnean Soc. N.S. Wales, (Ser. 2) 2: 946.

I904, Proc. Linnean Soc. N.S. Wales, 29: 280.

I9I8, Exotic Microlepidoptera, 2: I59. (BM)

I9I7, Trans. Ent. Soc. London, I9I7: 34. (BM)

I923, The Entomologist, 56:278. (BM)

I938, in Caradja and Meyrick, Deuts. Ent. Zeit. Iris, 52:22. (BM)

I926, Trans. Ent. Soc. London, 74: 274.

I902, Trans. Ent. Soc. London, I902: 578.

I925, Bull. Soc. R. Ent. Egypte, 9: 210.

I889, Proc. Linnean Soc. N.S. Wales, (Ser. 2) 3: I677.

I93I, Exotic Microlepidoptera, 4: I52. (v)

I883, Proc. Linnean Soc. N.S. Wales, 8: $33 \mathrm{I}$.

I9I5, Exotic Microlepidoptera, I: 369.

I897, Proc. Linnean Soc. N.S. Wales, 22: 392.

I933, Exotic Microlepidoptera, 4: 364.

I9I8, Ann. Transvaal Mus. 6: 39. (T)

I927, Insects of Samoa, 3, Lepidoptera, fasc. 2, p. 70.

I922, Exotic Microlepidoptera, 2: 48I. (BM)

I935, Arb. morph. taxon. Ent. Berl. 3: Iog. (D)

I9I5, Exotic Microlepidoptera, I: 407. (BM)

I929, Trans. Ent. Soc. London, 76: 5I5. (BM)

I923, Exotic Microlepidoptera, 3: 7. (BM)

I897, Proc. Linnean Soc. N.S. Wales, 22: 43 I.

I930, Exotic Microlepidoptera, 3: 572.

I883, New Zealand Journ. Sci. I: 525.

I9I8, Exotic Microlepidoptera, 2: 208. (BM)

I897, Proc. Linnean Soc. N.S. Wales, $22: 33$ I.

I92I, Ann. Transvaal Mus. 8: 64. (T)

I893, Proc. Linnean Soc. N.S. Wales, 7: 584.

err. pro aphrosticha q.v. 
aphrosticha

aphrotis

aphypnota

apiculata

apiletica

aplasta

aplytopis

apochranta

apochroa

apoclina

apocrypta

apodoxa

apoplectica

apora

aporodes

aporopis

aposema

apostatis

apothyma

apparitrix

approbata

apracta

aprica

aprica

apricata

apricata

aprobola

apsorrhoa

aptata

aptila

aquaria

aquaria

aquila

aquilana

aquilex

aquilifera

aquilina

aquosa

arabarcha

arachnia

arachnias

arachniota

arachniotis

arachnitis

arachnodes

arachnodeta

arachnogramm
TALEPORIA

TORTRIX

COLEOPHORA

COSMOPTERYX

HOMONA

PHILOBOTA

STENOMA

TORTRIX

XYSMATODOMA

PTILOGENES

PHAEOSACES*

SEMIOCOSMA

LACTURA

NEPHOGENES

ANTAEOTRICHA

CRYPTOLECHIA

STATHMOPODA

LECITHOCERA

COESYRA

CHELARIA

CEROSTOMA

MELASINA

[PROSPLOCAMIS*]

PARASPISTES

STATHMOPODA

BRACHMIA

PHILOBOTA

ECCOPSIS

STENOMA

ACROCERCOPS

STENOMA

NEPHOGENES

SCYTHRIS

PTEROPHORUS

PARAMORPHA *

SOPHRONIA

PERSICOPTILA

PHTHORIMAEA

ARISTOTELIA

ADELA

STENOMA

COLEOPHORA

ANARSIA

ANTAEOTRICHA

STAGMATOPHORA

TETRASCHALIS*

ARRHETOPISTA*

EULIA
I9I2, Trans. Proc. New Zealand Inst. 44: I23.

I9Io, Proc. Linnean Soc. N.S. Wales, 35: 242.

I9I7, Exotic Microlepidoptera, 2: 73.

1922, Exotic Microlepidoptera, 2: 568. (BM)

I934, in Caradja and Meyrick, Deuts. Ent. Zeit. Iris, 48: 29 .

I9I4, Exotic Microlepidoptera, I: 249.

I930, Ann. Naturhist. Mus. Wien, 44:253. (v)

I930, Exotic Microlepidoptera, 3: 609.

I893, Proc. Linnean Soc. N.S. Wales, 7: 50I.

1929, Trans. Ent. Soc. London, 76: 5I6. (BM)

1886, Trans. Proc. New Zealand Inst. I8: I72.

I888, Trans. Proc. New Zealand Inst. 20: 79.

I930, Exotic Microlepidoptera, 3: 594. (BM)

I883, Proc. Linnean Soc. N.S. Wales, 8: $38 \mathrm{I}$.

I9I5, Exotic Microlepidoptera, I: 400. (BM)

I928, Exotic Microlepidoptera, 3: 477. (BM)

I902, Trans. Ent. Soc. London, I902: 575.

I932, Exotic Microlepidoptera, 4: 205. (BM)

I864, Proc. Linnean Soc. N.S. Wales, 9: 787.

I92I, Zool. Meded. 6: I64. (L)

1936, Exotic Microlepidoptera, 4:623. (Sub.)

I908, Rec. Indian Mus. 2: 400. (K)

I9I3, Journ. Bombay Nat. Hist. Soc. 22: I70. (BM)

I9I3, Exotic Microlepidoptera, I: 86.

I9I3, Ann. Transvaal Mus. 3: 295. (T)

I9I3, Exotic Microlepidoptera, I : I27.

I886, Trans. Ent. Soc. London, I886: 275. (BM)

I9I5, Exotic Microlepidoptera, I: 4I3. (BM)

I9I4, Ann. Transvaal Mus. 4: 202. (T)

I9I5, Exotic Microlepidoptera, I: 467. (BM)

I9I3, Exotic Microlepidoptera, I: I32.

I9I3, Ann. Transvaal Mus. 3: 3I3. (T)

I908, Trans. Ent. Soc. London, I907: 503.

I88I, Proc. Linnean Soc. N.S. Wales, 6: 697.

I926, Ann. South African Mus. 23: 33I. (SA)

I932, Exotic Microlepidoptera, 4: 2I4.

I9I7, Trans. Ent. Soc. London, I9I7: 44. (BM)

I925, in Wytsman, Genera Insectorum, fasc. I84, p. 46.

(Sub.)

I930, Exotic Microlepidoptera, 3: 572. (D)

I9I5, Exotic Microlepidoptera, I: 429. (BM)

I922, Exotic Microlepidoptera, 2: 556. (BM)

I925, Bull. Soc. R. Ent. Egypte, 9: 2I0.

I930, Ann. Naturhist. Mus. Wien, 44: 233. (v)

I907, Journ. Bombay Nat. Hist. Soc. I7: 744 .

I887, Trans. Ent. Soc. London, I887:267. (A)

I936, Exotic Microlepidoptera, 4: 623 .

I926, Exotic Microlepidoptera, 3: 252. (BM) 


\begin{tabular}{|c|c|c|}
\hline araea & PICROSPORA* & I9I2, Ann. South African Mus. I0: 69. (SA) \\
\hline odes & ZELLERIA & I893, Proc. Linnean Soc. N.S. Wales, $7: 5^{82}$. \\
\hline opis & MELASINA & I926, Ann. South African Mus. 23: 349 . (SA) \\
\hline eosa & MALLOBATHRA & I9I4, Trans. Proc. New Zealand Inst. 46: II7. \\
\hline ata & CEROMITIA & I920, Ann. South African Mus. I7: 3II. (SA) \\
\hline bitra & EULECHRIA & I9I4, Exotic Microlepidoptera, I: I67. (MA) \\
\hline cana & THEREUTIS & I893, Proc. Linnean Soc. N.S. Wales, 7: 595. \\
\hline caria & TORTRIX & I9Io, Proc. Linnean Soc. N.S. Wales, 35:231. \\
\hline & STATHMOPODA & I9I3, Ann. Transvaal Mus. 3: 3II. (T) \\
\hline aeodes & TRICHOPTILUS & I9I3, Exotic Microlepidoptera, I: I05. \\
\hline aeonoma & ELACHISTA & I889, Trans. Proc. New Zealand Inst. 2 I : I79. \\
\hline ica & CHOLOTIS & I9I7, Exotic Microlepidoptera, 2: 44. \\
\hline epeda & EULECHRIA & I888, Proc. Linnean Soc. N.S. Wales, (Ser. 2) 2: 960. \\
\hline polis & CONOPOMORPHA & I907, Proc. Linnean Soc. N.S. Wales, 32: 56. \\
\hline scia & EOCHROIS & I888, Proc. Linnean Soc. N.S. Wales, (Ser. 2) 2: 932. \\
\hline rapha & ONEBALA & I929, Trans. Ent. Soc. London, 76: 508. (BM) \\
\hline chimedias & AGRYROPLOCE & I9I2, Exotic Microlepidoptera, I: 63. (BM) \\
\hline chimedica & GLYPHIPTERYX & I92I, Ann. Transvaal Mus. 8: II3. (T) \\
\hline ledon & PLATYPTILIA & I938, Trans. R. Ent. Soc. London, 87: 503. \\
\hline henax & NEOPSEUSTIS & I928, Exotic Microlepidoptera, 3: 404. \\
\hline era & BRACHMIA & I907, Journ. Bombay Nat. Hist. Soc. I7: 738. (вм) \\
\hline aga & IMMA & I9I4, Supplementa Entomologica, No. 3, p. 57. (D) \\
\hline nens & ANCYLIS & I922, Exotic Microlepidoptera, 2: 5I4. (P) \\
\hline cephala & AMYDRIA & I909, Ann. South African Mus. 5:363. (SA) \\
\hline les & NARYCIA & I920, Exotic Microlepidoptera, $2: 357$. \\
\hline Ayta & ELACHISTA & I897, Proc. Linnean Soc. N.S. Wales, $22: 336$. \\
\hline sceles & ARGYROPLOCE & I93I, Exotic Microlepidoptera, 4: І36. \\
\hline haea & CAPUA & I924, Arkiv för Zool. vol. I6, part I4, p. 3 . \\
\hline & BRENTHIA & I9I2, Exotic Microlepidoptera, I: 52. (BM) \\
\hline cola & PHTHORIMAEA & I931, Journ. Linnean Soc. London, 37: 280. (BM) \\
\hline cens & HEMIMENE & I9I6, Exotic Microlepidoptera, 2:26. (BM) \\
\hline phora & BATTARISTIS & I9I4, Trans. Ent. Soc. London, I9I4: 247 . (BM) \\
\hline & ONEBALA & \\
\hline & {$[$ EPHARMONIA $*]$} & I9Io, Journ. Bombay Nat. Hist. Soc. 20: $45^{8} .^{\circ}$ (Bм) \\
\hline & LASPEYRESIA & I9I8, Ann. Transvaal Mus. 6: I3. (T) \\
\hline & STRUTHISCA & I908, Proc. Zool. Soc. London, p. 742 . \\
\hline acea & POLYCHROSIS & I9I7, Trans. Ent. Soc. London, I9I7: 23. (Bм) \\
\hline vaga & COESYRA & I884, Proc. Linnean Soc. N.S. Wales, 9: 790. \\
\hline & STENOMA & I9I6, Exotic Microlepidoptera, I: 5I9. (BM) \\
\hline & EPIBACTRA & \\
\hline & {$[$ PARABACTRA $*]$} & I909, Journ. Bombay Nat. Hist. Soc. I9: 582. (вм) \\
\hline & PACHNISTIS & I9I3, Exotic Microlepidoptera, I: 66 . (BM) \\
\hline s & PACHNISTIS & I9I4, Journ. Bombay Nat. Hist. Soc. 22: 773. (вм) \\
\hline husa & LEUCOPTERA & I9I5, Exotic Microlepidoptera, I: 346. \\
\hline lea & GRACILARIA & I9o8, Journ. Bombay Nat. Hist. Soc. I8: 828 . \\
\hline inthes & PROTOLECHIA & I904, Proc. Linnean Soc. N.S. Wales, 29: 377. \\
\hline Iula & AGONOXENA* & I92I, Exotic Microlepidoptera, 2: 472. \\
\hline entaria & DICHOMERIS & I9I3, Ann. Transvaal Mus. 3: 304. (T) \\
\hline ntata & PSITTACASTIS & I92I, Exotic Microlepidoptera, 2: 399. (BM) \\
\hline & SYNCATHARTIS* & I92I, Zool. Meded. 6: I88. (L) \\
\hline
\end{tabular}




$\begin{array}{ll}\text { argicerauna } & \text { STENOMA } \\ \text { argillacea } & \text { BLABOPHANES } \\ \text { argillacea } & \text { EUCOSMA } \\ \text { argillosa } & \text { PHYLLOBROSTIS } \\ \text { argillosana } & \text { DICHELIA } \\ \text { argo } & \text { HEXERETMIS* } \\ \text { argobalana } & \text { PYRODERCES } \\ \text { argobathra } & \text { TELPHUSA }\end{array}$

argobela

argocentra

argoceros

argochalca

argocoma

argocorys

argocosma

argocosma

argocrossa

argocymba

argodecta

argodelta

argodesma

argodonta

argodora

argodoris

argodoxa

argolina

argolitha

argolyca

argomacha

argometra

argomicta

argomitra

argonias

argonota

argopa

argophanes

argophanta

argophracta

argophthalma

argopis

argopis

argoplaca

argoplecta

argopleura

argoptera

argoschista

argosema
SPHALEROSTOLA PROTOLECHIA

CALAMOGRAPTIS*

PROPHYLACTIS*

TINEOLA

STENOMA

ACROCERCOPS

PARAPTILA*

BRACHMIA

PTILOGENES

ARISTOTELIA

TINEA

ACROCERCOPS

PLEUROTA

IDIOGLOSSA

GELECHIA

PTERNOZYGA

LINOSTICHA

ACROLEPIA

LISSOCNEMITIS*

HYPOSMOCOMA

CRYPTOLECHIA

ETHMIA

LECITHOCERA

STENOMA

MACROBATHRA

ETHMIA

PYROZELA

CRYPTOPHASA

GLYPHIPTERYX

SYNCAMARIS*

DIPLOTYLA

ELACHISTA

AGRIOPHARA

CHALCOCRATES*

COLEOPHORA

PLEUROTA

DICHELOPA

GELECHIA
I925, Exotic Microlepidoptera, 3: 22I. (BM)

I893, Proc. Linnean Soc. N.S. Wales, 7: 528.

IgI2, Ent. Mon. Mag. 48: 34. (Sub.)

I9II, Ann. Transvaal Mus. 2: 237. (T)

I88I, Proc. Linnean Soc. N.S. Wales, 6: 479.

I929, Exotic Microlepidoptera, 3: 540. (BM)

I9I5, Exotic Microlepidoptera, I: 308.

I935, in Caradja and Meyrick, Materialien zu einer

Microlepidopteren Fauna der Chinesischen Provinzen

Kiangsu, Chekiang und Hunan, p. 66. (BM)

I93I, Exotic Microlepidoptera, 4: 45.

I904, Proc. Linnean Soc. N.S. Wales, 29: 350.

I937, Exotic Microlepidoptera, 5: Ioo. (BM)

I897, Proc. Linnean Soc. N.S. Wales, 22: 408.

I9I4, Bull. Mus. Nat. Hist. Nat. Paris, 20: I22. (P)

I93I, Exotic Microlepidoptera, 4: 34. (v)

I9I 5, Trans. Ent. Soc. London, I9I5: 225.

I9I2, Trans. Ent. Soc. London, I9I I : 677. (BM)

IgI I, Journ. Bombay Nat. Hist. Soc. 20: 7IO. (BM)

I926, Exotic Microlepidoptera, 3: 232. (Bм)

I9I8, Exotic Microlepidoptera, 2: I 18. (BM)

I9I5, Trans. Proc. New Zealand Inst. 47: 204.

I936, Exotic Microlepidoptera, 5: 34 .

I9I3, Ent. Mitteil. 2: 300. (D)

I9I3, Exotic Microlepidoptera, I: 78 .

I936, Exotic Microlepidoptera, 5: 43. (BM)

I922, Exotic Microlepidoptera, 2: 498. (P)

I889, Proc. Linnean Soc. N.S. Wales, (Ser. 2) 3: I578.

I932, Exotic Microlepidoptera, 4:227.

I934, Pacific Ent. Surv. Publ. 7, art. 28, p. 352. (вв)

I935, Proc. Hawaiian Ent. Soc. 9: 67. (SP)

I935, Exotic Microlepidoptera, 4:567. (BM)

I920, Voyage de Ch. Alluaud et R. Jeannel en Afrique

Oriental, II, Microlepidoptera, p. go. (P)

I925, Treubia, 6: 430 .

I925, Exotic Microlepidoptera, 3: 224. (BM)

I886, Proc. Linnean Soc. N.S. Wales, Io: 8 II.

I9Io, Trans. Ent. Soc. London, I9Io: 46I. (BM)

I907, Journ. Bombay Nat. Hist. Soc. I7: 747.

I9I7, Exotic Microlepidoptera, 2: 55 .

I926, Ann. South African Mus. 23: 338. (SA)

1932, Exotic Microlepidoptera, 4:262. (v)

I886, Trans. Ent. Soc. London, I886: 248.

I897, Proc. Linnean Soc. N.S. Wales, 22: 337.

I907, Journ. Bombay Nat. Hist. Soc. I7: 743. (вм)

I937, Exotic Microlepidoptera, 5: 84. (T)

I9I7, Exotic Microlepidoptera, 2: 7I.

I884, Proc. Linnean Soc. N.S. Wales, 9: 758 .

I929, Trans. Ent. Soc. London, 76: 492. (BM)

I9I7, Trans. Ent. Soc. London, I9I7: 50. (BM) 


\begin{tabular}{|c|c|}
\hline argosphena & DICHELOPA \\
\hline argospila & SCOLIOGRAPHA * \\
\hline argospora & STENOMA \\
\hline argostoma & ANTISPILA \\
\hline argoteles & PTEROPHORUS \\
\hline argothea & PHYLLOCNISTIS \\
\hline argotoma & STENOMA \\
\hline argotoxa & PHILOBOTA \\
\hline argoxantha & SIMAETHIS \\
\hline argoxantha & THIOTRICHA \\
\hline arguta & BLASTOBASIS \\
\hline argutus & PTEROPHORUS \\
\hline argyracma & COMPSOLECHIA \\
\hline argyracta & HAPALONOMA* \\
\hline argyractis & ARISTOTELIA \\
\hline argyraspis & ACROCERCOPS \\
\hline argyrastra & CHOREUTIS \\
\hline argyraula & EUCOSMA \\
\hline argyritis & STILBOSIS \\
\hline argyrocarpa & LOPHOPEPLA \\
\hline argyrocentra & TINEA \\
\hline argyrocyma & EUCOSMA \\
\hline argyrodesma & GRACILARIA \\
\hline argyrodoxa & NEPTICULA \\
\hline argyromis & GLYPHIPTERYX \\
\hline argyromitra & SYMBOLISTIS \\
\hline argyropa & HYPERCALLIA \\
\hline argyrophaea & TISIS \\
\hline argyrophanes & ENCOLPOTIS \\
\hline argyropis & LEPIDOTARSA \\
\hline argyropla & LABDIA \\
\hline argyroplaca & EUXANTHIS \\
\hline argyroplintha & ANTICRATES \\
\hline argyrosema & APISTOMORPHA $*$ \\
\hline argyrospila & NEMOTOIS \\
\hline argyrospora & OINOPHILA \\
\hline argyrostrepta & PYRODERCES \\
\hline argyrota & CHOREUTIS \\
\hline argyroxantha & CHOREUTIS \\
\hline argyrozona & ANTISPILA \\
\hline ariana & GRACILARIA \\
\hline arida & HESPEROPTILA* \\
\hline aridula & CRYPTOLECHIA \\
\hline arignota & CHELARIA \\
\hline arimaspia & PYRODERCES \\
\hline arisema & LICHENAULA \\
\hline aristarcha & ANTISPILA \\
\hline aristat & STATHMOPODA \\
\hline aristocratica & PILOPREPES \\
\hline
\end{tabular}

I934, Pacific Ent. Surv. Publ. 7, art. 28, p. 343. (ВB)

I9I6, Exotic Microlepidoptera, I: 554. (BM)

I9I5, Exotic Microlepidoptera, I: 443. (BM)

I9I6, Exotic Microlepidoptera, 2: 8.

I922, Exotic Microlepidoptera, 2: 549. (P)

I933, Exo tic Microlepidoptera, 4: 36r.

I9I5, Exotic Microlepidoptera, I: 4I2. (BM)

I889, Proc. Linnean Soc. N.S. Wales, (Ser. 2) 3: I608.

I934, Exotic Microlepidoptera, 4: 458. (BM)

I 9I4, Trans. Ent. Soc. London, I9I4: 237.

I9I8, Ann. Transvaal Mus. 6: 36. (T)

I926, Exotic Microlepidoptera, 3: 299.

I922, Trans. Ent. Soc. London, I922: 83. (BM)

I9I4, Trans. Ent. Soc. London, I9I4: 244. (BM)

I923, Exotic Microlepidoptera, 3: 8. (BM)

I908, Journ. Bombay Nat. Hist. Soc. I8: 820.

I932, Trans. Ent. Soc. London, 80: II5. (BM)

I9I2, Ent. Mon. Mag. 48: 34. (Sub.)

I922, Exotic Microlepidoptera, 2: 577. (BM)

I9I4, Exotic Microlepidoptera, I: 220.

I934, Exotic Microlepidoptera, 4: 48I.

I92 I, Exotic Microlepidoptera, 2: 447. (BM)

I883, Proc. Linnean Soc. N.S. Wales, 7: I94.

I9I8, Exotic Microlepidoptera, 2: I8I.

I907, Journ. Bombay Nat. Hist. Soc. I7: 985. (вм)

I904, Proc. Linnean Soc. N.S. Wales, 29:4I4.

I9I4, Exotic Microlepidoptera, I: I92. (вм)

I9Io, Trans. Ent. Soc. London, I9IO: 439. (BM)

I937, Exotic Microlepidoptera, 5:98. ( $($ )

I902, Trans. R. Soc. S. Australia, 26: I45.

I927, Insects of Samoa, 3, Lepidoptera, fasc. 2, p. 85.

I93I, Exotic Microlepidoptera, 4: I57. (BM)

I938, Trans. R. Ent. Soc. London, 87: 524. (BM)

I88I, Proc. Linnean Soc. N.S. Wales, 5: 247.

I923, Exotic Microlepidoptera, 3: 52. (BM)

I93I, Exotic Microlepidoptera, 4: I64.

I897, Proc. Linnean Soc. N.S. Wales, 22: 353.

I9I2, Exotic Microlepidoptera, I: 55. (BM)

I938, in Caradja and Meyrick, Deuts. Ent. Zeit. Iris, $52: 87$. (BM)

I9I8, Ann. Transvaal Mus. 6: 35. (T)

I9I4, Journ. Bombay Nat. Hist. Soc. 23 : I22.

I902, Trans. R. Soc. S. Australia, 26: I37.

I9I0, Trans. Ent. Soc. London, I9IO: 456. (Вм)

I9I6, Exotic Microlepidoptera, I: 579. (BM)

I897, Proc. Linnean Soc. N.S. Wales, 22: 347.

I89o, Trans. R. Soc. S. Australia, I3: 48.

I9I6, Exotic Microlepidoptera, 2: 8.

I9I3, Exotic Microlepidoptera, I: 89.

I889, Proc. Limnean Soc. N.S. Wales, (Ser. 2) 3: 1598. 


\begin{tabular}{|c|c|c|}
\hline aristodoxa & STATHMOPODA & I926, Trans. Proc. New Zealand Inst. 56: 416. \\
\hline aristogona & IOPTERA* & I883, Proc. Linnean Soc. N.S. Wales, 8: 345. \\
\hline aristomorpha & ARGYROPLOCE & I93I, Exotic Microlepidoptera, 4: I38. (BM) \\
\hline aristonica & EUPSELIA & I881, Proc. Linnean Soc. N.S. Wales, 5: 218. \\
\hline aristopa & IMMA & I924, Exotic Microlepidoptera, 3: I34. (BM) \\
\hline aristura & TIPULOMIMA & I93I, Exotic Microlepidoptera, 4: 49 \\
\hline arithmetica & ARGYROPLOCE & I92I, Exotic Microlepidoptera, 2: 448. \\
\hline rithmetis & HAPSIFERA & I933, Exotic Microlepidoptera, 4: 4I4. \\
\hline arithmologa & CRYPTOPHASA & I938, Trans. R. Ent. Soc. London, 87: 517. \\
\hline arizela & MICROCOLONA & I897, Proc. Linnean Soc. N.S. Wales, 22: 378. \\
\hline armata & STROBISIA & I9II, Journ. Bombay Nat. Hist. Soc. 20: 728. (BM) \\
\hline armifera & TINEA & I932, Exotic Microlepidoptera, 4: 326. \\
\hline armigera & ASCALENA & I923, Exotic Microlepidoptera, 3: 60. (BM) \\
\hline armillata & GORGOPIS & I92I, Ann. Transvaal Mus. 8: I4I. (T) \\
\hline rmilligera & ODITES & I922, Ent. Mitteil. II: 45. (D) \\
\hline neutis & MACROBATHRA & I9I4, Exotic Microlepidoptera, I: 2I8. (BM) \\
\hline romatias & ANCYLIS & I9I2, Exotic Microlepidoptera, I: 3I. (BM) \\
\hline omatica & STENOMA & I9I5, Exotic Microlepidoptera, I: 449. (BM) \\
\hline aea & CLADODES & I894, Trans. Ent. Soc. London, I894: I5. (вм) \\
\hline cotrias & GELECHIA & I9o8, Proc. Zool. Soc. London, p. 725. \\
\hline arquata & USSARA & I926, Exotic Microlepidoptera, 3: 307. (BM) \\
\hline cta & CAPUA & I9I7, Trans. Ent. Soc. London, I9I7: 5. (BM) \\
\hline recta & MELITTIA & I9I8, Exotic Microlepidoptera, 2: I8I. \\
\hline arridens & STENOMA & I9I3, Exotic Microlepidoptera, 4: 36. (вм) \\
\hline senica & PLATYPTILIA & I92I, Exotic Microlepidoptera, 2: 420. \\
\hline opa & ANARSIA & $\begin{array}{l}\text { I920, Voyage de Ch. Alluaud et } R \text {. Jeannel en Afrique } \\
\text { Oriental, II, Microlepidoptera, p. } 72 \text {. (P) }\end{array}$ \\
\hline era & ARGYROPLOCE & I92I, Ann. Transvaal Mus. 8: 59. (т) \\
\hline siptila & HAPSIFERA & I93I, Exotic Microlepidoptera, 4:98. \\
\hline sisceles & IMMA & I937, Exotic Microlepidoptera, 5: I23. (T) \\
\hline rsitricha & LIMNOECIA & I927, Insects of Samoa, 3, Lepidoptera, fasc. 2, p. 93 . \\
\hline tamota & LYONETIA & I9I5, Exotic Microlepidoptera, I: 350. \\
\hline tasyras & MONOPIS & I93I, Exotic Microlepidoptera, 4: 93. \\
\hline temidora & COSMOPTERYX & I909, Journ. Bombay Nat. Hist. Soc. I9: 4I5. \\
\hline arthrodes & THIOTRICHA & I904, Proc. Linnean Soc. N.S. Wales, 29: 295. \\
\hline arthrota & OPOSTEGA & I9I5, Exotic Microlepidoptera, I: 352. \\
\hline artiasta & BRACHMIA & I9I I, Journ. Bombay Nat. Hist. Soc. 20: 7I5. (BM) \\
\hline ticulata & ARISTOTELIA & I9I8, Exotic Microlepidoptera, 2: I I9. (BM) \\
\hline articulosa & ERECHTHIAS & I921, Exotic Microlepidoptera, $2: 45^{8}$ \\
\hline artifica & ACROCLITA & I9I I, Proc. Linnean Soc. N.S. Wales, 36: 237. \\
\hline ifica & COSMOPTERYX & I909, Journ. Bombay Nat. Hist. Soc. I9: 42I. \\
\hline artificiosa & TINEA & I9II, Journ. Bombay Nat. Hist. Soc. 2I: IIg. \\
\hline tigena & XYLORYCTA & I9I4, Ann. South African Mus. Io: 250. (SA) \\
\hline ionoma & PLACOPTILA & I9I9, Exotic Microlepidoptera, $2: 284$. \\
\hline artiphanes & HIEROXESTIS & I9I5, Exotic Microlepidoptera, I: 363 . \\
\hline artocopa & TORTRIX & I932, Exotic Microlepidoptera, 4: 255. (v) \\
\hline 1oma & COPHOMANTIS (?) & I936, Exotic Microlepidoptera, 5: 48. (c) \\
\hline ritis & BRACHMIA & I9I I, Journ. Bombay Nat. Hist. Soc. 20:723. (BM) \\
\hline valis & CRYPTOLECHIA & I9Io, Journ. Bombay Nat. Hist. Soc. 20: I63. (BM) \\
\hline arvicola & PROTHINODES & I924, Exotic Microlepidoptera, 3: 70. (T) \\
\hline
\end{tabular}




$\begin{array}{ll}\begin{array}{l}\text { arystis } \\ \text { arystis }\end{array} & \text { ANTAEOTRICHA } \\ \text { asaphaula } & \text { HOMALERNIS } \\ \text { asaphochalca } & \text { ZYGOGRAPHA* } \\ \text { asaphochra } & \text { PERIACMA } \\ \text { asaphocosma } & \text { EPORGASTIS } \\ \text { asaphogramma } & \text { ACROCERCOPS } \\ \text { asaphoneura } & \text { IMMA } \\ \text { asaphopis } & \text { SPHETERISTA } \\ \text { asarota } & \text { LECITHOCERA } \\ \text { asbolaea } & \text { PHLOEOPOLA } \\ \text { asbolandra } & \text { SPILONOTA } \\ \text { asbolarcha } & \text { ETHMIA }\end{array}$

asbolodes

CNEPHASIA

asbolodes

asbolopa

asbolopis

ascalopa

ascendens

ascetica

ascodes

ascomorpha

asemodes

asmenopa

asodes

asparta

aspasia

aspasta

aspasta

aspera

asperana

asperata

asperula

aspetodes

aspetura

asphalopis

asphaltis

asphaltitis

asphyctella

aspidephora

aspidias

aspirans

aspista

aspistana

assecula.

assidua

\section{GELECHIA}

ANCYLOMETIS

CARPOSINA

HOMILOSTOLA

PORINA

DEPRESSARIA

STENOMA

TORTRIX

CHELARIA

IMMA

DICHOMERIS

BORKHAUSENIA

COESYRA

AUTOSTICHA

ODITES

AGORAULA*

TINEA

PTOCHOGLYPTIS*

PROTOLECHIA

PSEUDALCATHOE

STENOMA

BORKHAUSENIA

DISSOPTILA

BUCCULATRIX

TRACHYPEPLA

ARGYROPLOCE

TINEA

EUCOSMA

PROSELENA

PHARMACIS

ARGYROPLOCE
PHRICANTHES*
I9I5, Exotic Microlepidoptera, I: 402. (BM) I9I8, Exotic Microlepidoptera, 2: I69. (BM) I92I, Exotic Microlepidoptera, 2: 439. (BM) I9I7, Ann. South African Mus. I7: I2. (SA) I93I, Exotic Microlepidoptera, 4: I92. (BM) I934, Exotic Microlepidoptera, 4: 452. (v) I920, Exotic Microlepidoptera, 2: $29 \mathrm{I}$. I934, Exotic Microlepidoptera, 4: 457. 1928, Proc. Hawaiian Ent. Soc. 7: 96. (SP) I925, Treubia, 6:430.

I883, Proc. Linnean Soc. N.S. Wales, 8: 349. I938, Trans. R. Ent. Soc. London, 87: 509. (BM) I938, in Caradja and Meyrick, Deuts. Ent. Zeit. Iris, 52: I8. (BM)

I920, Voyage de Ch. Alluaud et R. Jeannel en Afrique Oriental, II, Microlepidoptera, P. 55. (P) I927, Exotic Microlepidoptera, 3: 349. (BM) I923. Exotic Microlepidoptera, 3: 5I. (BM) I928, Exotic Microlepidoptera, 3: 403. (BM) I9I7, Exotic Microlepidoptera, 2: 93. (BM) I92I, Trans. Proc. New Zealand Inst. 53: 336. I926, Exotic Microlepidoptera, 3: 3II. (BM) I9I5, Exotic Microlepidoptera, I: 44I. (BM) I934, Trans. roy. Soc. N.Z. 64: I53.

I938, Trans. R. Ent. Soc. London, 87:5I3. (BM) I924, Exotic Microlepidoptera, 3: I33. (BM) I939, Trans. R. Ent. Soc. London, 89: 54. (BM) I906, Trans. R. Soc. S. Australia, 30: 36 . I884, Proc. Linnean Soc. N.S. Wales, 9: 783 . I908, Journ. Bombay Nat. Hist. Soc. I8: 457. (BM) I908, Journ. Bombay Nat. Hist. Soc. I8: 632. (BM) I9I9, Exotic Microlepidoptera, 2: 242.

I88I, Proc. Linnean Soc. N.S. Wales, 6: 636. I9I8, Ann. Transvaal Mus. 6: 44. (T) I938, Institut des Parcs Nationaux du Congo Belge, fasc. I4, p. 27. (c) I904, Proc. Linnean Soc. N.S. Wales, 29: 386. I932, Exotic Microlepidoptera, 4: 229. I925, Exotic Microlepidoptera, 3: I88. (BM) I9II, Trans. New Zealand Inst. 43: 65. I9I4, Trans. Ent. Soc. London, I9I4: 234. (BM) I88I, Proc. Linnean Soc. N.S. Wales, 5: I8I. I883, New Zealand Journ. Sci. I: 523. I9o9, Journ. Bombay Nat. Hist. Soc. I9: 599. (Bм) I920, Voyage de Ch. Alluaud et $R$. Jeannel en Afrique

Oriental, II, Microlepidoptera, p. IOI. (P) I9I2, Ent. Mon. Mag. 48: 35. (Sub.) I883, Trans. Proc. New Zealand Inst. I5: 42. I909, Ann. Transvaal Mus. 2: 5, pl. 2, fig. 5. (T) I9I4, Supplementa Entomologica, No. 3, p. 49. (D) 


\begin{tabular}{|c|c|c|}
\hline assidua & ODITES & IgI4, Ann. Transvaal Mus. 4: I99. (T) \\
\hline assignata & STENOMA & I9I8, Exotic Microlepidoptera, 2: 208. (BM) \\
\hline associata & STENOMA & I925, Exotic Microlepidoptera, 3: 2I4. (BM) \\
\hline astacopis & STENOMA & I930, Ann. Naturhist. Mus. Wien, 44:242. (v) \\
\hline astathma & BATRACHEDRA & I897, Proc. Linnean Soc. N.S. Wales, 22: 307. \\
\hline astath & LIMNOECIA & I934, Pacific Ent. Surv. Publ. 7, art. 28, 348. (Bв) \\
\hline a & MYSTOGENES* & I930, Trans. Ent. Soc. London, 78:3I2. (P) \\
\hline ta & ACROCERCOPS & I922, Exotic Microlepidoptera, $2: 562$. \\
\hline hella & MEGACRASPEDUS & Igo4, Proc. Linnean Soc. N.S. Wales, 29: 275. \\
\hline $\mathbf{s}$ & ANTICRATES & Igo7, Journ. Bombay Nat. Hist. Soc. I8: I54. (Bм) \\
\hline lla & GLYPHIPTERYX & I88I, Proc. Linnean Soc. N.S. Wales, 5: 235. \\
\hline les & LIMNOECIA & I9I5, Exotic Microlepidoptera, I: 3I9. \\
\hline loxa & NEMOTOIS & I928, Exotic Microlepidoptera, 3: 462 . \\
\hline euca & LIMNOECIA & I9I7, Exotic Microlepidoptera, $2: 43$. \\
\hline lota & GLYPHIPTERYX & I88I, Proc. Linnean Soc. N.S. Wales, 5: 240. \\
\hline pa & HETEROCHYTA & Igo6, Trans. R. Soc. S. Australia, 30: 48 . \\
\hline pis & DICHOMERIS & I92I, Ann. Transvaal Mus. 8: 83. (T) \\
\hline ta & ARGYROPLOCE & I9I8, Ann. Transvaal Mus. 6: I2. (т) \\
\hline odes & ARISTOTELIA & I923, Exotic Microlepidoptera, 3:6. (BM) \\
\hline opa & STENOMA & I9I6, Exotic Microlepidoptera, I: 5I7. (BM) \\
\hline opis & COESYRA & I888, Proc. Linnean Soc. N.S. Wales, (Ser. 2) 3: I658. \\
\hline atica & PARATHETA & I928, Exotic Microlepidoptera, 3: 467. (BM) \\
\hline ast & ACROCERCOPS & I930, Exotic Microlepidoptera, 3: 581. \\
\hline ast & TINEA & IgI I, Trans. New Zealand Inst. 43: 68. \\
\hline ast & POROTICA ${ }^{*}$ & I9I3, Ann. Transvaal Mus. 3: 324. (т) \\
\hline alitis & CEROSTOMA & I9I3, Exotic Microlepidoptera, I: I52. \\
\hline alodes & OPOGONA & I922, Exotic Microlepidoptera, 2: 559. (BM) \\
\hline alopa & HORMANTRIS* & I927, Exotic Microlepidoptera, 3: 327. (Bм) \\
\hline lota & TRACHYDORA & I897, Proc. Linnean Soc. N.S. Wales, 22: 40I. \\
\hline otes & PSELNOPHORUS & Igog, Ann. South African Mus. 5: 366. (sA) \\
\hline ea & CHALINIASTIS* & I904, Proc. Linnean Soc. N.S. Wales, 29:302. \\
\hline ea & GLYPHIPTERYX & I88I, Proc. Linnean Soc. N.S. Wales, 5: 245. \\
\hline ast & STATHMOPODA & 1897, Proc. Linnean Soc. N.S. Wales, 22: 320. \\
\hline as & ANCYLOMETIS & I887, Trans. Ent. Soc. London, I887: 278 . \\
\hline ast & STATHMOPODA & I9I3, Exotic Microlepidoptera, I: 86. \\
\hline laris & PANTHYTARCHA * & I922, Exotic Microlepidoptera, 2: $58 \mathrm{~g}$. \\
\hline onis & COMPSOLECHIA & I9I8, Exotic Microlepidoptera, 2: I39. (BM) \\
\hline $\mathrm{ma}$ & TETRACMANTHES* & I924, Exotic Microlepidoptera, 3: I36. (BM) \\
\hline nes & ARGYROPLOCE & I934, Exotic Microlepidoptera, $4: 485$. (v) \\
\hline gana & CACOECIA & I889, Trans. Proc. New Zealand Inst. 2I: I56. \\
\hline ma & STENOMA & Igog, Trans. Ent. Soc. London, Igog: 34. (вм) \\
\hline lanes & CAPANICA $*$ & I9I7, Exotic Microlepidoptera, 2: 63. \\
\hline ema & ARGYROPLOCE & Igog, Journ. Bombay Nat. Hist. Soc. I9: 594. (Bм) \\
\hline & MACROBATHRA & IgI4, Exotic Microlepidoptera, I: 2 I6. \\
\hline & PIGRITIA & I9I8, Exotic Microlepidoptera, 2: I6о. (вм) \\
\hline & TYMBARCHA & I9I2, Exotic Microlepidoptera, I: I4. (BM) \\
\hline cha & AEGERIA & I930, Exotic Microlepidoptera, 3: 584. (BM) \\
\hline ma & APHANOXENA & I9I5, Exotic Microlepidoptera, I: 388. (BM) \\
\hline & STEMAGORIS* & I9I2, Ann. Transvaal Mus. 3: 79. (T) \\
\hline & PARECTOPA & I9I8, Exotic Microlepidoptera, 2: I78. \\
\hline
\end{tabular}




\begin{tabular}{|c|c|}
\hline asyneta & CROSSOPHORA \\
\hline asyntacta & TINEA \\
\hline atacta & ARISTOTELIA \\
\hline atacta & EUCOSMA \\
\hline atactopis & TALAEPORIA \\
\hline atalodes & EULIA \\
\hline atalopis & PHTHORIMAEA \\
\hline ataracta & PHRYGANOSTOLA \\
\hline atechna & BYTHOGENES* \\
\hline atelacma & EXANTHICA \\
\hline atelactis & XYLORYCTA \\
\hline atelarga & AUTODECTIS* \\
\hline atelesta & BATTARISTIS \\
\hline atelogramma & TRYPHERANTIS* \\
\hline atelopis & CEROMITIA \\
\hline atelosticta & EUCOSMA \\
\hline atelura & GLYPHIPTERYX \\
\hline athleta & SCYROTIS* \\
\hline athletis & EULECHRIA \\
\hline athliopa & HARMOLOGA \\
\hline athlophora & NEMOTOIS \\
\hline athymopa & BLASTOBASIS \\
\hline athyra & AMATHYNTIS \\
\hline athyris & HYPONOMEUTA \\
\hline atimana & AROTROPHORA \\
\hline atlantis & ACROCERCOPS \\
\hline atmanthes & ENDOZESTIS* \\
\hline atmastra & COMPSOLECHIA \\
\hline atmobola & PHILOBOTA \\
\hline atmochlora & ARGYROPLOCE \\
\hline atmocycla & GYMNOGRAMMA \\
\hline atmodes & STENOMA \\
\hline atmodesma & CHOREUTIS \\
\hline atmogramma & TINEA \\
\hline atmopa & ODITES \\
\hline atmopis & NEPHOGENES \\
\hline atmosema & BLASTOBASIS \\
\hline atmospila & PHALONIA \\
\hline atmospora & STENOMA \\
\hline atmozona & BLASTOBASIS \\
\hline atoecha & HELIOCAUSTA \\
\hline atomarcha & ARCHYALA \\
\hline atomatma & PHTHORIMAEA \\
\hline atomoclasta & ORNEODES \\
\hline atomogypsa & GAESA \\
\hline atomophora & DORATA \\
\hline atomosperma & ODITES \\
\hline atonopa & ODITES \\
\hline
\end{tabular}

I886, Proc. Linnean Soc. N.S. Wales, Io: 795. I9I7, Exotic Microlepidoptera, 2: 76 .

I927, Exotic Microlepidoptera, 3: 343. (вм)

1912, Ent. Mon. Mag. 48: 34. (Sub.)

I936, Exotic Microlepidoptera, 5: 56.

I9I7, Trans. Ent. Soc. London, I9I7: 9. (вм)

I9I8, Exotic Microlepidoptera, 2: I35.

I888, Trans. Proc. New Zealand Inst. 20: 88.

I937, Exotic Microlepidoptera, 5: II6. (T)

I926, Ann. South African Mus. 23: 347. (SA)

I918, Exotic Microlepidoptera, 2: 197.

I937, Exotic Microlepidoptera, 5: 90.

I9I4, Trans. Ent. Soc. London, I9I4: 248. (BM)

I907, Journ. Bombay Nat. Hist. Soc. I7: 740. (вм)

I938, Institut des Parcs Nationaux du Congo Belge,

fasc. I4, p. 28, pl. 3 , fig. 8 . (c)

I922, Exotic Microlepidoptera, 2: 516. (P)

I920, Exotic Microlepidoptera, 2: 33I. (BM)

I909, Ann. South African Mus. 5: 377.

I888, Proc. Linnean Soc. N.S. Wales, (Ser. 2) 2: 96I.

I938, Trans. R. Ent. Soc. London, 87: 506. (вм)

I9I 2, in Wyțsman, Genera Insectorum, fasc. I33, p. 7.

I932, Exotic Microlepidoptera, 4: 3I6. (v)

I9I I, Journ. Bombay Nat. Hist. Soc. 2I I II3.

I928, Exotic Microlepidoptera, 3: 4I5. (BM)

I88I, Proc. Linnean Soc. N.S. Wales, 6: 533.

1924, Exotic Microlepidoptera, 3:85.

I933, Exotic Microlepidoptera, 4: 447. (вм)

I929, Exotic Microlepidoptera, 3: 507. (BM)

I884, Proc. Linnean Soc. N.S. Wales, 8: 486.

I930, Exotic Microlepidoptera, 3: 603.

I9I8, Exotic Microlepidoptera, 2: Igo.

I9I5, Exotic Microlepidoptera, I: 45I. (Bм)

I933, Exotic Microlepidoptera, 4: 37I. (вм)

I927, Trans. Proc. New Zealand Inst. 58: 3I6.

I9I4, Journ. Bombay Nat. Hist. Soc. 22: 780 .

I889, Proc. Linnean Soc. N.S. Wales, (Ser. 2) 3: I605.

I930, Ann. Naturhist. Mus. Wien, 44: 230. (v)

I937, in Caradja and Meyrick, Deuts. Ent. Zeit. Iris,

5I: I70. (BM)

1925, Exotic Microlepidoptera, 3: 209. (BM)

I939, Trans. R. Ent. Soc. London, 89: 58.

I886, Proc. Linnean Soc. N.S. Wales, Io: 830.

I917, Exotic Microlepidoptera, 2: 86.

I932, Exotic Microlepidoptera, 4: I96. (вм)

I934, Ann. Mag. Nat. Hist. I4: 4 OI.

I932, Exotic Microlepidoptera, 4: 202. (вм)

I928, Exotic Microlepidoptera, 3: 429. (Bм)

I933, Exotic Microlepidoptera, 4: 433. (v)

I9I8, Exotic Microlepidoptera, 2: I97. (BM) 


\begin{tabular}{|c|c|c|}
\hline atractias & MACHLOTICA & I909, Trans. Ent. Soc. London, I909: 38. (BM) \\
\hline atractias & PHYLLOCNISTIS & I906, Trans. R. Soc. S. Australia, 30: 64 . \\
\hline atractodes & THIOTRICHA & I922, Exotic Microlepidoptera, 2: 502. \\
\hline anota & PHYLLOCNISTIS & I9o6, Trans. R. Soc. S. Australia, 30: 64 . \\
\hline rescens & CORYPTILUM & I9Io, Trans. Ent. Soc. London, I9I0: 475. \\
\hline atricapilla & EUCOSMA & I9I7, Trans. Ent. Soc. London, I9I7:2I. (BM) \\
\hline atricapsis & ARGYROPLOCE & I930, Exotic Microlepidoptera, 3: 602. (BM) \\
\hline atricassis & STENOMA & I9I6, Exotic Microlepidoptera, I: 530. (BM) \\
\hline atricollis & PELTOPHORA & I884, Proc. Linnean Soc. N.S. Wales, 9: 726. \\
\hline ricoma & TINEOLA & I93I, Exotic Microlepidoptera, 4:94. \\
\hline ricordis & TRICHOTAPHE & I934, Exotic Microlepidoptera, 4: 452. (BM) \\
\hline atriensis & TINEOLA & I925, Bull. Soc. R. Ent. Egypte, 9:217. \\
\hline rifascis & PHTHORIMAEA & I9I7, Trans. Ent. Soc. London, I9I7: 45. (BM) \\
\hline atriflora & XENOTHICTIS & I930, Exotic Microlepidoptera, 3: 609. (Bм) \\
\hline atriflua & TINEA & I9I9, Exotic Microlepidoptera, 2: 247. (BM) \\
\hline atriguttata & TRICHOTAPHE & I93I, Exotic Microlepidoptera, 4:68. (v) \\
\hline atriloqua & BATRACHEDRA & I93I, Exotic Microlepidoptera, 4: 56. \\
\hline ervis & SCAEOSOPHA & $\begin{array}{l}\text { I93I, in Joannis, Ann. Soc. Ent. France, } 98 \text { (Supp.): } 729 \\
\text { [49I]. (P) }\end{array}$ \\
\hline nodis & ACHARNEODES* & I926, Ann. South African Mus. 23: 327. (SA) \\
\hline otata & STATHMOPODA & I929, Exotic Microlepidoptera, 3: 542. \\
\hline lpis & APHANOSTOLA* & I93I, Exotic Microlepidoptera, 4:57. (BM) \\
\hline ivis & DECADARCHIS & I93I, Exotic Microlepidoptera, 4: I66. \\
\hline atrisecta & HAPSIFERA & I9I 8, Ann. Transvaal Mus. 6: 58 . (T) \\
\hline atristrigana & DICHELIA & I88I, Proc. Linnean Soc. N.S. Wales, 6: 478. \\
\hline allata & HALIMARMARA* & I93I, Exotic Microlepidoptera, 4: I90. (BM) \\
\hline attenta & DICHOMERIS & I92I, Ann. Transvaal Mus. 8: 84. (T) \\
\hline attenuata & CHELARIA & I920, Exotic Microlepidoptera, 2: 300. \\
\hline uatana & BONDIA & I883, Proc. Linnean Soc. N.S. Wales, 7: I83. \\
\hline attica & EUCOSMA & I907, Journ. Bombay Nat. Hist. Soc. I8: I37. (BM) \\
\hline attonita & OXYCRYPTIS* & I9I2, Trans. Ent. Soc. London, IgII: 692. (BM) \\
\hline atyphella & ATALOPSYCHA* & I881, Proc. Linnean Soc. N.S. Wales, 5: I77. \\
\hline auantis & PELTOPHORA & I889, Proc. Linnean Soc. N.S. Wales, (Ser. 2) 3: I642. \\
\hline auchetidella & GRACILARIA & I88I, Proc. Linnean Soc. N.S. Wales, 5: I43. \\
\hline aucupatrix & THIOTRICHA & I929, Exotic Microlepidoptera, 3: 499. (BM) \\
\hline audax & ERECHTHIODES* & IgI4, Ann. Transvaal Mus. 4: I95. (T) \\
\hline audens & TINEA & I921, Ann. Transvaal Mus. 8: I29. (T) \\
\hline augantha & ADELA & I907, Journ. Bombay Nat. Hist. Soc. I7: 989. (BM) \\
\hline gescens & STENOMA & I925, Exotic Microlepidoptera, 3: 206. (BM) \\
\hline rites & NEMOTOIS & $\begin{array}{l}\text { I938, in Caradja and Meyrick, Deuts. Ent. Zeit. Iris, } \\
\text { 52: } 29 \text {. }\end{array}$ \\
\hline ralis & LETOGENES* & I92I, Zool. Meded. 6: I73. (L) \\
\hline gusta & STROBISIA & I9I I, Journ. Bombay Nat. Hist. Soc. 20: 727. (BM) \\
\hline lacana & TORTRIX & I881, Proc. Linnean Soc. N.S. Wales, 6: 5 I3. \\
\hline lacitis & TRACHYCENTRA & I907, Proc. Linnean Soc. N.S. Wales, 32: I43. \\
\hline acodes & PATNA & I933, Exotic Microlepidoptera, 4:385. (c) \\
\hline lacois & PALPARIA & I883, Proc. Linnean Soc. N.S. Wales, 7: 438. \\
\hline acopis & ARISTOTELIA & I923, Exotic Microlepidoptera, 3: 6. (BM) \\
\hline aeodes & EULIA & I926, Exotic Microlepidoptera, 3:254. (BM) \\
\hline laria & COLEOPHORA & I924, Exotic Microlepidoptera, 3: 66. \\
\hline
\end{tabular}


aulata

aulias

aulica

aulodocha

aulodoma

aulogramma

aulograpta

aulonias

aulonitis

aulonota

aulophora

aulorrhoa

aulosema

aulotes

aulotoma

aureolata

auricoma

auricrinis

auriculata

aurisulcata

aurita

aurorella

auspex

austalea

austera

austera

austerodes

austeropa

austrina

autadelpha

autadelpha

autampyx

autarcha

autarithma

authaema

autoberylla

autobrocha

autocasis

autocentra

autochalca

autocharacta

autochlora

autochroa

autochthonia

autoclina

autocnista

autoconis

autocosma

autocrena
PARAMORPHA

LECITHOCERA

EPIPHRACTIS

CROCANTHES

[SPHENOCRATES*]

MELASINA

GLYPHIPTERYX

AEGERIA

IMMA

TIMYRA

ARISTOTELIA

PARAPHYLLIS

PHTHORIMAEA

MACAROPHANTA*

PSELNOPHORUS

DICHOMERIS

AUTOSTICHA

STENOMA

CEROMITIA

CRYPSITHYRIS

TRICHOTAPHE

EUCOSMA

ECLECTA*

GRACILARIA

COESYRA

SEMIOCOSMA

CENOPIS

CHELARIA

ACROCERCOPS

SAROPLA

GRACILARIA

MELASINA

PROMENESTA

TIMYRA

ACROCERCOPS

EPICOENIA

ARGYROPLOCE

ANTICRATES

PRAYS

METACHANDA

MARMAROXENA*

BONDIA

PHILARGA*

SYNTOMACTIS

MELASINA

PROMALACTIS

DEPRESSARIA

BEDELLIA

LACTURA

HYPERCALLIA
I9I3, Exotic Microlepidoptera, I: 7I. (BM)

I9Io, Journ. Bombay Nat. Hist. Soc. 20: 447. (BM)

I9I2, Ann. South African Mus. Io: 66. (SA)

I9I8, Exotic Microlepidoptera, 2: 98.

1924, Exotic Microlepidoptera, 3: 76. (вм)

I907, Trans. Proc. New Zealand Inst. 39: I20.

I934, Exotic Microlepidoptera, 4: 455. (вм)

I906, Trans. Ent. Soc. London, I906: I79. (BM)

I9o8, Journ. Bombay Nat. Hist. Soc. I8: 447 . (BM)

I9I7, Trans. Ent. Soc. London, I9I7: 30. (BM)

19I8, Exotic Microlepidoptera, 2: 224.

I935, Exotic Microlepidoptera, 4: 560. (BM)

I932, Exotic Microlepidoptera, 4: 339.

I9II, Ann. Transvaal Mus. 2: 2I9. (T)

I9I7, Ann. South African Mus. I7: 5. (SA)

I9o8, Journ. Bombay Nat. Hist. Soc. I8: 455. (Bм)

I930, Exotic Microlepidoptera, 4: 32. (v)

I933, Exotic Microlepidoptera, 4: 364.

I9I7, Exotic Microlepidoptera, 2: 75.

I922, Trans. Ent. Soc. London, I922: Io8. (BM)

I920, Exotic Microlepidoptera, 2: 345.

I883, Proc. Linnean Soc. N.S. Wales, $7: 445$.

I9I2, in Wytsman, Genera Insectorum, fasc. I28, p. 29.

I884, Proc. Linnean Soc. N.S. Wales, 9: 789 .

I883, New Zealand Journ. Sci. I: 523.

I9I2, Ent. Mon. Mag. 48: 34. (Sub.)

I9I8, Ann. Transvaal Mus. 6:22. (T)

I9I4, Journ. Bombay Nat. Hist. Soc. 23: I2I.

I9I4, Trans. Proc. New Zealand Inst. 46: Io7.

I88I, Proc. Linnean Soc. N.S. Wales, 5: I47.

I905, Journ. Bombay Nat. Hist. Soc. I6: 6I6.

I925, Exotic Microlepidoptera, 3: I6I. (вм)

Igo8, Journ. Bombay Nat. Hist. Soc. I8: 445. (BM)

I934, Exotic Microlepidoptera, 4:472.

I906, Journ. Bombay Nat. Hist. Soc. I7: I4I. (BM)

I932, Exotic Microlepidoptera, 4: 226. (BM)

I922, Exotic Microlepidoptera, 2: 553. (BM)

I907, Proc. Linnean Soc. N.S. Wales, 32: 77.

I9II, Trans. Linnean Soc. London, I4: 280. (BM)

I927, Insects of Samoa, 3, Lepidoptera, fasc. 2, p. II5.

I932, Exotic Microlepidoptera, 4: 3I2. (BM)

I9I8, Exotic Microlepidoptera, 2: 222. (BM)

I9I5, Exotic Microlepidoptera, I: 333. (MA)

I93I, Exotic Microlepidoptera, 4: I04.

I935, Exotic Microlepidoptera, 4: 592. (вм)

I92I, The Entomologist, 54: 76. (Sub.)

I930, Trans. Ent. Soc. London, 78: 320. (P)

I924, Exotic Microlepidoptera, 3: I24.

I930, Exotic Microlepidoptera, 3:575. (P) 


\begin{tabular}{|c|c|c|}
\hline autocrossa & GELECHIA & $\begin{array}{l}\text { I936, in Caradja and Meyrick, Deuts. Ent. Zeit. Iris, } \\
\text { 50: I57. (BM) }\end{array}$ \\
\hline autocrypta & OCNOPHILA* & I926, Ann. South African Mus. 23:345. (SA) \\
\hline autoderma & MELASINA & I9I4, Ann. South African Mus. Io: 253. (SA) \\
\hline autodesma & ZALITHIA & I9I8, Exotic Microlepidoptera, 2: I43. (вм) \\
\hline autodoxa & THYLACOPLEURA* & I886, Trans. Ent. Soc. London, I886:285. (BM) \\
\hline autodyas & LECITHOCERA & I929, Exotic Microlepidoptera, 3: 525. \\
\hline autogama & HIEROXESTIS & I9I5, Exotic Microlepidoptera, I: 362 . \\
\hline autogenes & HELIOZELA & I897, Proc. Linnean Soc. N.S. Wales, 22: 406. \\
\hline autoglypta & EUPROPHANTIS* & I92I, Zool. Meded. 6: I9I. (L) \\
\hline autogramma & EULECHRIA & I905, Journ. Bombay Nat. Hist. Soc. I6: 603. (BM) \\
\hline autographa & EULECHRIA & I9I5, Exotic Microlepidoptera, I: 307. \\
\hline autographa & LINOSTICHA & I902, Trans. R. Soc. S. Australia, 26: I52. \\
\hline autograpta & LEUCOPTERA & I9I8, Ann. Transvaal Mus. 6: 40. (T) \\
\hline autoleuca & EPIPHTHORA & I904, Proc. Linnean Soc. N.S. Wales, $29: 264$. \\
\hline autolitha & EUCOSMA & I93I, Exotic Microlepidoptera, 4: I45. (BM) \\
\hline autologa & CARPOSINA & I9Io, Proc. Linnean Soc. N.S. Wales, 35: I49. \\
\hline loga & LECITHOCERA & I910, Journ. Bombay Nat. Hist. Soc. $20: 446$. (вм) \\
\hline hacha & EURYTYLA* & I893, Proc. Linnean Soc. N.S. Wales, 7: 566. \\
\hline etra & CYMOTRICHA & $\begin{array}{l}\text { I934, in Caradja and Meyrick, Deuts. Ent. Zeit. Iris, } \\
\text { 48: 34. (BM) }\end{array}$ \\
\hline omima & PHILOBOTA & I889, Proc. Linnean Soc. N.S. Wales, (Ser. 2) 3: I625. \\
\hline orpha & SYRMADAULA* & I9I8, Ann. Transvaal Mus. 6:26. (T) \\
\hline autonephes & TAPINODOXA* & I93I, Exotic Microlepidoptera, 4: I54. (v) \\
\hline autonoma & BRACHMIA & I9I0, Trans. Ent. Soc. London, I9Iо: 369. (вм) \\
\hline al & ANODITICA* & $\begin{array}{l}\text { I938, Institut des Parcs Nationaux du Congo Belge, } \\
\text { fasc. I4, p. } 2 \mathrm{I} \text {. (c) }\end{array}$ \\
\hline autopetes & GLYPHIPTERYX & I907, Proc. Linnean Soc. N.S. Wales, $32:$ II8. \\
\hline autopetra & MELASINA & I907, Journ. Bombay Nat. Hist. Soc. I8: I57. \\
\hline autophaea & ALLOTALANTA* & I9I3, Exotic Microlepidoptera, I: II4. (BM) \\
\hline autophanta & PACHNISTIS & I92I, Ann. Transvaal Mus. 8: 89. (T) \\
\hline autophylla & EULECHRIA & I888, Proc. Linnean Soc. N.S. Wales, (Ser. 2) 2: 947. \\
\hline autophyta & OPOGONA & I9I5, Exotic Microlepidoptera, I: 359. \\
\hline autopis & PROTOLECHIA & I904, Proc. Linnean Soc. N.S. Wales, $29: 348$. \\
\hline oplecta & HOMILOSTOLA & I9I7, Exotic Microlepidoptera, 2: 92. (BM) \\
\hline autoschista & ETHMIA & I932, Exotic Microlepidoptera, 4: 347. (BM) \\
\hline autoscia & EXORECTIS* & I906, Trans. R. Soc. S. Australia, 30: 65 . \\
\hline autotoma & STAGMATOPHORA & I9I9, Exotic Microlepidoptera, $2: 284$. \\
\hline autotypa & MICROCOLONA & I922, Exotic Microlepidoptera, 2: 575. \\
\hline autoxantha & STATHMOPODA & I9I3, Ann. Transvaal Mus. 3: 3II. (T) \\
\hline urga & PYCNOSTOLA & I92I, Ann. Transvaal Mus. 8: 65. (T) \\
\hline auxileuca & DIACROTRICHA & I908, Trans. Ent. Soc. London, I907: 47I. \\
\hline auxiliaris & ZALITHIA & I9I8, Exotic Microlepidoptera, 2: I43. (BM) \\
\hline imena & LIMNOECIA & I915, Exotic Microlepidoptera, I: 318. \\
\hline obathra & IMMA & I906, Trans. Ent. Soc. London, I906: I88. (BM) \\
\hline rodelta & AUTOSTICHA & I9I6, Exotic Microlepidoptera, I: 585 . (BM) \\
\hline soleuca & STENOMA & I925, Exotic Microlepidoptera, 3: 223. (BM) \\
\hline solyca & PHILOBOTA & I889, Proc. Linnean Soc. N.S. Wales, (Ser. 2) 3: I6 Io. \\
\hline oplaca & EULIA & I926, Exotic Microlepidoptera, 3:255. (BM) \\
\hline auxoptila & TELPHUSA & I926, Exotic Microlepidoptera, 3:276. (BM) \\
\hline
\end{tabular}




$\begin{array}{ll}\text { auxoscia } & \text { PHILOBOTA } \\ \text { auxozona } & \text { NEPTICULA } \\ \text { avara } & \text { MONOPIS } \\ \text { avida } & \text { HERPYSTIS* } \\ \text { avida } & \text { STENOMA } \\ \text { axena } & \text { ANTAEOTRICHA } \\ \text { axenana } & \text { EPALXIPHORA* } \\ \text { axenopa } & \text { SCYTHRIS } \\ \text { axenopis } & \text { PHTHORIMAEA } \\ \text { axesta } & \text { AGRIOPHARA } \\ \text { axiota } & \text { NEPHOGENES } \\ \text { axiotima } & \text { EUCOSMA } \\ \text { axiurga } & \text { CRONODOXA* } \\ \text { axysta } & \text { EUPETOCHIRA } \\ \text { azyga } & \text { PHALONIA }\end{array}$

babylonica baccata bacchata baccheutis bacchias bacterias bacteriopa bacteriopis bacteriota bactrias bactropa bactrophora bactrospila baeopis balanacma balanarcha balanaspis

balanistis balanitis balanitis balanobola balanocentra balanocentra balanochrysa balanophora balanophora balanopa balanopis balanoptis
SPILONOTA DICHOMERIS PERITORNEUTA PSEUDATTERIA SPANIACMA* PACHYRHABDA ALUCITA MICROPHIDIAS* BALIONEBRIS* GLYPHIPTERYX EUCOSMA COSMOPTERYX PICROGENES* LECITHOCERA ARGYROPLOCE STATHMOPODA CHELARIA

STATHMOPODA IDIOCRATES* ONEBALA ISCHNOBATHRA* APHANOXENA ARISTOTELIA XESTOCASIS SCYTHRIS SEMIOCOSMA THIOTRICHA PLUTELLA STENOMA
I922, Exotic Microlepidoptera, 2: 5I2.

I934, Exotic Microlepidoptera, 4: 468. (BM) I9I9, Exotic Microlepidoptera, 2: 239. I9I I, Proc. Linnean Soc. N.S. Wales, 36: 245. I9I5, Exotic Microlepidoptera, I: 435. (BM) I9I5, Exotic Microlepidoptera, I: 50I. (BM) I88I, Proc. Linnean Soc. N.S. Wales, 6: 648. I9I8, Exotic Microlepidoptera, 2: I57. I9I9, Exotic Microlepidoptera, 2: 234. (Sub.) I89o, Trans. R. Soc. S. Australia, I3: 79 . I889, Proc. Linnean Soc. N.S. Wales, (Ser. 2) 3: I604. I937, in Caradja and Meyrick, Deuts. Ent. Zeit. Iris, 5I: $\mathrm{I}^{8} 8$. (BM) I922, Exotic Microlepidoptera, 2: 602. (P) I927, Exotic Microlepidoptera, 3: 363. (T) I935, in Caradja and Meyrick, Materialien zu einer Microlepidopteren Fauna der Chinesischen Provinzen Kiangsu, Chekiang und Hunan, p. 47.

I9I2, Journ. Bombay Nat. Hist. Soc. 2I: 854. (BM) I923, Exotic Microlepidoptera, 2: 62I. (BM)

I9I4, Exotic Microlepidoptera, I: 225. I924, Exotic Microlepidoptera, 3: Io9. (BM) I9I3, Exotic Microlepidoptera, I: I29. I9I3, Exotic Microlepidoptera, I: 95. I922, Ent. Mitteil. II : 44. (D)

I937, Exotic Microlepidoptera, 5: I5I. (T) I935, Exotic Microlepidoptera, 4: 573. I9I I, Trans. New Zealand Inst. 43: 67. I927, Exotic Microlepidoptera, 3: 336. (BM) r9o8, Proc. Zool. Soc. London, p. 733. I9I7, Ann. South African Mus. I7: 5. (SA) I929, Exotic Microlepidoptera, 3: 523. (BM) I9I4, Exotic Microlepidoptera, I: 275. (BM) I921, Exotic Microlepidoptera, 2: 46I. I93I, in Joannis, Ann. Soc. Ent. France, 98 (Supp.): 723 [485]. I9I3, Exotic Microlepidoptera, I: 89. I9o9, Trans. Ent. Soc. London, Igo9: I9. (BM) I9Io, Journ. Bombay Nat. Hist. Soc. 20: 452. (BM) 1937, Exotic Microlepidoptera, 5: 8I. (T) I9I5, Exotic Microlepidoptera, I: 387. (BM) I9I4, Ann. Transvaal Mus. 4: I9o. (T) I9I5, Exotic Microlepidoptera, I : 3I6. I9I6, Exotic Microlepidoptera, 2: I5. (BM) I897, Trans. Ent. Soc. London, I897: 389. I9I8, Exotic Microlepidoptera, 2: I23. (BM) I909, Ann. South African Mus. 5: 376. (SA) I932, Exotic Microlepidoptera, 4: 293. (v) 


\begin{tabular}{|c|c|c|}
\hline balanoptycha & EUCOSMA & I9Io, Rec. Indian Mis. 5: 218. (K) \\
\hline balanosema & CHRYSORYCTIS & I893, Proc. Linnean Soc. N.S. Wales, $7: 553$. \\
\hline balanospila & ODITES & I930, Exotic Microlepidoptera, 3: 555. (BM) \\
\hline balanota & ATTEVA & I9Io, Trans. Ent. Soc. London, I9IO: 47I. (BM) \\
\hline balanota & PELTOPHORA & I889, Proc. Linnean Soc. N.S. Wales, (Ser. 2) 3: I643. \\
\hline balanotes & PTEROPHORUS & I908, Trans. Ent. Soc. London, I907: 503. \\
\hline balanotis & ASYMPHORODES & I934, Pacific Ent. Surv. Publ. 7, art. 28, p. 351. (вв) \\
\hline balantias & COESYRA & I908, Proc. Zool. Soc. London, p. 73 I. \\
\hline balbidota & ANTIOCHTHA* & I905, Journ. Bombay Nat. Hist. Soc. I6: 598 . (Bм) \\
\hline baliandra & STENOMA & I9I5, Exotic Microlepidoptera, I: 442. (BM) \\
\hline baliochlora & ORNEODES & I929, Exotic Microlepidoptera, 3: 535. (вм) \\
\hline baliodes & CTENOCOMPA* & I893, Proc. Linnean Soc. N.S. Wales, (Ser. 2) 7: 489. \\
\hline baliomicta & TINISSA & I928, Exotic Microlepidoptera, 3: 424 . \\
\hline balioneura & ANARSIA & I921, Ann. Transvaal Mus. 8: 79. (T) \\
\hline baliopa & LINDERA & I9I7, Exotic Microlepidoptera, $2: 84$. \\
\hline baliopsamma & HAPSIFERA & I921, Exotic Microlepidoptera, 2: 475 . \\
\hline balioxantha & ORNEODES & I921, Exotic Microlepidoptera, 2: 406. \\
\hline ballista & STENOMA & I9I6, Exotic Microlepidoptera, I: 5I6. (BM) \\
\hline ballistis & ETHMIA & I908, Proc. Zool. Soc. London, p. 732. \\
\hline balsamias & ODITES & I9I2, Ann. Transvaal Mus. 3: 75 . (T) \\
\hline balsamodes & AROTROPHORA & $\begin{array}{l}\text { I93I, Ann. Mus. Nac. Hist. Nat. Buenos Aires, 36: } 38 \mathrm{r} \text {. } \\
\text { (BM) }\end{array}$ \\
\hline balsamopa & EPITHECTIS & I923, Exotic Microlepidoptera, 3: I6. (Bм) \\
\hline balteata & STROBISIA & I9I I, Journ. Bombay Nat. Hist. Soc. 20: 732. (BM) \\
\hline bambusae & COSMOPTERYX & I9I7, Ent. Mon. Mag. 53: $25^{8 .}$ \\
\hline banausa & PHLOEOPOLA & I883, Proc. Linnean Soc. N.S. Wales, $8: 356$. \\
\hline banausodes & PROTOLECHIA & I904, Proc. Linnean Soc. N.S. Wales, 29: 384. \\
\hline banausopa & PACHNISTIS & I929, Exotic Microlepidoptera, 3: 526. (BM) \\
\hline banausopis & ARGYROPLOCE & $\begin{array}{l}\text { I938, in Caradja and Meyrick, Deuts. Ent. Zeit. Iris, } \\
52: 2 \text {. (BM) }\end{array}$ \\
\hline barathrias & HERMOGENES & I9o8, Journ. Bombay Nat. Hist. Soc. I8: 629. (вм) \\
\hline barathrodes & PARISTHMIA* & I909, Ann. Transvaal Mus. 2: I3, pl. 5, fig. I. (T) \\
\hline throta & SEMNOSTOMA & I9I8, Exotic Microlepidoptera, 2: I28. (Lost) \\
\hline barbara & HEDROXENA* & I924, Exotic Microlepidoptera, 3:92. (BM) \\
\hline barbata & LECITHOCERA & I933, Exotic Microlepidoptera, $4: 35^{6}$. (c) \\
\hline barbifera & LECITHOCERA & I922, Zool. Meded. $7: 84$. (L) \\
\hline barbigera & SIMAETHIS & I9I5, Trans. Proc. New Zealand Inst. 47: 203. \\
\hline arbitias & EPICEPHALA & I9o9, Ann. Transvaal Mus. 2: 24, pl. 7, fig. Io. (T) \\
\hline ydelta & EPITHECTIS & I923, Exotic Microlepidoptera, 3: I5. (BM) \\
\hline arydesma & ZALITHIA & I9I8, Exotic Microlepidoptera, 2: I42. (BM) \\
\hline arygrapta & TELPHUSA & I932, Exotic Microlepidoptera, 4: I93. (BM) \\
\hline arymochla & DESMOPHYLAX* & I935, Exotic Microlepidoptera, 4:588. (BM) \\
\hline yphragma & EUCOSMA & I937, Exotic Microlepidoptera, 5: I59. (BM) \\
\hline Iryscias & METACHANDA & I930, Trans. Ent. Soc. London, $78: 315$. (P) \\
\hline arysoma & ANTIDICA & I883, Proc. Linnean Soc. N.S. Wales, 8: 383 . \\
\hline ysphena & CROSSOBELA* & I923, Exotic Microlepidoptera, 3: 34. (BM) \\
\hline ryspila & ACROLOPHUS & I93I, Exotic Microlepidoptera, 4: I03. (v) \\
\hline yspilas & TINEA & I937, Exotic Microlepidoptera, 5: II2. \\
\hline stacta & PROTEROSPASTIS* & I937, Exotic Microlepidoptera, 5: 83. (T) \\
\hline arysticta & TINEA & I927, Exotic Microlepidoptera, 3: 322. (BM) \\
\hline
\end{tabular}




\begin{tabular}{|c|c|c|}
\hline barythyma & GLYPHIDOCERA & I929, Exotic Microlepidoptera, 3: 530. (BM) \\
\hline baryzela & PHALONIA & I912, Ent. Mon. Mag. 48: 35. (Sub.) \\
\hline basanista & MELEONOMA & I922, Exotic Microlepidoptera, 2: 5 I3. \\
\hline basanistis & STAGMATOPHORA & I909, Ann. South African Mus. 5: 358. (SA) \\
\hline asanistis & TINGENTERA & I9o8, Journ. Bombay Nat. Hist. Soc. I8: 453. (вм) \\
\hline ascanaula & LITHOCOLLETIS & I936, Exotic Microlepidoptera, 5: 32 . \\
\hline Jascanopa & LEPOCNEMIS* & I9I3, Ann. Transvaal Mus. 3: 325. (T) \\
\hline asichlora & PROCHOLA & I922, Exotic Microlepidoptera, 2: 582. (BM) \\
\hline asilica & COESYRA & I884, Proc. Linnean Soc. N.S. Wales, 9: 770. \\
\hline basilisca & COSMOPTERYX & I909, Journ. Bombay Nat. Hist. Soc. I9: 4I6. \\
\hline asilissa & OPOSTEGA & I893, Proc. Linnean Soc. N.S. Wales, 7: 606. \\
\hline asiplectra & STATHMOPODA & I9I3, Exotic Microlepidoptera, I: 97. \\
\hline athracma & PARECTOPA & I9I2, Exotic Microlepidoptera, I: 25. (T) \\
\hline athrarcha & STOMOPTERYX & I921, Ann. Transvaal Mus. 8: 76. (т) \\
\hline throcentra & STENOMA & I9I5, Exotic Microlepidoptera, I: 462. (BM) \\
\hline athrochlora & GELECHIA & I932, Exotic Microlepidoptera, 4: 352. (v) \\
\hline athroclina & PROMALACTIS & I9I8, Exotic Microlepidoptera, 2: 212. (BM) \\
\hline athrodelta & STATHMOPODA & I92I, Exotic Microlepidoptera, 2: 46I. \\
\hline throdyas & STENOLECHIA & I935, Exotic Microlepidoptera, 4: 583. (BM) \\
\hline athrogramma & ORPHNOLECHIA & I9I2, Trans. Ent. Soc. London, IgII: 7II. (BM) \\
\hline athropis & GELECHIA & I904, Proc. Linnean Soc. N.S. Wales, 29: 3Io. \\
\hline hroptila & HETERALCIS & I929, Exotic Microlepidoptera, 3: 5I7. (BM) \\
\hline athrosema & PYRODERCES & I897, Proc. Linnean Soc. N.S. Wales, 22: 353. \\
\hline athrosticta & GELECHIA & I936, Exotic Microlepidoptera, 5: 43 . \\
\hline throta & MOMPHA & I9II, Ann. Transvaal Mus. 3: 7I. (BM) \\
\hline hrotoma & STENOMA & I925, Exotic Microlepidoptera, 3: I89. (Bм) \\
\hline thyglypta & CACOECIA & I932, in Caradja, Bull. Sect. sci. Acad. roum. I5: 23. \\
\hline thyntis & STENOMA & I93I, Exotic Microlepidoptera, 4: 39. (v) \\
\hline thyphaea & STENOMA & I932, Exotic Microlepidoptera, 4: 297. (v) \\
\hline trachodes & ARGYROPLOCE & I9I I, Proc. Linnean Soc. N.S. Wales, 36: 274 . \\
\hline trachopa & ENARMONIA & I9o8, Proc. Zool. Soc. London, p. 7I8. (вм) \\
\hline rachopis & AGRIOPHARA & I9I3, Trans. Ent. Soc. London, I9I3: I82. (Bм) \\
\hline linda & ACROCLITA & I9I 2, Journ. Bombay Nat. Hist. Soc. 21: 858 . (BM) \\
\hline ellica & HILAROGRAPHA & I9I2, Exotic Microlepidoptera, I: 37. (BM) \\
\hline licosa & OPOSTEGA & I9II, Ann. Transvaal Mus. 2: 236. (T) \\
\hline lonacma & COSMOPTERYX & I909, Journ. Bombay Nat. Hist. Soc. I9: 4I6. \\
\hline lonodes & EPIPHTHORA & I904, Proc. Linnean Soc. N.S. Wales, 29:26I. \\
\hline lonota & TINEA & I888, Trans. Proc. New Zealand Inst. 20: 99. \\
\hline matica & ADOXOPHYES & I9Io, Proc. Limnean Soc. N.S. Wales, 35: 206. \\
\hline enedicta & CEROMITIA & I9I8, Ann. Transvaal Mus. 6: 47. (т) \\
\hline Senevola & EPICHORISTA & $\begin{array}{l}\text { I920, Voyage de Ch. Alluand et } R \text {. Jeannel en Afrique } \\
\text { Oriental, II, Microlepidoptera, p. } 5 \mathrm{I} . \text { ( }(\mathrm{P})\end{array}$ \\
\hline enigna & CARPOSINA & I9I3, Exotic Microlepidoptera, I: 76 . (вм) \\
\hline igna & STENOMA & I9I6, Exotic Microlepidoptera, I: 540. (Bм) \\
\hline erecynthia & NARYCIA & I93I, Exotic Microlepidoptera, 4: 99. \\
\hline erenice & BORKHAUSENIA & I929, Trans. Proc. New Zealand Inst. 60: 488. \\
\hline yllina & EUCOSMA & I927, Exotic Microlepidoptera, 3:335. (BM) \\
\hline yllina & SPILONOTA & I925, Treubia, 6: 428. (вм) \\
\hline ryllitis & TORTYRA & I9Io, Trans. Ent. Soc. London, I9Io: 462. (BM) \\
\hline ryllopa & NEMOTOIS & I935, Exotic Microlepidoptera, 4: 595. (м) \\
\hline
\end{tabular}




\begin{tabular}{|c|c|}
\hline bian & STOMOPTERYX \\
\hline biannulata & STENOMA \\
\hline biarcuata & ANTAEOTRICHA \\
\hline bibula & EXOSPHRANTIS* \\
\hline bicensa & STENOMA \\
\hline biclavata & GELECHIA \\
\hline biclavis & STATHMOPODA \\
\hline bicornis & GLYPHIPTERYX \\
\hline bicristata & LEPTOGENEIA* \\
\hline bicunea & STROBISIA \\
\hline bicuneata & PROTOCHANDA* \\
\hline bidens & TRICHOPTILUS \\
\hline bidentifera & DIACTENIS \\
\hline bifida & AGRIOPHARA \\
\hline bifissa & STIBARENCHES* \\
\hline biforata & CACOECIA \\
\hline biformis & TORTRIX \\
\hline bifrenata & ONEBALA \\
\hline bifrenis & ACROCERCOPS \\
\hline bifulminata & IMMA \\
\hline $\operatorname{mir}$ & LASPEYRESIA \\
\hline
\end{tabular}

$\begin{array}{ll}\text { bigenita } & \text { PENTHOCRATES* } \\ \text { bilinguis } & \text { STENOMA } \\ \text { bimarginata } & \text { DOLIOTECHNA } \\ \text { binaria } & \text { AUTOSTICHA } \\ \text { binigrata } & \text { CACOECIA } \\ \text { binocularis } & \text { ANTHISTARCHA } \\ \text { binodis } & \text { STENOMA } \\ \text { binotata } & \text { LECITHOCERA } \\ \text { binummulata } & \text { CHELARIA } \\ \text { biophora } & \text { PHILOBOTA } \\ \text { bipinnata } & \text { CHELARIA } \\ \text { biplagata } & \text { CATELAPHRIS } \\ \text { bipupillata } & \text { ANTAEOTRICHA } \\ \text { biremis } & \text { EULIA } \\ \text { biscissana } & \text { HOLOCOLA } \\ \text { biscutata } & \text { HOMONA } \\ \text { bisecta } & \text { EUCOSMA } \\ \text { bisepta } & \text { TINEA } \\ \text { biserialis } & \text { LASPEYRESIA }\end{array}$

$\begin{array}{ll}\text { biseriata } & \text { MACHIMIA } \\ \text { bisignata } & \text { STENOMA } \\ \text { bisignis } & \text { LIMNOECIA }\end{array}$

I921, Ann. Transvaal Mus. 8: 77. (T)

I930, Ann. Naturhist. Mus. Wien, 44:248. (v)

I926, Exotic Microlepidoptera, 3: 238. (BM)

I931, Ann. Mus. Nac. Hist. Nat. Buenos Aires, 36: 389. (BM)

I9I 5, Exotic Microlepidoptera, I : 464. (BM)

I934, Exotic Microlepidoptera, 4: 5I I. (BM)

I9I I, Trans. Linnean Soc. London, I4: 286.

I909, Journ. Bombay Nat. Hist. Soc. I9: 432. (BM)

I904, Proc. Linnean Soc. N.S. Wales, 29:4I3.

I9I I, Journ. Bombay Nat. Hist. Soc. 20: 73I. (BM)

I935, in Caradja and Meyrick, Materialien zu einer

Microlepidopteren Fauna der Chinesischen Provinzen

Kiangsu, Chekiang und Hunan, p. 76. (BM)

I930, Exotic Microlepidoptera, 3:564.

I 928, Exotic Microlepidoptera, 3: 458. (BM)

I9I6, Exotic Microlepidoptera, I : 54I. (BM)

I930, Ann. Naturhist. Mus. Wien, 44: 229. (v)

I930, Amn. Naturhist. Mus. Wien, 44: 224. (v)

I920, Ann. South African Mus. I7: 275. (SA)

I921, Ann. Transvaal Mus. 8: 89. (T)

I9I8, Exotic Microlepidoptera, 2: I76.

I930, Exotic Microlepidoptera, 4: 2. (BM)

I935, in Caradja and Meyrick, Materialien zu einer

Microlepidopteren Fauna der Chinesischen Provinzen

Kiangsu, Chekiang und Hunan, p. 63. (BM)

I934, Exotic Microlepidoptera, 4:523.

I9I8, Exotic Microlepidoptera, 2: 202. (BM)

I929, Trans. Ent. Soc. London, 76: 5I2. (BM)

I908, Journ. Bombay Nat. Hist. Soc. I8: 456. (Вм)

I928, Exotic Microlepidoptera, 3: 456. (BM)

I929, Exotic Microlepidoptera, 3: 484. (BM)

I9I 5, Exotic Microlepidoptera, I: 472. (Bм)

I9I8, Ann. Transvaal Mus. 6:24. (T)

I929, Exotic Microlepidoptera, 3:5 53. (BM)

I884, Proc. Linnean Soc. N.S. Wales, 8: 474.

I932, Exotic Microlepidoptera, 4: 200. (BM)

I93I, Exotic Microlepidoptera, 4: 69. (BM)

I930, Exotic Microlepidoptera, 4: I9. (v)

I926, Exotic Microlepidoptera, 3: 256. (вм)

I88I, Proc. Linnean Soc. N.S. Wales, 6: 674.

I93I, Exotic Microlepidoptera, 4: I49. (BM)

I9I8, Ann. Transvaal Mus. 6: Io. (T)

I893, Proc. Linnean Soc. N.S. Wales, 7: 532.

I935, in Caradja and Meyrick, Materialien zu einer

Microlepidopteren Fauna der Chinesischen Provinzen

Kiangsu, Chekiang und Hunan, p. 64. (BM)

I920, Exotic Microlepidoptera, 2: 375.

I9I6, Exotic Microlepidoptera, I : 523. (вм)

I92I, Exotic Microlepidoptera, 2: 54 . 


\begin{tabular}{|c|c|}
\hline bisinuata & ACROCERCOPS \\
\hline bispiculata & GELECHIA \\
\hline bistrigata & PROTOLECHIA \\
\hline isulcata & PROCOMETIS \\
\hline bitabulata & LABDIA \\
\hline bivia & DRAGMATUCHA \\
\hline blanda & PHTHORACMA* \\
\hline blandula & STENOMA \\
\hline blapsigona & PHTHORIMAEA \\
\hline blepharacma & TELETHERA* \\
\hline blepharopa & ANACAMPSIS \\
\hline blepharopis & BACTRA \\
\hline bletrias & GELECHIA \\
\hline bolidias & HYPONOMEUTA \\
\hline bolistis & STENOMA \\
\hline bombaulia & ACROLOPHUS \\
\hline borboropis & TINEA \\
\hline bostrychota & MELASINA \\
\hline botanodes & CHANYSTIS \\
\hline botryitis & PAROCYSTOLA \\
\hline bovina & THISIZIMA \\
\hline brabylitis & STROBISIA \\
\hline bracculata & BRACHMIA \\
\hline brachiata & MELASINA \\
\hline brachyacma & BORKHAUSENIA \\
\hline brachyanches & URODUS \\
\hline brachyaspis & MONOPIS \\
\hline brachyaula & GLYPHIPTERYX \\
\hline brachycasis & AMIANTASTIS \\
\hline brachycentra & CARPOSINA \\
\hline brachychlaena & METACHANDA \\
\hline brachychlora & TINEA \\
\hline brachyclina & COSMOPTERYX \\
\hline brachyclista & ODITES \\
\hline brachyctenis & MELASINA \\
\hline brachydelta & GLYPHIPTERYX \\
\hline brachyglypta & ACROCERCOPS \\
\hline brachygramma & STATHMOPODA \\
\hline brachygrapha & DICHOMERIS \\
\hline brachygrapta & ALLOCLITA \\
\hline brachyloga & LINOCLOSTIS \\
\hline brachymetra & DICHOMERIS \\
\hline brachymochla & STATHMOPODA \\
\hline brachymorpha & PLATYPTILIA \\
\hline achymorpha & SIMAETHIS \\
\hline brachynta & PHLOEOGRAPTIS \\
\hline chyomis & NEPHOGENES \\
\hline brachyota & SAROPLA \\
\hline
\end{tabular}

[PATTALODES*]
I921, Exotic Microlepidoptera, 2: 4I6. (BM) 1923, Exotic Microlepidoptera, 3: 23. (BM) I92I, Exotic Microlepidoptera, 2: 427. (BM) I890, Trans. R. Soc. S. Australia, I3: 7 I. I935, Exotic Microlepidoptera, 4: 605. I9I8, Ann. Transvaal Mus. 6: 25. (T) I921, Ann. Transvaal Mus. 8: 87. (T) I9I5, Exotic Microlepidoptera, I: 456. (Bм) I916, Exotic Microlepidoptera, I: 569. (вм) I9I3, Exotic Microlepidoptera, I: I55. I9I4, Trans. Ent. Soc. London, I9I4: 259. (BM) I9II, Proc. Linnean Soc. N.S. Wales, 36: 255. I9I3, Ann. Transwaal Mus. 3: 292. (T) I9I3, Exotic Microlepidoptera, I: I37. (вм) I925, Exotic Microlepidoptera, 3: 203. (BM) I922, Exotic Microlepidoptera, 2: 608. (P) I9I9, Exotic Microlepidoptera, 2: 275. (BM) I920, Voyage de Ch. Aliuaud et $R$. Jeannel en Afrique Oriental, II, Microlepidoptera, p. II3. (P) I9II, Trans. Linnean Soc. London, I4:28I. (BM) I9I4, Exotic Microlepidoptera, I : 245. I928, Exotic Microlepidoptera, 3: 428.

I9I I, Journ. Bombay Nat. Hist. Soc. 20: 729. (Bм) I9II, Journ. Bombay Nat. Hist. Soc. 20: 7IO. (BM) I9I9, Exotic Microlepidoptera, 2: 267. I909, Trans. New Zealand Inst. 4I: I3. I93I, Exotic Microlepidoptera, 4:89. (v) I921, Zool. Meded. 6: I97. (L) I907, Proc. Linnean Soc. N.S. Wales, 32: I27. I932, Exotic Microlepidoptera, 4: 348. (BM) I9I4, Ann. Transvaal Mus. 4: I88. (T) I930, Trans. Ent. Soc. London, 78: 3I4. (P) I905, Journ. Bombay Nat. Hist. Soc. I6: 6I8. I933, Exotic Microlepidoptera, 4: 426. I928, Exotic Microlepidoptera, 3: 434. (Bм) I909, Ann. South African Mus. 5: 364. (SA) I9I6, Trans. Proc. New Zealand Inst. 48: 4 I8. I93I, Exotic Microlepidoptera, 4: 48. I9I3, Exotic Microlepidoptera, I: 88. I920, Exotic Microlepidoptera, 2: 305. (BM) I925, Bull. Soc. R. Ent. Egypte, 9: 213. I9I7, Exotic Microlepidoptera, 2: 54. (BM) 1923, Exotic Microlepidoptera, 2: 620. (BM) 1937, Exotic Microlepidoptera, 5: 89. I888, Trans. Ent. Soc. London, I888: 240. I9I5, Trans. Ent. Soc. London, I9I5: 216. (BM) I904, Proc. Linnean Soc. N.S. Wales, 29: 394. I889, Proc. Linnean Soc. N.S. Wales, (Ser. 2) 3: 1603. I889, Proc. Linnean Soc. N.S. Wales, (Ser. 2) 3: I648. 
brachypepla brachypetala brachyplaca brachyplecta brachyplectra brachypogon brachyptila brachyptycha brachyptycta

brachyrrhiza brachysaris brachyscia brachysema

brachysticta

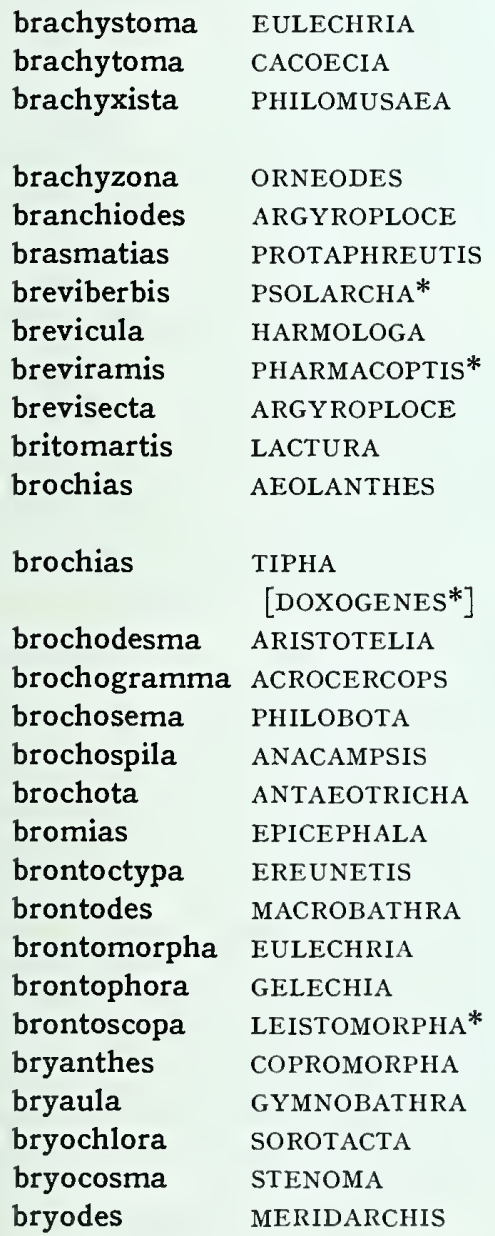

I883, Proc. Linnean Soc. N.S. Wales, 7:524.

I9I2, in Wytsman, Genera Insectorum, fasc. I33, p. 6. 1926, Exotic Microlepidoptera, 3: 228. (BM)

I928, Exotic Microlepidoptera, 3:4I2.

I921, Zool. Meded. 6: I86. (L)

1937, Exotic Microlepidoptera, 5:9I. (T)

I9I6, Exotic Microlepidoptera, I: 584. (BM)

I9I I, Proc. Linnean Soc. N.S. Wales, 36:246. (BM)

I938, Institut des Parcs Nationaux du Congo Belge,

fasc. I4, p. 6. (c)

I92I, Exotic Microlepidoptera, 2: 43I. (BM)

I9I6, Exotic Microlepidoptera, I: 504. (BM)

I934, Exotic Microlepidoptera, 4: 46I.

1938, Institut des Parcs Nationaux du Congo Belge, fasc. I4, p. I8. (c)

I935, in Caradja and Meyrick, Materialien zu einer Microlepidopteren Fauna der Chinesischen Provinzen Kiangsu, Chekiang und Hunan, p. 55.

I9I5, Exotic Microlepidoptera, I: 299.

I932, Exotic Microlepidoptera, 4: 34I. (BM)

I931, Ann. Mus. Nac. Hist. Nat. Buenos Aires, 36: 395.

(BM)

I920, Ann. South African Mus. I7: 293. (SA)

I9Io, Trans. Ent. Soc. London, I9Io: 435. (BM)

I930, Trans. Ent. Soc. London, 78: 323. (P)

I933, Exotic Microlepidoptera, 4: 4I2. (C)

I92I, Trans. Proc. New Zealand Inst. 53: 334.

I932, Exotic Microlepidoptera, 4:277. (v)

I930, Exotic Microlepidoptera, 3: 6оI. (BM)

I924, Exotic Microlepidoptera, 3: I26.

I938, in Caradja and Meyrick, Deuts. Ent. Zeit. Iris, 52: II. (BM)

I905, Journ. Bombay Nat. Hist. Soc. I6: 594. (BM) I908, Journ. Bombay Nat. Hist. Soc. I8: 438. (BM) I9I4, Exotic Microlepidoptera, I: 285.

I884, Proc. Linnean Soc. N.S. Wales, 8: 500. I9I4, Trans. Ent. Soc. London, I9I4: 265. (BM) I9I5, Exotic Microlepidoptera, I: 396. (BM) I9Io, Rec. Indian Mus. 5:227. (K)

I88I, Proc. Linnean Soc. N.S. Wales, 5: 259. I886, Proc. Linnean Soc. N.S. Wales, Io: 82 I. 1883, Proc. Linnean Soc. N.S. Wales, $7: 535$. I886, Trans. Proc. New Zealand Inst. I8: I68. I884, Proc. Linnean Soc. N.S. Wales, 8: 510. I926, Exotic Microlepidoptera, 3: 245. (BM) I905, Trans. Ent. Soc. London, I905: 238. I922, Trans. Ent. Soc. London, I922: 76. (BM) I9I6, Exotic Microlepidoptera, I: 535. (BM) I907, Journ. Bombay Nat. Hist. Soc. I7: 98I. (BM) 


$\begin{array}{ll}\text { bryographa } & \text { PERONEA } \\ \text { bryoleuca } & \text { CEROSTOMA } \\ \text { bryometalla } & \text { POLYORTHA } \\ \text { bryomima } & \text { PYRODERCES } \\ \text { bryomorpha } & \text { ACROCLITA } \\ \text { bryonephela } & \text { MERIDARCHIS } \\ & \\ \text { bryonota } & \text { HILAROGRAPHA } \\ \text { bryopa } & \text { ACROCLITA } \\ \text { bryophanes } & \text { STENOMA } \\ \text { bryophylla } & \text { PHYCOMORPHA } \\ \text { bryoscopa } & \text { ARGYROPLOCE } \\ \text { bryoxyla } & \text { STENOMA } \\ \text { bubalopa } & \text { THISIZIMA } \\ \text { bucolica } & \text { GNORIMOSCHEMA } \\ \text { bullata } & \text { CARPOSINA } \\ \text { bullata } & \text { THIOTRICHA } \\ \text { bullifera } & \text { CHRYSONOMA } \\ \text { bullifera } & \text { PLATYPTILIA } \\ \text { bulligera } & \text { TERTHREUTIS } \\ \text { bullulata } & \text { LECITHOCERA } \\ \text { butyracma } & \text { STATHMOPODA } \\ \text { butyranthes } & \text { TIQUADRA } \\ \text { butyropa } & \text { IDIOPHANTIS } \\ & \text { ONEBALA } \\ & \text { SHYPEROCHI }\end{array}$

butyropis

butyrota

byblinopa

byrseis

byrseuta

byrsina

byrsinitis

byrsinopa

brysochra

byrsocyma

byrsodepta

byrsodes

byrsopola

byrsostola

byrsoxantha

byssina

byssinodes

byssophanes

bythitis

bythochroa

bythodes

bythota
[HYPEROCHTHA*]

CONOPOTARSA*

STENOMA

SCIEROPEPLA

MELASINA

ECLACTISTIS*

AGRIOPHARA

STENOMA

TINEA

EULECHRIA

HYPERCALIIA

BLASTOBASIS

PERIACMA

PROTERODESMA*

MACROCORYSTIS*

TRICHOTAPHE

ONEBALA

CROBYLOPHORA

PTILOGENES

STENOMA

GONIOTERMA

ACROLEPIA

ONEBALA
Igog, Trans. Ent. Soc. London, Igog: I6. (BM)

I921, Exotic Microlepidoptera, 2: 440.

I932, Exotic Microlepidoptera, 4: 343. (BM)

I897, Proc. Linnean Soc. N.S. Wales, 22: 354.

I93 I, Exotic Microlepidoptera, 4: I26. (v)

I938, in Caradja and Meyrick, Deuts. Ent. Zeit. Iris,

52: I4.

I92I, Exotic Microlepidoptera, 2: 479. (BM)

I9I I, Proc. Linnean Soc. N.S. Wales, 36: 242.

I9I5, Exotic Microlepidoptera, I: 477.

I927, Insects of Samoa, 3, Lepidoptera, fasc. 2, p. 96. (BM)

I928, Exotic Microlepidoptera, 3: 445. (BM)

I9I5, Exotic Microlepidoptera, I: 437. (BM)

I9I I, Journ. Bombay Nat. Hist. Soc. 2I : I25.

I904, Proc. Linnean Soc. N.S. Wales, 29: 316.

I9I3, Exotic Microlepidoptera, I: 98. (BM)

I904, Proc. Linnean Soc. N.S. Wales, 29: 296.

I920, Exotic Microlepidoptera, 2: 3I4.

I9I8, Ann. Transvaal Mus. 6: 7. (T)

I928, Exotic Microlepidoptera, 3: 459. (BM)

I9Io, Journ. Bombay Nat. Hist. Soc. 20: 443. (BM)

I9I3, Exotic Microlepidoptera, I : 92.

I93I, Exotic Microlepidoptera, 4:97. (v)

I9I3, Ann. Transvaal Mus. 3:285. (T)

I9Io, Journ. Bombay Nat. Hist. Soc. 20: 458. (BM)

I9I3, Exotic Microlepidoptera, I : I52.

I929, Trans. Ent. Soc. London, 76: 5I6. (BM)

I925, Exotic Microlepidoptera, 3: I54.

I934, Exotic Microlepidoptera, 4: 5I8. (c)

I9I3, Exotic Microlepidoptera, I: I34.

I907, Journ. Bombay Nat. Hist. Soc. I8: I5I. (BM)

I9I2, Trans. Ent. Soc. London, I9II: 7I3. (BM)

I933, Exotic Microlepidoptera, 4: 4I3.

I9I5, Exotic Microlepidoptera, I: 30I.

I92 I, Exotic Microlepidoptera, 2:388. (BM)

I9I3, Ann. Transvaal Mus. 3: 314. (T)

I9Io, Journ. Bombay Nat. Hist. Soc. 20: I58. (BM)

I909, Subantarctic Is. New Zealand, I: 74.

I93I, Exotic Microlepidoptera, 4: 49.

I9I8, Ann. Transvaal Mus. 6: 23. (T)

I9Io, Journ. Bombay Nat. Hist. Soc. 20: 457. (BM)

I9I4, Exotic Microlepidoptera, I: 208.

I926, Exotic Microlepidoptera, 3: 230. (BM)

I9I5, Exotic Microlepidoptera, I: 443. (BM)

I9I5, Exotic Microlepidoptera, I: 385. (BM)

I9I9, Exotic Microlepidoptera, 2: 227. (BM)

I9I6, Exotic Microlepidoptera, I : 576. (BM) 


\begin{tabular}{|c|c|c|}
\hline cachrydias & DICHOMERIS & I9I4, Trans. Ent. Soc. London, I9I4: 283. \\
\hline aduca & ONEBALA & I9Iо, Journ. Bombay Nat. Hist. Soc. 20: 454. (вм) \\
\hline caeca & MACHLOTRICHA* & I9I2, Ann. South African Mus. Io: 62. (SA) \\
\hline aecata & AGRIOPHARA & I9I6, Exotic Microlepidoptera, I: 487. (Вм) \\
\hline lecigena & GELECHIA & I9I8, Exotic Microlepidoptera, 2: I34. \\
\hline aecilia & LECITHOCERA & I9I8, Exotic Microlepidoptera, 2: IIO. (BM) \\
\hline aecinervis & HYPOSMOCOMA & I928, Proc. Hawaiian Ent. Soc. 7: I03. (sP) \\
\hline lecivaga & APATETRIS & I928, Exotic Microlepidoptera, 3: 48o. (Bм) \\
\hline aelata & TELPHUSA & I9I3, Ann. Transvaal Mus. 3:287. (т) \\
\hline aelatella & SAROPLA* & I884, Proc. Linnean Soc. N.S. Wales, 9: 745. \\
\hline elebs & ELACHISTA & I933, Exotic Microlepidoptera, 4:360. \\
\hline estis & IMMA & I9o6, Trans. Ent. Soc. London, I906: I82. (BM) \\
\hline elicola & BRENTHIA & I9Io, Trans. Ent. Soc. London, I9Io: 468. \\
\hline teligena & ZALITHIA & I922, Trans. Ent. Soc. London, I922: IOI. (BM) \\
\hline ementaria & MERIDARCHIS & I9I I, Trans. Linnean Soc. London, I4: 266. (Bм) \\
\hline lementosa & ACROCERCOPS & I9I5, Trans. Ent. Soc. London, I9I5: 228. \\
\hline ementosa & CACOECIA & I9o8, Journ. Bombay Nat. Hist. Soc. I8: 6I5. (Bм) \\
\hline ochytis & STENOMA & I9I5, Exotic Microlepidoptera, I: 4I5. (BM) \\
\hline aenodora & NEPTICULA & I9o6, Trans. R. Soc. S. Australia, 3o: 58. \\
\hline aenologa & HIEROXESTIS & I9I5, Exotic Microlepidoptera, I: $36 \mathrm{I}$. \\
\hline aenotheta & GRACILARIA & I88I, Proc. Linnean Soc. N.S. Wales, 5: I48. \\
\hline la & TINEA & I927, Exotic Microlepidoptera, 3: 323. (BM) \\
\hline saerulea & CYPHOSTICHA & I9I2, Exotic Microlepidoptera, I: 26. \\
\hline aerulescens & TRICHOTAPHE & I9I3, Journ. Bombay Nat. Hist. Soc. 22: I8o. (BM) \\
\hline caerulipalpis & COMPSISTIS & I92I, Exotic Microlepidoptera, 2: 396 . (BM) \\
\hline caesarea & STENOMA & I9I5, Exotic Microlepidoptera, I: 450. (BM) \\
\hline caesia & STENOMA & I9I5, Exotic Microlepidoptera, I: 449. (BM) \\
\hline calais & PTEROPHORUS & I930, Exotic Microlepidoptera, 3: 570. (вм) \\
\hline calamaea & PHILOBOTA & I884, Proc. Linnean Soc. N.S. Wales, 8: 492. \\
\hline lamias & TRACHYCENTRA* & I886, Trans. Ent. Soc. London, I886: 288 . \\
\hline lamitosa & STICHOTACTIS* & I930, Exotic Microlepidoptera, 3: 563. (вм) \\
\hline calamogonus & MEGACRASPEDUS & I886, Trans. Proc. New Zealand Inst. I8: I63. \\
\hline & ERIDACHTHA & $\begin{array}{l}\text { I920, Voyage de Ch. Alluaud et } R \text {. Jeannel en Afrique } \\
\text { Oriental, II, Microlepidoptera, p. } 76 . \text { ( }(\mathrm{P})\end{array}$ \\
\hline calamoplecta & BACTRA & $\begin{array}{l}\text { I934, in Caradja and Meyrick, Deuts. Ent. Zeit. Iris, } \\
\text { 48: 33. }\end{array}$ \\
\hline iris & EPICEPHALA & I9o8, Journ. Bombay Nat. Hist. Soc. I8: 8I3. \\
\hline aea & TELPHUSA & I9I3, Ann. Transvaal Mus. 3: 286. (т) \\
\hline alathisca & HILAROGRAPHA & I9o9, Journ. Bombay Nat. Hist. Soc. I9: 427. (BM) \\
\hline alathiscias & PROMALACTIS & I937, Exotic Microlepidoptera, 5: I02. (BM) \\
\hline alcarata & HYPONOMEUTA & I924, Exotic Microlepidoptera, 3: II7. (BM) \\
\hline calcaria & PHYLLOBROSTIS & I9II, Ann. Transvaal Mus. 2: 237 . (T) \\
\hline calceata & AUTOSTICHA & I9o8, Journ. Bombay Nat. Hist. Soc. I8: 456. (Bм) \\
\hline ceata & TMETOCERA & I907, Journ. Bombay Nat. Hist. Soc. I8: I4I. (BM) \\
\hline chantis & ARGYROPLOCE & I9I4, Exotic Microlepidoptera, I: I96. (BM) \\
\hline calciflua & SCYTHRIS & I92I, Ann. Transvaal Mus. 8: I I5. (T) \\
\hline ularis & SETOMORPHA & I906, Trans. R. Soc. S. Australia, 30: 66. \\
\hline culata & OPOGONA & I9I9, Exotic Microlepidoptera, 2: 287. \\
\hline culata & TORTRIX & I9Io, Proc. Linnean Soc. N.S. Wales, 35: 223. \\
\hline culatrix & ARISTOTELIA & I923, Exotic Microlepidoptera, 3: 7. (BM) \\
\hline
\end{tabular}


calculosa

calculosa

calefacta

calens

calida

calidaria

caligata

caliginea

caliginosa

caligula

calliactis

callianassa

callianthes

calliarcha

calliarma

callibrocha

callicarpa

callichalca

callichalca

callicharis

callichlora

callichlora

callichora

callichroa

callichroa

callichroma

callichrysa

callicirrha

callicitra

callicoma

callicosma

callicratis

callicrossa

callida

callidelta

callidora

callidora

callidoxa

callidus

callidyas

callierastis

callierga

calligastra

calligenes

calliglauca

calliglypta

calligramma

calligrapha
EUCOSMA

HYPONOMEUTA

PYRODERCES

ARISTOTELIA

LABDIA

HYPERCALLIA

AMYDRIA

STENOMA

NEPTICULA

LECITHOCERA

GLYPHIPTERYX

EOCHROA

HOPLITICA

SABATINCA

EUCOSMA

PYRODERCES

IDIOPHANTIS

COSMOPTERYX

MONOPIS

MICTOPSICHIA

ARGYROPLOCE

PROMENESTA

GRACILARIA

DIOCOSMA*

TORTRICOPSIS

ZALITHIA

ANASTATHMA*

TIMODORA

TONZA

STENOMA

LIMNOECIA

ARGYROPLOCE

GLYPHIPTERYX

CROCIDOSEMA

GLYPHIPTERYX

AEOLANTHES*

SETIOSTOMA

ZELLERIA

PTEROPHORUS

EUPSELIA

MACHIMIA

NOTODRYAS

LITHOTACTIS*

MACHAERITIS

NEOPSEUSTIS*

LASPEYRESIA

TELECRATES

EUCOSMA
I9I3, Ann. Transvaal Mus. 3: 27I. (T)

I9I4, Journ. Bombay Nat. Hist. Soc. 23: I27. (BM)

I922, Exotic Microlepidoptera, 2: 57I.

1923, Exotic Microlepidoptera, 3: 9. (BM)

I92I, Exotic Microlepidoptera, 2: 4II. (BM)

I92I, Exotic Microlepidoptera, 2: 388. (BM)

I9I3, Ann. Transvaal Mus. 3: 335. (T)

I930, Exotic Microlepidoptera, 4: 30. (V)

I92I, Ann. Transvaal Mus. 8: I40. (T)

I9I8, Exotic Microlepidoptera, 2: I04.

I9I4, Trans. Proc. New Zealand Inst. 46: II2.

I883, Proc. Linnean Soc. N.S. Wales, 7: $45^{\circ}$.

I889, Proc. Linnean Soc. N.S. Wales, (Ser. 2) 3: I595.

I9I2, Trans. Proc. New Zealand Inst. 44: I24.

I909, Ann. Transvaal Mus. 2: 8, pl. 3, fig. 5. (T)

I9I5, Exotic Microlepidoptera, I: 312.

I927, Insects of Samoa, 3, Lepidoptera, fasc. 2, p. 82. (BM)

I922, Exotic Microlepidoptera, 2: 568. (BM)

I9Io, Trans. Ent. Soc. London, I9Io: 474. (BM)

I92I, Exotic Microlepidoptera, 2: 477. (BM)

I909, Journ. Bombay Nat. Hist. Soc. I9: 603. (BM)

I9I5, Exotic Microlepidoptera, I: 4II. (BM)

I9I5, Trans. Ent. Soc. London, I9I5: 237.

I909, Ann. South African Mus. 5: 353. (SA)

I902, Trans. R. Soc. S. Australia, 26: I44.

I9I4, Trans. Ent. Soc. London, I9I4: 269. (BM)

I886, Trans. Ent. Soc. London, I886: 290.

I924, Exotic Microlepidoptera, 3:86. (BM)

I9I3, Exotic Microlepidoptera, I: I46.

I9I6, Exotic Microlepidoptera, I: 528. (BM)

I9I5, Exotic Microlepidoptera, I: 3 I8.

I909, Journ. Bombay Nat. Hist. Soc. I9: 605. (BM)

I907, Proc. Linnean Soc. N.S. Wales, 32: II9.

I9I7, Trans. Ent. Soc. London, I9I7: I8. (BM)

I922, Exotic Microlepidoptera, 2: 492. (BM)

I907, Journ. Bombay Nat. Hist. Soc. I7: 739. (BM)

I909, Trans. Ent. Soc. London, I909: 36. (BM)

I893, Proc. Linnean Soc. N.S. Wales, $7: 584$.

I9I3, Ann. Transvaal Mus. 3: 268. (T)

I9I5, Exotic Microlepidoptera, I: 306.

I920, Exotic Microlepidoptera, 2: 378. (BM)

I906, Trans. R. Soc. S. Australia, 30:56.

I938, in Caradja and Meyrick, Deuts. Ent. Zeit. Iris, $52: 16$.

I886, Proc. Linnean Soc. N.S. Wales, Io: 768 .

I909, Journ. Bombay Nat. Hist. Soc. I9: 436.

I932, Trans. Ent. Soc. London, 80: II3. (BM)

I89o, Trans. R. Soc. S. Australia, I3: 64 .

I9I2, Journ. Bombay Nat. Hist. Soc. 2I: 865. (BM) 


\begin{tabular}{|c|c|c|}
\hline calligrapha & LICHENAULA & I89o, Trans. R. Soc. S. Australia, I3: 48 . \\
\hline calligypsa & CATAMACTA & I926, Trans. Proc. New Zealand Inst. 56: 4I5. \\
\hline callimacha & ACROCERCOPS & I920, Exotic Microlepidoptera, 2: 293. (BM) \\
\hline callimeris & EULECHRIA & I888, Proc. Linnean Soc. N.S. Wales, (Ser. 2) 2: 958. \\
\hline callimetalla & PROMALACTIS & I9o8, Journ. Bombay Nat. Hist. Soc. I8: 806. (Bм) \\
\hline callimitris & LIMNOECIA & I897, Proc. Linnean Soc. N.S. Wales, $22: 365$. \\
\hline callimnestra & RHODANASSA* & I9I5, Exotic Microlepidoptera, I: 480. (BM) \\
\hline callinympha & COSMOPTERYX & I9I3, Ann. Transvaal Mus. 3: 307. (T) \\
\hline calliophthalma & PELTOPHORA & I889, Proc. Linnean Soc. N.S. Wales, (Ser. 2) 3: I639. \\
\hline callipetala & FABIOLA & I92I, Ann. Transvaal Mus. 8: 98. (T) \\
\hline calliphana & ZAPYRASTRA* & I889, Trans. Proc. New Zealand Inst. 2I: I72. \\
\hline calliphanes & ARGYRESTHIA & I9I3, Exotic Microlepidoptera, I: I35. (BM) \\
\hline calliphrontis & THUDACA & I893, Proc. Linnean Soc. N.S. Wales, 7: 576. \\
\hline calliplaca & PALAEOMIÇRA & I902, Ent. Mon. Mag. $38: 60$. \\
\hline callipleura & EUHAGENA & r932, Exotic Microlepidoptera, 4: 338 . \\
\hline loca & GYMNOBATHRA & r883, New Zealand Journ. Sci. I: 523. \\
\hline callipyra & LACTURA & r928, Exotic Microlepidoptera, 3: 4I6. \\
\hline callirrhabda & LECITHOCERA & $\begin{array}{l}\text { I936, in Caradja and Meyrick, Deuts. Ent. Zeit. Iris, } \\
\text { 50: I58. }\end{array}$ \\
\hline callirrhoa & PROCORONIS & I9II, Proc. Linnean Soc. N.S. Wales, 36: 250. \\
\hline callir & ARISTOTELIA & I923, Exotic Microlepidoptera, 3:9. (BM) \\
\hline callisarca & CRYPTOLECHIA & I924, Exotic Microlepidoptera, 3: I05. \\
\hline calliscelis & COPOCENTRA* & I909, Trans. Ent. Soc. London, r909: 34 . \\
\hline callisceptra & EULECHRIA & I 888, Proc. Linnean Soc. N.S. Wales, (Ser. 2) 2: 964. \\
\hline callischema & MACAROSTOLA & I908, Journ. Bombay Nat. Hist. Soc. I8: 827 . \\
\hline callisphena & LASPEYRESIA & I907, Journ. Bombay Nat. Hist. Soc. I8: I44. (BM) \\
\hline callispora & HELIOSTIBES & I9I2, Exotic Microlepidoptera, I: 4I. \\
\hline & $\begin{array}{l}\text { OCYSTOLA } \\
\text { [HIPPOMACHA*] }\end{array}$ & I885, Proc. Linnean Soc. N.S. Wales, 9: ro67. \\
\hline callistis & MESOLECTA & I889, Proc. Linnean Soc. N.S. Wales, (Ser. 2) 3: 1062. \\
\hline callistrepta & PYRODERCES & I9I7, Exotic Microlepidoptera, 2: 38 . \\
\hline callitechna & TELPHUSA & I9I4, Trans. Ent. Soc. London, I9I4: 236. (BM) \\
\hline calliteucha & EPICROESA & I9I2, Exotic Microlepidoptera, I: 58. \\
\hline callithea & GLYPHIPTERYX & I92I, Ann. Transvaal Mus. 8: II3. (T) \\
\hline callitricha & HOMALOXESTIS & r9Io, Journ. Bombay Nat. Hist. Soc. $20: 440$. (BM) \\
\hline callixantha & OCYSTOLA & I889, Proc. Linnean Soc. N.S. Wales, (Ser. 2) 3: I663. \\
\hline callixyla & LEPTOSACES* & r888, Trans. Proc. New Zealand Inst. 20: 78 . \\
\hline callizona & LASPE YRESIA & I9II, Proc. Linnean Soc. N.S. Wales, 36: 290. \\
\hline callizona & PLEUROTA & r884, Proc. Linnean Soc. N.S. Wales, 9: 753. \\
\hline callopis & STATHMOPODA & I9I3, Exotic Microlepidoptera, I: 9I. \\
\hline call & $\begin{array}{l}\text { OCYSTOLA } \\
\text { [OLBONOMA*] }\end{array}$ & rolepidoptera, \\
\hline calotropha & EULECHRIA & I883, Proc. Linnean Soc. N.S. Wales, 7: 536. \\
\hline calpidias & NEOSPASTIS & I9I7, Exotic Microlepidoptera, 2: 59. (BM) \\
\hline nnians & MOLOPOSTOLA & I926, Exotic Microlepidoptera, 3: 284. (BM) \\
\hline calycias & PRAYS & I907, Proc. Linnean Soc. N.S. Wales, 32: 76. \\
\hline calycocentra & HOROMERISTIS* & I93I, Exotic Microlepidoptera, 4: I87. (BM) \\
\hline calycodes & TINEA & I9Io, Trans. Ent. Soc. London, I9Io: 474. \\
\hline calycopa & ANASTREBLOTIS* & I927, Insects of Samoa, 3, Lepidoptera, fasc. 2 \\
\hline calycophtl & ACROCERCOPS & Exotic Microlepidoptera, 3 \\
\hline
\end{tabular}




\begin{tabular}{|c|c|}
\hline pis & LYONETIA \\
\hline calycospila & UTUCA \\
\hline calypso & COSMOPTERYX \\
\hline calypta & EREUNETIS \\
\hline calyptraea & STATHMOPODA \\
\hline yptrodes & TRICLONELLA \\
\hline alyptrophanes & PHTHEOCHROA \\
\hline camacinana & PROSELENA \\
\hline marina & ANTAEOTRICHA \\
\hline marodes & STENOMA \\
\hline camaropa & LASIOCHIRA* \\
\hline marota & TINEA \\
\hline amarotis & ARGYROPLOCE \\
\hline amatodes & PSEUDODOXIA \\
\hline melaea & EULECHRIA \\
\hline amelina & ADOXOPHYES \\
\hline amelodes & AMPHORITIS* \\
\hline amelopis & ATELOSTICHA \\
\hline ameraria & ACROCLITA \\
\hline camerata & EULIA \\
\hline camerata & SAPHENEUTIS* \\
\hline aminaea & ENAEMIA \\
\hline caminias & PHILOBOTA \\
\hline caminites & OXYPTILUS \\
\hline caminodes & HILAROGRAPHA \\
\hline caminopa & SISYROTARSA* \\
\hline caminopis & PROTOLECHIA \\
\hline caminora & STATHMOPODA \\
\hline mpestris & LASPEYRESIA \\
\hline campestris & MELASINA \\
\hline campsigramma & IMMA \\
\hline campsigrapha & PHRIXOSCELES \\
\hline ampsiptera & PLATYPTILIA \\
\hline campsiptila & XYROSARIS \\
\hline mptochrysa & ACROCERCOPS \\
\hline mptogramma & GELECHIA \\
\hline amptosema & LIMNOECIA \\
\hline mptosphena & PLATYPTILIA \\
\hline mptospila & STENOMA \\
\hline campyla & PHILOBOTA \\
\hline mpylocha & STATHMOPODA \\
\hline mpylodes & ANTAEOTRICHA \\
\hline ampylota & THUDACA \\
\hline mpylotis & COESYRA \\
\hline anachodes & GLYPHIPTERYX \\
\hline nachopis & ACROLEPIA \\
\hline ncanodes & MACHIMIA \\
\hline ncanopis & IMMA \\
\hline Incellata & CHELARIA \\
\hline
\end{tabular}

I920, Exotic Microlepidoptera, 2: 358. I932, Exotic Microlepidoptera, 4: 336. I9I9, Exotic Microlepidoptera, 2: 283. I9I I, Trans. Linnean Soc. London, I4: 30 I. I908, Rec. Indian Mus. 2: 397. (к) I922, Exotic Microlepidoptera, 2: 5IO. (BM) I932, Exotic Microlepidoptera, 4: 266. (v) I883, Proc. Linnean Soc. N.S. Wales, 7: I72. I9I5, Exotic Microlepidoptera, I: 40I. (BM) I9I5, Exotic Microlepidoptera, I: 478. (вM) I93I, in Caradja, Bull. Sect. sci. Acad. roum. I4: 7I. (BM) I9I I, Journ. Bombay Nat. Hist. Soc. 2I : II7. I936, Exotic Microlepidoptera, 4:6I2. (BM) I9I4, Exotic Microlepidoptera, I: 264. (BM) I888, Proc. Limnean Soc. N.S. Wales, (Ser. 2) 2 : 943. I89I, Trans. Proc. New Zealand Inst. 23: 97. 1905, Journ. Bombay Nat. Hist. Soc. I6: 60I. I920, Exotic Microlepidoptera, 2:378. (BM) I932, Exotic Microlepidoptera, 4: 221. (BM) I9I2, Ent. Mon. Mag. 48: 35. (Sub.) I907, Journ. Bombay Nat. Hist. Soc. I8: I55. I887, Proc. Linnean Soc. N.S. Wales, (Ser. 2) I: I044. (A) I889, Proc. Linnean Soc. N.S. Wales, (Ser. 2) 3: I624. I9o8, Trans. Ent. Soc. London, I907: 478.

I905, Journ. Bombay Nat. Hist. Soc. I6: 6ro. (BM) I937, Exotic Microlepidoptera, 5: 89.

I904, Proc. Linnean Soc. N.S. Wales, 29:340. I890, Trans. Proc. New Zealand Inst. 22: 2 I9. I9I4, Ann. Transvaal Mus. 4: I89. (T) I9I6, Exotic Microlepidoptera, I: 6II. I938, Trans. R. Ent. Soc. London, 87: 522. I908, Journ. Bombay Nat. Hist. Soc. I8: 8I4. 1907, Trans. Proc. New Zealand Inst. 39: II2. I9I4, Journ. Bombay Nat. Hist. Soc. 23: I24. (BM) I92I, Exotic Microlepidoptera, 2: 466. I931, Exotic Microlepidoptera, 4: 58. (BM) I897, Proc. Linnean Soc. N.S. Wales, 22: 366 . (MA) 1931, Ann. Mus. Nac. Hist. Nat. Buenos Aires, 36: 379. I925, Exotic Microlepidoptera, 3: 202. (BM) 1889, Proc. Limnean Soc. N.S. Wales, (Ser. 2) 3: I6I7. I889, Trans. Proc. New Zealand Inst. 21: I68. I9I6, Exotic Microlepidoptera, I: 494. (BM) I893, Proc. Linnean Soc. N.S. Wales, 7: 572. I9I4, Ann. South African Mus. Io: 248. (SA) Igo9, Journ. Bombay Nat. Hist. Soc. I9: 434. (BM) I9I3, Ann. Transvaal Mus. 3:325. (T) I9I8, Exotic Microlepidoptera, 2: 2I7. (BM) I906, Trans. Ent. Soc. London, I906: 204. (BM) I9I4, Trans. Ent. Soc. London, I9I4: 255. (BM) 


$\begin{array}{ll}\text { cancellata } & \text { ORNEODES } \\ \text { candace } & \text { CARPOSINA } \\ \text { candescens } & \text { NEMATOBOLA } \\ \text { candidata } & \text { BLASTOBASIS } \\ \text { caneodes } & \text { ANACAMPSIS } \\ \text { canephora } & \text { LINOSTICHA } \\ \text { canicoma } & \text { TINEA } \\ \text { canispersa } & \text { SCYTHRIS } \\ \text { canonias } & \text { STENOMA } \\ \text { canonica } & \text { STATHMOPODA } \\ \text { canonitis } & \text { PALAMERNIS* } \\ \text { cantatrix } & \text { STENOMA } \\ \text { canthararcha } & \text { ARGYROTOXA } \\ \text { cantharites } & \text { NEMOTOIS } \\ \text { cantharitis } & \text { APHANOXENA } \\ \text { cantharodes } & \text { TORTYRA } \\ \text { cantharopa } & \text { ATTERIA } \\ \text { canthelias } & \text { AROTROPHORA } \\ \text { canthonias } & \text { ACROCLITA } \\ \text { canthopa } & \text { OINOPHILA } \\ \text { capax } & \text { ACROLOPHUS } \\ \text { capitolina } & \text { CENARCHIS } \\ \text { capitulata } & \text { CYDIA } \\ \text { capnanthes } & \text { STRYPHNAULA* }\end{array}$

capnarcha capnaula capnias capnites capnobactra

$\begin{array}{ll}\begin{array}{l}\text { capnobaphes } \\ \text { capnobathra }\end{array} & \text { LABDIA } \\ \text { capnobola } & \text { STENOMA } \\ \text { capnochalca } & \text { POLYMNESTRA } \\ \text { capnocoma } & \text { STENOMA } \\ \text { capnocrossa } & \text { STENOMA } \\ \text { capnodes } & \text { AGRIOPHARA } \\ \text { capnodesma } & \text { ARGYROPLOCE } \\ \text { capnodyta } & \text { BORKHAUSENIA } \\ \text { capnogramma } & \text { TINEOLA } \\ \text { capnographa } & \text { DECADARCHIS } \\ \text { capnoleuca } & \text { EUCOSMA } \\ \text { capnomicta } & \text { HYPOPHRICTIS } \\ \text { capnopis } & \text { TINEOLA } \\ \text { capnopora } & \text { SYNTOMACTIS } \\ \text { capnoscia } & \text { CTENOCOMPA } \\ \text { capnosphaera } & \text { STENOMA } \\ \text { capnospila } & \text { PHALONIA } \\ \text { capnosticha } & \text { TORTRIX }\end{array}$

Igo8, Trans. Ent. Soc. London, Igo7: 5I0.

I932, Trans. Ent. Soc. London, 80: II4.

1893, Proc. Linnean Soc. N.S. Wales, 7: 593.

I922, Exotic Microlepidoptera, 2: 539. (BM)

I922, Trans. Ent. Soc. London, I922: 79. (Вм)

1883, Proc. Linnean Soc. N.S. Wales, 8: 339.

I9I I, Journ. Bombay Nat. Hist. Soc. 2 I : I20.

I9I3, Ann. Transvaal Mus. 3: 3I2. (T)

I9I3, Trans. Ent. Soc. London, I9I3: I87. (BM)

I897, Proc. Linnean Soc. N.S. Wales, 22: 326.

Igo6, Trans. Ent. Soc. London, Igo6: 206. (BM)

I925, Exotic Microlepidoptera, 3: 22I. (BM)

I937, Exotic Microlepidoptera, 5: I28. (c)

I928, Exotic Microlepidoptera, 3: 464 .

I9I6, Exotic Microlepidoptera, I : 490. (Вм)

I922, Exotic Microlepidoptera, 2: 484. (BM)

Igo9, Trans. Ent. Soc. London, Igog: I4. (BM)

Igro, Proc. Linnean Soc. N.S. Wales, 35: 263.

I920, Exotic Microlepidoptera, 2: 343. (BM)

I924, Trans. Ent. Soc. London, I923: 555.

I929, Trans. Ent. Soc. London, 76: 521. (BM)

I924, Trans. Ent. Soc. London, I923: 550. (BM)

I907, Journ. Bombay Nat. Hist. Soc. I8: I42. (BM)

I938, in Caradja and Meyrick, Deuts. Ent. Zeit. Iris,

$52: 20$.

I938, Trans. R. Ent. Soc. London, 87: 5I9.

I9I I, Journ. Bombay Nat. Hist. Soc. 20: 7I9. (BM)

I908, Journ. Bombay Nat. Hist. Soc. I 8: 826 .

I904, Proc. Linnean Soc. N.S. Wales, 29: 435.

1938, Institut des Parcs Nationaux du Congo Belge,

fasc. I4, p. 7 .

I935, Exotic Microlepidoptera, 4:605.

I930, Exotic Microlepidoptera, 3: 605. (BM)

I9I3, Trans. Ent. Soc. London, I913: I86. (BM)

I932, Exotic Microlepidoptera, 4: 207.

I93I, Exotic Microlepidoptera, 4: 37. (v)

I925, Exotic Microlepidoptera, 3: I97. (BM)

I89o, Trans. R. Soc. S. Australia, I3: 77 .

I922, Exotic Microlepidoptera, 2: 525. (P)

I906, Trans. R. Soc. S. Australia, 30: 35 .

I922, Exotic Microlepidoptera, 2: 594. (P)

I928, Exotic Microlepidoptera, 3: 402. (BM)

I932, Exotic Microlepidoptera, 4: 223.

I934, Exotic Microlepidoptera, 4: 48I.

I922, Exotic Microlepidoptera, 2: 593.

I897, Proc. Linnean Soc. N.S. Wales, 22: 384.

I9I9, Exotic Microlepidoptera, 2: 263.

I9I6, Exotic Microlepidoptera, I: 5I6. (BM)

I9I2, Exotic Microlepidoptera, I: 20. (BM)

I9I7, Trans. Ent. Soc. London, I9I7:8 (BM) 


\begin{tabular}{|c|c|}
\hline capnota & TISCHERIA \\
\hline capnozona & SYNCOPACMA* \\
\hline capraria & GLYPHIDOCERA \\
\hline capsaria & LYSIGRAPHA* \\
\hline capsellata & EULECHRIA \\
\hline capsiformis & STENOMA \\
\hline capsigera & LIMNOECIA \\
\hline capsulata & ANTAEOTRICHA \\
\hline captans & HYPERCALLIA \\
\hline captatrix & LACTURA \\
\hline captiva & CNEPHASIA \\
\hline capyra & ACROCLITA \\
\hline capyrodes & ANACAMPSIS \\
\hline capyrota & MELASINA \\
\hline carabodes & STENOMA \\
\hline carabopa & URODUS \\
\hline carabophanes & ANTAEOTRICHA \\
\hline caranaea & SPIROTERMA* \\
\hline carbanaria & ANARSIA \\
\hline carbasea & STENOMA \\
\hline carbunculata & ACROCERCOPS \\
\hline carceraria & ARGYROPLOCE \\
\hline carcerata & LECITHOCERA \\
\hline carcharias & CROCANTHES \\
\hline carcharias & EUCOSMA \\
\hline carcharitis & EUCOSMA \\
\hline carcharodes & EPICHORISTA \\
\hline carcharopa & ODITES \\
\hline carcharota & ACROCERCOPS \\
\hline carcinopa & CARPOSINA \\
\hline carcinopis & LECITHOCERA \\
\hline carcinota & LYONETIA \\
\hline cardamitis & PARECTOPA \\
\hline cardinalis & NEPHOGENES \\
\hline cardinata & CHOLOTIS \\
\hline cardinata & TREPSITYPA* \\
\hline carenota & GLYPHIPTERYX \\
\hline carinaria & PHILOBOTA \\
\hline carinata & EPITHECTIS \\
\hline lotta & XYSMATODOMA \\
\hline eola & HEMIMENE \\
\hline nicolor & ARGYROPLOCE \\
\hline rola & BRENTHIA \\
\hline paea & PSAMMORIS* \\
\hline carpalima & ACROCLITA \\
\hline alima & ANCYLIS \\
\hline phalea & PELTOPHORA \\
\hline hitis & ANTAEOTRICHA \\
\hline & APETHISTIS \\
\hline olog & ACROLOPHUS \\
\hline
\end{tabular}

I9I 5, Trans. Ent. Soc. London, I9I5: 247.

I938, Trans. R. Ent. Soc. London, 87:527.

1929, Trans. Ent. Soc. London, 76: 5II.

I9I4, Exotic Microlepidoptera, I: I85. (BM)

I9I3, Exotic Microlepidoptera, I: I57.

I930, Exotic Microlepidoptera, 4: 25. (BM)

I92I, Exotic Microlepidoptera, 2: 454.

I9I8, Exotic Microlepidoptera, 2: I99. (BM)

I93I, Exotic Microlepidoptera, 4: I2I. (v)

I924, Exotic Microlepidoptera, 3: I26.

I9I I, Ann. Transvaal Mus. 2: 224.

I9II, Proc. Linnean Soc. N.S. Wales, 36: 24 I.

I922, Trans. Ent. Soc. London, I922: 8o. (BM)

I9I4, Exotic Microlepidoptera, I: 2 I3.

I9I5, Exotic Microlepidoptera, I: 435. (BM)

I925, Exotic Microlepidoptera, 3: I29. (BM)

I932, Exotic Microlepidoptera, 4:289. (US)

I9I5, Exotic Microlepidoptera, I: 324.

I9I3, Ann. Transvaal Mus. 3: 299.

I9I5, Exotic Microlepidoptera, I: 426. (BM)

I9I2, Exotic Microlepidoptera, I: 24. (T)

I9I3, Ann. Transvaal Mus. 3: 276. (T)

I923, Exotic Microlepidoptera, 3: 39. (BM)

I9I0, Trans. Ent. Soc. London, I9Io: 444.

I9I2, Ent. Mon. Mag. 48: 35. (Sub.)

I923, Bull. Mus. Nat. Hist. Nat. Paris, 29:563.

I9I4, Trans. Proc. New Zealand Inst. 46: I04.

I9I4, Ann. Transvaal Mus. 4: I98. (T)

I912, Exotic Microlepidoptera, I: 22. (T)

1927. Insects of Samoa, 3, Lepidoptera, fasc. 2, p. 95. (BM)

I929, Exotic Microlepidoptera, 3: 522. (BM)

I9ro, Trans. Ent. Soc. London, I9Io: 374.

I92I, Ann. Transvaal Mus. 8: I22. (T)

I9I3, Exotic Microlepidoptera, I: I3I. (MA)

I9I8, Ann. Transvaal Mus. 6:28. (T)

I9I3, Exotic Microlepidoptera, I: 73. (BM)

I909, Journ. Bombay Nat. Hist. Soc. I9: 43I. (BM)

I9I3, Exotic Microlepidoptera, I: I25. (MA)

I9I2, Ann. Transvaal Mus. 3:64. (T)

I893, Proc. Linnean Soc. N.S. Wales, 7: 501.

I9I6, Exotic Microlepidoptera, 2: 29. (BM)

I93I, Exotic Microlepidoptera, 4: I33.

I9I2, Exotic Microlepidoptera, I: 53. (ВM)

I9o6, Journ. Bombay Nat. Hist. Soc. I7: I49. (BM)

I9I I, Proc. Linnean Soc. N.S. Wales, 36: 244.

I9I 2, Journ. Bombay Nat. Hist. Soc. 2I: 86I.

I884, Proc. Linnean Soc. N.S. Wales, 9: 73I.

I9I2, Trans. Ent. Soc. London, I9II : 7 IO. (BM)

I9o8, Journ. Bombay Nat. Hist. Soc. I8: 459. (BM)

I9I9, Exotic Microlepidotpera, 2: 280. (BM) 


carphologa
carphota
carpophthora
carpotoma
caryactis
carycastis
carycina
carycodes
caryocephala
caryochroa
caryocoma
caryocrossa
caryocryptis
caryodesma
caryodora
caryograpta
caryophragma
caryoplecta
caryornis

caryosema caryosphena caryospila caryoterma caryothicta

$\begin{array}{ll}\text { caryotis } & \text { TORTRIX } \\ \text { caryotrota } & \text { SCHOENOTENES } \\ \text { caseata } & \text { BONDIA } \\ \text { cassandra } & \text { XESTOCASIS } \\ \text { [CALIIXESTIS } & \text { ANACAMPSIS } \\ \text { cassidata } & \text { STENOMA } \\ \text { cassigera } & \text { ETHMIA } \\ \text { cassiopeia } & \text { FILINOTA } \\ \text { cassiteranthes } & \text { ARGYROPLOCE } \\ \text { cassiteris } & \text { LAMPROTEUCHA* } \\ \text { cassiterites } & \text { NEMOTOIS } \\ \text { cassiterota } & \text { LECITHOCERA } \\ \text { casta } & \text { PYROZELA } \\ \text { castalia } & \text { COLEOPHORA } \\ \text { castanea } & \text { AROTROPHORA } \\ \text { castanopis } & \text { DICHELOPA } \\ \text { castellana } & \text { STENOMA } \\ \text { castellata } & \text { ACROCERCOPS } \\ \text { castigata } & \text { CRYPTOLECHIA } \\ \text { castrigera } & \text { TELPHUSA } \\ \text { catacaracta } & \text { TRYMALITIS } \\ & \end{array}$

ARGYROPLOCE

TRICHOTAPHE

[MYROPHILA*]

EULIA

TINEOLA

EUTORNA*

ARGYROPLOCE

EUCOSMA

TORTRIX

STENOMA

CHELARIA

STENOMA

MYROPHILA

MYROPHILA

OXYPTILUS

PERONEA

OPOGONA

COMPSOLECHIA

ARGYROPLOCE
I9I2, Ent. Mon. Mag. 48: 35. (Sub.)

I9I5, Trans. Ent. Soc. London, I9I5: 253.

I932, Exotic Microlepidoptera, 4: 232.

I9I6, Exotic Microlepidoptera, I: 567. (BM)

I909, Journ. Bombay Nat. Hist. Soc. I9: 596. (BM)

helcystogramma I922, Trans. Ent. Soc. London, I922: I04. (BM)

ARGYROPLOCE

I9I4, Trans. Ent. Soc. London, I9I4: 280. (BM)

I926, Exotic Microlepidoptera, 3:256. (BM)

1937, Exotic Microlepidoptera, 5: 77. ( $(\mathrm{)}$

I889, Trans. Proc. New Zealand Inst. 2I: I58.

I9I8, Ann. Transvaal Mus. 6: II. (T)

I932, Exotic Microlepidoptera, 4: 307. (v)

I932, Exotic Microlepidoptera, 4:342. (BM)

I925. Exotic Microlepidoptera, 3: I84. (BM)

I9I3, Journ. Bombay Nat. Hist. Soc. 22: I64. (BM)

I930, Exotic Microlepidoptera, 4: 26. (v)

1923, Exotic Microlepidoptera, 2: 624. (BM)

I930, Ann. Naturhist. Mus. Wien, 44: 227. (v)

I935, in Caradja and Meyrick, Materialien zu einer

Microlepidopteren Fauna der Chinesischen Provinzen

Kiangsu, Chekiang und Hunan, p. 46.

I93I, Exotic Microlepidoptera, 4: I38. (BM)

I937, Exotic Microlepidoptera, 5: I57. (o)

I920, Exotic Microlepidoptera, 2: 360. (BM)

I922, Trans. Ent. Soc. London, I922: 88. (Bм)

I920, Voyage de Ch. Alluaud et $R$. Jeannel en Afrique

Oriental, II, Microlepidoptera, p. 65. (P)

I9Io, Proc. Linnean Soc. N.S. Wales, 35:227. (MA)

I938, Trans. R. Ent. Soc. London, 87: 508. (BM)

I9Io, Proc. Linnean Soc. N.S. Wales, 35: I43.

I9I5, Exotic Microlepidoptera, I: 3 I7.

I9I4, Trans. Ent. Soc. London, I9I4: 257. (BM)

I9I5, Exotic Microlepidoptera, I: 479. (BM)

I927, Exotic Microlepidoptera, 3: 362.

I932, Exotic Microlepidoptera, 4: 279. (v)

I93I, Exotic Microlepidoptera, 4: I30. (BM)

I922, Exotic Microlepidoptera, 2: 586.

I907, Journ. Bombay Nat. Hist. Soc. I7: 99I.

I923, Exotic Microlepidoptera, 3: 40. (BM)

I907, Journ. Bombay Nat. Hist. Soc. I7: 747.

I930, Exotic Microlepidoptera, 3:561.

I9Io, Proc. Limnean Soc. N.S. Wales, 35: 263.

I934, Pacific Ent. Surv. Publ. 7, art. 28, p. 345. (вВ)

I916, Exotic Microlepidoptera, I: 516. (BM)

Igo8, Journ. Bombay Nat. Hist. Soc. I8: 8I9.

I926, Exotic Microlepidoptera, 3: 318. (BM)

I9I3, Ann. Transvaal Mus. 3: 287. (T)

I907, Journ. Bombay Nat. Hist. Soc. I8: I53. 


$\begin{array}{ll}\text { catacentra } & \text { PROCHOLA } \\ \text { catachalca } & \text { PHILOCORISTIS* } \\ \text { catachlora } & \text { EXINOTIS } \\ \text { catachrysa } & \text { PHILOBOTA } \\ \text { cataclasta } & \text { OPOGONA } \\ \text { cataclina } & \text { ONEBALA } \\ \text { catacrossa } & \text { GELECHIA } \\ \text { catadryas } & \text { TORTRIX } \\ \text { cataglypta } & \text { EUCOSMA }\end{array}$

catagnampta ACROLOPHUS

catagrapha catagrapta catalampra cataleuca catalina catalytica catamochla catapachna catapasta catapeltica cataphracta cataphracta cataplasta cataplecta catapneusta catapsecta catapsesta cataptila catapyrrha cataracta cataractias cataractis catarata catarrhacta catarrhactopa catascia cataspoda catasticta catastrepta catathectes catausta cataxantha cataxera cataxeranta cataxesta catectis
TINEA

PHILOBOTA

SCAEOPHANES*

SYNANTHEDON

TINEA

EUCOSMA

ARCATAULA*

HELICACMA*

ETHMIA

ARGYROPLOCE

XYSMATODOMA

EULECHRIA

DELOSCOPA*

PYRODERCES

STENOMA

IMMA

ELACHISTA

EUPERISSUS

TRYMALITIS

GRACILARIA

TORTRIX

ELACHISTA

PROTOLECHIA

ARGYROPLOCE

PHILOBOTA

SYNTOMACTIS

ATELOSTICHA

CNEPHASIA

OXYPTILUS

HYPERCALLIA

ODITES

PELTOPHORA

CHERSOMORPHA PANDEMIS

GLYPHIDOCERA
ENCENTROTIS*
I922, Exotic Microlepidoptera, 2: 582. (BM)

I927, Insects of Samoa, 3, Lepidoptera, fasc. 2, p. IO2. I9I6, Exotic Microlepidoptera, I: 599.

I889, Proc. Linnean Soc. N.S. Wales, (Ser. 2) 3: I625.

I9I5, Exotic Microlepidoptera, I: 358.

1923, Exotic Microlepidoptera, 3: 44. (BM)

I927, Exotic Microlepidoptera, 3: 347. (BM)

I937, Exotic Microlepidoptera, 5: I27. (T)

I935, in Caradja and Meyrick, Materialien zu einer

Microlepidopteren Fauna der Chinesischen Provinzen

Kiangsu, Chekiang und Hunan, p. 55. (BM)

I930, Ann. Naturhist. Mus. Wien, 44:267, pl. I, fig. 40.

( $\mathrm{v})$

I921, Ann. Transvaal Mus. 8: 65. (T)

I927, Exotic Microlepidoptera, 3: 332. (T)

I884, Proc. Linnean Soc. N.S. Wales, 8: 478.

1932, Exotic Microlepidoptera, 4:227.

I926, Exotic Microlepidoptera, 3: 267.

I9I9, Exotic Microlepidoptera, 2: 247. (BM)

I932, Exotic Microlepidoptera, 4: 223. (BM)

I921, Ann. Transvaal Mus. 8: II8. (T)

I9I4, Exotic Microlepidoptera, I: 233. (вм)

I924, Exotic Microlepidoptera, 3: I I9. (BM)

I9I7, Trans. Ent. Soc. London, I9I7: 25. (BM)

I893, Proc. Linnean Soc. N.S. Wales, 7: 504.

I888, Proc. Linnean Soc. N.S. Wales, (Ser. 2) $2: 957$.

I934, Exotic Microlepidoptera, 4:5I7. (C)

I9I7, Exotic Microlepidoptera, 2: 39.

I9I5, Exotic Microlepidoptera, I: 452. (BM)

I934, Pacific Ent. Surv. Publ. 7, art. 28, p. 352. (вB)

I897, Proc. Linnean Soc. N.S. Wales, 22: 33.

I935, Proc. Hawaiian Ent. Soc. 9: 64. (SP)

I907, Journ. Bombay Nat. Hist. Soc. I8: I53. (BM)

I921, Ann. Transvaal Mus. 8: I22. (T)

I9Io, Proc. Linnean Soc. N.S. Wales, 35: 246.

I897, Proc. Linnean Soc. N.S. Wales, 22: 338.

I904, Proc. Linnean Soc. N.S. Wales, 29: 366.

I938, Trans. R. Ent. Soc. London, 87: 5 I2. (BM)

I884, Proc. Linnean Soc. N.S. Wales, 8: 476.

I897, Proc. Linnean Soc. N.S. Wales, 22: 383 .

1920, Exotic Microlepidoptera, 2: 379. (BM)

I926, Ann. South African Mus. 23: 326. (SA)

I933, Exotic Microlepidoptera, 4: 425.

I906, Journ. Bombay Nat. Hist. Soc. I7: 406. (BM)

I9I5, Exotic Microlepidoptera, I: 379.

I884, Proc. Linnean Soc. N.S. Wales, 9: 736.

I938, Trans. R. Ent. Soc. London, 87: 5I9. (BM)

I937, in Caradja and Meyrick, Deuts. Ent. Zeit. Iris,

5I: I74.

I923, Exotic Microlepidoptera, 3: 49. (BM) 


\begin{tabular}{|c|c|}
\hline categorica & RHYNCHOPHYLLIS* \\
\hline cateiata & COLONOPHORA* \\
\hline catenata & BRENTHIA \\
\hline catenata & CNEPHASIA \\
\hline catericta & GNOSIMACHA* \\
\hline cathagrista & ANTAEOTRICHA \\
\hline catharacma & COSMOPTERYX \\
\hline catharactis & ANTAEOTRICHA \\
\hline catharaspis & ANCYLIS \\
\hline catharausta & PACHYPSALTIS \\
\hline cathareuta & BEDELLIA \\
\hline cathareutis & EUCOSMA \\
\hline catharmosta & STENOMA \\
\hline catharodes & GELECHIA \\
\hline catharopa & AMATHYNTIS \\
\hline catharopis & IDIOSTYLA \\
\hline catharosema & STAGMATURGIS* \\
\hline catharostoma & ARGYROPLOCE \\
\hline catharota & STATHEROTIS \\
\hline catharotes & PSELNOPHORUS \\
\hline catharotis & HYPONOMEUTA \\
\hline
\end{tabular}

catharotorna ACROCLITA

catharurga
cathedraea
cathidrota
catholica
cathosiota
cato
catochopis
catoptrias
catoptrina
catorthota
caudata
caulota
caumatias
causidica
causodes
causterias
caustica
causticopis
caustocoma
caustogramma
caustonota

SPHALEROSTOLA*

TRICHOTAPHE
I932, Exotic Microlepidoptera, 4:289. (v)

I9I4, Exotic Microlepidoptera, I: 28I.

I907, Journ. Bombay Nat. Hist. Soc. I7: 748. (BM)

I9Io, Proc. Linnean Soc. N.S. Wales, 35:272. (MA)

I927, Exotic Microlepidoptera, 3: 354. (T)

1925, Exotic Microlepidoptera, 3: I65. (BM)

I909, Journ. Bombay Nat. Hist. Soc. I9: 4I5.

I930, Exotic Microlepidoptera, 4: 22.

I922, Exotic Microlepidoptera, 2: 5I4. (P)

I938, in Caradja and Meyrick, Deuts. Ent. Zeit. Iris, $52: 26$.

I9I I, Ann. Transvaal Mus. 2: 237. (T)

I938, Institut des Parcs Nationaux $d u$ Congo Belge,

fasc. I 4, p. 8.

I9I5, Exotic Microlepidoptera, I : 445. (BM)

I920, Ann. South African Mus. I7: 284. (SA)

IgI I, Journ. Bombay Nat. Hist. Soc. 2I : II3.

I922, Exotic Microlepidoptera, 2: 573. (BM)

I923, Exotic Microlepidoptera, 3: 25. (BM)

I921, Zool. Meded. 6: I57. (L)

I928, Exotic Microlepidoptera, 3: 443. (BM)

I908, Trans. Ent. Soc. London, I907: 49I.

I935, in Caradja and Meyrick, Materialien zu einer

Microlepidopteren Fauna der Chinesischen Provinzen

Kiangsu, Chekiang und Hunan, p. 88. (BM)

I935, in Caradja and Meyrick, Materialien zu einer

Microlepidopteren Fauna der Chinesischen Provinzen

Kiangsu, Chekiang und Hunan, p. 53. (BM)

I923, Exotic Microlepidoptera, 3: 34. (BM)

I908, Journ. Bombay Nat. Hist. Soc. I8: 8I7.

I9I5, Exotic Microlepidoptera, I : 329. (BM)

I9I7, Exotic Microlepidoptera, 2: 46. (BM)

I925, Exotic Microlepidoptera, 3: 200. (BM)

1927, Exotic Microlepidoptera, 3: 374. (T)

I920, Exotic Microlepidoptera, 2: 307 . (BM)

I897, Proc. Linnean Soc. N.S. Wales, 22: 403.

I884, Proc. Linnean Soc. N.S. Wales, 9: 776.

I9I7, Exotic Microlepidoptera, 2: 80.

.I9I7, Exotic Microlepidoptera, 2: 37.

I9I 8, Ann. Transvaal Mus. 6: 27. (T)

I929, Trans. Ent. Soc. London, 76:5I3. (BM)

I9I0, Journ. Bombay Nat. Hist. Soc. 20: 45I. (BM)

I905, Journ. Bombay Nat. Hist. Soc. I6: 582.

I927, Insects of Samoa, 3, Lepidoptera, fasc. 2, p. 7 I.

I9I2, Trans. Proc. New Zealand Inst. 44: I24.

I937, Exotic Microlepidoptera, 5: 77. (c)

I928, Exotic Microlepidoptera, 3: 425.

I927, Exotic Microlepidoptera, 3: 366.

[COTYLOSCIA*] 


\begin{tabular}{|c|c|c|}
\hline caustopa & SEMIOCOSMA & I892, Trans. Proc. New Zealand Inst. 24: 219. \\
\hline :austopis & FALCULINA & I932, Exotic Microlepidoptera, 4: 288. (v) \\
\hline caustopola & EPICHTHONODES* & I938, Trans. R. Ent. Soc. London, 87: 525 . (BM) \\
\hline caustospila & LECITHOCERA & I9I8, Exotic Microlepidoptera, 2: I09. (BM) \\
\hline cauta & PHYLACODES* & I905, Trans. Ent. Soc. London, I905: 242. \\
\hline aveata & ARGYROPLOCE & I9I 2, Trans. Ent. Soc. London, I9I I: 69I. (BM) \\
\hline caveata & STATHMOPODA & I9I3, Exotic Microlepidoptera, I: 92. \\
\hline caveiformis & LECITHOCERA & I93I, Exotic Microlepidoptera, 4: 82. (BM) \\
\hline avillata & TORTRIX & I922, Exotic Microlepidoptera, 2: 497. (BM) \\
\hline cecropia & STENOMA & I9I6, Exotic Microlepidoptera, I: 509. (BM) (Sub.) \\
\hline cedrinopa & LABDIA & I928, Exotic Microlepidoptera, 3: 386. (вм) \\
\hline edrota & TORTRIX & I908, Proc. Zool. Soc. London, p. 722. (вм) \\
\hline edroxyla & ANTAEOTRICHA & I930, Exotic Microlepidoptera, 4: 23. (BM) \\
\hline celata & EULECHRIA & I9I3, Exotic Microlepidoptera, I: I60. (MA) \\
\hline elebrata & CENARCHIS & I924, Trans. Ent. Soc. London, I923: 55I. (Bм) \\
\hline elebratus & OXYPTILUS & I932, Exotic Microlepidoptera, 4: 249 . \\
\hline elerata & EUCOSMA & I9I2, Journ. Bombay Nat. Hist. Soc. 21: 863. (BM) \\
\hline eleris & PYCNOSTOLA & I920, Ann. South African Mus. I7: 280. (SA) \\
\hline eletica & SPHENOGRAPTIS* & I9I3, Exotic Microlepidoptera, I: I45. \\
\hline lidopa & SCYTHRIS & I92I, Exotic Microlepidoptera, 2: 44I. \\
\hline elidotis & ANTAEOTRICHA & I925, Exotic Microlepidoptera, 3: I69. (BM) \\
\hline elidotus & LIOPTILUS & I885, Trans. Proc. New Zealand Inst. I7: I25. \\
\hline cellaria & TRICHOTAPHE & I9I3, Joum. Bombay Nat. Hist. Soc. 22: I8o. (BM) \\
\hline cellicoma & ETHMIA & I93I, Exotic Microlepidoptera, 4: 89. (v) \\
\hline ellifera & POLYCHROSIS & I9I2, Journ. Bombay Nat. Hist. Soc. 21: 869. (BM) \\
\hline elligera & EBODA & I9I8, Exotic Microlepidoptera, 2: I7o. (BM) \\
\hline cellulata & MALLOBATHRA & I9I I, Journ. Bombay Nat. Hist. Soc. 21 : I25. \\
\hline cellulosa & CEROSTOMA & I928, Bull. Hill Mus. 2: 238. \\
\hline hora & TAENIOSTOLA* & I920, Exotic Microlepidoptera, 2: 327. (Bм) \\
\hline elyphodes & SITOTROGA & I909, Ann. Transvaal Mus. 2: Io. \\
\hline nchrias & BORKHAUSENIA & I909, Trans. New Zealand Inst. 4I: I3. \\
\hline chritis & BRACHMIA & I9I I, Journ. Bombay Nat. Hist. Soc. 20: 72I. (BM) \\
\hline hropis & ARGYROPLOCE & I920, Exotic Microlepidoptera, 2: 349 . (BM) \\
\hline Isoria & PLATYPTILIA & I9Io, Trans. Ent. Soc. London, I9Io: 367. \\
\hline oria & STENOMA & I9I5, Exotic Microlepidoptera, I: 460 . (BM) \\
\hline taurica & GORGOPIS & I92I, Ann. Transvaal Mus. 8: I4I. (T) \\
\hline etes & TRICHOPTILUS & I886, Trans. Ent. Soc. London, I886: i6. \\
\hline acma & TRICHOTAPHE & I923, Exotic Microlepidoptera, 3: 4. (Bм) \\
\hline raspis & EUCOSMA & I926, Sarawak Mus. Journ. 3: I5I. \\
\hline ntrias & TRICHERNIS* & I894, Trans. Ent. Soc. London, I894: 20. \\
\hline idias & EULECHRIA & I920, Exotic Microlepidoptera, 2: 373. \\
\hline -ifuga & TORTYRA & I9Io, Trans. Ent. Soc. London, I9Iо: 463. (вм) \\
\hline entritis & ARGYROPLOCE & I912, Journ. Bombay Nat. Hist. Soc. 21: 872. (Bм) \\
\hline entritis & THIOTRICHA & I908, Joum. Bombay Nat. Hist. Soc. I8: 439. (BM) \\
\hline ntrobola & COESYRA & I9I4, Ann. South African Mus. Io: 247. (SA) \\
\hline itrocrates & ALUCITA & I933, Exotic Microlepidoptera, 4: 425. (c) \\
\hline rocrossa & CALLIPRORA & I922, Trans. Ent. Soc. London, I922: 67. (BM) \\
\hline rodina & STENOMA & I9I6, Exotic Microlepidoptera, I: 53I. (Bм) \\
\hline rodoxa & METASTICHA* & I921, Zool. Meded. 6: Ig6. (L) \\
\hline trogramma & PSEPHOLOGA* & I92I, Exotic Microlepidoptera, \\
\hline
\end{tabular}




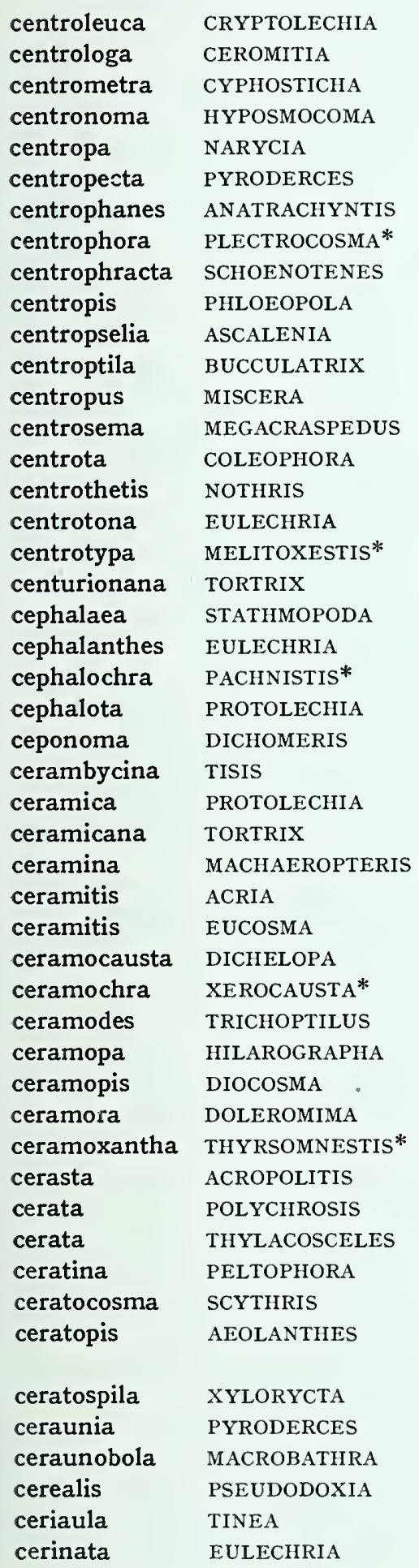

1922, Exotic Microlepidoptera, 2: 513. (P)

I937, Exotic Microlepidoptera, 5: I46. (T)

I920, Exotic Microlepidoptera, 2: 296. (BM)

1935, Proc. Hawaiian Ent. Soc. 9: 66. (SP)

1922, Exotic Microlepidoptera, 2: 605.

193I, Exotic Microlepidoptera, 4: 52 .

19I5, Exotic Microlepidoptera, I: 325.

I921, Ann. Transvaal Mus. 8: 75. (T)

I924, Exotic Microlepidoptera, 3: I 6 .

1889, Proc. Linnean Soc. N.S. Wales, (Ser. 2) 3: 1592.

1931, Exotic Microlepidoptera, 4: 54 .

1934, Exotic Microlepidoptera, 4: 462.

1907, Proc. Linnean Soc. N.S. Wales, 32 : I04.

I904, Proc. Linnean Soc. N.S. Wales, 29: 275.

19I7, Exotic Microlepidoptera, 2: $7 \mathrm{I}$.

I904, Proc. Linnean Soc. N.S. Wales, 29: 422.

I9I5, Exotic Microlepidoptera, I: 300.

I921, Ann. Transvaal Mus. 8: 76. (T)

I881, Proc. Linnean Soc. N.S. Wales, 6: 518 .

I897, Proc. Linnean Soc. N.S. Wales, 22: 319.

I888, Proc. Linnean Soc. N.S. Wales, (Ser. 2) 2: 949.

1907, Journ. Bombay Nat. Hist. Soc. 17: 737. (BM)

1904, Proc. Linnean Soc. N.S. Wales, 29: 373.

I9r8, Exotic Microlepidoptera, 2: I5I. (BM)

1926, Sarawak Mus. Journ. 3: I54.

1904, Proc. Linnean Soc. N.S. Wales, 29: 362.

I88I, Proc. Linnean Soc. N.S. Wales, 6: 5I2.

I9II, Journ. Bombay Nat. Hist. Soc. 2I: I 29.

r908, Journ. Bombay Nat. Hist. Soc. I8: 636. (BM)

I9I2, Ent. Mon. Mag. 48: 34. (Sub.)

1926, Trans. Ent. Soc. London, 74: 272. (Bм)

I929, Trans. Ent. Soc. London, $76: 520$.

I886, Trans. Ent. Soc. London, I886: 14.

1920, Exotic Microlepidoptera, 2: 329. (BM)

I909, Ann. South African Mus. 5: 353. (SA)

I902, Trans. R. Soc. S. A ustralia, 26: 160.

I929, Trans. Ent. Soc. London, 76: 5 I4.

I9ıо, Proc. Linnean Soc. N.S. Wales, 35: I76.

r909, Journ. Bombay Nat. Hist. Soc. I9: 587. (BM)

I913, Exotic Microlepidoptera, I : 95.

1884, Proc. Linnean Soc. N.S. Wales, 9: 737.

1897, Proc. Linnean Soc. N.S. Wales, 22: 424.

I934, in Caradja and Meyrick, Deuts. Ent. Zeit. Iris, 48: 39 .

I9I5, Exotic Microlepidoptera, I: 375.

1897, Proc. Linnean Soc. N.S. Wales, 22: 346.

I886, Proc. Linnean Soc. N.S. Wales, ro: 8 I8.

I9I4, Exotic Microlepidoptera, r: 263. (BM)

r914, Exotic Microlepidoptera, I: 2I I.

I9I4, Exotic Microlepidoptera, I: 239. (BM) 


\begin{tabular}{|c|c|}
\hline cerinaula & COLEOPHORA \\
\hline Eerinopa & TINEA \\
\hline cerinopa & TYMBARCHA $*$ \\
\hline cerinura & BRACHMIA \\
\hline ceriochranta & ANATHYRSOTIS* \\
\hline iocosma & LABDIA \\
\hline ceriodes & EUCOSMA \\
\hline cerioschema & HOMONA \\
\hline rochlora & CEROMITIA \\
\hline erochra & GLYPHIDOCERA \\
\hline cerocrossa & PARALECTA \\
\hline rodectis & CRYPSITHYRIS \\
\hline rodelta & OPOGONA \\
\hline rographa & EUCOSMA \\
\hline erograpta & CALLICOPRIS* \\
\hline romorpha & PTOCHORYCTIS \\
\hline rophaea & MACHIMIA \\
\hline rophanes & ISOCHORISTA \\
\hline roplasta & PERIACMA \\
\hline roxesta & HOMALOXESTIS \\
\hline rozona & PSALTICA \\
\hline rta & OINOPHILA \\
\hline rtatrix & MELASINA \\
\hline rtifica & ORNEODES \\
\hline rtificata & SAPHENEUTIS \\
\hline rtiorata & STENOMA \\
\hline russata & TORTRIX \\
\hline rvicalis & PTEROPHORUS \\
\hline rvicolor & ACROLOPHUS \\
\hline estrota & GLYPHIPTERYX \\
\hline trata & TORTRIX \\
\hline utholychna & BRENTHIA \\
\hline alarodes & PARALECTA \\
\hline halarodesma & ACROLEPIA \\
\hline ralastis & STENOMA \\
\hline halazombra & SPILOGENES* \\
\hline alazopa & PTOCHORYCTIS \\
\hline alcantha & PHARMACIS \\
\hline halcanthes & GRACILARIA \\
\hline chalcaspis & PROTOMACHA* \\
\hline alcias & PSEUDODOXIA \\
\hline alcistis & CHEREUTA \\
\hline lcitis & NEPTICULA \\
\hline lcitis & SPILONOTA \\
\hline 1cobathra & TORTYRA \\
\hline lcocapna & CEROMITIA \\
\hline lcocausta & ARGYRESTHIA \\
\hline
\end{tabular}

I936, Exotic Microlepidoptera, 5:63. I9I9, Exotic Microlepidoptera, 2: 245.

I908, Journ. Bombay Nat. Hist. Soc. I8: 622. (BM) I923, Exotic Microlepidoptera, 3: 47. (BM) I939, Trans. R. Ent. Soc. London, 89: 55. (вм) I934, Pacific Ent. Surv. Publ. 7, art. 28, p. 347. (Bв) I909, Journ. Bombay Nat. Hist. Soc. I9: 607. (Bм) I934, Exotic Microlepidoptera, 4: 526. (BM) I921, Ann. Transvaal Mus. 8: I39. (T) I929, Trans. Ent. Soc. London, 76: 5II. I938, Trans. R. Ent. Soc. London, 87: 5I4. I921, Ann. Transvaal Mus. 8: I26. (T) I9I I, Journ. Bombay Nat. Hist. Soc. 2 I : II2. I907, Journ. Bombay Nat. Hist. Soc. I7: 977. (Bм) I938, Trans. R. Ent. Soc. London, 87:5I5. I923, Exotic Microlepidoptera, 2:6I2. (BM) I9I4, Exotic Microlepidoptera, I: I82. (BM) I9Io, Proc. Linnean Soc. N.S. Wales, 35: I65. I9Io, Journ. Bombay Nat. Hist. Soc. 20: I59. (BM) I9I8, Exotic Microlepidoptera, 2: I02. (BM) I9I4, Exotic Microlepidoptera, I: 262. (Bм) I9I8, Ann. Transvaal Mus. 6:57. (T) I9I6, Exotic Microlepidoptera, I: 6I2. I9o9, Ann. Transvaal Mus. 2: 4, pl. 2, fig. 4. (T) I9I8, Ann. Transvaal Mus. 6: 45. (T) I932, Exotic Microlepidoptera, 4: 296. (v) I9Io, Proc. Linnean Soc. N.S. Wales, 35:234. (MA) I932, Exotic Microlepidoptera, 4: 25I. (v) I93I, Mitteil. Münch. Ent. Gesells. 2I: 39. I9I5, Trans. Ent. Soc. London, I9I5: 22I. (BM) I9Io, Proc. Linnean Soc. N.S. Wales, 35: 230. I9I5, Trans. Ent. Soc. London, p. 2 I8. (BM) I925, Exotic Microlepidoptera, 3: I53. I927, Exotic Microlepidoptera, 3: 359. (T) I9I5, Exotic Microlepidoptera, I: 4I3. (BM) I938, in Caradja and Meyrick, Deuts. Ent. Zeit. Iris, 52: I9. (BM) I920, Exotic Microlepidoptera, 2: 321. (BM) I9I2, Exotic Microlepidoptera, I: 20. (BM) I894, Trans. Ent. Soc. London, I894: 25. I884, Proc. Linnean Soc. N.S. Wales, 9: 740. I906, Journ. Bombay Nat. Hist. Soc. I7: 407. (BM) I906, Trans. R. Soc. S. Australia, 30: 34 . I9o6, Trans. R. Soc. S. Australia, 30: 60. I9II, Proc. Linnean Soc. N.S. Wales, 36:23I. I922, Exotic Microlepidoptera, 2: 485. (BM) I933, Exotic Microlepidoptera, 4: 364. (c) 1935, in Caradja and Meyrick, Materialien zu einer Microlepidopteren Fauna der Chinesischen Provinzen Kiangsu, Chekiang und Hunan, p. 9I. (BM) 


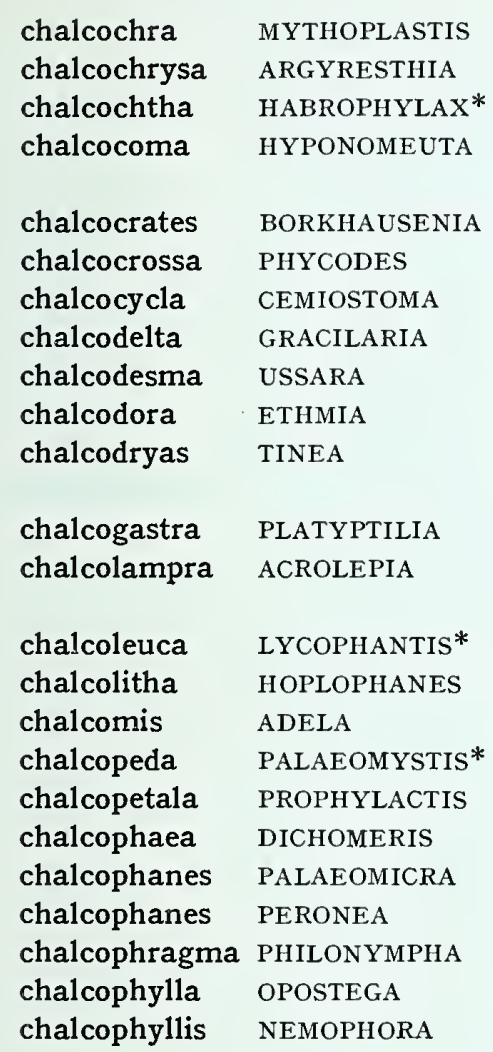

chalcoptera

chalcoptila

chalcoschista

chalcoscia

chalcospila

chalcostrepta

chalcotechna

chalcothorax

chalcotoxa

chalcotypa

chalcoxantha

chalcoxesta

chalcozela

chalcozona

chalcura

chaldaica

chalicitis

chalicodes

chalinaea

chalinias
GRACILARIA

SYNTOMACTIS

CRYSTALLOGENES*

MEGACRASPEDUS

PHALONIA

GLYPHIPTERYX

NEMOTOIS

PROCHOLA

SIMAETHIS

STATHMOPODA

PHILOBOTA

TINEA

CYPHACMA*

ASYMPHORODES

IDIOPHANTIS

COPTOTELIA

ECHYROTA*

TORTRIX

PITHARCHA*

OPOSTEGA
I93I, Exotic Microlepidoptera, 4:98. (v)

I9I8, Exotic Microlepidoptera, 2: I87. (BM)

I93I, Exotic Microlepidoptera, 4: I23. (v)

I938, in Caradja and Meyrick, Deuts. Ent. Zeit. Iris, 52: I7. (BM)

I930, Exotic Microlepidoptera, 3: 574. (D)

I9o9, Journ. Bombay Nat. Hist. Soc. I9: 424. (BM)

I883, Proc. Linnean Soc. N.S. Wales, 7: 201.

I889, Trans. Proc. New Zealand Inst. 2I: I83.

I9I3, Exotic Microlepidoptera, I: 67. (BM)

I9I2, Trans. Ent. Soc. London, I9II: 7I8.

1938, Institut des Parcs Nationaux du Congo Belge,

fasc. I4, p. 26, pl. 3, fig. 4 .

I92I, Exotic Microlepidoptera, 2: 420.

I93I, Ann. Mus. Nac. Hist. Nat. Buenos Aires, 36: 403.

(BM)

I9I 4, Journ. Bombay Nat. Hist. Soc. 23: I23. (BM)

I897, Proc. Linnean Soc. N.S. Wales, 22: $4 \mathrm{I} 3$.

I907, Journ. Bombay Nat. Hist. Soc. I7: 990. (BM)

I93I, Exotic Microlepidoptera, 4: 55. (P)

I897, Proc. Linnean Soc. N.S. Wales, 22: 408.

I92I, Exotic Microlepidoptera, 2: 434. (BM)

I886, Trans. Proc. New Zealand Inst. I8: I82.

I93I, Exotic Microlepidoptera, 4: I56. (BM)

I889, Proc. Linnean Soc. N.S. Wales, (Ser. 2) 3: I680.

I9I0, Rec. Indian Mus. 5: 229. (K)

I935, in Caradja and Meyrick, Materialien zu einer

Microlepidopteren Fauna der Chinesischen Provinzen

Kiangsu, Chekiang und Hunan, p. 96.

I88I, Proc. Linnean Soc. N.S. Wales, 5: I5I.

I922, Exotic Microlepidoptera, 2: 577. (BM)

I937, Exotic Microlepidoptera, 5: 83. (T)

I904, Proc. Linnean Soc. N.S. Wales, 29: 276.

I932, Exotic Microlepidoptera, 4: 265. (v)

I907, Proc. Linnean Soc. N.S. Wales, 32, I28.

I937, Arbeit. morph. taxon. Ent. Berl. 4: 204. (D)

I932, Exotic Microlepidoptera, 4: 3I5. (v)

I886, Trans. Ent. Soc. London, I886: 287.

I897, Proc. Linnean Soc. N.S. Wales, 22: 318.

I889, Proc. Linnean Soc. N.S. Wales, (Ser. 2) 3: I622.

I920, Ann. South African Mus. I7: 303. (SA)

I9I5, Trans. Ent. Soc. London, I9I5: 2I3.

I934, Pacific Ent. Surv. Publ. 6, art. 22, p. Iog. (вв)

I907, Journ. Bombay Nat. Hist. Soc. I8: I48. (BM)

I9I3, Trans. Ent. Soc. London, I9I3: I8I. (BM)

I9I6, Exotic Microlepidoptera, I : 60I.

I920, Voyage de Ch. Alluaud et $R$. Jeannel en Afrique

Oriental, II, Microlepidoptera, p. 48. (P)

I9o8, Proc. Zool. Soc. London, p. 75I.

I893, Proc. Linnean Soc. N.S. Wales, 7: 607. 


\begin{tabular}{|c|c|c|}
\hline chalinitis & PHILOBOTA & I9o9, Ann. South African Mus. 5: 355. (SA) \\
\hline chalinopa & ACROCERCOPS & I920, Exotic Microlepidoptera, 2: 294 . \\
\hline chalinophanes & STENOMA & I931, Exotic Microlepidoptera, 4: 42. (Bм) \\
\hline chalinopis & TRICHOTAPHE & I935, Exotic Microlepidoptera, 4: 562. (BM) \\
\hline chalinosema & ACROCERCOPS & I936, Exotic Microlepidoptera, 5: 36. \\
\hline chalinota & OPOGONA & I9Io, Rec. Indian Mus. 5: 230. (K) \\
\hline chalybacma & EPICEPHALA & r9o8, Journ. Bombay Nat. Hist. Soc. I8: 8I I. \\
\hline chalybanthes & TRACHYDORA & I897, Proc. Linnean Soc. N.S. Wales, 22: 393. \\
\hline chalybastra & PROTANYSTIS* & I92I, Zool. Meded. 6: I77. (L) \\
\hline chalybdica & LASPEYRESIA & I922, Exotic Microlepidoptera, 2: 533. (BM) \\
\hline chalybeis & STATHMOPODA & I897, Proc. Linnean Soc. N.S. Wales, 22: 322. \\
\hline chalybias & PROTOPTERNA* & Igo8, Journ. Bombay Nat. Hist. Soc. I8: 621. (Вм) \\
\hline chalybitis & TRICHOTAPHE & $\begin{array}{l}\text { I920, Voyage de Ch. Alluaud et } R \text {. Jeannel en Afrique } \\
\text { Oriental, II, Microlepidoptera, p. } 75 . \text { (P) }\end{array}$ \\
\hline chalybophanes & ACROCERCOPS & I937, Exotic Microlepidoptera, 5: 98. \\
\hline burga & HELCYSTOGRAMMA & I922, Trans. Ent. Soc. London, I922: I03. (Bм) \\
\hline chaodes & ISOCHORISTA & I9Io, Proc. Linnean Soc. N.S. Wales, 35: I68. \\
\hline chaomorpha & EUCOSMA & I929, Trans. Ent. Soc. London, 76: 495. (BM) \\
\hline hila & STREPSICRATES & I909, Trans. New Zealand Inst. 4I : Io. \\
\hline ica & TINEA & I893, Proc. Linnean Soc. N.S. Wales, 7: 538. \\
\hline haracias & THIOTRICHA & I9o8, Journ. Bombay Nat. Hist. Soc. I8: 440. (вм) \\
\hline copa & ORGANITIS* & I906, Journ. Bombay Nat. Hist. Soc. I7: I5I. \\
\hline cota & XYSMATODOMA & I893, Proc. Linnean Soc. N.S. Wales, 7: 504. \\
\hline acotis & CROCANTHES & I9I6, Exotic Microlepidoptera, I: 592. \\
\hline cta & MICROCOLONA* & I897, Proc. Linnean Soc. N.S. Wales, $22: 374$. \\
\hline ctana & CACOECIA & I88I, Proc. Linnean Soc. N.S. Wales, 6: 492. \\
\hline acterias & BONDIA & I932, Exotic Microlepidoptera, 4: 3I2. (BM) \\
\hline characteris & TORODORA* & I894, Trans. Ent. Soc. London, I894: I6. (BM) \\
\hline actis & LIMNOECIA & I897, Proc. Linnean Soc. N.S. Wales, $22: 363$. \\
\hline charadraea & ARGYROPLOCE & I9o9, Journ. Bombay Nat. Hist. Soc. I9: 594. (вм) \\
\hline adraea & TISIS & I9Io, Trans. Ent. Soc. London, I9Io: 439. (Bм) \\
\hline drias & STRUTHISCA & I9o7, Journ. Bombay Nat. Hist. Soc. I8: I55. \\
\hline drias & MIMAESEOPTILUS & I885, Trans. Proc. New Zealand Inst. I7: I26. \\
\hline adritis & ISORRHOA & I924, Exotic Microlepidoptera, 3: 65. \\
\hline dropis & EUPLOCAMUS & I934, Exotic Microlepidoptera, 4: 5I7. \\
\hline drota & ERECHTHIAS* & I88I, Proc. Linnean Soc. N.S. Wales, 5: 268. \\
\hline axias & HETEROCROSSA & I89I, Trans. Proc. New Zealand Inst. 23: 98. \\
\hline axias & PELTOPHORA & I889, Proc. Linnean Soc. N.S. Wales, (Ser. 2) 3: I64I. \\
\hline oti & PHTHORIMAEA & I934, The Entomologist, $67: 59$. \\
\hline idotis & COMPSOTROPHA & I884, Proc. Linnean Soc. N.S. Wales, $8: 5 \mathrm{I} 3$. \\
\hline ierga & EULECHRIA & I889, Proc. Linnean Soc. N.S. Wales, (Ser. 2) 3: I566. \\
\hline igramma & POLYHYMNO & I929, Exotic Microlepidoptera, 3: 502. (BM) \\
\hline ripepla & POMPOSTOLA* & I927, Exotic Microlepidoptera, 3: 326. (вм) \\
\hline phanes & ACROLEPIA & $\begin{array}{l}\text { I93I, Ann. Mus. Nac. Hist. Nat. Buenos Aires, 36: } 402 . \\
\text { (BM) }\end{array}$ \\
\hline iphila & PITHANURGA* & I921, Ann. Transvaal Mus. 8: 68. (T) \\
\hline risema & ANCYLIS & I934, Exotic Microlepidoptera, $4: 484$. \\
\hline risia & PYRODERCES & I897, Proc. Linnean Soc. N.S. Wales, $22: 350$. \\
\hline istis & AROTROPHORA & I9Io, Proc. Linnean Soc. N.S. Wales, 35: $26 \mathrm{I}$. \\
\hline & COLEOPHORA & I928, Bull. Hill \\
\hline
\end{tabular}


charitanthes

charitarcha

charitodes

charitopa

charitopa

charitopis

charixantha

charmatica

charmosyna

charon

charonaea

charopa

chartaria

chartularia

chasmatias

chasmatica

chasmodes

chelacma

chelaspis

chelias

chelidonia

chelobathra

chelograpta

chelonaea

chelonias

chelonitis

chelophora

chelophora

chelota

chelyodes

chelyodes

chenias

chenopa

cheradias

cheradota

cheramopis

cherandra

chernetis

cherota

chersadacta

chersaea

chersaea

chersitis

chersochlora

chersodes

chersodes

chersomicta
STATHMOPODA

STENOMA

XYSMATODOMA

OCTASPHALES*

PLATYPTILIA

ACROCERCOPS

CLYSIA

TINEA

SYNTOMACTIS

SCYTHRIS

HYPELICTIS

STREPSICRATES

TRICHOTAPHE

[MYTHOGRAPHA*]

ORTHENCHES

ERECHTHIAS

[HECTACMA $*$ ]

IMMA

ARGYROPLOCE

IMMA

SIMAETHIS

LASPEYRESIA

COMPSOLECHIA

ANTAEOTRICHA

CACOECIA

CRITICONOMA*

PERIACMA

ELACHISTA

[CLEROPTILA*]

PROCALYPTIS

THYRSOSTOMA

SCYTHRIS

TINEA

EPERMENIA

PROTOLECHIA

ACROCERCOPS

GELECHIA

ACROCLITA

TELEPHATA*

BRACHMIA

EPICOENIA*

TINEA

HOMOTINEA

EPHYSTERIS*

EUCOSMA

LECITHOCERA

PHTHORIMAEA

EULECHRIA

CARPOSINA

LEPTOCROCA
I933, Exotic Microlepidoptera, 4: 43I.

I9I5, Exotic Microlepidoptera, I: 438. (BM)

I893, Proc. Linnean Soc. N.S. Wales, 7: 497.

I886, Trans. Ent. Soc. London, I886: 284. (BM)

I9o8, Trans. Ent. Soc. London, I907: 483.

I9I5, Trans. Ent. Soc. London, I9I5: 228.

I928, Exotic Microlepidoptera, 3: 435. (BM)

I9II, Journ. Bombay Nat. Hist. Soc. 21 : II8.

I92I, Exotic Microlepidoptera, 2: 456.

I9I8, Exotic Microlepidoptera, 2: I56. (BM)

I9I3, Journ. Bombay Nat. Hist. Soc. 22: I72. (BM)

I 888, Trans. Proc. New Zealand Inst. 20: 73 .

I9I3, Journ. Bombay Nat. Hist. Soc. 22: I78. (BM)

I924, Trans. Proc. New Zealand Inst. 55: 205.

I88I, Proc. Linnean Soc. N.S. Wales, 5: 264.

I906, Trans. Ent. Soc. London, I906: I99. (BM)

I9I I, Proc. Linnean Soc. N.S. Wales, 36: 277.

I927, Insects of Samoa, 3, Lepidoptera, fasc. 2, p. Iо2. (Вм)

I929, Trans. Ent. Soc. London, 76: 504. (BM)

I907, Journ. Bombay Nat. Hist. Soc. I4: I45. (BM)

I922, Trans. Ent. Soc. London, I922: 82. (BM)

I9I6, Exotic Microlepidoptera, I: 498. (BM)

I9I7, Trans. Ent. Soc. London, I9I7: 7.

I9I0, Ann. South African Mus. 5: 4I5. (SA)

I9Io, Journ. Bombay Nat. Hist. Soc. 20: I59. (BM)

I909, Ann. Transvaal Mus. 2: 25, pl. 8, fig. 2. (T)

I9Io, Trans. Ent. Soc. London, I9Io: 43I. (BM)

I9I8, Exotic Microlepidoptera, 2: I2I. (BM)

I906, Journ. Bombay Nat. Hist. Soc. I7: 4II.

I9I I, Journ. Bombay Nat. Hist. Soc. 2I: II9.

I9Io, Rec. Indian Mus. 2: 228. (K)

I904, Proc. Linnean Soc. N.S. Wales, 29: 359.

I932, Exotic Microlepidoptera, 4: 270.

I909, Trans. New Zealand Inst. 4I: I2.

I9I2, Journ. Bombay Nat. Hist. Soc. 2I: 856. (BM)

I9I6, Exotic Microlepidoptera, I: 593.

I906, Journ. Bombay Nat. Hist. Soc. I7: I5I. (BM)

I9o6, Journ. Bombay Nat. Hist. Soc. I7: I4I. (BM)

I9I9, Exotic Microlepidoptera, 2: 246.

I932, Exotic Microlepidoptera, 4: 210.

I908, Proc. Zool. Soc. London, p. 725. (BM)

I9I2, Ent. Mon. Mag. 48: 34. (Sub.)

I9I 8, Exotic Microlepidoptera, 2: I06. (BM)

I922, Arkiv för Zool. vol. I4, part I5, p. 3.

I9I4, Exotic Microlepidoptera, I: 2 I9.

I9I5, Boll. Lab. Zool. Gen. Agr. 9: 333 .

I920, Exotic Microlepidoptera, 2: 308. 


$\begin{array}{ll}\begin{array}{l}\text { chersopa } \\ \text { chersophila } \\ \text { chersopis }\end{array} & \begin{array}{l}\text { SYRMOLOGA } \\ \text { chersopsamma }\end{array} \\ \text { chersopsamma } & \text { ANAPATRIS* } \\ \text { chersota } & \text { CHARADRAULA } \\ \text { chiasta } & \text { PERSICOPTILA } \\ \text { chiastis } & \text { PHILOBOTA } \\ \text { chiloptila } & \text { HOMALOXESTIS } \\ \text { chilosema } & \text { STENOMA } \\ \text { chilota } & \text { OXYLYCHNA } \\ \text { chimerina } & \text { TALANTIS* } \\ \text { chimerinana } & \text { CAPUA } \\ \text { chionacma } & \text { OCHMASTIS* } \\ \text { chionadelpha } & \text { ALUCITA }\end{array}$

chionarcha

chionardis

chionarga

chionastes

chionastra

chionaula

chionaula

chionea

chionites

chionitis

chionocarpa

chionocentra

chionochalca

chionochlaena

chionochrysa

chionochtha

chionocrossa

chionodelta

chionodira

chionodora

chionogramma

chionolitha

chionomima

chionopa

chionophanes

chionophricta

chionophthalmaSPARGANOTHIS

chionopis

chionoplecta

chionopsamma

chionoptera

chionoptila

chionopyrrha
POLYHYMNO

PHYCODES

CYLICOBATHRA*

PTEROPHORUS

HYPERCALLIA

AROTROPHORA

EPORYCTA

OCYSTOLA

NEMOTOIS

NOTHRIS

EULIA

ARGYROTOXA

DIPLOTHECTIS*

TRACHYBYRSIS

ARGYRESTHIA

CONOPOMORPHA

CEROMITIA

ARGYROPLOCE

ERECHTHIAS

STENOMA

ORPHNOLECHIA

POLYCHROSIS

GELECHIA

CONCHYLIS

PTEROPHORUS

EUCOSMA

HYPERCALLIA

GRACILARIA

PROTEROCOSMA

PHILOBOTA

PTILOGENES

AELOSCELIS [sic!]
I9I9, Exotic Microlepidopiera, 2: 273. (BM)

I909, Ann. South African Mus. 5: 351. (SA)

I933, Exotic Microlepidoptera, 4: 423. (v)

1932, Exotic Microlepidoptera, 4:287. (Us)

I93I, Exotic Microlepidoptera, 4: 124.

I9I5, Exotic Microlepidoptera, I: 330. (BM)

r9o9, Journ. Bombay Nat. Hist. Soc. r9: 42 I.

1889, Proc. Linnean Soc. N.S. Wales, (Ser. 2) 3: I606.

I92I, Exotic Microlepidoptera, 2: 435. (BM)

I9I8, Exotic Microlepidoptera, 2: 208. (BM)

I9I9, Exotic Microlepidoptera, 2: 250.

I889, Proc. Linnean Soc. N.S. Wales, (Ser. 2) 3: I6or.

r881, Proc. Linnean Soc. N.S. Wales, 6: $45^{2}$.

I908, Rec. Indian Mus. 2: 396. (K)

r93x, in Joannis, Ann. Soc. Ent. France, $9^{8}$ (Supp.): 708

[470].

I9I3, Ann. Transvaal Mus. 3:282. (T)

I909, Journ. Bombay Nat. Hist. Soc. 19: 424. (BM)

1920, Voyage de Ch. Alluaud et $R$. Jeannel en Afrique

Oriental, II, Microlepidoptera, p. Ioo. (P)

I908, Trans. Ent. Soc. London, r9o7: 50r.

I926, Exotic Microlepidoptera, 3: 3I3. (BM)

I9Io, Proc. Linnean Soc. N.S. Wales, 35: 262.

I 920, Ann. South African Mus. I7: 29I. (SA)

I885, Proc. Linnean Soc. N.S. Wales, 9: 1076.

I907, Journ. Bombay Nat. Hist. Soc. 17: 990.

I9I0, Ann. South African Mus. 5: 4I4. (SA)

1932, Exotic Microlepidoptera, 4: 257. (v)

I908, Journ. Bombay Nat. Hist. Soc. I8: 623. (BM)

1893, Proc. Linnean Soc. N.S. Wales, $7: 599$.

1932, Trans. Ent. Soc. London, 80: IIO. (BM)

I93I, Exotic Microlepidoptera, 4:92. (BM)

I907, Proc. Linnean Soc. N.S. Wales, 32: 59.

I92I, Exotic Microlepidoptera, 2: 405.

I9I I, Proc. Linnean Soc. N.S. Wales, 36: 275.

I88I, Proc. Limnean Soc. N.S. Wales, 5: 268.

I9I5, Exotic Microlepidoptera, I: 464. (BM)

rgog, Trans. Ent. Soc. London, rgo9: 29. (BM)

I938, Trans. R. Ent. Soc. London, 87: 5II. (BM)

I929, Exotic Microlepidoptera, 3: 488. (BM)

189x, Ent. Mon. Mag. 27: 55. (BM)

I930, Exotic Microlepidoptera, 3:569. (BM)

1922, Exotic Microlepidoptera, 2: 5I5. (P)

I932, Exotic Microlepidoptera, 4: 263. (v)

19I6, Exotic Microlepidoptera, I: 553. (BM)

I883, Proc. Linnean Soc. N.S. Wales, 7: I95.

1886, Trans. Ent. Soc. London, r886: 294.

I884, Proc. Linnean Soc. N.S. Wales, 8: 494.

1926, Exotic Microlepidoptera, 3: 229. (BM)

r927, Insects of Samoa, 3, Lepidoptera, fasc. 2, p. 98. 


\begin{tabular}{|c|c|c|}
\hline chionoscia & SCAEOSOPHA & I933, Exotic Microlepidoptera, 4: 374 . \\
\hline chionosema & CRYPTOPHASA & I938, Trans. R. Ent. Soc. London, 87: 517. \\
\hline hionosphena & PTILOGENES & I93I, Exotic Microlepidoptera, 4: 45 . \\
\hline hionospila & LIMNOECIA & I897, Proc. Linnean Soc. N.S. Wales, $22: 365$. \\
\hline hionostoma & TRITOPTERNA* & I92I, Zool. Meded. 6: I52. (L) \\
\hline onotarsa & CRYPTOPHASA & I925, Exotic Microlepidoptera, 3: I48. \\
\hline lionoxantha & ocySTOLA & I93I, Exotic Microlepidoptera, 4: I89. (BM) \\
\hline hionozona & TRICLONELLA & I93I, Exotic Microlepidoptera, 4: iI7. (v) \\
\hline ionozyga & THEREUTIS & I893, Proc. Linnean Soc. N.S. Wales, 7: 597. \\
\hline ura & COMMATICA & I9I4, Trans. Ent. Soc. London, I9I4: 240. (BM) \\
\hline dia & PROTOLECHIA & I904, Proc. Linnean Soc. N.S. Wales, 29: 380 . \\
\hline iridota & IDIOPHANTIS & I9I4, Exotic Microlepidoptera, I: 20I. (BM) \\
\hline irista & THAMNOSARA* & I883, New Zealand Journ. Sci. I: 523 . \\
\hline lota & HYPERTROPHA & I886, Proc. Linnean Soc. N.S. Wales, (Ser. 2) I: I042. \\
\hline laenota & SARISOPHORA & I904, Proc. Linnean Soc. N.S. Wales, 29: 404. \\
\hline lamydata & LASPEYRESIA & I924, Ent. Mitteil. I3: II7. (D) \\
\hline dota & TRICHOTAPHE & I927, Exotic Microlepidoptera, 3: 355. (BM) \\
\hline lidanopa & MOMETA & I927, Exotic Microlepidoptera, 3: 35I. (BM) \\
\hline loracma & OPOGONA & I907, Journ. Bombay Nat. Hist. Soc. I7: 987. (BM) \\
\hline oracta & ORNEODES & I908, Trans. Ent. Soc. London, I907: 507. \\
\hline oradelpha & EPICHORISTA & I9I2, Exotic Microlepidoptera, I: 8. (T) \\
\hline loradelpha & BORKHAUSENIA & I905, Trans, Ent. Soc. London, I905: 239. \\
\hline loraema & BUTALIS & I887, Trans. Ent. Soc. London, I887: 279. \\
\hline atha & ERINAEA* & I907, Journ. Bombay Nat. Hist. Soc. I8: I4I. \\
\hline itis & ECTOEDEMIA & I928, Exotic Microlepidoptera, $3: 462$. \\
\hline oraspis & CAPUA & I924, Exotic Microlepidoptera, 3: Io6. (вм) \\
\hline spis & CAPUA & I927, Exotic Microlepidoptera, 3: 369 . \\
\hline $\operatorname{tma}$ & TRACHYPEPLA & I9I6, Trans. Proc. New Zealand Inst. 48: 4I6. \\
\hline loraula & HYPOSMOCOMA & I928, Proc. Hawaiian Ent. Soc. 7: I03. (SP) \\
\hline $11 \mathrm{a}$ & EULECHRIA & I883, Proc. Linnean Soc. N.S. Wales, 7: 526. \\
\hline as & PTEROPHORUS & I9o8, Trans. Ent. Soc. London, I907: 497. \\
\hline pa & EULIA & I928, Proc. Hawaiian Ent. Soc. 7: 98. (SP) \\
\hline ssa & ACROCLITA & I9I2, Journ. Bombay Nat. Hist. Soc. 2I: 859 . (BM) \\
\hline ta & NEMOTOIS & I9I2, in Wytsman, Genera Insectorum, fasc. I33, p. 5. \\
\hline tis & NOTHRIS & I904, Proc. Linnean Soc. N.S. Wales, 29: 421. \\
\hline oristis & TINEOLA (?) & I9o8, Proc. Zool. Soc. London, p. 740. \\
\hline is & OECOPHORA & I883, New Zealand Journ. Sci. I: 524 . \\
\hline apta & LECITHOCERA & I93I, Exotic Microlepidoptera, 4: 82. (BM) \\
\hline robathra & EUCOSMA & I9I I, Trans. Linnean Soc. London, I4: 268. (Bм) \\
\hline cobela & HELIOSTIBES & I92I, Trans. Proc. New Zealand Inst. 53: 334. \\
\hline ocephala & GELECHIA & I932, Exotic Microlepidoptera, 4:35I. (v) \\
\hline roceros & TINEA & I9I9, Exotic Microlepidoptera, 2: 275. (BM) \\
\hline ochalca & COSMOPTERYX & I9I5, Exotic Microlepidoptera, I: 322. \\
\hline ochroa & MACHIMIA & I9I2, Trans. Ent. Soc. London, I9I I : 697. (Bм) \\
\hline rochrysa & HOPLOPHANES & I897, Proc. Linnean Soc. N.S. Wales, 22: 4Io. \\
\hline chyta & PLEUROTA & I884, Proc. Limnean Soc. N.S. Wales, 9: 757 . \\
\hline orocitra & NARYCIA & I934, Exotic Microlepidoptera, 4: 5I8. (c) \\
\hline orocoma & ORTHENCHES* & I886, Trans. Proc. New Zealand Inst. I8: I75. \\
\hline osma & NEMOTOIS & I9I5, Exotic Microlepidoptera, I: 294. \\
\hline crana & GELECHIA & I027, Exotic Microlepidoptera, 3: 348. (BM) \\
\hline
\end{tabular}




\begin{tabular}{|c|c|}
\hline chlo & CNEPHASIA \\
\hline chlorodecta & COMPSOLECHIA \\
\hline hlorodelta & EPICOENIA \\
\hline roderces & TELPHUSA \\
\hline rodesma & PERIACMA \\
\hline odeta & LIMNOECIA \\
\hline rodoxa & TORTRIX \\
\hline rogastra & LECITHOCERA \\
\hline rogenes & EPIMECYNTIS \\
\hline ogramma & TRACHYCENTRA \\
\hline ographa & ARISTOTELIA \\
\hline ograpta & PANDEMIS \\
\hline roleuca & DOLICHERNIS* \\
\hline oleuca & EUCOSMA \\
\hline olitha & PHALONIA \\
\hline oloba & STENOMA \\
\hline lyca & AMORBIA \\
\hline rometalla & HAPLOCHROIS* \\
\hline romima & EUCOSMA \\
\hline romis & STENOMA \\
\hline omydra & ADOXOPHYES \\
\hline nephes & LIMNOECIA \\
\hline neura & ARISTOTELIA \\
\hline ronota & ATOMOTRICHA \\
\hline ronympha & ACROCERCOPS \\
\hline ropa & ARISTEIS \\
\hline ropeda & HOLCOCERA \\
\hline ropelta & ACROLOPHUS \\
\hline ropepla & ELACHISTA \\
\hline opetala & LEPIDOSCIA \\
\hline phaea & OPOGONA \\
\hline ophanes & OPOGONA \\
\hline ophracta & DICHOMERIS \\
\hline rophthalma & METABOLAEA* \\
\hline ropis & PROCHOLA \\
\hline roplaca & GONIOTERMA \\
\hline oplintha & IMMA \\
\hline oplocama & TINISSA \\
\hline & THEATRISTA \\
\hline & GRACILARIA \\
\hline optris & BLASTOBASIS \\
\hline rorrhabda & ERYSIMAGA* \\
\hline rosaris & ARGYROPLOCE \\
\hline roschema & GELECHIA \\
\hline . & LECITHOCERA \\
\hline & ANTISPILA \\
\hline & IMMA \\
\hline & MACROBATHF \\
\hline
\end{tabular}

I926, Ann. South African Mus. 23: 326. (SA) I932, Exotic Microlepidoptera, 4: I97. (BM) I906, Journ. Bombay Nat. Hist. Soc. I7: I40. (вM) I929, Exotic Microlepidoptera, 3: 487. (BM) I894, Trans. Ent. Soc. London, I894: 22. (BM) I928, Exotic Microlepidoptera, 3: 393. (T) I920, Exotic Microlepidoptera, 2: 34I. (BM) I922, Zool. Meded. 7: 84. (L) I928, Exotic Microlepidoptera, 3: 470. (BM) 1907, Proc. Linnean Soc. N.S. Wales, 32: I42. I9I4, Ann. Transvaal Mus. 4: Igo. (T) I93I, Exotic Microlepidoptera, 4: I50. I89I, Trans. Proc. New Zealand Inst. 23: 99. I9I2, Ent. Mon. Mag. 48: 34. (Sub.) I93I, Exotic Microlepidoptera, 4: I57. (BM) I9I5, Exotic Microlepidoptera, I : 439. (BM) I93I, Exotic Microlepidoptera, 4: I55. (v) I897, Proc. Linnean Soc. N.S. Wales, 22:3Io. I93I, in Caradja, Bull. Sect. sci. Acad. roum. I4: 65. (вм) I9I5, Exotic Microlepidoptera, I: 433. (BM) I926, Sarawak Mus. Journ. 3: I47. I924, Exotic Microlepidoptera, 3:9I. (T) 1923, Exotic Microlepidoptera, 3: 6. (BM) I9I4, Trans. Proc. New Zealand Inst. 46: IIo. I92I, Exotic Microlepidoptera, 2: 467 . I9I4, Exotic Microlepidoptera, I: 244. (BM) I922, Exotic Microlepidoptera, 2: 54I. (BM) I923, Exotic Microlepidoptera, 2: 609. (BM) 1897, Proc. Linnean Soc. N.S. Wales, 22: 334. I893, Proc. Linnean Soc. N.S. Wales, 7: 5 II. I921, Zool. Meded. 6: I93. (L) I908, Proc. Zool. Soc. London, p. 737. I921, Ann. Transvaal Mus. 8: 82. (T) I923, Exotic Microlepidoptera, 3: 32. (BM) I922, Exotic Microlepidoptera, 2: 580. (BM) I915, Exotic Microlepidoptera, I: 383. I929, Trans. Ent. Soc. London, 76: 503. (вм) I938, Trans. R. Ent. Soc. London, 87: 526. I920, Voyage de Ch. Alluaud et $R$. Jeannel en Afrique Oriental, II, Microlepidoptera, p. I02. (P) I9I5, Trans. Ent. Soc. London, I9I5: 238. I93I, Exotic Microlepidoptera, 4: I78. I938, Trans. R. Ent. Soc. London, 87: 526. I9I4, Trans. Proc. New Zealand Inst. 46: 106. I923, Exotic Microlepidoptera, 3: 2I. (BM) I938, Institut des Parcs Nationaux du Congo Belge, fasc. I4, P. I4. I931, Ann. Mus. Nac. Hist. Nat. Buenos Aires, 36: 397. I906, Trans. Ent. Soc. London, I906: I97. (BM) I886, Proc. Linnean Soc. N.S. Wales, Io: 8Io. 
chlorosperma - ISCHNODORIS

chlorosphena

IMMA

IMMA

chlorospila

chlorospora

chlorospora

chlorostaura

chlorosticha

chlorosticta

chlorostola

chlorostoma

chloroterma

chlorothorax

chlorothyris

chlorotoma

chlorotoma

chlorotribes

chlorotricha

chlorotricha

chlorotripta

chlorotrota

chlorotypa

chloroxantha

chlorozona

chlorozyga

cholaea

choleranthes

cholerocrossa

cholerodes

choleroleuca

choleropa

choleropis

choleroptila

cholerota

cholodella

cholopis

chomatias

chonactis

choneuta

chordacta

chorderes

chordites

chordoscelis

chordostoma

chordotona

chorias

chorica
TINEA

ERNOLYTIS*

DELOSAPHES*

EUCOSMA

AGRIOPHARA

LASPEYRESIA

PSEUDODOXIA

EUCOSMA

DEPRESSARIA

AEGERIA

LEURONOMA*

ONEBALA

OSIDRYAS

CORTHYNTIS*

MELASINA

SPILONOTA

STENOMA

CACOECIA

STENOMA

TRACHYDORA

CRYPTOLECHIA

TINEA

DICHELOPA

STENOMA

EULECHRIA

LECITHOCERA

LASPEYRESIA

OPOGONA

STENOMA

LABDIA

HOPLITICA

LECITHOCERA

OSPHRETICA*

ACROLOPHUS

HELIODINES

OPOSTEGA

OCTASPHALES

OXYPTILUS

COLEOPHORA

CRYPTOLECHIA

CROCANTHES

ATHEROPLA

TORTYA
I929, Exotic Microlepidoptera, 3: 528. (BM)

I906, Trans. Ent. Soc. London, I906: г86. (вм)

I923, Exotic Microlepidoptera, 2: 6I6. (BM)

I924, Exotic Microlepidoptera, 3: 7I. (BM)

I922, Exotic Microlepidoptera, 2: 488 . (BM)

I938, in Caradja and Meyrick, Deuts. Ent. Zeit. Iris,

52: 9. (BM)

I934, in Caradja and Meyrick, Deuts. Ent. Zeit. Iris,

48:3I.

I9I3, Trans. Ent. Soc. London, I9I3: I84. (BM)

I932, Exotic Microlepidoptera, 4:226. (BM)

I9I4, Exotic Microlepidoptera, I: 264. (вM)

I9I3, Ann. Transvaal Mus. 3:272. (BM)

I92I, Exotic Microlepidoptera, 2: 392. (BM)

I935, Exotic Microlepidoptera, 4:557.

1918, Ann. Transvaal Mus. 6: 16.

I9I4, Exotic Microlepidoptera, I: 200. (BM)

I939, Trans. R. Ent. Soc. London, 89:58. (Bм)

I9I6, Exotic Microlepidoptera, I: 575 .

I9I6, Exotic Microlepidoptera, I: 608.

I921, Zool. Meded. 6: I5I. (L)

1932, Exotic Microlepidoptera, 4:296. (v)

I934, in Caradja and Meyrick, Deuts. Ent. Zeit. Iris, 48: 30 .

1925, Exotic Microlepidoptera, 3: 216. (BM)

I897, Proc. Linnean Soc. N.S. Wales, 22: 399.

I938, in Caradja and Meyrick, Deuts. Ent. Zeit. Iris,

52: Io. (BM)

I9II, Journ. Bombay Nat. Hist. Soc. 2I : I23.

I929, Trans. Ent. Soc. London, 76: 493. (BM)

I930, Exotic Microlepidoptera, 4:32. (v)

I888, Proc. Linnean Soc. N.S. Wales, 2: 963.

I93I, Exotic Microlepidoptera, 4: 79. (BM)

I9I3, Ann. Transvaal Mus. 3: 279. (T)

1920, Exotic Microlepidoptera, 2: 360.

I9I5, Exotic Microlepidoptera, I: 467. (Bм)

I928, Exotic Microlepidoptera, 3: 387.

1883, Proc. Linnean Soc. N.S. Wales, 7: 507.

I906, Journ. Bombay Nat. Hist. Soc. I7: I49. (BM)

I9Io, Trans. Ent. Soc. London, I9Io: 475.

1932, Exotic Microlepidoptera, 4: 33I.

I9I5, Trans. Ent. Soc. London, I9I5: 212.

I9I5, Exotic Microlepidoptera, I: 35I.

I902, Trans. R. Soc. S. Australia, 26: I6I.

I9I3, Exotic Microlepidoptera, I: Io6.

I9I7, Exotic Microlepidoptera, 2: 73.

I9I2, Trans. Ent. Soc. London, I9I I: 704. (вм)

I9I6, Exotic Microlepidoptera, I: 592.

I902, Trans. R. Soc. S. Australia, 26: I40.

I926, Exotic Microlepidoptera, 3: 302. (BM) 


\begin{tabular}{|c|c|}
\hline choricopa & ODITES \\
\hline odes & LICHENAULA \\
\hline choristis & DECTOBATHRA* \\
\hline choritica & CNISMORECTIS* \\
\hline ritis & LECITHOCERA \\
\hline rodoxa & EULECHRIA \\
\hline omima & PLACOPTILA \\
\hline ropepla & ELACHISTA \\
\hline ospila & HETEROGYMNA \\
\hline taea & EUCOSMA \\
\hline matistis & SPHAEROLBIA* \\
\hline tocoma & ANTAEOTRICHA \\
\hline latarcha & BORKHAUSENIA \\
\hline omataspis & LASPEYRESIA \\
\hline omatica & $\begin{array}{l}\text { CHALINIASTIS } \\
{\left[\text { APOCRITICA }{ }^{*}\right]}\end{array}$ \\
\hline omatopa & STENOMA \\
\hline omaturga & XESTOCASIS \\
\hline molitha & STENOMA \\
\hline nophanes & ARISTOTELIA \\
\hline & STENOMA \\
\hline ysallacta & GLYPHIPTERYX \\
\hline ampyx & PROMENESTA \\
\hline anches & BONDIA \\
\hline gela & USSARA \\
\hline tha & ANTICRATES* \\
\hline antha & THIOTRICHA \\
\hline santhema & ATTERIA \\
\hline inthes & STROMATITICA* \\
\hline sargyra & ACROCERCOPS \\
\hline chrysargyra & PALAEOMICRA* \\
\hline chr & HIPPOCHAETES* \\
\hline saula & STATHMOPODA \\
\hline chryselectra & PYRODERCES \\
\hline chrysias & CASTORURA* \\
\hline idias & NEMOTOIS \\
\hline chrysidiella & CROBYLOPHORA* \\
\hline sidota & CALLIXESTIS \\
\hline sippa & CRYPSITHYRIS \\
\hline ysobela & COSMOPTERYX \\
\hline carpa & STATHMOPODA \\
\hline sochalca & ACOROSTOMA \\
\hline sochoa & TIMODORA* \\
\hline & PHAEOSACES \\
\hline soconis & URODUS \\
\hline & ACROCERCOPS \\
\hline ocosm & LASPEYRESIA \\
\hline
\end{tabular}

I93I, in Caradja, Bull. Sect. sci. Acad. roum. I4: 74. (BM) I89o, Trans. R. Soc. S. Australia, I3: 50.

1904, Proc. Linnean Soc. N.S. Wales, 29: 300. 1935, Arb. morph. taxon. Ent. Berl. 3: I09. (D) I9Io, Journ. Bombay Nat. Hist. Soc. 20: 448. (BM) 1920, Exotic Microlepidoptera, 2: 372. I93I, Exotic Microlepidoptera, 4: I74. I897, Proc. Linnean Soc. N.S. Wales, 22: 334. I922, Zool. Meded. 7 : 8o. (Lost.) I9I2, Ent. Mon. Mag. 48: 35. (Sub.) I934, Exotic Microlepidoptera, 4: 513. (BM) I9I5, Exotic Microlepidoptera, I: 398. (BM) I9I4, Exotic Microlepidoptera, I: 232. I92I, Ann. Transvaal Mus. 8: 62. (T)

I9I I, Trans. Linnean Soc. London, I4: 272. (BM) I930, Exotic Microlepidoptera, 4: 29. (v)

I9I5, Exotic Microlepidoptera, I: 316. I925, Exotic Microlepidoptera, 3: 203. (BM) I938, in Caradja and Meyrick, Deuts. Ent. Zeit. Iris, $52: 3$. (BM)

I925, Exotic Microlepidoptera, 3: 2I2. (BM) I922, Exotic Microlepidoptera, 2: 49I. (BM) I9I5, Exotic Microlepidoptera, I: 4II. (BM) I938, in Caradja and Meyrick, Deuts. Ent. Zeit. Iris, 52: I5. I922, Exotic Microlepidoptera, 2: 488. (BM) I905, Journ. Bombay Nat. Hist. Soc. I6: 6I2. (BM) Igo8, Journ. Bombay Nat. Hist. Soc. I8: 443. (BM) I9I2, Trans. Ent. Soc. London, I9II: 676. (BM) I93I, Exotic Microlepidoptera, 4: 53 . I908, Journ. Bombay Nat. Hist. Soc. I8: 8I8. I886, Trans. Proc. New Zealand Inst. I8: I82. I88I, Proc. Linnean Soc. N.S. Wales, 5: 253. 1927, Exotic Microlepidoptera, 3: 377. 1897, Proc. Linnean Soc. N.S. Wales, 22: 344. I886, Proc. Linnean Soc. N.S. Wales, (Ser. 2) I: I047. I928, Exotic Microlepidoptera, 3: 463. I88I, Proc. Linnean Soc. N.S. Wales, 5: I78. I9I7, Exotic Microlepidoptera, 2: 4I. I9I7, Exotic Microlepidoptera, 2: 76 . I928, Exotic Microlepidoptera, 3: 395. I927, Exotic Microlepidoptera, 3: 378. I926, Ann. South African Mus. 23: 346. (SA) I886, Trans. Ent. Soc. London, I886: 296. I905, Journ. Bombay Nat. Hist. Soc. I6: 605. (BM) I932, Exotic Microlepidoptera, 4:285. (v) I9I5, Trans. Ent. Soc. London, I9I5: 225. I920, Voyage de Ch. Alluaud et $R$. Jeannel en Afrique Oriental, II, Microlepidopiera, p. 68. (P) 
chrysocosma chrysocrana chrysocrates chrysocrossa chrysodesma chrysodeta chrysodetis chrysodora chrysodoxa chrysogastra chrysogona chrysogramma chrysograpta chrysolampra chrysoleuca chrysolithella chrysomela chrysomelopa chrysometra chrysomitra chrysomochla chrysonesa chrysonympha chrysopa chrysopetala chrysophanes chrysophanes chrysophila chrysophoenicea chrysophthalma chrysophthalma chrysopis chrysoplaca chrysoplaca chrysoplanetis chrysoplitis chrysopoca chrysopotama chrysoprasias chrysoprasitis chrysorma chrysorrhabda chrysorrhoda chrysoschista chrysosema chrysosoma chrysosperma chrysospila chrysospila chrysostacta
MAGONYMPHA* HIEROPHANES * COSMOPTERYX

NEMOTOIS TRYPHEROGENES* MACHLOTICA* TORTRIX HIEROXESTIS TRACHYDORA STENOMA NEMOTOIS OECOPHORA ASTROGENES* GRACILARIA HIEROMANTIS GLYPHIPTERYX CATAMACTA ZACORISCA ARISTOTELIA CEROMITIA DORODOCA* LIMNOECIA

AEGERIA THIOTRICHA XYSMATODOMA OPOGONA SESIA ACROCERCOPS AEOLOSCELIS OPOGONA PHYLLOCNISTIS OCYSTOLA ANACAMPSIS IMMA GLYPHIPTERYX ACROCERCOPS LEPIDOTARSA* PHILOBOTA NEMOTOIS TORTYRA PHALERARCHA* STILBOSIS EUPHILTRA BRANCHOPHANTIS* METAPODISTIS* LABDIA BRENTHIA MACROBATHRA TORNOCOMETIS* CHOREUTIS
I9I6, Exotic Microlepidoptera, I: 572.

I930, Exotic Microlepidoptera, 3:586. (BM)

I9I9, Exotic Microlepidoptera, 2: 232. (BM)

I922, Exotic Microlepidoptera, 2: 537.

I93I, Exotic Microlepidoptera, 4: 76. (BM)

I9o9, Trans. Ent. Soc. London, I909: 37. (BM)

I926, Exotic Microlepidoptera, 3: 248. (BM)

1924, Trans. Ent. Soc. London, I923: 555.

I9I5, Exotic Microlepidoptera, I: 332.

I9I5, Exotic Microlepidoptera, I: 476 . (BM)

I922, Exotic Microlepidoptera, 2: 536.

I883, New Zealand Journ. Sci. I : 525 .

I921, Trans. Proc. New Zealand Inst. 53: 335.

I936, Exotic Microlepidoptera, 5: $3^{8 .}$

I9I3, Exotic Microlepidoptera, I: 82.

I88I, Proc. Linnean Soc. N.S. Wales, 5: 229.

I9I4, Trans. Proc. New Zealand Inst. 46: ro3.

I927, Exotic Microlepidoptera, 3: 369 .

I926, Exotic Microlepidoptera, 3: 273. (BM)

I937, Exotic Microlepidoptera, 5: I46.

I9I5, Exotic Microlepidoptera, I: 324 .

I897, Proc. Linnean Soc. N.S. Wales, 22: 367 .

I932, Exotic Microlepidoptera, 4: 336.

1904, Proc. Linnean Soc. N.S. Wales, 29: 293.

I893, Proc. Linnean Soc. N.S. Wales, 7: 502.

I9I5, Exotic Microlepidoptera, I : 359.

I886, Proc. Linnean Soc. N.S. Wales, (Ser. 2) I : 689.

I937, Exotic Microlepidoptera, 5: I 1 o.

I897, Proc. Linnean Soc. N.S. Wales, 22: 328.

I934, Exotic Microlepidoptera, 4: 462.

I9I5, Exotic Microlepidoptera, I: 347.

I902, Trans. R. Soc. S. Australia, 26: I35.

I9I 2, Trans. Ent. Soc. London, I9I I: 694. (BM)

I906, Trans. Ent. Soc. London, I906: I95. (BM)

I881, Proc. Linnean Soc. N.S. Wales, 5: 238.

I937, Exotic Microlepidoptera, 5: III.

I883, Proc. Linnean Soc. N.S. Wales, 7: 447.

I884, Proc. Linnean Soc. N.S. Wales, 8: 476.

I907, Journ. Bombay Nat. Hist. Soc. I7: 992. (вм)

I936, Exotic Microlepidoptera, 4:6I7.

I9I3, Exotic Microlepidoptera, I: IOI. (BM)

I922, Exotic Microlepidoptera, 2: 576. (BM)

I902, Trans. R. Soc. S. Australia, 26: I45.

I938, Trans. R. Ent. Soc. London, 87: 5I2. (BM)

I933, Exotic Microlepidoptera, 4:372. (BM)

I928, Exotic Microlepidoptera, 3: 387.

I93I, Exotic Microlepidoptera, 4: I83. (BM)

I886, Proc. Linnean Soc. N.S. Wales, го: 822.

I934, Exotic Microlepidoptera, 4: 496.

I933, Exotic Microlepidoptera, 4: 37I. (BM) 
chrysostoma chrysostoma chrysota chrysoterma chrysoteuches chrysothorax chrysotoxa chrysotypa chrysoxantha chrysoxantha chrysoxesta chrysozona chrysura chthoniopa cianolitha

cicatricosa cicinnota cimmeria

cimmeriella cincinnata cinclidias cinefacta

cineracea cinerascens cinerata cinerea cinetica cingulata cinnabarina cinnabaritis cionophora circaea circe circensis circiformis circographa circophora circopis circulata circumclusa circumflua circumfusa circumplexa cirrhacma cirrhaea cirrhantha
CAPUA

DESMAUCHA* SCHENDYLOTIS* CHOREUTIS

ARISTEIS*

LIMNOECIA MACROBATHRA * ENARMONIA

ANTHOZELA *

LEUCOPTERA

STATHMOPODA

GLYPHIPTERYX

XYSMATODOMA

ASBOLISTIS *

AEOLANTHES

TRACHYCENTRA

TIMYRA.

ERECHTHIAS

[TEPHROSARA*]

EULECHRIA

OINOPHILA

NOEZA

HYPONOMEUTA

\section{SAPHENEUTIS}

TINISSA

EPICHORISTA

PHYRGANEUTIS*

OROPHIA*

BRACHYNEMATA*

MUSURGA

LASPEYRESIA

CIRCICA *

STOMOPTERYX

COSMOPTERYX

SCHOENOTENES

ODITES

PARASPASTIS*

MELASINA

ASYMPHORODES

AETHERASTIS

PERIACMA

ASYMPLECTA *

BRACHMIA

ARGYROPLOCE

OPOSTEGA

EPIPHTHORA

ACROCERCOPS
I9I2, Trans. Ent. Soc. London, I9II: 675. (BM)

I9I8, Exotic Microlepidoptera, 2: I47. (BM)

I9Io, Rec. Indian Mus. 5: 226. (K)

I932, Exotic Microlepidoptera, 4:275. (v)

I884, Proc. Linnean Soc. N.S. Wales, 9: 762.

I920, Exotic Microlepidoptera, 2: 320.

I886, Proc. Linnean Soc. N.S. Wales, Io: 804.

I9I2, Ent. Mon. Mag. 48: 34. (Sub.)

I9I3, Ann. Transvaal Mus. 3: 28o. (T)

1920, Exotic Microlepidoptera, $2: 358$.

I924, Exotic Microlepidoptera, 3: 96.

I92I, Ann. Transvaal Mus. 8: II2. (T)

I893, Proc. Linnean Soc. N.S. Wales, 7: 506.

1936, Exotic Microlepidoptera, 5: 49.

I938, in Caradja and Meyrick, Deuts. Ent. Zeit. Iris, 52: I2. (BM)

I922, Exotic Microlepidoptera, 2: 598. (P)

I9I6, Exotic Microlepidoptera, I: 570. (BM)

I9I4, Trans. Proc. New Zealand Inst. 4: II3.

I883, Proc. Linnean Soc. N.S. Wales, 7: 543.

I9I5, Exotic Microlepidoptera, I: 355.

I9I8, Exotic Microlepidoptera, 2: I50. (BM)

I935, in Caradja and Meyrick, Materialien zu einer Microlepidopteren Fauna der Chinesischen Provinzen Kiangsu, Chekiang und Hunan, p. 89. (BM) I9I4, Supplementa Entomologica, No. 3, p. 59. (D) I9Io, Trans. Ent. Soc. London, I9Io: 476. I920, Ann. South African Mus. I7: 276. (SA) I884, Proc. Linnean Soc. N.S. Wales, 9: 742. (MA) I884, Proc. Linnean Soc. N.S. Wales, 9: 738. 1885, Proc. Linnean Soc. N.S. Wales, 9: 1045. 1923, Exotic Microlepidoptera, 3: 3. (BM) I928, Exotic Microlepidoptera, 3: 45I. I888, Trans. Proc. New Zealand Inst. 20: 88. I9I2, Ann. Transvaal Mus. 3:67. (BM) I921, Ann. Transvaal Mus. 8: 94. (T) I928, Exotic Microlepidoptera, 3: 459. I930, Exotic Microlepidoptera, 4: I8. (BM) I9I5, Exotic Microlepidoptera, I: 480. (BM) I909, Ann. South African Mus. 5: 364. (SA) I929, Trans. Ent. Soc. London, 76: 499. I9I8, Exotic Microlepidoptera, 2: I90. (BM) I922, Exotic Microlepidoptera, 2: 546. (BM) I921, Zool. Meded. 6: I94. (L) I922, Exotic Microlepidoptera, 2: 506. (P) I922, Exotic Microlepidoptera, 2: 523. (BM) I9II, Ann. Transvaal Mus. 2: 237. (T) I9I4, Ann. Transvaal Mus. 4: I9o. (T) I9I5, Trans. Ent. Soc. London, I9I5: 23 I. 


\begin{tabular}{|c|c|c|}
\hline cirrhanthes & CAPUA & I92I, Zool. Meded. 6: I46. (L) \\
\hline cirrhas & ENARMONIA & I9I 2, Ent. Mon. Mag. 48: 34. (Sub.) \\
\hline cirrhaspis & STATHMOPODA & I922, Exotic Microlepidoptera, 2: 585 . (P) \\
\hline cirrhocephala & MELASINA & I937, Exotic Microlepidoptera, 5: II4. \\
\hline hochrosta & LIMNOECIA & I933, Exotic Microlepidoptera, 4: 429 . \\
\hline cirrhocoma & ANACAMPSIS & I9I4, Ann. Transvaal Mus. 4: I93. (T) \\
\hline cirrhocosma & HILAROGRAPHA & I930, Exotic Microlepidoptera, 4: I. \\
\hline cirrhocrena & HYPOCHASMIA* & I929, Exotic Microlepidoptera, 3: 5I7. (BM) \\
\hline cirrhocrossa & CACOECIA & I926, Sarawak Mus. Journ. 3: I48. \\
\hline cirrhocrotala & GRACILARIA & I928, Exotic Microlepidoptera, 3: 4IO. \\
\hline cirrhodes & PHILOBOTA & I9I3, Exotic Microlepidoptera, I: I26. \\
\hline cirrhodora & MACROBATHRA & I9I5, Exotic Microlepidoptera, I : 296. \\
\hline odoris & DICHELOPA & I934, Pacific Ent. Surv. Publ. 7, art. 28, p. 344. (вв) \\
\hline ogramma & STENOMA & I93o, Exotic Microlepidoptera, 4:27. (BM) \\
\hline cirrhographa & BUCCULATRIX & I9I5, Trans. Ent. Soc. London, I9I5: 247 . \\
\hline cirrholeuca & IMMA & I928, Exotic Microlepidoptera, 3: 42I. (BM) \\
\hline $\mathrm{pa}$ & PROTEODOXA* & $\begin{array}{l}\text { I938, Institut des Parcs Nationaux du Congo Belge, } \\
\text { fasc. I4, p. I5. (c) }\end{array}$ \\
\hline cirrhophanes & PHYLLOCNISTIS & I9I5, Exotic Microlepidoptera, I : 348 . \\
\hline hragma & XYLORYCTA & I921, Exotic Microlepidoptera, 2: 445. \\
\hline opis & GRACILARIA & I907, Proc. Linnean Soc. N.S. Wales, 32: 66. \\
\hline cir & ZELLERIA & $\begin{array}{l}\text { I93I, Ann. Mus. Nac. Hist. Nat. Buenos Aires, } 36: 398 . \\
\text { (BM) }\end{array}$ \\
\hline cirrhosoma & ETHMIA & $\begin{array}{l}\text { I920, Voyage de Ch. Alluaud et } R \text {. Jeannel en Afrique } \\
\text { Oriental, II, Microlepidoptera, p. } 88 \text {. ( } \mathrm{P})\end{array}$ \\
\hline cirrhospila & CHELARIA & I920, Exotic Microlepidoptera, 2: 302. (вM) \\
\hline cirrhoxantha & STENOMA & I9I5, Exotic Microlepidoptera, I : 477. (BM) \\
\hline cirrhozona & TARPHYSCELIS & I921, Zool. Meded. 6: I89. (L) \\
\hline cirrophaea & PHALAROTARSA* & I924. Exotic Microlepidoptera, 3: I02. (BM) \\
\hline des & ASYMPHORODES & I929, Trans. Ent. Soc. London, 76: 500. \\
\hline cis: & STATHMOPODA & I9I3, Exotic Microlepidoptera, I: 84 . \\
\hline cistulata & ANACAMPSIS & I9I4, Trans. Ent. Soc. London, I9I4: 258. (Bм) \\
\hline citharista & NOTHRIS & I9I3, Journ. Bombay Nat. Hist. Soc. 22: I7o. (вм) \\
\hline citharistis & ARGYROPLOCE & I909, Journ. Bombay Nat. Hist. Soc. I9: 595. (K) \\
\hline citharistis & HILAROGRAPHA & I9o9, Journ. Bombay Nat. Hist. Soc. I9: 428. (BM) \\
\hline citharoda & PARECTOPA & I9I6, Trans. Proc. New Zealand Inst. 48: 4I8. \\
\hline aroeda & ORTHIOSTOLA & I928, Exotic Microlepidoptera, 3: 479. (вм) \\
\hline citracma & PYRODERCES & I9I5, Exotic Microlepidoptera, I: 3 I0. \\
\hline ntha & ODITES & I9o8, Proc. Zool. Soc. London, p. 729. (Bм) \\
\hline citranthes & EPITHECTIS & I923, Exotic Microlepidoptera, 3: I5. (BM) \\
\hline citrarga & COLEOPHORA & I934, Exotic Microlepidoptera, 4: 460. \\
\hline ula & PAMMECES & I922, Exotic Microlepidoptera, 2: 584. (BM) \\
\hline ulax & NEMATOCHARES* & I93I, Exotic Microlepidoptera, 4:83. (v) \\
\hline citricula & GRACILARIA & I9I2, Exotic Microlepidoptera, I: 26. (T) \\
\hline citrina & ZELLERIA & I893, Proc. Linnean Soc. N.S. Wales, 7: 586. \\
\hline citrinacma & PACHYRHABDA & I936, Exotic Microlepidoptera, 4: 6I7. \\
\hline citrinodes & HIEROXESTIS & I922, Exotic Microlepidoptera, 2: 559. (BM) \\
\hline nopa & COSMOPTERYX & I9I5, Trans. Ent. Soc. London, I9I5: 204. (BM) \\
\hline opis & STATHMOPODA & I927, Exotic Microlepidoptera, 3: 378. (T) \\
\hline citriseca & OPOGONA & I928, Exotic Microlepidoptera, 3: 400. \\
\hline
\end{tabular}


citrites

citrocarpa

citrocephala

citrochrysa

citrochyta

citroclista

citrocoma

citrocosma

citrocrana

citrodesma

citrodeta

citrodora

citroglypta

citrogramma

citrogramma

citroleuca

citrolopha

citromela

citromicta

citromitra

citronota

citronympha

citrophaea

citrophanes

citrophricta

citroplecta

citropleura

citroptila

citrorrhoa

citroscia

citrostrota

citroxantha

citrozona

citrozona

citrura

civica

civilis

cladara

cladophora

cladophragma

clandestina

clara

clarana

clarescens

clarescens

claricoma

clarifica

clarifica
PTEROPHORUS

SCAEOSOPHA

COPHOSOPHA*

GRACILARIA

PHILEDONE

HYPERCALLIA

TINEA

ARISTOTELIA

NOTHOGENES*

MACROSACES

CRYPTOLECHIA

ACROCERCOPS

LABDIA

DECADARCHIS

DELARCHIS*

CNEPHASIA

OPOGONA

ODITES

PROTOGRYPA*

ANARSIA

HYPERCALLIA

PHYLLOCNISTIS

STENOMA

PROTOPTERNA

HEMIMENE

MICROCOLONA

PLATYPTILIA

APOTACTIS

TONZA

PROMENESTA

BRACHMIA

STENOMA

CHARICRITA*

LEPTONOMA*

AEGERIA

ACROCERCOPS

HEXADACTYLIA

PROTOLECHIA

AEOLANTHES

IMMA

GELECHIA

HAPSIFERA

DICHELIA

BACTRA

DICHOMERIS

DIOPHILA *

EUCOSMA

HETEROZYGA
I908, Trans. Ent. Soc. London, I907: 502.

I93I, Exotic Microlepidoptera, 4: II8. (v)

I937, Exotic Microlepidoptera, 5: II7.

1930, Exotic Microlepidoptera, 3: 582.

1926, Exotic Microlepidoptera, 3: 247. (Bм)

I930, Exotic Microlepidoptera, 3:576. (P)

1924, Exotic Microlepidoptera, 3: 72.

rgo8, Journ. Bombay Nat. Hist. Soc. I8: 437. (BM)

I932, Exotic Microlepidoptera, 4: 2II.

I9II, Ann. Transvaal Mus. 3: 74. (BM)

I92I, Exotic Microlepidoptera, 2: 394. (BM)

I9I4, Journ. Bombay Nat. Hist. Soc. 23: I2I.

1928, Exotic Microlepidoptera, 3: 386.

I93I, Exotic Microlepidoptera, 4: I65.

r938, in Caradja and Meyrick, Deuts. Ent. Zeit. Iris, 52: 15 .

r9I2, Trans. Ent. Soc. London, I9I I: 683.

I932, Exotic Microlepidoptera, 4: 23I.

1923, Bull. Mus. Nat. Hist. Nat. Paris, 29: 564.

I9I4, Exotic Microlepidoptera, I: 234. (BM)

I921, Ann. Transvaal Mus. 8: 8o. (T)

I932, Exotic Microlepidoptera, 4: 279.

I926, Exotic Microlepidoptera, 3: 262.

I93I, Exotic Microlepidoptera, 4: 42. (v)

I921, Zool. Meded. 6: I50. (L)

1922, Exotic Microlepidoptera, 2: 528. (BM)

I9I7, Exotic Microlepidoptera, 2: 49.

rgo8, Trans. Ent. Soc. London, r907: 482.

1933, Exotic Microlepidoptera, 4: 353. (v)

igo5, Journ. Bombay Nat. Hist. Soc. r6: 6r.4.

I93I, Exotic Microlepidoptera, 4:46. (v)

rgI , Journ. Bombay Nat. Hist. Soc. 20: 72r. (BM)

I9I6, Exotic Microlepidoptera, I: 5I5. (BM)

I9I3, Exotic Microlepidoptera, I: I43.

I9I6, Exotic Microlepidoptera, I: 607.

1927, Exotic Microlepidoptera, 3: 73I. (BM)

r9I4, Journ. Bombay Nat. Hist. Soc. 23: II9.

r92 I, Exotic Microlepidoptera, 2: 4I7. (BM)

I904, Proc. Linnean Soc. N.S. Wales, 29:346.

I938, in Caradja and Meyrick, Deuts. Ent. Zeit. Iris,

$52: \mathrm{I}_{3}$.

I906, Trans. Ent. Soc. London, I9o6: I73. (BM)

1923, Exotic Microlepidoptera, 3: I9. (BM)

1934, Exotic Microlepidoptera, 4: 5I6. (c)

I881, Proc. Linnean Soc. N.S. Wales, 6: 475.

Igr2, Trans. Ent. Soc. London, IgI I : 689. (BM)

I9I3, Journ. Bombay Nat. Hist. Soc. 22: I74. (BM)

I937, Exotic Microlepidoptera, 5: I49.

I913, Ann. Transvaal Mus. 3:274. (T)

I9I4, Exotic Microlepidoptera, r: 243. (BM) 


$\begin{array}{ll}\text { clarisecta } & \text { LOBESIA } \\ \text { clarisignis } & \text { OXYPTILUS } \\ \text { clarisona } & \text { LITHOCOLLETIS } \\ \text { clarissa } & \text { ACROCLITA } \\ \text { clasmatica } & \text { CLEPTICODES } \\ \text { classica } & \text { METACHANDA } \\ \text { clastopetra } & \text { GRACILARIA } \\ \text { clastozona } & \text { OPOSTEGA } \\ \text { claudescens } & \text { TIMOCRATICA } \\ \text { clausa } & \text { DRIMYLASTIS } \\ \text { claustrata } & \text { LECITHOCERA } \\ \text { claustrifera } & \text { TELPHUSA }\end{array}$

clausulata

clavaria

clavata.

clavata

clavicularis

claviculata

claviculata

clavifera

clavifera

claviformis

clavigera

clearcha

clematias

clemens

cleodora

cleodoxa

cleomorpha

cleonyma

cleopatra

cleophanes

cleophanta

clepsidoma

clepsidoxa

clepsinoma

clepsiphaga

clerodotis

cleronoma

cleronoma

clerota

clerotheta

clerotoma

clidias

climacaspis

climacias

EULECHRIA

TRICHOTAPHE

ARGYROPLOCE

STENOMA

EMMOCHLISTA *

PRYTANEUTIS*

GLYPHIPTERYX

HYPTIASTIS*

SCYTHRIS

ARISTOTELIA

NEMOTOIS

CROCANTHES

LOZOSTOMA

ANTAEOTRICHA

COSMOPTERYX

SYSCALMA

EUCOSMA

THIOTRICHA

ACROCERCOPS

LITHOCOLLETIS

ONEBALA

ALUCITA

SAROPLA

ZONOPETALA*

BUCCULATRIX

LITHOCOLLETIS

THIOTRICHA

TRYMALTIS
1932, Exotic Microlepidoptera, 4: 308. (BM)

1924, Exotic Microlepidoptera, 3: 93.

I9I6, Exotic Microlepidoptera, I: 622.

I921, Zool. Meded. 6: I52. (L)

I934, Exotic Microlepidoptera, 4: 479.

I9I I, Trans. Linnean Soc. London, I4:278. (Bм)

I928, Exotic Microlepidoptera, 3: 4I2.

I9I3, Ann. Transvaal Mus. 3:327. (T)

I925, Exotic Microlepidoptera, 3: I77. (BM)

I9I9, Exotic Microlepidoptera, 2: 242.

I9I0, Trans. Ent. Soc. London, I9Io: 448. (вм)

I935, in Caradja and Meyrick, Materialien zu einer

Microlepidopteren Fauna der Chinesischen Provinzen

Kiangsu, Chekiang und Hunan, p. 66. (BM)

I9I2, Ann. Transvaal Mus. 3: 74. (T)

I9I4, Exotic Microlepidoptera, I: 284.

I9I4, Exotic Microlepidoptera, I: I97. (MA)

I9I4, Exotic Microlepidoptera, I: 166.

$\begin{array}{ll}\text { GLYCEROPHTHORA* I935, Exotic Microlepidoptera, 4: 590. (BM) } \\ \text { ARGYROPLOCE } & \text { I909, Journ. Bombay Nat. Hist. Soc. I9: } 600 .\end{array}$

I909, Ann. Transvaal Mus. 2: I7, pl. 5, fig. Io. (T)

I920, Voyage de Ch. Alluaud et R. Jeannel en Afrique

Oriental, II, Microlepidoptera, p. 65. (P)

I9I2, Trans. Ent. Soc. London, I9I I : 7 I5. (BM)

I93I, Exotic Microlepidoptera, 4: Io6.

I9I I, Journ. Bombay Nat. Hist. Soc. 2I : Iog.

I921, Zool. Meded. 6: I85. (L)

I9I I, Journ. Bombay Nat. Hist. Soc. 20: 734. (Bм)

I92I, Ann. Transvaal Mus. 8: II4. (T)

I935, Exotic Microlepidoptera, 4: 583. (вм)

1922, Exotic Microlepidoptera, 2: 535.

I93I, Exotic Microlepidoptera, 4: 74. (BM)

I897, Proc. Linnean Soc. N.S. Wales, 22: 4I6.

1925, Exotic Microlepidoptera, 3: I66. (BM)

1937, Exotic Microlepidoptera, 5: I47. (T)

I922, Arkiv för Zool. vol. I4, part I5, p. 7 .

I9I6, Exotic Microlepidoptera, 2: I8. (BM)

1929, Exotic Microlepidoptera, 3: 499. (Bм)

I9I6, Exotic Microlepidoptera, $2: 2$.

I922, Exotic Microlepidoptera, 2: 561.

I9Io, Journ. Bombay Nat. Hist. Soc. 20: 450. (BM)

I920, Voyage de Ch. Alluaud et $R$. Jeannel en Afrique

Oriental, II, Microlepidoptera, p. 4I. (P)

GLYPHIPTERYX
I884, Proc. Linnean Soc. N.S. Wales, 9: 746.

I883, Proc. Linnean Soc. N.S. Wales, $7: 460$.

I9I5, Exotic Microlepidoptera, I: 370.

I9I5, Trans. Ent. Soc. London, I9I5: 222.

Igo8, Journ. Bombay Nat. Hist. Soc. I8: 439. (вм)

1920, Ann. South African Mus. I7: 296. (SA)

I9I I, Proc. Limnean Soc. N.S. Wales, 36: 295. 


\section{climacista \\ climacodes \\ climacosema \\ climacota \\ clinacta}

clinarcha

clinocosma

clinodesma

clinogramma

clinometra

clinomima

clinomochla

clinopeda

clinosema

clinozona

clisias

clisiopa

clistogramma

clistopa

clistrodoma

clitarcha

clitellaria

clitozona

clivigera

clivosa

clivosa

clodiana

clonodes

clonomicta

clopaea

clopica

closterias

clotho

clydonias

clysmographa

clytandra

clytemnestra

clytocarpa

clytosema

clytozona

cnaphalodes

cnaphalodes

cnecodes

cnemoptila

cnemosaris

cnephaea

coacervata

coagulata
PTILOTHYRIS

PSEPHOLOGA (?)

EUCOSMA

PROMALACTIS

AEOLANTHES

STAGMATOPHORA

OXYLYCHNA

LIMNOECIA

ACROCERCOPS

PROMALACTIS

HIEROXESTIS

NEPTICULA

THIOTRICHA

ARISTOTELIA

ACROCERCOPS

CNEPHASIA

ACROCERCOPS

CALLIPRORA

DECADARCHIS

GELECHIA

TINAEGERIA

PACHYGENEIA*

PACHYDYTA*

CACOECIA

ACROCLITA

ANTAEOTRICHA

LABDIA

SCARDIA

ALLOTALANTA

PYRODERCES

GELECHIA

CHARIPHYLLA*

ASTOXENA*

CYDIA

STENOMA

ORTHENCHES

LABDIA

ARGYROPLOCE

ACROCERCOPS

DINOCHORA*

CAPUA

MELASINA

ATELOSTICHA

ACHARNEODES

STENOMA

PLEUROTA

LACTURA

MELASINA
I926, Exotic Microlepidoptera, 3: 289.

I925, Bull. Soc. R. Ent. Egypte, 9: 2I7.

I938, Trans. R. Ent. Soc. London, 87:5II. (BM)

I9I4, Exotic Microlepidoptera, I : 229. (BM)

I925, in Caradja, Mem. Sect. Sti. Acad. Românä. 3: 382.

(BM)

I92I, Exotic Microlepidoptera, 2: 45I.

I9I9, Exotic Microlepidoptera, $2: 249$.

I932, Exotic Microlepidoptera, 4: 2I5.

I93I, in Joannis, Ann. Soc. Ent. France, 98 (Supp.): 739

$[5 \mathrm{OI}] .(\mathrm{P})$

I908, Journ. Bombay Nat. Hist. Soc. I8: 809. (BM)

I9I8, Exotic Microlepidoptera, 2: I86.

I934, Exotic Microlepidoptera, 4: 468.

I9I8, Exotic Microlepidoptera, 2: I24. (BM)

I935, The Entomologist, 68: 285.

I920, Exotic Microlepidoptera, 2: 29I. (BM)

I9I 2, Trans. Ent. Soc. London, I9I I: 685. (BM)

I935, Exotic Microlepidoptera, 4:598.

I926, Exotic Microlepidoptera, 3: 28I. (Bм)

1929, Trans. Ent. Soc. London, 76: 506.

I923, Exotic Microlepidoptera, 3: 2I. (BM)

I92I, Exotic Microlepidoptera, 2: 442.

1923, Exotic Microlepidoptera, 3: I I. (BM)

I922, Exotic Microlepidoptera, 2: 592. (BM)

1932, Exotic Microlepidoptera, 4: 253. (v)

I9I2, Journ. Bombay Nat. Hist. Soc. 2I: 855. (BM)

I9I8, Exotic Microlepidoptera, 2: I99. (BM)

I927, Exotic Microlepidoptera, 3: 384. (BM)

I893, Proc. Linnean Soc. N.S. Wales, 7: 523.

I927, Exotic Microlepidoptera, 3: 38I. (T)

I9I7, Exotic Microlepidoptera, 2: 40.

I931, Ann.Mus. Nac. Hist. Nat. Buenos Aires, 36:384. (вм)

I92I, Exotic Microlepidoptera, 2: 388. (BM)

I930, Exotic Microlepidoptera, 3: 593. (BM)

I907, Journ. Bombay Nat. Hist. Soc. I7: 734. (BM)

1925, Exotic Microlepidoptera, 3: I97. (BM)

I93I, Ann. Mus. Nac. Hist. Nat. Buenos Aires, 36: 406.

1923, Exotic Microlepidoptera, 3: 56. (BM)

1920, Exotic Microlepidoptera, 2: 349. (BM)

I920, Exotic Microlepidoptera, 2: 294.

1924, Exotic Microlepidoptera, 3: 69.

I9Io, Proc. Linnean Soc. N.S. Wales, 35: I87. (MA)

I9I7, Ann. Natal Mus. 3:6I5. (T)

I920, Exotic Microlepidoptera, 2: 378. (BM)

1930, Ann. Naturhist. Mus. Wien, 44:225. (v)

I925, Exotic Microlepidoptera, 3: I8I. (BM)

I889, Proc. Linnean Soc. N.S. Wales, (Ser. 2) 3: I650.

1938, Trans. R. Ent. Soc. London, 87: 523. (BM)

I9I9, Exotic Microlepidoptera, 2: 267. 
cochlias cocta coctilis cocytias codicaria codicata codonectis codonias coelocrossa

coenodes coenographa coenomorpha coenosa coenotypa coenulenta coercita coesyrias colabristis

colasta coleasta coleosema coleoxantha colivora collapsa collecta collega collegialis colleta colligata collina collinearis colluta collybista colobota colocynthia colometra colonaea colonialis colonias colonica colonota coloptila colorata colposaris colpota colubratus colubrina
BERYLLOPHANTIS* TRICHOTAPHE TORTRIX SCALIDEUTIS SPANIOPTILA STENOMA ARGYROPLOCE GLYPHIPTERXX CRYPTOLECHIA

TRACHYNTIS TORTRIX BLASTOBASIS HOPLITICA PROTOBATHRA CYMOTRICHA ONEBALA ASYMPHORODES MIEZA

[ERIOPYRRHA *] PHTHORIMAEA LECITHOCERA DECADARCHIS

LACTURA ARGYROPLOCE SIMAETHIS APATETRIS ODITES HETEROGYMNA TINEA STENOMA PACHYSARIS GELECHIA THRYPSIGENES* GONIOTERMA PLATYNOTA SAPHENEUTIS XESTOCASIS COESYRA EULECHRIA HOPLITICA MELASINA ACROCLITA ACROCERCOPS GLYPHIPTERYX STENOMA SIMAETHIS PTEROPHORUS OINOPHILA
1938, Trans. R. Ent. Soc. London, 87: 509. (BM) I9I3, Journ. Bombay Nat. Hist. Soc. 22: I79. (BM) I922, Exotic Microlepidoptera, 2: 497. I9I5, Exotic Microlepidoptera, I: 307. 1920, Exotic Microlepidoptera, 2: 290. I9I6, Exotic Microlepidoptera, I: 530. (BM) I927, Exotic Microlepidoptera, 3: 339. (BM) I909, Trans. New Zealand Inst. 4I: I5. I935, in Caradja and Meyrick, Materialien zu einer Microlepidopteren Fauna der Chinesischen Provinzen Kiangsu, Chekiang und Hunan, p. 82. (BM) 1889, Proc. Linnean Soc. N.S. Wales, (Ser. 2) 3: 1588. 1938, Trans. R. Ent. Soc. London, 87: 506. (BM) I93I, Exotic Microlepidoptera, 4: I77. (BM) 1883, Proc. Linnean Soc. N.S. Wales, 7: 506. I9I8, Exotic Microlepidoptera, 2: I55. (BM) 1927, Exotic Microlepidoptera, 3: 355. (T) I923, Exotic Microlepidoptera, 3: 42. (BM) I929, Trans. Ent. Soc. London, 76: 498.

I907, Proc. Linnean Soc. N.S. Wales, 32: 89. I92I, Ann. Transvaal Mus. 8: 74. (T) I9I8, Exotic Microlepidoptera, 2: 103.

I934, Pacific Ent. Surv. Publ. 7, art. 28, p. 354. (Bв) I924, Exotic Microlepidoptera, 3: I22. (Lost) I932, Exotic Microlepidoptera, 4: 31о. (BM) I934, Exotic Microlepidoptera, 4: 457. (BM) I921, Ann. Transvaal Mus. 8: 64. (T) I927, Exotic Microlepidoptera, 3:364. 1925, Exotic Microlepidoptera, 3: г38. (вм) I893, Proc. Linnean Soc. N.S. Wales, 7: 539 . I9I5, Exotic Microlepidoptera, I: 46I. (BM) I9I4, Trans. Ent. Soc. London, I9I4: 277. (BM) 1927, Exotic Microlepidoptera, 3: 349. (BM) I9I4, Trans. Ent. Soc. London, I9I4: 272. (BM) I9I5, Exotic Microlepidoptera, I: 384 . (BM) I926, Trans. Ent. Soc. London, 74: 276. (BM) I9I6, Exotic Microlepidoptera, I: 607. I9I5, Exotic Microlepidoptera, I: 3 I7. I902, Trans. R. Soc. S. Australia, 26: I39. I936, Exotic Microlepidoptera, 5: 50. (BM) I 888, Proc. Linnean Soc. N.S. Wales, (Ser. 2) 2: 942. I9I6, Exotic Microlepidoptera, I: 6II.

I9I I, Proc. Linnean Soc. N.S. Wales, 36: 238.

I937, Exotic Microlepidoptera, 5: III.

I9I3, Exotic Microlepidoptera, I: I03. (BM) I925, Exotic Microlepidoptera, 3: I85. (BM) I9II, Trans. New Zealand Inst. 43:67. I909, Ann. Transvaal Mus. 2: 3, pl. I, fig. 4. (T) I9I4, Exotic Microlepidoptera, I: 288. 


$\begin{array}{ll}\text { columbaris } & \text { STENOMA } \\ \text { columella } & \text { HARMOLOGA } \\ \text { columella } & \text { MICROSCHISMUS } \\ \text { columnaris } & \text { GLYPHIPTERYX } \\ \text { columnata } & \text { METACHANDA } \\ \text { colymbetella } & \text { EPICEPHALA* } \\ \text { comarcha } & \text { BORKHAUSENIA } \\ \text { comastis } & \text { GONIONOTA } \\ \text { comata } & \text { AGROECODES } \\ \text { combota } & \text { LIMNOECIA } \\ \text { combusta } & \text { LECITHOCERA } \\ \text { cometa } & \text { SCYTHRIS } \\ \text { cometophora } & \text { GLYPHIPTERYX } \\ \text { comis } & \text { ARISTOTELIA } \\ \text { comissata } & \text { STEGASTA } \\ \text { comitialis } & \text { HETEROGYMNA } \\ \text { commaculata } & \text { TELPHUSA } \\ \text { commatias } & \text { HIEROXESTIS }\end{array}$

[MACHIMOSTOLA*]

$\begin{array}{ll}\text { commatopa } & \text { DELTOPLASTIS } \\ \text { commendata } & \text { BLASTOBASIS } \\ \text { commensalis } & \text { BACTRA } \\ \text { commixta } & \text { BORKHAUSENIA } \\ \text { commoda } & \text { STATHMOPODA } \\ \text { commodata } & \text { ONEBALA } \\ \text { commota } & \text { SCYTHRIS } \\ \text { commotica } & \text { PROMALACTIS } \\ \text { communicata } & \text { ENCRASIMA } \\ \text { communis } & \text { DEPRESSARIA } \\ \text { communita } & \text { EUMASIA } \\ \text { commutata } & \text { EUMITURGA } \\ \text { comochora } & \text { LEPIDOSCIA } \\ \text { comosaris } & \text { BORKHAUSENIA } \\ \text { comoxantha } & \text { COESYRA } \\ \text { compacta } & \text { DEPRESSARIA } \\ \text { compacta } & \text { CACOECIA } \\ \text { compar } & \text { LABDIA } \\ \text { comparanda } & \text { COMPSOLECHIA } \\ \text { compastis } & \text { GLYPHIPTERYX } \\ \text { complanata } & \text { LIOCLEPTA* } \\ \text { complexa } & \text { AGRIOPHARA } \\ \text { complicata } & \text { THIOTRICHA } \\ \text { composita } & \text { STRENIASTIS } \\ \text { comprehensa } & \text { LATYPICA } \\ \text { comprobata } & \text { TELPHUSA } \\ \text { compsacma } & \text { DIATAGA } \\ \text { compsasis } & \text { LIMNOECIA } \\ & \end{array}$

compseuta
I9o9, Trans. Ent. Soc. London, I9o9: 3I. (BM) I927, Trans. Proc. New Zealand Inst. 57: 699. I927, Exotic Microlepidoptera, 3: 373. (T) I9I3, Exotic Microlepidoptera, I: 69. (BM) I9I I, Trans. Linnean Soc. London, I4:276. (вм) I88I, Proc. Linnean Soc. N.S. Wales, 5: I69. I920, Exotic Microlepidoptera, 2: 367. I909, Trans. Ent. Soc. London, I909: 25. (BM) I937, Exotic Microlepidoptera, 5: 85. (T) I92I, Exotic Microlepidoptera, 2: 454 . I9I8, Exotic Microlepidoptera, 2: IIO. (BM) Igog, Ann. Transvaal Mus. 2: 2I, pl. 7, fig. 3. (T) I88I, Proc. Linnean Soc. N.S. Wales, 5: 23I. I9I3, Ann. Transvaal Mus. 3: 282. (T) I923, Exotic Microlepidoptera, 3: I8. (BM) I925, Exotic Microlepidoptera, 3: I38. I921, Ann. Transvaal Mus. 8: 69. (T)

I9I5, Exotic Microlepidoptera, I: 365 . I932, Exotic Microlepidoptera, 4: 205. (BM) I922, Exotic Microlepidoptera, 2: 539. (вм) I922, Exotic Microlepidoptera, 2: 522. (BM) I928, Exotic Microlepidoptera, 3: 468. (BM) I9I3, Exotic Microlepidoptera, I: 85. I923, Exotic Microlepidoptera, 3: 44. (BM) I9I8, Exotic Microlepidoptera, 2: I57. I923, Exotic Microlepidoptera, 3: 6I. (BM) I9I8, Exotic Microlepidoptera, 2: I 54. I920, Ann. South African Mus. I7: 288. (SA) I928, Bull. Hill Mus. 2: 240. I926, Exotic Microlepidoptera, 3: 233. (BM) I893, Proc. Linnean Soc. N.S. Wales, 7: 508. I93I, Trans. Proc. New Zealand Inst. 62: 95. I889, Proc. Linnean Soc. N.S. Wales, (Ser. 2) 3: I657. I9I4, Ann. South African Mus. Io: 249. (SA) I9I8, Exotic Microlepidoptera, 2: I64. (BM) I92I, Zool. Meded. 6: I68. (L) I929, Exotic Microlepidoptera, 3: 506. (BM) I923, Exotic Microlepidoptera, 2: 6I8. (BM) I922, Trans. Ent. Soc. London, I922: II6. (BM) I9I6, Exotic Microlepidoptera, I: 542. I9I8, Exotic Microlepidoptera, 2: I22. (BM) I922, Exotic Microlepidoptera, 2: 503. I92I, Ann. Transvaal Mus. 8: I3I. (T) I935, Exotic Microlepidoptera, 4:584. (BM) I9I9, Exotic Microlepidoptera, 2: 270. (BM) 1935, in Caradja and Meyrick, Materialien zu einer Microlepidopteren Fauna der Chinesischen Provinzen Kiangsu, Chekiang und Hunan, p. 77. I889, Trans. Proc. New Zealand Inst. 2I: I6I. 


\begin{tabular}{|c|c|}
\hline compsitis & ARGYROPLOCE \\
\hline compsochares & TRICHOPTILUS \\
\hline compsocharis & STENOMA \\
\hline compsochroa & PROTOLECHIA \\
\hline compsocoma & STENOMA \\
\hline compsodelta & ARISTOTELIA \\
\hline compsogramma & BORKHAUSENIA \\
\hline compsographa & ANTAEOTRICHA \\
\hline compsomorpha & GNORIMOSCHEMA \\
\hline compsoneura & STENOMA \\
\hline compsophila & BRACHMIA \\
\hline compsoptila & CROCIDOSEMA \\
\hline compsoptila & PERONEA \\
\hline compsostoma & FRISILIA \\
\hline compsotoma & COMMATICA \\
\hline compsotypa & PHAEOSACES \\
\hline compsoxantha & ORNEODES \\
\hline compta & TISCHERIA \\
\hline compulsa & PYRAMIDOBELA \\
\hline concava & ODITES \\
\hline concavata & CACOECIA \\
\hline conchifera & ARGYROPLOCE \\
\hline conchitis & ACROLEPIA \\
\hline conchitis & EULIA \\
\hline conchodes & SPATALISTIS \\
\hline conchophanes & SOBAREUTIS* \\
\hline conchopleura & ARGYROPLOCE \\
\hline conchylias & HECTACMA \\
\hline conchylitis & TINEA \\
\hline conciliata & AUTOSTICHA \\
\hline conciliata & EUCOSMA \\
\hline concinerata & PARAPTICA* \\
\hline concinna & MACHIMIA \\
\hline concinna & MERIDARCHIS \\
\hline concisa & BATTARISTIS \\
\hline concitata & BATRACHEDRA \\
\hline conclusa & TRICHOTAPHE \\
\hline concolorana & TORTRIX \\
\hline concordana & TORTRIX \\
\hline concors & BATRACHEDRA \\
\hline concreta & PHTHORIMAEA \\
\hline concurrens & SCYTHRIS \\
\hline concussa & ORNIX \\
\hline condemnatrix & STENOMA \\
\hline condensata & ARISTOTELIA \\
\hline condylodes & TRICHOTAPHE \\
\hline condylota & STILBOSIS \\
\hline conferta & TINEA \\
\hline $\begin{array}{l}\text { confirmata } \\
\text { M.T.M. I-8 }\end{array}$ & ELACIIISTA \\
\hline
\end{tabular}

I9I 2, Journ. Bombay Nat. Hist. Soc. 2I: 870. (BM) I886, Trans. Ent. Soc. London, I886: I6. 1925, Exotic Microlepidoptera, 3: 218. (BM) I904, Proc. Linnean Soc. N.S. Wales, 29:370. I930, Ann. Naturhist. Mus. Wien, 44: 249. (v) I928, Proc. Hareaiian Ent. Soc. 7: 99. (SP) I920, Trans. Proc. New Zealand Inst. 52: 31. I9I6, Exotic Microlepidoptera, I: 49I. (BM) I929, Exotic Microlepidoptera, 3: 49I. (Bм) 1925, Exotic Microlepidoptera, 3: 2I7. (BM) I9I I, Journ. Bombay Nat. Hist. Soc. 20: 709. (вм) I936, Veröff. Deutschen Kol.-Mus. I: 33I, pl. I3, fig. 2 I. (H)

I923, Exotic Microlepidoptera, 3:56. (вм) I921, Ann. Transvaal Mus. 8:87. (T) I92I, Ann. Transvaal Mus. 8: 77. (T) I886, Trans. Proc. New Zealand Inst. I8: I72. 1924, Exotic Microlepidoptera, 3: 95. (T) I9I5, Exotic Microlepidoptera, I: 354. I931, Ann. Mus. Nac. Hist. Nat. Buenos Aires, 36: 399. I922, Zool. Meded. 7: 88. (L) I930, Exotic Microlepidoptera, 3: 6I4. (вм) I93I, Exotic Microlepidoptera, 4: 130 . I9I3, Exotic Microlepidoptera, I: I50. I9I2, Trans. Ent. Soc. London, I9Ir: 68r. (вм) I9Io, Proc. Linnean Soc. N.S. Wales, 35: 287. r9Io, Trans. Ent. Soc. London, r9ro: 470. I9II, Trans. Linnean Soc. London, I4:27I. (BM) I9I6, Exotic Microlepidoptera, I: 621. I9I9, Exotic Microlepidoptera, 2: 249. (Вм) I9I8, Exotic Microlepidoptera, 2: I54. (вм) 1920, Exotic Microlepidoptera, 2: 345. (вм) I9I7, Ann. South African Mus. I7: I5. (SA) r9I2, Trans. Ent. Soc. London, r9II: 697. (BM) I9I3, Exotic Microlepidoptera, I: 7I. (BM) I929, Exotic Microlepidoptera, 3: 505. (BM) I928, Exotic Microlepidoptera, 3: 395. (вм) I9I8, Ann. Transvaal Mus. 6: 23. (T) I88I, Proc. Linnean Soc. N.S. Wales, 6: 522. I88I, Proc. Linnean Soc. N.S. Wales, 6: 519. I9I7, Exotic Microlepidoptera, 2: 33. (BM) I9I4, Ann. Transvaal Mus. 4: I9I. (T) I92I, Ann. Transvaal Mus. 8: II5. (T) I933, Exotic Microlepidoptera, 4:363. I930, Exotic Microlepidoptera, 4: 3I. r928, Bull. Hill Mus. 2: 234. I92I, Ann. Transvaal Mus. 8: 85. (T) 1917, Exotic Microlepidoptera, 2: 47. (вм) I.9I4, Trans. Proc. Nere Zealand Inst. 46: II5. I93I, Exotic Microlepidoptera, 4: 9I. 


\begin{tabular}{|c|c|}
\hline confirmata & OXYLECHIA* \\
\hline confixa & TELPHUSA \\
\hline conflicta & POLYHYMNO \\
\hline conflua & ACROCERCOPS \\
\hline confluens & IMMA \\
\hline conformata & ASCALENIA \\
\hline conformis & LITHOCOLLETIS \\
\hline confragosa & CAPUA \\
\hline congelata & ANTAEOTRICHA \\
\hline conglobata & ETHMIA \\
\hline conglomerata & SCARDIA \\
\hline congrua & STENOMA \\
\hline coniata & GYPSOSARIS* \\
\hline coniata & OCYSTOLA \\
\hline conica & SPILONOTA \\
\hline conifera & CHELARIA \\
\hline coniochersa & PROCOMETIS \\
\hline coniochra & DECADARCHIS \\
\hline coniodes & MEGACRASPEDUS \\
\hline coniodina & MONOPIS \\
\hline coniodoxa & ERETMOGRAPTIS* \\
\hline coniogramma & MEGACRASPEDUS \\
\hline coniographa & EMPHANTICA* \\
\hline coniographa & GNATHOSARISTIS* \\
\hline conioleuca & DEROXENA \\
\hline conioma & STATHMOPODA \\
\hline coniombra & EPIPHTHORA \\
\hline coniomicta & EPISYRTA* \\
\hline coniopa & STENOMA \\
\hline coniophaea & STENOMA \\
\hline coniopis & TRIPTOLOGA* \\
\hline conioptila & EPERMENIA \\
\hline coniortia & PELTOPHORA \\
\hline coniosema & BATTARISTIS \\
\hline coniostrepta & ZELLERIA \\
\hline
\end{tabular}

conioxantha

conisalia

conista

conjuncta

conjurata

conobathra

conomochla

conophanta

conosema

conoterma

conotoma

conscia

TINGENTERA

THEREUTIS
I9I7, Trans. Ent. Soc. London, I9I7: 39. (BM)

I9I8, Ann. Transvaal Mus. 6: 5I.

I9I7, Trans. Ent. Soc. London, I9I7: 5I.

I9I4, Ann. Transvaal Mus. 4: 20I. (T)

I93I, Exotic Microlepidoptera, 4: I8I. (BM)

I921, Ann. Transvaal Mus. 8: 97. (T)

I9Io, Rec. Indian Mus. 2: 226. (k)

I922, Exotic Microlepidoptera, 2: 494.

I926, Exotic Microlepidoptera, 3: 236. (BM)

I9I2, Trans. Ent. Soc. London, I9II: 7I7. (BM)

I922, Arkiv för Zool. vol. I4, part I5, p. I2.

I925, Exotic Microlepidoptera, 3:2I3. (BM)

I9o9, Ann. South African Mus. 5: 375. (SA)

I885, Proc. Linnean Soc. N.S. Wales, 9: Io69.

I9II, Ann. Transvaal Mus. 2: 225. (T)

I9I6, Exotic Microlepidoptera, I: 582. (BM)

I922, Arkiv för Zool. vol. I4, part 15, p. Io.

I9I5, Exotic Microlepidoptera, I: 368.

I904, Proc. Linnean Soc. N.S. Wales, 29:28I.

I93I, Exotic Microlepidoptera, 4: 93.

I938, Institut des Parcs Nationaux du Congo Belge,

fasc. I4, p. 22.

I92I, Exotic Microlepidoptera, 2: 424 .

I928, Exotic Microlepidoptera, 3: 430. (Bм)

I936, Exotic Microlepidoptera, 5: 54.

I926, Exotic Microlepidoptera, 3: 29I. (BM)

I93I, Exotic Microlepidoptera, 4: I75.

I904, Proc. Linnean Soc. N.S. Wales, 29: 267.

I929, Trans. Ent. Soc. London, 76: 520.

I925, Exotic Microlepidoptera, 3: I84. (BM)

I930, Ann. Naturhist. Mus. Wien, 44: 246. (v)

I9I4, Exotic Microlepidoptera, I: 257. (BM)

I921, Ann. Transvaal Mus. 8: I22. (T)

I884, Proc. Linnean Soc. N.S. Wales, 9: 725 .

I922, Trans. Ent. Soc. London, I922: 74. (BM)

I938, in Caradja and Meyrick, Deuts. Ent. Zeit. Iris, 52: I7. (BM)

I93I, Exotic Microlepidoptera, 4: I92. (BM)

I888, Trans. Proc. New Zealand Inst. 20: I02.

IgII, Ent. Mon. Mag. 47:2I2.

I92I, Ann. Transvaal Mus. 8: 95. (T)

I920, Exotic Microlepidoptera, 2: 36I. (BM)

I928, Exotic Microlepidoptera, 3: 403. (T)

I933, Exotic Microlepidoptera, 4: 372. (BM)

I9Io, Journ. Bombay Nat. Hist. Soc. 20: I6I. (BM)

I9I3, Exotic Microlepidoptera, I: 69. (Bм)

I922, Exotic Microlepidoptera, 2: 524. (P)

[DINOChares*] Igo8, Journ. Bombay Nat. Hist. Soc. I8: 453. (BM)
I92I, Exotic Microlepidoptera, 2: 437. (BM) 
conscius

consecrata

conserva

conservata

considerata

consignata

consobrina

consociata

consona

consors

conspersa

constellaris

constellata

constellata

constituta

constrata

constricta

constrictana

constructa

consularis

consummata

contempta

contemptrix

contenta

contermina

contestata

contexta

contigua

continua

continuata

contophora

contorta

contracta

contraria

contributa

contrita

contubernalis

contumax

contumescens

conturbata

convallata

conveniens

convergens

conversa

convexa

conviciata
PTEROPHORUS

ODITES

MACHIMIA

PROTOMACHA

ANACAMPSIS

ODITES

STENOMA

EPITHECTIS

GELECHIA

PACHNISTIS

BATRACHEDRA

MOMPHA

AETHERASTIS

COPTOTELIA

STENOMA

LATYPICA

STENOMA

BATHROTOMA*

ARGY ROPLOCE

COESYRA

BRACHMIA

CONIOGENES*

CACOECIA

IMMA

ERETMOCERA

SETOMORPHA

HAPSIFERA

COELOSTATHMA

ODITES

PERIACMA

STENOMA

ACROCERCOPS

LECITHOCERA

PERIACMA

TINEA

PACHYSARIS

ACROLOPHUS

STENOMA

PHALONIA

BRACHMIA

MYRMECOZELA

STENOMA

ACROCLITA

PHELOTROPA

MNESISTEGA

TELPHUSA
I920, Voyage de Ch. Alluaud et R. Jeannel en Afrique Oriental, II, Microlepidoptera, p. 4I. (P)

I9I7, Exotic Microlepidoptera, 2: 57. (BM)

I9I4, Exotic Microlepidoptera, I: I83. (BM)

I9I8, Ann. Transvaal Mus. 6: 3I. (T)

I922, Trans. Ent. Soc. London, I922: 78. (BM)

I92I, Ann. Transvaal Mus. 8: I07. (T)

I9I5, Exotic Microlepidoptera, I: 460. (BM)

I9I4, Trans. Ent. Soc. London, I9I4: 232. (BM)

I9I7, Trans. Ent. Soc. London, I9I7: 5O. (BM)

I92I, Ann. Transvaal Mus. 8: 89. (T)

I9I6, Exotic Microlepidoptera, 2: 3I. (BM)

I93I, Ann. Mus. Nac. Hist. Nat. Buenos Aires, 36: 388.

I9I4, Journ. Bombay Nat. Hist. Soc. 23: I29. (BM)

I9I2, Trans. Ent. Soc. London, I9II: 70I. (BM)

1925, Exotic Microlepidoptera, 3: I9I. (BM)

I9I9, Exotic Microlepidoptera, 2: 258.

1926, Exotic Microlepidoptera, 3: 226. (BM)

I88I, Proc. Linnean Soc. N.S. Wales, 6: 675.

I922, Exotic Microlepidoptera, 2: 526. (BM)

I913, Exotic Microlepidoptera, I: II9.

I923, Exotic Microlepidoptera, 3: 47. (BM)

I936, Exotic Microlepidoptera, 5: 4I.

I925, in Caradja, Bull. Sect. sci. Acad. roum. $3: 378$.

I9I6, Exotic Microlepidoptera, I: 560. (BM)

I926, Ann. South African Mus. 23: 337. (SA)

I922, Exotic Microlepidoptera, 2: 598.

I9I6, Exotic Microlepidoptera, I: 6I7.

I926, Exotic Microlepidoptera, 3:259. (Вм)

I935, in Caradja and Meyrick, Materialien zu einer

Microlepidopteren Fauna der Chinesischen Provinzen

Kiangsu, Chekiang und Hunan, p. 84. (BM)

I922, Exotic Microlepidoptera, 2: 546. (BM)

I9I5, Exotic Microlepidoptera, I: 472. (BM)

I920, Exotic Microlepidoptera, 2: 292.

I9I8, Exotic Microlepidoptera, 2: I07. (BM)

I9Io, Journ. Bombay Nat. Hist. Soc. 20: I56. (BM)

I932, Exotic Microlepidoptera, 4: 209.

1922, Trans. Ent. Soc. London, I922: I05. (BM)

I933, Exotic Microlepidoptera, 2: 6IO. (P)

I9I6, Exotic Microlepidoptera, I: 522. (BM)

I93I, Exotic Microlepidoptera, 4: I57. (BM)

1933, Exotic Microlepidoptera, 4: 359. (BM)

I9I8, Ann. Transvaal Mus. 6:57. (T)

1925, Exotic Microlepidoptera, 3:217. (BM)

I93I, in Joannis, Ann. Soc. Ent. France, 98 (Supp.): 7 I5

[477]. (P)

I923, Exotic Microlepidoptera, 2: 6I4. (P)

I923, Exotic Microlepidoptera, 3: 36. (BM)

I929, Exotic Microlepidoptera, 3: 487. (BM) 


$\begin{array}{ll}\text { convicta } & \text { HYLOPHYGAS* } \\ \text { conviva } & \text { MOMPHA } \\ \text { convoluta } & \text { ACROCERCOPS } \\ \text { copeuta } & \text { BUCCULATRIX } \\ \text { cophias } & \text { GELECHIA } \\ \text { cophodes } & \text { BLASTOBASIS } \\ \text { copidobathra } & \text { LIMNOECIA } \\ \text { copidora } & \text { HIEROXESTIS } \\ \text { copidota } & \text { CIRCOSTOLA* } \\ \text { copidotis } & \text { BACTRA } \\ \text { copiosa } & \text { NARYCIA } \\ \text { coppatias } & \text { HETEROZYGA } \\ \text { copromima } & \text { ANTAEOTRICHA } \\ \text { copularis } & \text { PORINA } \\ \text { coracina } & \text { STOMOPTERYX } \\ \text { coracodes } & \text { STATHMOPODA } \\ \text { coracophila } & \text { TIQUADRA } \\ \text { coracopis } & \text { TINEA } \\ \text { corallina } & \text { MACHIMIA } \\ \text { corculata } & \text { COMOTECHNA } \\ \text { cordata } & \text { BRACHMIA } \\ \text { cordelia } & \text { ACROCLITA }\end{array}$

cordifera

coriaria

coriata

coricopa

coridarcha

coridophaga

corinthia

coriodes

cornicina

corniculata

corniculata

corniculata

cornigera

cornigera

cornuta

corollata

coronata

coronias

coronifera

coronigera

coronis

coronopa

coronopis

corporalis
PHTHEOCHROA

CRYPTOLECHIA

CRYPTOLECHIA

HYPEURYNTIS*

TRICHOTHYRSA

PYRODERCES

ACROCLITA

ANTAEOTRICHA

ACROCERCOPS

MELASINA

PLATYPTILIA

TRICHOTAPHE

CAPUA

PROMALACTIS

CHRYSONOMA

GRACILARIA

TINEA

CORSOCASIS*

AEOLANTHES

BRENTHIA

EUCLEODORA

LACTURA

AEGERIA
ACROCLITA
I932, Trans. Ent. Soc. London, 80: II9.

I93 I, Ann. Mus. Nac. Hist. Nat. Buenos Aires, 36: 388.

I908, Journ. Bombay Nat. Hist. Soc. I8: 824 .

I9I9, Exotic Microlepidoptera, 2: 288.

I9I3, Ann. Transvaal Mus. 3: 292. (T)

I9I8, Exotic Microlepidoptera, 2: I58. (BM)

I936, Exotic Microlepidoptera, 5: 30.

I9I5, Exotic Microlepidoptera, I: 363.

I889, Trans. Proc. New Zealand Inst. 21 : I63.

I909, Journ. Bombay Nat. Hist. Soc. I9: 584. (BM)

I92I, Ann. Transvaal Mus. 8: I33. (T)

1885, Proc. Linnean Soc. N.S. Wales, 9: I049.

I930, Exotic Microlepidoptera, 4: 22. (BM)

I9I2, Trans. Proc. New Zealand Inst. 44: I23.

I921, Exotic Microlepidoptera, 2: 427. (BM)

I923, Trans. Proc. New Zealand Inst. 54: I67.

I932, Exotic Microlepidoptera, 4: 328. (BM)

I909, Trans. Ent. Soc. London, I909: 42. (BM)

I9I4, Exotic Microlepidoptera, I: I75. (BM)

I92I, Exotic Microlepidoptera, 2: 400. (BM)

IgII, Journ. Bombay Nat. Hist.Soc. 20: 720. (BM)

I935, in Caradja and Meyrick, Materialien zu einer

Microlepidopteren Fauna der Chinesischen Provinzen

Kiangsu, Chekiang und Hunan, p. 52. (BM)

I932, Exotic Microlepidoptera, 4:267. (v)

I9I4, Exotic Microlepidoptera, I: I73. (MA)

I9I4, Supplementa Entomologica, No. 3, p. 53. (D)

I897, Trans. Ent. Soc. London, I897: 389.

I9I2, Exotic Microlepidoptera, I: 6I.

I925, Bull. Soc. R. Ent. Egypte, 9: 2II.

I9I2, Journ. Bombay Nat. Hist. Soc. 2I: 858. (BM)

I9I5, Exotic Microlepidoptera, I: 397. (BM)

I908, Journ. Bombay Nat. Hist. Soc. I8: 822.

I93I, in Joannis, Ann. Soc. Ent. France, 98 (Supp.): 746 [508]. (P)

I9I3, Ann. Transvaal Mus. 3:267. (T)

I9I3, Journ. Bombay Nat. Hist. Soc. 22: I77. (BM)

I9I2, Exotic Microlepidoptera, I: 2. (BM)

I9I8, Exotic Microlepidoptera, 2: 213. (BM)

I9I4, Exotic Microlepidoptera, I: 25I. (BM)

I933, Exotic Microlepidoptera, 4:362.

I9I I, Trans. Linnean Soc. London, I4: 304.

I9I2, Exotic Microlepidoptera, I: 59.

I938, in Caradja and Meyrick, Deuts. Ent. Zeit. Iris, 52: I2. (BM)

I9I8, Exotic Microlepidoptera, 2: I94. (BM)

I9I4, Exotic Microlepidoptera, I: 258. (BM)

I9I I, Proc. Linnean Soc. N.S. Wales, 36: 237. (MA)

I928, Exotic Microlepidoptera, 3: 4I6.

I930, Ann. Naturhist. Mus. Wien, 44:26o. (v) 


\begin{tabular}{|c|c|c|}
\hline corpuscularis & YPONOMEUTA & I907, Journ. Bombay Nat. Hist. Soc. I8: I53. (BM) \\
\hline correcta & ZONOPETALA & I9I5, Exotic Microlepidoptera, I: 304. \\
\hline correpta & HAPSIFERA & $\begin{array}{l}\text { I93I, in Joannis, Ann. Soc. Ent. France, } 98 \text { (Supp.): } 746 \\
\text { [508]. (P) }\end{array}$ \\
\hline orroborata & TORTRIX & I9I8, Ann. Transvaal Mus. 6: 8. (T) \\
\hline corrugata & GRACILARIA & I9I8, Ann. Transvaal Mus. 6: 40. \\
\hline corrupta & TRIPTOLOGA & I931, Exotic Microlepidoptera, 4: I26. \\
\hline corsota & BRACHMIA & I9I I, Journ. Bombay Nat. Hist. Soc. 20: 7І2. (BM) \\
\hline corsota & EULECHRIA & I9I4, Exotic Microlepidoptera, I: I64. \\
\hline corthyntis & ARGYROPLOCE & I9o9, Journ. Bombay Nat. Hist. Soc. I9: 596. (BM) \\
\hline corticea & LATYPICA & I925, Bull. Soc. R. Ent. Egypte, 9: 218. \\
\hline orticina & TINEOLA & I9I7, Exotic Microlepidoptera, 2:96. \\
\hline orticivora & DELORYCTIS* & I934, Exotic Microlepidoptera, 4: 464. (BM) \\
\hline corticosa & ACROLEPIA & I9I3, Exotic Microlepidoptera, I: I49. \\
\hline corusca & CNEPHASIA & I9I2, Exotic Microlepidoptera, I: II. (BM) \\
\hline corusca & HYPONOMEUTA & I9I4, Journ. Bombay Nat. Hist. Soc. 23: I27. (BM) \\
\hline corvifera & METATHEORA & I920, Ann. South African Mus. I7: 3I5. (SA) \\
\hline corvigera & ANTAEOTRICHA & I9I5, Exotic Microlepidoptera, I: 390. (BM) \\
\hline corvula & ANTAEOTRICHA & I9I2, Trans. Ent. Soc. London, I9II: 7Io. (BM) \\
\hline corybantis & NEMOTOIS & I905, Journ. Bombay Nat. Hist. Soc. I6: 6I9. \\
\hline corymbas & COMPSOLECHIA & I9I8, Exotic Microlepidoptera, 2: I39. (BM) \\
\hline corymbitis & SYMMOCA & I926, Exotic Microlepidoptera, 3: 292. (BM) \\
\hline corymbota & MYRMECOZELA & I9I9, Exotic Microlepidoptera, 2: 269. (BM) \\
\hline corynetis & CHELARIA & I913, Journ. Bombay Nat. Hist. Soc. 22: 162. (BM) \\
\hline coryphaea & THRIAMBEUTIS & I9I2, Exotic Microlepidoptera, I: 6I. \\
\hline corysta & TRACHYDORA & I897, Proc. Linnean Soc. N.S. Wales, 22: 396. \\
\hline corystes & MACHIMIA & I9I4, Exotic Microlepidoptera, I: I8I. (BM) \\
\hline corystopa & DEPRESSARIA & I927, Exotic Microlepidoptera, 3: 382. (BM) \\
\hline corythaeola & LECITHOCERA & I93I, Exotic Microlepidoptera, 4: 80. \\
\hline corythista & TIMYRA & I9I8, Exotic Microlepidoptera, 2: 98 . (BM) \\
\hline corythota & HOMALOXESTIS & I929, Exotic Microlepidoptera, 3: 5I9. (BM) \\
\hline coscineutis & ETHMIA & I9II, Ann. Transvaal Mus. 3:76. \\
\hline cosmangela & LABDIA & I923, Exotic Microlepidoptera, 3: 57. \\
\hline cosmanthes & OECOPHORA & I889, Proc. Linnean Soc. N.S. Wales, (Ser. 2) 3: I671. \\
\hline cosmia & ANACAMPSIS & I92I, Ann. Transvaal Mus. 8: 77. (T) \\
\hline cosmocrates & PHILOBOTA & I889, Proc. Linnean Soc. N.S. Wales, (Ser. 2) 3: I6I5. \\
\hline cosmocrossa & ACROPHILETIS* & I932, Exotic Microlepidoptera, 4: 348. (v) \\
\hline cosmodoxa & PSITTACASTIS & I92I, Exotic Microlepidoptera, 2: 398. (BM) \\
\hline cosmogona & ATTEVA & I93I, Exotic Microlepidoptera, 4:87. (v) \\
\hline cosmographa & ARISTOTELIA & I9I7, Trans. Ent. Soc. London, I9I7:36. (BM) \\
\hline cosmolitha & EUXANTHIS & I93 I, Exotic Microlepidoptera, 4: I58. (Bм) \\
\hline cosmopa & HOMOSETIA & I9I9, Exotic Microlepidoptera, 2: 252. (BM) \\
\hline cosmophanes & THEATROCHORA* & I921, Ann. Transvaal Mus. 8: I28. (T) \\
\hline cosmophragma & ATHRINACIA & I922, Trans. Ent. Soc. London, I922: II6. (Bм) \\
\hline cosmopis & XYLORYCTA & I89o, Trans. R. Soc. S. Australia, I3: 60. \\
\hline cosmoplaca & IMMA & I93o, Exotic Microlepidoptera, 4: 3. (Bм) \\
\hline cosmoptila & CROCIDOSEMA & I9I7, Trans. Ent. Soc. London, I9I7: I7. (BM) \\
\hline cosmoscelis & TORTRIX & I932, Exotic Microlepidoptera, 4: 255. (v) \\
\hline cosmota & ISOCHORISTA & I886, Proc. Linnean Soc. N.S. Wales, (Ser. 2) I: 1oz8. \\
\hline cosmoterma & ANTAEOTRICHA & I930, Exotic Microlepidoptera, 4: I9. \\
\hline
\end{tabular}




\begin{tabular}{ll} 
cosmotis & PROTOLECHIA \\
cosmozona & OXYLYCHNA \\
costimacula & HOPLITICA \\
cosymbota & ERIDACHTHA \\
cothurnata & SIMAETHIS \\
cotifera & DICHOMERIS \\
cotifraga & LYONETIA \\
crambalea & EPIMACTIS \\
crambalaeas & TRICHOTAPHE \\
cranaodes & PHTHEOCHROA \\
craniota & LECITHOCERA \\
crasiphila & GRACILARIA \\
craspedota & CHALAROTONA \\
craspedotis & TRICHOTAPHE \\
crassa & TIMOCRATICA \\
crassata & PSEUDODOXIA \\
crassifica & BLASTOBASIS \\
crassinervis & PLEUROTA \\
crataea & MALLOBATHRA* \\
crateracma & BUCCULATRIX \\
craterana & ANATROPIA* \\
crateraula & PYCNOSTOLA \\
craterias & PHILOMUSAEA* \\
& \\
\hline
\end{tabular}

craterocrossa craterodes craterombra crateropa crateropis crateroptila craterosema craterospila craterota craterotypa crateroxantha craterozona craticula cratophanes crauropa craurota crebata crebra crebrescens credula cremantis cremasta cremastis cremastropis cremata cremnarcha
1904, Proc. Linnean Soc. N.S. Wales, 29:35I. I9I9, Exotic Microlepidoptera, 2: 250.

I883, Proc. Linnean Soc. N.S. Wales, 7: 502. I920, Ann. South African Mus. I7: 285. (SA) I9I2, Exotic Microlepidoptera, I: 45. (BM) I913, Ann. Transvaal Mus. 3: 303. (T) I909, Ann. South African Mus. 5: 360. (SA) I9I0, Trans. Ent. Soc. London, I9I0: 458. (вM) I913, Journ. Bombay Nat. Hist. Soc. 22: I78. (BM) 1937, Exotic Microlepidoptera, 5: I49. (BM) I9I3, Ann. Transvaal Mus. 3: 293. (T) I9I2, in Wytsman, Genera Insectorum, fasc. I28: p. 27. I89o, Trans. R. Soc. S. A ustralia, I3: 66. 1937, Exotic Microlepidoptera, 5:95. (c) I925, Exotic Microlepidoptera, 3: I77. (BM) I9I4, Exotic Microlepidoptera, I: 265. (BM) I9I6, Exotic Microlepidoptera, I : 595. I884, Proc. Linnean Soc. N.S. Wales, 9: $75^{2}$. I888, Trans. Proc. New Zealand Inst. 20: I02. I9I8, Exotic Microlepidoptera, 2: I84. I88I, Proc. Linnean Soc. N.S. Wales, 6: 464. I9I8, Ann. Transvaal Mus, 6: I4. (T) I93I, Ann. Mus. Nac. Hist. Nat. Buenos Aires, 36: 394. (BM)

1934, Exotic Microlepidoptera, 4: 449. (v)

I9I7, Ann. Natal Mus. 3:6I7. (T)

I920, Exotic Microlepidoptera, 2: 3I4. I930, Exotic Microlepidoptera, 3: 555. I92I, Ann. Transvaal Mus. 8: 9I. (T) I9I8, Exotic Microlepidoptera, 2: 2II. (BM) I9I2, Trans. Ent. Soc. London, I9II: 678. (BM) I923, Exotic Microlepidoptera, 3: 46. (BM) I9I3, Ann. Transvaal Mus. 3:287. (T) I939, Rev. Franç. Lépidopterologie, 9:70. (ML) I927, Exotic Microlepidoptera, 3: 32I. I932, Exotic Microlepidoptera, 4: 2 Iо. I92I, Ann. Transvaal Mus. 8: 90. (T) I929, Exotic Microlepidoptera, 3: 522. (P) I9I8, Exotic Microlepidoptera, 2: І30. (вм) I920, Ann. South African Mus. I7: 303. (SA) I9Io, Journ. Bombay Nat. Hist. Soc. 20: 447. (BM) Igo6, Trans. R. Soc. S. Australia, 30: 53. I921, Zool. Meded. 6: I99. (L) I927, Exotic Microlepidoptera, 3: 343. (T) I889, Proc. Linnean Soc. N.S. Wales, (Ser. 2) 3: 1638. I9I5, Exotic Microlepidoptera, I: 3II. I925, Exotic Microlepidoptera, 3: I94. (BM) I930, Exotic Microlepidoptera, 3: 599. (BM) I9I6, Exotic Microlepidoptera, I: 609. I932, Exotic Microlepidoptera, 4:323. 


\begin{tabular}{|c|c|}
\hline cremnaspis & ONEBALA \\
\hline cremnitis & EUCOSMA \\
\hline cremnobathra & PACHNISTIS \\
\hline cremnocrates & ARGYROPLOCE \\
\hline cremnodes & EULECHRIA \\
\hline remnotoma & SCHOENOTENES \\
\hline cremodelta & ARGYROPLOCE \\
\hline crenoides & HYODECTIS* \\
\hline crenopa & PROMALACTIS \\
\hline crepera & PHILOBOTA \\
\hline crepitans & STENOMA \\
\hline crepitatrix & DICHOMERIS \\
\hline crepusculana & PALAEOBIA \\
\hline crepuscularis & OLETH REUTES \\
\hline crepuscularis & PTEROPHORUS \\
\hline crepuscularis & XYSMATODOMA \\
\hline crescens & PTEROPHORUS \\
\hline creserias & PLATYPEPLUS \\
\hline creseritis & MYLOTHRA* \\
\hline cretacea & PHILOBOTA \\
\hline cretalis & PLATYPTILIA \\
\hline cretata & PSEUDODOXIA \\
\hline cretea & OPOSTEGA \\
\hline cretifera & AGDISTIS \\
\hline cretiflua & SCYTHRIS \\
\hline cretigena & PHTHORIMAEA \\
\hline cretiseca & PLATACMAEA* \\
\hline cretosa & DECADARCHIS \\
\hline cretulata & GELECHIA \\
\hline cribanota & HOMALOXESTIS \\
\hline cricopa & BRACHMIA \\
\hline cricota & MICROCOLONA \\
\hline cricota & PHALONIA \\
\hline criminata & SYNCATHEDRA* \\
\hline criminosa & THYROCOPA \\
\hline crimnodes & BORKHAUSENIA \\
\hline crinita & GLYPHIPTERYX \\
\hline criocephala & AGDISTIS \\
\hline criodes & ERIPNURA* \\
\hline criopis & ARGYROPLOCE \\
\hline crispa & LATYPICA \\
\hline crispata & TORTRIX \\
\hline crithina & STEREODYTIS* \\
\hline critica & EUCELIS \\
\hline iticodes & EPERMENIA \\
\hline criticopa & BUCCULATRIX \\
\hline obylistis & LEUCOPTERA \\
\hline ylora & OINOPHILA \\
\hline bylota & HARMOLOGA \\
\hline
\end{tabular}

1905, Journ. Bombay Nat. Hist. Soc. 16:598. (BM) I912, Journ. Bombay Nat. Hist. Soc. 21: 864. (BM) 1922, Zool. Meded. 7: 86. (L)

1932, Exotic Microlepidoptera, 4: 225. (BM) 1883, Proc. Linnean Soc. N.S. Wales, 7: 5I4. 1936, Exotic Microlepidoptera, 5: 62. (BM) I93I, in Caradja, Bull. Sect. sci. Acad. roum. I4: 65. I904, Proc. Linnean Soc. N.S. Wales, 29: 4II. 1908, Journ. Bombay Nat. Hist. Soc. I8: 808. (Bм) 1884, Proc. Linnean Soc. N.S. Wales, 8: 484. I9I8, Exotic Microlepidoptera, 2: 204. (BM) 1913, Journ. Bombay Nat. Hist. Soc. 22: I73. (BM) 188I, Proc. Linnean Soc. N.S. Wales, 6: 668. I9I2, Ent. Mon. Mag. 48: 35. (Sub.) I909, Ann. Transvaal Mus. 2: 4, pl. 2, fig. 2. (T) 1893, Proc. Linnean Soc. N.S. Wales, 7: 498. 1926, Exotic Microlepidoptera, 3: 300. 1905, Journ. Bombay Nat. Hist. Soc. I6: 585. (вм) I907, Journ. Bombay Nat. Hist. Soc. I7: 743. (BM) I884, Proc. Linnean Soc. N.S. Wales, 8: 49I. 1908, Trans. Ent. Soc. London, I907: 487. 1906, Journ. Bombay Nat. Hist. Soc. I7: 407. (BM) I920, Exotic Microlepidoptera, 2: 358. I909, Ann. South African Mus. 5:367. (SA) I913, Ann. Transvaal Mus. 3: 313. (T) I9I4, Ann. Transvaal Mus. 4: I9I. (T) 1920, Voyage de Ch. Alluaud et R. Jeannel en Afrique Oriental, II, Microlepidoptera, p. 97. (P) I9I5, Exotic Microlepidoptera, I: 367. 1927, Exotic Microlepidoptera, 3: 345. (BM) I9I0, Journ. Bombay Nat. Hist. Soc. 20: 442. (BM) I9II, Trans. Linnean Soc. London, I4: 274. (BM) I9I7, Exotic Microlepidoptera, 2: 50. I9I2, Ent. Mon. Mag. 48: 35. (Sub.) I923, Exotic Microlepidoptera, 3: 37. (Bм) I9I5, Exotic Microlepidoptera, I: 372. (BM) I9I2, Trans. Ent. Soc. London, IgI : 695. (BMI) I913, Exotic Microlepidoptera, I: I02. (BM) I909, Ann. South African Mus. 5 (7): 349. (SA) I9I4, Trans. Ent. Soc. London, I9I4: 242. (BM) I928, Exotic Microlepidoptera, 3: 445. (T) I920, Ann. South African Mus. I7: 306. (SA) I912, Ann. South African Mus. I0: 56. (SA) I9I4, Exotic Microlepidoptera, I: 239. (BM) 1905, Journ. Bombay Nat. Hist. Soc. I6: 587. (BM) 1913, Ann. Transvaal Mus. 3: 317. (T) I9I5, Trans. Ent. Soc. London, I9I5: 248. I926, Exotic Microlepidoptera, 3: 265. I9II, Trans. Linnean Soc. London, I4: 299. I910, Proc. Linnean Soc. N.S. Wales, 35: 270. 
crocacma

crocanthopa

crocidura

crocina

crocinacma

crocinastis

crocobapta

crococarpa

crococephala

crocochorda

crococoma

crococosma

crococrossa

crococyma

crocodelta

crocodesma

crocodeta

crocodilitis

crocodilopa

crocodora

crocogastra

crocogramma

crocograpta

crocoleuca

crocomis

crocomitra

croconeura

croconota

croconympha

crocopa

crocopepla

crocophaea

crocophanes

crocophracta

crocophragma

crocoplecta

crocoptila

crocoptycha

crocorrhoa

crocosema

crocosoma

crocospila

crocostacta

crocosticta

crocostoma

crocota

crocothicta
STATHMOPODA

TANYZANCLA

TIQUADRA

NOTHRIS

[XEROMETRA*]

ORTHOCHTHA

COESYRA

PHILOBOTA

LABDIA

MACROBATHRA

CRYPTOPHASA

CRYPSITHYRIS

MACROBATHRA

PHTHINOSTOMA

ORNEODES

XESTOCASIS

SYMMOCA

TINEA

NARYCIA

COMPSOLECHIA

DISSOPTILA

ELACHISTA

GLYPHIDOCERA

CAPUA

PHTHORIMAEA

TORTRIX

PSEUDODOXIA

PARANTHRENE

IDIOPHANTIS

TINAEGERIA

LASPEYRESIA

PERONEA

ANTICRATES

STATHMOPODA

PTILOTHYRIS

IMMA

EPIPHRACTIS

STENOMA

PERONEA

HYBROMA

LECITHOCERA

ETHMIA

ARGYROPLOCE

SCYTHROPIA

STENOMA

TAOSCELIS*

ODITES

AUTOSTICHA
I929, Exotic Microlepidoptera, 3: 542.

I9I 8, Exotic Microlepidoptera, 2: 2 I8.

I922, Exotic Microlepidoptera, 2: 599.

I904, Proc. Linnean Soc. N.S. Wales, 29: 423.

I930, Trans. Ent. Soc. London, 78: 321. (P)

I889, Proc. Linnean Soc. N.S. Wales, (Ser. 2) 3: I656.

I884, Proc. Linnean Soc. N.S. Wales, 8: 498.

I928, Exotic Microlepidoptera, 3: 385 . (BM)

I936, Exotic Microlepidoptera, 5: 50. (c)

1925, Exotic Microlepidoptera, 3: I5I.

I934, Exotic Microlepidoptera, 4: 479.

I922, Exotic Microlepidoptera, 2: 509.

I934, Exotic Microlepidoptera, 4: 528.

I937, Exolic Microlepidoptera, 5: II9. (T)

I9I5, Exotic Microlepidoptera, I: 3I6. (BM)

I9I I, Ann. Transvaal Mus. 3: 70. (вм)

I927, Exotic Microlepidoptera, 3: 323. (BM)

I930, Exotic Microlepidoptera, 3: 554. (BM)

I922, Trans. Ent. Soc. London, I922: 86. (BM)

I922, Trans. Ent. Soc. London, I922: 65. (вм)

I908, Proc. Zool. Soc. London, p. 733.

I923, Exotic Microlepidoptera, 3: 49. (BM)

I933, Exotic Microlepidoptera, 4: 422. (v)

I923, Exotic Microlepidoptera, 3: 5I.

I9o8, Journ. Bombay Nat. Hist. Soc. I8: 6I9. (BM)

I9I4, Exotic Microlepidoptera, I : 266. (BM)

I926, Exotic Microlepidoptera, 3:267.

I9I8, Exotic Microlepidoptera, 2: I29.

I92I, Exotic Microlepidoptera, 2: 44I.

I907, Journ. Bombay Nat. Hist. Soc. I8: I46. (BM)

1922, Exotic Microlepidoptera, 2: 500. (P)

I92I, Ann. Transvaal Mus. 8: I20. (T)

I897, Proc. Linnean Soc. N.S. Wales, 22: 324.

1938, Institut des Parcs Nationaux du Congo Belge,

fasc. I4, p. I8, pl. 2, fig. 4. (c)

I93I, in Joannis, Ann. Soc. Ent. France, 98 (Supp.): 736

[498]. (P)

I9I3, Ann. Transvaal Mus. 3: 32I. (T)

I9I5, Exotic Microlepidoptera, I : 466. (BM)

I93I, Ann. Mus. Nac. Hist. Nat. Buenos Aires, 36: 383.

I9I9, Exotic Microlepidoptera, 2: 240. (BM)

I929, Exotic Microlepidoptera, 3: 524. (BM)

I9I4, Exotic Microlepidoptera, I : I73. (BM)

I939, Trans. R. Ent. Soc. London, 89: 50. (BM)

I9I2, Ann. South African Mus. Io: 68. (SA)

1925, Exotic Microlepidoptera, 3: 219. (BM)

I938, Trans, roy. Soc. N.Z. $67: 428$.

I9I2, Ann. South African Mus. I0: 62. (SA)

I9I6, Exotic Microlepidoptera, I: 588. (BM) 
CROCOTRICHA

crocotricha
crocoxantha
crocoxysta
crocozela
crocozona
croniopa
crossanthes

\section{crossoceros} crossogramma crossoleuca crossophaea crossophanes crossophanta crossosema crossospila crossosticta crossota crossotis crossotoma crossotorna crotala crotala crotalista crotalistis crotalodes crotalopis crotalotis crotolitha crotospila cruciata

\begin{tabular}{|c|c|}
\hline crucifera & CALICOTIS* \\
\hline formi & TRICLONELLA \\
\hline cigera & ACROCERCOPS \\
\hline uda & MACHIMIA \\
\hline rudescens & GELECHIA \\
\hline udipennis & MARASMARCHA \\
\hline uenta & PLATYEDRA \\
\hline ustaria & PHTHORIMAEA \\
\hline ustata & AROTROPHORA \\
\hline crustulata & PHILOBOTA \\
\hline cryeranthes & XYLODRYAS* \\
\hline tyeropis & ANTAEOTRICHA \\
\hline crymalea & MACROBATHRA \\
\hline ymodes & HYOPRORA* \\
\hline ymorrhoa & PHALANGITIS \\
\hline ymorrhoa & PROTEROMICTA* \\
\hline odana & HETEROCROSSA \\
\hline
\end{tabular}

SAPHENEUTIS HELIOCAUSTA PAMMECES

IMMA

MACROSACES

TINEA

COLEOPHORA

PTILOTHYRIS

CORTHYNTIS

ARGYROPLOCE

LECITHOCERA

COLEOPHORA

ODITES

DALACA

DICHOMERIS

CRYPTOPHASA

ARGYROPLOCE

HYPOSMOCOMA

ARGYROPLOCE

COMMATICA

BORKHAUSENIA

CNEPHASIA

ORTHIOSTOLA

ACROCERCOPS

PROTOLECHIA

MACRAEOLA

GLYPHIPTERYX

ZARATHA

BLASTOBASIS

DIOCOSMA

CALICOTIS*

TRICLONELLA

TEROCROSSA
I910, Rec. Indian Mus. 5: 232. (Destroyed)

I 888, Proc. Linnean Soc. N.S. Wales, (Ser.2) 2: 934.

I922, Exotic Microlepidoptera, 2: 584. (BM)

I9o6, Trans. Ent. Soc. London, I906: I94. (BM)

I9I0, Journ. Bombay Nat. Hist. Soc. 20: I50. (BM)

I934, Exotic Microlepidoptera, 4:480.

1938, Institut des Parcs Nationaux du Congo Belge,

fasc. I4, p. 22.

I934, Exotic Microlepidoptera, 4:452. (v)

I92I, Ann. Transvaal Mus. 8: 87. (T)

I933, Exotic Microlepidoptera, 4: 420. (BM)

I93I, Exotic Microlepidoptera, 4: 80. (BM)

I9I7, Exotic Microlepidoptera, 2: 70.

I930, Exotic Microlepidoptera, 4: I7. (BM)

I921, Ann. Transvaal Mus. 8: I43.

I933, Exotic Microlepidoptera, 4: 353. (v)

I938, Trans. R. Ent. Soc. London, 87: 517.

I9I I, Proc. Limnean Soc. N.S. Wales, 36: 266.

I915, Exotic Microlepidoptera, I : 342. (BM)

I93I, Exotic Microlepidoptera, 4: I29.

I929, Exotic Microlepidoptera, 3: 503. (BM)

I9I 5, Trans. Proc. New Zealand Inst. 47: 213. (Sub.)

I9Io, Proc. Linnean Soc. N.S. Wales, 35: 276.

I928, Exotic Microlepidoptera, 3: 478. (BM)

I9I5, Trans. Ent. Soc. London, I9I 5: 229.

I904, Proc. Linnean Soc. N.S. Wales, 29: 385 .

I921, Ann. Transvaal Mus. 8: 127. (T)

I909, Journ. Bombay Nat. Hist. Soc. I9: 432. (BM)

I9I 5, Exotic Microlepidoptera, I: 336.

1926, Trans. Ent. Soc. London, 74: 278.

1938, Institut des Parcs Nationaux du Congo Belge,

fasc. I4, p. I9, pl. 2, fig. 7 .

I889, Trans. Proc. New Zealand Inst. 2I: I7o.

I93I, Exotic Microlepidoptera, 4: II7. (v)

I920, Exotic Microlepidoptera, 2: 295. (BM)

1926, Exotic Microlepidoptera, 3: 3II. (BM)

I920, Voyage de Ch. Alluaud et R. Jeannel en Afrique

Oriental, II, Microlepidoptera, p. 70. (P)

I932, Exotic Microlepidoptera, 4:252.

I920, Exotic Microlepidoptera, 2: 298. (BM)

I9I7, Trans. Ent. Soc. London, I9I7: 42. (BM)

I9I2, Exotic Microlepidoptera, I: 9. (BM)

I9I3, Exotic Microlepidoptera, I: I24. (MA)

1925, Exotic Microlepidoptera, 3: I52.

I925, Exotic Microlepidoptera, 3: I67. (BM)

I886, Proc. Linnean Soc. N.S. Wales, Io: 8I6.

I9o8, Proc. Zool. Soc. London, p. 754.

1907, Proc. Linnean Soc. N.S. Wales, 32: 136.

I889, Proc. Linnean Soc. N.S. Wales, (Ser. 2) 3: I669.

I885, Trans. Proc. New Zealand Inst. I7: I48. 


\begin{tabular}{|c|c|c|}
\hline cryolopha & EPIPHTHORA & I904, Proc. Linnean Soc. N.S. Wales, 29: 265. \\
\hline cryphaea & HERMOGENES & I9o8, Journ. Bombay Nat. Hist. Soc. I8: 63o. (вм) \\
\hline cryphias & SYMBATICA* & I9Io, Ann. South African Mus. 5: 4I3. (SA) \\
\hline cryphias & TRICHOPTILUS & I912, Ann. South African Mus. IO: 53. (SA) \\
\hline cryphiodes & HARPAGANDRA* & I9I8, Exotic Microlepidoptera, 2: 210. (BM) \\
\hline crypsangela & STENOMA & I932, Exotic Microlepidoptera, 4: 294. (v) \\
\hline crypsastra & STENOMA & I9I5, Exotic Microlepidoptera, I: 4I4. (BM) \\
\hline crypsetaera & STENOMA & I925, Exotic Microlepidoptera, 3: I83. (Bм) \\
\hline crypsibatis & PROTOLECHIA & I904, Proc. Linnean Soc. N.S. Wales, 29: 344. \\
\hline crypsicasis & MICROCOLONA & I897, Proc. Linnean Soc. N.S. Wales, 22: 373. \\
\hline crypsicentra & PHLOEOPOLA & I9I4, Exotic Microlepidoptera, I: I7o. \\
\hline crypsichlora & THIOTRICHA & I927, Insects of Samoa, 3, Lepidoptera, fasc. 2, p. 8I. (вм) \\
\hline crypsichola & CARPOSINA & I9Io, Trans. Ent. Soc. London, I9Io: 431. (BM) \\
\hline crypsichola & PHILOBOTA & I884, Proc. Linnean Soc. N.S. Wales, 6: 482. \\
\hline crypsicosma & DEPRESSARIA & I920, Ann. South African Mus. I7: 287. (SA) \\
\hline crypsicroca & PRYMNOTOMIS* & I93I, Exotic Microlepidoptera, 4:86. (v) \\
\hline crypsidelta & GRACILARIA & I926, Trans. Ent. Soc. London, 74: 275. \\
\hline crypsidesma & THUDACA & I893, Proc. Linnean Soc. N.S. Wales, 7: 572. \\
\hline crypsidora & DIPTERINA & I909, Trans. New Zealand Inst. 4I: II. \\
\hline crypsigenes & LECITHOCERA & I929, Exotic Microlepidoptera, 3: 523. (вм) \\
\hline crypsigramma & SCYTHRIS & I897, Proc. Linnean Soc. N.S. Wales, 22: 424. \\
\hline crypsigrapha & ACROCERCOPS & I930, Exotic Microlepidoptera, 3: 58I. \\
\hline crypsileuca & PELTOPHORA & I884, Proc. Linnean Soc. N.S. Wales, 9: 732. \\
\hline crypsilitha & POLYORTHA & I932, Exotic Microlepidoptera, 4:264. (v) \\
\hline crypsiloga & CHOLOTIS & I9I5, Exotic Microlepidoptera, I: 329. \\
\hline crypsilychna & BRACHMIA & I9I4, Journ. Bombay Nat. Hist. Soc. 22: 773. (Bм) \\
\hline crypsimetalla & HELIOZELA & I897, Proc. Linnean Soc. N.S. Wales, 22: 404. \\
\hline crypsimima & BORKHAUSENIA & I920, Trans. Proc. New Zealand Inst. 52: 31. \\
\hline crypsimorpha & BLASTOBASIS & I922, Exotic Microlepidoptera, $2: 538$. \\
\hline crypsinoma & BRACHMIA & I929, Exotic Microlepidoptera, 3: 527. (вм) \\
\hline crypsiphaea & STENOMA & I925, Exotic Microlepidoptera, 3: I9o. (BM) \\
\hline crypsiphanes & COLEOPHORA & I9I7, Exotic Microlepidoptera, 2: 70. \\
\hline crypsiphila & EUMASIA & I9I9, Exotic Microlepidoptera, 2: 26I. \\
\hline crypsiphragma & ORPHNOLECHIA* & I909, Trans. Ent. Soc. London, I909: 29. (вм) \\
\hline crypsiptila & CTENIOXENA* & I923, Exotic Microlepidoptera, 2:6 12. (BM) \\
\hline crypsirias & OINOPHILA & I930, Trans. Ent. Soc. London, 78: 320. (P) \\
\hline crypsithias & STENOMA & I930, Exotic Microlepidoptera, 3: 558. (BM) \\
\hline crypsitricha & METACHANDA & I9II, Trans. Linnean Soc. London, I4: 280. \\
\hline crypsixantha & NEPTICULA & I9I8, Ann. Transvaal Mus. 6: 43. (T) \\
\hline crypsizyga & ONEBALA & I9I4, Exotic Microlepidoptera, I: 278. (вм) \\
\hline cryptias & PSEUDODOXIA & I9I0, Journ. Bombay Nat. Hist. Soc. 20: I52. (BM) \\
\hline cryptica & ZELLERIA & I913, Exotic Microlepidoptera, I: г36. \\
\hline crypticopa & GELECHIA & $\begin{array}{l}\text { I93I, Ann. Mus. Nac. Hist. Nat. Buenos Aires, } 36: 384 . \\
\text { (BM) }\end{array}$ \\
\hline cryptochlora & COPROMORPHA & I930, Exotic Microlepidoptera, 3: 590. (Bм) \\
\hline cryptogramma & SPILONOTA & I922, Exotic Microlepidoptera, 2: 520. (BM) \\
\hline cryptomorpha & STASIPHRON* & I93I, Exotic Microlepidoptera, 4: I72. (BM) \\
\hline cryptophaea & STATHMOPODA & I922, Exotic Microlepidoptera, 2: $585 . \quad(\mathrm{P})$ \\
\hline cryptosema & CORSOCASIS & I929, Exotic Microlepidoptera, 3: 544 . \\
\hline cryptosperma & PROTOLECHIA & I92I, Exotic Microlepidoptera, 2: 428. \\
\hline
\end{tabular}




$\begin{array}{ll}\begin{array}{l}\text { crystallina } \\ \text { crystallista }\end{array} & \text { OCYSTOLA } \\ \text { crystallodes } & \text { APATETRIS } \\ \text { crystallopa } & \text { HEBDOMACTIS* } \\ \text { crystanta } & \text { ACROCERCOPS } \\ \text { ctenias } & \text { MICOROTRICHA* } \\ \text { cubiculata } & \text { ONEBALA } \\ \text { cubitalis } & \text { SAPHENEUTIS } \\ \text { cuculans } & \text { ODITES } \\ \text { cucullata } & \text { LECITHOCERA } \\ & \text { [ASMENISTIS } \\ \text { culmicola } & \text { SCYTHRIS } \\ \text { culminata } & \text { PLUTELLA } \\ \text { cultrata } & \text { GLYPHIPTERYX } \\ \text { cultrifera } & \text { TIOUADRA } \\ \text { cultrix } & \text { CEROSTOMA } \\ \text { cumulata } & \text { CACOECIA } \\ & \text { [PLANOSTOCHA }\end{array}$

cunctatrix

cuneata

cuneiformis

cuneigera

cunicularis

cuniculata

cupida

cupidinea

cuprata

cuprata

cuprea

curialis

curiata

curiosa

curriculata

curta

curulis

curvisignis

custodita

custos

cyamitis

cyamitis

cyanacma

cyanactis

cyananthes

cyanarcha

cyanaspis

cyanastra

cyanastra

cyanaula

cyanea

[PLANOSTOCHA*]

PLATYEDRA

IMMA

THIOTRICHA

BUCCULATRIX

ELEGISTIS*

STEMAGORIS

ENARMONIA

MACHIMIA

NEPTICULA

PERONEA

YPONOMEUTA

CRYPTOPHASA

STENOMA

OXYGRAPHA

PHILOBOTA

BLASTOBASIS

PRAYS

PHRATRIODES*

MACHIMIA

BRACHMIA

MACROTONA

PHALONIA

LAMACHAERA*

PESSOGRAPTIS

STENOMA

DOLEROMIMA

BRENTHIA

DACRYPHANES*

BRENTHIA

GYMNOGRAMMA
CNEMIDOLOPHUS

[CHALCoColona*] I9r2, Ann. Transvaal Mus. 3: 73. (T)

I93I, Exotic Microlepidoptera, 4: 6r.

I9I9, Exotic Microlepidoptera, 2: 288.

I9I9, Exotic Microlepidoptera, 2: 242.

I9I2, Ent. Mon. Mag. 48: 34. (Sub.)

I925, Exotic Microlepidoptera, 3: I49.

I920, Exotic Microlepidoptera, 2: 384 .

I9I6, Exotic Microlepidoptera, r: 596.

I9I2, Ent. Mon. Mag. 48: 35. (Sub.)

I9I5, Exotic Microlepidoptera, r: 338.
I885, Proc. Linnean Soc. N.S. Wales, 9: ro77.

I9II, Ann. Transvaal Mus. 2: 229. (T)

1929, Exotic Microlepidoptera, 3: 539. (BM)

I916, Exotic Microlepidoptera, I: 627.

I9I3, Exotic Microlepidoptera, I: I2I.

I9II, Ann. Transvaal Mus. 2: 222. (T)

I9I I, Trans. Linnean Soc. London, 14:273. (BM)

rgro, Trans. Ent. Soc. London, r9Io: 377.

I918, Ann. Transvaal Mus. 6:54. (T)

I9I4, Exotic Microlepidoptera, I: I99. (BM)

I9I6, Exotic Microlepidoptera, 2: II.

1931, Ann. Mus. Nac. Hist. Nat. Buenos Aires, 36: 409.

I9I2, Exotic Microlepidoptera, I: 56. (BM)

I9I4, Exotic Microlepidoptera, I: 2I3.

1924, Exotic Microlepidoptera, 3: 87.

1907, Journ. Bombay Nat. Hist. Soc. I7: 977. (BM)

I906, Trans. Ent. Soc. London, I906: 202. (BM)

r9I8, Exotic Microlepidoptera, 2: I24. (BM)

I9I I, Journ. Bombay Nat. Hist. Soc. 21: I25.

r9I4, Exotic Microlepidoptera, I: I75. (BM)

I9I5, Trans. Ent. Soc. London, I9r5: 255.

I9I7, Trans. Ent. Soc. London, I9I7: I4. (BM)

I902, Trans. Ent. Soc. London, I902: 575 .

1929, Trans. Ent. Soc. London, 76: 5I5.

I908, Journ. Bombay Nat. Hist. Soc. I8: 625.

I9I4, Joum. Bombay Nat. Hist. Soc. 23: I25. (BM)

I926, Ann. South African Mus. 23: 336. (SA)

I928, Exotic Microlepidoptera, 3: 470. (BM)

I9I I, Journ. Bombay Nat. Hist. Soc. 20: 725. (Bм)

I904, Proc. Limnean Soc. N.S. Wales, 29: 407.

1930, Ann. Naturhist. Mus. Wien, 44:226. (v)

I9I5, Exotic Microlepidoptera, I: 4I4. (BM)

I909, Trans. Ent. Soc. London, I909: 23. (BM)

I909, Trans. Ent. Soc. London, I909: 40. (BM)

I907, Journ. Bombay Nat. Hist. Soc. I8: I54.

I912, Exotic Microlepidoptera, I: 53. (BM)

I9I2, Amn. South African Mus. Io: 66. (SA) 


$\begin{array}{ll}\text { cyanitis } & \text { EPISIMUS } \\ \text { cyanobactra } & \text { COMOCRITIS } \\ \text { cyanocephala } & \text { LASPEYRESIA } \\ \text { cyanochalca } & \text { GLYPHIPTERYX } \\ \text { cyanochlora } & \text { ENTEUCHA* } \\ \text { cyanochra } & \text { EMBLEMATODES* } \\ \text { cyanochrysa } & \text { NEMOTOIS } \\ \text { cyanocoma } & \text { LABDIA } \\ \text { cyanocorys } & \text { SCLEROPHANTIS* } \\ \text { cyanodesma } & \text { HIEROXESTIS } \\ \text { cyanodeta } & \text { ACROCERCOPS } \\ \text { cyanodora } & \text { LABDIA } \\ \text { cyanodoxa } & \text { TONICA }\end{array}$

cyanogama cyanogona cyanogramma cyanograpta cyanolampra cyanolychna cyanombra cyanombra cyanomyia cyanoneura cyanopa cyanophaea cyanophanes cyanophracta cyanopis cyanopla cyanoplaca cyanoplecta cyanorma cyanorrhoa cyanosceptra cyanoschista cyanoscia cyanosoma cyanospila cyanospira cyanospora cyanostacta cyanostola

cyanostoma cyanotoxa cyanoxantha cyanoxantha cyanozona cyanura cyathodes

TIMODORA

HELCYSTOGRAMMA

ARGYROPLOCE

ANALYTARCHA *
I932, Exotic Microlepidoptera, 4: 308.

I922, Exotic Microlepidoptera, 2: 552. (BM)

I92I, Ann. Transvaal Mus. 8: 62. (T)

I883, Proc. Linnean Soc. N.S. Wales, 7: I85.

I9I5, Trans. Ent. Soc. London, I9I5: 24I.

I9I4, Exotic Microlepidoptera, I: 288.

I930, Exotic Microlepidoptera, 3: 57I.

I922, Exotic Microlepidoptera, 2: 569. (BM)

I935, Exotic Microlepidoptera, 4:587. (BM)

I9I I, Trans. Linnean Soc. London, I4: 294.

I9I8, Exotic Microlepidoptera, 2: I77.

I935, Exotic Microlepidoptera, 4: 604.

I924, Exotic Microlepidoptera, 3: I05.

I930, Exotic Microlepidoptera, 3:584. (BM)

Igo7, Journ. Bombay Nat. Hist. Soc. I8: I46. (вм)

I897, Proc. Linnean Soc. N.S. Wales, 22: 346.

I928, Exotic Microlepidoptera, 3: 449.

I935, Arb. morph. taxon. Ent. Berl. 3: I05. (D)

I9ro, Trans. Ent. Soc. London, I9Io: 453 .

I935, Exotic Microlepidoptera, 4:569. (c)

I9I3, Trans. Ent. Soc. London, I9I3: I90. (вм)

I930, Ann. Naturhist. Mus. Wien, 44: 260. (v)

I922, Trans. Ent. Soc. London, I922: I09. (BM)

I9I8, Exotic Microlepidoptera, 2: 99. (BM)

I927, Exotic Microlepidoptera, 3: 339. (BM)

I934, Exotic Microlepidoptera, 4:506.

I883, Proc. Linnean Soc. N.S. Wales, 7: г86.

I9I2, Journ. Bombay Nat. Hist. Soc. 2I: 866. (BM)

I897, Proc. Linnean Soc. N.S. Wales, 22: 3 I9.

I9I0, Trans. Ent. Soc. London, I9Io: 44I.

I9I4, Exotic Microlepidoptera, I: 224. (BM)

I934, Exotic Microlepidoptera, 4: 449.

I9I4, Trans. Ent. Soc. London, I9I4: 24I. (BM)

I93I, Exotic Microlepidotera, 4:66.

I926, Exotic Microlepidoptera, 3: 289.

I9I8, Ann. Transvaal Mus. 6:46. (T)

I9Io, Trans. Ent. Soc. London, I9Io: 454.

I886, Trans. Proc. New Zealand Inst. I8: I83.

I928, Exotic Microlepidoptera, 3: 465. (BM)

I926, Exotic Microlepidoptera, 3: 302. (BM)

I930, Exotic Microlepidoptera, 3: 6I I. (BM)

I937, in Caradja and Meyrick, Deuts. Ent. Zeit. Iris, 5I: I82.

I9I6, Exotic Microlepidoptera, 2: I6. (BM)

I907, Proc. Linnean Soc. N.S. Wales, 32: II3.

I907, Journ. Bombay Nat. Hist. Soc. I7: 979. (BM)

I920, Exotic Microlepidoptera, 2: 297. (BM)
I923, Exotic Microlepidoptera, 3: 26. (BM)

I909, Journ. Bombay Nat. Hist. Soc. I9: 598. (BM)

I92I, Exotic Microlepidoptera, 2: 474. 


\section{CYATHOPA}

cyathopa
cybicopa
cybophora
cyceonodes
cycladica
cycladopa
cyclantha
cyclas
cyclas
cyclatma
cyclidias
cyclobasis
cyclobathra
cyclocosma
cyclogramma
cyclometra
cyclonia
cyclonitis
cyclopa
cyclopaea
cyclophora
cyclophragma
cyclophthalma
cyclopica

COPTOTELIA

ARGYROPLOCE

LIMNOECIA

PHTHORIMAEA

ARGYROPLOCE

SYRMOLOGA

AEOLANTHES

DEPRESSARIA

PLACOPTILA

MELASINA

ARTICOLLA*

ANTAEOTRICHA

EPAGOGE

MOMPHA

ACROCERCOPS

NIPHODIDACTIS*

SYNTOMACTIS

STYLOCEROS*

ACROCERCOPS

COSMOPTERYX

ACROLOPHUS

LINOSTICHA

ATELOSTICHA

URODUS

cyclopis

cyclopodes

cycloptila

cyclosema

cyclospila

cyclostoma

cyclotoma

cycloxantha

cycnodes

cycnographa

cycnolopha

cycnomorpha

cycnoptera

cycota

cycotis

cydrota

cylicopa

cylicota

cylindraula

cylindrica

cylindrodes

cylindrota

cymataula

cymatias

cymatistis
BAREA

ONEBALA

STENOMA

ANTISPILA

TRICHOTAPHE

IMMA

COESYRA*

AEOLOCOSMA

LINOSTICHA

STENOMA

STENOMA

ANTAEOTRICHA

EULECHRIA

GELECHIA

ELACHISTA

CHOREUTIS

HETEROZYGA

ACROCERCOPS

MELASINA

PARAMORPHA

COLOBOCROSSA *

HOLCOCERA

SPORADARTHRA

MICROSCHISMUS

THUDACA
I9I3, Trans. Ent. Soc. London, I9I3: I79. (BM)

I933, Exotic Microlepidoptera, 4:42I. (BM)

1897, Proc. Linnean Soc. N.S. Wales, 22: 364.

I926, Exotic Microlepidoptera, 3:278. (BM)

I9I7, Trans. Ent. Soc. London, IOI7: 27. (BM)

I927, Exotic Microlepidoptera, 3: 326. (BM)

I923, Exotic Microlepidoptera, 2: 6II. (BM)

I9Io, Journ. Bombay Nat. Hist. Soc. 20: I66. (BM)

I937, Exotic Microlepidoptera, 5: 79.

I908, Proc. Zool. Soc. London, p. 746.

I907, Journ. Bombay Nat. Hist. Soc. I7: 976. (BM)

I930, Exotic Microlepidoptera, 4:23. (v)

I907, Trans. Proc. New Zealand Inst. 39: II3.

I92I, Ann. Transvaal Mus. 8: 97. (T)

I921, Exotic Microlepidoptera, 2: 466.

I938, Trans. R. Ent. Soc. London, 87: 525.

I897, Proc. Linnean Soc. N.S. Wales, 22: 383.

I904, Proc. Linnean Soc. N.S. Wales, 29: 409.

I908, Rec. Indian Mus. 2: 398. (K)

I909, Journ. Bombay Nat. Hist. Soc. I9: 4I3.

I931, Journ. Linnean Soc. London, 37: 283.

I889, Proc. Linnean Soc. N.S. Wales, (Ser. 2) 3: I58I.

I9I6, Exotic Microlepidoptera, I: 546. (BM)

I930, Ann. Naturhist. Mus. Wien, 44: 263, pl. 2, fig. 25.

(v)

I9I5, Exotic Microlepidoptera, I: 307.

I922, Ent. Mitteil. I I : 44. (D)

I9I5, Exotic Microlepidoptera, I: 446. (BM)

I921, Exotic Microlepidoptera, 2: 404.

I9I8, Exotic Microlepidoptera, 2: I5I. (BM)

I906, Trans. Ent. Soc. London, I906: I76. (BM)

I884, Proc. Linnean Soc. N.S. Wales, 9: 77 I.

I906, Trans. R. Soc. S. Australia, 30:38.

I889, Proc. Linnean Soc. N.S. Wales, (Ser. 2) 3: 582.

1930, Ann. Naturhist. Mus. Wien, 44: 254. (v)

I925, Exotic Microlepidoptera, 3: I86. (BM)

1925, Exotic Microlepidoptera, 3: I69. (вм)

I888, Proc. Linnean Soc. N.S. Wales, (Ser. 2) 2: 966.

I9I2, Ann. Transvaal Mus. 3:65. (T)

I897, Proc. Linnean Soc. N.S. Wales, 22: 335.

I9I5, Trans. Ent. Soc. London, I9I5: 220. (BM)

I9I4, Exotic Microlepidoptera, I: 243. (BM)

I9I4, Journ. Bombay Nat. Hist. Soc. 23: II9.

I920, Ann. South African Mus. I7: 309. (SA)

I92I, Exotic Microlepidoptera, 2: 409.

I924, Exotic Microlepidoptera, 3:84.

I9I8, Exotic Microlepidoptera, 2: I62. (BM)

I926, Exotic Microlepidoptera, 3:320. (T)

I9I8, Ann. Transvaal Mus. 6: 35. (T)

1893, Proc. Linnean Soc. N.S. Wales, $7: 577$. 


$\begin{array}{ll}\text { cymatodes } & \text { TRICHOTAPHE } \\ \text { cymbalista } & \text { STENOMA } \\ \text { cymbalistis } & \text { TINTHIA } \\ \text { cymbalodes } & \text { IMMA } \\ \text { cymbalora } & \text { COPROMORPHA } \\ \text { cymineuta } & \text { PHYCODES } \\ \text { cymodoce } & \text { TINEA } \\ \text { cymogramma } & \text { STENOMA } \\ \text { cymographa } & \text { ORNEODES } \\ \text { cymopelta } & \text { TINEA } \\ \text { cymoptila } & \text { CHELARIA } \\ \text { cymotoma } & \text { ARGYROPLOCE } \\ \text { cymotrocha } & \text { NOTHRIS } \\ \text { cynarivora } & \text { DEPRESSARIA } \\ \text { cynegetis } & \text { PHYTOMIMIA } \\ \text { cynetica } & \text { ZELLERIA } \\ \text { cynica } & \text { AMPHITRIAS* } \\ \text { cynicopis } & \text { LASPEYRESIA } \\ \text { cynopa } & \text { ELACHISTA } \\ \text { cynopis } & \text { STENOMA } \\ \text { cynthia } & \text { ARISTOTELIA } \\ \text { cypellias } & \text { SARIDOCOMPSA } \\ \text { cyphoma } & \text { EULECHRIA } \\ \text { cyphonias } & \text { ODITES } \\ \text { cyphospila } & \text { TRACHYPEPLA } \\ & \text { ARGYROPLOCE } \\ & \end{array}$

$\begin{array}{ll}\begin{array}{l}\text { cyphostacta } \\ \text { cyphoxantha }\end{array} & \text { ACROCERCOPS } \\ \text { cyphozona } & \text { NEMOTOIS } \\ \text { cypracma } & \text { NEPTICULA } \\ \text { cyprantha } & \text { ZACORISCA } \\ \text { cypraspis } & \text { ETHMIA } \\ \text { cypridia } & \text { TORTYRA } \\ \text { cypris } & \text { OEDEMATOPODA } \\ \text { cyprodeta } & \text { ANTAEOTRICHA } \\ \text { cyprophanes } & \text { DAEMONARCHA } \\ \text { cyrota } & \text { OEGOCONIA } \\ \text { cyrtomis } & \text { OPOGONA } \\ \text { cyrtosema } & \text { ADOXOPHYES } \\ \text { cystiodes } & \text { AGRIOPHARA } \\ \text { cytheraea } & \text { ARISTOTELIA }\end{array}$

dacryodes dactylia dactylias
I9I6, Exotic Microlepidoptera, I: 584. (BM) I9I8, Exotic Microlepidoptera, 2: 204. (BM) I926, Exotic Microlepidoptera, 3: 268. I906, Trans. Ent. Soc. London, I906: I93. (Bм) I907, Journ. Bombay Nat. Hist. Soc. I8: I52. (BM) I9o9, Journ. Bombay Nat. Hist. Soc. I9: 424. I924, Trans. Proc. New Zealand Inst. 55: 206. I925, Exotic Microlepidoptera, 3: I93. (BM) I929, Exotic Microlepidoptera, 3: 536. (BM) I925, Treubia, 6: 432. I 929, Exotic Microlepidoptera, 3: 5I4. (BM) I9I7, Trans. Ent. Soc. London, I9I7: 26. (BM) I9I3, Ann. Transvaal Mus. 3: 300. (T) I932, Exotic Microlepidoptera, 4:280. (BM) I932, Exotic Microlepidoptera, 4: 278.

I893, Proc. Linnean Soc. N.S. Wales, 7: 582. I908, Journ. Bombay Nat. Hist. Soc. I8: 63I. (Вм) 1938, Institut des Parcs Nationaux du Congo Belge, fasc. I4, p. II, pl. I, fig. 9. (c) I897, Proc. Linnean Soc. N.S. Wales, 22: 334. I9I5, Exotic Microlepidoptera, I: 434. (BM) I9I7, Trans. Ent. Soc. London, I9I7: 32. (BM) I937, Exotic Microlepidoptera, 5: II3. I922, Exotic Microlepidoptera, 2: 5II. I9I5, Exotic Microlepidoptera, I: 406. I927, Trans. Proc. New Zealand Inst. 58: 3 I4. I93I, Exotic Microlepidoptera, 4: I39. (v) I920, Voyage de Ch. Alluaud et $R$. Jeannel en Afrique Oriental, II, Microlepidoptera, p. 58. (P) I92I, Zool. Meded. 6: I89. (L) I93I, Exotic Microlepidoptera, 4:36. (v) I922, Exotic Microlepidoptera, 2: 537. I9I6, Trans. Proc. New Zealand Inst. 48: 4 I9. I924, Exotic Microlepidoptera, 3: III. I930, Ann. Naturhist. Mus. Wien, 44:263, pl. I, fig. 3I. (v)

IgIo, Trans. Ent. Soc. London, I9Io: 462. (BM) I905, Journ. Bombay Nat. Hist. Soc. I6: 608. I930, Exotic Microlepidoptera, 3:556. (Lost) I9I8, Ann. Transwaal Mus. 6:27. (T) I921, Ann. Transvaal Mus. 8: 94. (T) I9I5, Exotic Microlepidoptera, I: 360 . I886, Trans. Ent. Soc. London, I886: 276. (BM) I9I6, Exotic Microlepidoptera, I: 489 . I9I7, Trans. Ent. Soc. London, IgI7: 32. (BM)

IgIo, Trans. Ent. Soc. London, I9Io: 372.

I9I2, Ann. Transvaal Mus. 3: 76. (T) I92I, Zool. Meded. 6: I75. (L) 


\begin{tabular}{|c|c|}
\hline dactyliota & PYRODERCES \\
\hline dactylota & PARECTOPA \\
\hline daedalopis & HEMIMENE \\
\hline daedalota & LASPEYRESIA \\
\hline emonica & PLATYPTILIA \\
\hline daphnaea & CHRESMARCHA \\
\hline daricella & CROBYLOPHORA \\
\hline dasmophora & ACROCERCOPS \\
\hline dasychiras & RHINOPHYLLIS* \\
\hline dasyleuca & ORDRUPIA \\
\hline dasyneura & STENOMA \\
\hline dasyptera & EULACHNA* \\
\hline dasystola & HOMALOPSYCHA \\
\hline dasytricha & LIMNOECIA \\
\hline dealbata & OXYSCOPA* \\
\hline debilis & OPOGONA \\
\hline decachrysa & GLYPHIPTERYX \\
\hline decagramma & PHILEDONE \\
\hline decapitata & IPHIMACHAERA* \\
\hline decaplaca & ANTICRATES \\
\hline decaspila & ATHEROPLA \\
\hline decataea & MACROBATHRA \\
\hline decens & URODUS \\
\hline eptoria & PROTANYSTIS \\
\hline ceptrix & ALLOTALANTA \\
\hline cidua & ARGYROPLOCE \\
\hline linata & METACHANDA \\
\hline eclivis & PTEROPHORUS \\
\hline decolor & BLASTOBASIS \\
\hline decoranda & HECTACMA \\
\hline orata & PELECYSTOLA* \\
\hline rata & STATHEROTIS* \\
\hline currens & CHIROCOMPA \\
\hline dedecorata & PHILOBOTA \\
\hline edicata & IMMA \\
\hline Eensa & EUCOSMA \\
\hline deferens & DEMOBROTIS \\
\hline fessa & MACHIMIA \\
\hline iciens & ELACHISTA \\
\hline defigurata & ACROCERCOPS \\
\hline definita & PHALONIA \\
\hline efixa & ARISTOTELIA \\
\hline deflexa & AGRIOPHARA \\
\hline deflorescens & HYPERCALLIA \\
\hline flua & PTILOGENES \\
\hline ffuescens & TINEA \\
\hline eformata & DIACTENIS \\
\hline ormis & PARADOXUS \\
\hline . & EULIA \\
\hline
\end{tabular}

I93I, Exotic Microlepidoptera, 4:52.

I9I5, Trans. Ent. Soc. London, I9I 5: 236.

I922, Exotic Microlepidoptera, 2: 530. (BM)

I916, Exotic Microlepidoptera, 2: 23. (BM)

I932, Trans. Ent. Soc. London, 80: Iog.

I924, Exotic Microlepidoptera, 3: IIo.

I88I, Proc. Linnean Soc. N.S. Wales, 5: I78.

Igo8, Proc. Zool. Soc. London, p. 735.

I936, Exotic Microlepidoptera, 5: 55.

I926, Exotic Microlepidoptera, 3: 242. (BM)

I923, Exotic Microlepidoptera, 2: 6I4. (P)

I884, Proc. Linnean Soc. N.S. Wales, 9: 761.

I922, Exotic Microlepidoptera, 2: 60I. (BM)

I9I7, Exotic Microlepidoptera, 2: 43. (BM)

I926, Ann. South African Mus. 23: 335. (SA)

I92I, Zool. Meded. 6: I93. (L)

I918, Ann. Transvaal Mus. 6:36. (T)

I932, Exotic Microlepidoptera, 4:252. (v)

I93I, Exotic Microlepidoptera, 4: 83. (v)

I9I4, Journ. Bombay Nat. Hist. Soc. 23: I27. (BM)

I889, Proc. Linnean Soc. N.S. Wales, (Ser. 2) 3: I653.

I9I4, Exotic Microlepidoptera, I: 216.

I925, Exotic Microlepidoptera, 3: I29. (BM)

I938, Trans. R. Ent. Soc. London, 87: 52I.

I925, in Caradja, Mem. Sect. Sti. Acad. Românā, 3: 380.

I934, Ann. Mag. Nat. Hist. I4: 406. (BM)

I924, Trans. Ent. Soc. London, I923: 548. (BM)

I9I3, Exotic Microlepidoptera, I: II2.

I907, Journ. Bombay Nat. Hist. Soc. I8: I50.

I925, Rec. Cant. Mus. 2: 274.

1920, Voyage de Ch. Alluaud et $R$. Jeannel en Afrique

Oriental, II, Microlepidoptera, p. I04. (P)

I909, Journ. Bombay Nat. Hist. Soc. I9: 59I. (вм)

I9I8, Ann. Transvaal Mus. 6: 30. (T)

I9I5, Exotic Microlepidoptera, I: 302.

I925, Exotic Microlepidoptera, 3: I33. (Bм)

I922, Exotic Microlepidoptera, 2: 5I7. (BM)

I927, Exotic Microlepidoptera, 3: 324.

I920, Exotic Microlepidoptera, 2: 376.

I922, Exotic Microlepidoptera, 2: 508.

I928, Exotic Microlepidoptera, 3:407.

I928, Exotic Microlepidoptera, 3: 438. (вм)

I929, Exotic Microlepidoptera, 3: 48I. (BM)

I9I6, Exotic Microlepidoptera, I: 489. (Bм)

I930, Exotic Microlepidoptera, 3: 621. (вм)

I9I8, Exotic Microlepidoptera, 2: 210. (вм)

I934, Exotic Microlepidoptera, 4: 48o.

I928, Exotic Microlepidoptera, 3: 458. (вм)

I9I4, Journ. Bombay Nat. Hist. Soc. 23: I24. (BM)

I926, Exotic Microlepidoptera, 3: 253. (BM) 


\begin{tabular}{|c|c|c|}
\hline degenerans & TORTRIX & I930, Ann. Naturhist. Mus. Wien, 44: 224. (v) \\
\hline dehiscens & ETHMIA & I924, Exotic Microlepidoptera, 3: I20. (BM) \\
\hline deianira & LABDIA & I927, Insects of Samoa, 3, Lepidoptera, fasc. 2, p. 86. \\
\hline dejecta & PLATYPTILIA & I932, Exotic Microlepidoptera, 4: 334. \\
\hline dejiciens & ACROCLITA & I932, Exotic Microlepidoptera, 4: 22I. (BM) \\
\hline delacma & THIOTRICHA & I923, Exotic Microlepidoptera, 3: 24. (BM) \\
\hline delapsa & GELECHIA & I93I, Exotic Microlepidoptera, 4: 60. (v) \\
\hline delatrix & TELPHUSA & I923, Exotic Microlepidoptera, 3: I7. (BM) \\
\hline leastra & BRACHMIA & I9I I, Journ. Bombay Nat. Hist. Soc. 20: 7 II. (BM) \\
\hline delegata & PERIACMA & I9I4, Supplementa Entomologica, No. 3, p. 52. (D) \\
\hline delinita & STENOMA & I9I5, Exotic Microlepidoptera, I: 473 (BM) \\
\hline elia & CHRESMARCHA & I924, Exotic Microlepidoptera, 3: IIo. \\
\hline leliarcha & CHRYSOLYTIS* & I937, Exotic Microlepidoptera, 5: 88. (T) \\
\hline Aelias & CALANTICA & I9I3, Exotic Microlepidoptera, I: I48. \\
\hline elicata & ACROCERCOPS & I921, Zool. Meded. 6: I89. (L) \\
\hline gata & $\begin{array}{l}\text { CRYPTOLECHIA } \\
\text { [SELIDORIS*] }\end{array}$ & I92I, Ann. Transvaal Mus. 8: IOI. (T) \\
\hline eligata & MACHIMIA & I9I4, Exotic Microlepidoptera, I: I80. (BM) \\
\hline eliquescens & TISCHERIA & I9I5, Trans. Ent. Soc. London, I9I5: 246. \\
\hline centra & CRYPTOPHAGA & I89o, Trans. R. Soc. S. Australia, I3: 39. \\
\hline elocharis & ELACHISTA & I932, Trans. Ent. Soc. London, 80: II5. \\
\hline locosma & ONEBALA & I936, Exotic Microlepidoptera, 5: 46. (Bм) \\
\hline lelocrossa & MELASINA & I92I, Ann. Transvaal Mus. 8: I36. (T) \\
\hline delodectis & GELECHIA & $\begin{array}{l}\text { I938, in Caradja and Meyrick, Deuts. Ent. Zeit. Iris, 52: } \\
\text { 3. (Bм) }\end{array}$ \\
\hline delodelta & SCYTHRIS & I930, Exotic Microlepidoptera, 3: $5^{6} \mathrm{I}$. (BM) \\
\hline elographa & ACROCERCOPS & I939, Trans. R. Ent. Soc. London, 89: 60. \\
\hline elophanes & TRACHYNTIS & I889, Proc. Linnean Soc. N.S. Wales, (Ser. 2) 3: I587. \\
\hline lophanta & PERIACMA & I930, Exotic Microlepidoptera, 3: 623. (Bм) \\
\hline elospilus & PTEROPHORUS & I92I, Exotic Microlepidoptera, 2: 42I. \\
\hline lelotis & EULECHRIA & I888, Proc. Linnean Soc. N.S. Wales, (Ser. 2) 2: 952. \\
\hline lotoma & TINEA & I9I9, Exotic Microlepidoptera, 2: 248. (BM) \\
\hline delozona & ALLOCLITA & I9I9, Exotic Microlepidoptera, 2: 238. (BM) \\
\hline delphica & CHRESMARCHA & I9Io, Proc. Linnean Soc. N.S. Wales, 35: 2 Ig. \\
\hline delphinias & DEPRESSARIA & I936, Exotic Microlepidoptera, 4:623. \\
\hline delphinodes & STENOMA & I925, Exotic Microlepidoptera, 3: 222. (BM) \\
\hline delphinopa & GEMORODES & I930, Exotic Microlepidoptera, 4: I3. (BM) \\
\hline deltanthes & GRACILARIA & I934, Pacific Ent. Surv. Publ. 7, art. 28, p. 354. (Bв) \\
\hline deltaspis & THUBANA & I935, Exotic Microlepidoptera, 4: 563. (BM) \\
\hline deltaspis & YPSOLOPHUS & I905, Journ. Bombay Nat. Hist. Soc. I6: 601. \\
\hline Itidias & LEUCOPTERA & I906, Trans. R. Soc. S. Australia, 30:6I. \\
\hline 1tocausta & STENOLECHIA & I929, Exotic Microlepidoptera, 3:484. (Bм) \\
\hline deltochlaena & PHTHEOCHROA & I930, Exotic Microlepidoptera, 3: 59I. (BM) \\
\hline deltochlora & ALSODRYAS & I922, Trans. Ent. Soc. London, I922: 76 . (BM) \\
\hline 1tocrates & NOTHRIS & I927, Exotic Microlepidoptera, 3: 352. (T) \\
\hline eltocycla & METROGENES* & I926, Sarawak Mus. Journ. 3: I6I. \\
\hline eltodoma & PLUTELLA & I93I, Ann. Mus. Nac. Hist. Nat. Buenos Aires, 36: 409. \\
\hline togramma & AEOLANTHES & I923, Exotic Microlepidoptera, 2: 6II. (BM) \\
\hline Itographa & HYDARANTHES* & I928, Exotic Microlepidoptera, 3: 460 . \\
\hline Itomis & STENOMA & I925, Exotic Microlepidoptera, 3: 206. (BM) \\
\hline
\end{tabular}


deltopa DEPRESSARIA

deltophanes

PORTHMOLOGA

deltophora

deltophracta

deltopis

deltopis

deltoplaca

deltoptilus

deltosema

deltospila

deltosticta

deltoxyla

deltozancla

deltozela

deltozona

deltozyga

demarcha

demens

demetrias

demiana

demias

demica

demiurga

democratica

demodes

demogenes

demonstrata

demophila

demotarcha

demotica

demotica

dendronympha

dendrophaga

densata

dentata

dentata

denticulata

dentifera

dentiger

dentigera

dependens

deplanata

deposita

depressa

deprivata
POLYCHROSIS

ELATOBIA

ANTAEOTRICHA

BRACHMIA

EUCOSMA

DEUTEROCOPUS

COESYRA

LECITHOCERA

GRACILARIA

CYMOTRICHA

DICHELOPA

TETRASCHALIS

MACROBATHRA

EUCOSMA

HELIODINES

ANOMOLOGA

AUTOSTICHA

TORTRIX

AUTOSTICHA

IOPTERA

TINEA

GLYPHIDOCERA

EUPLOCAMUS

ELACHISTA

CHELARIA

GELECHIA

LACTISTICA

AUTOSTICHA

EURYPLACA

BRENTHIA

PYRODERCES

PHTHORIMAEA

CNEPHASIA

NOTHRIS

IMMA

COMOTECHNA

OXYPTILUS

VANICELA

ARGYROPLOCE

SCYTHRIS

MELASINA

SCYTHRIS

HIEROMANTIS
1935, in Caradja and Meyrick, Materialien zu einer Microlepidopteren Fauna der Chinesischen Provinzen Kiangsu, Chekiang und Hunan, p. 8o. (Bм)

rgr8, Ann. Transvaal Mus. 6: 53. (T)

1921, Ann. Transvaal Mus. 8: 55. (T)

r926, Sarawak Mus. Journ. 3: 165.

I9I5, Exotic Microlepidoptera, I:39o. (Bм)

r920, Voyage de Ch. Alluaud et $R$. Jeannel en Afrique

Oriental, $I I$, Microlepidoptera, p. 79. (P)

I921, Zool. Meded. 6: I54. (L)

1930, Exotic Microlepidoptera, 3: 565. (BM)

I884, Proc. Linnean Soc. N.S. Wales, 9: 782 .

rgrr, Journ. Bombay Nat. Hist. Soc. 20: 706. (BM)

1933, Exotic Microlepidoptera, 4:362.

I934, in Caradja and Meyrick, Deuts. Ent. Zeit. Iris, 48:

35 .

1926, Trans. Ent. Soc. London, 74: 272. (BM)

1924, Exotic Microlepidoptera, 3: 92.

1932, Exotic Microlepidoptera, 4:276. (Bм)

1928, Exotic Microlepidoptera, 3: 440. (T)

I917, Exotic Microlepidoptera, 2: 65.

1926, Exotic Microlepidoptera, 3: 309. (T)

I908, Journ. Bombay Nat. Hist. Soc. I8: 457. (BM)

r883, Trans. Proc. New Zealand Inst. r5: 51.

I886, Trans. Ent. Soc. London, r886: $28 \mathrm{I}$.

r889, Proc. Linnean Soc. N.S. Wales, (Ser. 2) 3: r589.

I920, Exotic Microlepidoptera, 2: 354.

1929, Exotic Microlepidoptera, 3: 531. (BM)

r924, Exotic Microlepidoptera, 3: 74.

1897, Proc. Linnean Soc. N.S. Wales, 22: 336.

1920, Exotic Microlepidoptera, 2: 303.

r936, in Caradja and Meyrick, Deuts. Ent. Zeit. Iris, 50:

I57. (BM)

1907, Journ. Bombay Nat. Hist. Soc. 17: 742. (Bм)

rgo8, Journ. Bombay Nat. Hist. Soc. I8: 458. (BM)

r883, Proc. Linnean Soc. N.S. Wales, 7: 489.

1937, Exotic Microlepidoptera, 5: 125. (BM)

1920, Exotic Microlepidoptera, 2: 318. (BM)

r9r7, Trans. Ent. Soc. London, r9r7: 42. (BM)

I9I7, Trans. Ent. Soc. London, I9I7: II. (BM)

r904, Proc. Linnean Soc. N.S. Wales, 29: 427.

I9Io, Trans. Ent. Soc. London, I910: 464. (BM)

r92r, Exotic Microlepidoptera, 2: 40r. (BM)

rgr6, Exotic Microlepidoptera, r: 557.

I9I3, Exotic Microlepidoptera, I: 81.

1922, Exotic Microlepidoptera, 2: 524. (P)

I928, Exotic Microlepidoptera, 3: 4I3.

I919, Exotic Microlepidoptera, 2: 266.

r931, Journ. Linnean Soc. London, 37: 282. (BM)

I927, Insects of Samoa, 3, Lepidoptera, fasc. 2, p. 98. 
derelicta

derelictus

deridens

dermatica

dermatodes

dermatopa

derogata

descripta

desecta

desiccata

desiccata

desidiosa

designata

designata

desiliens

desipiens

desmochares

desmophanes

desmophora

desmophthora

desmotana

desmoteles

desmotoma

desolata

despecta

despotica

destillans

determinata

detestata

detrita

deuterarcha

deuterastis

deuterastis

deuteraula

deuteropa

deversa

devexa

devia

devincta

devincta

devoluta

devota

diacapna

diacentra

diacentrota

diachelota

diachordá

diacma

diacmota
SPILADARCHA* TRICHOPTILUS

ANTAEOTRICHA

CHELARIA

MELASINA

EULIA

BRACHMIA

CEROMITIA

STENOMA

ACROCERCOPS

BORKHAUSENIA

STENOMA

CNEPHASIA

DOLIOTECHNA

GELECHIA

EUCOSMA

ACROCERCOPS

MICROPLITIS*

LOCHEUTIS

LEPIDOSCIA

CACOECIA

STATHMOPODA

MACROBATHRA

LECITHOCERA

TINEA

ANTICRATES

TELPHUSA

BLASTOBASIS

SCYTHRIS

LASPEYRESIA

THRIAMBEUTIS

CAPUA

GLYPHIPTERYX

POLYHYMNO

STENOMA

ACROCERCOPS

TORTRIX

GELECHIA

AMALLECTIS*

MELASINA

STILBOSIS

CALANTICA

BUCCULATRIX

ODITES

ACROCERCOPS

ACROLOPHUS

BACTRA

TIPHA

[OXYGNOSTIS*]

GELECHIA
I9I3, Exotic Microlepidoptera, I: I39. (BM)

I926, Trans. Ent. Soc. London, 74: 276.

I925, Exotic Microlepidoptera, 3: I62. (BM)

I92I, Exotic Microlepidoptera, 2: 432. (BM)

I9I4, Ann. South African Mus. Io: 253. (SA)

1928, Proc. Hawaiian Ent. Soc. 7: 96. (SP)

I921, Ann. Transvaal Mus. 8: 9I. (T)

I924, Exotic Microlepidoptera, 3: 8o. (T)

I9I8, Exotic Microlepidoptera, 2: 203. (BM)

I9I6, Exotic Microlepidoptera, 2: 4. (BM)

I9I5, Exotic Microlepidoptera, I: 296.

1925, Exotic Microlepidoptera, 3:20I. (BM)

I92I, Ann. Transvaal Mus. 8: 52. (T)

I9I4, Exotic Microlepidoptera, I: I88. (BM)

I923, Exotic Microlepidoptera, 3: 23. (BM)

I9I8, Ann. Transvaal Mus. 6: Io. (T)

I92I, Exotic Microlepidoptera, 2: 468.

I922, Exotic Microlepidoptera, 2: 555.

I883, Proc. Linnean Soc. N.S. Wales, 8: 343.

1893, Proc. Limnean Soc. N.S. Wales, 7: 5I2.

I88I, Proc. Linnean Soc. N.S. Wales, 6: 506.

I897, Proc. Linnean Soc. N.S. Wales, 22: 322.

I886, Proc. Linnean Soc. N.S. Wales, Io: 806.

I9I8, Exotic Microlepidoptera, 2: I05. (BM)

I9I9, Exotic Microlepidoptera, 2: 274. (BM)

I926, Sarawak Mus. Journ. 3: I63.

I9I8, Exotic Microlepidoptera, 2: I33. (BM)

I92I, Ann. Transvaal Mus. 8: II6. (T)

I922, Exotic Microlepidoptera, 2: 554 .

I928, Exotic Microlepidoptera, 3: 450. (BM)

I938, Trans. R. Ent. Soc. London, 87: 522.

I9Io, Proc. Linnean Soc. N.S. Wales, 35: I9I.

I907, Proc. Linnean Soc. N.S. Wales, 32: I2I.

I9I4, Ann. Transvaal Mus. 4: I93. (T)

I93I, Journ. Linnean Soc. London, 37: 28I. (BM)

1922, Exotic Microlepidoptera, 2: 563 .

I926, Exotic Microlepidoptera, 3: 248. (BM)

I9I3, Ann. Transvaal Mus. 3: 290. (T)

I9I7, Trans. Ent. Soc. London, I9I7: I. (BM)

I9I6, Exotic Microlepidoptera, I: 6Io.

I9I7, Exotic Microlepidoptera, 2: 48. (BM)

I9I3, Exotic Microlepidoptera, I: I48.

I920, Exotic Microlepidoptera, 2: 359. (BM)

I92I, Zool. Meded. 6: I74. (L)

I935, Exotic Microlepidoptera, 4: 597.

I93I, Mitteil. Mïnch. Ent. Gesells. 21: 39.

I932, Exotic Microlepidoptera, 4: 308. (v)

I906, Journ. Bombay Nat. Hist. Soc. I7: I42. (BM) I932, Exotic Microlepidoptera, 4: 350. (v) 
diacnista diaconalis diacritica diacta diagramma diagrapha diagrapta dialectica diallacta diallactis diametrica diametrica dianeura diantha dianthes diaphanta diaphora diaphracta diaplintha diarthra diarthrota diasticta diatonica diaugella diaula diaxantha diaxantha diaxesta diazeucta dicarpa dicastica dicastis dicausta dicax dicax dicellias dicentra dicentris

dicentropa

dicentrota dichalina dicharacta dichlora dichorda dichroanthes dicitra
MYROPHILA

NOTHRIS

AEOLANTHES

STENOMA

EULECHRIA

MACHIMIA

HEMIMENE

APOTHETODES*

ARGYROPLOCE

MELASINA

STENOMA

TORTRIX

AUTOSTICHA

PLACOCOSMA

EUCOSMA

STEREOPTILA

TINEA

TONICURGIS*

PLAESIOSTOLA*

LEPTOCHERSA*

TINEA

ISEMBOLA*

ACROCERCOPS

PHYLLOCNISTIS

EUTORNA

DOXOMERES*

LYSIPATHA

BORKHAUSENIA

COMPSOLECHIA

LABDIA

PAMMENE

CUPHODES

ZOMEUTIS*

ANACAMPSIS

HYSTEROSIA

ZARATHA

STENOMA

ANTISCLEROTA*

LECITHOCERA

BRENTHIA

GLYPHIPTERYX

TINEA

SIMAETHIS

GLYPHIPTERYX

BRITH YCEROS*

STATHMOPODA
I923, Exotic Microlepidoptera, 2: 624. (BM)

I929, Exotic Microlepidoptera, 3: 495. (BM)

I9I8, Exotic Microlepidoptera, 2: I96. (BM)

I9I6, Exotic Microlepidoptera, I: 5I3. (BM)

I888, Proc. Linnean Soc. N.S. Wales, (Ser. 2) 2: 96r.

I93I, Exotic Microlepidoptera, 4: I20. (v)

I9I6, Exotic Microlepidoptera, 2: 27. (BM)

I9I9, Exotic Microlepidoptera, 2: 233.

I932, Exotic Microlepidoptera, 4: 225.

I934, Exotic Microlepidoptera, 4: 520. (c)

I926, Exotic Microlepidoptera, 3: 239. (BM)

I932, Trans. Ent. Soc. London, 80: IIo. (BM)

I939, Trans. R. Ent. Soc. London, 89: 56. (BM)

I9I3, Exotic Microlepidoptera, I: I34.

I928, Exotic Microlepidoptera, 3: 439. (BM)

I93I, Exotic Microlepidoptera, 4: 9I. (BM)

I893, Proc. Linnean Soc. N.S. Wales, 7: 537.

I922, Exotic Microlepidoptera, 2: 603. (BM)

I926, Sarawak Mus. Journ. 3: I68.

I9I9, Exotic Microlepidoptera, 2: 272.

I936, Exotic Microlepidoptera, 5: 53.

I926, Exotic Microlepidoptera, 3: 27I. (BM)

I9I6, Exotic Microlepidoptera, I: 625.

I88I, Proc. Linnean Soc. N.S. Wales, 5: I73.

I9o6, Trans. R. Soc. S. Australia, 30:45.

I9I7, Ann. South African Mus. I7: 6. (SA)

I932, Exotic Microlepidoptera, 4: 203. (BM)

I922, Arkiv för Zool. vol. I4, part I5, p. 6 .

I9I8, Exotic Microlepidoptera, 2: I38. (Sub.)

I927, Insects of Samoa, 3, Lepidoptera, fasc. 2, p. 88.

I922, Exotic Microlepidoptera, 2: 526. (BM)

I905, Journ. Bombay Nat. Hist. Soc. I6: 606.

I9I3, Journ. Bombay Nat. Hist. Soc. 22: I82. (Bм)

I9I4, Trans. Ent. Soc. London, I9I4: 266. (BM)

I9I2, Ent. Mon. Mag. 48: 35. (Sub.)

I909, Journ. Bombay Nat. Hist. Soc. I9: 4Io.

I9I3, Ann. Transwaal Mus. 3: 3I7. (T)

I938, Institut des Parcs Nationaux du Congo Belge, fasc. I4, p. 20.

I938, Institut des Parcs Nationaux du Congo Belge, fasc. I4, p. I4.

I93I, Exotic Microlepidoptera, 4: I83. (BM)

I9I I, Trans. Linnean Soc. London, I4: 290.

I893, Proc. Linnean Soc. N.S. Wales, 7: 536.

I9I2, Exotic Microlepidoptera, I: 48. (BM)

I9I I, Trans. New Zealand Inst. 43: 76 .

I932, Exotic Microlepidoptera, 4: 2 I0.

I935, in Caradja and Meyrick, Materialien zu einer Microlepidopteren Fauna der Chinesischen Provinzen Kiangsu,

Chekiang und Hunan, p. 85. 


$\begin{array}{ll}\text { diclera } & \text { GEMORODES* } \\ \text { diclethra } & \text { OCYSTOLA } \\ \text { diclidias } & \text { STATHMOPODA } \\ \text { dicommatias } & \text { LAMPRONIA } \\ \text { dicraea } & \text { AEOLANTHES } \\ \text { dicremna } & \text { HERMOGENES } \\ \text { dictyanthes } & \text { PSEUDATTERIA } \\ \text { dictyarcha } & \text { ORTHENCHES } \\ \text { dictyodes } & \text { COESYRA }\end{array}$

$\begin{array}{ll}\text { dictyodes } & \text { OXYGRAPHA } \\ \text { dictyogramma } & \text { STENOMA } \\ \text { dictyophanes } & \text { EULIA } \\ \text { dictyopsamma } & \text { ACROLOPHUS } \\ \text { dictyotis } & \text { SCARDIA } \\ \text { dicyanitis } & \text { LABDIA } \\ \text { dicycla } & \text { MONOPIS } \\ \text { didyma } & \text { CHOANOGRAPTIS* } \\ \text { didymella } & \text { GRACILARIA } \\ \text { didymopa } & \text { ANARSIA } \\ \text { difficilis } & \text { CACOECIA } \\ \text { diffua } & \text { ANTICRATES }\end{array}$

$\begin{array}{ll}\text { diffracta } & \text { ANTAEOTRICHA } \\ \text { diffusa } & \text { COLEOPHORA } \\ \text { digesta } & \text { HOLCOCERA } \\ \text { digitata } & \text { ANACHASTIS } \\ \text { digitata } & \text { ONEBALA } \\ \text { diglypta } & \text { TRICLONELLA } \\ \text { digna } & \text { EUCOSMA } \\ \text { digna } & \text { LECITHOCERA } \\ \text { digramma } & \text { BONDIA } \\ \text { digrapta } & \text { GELECHIA } \\ \text { digressa } & \text { STEREOPTILA } \\ \text { dilacerata } & \text { SPILONOTA } \\ \text { dilecta } & \text { MACHIMIA } \\ \text { diligenda } & \text { CRYPTOLECHIA } \\ \text { diligens } & \text { LECITHOCERA } \\ \text { dilinopa } & \text { STENOMA } \\ \text { dilucescens } & \text { CONIOGYRA* } \\ \text { dilucida } & \text { ANCYLOMETIS } \\ \text { diluta } & \text { PLUTELLA } \\ \text { diluvialis } & \text { EUTORNA } \\ \text { diluviata } & \text { SYNNYMPHA } \\ \text { dimensa } & \text { SCYTHRIS } \\ \text { dimetra } & \text { AGDISTIS } \\ \text { dimetropis } & \text { STENOMA } \\ \text { dimochla } & \text { AUTOSTICHA }\end{array}$

1925, Exotic Microlepidoptera, 3: I55. (T)

I885, Proc. Linnean Soc. N.S. Wales, 9: ro78.

r921, Exotic Microlepidoptera, 2: 462.

r931, Ann. Mus. Nac. Hist. Nat. Buenos Aires, 36: 4 I4.

I908, Journ. Bombay Nat. Hist. Soc. I8: 637. (вм)

I908, Journ. Bombay Nat. Hist. Soc. 18: 629. (вм)

1935, Arb. morph. taxon. Ent. Berl. 3: I04. (D)

1927, Trans. Proc. New Zealand Inst. 58: 3I5.

I889, Proc. Linnean Soc. N.S. Wales, (Ser. 2) 3: I662.

(BM)

I907, Journ. Bombay Nat. Hist. Soc. I7: 734. (BM)

I932, Exotic Microlepidoptera, 4: 30I. (v)

1926, Exotic Microlepidoptera, 3: 254. (BM)

I93I, Exotic Microlepidoptera, 4: Ior.

1893, Proc. Linnean Soc. N.S. Wales, 7: 522.

I934, Pacific Ent. Surv. Publ. 7, art. 28, p. 347. (вв)

1905, Journ. Bombay Nat. Hist. Soc. 16: 618.

1938, Trans. R. Ent. Soc. London, 87: 504. (вм)

r881, Proc. Linnean Soc. N.S. Wales, 5: r64.

I9I6, Exotic Microlepidoptera, I: 583. (BM)

1928, Exotic Microlepidoptera, 3: 456. (вм)

1927, Insects of Samoa, 3, Lepidoptera, fasc. 2, p. ro6. (BM)

I9I6, Exotic Microlepidoptera, I: 500. (BM)

I9I3, Ann. Transvaal Mus. 3: 323. (T)

I922, Exotic Microlepidoptera, 2: 542. (BM)

Igr I, Trans. Linnean Soc. London, I4: 288.

I914, Exotic Microlepidoptera, I: 200. (BM)

I9r3, Exotic Microlepidoptera, 4: II7. (v)

I917, Trans. Ent. Soc. London, I9I7: 22. (вм)

I9I8, Exotic Microlepidoptera, 2: I05. (BM)

r9ro, Proc. Linnean Soc. N.S. Wales, 35: 145.

I936, Exotic Microlepidoptera, 5: 44. (BM)

I931, in Caradja, Bull. Sect. sci. Acad. roum. I4: 70. (Bм)

I929, Trans. Ent. Soc. London, 76: 494. (BM)

1920, Exotic Microlepidoptera, 2: 377. (BM)

I928, Exotic Microlepidoptera, 3: 477. (BM)

r922, Zool. Meded. 7:84.

I925, Exotic Microlepidoptera, 3: I98. (BM)

I921, Ann. Transvaal Mus. 8: 66. (T)

I9Io, Trans. Ent. Soc. London, x910: 37x.

I931, Ann. Mus. Nac. Hist. Nat. Buenos Aires, 36: 407.

I9I3, Ann. Transvaal Mus. 3: 3I6. (T)

I9I5, Exotic Microlepidoptera, I: 366.

1920, Ann. South African Mus. I7: 298. (SA)

1924, Exotic Microlepidoptera, 3: 95. (T)

I932, Exotic Microlepidoptera, 4: 297. (Us)

1935, in Caradja and Meyrick, Materialien zu einer Microlepidopteren Fauna der Chinesischen Provinzen Kiangsu,

Chekiang und Hunan, p. 76 . 
dimolybda PROMALACTIS

$\begin{array}{ll}\begin{array}{l}\text { dimorpha } \\ \text { dimorpha }\end{array} & \text { ARGYROPLOCE } \\ \text { dimota } & \text { SCYTHRIS } \\ \text { dinocosma } & \text { PHLOEOPOLA } \\ \text { dinodes } & \text { PORINA } \\ \text { dinosema } & \text { OENOCHROA } \\ \text { dinosticha } & \text { ACROCERCOPS } \\ \text { dinota } & \text { TORTRIX } \\ \text { dioctis } & \text { AERAULA* } \\ \text { diogma } & \text { EUCOSMA } \\ \text { dionysias } & \text { TRACHYDORA } \\ \text { diophanes } & \text { LABDIA } \\ \text { diopta } & \text { ODITES } \\ \text { dioptrias } & \text { IMMA } \\ \text { dioptrica } & \text { PHYLACTERITIS* } \\ \text { diorista } & \text { PTILOGENES } \\ \text { diortha } & \text { ANACAMPSIS }\end{array}$

[COMPSOLECHIA * I9I4, Trans. Ent. Soc. London, I9I4: 263. (BM)

diorthota diorycta dioryctis diphtherina diplaca diplacopa diplaneta diplanetis diplarcha diplardis diplaspis diplecta diplobathra diplobola diplocentra diplochorda diplocosma diplodelta diplodoxa diplogramma diplolychna diplonesa diplophaea diplophragma

diplopsamma diplorhiza diplosaris

\section{OPOSTEGA}

TANYZANCLA

ACOMPSOGMA* TORTRICOMORPHA PERILICMETIS* ACROCERCOPS SCORPIOPSIS PROTOLECHIA ANTAEOTRICHA OPOSTEGA PLACOSTOLA* PYRODERCES THYRSOSTOMA TINEA PROCOMETIS HARMOTONA* NEPTICULA ANACAMPSIS STOMOPTERYX SIMAETHIS COMPSOLECHIA PROTOLECHIA ANTAEOTRICHA NEMOTOIS

SAPHENEUTIS DECADARCHIS
I935, in Caradja and Meyrick, Materialien zu einer Microlepidopteren Fauna der Chinesischen Provinzen Kiangsu, Chekiang und Hunan, p. 78. (BM)

I909, Journ. Bombay Nat. Hist. Soc. I9: 606. (BM)

I907, Proc. Linnean Soc. N.S. Wales, 32: I41.

I931, Journ. Linnean Soc. London, 37:282. (BM)

1883, New Zealand Journ. Sci. I: 522.

I89o, Trans. Proc. New Zealand Inst. 22: 206.

1889, Proc. Linnean Soc. N.S. Wales, (Ser. 2) 3: I 575.

I936, Exotic Microlepidoptera, 5: 36.

I918, Exotic Microlepidoptera, 2: I68. (BM)

I897, Proc. Linnean Soc. N.S. Wales, 22: 370.

I927, Exotic Microlepidoptera, 3:336. (BM)

I921, Exotic Microlepidoptera, 2: 456.

1927, Exotic Microlepidoptera, 3:384. (T)

I9I7, Exotic Microlepidoptera, 2: 57. (BM)

I9o6, Trans. Ent. Soc. London, I906: I8I. (BM)

I922, Exotic Microlepidoptera, 2: 499. (BM)

I929, Trans. Ent. Soc. London, 76:5I7. (BM)

1893, Proc. Linnean Soc. N.S. Wales, 7: 607.

I920, Ann. South African Mus. I7: 286. (SA)

I938, Trans. R. Ent. Soc. London, 87: 518.

I905, Journ. Bombay Nat. Hist. Soc. I6: 6I I.

I932, Exotic Microlepidoptera, 4: 324. (v)

I936, Exotic Microlepidoptera, 5: 36.

I930, Exotic Microlepidoptera, 3: 6r9.

I904, Proc. Linnean Soc. N.S. Wales, 29: 373.

I9I5, Exotic Microlepidoptera, I: 403. (BM)

I921, Ann. Transvaal Mus. 8: I23. (T)

I887, Trans. Ent. Soc. London, I887: 280.

I935, Exotic Microlepidoptera, 4: 606.

I9I8, Exotic Microlepidoptera, 2: I20. (BM)

I93I, Exotic Microlepidoptera, 4: 95.

r89o, Trans. R. Soc. S. Australia, 13: 73 .

I9I9, Exotic Microlepidoptera, 2: 255.

I921, Exotic Microlepidoptera, 2: 4IO.

1922, Trans. Ent. Soc. London, I922: 76. (BM)

I936, Exotic Microlepidoptera, 4: 624. (BM)

I9I2, Exotic Microlepidoptera, I : 49. (BM)

I922, Trans. Ent. Soc. London, I922: 86. (Bм)

I904, Proc. Linnean Soc. N.S. Wales, 29: 344.

I9I6, Exotic Microlepidoptera, I : 494. (BM)

I938, in Caradja and Meyrick Deuts. Ent. Zeit. Iris, 52: 28.

I9I8, Ann. Transvaal Mus. 6: 45. (T)

I931, in Joannis, Ann. Soc. Ent. France, 98 (Supp.):

$74 \mathrm{I}[503] .(\mathrm{P})$

I9I5, Exotic Microlepidoptera, I: $4 \mathrm{I} S$. (BM) 


diplosema
diplospila
diplosticha
diplosticta
diplotaphra
diplotima
diplotoma
diplotoxa
diplotrocha
diploxantha
diplozona
dipsalea
dipselia
dipsia
diptila

directa

directa

directrix

dirupta

discerpta

dischema

discincta

discipula

discissa

disclusa

discors

discors

discosema

discrepans

discreta

discriminata

discruciata

discura

discussa

disema

disemanta

diserta

disjuncta

dislocata

disparata

dispensata

disperma

dispersa

dispertita

disphaerias

disposita

dispulsa

disputanda

disrupta
BATRACHEDRA

IMMA

CRYPTOLECHIA

LECITHOCERA

BRENTHIA

TRICHOCEROTA

PERIACMA

GLYPHIPTERYX

HYPERCALLIA

PETALANTHES

COSMOPTERYX

TIMYRA

IMMA

FRISILIA

COLPOCRITA*

EUCOSMA

TRICHOTAPHE

THIOSCELIS*

IECITHOCERA

GYMNELEMA

ONEBALA

COESYRA

EUCOSMA

CHELARIA

TELPHUSA

AGRIOPHARA

PTEROPHORUS

AXIARCHA*

STENOMA

DECADARCHIS

ARISTOTELIA

TORTRIX

IDIOPHANTIS

TALAEPORIA

COESYRA

HYPONOMEUTA

ARGYROPLOCE

DECADARCHIS

ARGYRESTHIA

IDIOPHANTIS

PHTHORIMAEA

LASPEYRESIA

IPHIERGA

HOMADAULA

CYPHOTHYRIS

CHELARIA

ANOMOLOGA*

AMPHIPSEUSTIS*

DISSOPTILA
I897, Proc. Linnean Soc. N.S. Wales, 22: 306.

I928, Exotic Microlepidoptera, 3: 420. (BM)

I926, Exotic Microlepidoptera, 3: 3I8. (BM)

I922, Zool. Meded. 7: 84. (L)

I938, Trans. R. Ent. Soc. London, 87: 523. (BM)

I926, Exotic Microlepidoptera, 3: 269.

I939, Trans. R. Ent. Soc. London, 89: 57. (BM)

I920, Ann. South African Mus. I7: 296. (SA)

I937, Exotic Microlepidoptera, 5:85.

I9I4, Exotic Microlepidoptera, I: 236.

I921, Ann. Transvaal Mus. 8: 95. (T)

I9o8, Journ. Bombay Nat. Hist. Soc. I8: 446. (BM)

I906, Trans. Ent. Soc. London, I906: I79. (BM)

I9Io, Journ. Bombay Nat. Hist. Soc. 20: 437. (BM)

I930, Ann. Naturhist. Mus. Wien, 44:264, pl. I, fig. 33.

(v)

I9I2, Exotic Microlepidoptera, I: 34.

I9I 2, Trans. Ent. Soc. London, I9I : 694. (BM)

Igo9, Trans. Ent. Soc. London, I909: 30. (BM)

I923, Exotic Microlepidoptera, 3: 39. (BM)

I924, Exotic Microlepidoptera, 3: 78 . (T)

I9I6, Exotic Microlepidoptera, I: 576. (BM)

I884, Proc. Linnean Soc. N.S. Wales, 9: 788.

I9I2, Ent. Mon. Mag. 48: 35. (Sub.)

I9I6, Exotic Microlepidoptera, I: 58I.

I923, Exotic Microlepidoptera, 3: I6. (BM)

I9I3, Trans. Ent. Soc. London, I9I3: I82.

I9I3, Exotic Microlepidoptera, I: II2.

I92I, Ann. Transvaal Mus. 8: 96. (T)

I925, Exotic Microlepidoptera, 3: I99. (BM)

I9Io, Trans. Ent. Soc. London, I9Io: 473.

I923, Exotic Microlepidoptera, 3: Io. (BM)

I930, Trans. Ent. Soc. London, 78: 3I0. (P)

I907, Journ. Bombay Nat. Hist. Soc. I8: I48. (Bм)

I92I, Ann. Transvaal Mus. 8: I33. (T)

I884, Proc. Linnean Soc. N.S. Wales, 9: 786.

I933, Exotic Microlepidoptera, 4: 373. (BM)

I909, Journ. Bombay Nat. Hist. Soc. I9: 593. (BM)

I922, Exotic Microlepidoptera, 2: 559.

I9I4, Journ. Bombay Nat. Hist. Soc. 23: I23. (BM)

I923, Exotic Microlepidoptera, 3: 24. (BM)

I921, Ann. Transvaal Mus. 8: 73. (T)

I93I, Exotic Microlepidoptera, 4: I43.

I9I7, Exotic Microlepidoptera, 2: 90.

I922, Exotic Microlepidoptera, 2: 55I (P)

I932, Exotic Microlepidoptera, 4:333. (cu)

I93I, Exotic Microlepidoptera, 4: 7I. (V)

I926, Exotic Microlepidoptera, 3: 309. (T)

I92I, Ann. Transvaal Mus. 8: I03. (T)

I9I4, Trans. Ent. Soc. London, I9I4: 235. (BM) 


\begin{tabular}{|c|c|c|}
\hline dissecta & EPIMACTIS & I921, Zool. Meded. 6: I73. (L) \\
\hline dissepta & DECADARCHIS & I93I, Exotic Microlepidoptera, 4: I66. \\
\hline dissias & LASPEYRESIA & I907, Journ. Bombay Nat. Hist. Soc. I8: I44. (Bм) \\
\hline dissidens & NOTHRIS & I9I3, Ann. Transvaal Mus. 3: 30I. (T) \\
\hline dissimulans & DECADARCHIS & I9I5, Exotic Microlepidoptera, I: 368. \\
\hline dissimulatrix & ORTHENCHES & I93 I, Ann. Mus. Nac. Hist. Nat. Buenos Aires, 36: 407. \\
\hline dissipata & TORTRIX & I922, Exotic Microlepidoptera, 2: 496. (BM) \\
\hline dissobola & THIOTRICHA & I935, Exotic Microlepidoptera, 4:56I. (вм) \\
\hline dissociata & TINEOLA & I922, Exotic Microlepidoptera, 2: 594. \\
\hline dissoluta & MELASINA & I908, Proc. Zool. Soc. London, p. 747. \\
\hline dissolutana & BONDIA & I883, Proc. Linnean Soc. N.S. Wales, 7: I82. \\
\hline dissona & STENOMA & I925, Exotic Microlepidoptera, 3: I9I. (BM) \\
\hline dissoplaca & ACROCLITA & I936, Exotic Microlepidoptera, 5: 23. (BM) \\
\hline dissota & CLEPSIGENES* & I93o, Exotic Microlepidoptera, 4: I6. \\
\hline dissotoma & ACROCERCOPS & I93I, Exotic Microlepidoptera, 4: I68. \\
\hline distactae & PERITRANA* & I907, Journ. Bombay Nat. Hist. Soc. I7: 988. \\
\hline istactica & SCYTHRIS & I92I, Ann. Transvaal Mus. 8: II5. (T) \\
\hline distephana & COESYRA & I884, Proc. Linnean Soc. N.S. Wales, 9: 768. \\
\hline listicha & AGRIOPHARA & I9I6, Exotic Microlepidoptera, I: 488. (BM) \\
\hline distorta & MACHIMIA & I920, Exotic Microlepidoptera, 2: 377 . (BM) \\
\hline distracta & TINISSA & I9I6, Exotic Microlepidoptera, I: 6I5. \\
\hline icta & TORTRIX & I920, Exotic Microlepidoptera, 2: 342. (BM) \\
\hline ditemenitis & BRACHMIA & I934, Ann. Mag. Nat. Hist. I4: 408. (BM) \\
\hline ditreta & ETHMIA & $\begin{array}{l}\text { I920, Voyage de Ch. Alluaud et } R \text {. Jeannel en Afrique } \\
\text { Oriental, } I I, \text { Microlepidoptera, p. } 88 \text {. (P) }\end{array}$ \\
\hline ditrocha & CIRCOXENA* & igi6, Trans. Proc. New Zealand Inst. 48: 4I9. \\
\hline trota & BATRACHEDRA & I897, Proc. Linnean Soc. N.S. Wales, 22: 305. \\
\hline liula & CROCANTHES & I904, Proc. Linnean Soc. N.S. Wales, 29: 398. \\
\hline dividua & SCYTHRIS & I9I6, Exotic Microlepidoptera, 2: I2. (BM) \\
\hline divulsa & HIEROXESTIS & I923, Exotic Microlepidoptera, 3: 63 . \\
\hline dochaea & CRYPTOLECHIA & I9Io, Journ. Bombay Nat. Hist. Soc. 20: I64. \\
\hline dochmia & PHTHINOCOLA* & I886, Trans. Ent. Soc. London, I886: 29I. \\
\hline docilis & MESOTHYRSA & I9I8, Exotic Microlepidoptera, 2: 22I. (BM) \\
\hline doctissima & TRISOPHISTA* & I924, Exotic Microlepidoptera, 3: II8. (BM) \\
\hline doctrinalis & ARGYROPLOCE & I934, Ann. Mag. Nat. Hist. I4: 407. (BM) \\
\hline dogmatica & GRACILARIA & Igo8, Journ. Bombay Nat. Hist. Soc. I8: 830. \\
\hline lolabrata & SYMMOCA & I9I6, Exotic Microlepidoptera, I: 589. (BM) \\
\hline & $\begin{array}{l}\text { CRYPTOPHAGA } \\
\left.\text { [ENTEREMNA }{ }^{*}\right]\end{array}$ & I89o, Trans. R. Soc. S. Australia, I3: 30. \\
\hline doleropa & HYPATIMA & I907, Journ. Bombay Nat. Hist. Soc. I8: I5I. \\
\hline pis & STENOMA & I9I5, Exotic Microlepidoptera, I: 42I. (BM) \\
\hline dolichaula & DICHOMERIS & I93I, Exotic Microlepidoptera, 4:67. (BM) \\
\hline dolichoscia & BATRACHEDRA & I928, Exotic Microlepidopterci, 3: 394. (BM) \\
\hline liodes & APODIA & I89I, Ent. Mon. Mag. 27: 55. (BM) \\
\hline doliopa & CROCANTHES & I92I, Exotic Microlepidoptera, 2: 434. \\
\hline doliopis & MICROSOPHISTA* & I932, Exotic Microlepidoptera, 4: 328. \\
\hline dolopaea & STREPSICRATES & I905, Trans. Ent. Sroc. London, I905: 232. \\
\hline doloploca & ACROCERCOPS & I92I, Exotic Microlepidoptera, 2: 469. \\
\hline orosa & PHTHEOCHROA & I932, Exotic Microlepidoptera, 4: 266. (v) \\
\hline dolosa & BRACHMIA & I9II, Journ. Bombay Nat. Hist. Soc. 20: 724. (BM) \\
\hline
\end{tabular}




\begin{tabular}{|c|c|c|}
\hline doratias & STATHMOPODA & I897, Proc. Linnean Soc. N.S. Wales, 22: 323. \\
\hline dorcadopa & STENOMA & I9I6, Exotic Microlepidoptera, I: 532. (BM) \\
\hline dorcas & PARATORNA* & I907, Journ. Bombay Nat. Hist. Soc. I7: 980. (вм) \\
\hline orcas & PHYLLOCNISTIS & I9I5, Trans. Ent. Soc. London, I9I5: 24I. \\
\hline lorinda & LITHOCOLLETIS & I9I2, Exotic Microlepidoptera, I: 2I. \\
\hline loris & MELODRYAS* & I9Io, Trans. Ent. Soc. London, I9Io: 472. \\
\hline ites & DEUTEROCOPUS & I913, Exotic Microlepidoptera, I: Io8. \\
\hline dormiens & ISCHNOBATHRA & I937, Exotic Microlepidoptera, 5: 8I. (T) \\
\hline dorochares & MICROCOLONA & I927, Insects of Samoa, 3, Lepidoptera, fasc. 2, p. 94. \\
\hline phanes & TORTRICOPSIS & I9I4, Exotic Microlepidoptera, I: I94. \\
\hline dorothea & SPHAERELICTIS* & I924, Exotic Microlepidoptera, 3: I03. (BM) \\
\hline doroxena & $\begin{array}{l}\text { PALAEOMICRA } \\
\text { [MICROPARDALIS*] }\end{array}$ & I888 Trans Pro \\
\hline oxanthes & HIERANGELA & $\begin{array}{l}\text { I888, Trans. Proc. New Zealand Inst. } 2 \\
\text { I929, Exotic Microlepidoptera, 3: } 498 \text {. }\end{array}$ \\
\hline rcha & HELCYSTOGRAMMA & I9I6, Exotic Microlepidoptera, I: 578 . (BM) \\
\hline oxastica & MACHAERITIS & I889, Proc. Linnean Soc. N.S. Wales, (Ser. 2) 3: 1667. \\
\hline oxasticana & PENTHINA & I88I, Proc. Linnean Soc. N.S. Wales, 6: 644 . \\
\hline oxochares & TINEA & I926, Ann. South African Mus. 23: 343. (SA) \\
\hline loxophanes & OPOGONA & I9I5, Exotic Microlepidoptera, I: 357. \\
\hline Iracaenopa & STATHMOPODA & I933, Exotic Microlepidoptera, 4: 430. \\
\hline racaenura & DECADARCHIS & I934, Ann. Mag. Nat. Hist. I4: 4II. \\
\hline hmaea & COMPSOLECHIA & I922, Trans. Ent. Soc. London, I922: 94. (Вм) \\
\hline Iracontias & EPERMENIA & I9I7, Exotic Microlepidoptera, 2:67. \\
\hline racopis & CELETODES* & I921, Zool. Meded. 6: I66. (L) \\
\hline cuncula & GERONTHA & I928, Exotic Microlepidoptera, 3: 428. (вм) \\
\hline Aramatica & OPOGONA & I9I I, Journ. Bombay Nat. Hist. Soc. 21: IIo. \\
\hline Hrapetica & TIQUADRA & I9I9, Exotic Microlepidoptera, 2: 276. (Bм) \\
\hline tica & EUCOSMA & I9I8, Ann. Transvaal Mus. 6: 49. (т) \\
\hline repanota & LITHOCOLLETIS & I928, Exotic Microlepidoptera, 3: 406. \\
\hline Arimylota & APOTACTIS* & I9I8, Ann. Transvaal Mus. 6: 52 . (T) \\
\hline romica & STENOMA & I925, Exotic Microlepidoptera, 3: I85. (вм) \\
\hline ractis & SPHENASPIS* & $\begin{array}{l}\text { I934, in Caradja and Meyrick, Deuts. Ent. Zeit. Iris, } 48 \text { : } \\
38 \text {. }\end{array}$ \\
\hline oserantha & SPATALISTIS & I930, Exotic Microlepidoptera, 3: 6II. (BM) \\
\hline rodes & TRACHYDORA & I897, Proc. Linnean Soc. N.S. Wales, 22: 395. \\
\hline roseropa & ADELA & I921, Ann. Transvaal Mus. 8: I37. (т) \\
\hline rosias & EPIPHTHORA & I904, Proc. Linnean Soc. N.S. Wales, 29: 262. \\
\hline ocapna & EULECHRIA & I920, Exotic Microlepidoptera, 2: 373. \\
\hline rosochalca & ORTHENCHES & I905, Trans. Ent. Soc. London, I905: 242. \\
\hline rosochlora & ANTICRATES & I907, Proc. Linnean Soc. N.S. Wales, 32: 84 . \\
\hline ocrypta & ARISTOTELIA & I926, Exotic Microlepidoptera, 3: 273. (Bм) \\
\hline rosocycla & BYTHOCRATES* & I9I9, Exotic Microlepidoptera, 2: 268. (BM) \\
\hline Irosodoxa & CHOREUTIS & I933, Exotic Microlepidoptera, 4: 370. (BM) \\
\hline rosophaea & GLYPHIDOCERA & I929, Trans. Ent. Soc. London, 76: 5II. (BM) \\
\hline rosophaes & PHRYGANOSTOLA* & I88I, Proc. Linnean Soc. N.S. Wales, $5: 249$. \\
\hline ophanes & STAGMATOPHORA & I92I, Exotic Microlepidoptera, 2: 4I2. \\
\hline optila & CATALECTIS & I924, Exotic Microlepidoptera, 3: 82. (Bм) \\
\hline lidica & HILAROGRAPHA & I9o9, Journ. Bombay Nat. Hist. Soc. I9: 428. (Bм) \\
\hline darcha & PERONEA & I9I2, Exotic Microlepidoptera, I: I7. (Bм) \\
\hline adopa & BRACHMIA & I9I8, Ann. Transvaal Mus. 6: 25. (т) \\
\hline
\end{tabular}




$\begin{array}{ll}\text { dryadopis } & \text { GELECHIA } \\ \text { dryadoxena } & \text { DEPRESSARIA } \\ \text { dryaula } & \text { STENOMA } \\ \text { dryina } & \text { CAPUA } \\ \text { dryinarma } & \text { MACHAEROPTERIS } \\ \text { dryinodes } & \text { ACROPOLITIS } \\ \text { dryinodes } & \text { EULECHRIA } \\ \text { dryinopa } & \text { CLERARCHA } \\ \text { dryinota } & \text { PHILOBOTA } \\ \text { dryites } & \text { TRICHOPTILUS } \\ \text { dryobathra } & \text { GELECHIA } \\ \text { dryocarpa } & \text { EUCOSMA } \\ \text { dryocausta } & \text { TORTRIX }\end{array}$

dryochra EUCOSMA

dryochyta TORTRIX

dryocoma

dryoconis

dryocosma

dryocrates

dryocremna

dryocrossa

dryocrypta

dryodesma

dryodora

dryoglypta

dryogramma

dryograpta

dryomorpha

dryonephela

dryonota

dryopa

dryopelta

dryophaea

dryophracta

dryophthalma

dryoptycha

dryoscia

dryosphaera

dryosyrta

dryotechna

dryoterma

dryotoma

dryotyphla

dryoxantha

dryoxesta

dryoxyla

dryozona
I926, Ann. South African Mus. 23: 330. (SA)

I920, Exotic Microlepidoptera, 2: 3I5. (ВM)

I925, Exotic Microlepidoptera, 3: 216. (вм)

I9I0, Proc. Linnean Soc. N.S. Wales, 35: I92.

I9I6, Exotic Microlepidoptera, I: 6I4.

I9Io, Proc. Linnean Soc. N.S. Wales, 35: I7I.

I 889, Proc. Linnean Soc. N.S. Wales, (Ser. 2) 3: 1565.

I890, Trans. R. Soc. S. Australia, I3: 54.

I9I4, Ann. South African Mus. I0: 248. (SA)

I936, Exotic Microlepidoptera, 4: 625.

I9I7, Trans. Ent. Soc. London, I9I7: 49. (вм)

I925, Exotic Microlepidoptera, 3: I42. (BM)

1938, Institut des Parcs Nationaux du Congo Belge,

fasc. I4, p. 6, pl. I, fig. 5 .

I937, Exotic Microlepidoptera, 5: 99. (BM)

I937, in Caradja and Meyrick, Deuts. Ent. Zeit. Iris, 5I:

I74.

I9I6, Exotic Microlepidoptera, 2:2I. (BM)

I930, Exotic Microlepidoptera, 4: 3I. (BM)

I9I8, Exotic Microlepidoptera, 2: 206. (BM)

I921, Ann. Transvaal Mus. 8: Ioo. (T)

I932, Exotic Microlepidoptera, 4:343. (Us)

I922, Trans. Ent. Soc. London, I922: 98. (BM)

I93I, Exotic Microlepidoptera, 4: I2I. (Us)

I9I6, Exotic Microlepidoptera, I: 552. (BM)

I92I, Ann. Transvaal Mus. 8: III. (T)

I9I3, Trans. Ent. Soc. London, I9I3: I72. (BM)

I930, Exotic Microlepidoptera, 3: 567 .

I930, Ann. Naturhist. Mus. Wien, 44: 265, pl. I, fig. 34.

(v)

I929, Trans. Ent. Soc. London, 76: 492. (BM)

I932, Exotic Microlepidoptera, 4: 256.

I926, Ann. South African Mus. 23: 328. (SA)

I907, Proc. Linnean Soc. N.S. Wales, 32: 72.

I932, Exotic Microlepidoptera, 4: 225. (BM)

I922, Exotic Microlepidoptera, 2: 585.

I9I7, Exotic Microlepidoptera, 2: 90.

I933, Exotic Microlepidoptera, 4:367. (ВM)

I922, Zool. Meded. 7: 8I. (L)

I932, Exotic Microlepidoptera, 4: 30I. (v)

I926, Exotic Microlepidoptera, 3: 23I. (BM)

I93I, Exotic Microlepidoptera, 4: 58. (BM)

I9I5, Exotic Microlepidoptera, I: 42I. (BM)

I920, Exotic Microlepidoptera, 2: 372.

I9I3, Ann. Transvaal Mus. 3: 320. (T)

I937, Exotic Microlepidoptera, 5： I23. (BM)

I922, Eni. Mitteil. II: 45. (D)

I920, Exotic Microlepidoptera, 2: 34I. (BM)

I932, Trans. Ent. Soc. London, 80: I I8.

I9I6, Exotic Microlepidoptera, I: 567. (вм) 


$\begin{array}{ll}\text { dryozona } & \text { SAROPLA } \\ \text { drypsolitha } & \text { METACHANDA } \\ \text { dubitans } & \text { LECITHOCERA } \\ \text { dubitatrix } & \text { TINEA } \\ \text { ducalis } & \text { PRAYS } \\ \text { ductaria } & \text { EULECHRIA } \\ \text { ductifera } & \text { ACROLOPHUS } \\ \text { dulcescens } & \text { NEPHOGENES } \\ \text { dulcicula } & \text { PSALTICA } \\ \text { dulcis } & \text { BUCCULATRIX } \\ \text { dulcivora } & \text { COSMOPTERYX } \\ \text { dumigera } & \text { ARGYROTOXA } \\ \text { duplicata } & \text { CALANTICA } \\ \text { duplicata } & \text { ETHMIA } \\ \text { durata } & \text { AGANOPTILA } \\ \text { dynastis } & \text { ANTAEOTRICHA } \\ \text { dyscheranta } & \text { CYPHOPHANES* } \\ \text { dyscrita } & \text { PENESTOGLOSSA } \\ \text { dystechna } & \text { OCYSTOLA }\end{array}$

eaolotechna ARGYROPLOCE

$\begin{array}{ll}\text { ebenina } & \text { ARGYROPLOCE } \\ \text { ebenocista } & \text { PTILOGENES } \\ \text { ebria } & \text { STENOMA } \\ \text { eburata } & \text { GELECHIA } \\ \text { eccentra } & \text { CEROMITIA } \\ \text { eccentropa } & \text { HOMALOXESTIS } \\ \text { eccephala } & \text { AMYDRIA } \\ \text { ecclesiastis } & \text { CRYPTOPHASA } \\ \text { echidnias } & \text { [THYSIARCHA } \\ \text { echidnias } & \text { PHILOBOTA } \\ \text { echinastra } & \text { TINEA } \\ \text { echinitis } & \text { TORTRIX } \\ \text { echinodes } & \text { PHYLLOCNISTIS } \\ \text { echinura } & \text { ACROLOPHUS } \\ \text { echioglossa } & \text { LABDIA } \\ \text { echo } & \text { GELECHIA } \\ \text { echodes } & \text { OPOGONA } \\ \text { echyropis } & \text { COLEOPHORA } \\ \text { ecliptica } & \text { COESYRA } \\ \text { ecliptica } & \text { MACROSACES } \\ \text { ecliptica } & \text { TINGENTERA } \\ \text { eclipticodes } & \text { ACROCLITA } \\ & \end{array}$

I9I3, Ann. Transvaal Mus. 3: 3I5. (T) I930, Trans. Ent. Soc. London, 78: 3I5. (P) I926, Sarawak Mus. Journ. 3: I57. I932, Exotic Microlepidoptera, 4: 327. (v) I9I4, Journ. Bombay Nat. Hist. Soc. 23: I25. (BM) I9I3, Exotic Microlepidoptera, I: I59. (MA) I927, Exotic Microlepidoptera, 3: 329. (BM) I9I3, Exotic Microlepidoptera, I: I3o. (MA) I9I4, Exotic Microlepidoptera, I: 262. (BM) I9I3, Ann. Transvaal Mus. 3: 33o. (т) IgI9, Exotic Microlepidoptera, 2: 233. (BM) Ig2I, Zool. Meded. 6: I5o. (L) I9I3, Exotic Microlepidoptera, I: I47.

I9I4, Journ. Bombay Nat. Hist. Soc. 23: гзо. (BM) I922, Exotic Microlepidoptera, 2: 576 .

I9I5, Exotic Microlepidoptera, I: 398. (BM) I937, Exotic Microlepidoptera, 5: I60. (Вм) I926, Ann. South African Mus. 23: 348. (SA) I889, Proc. Linnean Soc. N.S. Wales, (Ser. 2) 3: I664.

I935, in Caradja and Meyrick, Materialien zu einer Microlepidopteren Fauna der Chinesischen Provinzen Kiangsu, Chekiang und Hunan, p. 6o. (BM) I9I6, Exotic Microlepidoptera, 2: 20. (BM) I928, Exotic Microlepidoptera, 3: 433. (BM) I9I5, Exotic Microlepidoptera, I: 44I. (BM) I9I7, Trans. Ent. Soc. London, I9I7: 50. (BM) I92I, Exotic Microlepidoptera, 2: 405. (BM) I934, in Caradja and Meyrick, Deuts. Ent. Zeit. Iris, 48: 36. I9I4, Bull. Mus. Paris, 20: I22. (P)

I887, Proc. Linnean Soc. N.S. Wales, (Ser. 2) I: Io4o. I89x, Ent. Mon. Mag. 27: 6r.

I889, Proc. Linnean Soc. N.S. Wales, (Ser. 2) 3: I624. I93I, Exotic Microlepidoptera, 4: 94.

I9Io, Proc. Linnean Soc. N.S. Wales, 35: 249.

I926, Exotic Microlepidoptera, 3:263.

I9I 5, Trans. Ent. Soc. London, I9I5: 254. (BM)

I928, Exotic Microlepidoptera, 3: 385 . (BM)

I937, Exotic Microlepidoptera, 5: I22.

I936, Exotic Microlepidoptera, 4: 620 .

I930, Exotic Microlepidoptera, 3: 562 .

I884, Proc. Linnean Soc. N.S. Wales, 9: 775.

I9Io, Journ. Bombay Nat. Hist. Soc. 20: I49. (вм) Igo8, Journ. Bombay Nat. Hist. Soc. I8: 45I. (BM) I935, in Caradja and Meyrick, Materialien zu einer Microlepidopteren Fauna der Chinesischen Provinzen Kiangsu, Chekiang und Hunan, p. 52. (BM) 
eclipticopa

ecliptis

ecstatica

ecstaticus

edax

edocta

educta

effera

effervescens

effeta

efficax

efflorescens

efflua

effluxa

effossa

effrenata

effulgens

effulgens

effusa

egelida

egena

egens

ejaculata

ejiciens

elachistarcha

elaeanthes

elaearcha

elaeas

elaeocoma

elaeodes

elaeopa

elaeopetra

elaeophanes

elaeopis

elaeorrhoa

elaeostola

elaeota

elaeotropha

elaeoxyla

elaeurga

elaitis

elaphia

elaphodes

elaphopa

elaphopis

elaphrodes

elapsa

electracma
LACTURA

XENOPHANTA*

SYNTOMACTIS

PTEROPHORUS

THYESTARCHA *

BUCCULATRIX

NARYCIA

LECITHOCERA

MELASINA

HYPONOMEUTA

EUCOSMA

COPROMORPHA

COLEOPHORA

LYCHNOCRATES

STATHMOPODA

PHILOPTILA*

CAPUA

LIMNOECIA

CRYPSITHYRIS

NEPHOGENES

CYCLOTORNA

BLASTOBASIS

LABDIA

SCYTHRIS

NEPTICULA

ARGYROPLOCE

ARGYROTOXA

GRACILARIA

ANACAMPSIS

HELIOCAUSTA

ALUCITA

AMORBIA

BRACHMIA

ENCRASIMA

ERECHTHIAS

PTILOGENES

EULECHRIA

TRACHYCENTRA

LASIARACHIS*

STENOMA

OPOGONA

MACRONEMATA*

MACHIMIA

ACROCERCOPS

ONEBALA

[COPHOMANTIS*]

ACROLEPIA

PERONEA

ASTEROSTOMA*
I928, Exotic Microlepidoptera, 3: 4I5.

I9I4, Ann. Transvaal Mus. 4: I94. (T)

I897, Proc. Linnean Soc. N.S. Wales, 22: 386.

I932, Exotic Microlepidoptera, 4: 335.

I9I2, Ann. South African Mus. IO: 65. (SA)

I921, Ann. Transvaal Mus. 8: I24. (T)

I920, Exotic Microlepidoptera, $2: 356$.

I9I8, Exotic Microlepidoptera, 2: I04. (вм)

I9II, Ann. Transvaal Mus. 3: 8I.

I924, Exotic Microlepidoptera, 3: II7. (T)

I9I2, Ent. Mon. Mag. 48: 35. (Sub.)

I9o6, Journ. Bombay Nat. Hist. Soc. I7: 4I2. (BM)

I9II, Ann. Transvaal Mus. 3: 78 .

I930, Exotic Microlepidoptera, 4: I9. (BM)

I92I, Exotic Microlepidoptera, 2: 460.

I9I8, Exotic Microlepidoptera, 2: III. (BM)

I9Io, Proc. Linnean Soc. N.S. Wales, 35:202. (MA)

I9I8, Ann. Transvaal Mus. 6: 27. (T)

I9I7, Exotic Microlepidoptera, 2: 75.

I883, Proc. Linnean Soc. N.S. Wales, 8: 374.

I9I 2, Trans. Ent. Soc. London, I9II: 590.

I9I8, Ann. Transvaal Mus. 6:37. (T)

I92 I, Exotic Microlepidoptera, 2: 450 .

I928, Exotic Microlepidoptera, 3: 4I2. (BM)

I934, Exotic Microlepidoptera, 4: 467.

I927, Exotic Microlepidoptera, 3: 340. (BM)

I908, Journ. Bombay Nat. Hist. Soc. I8: 623. (Bм)

I9I I, Trans. New Zealand Inst. 43: 66.

I9I8, Ann. Transvaal Mus. 6: I9. (T)

I883, Proc. Linnean Soc. N.S. Wales, 7: 474.

I908, Trans. Ent. Soc. London, I907: 490.

I932, Exotic Microlepidoptera, 4:263. (v)

I931, in Caradja, Bull. Sect. sci. Acad. roum. I4: 70.

(BM)

I9I6, Exotic Microlepidoptera, I: 594. (Bм)

I88I, Proc. Linnean Soc. N.S. Wales, 5:267.

I930, Ann. Naturhist. Mus. Wien, 44:257. (v)

I888, Proc. Linnean Soc. N.S. Wales, (Ser. 2) 2: 957.

1933, Exotic Microlepidoptera, 4: 4I3.

1937, Exotic Microlepidoptera, 5: 92. (T)

I926, Exotic Microlepidoptera, 3: 226. (BM)

I9I I, Journ. Bombay Nat. Hist. Soc. 2 I: II 2.

I883, Proc. Linnean Soc. N.S. Wales, 8: 346.

1930, Exotic Microlepidoptera, 3: 6I8. (BM)

I9I4, Journ. Bombay Nat. Hist. Soc. 23: I2I.

I9Io, Journ. Bombay Nat. Hist. Soc. 20: 459. (BM)

I9I9, Exotic Microlepidoptera, 2: 226. (BM)

I922, The Entomologist, 55: 255. (Sub.)

I935, Exotic Microlepidoptera, 4: 607. 


electrantha
electrias
electrica
electrica
electrina
electritis
electrodes
electropa
electrophanes
electropis
elementaris
elephantias
elephantina
elephantodes

elephantopa

elephantopis

elevata
elimata
elissa

ellipsias

elliptica

ellipticopa

ellochistis

eloquens

elpistis

eludens

elytrata

emancipata

emblematica

embolaea

embolarcha

embolopis

embrithopa

embrocha

embrochota

embythia

emensa

ementita

emerita

emicans

emigrans

eminens

eminula

emitescens

emmetra

emmolybda
STATHMOPODA ARGYROPLOCE HELIOSTIBES PLACOPTILA* MEGALODORIS* HOPLOPHANES PHILOBOTA CEROSTOMA PARALECTA ANTICRATES HYPONOMEUTA EPICHOSTIS* PROSELENA PARATORNA

BRACHMIA GELECHIA

LASPEYRESIA SPARGANOTHIS PHILOMUSAEA

DICHOMERIS

TRICLONELLA

PAMMENE

EPERMENIA SCYTHRIS PROTOLECHIA LECITHOCERA MAGORRHABDA* ADULLAMITIS* EPIMIMASTIS HEMIMENE THIOTRICHA MICROCOLONA PHALONIA ANACAMPSIS PYRODERCES STENOMA ODITES CYCLOTORNA EPITHECTIS LOCHARCHA * LECITHOCERA STENOMA STENOMA CACOECIA

AUTOSTICHA COSMOPTERYX
I927, Exotic Microlepidoptera, 3: 377. (BM) I9I2, Trans. Ent. Soc. London, I9II: 69I. (BM) I889, Trans. Proc. New Zealand Inst. 21 : I57. I894, Trans. Ent. Soc. London, I894: 23. I9I2, Exotic Microlepidoptera, I: 5. (BM) I897, Proc. Linnean Soc. N.S. Wales, 22: 4 I3. I884, Proc. Linnean Soc. N.S. Wales, 8: 509. I9I4, Exotic Microlepidoptera, I: 228. (BM) I930, Exotic Microlepidoptera, 4: I2. I92I, Ann. Transvaal Mus. 8: II9. (T) I93I, Exotic Microlepidoptera, 4: I72. (BM) I906, Journ. Bombay Nat. Hist. Soc. I7: 405. (Bм) I885, Trans. Proc. New Zealand Inst. I7: I43. I938, in Caradja and Meyrick, Deuts. Ent. Zeit. Iris, 52: 84. (BM) I9IO, Rec. Indian Mus. 2: 222. (K) I936, Veröff. Deutschen Kol.-Mus. I: 332, pl. I3, fig. 27. (H) I9I6, Exotic Microlepidoptera, 2: 24. (BM) I930, Exotic Microlepidoptera, 3: 6II. (BM) I931, Ann. Mus. Nac. Hist. Nat. Buenos Aires, 36: 395. (BM)

I922, Trans. Ent. Soc. London, I922: I I4. (BM) I9I6, Exotic Microlepidoptera, I: 543. (BM) I934, Exotic Microlepidoptera, 4: 486. (BM) I9I7, Exotic Microlepidoptera, 2: 67. I92I, Ann. Transvaal Mus. 8: II5. (T) I904, Proc. Linnean Soc. N.S. Wales, 29: 349. I9I8, Exotic Microlepidoptera, 2: 108. (BM) I932, Exotic Microlepidoptera, 4: 273. I932, Exotic Microlepidoptera, 4: I98. I9I6, Exotic Microlepidoptera, I: 589. (BM) I9I8, Ann. Transvaal Mus. 6: 5I. (T) I929, Exotic Microlepidoptera, 3: 500. (BM) I897, Proc. Limnean Soc. N.S. Wales, 22: 372. I927, Exotic Microlepidoptera, 3:366. (BM) I9I4, Ann. Transvaal Mus. 4: Ig2. (T) I9I4, Exotic Microlepidoptera, I: 280. I9I6, Exotic Microlepidoptera, I: 529. (BM) I92I, Ann. Transvaal Mus. 8: Io6. (T) I92 I, Exotic Microlepidoptera, 2: 463. I92I, Ann. Transvaal Mus. 8: 67. (T) I923, Exotic Microlepidoptera, 3: I8. (BM) I92I, Exotic Microlepidoptera, 2: 435. (BM) I9I8, Exotic Microlepidoptera, 2: 206. (BM) I9I5, Exotic Microlepidoptera, I: 475. (BM) I931, in Joannis, Ann. Soc. Ent. France, 98 (Supp.): 7 I 2 [474].

I92I, Ann. Transvaal Mus. 8: 93. (т) I9I4, Exotic Microlepidoptera, I: 280. 
emollita empalacta empedarhtra empedocles empedota empedota empetra emphanes emphanes emphanista emphanopa emphatica emphytopa emplasta emplasta empolaea emporica emptycta empyrea empyrota encaeria encalypta encamina encarpa encarsia encausta encausta encaustica encentra encentris encharacta encharacta enchidias enchitis

enchorda enchrista enchytopa enclista enclitica enclitica encomias encotodes encotopa encratodes encratopis encryphias

encrypta encyclia
ANTAEOTRICHA CRYPTOLECHIA LACTURA ACROLOPHUS HYPOSMOCOMA PLATYPTILIA APHTHONETUS PROSELENA STENOMA TELPHUSA LABDIA STENOMA ACROLOPHUS COMMATICA STREPSICRATES LOCHEUTIS MICROCOLONA PANDEMIS MACHIMIA STENOMA LITHOCOLLETIS PSEUDODOXIA MACHIMIA ARGYROPLOCE ODITES

TINEA TORTRIX EUCOSMA DEPRESSARIA ACROCERCOPS ARGYROPLOCE OPOGONA PILOSTIBES* COLEOPHORA

COLEOPHORA TINEA TINEA LIMNOECIA GLYPHIPTERYX PHALONIA SYMPHOROSTOLA* ISOCHORISTA MACHAEROPTERIS EULECHRIA BATODES AGRIOPHARA [NEOSPASTIS*] EUMENODORA* ANTAEOTRICHA
1926, Exotic Microlepidoptera, 3: 234.

(BM) I9I5, Exotic Microlepidoptera, I: 305. I924, Exotic Microlepidoptera, 3: I24. I930, Exotic Microlepidoptera, 3: 552. I9I5, Exotic Microlepidoptera, I: 34I. (BM) I908, Trans. Ent. Soc. London, I907: 487. I9I5, Exotic Microlepidoptera, I: 339. (BM) I902, Trans. Ent. Soc. London, I902: 57 I. I9I7, Exotic Microlepidoptera, 2: 58. (BM) I92 I, Ann. Transvaal Mus. 8: 70. (T) I922, Exotic Microlepidoptera, 2: 570 . I9I6, Exotic Microlepidoptera, I: 539. (BM) I932, Exotic Microlepidoptera, 4: 2I3. I9I4, Trans. Ent. Soc. London, I9I4: 240. (BM) I9oI, Trans. Ent. Soc. London, p. 57I. I9I4, Exotic Microlepidoptera, I: 236. (BM) I9I7, Exotic Microlepidoptera, 2: 50. I937, Exotic Microlepidoptera, 5: I56. (BM) I920, Exotic Microlepidoptera, 2: 3II. (BM) I9I5, Exotic Microlepidoptera, I : 46I. I9II, Ann. Transvaal Mus. 2: 234. (T) I9I4, Exotic Microlepidoptera, I: 264. (BM) I9I2, Trans. Ent. Soc. London, I9II: 696. (Bм) I920, Exotic Microlepidoptera, 2: 349. (вм) I9o8, Journ. Bombay Nat. Hist. Soc. I8: 634. (вм) I9I2, Ann. Transvaal Mus. 3:8I. (T) I907, Journ. Bombay Nat. Hist. Soc. I7: 735. I922, Exotic Microlepidoptera, 2: 5I8. (BM) I9I4, Exotic Microlepidoptera, I: 252. (BM) I9I5, Trans. Ent. Soc. London, I9I5: 227. I9I8, Ann. Transvaal Mus. 6: 5o. (T) I9I5, Exotic Microlepidoptera, I: 359. I89o, Trans. R. Soc. S. Australia, I3: 27. I920, Voyage de Ch. Alluaud et R. Jeannel en Afrique Oriental, II, Microlepidoptera, p. 9I. (P)

I93I, Exotic Microlepidoptera, 4: I80. I9I7, Exotic Microlepidoptera, 2: 78. I931, Ann. Mus. Nac. Hist. Nat. Buenos Aires, 36: 4I2. I92I, Zool. Meded. 6: I68. (L) I9o9, Journ. Bombay Nat. Hist. Soc. I9: 430. (Bм) I9I7, Trans. Ent. Soc. London, I9I7: 3. (BM) I927, Exotic Microlepidoptera, 3: 376. (BM) I9Io, Proc. Linnean Soc. N.S. Wales, 35: I66. I922, Exotic Microlepidoptera, 2: 600. I922, Exotic Microlepidoptera, 2: 5 Io. I920, Exotic Microlepidoptera, 2: 322. (BM)

I9o7, Journ. Bombay Nat. Hist. Soc. I7: 743. (Bu) I906, Trans. R. Soc. S. Australia, 30: 55 . I9I5, Exotic Microlepidoptera, I: 403. (BM) 


encycota
endesma
endobela
endocapna
endocarpa
endocentra
endochlora
endochra
endochrysa
endoclina
endocoma
endocrossa
endocypha
endogramma
endoleuca
endoneurias
endopercna
endophaea
endophaga
endopyrrha
endotherma
endoxa
endrosias
endryas
endryopa
energa
enervata
engalactis
englypta
englyptopa

$\begin{array}{ll}\text { engrapta } & \text { BRACHMIA } \\ \text { engraptes } & \text { NEMOTOIS } \\ \text { enitescens } & \text { PERONEA } \\ \text { enixa } & \text { CRYPSITHYRIS } \\ \text { enneacentra } & \text { BORKHAUSENIA } \\ \text { enneametra } & \text { ANCYLIS } \\ \text { enneora } & \text { COMOCRITIS } \\ \text { ennephela } & \text { NEPHOGENES } \\ \text { ennomopis } & \text { NARYCIA } \\ \text { ennychodes } & \text { ACROCERCOPS } \\ \text { enodata } & \text { ANTAEOTRICHA } \\ \text { enochlodes } & \text { TORTRIX } \\ \text { enopias } & \text { AMBLYXENA* } \\ \text { enoplana } & \text { CACOECIA } \\ \text { enoplia } & \text { OCYSTOLA } \\ \text { enoptrias } & \text { STROBISIA } \\ \text { enormis } & \text { BHYPERECTA }{ }^{*} \text { BATRACHEDRA }\end{array}$

I922, Exotic Microlepidoptera, 2: 506. (P)

I884, Proc. Limean Soc. N.S. Wales, 9: 755.

1926, Exotic Microlepidoptera, 3: 296.

I9o6, Trans. R. Soc. S. Australia, 30: 60.

I9I9, Exotic Microlepidoptera, 2: 234. (BM)

I9I4, Ann. South African Mus. Io: 247. (SA)

I883, Proc. Limnean Soc. N.S. Wales, 8: 329.

I925, Exotic Microlepidoptera, 3: I98. (BM)

I935, Exotic Microlepidoptera, 4: 579 .

I928, Exotic Microlepidoptera, 3: 426.

I9Io, Journ. Bombay Nat. Hist. Soc, 20: 44I. (BM)

I921, Zool. Meded. 6: I8o. (L)

I93I, Exotic Microlepidoptera, 4: I48. (BM)

1922, Exotic Microlepidoptera, 2: 549. (BM)

I888, Proc. Linnean Soc. N.S. Wales, (Ser. 2) 2: 938.

I925, Exotic Microlepidoptera, 3: I34. (BM)

I936, Exotic Microlepidoptera, 5: 47.

I930, Exotic Microlepidoptera, 3: 567.

I9II, Proc. Linnean Soc. N.S. Wales, 36: 278.

I930, Exotic Microlepidoptera, 3: 609. (BM)

I93I, Rec. Cant. Mus. 3: 368.

I926, Exotic Microlepidoptera, 3: 263.

I907, Journ. Bombay Nat. Hist. Soc. I8: I45. (BM)

I9I5, Exotic Microlepidoptera, I: 343. (BM)

I9I8, Exotic Microlepidoptera, 2: 223. (BM)

I905, Journ. Bombay Nat. Hist. Soc. I6: 6 I6.

I929, Exotic Microlepidoptera, 3: 532. (BM)

I932, Exotic Microlepidoptera, 4: 3I3. (v)

I904, Proc. Limnean Soc. N.S. Wales, 29: 357.

I938, in Caradja and Meyrick, Deuts. Ent. Zeit. Iris, 52:

85. (BM)

I9I8, Exotic Microlepidoptera, 2: II4. (BM)

I907, Joum. Bombay Nat. Hist. Soc. I7: 993.

I9I2, Exotic Microlepidoptera, I: I6. (BM)

I921, Zool. Meded. 6: I97. (L)

I925, Treubia, 6: 43I.

I927, Insects of Samoa, 3, Lepidoptera, fasc. 2, p. 72.

I9I4, Journ. Bombay Nat. Hist. Soc. 23: гзо. (BM)

I883, Proc. Linnean Soc. N.S. Wales, 8: 374.

I934, Exotic Microlepidoptera, 4:482.

I92I, Exotic Microlepidoptera, 2: 467 .

I9I6, Exotic Microlepidoptera, I : 493. (BM)

I938, Institut des Parcs Nationaux du Congo Belge,

fasc. I4, p. 7, pl. I, fig. 6. (c)

I9I4, Exotic Microlepidoptera, I: 207.

I883, Trans. Proc. New Zealand Inst. I5: 49.

I885, Proc. Linnean Soc. N.S. Wales, 9: 1069.

I9II, Joum. Bombay Nat. Hist. Soc. 20: 728. (BM) I928, Exotic Microlepidoptera, 3: 394. (BM) 
ensifera

ensigera

enstacta

entaphrota

entechna

entephras

entherastis

entherma

enthetica

enthrypta

entomaula

entripta

entryphopa

entypa

enumerata

eoa

eochrodes

eocnephaea

eocrossa

eodora

eodryas

eolampis

eoleuca

eometalla

eonephella

eophaea

eophanes

eophthalma

eoplecta

eothina

eotrocha

eoxantha

epachyrota

epactaea

epactaea

epadelpha

epanthista

epaphria

eparches

epastra

epenthetica

epethistis

ephalta

ephaptis

ephedra

ephelota

ephelotis
HIEROXESTIS

[PROBLASTODES*] I9II, Trans. Linnean Soc. London, I4: 295.

CRYPTOPHASA

CYATHAULA

MACHIMIA

SIMAETHIS

STENOMA

BARYMOCHTHA *

TORTRIX

CRYPSICHARIS

BEDELLIA

ANEMERARCHA *

MESOPHERNA

COLEOSTOMA*

MICROLOCHA *

STENOMA

CRYPTOLECHIA

TRICHOPTILUS

ACROCLITA

EULECHRIA

SABATINCA

ACOMPSIA

GRACILARIA

ENARMONIA

DORODOCA

PALPARIA

METACHANDA

LITHOCOLLETIS

AEOLARCHIA*

POLYCHROSIS

MACHIMIA

DIOCOSMA

GYMNOGRAMMA

CRYPSITHYRIS

GELECHIA

OPOSTEGA

CRYPTOPHAGA

LASPEYRESIA

SYNCOLA*

PTEROPHORUS

GLYPHIPTERYX

SYMMOCA

PHLOEOPOLA

EULECHRIA

TISCHERIA

CAPUA

HYPERTRICHA *

ANAPTILORA
1925, Exotic Microlepidoptera, 3: I50.

I928, Exotic Microlepidoptera, 3: 429.

I9I5, Trans. Ent. Soc. London, I9I5: 209. (BM)

I920, Ann. South African Mus. I7: 295. (SA)

I9I5, Exotic Microlepidoptera, I: 453. (BM)

I922, Exotic Microlepidoptera, 2: 593. (BM)

I9I4, Exotic Microlepidoptera, I: I95. (BM)

I922, Arkiv för Zool. vol. I4, part I5, p. IO.

I928, Exotic Microlepidoptera, 3: 397.

I937, Exotic Microlepidoptera, 5: I54.

I9I7, Exotic Microlepidoptera, 2: 88.

I922, Trans. Ent. Soc. London, I922: 99. (BM)

I9I4, Exotic Microlepidoptera, I: 24I.

I932, Exotic Microlepidoptera, 4: 299.

I9Io, Journ. Bombay Nat. Hist. Soc. 20: I65. (BM)

I935, in Caradja and Meyrick, Materialien zu einer Micro-

lepidopteren Fauna der Chinesischen Provinzen Kiangsu,

Chekiang und Hunan, p. 45.

1934, Pacific Ent. Surv. Publ. 7, art. 28, p. 345. (BB)

I888, Proc. Linnean Soc. N.S. Wales, (Ser. 2) 2: 949.

I9I8, Trans. Proc. New Zealand Inst. 50: I34.

I9I8, Ann. Transvaal Mus. 6: 20. (T)

I9I5, Trans. Ent. Soc. London, I9I5: 238.

I9I2, Ent. Mon. Mag. 48: 34. (Sub.)

I926, Ann. South African Mus. 23: 334. (SA)

I883, Proc. Linnean Soc. N.S. Wales, 9: 432.

I930, Trans. Ent. Soc. London, 78: 3I6. (P)

I93I, Exotic Microlepidoptera, 4: 46.

I93I, in Caradja, Bull. Sect. sci. Acad. roum. I4: 2 I2.

I925, Exotic Microlepidoptera, 3: I42. (T)

I920, Exotic Microlepidoptera, 2: 376 . (BM)

I9I8, Ann. Transvaal Mus. 6: 33. (T)

I92I, Ann. Transvaal Mus. 8: II9. (T)

I9I7, Exotic Microlepidoptera, 2: 73.

1904, Proc. Linnean Soc. N.S. Wales, 29: 312.

I907, Journ. Bombay Nat. Hist. Soc. I7: 985.

I89o, Trans. R. Soc. S. Australia, I3: 36.

I922, Exotic Microlepidoptera, 2: 530.

I9I6, Exotic Microlepidoptera, I: 598.

I93I, Exotic Microlepidoptera, 4: I76.

I922, Exotic Microlepidoptera, 2: 490. (BM)

I93I, Exotic Microlepidoptera, 4: 72. (BM)

I902, Trans. R. Soc. S. Australia, 26: I54.

I9I5, Exotic Microlepidoptera, I: 299.

I9I5, Trans. Ent. Soc. London, I9I5: 246.

I9Io, Proc. Linnean Soc. N.S. Wales, 35: r93.

I890, Trans. R. Soc. S. Australia, I3: 74.

I9I6, Exotic Microlepidoptera, I: 59I. 
ephestris

ephippias

ephippias

ephippiastis

ephodophora

ephoria

ephorista

ephoropa

epibathra

epibryas

epibyrsa

epicapna

epicausta

epicentra

epicentra

epichalca

epichares

epichersa

epichlaena

epichlora

epichlora

epichorda

epichorda

epichrysa

epichthonia
TINGENTERA

ANARSIA

CHROSIS

CHELARIA

HIEROMANTIS*

ARISTOTELIA

THOMICTIS*

EUCOSMA

BUCCULATRIX

PYRAMIDOBELA

NARYCIA

SYNOMOTIS*

EULECHRIA

GELECHIA

STROBISIA

CREMNOGENES

LITHOCOLLETIS

DEPRESSARIA

IMMA

AUTOMACHAERIS*

STATHMOPODA

TORTRIX

TRICHEMBOLA

STACHYOTIS*

HOMOSHELAS*

MELASINA

ACROCERCOPS

CAPUA

THIOTRICHA

SPHALERACTIS

EUTORNA

STENOMA

ELACHISTA

[PROTERoChyta*] I9I4, Ann. Transvaal Mus. 4: Ig6. (T)

IMMA

CASMARA

MICROBELA

NEPTICULA

EULIA

STENOMA

CHOLOTIS

HARMOLOGA

CACOECIA

OXYPTILUS

PLATYPTILIA

HELIOCAUSTA

HERMENIAS*

ASTHENOPTYCHA
Igo8, Journ. Bombay Nat. Hist. Soc. I8: 454. (BM)

I908, Ent. Mon. Mag. 44: I97. (BM)

I907, Journ. Bombay Nat. Hist. Soc. I7: 73I.

1937, Exotic Microlepidoptera, 5: 95. (BM)

I897, Proc. Linnean Soc. N.S. Wales, 22: 315.

I9I7, Trans. Ent. Soc. London, I9I7: 36. (BM)

I920, Exotic Microlepidoptera, 2: 289 .

I93I, Exotic Microlepidoptera, 4: I46. (BM)

I934, Exotic Microlepidoptera, 4:462.

I93I, Exotic Microlepidoptera, 4: 88. (v)

I922, Exotic Microlepidoptera, 2: 604. (P)

I883, Ent. Mon. Mag. 20:33.

I883, Proc. Linnean Soc. N.S. Wales, 7: 525.

I909, Ann. South African Mus. 5: 370. (SA)

I9I I, Journ. Bombay Nat. Hist. Soc. 20: 730. (BM)

I886, Proc. Linnean Soc. N.S. Wales, Io: 792.

I928, Exotic Microlepidoptera, 3: 406.

I9I4, Exotic Microlepidoptera, I: 253. (BM)

I906, Trans. Ent. Soc. London, I906: I92. (BM)

I907, Journ. Bombay Nat. Hist. Soc. I7: 74.

I889, Trans. Proc. New Zealand Inst. 21: I69.

I9Io, Proc. Linnean Soc. N.S. Wales, 35: 249.

I9I8, Exotic Microlepidoptera, 2: II6. (BM)

I905, Journ. Bombay Nat. Hist. Soc. I6: 6I 2.

I935, in Caradja and Meyrick, Materialien zu einer Micro-

lepidopteren Fauna der Chinesischen Provinzen Kiangsu,

Chekiang und Hunan, p. 7I. (BM)

I907, Journ. Bombay Nat. Hist. Soc. I8: 156.

I9I8, Exotic Microlepidoptera, 2: I76.

I928, Exotic Microlepidoptera, 3: 452. (Bм)

I 908 , Journ. Bombay Nat. Hist. Soc. I8: 440. (BM)

I920, Exotic Microlepidoptera, 2: 299.

I9o6, Trans. $R$. Soc. S. A ustralia, 30: 46.

I9I5, Exotic Microlepidoptera, I: 46I. (BM)

I906, Trans. Ent. Soc. London, I906: I95. (BM)

I922, Exotic Microlepidoptera, 2: 544. (BM)

I885, Proc. Linnean Soc. N.S. Wales, 9: I046.

I9I5, Trans. Ent. Soc. London, I9I5: 255.

I926, Exotic Microlepidoptera, 3: 25I. (BM)

I932, Exotic Microlepidoptera, 4: 294. (v)

I9I5, Exotic Microlepidoptera, I: 327.

I9I I, Trans. New Zealand Inst. 43: 86.

I905, Journ. Bombay Nat. Hist. Soc. I6: 589. (BM)

I9o8, Trans. Ent. Soc. London, I907: 476.

I9o8, Trans. Ent. Soc. London, I907: 486.

I886, Proc. Linnean Soc. N.S. Wales, Io: 832 .

I9II, Proc. Linnean Soc. N.S. Wales, 36: 225.

I9I0, Proc. Linnean Soc. N.S. Wales, 35: I78. 
epignampta epigompha epigramma epigypsa epilampra epiloxa epimetalla epimicta epimictis epimochla epimylia epimyxa epinyctia epiochra epipacta epiphanes epiphanopa epiphanta epiphaula epiphloea epiphracta epiphragma epiphricta epiphrixa epipona epirota epirrhicna

episacta epischista episcopopa episcota episema episimbla epismaragda episotras epispila epispora epistacta episticta epistolaris epistolica epistrota epitoxa epitricha epitrocha epiula epixantha epixesta epixutha
ANTAEOTRICHA LECITHOCERA CRYPTOPHAGA GELECHIA STATHMOPODA ETHMIA ARISTOTELIA OECOPHORA LIMNOECIA TINEA OECOPHORA BATRACHEDRA ARCTOSCELIS* BRACHYACMA* STENOMA SEMIOCOSMA COLEOPHORA PROMOLOPICA* TRACHYNTIS GELECHIA SCHEDIASTIS * EULECHRIA ORTHENCHES SYNTOMACTIS TRACHYNTIS SPORADARTHRA EPERMENIA

MERIDARCHIS THERMOCRATES* AMPHITHALES* OCYSTOLA EULECHRIA APHANOXENA COSMOPTERYX BRENTHIA LITHOCOLLETIS EPERMENIA PROMALACTIS TORODORA OPOSTEGA GELECHIA BUTALIS PELTOPHORA PHTHORIMAEA CERATOPHYSETIS ANARSIA BATRACHEDRA LINOSTICHA MICROCOLONA
I9I5, Exotic Microlepidoptera, I: 395. (BM) I9Io, Journ. Bombay Nat. Hist. Soc. 20: 448. (BM) I890, Trans. R. Soc. S. Australia, I3: 3I. I927, Exotic Microlepidoptera, 3: 35I. (BM) I9II, Trans. Linnean Soc. London, I4: 286. I9I4, Bull. Mus. Nat. Hist. Nat. Paris, 20: I21. 1904, Proc. Linnean Soc. N.S. Wales, 29: 290. I886, Proc. Linnean Soc. N.S. Wales, Io: 786. 1897, Proc. Linnean Soc. N.S. Wales, 22: 36r. I893, Proc. Linnean Soc. N.S. Wales, 7: 543. I883, New Zealand Journ. Sci. I : 524 . 19I6, Exotic Microlepidoptera, 2: 32. I894, Trans. Ent. Soc. London, I894: 23. (BM) I886, Trans. Ent. Soc. London, I886: 279. I9I5, Exotic Microlepidoptera, I: 444. (BM) I883, New Zealand Journ. Sci. I: 523 . I937, Exotic Microlepidoptera, 5: I30. (c) I926, Exotic Microlepidoptera, 3: 284. (BM) I889, Proc. Linnean Soc. N.S. Wales, (Ser. 2) 3: I588. I9I3, Ann. Transvaat Mus. 3: 292. (T) I92I, Exotic Microlepidoptera, 2: 476. (BM) I888, Proc. Linnean Soc. N.S. Wales, 2: 954. I907, Proc. Linnean Soc. N.S. Wales, 32: I35. 1897, Proc. Linnean Soc. N.S. Wales, 22: 380. I902, Trans. R. Soc. S. Australia, 26: I54. I9I4, Exotic Microlepidoptera, I: 2 I0.

I938, Institut des Parcs Nationaux $d u$ Congo Belge, fasc. I4, p. 23. (c) I9o6, Journ. Bombay Nat. Hist. Soc. I7: I37. (BM) I936, Exotic Microlepidoptera, 4:62I. I926, Exotic Microlepidoptera, 3: 268. I889, Proc. Linnean Soc. N.S. Wales, (Ser. 2) 3: I664. I883, Proc. Linnean Soc. N.S. Wales, 7: 5I7. I9I5, Exotic Microlepidoptera, I: 389. (BM) I932, Trans. Ent. Soc. London, 80: II3. I920, Exotic Microlepidoptera, 2: 335. (BM) I9I5, Trans. Ent. Soc. London, I9I5: 223. I897, Proc. Linnean Soc. N.S. Wales, 22: 430. I908, Journ. Bombay Nat. Hist. Soc. I 8: 81о. (Bм) I905, Journ. Bombay Nat. Hist. Soc. I6: 599. (BM) I9II, Journ. Bombay Nat. Hist. Soc. 2I : Io8. I93I, Exotic Microlepidoptera, 4: 59. (BM) I889, Trans. Proc. New Zealand Inst. 21: I6r. I889, Proc. Linnean Soc. N.S. Wales, (Ser. 2) 3: I637, I9I7, Trans. Ent. Soc. London, I9I7: 47. (BM) I9I4, Supplementa Entomologica, No. 3, p. 54. I904, Proc. Linnean Soc. N.S. Wales, 29: 4 I8. I897, Proc. Linnean Soc. N.S. Wales, 22: 309. I889, Proc. Linnean Soc. N.S. Wales, (Ser. 2) 3: I5S2 1897, Proc. Linnean Soc. N.S. Wales, 22: 376. 


$\begin{array}{ll}\text { epixyla } & \text { LYSIPHRAGMA } \\ \text { epizeucta } & \text { ADOXOPHYES } \\ \text { epizona } & \text { COSMOPTERYX } \\ \text { epizona } & \text { PROTEROCOSMA } \\ \text { epochaea } & \text { SYNTOMACTIS } \\ \text { epombra } & \text { BATRACHEDRA } \\ \text { epomia } & \text { TORODORA } \\ \text { epomiana } & \text { HETEROCROSSA } \\ \text { epomias } & \text { NARYCIA } \\ \text { epophrysta } & \text { STENOMA } \\ \text { eporphna } & \text { PERIACMA } \\ \text { epotias } & \text { ANARSIA } \\ \text { epotis } & \text { PLATYPTILIA } \\ \text { epularis } & \text { PHILOBOTA } \\ \text { equestris } & \text { MACROBATHRA } \\ \text { eranista } & \text { ACROCERCOPS } \\ \text { erasa } & \text { BACTRA } \\ \text { erasicosma } & \text { ATELOSTICHA } \\ \text { erasmia } & \text { COSMOPTERYX } \\ \text { erastis } & \text { GLYPHIPTERYX } \\ \text { erastis } & \text { PHILOBOTA (?) } \\ \text { eratantha } & \text { LITHOCOLLETIS } \\ \text { eratopa } & \text { LYONETIA } \\ \text { eratopis } & \text { PROTOSYNAEMA } \\ \text { erebactis } & \text { DRYANASSA* } \\ \text { erebanassa } & \text { GLYPHIPTERYX } \\ & \end{array}$

$\begin{array}{ll}\text { erebantha } & \text { TRITHAMNORA } \\ \text { erebantra } & \text { EUCOSMA }\end{array}$

$\begin{array}{ll}\text { erebastra } & \text { LEUCOPTERA } \\ \text { erebaula } & \text { PYCNODYTIS* } \\ \text { erebenna } & \text { SMENODOCA* } \\ \text { erebinthia } & \text { STAGMATOPHORA } \\ \text { erebistis } & \text { ERECHTHIAS } \\ \text { erebites } & \text { OXYPTILUS } \\ \text { erebitis } & \text { PRAYS } \\ \text { erebocosma } & \text { TINEA } \\ \text { erebocrossa } & \text { EOCHROIS } \\ \text { erebodelta } & \text { COMPSOLECHIA } \\ \text { erebodes } & \text { PHILOBOTA } \\ \text { erebodoxa } & \text { PLATYEDRA } \\ \text { ereboglauca } & \text { PANSEPTA } \\ \text { erebogramma } & \text { NEELYSIA } \\ \text { erebolimnas } & \text { MERIDARCHIS } \\ \text { erebomicta }, & \text { AEOLANTHES } \\ \text { erebopa } & \\ \text { erebophthalma } & \text { ACROCERCOPS } \\ \text { erebopis } & \text { BLACHISTA }\end{array}$

I888, Trans. Proc. New Zealand Inst. 20: I05. I9Io, Proc. Linnean Soc. N.S. Wales, 35: 207. I897, Proc. Linnean Soc. N.S. Wales, 22: 340. I886, Trans. Ent. Soc. London, I886: 293.

I9I7, Exotic Microlepidoptera, 2: 5I. I9I4, Exotic Microlepidoptera, I: 206. I905, Journ. Bombay Nat. Hist. Soc. I6: 599. (BM) I885, Trans. Proc. New Zealand Inst. I7: I49. I92I, Zool. Meded. 6: 20I. (L) I909, Trans. Ent. Soc. London, I909: 33. I923, Exotic Microlepidoptera, 3: 62. (BM) I9I6, Exotic Microlepidoptera, I: 583. (BM) I905, Trans. Ent. Soc. London, I905: 23 I. I9I3, Exotic Microlepidoptera, I: I27. I9Io, Journ. Bombay Nat. Hist. Soc. 20: I43. (Bм) I9I8, Exotic Microlepidoptera, 2: I76. I928, Exotic Microlepidoptera, 3: 442. (вм) I9I6, Exotic Microlepidoptera, I: 548. (BM) I9I5, Trans. Ent. Soc. London, I9I 5: 206. (BM) I9I I, Trans. New Zealand Inst. 43: 76. I9Io, Ann. South African Mus. 5: 4I4. (SA) I922, Exotic Microlepidoptera, 2: 560 . I933, Exotic Microlepidoptera, 4:365. I886, Trans. Proc. New Zealand Inst. I8: I74. I936, Exotic Microlepidoptera, 5: 4I. I934, in Caradja and Meyrick, Deuts. Ent. Zeit. Iris, 48: $4 \mathrm{I}$.

I9I9, Exotic Microlepidoptera, 2: 260.

I937, in Caradja and Meyrick, Deuts. Ent. Zeit. Iris, 5I: I $8 \mathrm{I}$.

I934, Exotic Microlepidoptera, 4:460. I9I8, Ann. Transvaal Mus. 6: I5. (T) I904, Proc. Linnean Soc. N.S. Wales, 29: 303. I92I, Exotic Microlepidoptera, 2: 4I3. (BM) I892, Trans. Proc. New Zealand Inst. 24: 220. 1937, Exotic Microlepidoptera, 5: II8. (c) I9I4, Journ. Bombay Nat. Hist. Soc. 23: I24. (BM) I893, Proc. Linnean Soc. N.S. Wales, 7: 537. I93o, Exotic Microlepidoptera, 3: 574. (BM) I922, Trans. Ent. Soc. London, I922: 96. (BM) I884, Proc. Linnean Soc. N.S. Wales, 8: 487. I927, Exotic Microlepidoptera, 3: 35I. (BM) I926, Sarawak Mus. Journ. 3: I6o. I935, Proc. Hawaiian Ent. Soc. 9: 64. (SP) I938, Trans. R. Ent. Soc. London, 87: 520. I93I, in Caradja, Bull. Sect. sci. Acad. roum. I4: 73. (BM)

I936, Exotic Microlepidoptera, 5: 35 .

I897, Proc. Linnean Soc. N.S. Wales, 22: 337. I934, Exotic Microlepidoptera, 4: 509. 


\begin{abstract}
erebopleura erebornis
\end{abstract}

erebospila

erecta

eremaea

eremaea

eremana

eremarcha

eremasta

eremaula

eremias

eremitis

eremna

eremocentra

eremochtha

eremodelta

eremodes

eremodora

eremopa

eremopis

eremospora

eremota

erethismia

erethista

erethistis

erethopa

eretmota

ergasia

ergasima

ergasima

ergastica

ergastis

ergastularis

ergatica

ergatis

ergodes

ergophora

eriacma

eribapta

eribola

ericnista

ericta

ericydes

eridantis

eridarcha
LABDIA

AGRIOPLECTA*

SCYTHRIS

LECITHOCERA

OECOPHORA

PARAMERISTIS*

PROSELENA

CEROMITIA

ISOCRITA

GELECHIA

ANAPTILORA

TRIBONICA *

COMMATICA*

ISCHNANGELA*

BATRACHEDRA

ARGYROPLOCE

COLEOPHORA

EUCOSMA

PTOCHORYCTIS*

LEURONOMA

BUCCULATRIX

ONEBALA

ACROLOPHUS

COSMOPTERYX

CALLIPRORA

MELASINA

LIMNOECIA

TORTRICOMORPHA

ARGYROPLOCE

PHTHORIMAEA

EPERMENIA

ACHYROSTOLA*

CNEPHASIA

ADOXOPHYES

COESYRA

ASYMPHORODES

ACROPOLITIS

STENOMA

MACHAEROPTERIS

PROSELENA

PHTHORIMAEA

CHELARIA

HELIANGARA

YPSOLOPHUS

EUCOSMA
I922, Exotic Microlepidoptera, 2: 570.

I935, in Caradja and Meyrick, Materialien zu einer Micro-

lepidopteren Fauna der Chinesischen Provinzen Kiangsu,

Chekiang und Hunan, p. 8o.

1897, Proc. Linnean Soc. N.S. Wales, 22: 426.

I935, in Caradja and Meyrick, Materialien zu einer Micro-

lepidopteren Fauna der Chinesischen Provinzen Kiangsu,

Chekiang und Hunan, p. 74.

I886, Proc. Linnean Soc. N.S. Wales, Io: 783 .

I9I9, Exotic Microlepidoptera, 2: 257.

I885, Trans. Proc. New Zealand Inst. I7: I44.

I93I, Exotic Microlepidoptera, 4: I79. (v)

I9I4, Ann. South African Mus. Io: 25I. (SA)

I891, Ent. Mon. Mag. 27: 57. (BM)

I904, Proc. Linnean Soc. N.S. Wales, 29:39I.

1905, Journ. Bombay Nat. Hist. Soc. 16: 590. (BM)

I909, Trans. Ent. Soc. London, I909: I9. (BM)

I933, Exotic Microlepidoptera, 4: 429.

I897, Proc. Linnean Soc. N.S. Wales, 22: 304.

I93I, in Caradja, Bull. Sect. sci. Acad. roum. I4: 65.

I912, Ann. South African Mus. Io: 68. (SA)

I932, Exotic Microlepidoptera, 4:224.

I894, Trans. Ent. Soc. London, I894: I9. (BM)

I92I, Ann. Transvaal Mus. 8:67. (T)

I936, Exotic Microlepidoptera, 4: 6I9.

I9I I, Journ. Bombay Nat. Hist. Soc. 20: 706. (BM)

I930, Ann. Naturhist. Mus. Wien, 44:267, pl. I, fig. 4I.

(v)

I909, Journ. Bombay Nat. Hist. Soc. I9: 4I3.

I922, Trans. Ent. Soc. London, I922: 7O. (BM)

I934, Exotic Microlepidoptera, 4:520. (c)

I909, Ann. Transvaal Mus. 2: 20, pl. 6, fig. Io. (T)

I905, Journ. Bombay Nat. Hist. Soc. I6: 6I I. (BM)

I9I I, Proc. Linnean Soc. N.S. Wales, 36: 27I.

I9I6, Exotic Microlepidoptera, I: 568. (BM)

I9I7, Exotic Microlepidoptera, 2: 68.

I921, Zool. Meded. 6: I7I. (L)

I9II, Ann. Transvaal. Mus. 2: 225. (BM)

I9I I, Trans. Linnean Soc. London, I4:267. (BM)

I884, Proc. Linnean Soc. N.S. Wales, 9: 785 .

I934, Pacific Ent. Surv. Publ. 7, art. 28, p. 35I. (Bв)

I9Io, Proc. Linnean Soc. N.S. Wales, 35: I75.

I9I5, Exotic Microlepidoptera, I : 456. (BM)

I9I5, Exotic Microlepidoptera, I: 29I.

I889, Trans. Proc. New Zealand Inst. 21 : 156.

I9I4, Ann. South African Mus. Io: 245. (SA)

I9I3, Journ. Bombay Nat. Hist. Soc. 22: I62. (BM)

I9I6, Exotic Microlepidoptera, I: 573. (BM)

I907, Journ. Bombay Nat. Hist. Soc. I7: 98I. (BM)

I927, Exotic Microlepidoptera, 3: 336. (BM) 
eridopa

eridora

eridryas

eriglypta

eriochiras

eriochrysa

eriocnista

eriocrossa

eriogastra

eriomis

eriomorpha

eriopa

eriophysa

eriopis

erioplaca

erioptila

eriospila

eriozona

eriphaea

eriphila

eriphylla

eripias

eriptila

eriscota

erismatias

erista

eristica

eritima

erixantha

erotarcha

erotias

erotias

erotica

eroticella

erotopis

erratica

ersodes

erudita

erudita

erycina

erymnota

erysiphaea

erythractis

erythrana

erythranches

erythrantis
EPICEPHALA

SAROTORNA*

BLASTOBASIS

HILAROGRAPHA

HIERODRYAS*

TINEA

AGRIOTORNA*

CLEPSIMACHA*

DALACA

EUCOSMA

ANTIDICA*

ODITES

DECADARCHIS

BRENTHIA

ACROCERCOPS

PERONEA

IMMA

CHELARIA

BORKHAUSENIA

EULECHRIA

HETEROCROSSA

ENICOSTOMA

[ANACATHARTIS*]

MICROCOLONA

PHILOBOTA

HYPOSMOCOMA

PROTOBATHRA*

LIMNOECIA

COERANICA

TRICHOTAPHE

STENOMA

MACHIMIA

PLATYPEPLUS

STENOMA

EUPHILTRA*

HYPERCALLIA

NOSPHISTICA*

PHTHEOCHROA

PROTOLECHIA

SCYTHRIS

ARISTOTELIA

XESTOCASIS

PLUTELLA

ENAEMIA

PALAEOBIA

TRICHOCEROTA

AEOLANTHES
I928, Exotic Microlepidoptera, 3: 407.

1904, Proc. Linnean Soc. N.S. Wales, 29: 323.

I932, Trans. Ent. Soc. London, 80: II4.

I92I, Exotic Microlepidoptera, 2: 478. (BM)

I93I, Exotic Microlepidoptera, 4: 88. (BM)

I932, Exotic Microlepidoptera, 4: 209. (BM)

I93I, Exotic Microlepidoptera, 4: I88. (BM)

I934, Exotic Microlepidoptera, 4: 450. (BM)

I921, Ann. Transvaal Mus. 8: I43. (T)

I933, Exotic Microlepidoptera, 4: 4I9. (BM)

I883, Proc. Linnean Soc. N.S. Wales, 8: 382.

I9o8, Journ. Bombay Nat. Hist. Soc. I8: 633. (BM)

I927, Insects of Samoa, 3, Lepidoptera, fasc. 2, p. II2.

I920, Exotic Microlepidoptera, 2: 337. (BM)

I9I8, Exotic Microlepidoptera, 2: I75.

I9I2, Exotic Microlepidoptera, I: I6. (BM)

I922, Exotic Microlepidoptera, 2: 483. (BM)

I92I, Ann. Transvaal Mus. 8: 8o. (T)

I9I4, Trans. Proc. New Zealand Inst. 46: Io7.

I888, Proc. Linnean Soc. N.S. Wales, (Ser. 2) 2: 946.

I888, Trans. Proc. New Zealand Inst. 20: 76 .

I9I4, Exotic Microlepidoptera, I: 252.

I9I5, Exotic Microlepidoptera, I: 334.

(BM)

I889, Proc. Linnean Soc. N.S. Wales, (Ser. 2) 3: I6r2.

I928, Proc. Hawaiian Ent. Soc. 7: I02. (SP)

I9I6, Exotic Microlepidoptera, I: 595. (BM)

I9I9, Exotic Microlepidoptera, 2: 286.

I884, Proc. Linnean Soc. N.S. Wales, 9: 760 .

I9I4, Exotic Microlepidoptera, I: 279. (BM)

I9I5, Exotic Microlepidoptera, I: 47I. (BM)

I9I2, Trans. Ent. Soc. London, I9II: 698. (BM)

I905, Journ. Bombay Nat. Hist. Soc. I6: 584.

I9I6, Exotic Microlepidoptera, I: 534. (BM)

I883, Proc. Linnean Soc. N.S. Wales, 7: 458.

I926, Exotic Microlepidoptera, 3: 3I5. (BM)

I9II, Journ. Bombay Nat. Hist. Soc. 20: 733. (BM)

I927, Exotic Microlepidoptera, 3:367. (BM)

I9I6, Exotic Microlepidoptera, I: 59I.

I9I7, Ann. South African Mus. I7: IO. (SA)

I9I7, Trans. Ent. Soc. London, I9I7: 3I. (BM)

I9I7, Exotic Microlepidoptera, 2: 62.

I938, in Caradja and Meyrick, Deuts. Ent. Zeit. Iris, 52: 25.

I887, Proc. Linnean Soc. N.S. Wales, (Ser. 2) I : I043.

I882, Proc. Linnean Soc. N.S. Wales, 6: 664.

I926, Exotic Microlepidoptera, 3: 269.

I935, in Caradja and Meyrick, Materialien zu einer Micro-

lepidopteren Fauna der Chinesischen Provinzen Kiangsu,

Chekiang und Hunan, p. 83. (BM) 


\section{erythrastis}

PHILOBOTA

erythrites

erythrocosma

erythrocyma

erythrodesma

erythrogama

erythrogramma

erythroleuca

erythromima

erythromis

erythropa

erythropis

erythrorma

erythrosema

erythrota

erythrozona

erythrura

escharia

escharias

escharitis

escharitis

escharota

eschatias

eschatopa

eschatopis

esmeralda

esmodes

essedaria

esuriens

etearcha

eteoxantha

ethadopa

ethela

ethelinda

ethicodes

ethirastis

ethnitis

euacta

euanthes

euargyra

euarithma

euastera

eucallynta

eucalypta

eucalypti

eucarpa

eucausta

eucela
ARGYROTOXA

CORACISTIS*

ARGYROTOXA

LACTURA

AEGERIA

HIERANGELA*

HYPERCALLIA

PYROZELA

ZACORISCA

ARGYROPLOCE

ERISMATICA*

CREMBALASTIS*

ZONOPETALA

PERSICOPTILA*

EONYMPHA*

TORTRICOPSIS

SCALIDEUTIS*

SPHYRELATA

EPIMIMASTIS

PHYCOMORPHA

ARGYROPLOCE

BUCCULATRIX

EPIMECYNTIS*

GNORIMOSCHEMA

ACROCLITA

EUCOSMA

CRONICOMBRA

OXYPTILUS

TRICLONELLA

ORNEODES

TINEA

GRACILARIA

LASPEYRESIA

CHILOPSELAPHUS

IMMA

PHILOBOTA

AERONECTRIS*

OCYSTOLA

ACROCERCOPS

ETHMIA

GLYPHIPTERYX

PHYCODES

CLYSIA

BUCCULATRIX

HELIOZELA

GNORIMOSCHEMA

TORTRIX
I889, Proc. Linnean Soc. N.S. Wales, (Ser. 2) 3: I626.

I928, Exotic Microlepidoptera, 3: 46I. (BM)

1897, Proc. Linnean Soc. N.S. Wales, 22: 370. (MA)

I930, Exotic Microlepidoptera, 3: 6I2. (BM)

I930, Exotic Microlepidoptera, 3: 595. (BM)

I934, Exotic Microlepidoptera, 4: 455.

1894, Trans. Ent. Soc. London, I894: I5. (BM)

1928, Exotic Microlepidoptera, 3: 473. (BM)

I906, Journ. Bombay Nat. Hist. Soc. I7: 4I5. (BM)

1924, Exotic Microlepidoptera, 3: II I.

I9I8, Ann. Transvaal Mus. 6: II. (T)

I933, Exotic Microlepidoptera, 4: 4I5.

I9I5, Trans. Ent. Soc. London, I9I5: 2I4.

I886, Proc. Linnean Soc. N.S. Wales, Io: 829 .

I886, Trans. Ent. Soc. London, I886: 295.

I906, Journ. Bombay Nat. Hist. Soc. I7: 407. (BM)

I9I4, Exotic Microlepidoptera, I: 228. (MA)

I906, Journ. Bombay Nat. Hist. Soc. I7: 409. (BM)

I92I, Exotic Microlepidoptera, 2: 402. (BM)

I9I6, Exotic Microlepidoptera, I: 589. (BM)

I9I6, Exotic Microlepidoptera, I: 555. (BM)

I9Io, Trans. Ent. Soc. London, I9Io: 436. (BM)

I9I6, Exotic Microlepidoptera, I: 6I9.

I924, Exotic Microlepidoptera, 3: Ioo. (BM)

I904, Proc. Linnean Soc. N.S. Wales, 29:321.

I9I2, Journ. Bombay Nat. Hist. Soc. 21:858. (BM)

1937, Exotic Microlepidoptera, 5: 99.

I926, Exotic Microlepidoptera, 3: 306. (BM)

I932, Trans. Ent. Soc. London, 80: 108.

I920, Exotic Microlepidoptera, 2: 365. (BM)

I929, Exotic Microlepidoptera, 3: 533. (BM)

I938, Institut des Parcs Nationaux du Congo Belge,

fasc. I4, p. 27.

I88I, Proc. Linnean Soc. N.S. Wales, 5: I52.

I934, Exotic Microlepidoptera, 4: 486. (BM)

I920, Voyage de Ch. Alluaud et $R$. Jeannel en Afrique

Oriental, II, Microlepidoptera, p. 68. (P)

I922, Exotic Microlepidoptera, 2: 48I. (BM)

I920, Exotic Microlepidoptera, 2: 384 .

I9I7, Exotic Microlepidoptera, 2: 35.

I885, Proc. Linnean Soc. N.S. Wales, 9: Io72.

1934, Exotic Microlepidoptera, 4: 47I.

I924, Exotic Microlepidoptera, 3: I20. (BM)

I88I, Proc. Linnean Soc. N.S. Wales, 5: 236.

I937, Exotic Microlepidoptera, 5: I24. (T)

I928, Exotic Microlepidoptera, 3: 436. (BM)

I88I, Proc. Linnean Soc. N.S. Wales, 5: I79.

I897, Proc. Linnean Soc. N.S. Wales, 22: 404.

I929, Exotic Microlepidoptera, 3: 492. (BM)

I9I0, Proc. Linnean Soc. N.S. Wales, 35: 250. 


\begin{tabular}{|c|c|c|}
\hline eucentra & DOLIOTECHNA & I920, Exotic Microlepidoptera, 2: 38I. (BM) \\
\hline eucentrota & ELAPHRERGA & I930, Exotic Microlepidoptera, 3: 574. (BM) \\
\hline euchalina & EPICEPHALA & I922, Exotic Microlepidoptera, $2: 562$. \\
\hline & STOMOPTERYX & \\
\hline & [HARPOGRAPTIS $*$ ] & I922, Trans. Ent. Soc. London, I922: 66. (BM) \\
\hline eucharis & LECITHOCERA & I933, Exotic Microlepidoptera, 4:356. (BM) \\
\hline 1charistis & CRYPTOLECHIA & $\begin{array}{l}\text { I } 93 \text { I, Ann. Mus. Nac. Hist. Nat. Buenos Aires, } 36: 396 . \\
\text { (BM) }\end{array}$ \\
\hline Ichorda & CHELARIA & I923, Exotic Microlepidoptera, 3: 3I. (BM) \\
\hline Ichthonia & PHTHORIMAEA & I939, Trans. R. Ent. Soc. London, 89: 53. (BM) \\
\hline lera & LASPEYRESIA & I921, Ann. Transvaal Mus. 8: 63. (T) \\
\hline גclina & PROCHOLA & I922, Exotic Microlepidoptera, 2: 583 . (BM) \\
\hline uclista & SIMAETHIS & I9I8, Exotic Microlepidoptera, 2: I92. (BM) \\
\hline cola & BATRACHEDRÁ & I889, Trans. Proc. New Zealand Inst. 2I: I8o. \\
\hline colapta & COMMOTRIAS* & I924, Exotic Microlepidoptera, 3:73. (T) \\
\hline lcoma & ANTAEOTRICHA & I925, Exotic Microlepidoptera, 3: I68. (вм) \\
\hline 1comopa & DICHOMERIS & I939, Trans. R. Ent. Soc. London, 89: 54. (BM) \\
\hline dcorystis & STATHMOPODA & I936, Exotic Microlepidoptera, 4:6I8. (c) \\
\hline crossa & ARGYROPLOCE & I9I4, Supplementa Entomologica, No. 3, p. 49. (D) \\
\hline dorana & PYRGOTIS & I885, Trans. Proc. New Zealand Inst. I7: I43. \\
\hline doxa & HELIOCAUSTA & I886, Proc. Linnean Soc. N.S. Wales, I0: $83 \mathrm{I}$. \\
\hline dra & ARGYROPLOCE & $\begin{array}{l}\text { I936, in Caradja and Meyrick, Deuts. Ent. Zeit. Iris, 50: } \\
\text { I56. (BM) }\end{array}$ \\
\hline uglypta & IMMA & I93I, Exotic Microlepidoptera, 4: I8I. \\
\hline iglypta & TRACHYBYRSIS* & I927, Exotic Microlepidoptera, $3: 368$. (BM) \\
\hline Igramma & CATORYCTIS & I89o, Trans. R. Soc. S. Australia, I3: 43 . \\
\hline igrapta & LABDIA & I927, Insects of Samoa, 3, Lepidoptera, fasc. 2, p. 87 . \\
\hline lectra & ACRIA & I9o8, Journ. Bombay Nat. Hist. Soc. I8: 635. (Bм) \\
\hline ulidias & ANCHICREMNA* & I926, Exotic Microlepidoptera, 3:246. (BM) \\
\hline ulimna & ANTICRATES & I9I3, Exotic Microlepidoptera, I: I40. (BM) \\
\hline imaea & EUCOSMA & IgI2, Ent. Mon. Mag. 48: 34 . \\
\hline imarodes & EUCOSMA & I924, Exotic Microlepidoptera, 3: 68. (BM) \\
\hline umelaena & PYRODERCES & I897, Proc. Linnean Soc. N.S. Wales, $22: 356$. \\
\hline menica & ARGYROPLOCE & I929, Trans. Ent. Soc. London, $76: 496$. (BM) \\
\hline umenodora & STENOMA & I937, Exotic Microlepidoptera, 5: I52. (BM) \\
\hline umenopa & TRACHYPEPLA & I926, Trans. Proc. New Zealand Inst. 56: 4 I6. \\
\hline menopis & LECITHOCERA & I9I4, Exotic Microlepidoptera, I: I99. (BM) \\
\hline umeris & ARISTOTELIA & I923, Exotic Microlepidoptera, 3: 9. (BM) \\
\hline eumeristis & LIMNOECIA & I927, Insects of Samoa, 3, Lepidoptera, fasc. 2, p. 93. \\
\hline eumetalla & GRACILARIA & I881, Proc. Linnean Soc. N.S. Wales, 5: I6o. \\
\hline eumetra & SIMAETHIS & I9I2, Exotic Microlepidoptera, I: 43. (BM) \\
\hline umetrota & TINISSA & I926, Exotic Microlepidoptera, 3: 3I9. \\
\hline umolybda & PSITTACASTIS & I926, Exotic Microlepidoptera, 3: 3I6. (BM) \\
\hline morpha & DOLEROMIMA* & I902, Trans. R. Soc. S. Australia, 26: I59. \\
\hline ophthalma & DECADARCHIS & I924, Exotic Microlepidoptera, 3:83. (BM) \\
\hline parypha & TRICHOTAPHE & I922, Trans. Ent. Soc. London, I922: Io8. (BM) \\
\hline patris & CACOECIA & Igo8, Journ. Bombay Nat. Hist. Soc. I8: 6I4. (BM) \\
\hline tris & $\begin{array}{l}\text { LECITHOCERA } \\
{[\text { HABROGENES*] }}\end{array}$ & Is \\
\hline ecta & ANACAMPSIS & I9I4, Trans. Ent. Soc. London, I9I4: 265. (BM) \\
\hline
\end{tabular}




$\begin{array}{ll}\text { eupepla } & \text { CHRYSOCENTRIS } \\ \text { eupetala } & \text { GRACILARIA } \\ \text { euphanes } & \text { BAEONOMA } \\ \text { euphanopis } & \text { PACHYRHABDA } \\ \text { euphema } & \text { ODITES } \\ \text { euphona } & \text { CAPUA } \\ \text { euphorodes } & \text { ANARSIA } \\ \text { euphracta } & \text { ZALITHIA } \\ \text { euphragma } & \text { IPHIERGA } \\ \text { euphrantis } & \text { OENOE } \\ \text { euphronica } & \text { HILAROGRAPHA } \\ \text { euphronopa } & \text { SPARGANOTHIS } \\ \text { euphylla } & \text { ACROCLITA } \\ \text { euplecta } & \text { CYMATOMORPHA* } \\ \text { euplocamis } & \text { ARCHIMAGA } \\ \text { euplocamis } & \text { TINEA } \\ \text { euprosopa } & \text { GELECHIA } \\ \text { euprosopis } & \text { OPOGONA } \\ \text { eurhythmopa } & \text { ACROCERCOPS } \\ \text { eurrhoa } & \text { OECOPHORA } \\ \text { eurrhipis } & \text { GLYPHIDOCERA } \\ \text { euryacta } & \text { LYSITONA* } \\ \text { euryanthes } & \text { GELECHIA } \\ \text { euryarga } & \text { PHILONOME } \\ \text { euryatma } & \text { AEOLANTHES } \\ \text { euryaula } & \text { COLEOPHORA } \\ \text { eurybaphes } & \text { CHRYSORYCTIS } \\ \text { eurybathra } & \text { GELECHIA } \\ \text { eurybatis } & \text { MACRENCHES* } \\ \text { eurybias } & \text { EPERMENIA } \\ \text { eurycapna } & \text { CHEZALA } \\ \text { eurychalca } & \text { ACROCERCOPS } \\ \text { eurychlora } & \text { EULIA } \\ \text { eurychora } & \text { IRENICODES* } \\ \text { eurychrysa } & \text { PSITTACASTIS } \\ \text { eurycitra } & \text { NEMOTOIS } \\ \text { eurycrates } & \text { LACTURA } \\ \text { eurycryptis } & \text { GRACILARIA } \\ \text { eurydelta } & \text { CALLIPRORA } \\ \text { eurydesma } & \text { NEPTICULA } \\ \text { eurydoxa } & \text { POLYHYMNO } \\ \text { eurydryas } & \text { HYPERCALLIA } \\ \text { eurygramma } & \text { EUTORNA } \\ \text { THALLOSTOMA* } & \text { TISIS } \\ \text { euRPG } & \text { TACROBATHRA } \\ \text { TRACHYPEPLA* }\end{array}$

I930, Exotic Microlepidoptera, 4: 7 .

I881, Proc. Linnean Soc. N.S. Wales, 5: i6o. I9I6, Exotic Microlepidoptera, I: 507. (вм)

I927, Exotic Microlepidoptera, 3: 377. (Bм)

I9I4, Journ. Bombay Nat. Hist. Soc. 22: 780. (Bм)

I9Io, Proc. Linnean Soc. N.S. Wales, 35: I89.

I922, Exotic Microlepidoptera, 2: 503. (P)

I9I4, Trans. Ent. Soc. London, I9I4: 268.

I893, Proc. Linnean Soc. N.S. Wales, 7: 5 I8.

1927, Exotic Microlepidoptera, 3: 325. (BM)

I920, Exotic Microlepidoptera, 2: 328. (Bм)

1927, Exotic Microlepidoptera, 3: 370. (BM)

I926, Sarawak Mus. Journ. 3: I50.

I904, Proc. Linnean Soc. N.S. Wales, 29: 4 I2.

I9I8, Exotic Microlepidoptera, 2: I7I. (BM)

I9I8, Ann. Transvaal Mus. 6: 44. (T)

I926, Exotic Microlepidoptera, 3:276. (BM)

I927, Insects of Samoa, 3, Lepidoptera, fasc. 2, p. I Io.

I934, Exotic Microlepidoptera, 4: 47I.

I886, Proc. Linnean Soc. N.S. Wales, Io: 788 .

I929, Trans. Ent. Soc. London, 76: 5Iо. (BM)

I9I8, Ann. Transvaal Mus. 6:57. (T)

I922, Arkiv för Zool. vol. I4, part I 5, p. 3.

I9I 5, Trans. Ent. Soc. London, I9I 5: 250.

I908, Journ. Bombay Nat. Hist. Soc. I8: 638. (BM)

1925, Bull. Soc. R. Ent. Egypte, 9:215.

I893, Proc. Linnean Soc. N.S. Wales, 7: 550.

I93I, Rec. Cant. Mus. 3: 368.

I904, Proc. Linnean Soc. N.S. Wales, 29: 307.

I897, Proc. Linnean Soc. N.S. Wales, 22: 429.

I920, Exotic Microlepidoptera, 2:313.

I920, Exotic Microlepidoptera, 2: 290.

I926, Exotic Microlepidoptera, 3: 258. (BM)

I9I9, Trans. Proc. New Zealand Inst. 5I : $35^{2}$.

I909, Trans. Ent. Soc. London, I909: 22. (BM)

I928, Exotic Microlepidoptera, 3: 463.

I924, Exotic Microlepidoptera, 3: I2I.

I928, Exotic Microlepidoptera, 3: 4II.

I922, Trans. Ent. Soc. London, I922: 69. (ВM)

I9I5, Trans. Ent. Soc. London, I9I5: 255.

I909, Ann. Transvaal Mus. 2: I5, pl. 5, fig. 6. (T)

I926, Exotic Microlepidoptera, 3: 3I4. (BM)

I906, Trans. R. Soc. S. Australia, 30: 43 .

I9I3, Trans. Proc. New Zealand Inst. 45: 29.

I922, Trans. Ent. Soc. London, I922: 9I. (BM)

I9Iо, Trans. Ent. Soc. London, I9го: 438. (вм)

I9I2, Ent. Mon. Mag. 48: 35. (Sub.)

I886, Proc. Linnean Soc. N.S. Wales, , : 808 .

I884, Trans. Proc. New Zealand Inst. I6: I4.

I883, New Zealand Journ. Sci. I : 522 . 
I36

euryloxa

eurylyca

eurylyta

eurymenes

eurymolybda

eurynephela

eurynephes

euryntis

euryochtha

euryomis

euryopa

euryopis

eurypepla

eurypercna

euryphanella

euryphanta

euryphracta

eurypinax

euryplaca

euryplintha

euryptera

euryptera

eurypyra

euryscia

eurysema

euryspora

euryteles

euryterma

eurytricha

euryxantha

euryzancla

euryzeucta

euryzona

eusarca

eusaris

eusebasta

euselma

eustacta

eusticta

eustola

eustyla

eutacta

eutalanta
PTERIDOPORTHIS*

LACTURA

DECADARCHIS

TORTRIX

MACHLOTICA

ORNEODES

LOXOZYGA*

OPOSTEGA

CHIONOREAS*

ADOXOPHYES

EXACRISTIS*

ARGYROPLOCE

CHALCONYMPHA*

LACTURA

PALPARIA

ACROCERCOPS

ERIOCOTTIS

EUCOSMA

BATODES

CACOECIA

GRACILARIA

XYSMATODOMA

CROCANTHES

EPICNISTIS*

SPHALERACTIS

PYRODERCES

LASPEYRESIA

AUTOSTICHA

PERSICOPTILA

MACROBATHRA

OXYMACHAERIS

TELPHUSA

OXYLYCHNA

TORTRICOPSIS

[CLONITICA *]

HOLCOCERA

GELECHIA

HELIOCAUSTA

DICHOMERIS

STENOMA

BATRACHEDRA

CATERISTIS*

ANARSIA

PLATYPTILIA
EURYLOXA

1937, Exotic Microlepidoptera, 5: 156. (BM) I928, Exotic Microlepidoptera, 3: 4I7.

I9II, Trans. Linnean Soc. London, I4: 300.

I930, Exotic Microlepidoptera, 3: 608. (BM)

I926, Exotic Microlepidoptera, 3: 306. (BM)

I929, Exotic Microlepidoptera, 3: 534.

I938, in Caradja and Meyrick, Deuts. Ent. Zeit. Iris, 52:

20. (BM)

I907, Journ. Bombay Nat. Hist. Soc. I7: 985.

1926, Sarawak Mus. Journ. 3: I65.

I902, in Gardiner, The Fauna and Geography of the

Maldive and Laccadive Archipelagoes, I: I26. (CAM)

I921, Ann. Transvaal Mus. 8: 106. (T)

I937, in Caradja and Meyrick, Deuts. Ent. Zeit. Iris, 5I:

I82. (BM)

I93I, Ann. Mus. Nac. Hist. Nat. Buenos Aires, 36: $40 \mathrm{I}$. (BM)

1930, Exotic Microlepidoptera, 3: 597. (BM)

I883, Proc. Linnean Soc. N.S. Wales, 7: 435.

I9II, Trans. Linnean Soc. London, I4: 29I.

I893, Proc. Linnean Soc. N.S. Wales, 7: 5 I4.

r937, in Caradja and Meyrick, Deuts. Ent. Zeit. Iris, 51:

I78. (BM)

1933, Exotic Microlepidoptera, 4: 422. (BM)

1923, Exotic Microlepidoptera, 3: 53. (BM)

I908, Journ. Bombay Nat. Hist. Soc. I8: 829.

1893, Proc. Linnean Soc. N.S. Wales, 7: 494.

I9I8, Exotic Microlepidoptera, 2: 97.

I9o6, Trans. R. Soc. S. Australia, 30: 65.

I904, Proc. Linnean Soc. N.S. Wales, 29: 329.

1922, Exotic Microlepidoptera, 2: 571. (BM)

I936, Exotic Microlepidoptera, 5: 25. (c)

I920, Voyage de Ch. Alluaud et R. Jeannel en Afrique

Oriental, II, Microlepidoptera, p. 8o. (P)

I927, Insects of Samoa, 3, Lepidoptera, fasc. 2, p. 91.

I886, Proc. Linnean Soc. N.S. Wales, Io: 803 .

I918, Ann. Transvaal Mus. 6: 43. (T)

1922, Exotic Microlepidoptera, 2: 50I. (P)

I920, Exotic Microlepidoptera, 2: 355 .

I902, Trans. R. Soc. S. Australia, 26: 144.

I922, Exotic Microlepidoptera, 2: 542. (BM)

I926, Ann. South African Mus. 23: 329. (SA)

I883, Proc. Linnean Soc. N.S. Wales, 7: 482 .

1921, Ann. Transvaal Mus. 8: 84. (T)

I9I6, Exotic Microlepidoptera, I: 52I. (BM)

1897, Proc. Linnean Soc. N.S. Wales, 22: 308.

I889, Trans. Proc. New Zealand Inst. 21: I64.

I92 I, Zool. Meded. 6: I63. (L)

I931, Ann. Mus. Nac. Hist. Nat. Buenos Aires, 36: 379. 


\begin{tabular}{|c|c|c|}
\hline eutechna & EUCOSMA & I936, Exotic Microlepidoptera, 4: 6го. (вм) \\
\hline eutelopis & PHILOBOTA & 1920, Exotic Microlepidoptera, 2: 384 . \\
\hline euthalama & ZACORISCA & I924, Exotic Microlepidoptera, 3: II2. \\
\hline euthenopa & LASPEYRESIA & I92I, Zool. Meded. 6: I59. (L) \\
\hline euthetodes & SYNCRATOMORPHA* & I929, Exotic Microlepidoptera, 3: 509. (вм) \\
\hline euthrepta & GLAPHYRARCHA* & 1938, Trans. roy. Soc. N.Z. $67: 429$. \\
\hline euthrinca & ANTAEOTRICHA & I9I5, Exotic Microlepidoptera, I: 399. (BM) \\
\hline euthybelemna & PHRYGANOSTOLA & I88I, Proc. Linnean Soc. N.S. Wales, 5: 250. \\
\hline ycolona & ACROCERCOPS & I931, Exotic Microlepidoptera, 4: 46. \\
\hline euthydroma & ERECHTHIAS & I92I, Exotic Microlepidoptera, 2: 459. \\
\hline euthyrsa & HYPERCALLIA & I930, Exotic Microlepidoptera, 3: 577. (BM) \\
\hline euthysana & MACHAEROPTERIS & I93I, Exotic Microlepidoptera, 4: 98 . \\
\hline Intha & PHILOBOTA & I884, Proc. Linnean Soc. N.S. Wales, 8: 505. \\
\hline euxena & MEGACRASPEDUS & I904, Proc. Linnean Soc. N.S. Wales, 29: 277. \\
\hline euzela & TINEA & I9I6, Exotic Microlepidoptera, I: 603. \\
\hline eva & STENOMA & I9I5, Exotic Microlepidoptera, I: 447 . (BM) \\
\hline evagata & MELASINA & I92I, Zool. Meded. 6: 20I. (L) \\
\hline evelina & IMMA & I938, Trans. R. Ent. Soc. London, 87: 522. (вм) \\
\hline evidens & EUCOSMA & I9I7, Trans. Ent. Soc. London, I9I7: I9. \\
\hline evitans & ASCALENIA & I925, Bull. Soc. R. Ent. Egypte, 9: 212. \\
\hline evocata & CHALCEOPLA & I923, Exotic Microlepidoptera, 3: 53 . \\
\hline exacta & PHTHORIMAEA & I9I7, Trans. Ent. Soc. London, p. 46. (Bм) \\
\hline ma & $\begin{array}{l}\text { PACHNISTIS } \\
\text { [STELECHORIS*] }\end{array}$ & I9II, Journ. Bombay Nat. Hist. Soc. 20: 707. (Bм) \\
\hline exaequata & PHALONIA & I923, Exotic Microlepidoptera, 3:52. (вм) \\
\hline exaeta & PHYLLOCNISTIS & I926, Exotic Microlepidoptera, 3: 264 . \\
\hline exagitata & CRYPTOLECHIA & I926, Exotic Microlepidoptera, 3: 3I9. (BM) \\
\hline exagria & ANATRACHYNTIS & I9I5, Exotic Microlepidoptera, I: 326. \\
\hline exalbata & CEROMITIA & I921, Exotic Microlepidoptera, 2: 405. (BM) \\
\hline exalbescens & CAPUA & I922, Zool. Meded. 7: 8I. (L) \\
\hline exalbida & NARYCIA & $\begin{array}{l}\text { I920, Voyage de Ch. Alluaud et } R \text {. Jeannel en Afrique } \\
\text { Oriental, II, Microlepidoptera, p. I08. (P) }\end{array}$ \\
\hline exallacta & CYMOTRICHA & I926, Exotic Microlepidoptera, 3:287. (Bм) \\
\hline examinalis & DIPTYCHOPHORA & I93I, Exotic Microlepidoptera, 4: Io9. \\
\hline exanimata & EPICHORISTA & I920, Ann. South African Mus. I7: 276. (SA) \\
\hline exanimis & EULECHRIA* & r883, Proc. Linnean Soc. N.S. Wales, 7: 5I9. \\
\hline exanthema & CREPIDOSCELES & 1885, Proc. Linnean Soc. N.S. Wales, 9: ro57. \\
\hline exanthes & MYTHOPLASTIS* & I9I9, Exotic Microlepidoptera, 2: 277. (BM) \\
\hline exanthina & EULIA & I931, Exotic Microlepidoptera, 4: I52. (v) \\
\hline exanthista & TORTYRA & I9Io, Trans. Ent. Soc. London, I9Io: 464. \\
\hline exanthistis & SCORPIOPSIS & I930, Exotic Microlepidoptera, 3:6I9. \\
\hline exantlia & CROBYLOPHORA & I9I5, Exotic Microlepidoptera, I: 345 . \\
\hline exaphrista & ACROLOPHUS & I9I9, Exotic Microlepidoptera, 2: 279. (Bм) \\
\hline exarcha & PHLOEOPOLA & I883, Proc. Linnean Soc. N.S. Wales, 8: 357 . \\
\hline exarga & COLEOPHORA & I9I7, Exotic Microlepidoptera, 2: 7I. \\
\hline exarista & PROTOLECHIA & I904, Proc. Linnean Soc. N.S. Wales, 29: 339. \\
\hline arthra & SCHOENOTENES & I9I8, Exotic Microlepidoptera, 2: I69. (BM) \\
\hline rthrota & LYONETIA & I9I8, Exotic Microlepidoptera, 2: I83. \\
\hline sperata & STENOMA & r916, Exotic Microlepidoptera, I: 533. (BM) \\
\hline raula & ELACHISTA & I889, Trans. Proc. New Zealand Inst. 2I: I78. \\
\hline
\end{tabular}


excaecata

excavata

excelsa

excepta

excisa

excitata

excoriata

excoriata

excors

excreta

exculta

excurata

excurvata

exedra

exedra

exemplaris

exemplaris

exempta

exercitata

exeristis

exetastis

exhalata

exhausta

exhilarata

exigua

exilis

eximia

exocentra

exocha

exochana

exodias

exodroma

exoeca

exoenota

exolescens

exophthalma

exoria

exorycha

exospila

exosticha

exoterica

expansa

expedita

expers

experta

expilata

explanata

expleta
LECITHOCERA

TINEA

ACROPOLITIS

DICHOMERIS

ANTAEOTRICHA

DROMIAULIS*

DICHOMERIS

LASPEYRESIA

PTEROPHORUS

ADAINA

LACTISTICA

AGDISTIS

CACOECIA

BUCCULATRIX

TORTRIX

AUTOSTICHA

LASPEYRESIA

STENOMA

TIQUADRA

EBODA

EPICEPHALA

STENOMA

TINEA

ARGYROPLOCE

ACROLOPHUS

EPERMENIA

SYNTOMACTIS

LASPEYRESIA

SIMAETHIS

HETEROCROSSA

MOMPHA

PYRODERCES

HELIOCOSMA

GELECHIA

TINEA

BRACHMIA

EUMASIA

PARECTOPA

EREUNETIS

LASPEYRESIA

EUXANTHIS

STENOMA

MELASINA

MELASINA

CYCLOTORNA

STENOMA

LIMNOECIA

CACOECIA
I922, Zool. Meded. 7: 86. (L)

I9I4, Exotic Microlepidoptera, I: 2 Io.

I9I0, Proc. Linnean Soc. N.S. Wales, 35: I72. (MA)

I9I4, Exotic Microlepidoptera, I: 279. (BM)

I9I6, Exotic Microlepidoptera, I: 496. (BM)

I922, Exotic Microlepidoptera, 2: 575. (BM)

I9I3, Journ. Bombay Nat. Hist. Soc. 22: I74. (BM)

I9I8, Ann. Transvaal Mus. 6: I3. (T)

I930, Exotic Microlepidoptera, 3:568.

I930, Exotic Microlepidoptera, 3: 568. (вм)

I9I4, Exotic Microlepidoptera, I : 237. (BM)

I92 I, Exotic Microlepidoptera, 2: 423. (BM)

I93I, in Joannis, Ann. Soc. Ent. France, 98 (Supp.): 7 I2 [474].

I9I5, Exotic Microlepidoptera, I: 354.

I920, Voyage de Ch. Alluaud et R. Jeannel en Afrique

Oriental, II, Microlepidoptera, p. 50. (P)

I9I6, Exotic Microlepidoptera, I: 586. (BM)

I9I I, Proc. Linnean Soc. N.S. Wales, 36: 296.

1925, Exotic Microlepidoptera, 3: I79. (BM)

I922, Exotic Microlepidoptera, 2: 599. (P)

I9I0, Proc. Linnean Soc. N.S. Wales, 35: 290.

I908, Journ. Bombay Nat. Hist. Soc. I8: 8I I.

I9I5, Exotic Microlepidoptera, I: 474. (BM)

I9I7, Exotic Microlepidoptera, 2: 78.

I9I8, Ann. Transvaal Mus. 6: 50. (T)

I9I5, Trans. Ent. Soc. London, I9I5: 253.

I897, Proc. Linnean Soc. N.S. Wales, 22: 43 I.

I897, Proc. Linnean Soc. N.S. Wales, 22: 390.

I939, Trans. R. Ent. Soc. London, 89:52. (BM)

I907, Trans. Proc. New Zealand Inst. 39: I20.

I888, Trans. Proc. New Zealand Inst. 20: 76 .

1931, Ann. Mus. Nac. Hist. Nat. Buenos Aires, 36: 389.

I897, Proc. Linnean Soc. N.S. Wales, 22: 356.

I9Io, Proc. Linnean Soc. N.S. Wales, 35: 160.

I9I8, Ann. Transvaal Mus. 6: 52. (T)

I926, Ann. South African Mus. 23: 343. (SA)

IgII, Joum. Bombay Nat. Hist. Soc. 20:720. (BM)

I9I9, Exotic Microlepidoptera, 2: 26I.

I928, Exotic Microlepidoptera, 3: 409.

I902, Trans. Ent. Soc. London, I902: 577.

I936, Exotic Microlepidoptera, 5:25.

I924, Arkiv för Zool. vol. I6, part I4, p. 2.

I9I5, Exotic Microlepidoptera, I: 464. (BM)

I907, Journ. Bombay Nat. Hist. Soc. I8: I57.

I9I2, Ann. Transvaal Mus. 3: 82. (T)

I9I2, Trans. Ent. Soc. London, I9II: 589.

I9I5, Exotic Microlepidoptera, I: 469. (BM)

I92I, Ann. Transvaal Mus. 8: 95. (T)

I923, Exotic Microlepidoptera, 3: 54. (BM) 


$\begin{array}{ll}\text { explicata } & \text { GAESA } \\ \text { explicita } & \text { STENOMA } \\ \text { explorata } & \text { BLASTOBASIS } \\ \text { explosa } & \text { EUCOSMA } \\ \text { expolita } & \text { SCYTHRIS } \\ \text { exposita } & \text { TELPHUSA } \\ \text { expressa } & \text { MELASINA } \\ \text { expurgata } & \text { GLYPHIPTERYX } \\ \text { exquisitus } & \text { DEUTEROCOPUS } \\ \text { exsanguis } & \text { CARPOSINA } \\ \text { exsculpta } & \text { TINEOLA } \\ \text { exsecrata } & \text { MELASINA } \\ \text { exsecta } & \text { DICHOMERIS } \\ \text { exsiccata } & \text { GLYPHIDOCERA } \\ \text { exsignata } & \text { ARGYROPLOCE } \\ \text { exsoluta } & \text { SCYTHRIS } \\ \text { exsomnis } & \text { ATOMOTRICHA } \\ \text { exsors } & \text { NESOSCOPA* } \\ \text { exstans } & \text { CALAMOTYPA* } \\ \text { exstimulata } & \text { BORKHAUSENIA } \\ \text { exstincta } & \text { GELECHIA } \\ \text { exstinctrix } & \text { EULIA } \\ \text { exsularis } & \text { CEROSTOMA } \\ \text { exsulata } & \text { ANACAMPSIS } \\ \text { exsuperans } & \text { PISINIDEA } \\ \text { exsurgens } & \text { LASPEYRESIA } \\ \text { extensa } & \text { BLASTOBASIS } \\ \text { extenuata } & \text { ACROCERCOPS } \\ \text { exterrita } & \text { ODITES } \\ \text { extorris } & \text { PHTHORIMAEA } \\ \text { exulcerata } & \text { TINEA } \\ \text { ANTHELOPA } \\ \text { exta } & \\ \text { exHEORICHA }\end{array}$

$\begin{array}{ll}\text { fabicola } & \text { PHALONIA } \\ \text { fabivora } & \text { LASPEYRESIA } \\ \text { fabricata } & \text { ORDRUPIA } \\ \text { fabricata } & \text { TORTRIX } \\ \text { fabrilis } & \text { CAPUA } \\ \text { - fabulosa } & \text { ECHIOMIMA } \\ \text { faceta } & \text { ANACAMPSIS } \\ \text { faceta } & \text { ARGYROPLOCE } \\ \text { faceta } & \text { PYRODERCES } \\ \text { facilis } & \text { BUCCULATRIX } \\ \text { facilis } & \text { EBODA } \\ \text { factiosa } & \text { PHATNOTIS* } \\ \text { facunda } & \text { LEPTOSACES } \\ \text { faeculenta } & \text { SCYTHRIS } \\ \text { faeculosa } & \text { ADOXOPHYES }\end{array}$

1929, Exotic Microlepidoptera, 3: 510.

1930, Ann. Naturhist. Mus. Wien, 44: 247. (v)

I9I8, Exotic Microlepidoptera, 2: I58.

I9I2, Ent. Mon. Mag. 48: 35. (Sub.)

I9IO, Rec. Indian Mus. 2: 224. (К)

1926, Sarawak Mus. Journ. 3: I52. (Bм)

I9I6, Exotic Microlepidoptera, I: 6II.

I922, Exotic Microlepidoptera, 2: 493. (BM)

I92I, Exotic Microlepidoptera, 2: 4I9.

I9I8, Ann. Transvaal Mus. 6:8. (T)

I9I7, Exotic Microlepidoptera, 2: 96.

1937, Exotic Microlepidoptera, 5: II5.

1927, Exotic Microlepidoptera, 3: 354. (T)

I9I4, Trans. Ent. Soc. London, I9I4: 273. (BM)

I9I6, Exotic Microlepidoptera, 2: I9. (BM)

1920, Ann. South African Mus. I7: 298. (SA)

I9I3, Trans. Proc. New Zealand Inst. 45: 26.

I926, Trans. Ent. Soc. London, 74: 27I. (BM)

1926, Exotic Microlepidoptera, 3: 272. (BM)

I928, Exotic Microlepidoptera, 3: 468. (Bм)

I9II, Ann. Transvaal Mus. 2: 23I. (T)

193I, Exotic Microlepidoptera, 4: I53. (v)

1937, Exotic Microlepidoptera, 5: I44. (T)

I9I8, Ann. Transvaal Mus. 6: 20. (T)

I920, Ann. South African Mus. I7: 30I. (SA)

1922, Exotic Microlepidoptera, 2: 534. (вм)

I918, Ann. Transvaal Mus. 6: 55. (T)

I916, Exotic Microlepidoptera, I: 624 .

1937, Exotic Microlepidoptera, 5: I52. (T)

I923, Exotic Microlepidoptera, 3: 50.

I9I9, Exotic Microlepidoptera, 2: 274. (вм)

1926, Trans. Ent. Soc. London, 74: 273. (вм)

I9I6, Exotic Microlepidoptera, I: 492. (вм)

I9I2, Ent. Mon. Mag. 48: 35. (Sub.)

1928, Exotic Microlepidoptera, 3: 449.

I9I5, Trans. Ent. Soc. London, I9I5: 215. (BM)

I9IO, Proc. Linnean Soc. N.S. Wales, 35: 233. (ма)

I9I2, Exotic Microlepidoptera, I: I. (BM)

I9I5, Exotic Microlepidoptera, I: 373.

I9I4, Ann. Transvaal Mus. 4: I92. (T)

I9I7, Trans. Ent. Soc. London, I9I7: 25. (BM)

1917, Exotic Microlepidoptera, 2: 38 .

I9II, Ann. Transvaal Mus. 2: 237. (T)

I9I2, Exotic Microlepidoptera, I: I5. (BM)

I9I3, Journ. Bombay Nat. Hist. Soc. 22: I8I. (BM)

I9I0, Journ. Bombay Nat. Hist. Soc. 20: I55. (BM)

I9I2, Ann. South African Mus. Io: 64. (SA)

1928, Exotic Microlepidoptera, 3: 453. (BM) 
fagicola

fagoniae

falcifera

falciformis

falcigera
fallax
falsa
falsicoma
falsidica
falsifica
falsiloqua
familiaris
famosa
famosa
famula

famulata

famulata

famulus

fanatica

farinata

farinosa

farracea

farraginea

farraria

farrata

fasciculata

fascifera

fasciformis

fascigera

fascinatrix

fastidiosa

fastidiosa

fastigata

fastosa

fatalis

fatalis

fatigata

fatigata

fatima

fatua

fausta

favens

favicola

favicolor

favigera

favillacea
TINEA

PHLOEOCECIS

PALPARIA

[ZELOTECHNA*] RHIZOSTHENES*

GLYPHIPTERYX

POLYCHROSIS

ULODEMIS

ANCYLIS

STENOMA

CHOREUTIS

TRACHYPEPLA

EUCOSMA

THIODIA

TRICHOTAPHE

CTENOCOMPA

DICHOMERIS

MERIDARCHIS

DEUTEROCOPUS

PHTHORIMAEA

EPITHECTIS

CTENOCOMPA

ACROLOPHUS

ARGYROPLOCE

STENOMA

SCYTHRIS

ACROCERCOPS

AGRIOPHARA

LITHOCOLLETIS

OPOGONA

LECITHOCERA

PARAPSECTIS

THIODIA

STENOMA

ZANCLARCHES*

FUMEA

HOMONA

BLASTOBASIS

MOROTRIPTA*

OPOGONA

CRYPTOLECHIA

LECITHOCERA

MYRMECOZELA

TINEOLA

EUCOSMA

TRICHOSTIBAS

HYPONOMEUTA
I92 I, Trans. Proc. New Zealand Inst. 53:336.

I925, in Wytsman, Genera Insectorum, fasc. I84, p. 88.

I883, Proc. Linnean Soc. N.S. Wales, $7: 440$.

I935, in Caradja and Meyrick, Materialien zu einer Micro-

lepidopteren Fauna der Chinesischen Provinzen Kiangsu,

Chekiang und Hunan, p. 83. (BM)

I9I3, Exotic Microlepidoptera, I: I02. (BM)

I909, Journ. Bombay Nat. Hist. Soc. I9: 587. (BM)

I9I4, Journ. Bombay Nat. Hist. Soc. 22: 77I. (BM)

I9I4, Ann. Transvaal Mus. 4: I88. (T)

I9I5, Exotic Microlepidoptera, I: 426. (BM)

I927, Insects of Samoa, 3, Lepidoptera, fasc. 2, p. I04.

I932, Trans. Proc. New Zealand Inst. 63: 24.

I92I, Zool. Meded. 6: I53. (L)

I9I2, Ent. Mon. Mag. 48: 34. (Sub.)

I9I4, Exotic Microlepidoptera, I: 202. (BM)

I920, Voyage de Ch. Alluand et $R$. Jeannel en Afrique

Oriental, II, Microlepidoptera, p. IIO. (P)

I9I4, Trans. Ent. Soc. London, I9I4: 284. (BM)

I9I3, Exotic Microlepidoptera, I: 72. (BM)

Igo8, Trans. Ent. Soc. London, I907: 474 .

I92I, Ann. Transvaal Mus. 8: 73. (T)

I9I3, Ann. Transvaal Mus. 3: 285. (T)

I9I9, Exotic Microlepidoptera, 2: 264.

I93I, Exotic Microlepidoptera, 4: I00. (v)

I93I, Exotic Microlepidoptera, 4: I37.

I9I5, Exotic Microlepidoptera, I: 462. (BM)

I9I2, Ann. Transvaal Mus. 3: 3I2. (T)

I9I5, Trans. Ent. Soc. London, I9I5: 230.

I89o, Trans. R. Soc. S. Australia, I3:80.

1930, Exotic Microlepidoptera, 3: 582.

I9I5, Exotic Microlepidoptera, I: 358.

1935, Exotic Microlepidoptera, 4: 563. (BM)

I9I2, Ann. Transvaal Mus. 3: 66. (T)

I9I2, Ent. Mon. Mag. 48: 34. (Sub.)

I9I5, Exotic Microlepidoptera, I: 430. (BM)

I921, Zool. Meded. 6: I69. (L)

I926, Ann. South African Mus. 23: 349. (SA)

I936, Exotic Microlepidoptera, 5: 59. (BM)

I9I4, Ann. Transvaal Mus. 4: I95. (T)

I9I7, Ann. South African Mus. I7: II. (SA)

I92I, Exotic Microlepidoptera, 2: $45^{8}$.

I92I, Zool. Meded. 6: I72. (L)

I9Io, Trans. Ent. Soc. London, I9Io: 449. (BM)

I9I7, Exotic Microlepidoptera, 2: 87.

I933, Exotic Microlepidoptera, 4: 4I2.

I927, Insects of Samoa, 3, Lepidoptera, fasc. 2, p. 72. (Вм)

I9I3, Trans. Ent. Soc. London, I9I3: I9o. (BM)

I922, Z̈ool. Meded. 7:88. (L) 


\begin{tabular}{|c|c|c|}
\hline favillata & STENOMA & I9I5, Exotic Microlepidoptera, I: 430. (вм) \\
\hline fecunda & GELECHIA & I9I8, Ann. Transvaal Mus. 6: I7. (T) \\
\hline felina & TORTRIX & I926, Sarawak Mus. Journ. 3: I48. \\
\hline felix & SAGEPHORA & I9I4, Trans. Proc. New Zealand Inst. 46: II4. \\
\hline fenerata & CRYPTOLECHIA & I9I4, Supplementa Entomologica, No. 3, p. 53. (D) \\
\hline fenicoma & $\mathrm{PH}[\mathrm{L}]$ OEPOLA & I9I4, Exotic Microlepidoptera, I: I7o. \\
\hline feniseca & EULECHRIA & I9I5, Exotic Microlepidoptera, I: 300. \\
\hline ferax & GELECHIA & I9I3, Ann. Transvaal Mus. 3:289. (T) \\
\hline fercularia & GNORIMOSCHEMA & I929, Exotic Microlepidoptera, 3: 492. (вм) \\
\hline ferculata & STENOMA & I923, Exotic Microlepidoptera, 2: 6I5. (P) \\
\hline ferialis & PERIACMA* & I894, Trans. Ent. Soc. London, I894: 21. (BM) \\
\hline feriata & PHARMACIS & I9I3, Ann. Transvaal Mus. 3:27o. (T) \\
\hline fermentata & HYSTEROSIA & I9I2, Ent. Mon. Mag. 48: 35. (Sub.) \\
\hline fermentata & STENOMA & I9I6, Exotic Microlepidoptera, I: 539. (Вм) \\
\hline ferox & AEGERIA & I929, Proc. Ent. Soc. London, 3: 54 . \\
\hline ferox & HILAROGRAPHA & I921, Zool. Meded. 6: I79. (L) \\
\hline ferrata & DICHOMERIS & I9I3, Journ. Bombay Nat. Hist. Soc. 22: I74. (Bм) \\
\hline ferrata & HEMIMENE & I9I6, Exotic Microlepidoptera, 2: 28. (BM) \\
\hline ferrea & TORTRIX & I9Io, Proc. Linnean Soc. N.S. Wales, 35:239. (MA) \\
\hline ferreata & ANACAMPSIS & I9I4, Trans. Ent. Soc. London, I9I4: 257. (BM) \\
\hline ferruginea & CARPOSINA & I925, Exotic Microlepidoptera, 3: I37. \\
\hline ferruginosa & DICHOMERIS & I9I3, Journ. Bombay Nat. Hist. Soc. 22: I73. (Bм) \\
\hline ferulata & PARAPSECTRIS & I9I8, Ann. Transvaal Mus. 6: I7. (т) \\
\hline fervens & DEUTEROCOPUS & I913, Exotic Microlepidoptera, I: Io8. \\
\hline fervescens & AMPHICLADA* & I912, Exotic Microlepidoptera, I: 60. \\
\hline fervida & CACOECIA & I902, Trans. Ent. Soc. London, I902: 572. \\
\hline fessa & ODITES & I921, Ann. Transvaal Mus. 8: Iо7. (т) \\
\hline festa & IOCHARES* & I92I, Ann. Transvaal Mus. 8: 8I. (T) \\
\hline festalis & LETOGENES & I930, Exotic Microlepidoptera, 4: I6. (BM) \\
\hline festicola & HYPERCALLIA & I924, Exotic Microlepidoptera, 3: I04. (BM) \\
\hline festus & TRICHOPTILUS & I920, Ann. South African Mus. I7: 273. (SA) \\
\hline fetialis & HAPSIFERA & I9I7, Exotic Microlepidoptera, 2: 88. \\
\hline fetialis & POLYCHROSIS & I920, Exotic Microlepidoptera, 2: 346. (BM) \\
\hline fibrata & ARGYROPLOCE & I909, Journ. Bombay Nat. Hist. Soc. I9: 597. (Bм) \\
\hline fibriculata & ARSIRRHYNCHA* & $\begin{array}{l}\text { I938, Institut des Parcs Nationaux du Congo Belge, } \\
\text { fasc. I4, p. } 20 . \text { (c) }\end{array}$ \\
\hline ora & DECADARCHIS & I933, Exotic Microlepiäoptera, 4: 365. \\
\hline ris & $\begin{array}{l}\text { ONEBALA } \\
\text { [PROSODARMA*] }\end{array}$ & I92I, Zool. Meded. 6: I67. (L) \\
\hline lata & HIEROMANTIS & I9o6, Journ. Bombay Nat. Hist. Soc. I7: 4Iо. (Bм) \\
\hline fibulatrix & PHRIXOSCELES & I922, Exotic Microlepidoptera, 2: 56I. \\
\hline fibuligera & EUCOSMA & $\begin{array}{l}\text { I934, in Caradja and Meyrick, Deuts. Ent. Zeit. Iris, } 48 \text { : } \\
\text { 3I. }\end{array}$ \\
\hline fictilis & CHEZALA & I9I4, Exotic Microlepidoptera, I: 248. \\
\hline fictrix & TINEA & I9I4, Supplementa Entomologica, No. 3, p. 59. (D) \\
\hline fida & DICHOMERIS & I923, Exotic Microlepidoptera, 2: 620. (BM) \\
\hline fidana & PALAEOBIA & I88I, Proc. Linnean Soc. N.S. Wales, 6: 667. \\
\hline fidelis & BONDIA & I9I3, Exotic Microlepidoptera, I: 7o. (Bм) \\
\hline figularis & STENOMA & I9I8, Exotic Microlepidoptera, 2:204. (BM) \\
\hline figurata & ONEBALA & I9Io, Joum. Bombay Nat. Hist. Soc. 20: 453 . (BM) \\
\hline
\end{tabular}


filicicola

filicincta

filicivora

filicivora

filifera

fimbriata

finita

finitima

finitrix

firma

firmata

firmata

fiscinata

fissa

fissiculata

fissilis

fissirostris

fistularis

fistulata

flabellata

flaccescens

flaccida

flagellata

flagellatrix

flagelliformis

flagitiosa

flagrans

flaminia

flammifera

flammivola

flavescens

flavicoma

flavida

flavidorsis

flavifusa

flavisecta

flaviserta

flavispinis

flexa

flexanimana

flexibilis

flexilis

flexiloqua

flocculosa

flora

florea

florens

florescens
BATRACHEDRA

TARAGMARCHA

MNESIPATRIS

HYPOSMOCOMA

GRACILARIA

METACHANDA

CNEPHASIA

PACHNISTIS

STENOMA

SYNTOMACTIS

LYONETIA

TORTRIX

BRACHMIA

PACHYRHABDA

TORTRIX

THYRSOSTOMA

SCYTHRIS

LASPEYRESIA

HOMONA

ATTERIA

DURRANTIA

PHILOBOTA

EPICEPHALA

THIOTRICHA

PHOLCOBATES*

HOMALOXESTIS

[CARODISTA*]

ADOXOPHYES

EULIA

OEDEMATOPODA

TRICHOTHYRSA *

AUTOSTICHA

CEROMITIA

[AGISANA]

AUXIMOBASIS

GONADA

LECITHOCERA

CNEPHASIA

ORNEODES

ACANTHOCASIS*

STAGMATOPHORA

MICTONEURA*

STENOMA

PROTOLECHIA

ANACAMPSIS

EUMITURGA*

PARECTOPA

HIEROXESTIS

COMPSOCRITA*

EUCOSMA
I9I7, Trans. Proc. New Zealand Inst. 49: 247.

I930, Trans. Ent. Soc. London, 78: 313. (P)

I937, The Entomologist, 70: I95.

I935, Proc. Hawaiian Ent. Soc. 9:68. (sP)

I9I2, Exotic Microlepidoptera, I: 27. ( ( )

Igro, Trans. Ent. Soc. London, I9Io: 369.

I924, Exotic Microlepidoptera, 3: II6. (T)

I92I, Ann. Transvaal Mus. 8: 88. (T)

I925, Exotic Microlepidoptera, 3:208. (BM)

I9II, Trans. Linnean Soc. London, I4: 284.

I9I 5, Trans. Ent. Soc. London, I9r5: 243.

I9Io, Proc. Linnean Soc. N.S. Wales, 35: 237. (MA)

I9I8, Ann. Transvaal Mus. 6: 26. (T)

I92 I, Zool. Meded. 6: I75. (L)

I9I7, Trans. Ent. Soc. London, I9I7: 9. (BM)

I9I8, Exotic Microlepidoptera, 2: I2I. (BM)

I928, Exotic Microlepidoptera, 3: 4I3. (BM)

I928, Exotic Microlepidoptera, 3: 449. (BM)

r9io, Proc. Linnean Soc. N.S. Wales, 35:212.

I9I2, Trans. Ent. Soc. London, I9II: 676. (BM)

I925, Exotic Microlepidoptera, 3: I6I. (вм)

I9I3, Exotic Microlepidoptera, I: I26.

Igo8, Journ. Bombay Nat. Hist. Soc. I8: 8I2.

I929, Exotic Microlepidoptera, 3: 499. (Missing)

I93I, Exotic Microlepidoptera, 4: I22. (v)

I9I4, Exotic Microlepidoptera, I: I98. (BM)

I9I2, Exotic Microlepidoptera, I: 3. (BM)

I926, Exotic Microlepidoptera, 3: 255. (BM)

I9I5, Exotic Microlepidoptera, I: 388 .

I9I2, Exotic Microlepidoptera, r:6r.

I9r6, Exotic Microlepidoptera, I: 587. (BM)

I937, Exotic Microlepidoptera, 5: I46. (T)

1922, Exotic Microlepidoptera, 2: 540. (BM)

I930, Exotic Microlepidoptera, 3: 573. (P)

I926, Sarawak Mus. Journ. 3: I56.

I9I8, Ann. Transvaal Mus. 6: 9. (T)

I92I, Ann. Transwaal Mus. 8: 107. (T)

I92I, Zool. Meded. 6: I78. (L)

I92I, Exotic Microlepidoptera, 2: 4I3. (BM)

I88I, Proc. Linnean Soc. N.S. Wales, 6: 420.

I9I6, Exotic Microlepidoptera, I: 538. (BM)

I904, Proc. Linnean Soc. N.S. Wales, 29: 345.

I922, Trans. Ent. Soc. London, I922: 80. (BM)

1925, Exotic Micrclepidoptera, 3: I78. (BM)

I926, Ann. South African Mus. 23: 340. (SA)

I9I I, Trans. Linnean Soc. London, I4: 294.

I922, Exotic Microlepidoptera, 2: 589.

I925, Exotic Microlepidoptera, 3: I4I. 


$\begin{array}{ll}\text { floricolana } & \text { STIGMONOTA } \\ \text { floricoma } & \text { LACTURA } \\ \text { floridula } & \text { HELIOCAUSTA } \\ \text { florifera } & \text { ERETMOCERA } \\ \text { florilega } & \text { MONOPIS } \\ \text { florivora } & \text { PROSINTIS* } \\ \text { fluctuans } & \text { CYMOTRICHA } \\ \text { fluctuosa } & \text { SCYTHRIS } \\ \text { fluida } & \text { NEPTICULA } \\ \text { fluidana } & \text { STREPSICEROS } \\ \text { fluidescens } & \text { ARISTOTELIA } \\ \text { fluitans } & \text { DICHOMERIS } \\ \text { fluminata } & \text { STENOMA } \\ \text { fluorites } & \text { NEMOTOIS } \\ \text { fluvialis } & \text { SCYTHRIS } \\ \text { foederalis } & \text { ONEBALA } \\ \text { foederata } & \text { BACTRA } \\ \text { foliacea } & \text { IMMA } \\ \text { foliosa } & \text { ARCHISOPHA* } \\ \text { folligera } & \text { MELASINA } \\ \text { fonticola } & \text { SCYTHRIS } \\ \text { forcipata } & \text { AGRIOPHARA } \\ \text { formalis } & \text { POLYCHROSIS } \\ & \end{array}$

formidolosa formularis formulata fornacalis fornacaria fornicata

fornicata fortis fortis fortuita fortunata fortunatus fossoria fragilis fragosa fraudulenta frenata frenigera frenigera frequens fricata frigens frigescens
UNTOMIA

TRICHOTAPHE

BRACHMIA

GELECHIA

GENOSTELE

PSEUDATTERIA

LECITHOCERA

MICROSCHISMUS

. TINEA

METACHANDA

DEUTEROCOPUS

MELASINA

EULECHRIA

OINOPHILA

AMYDRIA

EPICEPHALA

HYPELICTIS

MACHAEROPTERIS

PHTHORIMAEA

HIEROXESTIS

ACOROSTOMA

EULECHRIA
CHELARIA
I881, Proc. Linnean Soc. N.S. Wales, 6: 656.

1924, Exotic Microlepidoptera, 3: I23.

I9I3, Exotic Microlepidoptera, I: I28. (MA)

I9o9, Ann. Transvaal Mus. 2: 2I, pl. 7, fig. 2. (T)

I9I I, Journ. Bombay Nat. Hist. Soc. 21: II4.

I9I6, Exotic Microlepidoptera, I: 598.

I923, Exotic Microlepidoptera, 3: 2. (BM)

I9I4, Ann. Transvaal Mus. 4: 197. (T)

I9II, Ann. Transvaal Mus. 2: 236. (T)

I88I, Proc. Linnean Soc. N.S. Wales, 6: 686.

I9I4, Ann. Transvaal Mus. 4: rgo. (T)

I920, Ann. South African Mus. I7: 284. (SA)

I9I2, Trans. Ent. Soc. London, I9I I 7I6. (BM)

I907, Journ. Bombay Nat. Hist. Soc. I7: 991.

I9I6, Exotic Microlepidoptera, 2: I5. (BM)

I923, Exotic Microlepidoptera, 3: 43. (Bм)

r9o9, Journ. Bomóay Nat. Hist. Soc. I9: 582. (BM)

I930, Exotic Microlepidoptera, 4: 4. (BM)

I9I8, Exotic Microlepidoptera, 2: 214. (BM)

I920, Voyage de Ch. Alluaud et R. Jeannel en Afrique

Oriental, II, Microlepidoptera, p. II5. (P)

IgII, Ann. Transvaal Mus. 3: 72. (BM)

I913, Trans. Ent. Soc. London, I9I3: I83. (Bu)

I935, in Caradja and Meyrick, Materialien zu einer Micro-

lepidopteren Fauna der Chinesischen Provinzen Kiangsu,

Chekiang und Hunan, p. 57. (BM)

I9I6, Exotic Microlepidoptera, I: $58 \mathrm{r}$.

I929, Exotic Microlepidoptera, 3: 503 .

I922, Trans. Ent. Soc. London, I922: IIo. (BM)

I9I I, Journ. Bombay Nat. Hist. Soc. 20: 7I9. (BM)

I9I3, Ann. Transvaal Mus. 3:289. (T)

I920, Voyage de Ch. Alluaud et R. Jeannel en Afrique

Oriental, $I I$, Microlepidoptera, p. 92. (P)

I9I7, Trans. Ent. Soc. London, I9I7: 6. (BM)

I9I8, Exotic Microlepidoptera, 2: II I. (BM)

I9I0, in Wytsman, Genera Insectorum, fasc. I08, p. Ij.

1920, Ann. South African Mus. I7: 303. (SA)

I9II, Trans. Linnean Soc. London, I4: 279. (BM)

I921, Exotic Microlepidoptera, 2: 4I8.

I920, Ann. South African Mus. I7: 3Io. (SA)

I9I4, Exotic Microlepidoptera, I: 27I.

I9Io, Trans. Ent. Soc. London, I9Io: 376.

I912, Ann. South African Mus. Io: 7I. (SA)

I908, Journ. Bombay Nat. Hist. Soc. I8: 812.

I913, Journ. Bombay Nat. Hist. Soc. 22: I7I. (Bu)

I9I I, Journ. Bombay Nat. Hist. Soc. 2I : 130.

I92I, Exotic Microlepidoptera, 2: 426. (BM)

I9II, Trans. Limnean Soc. London, I4: 297.

I921, Ann. Transvaal Mus. 8: I32. (T)

I9I3, Exotic Microlepidoptera, I: I60. (MA) 


$\begin{array}{ll}\text { frigida } & \text { OPOSTEGA } \\ \text { frivola } & \text { STOMOPTERYX } \\ \text { fructuosa } & \text { ODITES } \\ \text { frugalis } & \text { PROTOLECHIA } \\ \text { frugivora } & \text { TINEA } \\ \text { frustrata } & \text { LECITHOCERA } \\ \text { frustulenta } & \text { STENOLECHIA } \\ \text { fruticosa } & \text { ODITES } \\ \text { fucosa } & \text { ELACHISTA } \\ \text { fugax } & \text { NEPHOGENES } \\ \text { fugitivana } & \text { PROTITHONA* } \\ \text { fulcrata } & \text { STENOMA } \\ \text { fuliginea } & \text { GELECHIA } \\ \text { fulminalis } & \text { URODUS } \\ \text { fulminata } & \text { STENOMA } \\ \text { fulminatrix } & \text { IMMA } \\ \text { fulminea } & \text { SIMAETHIS } \\ \text { fulta } & \text { ANTAEOTRICHA } \\ \text { fulvicilia } & \text { TRICHOTAPHE } \\ \text { fulvicolor } & \text { XYSTROLOGA } \\ \text { fulvicoma } & \text { TINEA } \\ \text { fulvicrinis } & \text { METANOMEUTA* }\end{array}$

$\begin{array}{ll}\text { fulvipalpis } & \text { HEMIMENE } \\ \text { fulvistrigata } & \text { DICHELOPA } \\ \text { fumata } & \text { METACHANDA } \\ \text { fumicoma } & \text { NARYCIA } \\ \text { fundigera } & \text { MACHIMIA } \\ \text { funeralis } & \text { NEPTICULA } \\ \text { funerea } & \text { ARGYROPLOCE } \\ \text { funesta } & \text { HYPONOMEUTA } \\ \text { funesta } & \text { PHALONIA } \\ \text { fungifera } & \text { TRICHOTAPHE } \\ \text { fungiferana } & \text { HELICTOPHANES } \\ \text { fungivora } & \text { OXYLYCHNA } \\ \text { fungosa } & \text { IMMA } \\ \text { funicularis } & \text { PTILOGENES } \\ \text { furcifera } & \text { IDIOTECHNA* } \\ \text { furciformis } & \text { MELASINA } \\ \text { furcigera } & \text { DICELLITIS } \\ \text { furculata } & \text { ANCHINIA } \\ \text { furfurosa } & \text { MYRIOPLEURA } \\ \text { furfurosus } & \text { PTEROPHORUS } \\ \text { furiosa } & \text { LASPEYRESIA } \\ \text { furnaria } & \text { LECITHOCERA } \\ \text { furtiva } & \text { ARISTOTELIA } \\ \text { furtiva } & \text { TORTRIX } \\ \text { furva } & \text { MACHIMIA } \\ \text { furvescens } & \text { THRYPSIGENES }\end{array}$

I906, Journ. Bombay Nat. Hist. Soc. I7: 4I6. I926, Ann. South African Mus. 23: 330. (SA) I9I5, Exotic Microlepidoptera, I: 380. I904, Proc. Linnean Soc. N.S. Wales, 29:345. I9I7, Exotic Microlepidoptera, 2: 77.

I9I8, Exotic Microlepidoptera, 2: I07. I923, Exotic Microlepidoptera, 3: I3. (BM) I9I5, Exotic Microlepidoptera, I: 380. (BM) I922, Exotic Microlepidoptera, 2: 509. I9Io, Journ. Bombay Nat. Hist. Soc. 20: I44. (BM) I883, Trans. New Zealand Inst. I883: 62. I9I5, Exotic Microlepidoptera, I: 47I. (BM) I929, Exotic Microlepidoptera, 3: 490. (BM) I93I, Exotic Microlepidoptera, 4: 90. (v) I9I6, Exotic Microlepidoptera, I: 539. (BM) I934, Pacific Ent. Surv. Publ. 7, art. 28, p. 353. (вB) I9I2, Exotic Microlepidoptera, I: 48. (BM) I926, Exotic Microlepidoptera, 3: 234. (BM) I922, Trans. Ent. Soc. London, I922: Iog. (BM) I9I9, Exotic Microlepidoptera, 2: 272. (BM) I92I, Ann. Transvaal Mus. 8: I29. (T) I935, in Caradja and Meyrick, Materialien zu einer Microlepidopteren Fauna der Chinesischen Provinzen Kiangsu,

Chekiang und Hunan, p. 87. (BM) I922, Exotic Microlepidoptera, 2: 529. (BM) I929, Trans. Ent. Soc. London, 76: 494. (BM) I9II, Trans. Linnean Soc. London, I4: 279. (BM) I922, Exotic Microlepidoptera, 2: 604 . I9I2, Trans. Ent. Soc. London, I9II: 696. (BM) I906, Trans. R. Soc. S. Australia, 30: 59. I920, Exotic Microlepidoptera, 2: 350. (BM) I9I4, Exotic Microlepidoptera, I: I73. I9I2, Ent. Mon. Mag. 48: 36. I9I3, Journ. Bombay Nat. Hist. Soc. 22: I77. (BM) I88 I, Proc. Linnean Soc. N.S. Wales, 6: 640. I937, Exotic Microlepidoptera, 5: I54. I9I4, Supplementa Entomologica, No. 3, p. 56. (D) I926, Exotic Microlepidoptera, 3: 228. (BM) I920, Ann. South African Mus. I7: 305. (SA) I92I, Ann. Transvaal Mus. 8: I35. (T) I928, Exotic Microlepidoptera, 3: 460 . I924, Exotic Microlepidoptera, 3: IOI. (T) I906, Journ. Bombay Nat. Hist. Soc. I7: 405. I9II, Ann. Transvaal Mus. 2: 220. (T) I92I, Exotic Microlepidoptera, 2: 450 . I9I3, Ann. Transvaal Mus. 3: 294. (T) I904, Proc. Linnean Soc. N.S. Wales, 29: 288. I9II, Ann. Transvaal Mus. 2: 223. (BM) I9I6, Exotic Microlepidoptera, I: 549. (BM) I9I4, Trans. Ent. Soc. London, I9I4:272. (BM) 
fuscata fuscicoma fuscipalpis fusifera fusigera fusilis fustigera futilis futura

$\begin{array}{ll}\text { gabina } & \text { POLYCHROSIS } \\ \text { gaesata } & \text { INOTICA* } \\ \text { galactacma } & \text { NEPTICULA } \\ \text { galactaea } & \text { BORKHAUSENIA } \\ & \text { [PLESIOSTICHA*] } \\ \text { galactaea } & \text { THIOTRICHA } \\ \text { galactarcha } & \text { ETHMIA } \\ \text { galactitis } & \text { EUCOSMA } \\ \text { galactitis } & \text { ORTHROMICTA* } \\ \text { galactodes } & \text { SAPHENEUTIS } \\ \text { galactombra } & \text { SPORADARCHI* } \\ \text { galactopa } & \text { LEXIARCHA* } \\ \text { galactopis } & \text { CRITICONOMA } \\ \text { galactura } & \text { TIQUADRA } \\ \text { galatea } & \text { GELECHIA } \\ \text { galaxaea } & \text { EULECHRIA } \\ \text { galaxias } & \text { TRACHYPEPLA } \\ \text { galbanea } & \text { AMORBAEA } \\ \text { galbanea } & \text { OCYSTOLA } \\ \text { galbanea } & \text { PHALONIA } \\ \text { galeata } & \text { CORYTHANGELA* } \\ \text { galeata } & \text { EPICHORISTA } \\ \text { galenaea } & \text { MACROBATHRA } \\ \text { galenaea } & \text { THIOTRICHA } \\ \text { galenitis } & \text { PAUROPTILA* } \\ \text { galenopa } & \text { ANTIPHRASTIS* } \\ & \end{array}$

galeodes galeodes galeomorpha galeopa galeotis galeotis

galerita gamelia gamicopis ganodes ganota M.T.M. I-I I
TRICHEMBOLA CRYPSITHYRIS LIMNOECIA PHTHEOCHROA STENOMA STATHMOPODA ACROCERCOPS PHILOBOTA STENOMA

POLYCHROSIS BORKHAUSENIA [PLESIOSTICHA*] THIOTRICHA

ETHMIA

UCOSMA TA PHENEUTIS PORADARCHIS* LEXIARCHA* CRITICONOMA EULECHRIA BUCCULATRIX HOMALOXESTIS PTILOGENES ACROCERCOPS ARISTOTELIA CNEPHASIA

SAPHENEUTIS PARECTOPA BRENTHIA LITHOCOLLETIS PLATYBATHRA*
I9I8, Exotic Microlepidoptera, 2: II6. (Bм) I937, Exotic Microlepidoptera, 5: 77. (T) I92I, Exotic Microlepidoptera, 2: 455. I9I2, Trans. Ent. Soc. London, I9Ix: 674. (BM) I9I5, Exotic Microlepidoptera, I: 476. (BM) I9I4, Trans. Proc. New Zealand Inst. 46: I I I. I928, Exotic Microlepidoptera, 3: 408. (BM) I920, Exotic Microlepidoptera, 2: 383. I913, Trans. Ent. Soc. London, I9I3: I88. (Bм)

I909, Journ. Bombay Nat. Hist. Soc. I9: 588. (BM) I9I3, Exotic Microlepidoptera, I: 66. (BM) I924, Exotic Microlepidoptera, 3: II7. (BM)

I9o8, Proc. Zool. Soc. London, p. 732.

I908, Journ. Bombay Nat. Hist. Soc. I8: 44I. (BM) I928, Exotic Microlepidoptera, 3:4I8. (BM) I9I2, Ann. South African Mus. Io: 57. (SA) I897, Proc. Linnean Soc. N.S. Wales, 22: 401. I9I5, Exotic Microlepidoptera, I: 289. I935, Exotic Microlepidoptera, 4: 60I. I9I6, Exotic Microlepidoptera, I : 590. I92I, Ann. Transvaal Mus. 8: I30. (T) I93I, Exotic Microlepidoptera, 4:97. (V) I926, Exotic Microlepidoptera, 3: 278. (вм) I922, Exotic Microlepidoptera, 2: 544. (BM) 1883, New Zealand Journ. Sci. I : 522. I9I4, Journ. Bombay Nat. Hist. Soc. 22: 778. (вм) I9I3, Exotic Microlepidoptera, I： I I5. (MA) I9I7, Trans. Ent. Soc. London, I9I7: 2. (BM) I897, Proc. Linnean Soc. N.S. Wales, 22: 300. I921, Ann. Transvaal Mus. 8:52. (T) I902, Trans. R. Soc. S. Australia, 26: I67. I908, Journ. Bombay Nat. Hist. Soc. I8: 443. (вм) I9I3, Ann. Transvaal Mus. 3: 309. (T) I93I, in Joannis, Ann. Soc. Ent. France, 98 (Supp.): 7 I4 $[476]$.

I9I3, Ann. Transvaal Mus. 3: 329. (T) I9Io, Journ. Bombay Nat. Hist. Soc. 20: 442. (вм) I93I, Exotic Microlepidoptera, 4: 44. (BM) I 908 , Journ. Bombay Nat. Hist. Soc. I8: 82 I. I908, Journ. Bombay Nat. Hist. Soc. I8: 438. (BM) I920, Voyage de Ch. Alluaud et $R$. Jeannel en Afrique:

Oriental, II, Microlepidoptera, p. 56 . (P) I9II, Journ. Bombay Nat. Hist. Soc. 2I : I27. I936, Exotic Microlepidoptera, 5: 37. I930, Exotic Microlepidoptera, 4: 6. (BM) I9I8, Exotic Microlepidoptera, 2: I72. I9II, Ann. Transvaal Mus. 3: 7S. (BM) 
garrula

gastrocosma

gastroptila

gastrozona

gaulica

gelastis

gelida

gelophodes

gemellata

geminata

geminorum

gemistis

gemmaria

gemmulans

generalis

generatrix

generosa

generosa

genialis

genialis

geniculata

geniola

genitalis

genitrix

gennaea

gentilis

gentilis

genuina

geochrota

geodes

geographica

geologica

geometra

geometrica

geometropis

geomicta

gephyraea

gephyrias

gephyritis

gephyropa

gephyrota

geraea

geraeas

geraeopa

geranodes

geranomorpha

geranoptera

geraropa
NARYCIA

ASCALENIA

LABDIA

CORSOCASIS

PSITTACASTIS

CROCANTHES

ACROLEPIA

CACOECIA

STENOMA

CEROMITIA

SPARGANOTHIS

AMYDRIA

EUPHILTRA

THIOTRICHA

EUTORNA

ANTAEOTRICHA

AEOLOTROCHA*

ARGYROPLOCE

LOBESIA

PROCOMETIS

PHALONIA

CROCANTHES

SCARDIA

MACROBATHRA

PERITORNEUTA

ADAINA

MACROBATHRA

MACHIMIA

TRICHOTAPHE

BUCOLARCHA*

CACOECIA

ACROCERCOPS

ACROCERCOPS

PROMALACTIS

PARECTOPA

PHTHORIMAEA

OPOSTEGA

DECADARCHIS

ARGYRESTHIA

CALLIPHRACTIS

COESYRA

BRACHMIA

EPICHORISTA

COESYRA

LACTISTICA *

THIOSCELIS

SCAEOSTREPTA

BACTRA
I934, Exotic Microlepidoptera, 4: 48I.

I93I, Exotic Microlepidoptera, 4: 54 .

I93I, Exotic Microlepidoptera, 4: 52.

I932, Exotic Microlepidoptera, 4: 273.

I909, Trans. Ent. Soc. London, I909: 21. (вм)

I9I.8, Exotic Microlepidoptera, 2: 97.

I92I, Ann. Transvaal Mus. 8: I23. (T)

I936, Veröff. Deutschen Kol.-Mus. I: 329, pl. I3, fig. 22.

(H)

I9I6, Exotic Microlepidoptera, I: 524. (Bм)

I9I4, Ann. South African Mus. Io: 256. (SA)

1932, Exotic Microlepidoptera, 4: 263. (v)

I909, Trans. Ent. Soc. London, I909: 43. (BM)

I92I, Exotic Microlepidoptera, 2: 386.

I93I, Exotic Microlepidoptera, 4: 63. (Bм)

I92I, Zool. Meded. 6: I72. (L)

I926, Exotic Microlepidoptera, 3: 239. (вм)

I921, Ann. Transwaal Mus. 8: 78. (T)

I909, Journ. Bombay Nat. Hist. Soc. I9: 594. (вм)

I9I2, Journ. Bombay Nat. Hist. Soc. 2I: 869. (BM)

I89o, Trans. R. Soc. S. Australia, I3: 73 .

I930, Exotic Microlepidoptera, 3: 59I. (BM)

I93I, Exotic Microlepidoptera, 4: 74.

I9I3, Ann. Transvaal Mus. 3: 335 .

1927, Exotic Microlepidoptera, 3:380. (T)

I923, Exotic Microlepidoptera, 3: 62. (BM)

I9II, Ann. Transvaal Mus. 2: 2I9. (T)

I9I 8, Exotic Microlepidoptera, 2: 2I2. (Bм)

I9I4, Exotic Microlepidoptera, I: I80. (BM)

I9I4, Journ. Bombay Nat. Hist. Soc. 22: 775. (BM)

I929, Exotic Microlepidoptera, 3: 5I5. (BM)

I9I2, Trans. Ent. Soc. London, I9I I: 678. (вм)

I908, Journ. Bombay Nat. Hist. Soc. I8: 8I6.

I9I6, Exotic Microlepidoptera, I : 626.

I9I3, Ann. Transvaal Mus. 3: 3I5. (T)

1936, Exotic Microlepidoptera, 5: 37.

I9I8, Ann. Transwaal Mus. 6: I8. (T)

I881, Proc. Linnean Soc. N.S. Wales, 5: 176.

I907, Journ. Bombay Nat. Hist. Soc. I 7: 752. (вм)

I938, in Caradja and Meyrick, Dents. Ent. Zeit. Iris, 52:

22. (BM)

I937, Exotic Microlepidoptera, 5: I20. (c)

I884, Proc. Linnean Soc. N.S. Wales, 9: 788 .

I9II, Journ. Bombay Nat. Hist. Soc. 20: 7I7. (BM)

I909, Ann. Transvaal Mus. 2: 5, pl. 2, fig. 6. (T)

I9I3, Exotic Microlepidoptera, I: II8. (MA)

I907, Journ. Bombay Nat. Hist. Soc. I7: 74I. (BM)

I932, Exotic Microlepidoptera, 4: 287.

I93I, Exotic Microlepidoptera, 4: 77. (BM)

I93I, Exotic Microlepidoptera, 4: I47. (BM) 


\begin{tabular}{|c|c|}
\hline geraropa & MELISOPHISTA* \\
\hline gerasmia & ELACHISTA \\
\hline germinalis & PHILOBOTA \\
\hline germinans & STENOMA \\
\hline gilva & NEPTICULA \\
\hline glabra & ETHMIA \\
\hline glabrata & NEMOTOIS \\
\hline glacialis & OCYSTOLA \\
\hline & [ANTIPTERNA * \\
\hline glaciata & ANTAEOTRICHA \\
\hline glaciata & CYDIA \\
\hline glaciata & METACHANDA \\
\hline gladiata & POLYHYMNO \\
\hline gladiatrix & GRACILARIA \\
\hline glandifera & ETHMIA \\
\hline glandularis & CEROMITIA \\
\hline glandulata & PARAMORPHA \\
\hline glaphyra & ODITES \\
\hline glaphyrana & TORTRIX \\
\hline glaphyraspis & ARGYROPLOCE \\
\hline glaphyritis & LECITHOCERA \\
\hline glaphyrodes & STENOMA \\
\hline glaphyropa & SCYTHRIS \\
\hline glaphyropis & YPONOMEUTA \\
\hline glaphyropla & PELTOPHORA \\
\hline glaphyrota & EULECHRIA \\
\hline glaphyrotes & PTEROPHORUS \\
\hline glareosa & HAPSIFERA \\
\hline glauca & CARPOSINA \\
\hline glaucescens & STENOMA \\
\hline glaucias & TRACHYCENTRA \\
\hline glaucitis & THRYSOSTOMA* \\
\hline glaucodes & EPIMIMASTIS \\
\hline glaucogramma & COSMOPTERYX \\
\hline glaucomis & ARGYROTOXA \\
\hline glauconephela & ZONOPETALA \\
\hline glaucopa & STENOMA \\
\hline glaucophanes & GLYPHIPTERYX \\
\hline glaucopis & ENCHOCRATES* \\
\hline glaucoprosopis & PARATORNA \\
\hline glaucoptera & PHILOBOTA \\
\hline glaucoterma & GELECHIA \\
\hline glaucothoe & SPILONOTA \\
\hline glaucoxantha & LABDIA \\
\hline glebaria & MACROSACES \\
\hline glebata & HAPSIFERA \\
\hline glebifera & MYRMECOZELA \\
\hline glebifera & POLYCHROSIS \\
\hline glenias & THIOTRICHA \\
\hline
\end{tabular}

1927, Exotic Microlepidoptera, 3: 371. (BM)

I889, Trans. Proc. New Zealand Inst. 21: I77.

r913, Exotic Microlepidoptera, I: I26. (MA)

I925, Exotic Microlepidoptera, 3: 2rr. (BM)

1906, Trans. R. Soc. S. Australia, 30:59.

I920, Voyage de Ch. Alluaud et R. Jeannel en Afrique

Oriental, II, Microlepidoptera, p. 89. (P)

I922, Exotic Microlepidoptera, 2: 535. (Bм)

1885, Proc. Linnean Soc. N.S. Wales, 9: 1077.

Igo9, Trans. Ent. Soc. London, rgog: 30. (BM)

1907, Journ. Bombay Nat. Hist. Soc. I8: 142. (Вм)

I9II, Trans. Linnean Soc. London, I4:277. (BM)

I9I7, Trans. Ent. Soc. London, I9I7: 5I. (BM)

1922, Exotic Microlepidoptera, 2: 564. (P)

1918, Ann. Transvaal Mus. 6: 37. (T)

r9o8, Proc. Zool. Soc. London, p. 756.

1922, Zool. Meded. 7:80. (L)

I908, Journ. Bombay Nat. Hist. Soc. I8: 632. (BM).

I881, Proc. Linnean Soc. N.S. Wales, 6: 516.

I921, Ann. Transvaal Mus. 8: 59. (T)

I9I8, Exotic Microlepidoptera, 2: 106. (BM)

I9I3, Trans. Ent. Soc. London, r913: r86. (Bм)

r9I4, Ann. Transvaal Mus. 4: 196. (T)

I908, Proc. Zool. Soc. London, p. 736.

I884, Proc. Linnean Soc. N.S. Wales, 9: 735 .

1888, Proc. Linnean Soc. N.S. Wales, 2: 959.

1908, Trans. Ent. Soc. London, I907: 497.

I9I2, Ann. South African Mus. Io: 72. (SA)

I913, Exotic Microlepidoptera, I: 74. (BM)

I9I6, Exotic Microlepidoptera, I: 537. (BM)

1907, Proc. Linnean Soc. N.S. Wales, $32:$ I45.

I907, Journ. Bombay Nat. Hist. Soc. I 7: 736. (BM)

rgro, Journ. Bombay Nat. Hist. Soc. 20: 46r. (BM)

I934, Exotic Microlepidoptera, 4: 508.

1908, Journ. Bombay Nat. Hist. Soc. 18: 623. (Вм)

I883, Proc. Linnean Soc. N.S. Wales, 7: 462.

I9I2, Trans. Ent. Soc. London, I9I I: 7I2. (BM)

I922, Exotic Microlepidoptera, 2: 489. (BM)

I883, Proc. Linnean Soc. N.S. Wales, $7: 443$.

I93I, Exotic Microlepidoptera, 4: I55. (BM)

I884, Proc. Linnean Soc. N.S. Wales, 8: 490.

I9II, Trans. New Zealand Inst. 43: 63.

1927, Insects of Samoa, 3, Lepidoptera, fasc. 2, p. 70. (BM)

I92I, Exotic Microlepidoptera, 2: 45I.

Igro, Journ. Bombay Nat. Hist. Soc. 20: I48. (BM)

I908, Proc. Zool. Soc. London, p. 75I.

I9II, Journ. Bombay Nat. Hist. Soc. 2I: 126.

I9I2, Exotic Microlepidoptera, I: 34. (BM)

rgos, Journ. Bombay Nat. Hist. Soc. IS: 439. (BM) 
glischrodes

globifera

globigera

globigera

globulata

globulifera

globulosa

globulosa

glochinias

glomerata

glomeratrix

gloriosa

glossophora

glutinata

glycaea

glycera

glyceranthes

glyceropa

glycerostoma

glycina

glycinocoma

glycinopa

glycinopis

glycitis

glycyphaga

glycyphaga

glycystrota

glypharcha

glyphicodes

glyphidaula

glyphidopa

glyphidopis

glyphidota

glyptocosma

glyptosema

gnathodes

gnathodoxa

gnomica

gnomonica

gnomonodes

gomphacma

gomphias

gomphopis

gonatias

gongylopis

gonimodes

goniocentra
CRYPTOLECHIA

MERIDARCHIS

ARGYROPLOCE

LEPIDOSCIA

DACTYLETHRA

ACROCERCOPS

ALLOTALANTA

MALLOBATHRA

PTEROPHORUS

OINOPHILA

PROTONOMA*

COSMOPTERYX

HECTACMA

TARAGMARCHA

ANACOEMASTIS*

TYMBARCHA

ARGYROPLOCE

STATHMOPODA

ANTAEOTRICHA

CROCANTHES

TINEA

DRYADAULA*

EUNOMARCHA*

PHALONIA

ANCYLIS

OPOGONA

NEPTICULA

ORTHENCHES

EUCOSMA

AMPHISYNCENTRIS

DEPRESSARIA

GRACILARIA

SCYTHRIS

TANYARCHES*

LECITHOCERA

CYCLOPLASIS

GELECHIA

OCYSTOLA

STILBOSIS

EPICHARTA*

EUCOSMA

ODITES

GELECHIA

LINOCLOSTIS*

CTENOCOMPA

ETHMIA

ACROLOPHUS
I93I, Ann. Mus. Nac. Hist. Nat. Buenos Aires, 36: 396. (BM)

I938, Trans. R. Ent. Soc. London, 87: 5I9.

I9I4, Ann. South African Mus. Io: 243. (SA)

I9I I, Journ. Bombay Nat. Hist. Soc. 2I: I24.

I9Io, Journ. Bombay Nat. Hist. Soc. 20: 46I. (BM)

I93I, Exotic Microlepidoptera, 4: 48.

I9I4, Exotic Microlepidoptera, I: 234. (BM)

I9I4, Trans. Proc. New Zealand Inst. 46: II7.

Igo8, Trans. Ent. Soc. London, 40: 50I.

I9I I, Trans. Linnean Soc. London, I4: 298.

I938, in Caradja and Meyrick, Deuts. Ent. Zeit. Iris, 52:

I7.

I922, Exotic Microlepidoptera, 2: 567. (BM)

I926, Ann. South African Mus. 23: 34I. (SA)

1930, Trans. Ent. Soc. London, 78: 3I3. (P)

I9I4, Exotic Microlepidoptera, I: 229. (BM)

I9Io, Proc. Linnean Soc. N.S. Wales, 35: 286.

I928, Exotic Microlepidoptera. 3: 445. (BM)

I9I5, Exotic Microlepidoptera, I: 337.

I9I5, Exotic Microlepidoptera, I: 399. (BM)

I904, Proc. Linnean Soc. N.S. Wales, 29: 400.

I932, Trans. Ent. Soc. London, 80: I20.

I893, Proc. Linnean Soc. N.S. Wales, (Ser. 2) $7: 559$.

I923, Exotic Microlepidoptera, 3:26.

I928, Exotic Microlepidoptera, 3: 438. (BM)

I9I2, Exotic Microlepidoptera, I: 32. (BM)

I9I5, Ent. Mon. Mag. 5I : 29I.

I928, Exotic Microlepidoptera, 3:462.

I9I9, Trans. New Zealand Inst. I9I9: 353.

I9I8, Ann. Transvaal Mus. 6: Io. (T)

I933, Exotic Microlepidoptera, 4: 4I2.

I928, Exotic Microlepidoptera, 3: 475. (BM)

I934, Exotic Microlepidoptera, 4: 474 .

I9I6, Exotic Microlepidoptera, 2: Io.

I924, Exotic Microlepidoptera, 3: 99.

I938, in Caradja and Meyrick, Deuts. Ent. Zeit. Iris, 52:

6. (BM)

I9I7, Exotic Microlepidoptera, 2: 64 .

I926, Trans. Ent. Soc. London, 74: 277. (BM)

I885, Proc. Linnean Soc. N.S. Wales, 9: 1062.

I9I7, Exotic Microlepidoptera, 2: 47. (BM)

I926, Exotic Microlepidoptera, 3: 285. (T)

I928, Exotic Microlepidoptera, 3: 440. (T)

Igo8, Journ. Bombay Nat. Hist. Soc. I8: 633. (BM)

I927, Exotic Microlepidoptera, 3: 350. (BM)

Igo8, Journ. Bombay Nat. Hist. Soc. I8: 626. (BM)

I925, Treubia, 6: 433 .

1925, Treubia, 6: 43I.

I923, Exotic Microlepidoptera, 2: 609. (BM) 


goniodesma
goniometra
goniospila
goniozona
gonometra
gonoscia
gonosema
gonosemana
gonoteles
gorgonias
gorgopa
gracula
gradata

graminivora grammarcha grammatacma grammatica grammatistis grammatistis grammatopa grammatopis grammidias grammitis grammocentra grammocosma grammocrossa grammophanes grammophora grammotorna grammozona granata grandaevus grandinosa granifera granivora granosa granosa granularis granulata graphica graphicodes graphicopa graptosema grata gratifica gratiosa gratula gratulata
ACROCERCOPS LECITHOCERA GELECHIA

THEMELIOTIS HARPEDONISTIS* ALUCITA

EULECHRIA HETEROCROSSA GLYPHIPTERYX PROTOLECHIA ATASTHALISTIS NOTHRIS

BRACHMIA

[SCHEMATASPIS*] BACTRA TORTRICOMORPHA ACROCERCOPS EULECHRIA CLERARCHA IMMA DEPRESSARIA STATHMOPODA NEPHOGENES THIOTRICHA LEPTOCROCA TINEA

PERIACMA

LECITHOCERA

MACHAERITIS EULIA

IMMA ORNEODES PTEROPHORUS NEPTICULA OPOSTEGA DICHOMERIS BATRACHEDRA SAPHENEUTIS MELASINA CRONICOMBRA* EULECHRIA BRACHMIA HECTACMA CEROMITIA SECITIS* SCYTHRIS SIMAETHIS ARISTOTELIA LASPEYRESIA
1934, Exotic Microlepidoptera, 4: 47I.

1929, Exotic Microlepidoptera, 3: 522.

I931, Ann. Mus. Nac. Hist. Nat. Buenos Aires, 36: 385. (BM)

I922, Exotic Microlepidoptera, 2: 602. (BM)

I893, Proc. Linnean Soc. N.S. Wales, 7: 594.

I922, Exotic Microlepidoptera, 2: 549.

I888, Proc. Linnean Soc. N.S. Wales, (Ser. 2) 2: 953.

I883, Proc. Linnean Soc. N.S. Wales, 7: I79.

I907, Proc. Linnean Soc. N.S. Wales, 32: II9.

1904, Proc. Linnean Soc. N.S. Wales, 29: 380.

I9I8, Exotic Microlepidoptera, 2: II5.

I929, Exotic Microlepidoptera, 3: 495. (BM)

I9Io, Rec. Indian Mus. 2: 221. (K)

I922, Exotic Microlepidoptera, 2: 521. (BM)

I905, Journ. Bombay Nat. Hist. Soc. I6: 6II. (BM)

I92I, Exotic Microlepidoptera, 2: 468.

I883, Proc. Linnean Soc. N.S. Wales, 7: 545.

I89o, Trans. R. Soc. S. Australia, I3: 53 .

I906, Trans. Ent. Soc. London, I906: I9I. (BM)

I920, Ann. South African Mus. I7: 287. (SA)

I92I, Exotic Microlepidoptera, 2: 462.

I9I3, Exotic Microlepidoptera, I: I33.

I9o8, Journ. Bombay Nat. Hist. Soc. I8: 442. (BM)

I920, Exotic Microlepidoptera, 2: 367.

I888, Trans. Proc. New Zealand Inst. 20: 98.

I93I, in Caradja, Bull. Sect. sci. Acad. roum. I4: 72. (BM)

I926, Sarawak Mus. Journ. 3: I58.

I886, Proc. Linnean Soc. N.S. Wales, ro: 769 .

I926, Exotic Microlepidoptera, 3: 256. (BM)

I925. Exotic Microlepidoptera, 3: I35. (BM)

I921, Ann. Transvaal Mus. 8: Io8. (T)

I931, Ann. Mus. Nac. Hist. Nat. Buenos Aires, 36: 380.

I9II, Ann. Transvaal Mus. 2: 236. (T)

I9I3, Ann. Transvaal Mus. 3: 327. (T)

I932, Exotic Microlepidoptera, 4: 20I. (BM)

I9I I, Ann. Transvaal Mus. 2: 235. (T)

I9I2, Ann. South African Mus. I0: 70. (SA)

I916, Exotic Microlepidoptera, I: 609.

I920, Exotic Microlepidoptera, 2: 327. (BM)

I888, Proc. Linnean Soc. N.S. Wales, 2: 95I.

I9I4, Ann. Transvaal Mus. 4: I94. (T)

I921, Zool. Meded. 6: ig6.

I9I4, Ann. Transvaal Mus. 4: 205. (T)

I928, Exotic Microlepidoptera, 3: 472. (P)

I921, Exotic Microlepidoptera, 2: 440.

I9II, Trans. Linnean Soc. London, I4:290. (BM)

I928, Proc. Hawaiian Ent. Soc. 7: IOI. (SP)

I9I6, Exotic Microlepidoptera, 2: 23. (BM) 
gratus

gravata

gravescens

gravior

gravis

gravissima

gregalis

gregaria

griphodes

griseata

griseicoma

griseicornis

grypodes

grypodes

grypota

gubernata

gubernatrix

gummosa

guttifera

guttulata

gutturalis

gymnastica

gymnastis

gymnocentra

gymnolopha

gymnosopha

gymnota

gypsaspis

gypsatma

gypsina

gypsochra

gypsocoma

gypsodelta

gypsograpta

gypsolitha

gypsomicta

gypsomorpha

gypsopa

gypsopeda

gypsopetra

gypsophila

gypsopis

gypsopyga

gypsospora

gypsota

gypsoterma

gypsotes
DEUTEROCOPUS

ANARSIA

ANTAEOTRICHA

PLATYPTILIA

AGRIOPHARA

ACROCERCOPS

PHTHORIMAEA

MELASINA

EUCOSMA

OECOPHORA

TORTRIX

ELACHISTA

ACROCLITA

TRICHOTHYRSA

GRACILARIA

STENOMA

ANTAEOTRICHA

PHILOBOTA

LASPEYRESIA

AUTOSTICHA

ARGYROPLOCE

PHILOBOTA

STENOMA

PYRODERCES

ANTAEOTRICHA

METACHANDA

NEMOTOIS

HELCYSTOGRAMMA

MASTIGOSTOMA*

PLEUROTA

CRYPTOLECHIA

LINDERA

LABDIA

ADENONEURA

STENOMA

TINEA

SYMMOCA

OTOCHARES*

DELTOPLASTIS

MELASINA

ELACHISTA

TONICA

HELIOCAUSTA

AGRIOMELISSA *

COPROMORPHA*

STENOMA

PTEROPHORUS
I92I, Exotic Microlepidoptera, 2: 4I9.

I9I2, Ann. Transvaal Mus. 3:69. (T)

I926, Exotic Microlepidoptera, 3:237. (BM)

I932, Exotic Microlepidoptera, 4: 250. (v)

I89o, Trans. R. Soc. S. Australia, I3: 77.

I9I2, Exotic Microlepidoptera, I: 24. (T)

I9I7, Trans. Ent. Soc. London, I9I7: 43. (BM)

I9I6, Exotic Microlepidoptera, I: 6I3.

I9I2, Ent. Mon. Mag. 48: 34. (Sub.)

I884, Trans. Proc. New Zealand Inst. I6: 39.

I924, Exotic Microlepidoptera, 3: II5. (BM)

I932, Exotic Microlepidoptera, 4: 2 I8.

I9I2, Journ. Bombay Nat. Hist. Soc. 2I: 856. (BM)

I9I2, Exotic Microlepidoptera, I: 62.

I9I4, Ann. Transvaal Mus. 4:202. (T)

I9I5, Exotic Microlepidoptera, I: 465. (BM)

I925, Exotic Microlepidoptera, 3: I73. (BM)

I9I3, Exotic Microlepidoptera, I : I27.

I9I3, Trans. Ent. Soc. London, I9I3: I73. (BM)

I925, in Caradja, Mem. Sect. Sti. Acad. Românā. 3: 380.

I934, Ann. Mag. Nat. Hist. I4: 407. (BM)

I920, Exotic Microlepidoptera, 2: 3I4.

I9I5, Exotic Microlepidoptera, I: 453. (BM)

I937, Exotic Microlepidoptera, 5: I48. (C)

I925, Exotic Microlepidoptera, 3: I74. (BM)

I930, Trans. Ent. Soc. London, 78: 3I6. (P)

I9I2, Exotic Microlepidoptera, I: 30.

I921, Zool. Meded. 6: I63. (L)

I9I I, Trans. Linnean Soc. London, I4: 302.

I884, Proc. Linnean Soc. N.S. Wales, 9: 756.

I938, in Caradja and Meyrick, Deuts. Ent. Zeit. Iris, 52:

IO. (BM)

I93I, Exotic Microlepidoptera, 4: Io6.

I927. Insects of Samoa, 3, Lepidoptera, fasc. 2, p. 89.

I932, Exotic Microlepidoptera, 4: 222. (BM)

I93I, Exotic Microlepidoptera, 4:38. (v)

I93I, Ann. Mus. Nac. Hist. Nat. Buenos Aires, 36: 4II. (BM)

I928, Bull. Hill Mus. 2: 236. (Bм)

I9I9, Exotic Microlepidoptera, 2: 244. (BM)

I934, Exotic Microlepidoptera, 4: 5I4. (BM)

I937, Exotic Microlepidoptera, 5: II5. (T)

I9II, Ann. Transwaal Mus. 2: 233. (T)

I928, Exotic Microlepidoptera, 3: 474. (BM)

I9I4, Exotic Microlepidoptera, I: 220.

I93I, Exotic Microlepidoptera, 4: 5I.

I886, Trans. Ent. Soc. London, I886: 282.

I9I5, Exotic Microlepidoptera, I : 424. (BM)

I936, in Caradja and Meyrick, Deuts. Ent. Zeit. Iris, 50:

I55. 
gypsothicta

LASPEYRESIA

gypsozyga

gyracma

gyralea

gyritis

gyrospila

gyrotis

habilis

habrarcha

habrias

habristis

habrochroa

habrocosma

habrodes

habrophanes

habrophila

habropis

habroscia

habrotoma

habryntis

hadassa

hadromacha

haemanthes

haemanthes

haematantha

haemataula

haemataula

haematitis

\section{haematopa}

haematosema

haematospila

haemodryas

haemogastra

haemogastra

haemographa

haemograpta

haemoplecta

haemorrhanta

haemorrhoa

haemostacta

haemothicta

haemylopis

haeretica

hagnopa

hagnopis

halans
BORKHAUSENIA

HIEROXESTIS

NOEZA

PARAMORPHA

HETEROZYGA

ARGYROPLOCE

STENOMA

CYCLOPLASIS

IDIOPHANTIS*

HYPERCALLIA

PHYLLOCNISTIS

EUCHAETIS*

CONOPOMORPHA

EULECHRIA

ORNEODES

GYMNOBATHRA

ACROCERCOPS

EUCOSMA

MACHIMOSTOLA

AEGERIA

CRYPTOLECHIA

ACROPLECTIS*

PERSICOPTILA

ANTICRATES

MACHIMIA

PARECTOPA

EULIA

AEOLANTHES

STATHMOPODA

EUCOSMA

PYRODERCES

ERETMOCERA

PLATYPTILIA

CARCINA

LASPEY RESIA

TARUDA

CRYPTOLECHIA

THAMNOCRANA*

ARGYROPLOCE

EULIA

BRACHMIA

PTERNOZYGA*

PHYLLOCNISTIS

BUCCULATRIX

BATRACHEDRA
I938, Institut des Parcs Nationaux du Congo Belge, fasc. I4, P. II.

I93I, Exotic Microlepidoptera, 4: II8.

I9I5, Exotic Microlepidoptera, I: 365.

I922, Trans. Ent. Soc. London, I922: I06. (BM)

I9Io, Trans. Ent. Soc. London, I9I0: 431. (BM)

I9I8, Exotic Microlepidoptera, 2: 2I9.

I909, Journ. Bombay Nat. Hist. Soc. I9: 604. (вм)

I9I5, Exotic Microlepidoptera, I: 427. (BM)

I9I7, Exotic Microlepidoptera, 2: 64 .

1904, Proc. Linnean Soc. N.S. Wales, 29: 298.

I9I4, Exotic Microlepidoptera, I: I89. (BM)

I9I5, Exotic Microlepidoptera, I: 349.

I883, Proc. Linnean Soc. N.S. Wales, 7: 484.

I907, Proc. Linnean Soc. N.S. Wales, 32: 57.

I883, Proc. Linnean Soc. N.S. Wales, 7: 532.

I920, Ann. South African Mus. I7: 293. (SA)

I888, Trans. Proc. New Zealand Inst. 20: 80.

I921, Exotic Microlepidoptera, 2: 467. (BM)

I934, Exotic Microlepidoptera, 4:485.

I930, Exotic Microlepidoptera, 4:8.

1932, Exotic Microlepidoptera, 4: 336.

I937, Exotic Microlepidoptera, 5:87. (T)

I927, Exotic Microlepidoptera, 3: 370. (BM)

I935, Exotic Microlepidoptera, 4: 605 .

I924, Exotic Microlepidoptera, 3: I2I.

I9I2, Trans. Ent. Soc. London, I9I I: 699. (BM)

I9I2, in Wytsman, Genera Insectorum fasc. I28, p. $2 \mathrm{I}$.

I93I, Ann. Mus. Nac. Hist. Nat. Buenos Aires, 36: 38I.

(BM)

I93I, in Caradja, Bull. Sect. sci. Acad. roum. I4: 73. (Bu)

I933, Exotic Microlepidoptera, 4: 43I.

I92I, Ann. Transvaal Mus. 8: 55. (T)

I930, Exotic Microlepidoptera, 3:546.

1936, Exotic Microlepidoptera, 5: 62. (c)

I926, Exotic Microlepidoptera, 3: 297.

1937, Exotic Microlepidoptera, 5: 84. (c)

I928, Exotic Microlepidoptera, 3: 45I. (BM)

I93I, Exotic Microlepidoptera, 4: I22. (v)

I924, Exotic Microlepidoptera, 3: I05.

1927, Exotic Microlepidoptera, 3:383. (T)

I93I, Exotic Microlepidoptera, 4: I32.

I926, Exotic Microlepidoptera, 3: 257. (BM)

I9II, Journ. Bombay Nat. Hist. Soc. 20: 7I6. (BMI)

I908, Journ. Bombay Nat. Hist. Soc. I8: 621. (BMI)

I920, Exotic Microlepidoptera, 2: 359.

I930, Exotic Microlepiáptera, 4: 7 .

I924, Exotic Microlepidoptera, 3: 92. (BM) 


\begin{tabular}{|c|c|c|}
\hline halantha & ARGYROPLOCE & I909, Journ. Bombay Nat. Hist. Soc. I9: 6ог. (вм) \\
\hline halarcta & AGRIOPHARA & I9I7, Exotic Microlepidoptera, 2: 59. (BM) \\
\hline halata & OCYSTOLA & I9I3, Exotic Microlepidoptera, I: II7. (MA) \\
\hline halianassa & POLYORTHA & I932, Exotic Microlepidoptera, 4:264. (v) \\
\hline haliclysta & PROMALACTIS & I9o8, Journ. Bombay Nat. Hist. Soc. I8: 8o8. (BM) \\
\hline halidora & ACROLOPHUS & I9I5, Trans. Ent. Soc. London, I9I5: 253. (BM) \\
\hline halidora & OXYGRAPHA & I9o8, Journ. Bombay Nat. Hist. Soc. I8: 624. (BM) \\
\hline halieutica & MACROPIRATIS* & I932, Exotic Microlepidoptera, 4: 249 . \\
\hline halieutis & MELASINA & I9o8, Proc. Zool. Soc. London, p. 743. \\
\hline haligramma & CHELARIA & I926, Exotic Microlepidoptera, 3:282. (BM) \\
\hline halimora & SIMAETHIS & I9I2, Exotic Microlepidoptera, I: 50. \\
\hline haliphaea & PERIACMA & I9Io, Journ. Bombay Nat. Hist. Soc. 20: I6o. (BM) \\
\hline haliphanes & VANICELA & I927, Insects of Samoa, 3, Lepidoptera, fasc. 2, p. 97. \\
\hline haliplancta & DEMOBROTIS & I927, Exotic Microlepidoptera, 3: 325. (BM) \\
\hline halirrhothia & HARMOLOGA & I938, Trans. R. Ent. Soc. London, 87: 507. (BM) \\
\hline halisparta & ANCYLIS & I909, Ann. Transvaal Mus. $2: 6$, pl. 2, fig. 8. (T) \\
\hline halistrepta & MACHAEROPTERIS & I9II, Journ. Bombay Nat. Hist. Soc. 21 : I28. \\
\hline halithea & MASTIGOSTOMA & I927, Exotic Microlepidoptera, 3: 330. (BM) \\
\hline halixanta & ARGYROPLOCE & I9Io, Trans. Ent. Soc. London, I9Io: 435. (BM) \\
\hline halmas & STENOMA & I925, Exotic Microlepidoptera, 3: 200. (BM) \\
\hline halmeuta & POLYPSECTA* & $\begin{array}{l}\text { I930, Ann. Naturhist. Mus. Wien, 44: 265, pl. 2, fig. } 23 . \\
\text { (v) }\end{array}$ \\
\hline odes & COLEOPHORA & I9II, Ann. Transvaal Mus. 2: 235. (T) \\
\hline halmopeda & EULECHRIA & I888, Proc. Linnean Soc. N.S. Wales, $2: 963$. \\
\hline halmyra & ANACAMPSIS & I9I4, Trans. Ent. Soc. London, I9I4: 262. \\
\hline halmyris & LASPEYRESIA & I9o9, Ann. Transvaal Mus. 2: 9, pl. 3, fig. 9. (T) \\
\hline halmyrodes & SCYTHRIS & I92I, Ann. Transvaal Mus. 8: II6. (T) \\
\hline halmyropis & EPIDOLA & I926, Exotic Microlepidoptera, 3: 270. \\
\hline halobapta & HYPERCALLIA & I930, Exotic Microlepidoptera, 3: 575. (P) \\
\hline halodelta & AGDISTIS & I925, Bull. Soc. R. Ent. Egypte, 9:207. \\
\hline halogramma & APHANOPTIS* & I927, Boll. Soc. Ent. Italiana, 59: I6I. \\
\hline halonitis & IMMA & I920, Exotic Microlepidoptera, $2: 337$. (BM) \\
\hline halophanta & CTENOCOMPA & I92I, Ann. Transvaal Mus. 8: I34. (T) \\
\hline halosema & ACROLEPIA & $\begin{array}{l}\text { I93I, Ann. Mus. Nac. Hist. Nat. Buenos Aires, } 36: 404 \text {. } \\
\text { (BM) }\end{array}$ \\
\hline halosparta & ARCHYALA & I9I9, Trans. Proc. New Zealand Inst. 5I: 354. \\
\hline halosphora & ATELOSTICHA & I9I6, Exotic Microlepidoptera, I: $547 \cdot$ (BM) \\
\hline halospila & MONOPIS & I9I9, Exotic Microlepidoptera, 2: 239. (BM) \\
\hline halosticta & ACROLEPIA & I9I4, Exotic Microlepidoptera, I: 286. \\
\hline halticopa & LABDIA & I927, Insects of Samoa, 3, Lepidoptera, fasc. 2, p. 86 \\
\hline halurga & CROCANTHES & I904, Proc. Linnean Soc. N.S. Wales, 29:399. \\
\hline halycopa & GELECHIA & I927, Exotic Microlepidoptera, 3: 350. (BM) \\
\hline hamadelpha & MNESARCHAEA & I888, Trans. Proc. New Zealand Inst. 20: 9I. \\
\hline nalitha & XYSMATODOMA & I893, Proc. Linnean Soc. N.S. Wales, 7: 502. \\
\hline nata & MACROBATHRA & I93I, Exotic Microlepidoptera, 4: II6. (BM) \\
\hline hamaxastra & ETHMIA & I930, Exotic Microlepidoptera, 3: 563. (BM) \\
\hline naxitodes & MACROBATHRA & I886, Proc. Linnean Soc. N.S. Wales, Io: 802. \\
\hline nifera & COSMOPTERYX & I909, Journ. Bombay Nat. Hist. Soc. I9: 420. \\
\hline ulata & THIOTRICHA & I921, Zool. Meded. 6: I62. (L) \\
\hline alactis & PERONEA & I9I2, Exotic Microlepidoptera, I: I8. (BM) \\
\hline
\end{tabular}


hapalarga

hapalaspis

hapalocharis

hapalochra

hapalodes

hapalopa

hapalopis

hapalosarca

hapalotoxa

hapalyntis

haplistes

haplocentra

haplochrysa

haplocnista

haplocosma

haplodes

haplodora

haplodoxa

haplodryas

haplogramma

haplomorpha

haplonoma

haplonota

haplotherma

haploxyla

haplozona

hapsicora

hapsidota

hapsimacha

hapula

harmodes

harmodia

harmographa

harmonia

harmonia

harmonias

harmonica

harmonica

harmosta

harmosta

harmostis

harpactes

harpactis

harpagacma

harpalea

harpastis

harpobathra

harpoceros

harpodes
ACROCERCOPS

ACROCLITA

GRACILARIA

ERIDACHTHA

PHYLLOCNISTIS

HIEROXESTIS

PARAMORPHA

EUCOSMA

LITHOCOLLETIS

BRACHMIA

PTEROPHORUS

ANTAEOTRICHA

HOPLOPHANES

PSEUDODOXIA

ACROCERCOPS

TORTRIX

TINEA

PROMENESTA

CTENOPSEUSTIS

ODITES

CRYPHIOXENA*

ODITES

THUDACA

HAPSIFERA

STENOMA

DIACTENIS

ANTAEOTRICHA

ACROCERCOPS

AMPHIXYSTIS*

PHILOBOTA

DICHELOPA

ERIOPTRIS*

PHALAROCARPA*

EPAGOGE

POLYCHROSIS

DICHOMERIS

BRENTHIA

PLATYPEPLUS

PATANOTIS*

SYNTOMACTIS

MACROBATHRA

PTEROPHORUS

SAROPLA

IMMA

OPOGONA

EPIPHTHORA

ANTAEOTRICHA

STENOMA

PICRODOXA*
I9I6, Exotic Microlepidoptera, 2: I.

I93I, Exotic Microlepidoptera, 4: I44. (BM)

I935, Exotic Microlepidoptera, 4: 599.

I932, Exotic Microlepidoptera, 4: 203.

I906, Trans. R. Soc. S. Australia, 30:63.

I922, Exotic Microlepidoptera, 2: 559. (BM)

I9Io, Proc. Linnean Soc. N.S. Wales, 35: I56.

1924, Exotic Microlepidoptera, 3:67. (BM)

I92I, Exotic Microlepidoptera, 2: 4I5.

I9I I, Journ. Bombay Nat. Hist. Soc. 20: 724. (вм)

I936, Exotic Microlepidoptera, 4: 626.

1925, Exotic Microlepidoptera, 3: I70. (Bм)

I897, Proc. Linnean Soc. N.S. Wales, 22: 4 IO.

I939, Trans. R. Ent. Soc. London, 89: 58.

I936, Exotic Microlepidoptera, 5: 35.

I9Io, Proc. Linnean Soc. N.S. Wales, 35: 253.

19I7, Exotic Microlepidoptera, 2: 79. (MA)

I925, Exotic Microlepidoptera, 3: I62. (BM)

I920, Voyage de Ch. Alluand et $R$. Jeannel en Afrique

Oriental, II, Microlepidoptera, p. 54. (P)

I930, Exotic Microlepidoptera, 4: I7. (BM)

I921, Ann. Transvaal Mus. 8: I23. (T)

I9I5, Exotic Microlepidoptera, I: 379. (BM)

I893, Proc. Linnean Soc. N.S. Wales, 7: 573.

I934, Exotic Microlepidoptera, 4: 5I6. (c)

I9I5, Exotic Microlepidoptera, I: 420. (BM)

I92I, Zool. Meded. 6: I49. (L)

I9I5, Exotic Microlepidoptera, I: 399. (BM)

I9I5, Trans. Ent. Soc. London, I9I5: 229

I902, Trans. Ent. Soc. London, I902: 577.

I884, Proc. Linnean Soc. N.S. Wales, 8: 489.

I929, Trans. Ent. Soc. London, 76: 494. (BM)

I9I5, Trans. Ent. Soc. London, I9I 5: 244.

I937, Exotic Microlepidoptera, 5: I29. (BM)

I908, Journ. Bombay Nat. Hist. Soc. I8: 6г7. (вм)

I908, Proc. Zool. Soc. London, p. 7I6. (BM)

I922, Exotic Microlepidoptera, 2: 5I4. (P)

I9I8, Exotic Microlepidoptera, 2: I92. (BM)

I905, Journ. Bombay Nat. Hist. Soc. I6: 584. (вм)

I913, Exotic Microlepidoptera, I: 80.

I897, Proc. Linnean Soc. N.S. Wales, 22: 388.

I889, Proc. Linnean Soc. N.S. Wales, (Ser. 2) 3: I679.

I908, Trans. Ent. Soc. London, I907: 495.

I889, Proc. Linnean Soc. N.S. Wales, (Ser. 2) 3: I648.

1935, Exotic Microlepidoptera, 4: 574. (BM)

I9I I, Trans. Linnean Soc. London, I4: 293.

Ig04, Proc. Linnean Soc. N.S. Wales, 29: 267.

I9I6, Exotic Microlepidoptera, I : 499. (BM)

I930, Ann. Naturhist. Mus. Wien, 44:255. (v)

I923, Exotic Microlepidoptera, 2:6I7. (BM) 


\begin{tabular}{|c|c|c|}
\hline harpophora & CHELARIA & I92I, Exotic Microlepidoptera, 2: 43I. (вм) \\
\hline haruspex & EBODA & I9I2, Exotic Microlepidoptera, I: I5. (BM) \\
\hline hastata & DICHOMERIS & I9I8, Exotic Microlepidoptera, 2: I52. (вм) \\
\hline hastifera & LABDIA & I920, Exotic Microlepidoptera, 2: 3I8. (Bм) \\
\hline hastigera & ACROCERCOPS & I9I5, Trans. Ent. Soc. London, I9I5: 227. \\
\hline hebaea & PSYCHROMNESTRA & I924, Exotic Microlepidoptera, 3:89. \\
\hline hebetata & BACTRA & I9II, Ann. Transvaal Mus. 2:227. \\
\hline hebrus & PTEROPHORUS & I932, Exotic Microlepidoptera, 4: 25I. (v) \\
\hline hecataea & BRENTHIA & I907, Proc. Linnean Soc. N.S. Wales, 32: Iog. \\
\hline hectaea & ARGYROTOXA & I9II, Ann. Transvaal Mus. $2: 224$. (T) \\
\hline hectaea & IMMA & I9o6, Trans. Ent. Soc. London, I9o6: I89. (вм) \\
\hline hecteracma & DICHOMERIS & I923, Exotic Microlepidoptera, 2: 622. (Bм) \\
\hline hectorea & GONIOTERMA & I9I5, Exotic Microlepidoptera, I: 384 . (вм) \\
\hline hecuba & CROCANTHES & I93I, Exotic Microlepidoptera, 4: 74 . \\
\hline hedraea & PLATYPEPLUS & I905, Joum. Bombay Nat. Hist. Soc. I6: 584 (Bм) \\
\hline hedrastis & CACOECIA & Igo8, Proc. Zool. Soc. London, p. 722. \\
\hline hedrastis & MACROBATHRA & I9I5, Exotic Microlepidoptera, I: 295. (Bм) \\
\hline hedrotoma & ARGYROPLOCE & $\begin{array}{l}\text { I938, in Caradja and Meyrick, Deuts. Ent. Zeit. Iris, } 52 \text { : } \\
\text { I. (BM) }\end{array}$ \\
\hline helarcha & BATRACHEDRA & I897, Proc. Linnean Soc. N.S. Wales, 22: 305. \\
\hline helena & LABDIA & I928, Exotic Microlepidoptera, 3: 388. \\
\hline heliaca & MELOCHRYSIS* & I9I6, Exotic Microlepidoptera, I: 544. (вм) \\
\hline heliacma & ARTIASTIS & I889, Proc. Linnean Soc. N.S. Wales, (Ser. 2) 3: I674. \\
\hline heliactis & COSMOPTERYX & I897, Proc. Linnean Soc. N.S. Wales, 22: 340. \\
\hline heliadelpha & MERITASTIS & I932, Exotic Microlepidoptera, 4: 26I. (v) \\
\hline heliadopa & GNORISMONEURA & $\begin{array}{l}\text { I937, in Caradja and Meyrick, Deuts. Ent. Zeit. Iris, 5I: } \\
\text { I72. }\end{array}$ \\
\hline heliantha & LACTURA & I9I8, Exotic Microlepidoptera, 2: Igo. \\
\hline helianthes & SPARGANOTHIS & I932, Exotic Microlepidoptera, 4:264. (v) \\
\hline heliapta & MACRERNIS* & I887, Trans. Ent. Soc. London, I887: 275 . (вм) \\
\hline heliarcha & CROCANTHES & I886, Trans. Ent. Soc. London, I886: 278 . \\
\hline helias & PELTOPHORA & I884, Proc. Linnean Soc. N.S. Wales, 9: 733. \\
\hline heliaspis & CACOECIA & I909, Ann. South African Mus. 5: 349. (SA) \\
\hline heliaspis & TORTYRA & I926, Exotic Microlepidoptera, 3: 303. (BM) \\
\hline heliastis & PLATYPTILIA & I885, Trans. Proc. New Zealand Inst. I7: I29. \\
\hline heliatma & PERSICOPTILA & I935, Exotic Microlepidoptera, 4: 606. \\
\hline heliaula & ATTERIA & I9Io, Trans. Ent. Soc. London, I9Io: 433. (Bм) \\
\hline helica & PHLOEOPOLA & I883, Proc. Linnean Soc. N.S. Wales, 8: 35I. \\
\hline helicana & PENTHINA & I88I, Proc. Linnean Soc. N.S. Wales, 6: 645. \\
\hline helicaula & PALTODORA & I9I2, Ann. South African Mus. IO: 59. (SA) \\
\hline helicias & ANTAEOTRICHA & I9I6, Exotic Microlepidoptera, I: 502. (BM) \\
\hline helicodes & PHYLLOCNISTIS & I9I6, Exotic Microlepidoptera, I: 618. \\
\hline helicomitra & ACROCERCOPS & I924, Exotic Microlepidoptera, 3: 85. (BM) \\
\hline heliconia & COMOCRITIS & I933, Exotic Microlepidoptera, 4: 374. (BM) \\
\hline helicopa & ACROCERCOPS & I9I9, Exotic Microlepidoptera, 2: 23I. \\
\hline helicopis & STROBISIA & \\
\hline & [PARELECTRA*] & I922, Trans. Ent. Soc. London, I922: IOI. (BM) \\
\hline icosema & NEOPHYLARCHA* & I926, Exotic Microlepidoptera, 3:240. (вм) \\
\hline icosticta & GELECHIA & I929, Exotic Microlepidoptera, 3: 490. (вм) \\
\hline elictis & LINOSTICHA & I889, Proc. Linnean Soc. N.S. Wales, (Ser. 2) 3: I 584. \\
\hline
\end{tabular}


heliocephala

heliochalca

heliochares

heliochra

heliochrosta

helioclina

heliocoma

heliodepta

heliodora

heliodoxa

heliogramma

heliograpta

heliomima

heliopepta

heliophanes

heliopla

heliostoma

heliota

heliotoxa

helioxantha

heliozona

helminthias

helobia

helonoma

helonoma

helophaea

helota

helota

helotypa

hemera

hemerana

hemeris

hemeropa

hemiacma

hemiacta

hemiadelpha

hemiargus

hemiarthra

hemibathra

hemicalypta

hemicamina

hemicarpa

hemicasis

hemicatharta

hemicausta
LAMPRONIA

NEMOTOIS

XYSMATODOMA

SCLEROCOPA

EUXANTHIS

TIPHA

EULECHRIA

HYPERCALLIA

EULECHRIA

ATTEVA

HIEROXESTIS

CROCANTHES

HYPERCALLIA

ENCOLPOTIS

ARGYROPLOCE

CONOPOMORPHA

CHAMANTHEDON

BATRACHEDRA

DECADARCHIS

AMORBIA

SENTICA
I93I, Ann. Mus. Nac. Hist. Nat. Buenos Aires, 36: $4^{\text {I4 }}$.

I928, Exotic Microlepidoptera, 3: 464.

I893, Proc. Linnean Soc. N.S. Wales, 7: 498.

I937, Exotic Microlepidoptera, 5: 97.

I928, Exotic Microlepidoptera, 3: 437. (BM)

I894, Trans. Ent. Soc. London, I894: I9. (BM)

I888, Proc. Linnean Soc. N.S. Wales, (Ser. 2) 2: 948.

I932, Exotic Microlepidoptera, 4: 280.

I888, Proc. Linnean Soc. N.S. Wales, (Ser. 2) 2: 948.

I9Io, Trans. Ent. Soc. London, I9Io: 47 I.

I9II, Trans. Linnean Soc. London, I4: 295.

I929, Exotic Microlepidoptera, 3: 520. (BM)

I930, Exotic Microlepidoptera, 3:576. (BM)

I9I8, Exotic Microlepidoptera, 2: I53. (BM)

I922, Exotic Microlepidoptera, 2: 523. (BM)

I907, Proc. Linnean Soc. N.S. Wales, 32: 57.

I926, Exotic Microlepidoptera, 3:267. (BM)

I9I3, Ann. Transvaal Mus. 3: 323. (T)

I935, Exotic Microlepidoptera, 4:568.

I9I7, Trans. Ent. Soc. London, I9I7: I2. (BM)

I893, Proc. Linnean Soc. N.S. Wales, 7: 487.

SCHIFFermuelleria IgI8, Ann. Transvaal Mus. 6: 29. (T)

MACHIMIA

ELACHISTA

PHALONIA

BACTRA

ISOCHORISCA

NOTOCELIA

[ACHARNEODES*]

BAEONOMA

MACHAERITIS

AROTROPHORA

PHILOBOTA

ILINGIOTIS

LECITHOCERA

[BRACHYERGA*]

HYSTEROSIA

ROESLERSTAMMIA

PSELNOPHORUS

CRYPTOLECHIA

ANTAEOTRICHA

OCYSTOLA

SISYROCTENIS*

EULECHRIA

HYPOSMOCOMA

ANCYLIS

THRIAMBEUTIS*
I93I, Journ. Linnean Soc. London, 37: 28I.

I889, Trans. Proc. New Zealand Inst. 2I: I78.

I9I2, Ent. Mon. Mag. 48: 35. (Sub.)

I928, Exotic Microlepidoptera, 3: 442. (BM)

I9Io, Proc. Linnean Soc. N.S. Wales, 35: I68.

I905, Journ. Bombay Nat. Hist. Soc. I6: 586. (BM)

I9I6, Exotic Microlepidoptera, I: 508. (BM)

I886, Proc. Linnean Soc. N.S. Wales, Io: 77 I.

I883, Proc. Linnean Soc. N.S. Wales, 7: 176.

I9I5, Exotic Microlepidoptera, I: 303.

I923, Exotic Microlepidoptera, 3:2. (BM)

I9Io, Trans. Ent. Soc. London, I9IO: 448. (BM)

I920, Voyage de Ch. Alluand et R. Jeannel en Afrique

Oriental, II, Microlepidoptera, p. 45. (P)

I922, Exotic Microlepidoptera, 2: 553.

I908, Trans. Ent. Soc. London, I907: 49I.

I922, Exotic Microlepidoptera, 2: 546. (BM)

I932, Exotic Microlepidoptera, 4: 290.

I885, Proc. Linnean Soc. N.S. Wales, 9: Io6r.

I935, Arb. morph.taxon. Ent. Berlin-Dahlem, 3: I05. (D)

I888, Proc. Limnean Soc. N.S. Wales, (Ser. 2) 2: 954.

I935, Proc. Hawaiian Ent. Soc. 9: 68. (SP)

I935, in Caradja and Meyrick, Materialien zu einer Micro-

lepidopteren Fauna der Chinesischen Provinzen Kiangsu,

Chekiang und Hunan, p. 54. (BM)

I9Io, Trans. Ent. Soc. London, I9IO: 470. 


$\begin{array}{ll}\begin{array}{l}\text { hemichlora } \\ \text { hemichroa }\end{array} & \text { AGRIOPHARA } \\ \text { hemichrysa } & \text { ELECTRACMA* } \\ \text { hemicitra } & \text { MONOPIS } \\ \text { hemiclina } & \text { TRICHOTAPHE } \\ \text { hemiclista } & \text { DIPTERINA } \\ & \text { [ECCLITICA*] } \\ \text { hemiclistra } & \text { DECADARCHIS } \\ \text { hemiconis } & \text { ANTIOLOPHA } \\ \text { hemicopa } & \text { THRANITICA* } \\ \text { hemicosma } & \text { ETHMIA }\end{array}$

\section{hemicosmana CAPUA}

hemicrates

hemicrossa

hemicryptana

hemicycla

hemidictyas

hemidoma

hemidoxa

hemidryas

hemigastra

hemigenes

hemiglypta

hemigrapta

hemigymna

hemilampra

hemileuca

hemileuca

hemileucas

hemilyca

hemimetra

hemimochla

heminephela

hemionana

hemiopa

hemiopta

hemipelta

hemipempta

hemipercna

hemiphaea

hemiphanes

hemiphanta

hemiphara

hemiphracta

hemiphragma

hemiphragma

hemiplaca

hemiplecta
ANTIGRAPTIS*
GELECHIA

ASTHENOPTYCHA *

TELPHUSA

SCYTHRIS

LIMNOECIA

OPOGONA

HOMALOXESTIS

HOPLITICA

ACROCERCOPS

ARGYROPLOCE

ODITES

STENOMA

HYPONOMEUTA

TORTRIX

COMPSOLECHIA

MACROSACES

PLATYPTILIA

OECOPHORA

MACROBATHRA

PROSELENA

[EPICHORISTA*]

BRACHMIA

PLATYPEPLUS

ANATRACHYNTIS

GLYPHIPTERYX

ODITES

IDIOPHANTIS

EULECHRIA

STENOMA

DEMOBROTIS

CRYPSITHYRIS

COESYRA

HOPLOPHANES

ARGYROPLOCE

STATHMOPODA
LASPEYRESIA
I9I6, Exotic Microlepidoptera, I: 487. (BM)

I906, Journ. Bombay Nat. Hist. Soc. I7: 4I3. (Ві)

т9Iо, Joum. Bombay Nat. Hist. Soc. 20: 447. (BM)

I9o6, Journ. Bombay Nat. Hist. Soc. I7: 4I7.

I929, Exotic Microlepidoptera, 3: 5I2. (BM)

I905, Trans. Ent. Soc. London, I905: 233.

I9I I, Trans. New Zealand Inst. 43: 77.

I894, Trans. Ent. Soc. London, I894: 25.

I908, Proc. Zool. Soc. London, p. 743.

I920, Voyage de Ch. Alluand et $R$. Jeannel en Afrique

Oriental, II, Microlepidoptera, p. 89. (P)

I88I, Proc. Linnean Soc. N.S. Wales, 6: 449.

I930, Exotic Microlepidoptera, 3:6 13. (BM)

I927, Exotic Microlepidoptera, 3: 345. (BM)

I88I, Proc. Linnean Soc. N.S. Wales, 6: 46 I.

1932, Exotic Microlepidoptera, 4: 350. (v)

I928, Exotic Microlepidoptera, 3: 4I4. (BM)

I897, Proc. Linnean Soc. N.S. Wales, 22: 362.

I907, Journ. Bombay Nat. Hist. Soc. I8: I45. (BM)

I9I5, Trans. Ent. Soc. London, I9I5: 252.

I93I, Exotic Microlepidoptera, 4: 77. (BM)

I889, Proc. Linnean Soc. N.S. Wales, (Ser. 2) 3: 596.

I9I6, Exotic Microlepidoptera, 2: I.

I931, Exotic Microlepidoptera, 4: I33. (BM)

I930, Exotic Microlepidoptera, 4: I8. (P)

I9I5, Exotic Microlepidoptera, I: 470. (BM)

1932, Exotic Microlepidoptera, 4: 346. (BM)

I932, Exotic Microlepidoptera, 4: 256. (v)

I922, Trans. Ent. Soc. London, I922: 82. (BM)

I9Io, Journ. Bombay Nat. Hist. Soc. 20: I49. (BM)

I886, Trans. Ent. Soc. London, I886: I8.

I883, New Zealand Journ. Sci. I : 524 .

I886, Proc. Linnean Soc. N.S. Wales, го: 806.

I883, Trans. Proc. New Zealand Inst. I5: 43.

I92I, Ann. Transvaal Mus. 8: 9o. (T)

I905, Journ. Bombay Nat. Hist. Soc. I6: 586. (Bм)

I9I7, Exotic Microlepidoptera, 2: 44.

I909, Journ. Bombay Nat. Hist. Soc. I9: 429. (BM)

I9I4, Exotic Microlepidoptera, I: 282. (BM)

I907, Journ. Bombay Nat. Hist. Soc. I8: I49. (BM)

I883, Proc. Linnean Soc. N.S. Wales, 7: 529 .

1925, Exotic Microlepidoptera, 3: I98. (BM)

I893, Proc. Linnean Soc. N.S. Wales, 7: 557.

I926, Exotic Microlepidoptera, 3: 320. (T)

i889, Proc. Linnean Soc. N.S. Wales, (Ser. 2) 3: i659.

I897, Proc. Linnean Soc. N.S. Wales, 22: 4II.

I922, Exotic Microlepidoptera, 2: 525. (P)

I92I, Ann. Transvaal Mus. 8: Iog. (T) 


hemipyra
hemiscia
hemisema
hemisphaerica
hemispila
hemispora
hemistacta
hemiteles
hemitephras

hemithalama

hemithia

hemitorna

hemitropa

hemixantha

hemixysta

hemizona

hemizopha

heniocha

hepatica

hepatitis

hephaestea

heptacentra

heptacentra

heptachalca

heptachora

heptacopa

heptacosma

heptadeta

heptagramma

heptalitha

heptametra

heptanoma

heptarcha

heptaspila

heptasticta

heptazona

herbacea

herbicola

herbifera

herbosa

hercogramma

hercoptila

hercoscelis

hercotis

herifuga

hermaea

hermatias

hermatica

hermatodes
CROCANTHES

HELIOCAUSTA

OCYSTOLA

OECOPHORA

COMPSOTROPHA

LYONETIA

ACROCERCOPS

HELIOCAUSTA

ANTAEOTRICHA

MELASINA

ECHINOSCELIS*

STATHMOPODA

MACROBATHRA

CACOECIA

TISIS

LEUCOPTERA

ANATRACHYNTIS

MACHAERITIS

AMORBAEA*

HOPLITICA

PLACOCOSMA

METZNERIA

PARAMORPHA

HOPLOPHRACTIS*

MACHAERITIS

LASPEYRESIA

BRENTHIA

ACROCERCOPS

SCYTHOSTOLA*

SCHIFFERMUELLERIA I93I, Exotic Microlepidoptera, 4: I86. (BM)

PARECTOPA

HETEROGYMNA

OENOCHROA

MERIDARCHIS

ODITES

HAPSIFERA

STENOMA

LEPIDOSCIA

ARGYROPLOCE

ARGYROPLOCE

TRICHOTAPHE

ACROCLITA

GRACILARIA

CARPOSINA

STENOMA

SPHYRELATA

HIEROXESTIS

[ORTHOCHTHA*]

ODITES

HILAROGRAPHA
I938, Trans. R. Ent. Soc. London, 87: 5I3. (BM)

I883, Proc. Linnean Soc. N.S. Wales, 7: 472.

1885, Proc. Linnean Soc. N.S. Wales, 9: I063.

I886, Proc. Linnean Soc. N.S. Wales, Io: 780 .

I889, Proc. Linnean Soc. N.S. Wales, (Ser. 2) $3: 1636$.

I924, Exotic Microlepidoptera, 3: 8I.

I924, Exotic Microlepidoptera, 3: 85.

I883, Proc. Linnean Soc. N.S. Wales, $7: 475$.

I930, Ann. Naturhist. Mus. Wien, 44: 236. (v)

1935, Exotic Microlepidoptera, 4: 578.

I886, Trans. Ent. Soc. London, I886: 292.

I9I3, Exotic Microlepidoptera, I: 97.

I886, Proc. Linnean Soc. N.S. Wales, Io: 8I7.

I9I8, Exotic Microlepidoptera, 2: I65. (BM)

I9Io, Trans. Ent. Soc. London, I9Io: 438. (ВM)

I906, Trans. R. Soc. S. Australia, 30: 61.

I9I6, Exotic Microlepidoptera, I: 565.

I886, Proc. Linnean Soc. N.S. Wales, , Io: 769 .

I908, Journ. Bombay Nat. Hist. Soc. I8: 627. (BM)

I902, Trans. R. Soc. S. Australia, 26: I47. (MA)

I883, Proc. Linnean Soc. N.S. Wales, 8: 333 .

I9I2, Ann. Transvaal Mus. 3: 64. (T)

I931, Trans. Proc. New Zealand Inst. 62: 96.

I920, Exotic Microlepidoptera, 2: 326. (BM)

I9I5, Exotic Microlepidoptera, I: 30I.

I9I6, Exatic Microlepidoptera, I: 564. (BM)

I920, Exotic Microlepidoptera, 2: 336. (BM)

I936, Exotic Microlepidoptera, 5: 34.

I925, Treubia, 6: 429 .

I9I5, Trans. Ent. Soc. London, I9I5: 235.

I925, Exotic Microlepidoptera, 3: I38.

I889, Proc. Linnean Soc. N.S. Wales, (Ser. 2) 3: 1576.

I930, Exotic Microlepidoptera, 3: 589. (BM)

I9I4, Exotic Microlepidoptera, I: 28I. (BM)

I93I, Exotic Microlepidoptera, 4: I07.

I93I, Exotic Microlepidoptera, 4:38. (v)

I92I, Exotic Microlepidoptera, 2: 476. (BM)

I909, Journ. Bombay Nat. Hist. Soc. I9: 603. (BM)

I9o9, Journ. Bombay Nat. Hist. Soc. I9: 600. (BM)

I92I, Ann. Transvaal Mus. 8: 86. (T)

I927, Exotic Microlepidoptera, 3: 333. (BM)

I939, Trans. R. Ent. Soc. London, 89: 60.

I9I3, Exotic Microlepidoptera, I: 76. (BM)

I932, Exotic Microlepidoptera, 4: 298. (BM)

I9I4, Exotic Microlepidoptera, I: I72.

I9I I, Trans. Linnean Soc. London, I4: 296.

I9I5, Exotic Microlepidoptera, I: 378. (BM)

I909. Journ. Bombay Nat. Hist. Soc. I9: 426. (BM) 


\begin{tabular}{|c|c|c|}
\hline nermatopis & EULECHRIA & I922, Arkiv för Zool. vol. I4, part I5, p. 7 . \\
\hline hermione & CYCLOPONYMPHA & I92I, Ann. Transvaal Mus. 8: I25. (T) \\
\hline hermodora & COSMOPTERYX & I9I9, Exotic Microlepidoptera, 2: 282. (BM) \\
\hline hermophila & DEPRESSARIA & I922, Exotic Microlepidoptera, 2: 5I3. (P) \\
\hline heroica & PERSICOPTILA & I928, Exotic Microlepidoptera, 3: 39I. (BM) \\
\hline herpestica & PEUCOTELES* & I93I, Exotic Microlepidoptera, 4: 57. \\
\hline esmarcha & PTILOGENES & I930, Ann. Naturhist. Mus. Wien, 44: 258. (v) \\
\hline esperanthes & EROTIS & I92I, Ann. Transvaal Mus. 8: IO2. (T) \\
\hline esperias & STEGOMMATA & I893, Proc. Linnean Soc. N.S. Wales, 7: 603. \\
\hline esperidella & PALPARIA & I883, Proc. Linnean Soc. N.S. Wales, 7: 429. \\
\hline peris & PERSICOPTILA & I897, Proc. Linnean Soc. N.S. Wales, 22: 330. \\
\hline larcha & CHOREUTIS & I9I2, Exotic Microlepidoptera, I: 54. (вм) \\
\hline estias & TISCHERIA & I9I5, Exotic Microlepidoptera, I: 354. \\
\hline estiopa & SYNTOMACTIS & I897, Proc. Linnean Soc. N.S. Wales, $22: 380$. \\
\hline chaea & PALPARIA & I886, Proc. Linnean Soc. N.S. Wales, Io: 826 . \\
\hline era & PTOCHEUUSA & \\
\hline & [SYMPHANACTIS*] & I9I4, Trans. Ent. Soc. London, I9I4: 23I. (BM) \\
\hline taeropsis & CHELARIA & I935, Exotic Microlepidoptera, 4: 590. (BM) \\
\hline acma & DICHOMERIS & I923, Exotic Microlepidoptera, 2: 622. (BM) \\
\hline eteractis & ANTERETHISTA* & I9I4, Trans. Ent. Soc. London, I9I4: 237. (BM) \\
\hline anthes & NEPTICULA & I926, Exotic Microlepidoptera, 3: 296. \\
\hline aspis & ARGYROPLOCE & I936, Exotic Microlepidoptera, 4: 6I4. (BM) \\
\hline eterastis & THUDACA & I893, Proc. Linnean Soc. N.S. Wales, 7: 571. \\
\hline eraula & MELITONYMPHA* & I927, Exotic Microlepidoptera, 3: 360. (BM) \\
\hline ochrysa & TRICHOMOERIS & I922, Arkiv för Zool. vol. I4, part I5, p. 6 . \\
\hline oclina & HIEROXESTIS & I9I5, Exotic Microlepidoptera, I: 364 . \\
\hline terocosma & GRACILARIA & I93I, Exotic Microlepidoptera, 4: I7o. \\
\hline crossa & XYLOSCOPA* & I920, Exotic Microlepidoptera, 2: 354 . \\
\hline eterodesma & MELANOZESTIS* & I930, Exotic Microlepidoptera, 3: 547. (BM) \\
\hline eterodisca & SCYTHRIS & I929, Entomologist, $62:$ I 49. \\
\hline codoxa & ACROCERCOPS & I9I2, Exotic Microlepidoptera, I: 23. (T) \\
\hline leterogastra & STERRHOSTOMA* & I935, Exotic Microlepidoptera, 4: 587. (BM) \\
\hline eterogramma & DECADARCHIS & I92I, Exotic Microlepidoptera, $2: 459$. \\
\hline ograpta & TINISSA & I928, Exotic Microlepidoptera, 3: 425 . \\
\hline eroidana & ADOXOPHYES* & I88I, Proc. Linnean Soc. N.S. Wales, 6: 429. \\
\hline eterolicma & PLATYPTILIA & I936, Exotic Microlepidoptera, 5: 64. (c) \\
\hline eroloba & ACROCERCOPS & I932, Trans. Ent. Soc. London, 80: i 6 . \\
\hline olychna & MOMPHA & I922, Exotic Microlepidoptera, 2: 578 . (BM) \\
\hline eteromantis & MACROPIRATIS & I932, Exotic Microlepidoptera, 4: 249. \\
\hline leteromorpha & AMPHITHERA* & I893, Proc. Linnean Soc. N.S. Wales, 7: 597. \\
\hline ropa & LASPEYRESIA & I920, Exotic Microlepidoptera, 2: 35I. (BM) \\
\hline heteropa & STENOMA & I9I3, Trans. Ent. Soc. London, I9I3: I87. (BM) \\
\hline eropla & HAPLODYTA & I886, Proc. Linnean Soc. N.S. Wales, Io: 766. \\
\hline roplaca & ELACHISTA & I934, Exotic Microlepidoptera, 4: 528. \\
\hline heterosaris & STENOMA & I9I5, Exotic Microlepidoptera, I: 4I8. (BM) \\
\hline rosema & STENOMA & I930, Ann. Naturhist. Mus. Wien, 44: 244. (v) \\
\hline terospila & HOPLOPHANES & I897, Proc. Linnean Soc. N.S. Wales, 22: 4II. \\
\hline leterospora & PHTHORIMAEA & I924, Trans. Proc. New Zealand Inst. 44: 204. \\
\hline osticta & LIMNOECIA & I9I7, Exotic Microlepidoptera, 2: 42. \\
\hline erostoma & DECADARCHIS & I930, Exotic Microlepidoptera, 4: 9. \\
\hline
\end{tabular}




$\begin{array}{ll}\text { heterota } & \text { MELEONOMA } \\ \text { heteroxantha } & \text { STENOMA } \\ \text { heterozona } & \text { MACROBATHRA } \\ \text { hexabola } & \text { HYPONOMEUTA } \\ \text { hexacentra } & \text { COMMATICA } \\ \text { hexacentris } & \text { URODUS } \\ \text { hexachorda } & \text { ACROCERCOPS } \\ \text { hexachrysa } & \text { CHRYSOCLISTA } \\ \text { hexaclosta } & \text { ACROCERCOPS } \\ \text { hexacopa } & \text { ARISTOTELIA } \\ \text { hexactis } & \text { PARECTOPA } \\ \text { hexadyas } & \text { MACROBATHRA } \\ \text { hexagona } & \text { TETANOSTOLA* } \\ \text { hexagramma } & \text { APATETRIS } \\ \text { hexaleuca } & \text { CLEPTICODES } \\ \text { hexalocha } & \text { ACROCERCOPS } \\ \text { hexameris } & \text { GRACILARIA } \\ \text { hexametra } & \text { SPILONOTA } \\ \text { hexanesa } & \text { THIOTRICHA } \\ \text { hexanoma } & \text { ABACISTIS* } \\ \text { hexapeda } & \text { HILAROGRAPHA } \\ \text { hexaphala } & \text { MICTOPSICHIA } \\ & \text { PAMMENE } \\ & \end{array}$

$\begin{array}{ll}\text { hexascia } & \text { STENOMA } \\ \text { hexaselena } & \text { BRENTHIA } \\ \text { hexaspila } & \text { CTENOCOMPA } \\ \text { hexastera } & \text { PETALANTHES } \\ \text { hexatoma } & \text { LEUCOPTERA } \\ \text { hexatyla } & \text { STATHMOPODA } \\ \text { hians } & \text { TRACHYSCHISTIS* } \\ \text { hibbertiana } & \text { PALAEOBIA* } \\ \text { hiberna } & \text { PALTODORA } \\ \text { hiemalis } & \text { EULECHRIA } \\ \text { hieracitis } & \text { POLYHYMNO } \\ \text { hieranthes } & \text { CORISCIUM } \\ \text { hierarcha } & \text { PYRODERCES } \\ \text { hieraspis } & \text { COSMOPTERYX } \\ \text { hierastis } & \text { CRYPTOPHAGA } \\ & \text { [POTNIARCHA }{ }^{*} \text {. } \\ \text { hierocosma } & \text { ACROCERCOPS } \\ \text { hierodora } & \text { ERIOPTRIS } \\ \text { hieroglyphica } & \text { OXYTHECTA } \\ \text { hieroglypta } & \text { STAGMATOPHORA } \\ \text { hierographa } & \text { TINEA } \\ \text { hierograpta } & \text { PERIDAEDALA* } \\ \text { hierophanes } & \text { PANSEPTA } \\ \text { hierophanta } & \text { EUPLOCAMUS } \\ \text { hieropis } & \text { TRACHYPEPLA } \\ & \end{array}$

I9I4, Exotic Microlepidoptera, I: 256. (BM) I93I, Exotic Microlepidoptera, 4:37 (v) I889, Proc. Linnean Soc. N.S. Wales, (Ser. 2) 3: I676. 1935, Exotic Microlepidoptera, 4:602. (вм) I922, Trans. Ent. Soc. London, I922: 73. (BM) I93I, Exotic Microlepidoptera, 4: 90. (v)

I9I4, Journ. Bombay Nat. Hist. Soc. 23: II9.

1935, Exotic Microlepidoptera, 4:607.

1934, Exotic Microlepidoptera, 4: 470.

I929, Exotic Microlepidoptera, 3: 483. (вм)

I932, Trans. Ent. Soc. London, 80: II7.

I906, Trans. R. Soc. S. Australia, 30: 35.

I93I, Exotic Microlepidoptera, 4: 92. (P)

I921, Exotic Microlepidoptera, 2: 424. (BM)

I932, Exotic Microlepidoptera, 4: 324. (v)

I9I2, Exotic Microlepidoptera, I: 22. (T)

I92I, Exotic Microlepidoptera, 2: 470.

I920, Exotic Microlepidoptera, 2: 342. (вм)

I929, Exotic Microlepidoptera, 3: 501. (BM)

I9I3, Ann. Transvaal Mus. 3: 319. (T)

I913, Exotic Microlepidoptera, I: 99. (вм)

I912, Exotic Microlepidoptera, I: 36 . (вм)

I935, in Caradja and Meyrick, Materialien zu einer Microlepidopteren Fauna der Chinesischen Provinzen Kiangsu,

Chekiang und Hunan, p. 62. (BM)

1925, Exotic Microlepidoptera, 3: 215. (BM)

I909, Trans. Ent. Soc. London, I909: 4I. (BM)

I9I9, Exotic Microlepidoptera, 2: 265.

I883, Proc. Linnean Soc. N.S. Wales, 8: 336.

I9I5, Exotic Microlepidoptera, I: 346.

I907, Journ. Bombay Nat. Hist. Soc. I7: 744.

I92I, Exotic Microlepidoptera, 2: 448.

I88I, Proc. Linnean Soc. N.S. Wales, 6: 665.

-1912, Ann. South African Mus. Io: 6o. (SA)

I9I4, Exotic Microlepidoptera, I : 2I8.

I913, Ann. Transvaal Mus. 3: 283. (T)

I907, Journ. Bombay Nat. Hist. Soc. I7: 745.

I897, Proc. Linnean Soc. N.S. Wales, 22: 346.

1924, Exotic Microlepidoptera, 3:89.

I890, Trans. R. Soc. S. Australia, I3: 30.

I9I2, in Wytsman, Genera Insectorum, fasc. I28, p. IS.

I9I5, Trans. Ent. Soc. London, I915: 244.

I885, Proc. Linnean Soc. N.S. Wales, 9: 1052.

I9I I, Trans. Linnean Soc. London, I4: 282.

I9I I, Journ. Bombay Nat. Hist. Soc. 2I : I I6.

1925, Exotic Microlepidoptera, 3: I39.

1925, Exotic Microlepidoptera, 3: I53.

I9I6, Exotic Microlepidoptera, I: 6I 7 .

I892, Trans. Proc. New Zealand Inst. 24: 2I8. 


\begin{tabular}{|c|c|c|}
\hline hieropla & ATASTHALISTIS & I9I9, Exotic Microlepidoptera, 2: 235. (Bм) \\
\hline hilarantha & TORTRIX & I9I8, Exotic Microlepidoptera, 2: I69. \\
\hline hilaraspis & ARGYROPLOCE & I93I, Exotic Microlepidoptera, 4: I39. (Bм) \\
\hline hilarocrossa & EUCOSMA & $\begin{array}{l}\text { I93I, in Joannis, Ann. Soc. Ent. France, } 98 \text { (Supp.): } \\
\quad 7 \text { I6 [478]. (P) }\end{array}$ \\
\hline hilarodes & GLYPHIPTERYX & Igo9, Journ. Bombay Nat. Hist. Soc. I9: 430. \\
\hline hilarograpta & ARGYROPLOCE & I933, Exotic Microlepidoptera, 4: 42I. (BM) \\
\hline hilaropa & OECOPHORA & I889, Proc. Linnean Soc. N.S. Wales, (Ser. 2) 3: I672. \\
\hline hilaropis & GRACILARIA & I926, Trans. Ent. Soc. Lonàon, 74: 274 . \\
\hline hilda & ATMOZOSTIS* & I932, Exotic Microlepidoptera, 4:218. \\
\hline himaea & ANTAEOTRICHA & I9I6, Exotic Microlepidoptera, I: 505. (BM) \\
\hline himantias & PLEUTORA & I9I3, Exotic Microlepidoptera, I: I2I. (MA) \\
\hline himerodana & PALAEOBIA & I88r, Proc. Linnean Soc. N.S. Wales, 6: 666. \\
\hline himerodes & STENOMA & I9I6, Exotic Microlepidoptera, I: 535. (BM) \\
\hline hipparcha & AEOLOSCELIS* & I897, Proc. Linnean Soc. N.S. Wales, 22: 328. \\
\hline hipparchus & DEUTEROCOPUS & I92I, Exotic Microlepidoptera, 2: 420. \\
\hline hippastis & $\begin{array}{l}\text { TIMYRA } \\
{[\text { MONERISTA*] }}\end{array}$ & \\
\hline & $\begin{array}{l}\text { [MONERISTA*] } \\
\text { GELECHIA }\end{array}$ & I9o8, Journ. Bombay Nat. Hist. Soc. I8: 45I. (BM) \\
\hline hippeis & $\begin{array}{l}\text { GELECHIA } \\
\text { MELASINA }\end{array}$ & I902, Trans. Ent. Soc. London, I902: 573. \\
\hline hippias & $\begin{array}{l}\text { MELASINA } \\
\text { PALINTROPA* }\end{array}$ & I92I, Ann. Transvaal Mus. 8: I34. (T) \\
\hline hippica & $\begin{array}{l}\text { PALINTROPA* } \\
\text { ANARSIA }\end{array}$ & I9I3, Journ. Bombay Nat. Hist. Soc. 22: I6o. (вм) \\
\hline hippocoma & & I92I, Exotic Microlepidoptera, 2: 429. \\
\hline hippophylax & NEMOTOIS & I930, Exotic Microlepidoptera, 3: 572. (Bм) \\
\hline hipposaris & GELECHIA & I927, Exotic Microlepidoptera, 3: 348. (BM) \\
\hline hippotarcha & LECITHOCERA & I923, Exotic Microlepidoptera, 3: 41. \\
\hline hippuris & ACROCERCOPS & I9I5, Trans. Ent. Soc. London, I9I5: 232. \\
\hline hippurista & RECURVARIA & I932, Exotic Microlepidoptera, 4:349. (v) \\
\hline hiracistis & PHILOBOTA & I889, Proc. Linnean Soc. N.S. Wales, (Ser. 2) 3: Io69. \\
\hline hiracopis & EPORYCTA & I92I, Exotic Microlepidoptera, 2: 444. (BM) \\
\hline hirax & PALPARIA & I883, Proc. Linnean Soc. N.S. Wales, 7: 44r. \\
\hline hirundinae & TINEA & I928, Bull. Hill Mus. 2: 239. (P) \\
\hline historica & ACROCLITA & I920, Exotic Microlepidoptera, 2: 343 . (BM) \\
\hline histurga & MACHAEROPTERIS & I9I5, Exotic Microlepidoptera, I: 29I. \\
\hline hodias & MARASMARCHA & I9o8, Trans. Ent. Soc. London, I907: 492. \\
\hline holachyrma & SIMAETHIS & I9I2, Exotic Microlepidoptera, I: 47. (BM) \\
\hline holantha & ZACORISCA* & I9ro, Proc. Linnean Soc. N.S. Wales, 35:221. \\
\hline holarga & BAEONOMA & I9I6, Exotic Microlepidoptera, I: 509. (вм) \\
\hline holcadica & STENOMA & I9I6, Exotic Microlepidoptera, I: 53I. (Bм) \\
\hline holmodes & EULECHRIA & I922, Exotic Microlepidoptera, 2: 510. \\
\hline holocapna & TINEA & $\begin{array}{l}\text { I93r, Ann. Mus. Nac. Hist. Nat. Buenos Aires, 36: } 4 \text { I4. } \\
\text { (BM) }\end{array}$ \\
\hline holochalca & PANTOSPERMA* & I888, Trans. Proc. New Zealand Inst. 20: 89. \\
\hline holochlora & BATRACHEDRA & I897, Proc. Linnean Soc. N.S. Wales, 22: 306. \\
\hline holochorda & TRACHYDORA & I9I5, Exotic Microlepidoptera, I: 332. (MA) \\
\hline holochra & STATHMOPODA & I889, Trans. Proc. New Zealand Inst. 2I: I68. \\
\hline holocitra & ODITES & I925, Exotic Microlepidoptera, 3: I56. (BM) \\
\hline holoclera & EUCHAETIS & I888, Proc. Linnean Soc. N.S. Wales, (Ser. 2) 2: 940. \\
\hline holocnista & ANTICHLIDAS & $\begin{array}{l}\text { I93I, in Caradja, Bull. Sect. sci. Acad. roum. I4: } 66 . \\
\text { (BM) }\end{array}$ \\
\hline olc & TIMYRA & r9o8, Journ. Bombay Nat. Hist. Soc. 18: 446. (вм) \\
\hline
\end{tabular}


holocrossa

holocrypta

holodesma

holodisca

holodryas

hologramma

hololampra

hololeuca

holombra

holomorpha

holonota

holopercna

holopetra

holophaea

holophracta

holoporphyra

holopsamma

holopyrrha

holorphna

holoscia

holosticta

holotephras

holothecta

holotherma

holotorna

holoxantha

holoxesta

holoxyla

holoxytha

holozona

homadelpha

homalacta

homalistis

homalocrossa

homalodoxa

homalopa

homalopis

homalospora

homalota

homalotis

homaloxesta

homestia

homocentra

homochalca

homochares

homochlora

homochorda

homoclera

homocosma
PHILOBOTA

EUCOSMA

GLYPHIPTERYX

MISCERA

MELASINA

GLYPHIPTERYX

XESTOCASIS

ERIODYTA

METACHANDA

PROCHOLA

OCYSTOLA

GYMNELEMA

LABDIA

BAEONOMA

COSMOPTERYX

ASYMPHORODES

TINEOLA

CRYPTOLECHIA

CNEPHASIA

STRUTHISCA

CEROMITIA

SPILONOTA

STATHMOPODA

PYRODERCES

ODITES

OCYSTOLA

PLEUROTA

MELASINA

PHOLEUTIS

PROMALACTIS*

BLASTOBASIS

ACROCERCOPS

FRISILIA

ERETMOCERA

ASARISTA

MALLOBATHRA

MACHAERITIS

THURIOSTOMA*

PLEUROTA

IMMA

TRICHOTAPHE

TINEA

LECITHOCERA

EULECHRIA

CHARITOLEUCA*

FRISILIA

COMPSISTIS

ANAPTILORA

MACROBATHRA
I889, Proc. Linnean Soc. N.S. Wales, (Ser. 2) 3: I6I6.

I93I, Exotic Microlepidoptera, 4: I46. (BM)

I883, Proc. Linnean Soc. N.S. Wales, 7: 190.

I907, Proc. Linnean Soc. N.S. Wales, 32: 105.

1934, Exotic Microlepidoptera, 4:483. (c)

I920, Exotic Microlepidoptera, 2: 333. (BM)

I9I5, Exotic Microlepidoptera, I: 317.

I886, Proc. Linnean Soc. N.S. Wales, 8: 5 I8.

I930, Trans. Ent. Soc. London, 78: 3I5. (P)

I93I, Journ. Linnean Soc. London, 37: 282. (BM)

I889, Proc. Linnean Soc. N.S. Wales, (Ser. 2) 3: 1665.

I924, Exotic Microlepidoptera, 3: 78. (T)

I927, Insects of Samoa, 3, Lepidoptera, fasc. 2, p. 90.

I9I6, Exotic Microlepidoptera, I: 54I. (BM)

I909, Journ. Bombay Nat. Hist. Soc. I9: 4I4.

1934, Pacific Ent. Surv. Publ. 7, art. 28, p. 352. (Bв)

I908, Proc. Zool. Soc. London, p. 739.

rgI2, Trans. Ent. Soc. London, I9I I: 704. (BM)

I9II, Trans. New Zealand Inst. 43: 74.

1905, Journ. Bombay Nat. Hist. Soc. I6: 614.

1918, Ann. Transvaal Mus. 6: 59. (T)

I924, Exotic Microlepidoptera, 3:67. (вM)

1934, Ann. Mag. Nat. Hist. I4: 4Io.

1936, Exotic Microlepidoptera, 5: 29. (c)

1925, Exotic Microlepidoptera, 3: I57. (BM)

I902, Trans. R. Soc. S. Australia, 26: I36.

r889, Proc. Linnean Soc. N.S. Wales, (Ser. 2) 3: r652.

1936, Exotic Microlepidoptera, 5: 57. (c)

I9I5, Exotic Microlepidoptera, I: 307.

I908, Journ. Bombay Nat. Hist. Soc. I8: 8og. (Bм)

I902, Trans. R. Soc. S. Australia, 26: I7I.

1927, Insects of Samoa, 3, Lepidoptera, fasc. 2, p. I07.

1935, Exotic Microlepidoptera, 4:563. (вм)

I930, Exotic Microlepidoptera, 3:586. (BM)

I935, Exotic Microlepidoptera, 4: 59I. (BM)

I89I, Trans. Proc. New Zealand Inst. 23: Ioo.

I889, Proc. Linnean Soc. N.S. Wales, (Ser. 2) 3: I668.

1934, Exotic Microlepidoptera, 4:5I5.

I889, Proc. Linnean Soc. N.S. Wales, (Ser. 2) 3: 1649.

I906, Trans. Ent. Soc. London, 1906: 187. (BM)

I921, Ann. Transvaal Mus. 8: 86. (T)

I908, Proc. Zool. Soc. London, p. 74I.

I9Io, Journ. Bombay Nat. Hist. Soc. 20: 449. (BM)

1888, Proc. Linnean Soc. N.S. Wales, $2: 965$.

I938, in Caradja and Meyrick, Deuts. Ent. Zeit. Iris, 52:

26.

I9Io, Journ. Bombay Nat. Hist. Soc. 20: 437. (BM)

I921, Exotic Microlepidoptera, 2: 398. (BM)

I9I6, Exotic Microlepidoptera, I: 59I.

1902, Trans. R. Soc. S. Australia, 26: I67. 


\begin{tabular}{|c|c|c|}
\hline homocrossa & IMMA & I930, Exotic Microlepidoptera, 4: 3. (BM) \\
\hline homodoxa & OECOPHORA & I883, New Zealand Journ. Sci. I: 525 . \\
\hline homogama & HOMONA & I9I0, Proc. Linnean Soc. N.S. Wales, 35: 2 II. \\
\hline homogenes & DEPRESSARIA & I920, Ann. South African Mus. I7: 288. (SA) \\
\hline lomogramma & ONEBALA & I9I8, Ann. Transvaal Mus. 6: 25. (T) \\
\hline homograpta & DOLIOCHASTIS* & I920, Ann. South African Mus. I7: 277 . (SA) \\
\hline 1omoleuca & OCYSTOLA & I885, Proc. Linnean Soc. N.S. Wales, 9: 1о76. \\
\hline omologa & APHANOXENA & I9I5, Exotic Microlepidoptera, I: 388 . (BM) \\
\hline omomorpha & HETERODMETA* & I93I, Exotic Microlepidoptera, 4: I88. (BM) \\
\hline omopercna & MELASINA & I920, Ann. South African Mus. I7: 3Io. (SA) \\
\hline omophaea & NEPTICULA & I9I8, Exotic Microlepidoptera, 2: I8I. \\
\hline omophyla & TORTRIX & I9I7, Trans. Ent. Soc. London, I9I7: 8. (BM) \\
\hline omoplasta & COMPSOLECHIA & I932, Exotic Microlepidoptera, 4: I97. (BM) \\
\hline omopyrrha & PHTHORAULA* & I935, Proc. Hawaiian Ent. Soc. 9: 65. (sP) \\
\hline omora & OENOCHROA. & I902, Trans. R. Soc. S. Australia, 26: I52. \\
\hline omosacta & EULIA & I930, Exotic Microlepidoptera, 3: 61о. (BM) \\
\hline omospora & EULECHRIA & I9I3, Exotic Microlepidoptera, I: I58. \\
\hline omoteles & EULECHRIA & 1888, Proc. Linnean Soc. N.S. Wales, (Ser. 2) $2: 947$. \\
\hline omoterma & DALACA & I92I, Ann. Transvaal Mus. 8: I42. (T) \\
\hline omotona & PHILOBOTA & I884, Proc. Linnean Soc. N.S. Wales, 8: 508. \\
\hline omotorna & PAMMENE & I9I2, Journ. Bombay Nat. Hist. Soc. 2I: 874. (BM) \\
\hline lotrocha & ORNEODES & I921, Ann. Transvaal Mus. 8: Io8. (T) \\
\hline omotropha & LEPIDOPODA & I921, Exotic Microlepidoptera, 2: 443 . \\
\hline notypa & CHOREUTIS & I907, Proc. Linnean Soc. N.S. Wales, 32: Io9. \\
\hline loxantha & SCYTHRIS & I92I, Ann. Transvaal Mus. 8: II6. (T) \\
\hline lomoxesta & EULECHRIA & I888, Proc. Linnean Soc. N.S. Wales, (Ser. 2) 2: 965. \\
\hline omoxyla & ARISTOTELIA & I928, Proc. Hawaiian Ent. Soc. 7: IоI. (SP) \\
\hline esta & BACTRA & I909, Journ. Bombay Nat. Hist. Soc. I9: 585 . (Bм) \\
\hline onesta & SPILONOTA & I9I I, Proc. Linnean Soc. N.S. Wales, 36: 233. \\
\hline onoranda & DICHELOPA & I926, Trans. Ent. Soc. London, 74: 272. (BM) \\
\hline orata & ACROLEPIA & I92I, Exotic Microlepidoptera, 2: 440. \\
\hline onoratus & DEUTEROCOPUS & I92I, Exotic Microlepidoptera, 2: 4I8. \\
\hline oplanthes & PEZETAERA* & I924, Exotic Microlepidoptera, 3: 75. (Bм) \\
\hline lista & ARGYROPLOCE & I927, Exotic Microlepidoptera, 3: 340. (BM) \\
\hline Ioplitica & STENOMA & I925, Exotic Microlepidoptera, 3: 2I6. (BM) \\
\hline oplitis & MEGACRASPEDUS & I904, Proc. Linnean Soc. N.S. Wales, 29: 277. \\
\hline locala & GRACILARIA & I88I, Proc. Linnean Soc. N.S. Wales, 5: I49. \\
\hline loplocrates & TRICYANAULA & I932, Exotic Microlepidoptera, 4: I98. (BM) \\
\hline oplodesma & OECOPHORA & I883, New Zealand Journ. Sci. I: 525. \\
\hline loplodoxa & CHALCOMIMA* & I929, Exotic Microlepidoptera, 3: 507. (BM) \\
\hline 1oplomacha & THIOTRICHA & I9o8, Journ. Bombay Nat. Hist. Soc. I 8: 44I. (Bм) \\
\hline hoplometalla & NEPTICULA & I934, Exotic Microlepidoptera, 4: 467. \\
\hline oplophanes & PLEUROTA & I889, Proc. Linnean Soc. N.S. Wales, (Ser. 2) 3: I65I. \\
\hline oplophora & HELCYSTOGRAMMA & I9I6, Exotic Microlepidoptera, I: 577. (BM) \\
\hline hoplostola & CRYPTOLECHIA & $\begin{array}{l}\text { I938, in Caradja and Meyrick, Deuts. Ent. Zeit. Iris, } 52 \text { : } \\
\text { Io. (BM) }\end{array}$ \\
\hline oraea & OECOPHORA & I883, New Zealand Journ. Sci. I : 524 . \\
\hline aria & OPOSTEGA & I921, Exotic Microlepidoptera, 2: 457. \\
\hline archa & PERISCEPTIS & I931, Journ. Linnean Soc. London, 37:283. (BM) \\
\hline riaula & GELECHIA & I9I8, Exotic Microlepidoptera, 2: I33. (BM). \\
\hline
\end{tabular}




\begin{tabular}{|c|c|}
\hline horiodes & DICHOMERIS \\
\hline oristis & ONEBALA \\
\hline orizontias & STENOMA \\
\hline rmathota & PLATACTIS* \\
\hline rmista & ACROCERCOPS \\
\hline rmocrossa & CRYPTOPHASA \\
\hline modes & PROTOLECHIA \\
\hline lophora & ACROCERCOPS \\
\hline ta & SPATALISTIS \\
\hline erma & ARGYROPLOCE \\
\hline ris & STRUTHISCA \\
\hline ntra & CLEPTICODES* \\
\hline laris & STENOMA \\
\hline lora & HOMALOXESTIS \\
\hline compsa & DICHOMERIS \\
\hline cyma & STENOMA \\
\hline glypta & DICHOMERIS \\
\hline gramma & NESOLECHIA* \\
\hline tpha & ADOXOPHYES \\
\hline 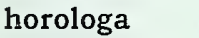 & HYPONOMEUTA \\
\hline 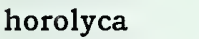 & HIEROXESTIS \\
\hline & STENOMA \\
\hline & PHTHORIMAEA \\
\hline & TINEA \\
\hline
\end{tabular}

horrealis

horrenda

horrens

horrida

horridula

horrifera

horrifica

hortaria

hortatrix

hortulana

hospitalis

hostilis

hotlias [sic]

humana

humata

humeratrix

humida

humida

hyacinthias

hyacinthina

hyacinthina
METARSIORA*

PTOCHOSARIS*

ACROCERCOPS

STATHMOPODA

AGRIOPHARA

MACHAEROPTERIS

ZATRICHODES

ACROCLITA

MELASINA

TRICHOTAPHE

STENOMA

POLYHYMNO

DOLICHOTORNA

TORTRIX

MACHIMIA

ATTEVA

LYTROPHILA

PLATYPTILIA

MELASINA

CNEPHASIA

TISIS
1923, Exotic Microlepidoptera, 2: 620. (BM)

I9Io, Journ. Bombay Nat. Hist. Soc. 20: 452. (BM)

1925, Exotic Microlepidoptera, 3: I88. (BM)

I9I I, Trans. Linnean Soc. London, I4: 287.

I9I6, Exotic Microlepidoptera, 2: 3.

I925, Exotic Microlepidoptera, 3: I48.

I904, Proc. Linnean Soc. N.S. Wales, 29: 363.

I9I2, Exotic Microlepidoptera, I: 23. (T)

I907, Journ. Bombay Nat. Hist. Soc. I7: 979. (Bм)

I938, Institut des Parcs Nationaux du Congo Belge, fasc. I4,

p. Io, pl. I, fig. 8.

I908, Proc. Zool. Soc. London, p. 742.

I927, Exotic Microlepidoptera, 3: 332. (T)

1930, Ann. Naturhist. Mus. Wien, 44:25I. (v)

I929, Exotic Microlepidoptera, 3: 5I9. (BM)

I933, Exotic Microlepidoptera, 4: 353. (v)

I925, Exotic Microlepidoptera, 3: 209. (BM)

1932, Exotic Microlepidoptera, 4: 202. (BM)

I92I, Exotic Microlepidoptera, 2: 425. (BM)

I928, Exotic Microlepidoptera, 3: 454 .

I935, Exotic Microlepidoptera, 4:556. (c)

I915, Exotic Microlepidoptera, I: 36I.

I925, Exotic Microlepidoptera, 3: 222. (BM)

I926, Exotic Microlepidoptera, 3: 279. (вм)

I93I, Ann. Mus. Nac. Hist. Nat. Buenos Aires, 36: 4I3.

(BM)

I937, Exotic Microlepidoptera, 5: 76 .

I907, Trans. R. Soc. S. Australia, 30: 37.

I932, Trans. Ent. Soc. London, 80: II7.

I936, Exotic Microlepidoptera, 4: 6I8.

I89o, Trans. R. Soc. S. Australia, I3: 77.

I9II, Journ. Bombay Nat. Hist. Soc. 2I: I29.

I922, Exotic Microlepidoptera, 2: 5I2.

I9II, Proc. Linnean Soc. N.S. Wales, 36: $24 \mathrm{I}$.

I924, Arkiv för Zool. vol. I6, part I4, p. 4.

I9I8, Ann. Transvaal Mus. 6:23. (T)

I9I5, Exotic Microlepidoptera, I: 463. (BM)

I9I8, Ann. Transvaal Mus. 6: I9. (T)

I9Io, Journ. Bombay Nat. Hist. Soc. 20: 439.

I9I2, Exotic Microlepidoptera, I: 6. (Bм)

I9I4, Exotic Microlepidoptera, I: I8I. (BM)

I930, Ann. Naturhist. Mus. Wien, 44: 262, pl. I, fig. 30.

(v)

I9I3, Ann. Transvaal Mus. 3: 319. (T)

I920, Voyage de Ch. Alluaud et R. Jeannel en Afrique

Oriental, II, Microlepidoptera, p. 39. (P)

I920, Voyage de Ch. Alluaud et $R$. Jeannel en Afrique

Oriental, II, Microlepidoptera, p. II4. (P)

I9I2, Trans. Ent. Soc. London, I9I I: 682. (BM)

I9Io, Trans. Ent. Soc. London, I9Io: 439. (Вм) 


\begin{tabular}{ll} 
hyacinthitis & STENOMA \\
hyacinthopa & HOMALOPSYCHA \\
hyalistis & MACROBATHRA \\
hyalitis & ARGYROPLOCE \\
hyalocryptis & STENOMA \\
hyalodes & TINEA \\
hyalombra & DICHOMERIS \\
hyalophaea & DOLIOTECHNA \\
hyalophanta & STENOMA \\
hyaloxantha & MELITTIA \\
hyalozona & TORTYRA \\
hybrista & LECITHOCERA \\
hybristis & PROTYPANTHES* \\
hydara & PHILOBOTA \\
hydractis & TORTRIX \\
hydraea & CEROSTOMA \\
hydraena & STENOMA \\
hydrargyra & ARGYROPLOCE \\
hydraula & METACHANDA \\
hydrelaeas & STENOMA \\
hydrochalca & OINOPHILA \\
hydrochoa & PARAPTILA \\
hydrochroa & PYCNOTARSA \\
hydrocosma & DOXOPHYRTIS* \\
hydrocosma & PHRIXOSCELES \\
hydrogramma & CNEPHASIA \\
hydrogramma & COPTOTELIA \\
hydrographa & AEOLOSCELIS \\
hydronoma & SCYTHRIS \\
hydrophora & ANTAEOTRICHA \\
hydrosema & TIMYRA \\
& [OLBOIHREPT \\
\hline
\end{tabular}

hyetodes

hygraema

hygrantis

hygroberylla

\section{hygrodes \\ hygropis \\ hygroscopa \\ hylactica}

hylaea

hylarcha

hylias

hylomaga

hylonoma

hylophanes

hylurga

hymenaea
I930, Exotic Microlepidoptera, 3: 559. (BM)

I932, Exotic Microlepidoptera, 4: 208.

I889, Proc. Linnean Soc. N.S. Wales, (Ser. 2) 3: I679.

I9o9, Journ. Bombay Nat. Hist. Soc. I9: 599. (BM)

I930, Ann. Naturhist. Mus. Wien, 44:250. (v)

I9I3, Ann. Transvaal Mus. 3: 332. (T)

I922, Exotic Microlepidoptera, 2: 503.

I9I4, Exotic Microlepidoptera, I: I88. (BM)

I932, Exotic Microlepidoptera, 4: 294. (v)

1928, Exotic Microlepidoptera, 3:467. (BM)

I9I2, Exotic Microlepidoptera, I: 38. (BM)

1922, Exotic Microlepidoptera, 2: 505. (P)

1933, Exotic Microlepidoptera, 4: 424. (BM)

I884, Proc. Linnean Soc. N.S. Wales, 8: 494.

19I0, Proc. Linnean Soc. N.S. Wales, 35: 244.

I9I9, Exotic Microlepidoptera, 2: 228. (BM)

I9I6, Exotic Microlepidoptera, I: 536. (BM)

I93I, Exotic Microlepidoptera, 4: I37.

I9II, Trans. Linnean Soc. London, I4:276. (BM)

I93I, Exotic Microlepidoptera, 4: 33. (BM)

1930, Trans. Ent. Soc. London, 78: 320. (P)

I930, Exotic Microlepidoptera, 3: 6ro. (BM)

I920, Exotic Microlepidoptera, 2: 374. (BM)

I9I4, Trans. Proc. New Zealand Inst. 46: II3.

I908, Journ. Bombay Nat. Hist. Soc. I8: 8I5.

I9I2, Trans. Ent. Soc. London, I9II: 683. (BM)

I9I2, Trans. Ent. Soc. London, I9I I: 702. (BM)

1897, Proc. Linnean Soc. N.S. Wales, 22: 327.

I930, Exotic Microlepidoptera, 3: 56r.

I925, Exotic Microlepidoptera, 3: I7I. (BM)

I9I6, Exotic Microlepidoptera, I: 572. (BM)

I883, New Zealand Journ. Sci. I: 523.

1927, Exotic Microlepidoptera, 3: 36I.

I911, Trans. Linnean Soc. London, 14: 270. (BM)

1937, in Caradja and Meyrick, Deuts. Ent. Zeit. Iris, 5I: I 80 .

I936, Exotic Microlepidoptera, 5:22. (BM)

1936, Exotic Microlepidoptera, 5: 57. (C)

1935, Proc. Hawaiian Ent. Soc. 9:66. (SP)

I938, in Caradja and Meyrick, Deuts. Ent. Zeit. Iris, 52:

87 .

I9I2, Exotic Microlepidoptera, I: 3I. (BM)

1928, Exotic Microlepidoptera, 3: 400.

1904, Proc. Linnean Soc. N.S. Wales, 29: 369.

I931, Ann. Mus. Nac. Hist. Nat. Buenos Aires, 36: 4I5.

I89o, Trans. R. Soc. S. Australia, I3: 72.

I928, Exotic Microlepidoptera, 3: 447. (BM)

I92I, Ann. Transvaal Mus. 8:83. (T)

Igo9, Trans. Ent. Soc. London, I909: 38. (BM) 


$\begin{array}{ll}\text { hymenaea } & \text { EULECHRIA } \\ \text { hymenopis } & \text { PROTOSYNAEMA } \\ \text { hypachroa } & \text { BATRACHEDRA } \\ \text { hypanthes } & \text { STILBOSIS } \\ \text { hyperacma } & \text { DECADARCHIS } \\ \text { hyperaesthetica } & \text { MONACHOPTILAS* } \\ \text { hyperarcha } & \text { PHLOEOPOLA } \\ \text { hyperbolacma } & \text { PHRIXOSCELES } \\ \text { hyperbolica } & \text { MACHIMIA } \\ \text { hyperchlora } & \text { EULECHRIA } \\ \text { hyperetana } & \text { DICHELIA } \\ \text { hyperocha } & \text { SAROPLA } \\ & \text { [ORTHIASTIS*] } \\ \text { hyperophrys } & \text { LEUCOPTERA } \\ \text { hyperopta } & \text { TRACHYNTIS } \\ \text { hyperptycha } & \text { TORTRIX } \\ \text { hyperythra } & \text { STOMOPTERYX } \\ \text { hyperythrota } & \text { GYMNOMACHA* }\end{array}$

hyphantica

hyphantis

hypnodes

hypnota

hypnotis

hypocausta

hypochalca

hypochloris

hypochrysa

hypocirrha

hypocosma

hypocritica

hypocypha

hypoleuca

hypopercna

hypophaea

hypopiasta

hyposcia

hypoxutha

hypsichora

hypsicrates

hypsicrates

hypsidryas

hypsiphila

hypsipola

hypsipora

hypsithrona

hyptiota

hysterota
ACROCERCOPS

IMMA

PSALTICA

CRYPSITHYRIS

LINOSTICHA

PHILOBOTA

OECOPHORA

EULIA

TINEA

STENOMA

ORNEODES

CHRYSORYCTIS

BUCCULATRIX

PROTOLECHIA

NIPHORYCTA*

ACROLOPHUS

MONOPIS

STATHMOPODA

BATRACHEDRA

EUPSELIA

PYRSARCHA*

URODUS

EUCOSMA

BUCCULATRIX

LECITHOCERA

PLATYPTILIA

EULIA

STRENOPHILA*

PLATYBATHRA
I902, Trans. R. Soc. S. Australia, 26: I49.

I935, Trans. roy. Soc. N.Z. 65:304.

I897, Proc. Linnean Soc. N.S. Wales, 22: 306.

I9I7, Exotic Microlepidoptera, 2: 48. (BM)

I9I5, Exotic Microlepidoptera, I: 367 .

1934, Exotic Microlepidoptera, 4: 482.

I889, Proc. Linnean Soc. N.S. Wales, (Ser. 2) 3: I59I.

I931, Exotic Microlepidoptera, 4: I67.

I928, Exotic Microlepidoptera, 3: 47I. (BM)

I888, Proc. Linnean Soc. N.S. Wales, 2: 962.

I88I, Proc. Linnean Soc. N.S. Wales, 6: 479.

I884, Proc. Linnean Soc. N.S. Wales, 9: 744 .

I939, Trans. R. Ent. Soc. London, 89: 6r.

I889, Proc. Linnean Soc. N.S. Wales, (Ser. 2) 3: 586.

I93I, in Caradja, Bull. Sect. sci. Acad. roum. I4: 63.

I92I, Ann. Transvaal Mus. 8: 76. (T)

I938, in Caradja and Meyrick, Deuts. Ent. Zeit. Iris, 52:

86.

I9I2, Exotic Microlepidoptera, I: 25.

I906, Trans. Ent. Soc. London, I906: I83. (BM)

I9I4, Exotic Microlepidoptera, I: 26I (BM)

1907, Journ. Bombay Nat. Hist. Soc. I7: 753.

I889, Proc. Linnean Soc. N.S. Wales, (Ser. 2) 3: 1583.

I884, Proc. Linnean Soc. N.S. Wales, 8: 48I.

I886, Proc. Linnean Soc. N.S. Wales, Io: 782.

I932, Exotic Microlepidoptera, 4:256. (v)

I927, Exotic Microlepidoptera, 3: 332.

I930, Exotic Microlepidoptera, 4: 30. (Bм)

I934, in Caradja and Meyrick, Deuts. Ent. Zeit. Iris, 48:

29.

I893, Proc. Linnean Soc. N.S. Wales, 7: 55I.

I936, Exotic Microlepidoptera, 4: 620.

I904, Proc. Linnean Soc. N.S. Wales, 29: 35I.

I938, Trans. R. Ent. Soc. London, 87: 5I8.

I923, Exotic Microlepidoptera, 2: 609. (BM)

I93I, Exotic Microlepidoptera, 4: 94.

I897, Proc. Linnean Soc. N.S. Wales, 22: 320.

I897, Proc. Linnean Soc. N.S. Wales, 22: 308.

I906, Trans. R. Soc. S. Australia, 30: 39 .

I932, Exotic Microlepidoptera, 4: 340. (BM)

I925, Exotic Microlepidoptera, 3: 130. (BM)

I925, Exotic Microlepidoptera, 3: I40. (BM)

I9I5, Trans. Ent. Soc. London, I9I5: 250.

I926, Exotic Microlepidoptera, 3: 290. (BM)

I9I6, Exotic Microlepidoptera, I: 559.

I926, Exotic Microlepidoptera, 3: 25I. (BM)

I9r3, Ann. Transvaal Mis. 3: 306. (T)

I9I8, Ann. Transwaal Mus. 6: 38. (T) 


$\begin{array}{ll}\text { ialeura } & \text { SIMAETHIS } \\ \text { iambica } & \text { HOMOSETIA } \\ \text { iantha } & \text { CNEPHASIA } \\ \text { ianthes } & \text { HYYSOLOPHUS } \\ \text { ianthodes } & \text { LECITHOCERA } \\ \text { iatma } & \text { STENOMA } \\ \text { ichnaea } & \text { PSEUDODOXIA } \\ \text { ichnaea } & \text { STENOMA } \\ \text { ichneuta } & \text { EULECHRIA } \\ \text { ichnitis } & \text { CRYPTOLECHIA } \\ \text { ichnographa } & \text { LIMNOECIA } \\ \text { ichnophora } & \text { MELASINA }\end{array}$

ichnora

ichnota

ichorodes

ichthyodes

ich thyopa

ichthyura

icmaea

icriodes

icriota

icterias

icterica

icterina

icteropa
icteropis
icterota
ictifera
ida
idalia
ideologa
idiarcha
idiastis
idiocentra
idiocoma
idiocrossa
idiocrossa
idiogama
idiograpta

idiomorpha
idioptila
idiospila
idiotricha
idolias
idonea

HIEROXESTIS

BATTARISTIS*

LECITHOCERA

PTILOGENES

IRIDOSTOMA*

LASPEYRESIA

EULECHRIA

GELECHIA

CHOLOTIS

ARGYRESTHIA

OPOGONA

EPIMACTIS

MACROSACES

STENOMA

MACHIMIA

COLONOPHORA

GRACILARIA

PLEUROTA

LECITHOCERA

TRICHEMBOLA

BRACHMIA

ANACAMPSIS

OPOSTEGA

ORNEODES BORKHAUSENIA

ARGYRESTHIA

GLYPHIPTERYX

ANARSIA

SEMOCHARISTA*

PHYLOMICTIS

LITHOCOLLETIS

ODITES
[PERIPHORECTIS*] i9io, Journ. Bombay Nat. Hist.Soc. 20: 445. (BM)

THRYSOCHARES* I9I I, Trans. Linnean Soc. London, I4: 297. I9I4, Trans. Ent. Soc. London, I9I4: 247. (BM) I926, Exotic Microlepidoptera, 3: 23I. (BM) I926, Ann. South African Mus. 23: 327. (SA) I9I5, Exotic Microlepidoptera, I: 299. I9I5, Exotic Microlepidoptera, I: 328.

I9o7, Journ. Bombay Nat. Hist. Soc. I7: 984. (BM) I9I5, Exotic Microlepidoptera, I : 360 . 73I [493]. (P) I925, Exotic Microlepidoptera, 3: 209. (BM) I9I4, Exotic Microlepidoptera, I: I78. (BM) I937, Exotic Microlepidoptera, 5: 80. (T) I88 I, Proc. Linnean Soc. N.S. Wales, 5: I55. 1923, Exotic Microlepidoptera, 3:62. (BM) I937, Exotic Microlepidoptera, 5: 96. (T) I93I, Journ. Linnean Soc. London, 37:278. (BM) I9I6, Exotic Microlepidoptera, I: 577. (BM) I 922, Trans. Ent. Soc. London, I922: 80. (BM) I9I8, Ann. Transvaal Mus. 6: 42. (T) I936, Exotic Microlepidoptera, 4: 625. I938, Trans. R. Ent. Soc. London, 87: 527. I924, Trans. Proc. New Zealand Inst. 55: 66I.

Chekiang und Hunan, p. 9I. (BM) I9I7, Ann. South African Mus. I7: Io. (SA) I9I6, Exotic Microlepidoptera, I: 582. (BM) I922, Arkiv för Zool. vol. I4, part I5, p. 4 . I921, Exotic Microlepidoptera, 2: 446. (BM) I891, Ent. Mon. Mag. 27: 61.
I9I2, Exotic Microlepidoptera, I: 46. (BM) I9I9, Exotic Microlepidoptera, 2: 252. (BM) I9I2, Trans. Ent. Soc. London, I9I I : 684. (BM) I887, Trans. Ent. Soc. London, I887: 273. (Lost) I931, Exotic Microlepidoptera, 4: 80. (BM) I9I5, Exotic Microlepidoptera, I: 474. (BM) I9Io, Journ. Bombay Nat. Hist. Soc. 20: I53. (BM) I9I4, Journ. Bombay Nat. Hist. Soc. 23: II8. (BM) I888, Proc. Linnean Soc. N.S. Wales, 2: 944. 1918, Exotic Microlepidoptera, 2: 222. (BM) I9o8, Proc. Zool. Soc. London, p. 734. I920, Voyage de Ch. Alluaud et R. Jeannel en Afrique Oriental, II, Microlepidoptera, p. III. (P) I9o9, Journ. Bombay Nat. Hist. Soc. I9: 425. (BM) I93I, Ann.Mus.Nac.Hist.Nat.BuenosAires, 36:384. (вм) I93I, in Joannis, Ann. Soc. Ent. France, 98 (Supp.): I9Io, Journ. Bombay Nat. Hist. Soc. 20: I47. (BM) I935, in Caradja and Meyrick, Materialien zu einer Microlepidopteren Fauna der Chinesischen Provinzen Kiangsu, I925, in Caradja, Mem. Sect. Sti. Acad. Românā, 3: 382. 


\begin{tabular}{|c|c|c|}
\hline ignara & DEMOBROTIS & I922, Exotic Microlepidoptera, 2: 60I. \\
\hline ignava & PHILOBOTA & I913, Exotic Microlepidoptera, I: I25. \\
\hline ignea & CROCANTHES & I925, Treubia, 6: 429 \\
\hline gnifera & PLATYPTILIA & I908, Trans. Ent. Soc. London, I907: 48I. \\
\hline igniflora & PSEUDATTERIA & I930, Exotic Microlepidoptera, 3: 606. (BM) \\
\hline ignigera & CROCANTHES & $\begin{array}{l}\text { I938, Trans. R. Ent. Soc. London, 87: 513. (вм) } \\
\text { I9I9, Exotic Microlepidoptera, 2: 259. (Bм) }\end{array}$ \\
\hline ignobilis & HAPSIFERA & $\left\{\begin{array}{l}\text { I920, Voyage de Ch. Alluaud et } R \text {. Jeannel en Afrique } \\
\text { Oriental, II, Microlepidoptera, p. Io6. (P) }\end{array}\right.$ \\
\hline gnominiosa & STATHMOPODA & I9I3, Exotic Microlepidoptera, I : 86. \\
\hline ata & DICHOMERIS & I921, Zool. Meded. 6: I65. (L) \\
\hline larcha & STAGMATOPHORA & I9II, Trans. Linnean Soc. London, I4: 282. \\
\hline 1lapsa & AGORARCHA* & I925, Treubia, 6: 433 . \\
\hline llaqueata & CAPUA & I9I7, Trans. Ent. Soc. London, I9I7: 6. (BM) \\
\hline illepida & ANTAEOTRICHA & I9I6, Exotic Microlepidoptera, I: 495. (BM) \\
\hline illiberalis & TORTRIX & I9I8, Exotic Microlepidoptera, 2: I68. (BM) \\
\hline illicita & CYMOTRICHA & I929, Exotic Microlepidoptera, 3: 5II. (BM) \\
\hline illita & PTILOGENES & I926, Exotic Microlepidoptera, 3: 232. (BM) \\
\hline illiterata & GLYPHIDOCERA & I929, Trans. Ent. Soc. London, 76: 509. (BM) \\
\hline illota & PHALONIA & I9I4, Bull. Mus. Paris, 20: I2I. (P) \\
\hline illucens & ERETMOCERA & I9I4, Ann. Transvaal Mus. 4: 200. (T) \\
\hline illucescens & TRICHOTAPHE & I9I8, Exotic Microlepidoptera, 2: I5I. (BM) \\
\hline illucida & EPICHORISTA & I9Io, Proc. Linnean Soc. N.S. Wales, 35: 257. \\
\hline illudens & ACROLOPHUS & I924, Exotic Microlepidoptera, 3:76. (BM) \\
\hline illuminata & PALTODORA & I9I3, Ann. Transvaal Mus. 3: 28I. (T) \\
\hline illuminata & SPARGANOTHIS & I9I7, Trans. Ent. Soc. London, I9I7: I3. \\
\hline illuminatrix & ORNEODES & I929, Exotic Microlepidoptera, 3: 537. \\
\hline illustrata & COLEOPHORA & I9I3, Ann. Transvaal Mus. 3: 324. (T) \\
\hline illustris & TRACHYDORA* & I897, Proc. Linnean Soc. N.S. Wales, 22: 397. \\
\hline illuta & OCYSTOLA & I885, Proc. Linnean Soc. N.S. Wales, 9: Io74. \\
\hline illutata & PSEUDODOXIA & I9I4, Exotic Microlepidoptera, I: 266. (BM) \\
\hline illutus & PTEROPHORUS & I9I7, Ann. South African Mus. I7: 2. (SA) \\
\hline illuvialis & PTOCHORYCTIS & I9I4, Journ. Bombay Nat. Hist. Soc. 22: 778. (BM) \\
\hline lyodes & CEROMITIA & I93I, Ann. Mus. Nac. Hist. Nat. Buenos Aires, 36: 4I 4. \\
\hline ilyopa & SCYTHRIS & I92I, Ann. Transvaal Mus. 8: II6. (T) \\
\hline ilyopis & SARIDACMA* & $\begin{array}{l}\text { I930, Ann. Naturhist. Mus. Wien, 44: 259, pl. I, fig. } \\
\text { 37. }\end{array}$ \\
\hline imbecilla & ALUCITA & I925, Bull. Soc. R. Ent. Egypte, 9: 206. \\
\hline ellis & EPIPHRACTIS & I9I4, Ann. South African Mus. Io: 252. (SA) \\
\hline mbricata & DICHOMERIS & I9I3, Journ. Bombay Nat. Hist. Soc. 22: I75. (BM) \\
\hline mbricata & TINEA & I9I I, Journ. Bombay Nat. Hist. Soc. 2I: II6. \\
\hline iculata & CATAMACTA & $\begin{array}{l}\text { I938, institut des Parcs Nationaux du Congo Belge, } \\
\text { fasc. I4, p. } 5 .\end{array}$ \\
\hline ifera & ORNEODES & I929, Exotic Microlepidoptera, 3: 538 . \\
\hline rifera & HERMENIAS & I9II, Proc. Linnean Soc. N.S. Wales, 36: 226. \\
\hline briferana & DIPTERINA* & I881, Proc. Linnean Soc. N.S. Wales, 6: 527. \\
\hline manis & CARPOCAPSA & I886, Trans. Ent. Soc. London, I886: 275. \\
\hline hanis & MELASINA & I9o8, Proc. Zool. Soc. London, p. 746. \\
\hline latura & STATHMOPODA & I922, Zool. Meded. $7: 88$. (L) \\
\hline nemor & NEPHOGENES & I9I3, Exotic Microlepidoptera, I: I30. \\
\hline
\end{tabular}


immerita

imminens

imminens

imminuta

immobilis

immodica

immolata

immortalis

immota

immunis

immuricata

immutabilis

imparata

impatiens

impavida

impedita

impendens

imperatrix

imperfecta

imperita

impervia

impigra

implacata

implexa

implexa

implicata

impolita

importuna

imposita

imposita

impotens

improba

improba

improbana

improbus

impropria

improvida

improvisa

imprudens

impulsa

impurata

inamoena

inanima

inanis

inardescens

inarticulata

inaugurata
TRICHOTAPHE

SPILONOTA

STENOMA

MELASINA

LECITHOCERA

EUSCELETAULA*

TINEA

COLEOPHORA

ANTAEOTRICHA

SCYTHRIS

GRACILARIA

COELOSTATHMA

MELASINA

EUCOSMA

CARPOSINA

STENOMA

CROCIDOSEMA

TISIS

MELASINA

TORTRIX

CACOECIA

DICHOMERIS

PLATYPTILIA

HERMENIAS

MELEONOMA

ISORRHOA

ARGYROPLOCE

TRACHYPEPLA

EUCOSMA

GAMBROSTOLA*

OPOGONA

CHELARIA

TRITHAMORA*

COSCINOPTYCHA

PTEROPHORUS

EUCOSMA

TELPHUSA

TINEA

BRACHMIA

MELEONOMA

STENOMA

PHTHINOSTOMA

SCYTHRIS

PACHYRHABDA

STENOMA

MELASINA

COSMOPTERYX
I9I3, Journ. Bombay Nat. Hist. Soc. 22: I78. (BM)

I9I7, Trans. Ent. Soc. London, I9I7: I5. (BM)

I9I5, Exotic Microlepidoptera, I: 43I. (BM)

I9I4, Exotic Microlepidoptera, I: 2II.

I9I8, Exotic Microlepidoptera, 2: I03. (BM)

I936, Exotic Microlepidoptera, 5: 56.

I93I, Exotic Microlepidoptera, 4: I05.

I922, Exotic Microlepidoptera, 2: 556. (BM)

I9I6, Exotic Microlepidoptera, I: 502. (BM)

I9I6, Exotic Microlepidoptera, 2: I3. (BM)

I9I5, Trans. Ent. Soc. London, I9I5: 238.

I926, Exotic Microlepidoptera, 3: 259. (вм)

I928, Exotic Microlepidoptera, 3: 430.

I92I, Ann. Transvaal Mus. 8: 54. (T)

I9I3, Exotic Microlepidoptera, I: 77.

I9I5, Exotic Microlepidoptera, I: 422. (BM)

I9I7, Trans. Ent. Soc. London, I9I7: I7. (BM)

I9I0, Trans. Ent. Soc. London, I9I0: 440. (BM)

I922, Exotic Microlepidoptera, 2: 607. (P)

I937, in Caradja and Meyrick, Deuts. Ent. Zeit. Iris, 5I :

I74. (BM)

I93I, in Joannis, Ann. Soc. Ent. France, 98 (Supp.):

7II [473]. (P)

I9I3, Ann. Transvaal Mus. 3: 305. (T)

I932, Trans. Ent. Soc. London, 80: Io9.

I9I2, Journ. Bombay Nat. Hist. Soc. 2I : 852.

I9I8, Exotic Microlepidoptera, 2: 220. (BM)

I920, Exotic Microlepidoptera, $2: 324$. (BM)

I9I7, Trans. Ent. Soc. London, I9I7: 28. (BM)

I9I4, Trans. Proc. New Zealand Inst. 46: I08.

I937, Exotic Microlepidoptera, 5: I58.

I926, Ann. South African Mus. 23: 332. (SA)

I9I5, Exotic Microlepidoptera, I: 359.

I9I3, Ann. Transvaal Mus. 3: 296.

I9I3, Trans. Proc. New Zealand Inst. 45: 29.

I88I, Proc. Linnean Soc. N.S. Wales, 6: 7oI.

I934, in Caradja and Meyrick, Deuts. Ent. Zeit. Iris, 48: 28.

I932, Exotic Microlepidoptera, 4: 224.

I926, Exotic Microlepidoptera, 3:275. (BM)

I926, Sarawak Mus. Journ. 3: I66.

I9I4, Exotic Microlepidoptera, I: 20I. (MA)

I934, Exotic Microlepidoptera, 4: 477. (v)

I9I5, Exotic Microlepidoptera, I: 468. (BM)

I922, Exotic Microlepidoptera, 2: 507.

I9I6, Exotic Microlepidoptera, 2: I3. (BM)

I936, Exotic Microlepidoptera, 4: 6I 7 .

I925, Exotic Microlepidoptera, 3:202. (Вм)

I935, Exotic Microlepidoptera, 4:578.

I922, Exotic Microlepidoptera, 2: 568. (Bм) 
inca incalescens incallida incandescens incanescens incantata incauta incendiaria incepta inceptrix inceptrix incertula inchoata incincta incinerata

incisa incisoria incitata incitatrix inclemens inclinata inclusa inclyta incola incolumis incommoda incompleta incomposita incompta inconcinna incondita incongrua incorrupta incrassata incredibilis increpans increpata increta increta

incretata incretata incrustata inculta incuriosa incurva incurvata incusa incusata
ARRHENOPHANES

HYPERCALLIA

ODITES

PTILOSTICHA

EPORYCTA

SCAEOSOPHA

MELASINA

ECCLITICA

CNEPHASIA

HYPOPHRICTIS*

PLATYPTILIA

OMICHLOSPORA*

BUCCULATRIX

DEMOBROTIS

CNEPHASIA

GONIONOTA

EPHEDROXENA*

ARISTOTELIA

STENOMA

THAMNOSCELIS*

DECADARCHIS

CYMOTRICHA

CAMPTRODOXA*

MEGACRASPEDUS

ODITES

PHILOMUSAEA

ANTAEOTRICHA

PELTOPHORA

PYRODERCES

LIMNOECIA

NOTHRIS

ANTAEOTRICHA

CAPUA

ANTAEOTRICHA

TINEA

EXONCOTIS*

BUCCULATRIX

EULECHRIA

HOLCOCERA

AUXIMOBASIS

SPILONOTA

HAPSIFERA

DECADARCHIS

BLASTOBASIS

ANACAMPSIS

LITHOCOLLETIS

CNEPHASIA

ODITES
I9I3, Trans. Ent. Soc. London, I9I3: I99.

I9I4, Exotic Microlepidoptera, I : I92. (BM)

I9I5, Exotic Microlepidoptera, I: 38I. (BM)

I9Io, Trans. Ent. Soc. London, I9Io: 44I.

I92I, Ann. Transvaal Mus. 8: I05. (T)

I928, Exotic Microlepidoptera, 3: 469. (BM)

I9I4, Exotic Microlepidoptera, I: 2 I2.

I923, Trans. Proc. New Zealand Inst. 54: I64.

I9I2, Exotic Microlepidoptera, I: Io. (T)

I9I6, Exotic Microlepidoptera, I: 605.

I9I3, Exotic Microlepidoptera, I : III.

I928, Bull. Hill Mus. 2: 239. (P)

I9I3, Ann. Transvaal Mus. 3: 329. (T)

I9I9, Exotic Microlepidoptera, 2: 252.

1920, Voyage de Ch. Alluaud et $R$. Jeannel en Afrique

Oriental, II, Microlepidoptera, p. 55. (P)

I909, Trans. Ent. Soc. London, I909: 27. (BM)

I9I9, Exotic Microlepidoptera, 2: 277. (Bм)

I9I8, Exotic Microlepidoptera, 2: I I7. (BM)

1925, Exotic Microlepidoptera, 3: 207. (BM)

I928, Exotic Microlepidoptera, 3: 466. (BM)

I92I, Zool. Meded. 6: I95. (L)

1927, Exotic Microlepidoptera, 3: 355. (T)

I925, Exotic Microlepidoptera, 3: I44. (BM)

I9I2, Ann. South African Mus. Io: 60. (SA)

I9I8, Ann. Transvaal Mus. 6: 54. (T)

I931, Ann. Mus. Nac. Hist. Nat. Buenos Aires, 36: 395.

I932, Exotic Microlepidoptera, 4: 293. (v)

I884, Proc. Linnean Soc. N.S. Wales, 9: 728 .

I9I7, Exotic Microlepidoptera, 2: 40.

I923, Exotic Microlepidoptera, 3: 59. (BM)

I9I3, Journ. Bombay Nat. Hist. Soc. 22: I70. (вм)

I932, Exotic Microlepidoptera, 4: 29I. (v)

I922, Exotic Microlepidoptera, 2: 494.

I9I6, Exotic Microlepidoptera, I: 504. (вм)

I920, Exotic Microlepidoptera, 2: 354.

I9I9, Exotic Microlepidoptera, 2: 269. (BM)

I9I5, Trans. Ent. Soc. London, I9I5: 249.

I93I, Exotic Microlepidoptera, 4: I87. (BM)

I931, in Joannis, Ann. Soc. Ent. France, 98 (Supp.):

$729[49 \mathrm{I}] .(\mathrm{P})$

I93I, Exotic Microlepidoptera, 4: I77. (BM)

I93I, Exotic Microlepidoptera, 4: I43.

I930, Exotic Microlepidoptera, 3: 553. (BM)

I927, Insects of Samoa, 3, Lepidoptera, fasc. 2, p. III.

I9I6, Exotic Micrnlepidoptera, I: 597.

I9I4, Trans. Ent. Soc. London, I9I4: 264. (BM)

I9I6, Exotic Microlepidoptera, I: 622.

I9I7, Trans, Ent. Soc. London, I9I7: I2. (BM)

I92I, Ann. Transvaal Mus. 8: Io7. (T) 


\begin{tabular}{|c|c|}
\hline indagata & SYMMOCA \\
\hline indecorella & SPHYRELATA* \\
\hline indefessa & PROADAMAS* \\
\hline indentatus & OXYPTILUS \\
\hline index & OPOSTEGA \\
\hline indicans & ERECHTHIAS \\
\hline indicata & TELEPHILA \\
\hline indicatrix & HECTACMA \\
\hline indigena & MELASINA \\
\hline indigens & FRISILIA \\
\hline indigesta & BLASTOBASIS \\
\hline indigestana & TORTRIX \\
\hline indigna & ULOMETRA* \\
\hline indirecta & BLASTOBASIS \\
\hline indiscreta & HIEROXESTIS \\
\hline indiserta & DICHOMERIS \\
\hline indocilis & GLYPHIDOCERA \\
\hline indocta & ALUCITA \\
\hline indocta & MACHAERITIS \\
\hline indolescens & TRACHYPEPLA \\
\hline indomita & GLYPHIPTERYX \\
\hline industria & BLASTOBASIS \\
\hline inepta & LECITHOCERA \\
\hline inelegans & MICROZESTIS* \\
\hline inermis & POLYHYMNO \\
\hline iners & STATHMOPODA \\
\hline inerudita & BRACHMIA \\
\hline inexperta & PHTHORIMAEA \\
\hline inexpleta & THEMELIOTIS \\
\hline infamis & BAEONOMA \\
\hline infanda & ATYCHIA \\
\hline infausta & ELLOCHOTIS* \\
\hline infecta & STENOMA \\
\hline infectana & PALAEOBIA \\
\hline infelix & ETHMIA \\
\hline infensa & MELASINA \\
\hline infensa & SPILONOTA \\
\hline infera & LEPIDOPODA \\
\hline inferialis & TELPHUSA \\
\hline infernus & TRICHOPTILUS \\
\hline infervescens & LACTURA \\
\hline infesta & HETEROBATHRA \\
\hline infesta & PLATYPTILIA \\
\hline infestata & EULECHRIA \\
\hline infibulata & HELCYSTOGRAMMA \\
\hline inficeta & MEGACRASPEDUS \\
\hline infida & ACROLOPHUS \\
\hline infima & IMMA \\
\hline irma & GNORIMOSCHEMA \\
\hline
\end{tabular}

I9I8, Exotic Microlepidoptera, 2: I55. (BM) I883, Proc. Linnean Soc. N.S. Wales, 8: 362. I929, Exotic Microlepidoptera, 3: 528 . I930, Exotic Microlepidoptera, 3: 564 . 1922, Exotic Microlepidoptera, 2: 557. 1923, Trans. Proc. New Zealand Inst. 54: I68. I93I, Exotic Microlepidoptera, 4:67. (BM) 1924, Exotic Microlepidoptera, 3: 84. (BM) I9I7, Ann. South African Mus. I7: I5. (SA) I9I4, Supplementa Entomologica, No. 3, p. 50. (D) I93I, Exotic Microlepidoptera, 4: I77. (T) I88I, Proc. Linnean Soc. N.S. Wales, 6: 520. I9I2, Exotic Microlepidoptera, I: 28. (T) I935, Exotic Microlepidoptera, 4:567. I9I7, Ann. South African Mus. I7: I3. (SA) I926, Exotic Microlepidoptera, 3: 285. (BM) I930, Ann. Naturhist. Mus. Wien, 44: 229. (v) I9I3, Exotic Microlepidoptera, I: III. I886, Proc. Linnean Soc. N.S. Wales, Io: 772. I927, Trans. Proc. New Zealand Inst. 57: 700. I922, Exotic Microlepidoptera, 2: 49I. (BM) I9I3, Ann. Transvaal Mus. 3: 3I4. (T) I926, Sarawak Mus. Journ. 3: I57. I929, Trans. Ent. Soc. London, 76: 502. I9I3, Ann. Transvaal Mus. 3: 284. (T) I9I3, Exotic Microlepidoptera, I: 87. I926, Exotic Microlepidoptera, 3: 290. (BM) I925, in Wytsman, Genera Insectorum, fasc. I84, p. 93. I9I9, Exotic Microlepidoptera, 2: 260. I925, Exotic Microlepidoptera, 3: I60. (BM) I920, Ann. South African Mus. I7: 294. (SA) I920, Ann. South African Mus. I7: 3II. (SA) I930, Ann. Naturhist. Mus. Wien, 44: 254. (v) I88I, Proc. Linnean Soc. N.S. Wales, 6: 663. I9I4, in Wagner, Lepidopterorum Catalogus, I9: 29. I9I6, Exotic Microlepidoptera, I: 6Io.

I9I I, Proc. Linnean Soc. N.S. Wales, 36: 228. I92I, Exotic Microlepidoptera, 2: 442. I9I8, Exotic Microlepidoptera, 2: I33. (BM) 1938, Institut des Parcs Nationaux $d u$ Congo Belge, fasc. I4, p. 3 . I930, Exotic Microlepidoptera, 3: 596. (BM) I921, Exotic Microlepidoptera, 2: 393. 1934, Ann. Mag. Nat. Hist. I4: 403. I9I4, Exotic Microlepidoptera, I : I66. I9I6, Exotic Microlepidoptera, I: 577. (BM) I904, Proc. Linnean Soc. N.S. Wales, 29: 277. I9I3, Trans. Ent. Soc. London, I9I3: I97. (BM) I930, Trans. Ent. Soc. London, 78: 3I9. (P) I9I2, Ann. South African Mus. IO: 6I. (SA) 
infixa

BRACHMIA

inflammata
inflata
inflicta
informalis

informis

infrenata

infricta

infulata

infumata

infumata

infusa

infusoria

ingeminans

ingeminata

ingeminata

ingeniosa

ingens

ingenua

ingenua

ingloria

ingrata

ingrata

ingravata

ingravescens

inhonesta

inhonorata

inimica

iniqua

initiata

injucunda

injusta

innexa

innocens

innocua

innumera

innupta

inodes

inodes

inophora

inopina

inops

inota

inquieta

inquinata

inquisitrix

insana
MACHIMIA

LASPEYRESIA

POLYCHROSIS

ARGYROPLOCE

STATHMOPODA

STENOMA

PARAPSECTRIS

EPIMACTIS

AGDISTIS

PHTHINOSTOMA*

STENOMA

PARAPTILA

CEROMITIA

LYTROPHILA

THYROCOPA

COSMOPTERYX

NEPTICULA

EPICHORISTA

TRACHYPEPLA

DICHOMERIS

COESYRA

EUCLEODORA

ARISTOTELIA

ASYMPHORODES

PACHNISTIS

HIEROMANTIS

MELASINA

ARGYROPLOCE

PAROCYSTOLA

STENOMA

PARAMORPHA

STENOMA

COESYRA

PALAETHETA

COESYRA

PHYLLOCNISTIS

ARGYROPLOCE

LABDIA

CYATHAULA

ACHORIA*

ACOROSTOMA

SCYTHRIS

AGRIASTIS

LOPHAEOLA*

MACRAEOLA

HELIOCAUSTA
I938, Institut des Parcs Nationaux du Congo Belge, fasc. I4, p. I7. (c)

I9I6, Exotic Microlepidoptera, I: 55I. (BM)

I9I6, Exotic Microlepidoptera, 2: 23. (BM)

I920, Exotic Microlepidoptera, 2: 347. (BM)

I935, in Caradja and Meyrick, Materialien zu einer Micro-

lepidopteren Fauna der Chinesischen Provinzen Kiangsu,

Chekiang und Hunan, p. 59. (BM)

I9I3, Exotic Microlepidoptera, I: 87 .

I9I8, Exotic Microlepidoptera, 2: 202. (BM)

I9I6, Exotic Microlepidoptera, I: 579. (BM)

I9I4, Journ. Bombay Nat. Hist. Soc. 22: 779. (BM)

I9I2, Ann. South African Mus. Io: 55. (SA)

I9I4, Ann. Transvaal Mus. 4: Ig6. (T)

I9I6, Exotic Microlepidoptera, I: 522. (BM)

I926, Exotic Microlepidoptera, 3: 259. (BM)

I935, Exotic Microlepidoptera, 4: 557. (C)

I9I4, Ann. Transvaal Mus. 4: 20I. (T)

I9I5, Exotic Microlepidoptera, I: 37I. (BM)

I909, Journ. Bombay Nat. Hist. Soc. I9: 42I.

I9I3, Ann. Transvaal Mus. 3: 327. (T)

I9I2, Exotic Microlepidoptera, I: 9. (BM)

I9I I, Trans. New Zealand Inst. 43: 65.

I923, Exotic Microlepidoptera, 2:621. (BM)

I9I3, Exotic Microlepidoptera, I: II7. (MA)

I9I3, Ann. Transvaal Mus. 3: 3I6. (T)

I9I8, Exotic Microlepidoptera, 2: II8.

I934, Pacific Ent. Surv. Publ. 7, art. 28, p. 350. (вв)

I9I6, Exotic Microlepidoptera, I: 579. (BM)

I927, Insects of Samoa, 3, Lepidoptera, fasc. 2, p. 99.

I908, Proc. Zool. Soc. London, p. 747.

I92I, Exotic Microlepidoptera, 2: 449.

I920, Exotic Microlepidoptera, 2: 382.

I925, Exotic Microlepidoptera, 3: 2I9. (BM)

I9I3, Exotic Microlepidoptera, I: I53.

I925, Exotic Microlepidoptera, 3: I90. (Bм)

I9I3, Exotic Microlepidoptera, I: II8.

I9I2, Ann. Transvaal Mus. 3: 76. (T)

I889, Proc. Linnean Soc. N.S. Wales, (Ser. 2) 3: I66o.

1922, Exotic Microlepidoptera, 2: 557.

I9II, Proc. Limnean Soc. N.S. Wales, 36: 269. (BM)

I922, Exotic Microlepidoptera, 2: 569.

I9I9, Exotic Microlepidoptera, 2: 254.

I904, Proc. Linnean Soc. N.S. Wales, 29: 405.

I921, Ann. Transvaal Mus. 8: I32. (T)

I924, Exotic Microlepidoptera, 3:87.

I9I4, Trans. Ent. Soc. London, I9I4: 253. (BM)

I932, Exotic Microlepidoptera, 4: I95. (BM)

I9I6, Exotic Microlepidoptera, I: 602.

I92I, Exotic Microlepidoptera, 2: 357 . 


\begin{tabular}{|c|c|c|}
\hline 172 & & INSCIA \\
\hline inscia & MENDESIA & I913, Ann. Transvaal Mus. 3: 322. (T) \\
\hline inscita & EUCOSMA & I9I3, Ann. Transvaal Mus. 3:272. (T) \\
\hline inscripta & PRAYS & I907, Proc. Linnean Soc. N.S. Wales, $32: 75$. \\
\hline insectivora & TINEA & I932, Exotic Microlepidoptera, $4: 325$. \\
\hline insecutrix & LACTURA & I924, Exotic Microlepidoptera, 3: I27. \\
\hline insellata & ARGYROPLOCE & $\begin{array}{l}\text { I920, Voyage de Ch. Alluand et } R \text {. Jeannel en Afrique } \\
\text { Oriental, II, Microlepidoptera, p. } 66 .(\mathrm{P})\end{array}$ \\
\hline insequens & RECURVARIA & I93I, Exotic Microlepidoptera, 4:56. (v) \\
\hline insidians & LECITHOCERA & I9I8, Exotic Microlepidoptera, 2: Io8. (BM) \\
\hline insidiata & STENOMA & I9I6, Exotic Microlepidoptera, I: 512. (BM) \\
\hline insidiatrix & AGDISTIS & I933, Exotic Microlepidoptera, 4: 426. (v) \\
\hline insidiosa & EUTORNA & I9Io, Journ. Bombay Nat. Hist. Soc. 20: I5o. (BM) \\
\hline insidiosa & THEREUTIS & I893, Proc. Linnean Soc. N.S. Wales, 7: 596. \\
\hline insignana & PYRGOTIS* & I88I, Proc. Linnean Soc. N.S. Wales, 6: 440. \\
\hline insignata & TINEA & I9I9, Exotic Microlepidoptera, $2: 246$. \\
\hline insignis & DECTOBATHRA & I904, Proc. Linnean Soc. N.S. Wales, 29: 301. \\
\hline insimulata & ANTAEOTRICHA & I926, Exotic Microlepidoptera, 3: 237. (BM) \\
\hline insincera & CHALAROTONA & I89o, Trans. R. Soc. S. Australia, I3: 66. \\
\hline insincera & TORTRIX & I9I2, Exotic Microlepidoptera, I: 7. (Bм) \\
\hline insinuans & DIPLONEARCHA* & I9I4, Exotic Microlepidoptera, I : 274 . \\
\hline insinuata & TACHYSTOLA & I9I4, Exotic Microlepidoptera, I: 24I. (BM) \\
\hline insinuatrix & HYPOSMOCOMA & I928, Proc. Hawaiian Ent. Soc. 7: I03. (SP) \\
\hline insipiens & EMPEDAULA* & I9I8, Exotic Microlepidoptera, 2: I49. (BM) \\
\hline insolens & EUCosma & I909, Ann. South African Mus. 5:367. (SA) \\
\hline insolens & PACHYPSALTIS* & I9I4, Supplementa Entorologica, No. 3, p. 6o. (D) \\
\hline insolita & CRYPSITHYRIS & I9I 8, Ann. Transvaal Mus. 6:57. (T) \\
\hline insomnis & COLOBODES* & I904, Proc. Linnean Soc. N.S. Wales, 29: 297. \\
\hline insons & ODITES & I912, Ann. South African Mus. I0: 62. (SA) \\
\hline inspectrix & AGRIOPHARA & I916, Exotic Microlepidoptera, I: 532. (BM) \\
\hline insperata & STENOPTILIA & I921, Exotic Microlepidoptera, 2: 422. \\
\hline inspersa & BRACHMIA & I92I, Exotic Microlepidoptera, 2: 436. (Bм) \\
\hline inspiciens & CYMOTRICHA & I93I, Exotic Microlepidoptera, 4:68. (v) \\
\hline inspirata & CHOREUTIS & I9I6, Exotic Microlepidoptera, I: 560. (BM) \\
\hline instabilis & TALAEPORIA & I924, Exotic Microlepidoptera, 3: 75. (BM) \\
\hline instans & DICHOMERIS & I923, Exotic Microlepidoptera, 2: 6I9. (BM) \\
\hline instaurata & CHELARIA & I921, Zool. Meded. 6: I65. (L) \\
\hline instigata & BUCCULATRIX & I915, Trans. Ent. Soc. London, I9I5: 249. \\
\hline instillata & ADOXOPHYES & I922, Arkiv för Zool. vol. I4, part I5, p. 2. \\
\hline instincta & GRACILARIA & I922, Exotic Microlepidoptera, 2: 564 . (Bм) \\
\hline instructa & BAREA & I920, Exotic Microlepidoptera, 2: 370. \\
\hline insuavis & BRACHMIA & I9I4, Supplementa Entomologica, No. 3, p. 5I. \\
\hline insulata & NEPTICULA & I9I2, Ann. Transwaal Mus. 3: 79. (T) \\
\hline insulata & TORTRIX & I9o8, Journ. Bombay Nat. Hist. Soc. I8: 6r 9. (Bм) \\
\hline insulicola & OLETHREUTES & I9I2, Ent. Mon. Mag. 48: 35. (Sub.) \\
\hline insulsa & BRACHMIA & I9I4, Journ. Bombay Nat. Hist. Soc. 22: 774. (вм) \\
\hline intabescens & CHALAROTONA* & I89o, Trans. R. Soc. S. Australia, I3: 65. \\
\hline intacta & PTOCHORYCTIS & $\begin{array}{l}\text { I938, in Caradja and Meyrick, Deuts. Ent. Zeit. Iris, } 52 \text { : } \\
\text { I4. (BM) }\end{array}$ \\
\hline & MACHIMIA & I922, Exotic Microlepidoptera, $2: 544 . \quad$ (P) \\
\hline & DOLIOTECHNA & I9I4, Exotic Microlepidoptera, I: I89. (BM) \\
\hline
\end{tabular}


integrata

integratus

intensa

intensa

intensa

interamna

intercepta

intercisus

interfracta

interfusa

interjecta

interjuncta

intermissa

internexa

interpolata

interpres

interscissa

intersecta

interstincta

intestina

intexta

intonans

intonsa

intorta

intractata

intrepida

intrepida

introspiciens

intuens

intumescens

inulivora

inumbrata

inurbana

inusta

invalida

inventrix

inversa

inveterata

invia

invicta

invida

invida

invida

invidiosa

invidiosus

invigilans

invigotata

inviolata

invisa
LECITHOCERA

PTEROPHORUS

COLEOPHORA

DICHOMERIS

TORTRIX

DICHOMERIS

APHANOSTOLA

OXYPTILUS

COSMOPTERYX

ACROLOPHUS

CACOECIA

PHTHORIMAEA

ARGYROPLOCE

LABDIA

ETHIROSTOMA

PLATYPTILIA

MELASINA

STENOMA

ASYMPHORODES

PHTHORIMAEA

COLEOPHORA

HYPERCALLIA

EUTORNA

POLYHYMNO

ILYGENES*

BLASTOBASIS

TORTRIX

TRICHOTAPHE

LABDIA

BORKHAUSENIA

EUCOSMA

ANACAMPSIS

GLYPHIDOCERA

ANACAMPSIS

PROTOLECHIA

CYANOCRATES

ODITES

MELASINA

ACOROSTOMA

GLYPHIPTERYX

MARASMARCHA

PALTODORA

PROSCHISTIS

XYSTROLOGA*

PTEROPHORUS

STENOMA

AUXIMOBASIS

PTOCHORYCTIS

SCYTHRIS
I9I8, Exotic Microlepidoptera, 2: I07. (BM)

I9I3, Exotic Microlepidoptera, I: II3.

I9I3, Ann. Transvaal Mus. 3: 323. (T)

I9I3, Journ. Bombay Nat. Hist. Soc. 22: I73. (BM)

I92I, Ann. Transvaal Mus. 8: 52. (T)

1926, Ann. South African Mus. 23: 333. (sA)

I932, Exotic Microlepidoptera, 4: 348. (BM)

1930, Exotic Microlepidoptera, 3: 565.

I922, Exotic Microlepidoptera, 2: 567. (BM)

I927, Exotic Microlepidoptera, 3: 330. (BM)

1922, Exotic Microlepidoptera, 2: 496. (P)

I93I, Exotic Microlepidoptera, 4: 62. (v)

I93I, Exotic Microlepidoptera, 4: I39. (v)

I927, Insects of Samoa, 3, Lepidoptera, fasc. 2, p. 88.

I922, Trans. Ent. Soc. London, p. 7 I. (BM)

I922, Exotic Microlepidoptera, 2: 548.

1924, Exotic Microlepidoptera, 3: 76. (T)

I9I6, Exotic Microlepidoptera, I: 5I5. (BM)

1929, Trans. Ent. Soc. London, 76: 500.

I921, Ann. Transvaal Mus. 8: 74. (T)

I9I7, Exotic Microlepidoptera, 2: 72.

I933, Exotic Microlepidoptera, 4: 368. (BM)

I9o6, Trans. R. Soc. S. Australia, 30: 42.

I9I8, Ann. Transvaal Mus. 6: i9. (T)

I938, in Caradja and Meyrick, Deuts. Ent. Zeit. Iris, 52: 27.

I9II, Trans. Linnean Soc. London, I4: 287.

I9I2, Exotic Microlepidoptera, I: 7. (BM)

I926, Exotic Microlepidoptera, 3: 287. (BM)

1923, Exotic Microlepidoptera, 3: 59. (BM)

I921, Ann. Transvaal Mus. 8: 99. (T)

I932, Exotic Microlepidoptera, 4:224.

I9I4, Ann. Transvaal Mus. 4: I93. (T)

I9I4, Trans. Ent. Soc. London, I9I4: 273. (BM)

I9I4, Trans. Ent. Soc. London, I9I4: 264. (BM)

I904, Proc. Linnean Soc. N.S. Wales, 29: 346.

I925, Exotic Microlepidoptera, 3: 156.

I9I4, Ann. South African Mus. I0: 250. (SA)

I9I5, Exotic Microlepidoptera, I: 290.

I92I, Ann. Transvaal Mus. 8: I32. (T)

I920, Exotic Microlepidoptera, 2: 334. (BM)

I908, Trans. Ent. Soc. London, I907: 493.

I9I2, Ann. Transvaal Mus. 3: 64. (T)

I909, Journ. Bombay Nat. Hist. Soc. I9: 590. (BM)

I9I9, Exotic Microlepidoptera, 2: 272. (BM)

I9I I, Ann. Transvaal Mus. 2: 220. (T)

I9I5, Exotic Microlepidoptera, I: 476. (BM)

I932, Exotic Microlepidoptera, 4: 3I6. (v)

I925, Exotic Microlepidopiera, 3: I52.

I920, Voyage de Ch. Alluaud et R. Jeannel en Afrique

Oriental, II, Microlepidoptera, p. 85. 
invita

involucralis

involucrata

involuta

invulgata

iobaphes

iobapta

iobola

iocapna

iochlaca

iocharis

iocheaera

iochlaena

iochondra

iochorda

iochrysa

iochrysis

ioclista

iocoma

iocoma

iocrossa

iocycla

iodes

iodes

iodesma

iodocella

iodorus

iodryas

iograpta

iolampra

ioleuca

iolitha

ioloncha

ioloxa

iolychna

iomarmara

iometalla

iomolybda

ionephela

ionota

iopercna

iopetra

iophaea

iophaea

iophana

iophanes
EPICEPHALA

STENOMA

EUCOSMA

PHTHORIMAEA

STENOMA

OENOCHROA

STOMOPTERYX

LEISTARCHA *

PARALECTA

HAPLODYTA

TRACHYDORA

GLYPHIPTERYX

PROTOLECHIA

SIMAETHIS

DICHELOPA

USSARA

LITHOCOLLETIS

GLYPHIPTERYX

STENOMA

TORTRIX

HEMIMENE

STATHMOPODA

EPICHORISTA

STATHMOPODA

PERIACMA

PHYLLOCNISTIS

YPSOLOPHUS

HERPYSTIS

EUCOSMA

LABDIA

COPTOTELIA

CEROMITIA

PARASPISTES*

DECADARCHIS

HIE ROXESTIS

ARISTOTELIA

GLYPHIPTERYX

BACTRA

PROSELENA

STENOPTILIA

STENOMA

STENOMA

ARGYROPLOCE

AROTRIA*

CHELARIA

GRACILARIA
I9I2, Exotic Microlepidoptera, I: 22. (T)

I93I, Exotic Microlepidoptera, 4: 40. (v)

I927, Insects of Samoa, 3, Lepidoptera, fasc. 2, p. 73. (BM)

I9I7, Trans. Ent. Soc. London, I9I7: 47. (BM)

I9I 5, Exotic Microlepidoptera, I: 458. (Bм)

I883, Proc. Linnean Soc. N.S. Wales, 8: 330.

1927, Exotic Microlepidoptera, 3: 352. (BM)

I883, Proc. Linnean Soc. N.S. Wales, 8: 326.

1925, Exotic Microlepidoptera, 3: I54.

I886, Proc Linnean Soc. N.S. Wales, Io: 766 .

I9I8, Ann. Transvaal Mus. 6: 28. (T)

I88I, Proc. Linnean Soc. N.S. Wales, 5: 243.

I904, Proc. Linnean Soc. N.S. Wales, 29: 353.

I9I I, Trans. New Zealand Inst. 32: 77.

I926, Trans. Ent. Soc. London, 74: 273. (BM)

I921, Zool. Meded. 6: I84. (L)

I93I, Exotic Microlepidoptera, 4: I67.

I9I3, Exotic Microlepidoptera, I: 70. (BM)

I9I5, Exotic Microlepidoptera, I: 450. (BM)

I908, Proc. Zool. Soc. London, p. 723. (BM)

I9I6, Exotic Microlepidoptera, 2: 25. (BM)

I933, Exotic Microlepidoptera, 4: 430.

I9ro, Proc. Linnean Soc. N.S. Wales, 35: 258.

I897, Proc. Linnean Soc. N.S. Wales, $22: 323$.

I9Io, Journ. Bombay Nat. Hist. Soc. 20: I6I. (BM)

I88I, Proc. Linnean Soc. N.S. Wales, 5: I74.

1904, Proc. Linnean Soc. N.S. Wales, 29: 432.

I937, in Caradja and Meyrick, Deuts. Ent. Zeit. Iris, 5I: I76. (BM)

I907, Journ. Bombay Nat. Hist. Soc. I8: I37. (BM)

I938, in Caradja and Meyrick, Deuts. Ent. Zeit. Iris, $52: 8$.

I9I2, Trans. Ent. Soc. London, I9I I: 703. (BM)

I9I4, Exotic Microlepidoptera, I: 2 I5.

I905, Journ. Bombay Nat. Hist. Soc. I6: 600.

I936, Exotic Microlepidoptera, 4:62I.

I920, Exotic Microlepidoptera, 2: 361.

I92I, Exotic Microlepidoptera, 2: 425. (BM)

I88I, Proc. Linnean Soc. N.S. Wales, 5: 232.

I932, Exotic Microlepidoptera, 4: 224. (BM)

I905, Ann. South African Mus. 5: 350. (SA)

I920, Voyage de Ch. Alluaud et R. Jeannel en Afrique

Oriental, II, Microlepidoptera, p. 43. (P)

I932, Exotic Microlepidoptera, 4: 300. (BM)

I932, Exotic Microlepidoptera, 4: 295. (BM)

I9I2, Journ. Bombay Nat. Hist. Soc. 2I: 873. (BM)

I904, Proc. Limnean Soc. N.S. Wales, 29: 387.

I9I3, Journ. Bombay Nat. Hist. Soc. 22: I62. (BM)

I9I2, in Wytsman, Genera Insectorum, fasc. I28, p. 27. 


\begin{tabular}{|c|c|}
\hline iophanes & HIERODORIS* \\
\hline ioplaca & BRACHMIA \\
\hline iopleura & ARGYRESTHIA \\
\hline ioplintha & EUCOSMA \\
\hline ioploca & ZALITHIA \\
\hline ioplocama & HAPALOTHYMA \\
\hline iopsamma & TORTRIX \\
\hline ioptila & STENOMA \\
\hline iopyrtha & DIACHOLOTIS* \\
\hline ioreas & EUCOSMA \\
\hline iorphna & GRACILARIA \\
\hline iorrhoa & ARCHEMITRA* \\
\hline iorrhoa & ARGYROPLOCE \\
\hline iosema & PHILOBOTA \\
\hline iospila & BALIOXENA* \\
\hline iospila & EUCHAETIS \\
\hline iospila & PALTODORA \\
\hline iospora & ARISTOTELIA \\
\hline iostalacta & STENOMA \\
\hline iostephana & CREPIDOSCELES* \\
\hline iosticta & TELPHUSA \\
\hline iostrota & $\begin{array}{l}\text { LIMNOECIA } \\
\text { [XESTOCASIS*] }\end{array}$ \\
\hline ioterma & ADOXOPHYES \\
\hline iothrinca & DELONOMA* \\
\hline ioxantha & ENARMONIA \\
\hline ioxantha & PYRODERCES \\
\hline ioxanthas & ACROCLITA \\
\hline ioxesta & LASPEYRESIA \\
\hline ioxysta & HIEROMANTIS \\
\hline iozona & COESYRA \\
\hline iphicleia & TRICLONELLA \\
\hline iphicrates & ETHMIA \\
\hline iphigenes & PHILOBOTA \\
\hline iphigenia & LYONETIA \\
\hline iphigona & COSMOPTERYX \\
\hline iphimacha & POLYHYMNO \\
\hline ipnitis & $\begin{array}{l}\text { DRACHMOBOLA } \\
\text { [CAPNOPTYCHA*] }\end{array}$ \\
\hline iracunda & ORSIMACHA \\
\hline ras & ANTAEOTRICHA \\
\hline ascens & STENOMA \\
\hline irata & CARPOSINA \\
\hline irenaea & EULECHRIA \\
\hline enias & STENOMA \\
\hline nica & HIEROXESTIS \\
\hline esia & BRACHMIA \\
\hline
\end{tabular}

I9I2, Exotic Microlepidoptera, I: 42.

I934, Exotic Microlepidoptera, 4: 543.

I918, Exotic Microlepidoptera, 2: 187. (вм)

I930, Exotic Microlepidoptera, 3: 600. (BM)

I922, Trans. Ent. Soc. London, I922: I03. (вм)

I920, Exotic Microlepidoptera, 2: 289.

I93I, Exotic Microlepidoptera, 4: I50.

1915, Exotic Microlepidoptera, I: 433. (вм)

1937, Exotic Microlepidoptera, 5: 79.

I920, Voyage de Ch. Alluaud et $R$. Jeannel en Afrique

Oriental, II, Microlepidoptera, p. 59. (P)

1939, Trans. R. Ent. Soc. London, 89: 60.

I920, Voyage de Ch. Alluaud et $R$. Jeannel en Afrique

Oriental, II, Microlepidoptera, p. 96. (P)

1914, Exotic Microlepidoptera, I: I96. (BM)

I889, Proc. Linnean Soc. N.S. Wales, (Ser. 2) 3: I618.

I9I2, Exotic Microlepidoptera, I: I3. (BM)

I888, Proc. Linnean Soc. N.S. Wales, (Ser. 2) 2: 938.

I909, Ann. Transvaal Mus. 2: Io, pl. 4, fig. 2. (T)

1929, Exotic Microlepidoptera, 3: 482. (BM)

I925, Exotic Microlepidoptera, 3: 2I2. (BM)

I885, Proc. Linnean Soc. N.S. Wales, 9: 1056.

1937, Exotic Microlepidoptera, 5:92. (T)

I9Io, Trans. Ent. Soc. London, I9Io: 454.

I910, Proc. Linnean Soc. N.S. Wales, 35: 205.

I9I4, Exotic Microlepidoptera, I: I93.

I907, Journ. Bombay Nat. Hist. Soc. I8: I39. (BM)

I915, Exotic Microlepidoptera, I : 3 I3.

I930, Exotic Microlepidoptera, 3: 599. (вм)

1922, Exotic Microlepidoptera, 2: 532. (вм)

I913, Exctic Microlepidoptera, I: 82.

I884, Proc. Linnean Soc. N.S. Wales, 9: 768.

1924, Exotic Microlefidoptera, 3: 98. (BM)

I92 I, Exotic Microlepidoptera, 2: 473. (BM)

I889, Proc. Linnean Soc. N.S. Wales, (Ser. 2) 3: I6I4.

1932, Exotic Microlepidoftera, 4: 230.

1915, Exotic Microlepidoptera, I : 323.

1933, Exotic Microlepidoptera, 4: 448. (вм)

I910, Proc. Linnean Soc. N.S. Wales, 35:285.

I9I4, Exotic Microlepidoptera, I: I87. (BM)

1926, Exotic Microlepidoptera, 3: 237. (вм)

I930, Ann. Naturhist. Mus. Wien, 44: 252. (v)

I9I4, Ann. Transvaal Mus. 4: I87. (T)

I888, Proc. Linnean Soc. N.S. Wales, (Ser. 2) 2: 962.

(MA)

I916, Exotic Microlepidoptera, I: 537 . (вм)

I9II, Trans. Linnean Soc. London, I4: 296.

I9II, Journ. Bombay Nat. Hist. Soc. 20: 709. (BM) 


iresiarcha
iriantha
irianthes
iriarcha
iriastis
iricharis
iricolor
iridescens
iridesma
iridias
iriditus
iridocrossa
iridogramma
iridopa
iridophanes
iridoptila
iridorphna
iridosoma
iridosoma
iridoxa

\section{iridozona}

irigramma

irimetalla

irimochla

irina

iriodes

iriphaea

iriphanes

iritis

ironica

irradians

irradiata

irridens

irrigata

irrigua

irrisoria

irrita

irrita

irritabilis

irritata

isacma

isacta

isalopex
CHOLOTIS

ZALITHIA

PASSALOTIS*

COCONYMPHA*

LIMNOECIA

HIEROXESTIS

PROSCHISTIS

STIGMONOTA

CARMENTINA*

CRYPTOLECHIA

TELPHUSA

ACROCERCOPS

OPOGONA

TORTYRA

GRACILARIA

TRACHYDORA

ACROCLITA

ARGYROPLOCE

HELCYSTOGRAMMA PHILPOTTIA*

[CHARIXENA*]

AEOLOCOSMA*

LABDIA

LABDIA

SIMAETHIS

ACTINOSCELIS*

PILOPREPES

[HABROSCOPA*]

LABDIA

LITHOCOLLETIS

TINEA

TRICHOTAPHE

ACROCERCOPS

CHOREUTIS

SIMAETHIS

PYCNODYTIS

PYRODERCES

ACROLOPHUS

LATHRONYMPHA

TINEA

MACHAEROPTERIS

TINEA

LASPEYRESIA

EUPSELIA

ORIDRYAS*
I9I5, Exotic Microlepidoptera, I: 329. (BM)

I9I4, Trans. Ent. Soc. London, I9I4: 270. (BM)

I932, Exotic Microlepidoptera, 4:215.

I93I, Exotic Microlepidoptera, 4: 66. (BM)

I897, Proc. Linnean Soc. N.S. Wales, 22: 367.

1926, Ann. South African Mus. 23: 34I. (SA)

I930, Exotic Microlepidoptera, 3: 60I. (BM)

I88I, Proc. Linnean Soc. N.S. Wales, 6: 655.

I930, Exotic Microlepidoptera, 3: 598. (BM)

I9Io, Journ. Bombay Nat. Hist. Soc. 20: I63. (BM)

I920, Ann. South African Mus. I7:282. (SA)

I938, in Caradja and Meyrick, Deuts. Ent. Zeit. Iris, 52:

$2 \mathrm{I}$.

I924, Trans. Ent. Soc. London, I923: 555.

1907, Proc. Linnean Soc. N.S. Wales, 32: 97.

I935, Exotic Microlepidoptera, 4: 599 .

I92I, Exotic Microlepidoptera, 2: 4I4.

I936, Exotic Microlepidoptera, 4: 609. (вм)

I9I I, Proc. Linnean Soc. N.S. Wales, 36: 264.

I9I8, Exotic Microlepidoptera, 2: I44.

I9I6, Trans. Proc. New Zealand Inst. 48: 4 I7.

I881, Proc. Linnean Soc. N.S. Wales, 5: 225.

I927, Insects of Samoa, 3, Lepidoptera, fasc. 2, p. 85 .

I933, Exotic Microlepidoptera, 4: 427.

I921, Zool. Meded. 6: I82. (L)

I9I2, Exotic Microlepidoptera, I: 59.

I883, Proc. Linnean Soc. N.S. Wales, 8: 365.

1932, Exotic Microlepidoptera, 4: 213.

I9I5, Trans. Ent. Soc. London, I9I5: 223.

I9II, Journ. Bombay Nat. Hist. Soc. 21 : I22.

I9o9, Ann. Transvaal Mus. 2: I7, pl. 6, fig. I. (T)

I93I, Exotic Microlepidoplera, 4: I70.

I9I3, Exotic Microlepidoptera, I: 67. (BM)

I92I, Ann. Transvaal Mus. 8: III. (T)

I9I8, Exotic Microlepidoptera, 2: I20.

I9I5, Exotic Microlepidoptera, I: 3I5.

1924, Exotic Microlepidoptera, 3: 76. (BM)

I935, in Caradja and Meyrick, Materialien zu einer Micro-

lepidopteren Fauna der Chinesischen Provinzen Kiangsu,

Chekiang und Hunan, p. 6I. (BM)

I936, in Caradja and Meyrick, Deuts. Ent. Zeit. Iris, 50: I59.

1932, Exotic Microlepidoptera, 4: 329.

I9I9, Exotic Microlepidoptera, 2: 245.

I907, Journ. Bombay Nat. Hist. Soc. I8: I44. (BM)

I9Io, Journ. Bombay Nat. Hist. Soc. 20: I54. (Вм)

I938, in Caradja and Meyrick, Deuts. Ent. Zeit. Iris, 52: I8. 
isanema

isarga

isarithma

isastra

ischalea

ischalea

ischiastris

ischioptila

ischnites

ischnobathra

ischnodes

ischnodesma

ischnophaea

ischnoptera

ischnoscia

ischnoscia

ischnota

ischnotis

ischnotoma

ischnozona

iselaea

isemera

isidora

isocampta

isocentra

isochalca

isocharis

isochlora

isochorda

isochorda

isochra

isochroa

isochroa

isochroa

isochrysa

isochrysa

isochtha

isochyta

isoclera

isoclera

isoclina

isoclista

isocola

isocosma

isocrates

isocrita

isocrossa

isocrypta

isocycla
ANTICRATES

[CHIONOGENES*]

STENOMA

OCYSTOLA

HYPERCALLIA

ACROCLITA

ORNEODES

GRACILARIA

STENOMA

TETRASCHALIS

ACHARNEODES

EULECHRIA

NEMOTOIS

OPOSTEGA

COMPSOLECHIA

OPOGONA

STENOMA

MEGACRASPEDUS

STATHMOPODA

PARECTOPA

PALAETHETA*

GRACILARIA

HEMICALYPTRIS*

PROMALACTIS

PAMMENE

MYRIOPLEURA

NEPTICULA

TINEA

PHTHORIMAEA

CONOPOMORPHA

GELECHIA

TIMYRA

EUDEMIS

HELIOZELA

TORTRIX

ANAPHANTIS*

GRACILARIA

BATRACHEDRA

STENOMA

AEOLOSCELIS

HOLAXYRA

OPOGONA

GLYPHIPTERYX

CROCOGMA*

ANAPTILORA*

PLATYPTILIA

MOMPHA

GELECHIA

BRACHMIA

CHEZALA
1907, Proc. Linnean Soc. N.S. Wales, 32: 84. I925, Exotic Microlepidoptera, 3: 224. (BM) 1885, Proc. Linnean Soc. N.S. Wales, 9: 1065. I926, Exotic Microlepidoptera, 3: 3I2. (BM) I9II, Proc. Linnean Soc. N.S. Wales, 36: 240. 1905, Journ. Bombay Nat. Hist. Soc. I6: 583. 1907, Proc. Linnean Soc. N.S. Wales, 32: 66. I925, Exotic Microlepidoptera, 3: 2II. (BM) I9o8, Trans. Ent. Soc. London, I907: 474. I938, Trans. R. Ent. Soc. London, 87: 5II. (BM) I902, Trans. R. Soc. S. Australia, 26: I50. I928, Exotic Microlepidoptera, 3: 464 . I930, Exotic Microlepidoptera, 4: 7 . I922, Trans. Ent. Soc. London, I922: 85. (BM) 1928, Exotic Microlepidoptera, 3:40I. (T) I932, Exotic Microlepidoptera, 4: 298. (v) I904, Proc. Linnean Soc. N.S. Wales, 29: 282. I897, Proc. Linnean Soc. N.S. Wales, 22: 324. I9I5, Trans. Ent. Soc. London, I9I5: 233.

I909, Ann. Transvaal Mus. 2: 24, pl. 7, fig. 9. (T) I9I4, Exotic Microlepidoptera, I: 286.

I933, Exotic Microlepidoptera, 4:432.

I908, Journ. Bombay Nat. Hist. Soc. I8: 8og. (BM)

I9I4, Exotic Microlepidoptera, I : I96. (Bм)

I9o6, Journ. Bombay Nat. Hist. Soc. I7: 406. (BM) I9I6, Exotic Microlepidoptera, 2: 6.

I930, Exotic Microlepidoptera, 3: 547.

I93I, Journ. Linnean Soc. London, 37: 280. (BM)

I907, Journ. Bombay Nat. Hist. Soc. I7: 746.

I921, Ann. Transvaal Mus. 8: 72. (T)

I9o8, Journ. Bombay Nat. Hist. Soc. I8: 444. (Bм) I89I, Ent. Mon. Mag. 27: I2.

I897, Proc. Linnean Soc. N.S. Wales, 22: 406. I9Io, Proc. Linnean Soc. N.S. Wales, 35:252.

I907, Proc. Linnean Soc. N.S. Wales, 32: 9I. I9o8, Journ. Bombay Nat. Hist. Soc. I 8: 829.

I9I4, Ann. Transvaal Mus. 4: 203. (T)

I9I5, Exotic Microlepidoptera, I: 420. (BM)

I897, Proc. Linnean Soc. N.S. Wales, 22: 328.

I9I3, Journ. Bombay Nat. Hist. Soc. 22: I76. (Вм) 1907, Journ. Bombay Nat. Hist. Soc. I7: 986.

I925, Exotic Microlepidoptera, 3: I36.

I9I8, Exotic Microlepidoptera, 2: Ioo. (BM)

I904, Proc. Linnean Soc. N.S. Wales, 29: 390.

I924, Exotic Microlepidoptera, 3: 94.

1935, Exotic Microlepidoptera, 4:607.

I927, Exotic Microlepidoptera, 3: 346. (BM)

I9II, Journ. Bombay Nat. Hist. Soc. 20: 709. (BM)

I922, Arkiv för Zool. vol. I4, part I5, p. S. 
isocyrta
isodelta
isodelta
isodina

isodisca

isodonta

isodoxa

isodryas

isogama

isogona

isogramma

isogramma

isographa

isographa

isograpta

isoletras

isoleuca

isoleura

isolitha

isoloxa

isomacha

isomacra

isomalla

isomeris

isometra

isomichla

isomila

isomima

isomitra

isomora

isomorpha

isonephela

isonephes

isoniphas

isonira

isonoma

isopeda

isopela

isopela

isopelta

isopercna

isopetra

isophaea

isophaula

isophylla

isoplaca
CACOECIA

ACROCERCOPS

EULIA

ORNEODES

CHOROPLECA

TINEA

ARGYROPLOCE

HYPERCALLIA

ATOMOTRICHA

ANARSIA

COESYRA

CYDIA

CAPUA

TIMOCRATICA*

GRACILARIA

TORTRIX

STATHMOPODA

HELIODINES

PHILOBOTA

EPIMARPTIS

SAGALASSA

MESOPHERNA

EUCOSMA

STENOMA

ACOROSTOMA

CRYPTOLECHIA

BRACHMIA

PAREXAULA*

LECITHOCERA

PERIACMA

PEDIOXESTIS*

MELASINA

GLYPHIDOCERA

PSYCHROMNESTRA*

EPIPHTHORA

ACROCERCOPS

MELASINA

PARALECTA

TINEA

ARISTOTELIA

ARGYROPLOCE

MELASINA

AMBLYSCOPA*

ANCYLOMETIS

GONIONOTA

TINEA
I920, Exotic Microlepidoptera, 2: 340. (BM)

I908, Journ. Bombay Nat. Hist. Soc. I8: 820.

I9I2, Trans. Ent. Soc. London, I9I I: 68I. (BM)

I920, Voyage de Ch. Alluaud et $R$. Jeannel en Afrique

Oriental, II, Microlepidoptera, p. 83. (P)

I9I7, Exotic Microlepidoptera, 2:80.

I93I, Ann. Mus. Nac. Hist. Nat. Buenos Aires, 36: 4II.

(BM)

I928, Exotic Microlepidoptera, 3: 444. (BM)

I92I, Exotic Microlepidoptera, 2:389 (BM)

Igo9, Trans. New Zealand Inst. 4I: I3.

I9I3, Journ. Bombay Nat. Hist. Soc. 22: I69. (BM)

I884, Proc. Linnean Soc. N.S. Wales, 9: 779 .

I908, Proc. Zool. Soc. London, p. 720. (BM)

I9Io, Proc. Linnean Soc. N.S. Wales, 35: I87. (MA)

I9I2, Trans. Ent. Soc. London, I9II: 707. (BM)

I928, Exotic Microlepidoptera, 3: 4IO.

I934, Ann. Mag. Nat. Hist. I4: 403.

I9I3, Exotic Microlepidoptera, I: 93.

I9I7, Exotic Microlepidoptera, 2: 65.

I9I3, Exotic Microlepidoptera, I: I25. (MA)

I93I, Exotic Microlepidoptera, 4: I6I.

I925, Exotic Microlepidoptera, 3: I32.

I893, Proc. Linnean Soc. N.S. Wales, 7: 5 I6.

1927, Insects of Samoa, 3, Lepidoptera, fasc. 2, p. 73.

(BM)

I9I2, Trans. Ent. Soc. London, I9II: 7II. (BM)

1926, Ann. South African Mus. 23: 346. (SA)

I938, in Caradja and Meyrick, Deuts. Ent. Zeit. Iris, 52:

II.

I9I I, Journ. Bombay Nat. Hist. Soc. 20: 7I8. (BM)

I909, Ann. South African Mus. 5: 356. (SA)

I9I4, Exotic Microlepidoptera, I: 277. (BM)

I9I0, Journ. Bombay Nat. Hist. Soc. 20: I6o. (BM)

1932, Exotic Microlepidoptera, 4: I99. (BM)

I934, Exotic Microlepidoptera, 4: 5I8. (C)

I929, Exotic Microlepidoptera, 3: 53I. (BM)

I924, Exotic Microlepidoptera, 3: 88.

I904, Proc. Linnean Soc. N.S. Wales, 29: 264.

I9I6, Exotic Microlepidoptera, I: 625.

I907, Journ. Bombay Nat. Hist. Soc. I8: I58.

1925, Exotic Microlepidoptera, 3: I53.

I9I7, Exotic Microlepidoptera, 2: 77.

I929, Exotic Microlepidoptera, 3: 482. (BM)

I927, Exotic Microlepidoptera, 3:340. (BM)

I92I, Ann. Transvaal Mus. 8: I36. (T)

I922, Exotic Microlepidopiera, 2: 588.

I934, Exotic Microlepidoptera, 4:466. (v)

I909, Trans. Ent. Soc. London, I909: 25. (BM)

I9I I, Journ. Bombay Nat. Hist. Soc. 21: I I6. 


\begin{tabular}{|c|c|c|}
\hline isoplasta & TISIS & I929, Exotic Microlepidoptera, 3: 5I6. (BM) \\
\hline isoplintha & STENOMA & I925, Exotic Microlepidoptera, 3: I93. (вм) \\
\hline isoploca & DEMOBROTIS & I9I9, Exotic Microlepidoptera, $2: 253$. \\
\hline isopoca & MELASINA & I930, Exotic Microlepidoptera, 3:55I. (вм) \\
\hline isopogon & CHELARIA & I929, Exotic Microlepidoptera, 3: 5I3. (BM) \\
\hline isoporphyra & ASAPHARCHA & I932, Exotic Microlepidoptera, 4: 286. \\
\hline isopsamma & MYRMECOZELA & $\begin{array}{l}\text { I920, Voyage de Ch. Alluaud et } R \text {. Jeannel en Afrique } \\
\text { Oriental, II, Microlepidoptera, p. } 97 .(\mathrm{P})\end{array}$ \\
\hline isopselia & EPICALLIMA & I9o6, Journ. Bombay Nat. Hist. Soc. I7: 408. (вм) \\
\hline isopsepha & EULECHRIA & I9I3, Exotic Microlepidoptera, I: I58. \\
\hline isoptila & CHELARIA & I9I3, Journ. Bombay Nat. Hist. Soc. 22: I63. (Bм) \\
\hline sopyrrha & ZELLERIA & I92I, Exotic Microlepidoptera, $2: 437$. \\
\hline sorista & NEMATOBOLA & I893, Proc. Linnean Soc. N.S. Wales, 7: 592. \\
\hline orrhoa & DALACA & I921, Ann. Transvaal Mus. 8: I42. (Lost?) \\
\hline sorrhythma & ANTISPILA & I926, Exotic Microlepidoptera, 3: 262. \\
\hline ha & PARECTOPA & I9I5, Trans. Ent. Soc. London, I9I5: 233. \\
\hline orthota & OPOGONA & I925, The Entomologist, 58: I85. \\
\hline soscelana & DICHELIA & I88I, Proc. Linnean Soc. N.S. Wales, 6: 470. \\
\hline sosema & CHELARIA & I92I, Ann. Transvaal Mus. 8: 8I. \\
\hline ospila & MELASINA & I9o8, Proc. Zool. Soc. London, p. 746. \\
\hline ospora & EUCOSMA & I9I2, Ent. Mon. Mag. 48: 34. (Sub.) \\
\hline acta & ARISTOTELIA & I926, Exotic Microlepidoptera, 3: 273. (BM) \\
\hline cha & ODITES & I9I5, Exotic Microlepidoptera, I:38I. \\
\hline osticta & STENOMA & I932, Exotic Microlepidoptera, 4: 299. (v) \\
\hline tacta & CHOLOTIS & I9II, Trans. Linnean Soc. London, I4: 284 . \\
\hline lanta & OPOGONA & I930, Exotic Microlepidoptera, $4: 8$. \\
\hline isoteles & COSMOPTERYX & I9I9, Exotic Microlepidoptera, 2: 282. \\
\hline soterma & PLATYPTILIA & I909, Trans. New Zealand Inst. 4I: Io. \\
\hline otetras & TORTRIX & I934, Ann. Mag. Nat. Hist. I4: 403. (BM) \\
\hline othea & PROMALACTIS & I930, Exotic Microlepidoptera, 3:6I5. (Bм) \\
\hline otherma & PYROPHRACTIS* & I930, Exotic Microlepidoptera, 3: 6I7. \\
\hline otis & MEGACRASPEDUS & I904, Proc. Linnean Soc. N.S. Wales, 29: 280. \\
\hline otoma & COSMOPTERYX & I9I5, Trans. Ent. Soc. London, I9I5: 205. (BM) \\
\hline otoma & GRACILARIA & I9I4, Ann. Transvaal Mus. 4:202. (T) \\
\hline isotona & ANTAEOTRICHA & I932, Exotic Microlepidoptera, 4:29I. (US) \\
\hline tricha & CHELARIA & I92I, Zool. Meded. 6: I64. (L) \\
\hline otrocha & PROMENESTA & I9I8, Exotic Microlepidoptera, 2: 209. (BM) \\
\hline isotyra & CRYPTOLECHIA & $\begin{array}{l}\text { I938, in Caradja and Meyrick, Deuts. Ent. Zeit. Iris, } 52 \text { : } \\
\text { Io. (вм) }\end{array}$ \\
\hline isoxantha & NARYCIA & I920, Ann. South African Mus. I7: 308 . (SA) \\
\hline oxesta & URODUS & I932, Exotic Microlepidoptera, 4:284. (v) \\
\hline oxysta & CHORONOMA* & I926, Exotic Microlepidoptera, 3: 3I7. (T) \\
\hline isozela & GLYPHIPTERYX & I907, Proc. Linnean Soc. N.S. Wales, 32: I24. \\
\hline ozona & CACOECIA & I9o8, Journ. Bombay Nat. Hist. Soc. I8: 6I5. (BM) \\
\hline zzona & LIMNOECIA & I897, Proc. Limnean Soc. N.S. Wales, $22: 363$. \\
\hline isozona & SESIA & I886, Proc. Linnean Soc. N.S. Wales, (Ser. 2) I:689. (A) \\
\hline isozopha & TALAEPORIA & $\begin{array}{l}\text { I936, in Caradja and Meyrick, Deuts. Ent. Zeit. Iris, } 50 \text { : } \\
\text { I59. }\end{array}$ \\
\hline & OEGOCONIA & icrolepidoptera, 2: 506. \\
\hline zina & LITHOCOLLETIS & I9I8, Exotic Microlepidoptera, 2 \\
\hline
\end{tabular}


iterata

ithyaula

ithycentra

ithyclina

ithycosma

ithycypha

ithydoxa

ithygramma

ithygramma

ithymetra

ithytona

ithyxyla

itrinea

itriodes

itygramma

iuloptera

ixeuta

ixeuta

ixota

jactata

jactatrix

jaculatrix

janitrix

jason

jaspidata

jejuna

jocosa

jucunda

jucundella

judex

judicialis

jugalis

jugata

jugicolana

jugifera

julia

juncta

juridica

justa

justifica

justificata

juvenalis

juvenilis

labyrinthias

labyrinthica

labyrinthopa
AUTOSTICHA

DICHOMERIS

EPERMENIA

SPARGANOTHIS

STROBISIA

ORNEODES

ISOCRITA

ANTEIA

ELACHISTA

FILINOTA

ANTAEOTRICHA

IULOTA*

LECITHOCERA

SIMAETHIS

IMMA

EREUNETIS*

PHALONIA

XYLORYCTA

PERILACHNA*

MELASINA

ARISTOTELIA

LASPEYRESIA

THIOTRICHA

PTEROPHORUS

ACROLEPIA

HERPYSTIS

PORINA

STENOMA

HIEROPLA*

THYLACOSCELES

ETHMIA

LECITHOCERA

EULECHRIA

CACOECIA

HYPOSMOCOMA

CYCLOPONYMPHA*

EULIA

BRACHMIA

ONEBALA

SCYTHRIS

ELACHISTA

STENOMA

ARISTOTELIA
I9I6, Exotic Microlepidoptera, I: 585. (BM)

I926, Ann. South African Mus. 23: 333. (SA)

1926, Ann. South African Mus. 23: 34I. (SA)

I926, Exotic Microlepidoptera, 3:260. (BM)

I9I4, Trans. Ent. Soc. London, I9I4: 267. (BM)

1927, Exotic Microlepidoptera, 3: 373. (BM)

I920, Ann. South African Mus. I7: 29I. (SA)

I886, Trans. Ent. Soc. London, I886: $20 \mathrm{I}$.

I9Io, Rec. Indian Mus. 2: 225. (K)

I926, Exotic Microlepidoptera, 3: 312. (BM)

1929, Trans. Ent. Soc. London, 76: 5I4. (BM)

1904, Proc. Linnean Soc. N.S. Wales, 29: 283.

I9Io, Journ. Bombay Nat. Hist. Soc. 20: 444. (BM)

I9I2, Exotic Microlepidoptera, I: 49. (BM)

I928, Exotic Microlepidoptera, 3: 422. (BM)

I88I, Proc. Linnean Soc. N.S. Wales, 5: 260.

I9I2, Ent. Mon. Mag. 48: 35. (Sub.)

I9I5, Exotic Microlepidoptera, I: 374 .

I9I4, Exotic Microlepidoptera, I: 230. (BM)

I937, Exotic Microlepidopiera, 5: II5.

I926, Exotic Microlepidoptera, 3: 272. (BM)

I9I0, Rec. Indian Mus. 2: 219. (K)

I9I2, Exotic Microlepidoptera, I: 64. (BM)

I930, Exotic Microlepidoptera, 3: 568. (BM)

I9I9, Exotic Microlepidoptera, 2: 227. (BM)

I9I6, Exotic Microlepidoptera, 2: I6. (BM)

I9I2, Trans. Proc. New Zealand Inst. 44: I24.

I9I5, Exotic Microlepidoptera, I: 429. (BM)

I883, Proc. Linnean Soc. N.S. Wales, 8: 364.

I9I3, Exotic Microlepidoptera, I : 95.

I92I, Ann. Transvaal Mus. 8: II9. (T)

I9I8, Exotic Microlepidoptera, 2: I09. (BM)

I9I4, Exotic Microlepidoptera, I: I6I. (MA)

I88I, Proc. Linnean Soc. N.S. Wales, 6: 499.

I928, Proc. Hawaiian Ent. Soc. 7: I02. (SP)

I9I3, Ann. Transvaal Mus. 3: 328. (T)

I926, Exotic Microlepidoptera, 3: 258. (BM)

I9I I, Journ. Bombay Nat. Hist. Soc. 20: 7I6. (BM)

I9Io, Journ. Bombay Nat. Hist. Soc. 20: 458. (BM)

I9II, Ann. Transvaal Mus. 2: 233. (T)

I926, Ann. South African Mus. 23: 340. (SA)

I930, Ann. Naturhist. Mus. Wien, 44:240.

I929, Exotic Microlepidoptera, 3:48I. (BM)

I92I, Exotic Microlepidoptera, 2: 396. (вм)

I9I8, Exotic Microlepidoptera, 2: I77.

I932, Exotic Microlepidoptera, 4: 253. (v)
ACROCERCOPS

CACOECIA 
lacerata

lacerata

lacertosa

lachanitis

laciniella

laciniosa

laconica

lacrimosa

lactanea

lactaria

lacteata

lacteata

lacticoma

lactifera

lactiflora

lactiflua

lactipalpis

lactirivis

lacunata

lacunosa

laetifica

laevis

-laganodes

laganopa

lagara

lagaropis

lagodes

lagophthalma

laica

lainodes

lampadacma

lampadias

lampadura

lampetis

lamprochalca

lamprocoma

lamprocosma

lamprocrossa

lamprodes

lamprodesma

lamprodeta

lamprodoxa

lamprolitha

lampropeda

lamprophanes

lamprostola

lampryntis

lamyra

languens
ARDIOSTERES*

CARPOSINA

ANTAEOTRICHA

OPOGONA

GRACILARIA

PHTHORIMAEA

ODITES

EPITHECTIS

SCYTHRIS

ALSODRYAS*

ALLOTALANTA

AMALTHINA*

GELECHIA

CHELARIA

GELECHIA

HIEROXESTIS

COESYRA

SYRRHOAULA*

MACHIMIA

EPITHECTIS

COSMOPTERYX

MITROGONA*

TORTRIX

PERIACMA

OECOPHORA

TONICA

PHTHORIMAEA

PERIACMA

CRY PTOLECHIA

LEPIDOSCIA

ERECHTHIAS

CHOREUTIS

SURA

HELIANGARA*

SCYTHRIS

GLYPHIPTERYX

FILINOTA

OPOGONA

NEMOTOIS

LECITHOCERA

DEMOBROTIS

XESTOCASIS

CAPANICA

PYRODERCES

OPOGONA

MONOPIS

STATHMOPODA

ARGYROPLOCE

ANACAMPSIS
19I7, Exotic Microlepidoptera, 2: 9I.

I9I3, Exotic Microlepidoptera, I: 74. (BM)

I915, Exotic Microlepidoptera, I: 404. (BM)

Igo6, Journ. Bombay Nat. Hist. Soc. I7: 4I6.

I88I, Proc. Linnean Soc. N.S. Wales, 5: I64.

1931, Ann. Mus. Nac. Hist. Nat. Buenos Aires, 36: 386.

I927, Exotic Microlepidoptera, 3: 365. (T)

I9I3, Ent. Mitteil. 2: 299. (D)

I9I3, Ann. Transvaal Mus. 3: 3I3. (T)

I9I4, Trans. Ent. Soc. London, I9I4: 250. (BM)

I9I4, Exotic Microlepidoptera, I: 235. (BM)

I9I4, Ann. Transvaal Mus. 4: 200. (T)

I9I7, Trans. Ent. Soc. London, I9I7: 48. (BM)

I9I3, Journ. Bombay Nat. Hist. Soc. 22: I6I. (BM)

I92I, Ann. Transvaal Mus. 8: 7I. (T)

I9II, Trans. Linnean Soc. London, I4: 298.

1920, Exotic Microlepidoptera, 2: 382.

1932, Exotic Microlepidoptera, 4: 207. (BM)

I9I4, Exotic Microlepidoptera, I: I78. (BM)

I9I8, Ann. Transvaal Mus. 6: I6. (T)

I909, Journ. Bombay Nat. Hist. Soc. I9: 4I8.

I920, Voyage de Ch. Alluaud et R. Jeannel en Afrique

Oriental, II, Microlepidoptera, p. 95. (P)

I9IO, Proc. Linnean Soc. N.S. Wales, 35: 238. (мA)

I910, Journ. Bombay Nat. Hist. Soc. 20: 160. (BM)

I886, Proc. Linnean Soc. N.S. Wales, Io: 783 .

I928, Exotic Microlepidoptera, 3: 474. (вм)

I926, Exotic Microlepidoptera, 3: 278. (BM)

I932, Exotic Microlepidoptera, 4: 28I. (BM)

I9I0, Trans. Ent. Soc. London, I9I0: 456. (Bм)

I92I, Exotic Microlepidoptera, 2: 476. (BM)

I92I, Exotic Microlepidoptera, 2: 458.

1907, Proc. Linnean Soc. N.S. Wales, 32: IIO.

I935, Exotic Microlepidoptera, 4: 558.

I906, Journ. Bombay Nat. Hist Soc. I7： I47. (BM)

I93I, Exotic Microlepidoptera, 4: I79.

I907, Proc. Linnean Soc. N.S. Wales, 32: I27.

I9I6, Exotic Microlepidoptera, I: 553. (BM)

I928, Exotic Microlepidoptera, 3: 400.

I9I4, Exotic Microlepidoptera, I: 215.

I922, Zool. Meded. 7: 85. (L)

I9I9, Exotic Microlepidoptera, 2: 253.

I922, Exotic Microlepidoptera, 2: 586.

I9I7, Exotic Microlepidoptera, 2: 63.

I9I7, Exotic Microlepidoptera, 2: 39.

I9I5, Exotic Microlepidoptera, I: 357.

I9I8, Ann. Transvaal Mus. 6: 43. (Т)

I927, Insects of Samoa, 3, Lepidoptera, fasc. 2, p. Ioo.

I9II, Proc. Limnean Soc. N.S. Wales, 36: 268.

I9I8, Exotic Microlepidoptera, 2: I42. (BM) 


\section{languescens \\ languida \\ languidula \\ laniata \\ lanista \\ lapidaria \\ lapidea \\ lapidescens \\ lapidosa \\ lapillata \\ lapillosa \\ laquearia}

laqueata

laqueatus

larochroa

lasiandra

lasiocharis

lasiocola

lasiopa

lasiosoma

lasiura

lassella

latebrata

latebricola

latebrivora

latebrosa

latebrosa

latens

lathicentra

lathidora

lathiptila

lathraea

lathraea

lathraeopa

lathridia

lathriopa

lathroea

latiberbis

latibularis

laticivora

laticlavia

latiflua

latisecta

lativagans

latomana

latriodes

latro

laudata

laureata
STENOMA

MACHIMIA

CRYPTOLECHIA

LICHENAULA

GLYPHIPTERYX

CHOREUTIS

STENOMA

GELECHIA

MALLOBATHRA

NOSYMNA

GELECHIA

DECADARCHIS

[EUGENNAEA*]

TARAGMARCHA*

PTEROPHORUS

METACHANDA

ARGYROPLOCE

COLEOPHORA

XYSMATODOMA

COLPOTORNA*

ARGYROPLOCE

EUCOSMA

BUCCULATRIX

HEMIMENE

TELPHUSA

TINEA

CARPOSINA

SCYTHRIS

DAULOCOMA*

PHILOBOTA

IMMA

STENOMA

SANDALOECA*

TRISSONCA

LASPEYRESIA

TELPHUSA

TRACHYPEPLA

TORTRIX

ACROLOPHUS

MYRMECOZELA

AEGERIA

NEMOTOIS

HYPOSMOCOMA

PARECTOPA

MELASINA

HARMOLOGA

HAPSIFERA

TINEA

ANTAEOTRICHA

CEROMITIA
I9I5, Exotic Microlepidoptera, I : 458. (BM)

I9I2, Trans. Ent. Soc. London, I9II : 698. (BM)

I92I, Ann. Transvaal Mus. 8: IOI.

I89o, Trans. R. Soc. S. Australia, I3: 47.

I9I8, Exotic Microlepidoptera, 2: I95. (BM)

I909, Trans. Ent. Soc. London, I909: 39. (BM)

I9I6, Exotic Microlepidoptera, I: 54I. (BM)

I9I6, Exotic Microlepidoptera, I: 509. (Sub.)

I9I4, Trans. Proc. New Zealand Inst. 46: II7.

I9I4, Journ. Bombay Nat. Hist. Soc. 23: I27. (BM)

I924, Trans. Proc. New Zealand Inst. 55: 203.

I9I4, Trans. Proc. New Zealand Inst. 46: II3.

I9Io, Trans. Ent. Soc. London, I9Io: 370.

I9I3, Ann. Transvaal Mus. 3: 268. (T)

I930, Trans. Ent. Soc. London, 78: 3I4. (P)

I9o9, Journ. Bombay Nat. Hist. Soc. I9: 592. (BM)

I93I, Exotic Microlepidoptera, 4: I80.

I893, Proc. Linnean Soc. N.S. Wales, $7: 495$.

I920, Exotic Microlepidoptera, 2: 326. (BM)

I92I, Exotic Microlepidoptera, 2: 449.

I9I2, Journ. Bombay Nat. Hist. Soc. 2I: 868. (BM)

I88I, Proc. Linnean Soc. N.S. Wales, 5: I80.

I9I6, Exotic Microlepidoptera, 2: 26. (BM)

I932, Exotic Microlepidoptera, 4: 349. (v)

I935, Exotic Microlepidoptera, 4:575.

I9Io, Proc. Linnean Soc. N.S. Wales, 35: I53.

I9I3, Ann. Transvaal Mus. 3: 3I4. (T)

I921, Zool. Meded. 6: I92. (L)

I889, Proc. Linnean Soc. N.S. Wales, (Ser. 2) 3: I6I8. I9I4, Supplementa Entomologica, No. 3, p. 56. (D)

I9I5, Exotic Microlepidoptera, I: 425.

1920, Ann. South African Mus. I7:300. (SA)

I934, Exotic Microlepidoptera, 4: 494.

I922, Exotic Microlepidoptera, 2: 532. (BM)

I9o9, Ann. Transvaal Mus. 2: II, pl. 4. figs. 5-6. (T)

I905, Trans. Ent. Soc. London, I905: 237.

I9Io, Proc. Linnean Soc. N.S. Wales, 35: 248.

I93I, Exotic Microlepidoptera, 4: I02.

I9I6, Exotic Microlepidoptera, I: 605.

I927, Exotic Microlepidoptera, 3:372. (BM)

I9I2, in Wytsman, Genera Insectorum, fasc. I33, p. 6.

I9I5, Exotic Microlepidoptera, I: 344. (BM)

I922, Exotic Microlepidoptera, 2: 564. (P)

I934, Exotic Microlepidoptera, 4: 5 I9.

I885, Trans. Proc. New Zealand Inst. I7: I45.

I9I7, Exotic Microlepidoptera, 2: 88.

I93I, Exotic Microlepidoptera, 4: 95.

I9I6, Exotic Microlepidoptera, I: 496. (BM)

I9II, Ann. Transvaal Mus. 2: 239. 


$\begin{array}{ll}\text { lauta } & \text { CHRYSOXESTIS* } \\ \text { lavata } & \text { MELASINA } \\ \text { laxa } & \text { STENOMA } \\ \text { laxata } & \text { BRACHMIA } \\ \text { laxeuta } & \text { NEPHOGENES } \\ \text { laxeuta } & \text { PARAMORPHA } \\ \text { lebetias } & \text { STENOMA } \\ \text { lechriaspis } & \text { SPILONOTA } \\ \text { lechriocentra } & \text { IMMA }\end{array}$

lechriosema DEPRESSARIA

lecithaula ANTAEOTRICHA

lecithitis

lecticaria

lecticata

lectulifera

lecythocera

lecythophora

legalis

legata

legitima

legitima

lembifera

lembula

lemniscata

lemurella

lemurias

lemurodes

leniflua

lenis

lenita

lenobapta

lenoea

lenta

lenticulata

leontina

leontodes

leontopa

lepidella

lepidocarpa

lepidocyma

lepidota

leporina

lepras

leptacma

leptancistra

leptarga

leptaula

leptobela

leptobelisca
ANTAEOTRICHA

TIMYRA

CHELARIA

GNORIMOSCHEMA

ARGYROPLOCE

ARGYROPLOCE

BRACHMIA

BACTRA

EUCOSMA

STENOMA

MERIDARCHIS

PRONOMEUTA

EPIPHTHORA

ESCHATURA*

TETRASCHALIS

IMMA

BUCCULATRIX

ELAEONOMA

ORYGOCERA

CNEPHASIA

PHTHORIMAEA

ACROCERCOPS

MYRMECOZELA

GONIOTERMA

AMYDRIA

GRACILARIA

STENOMA

GLYPHIDOCERA

FALCULINA

TRACHYNTIS

HYPOPHRICTIS

TRITHAMNORA

EUCOSMA

STAGMATOPHORA

CEROSTOMA

EULECHRIA

PTILOGENES
PHATNOTIS
I921, Zool. Meded. 6: I76. (L)

I930, Exotic Microlepidoptera, 3: 550.

I9I5, Exotic Microlepidoptera, I: 428. (BM)

I9I I, Journ. Bombay Nat. Hist. Soc. 20: 7I3. (BM)

I9I3, Exotic Microlepidoptera, I: I30.

I906, Journ. Bombay Nat. Hist. Soc. I7: I38. (Bм)

I9I5, Exotic Microlepidoptera, I: 433. (BM)

I932, Exotic Microlepidoptera, 4: 306. (BM)

I938, in Caradja and Meyrick, Deuts. Ent. Zeit. Iris, 52:

86. (BM)

1928, Exotic Microlepidoptera, 3: 475. (BM)

I9I5, Exotic Microlepidoptera, I: 40I. (BM)

I9I2, Trans. Ent. Soc. London, I9II: 703. (BM)

I9I6, Exotic Microlepidoptera, I: 57I. (BM)

I926, Exotic Microlepidoptera, 3: 282.

1929, Exotic Microlepidoptera, 3: 492. (BM)

I937, Exotic Microlepidoptera, 5: I60. (BM)

I939, Trans. R. Ent. Soc. London, 89: 49.

I92I, Ann. Transvaal Mus. 8: 9I. (T)

I9I3, Journ. Bombay Nat. Hist. Soc. 22: I8I. (BM)

I9I I, Trans. Linnean Soc. London, I4:269. (BM)

I9I2, Journ. Bombay Nat. Hist. Soc. 2I: 865. (BM)

I9I5, Exotic Microlepidoptera, I: 440. (BM)

I9I0, Trans. Ent. Soc. London, I9IO: 430. (BM)

I922, Zool. Meded. 7: 89. (L)

I904, Proc. Linnean Soc. N.S. Wales, 29: 262.

I897, Trans. Ent. Soc. London, I897: 382.

I908, Trans. Ent. Soc. London, 40: 476.

I93I, Exotic Microlepidoptera, 4: I82. (BM)

I9I3, Ann. Transvaal Mus. 3: 330. (T)

I920, Exotic Microlepidoptera, 2: 308.

I924, Trans. Ent. Soc. London, I923: 553.

I9Io, Proc. Linnean Soc. N.S. Wales, 35: 273.

I9I7, Trans. Ent. Soc. London, I9I7: 4I. (BM)

I922, Exotic Microlepidoptera, 2: 563 .

I9I I, Journ. Bombay Nat. Hist. Soc. 21 : I26.

I9I5, Exotic Microlepidoptera, I: 384. (BM)

I908, Proc. Zool. Soc. London, p. 752.

I88I, Proc. Linnean Soc. N.S. Wales, 5: I45.

I930, Ann. Naturhist. Mus. Wien, 44:239. (v)

I929, Trans. Ent. Soc. London, 76: 509. (BM)

I9I6, Exotic Microlepidoptera, I: 482. (BM)

I9I4, Exotic Microlepidoptera, I: I69.

I9I7, Exotic Microlepidoptera, 2: 85 .

I9I9, Exotic Microlepidoptera, 2: 260.

I925, Treubia, 6: 428 .

I9I4, Supplementa Entomologica, No. 3, p. 54. (D)

I927, Exotic Microlepidoptera, 3: 360. (BM)

I883, Proc. Linnean Soc. N.S. Wales, 7:521.

I929, Trans. Ent. Soc. London, 76:5I8. (BM) 


\begin{tabular}{|c|c|}
\hline leptobrocha & ONEBALA \\
\hline leptocentra & ARISTOTELIA \\
\hline leptochorda & ALUCITA \\
\hline leptochorda & PSALTICA \\
\hline leptoclista & STATHMOPODA \\
\hline leptocona & GLYPHIPTERYX \\
\hline leptocosma & BRENTHIA \\
\hline leptocrossa & ARISTOTELIA \\
\hline leptodesma & MARMARA \\
\hline leptodeta & CERATOSTICHA* \\
\hline leptoglypta & SARISOPHORA* \\
\hline leptogma & STENOMA \\
\hline leptogramma & LASPEY RESIA \\
\hline leptogramma & PSEPHOMERES* \\
\hline leptographa & EUTORNA \\
\hline leptograpta & TORTRIX \\
\hline leptograpta & TORTRIX \\
\hline leptomeres & TRICHOPTILUS \\
\hline leptomeris & LIMNOECIA \\
\hline leptomitella & STEGOMMATA* \\
\hline leptomorpha & AEGERIA \\
\hline leptoneura & CARPOSINA \\
\hline leptonoma & LABDIA \\
\hline leptopalta & CHELARIA \\
\hline leptophanes & GRACILARIA \\
\hline leptophracta & CAPUA \\
\hline leptophragma & SIDEROGRAPTIS* \\
\hline leptopis & MICROCOLONA \\
\hline leptopsamma & ALUCITA \\
\hline leptorrhiza & OINOPHILA \\
\hline leptorthra & EPICHOSTIS \\
\hline leptosaris & DICHOMERIS \\
\hline leptosema & GLYPHIPTERYX \\
\hline leptospila & COESYRA \\
\hline leptospora & GELECHIA \\
\hline leptostola & $\begin{array}{l}\text { ERIODYTA } \\
{[\text { LAXONOMA*] }}\end{array}$ \\
\hline leptotechna & ARISTOTELIA \\
\hline leptozona & EUCOSMA \\
\hline leptura & GNAMPTONOMA* \\
\hline leptynta & OPOGONA \\
\hline lepyropis & COLEOPHORA \\
\hline lethaea & SIMAETHIS \\
\hline letharga & OECOPHORA \\
\hline lethonoa & STATHMOPODA \\
\hline leucactis & PETASANTHES* \\
\hline leucadelpha & CRYPTOPHASA \\
\hline leucallactis & ACROLOPHUS \\
\hline leucantha & EUCOSMA \\
\hline leucanthes & TINAGMA \\
\hline
\end{tabular}

1923, Exotic Microlepidoptera, 3: 44. (вм)

I9I2, Exotic Microlepidoptera, I: 64. (BM)

I9I3, Exotic Microlepidoptera, I: III.

I9I4, Exotic Microlepidoptera, I: 263. (вм)

I929, Exotic Microlepidoptera, 3: 54I.

I922, Exotic Microlepidoptera, 2: 493. (Вм)

I9I6, Exotic Microlepidoptera, I: 560. (вм)

I926, Exotic Microlepidoptera, 3: 273. (вм)

I928, Exotic Microlepidoptera, 3: 480.

I935, Exotic Microlepidoptera, 4: 580.

1904, Proc. Linnean Soc. N.S. Wales, 29: 404.

I925, Exotic Microlepidcptera, 3:217. (BM)

I913, Ann. Transvaal Mus. 3: 278. (т)

I9I6, Exotic Microlepidoptera, I: 506. (Вм)

I906, Trans. R. Soc. S. Australia, 30: 4I.

I924, Exotic Microlepidoptera, 3: II5. (вм)

1927, Exotic Microlepidoptera, 3: 369.

I886, Trans. Ent. Soc. London, I886: I5.

I897, Proc. Linnean Soc. N.S. Wales, 22: 362.

I88I, Proc. Linnean Soc. N.S. Wales, 5: I72.

I931, Exotic Microlepidoptera, 4:50.

I920, Exotic Microlepidoptera, 2: 338. (BM)

I927, Insects of Samoa, 3, Lepidoptera, fasc. 2, p. 89.

I934, Exotic Microlepidoptera, 4: 45I.

I928, Exotic Microlepidoptera, 3: 4II. (T)

I931, Exotic Microlepidoptera, 4: 147. (v)

I920, Exotic Microlepidoptera, 2: 3II. (вM)

I897, Proc. Linnean Soc. N.S. Wales, 22: 374.

I925, Bull. Soc. R. Ent. Egypte, 9: 206.

I921, Zool. Meded. 6: I93. (L)

I93I, in Caradja, Bull. Sect. sci. Acad.roum. I4: 74. (BM)

1932, Exotic Microlepidoptera, 4: 202. (BM)

I888, Trans. Proc. New Zealand Inst. 20: 87.

I889, Proc. Linnean Soc. N.S. Wales, (Ser. 2) 3: I654.

I932, Exotic Microlepidoptera, 4:350. (v)

I884, Proc. Linnean Soc. N.S. Wales, 8: 5 I7.

1937, Exotic Microlepidoptera, 5: I2I. (LE)

I92I, Ann. Transvaal Mus. 8: 54. (T)

I9I7, Exotic Microlepidoptera, 2: 65 .

I9I5, Trans. Ent. Soc. London, I9I5: 252.

I92I, Exotic Microlepidoptera, 2: 472.

I9I2, Exotic Microlepidoptera, I: 47. (вм)

I883, New Zealand Journ. Sci. I: 524.

I897, Proc. Linnean Soc. N.S. Wales, 22: 322.

I925, Exotic Microlepidoptera, 3: I58. (BM)

I887, Proc. Linnean Soc. N.S. Wales, (Ser. 2) I: I040.

I9I9, Exotic Microlepidoptera, 2: 28I. (BM)

I93I, Exotic Microlepidoptera, 4: I45. (BM)

I897, Proc. Linnean Soc. N.S. Wales, 22: 422. 


\begin{tabular}{|c|c|c|}
\hline leucargyra & NEPTICULA & I906, Trans. R. Soc. S. Australia, 30: 57. \\
\hline leucaspis & EUPSELIA & I906, Trans. R. Soc. S. Australia, 30: 38. (вм) \\
\hline leucaspis & EUCOSMA & $\begin{array}{l}\text { I902, in Gardiner, The Fauna and Geography of the } \\
\text { Maldive and Laccadive Archipelagoes, I: I26. (CAM) }\end{array}$ \\
\hline leucastis & TINEOLA & I9o8, Proc. Zool. Soc. London, p. 738. \\
\hline leucastra & DICASTERIS* & I9o6, Trans. R. Soc. S. Australia, 30: 55. \\
\hline leucatma & LASPEYRESIA & I925, Exotic Microlepidoptera, 3: I44. (T) \\
\hline leucatoma & BRENTHIA & I9I8, Ann. Transvaal Mus. 6:36. (т) \\
\hline leucaula & COLEOPHORA & I9I2, Ann. Transvaal Mus. 3: 78 . (т) \\
\hline leucerythra & HOPLITICA & I883, Proc. Linnean Soc. N.S. Wales, 7: 50I. \\
\hline leucitis & LASPEYRESIA & I907, Journ. Bombay Nat. Hist. Soc. I8: I43. (BM) \\
\hline leucobactra & EULIA & I926, Exotic Microlepidoptera, 3: 256. (BM) \\
\hline leucobathra & ELACHISTA & I923, Exotic Microlepidoptera, 3:6I. \\
\hline leucobela & ENSCEPASTRA & I934, Exotic Microlepidoptera, 4: 460. (v) \\
\hline leucocapna & LYCHNOCRATES* & 1926, Exotic Microlepidoptera, 3: 227. (BM) \\
\hline leucocentra & LATHICROSSA* & I883, New Zealand Journ. Sci. I: 523. \\
\hline leucocerastes & GLYPHIPTERYX & I881, Proc. Linnean Soc. N.S. Wales, 5: 239. \\
\hline leucoceros & LECITHOCERA & I932, Exotic Microlepidoptera, 4: 204. (BM) \\
\hline leucochalca & SEMNOSTOMA* & I9I8, Exotic Microlepidoptera, 2: I27. (BM) \\
\hline leucochares & COLEOPHORA & I922, Exotic Microlepidoptera, 2: 556 . \\
\hline leucocharis & TORTRIX & I933, Exotic Microlepidoptera, 4: 423. (BM) \\
\hline leucochlaena & OTONOMA & I9I9, Exotic Microlepidoptera, 2: 285. (BM) \\
\hline leucochlora & LECITHOCERA & I9Io, Trans. Ent. Soc. London, I9Io: 448. (BM) \\
\hline leucochna & TARUDA & I921, Exotic Microlepidoptera, 2: 398. (BM) \\
\hline leucochorda & MACAROSTOLA & I9o8, Journ. Bombay Nat. Hist. Soc. I8: 826. \\
\hline leucochrysa & MACARANGELA* & I893, Proc. Linnean Soc. N.S. Wales, 7: 588. \\
\hline leucochrysis & MOMPHA & I935, Exotic Microlepidoptera, 4: 573. (BM) \\
\hline leucochtha & MICROCOLONA & I897, Proc. Linnean Soc. N.S. Wales, 22: 377. \\
\hline leucocirrha & ETHMIA & 1926, Ann. South African Mus. 23: 339. (SA) \\
\hline leucoclista & EOMICHLA & I930, Ann. Naturhist. Mus. Wien, 44: 23I. (v) \\
\hline leucoclistra & SYRMOLOGA* & I9I9, Exotic Microlepidoptera, 2: 244. (BM) \\
\hline leucoconis & MELASINA & I926, Ann. South African Mus. 23: 348. (SA) \\
\hline leucocosma & TRICHOTAPHE & I9I6, Exotic Microlepidoptera, I: 584 . (BM) \\
\hline leucocrossa & LINOSTICHA & I889, Proc. Linnean Soc. N.S. Wales, (Ser. 2) 3: I583. \\
\hline leucocrossa & MARASMARCHA & I936, Exotic Microlepidoptera, 5: 64 . \\
\hline leucocryptis & STENOMA & I932, Exotic Microlepidoptera, 4: 295. \\
\hline leucocyma & GRACILARIA & I889, Trans. Proc. New Zealand Inst. 2 I : I84. \\
\hline leucocymba & CACOECIA & I9I2, Exotic Microlepidoptera, I: 4. \\
\hline leucodelta & MACHIMIA & I9I4, Exotic Microlepidoptera, I: I84. (BM) \\
\hline leucodesma & EUCOSMA & I9I2, Trans. Ent. Soc. London, I9II: 688. (BM) \\
\hline leucodeta & OPOGONA & I9I4, Supplementa Entomologica, No. 3, p. 59. (D) \\
\hline leucodetis & LEPIDOTARSA & I888, Froc. Linnean Soc. N.S. Wales, (Ser. 2) 2: 931. \\
\hline leucodora & PAMMENE & I928, Exotic Microlepidoptera, 3: 447. (BM) \\
\hline leucodoxa & GELECHIA & I920, Ann. South African Mus. I7:283. (SA) \\
\hline leucogaea & APATETRIS & I92I, Ann. Transvaal Mus. 8:65. (т) \\
\hline leucogama & BACTRA & I9o9, Journ. Bombay Nat. Hist. Soc. I9: 584 . (BM) \\
\hline leucoglypta & APATETRIS & I9I8, Exotic Microlepidoptera, 2: II7. (BM) \\
\hline leucogramma & ANTAEOTRICHA & I9I5, Exotic Microlepidoptera, I: 404. (BM) \\
\hline leucograpta & ONEBALA & I923, Exotic Microlepidoptera, 3: 45. (BM) \\
\hline leucolitha & GRACILARIA & I9r 2 , in Wytsman, Genera Insectorum, fasc. I2S, p. 30. \\
\hline
\end{tabular}




\section{leucomalla leucomantis leucombra leucomias leucomichla leucomima leucomitra leucomochla leucomystis leuconephes leuconympha leucopeda leucopelta leucopetala leucopetra leucophaea leucophanes leucophanta leucophora leucophracta}

leucophragma leucophthalma leucophyta leucopis leucopla

$\begin{array}{ll}\text { leucoplanetis } & \text { TRACHYPEPLA } \\ \text { leucoplasta } & \text { PTILOGENES } \\ \text { leucoplecta } & \text { STROBISIA } \\ \text { leucopleura } & \text { TEUCHOPHANES* } \\ \text { leucopogon } & \text { DECADARCHIS } \\ \text { leucoporpa } & \text { HYPERCALLIA } \\ \text { leucopsamma } & \text { PROTAPHREUTIS } \\ \text { leucopselia } & \text { LAMYRISTIS* } \\ \text { leucoptila } & \text { PTILOGENES } \\ \text { leucopyrga } & \text { HILAROGRAPHA } \\ \text { leucorectis } & \text { STENOMA } \\ \text { leucoritis } & \text { BORKHAUSENIA } \\ \text { leucorma } & \text { HERMOGENES } \\ \text { leucorrhapta } & \text { ANACAMPSIS } \\ \text { leucorrhoa } & \text { OINOPHILA } \\ \text { leucorthyncha } & \text { PLATYPTILIA } \\ \text { leucosarca } & \text { PERSICOPTILA } \\ \text { leucosaris } & \text { STENOMA } \\ \text { leucosceptra } & \text { MELASINA } \\ \text { leucoschista } & \text { ZELLERIA }\end{array}$

leucoscia leucosema
EUCOSMA EPERMENIA PYRODERCES PROMENESTA EPIPHTHORA TINEA PHILOBOTA DORODOCA IMMA MIMESEOPTILUS SETIOSTOMA MACROBATHRA EULECHRIA GORGOPIS EUCOSMA ACROCERCOPS EULECHRIA ARISTOTELIA ANARSIA ARGYROTOXA

GLYPHIPTERYX MIEZA BATRACHEDRA MISCERA BRACHMIA

TRACHYPEPLA RTOBISIA TEUCHOPHANES* DECADARCHIS HYPERCALLIA PROTAPHREUTIS LAMYRISTIS* STENOMA BORKHAUSENIA HERMOGENES ANACAMPSIS OINOPHILA PLATYPTILIA MELASINA PSEUDURGIS
I9I2, Ent. Mon. Mag. 48: 35. (Sub.) I9I7, Exotic Microlepidoptera, 2: 67. I897, Proc. Linnean Soc. N.S. Wales, 22: 345. I925, Exotic Microlepidoptera, 3: I62. (BM) I904, Proc. Linnean Soc. N.S. Wales, 29:265. I921, Ann. Transvaal Mus. 8: I29. (T) I884, Proc. Linnean Soc. N.S. Wales, 8: 488. I922, Exotic Microlepidoptera, 2: 572. I923, Exotic Microlepidoptera, 2: 6I6. (Bм) I886, Trans. Ent. Soc. London, I886: 20. I921, Exotic Microlepidoptera, 2: 477. (BM) I886, Proc. Linnean Soc. N.S. Wales, Io: 8I3. I883, Proc. Linnean Soc. N.S. Wales, 7:530. I92I, Ann. Transvaal Mus. 8: I4I. (T) I9o8, Proc. Zool. Soc. London, p. 7I9. (BM) I9I9, Exotic Microlepidoptera, 2: 23I. I883, Proc. Limnean Soc. N.S. Wales, 8: 320. I9o8, Journ. Bombay Nat. Hist. Soc. I8: 438. (BM) I904, Proc. Limnean Soc. N.S. Wales, 29: 4I7. I937, in Caradja and Meyrick, Deuts. Ent. Zeit. Iris, 5I: I75. (BM) 1923, Bull. Mus. Nat. Hist. Nat. Paris, 29:564. I907, Proc. Linnean Soc. N.S. Wales, 32: 89. I897, Proc. Limnean Soc. N.S. Wales, 22: 307. I907, Proc. Linnean Soc. N.S. Wales, 32: I02. I938, Institut des Parcs Nationaux du Congo Belge, fasc. I4, p. I6. I883, New Zealand Journ. Sci. I: 522. I926, Exotic Microlepidoptera, 3: 232. (BM) I9I I, Journ. Bombay Nat. Hist. Soc. 20: 729. (BM) I9I4, Trans. Ent. Soc. London, I9I4: 274. (BM) I924, Exotic Microlepidoptera, 3:83. (BM) I926, Exotic Microlepidoptera, 3: 3I3. (BM) I930, Trans. Ent. Soc. London, 78: 322. (P) I9I I, Journ. Bombay Nat. Hist. Soc. 2I: I3I. (BM) I9I8, Exotic Microlepidoptera, 2: 210. (BM) I9I2, Exotic Microlepidoptera, I: 36. I925, Exotic Microlepidoptera, 3: 223. (BM) I927, Exotic Microlepidoptera, 3: 38I. (вм) Igo8, Journ. Bombay Nat. Hist. Soc. I8: 627. (Bм) I9I4, Trans. Ent. Soc. London, I9I4: 26I. (BM) I9I5, Exotic Microlepidoptera, I: 355. I902, Ent. Mon. Mag. 38:2I7. I936, Exotic Microlepidoptera, 5: 30. I925, Exotic Microlepidoptera, 3: I96. (BM) I907, Journ. Bombay Nat. Hist. Soc. I8: I57. (BM) I93I, Ann. Mus. Nac. Hist. Nat. Buenos Aires, 36: 399. (BM) I927, Exotic Microlepidoptera, 3: 359. (BM) I9I4, Ann. South African Mus. IO: 254. (SA) 


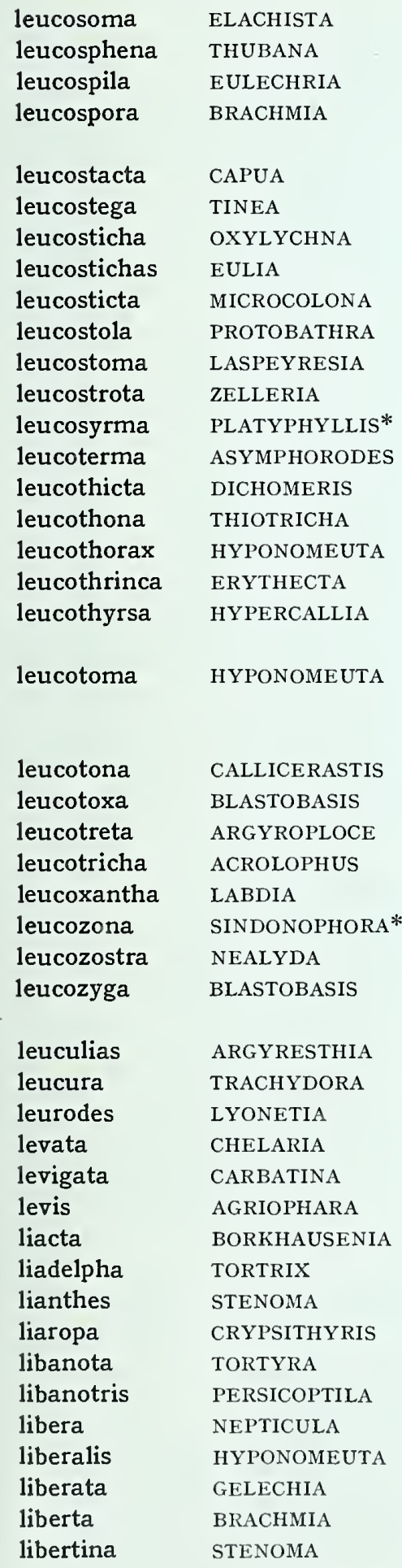

I922, Exotic Microlepidoptera, 2: 508.

I931, in Caradja, Bull. Sect. sci. Acad. roum. I4: 69. (вм) I920, Exotic Microlepidoptera, 2: 370 .

1938, Institut des Parcs Nationaux du Congo Belge,

fasc. I4, p. I7.

I910, Proc. Linnean Soc. N.S. Wales, 35: 202.

I932, Exotic Microlepidoptera, 4: 325.

I9I9, Exotic Microlepidoptera, 2: 250.

1932, Exotic Microlepidoptera, 4: 258. (Bм)

1928, Exotic Microlepidoptera, 3: 39I.

I92I, Exotic Microlepidoptera, 2: 436. (BM)

I9I2, Journ. Bombay Nat. Hist. Soc. 2I: 876. (Вм)

I929, Trans. Ent. Soc. London, 76: 504.

I932, Exotic Microlepidoptera, 4: 216.

I929, Trans. Ent. Soc. London, 76: 499.

I9I9, Exotic Microlepidoptera, 2: 235. (вм)

I904, Proc. Linnean Soc. N.S. Wales, 29: 294.

I913, Exotic Microlepidoptera, I: 138.

I93I, Rec. Cant. Mus. 3:367.

I938, in Caradja and Meyrick Dents. Ent. Zeit. Iris, 52:

8. (вм)

I935, in Caradja and Meyrick, Materialien zu einer Micro-

lepidopteren Fauna der Chinesischen Provinzen Kiangsu,

Chekiang und Hunan, p. 88.

I917, Exotic Microlepidoptera, 2: 83.

I902, Trans. R. Soc.S. Australia, 26: I71.

I913, Ann. Transvaal Mus. 3: 276. (BM)

I93I, Journ. Linnean Soc. London, 37: 284.

I927, Insects of Samoa, 3, Lepidoptera, fasc. 2, p. 87.

I9I7, Ann. South African Mus. I7: I6. (SA)

I923, Exotic Microlepidoptera, 3: 5. (BM)

I936, Veröff. Deutschen Kol.-Mus. I: 332, pl. I3, fig. 3I.

(H)

I9I4, Journ. Bombay Nat. Hist. Soc. 23: I23. (BM)

I897, Proc. Linnean Soc. N.S. Wales, 22: 398.

I9I5, Exotic Microlepidoptera, I: 350.

I920, Exotic Microlepidoptera, 2: 304.

I913, Journ. Bombay Nat. Hist. Soc. 22: I82. (Вм)

I92I, Exotic Microlepidoptera, 2: 446. (BM)

I920, Exotic Microlepidoptera, 2: 367 .

I910, Proc. Linnean Soc. N.S. Wales, 35: 227.

I932, Exotic Microlepidoptera, 4: 30I. (v)

I924, Exotic Microlepidoptera, 3: 7 I.

I9I0, Trans. Ent. Soc. London, I9Io: 463.

I906, Journ. Bombay Nat. Hist. Soc. I7 : 4II.

I906, Trans. R. Soc.S. Australia, 30:6I.

I9I3, Exotic Microlepidoptera, I: I38.

I9I0, Ann. South African Mus. 5: 4I4. (sa)

I926, Exotic Microlepidoptera, 3: 29I.

I9I6, Exotic Microlepidoptera, I: 526. (Вм) 


\begin{tabular}{|c|c|c|}
\hline libidinosa & MACHIMIA & I926, Exotic Microlepidoptera, 3: 3II. (BM) \\
\hline libralis & ADOXOPHYES & I927, Insects of Samoa, 3, Lepidoptera, fasc. 2, p. 69. \\
\hline libraria & ORNEODES & I9II, Ann. Transvaal Mus. 2: 22I. (T) \\
\hline librata & PARELLIPTIS & I9Io, Trans. Ent. Soc. London, I9Io: 445. (Bм) \\
\hline librodes & THYROCOPA & I9I5, Exotic Microlepidoptera, I: 370. (BM) \\
\hline libropis & CEROMITIA & Igo8, Proc. Zool, Soc. London, p. 755. \\
\hline lichenea & LICHENAULA* & I89o, Trans. R. Soc. S. Australia, I3: 49. \\
\hline lichenias & AGRIOPHARA & I9I6, Exotic Microlepidoptera, I: 486. (BM) \\
\hline lichenista & HYPERCALLIA & I926, Exotic Microlepidoptera, 3: 3I2. (BM) \\
\hline lichenodes & TRACHYPEPLA & 1883, New Zealand Journ. Sci. I: 523. \\
\hline lichmodes & TINEA & I92I, Ann. Transvaal Mus. 8: I3о. (T) \\
\hline lichneuta & XYROSARIS & I9I8, Exotic Microlepidoptera, 2: I88. (BM) \\
\hline liciata & HOMALOXESTIS & I922, Zool. Meded. 7: 83. (L) \\
\hline licmaea & EPICHORISTA & $\begin{array}{l}\text { I920, Voyage de Ch. Alluaud et } R \text {. Jeannel en Afrique } \\
\text { Oriental, II, Microlepidoptera, p. } 53 . \text { (P) }\end{array}$ \\
\hline licmaea & STENOMA & I9I5, Exotic Microlepidoptera, I: 462. (BM) \\
\hline licnura & COSMOPTERYX & I909, Journ. Bombay Nat. Hist. Soc. I9: 4I4. \\
\hline ligata & GRACILARIA & I9I2, Exotic Microlepidoptera, I: 26. (T) \\
\hline lignaria & COMPSOLECHIA & I926, Exotic Microlepidoptera, 3: 292. (BM) \\
\hline lignatrix & NARYCIA & I92I, Exotic Microlepidoptera, 2: 477 . (BM) \\
\hline lignea & TORTRIX & I9I7, Trans. Ent. Soc. London, I9I7: 9. (BM) \\
\hline lignosa & MELASINA & I9I7, Exotic Microlepidoptera, 2: 94 \\
\hline lignyodes & SYNDROMA* & I9I4, Exotic Microlepidoptera, I: 27I. (BM) \\
\hline ligyra & TRICHOTAPHE & I9I3, Ann. Transvaal Mus. 3: 3oI. (T) \\
\hline ligyrodes & COSMOPTERYX & I9I5, Exotic Microlepidoptera, I: 322. \\
\hline ligyropa & OINOPHILA & I9I5, Exotic Microlepidoptera, I: 356. \\
\hline ligyropis & ACROCLITA & $\begin{array}{l}\text { 1937, in Caradja and Meyrick, Deuts. Ent. Zeit. Iris, 5I: } \\
\text { I76. (Bм) }\end{array}$ \\
\hline liliacea & ORYGOCERA & I930, Exotic Microlepidoptera, 3: 622. (BM) \\
\hline lilitha & GYMNOGELASTIS & I930, Exotic Microlepidoptera, 3: 587. (Bм) \\
\hline limata & EULECHRIA & I9I4, Exotic Microlepidoptera, I: I68. (MA) \\
\hline limatula & LASIOTHYRIS* & I9I7, Trans. Ent. Soc. London, I9I7: 4. (BM) \\
\hline limatula & MACHAEROPTERIS & I9I I, Journ. Bombay Nat. Hist. Soc. 21 : I30. \\
\hline limbata & HELIOCAUSTA & I883, Proc. Linnean Soc. N.S. Wales, 7: 47I. \\
\hline limenaea & TELPHUSA & I920, Ann. South African Mus. I7: 282 (SA) \\
\hline limenarcha & PHILOBOTA & I913, Exotic Microlepidoptera, I: I23. \\
\hline limenias & EUXANTHIS & I928, Exotic Microlepidoptera, 3:437. (BM) \\
\hline limenita & HEMIMENE & I922, Exotic Microlepidoptera, 2: 530. (Bм) \\
\hline limenites & NEMOTOIS & I9I4, Supplementa Entomologica, No. 3, p. 6I. (D) \\
\hline limenitis & TINEA & I935, Exotic Microlepidoptera, 4: 575. \\
\hline limenodes & DECADARCHIS & I9I5, Exotic Microlepidoptera, I: 368. \\
\hline limenosema & ARGYROPLOCE & I93I, Exotic Microlepidoptera, 4: I35. \\
\hline limicola & HOLCOCERA & I9I8, Exotic Microlepidoptera, 2: I62. (BM) \\
\hline limigena & EUCOSMA & I9I2, Ent. Mon. Mag. 48: 35. (Sub.) \\
\hline limitaris & CHEZALA & I915, Exotic Microlepidoptera, I: 302. \\
\hline limitata & PROCOMETIS & I9I2, Ann. Transvaal Mus. 3:75. (T) \\
\hline limnephilana & STREPSICEROS & I88I, Proc. Linnean Soc. N.S. Wales, 6: 680. \\
\hline limodes & MICROCOLONA & 1897, Proc. Linnean Soc. N.S. Wales, 22: 372. \\
\hline limonia & PHILOBOTA & I9I3, Exotic Microlepidoptera, I: I25. \\
\hline limonias & SIMAETHIS & I907, Proc. Linnean Soc. N.S. Wales, 32: III. \\
\hline
\end{tabular}




\begin{tabular}{|c|c|}
\hline limosa & PERONEA \\
\hline limosa & PERIACMA \\
\hline lingulata & COMPSOLECHIA \\
\hline linicoma & MELASINA \\
\hline linobola & MACRAEOLA* \\
\hline linocoma & LECITHOCERA \\
\hline linodyta & MELASINA \\
\hline linoplecta & COLEOPHORA \\
\hline linozona & TRICHOCEROTA \\
\hline lintearia & PHYLOMICTIS \\
\hline linteata & STENOMA \\
\hline liobathra & TORTRIX \\
\hline liochalca & NEPTICULA \\
\hline liochlaena & HOMALOXESTIS \\
\hline liochlora & ARGYROPLOCE \\
\hline liochra & MELASINA \\
\hline liochroa & PHAEOSACES \\
\hline liodryas & DEPRESSARIA \\
\hline liolitha & LABDIA \\
\hline liomorpha & TINEA \\
\hline liopercna & CTENOCOMPA \\
\hline liophaea & PRAYS \\
\hline liophanes & MARASMARCHA \\
\hline liophanes & SCIEROPEPLA \\
\hline liopis & BATRACHEDRA \\
\hline lioplintha & EUCOSMA \\
\hline liopsamma & CENARCHIS \\
\hline liosarca & HOPLITICA \\
\hline lioscelis & MACROTARSIPUS \\
\hline liota & PROTOLECHIA \\
\hline liotoma & TORTRIX \\
\hline lioxantha & OTOPTRIS* \\
\hline lioxera & LIPARISTIS* \\
\hline lioxesta & ODITES \\
\hline lipara & PROCOMETIS* \\
\hline liparochra & CAPUA \\
\hline liparodes & ARGYRESTHIA \\
\hline liparophanes & ASCALENIA \\
\hline liparopis & OPOGONA \\
\hline liparota & MONOPIS \\
\hline liparoxantha & GRACILARIA \\
\hline liporrhoa & STATHMOPODA \\
\hline liquefacta & TORTRIX \\
\hline liquescens & MYSAROMIMA* \\
\hline liquida & EULECHRIA \\
\hline liquida & PHALONIA \\
\hline liquidana & CACOECIA \\
\hline lirinopa & XYROSARIS \\
\hline lissochlora & TINEA \\
\hline
\end{tabular}

I9I2, Trans. Ent. Soc. London, I9II: 688. (BM) I9Io, Journ. Bombay Nat. Hist. Soc. 20: I57. (BM) I9I8, Exotic Microlepidoptera, 2: I40. (BM) I9I4, Exotic Microlepidoptera, I : 2 I2. I893, Proc. Linnean Soc. N.S. Wales, 7: 555. I9I6, Exotic Microlepidoptera, I: 593. I921, Ann. Transvaal Mus. 8: I35. (T) I924, Exotic Microlepidoptera, 3:67. (BM) I926, Exotic Microlepidoptera, 3: 269. I921, Exotic Microlepidoptera, 2: 445. (BM) I9I6, Exotic Microlepidoptera, I: 525. (BM) I923, Exotic Microlepidoptera, 3:55. (BM) I9I6, Exotic Microlepidoptera, 2: 6 . I93I, in Caradja, Bull. Sect. sci. Acad. roum. I4: 68. (Вм) I9I4, Journ. Bombay Nat. Hist. Soc. 22: 77I. (BM) I908, Proc. Zool. Soc. London, p. 748. I89I, Trans. Proc. New Zealand Inst. 23: 98. I92I, Exotic Microlepidoptera, 2: 39I. (BM) I922, Exotic Microlepidoptera, 2: 569 . I894, Trans. Ent. Soc. London, I894: 28. I9I7, Exotic Mirrolepidoptera, 2: 93 . I927, Exotic Microlepidoptera, 3:36I. (T) I886, Trans. Ent. Soc. London, I886: I9. I89o, Trans. R. Soc. S. Australia, I3: 68. I897, Proc. Linnean Soc. N.S. Wales, 22: 308. I920, Exotic Microlepidoptera, 2: 344. (BM) I924, Trans. Ent. Soc. London, I923: 550. (BM) I888, Proc. Linnean Soc. N.S. Wales, (Ser. 2) 2: $94 \mathrm{I}$. I935, Exotic Microlepidoptera, 4: 557. I904, Proc. Linnean Soc. N.S. Wales, 29: 350. I936, Exotic Microlepidoptera, 5: 60. (v) I9I5, Trans. Ent. Soc. London, I9I5: 245. I9I5, Exotic Microlepidoptera, I: 376. I933, Exotic Microlepidoptera, 4: 434. (v) I89o, Trans. R. Soc. S. Australia, I3: 72 . I928, Exotic Microlepidoptera, 3: 452. (BM) I9I4, Exotic Microlepidoptera, I: 205. I932, Exotic Microlepidoptera, 4:215. I922, Exotic Microlepidoptera, 2: 558 . I920, Voyage de Ch. Alluaud et R. Jeannel en Afrique Oriental, II, Microlepidoptera, p. 99. (P) I920, Exctic Microlepidoptera, 2: 297. (BM) I897, Proc. Linnean Soc. N.S. Wales, 22: 326. I908, Journ. Bombay Nat. Hist. Soc. I8: 6 I9. 1926, Exotic Microlepidoptera, 3: 227. (Lost) I9I4, Exotic Microlepidoptera, I: I64. I9I2, Ent. Mon. Mag. 48: 35. (Sub.) I881, Proc. Limnean Soc. N.S. Wales, 6: 505. I922, Exotic Microlepidoptera, 2: 55I. (P) I92I, Ann. Transvaal Mus. 8: I30. (T) 


$\begin{array}{ll}\text { lissopeda } & \text { PYGMOCRATES* } \\ \text { lissota } & \text { TRICHOTAPHE } \\ \text { literaria } & \text { PHRIXOSCELES } \\ \text { literata } & \text { CADMOGENES* } \\ \text { lithacma } & \text { HIEROXESTIS } \\ \text { lithacopa } & \text { PICROSPORA } \\ \text { lithina } & \text { LICHENAULA } \\ \text { lithinopa } & \text { GLAPHYRISTIS } \\ \text { lithocentra } & \text { HAPSIFERA }\end{array}$

lithochalca

lithochlora

lithochrysa

lithocosma

lithocrossa

lithodelta

lithodes

lithodoxa

lithoglypta

lithogramma

lithographa

lithograpta

lithogypsa

litholeuca

lithomacha

lithomorpha

lithophaea

lithophanes

lithosema

lithostrota

lithoxesta

lithoxestus

litigatrix

litigiosa

litigiosa

litoxyla

lividana

lividella

lividula

lobocasis

lobostola

lobotona

lochaea

locharcha

lochistis

lochitis

lochmaea

loculata

loculosa
ACROCERCOPS

PHILOBOTA

ACROCERCOPS

PALPARIA

BRENTHIA

ANACAMPSIS

GELECHIA

GLYPHIDOCERA

PHLOEOPOLA

ACROCERCOPS

PHRIXOSCELES

AGRIOPHARA

STENOMA

HOMOTHAMNIS*

PARECTOPA

ANACAMPSIS

TRICHOSTIBAS

OCYSTOLA

BACTROPALTIS*

HELCYSTOGRAMMA

STENOMA

MIMAESEOPTILUS

BACTRA

EUCOSMA

GELECHIA

DICHOMERIS

AROTROPHORA

EULECHRIA

ODITES

ARGYROPLOCE

EUCOSMA

ARGYROPLOCE

SCARDIA

PLATYPTILIA

BRACHMIA

HOMALOXESTIS

EUCOSMA

OPOGONA

CEROSTOMA
I932, Exotic Microlepidoptera, 4: 283. (v)

I9I3, Journ. Bombay Nat. Hist. Soc. 22: I77. (BM)

I908, Journ. Bombay Nat. Hist. Soc. I8: 8I4.

I923, Trans. Proc. New Zealand Inst. 54: I68.

I92I, Ann. Transvaal Mus. 8: I25. (T)

I920, Ann. South African Mus. I7: 307. (SA)

I89o, Trans. R. Soc. S. Australia, I3: 49.

I9I7, Exotic Microlepidoptera, 2: 40.

I920, Voyage de Ch. Alluaud et R. Jeannel en Afrique

Oriental, II, Microlepidoptera, p. I05. (P)

I930, Exotic Microlepidoptera, 3: 58I.

I889, Proc. Linnean Soc. N.S. Wales, (Ser. 2) 3: 1606.

I930, Exotic Microlepidoptera, 3: 580. (BM)

I886, Proc. Linnean Soc. N.S. Wales, Io: 827 .

I922, Exotic Microlepidoptera, 2: 487. (P)

I922, Trans. Ent. Soc. London, I922: 77. (BM)

I886, Trans. Proc. New Zealand Inst. I8: I7o.

I929, Exotic Microlepidoptera, 3: 529. (BM)

I883, Proc. Linnean Soc. N.S. Wales, 8: 358.

I920, Exotic Microlepidoptera, 2: 296. (BM)

I9I2, in Wytsman, Genera Insectorum, fasc. I28, p. I3.

I9I3, Trans. Ent. Soc. London, I9I3: I84.

I932, Exotic Microlepidoptera, 4: 299. (BM)

I92I, Ann. Transvaal Mus. 8: II7. (T)

I9I5, Trans. Ent. Soc. London, I9I5: 234.

I9I4, Trans. Ent. Soc. London, I9I4: 256. (BM)

I9I3, Exotic Microlepidoptera, I: I40.

1885, Proc. Linnean Soc. N.S. Wales, 9: I075.

I939, Trans. R. Ent. Soc. London, 89:56.

I9I6, Exotic Microlepidoptera, I: 578. (BM)

I9I5, Exotic Microlepidoptera, I: 432. (BM)

I885, Trans. Proc. New Zealand Inst. I7: I27.

1929, Trans. Ent. Soc. London, 76: 495. (BM)

I9I2, Journ. Bombay Nat. Hist. Soc. 2I : 867. (BM)

I9I7, Trans. Ent. Soc. London, I9I7: 49. (BM)

I937, Exotic Microlepidoptera, 5: I23. (BM)

I881, Proc. Linnean Soc. N.S. Wales, 6: 53I.

I883, Proc. Linnean Soc. N.S. Wales, $7: 533$.

I932, Exotic Microlepidoptera, 4:286. (BM)

I936, in Caradja and Meyrick, Deuts. Ent. Zeit. Iris, 50: I56. (BM)

I9I8, Ann. Transvaal Mus. 6:49. (T)

I921, Ann. Transvaal Mus. 8: 59. (T)

I9II, Trans. Linnean Soc. London, I4: 307.

I924, Exotic Microlepidoptera, 3: 94. (T)

I9II, Journ. Bombay Nat. Hist. Soc. 20: 723. (BM)

I9I8, Exotic Microlepidoptera, 2: IOI. (BM)

I920, Ann. South African Mus. I7:278. (SA)

I9I5, Exotic Microlepidoptera, I: 357.

I922, Exotic Microlepidoptera, 2: 565 . 


\section{locuples \\ loemias \\ logica \\ logica \\ logistes}

logistica

logistis

longa

longinqua

longipalpis

longirostris

lopelictes

lophandra

lophonota

lophoptycha

lophosaris

loquax

loranthivora

loranthivora

loraria

loricata

loriculata

lorigera

lotina

lotinana

lotoxantha

loxaspis

loxaula

loxias

loxias

loxobathra

loxocasis

loxochorda

loxoclista

loxodes

loxodesma

loxogramma

loxonephes

loxonoma

loxopa

loxophanta

loxophracta

loxophragma

loxophthalma

loxopis
BUCCULATRIX

PROTOLECHIA

ARGYROPLOCE

NARTHECOCEROS

PTEROPHORUS

COLONOPHORA

BRENTHIA

BORKHAUSENIA

STIPHROSTOLA *

BORKHAUSENIA

ENSCEPASTRA

MACRONEMATA

GLYPHIDOCERA

ACROCERCOPS

STENOMA

STENOMA

PHTHORIMAEA

PTOCHORYCTIS

ZELLERIA

RHADINASTIS

DICHELOPA

HELIODINES

PTILODOXA*

EPACTRIS

ADOXOPHYES

OPOGONA

EUCOSMA

LEUCOPTERA

ACROCERCOPS

PROSELENA

CRYPTOLECHIA

PTILOTHYRIS

HYPERCALLIA

LEUCOPTERA

CERACE

PRODOSIARCHA*

COMPSOLECHIA

EULIA

DICHOMERIS

AMYDRIA

OPOGONA

CATANOMISTIS*

BORKHAUSENIA

LECITHOCERA

IMMA
I9I9, Exotic Microlepidoptera, 2: 287.

I904, Proc. Linnean Soc. N.S. Wales, 29: 383 .

I9I2, Trans. Ent. Soc. London, I9II: 692.

I9I0, Journ. Bombay Nat. Hist. Soc. 20: 435. (BM)

I935, in Caradja and Meyrick, Materialien zu einer Micro-

lepidopteren Fauna der Chinesischen Provinzen Kiangsu,

Chekiang und Hunan, p. 46.

I931, Ann. Mus. Nac. Hist. Nat. Buenos Aires, 36: 387.

I909, Trans. Ent. Soc. London, I909: 4I. (BM)

I927, Exotic Microlepidoptera, 3:38I. (BM)

1923, Exotic Microlepidoptera, 3: 25. (BM)

I931, Ann. Mus. Nac. Hist. Nat. Buenos Aires, 36: 392. (BM)

I926, Ann. South African Mus. 23: 339. (SA)

I883, Proc. Linnean Soc. N.S. Wales, 8: 346.

I929, Exotic Microlepidoptera, 3: 530. (BM)

I921, Zool. Meded. 6: igo. (L)

I925, Exotic Microlepidoptera, 3: I88. (BM)

1925, Exotic Microlepidoptera, 3: I86. (BM)

I9I7, Trans. Ent. Soc. London, I9I7: 45. (BM)

I937, Exotic Microlepidoptera, 5: I50. (BM)

I930, Exotic Microlepidoptera, 3: 59I. (Bм)

I9I7, Exotic Microlepidoptera, 2: 43.

I9ro, Proc. Linnean Soc. N.S. Wales, 35: 283.

I932, Exotic Microlepidoptera, 4: 272. (v)

I921, Zool. Meded. 6: I86. (L)

I9I6, Exotic Microlepidoptera, I: 600.

I883, Trans. Proc. New Zealand Inst. I5: 40.

I9I5, Trans. Ent. Soc. London, I9I5: 25 I.

I93I, Exotic Microlepidoptera, 4: I27. (BM)

I928, Exotic Microlepidoptera, 3: 396. (T)

I9I8, Exotic Microlepidoptera, 2 : I74.

I888, Trans. Proc. New Zealand Inst. 20: 74.

I9I 5, Trans. Ent. Soc. London, I9I5: 2II. (BM)

I938, Institut des Parcs Nationaux du Congo Belge, fasc. I4,

p. I7.

1926, Exotic Microlepidoptera, 3: 315. (BM)

I934, Exotic Microlepidoptera, 4: 46I.

I9I2, Exotic Microlepidoptera, I: I9.

I904, Proc. Linnean Soc. N.S. Wales, 29:330.

I922, Trans. Ent. Soc. London, I922: 97. (BM)

I937, Exotic Microlepidoptera, 5: I28. (FB)

I937, Exotic Microlepidoptera, 5: I23. (c)

I9I4, Ann. South African Mus. IO: 255. (SA)

I936, Exotic Microlepidoptera, 4: 620.

I933, Exotic Microlepidoptera, 4: 433.

I922, Exotic Microlepidoptera, 2: 543. (BM)

I934, Exotic Microlepidoptera, 4: 453 .

I909, Journ. Bombay Nat. Hist. Soc. I9: 425. (Bu) 
loxoplecta

ACROCLITA

$\begin{array}{ll}\text { loxoptila } & \text { BUCCULATRIX } \\ \text { loxosaris } & \text { CHELARIA } \\ \text { loxoschista } & \text { ORNEODES } \\ \text { loxoscia } & \text { MNESARCHAEA } \\ \text { loxoscia } & \text { OXYGRAPHA } \\ \text { loxosema } & \text { PONTODRYAS } \\ \text { loxospila } & \text { CYMOTRICHA } \\ \text { loxotis } & \text { BORKHAUSENIA } \\ \text { loxozona } & \text { LITHOCOLLETIS } \\ \text { lubrica } & \text { ONEBALA } \\ \text { lubricata } & \text { CALLIXESTIS } \\ \text { lucens } & \text { CHEZALA } \\ \text { lucernata } & \text { LECITHOCERA } \\ \text { lucernifera } & \text { PHYLLOCNISTIS } \\ \text { lucescens } & \text { LANGASTIS } \\ \text { lucicincta } & \text { PLACOPTILA } \\ \text { lucida } & \text { LASPEYRESIA } \\ \text { lucifera } & \text { ANTICRATES } \\ \text { lucifera } & \text { PROSCHISTIS } \\ \text { luciflua } & \text { CRYPTOPHASA } \\ \text { lucifuga } & \text { PHRICONYMA* } \\ \text { lucinda } & \text { COMODICA } \\ \text { lucrifuga } & \text { DICHOMERIS } \\ \text { lucrosa } & \text { STENOMA } \\ \text { luctifica } & \text { HEMIMENE } \\ \text { luctuosa } & \text { ACROCERCOPS } \\ \text { lucubrata } & \text { MACROSACES } \\ \text { luculenta } & \text { STATHMOPODA } \\ \text { ludicra } & \text { COMOTECHNA* } \\ \text { ludicra } & \text { EUCOSMA } \\ \text { ludifica } & \text { SIMAETHIS } \\ \text { luminata } & \text { STATHMOPODA } \\ \text { luminifera } & \text { BRENTHIA } \\ \text { lunatica } & \text { AMBLYTENES* } \\ \text { lunisequa } & \text { PROMALACTIS } \\ \text { lunularis } & \text { CHEZALA } \\ \text { lunulifera } & \text { PYRODERCES }\end{array}$

lupata

lupata

lupata

lurida

lurida

luridula

lusciosa

lusoria
DEPRESSARIA

BRACHMIA
I935, in Caradja and Meyrick, Materialien zu einer Microlepidopteren Fauna der Chinesischen Provinzen Kiangsu, Chekiang und Hunan, p. 53. (BM)

I9I4, Exotic Microlepidoptera, I: 209.

I9I8, Ann. Transvaal Mus. 6:2I. (T)

I93I, Exotic Microlepidoptera, 4: 86.

I888, Trans. Proc. New Zealand Inst. 20: 90.

I907, Journ. Bombay Nat. Hist. Soc. I7: 735. (Bм)

I920, Exotic Microlepidoptera, 2: 362. (BM)

I932, Exotic Microlepidoptera, 4: 203. (BM)

I905, Trans. Ent. Soc. London, I905: $24 \mathrm{I}$.

I936, Exotic Microlepidoptera, 5: 33.

I9I0, Journ. Bombay Nat. Hist. Soc. 20: 460.

I9I7, Exotic Microlepidoptera, 2: 4I.

I9I5, Exotic Microlepidoptera, I: 302.

I9I3, Ann. Transvaal Mus. 3: 294.

I935, Exotic Microlepidoptera, 4: 595 .

I9I4, Exotic Microlepidoptera, I: 267. (BM)

I920, Exotic Microlepidoptera, 2: 323.

I9I6, Exotic Microlepidoptera, 2: 22. (BM)

I9I4, Journ. Bombay Nat. Hist. Soc. 23: I28. (BM)

Igog, Journ. Bombay Nat. Hist. Soc. Ig: 589. (BM)

I938, Trans. R. Ent. Soc. London, 87: 5I6.

I883, Proc. Linnean Soc. N.S. Wales, 8: 340.

I927, Insects of Samoa, 3, Lepidoptera, fasc. 2, p. II4.

r923, Exotic Microlepidoptera, 2: 620. (BM)

I925, Exotic Microlepidoptera, 3: I84. (BM)

I9I6, Exotic Microlepidoptera, 2: 27. (BM)

I9I5, Trans. Ent. Soc. London, I9I5: 228.

I9I0, Journ. Bombay Nat. Hist. Soc. 20: I48. (BM)

I9I3, Exotic Microlepidoptera, I: 88.

I920, Exotic Microlepidoptera, 2: 3I7. (BM)

I9I2, Journ. Bombay Nat. Hist. Soc. 2I: 867. (BM)

I9I4, Exotic Microlepidoptera, I: 283. (BM)

IgII, Ann. Transvaal Mus. 2: 232. (T)

I9I2, Exotic Microlepidoptera, I: 5I. (BM)

I930, Ann. Naturhist. Mus. Wien, 44: 230. (v)

I93I, Exotic Microlepidoptera, 4: I86.

I926, Exotic Microlepidoptera, 3: 3Iо. (BM)

1934, Pacific Ent. Surv. Publ. 7, art. 28, p. 347.

(BB)

I9I2, Trans. Ent. Soc. London, I9I I: 690. (BM)

I9I4, Trans. Ent. Soc. London, I9I4: 239. (BM)

I9I3, Journ. Bombay Nat. Hist. Soc. 22: I7I. (BM)

I89o, Trans. R. Soc. S. Australia, I3: 37 .

IgI2, Trans. Ent. Soc. London, I9II: 68I. (BM)

I932, Trans. Ent. Soc. London, 80: I20.

I9I5, Trans. Ent. Soc. London, I9I5:2II. (BM) 


$\begin{array}{ll}\text { lustralis } & \text { ARGYRESTHIA } \\ \text { lutata } & \text { PROTHINODES* } \\ \text { lutescens } & \text { ANCYLIS } \\ \text { luticilia } & \text { OPOSTEGA } \\ \text { luticoma } & \text { GELECHIA } \\ \text { lutiflua } & \text { BLASTOBASIS } \\ \text { lutigena } & \text { HIEROXESTIS } \\ \text { lutipalpis } & \text { MYRMECOZELA } \\ \text { lutipennis } & \text { ARGYROPLOCE } \\ \text { lutivittata } & \text { DICHOMERIS } \\ \text { lutosa } & \text { OXYCHARIS } \\ \text { lutraula } & \text { GELECHIA } \\ \text { lutrocopa } & \text { EUCOSMA } \\ \text { lutulenta } & \text { NEPHOGENES } \\ \text { luxurians } & \text { LYONETIA } \\ \text { luxuriosa } & \text { CUPHODES } \\ \text { lychnacma } & \text { STATHMOPODA } \\ \text { lychnidias } & \text { AEOLANTHES } \\ \text { lychnitis } & \text { SCYTHRIS } \\ \text { lychnocentra } & \text { STYLOCEROS } \\ \text { lychnopa } & \text { ERECHTHIAS } \\ \text { lychnophanes } & \text { HARMOLOGA } \\ \text { lychnopis } & \text { MOMPHA } \\ \text { lychnosema } & \text { OECOPHORA } \\ \text { lycocephala } & \text { MYRMECOZELA } \\ \text { lycodes } & \text { TORTRIX } \\ \text { lycophanes } & \text { MELASINA } \\ \text { lycopis } & \text { BRACHMIA } \\ \text { lycosema } & \text { ACIPTILIA } \\ \text { lygaea } & \text { PACHNISTIS } \\ \text { lyginella } & \text { GRACILARIA } \\ \text { lygrodes } & \text { PETALOSTOMA* } \\ \text { lygrodoxa } & \text { HAPLOSCOPA* } \\ \text { lygrophthalma } & \text { SARISOPHORA }\end{array}$

\section{lygrosema}

lymphatica

lyncodes

lyrica

lyrifera

lyristis

lyroda

iysibathra

lysidesma

lysigama

lysimacha

lysimeris

lysimopa

lysiphracta
OXYTHECTA

OECOPHORA

MYRMECOZELA

ACROCERCOPS

IMMA

ORNEODES

ORTHIOSTOLA*

ACROCERCOPS

IMMA

TANYCHASTIS*

AGRIOPHARA

ANTAEOTRICHA

COPHOMANTIS

IPHIERGA
IgII, Trans. Linnean Soc. London, I4: 292.

I9I4, Trans. Proc. New Zealand Inst. 46: I 6 .

I9I2, Exotic Microlepidoptera, I: 32. (BM)

I9I5, Exotic Microlepidoptera, I: 35I.

1929, Exotic Microlepidoptera, 3: 489. (BM)

I922, Exotic Microlepidoptera, 2: 539.

I9I5, Exotic Microlepidoptera, I : 362 .

I922, Exotic Microlepidoptera, 2: 59I. (P)

I921, Ann. Transvaal Mus. 8: 6I. (T)

I92I, Exotic Microlepidoptera, 2: 434. (BM)

I939, Trans. R. Ent. Soc. London, 89: 57. (Bм)

I923, Exotic Microlepidoptera, 3: 20.

I9I4, Ann. Transvaal Mus. 4: I88. (T)

I9I3, Exotic Microlepidoptera, I : I3I.

I922, Exotic Microlepidoptera, 2: 558. (BM)

I9II, Trans. Linnean Soc. London, I4: 285 .

1927, Exotic Microlepidoptera, 3:378. (T)

Igo8, Journ. Bombay Nat. Hist. Soc. I8: 638. (вм)

I9I8, Exotic Microlepidoptera, 2: I57.

I904, Proc. Linnean Soc. N.S. Wales, 29: 409.

1927, Trans. Proc. New Zealand Inst. 57: 702.

I9I6, Trans. Proc. New Zealand Inst. 48: 4I5.

I933, Exotic Microlepidoptera, 4: 429.

I886, Proc. Linnean Soc. N.S. Wales, 10: 787.

I920, Voyage de Ch. Alluaud et $R$. Jeannel en Afrique

Oriental, $I I$, Microlepidoptera, p. 98. (P)

I9ıo, Proc. Linnean Soc. N.S. Wales, 35: 232.

I924, Exotic Microlepidoptera, 3: 78. (T)

I9I I, Journ. Bombay Nat. Hist. Soc. 20: 7I7. (BM)

I885, Trans. Proc. New Zealand Inst. I7: I24.

I9I I, Journ. Bombay Nat. Hist. Soc. 20: 707. (BM)

I88I, Proc. Linnean Soc. N.S. Wales, 5: I57.

I93I, Exotic Microlepidoptera, 4: I23. (BM)

1939, Trans. R. Ent. Soc. London, 89: 57. (BM)

I934, in Caradja and Meyrick, Deuts. Ent. Zeit. Iris, 48:

36.

1885, Proc. Limnean Soc. N.S. Wales, 9: x054.

I886, Proc. Linnean Soc. N.S. Wales, ro: 785 .

I921, Ann. Transvaal Mus. 8: 126. (T)

I908, Journ. Bombay Nat. Hist. Soc. I 8: 820.

I9Io, Trans. Ent. Soc. London, I9Io: 466. (BM)

I9II, Journ. Bombay Nat. Hist. Soc. 2I : I07.

1927, Exotic Microlepidoptera, 3: 357. (BM)

I9I6, Exotic Microlepidoptera, I: 626.

I906, Trans. Ent. Soc. London, I906: I77. (BM)

I9I0, Trans. Ent. Soc. London, I9I0: 37 I.

I9I5, Exotic Microlepidoptera, I: 4 IO.

I9I5, Exotic Microlepidoptera, I: 39I. (BM)

I933, Exotic Microlepidoptera, 4: 357. (BM)

I9I7, Exotic Microlepidoptera, 2: 89. 
lysizona

lyssodes

lythrodana

\begin{tabular}{|c|c|}
\hline macarella & OECOPHORA \\
\hline macarista & TRYMALITIS \\
\hline macaritis & HELIANGARA \\
\hline macella & LECITHOCERA \\
\hline maceratus & TRICHOPTILUS \\
\hline macescens & EPERMENIA \\
\hline machaerias & OPOSTEGA \\
\hline machinata & TELPHUSA \\
\hline machinatrix & STENOMA \\
\hline machinopis & ENSCEPASTRA \\
\hline machinosa & EULECHRIA \\
\hline machlas & TIMYRA \\
\hline machlopis & CACOECIA \\
\hline macra & PERIACMA \\
\hline macranthes & GRACILARIA \\
\hline macraula & GLYPHIPTERYX \\
\hline macraulax & STENOMA \\
\hline macrobela & LABDIA \\
\hline macrochaeta & NEPTICULA \\
\hline macrochalca & ACROCERCOPS \\
\hline macrochorda & COMPSISTIS \\
\hline macroclina & ACROCERCOPS \\
\hline macroctenis & ORDRUPIA \\
\hline macrodelta & THYRSOSTOMA \\
\hline macroglena & DIAPHRAGMISTIS* \\
\hline macroglossa & COSMOPTERYX \\
\hline macrogona & ORSOCOMA* \\
\hline macrogona & PARASTRANGA* \\
\hline macrogramma & DIATONICA* \\
\hline macrograpta & CEROMITIA \\
\hline macroleuca & STENOMA \\
\hline macroloncha & BATRACHEDRA \\
\hline macronota & STENOMA \\
\hline macropetana & STREPSICEROS \\
\hline macrophaea & TERTHROTICA * \\
\hline macroplaca & ACROCERCOPS \\
\hline macropleura & GRACILARIA \\
\hline macropodias & PHILOBOTA \\
\hline macroptera & DICHOMERIS \\
\hline macroptycha & STENOMA \\
\hline macrornis & PLATYPTILIA \\
\hline macrorrhyncha & NOSYMNA \\
\hline hacrosaris & EUCOSMA \\
\hline
\end{tabular}

I889, Proc. Linnean Soc. N.S. Wales, (Ser. 2) 3: I6og. I9IO, Proc. Linnean Soc. N.S. Wales, 35: 282. I881, Proc. Linnean Soc. N.S. Wales, 6: 497.

I883, New Zealand Journ. Sci. I: 524. I934, Exotic Microlepidoptera, 4: 489. (BM) I9Io, Rec. Indian Mus. 2: 22I. (K) I9I8, Exotic Microlepidoptera, 2: I05. (BM) I909, Ann. Transvaal Mus. 2: 2, pl. I, fig. 3. (T) I9I7, Exotic Microlepidoptera, 2: 66. I907, Journ. Bombay Nat. Hist. Soc. I7: 985 . I929, Exotic Microlepidoptera, 3: 488. (BM) I925, Exotic Microlepidoptera, 3: 208. (BM) I936, Exotic Microlepidoptera, 5: 63. I9I3, Exotic Microlepidoptera, I: I59. (MA) I905, Journ. Bombay Nat. Hist. Soc. I6: 595. (BM) I9I4, Exotic Microlepidoptera, I: 4. (BM) I9Io, Trans. Ent. Soc. London, I9Io: 455. (BM) I928, Exotic Microlepidoptera, 3: 480. I907, Proc. Linnean Soc. N.S. Wales, 32: I20. I930, Exotic Microlepidoptera, 3: 559. 19I8, Ann. Transvaal Mus. 6: 52. (T) I92I, Ann. Transvaal Mus. 8: I40. (T) I9Io, Trans. Ent. Soc. London, I9Io: 373. I92I, Exotic Microlepidoptera, 2: 397. (BM) I9I6, Exotic Microlepidoptera, 2: 2. I926, Exotic Microlepidoptera, 3: 243. (BM) I9I8, Exotic Microlepidoptera, 2: I2I. (BM) I9I4, Journ. Bombay Nat. Hist. Soc. 23: I26. (BM) I9I3, Ann. Transvaal Mus. 3: 3o6. (T) I92I, Exotic Microlepidoptera, 2: 438. (BM) I9Io, Proc. Linnean Soc. N.S. Wales, 35: 289. I921, Exotic Microlepidoptera, 2: 453. I934, Exotic Microlepidoptera, 4: 453. (c) I932, Exotic Microlepidoptera, 4: 304. (Us) I9I6, Exotic Microlepidoptera, 2: 32. I9I2, Trans. Ent. Soc. London, I9II: 7I6. (BM) I88I, Proc. Linnean Soc. N.S. Wales, 6: 683. I9I4, Exotic Microlepidoptera, I: 23I. (BM) I908, Journ. Bombay Nat. Hist. Soc. I8: 823. I932, Trans. Ent. Soc. London, 80: II7. I9I3, Exotic Microlepidoptera, I: I27. I9I4, Trans. Ent. Soc. London, I9I4: 283 . I930, Exotic Microlepidoptera, 4:28. (BM) I930, Exotic Microlepidoptera, 3:567. (BM) I930, Exotic Microlepidoptera, 3: 592. (BM) I938, in Caradja and Meyrick, Deuts. Ent. Zeit. Iris, 52 :

84. (BM) I889, Proc. Linnean Soc. N.S. Wales, (Ser. 2) 3:165I. 


$\begin{array}{ll}\text { macroscopa } & \text { BRACHMIA } \\ \text { macrosphena } & \text { TRICHOTAPHE } \\ \text { macrostoma } & \text { CNEPHASIA } \\ \text { macrostoma } & \text { PARAXENISTIS } \\ \text { macrothecta } & \text { ARISTOTELIA } \\ \text { macrotoma } & \text { LECITHOCERA } \\ \text { macrotona } & \text { HOLCOCERA } \\ \text { macrotrachela } & \text { OMIOSTOLA } \\ \text { macroxyla } & \text { ANATHYRSA* } \\ \text { macroxyla } & \text { TRICHOTAPHE } \\ \text { macrozancla } & \text { ACROLOPHUS } \\ \text { macrozyga } & \text { ERECHTHIAS } \\ \text { macrula } & \text { COSMOPTERYX } \\ \text { macrynta } & \text { PHLOEOGRAPTIS } \\ \text { maculata } & \text { CYATHAULA* } \\ \text { madens } & \text { ACROCLITA } \\ \text { madida } & \text { PHILOBOTA } \\ \text { maeandria } & \text { XYLORYCTA } \\ \text { maenas } & \text { PSEUDATTERIA } \\ \text { magadis } & \text { ORNEODES } \\ \text { magdalena } & \text { ORYGOCERA(?) } \\ \text { magica } & \text { PYRODERCES } \\ \text { magicosema } & \text { ASTAROPOLA* } \\ \text { magnanima } & \text { PALTODORA } \\ \text { magnetica } & \text { LASPEYRESIA } \\ \text { magocantiaca } & \text { LEPIDOSCIA } \\ \text { magosias } & \text { ANTICRATES } \\ \text { MTATHMOPODA } \\ \text { OCYSTOLA } \\ \text { TRICHOTAPHE }\end{array}$

$\begin{array}{ll}\text { malachita } & \text { ANTAEOTRICHA } \\ \text { malachitis } & \text { BRENTHIA } \\ \text { malacista } & \text { LATYPICA } \\ \text { malacobryas } & \text { SYNCHALARA } \\ \text { malacobyrsa } & \text { CRYPTOLECHIA } \\ \text { malacodepta } & \text { NARYCIA } \\ \text { malacodes } & \text { ACROPOLITIS } \\ \text { malacodes } & \text { EUCOSMA } \\ \text { malacodes } & \text { NOTHRIS } \\ & \text { [ACRIBOLOGA*] }\end{array}$

malacodoxa CEROSTOMA malacogramma BRACHMIA malacolectra ACRIA malacopa malacopis malacoptera malacoscia
I932, Exotic Microlepidoptera, 4: 206. (Вм)

I9I3, Trans. Ent. Soc. London, I9I3: I75. (BM)

I920, Ann. South African Mus. I7: 277. (SA)

I9I9, Exotic Microlepidoptera, 2: 225.

rgo4, Proc. Linnean Soc. N.S. Wales, 29: 288.

I934, in Caradja and Meyrick, Deuts. Ent. Zeit. Iris, 48: 36.

I9I6, Exotic Microlepidoptera, I: 599.

I922, Exotic Microlepidoptera 2: 5I9. (BM)

I920, Ann. South African Mus. I7: 299. (SA)

I9I3, Journ. Bombay Nat. Hist. Soc. 22: I8o.

1922, Exotic Microlepidoptera, 2: 608. (Bм)

I9I6, Trans. Proc. New Zealand Inst. 48: 4I9.

I897, Proc. Linnean Soc. N.S. Wales, 22: 339.

1904, Proc. Linnean Soc. N.S. Wales, 29: 394.

I886, Trans. Ent. Soc. London, I886: 289.

I921, Zool. Meded. 6: I53. (L)

I920, Exotic Microlepidoptera, 2: 384 .

I9I5, Exotic Microlepidoptera, I: 375 .

I924, Exotic Microlepidoptera, 3: I09. (BM)

Igo8, Trans. Ent. Soc. London, I907: 510.

I930, Exotic Microlepidoptera, 3: 622. (вм)

I905, Journ. Bombay Nat. Hist. Soc. I6: 607.

1935, Arb. morph. taxon. Ent. Berl., 3: 107.

I9I2, Ann. South African Mus. I0: 59. (SA)

I928, Exotic Microlepidoptera, 3: 45I. (BM)

I893, Proc. Linnean Soc. N.S. Wales, $7: 508$.

I930, Exotic Microlepidoptera, 3: 594. (вM)

I9I9, Exotic Microlepidoptera, 2: 282. (BM)

I938, Trans. R. Ent. Soc. London, 87: 520.

I885, Proc. Linnean Soc. N.S. Wales, 9: I064.

I913, Journ. Bombay Nat. Hist. Soc. 22: I79.

I9I5, Exotic Microlepidoptera, I: 404. (BM)

I909, Trans. Ent. Soc. London, I909: 40. (Вм)

1924, Trans. Ent. Soc. London, I923: 557.

I938, Trans. R. Ent. Soc. London, 87: 518.

I921, Exotic Microlepidoptera, 2: 394. (BM)

I933, Exotic Microlepidoptera, 4: 4I5.

I9I0, Proc. Linnean Soc. N.S. Wales, 35: I73.

I9II, Ann. Transvaal Mus. 2: 226. (T)

I9Io, Trans. Ent. Soc. London, I9Io: 45I. (BM)

I932, Exotic Microlepidoptera, 4: 340.

I909, Ann. Transvaal Mus. 2: I4, pl. 5, fig. 2. (T)

I930, Exotic Microlepidoptera, 4: I3.

I9I5, Exotic Microlepidoptera, I: 343. (BM)

I889, Proc. Linnean Soc. N.S. Wales, (Ser. 2) 3: I643.

I888, Proc. Linnean Soc. N.S. Wales, (Ser. 2) 2: 949.

I92I, Exotic Microlepidoptera, 2: 397. (BM) 


malacotorna
malacoxesta
malacozona
malacta
maledicta
maleficana
maleficus
maleropa
malesana
malesanus
malescripta

malevola malevolens malicola maligna maligna maligna malitiosa malivora mallodeta malthaca malthacodes malthacopa malthacopis mamertina manceps mancipata manganeutis manicola manifesta manipularis manniana

mansueta mantica manticodes manticopa manubriata mappigera maranta marcens marcescens margarias margaritacea margaritis margarodes marileutis marina
CAPUA

STENOMA

TORTYRA

LECITHOCERA

STOMOPTERYX

BONDIA

OXYPTILUS

EUXANTHIS

LASPEYRESIA

PTEROPHORUS

MONOPIS

METACHANDA CRYPTOPHASA ACROCERCOPS PHYLOMICTIS* PLATYPTILIA

XYROSARIS

AGDISTIS

ODITES

TIQUADRA

DECADARCHIS

TONICA

TAPHROSARIS*

TINEA

LASPEYRESIA

ANTAEOTRICHA

CHELARIA

ACROLEPIA

AMIANTASTIS*

PSEUDODOXIA

COSMOPTERYX

PHALONIA

CACOECIA

ACROCERCOPS

ACROLOPHUS

CATAMACTA

IZATHA

PARAPSECTRIS

OECOPHORA

PHILOBOTA

TINEOLA

TRYMALITIS*

HYPERCALLIA

TINEA

THIOTRICHA

IMMA

GNORIMOSCHEMA
I93I, Exotic Microlepidoptera, 4: I48. (BM)

I930, Ann. Naturhist. Mus. Wien., 44: 252.

I922, Exotic Microlepidoptera, 2: 485. (BM)

I9I8, Exotic Microlepidoptera, 2: IIO.

I92I, Zool. Meded. 6: I62. (L)

I883, Proc. Linnean Soc. N.S. Wales, 7: I93.

I926, Exotic Microlepidoptera, 3: 296.

I937, in Caradja and Meyrick, Deuts. Ent. Zeit. Iris, 5I: I72. (BM)

I920, Exotic Microlepidoptera 2: 352. (BM)

I92I, Exotic Microlepidoptera, 2: 422.

I938, Institut des Parcs Nationaux du Congo Belge,

fasc. I4, p. 26.

I924, Trans. Ent. Soc. London, I923: 547. (вм)

I928, Exotic Microlepidoptera, 3: 433 .

I92I, Exotic Microlepidoptera, 2: 4I6.

I89o, Trans. R. Soc. S. Australia, I3: 75.

I93I, Exotic Microlepidoptera, I : Iro.

I907, Journ. Bombay Nat. Hist. Soc. I7: 984. (вм)

I9o9, Ann. Transvaal Mus. 2: 4, pl. 2, fig. 3. (T)

I930, Exotic Microlepidoptera, 3: 555. (BM)

I924, Exotic Microlepidoptera, 3: 73. (BM)

I928, Exotic Microlepidoptera, 3: 40I. (ВM)

I9I4, Exotic Microlepidoptera, I : 226.

I922, Trans. Ent. Soc. London, I922: I04. (вм)

I936, Exotic Microlepidoptera, 5: 53 .

I920, Exotic Microlepidoptera, 2: 353. (ВM)

I925, Exotic Microlepidoptera, 3: I72. (BM)

I9I3, Ann. Transvaal Mus. 3: 297. (T)

I9I3, Exotic Microlepidoptera, I: I49.

I932, Exotic Microlepidoptera, 4: 347. (Bм)

I9I4, Exotic Microlepidoptera, I: 265.

I909, Journ. Bombay Nat. Hist. Soc. I9: 4I9.

IgII [F. R., Meyr. nec F. R.], Proc. Linnean Soc.

N.S. Wales, 36: 297.

I923, Exotic Microlepidoptera, 3: 54. (P)

I9o8, Journ. Bombay Nat. Hist. Soc. I8: 822.

I9I9, Exotic Microlepidoptera, 2: 280. (BM)

I934, Exotic Microlepidoptera, 4:525. (C)

I923. Trans. Proc. New Zealand Inst. 54: I65.

I9I4, Ann. Transvaal Mus. 4: I9I. (T)

I886, Proc. Linnean Soc. N.S. Wales, ro: 79 I.

I9I4, Exotic Microlepidoptera, I: 220.

I9o8, Proc. Zool. Soc. London, p. 739.

I905, Journ. Bombay Nat. Hist. Soc. I6: 590. (вм)

I924, Exotic Microlepidoptera, 3: I04. (ВM)

I9I4, Trans. Proc. New Zealand Inst. 46: II6.

I904, Proc. Linnean Soc. N.S. Wales, 29: 294.

I906, Trans. Ent. Soc. London, I906: I75. (BM)

I904, Proc. Linnean Soc. N.S. Wales, 29: 3I9. 


$\begin{array}{ll}\text { marmara } & \text { EUCOSMA } \\ \text { marmarantha } & \text { PSEUDATTERIA } \\ \text { marmaranthes } & \text { PAMMENE } \\ \text { marmaraspis } & \text { AEOLOCOSMA } \\ \text { marmarastra } & \text { MICTOPSICHIA } \\ \text { marmarauges } & \text { ACROCERCOPS } \\ \text { marmarea } & \text { GLAPHYRISTIS } \\ \text { marmarea } & \text { SIMAETHIS } \\ \text { marmarias } & \text { XYROPTILA } \\ \text { marmaritis } & \text { TIMYRA } \\ \text { marmarocyma } & \text { ENDOTHAMNA* } \\ \text { marmarocyma } & \text { HERMIMENE } \\ \text { marmarodes } & \text { MELASINA } \\ \text { marmarodes } & \text { PERONEA } \\ \text { marmaropa } & \text { SPOROCELIS* } \\ \text { marmarophanes } & \text { EUCOSMA }\end{array}$

marmaropis ACROLEPIA marmarota" HAPSIFERA marmaroxantha EUCOSMA

$\begin{array}{ll}\text { marmarozona } & \text { IMMA } \\ \text { marmarurga } & \text { SAPHENEUTIS } \\ \text { marmorata } & \text { PHILOBOTA } \\ \text { marsyas } & \text { LACTURA } \\ \text { martia } & \text { LASPEYRESIA } \\ \text { martialis } & \text { ATHYMORIS* } \\ \text { martyranthes } & \text { TORTRIX } \\ \text { maschalis } & \text { GLYPHIPTERYX } \\ \text { maschalista } & \text { ARGYROPLOCE } \\ \text { maschalista } & \text { SYMMOCA } \\ \text { masinissa } & \text { STATHMOPODA } \\ \text { mastodes } & \text { BAEONOMA* } \\ \text { mastopis } & \text { GRACILARIA } \\ \text { mataea } & \text { ATELOPSYCHA } \\ \text { mataea } & \text { LEPTOSACES } \\ \text { mathematica } & \text { NEPHOGENES } \\ \text { matura } & \text { ODITES } \\ \text { maturata } & \text { EPORGASTI* } \\ \text { maturescens } & \text { STENOMA } \\ \text { matutina } & \text { EVETRIA } \\ \text { matutina } & \text { GELECHIA } \\ \text { maxima } & \text { CARPOSINA } \\ \text { mazina } & \text { LECITHOCERA } \\ \text { mechanica } & \text { IIILAROGRAPHA } \\ \text { mechanica } & \text { LINOSTICHA } \\ \text { mechanistis } & \text { PROTOLECHIA } \\ \text { mechanodes } & \text { POLYCHROSIS } \\ \text { mechanopla } & \text { ACROCERCOPS } \\ \text { meconitis } & \text { TRICHOTAPHE }\end{array}$

I9o9, Ann. Transvaal Mus. 2: 8, pl. 3, fig. 6. (T) I924, Exotic Microlepidoptera, 3: I09. (BM) I933, Exotic Microlepidoptera, 4: 422. (BM) I88I, Proc. Linnean Soc. N.S. Wales, 5: 225. I932, Exotic Microlepidoptera, 4:273. (BM) I936, Exotic Microlepidoptera, 5: 36. I897, Proc. Linnean Soc. N.S. Wales, 22: 357. I888, Trans. Proc. New Zealand Inst. 20: 85 . I9o8, Trans. Ent. Soc. London, I907: 480 . I906, Journ. Bombay Nat. Hist. Soc. I7: I44. (BM) I922, Ent. Mitteil. II: 46. (D) I93I, in Caradja, Bull. Sect. sci. Acad. roum. I4:66. (Bм) I920, Ann. South African Mus. I7: 309. (SA) I9I2, Trans. Ent. Soc. London, I9II: 687. (BM) I907, Journ. Bombay Nat. Hist. Soc. I7: 732. (вм) I934, in Caradja and Meyrick, Deuts. Ent. Zeit. Iris, 48: $3 \mathrm{I}$. I9I9, Exotic Microlepidoptera, 2: 226. (BM) I9I4, Exotic Microlepidoptera, I : 2 I4. I937, in Caradja and Meyrick, Deuts. Ent. Zeit. Iris, 5I: I80. (вм) I928, Exotic Microlepidoptera, 3: 42I. (BM) I907, Journ. Bombay Nat. Hist. Soc. I8: I56. I889, Proc. Linnean Soc. N.S. Wales, (Ser. 2) 3: I6I . I938, Trans. R. Ent. Soc. London, 87: 524. (BM) I9I I, Proc. Linnean Soc. N.S. Wales, 36: 291. I935, Exotic Microlepidoptera, 4: 564. (BM) I938, Trans. R. Ent. Soc. London, 87: 505. (BM) I9o9, Journ. Bombay Nat. Hist. Soc. I9: 433. (BM) I932, Trans. Ent. Soc. London, 80: II2. (BM) I926, Exotic Microlepidoptera, 3:292. (BM) I906, Journ. Bombay Nat. Hist. Soc. I 7: 4IO. I9I6, Exotic Microlepidoptera, I: 508. (BM) I9I 8, Exotic Microlepidoptera 2: I79. I937, Exotic Microlepidoptera, 5: II8. I9I0, Journ. Bombay Nat. Hist. Soc. 20: I56. (BM) I883, Proc. Linnean Soc. N.S. Wales, 8: 375 . I9I4, Exotic Microlepidoptera, I: 282. (BM) I921, Ann. Transvaal Mus. 8: 82. (T) I925, Exotic Microlepidoptera, 3: 223. (BM) I9I2, Ent. Mon. Mag. 48: 35. (Sub.) I9I3, Ann. Transvaal Mus. 3: 290. (T) I9I2, Trans. Ent. Soc. London, I9II: 673. (BM) I9Io, Journ. Bombay Nat. Hist. Soc. 20: 445. (Вi) I909, Journ. Bombay Nat. Hist. Soc. I9: 427. (BM) I889, Proc. Linnean Soc. N.S. Wales, (Ser. 2) 3: I58 I. I904, Proc. Linnean Soc. N.S. Wales, 29:360. I936, Exotic Microlepidoptera, 4: 6II. I934, Exotic Microlepidoptera, 4:472. I9I3, Trans. Ent. Soc. London, I9I3: I76. (BM) 
medica

medicata

mediocris

meditans

medullata

megacentra

megalarthra

megalastra

megaleuca

megalocentra

megalochra

megalodelta

megalodoxa

megalommata

megalopa

megalophthalma

megalopis

megaloplaca

megaloptila

megalorma

megalornis

megalotis

megalozona

megalynta

megalyntis

megaspila

megathyma

megophthalma

melanachaeta

melanacma

melanacta

melanactis

melanaema

melanaleuca

melanamba

melanamma

melanancalis

melananchis

melanaphra

melanarcha

melanarches

melanardis

melanargyra

melanarma

melanarthra

melanaspis

melanaster

melanastra

melanatma

melanatracta
GLYPHIPTERYX

ACOROSTOMA*

CNEPHASIA

TOXOTACMA*

SCYTHRIS

PYRODERCES

EPHELICTIS

ATTEVA

STENOMA

PHILOBOTA

STENOPTILIA

MONOPIS

BATRACHEDRA

PROTOLECHIA

PACHYPSALTIS

AEOLANTHES

LECITHOCERA

EULIA

COLEOPHORA

CRYPTOPHASA

EPIPHTHORA

GRACILARIA

ANATHAMNA

PLUTELLA

IMMA

TINEA

STATHMOPODA

CTENOCOMPA

EPACTRIS*

AGRIOTHERA*

ENARMONIA

ACROCERCOPS

PSEUDODOXIA

SPHYRELATA

BATTARISTIS

BORKHAUSENIA

CAENOGENES*

TORTRIX

ARISTOTELIA

DEPRESSARIA

COSMOPTERYX

FRISILIA

MACROBATHRA

ANTAEOTRICHA

XYSMATODOMA

EPISIMUS

YPONOMEUTA

DECADARCHIS*

CAPUA

PALTODORA
I9II, Trans. Linnean Soc. London, I4: 29I.

I9I4, Ann. South African Mus. Io: 256. (SA)

I9I4, Bull. Mus. Paris, 20: I2I. (P)

I929, Exotic Microlepidoptera, 3: 504. (BM)

I9I6, Exotic Microlepidoptera, 2: I3. (BM)

I923, Exotic Microlepidoptera, 3: 59. (BM)

I904, Proc. Linnean Soc. N.S. Wales, 29: 388.

I907, Proc. Linnean Soc. N.S. Wales, 32: 8I.

I9I 2, Trans. Ent. Soc. London, I9II: 7II. (BM)

I889, Proc. Linnean Soc. N.S. Wales, (Ser. 2) 3: I6r9.

I930, Exotic Microlepidoptera, 3: 570.

I 9o8, Proc. Zool. Soc. London, p. 737.

I897, Proc. Linnean Soc. N.S. Wales, 22: 308.

I904, Proc. Linnean Soc. N.S. Wales, 29: 384.

I9I5, Exotic Microlepidoptera, I : $29 \mathrm{I}$.

I930, Exotic Microlepidoptera, 4: II. (BM)

I9I6, Exotic Microlepidoptera, I: 575. (BM)

I932, Exotic Microlepidoptera, 4: 258. (v)

I909, Ann. South African Mus. 5: 360. (SA)

I9Io, Trans. Ent. Soc. London, I9Io: 457.

I904, Proc. Linnean Soc. N.S. Wales, 29: 26 I.

I 908, Journ. Bombay Nat. Hist. Soc. I8: 830.

I9I6, Exotic Microlepidoptera, I: 562. (BM)

I9I5, Trans. Proc. New Zealand Inst. 47: 203.

I906, Trans. Ent. Soc. London, I906: I88. (BM)

I9I3, Ann. Transvaal Mus. 3: 332. (T)

I897, Proc. Linnean Soc. N.S. Wales, 22: 325.

I932, Exotic Microlepidoptera, 4: 2I2.

I905, Joum. Bombay Nat. Hist. Soc. I6: 6I7.

I907, Journ. Bombay Nat. Hist. Soc. I7: $75^{\circ}$.

I907, Journ. Bombay Nat. Hist. Soc. I8: I40. (BM)

I9I5, Trans. Ent. Soc. London, I9I5: 226.

I9Io, Journ. Bombay Nat. Hist. Soc. 20: I5I. (BM)

I883, Proc. Linnean Soc. N.S. Wales, 8: 363.

I9I4, Trans. Ent. Soc. London, I9I4: 249.

I905, Trans. Ent. Soc. London, I905: 240.

I937, Exotic Microlepidoptera, 5: I60. (BM)

I933, Exotic Microlepidoptera, 4: 424. (BM)

I923, Exotic Microlepidoptera, 3: IO. (BM)

I9I3, Ann. Transvaal Mus. 3:3I6. (T)

I929, Trans. Ent. Soc. London, 76: 497.

I9Io, Trans. Ent. Soc. London, I9Io: 446. (BM)

I886, Proc. Linnean Soc. N.S. Wales, Io: 8 I7.

I9I6, Exotic Microlepidoptera, I: 500. (BM)

I893, Proc. Linnean Soc. N.S. Wales, 7: 505.

I927, Exotic Microlepidoptera, 3: 337. (BM)

I907, Journ. Bombay Nat. Hist. Soc. I7: 746. (BM)

I886, Trans. Ent. Soc. London, I886: 29I.

I9o8, Journ. Bombay Nat. Hist. Soc. I8: 6I4. (BM)

I9IO, Ann. South African Mus. 5: 4I3. (SA) 


\begin{tabular}{|c|c|}
\hline mel & EUCOSMA \\
\hline melanchares & ZULARCHA* \\
\hline melanchlora & NOTHRIS \\
\hline melancholica & COESYRA \\
\hline melanchrodes & EUPLOCAMUS \\
\hline melanchropa & ANARSIA \\
\hline melancrocana & CAPUA \\
\hline melanecta & CHELARIA \\
\hline melanecta & EULIA \\
\hline melanesia & STENOMA \\
\hline melanixa & STENOMA \\
\hline melanobathra & UNTOMIA \\
\hline melanocampta & PHTHORIMAEA \\
\hline melanocarpa & BRACHMIA \\
\hline melanocentra & ZONOPETALA \\
\hline melanocephala & TINEA \\
\hline melanochalca & LYONETIA \\
\hline melanocharis & CHELARIA \\
\hline melanochlaena & EUCOSMA \\
\hline melanochlora & EULIA \\
\hline melanochra & STATHMOPODA \\
\hline melanochrysa & TINEA \\
\hline melanoclera & ISOTENES* \\
\hline melanoclista & PROBOLACMA* \\
\hline melanocnista & CEROSTOMA \\
\hline melanocona & HERMOGENES \\
\hline melanocosma & ACROCERCOPS \\
\hline melanocrates & ЕTHMIA \\
\hline melanocrossa & PELTOPHORA \\
\hline melanocrypta & ISOCHORISTA \\
\hline melanocrypta & STENOMA \\
\hline melanodelta & PHLOEOPOLA \\
\hline melanodesma & CEROMITIA \\
\hline melanodora & SCYTHRIS \\
\hline melanodoxa & PSEPHOCRITA* \\
\hline melanogastra & CHOLOTIS \\
\hline melanoglypta & PHILOBOTA \\
\hline melanogma & IRIOTHYRSA* \\
\hline melanograpta & COLEOPHORA \\
\hline
\end{tabular}

melanoloncha melanoma melanombra melanometra melanomitra melanomochla melanonca melanoneura
STENOPTILIA ZONOPETALA EPIPHTHORA* PTILOGENES MACROBATHRA ACROCLITA AGRIOPHARA EUCOSMA
I9I6, Exotic Microlepidoptera, 2: I7. (вM)

I937, Exotic Microlepidoptera, 5: 76. (T) I929, Exotic Microlepidoptera, 3: 496. (BM) I9I8, Exotic Microlepidoptera, 2: 2I8. (BM) I9I6, Exotic Microlepidoptera, I: 6I7. I926, Exotic Microlepidoptera, 3: 28I. I88I, Proc. Linnean Soc. N.S. Wales, 6: $45^{\circ}$. I9I4, Ann. South African Mus. I0: 246. (SA) I9I7, Trans. Ent. Soc. London, I9I7: Io. (BM) I9I2, Trans. Ent. Soc. London, I9II: 7I2. (BM) I9I2, Trans. Ent. Soc. London, I9II: 7 I5. (BM) I9I8, Exotic Microlepidoptera, 2: I46. I9I7, Trans. Ent. Soc. London, p. 44. (Bм) I929, Exotic Microlepidoptera, 3: 527. (BM) I889, Proc. Linnean Soc. N.S. Wales, (Ser. 2) 3: I679. I933, Exotic Microlepidoptera, 4: 4I3. (C) I9I I, Journ. Bombay Nat. Hist. Soc. 2I: I08. I934, Exotic Microlepidoptera, 4: 5II. (BM) I936, Exotic Microlepidoptera, 4:6II. (BM) I93I, Exotic Microlepidoptera, 4: I5I. (v) I897, Proc. Linnean Soc. N.S. Wales, 22: 32 I. I9I I, Journ. Bombay Nat. Hist. Soc. 21 : I 20. I938, Trans. R. Ent. Soc. London, 87:507. (BM) I927, Exotic Microlepidoptera, 3:362. (BM) I938, in Caradja and Meyrick, Deuts. Ent. Zeit. Iris, 52: 24. I9o8, Journ. Bombay Nat. Hist. Soc. I8: 629. (BM) I920, Exotic Microlepidoptera, 2: 29I. I923, Bull. Mus. Nat. Hist. Nat. Paris, 29: 565. I889, Proc. Linnean Soc. N.S. Wales, (Ser. 2) 3: I640. I9I0, Proc. Linnean Soc. N.S. Wales, 35: I66.

I9I5, Exotic Microlepidoptera, I: 455.

I883, Proc. Linnean Soc. N.S. Wales, 8: 359.

I9I4, Exotic Microlepidoptera, I: 215.

I9I2, Ann. South African Mus. I0: 63. (SA)

I9I9, Exotic Microlepidoptera, 2: 255. (BM)

I9I8, Ann. Transvaal Mus. 6:28. (T) I889, Proc. Linnean Soc. N.S. Wales, (Ser. 2) 3: i6 14. I908, Proc. Zool. Soc. London, p. 736.

I935, in Caradja and Meyrick, Materialien zu einer

Microlepidopteren Fauna der Chinesischen Provinzen

Kiangsu, Chekiang und Hunan, p. 90.

I926, Exotic Microlepidoptera, 3: 301.

I883, Proc. Linnean Soc. N.S. Wales, $7: 465$.

I888, Trans. Proc. New Zealand Inst. 20: 77.

I926, Exotic Microlepidoptera, 3: 229. (BM)

I886, Proc. Linnean Soc. N.S. Wales, ro: 807.

I936, Exotic Microlepidoptera, 5:24. (BM)

I9I5, Exotic Microlepidoptera, I: 408. (BM)

I9I2, Journ. Bombay Nat. Hist. Soc. 2I: 866. 


\begin{tabular}{|c|c|}
\hline melanoneura & SAROPLA \\
\hline melanopa & SPILONOTA \\
\hline melanopa & SYNTOMACTIS \\
\hline melanopepla & SIMAETHIS \\
\hline 1ophaea & CNEPHASIA \\
\hline hragma & CAPUA \\
\hline & a $\mathrm{CR}$ \\
\hline ophyta & ARGYROTOXA \\
\hline lopis & ANTAEOTRICHA \\
\hline loplecta & ACROCERCOPS \\
\hline noplecta & ANARSIA \\
\hline Iopleura & SCYTHRIS \\
\hline soplintha & PHTHORIMAEA \\
\hline oploca & PHILOBOTA \\
\hline opsamma & XYROSARIS \\
\hline optila & TRACHYPEPLA \\
\hline orma & CHOROCOSMA* \\
\hline lortha & DICHOMERIS \\
\hline osacta & IDIOPHANTIS \\
\hline losarca & PYRODERCES \\
\hline oscia & COESYRA \\
\hline soma & TRICHOTAPHE \\
\hline sparta & LITHOCOLLETIS \\
\hline sperma & TORTRIX \\
\hline losphena & IMMA \\
\hline ospila & NEPTICULA \\
\hline ospora & MACHAERITIS \\
\hline lostola & PHRIXOSCELES \\
\hline stoma & TINEOLA \\
\hline strepta & EUPSELIA \\
\hline ostropha & DECADARCHIS \\
\hline strota & CEROMITIA \\
\hline ota & MACROBATHRA \\
\hline lothicta & PHALONIA \\
\hline lotis & NEPTICULA \\
\hline otreta & SPILONOTA \\
\hline & ERECHTHIAS \\
\hline oxantha & PHILOBOTA \\
\hline zestas & ZARCINIA \\
\hline & TELPHUSA \\
\hline & ATALOPSYCHA \\
\hline pula & TELECRATES \\
\hline nura & ELACHISTA \\
\hline anocopa & SPILONOTA \\
\hline & CLEROGENES* \\
\hline & MONOPIS \\
\hline & MACHIMIA \\
\hline & GELECHIA \\
\hline & BRACHMIA \\
\hline
\end{tabular}

I884, Proc. Limnean Soc. N.S. Wales, 9: 744. I9I2, Journ. Bombay Nat. Hist. Soc. 2I: 853. (BM) I897, Proc. Linnean Soc. N.S. Wales, 22: 384. I88I, Proc. Linnean Soc. N.S. Wales, 5:212. 1927, Trans. Proc. New Zealand Inst. 57: 698. I936, Exotic Microlepidoptera, 5: 59. (c) I929, Trans. Ent. Soc. London, 76: 5I3. (BM) I9I3, Trans. Ent. Soc. London, I9I3: I72. (BM) I9o9, Trans. Ent. Soc. London, I909: 3I. (BM) I908, Journ. Bombay Nat. Hist. Soc. I8: 823. I9I4, Journ. Bombay Nat. Hist. Soc. 22: 774. (BM) I9I4, Ann. South African Mus. Io: 246. (SA) I926, Exotic Microlepidoptera, 3: 279. I884, Proc. Linnean Soc. N.S. Wales, 8: 508. I93I, Exotic Microlepidoptera, 4: I7I. (BM) I883, Proc. Linnean Soc. N.S. Wales, 8: 370. 1893, Proc. Linnean Soc. N.S. Wales, 7: 560. I929, Exotic Microlepidoptera, 3: 5IO. (BM) I907, Journ. Bombay Nat. Hist. Soc. I8: I48. (BM) I937, Exotic Microlepidoptera, 5: I48. I889, Proc. Linnean Soc. N.S. Wales, (Ser. 2) 3: I66o. 1920, Voyage de Ch. Alluand et $R$. Jeannel en Afrique Oriental, II, Microlepidoptera, p. 75. (P) I9I2, Exotic Microlepidoptera, I: 2I. (T) I9I6, Trans. Proc. New Zealand Inst. 48: 4I4. I9I8, Exotic Microlepidoptera, 2: I92.

I934, Exotic Microlepidoptera, 4: 468. I886, Proc. Linnean Soc. N.S. Wales, Io: 770. I9I8, Ann. Transvaal Mus. 6: 39. (T) I9o8, Proc. Zool. Soc. London, p. 739. I88I, Proc. Linnean Soc. N.S. Wales, 5: 223. I93I, Exotic Microlepidoptera, 4: I65. I9I2, Ann. South African Mus. Io: 72. (SA) I886, Proc. Linnean Soc. N.S. Wales, Io: 809. I927, Exotic Microlepidoptera, 3: 367. I906, Trans. R. Soc. S. Australia, 30: 58 . I9Io, Trans. New Zealand Inst. 42: 72. I888, Trans. Proc. New Zealand Inst. 20: 93. I889, Proc. Linnean Soc. N.S. Wales, (Ser. 2) 3: I6o8. I935, Exotic Microlepidoptera, 4: 556. (BM)

I9I3, Exotic Microlepidoptera, I: 65. (BM) I887, Proc. Linnean Soc. N.S. Wales, (Ser. 2) I : 1048. I89o, Trans. R. Soc. S. Australia, I3: 62 . I889, Trans. Proc. New Zealand Inst. I889: I77. I9I2, Journ. Bombay Nat. Hist. Soc. 2I: 853 . I92I, Ann. Transwaal Mus. 8: 93. (T) I9I7, Exotic Microlepidoptera, 2:84. I9I4, Exotic Microlepidoptera, I: I84. (BM) I92I, Ann. Transvaal Mus. 8: 7I. (T) I9I8, Exotic Microlepidoptera, 2: II4. (BM) 


$\begin{array}{ll}\text { melicera } & \text { MACHAEROPTERIS } \\ \text { meliceris } & \text { HAPSIFERA } \\ \text { melichlora } & \text { ATHEROPLA } \\ \text { melichlorias } & \text { NEMOTOIS } \\ \text { melichroa } & \text { YPSOLOPHUS } \\ \text { melichrosta } & \text { TIOUADRA } \\ \text { melichrysa } & \text { ESCHATOTYPA } \\ \text { melicoma } & \text { CRYPTOLECHIA } \\ \text { melicosma } & \text { EMMETOECA* } \\ \text { melicrana } & \text { MELASINA } \\ \text { melicrana } & \text { PSEUDODOXIA } \\ \text { melicrata } & \text { HOMALOXESTIS } \\ \text { melicrossa } & \text { PERIACMA } \\ \text { melicrossis } & \text { LASPEYRESIA } \\ \text { meligastra } & \text { SCYTHRIS } \\ \text { meligrapta } & \text { STENOMA } \\ \text { melileuca } & \text { LIMNOECIA } \\ \text { melinopa } & \text { STENOMA } \\ \text { meliochra } & \text { MELASINA } \\ \text { melipecta } & \text { BUCCULATRIX } \\ \text { melipecta } & \text { PARACLYSTIS } \\ \text { meliphaea } & \text { MELASINA } \\ \text { meliphaga } & \text { PHRURIASTIS* } \\ \text { meliphanes } & \text { CHRYSORYCTIS } \\ \text { melipnoa } & \text { CHALAROTONA } \\ \text { meliptila } & \text { CHELARIA } \\ \text { melirrhoa } & \text { PHILOBOTA } \\ \text { meliscelis } & \text { STATHMOPODA } \\ \text { meliscia } & \text { POLYCHROSIS } \\ \text { melissa } & \text { CAPUA } \\ \text { melistoma } & \text { LASIODICTIS* } \\ \text { melitacma } & \text { THYMIATRIS* } \\ \text { melitardis } & \text { OPOSTEGA } \\ \text { melitaula } & \text { ARGYRESTHIA } \\ \text { meliteucta } & \text { PERSICOPTILA } \\ \text { melithorax } & \text { EPIMACTIS } \\ \text { melithrepta } & \text { GLYPHIDOCERA } \\ \text { melitocoma } & \text { TINEA } \\ \text { melitocosma } & \text { RHADINASTIS } \\ \text { melitocrossa } & \text { EULIA } \\ & \text { TELPHUSA } \\ & \end{array}$

melitoleuca melitolicna

melitophanes melitopis
CHALAROTONA GELECHIA

GELECHIA MEGACRASPEDUS
I9I I, Journ. Bombay Nat. Hist. Soc. 21 : I3o.

I908, Proc. Zool. Soc. London, p. 750.

I884, Proc. Linnean Soc. N.S. Wales, 9: 759.

1907, Journ. Bombay Nat. Hist. Soc. I7: 992.

I904, [Meyr. nec Low], Proc. Linnean Soc. N.S. Wales, 29: 43I.

1922, Exotic Microlepidoptera, 2: 598. (BM)

I88I, Proc. Linnean Soc. N.S. Wales, 5: 257.

I93I, Exotic Microlepidoptera, 4: I25.

I921, Ann. Transvaal Mus. 8: I27.

I930, Exotic Microlepidoptera, 3:550.

I928, Exotic Microlepidoptera, 3: 478. (BM)

I9Io, Journ. Bombay Nat. Hist. Soc. 20: 442. (Bм)

I9I0, Journ. Bombay Nat. Hist. Soc. 20: I6o. (BM)

I932, Exotic Microlepidoptera, 4: 3II. (BM)

I920, Ann. South African Mus. I7: 298. (SA)

1925, Exotic Microlepidoptera, 3: I83. (BM)

I937, Exotic Microlepidoptera, 5: I48.

1925, Exotic Microlepidoptera, 3:222. (BM)

I9I5, Exotic Microlepidoptera, I: 290.

I9I4, Ann. Transvaal Mus. 4: 204. (T)

I9I5, Exotic Microlepidoptera, I: 293.

I9I6, Exotic Microlepidoptera, I: 609.

I923, Exotic Microlepidoptera, 3: 64. (BM)

I893, Proc. Linnean Soc. N.S. Wales, $7: 553$.

I89o, Trans. R. Soc. S. Australia, I3: 65 .

I926, Exotic Microlepidoptera, 3: 283.

I884, Proc. Linnean Soc. N.S. Wales, 8: 498.

1929, Exotic Microlepidoptera, 3: 542.

I9I0, Trans. New Zealand Inst. 42: 72.

I908, Journ. Bombay Nat. Hist. Soc. I8: 6rз. (Bм)

I9I2, Exotic Microlepidoptera, I : 4I. (BM)

I907, Journ. Bombay Nat. Hist. Soc. I7: 738. (вм)

I9I8, Ann. Transvaal Mus. 6: 4I. (T)

I9I8, Exotic Microlepidoptera, 2: I87. (BM)

I9I5, Exotic Microlepidoptera, I: 3 I9.

I923, Exotic Microlepidoptera, 2: 6I3. (BM)

I929, Trans. Ent. Soc. London, 76: 5IO. (BM)

I893, Proc. Linnean Soc. N.S. Wales, 7: 542.

I93I, Exotic Microlepidoptera, 4: 55 .

I926, Exotic Microlepidoptera, 3: 253. (BM)

I935, in Caradja and Meyrick, Materialien zu einer Micro-

lepidopteren Fauna der Chinesischen Provinzen Kiangsu,

Chekiang und Hunan, p. 65.

I89o, Trans. R. Soc. S. Australia, I3: 66.

I935, in Caradja and Meyrick, Materialien zu einer Micro-

lepidopteren Fauna der Chinesischen Provinzen Kiangsu,

Chekiang und Hunan, p. 67. (BM)

I93I, Exotic Microlepidoptera, 4:85. (BM)

I904, Proc. Linnean Soc. N.S. Wales, 29: 276. 


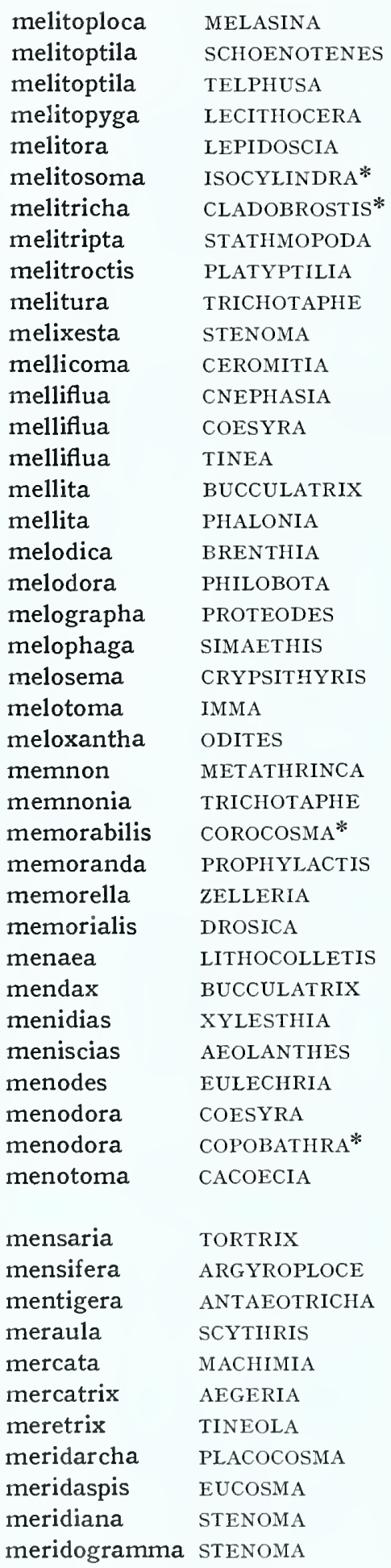

I927, Exotic Microlepidoptera, 3: 329. (T)

1938, Trans. R. Ent. Soc. London, 87: 508. (BM)

I923, Exotic Microlepidoptera, 3: I7. (BM)

I923, Exotic Microlepidoptera, 3: 4I. (BM)

I893, Proc. Linnean Soc. N.S. Wales, 7: 509.

I930, Exotic Microlepidoptera, 3: 584. (BM)

I92I, Exotic Microlepidoptera, 2: 4IO.

I933, Exotic Microlepidoptera, 4: 43I.

I924, Arkiv för Zool. vol. I6, part I4, p. 2.

I9I6, Exotic Microlepidoptera, I: 585. (BM)

1925, Exotic Microlepidoptera, 3: 2IO. (BM)

I9I2, Exotic Microlepidoptera, I: 29. (Т)

I9I4, Exotic Microlepidoptera, I: I95. (BM)

I884, Proc. Limnean Soc. N.S. Wales, 9: 78I.

I9I I, Journ. Bombay Nat. Hist. Soc. 2 I : I2I.

I9I5, Trans. Ent. Soc. London, I9I5: 248.

I9I6, Ent. Mon. Mag. 52: 277.

1922, Exotic Microlepidoptera, 2: 487. (BM)

I889, Proc. Linnean Soc. N.S. Wales, (Ser. 2) 3: I627.

1927, Trans. Proc. New Zealand Inst. 57: 701.

I93I, Exotic Microlepidoptera, 4: I82. (BM)

I9I7, Exotic Microlepidoptera, 2: 75.

I9o6, Trans. Ent. Soc. London, Ig06: I94. (BM)

1927, Exotic Microlepidoptera, 3: 364. (T)

I9I4, Journ. Bombay Nat. Hist. Soc. 22: 778. (BM)

I9I3, Trans. Ent. Soc. London, I9I3: I76. (BM)

1927, Trans. Proc. New Zealand Inst. 57: 700.

I922, Exotic Microlepidoptera, 2: 554.

I893, Proc. Linnean Soc. N.S. Wales, 7: 583 .

I921, Zool. Meded. 6: 200. (L)

I9I8, Exotic Microlepidoptera, 2: I72.

I9I8, Exotic Microlepidoptera, 2: I85.

I922, Exotic Microlepidoptera, 2: 597.

19I8, Exotic Microlepidoptera, 2: I96. (BM)

I888, Proc. Linnean Soc. N.S. Wales, (Ser. 2) 2: 945.

I889, Proc. Linnean Soc. N.S. Wales, (Ser. 2) 3: 1657.

I9I I, Ann. Transvaal Mus. 2: 238. (T)

I937, in Caradja and Meyrick, Deuts. Ent. Zeit. Iris, 5I:

I73.

I9I2, Ann. South African Mus. Io: 56. (SA)

I9I6, Exotic Microlepidoptera, 2: I9. (BM)

I926, Exotic Microlepidoptera, 3:236. (BM)

I9I6, Exotic Microlepidoptera, 2: II.

I9I4, Exotic Microlepidoptera, I: I83. (BM)

I93I, Exotic Microlepidoptera, 4: 50.

I9o8, Proc. Zool. Soc. London, p. 739.

I889, Proc. Linnean Soc. N.S. Wales, (Ser. 2) 3: I576.

I922, Exotic Microlepidoptera, 2: 5I6. (P)

I9I5, Exotic Microlepidoptera, I: 435. (BM)

I930, Ann. Naturhist. Mus. Wien, 44: 242. (v) 


\begin{tabular}{|c|c|c|}
\hline meridospila & EUCOSMA & I922, Exotic Microlepidoptera, 2: 5I8. (BM) \\
\hline merimnaea & ELACHISTA & I920, Ann. South African Mus. I7: 297. (SA) \\
\hline merinthias & HILAROGRAPHA & I909, Journ. Bombay Nat. Hist. Soc. I9: 426. (BM) \\
\hline merista & PYCNOSTOLA & I9I8, Ann. Transvaal Mus. 6: I4. (T) \\
\hline mermera & CNEPHASIA & I9Io, Proc. Linnean Soc. N.S. Wales, 35: 277. \\
\hline mermerodes & HOMONA & I910, Proc. Linnean Soc. N.S. Wales, 35: 213. \\
\hline merocentra & CRYPTOPHASA & I925, Exotic Microlepidoptera, 3: I47. \\
\hline merocoma & ACROLOPHUS & I922, Exotic Microlepidoptera, 2: 607. (BM) \\
\hline eryntis & $\begin{array}{l}\text { TINGENTERA } \\
\text { [PHANOSCHISTA*] }\end{array}$ & I9o8, Journ. Bombay Nat. Hist. Soc. I8: 455. (BM) \\
\hline mesacta & GELECHIA & Igo9, Ann. Transvaal Mus. 2: I2, pl. 4, fig. 8. (T) \\
\hline nesarotra & ARGYROPLOCE & I9II, Proc. Linnean Soc. N.S. Wales, 36: 273. \\
\hline mesatma & TINTHIA & I926, Exotic Microlepidoptera, 3: 269 . \\
\hline mesaula & GLYPHIPTERYX & I907, Proc. Linnean Soc. N.S. Wales, 32: I2I. \\
\hline neselectra & EULECHRIA & I902, Trans. R. Soc. S. Australia, 26: I48. \\
\hline lesobactris & COPROMORPHA & I930, Exotic Microlepidoptera, 3: 590. (BM) \\
\hline nesocausta & ARGYRESTHIA & I9I3, Exotic Microlepidoptera, I: I35. (BM) \\
\hline nesocentra & PHLOEOPOLA & I889, Proc. Linnean Soc. N.S. Wales, (Ser. 2) 3: I590. \\
\hline lesocentra & STRUTHISCA & I905, Journ. Bombay Nat. Hist. Soc. I6: 6I 4 . \\
\hline nesochaeta & ACROCERCOPS & I920, Exotic Microlepidoptera, 2: 294. (вм) \\
\hline zesochlora & TALAEPORIA & I9I8, Ann. Transvaal Mus. 6: 44. (T) \\
\hline lesochorda & IMMA & I906, Trans. Ent. Soc. London, I906: I89. (BM) \\
\hline mesoclasta & CERACE & I9o8, Rec. Indian Mus. $2: 395$. (K) \\
\hline mesocrossa & STATHMOPODA & I927, Exotic Microlepidoptera, 3: 379. (BM) \\
\hline mesoctenis & DICHOMERIS & I92I, Exotic Microlepidoptera, 2: 434. (BM) \\
\hline lesodelta & COMPSOLECHIA & I922, Trans. Ent. Soc. London, I922: 83. (Bм) \\
\hline nesodesma & PELTOPHORA & I889, Proc. Linnean Soc. N.S. Wales, (Ser. 2) 3: I642. \\
\hline nesodyas & CRYPSITHYRIS* & I907, Journ. Bombay Nat. Hist. Soc. I7: 753 . \\
\hline esoglena & DICHOMERIS & I923, Exotic Microlepidoptera, 2: 6I9. (BM) \\
\hline nesogramma & ANTISPILA & I921, Exotic Microlepidoptera, 2: 403. \\
\hline nesogypsa & ERIOGENES* & I925, Exotic Microlepidoptera, 3: I59. \\
\hline lesogypsa & NARYCIA & I932, Exotic Microlepidoptera, 4:2I2. (BM) \\
\hline nesolampra & IMMA & I927, Exotic Microlepidoptera, 3: 376. (Bм) \\
\hline mesoloba & CAPUA & $\begin{array}{l}\text { I937, in Caradja and Meyrick, Deuts. Ent. Zeit. Iris, 5I: } \\
\text { I72. (Bм) }\end{array}$ \\
\hline resolychna & ORNEODES & I908, Trans. Ent. Soc. London, I907: 508. \\
\hline lesombra & STATHMOPODA & I897, Proc. Linnean Soc. N.S. Wales, 22: 320. \\
\hline lesonyctia & ZARATHA & I909, Trans. Ent. Soc. London, I909: I7. \\
\hline esopercna & ANTICRATES & I926, Sarawak Mus. Journ. 3: I64. \\
\hline mesophragma & EULECHRIA & I888, Proc. Linnean Soc. N.S. Wales, (Ser. 2) 2: 950. \\
\hline lesophthora & PALPARIA & I886, Proc. Linnean Soc. N.S. Wales, I0: 825 . \\
\hline nesoplaca & TINEA & I9I9, Exotic Microlepidoptera, 2: 246. \\
\hline mesoplecta & SCYTHRIS & I92I, Ann. Transvaal Mus. 8: II6. (т) \\
\hline mesopora & MACROBATHRA & I886, Proc. Linnean Soc. N.S. Wales, Io: 804. \\
\hline nesoptila & PYRODERCES & I897, Proc. Linnean Soc. N.S. Wales, 22: 347. \\
\hline mesorectis & HYPOSMOCOMA & I9I5, Exotic Microlepidoptera, I: 342 . (BM) \\
\hline mesosaris & STENOMA & I925, Exotic Microlepidoptera, 3: I78. (BM) \\
\hline mesosceptra & DEPRESSARIA & I9I5, Trans. Ent. Soc. London, I9I5: 210. (BM) \\
\hline esosceptra & EPICHORISTA & $\begin{array}{l}\text { I920, Voyage de Ch. Alluand et R. Jeannel en Afrique } \\
\text { Oriental, II, Microlepidoptera, p. } 53 . \text { ( }(\mathrm{P})\end{array}$ \\
\hline
\end{tabular}




\begin{tabular}{|c|c|}
\hline mesoschista & IZATHA \\
\hline mesoscia & ACROCLITA \\
\hline mesosema & CEROMITIA \\
\hline mesosphena & IMMA \\
\hline mesospila & CARPOSINA \\
\hline mesostrepta & GELECHIA \\
\hline mesostrota & ANTAEOTRICHA \\
\hline mesotoma & CRYPTOPHASA \\
\hline mesotricha & ANTICTENISTA* \\
\hline mesotypa & $\begin{array}{l}\text { ENDOPHTHORA } \\
{[\text { CRYPSITRICHA*] }}\end{array}$ \\
\hline mesoxantha & OCYSTOLA \\
\hline mesoxysta & ARISTOTELIA \\
\hline mesozona & MYCHONOA* \\
\hline mesozosta & TISIS \\
\hline mesozyga & ETHMIA \\
\hline messoria & CORMOTYPA \\
\hline metabapta & EULECHRIA \\
\hline metacapna & PSEUDATTERIA \\
\hline metacausta & LECITHOCERA \\
\hline metacentra & CAPUA \\
\hline metacentra & CRYPTOLECHIA \\
\hline metacentra & SAPHENEUTIS \\
\hline metachalca & THIOGNATHA* \\
\hline
\end{tabular}

metachlora metachlora metachroa metachrysa metacirrha metaclina metaclista metacritica metacrocota metactenis metacymba metacypha metacystis metadelta

metadesma metadesmia metadoxa metagramma metagrapta metagypsa metallanthes metallastra
IMMA

POLYCHROSIS

PHILOBOTA PHYCOMORPHA * CLEMMATISTA* BARBAROSCARDIA ODITES EUCOSMA ANCYLOMETIS ARGYROPLOCE AGRIOPHARA LIMNOECIA PTILOGENES IZATHA

[SEMIOCOSMA] STOMOPTERYX MICROPLITIS GRACILARIA COMPSOLECHIA EUCOSMA EUCOSMA

TIMYRA ARGYROTOXA
I93I, Trans. Proc. New Zealand Inst. 62: 96. I9I I, Proc. Linnean Soc. N.S. Wales, 36: 235. I92 I, Ann. Transvaal Mus. 8: I39. (T) I936, Exotic Microlepidoptera, 5: 39. (v) I920, Voyage de Ch. Alluaud et R. Jeannel en Afrique

Oriental, II, Microlepidoptera, p. 45. (P) I932, Exotic Microlepidoptera, 4: I95. (BM) I9I2, Trans. Ent. Soc. London, I9I : 708. (BM) I925, Exotic Microlepidoptera, 3: I49. I927, Exotic Microlepidoptera, 3: 337. (BM)

I888, Trans. Proc. New Zealand Inst. 20: 94. I885, Proc. Linnean Soc. N.S. Wales, 9: I073. I9I3, Ent. Mitteil. 2: 299. (D) I893, Proc. Linnean Soc. N.S. Wales, $7: 559$. I9I4, Supplementa Entomologica, No. 3, p. 50. (D) 1935, Exotic Microlepidoptera, 4: 603. (BM) I9I4, Exotic Microlepidoptera, I: 250. I9I4, Exotic Microlepidoptera, I: I64. 1924, Exotic Microlepidoptera, 3: I08. (BM) I9Io, Journ. Bombay Nat. Hist. Soc. 20: 446. (BM) I9I 8, Exotic Microlepidoptera, 2: I63. I9I4, Supplementa Entomologica, No. 3, p. 52. (D) I907, Journ. Bombay Nat. Hist. Soc. I8: I56. I920, Voyage de Ch. Alluaud et R. Jeannel en Afrique Oriental, II, Microlepidoptera, p. 74. (P) I906, Trans. Ent. Soc. London, I906: 203. (BM) I9I3, Ann. Transvaal Mus. 3: 275. (T) I889, Proc. Linnean Soc. N.S. Wales, (Ser. 2) 3: I6I6. I9I4, Trans. New Zealand Inst. I9I4: Io6. I92I, Exotic Microlepidoptera, 2: 4I4. I920, Ann. South African Mus. I7: 308. (SA) I9I5, Exotic Microlepidoptera, I: 379. (BM) I922, Exotic Microlepidoptera, 2: 5I5. (P) I930, Trans. Ent. Soc. London, 78: 317. (P) I9o9, Journ. Bombay Nat. Hist. Soc. I9: 597. (BM) I9I6, Exotic Microlepidoptera, I: 489. (BM) I9I4, Exotic Microlepidoptera, I: 203. I9I8, Exotic Microlepidoptera, 2: 2I I. (BM)

I905, Trans. Ent. Soc. London, I905: 238. I927, Exotic Microlepidoptera, 3: 352. (Bм) I934, Exotic Microlepidoptera, 4: 467.

I908, Journ. Bombay Nat. Hist. Soc. I8: 83I. (BM) I9I8, Exotic Microlepidoptera, 2: I38. (BM) I932, Exotic Microlepidoptera, 4:224. (BM) I920, Voyage de Ch. Alluaud et R. Jeannel en Afrique Oriental, II, Microlepidoptera, p. 6r. (P) I905, Journ. Bombay Nat. Hist. Soc. I6: 596. (BM) I933, Exotic Microlepidoptera, 4: 424. 


\begin{abstract}
metallidias metallifera metallitis metallophora metallota metalychna
\end{abstract}

metamelopa
metamochla
metamorphica
metanocona
metapelista
metaphorica
metaphracta
metaplastica
metaplecta

metapyrrha metasaris metaschista metascia metaspila metasticta metata metathyris metatoxa metaxantha metaxias metazona meteora meteoris meteoropa metherca methodica methystica methystis metochra metoeca metopias metorcha metriacma metrica metriodes metriodoxa metrionoma metriopa metriopis metrodelta metrodes
PATANOTIS EPICROESA COPROMORPHA MOLYBDURGA* EUCHAETIS PHILOPTILA

DEPRESSARIA PTILOGENES EUCOSMA HERMOGENES LEPOCNEMIS CHELARIA ODITES ROESLERSTAMMIA ARGYROPLOCE

TORTRIX

BRACHMIA EUCOSMA ODITES ATYCHIA GLYPHIPTERYX EULECHRIA

TINEA CYMOTRICHA TYROMANTIS* BATRACHEDRA EPIMACTIS GLYPHIPTERYX ETHMIA DINOGENES* MELASINA DECADARCHIS STENOMA HILAROGRAPHA COMMATICA APETHISTIS* STATHMOPODA ENCOLAPTA* BACTRA PERIACMA ONEBALA IMMA PARACHARACTIS ZELLERIA HOPLITICA CHALINIASTIS DICHOMERIS
I9I3, Exotic Microlepidoptera, I: 8 o. 1907, Proc. Linnean Soc. N.S. Wales, 32: 96. I906, Journ. Bombay Nat. Hist. Soc. I7: 4I I. (BM) I897, Proc. Linnean Soc. N.S. Wales, 22: 369. 1883, Proc. Linnean Soc. N.S. Wales, 7: 486. I935, in Caradja and Meyrick, Materialien zu einer Microlepidopteren Fauna der Chinesischen Provinzen Kiangsu, Chekiang und Hunan, p. 73.

I93I, in Caradja, Bull. Sect. sci. Acad. roum. I4: 72. (BM) I93I, Exotic Microlepidoptera, 4: 45. (v) I928, Exotic Microlepidoptera, 3: 439. (BM) 1908, Journ. Bombay Nat. Hist. Soc. I8: 629. I932, Trans. Ent. Soc. London, 80: II7. I921, Exotic Microlepidoptera, 2: 430. (BM) I909, Ann. South African Mus. 5: 354. (SA) I92 I, Exotic Microlepidoptera, 2: 439. I920, Voyage de Ch. Alluaud et $R$. Jeannel en Afrique Oriental, II, Microlepidoptera, p. 63. (P) 19I8, Ann. Transvaal Mus. 6:8. (T) I9II, Journ. Bombay Nat. Hist. Soc. 20: 7 I2. (BM) I912, Ent. Mon. Mag. 48: 35. (Sub.) I937, Exotic Microlepidoptera, 5： I5I. (T) I926, Exotic Microlepidoptera, 3: 302. (T) 1907, Trans. Proc. New Zealand Inst. 39: I 9. I9I4, Exotic Microlepidoptera, I: I68. (MA) 1935, Exotic Microlepidoptera, 4: 575 . I935, Exotic Microlepidoptera, 4: 588. I9I8, Exotic Microlepidoptera, 2: 217. 1897, Proc. Linnean Soc. N.S. Wales, 22: 303. I908, Proc. Zool. Soc. London, p. 730. I88I, Proc. Linnean Soc. N.S. Wales, 5: 237. I9I I, Trans. Linnean Soc. London, I4:289. (BM) I934, Exotic Microlepidoptera, 4: 486. (BM) I9I6, Exotic Microlepidoptera, I: 608.

I9II, Trans. Linnean Soc. London, I4: 299. I930, Ann. Naturhist. Mus. Wien, 44:25I. (v) I92I, Exotic Microlepidoptera, 2: 479. (BM) I9I4, Trans. Ent. Soc. London, I9I4: 238. (Bм) I908, Journ. Bombay Nat. Hist. Soc. I8: 460. (BM) I920, Exotic Microlepidoptera, 2: 324. (BM) I913, Journ. Bombay Nat. Hist. Soc. 22: 167. (Bм) I909, Journ. Bombay Nat. Hist. Soc. I9: 583. (BM) I9Io, Journ. Bombay Nat. Hist. Soc. 20: I57. (BM) I9I8, Exotic Microlepidoptera, 2: II2. I906, Trans. Ent. Soc. London, I906: I77. I928, Exotic Microlepidoptera, 3: 470. 1933, Exotic Microlepidoptera, 4: 373. (BM) I888, Proc. Linnean Soc. N.S. Wales, (Ser. 2) 2: 94 I. I905, Journ. Bombay Nat. Hist. Soc. I6: 59I. (BM) I9I3, Journ. Bombay Nat. Hist. Soc. 22: I72. (BM) 


$\begin{array}{ll}\text { metrodoxa } & \text { OPSODOCA* } \\ \text { metroleuca } & \text { STENOMA } \\ \text { metronoma } & \text { GLYPHIPTERYX } \\ \text { metrophora } & \text { MYRMECOZELA } \\ \text { metrosema } & \text { MALLOBATHRA } \\ \text { metrospila } & \text { TRACHYNTIS } \\ \text { metrothetis } & \text { EPERMENIA } \\ \text { metuens } & \text { DICHOMERIS } \\ \text { miarodes } & \text { EPIPHTHORA } \\ \text { micans } & \text { EPACTRIS } \\ \text { micracma } & \text { CRYPTOLECHIA } \\ \text { micracma } & \text { TELECRATES } \\ \text { micranthes } & \text { LOZOSTOMA } \\ \text { micranthis } & \text { SYNACTIAS* } \\ \text { micrarcha } & \text { ANTISPILA } \\ \text { micrastra } & \text { MISCERA } \\ \text { micrastrella } & \text { PALPARIA }\end{array}$

microbathra microbias microbolista microcasis microcentra microchalca microchlora microctenis microctenota microdelta microdesma microdictyas microdora microdoxa microglena microglypta microglyptis

microgramma micrographa micrograpta microlepta microlina microlitha microlychna micromacha micrometra micronca

micropa microphanes microphanta
[ARACHNOGRAPHA * CNEPHASIA BATRACHEDRA ODITES PHTHORIMAEA STREPSIMELA (?) LABDIA PHILOBOTA MELASINA MICTOPSICHIA EPICHOSTIS ORNEODES

LABDIA PROTOLECHIA GAESA PYRODERCES LIMNOECIA CRYPTOLECHIA

CHELARIA PHYLLOCNISTIS ARGYROPLOCE OPOSTEGA ISOPHRICTIS SIMAETHIS RHADINASTIS* ACROLOPHUS ARISTOTELIA CAPUA

PROTOLECHIA MALLOBATHRA PACHNISTIS
I9I9, Exotic Microlepidoptera, 2: 27I. (BM) I930, Ann. Naturhist. Mus. Wien, 44:256. (v) I907, Proc. Linnean Soc. N.S. Wales, 32: I23. I9II, Journ. Bombay Nat. Hist. Soc. 21 : I26. I888, Trans. Proc. New Zealand Inst. 20: I03. I889, Proc. Linnean Soc. N.S. Wales, (Ser. 2) 3: 1587. I92I, Exotic Microlepidoptera, 2: 464. (BM) I932, Exotic Microlepidoptera, 4: 20I. (BM) I904, Proc. Linnean Soc. N.S. Wales, 29: 263. I921, Zool. Meded. 6: I98. (L) I9Io, Journ. Bombay Nat. Hist. Soc. 20: I62. (BM) I89o, Trans. R. Soc. S. Australia, I3: 64. I897, Proc. Linnean Soc. N.S. Wales, 22: 4I9. I93I, Journ. Linnean Soc. London, 37: 278. (BM) 1926, Exotic Microlepidoptera, 3:261. 1907, Proc. Linnean Soc. N.S. Wales, 32: 105.

I883, Proc. Linnean Soc. N.S. Wales, 7: 433. I9II, Trans. New Zealand Inst. 43: 62. I9I4, Ann. Transvaal Mus. 4: 203. (T) I937, Exotic Microlepidoptera, 5: I52. I929, Exotic Microlepidoptera, 3: 532. (Sub.) I886, Trans. Ent. Soc. London, I886: 249. I92I, Exotic Microlepidoptera, 2: 4I2. (BM) I9I5, Exotic Microlepidoptera, I: 303. I9I4, Ann. South African Mus. Io: 253. (SA) 1933, Exotic Microlepidoptera, 4: 370. I927, in Caradja, Mem. Sect.Sti. Acad.Românā, 4:422. (BM) I929, Exotic Microlepidoptera, 3: 534. I923, Exotic Microlepidoptera, 3: 58. (BM) I904, Proc. Linnean Soc. N.S. Wales, 29:346. 1933, Exotic Microlepidoptera, 4: 357. (BM) I9I5, Exotic Microlepidoptera, I: 3I2. I928, Exotic Microlepidoptera, 3: 393. I936, Veröff. Deutschen Kol.-Mus. I: 333, pl. I3, fig. 26. (H) I920, Exotic Microlepidoptera, 2: $30 \mathrm{I}$. I9I6, Exotic Microlepidoptera, I : 6I9. I921, Ann. Transvaal Mus. 8: 56. (T) I9I5, Trans. Ent. Soc. London, I9I5: 239. I935, Exotic Microlepidoptera, 4: 582. (M) I888, Trans. Proc. New Zealand Inst. 20:84. I897, Proc. Linnean Soc. N.S. Wales, 22: 3II. I932, Exotic Microlepidoptera, 4: 330. (v) 1935, The Entomologist, 68: I2I. (BM) I937, in Caradja and Meyrick, Deuts. Ent. Zeit. Iris, 5I: I73. (BM) I904, Proc. Linnean Soc. N.S. Wales, 29:37I. I888, Trans. Proc. Nere Zealand Inst. 20: I03. I921, Ann. Transwaal Mus. 8: 89. 


\begin{tabular}{|c|c|c|}
\hline microphis & ACROCERCOPS & I921, Exotic Microlepidoptera, 2: 466. \\
\hline microphthalma & PETALOTHYRSA & I93I, Exotic Microlepidoptera, 4: I62. (вм) \\
\hline microphthora & ANCYLIS & I936, Exotic Microlepidoptera, 4: 609. (вм) \\
\hline ophylla & HELIOZELA & I897, Proc. Linnean Soc. N.S. Wales, 22: 403. \\
\hline opis & PHLOEOPOLA & I889, Proc. Linnean Soc. N.S. Wales, (Ser. 2) 3: I593. \\
\hline microplaca & ARGYROPLOCE & I9I2, Journ. Bombay Nat. Hist. Soc. 21 : 873. (вм) \\
\hline micropsycha & CTENOCOMPA & I9I9, Exotic Microlepidoptera, 2: 264 . \\
\hline coptila & TRITADELPHA* & I904, Proc. Linnean Soc. N.S. Wales, 29: 323. \\
\hline microrrhoda & THIOTRICHA & I935, Exotic Microlepidoptera, 4: 586. (BM) \\
\hline microrrhyncha & ACROCLITA & I93I, Exotic Microlepidoptera, 4: I27. (BM) \\
\hline microsacta & ACARTOPHILA* & I932, Exotic Microlepidoptera, 4: 282. (v) \\
\hline microschema & NEPHOGENES & I883, Proc. Linnean Soc. N.S. Wales, 8: 380. \\
\hline microsema & BRACHMIA & I9I I, Trans. Linnean Soc. London, I4: 274. (BM) \\
\hline microsperma & TELPHUSA & $\begin{array}{l}\text { I920, Voyage de Ch. Alluaud et } R \text {. Jeannel en Afrique } \\
\text { Oriental, } I I \text {, Microlepidoptera, p. } 69 . \text { (P) }\end{array}$ \\
\hline microsphena & DICHOMERIS & I92I, Zool. Meded. $6:$ I66. (L) \\
\hline ospora & TINEA & I893, Proc. Linnean Soc. N.S. Wales, $7: 545$. \\
\hline microsticha & LEPIDOSCIA & I893, Proc. Linnean Soc. N.S. Wales, 7: 510. \\
\hline ostictis & COESYRA & I902, Trans. R. Soc. S. Australia, 26: г39. \\
\hline microstictis & EPICHORISTA & I9Io, Proc. Linnean Soc. N.S. Wales, $35: 260$. \\
\hline microtima & EPIPHTHORA & I904, Proc. Linnean Soc. N.S. Wales, 29: 267. \\
\hline otoma & BATRACHEDRA & I897, Proc. Linnean Soc. N.S. Wales, $22: 303$. \\
\hline ipta & BACTRA & $\begin{array}{l}\text { I927, Insects of Samoa, 3, Lepidoptera, fasc. 2, p. } 75 \text {. } \\
\text { (BM) }\end{array}$ \\
\hline microtypa & STENOMA & I9I5, Exotic Microlepidoptera, I: 422. (BM) \\
\hline xantha & PHILOBOTA & I 889, Proc. Linnean Soc. N.S. Wales, (Ser. 2) 3: I6 I5. \\
\hline mictopsamma & BATRACHEDRA & I932, Exotic Microlepidoptera, 4: 3I6. \\
\hline milichia & OCYSTOLA & I885, Proc. Linnean Soc. N.S. Wales, 9: I07I. \\
\hline hodes & STENOMA & I9I5, Exotic Microlepidoptera, I: 448. (BM) \\
\hline milichopa & TINEA & I9II, Trans. Linnean Soc. London, I4: 304. \\
\hline milichopis & ARGYROPLOCE & I93I, Exotic Microlepidoptera, 4: I32. (BM) \\
\hline milictis & ANTAEOTRICHA & I925, Exotic Microlepidoptera, 3: I63. (вм) \\
\hline aris & HEMIMENE & I9I6, Exotic Microlepidoptera, 3: 28. (BM) \\
\hline militaris & HYPERCALLIA & I9I4, Exotic Microlepidoptera, I: I9I. (BM) \\
\hline milliaria & SIMAETHIS & I922, Exotic Microlepidoptera, 2: 486. (BM) \\
\hline miltochorda & ANTICRATES & I9I4, Journ. Bombay Nat. Hist. Soc. 23: І28. (Bм) \\
\hline miltocosma & POLEMOGRAPTIS* & I9Io, Trans. Ent. Soc. London, I9IO: 432. (BM) \\
\hline miltogramma & PYRETAULAX* & I92I, Zool. Meded. 6: I7o. (L) \\
\hline miltographa & EUCOSMA & I907, Journ. Bombay Nat. Hist. Soc. I8: I39. (вм) \\
\hline miltopa & COPTOTELIA & I9I2, Trans. Ent. Soc. London, I9I I: 700. (BM) \\
\hline ragma & $\begin{array}{l}\text { DICHOMERIS } \\
{[\text { [CYMOTRICHA } *]}\end{array}$ & rans. Ent. Soc. London, I922: II5. (BM) \\
\hline miltospila & METACHANDA & I9II, Trans. Linnean Soc. London, I4: 279. (BMI) \\
\hline milvina & PROCOMETIS & I9I4, Ann. Transvaal Mus. 4: I99. (T) \\
\hline mimantha & STATHMOPODA & I9I3, Exotic Microlepidoptera, I: 92. \\
\hline mimetis & COSMOPTERYX & I897, Proc. Linnean Soc. N.S. Wales, 22: 339. \\
\hline mimica & HELIOCAUSTA & I888, Proc. Linnean Soc. N.S. Wales, (Ser. 2) 2: 934. \\
\hline mimobathra & ATELOSTICHA & I920, Exotic Microlepidoptera, 2: 379. (вм) \\
\hline charis & APREPODOXA* & $\begin{array}{l}\text { I937, in Caradja and Meyrick, Deuts. Ent. Zeit. Iris, }{ }^{\mathrm{I}} \text { : } \\
\text { I7I. (BM) }\end{array}$ \\
\hline
\end{tabular}




\begin{tabular}{|c|c|c|}
\hline mimochora & PERSICOPTILA & I897, Proc. Linnean Soc. N.S. Wales, 22: 330. \\
\hline mimodes & CARPOSINA & I9I0, Proc. Linnean Soc. N.S. Wales, 35: I49. \\
\hline mimodora & THUDACA & I893, Proc. Linnean Soc. N.S. Wales, $7: 574$. \\
\hline mimopa & COESYRA & I902, Trans. R. Soc. S. Australia, 26: I39. \\
\hline minans & PERITORNEUTA & I92I, Exotic Microlepidoptera, 2: 39I. \\
\hline minatrix & IMMA & I906, Trans. Ent. Soc. London, I906: I9I. (Bм) \\
\hline $\operatorname{minax}$ & AGRIOPHARA & I907, Journ. Bombay Nat. Hist. Soc. I8: I52. (Bм) \\
\hline minerva & CRYPTOLECHIA & I928, Exotic Microlepidoptera, $3: 477$. (BM) \\
\hline miniana & HAEMOLYTIS* & I926, Exotic Microlepidoptera, 3: 246. \\
\hline minima & BACTRA & I9o9, Journ. Bombay Nat. Hist. Soc. I9: 586 . \\
\hline ministra & SIMAETHIS & I9I2, Trans. Proc. New Zealand Inst. 44: I2I. \\
\hline minyra & CITHARODICA* & I9I4, Exotic Microlepidoptera, I: 22I. (MA) \\
\hline miocentra & MICTOPSICHIA & I920, Exotic Microlepidoptera, 2: 330. (вм) \\
\hline mioclina & CEROMITIA & I921, Ann. Transvaal Mus. 8: I38. (т) \\
\hline miraculosa & $\begin{array}{l}\text { OLINDIA } \\
{[\text { OCHETARCHA } *]}\end{array}$ & I9I7, Trans. Proc. New Zealand Inst. 49: 246. \\
\hline miranda & LASPEYRESIA & I9II, Trans. Linnean Soc. London, I4: 27I. (BM) \\
\hline miranda & $\begin{array}{l}\text { MELODRYAS } \\
\text { [GYMNOGELASTIS* }]\end{array}$ & ] I9I3, Exotic Microlepidoptera, I: I42. \\
\hline mirifica & MORPHOTICA $*$ & I9I5, Exotic Microlepidoptera, I: 297. \\
\hline miseranda & PSEUDODOXIA & I923, Exotic Microlepidoptera, 3: 62. (Bм) \\
\hline mistharna & ODITES & I908, Journ. Bombay Nat. Hist. Soc. I8: 634. (BM) \\
\hline misthota & OCYSTOLA & I902, Trans. R. Soc. S. Australia, 26: I35. \\
\hline mitescens & ANARSIA & I9I3, Ann. Transvaal Mus. 3: 299. (T) \\
\hline mitescens & MACHIMIA & I9I4, Exotic Microlepidoptera, I: I74. \\
\hline mitigata & PACHNISTIS & I9I4, Exotic Microlepidoptera, I: 278. (BM) \\
\hline mitis & CRYPTOLECHIA & I9I4, Supplementa Entomologica, No. 3, p. 52. (D) \\
\hline mitis & HYPERCALLIA & I9I4, Exotic Microlepidoptera, I: Igo. (BM) \\
\hline mitrata & CEROMITIA & I9I7, Ann. South African Mus. I7: I7. (SA) \\
\hline mitrodeta & IMMA & I922, Exotic Microlepidoptera, 2: 482 . (Bм) \\
\hline mitrota & TORTRIX & $\begin{array}{l}\text { I920, Voyage de Ch. Alluaud et } R \text {. Jeannel en Afrique } \\
\text { Oriental, II, Microlepidoptera, p. } 5^{\circ} \text {. (P) }\end{array}$ \\
\hline mixadelpha & PELOMIMAS* & I9I4, Exotic Microlepidoptera, I: I86. (BM) \\
\hline mixanthes & ARGYROPLOCE & 1932, Exotic Microlepidoptera, 4: 309. (Bм) \\
\hline mixaula & SCYTHRIS & I9I6, Exotic Microlepidoptera, 2: I4. (BM) \\
\hline mixochlora & LYSIPHRAGMA* & I888, Trans. Proc. New Zealand Inst. 20: I05. \\
\hline mixogama & TONICA & I928, Exotic Microlepidoptera, 3: 474. (BM) \\
\hline mixographa & LASPEYRESIA & I939, Trans. R. Ent. Soc. London, 89: 5I. (c) \\
\hline mixolitha & PHTHORIMAEA & I9I8, Exotic Microlepidoptera, 2: I35. (BM) \\
\hline mixophanes & LITHOPSAESTIS* & I932, Exotic Microlepidoptera, 4:330. (v) \\
\hline mixoscia & NARYCIA & I922, Exotic Microlepidoptera, 2: 604. \\
\hline mixotypa & ACROLEPIA & $\begin{array}{l}\text { I931, Ann. Mus. Nac. Hist. Nat. Buenos Aires, 36: } 403 . \\
\text { (BM) }\end{array}$ \\
\hline mnemonica & PERIACMA (?) & I9Io, Rec. Indian Mus. 5:224. (K) \\
\hline mnemosynana & CACOECIA & I881, Proc. Linnean Soc. N.S. Wales, 6: 504. \\
\hline mnesicala & GRACILARIA & I881, Proc. Limnean Soc. N.S. Wales, 5: I56. \\
\hline mnesicentra & XYROSARIS & I913, Exotic Microlepidoptera, I: І 36. (вм) \\
\hline mnesicosma & ETHMIA & I924, Exotic Microlepidoptera, 3: II9. (BM) \\
\hline mnesileuca & BEDELLIA & I928, Exotic Microlepidoptera, 3: 397. \\
\hline mniochlora & EUCOSMA & I907, Journ. Bombay Nat. Hist. Soc. I8: I38. (вм) \\
\hline
\end{tabular}




$\begin{array}{ll}\text { mniocosma } & \text { COMPSOLECHIA } \\ \text { mniodora } & \text { STENOMA } \\ \text { mniograpta } & \text { IMMA } \\ \text { mniopyrrha } & \text { ARGYROPLOCE } \\ \text { mobilis } & \text { EULECHRIA } \\ \text { mochlacma } & \text { MICRERETHISTA* } \\ \text { mochlaspis } & \text { ARGYROPLOCE } \\ \text { mochlastis } & \text { EULECHRIA } \\ \text { mochlias } & \text { LICHENAULA } \\ \text { mochlocrossa } & \text { HYPONOMEUTA }\end{array}$

I922, Trans. Ent. Soc. London, I922: 89. (BM)

1925, Exotic Microlepidoptera, 3: 204. (BM)

I93I, Exotic Microlepidoptera, 4: I8I. (BM)

I93I, Exotic Microlepidoptera, 4: 134. (BM)

I9I5, Exotic Microlepidoptera, I: 300.

I938, Trans. R. Ent. Soc. London, 87: 527.

I921, Ann. Transvaal Mus. 8: 58. (T)

I888, Proc. Linnean Soc. N.S. Wales, (Ser. 2) 2: 960.

I89o, Trans. R. Soc. S. Australia, I3: 52.

I935, Exotic Microlepidoptera, 4:602. (BM)

mochlodroma

SPHAERICOBATHRA* I933, Exotic Microlepidoptera, 4: 4I4.

mochlopa

AGRIOPHARA

mochlophanes PHYCODES

mochlophorana EXORIA*

mochlopis CYMOTRICHA

mochlota TINEA

modica

LASPEYRESIA

modicola

STENOMA

modularis

CRYPTOLECHIA

STENOMA

modulata

ARISTOTELIA

modulatrix

9I5, Exotic Microlepidoptera, I: 409. (BM)

I92I, Ann. Transvaal Mus. 8: I Iо. (T)

I883, Trans. Proc. New Zealand Inst. I5: 65 .

I923, Exotic Microlepidoptera, 3: I. (BM)

I888, Trans. Proc. New Zealand Inst. 20: roo.

I9I3, Ann. Transvaal Mus. 3:280.

I9II, Ann. Transwaal Mus. 3: 75 .

I921, Zool. Meded. 6: 172. (L)

I9I5, Exotic Microlepidoptera, I: 436. (BM)

1938, Institut des Parcs Nationaux du Congo Belge,

fasc. I4, p. II.

I92I, Ann. Transvaal Mus. 8: I20. (T)

I9ro, Proc. Linnean Soc. N.S. Wales, 35: 222.

I9I5, Exotic Microlepidoptera, I: 327.

I9I4, Exotic Microlepidoptera, I: I82. (BM)

I9I4, Exotic Microlepidoptera, I : $22 \mathrm{I}$.

1925, Exotic Microlepidoptera, 3: I45.

I927, Exotic Microlepidoptera, 3: 373. (вм)

I93I, Exotic Microlepidoptera, 4: I47. (BM)

I906, Journ. Bombay Nat. Hist. Soc. I7: I35.

I930, Exotic Microlepidoptera, 3: 603. (BM)

I9ro, Proc. Linnean Soc. N.S. Wales, 35:288. (мA)

SCOLIOPLECTA

TINGENTERA

[ALCIPHANES*]

molybdanthes

PHTHEOCHROA

molybdaspis

molybdastra

molybdaula

molybdela

molybdias

PSITTACASTIS

GLYPHIPTERYX

PYRODERCES

DIOCOSMA

ONEBALA

[THYMBRITIS*]

molybdis

OPOGONA

molybditis

TORTRIX

molybdopa

molybdora

molybdota

SIDEROGRAPTIS

GLYPHIPTERYX

ANARSIA

CYMOTRICHA

molybdoterma

molynta

DECADARCHIS

molyntis

PROTOLECHIA

Igo8, Journ. Bombay Nat. Hist. Soc. 10: 454. (BM)

1932, Exotic Microlepidoptera, 4: 266. (v)

I926, Exotic Microlepidoptera, 3: 316. (BM)

I923, Bull. Mus. Nat. Hist. Nat. Paris, 29: 564.

I9I5, Exotic Microlepidoptera, I: 309.

I917, Ann. South African Mus. I7: 8. (sA)

I9Io, Journ. Bombay Nat. Hist. Soc. 20: 456 . (Вм)

I9I5, Exotic Microlepidoptera, I: 360.

I907, Trans. Proc. New Zealand Inst. 39: II5.

I920, Exotic Microlepidoptera, 2: 380. (BM)

I9I2, Exotic Microlepidoptera, I: 57 . (BM)

I904, Proc. Limnean Soc. N.S. Wales, 29: 4 I7.

I933, Exotic Microlepidopter., 4: 353. (BM)

I9Ir, Trans. Linnean Soc. London, I4: 300.

I904, Proc. Linnean Soc. N.S. Wales, 29: 385. 


$\begin{array}{ll}\text { monachopis } & \text { LITHOPLEUROTA* } \\ \text { monactis } & \text { ARISTOTELIA } \\ \text { monastica } & \text { IMMA } \\ \text { monastra } & \text { DECADARCHIS } \\ \text { monastra } & \text { STENOMA } \\ \text { monelpis } & \text { BUCCULATRIX } \\ \text { monerythra } & \text { ATTEVA } \\ \text { moniliata } & \text { EULIA }\end{array}$

moniligera

monitrix

monobathra

monobyrsa

monocalama

monocentra

monocentra

monochlora

monochorda

monoclina

monococca

monocolona

monoctenis

monodecta

monodesma

monodonta

monodoxa

monodryas

monodyas

monogama

monoglossa

monogona

monogramma

monolitha

monoloncha

monolychna

monopa

monophaea

monophaes

monophragma

monophthalma

monoplanetis

monoplecta

monosaris

monosema

monosperma

monospila

monospora

monostadia

monosticta

\section{CHOREUTIS}

EUCOSMA

STATHMOPODA

LECITHOCERA

PROCOMETIS

CYCLOTORNA*

ISCHNOPHANES*

PLUTELLA

PSALTICA*

MACROBATHRA

TRICHOTAPHE

ANTAEOTRICHA

ACROLOPHUS

ACROCERCOPS

CROCANTHES

CREMNOGENES

EPIMACTIS*

PACHNISTIS

MICROBELA

AEGERIA

PROTOTHEORA

ODITES

PHILOBOTA

PHILOBOTA

PHILOBOTA

BRENTHIA

GELECHIA

ERETMOCERA

PHILOBOTA

GELECHIA

TINEA

ATTEVA

ACOROSTOMA

STENOMA

HOPLOPHANES

OPOSTEGA

TINEA

ARGYROPLOCE

MACROBATHRA

HIEROXESTIS
I939, Trans. R. Ent. Soc. London, 89: 6I. (T)

I923, Exotic Microlepidoptera, 3: Io. (BM)

I9Io, Trans. Ent. Soc. London, I9Io: 465. (BM)

I89I, Trans. Proc. New Zealand Inst. 23: Ioo.

Igo9, Trans. Ent. Soc. London, I909: 33. (BM)

I928, Exotic Microlepidoptera, 3: 398. (T)

I926, Trans. Ent. Soc. London, 74:278. (BM)

I936, Veröff. Deutschen Kol.-Mus. I: 330, pl. I3, fig. 20.

(H)

I9I3, Exotic Microlepidoptera, I: 54. (BM)

I9o9, Ann. Transvaal Mus. 2: 7, pl. 3, fig. 2. (T)

I937, Exotic Microlepidoptera, 5: I50.

I93I, Exotic Microlepidoptera, 4: 80. (BM)

I890, Trans. R. Soc. S. Australia, I3: 72.

I907, Proc. Linnean Soc. N.S. Wales, 32: 72.

I89I, Ent. Mon. Mag. 27: 60.

I9I4, Exotic Microlepidoptera, I: 228.

I905, Journ. Bombay Nat. Hist. Soc. I6: 604. (BM)

I9I5, Exotic Microlepidoptera, I: 294. (BM)

I92I, Ann. Transvaal Mus. 8: 85. (T)

I932, Exotic Microlepidoptera, 4: 293. (US)

I93I, Journ. Linnean Soc. London, 37:284.

I9I2, Exotic Microlepidoptera, I: 23. (T)

I93I, Exotic Microlepidoptera, 4: 73. (BM)

I9II, Trans. New Zealand Inst. 43: 75 .

I907, Journ. Bombay Nat. Hist. Soc. I7: 74I. (Lost)

I9I4, Journ. Bombay Nat. Hist. Soc. 22: 773. (BM)

I885, Proc. Linnean Soc. N.S. Wales, 9: 1048.

I932, Exotic Microlepidoptera, 4: 337.

1924, Exotic Microlepidoptera, 3: 80. (T)

I938, Institut des Parcs Nationaux du Congo Belge,

fasc. I4, p. 2 I, pl. 2, fig. 8.

I884, Proc. Linnean Soc. N.S. Wales, 8: 493.

I884, Proc. Linnean Soc. N.S. Wales, 8: 477.

I889, Proc. Linnean Soc. N.S. Wales, (Ser. 2) 3: I607.

I9I5, Trans. Ent. Soc. London, I9I5: 2I8. (BM)

I927, Exotic Microlepidoptera, 3: 350. (BM)

I927, Exotic Microlepidoptera, 3: 38o. (T)

I884, Proc. Linnean Soc. N.S. Wales, 8: 504.

I886, Trans. Proc. New Zealand Inst. I8: I69.

I893, Proc. Linnean Soc. N.S. Wales, 7: 543.

I9Io, Trans. Ent. Soc. London, I9I0: 470.

I92I, Ann. Transvaal Mus. 8: I32. (T)

I9I5, Exotic Microlepidoptera, I: 4I9. (BM)

I897, Proc. Linnean Soc. N.S. Wales, 22: 4I2.

I93I, Exotic Microlepidoptera, 4: I62. (v)

I929, Trans. Ent. Soc. London, 76:507.

I939, Trans. R. Ent. Soc. London, 89:50. (c)

I886, Proc. Linnean Soc. N.S. Wales, Io: 803.

I9I5, Exotic Microlepidoptera, I: 362. 


\begin{tabular}{|c|c|c|}
\hline pha & OCYSTOLA & I885, Proc. Linnean Soc. N.S. Wales, 9: Io75. \\
\hline monotyla & CHRYSOCLISTA & I92I, Exotic Microlepidoptera, 2: 4I3. (BM) \\
\hline monoxantha & MACROBATHRA & I922, Exotic Microlepidoptera, 2: 509. \\
\hline onoxesta & ASYMPHORODES & I929, Trans. Ent. Soc. London, 76: 50I. \\
\hline monozona & LINOSTICHA & I889, Proc. Linnean Soc. N.S. Wales, (Ser. 2) 3: I582. \\
\hline monozona & TINEA & I893, Proc. Linnean Soc. N.S. Wales, 7: 533 . \\
\hline onstruosa & DECADARCHIS & I930, Exotic Microlepidoptera, 4: 9 . \\
\hline ontanana & CAPUA & I88I, Proc. Linnean Soc. N.S. Wales, 6: 45I. \\
\hline montezuma & TINEA & I927, Exotic Microlepidoptera, 3: 323. (BM) \\
\hline montigena & PSEUDODOXIA & I9I4, Exotic Microlepidoptera, I: 273. (BM) \\
\hline ontivagana & DICHELIA & I88I, Proc. Linnean Soc. N.S. Wales, 6: 477. \\
\hline Iontivola & ADAINA & $\begin{array}{l}\text { I937, in Caradja and Meyrick, Deuts. Ent. Zeit. Iris, 5I: } \\
\text { I70. }\end{array}$ \\
\hline contivola & DURRANTIA & I927, Exotic Microlepidoptera, 3: 364. (BM) \\
\hline norata & MACHIMIA & I9I2, Trans. Ent. Soc. London, I9I I: 697. (BM) \\
\hline norbida & CARPOSINA & I912, Trans. Proc. New Zealand Inst. 44: I20. \\
\hline norbida & MELASINA & I908, Proc. Zool. Soc. London, p. 744. \\
\hline morbidula & ACROLOPHUS & I931, Exotic Microlepidoptera, 4: Io2. (v) \\
\hline mordax & PHALONIA & I9I7, Trans. Ent. Soc. London, I9I7: 4. (BM) \\
\hline moretonella & ARDIOSTERES & $\begin{array}{l}\text { I893 [Meyr. nec Walk.], Proc. Linnean Soc. N.S. Waies, } \\
\text { 7: 5I9. }\end{array}$ \\
\hline lodes & METACHANDA & I9I I, Trans. Linnean Soc. London, I4: 277. (BM) \\
\hline mormopa & IMMA & I9Io, Trans. Ent. Soc. London, I9Io: 467. (BM) \\
\hline normopa & PLATYPEPLUS & I9o6, Journ. Bombay Nat. Hist. Soc. I7: г36. (BM) \\
\hline mormopica & NEELYSIA & 1935, Proc. Hawaiian Ent. Soc. 9: 64. (SP) \\
\hline mormopis & CROTHAEMA & I934, Exotic Microlepidoptera, 4: 528 . \\
\hline mormopis & TRICHOPHAGA & 1935, Exotic Microlepidoptera, 4: 575. (c) \\
\hline morologa & PACHNISTIS. & 1923, Bull. Mus. Nat. Hist. Nat. Paris, 29: 564 . \\
\hline morophaea & PLATYPTILIA & $\begin{array}{l}\text { I920, Voyage de Ch. Alluaud et } R \text {. Jeannel en Afrique } \\
\text { Oriental, II, Microlepidoptera, p. } 38 \text {. (P) }\end{array}$ \\
\hline morosa & SPILONOTA & I9II, Proc. Linnean Soc. N.S. Wales, 36: 232. \\
\hline mosaica & EUCOSMA & I9o7, Journ. Bombay Nat. Hist. Soc. I8: I38. \\
\hline moschias & TINEOLA & I9I4, Exotic Microlepidoptera, I: 2 Io. \\
\hline mucronata & ZANCLOPSEUSTIS* & I921, Zool. Meded. 6: I97. (L) \\
\hline mulciber & ORNEODES & I932, Exotic Microlepidoptera, 4: 333. (v) \\
\hline muliebris & EUCOSMA & I922, Exotic Microlepidoptera, 2: 5I5. (P) \\
\hline multifida & POLYHYMNO & I9I7, Ann. South African Mus. I7: 4. (SA) \\
\hline multiformis & ARISTOTELIA & 1928, Proc. Hawaiian Ent. Soc. 7: IOI. (SP) \\
\hline multifurcata & EULIA & I932, Exotic Microlepidoptera, 4:260. (v) \\
\hline multinotata & GELECHIA & I9I8, Exotic Microlepidoptera, 2: I34. (BM) \\
\hline multiplex & ACROCLITA & I912, Journ. Bombay Nat. Hist. Soc. 21: 86o. (BM) \\
\hline multiplex & MELASINA & I9I7, Exotic Microlepidoptera, $2: 94$. \\
\hline munda & HYPONOMEUTA & I92I, Exotic Microlepidoptera, 2: 438 . (BM) \\
\hline & $\begin{array}{l}\text { CHELARIA } \\
\text { [HAPLOCHEL }\end{array}$ & , \\
\hline data & NOTHRIS & I929, Exotic Microlepidoptera, 3: 495. (BM) \\
\hline undula & STENOMA & I9I6, Exotic Microlepidoptera, I: 528. (BM) \\
\hline nerata & HIEROMANTIS & I924, Exotic Microlepidoptera, 3: 66. \\
\hline nicipalis & CRYPTOLECHIA & I920, Exotic Microlepidoptera, 2: 3I6. (B.I) \\
\hline unita & TINEA & I932, Trans. Proc. New Zealand Inst. 63: 24. \\
\hline
\end{tabular}




\begin{tabular}{|c|c|c|}
\hline iula & CHOROPLECA & I924, Exotic Microlepidoptera, 3: 65. \\
\hline muricata & TINEA & I893, Proc. Linnean Soc. N.S. Wales, $7: 542$. \\
\hline murifica & MELASINA & r922, Exotic Microlepidoptera, 2: 606. (P) \\
\hline murmurans & DEPRESSARIA & I927, Exotic Microlepidoptera, 3: 382. (BM) \\
\hline murmurata & HELENODES* & I9I3, Exotic Microlepidoptera, I: I5I. \\
\hline musaea & TRACHYDORA & I897, Proc. Linnean Soc. N.S. Wales, 22: 398. \\
\hline muscicolor & AGRIOPHARA & I930, Exotic Microlepidoptera, 3: 560. \\
\hline muscosa & EUCOSMA & I927, Exotic Microlepidoptera, 3: 335. (BM) \\
\hline usica & LICHENAULA & I89o, Trans. R. Soc. S. Australia, I3: 5I. \\
\hline musicodes & LINOCLOSTIS & I9Io, Trans. Ent. Soc. London, I9Io: $45^{8 .}$ \\
\hline usicodora & MONACHOPTILAS & I934, Exotic Microlepidoptera, 4: 482 . \\
\hline usicopa & BRACHMIA & I9o8, Proc. Zool. Soc. London, p. 727. \\
\hline musicosema & SIMAETHIS & I926, Exotic Microlepidoptera, 3: 305. (Lost) \\
\hline musochares & ARGYROPLOCE & I925, in Caradja, Bull. Sect. sci. Acad. roum. 3: 379. \\
\hline usota & MOMPHA & I9I7, Exotic Microlepidoptera, 2: 52 . (BM) \\
\hline mutabilis & DISSOPTILA* & I9I4, Trans. Ent. Soc. London, I9I4: 235. (вм) \\
\hline myadelpha & ONEBALA & I9Io, Journ. Bombay Nat. Hist. Soc. 20: 459. (BM) \\
\hline mycetinopa & CHELARIA & I934, Exotic Microlepidoptera, 4: 45I. (BM) \\
\hline mychias & PELTOPHORA & I889, Proc. Linnean Soc. N.S. Wales, (Ser. 2) 3: 1639. \\
\hline mycites & OXYPTILUS & I9I4, Supplementa Entomologica, No. 3, p. 46. (D) \\
\hline mydopis & MACHIMIA & I9I4, Exotic Microlepidoptera, I: I79. (BM) \\
\hline myelopis & TINEA & I927, Exotic Microlepidoptera, 3: 322. (T) \\
\hline myeloxesta & HOMALOXESTIS & I932, Exotic Microlepidoptera, 4: 203. (BM) \\
\hline mylephata & BATRACHEDRA & I897, Proc. Linnean Soc. N.S. Wales, 22: 304. \\
\hline mylias & IMMA & I906, Trans. Ent. Soc. London, I906: I73. (BM) \\
\hline mylica & MELASINA & I9o8, Proc. Zool. Soc. London, p. 748 . \\
\hline mylicella & THALEROTRICHA* & I884, Proc. Linnean Soc. N.S. Wales, 9:74I. \\
\hline mylicopa & ZYMOLOGA* & I9I9, Exotic Microlepidoptera, 2: 275. (BM) \\
\hline mylicotis & NOTHRIS & I904, Proc. Linnean Soc. N.S. Wales, 29: 426. \\
\hline mylitis & ACRIDOTARSA* & I893, Proc. Linnean Soc. N.S. Wales, 7: 5I7. \\
\hline myllerana & CHOREUTIS & Igo3, Ent. Mon. Mag. 39: 5. (BM) \\
\hline myloclasta & MONOPIS & I927, Exotic Microlepidoptera, 3: 32I. (Sub.) \\
\hline mylothris & PHILOBOTA & I921, Exotic Microlepidoptera, 2: 385 . \\
\hline myodes & HOPLITICA & I883, Proc. Linnean Soc. N.S. Wales, 7: 496. \\
\hline myopa & LECITHOCERA & I9I3, Ann. Transvaal Mus. 3: 294. (T) \\
\hline myriastra & ATTEVA & I907, Proc. Linnean Soc. N.S. Wales, $32: 82$. \\
\hline myrinitis & PHALONIA & I9I2, Ent. Mon. Mag. 48: 35. (Sub.) \\
\hline myriocosma & PSEUDATTERIA & I930, Exotic Microlepidoptera, 3: 608. (вм) \\
\hline myriodesma & ORNEODES & I929, Exotic Microlepidoptera, 3: 533 . \\
\hline myriogramma & ACROCERCOPS & I937, Exotic Microlepidoptera, 5: III. \\
\hline myriopa & CERACE & I922, Exotic Microlepidoptera, $2: 497$. (P) \\
\hline myriophthalma & MACROBATHRA & I886, Proc. Linnean Soc. N.S. Wales, Io: 822 . \\
\hline myriopleura & CTENOCOMPA & I931, Exotic Microlepidoptera, 4: 99. \\
\hline myriosema & HOMONA & I936, Exotic Microlepidoptera, 5: 60. (c) \\
\hline myriospila & HOMADAULA* & I907, Proc. Limean Soc. N.S. Wales, $32: 73$. \\
\hline myrmecias & COPROMORPHA & I930, Exotic Microlepidoptera, 3: 590. (BM) \\
\hline myrochrista & EULECHRIA & I920, Exotic Microlepidoptera, 2: 37I. \\
\hline myrochroa & STENOMA & I9I5, Exotic Microlepidoptera, I: 448. (BM) \\
\hline myrocoma & MACROBATHRA & I9I4, Exotic Microlepidoptera, I: 2I8. (BM) \\
\hline myrodora & STENOMA & 1925, Exotic Microlepidoptera, 3: I97. (BM) \\
\hline
\end{tabular}




myronota
myrota
myroxesta
myrrhicoma
myrrhina
myrrhinopa
myrrhophanes
mystarcha
mysteriastis
mysteriodes

mysteris

mystica

mystica

mysticopa

mysticopis

mystis

mystodoxa

mystolitha

mythica

mythologica

myura

myxodes

$\begin{array}{ll}\text { naevana } & \text { ACROCLITA } \\ \text { naiadopa } & \text { ADAINA } \\ \text { naias } & \text { MACHAERITIS } \\ \text { nanodes } & \text { AGDISTIS } \\ \text { napaea } & \text { DRYADAULA } \\ \text { napaea } & \text { PERONEA } \\ \text { narcodes } & \text { COPROMORPHA } \\ \text { narcota } & \text { STAGMATOPHORA } \\ \text { nardinopa } & \text { LECITHOCERA } \\ \text { nasuta } & \text { EUCOSMA } \\ \text { nauarches } & \text { PTEROPHORUS } \\ \text { naufraga } & \text { GELECHIA } \\ \text { naulychna } & \text { AUTOSTICHA } \\ \text { nausicaa } & \text { PTILOTHYRIS } \\ \text { nautica } & \text { AEGERIA } \\ \text { navalis } & \text { PROTEOPTERYX } \\ \text { navicularis } & \text { STENOMA } \\ \text { navigatrix } & \text { XYLORYCTA } \\ \text { naxia } & \text { ARISTOTELIA } \\ \text { neaera } & \text { ACROCLITA } \\ \text { nearcha } & \text { ELACHISTA } \\ \text { neastra } & \text { STENOMA } \\ \text { neatodes } & \text { DICHOMERIS } \\ & \end{array}$

STATHMOPODA

STRUTHISCA

COSMOPTERYX

CROTHAEMA

[PRODIDACTIS*]

TINEA

PTILOGENES

SEMIOCOSMA

HYPOSMOCOMA

CAMINOPHANTIS*

MAROGA

[ECHIOMIMA*]

ANATRACHYNTIS

LYONETIA

OPOSTEGA
I929, Trans. Ent. Soc. London, 76: 500.

I9I2, Trans. Ent. Soc. London, I9II: 686. (Bм)

I924, Exotic Microlepidoptera, 3: II4. (T)

I9I7, Exotic Microlepidoptera, 2: 40.

I905, Trans. Ent. Soc. London, I905: 243.

I932, Exotic Microlepidoptera, 4: 300. (BM)

I93I, in Caradja, Bull. Sect. sci. Acad. roum. I4: 63.

I893, Proc. Linnean Soc. N.S. Wales, 7: 586.

I902, Trans. Ent. Soc. London, I902: 575 .

thalassonympha* I93I, Ann. Mus. Nac. Hist. Nat. Buenos Aires, 36:405.

(BM)

I907, Journ. Bombay Nat. Hist. Soc. I8: I54.

I897, Proc. Linnean Soc. N.S. Wales, 22: 338.

I9I8, Exotic Microlepidoptera, 2: I63.

I9I4, Trans. Proc. New Zealand Inst. 46: II5.

I926, Exotic Microlepidoptera, 3: 233. (BM)

I888, Trans. Proc. New Zealand Inst. 20: 79.

I9I5, Exotic Microlepidoptera, I: 344. (BM)

I933, Exotic Microlepidoptera, 4:373.

I89o, Trans. R. Soc. S. Australia, I3:4I.

I9I7, Exotic Microlepidoptera, 2: 45.

I93I, Exotic Microlepidoptera, 4: I63.

I9I6, Exotic Microlepidoptera, I: 6I9.

I9I2, Journ. Bombay Nat. Hist. Soc. 2I : 857.

I93I, Journ. Linnean Soc. London, 37: 277.

I902, Trans. R. Soc. S. Australia, 26: I34.

I9o6, Journ. Bombay Nat. Hist. Soc. I7: I36.

I905, Trans. Ent. Soc. London, I905: 244.

I9I2, Exotic Microlepidoptera, I: I8. (BM)

I9I6, Exotic Microlepidoptera, I: 556 .

I9o9, Ann. Transvaal Mus. 2: I9, pl. 6, fig. 5. (T)

I9I8, Exotic Microlepidoptera, 2: 103. (BM)

I9I2, Ann. Transvaal Mus. 3:63. (T)

I930, Exotic Microlepidoptera, 3: 569. (BM)

I9I2, Ann. Transvaal Mus. 3:66. (T)

I go8, Journ. Bombay Nat. Hist. Soc. I8: 456. (вм)

I926, Exotic Microlepidoptera, 3: 288.

I932, Exotic Microlepidoptera, 4: 229.

I9I2, Ent. Mon. Mag. 48: 34. (Sub.)

I930, Exotic Microlepidoptera, 4: 24. (v)

I9I2, Trans. Ent. Soc. London, I9II: 705. (BM)

I926, Trans. Ent. Soc. London, 74: 277.

I9I2, Journ. Bombay Nat. Hist. Soc. 2 I : 859. (BM)

I9I0, Rec. Indian Mus. 2: 225. (K)

I9I5, Exotic Microlepidoptera, I: 4I2. (BM)

I923, Exotic Microlepidoptera, 3: 35. (BM) 
nebras

nebrias

nebritis

nebrodes

nebropa

nebularis

nebulosa

necromantis

necromantis

nectarea

nectaritis

nectaritis

nectarodes

nectaropa

nefanda

nefasta

negotiosa

negotiosus

nematodes

nemeseta

neochalca

neochlora

neochorda

neocompsa

neocosma

neocrates

neocrossa

neodesma

neodora

neodoxa

neograpta

neolecta

neoleuca

neolopha

neolopha

neomeris

neopercna

neoptila

neoris

neospila

neosticta

neotricha

neoxanta

neoxesta

neozona

nephalia

nephallactis

nephanthes

nephaula

nephelaegis
MACHLOTICA

PROMALACTIS

EULECHRIA

ADOXOPHYES

ACROCERCOPS

LOZOSTOMA

BUCCULATRIX

SEMNOCOSMA*

TELPHUSA

TINEA

MELASINA

PERONEA

ARGYROPLOCE

ODITES

SPHYRELATA

LECITHOCERA

STENOMA

TRICHOPTILUS

CAPUA

SPANIOPTILA

EPHELICTIS*

HOPLITICA

GLYPHIPTERYX

SYNTETRERNIS

CRYPSICHARIS*

CRYPTOPHASA

STENOMA

COSMOPTERYX

NEPTICULA

LITHOCOLLETIS

PARAPSECTRIS

PHOLEUTIS*

MONACHOZELA*

ARGYROPLOCE

EULIA

STATHMOPODA

STENOMA

STENOMA

EPIRRHOECA*

MACHIMIA

LECITHOCERA

HEMIMENE

AGRIOPHARA

DEPRESSARIA

EXINOTIS

HIEROXESTIS

IMMA

NOTHRIS

TORTRIX

PLUTELLA
I909, Trans. Ent. Soc. London, I909: 37. (BM)

I908, Rec. Indian Mus. 2: 397. (K)

I9I4, Exotic Microlepidoptera, I: I62. (MA)

I920, Exotic Microlepidoptera, 2: 339.

I927, Insects of Samoa, 3, Lepidoptera, fasc. 2, p. Io7.

I897, Proc. Linnean Soc. N.S. Wales, 22: 420.

I9I5, Trans. Ent. Soc. London, I9I5: 249.

I924, Trans. Ent. Soc. London, I923: 549. (BM)

I932, Exotic Microlepidoptera, 4: I94.

I893, Proc. Linnean Soc. N.S. Wales, 7: 546.

I9I5, Exotic Microlepidoptera, I: 290.

I9I2, Exotic Microlepidoptera, I: I8. (BM)

I92I, Ann. Transvaal Mus. 8: 6I. (T)

I9I4, Exotic Microlepidoptera, I: 282.

I9I4, Exotic Microlepidoptera, I: I7I.

I9I6, Exotic Microlepidoptera, I: 575.

I925, Exotic Microlepidoptera, 3: 201. (BM)

1926, Ann. South African Mus. 23: 325. (SA)

I928, Exotic Microlepidoptera, 3: 452. (BM)

I920, Exotic Microlepidoptera, 2: 290.

I904, Proc. Linnean Soc. N.S. Wales, 29: 388.

I883, Proc. Linnean Soc. N.S. Wales, 7: 495.

I922, Exotic Microlepidoptera, 2: 493. (BM)

I933, Exotic Microlepidoptera, 4: 428. (BM)

I89o, Trans. R. Soc. S. Australia, I3: 45.

I925, Exotic Microlepidoptera, 3: I46.

I925, Exotic Microlepidoptera, 3: I93. (BM)

I9I5, Exotic Microlepidoptera, I: 323.

I9I8, Exotic Microlepidoptera, 2: I82.

I9I6, Exotic Microlepidoptera, I: 62I.

I9I4, Ann. Transvaal Mus. 4: I92. (T)

I906, Trans. R. Soc. S. Australia, 30:50.

I93I, Exotic Microlepidoptera, 4: I85.

I93I, Exotic Microlepidoptera, 4: I3I.

I926, Exotic Microlepidoptera, 3: 259. (BM)

I9I3, Exotic Microlepidoptera, I: 88.

I930, Ann. Naturhist. Mus. Wien, 44:245. (v)

I925, Exotic Microlepidoptera, 3: I95. (BM)

I9II, Proc. Linnean Soc. N.S. Wales, 36: 293.

I928, Exotic Microlepidoptera, 3:47I. (BM)

I9I8, Exotic Microlepidoptera, 2: I07. (BM)

I922, Exotic Microlepidoptera, 2: 527. (BM)

I9I5, Exotic Microlepidoptera, I: 4IO.

I9I8, Ann. Transvaal Mus. 6: 3I. (T)

I9I8, Exotic Microlepidoptera, 2: I59. (BM)

I9II, Trans. Linnean Soc. London, I4: 297.

I906, Trans. Ent. Soc. London, I906: 20I. (BM)

1929, Exotic Microlepidoptera, 3: 497. (BM)

I9Io, Proc. Linnean Soc. N.S. Wales, 35: 25 I.

I93I, Ann. Mus. Nac. Hist. Nat. Buenos Aires, 36: 408. 


$\begin{array}{ll}\text { nephelarcha } & \text { PHILOBOTA } \\ \text { nephelaspis } & \text { TELPHUSA } \\ \text { nephelastra } & \text { IMMA } \\ \text { nephelatma } & \text { IMMA } \\ \text { nephelias } & \text { BLASTOBASIS } \\ \text { nephelicta } & \text { PSEUDURGIS } \\ \text { nephelissa } & \text { ELAEONOMA } \\ \text { nephelista } & \text { COMOCRITIS } \\ \text { nephelitis } & \text { HELIOZELA } \\ \text { nephelobathra } & \text { ISOTORNIS* }\end{array}$

nephelocentra nephelochtha

nephelocrana nephelocyma nephelodelta nephelodes nephelodesma nepheloleuca nephelombra nephelomicta nephelomima nephelomorpha nephelonota nephelopa nephelopa nephelophaea nephelophracta nephelopis nephelopsycha nephelopyrga

nephelopyrrha nephelora nepheloscopa nephelospila nephelota nephelotana nephelotis nephelotorna nephelotoxa nephelozona nephelozyga nephocentra nephocirca nephodesma nephodryas nephogenes
SCIEROPEPLA THYRSOSTOMA

NARYCIA

STENOMA

PARALECTA

XYSMATODOMA

THIOTRICHA

STENOMA

GELECHIA

ASAPHARCHA

PRAYS

MACROBATHRA

OXYTHECTA

DIPSOCHARES

EULECHRIA

BLASTOBASIS

GELECHIA

BRACHMIA

ARGYROPLOCE

ARGYROPLOCE

ANATRACHYNTIS MACHAERITIS MONOPIS JONACA PROTOLECHIA PROTHELYMNA* NOTOSARA*

TINEA ORNEODES OPOSTEGA TINAEGERIA STATHMOPODA ASYMPHORODES THIOTRICHA IMMA PTEROPHORUS
I884, Proc. Linnean Soc. N.S. Wales, 8: 483. I926, Exotic Microlepidoptera, 3: 276. I906, Trans. Ent. Soc. London, I906: 200. (Bм) I927, Exotic Microlepidoptera, 3: 375. (BM) I902, Trans. R. Soc. S. Australia, 26: I70. I9I3, Ann. Transvaal Mus. 3: 333. (T) I9I4, Exotic Microlepidoptera, I: 238. I9I4, Journ. Bombay Nat. Hist. Soc. 23: I29. (BM) I897, Proc. Linnean Soc. N.S. Wales, 22: 405.

I935, in Caradja and Meyrick, Materialien zu einer Micro-

lepidopteren Fauna der Chinesischen Provinzen Kiangsu,

Chekiang und Hunan, p. 89.

I933, Exotic Microlepidoptera, 4:432. (v)

1927, Insects of Samoa, 3, Lepidoptera, fasc. 2, p. 79. (BM)

I933, Exotic Microlepidoptera, 4: 4I4. (c) I930, Exotic Microlepidoptera, 4:27. (v)

I930, Exotic Microlepidoptera, 4: I2.

I893, Proc. Linnean Soc. N.S. Wales, 7: 500.

1926, Exotic Microlepidoptera, 3:280.

I932, Exotic Microlepidoptera, 4: 298. (v)

I904, Proc. Linnean Soc. N.S. Wales, 29: 309.

I930, Exotic Microlepidoptera, 3: 555. (BM)

1907, Proc. Linnean Soc. N.S. Wales, 32: 76.

I886, Proc. Linnean Soc. N.S. Wales, Io: 820 . 1885, Proc. Linnean Soc. N.S. Wales, 9: I05I.

I937, Exotic Microlepidoptera, 5: 72

1883, Proc. Linnean Soc. N.S. Wales, 7: 5I2.

I93I, Exotic Microlepidoptera, 4: I78.

I932, Exotic Microlepidoptera, 4: 352. (v)

I92I, Ann. Transvaal Mus. 8: 9I. (T)

I934, Exotic Microlepidoptera, 4: 527. (C)

I938, Institut des Parcs Nationaux du Congo Belge, fasc. I4, p. Io. I9I7, Exotic Microlepidoptera, 2: 45.

I889, Proc. Linnean Soc. N.S. Wales, (Ser. 2) 3: 1668.

I928, Exotic Microlepidoptera, 3: 426. (T)

I9I2, Exotic Microlepidoptera, I: 38. (BM)

I904, Proc. Linnean Soc. N.S. Wales, 29: 354 .

1883, Trans. Proc. New Zealand Inst. I 5: 57.

I89o, Trans. R. Soc. S. Australia, I3: 52.

I932, Trans. Ent. Soc. London, 80: I20.

I9o8, Trans. Ent. Soc. London, I907: 505.

I9I5, Exotic Microlepidoptera, I: 352.

I930, Exotic Microlepidoptera, 3: 586. (P)

I921, Exotic Microlepidoptera, 2: $46 \mathrm{I}$.

I929, Trans. Ent. Soc. London, 76: 500.

I9I8, Exotic Microlepidopteia, 2: I25. (BM)

I928, Exotic Microlepidoptera, 3: 420. (B.1I)

I926, Trans. Ent. Soc. London, 74: 276. 
nephomicta

nephoptera

nephospora

nereidopa

nereis

nereitis

nerina

neritis

nerteria

nerterodes

nerteropa

nesiastis

nesidias

nesocharis

nesoclera

nesographa

nesograpta

nesolocha

nesophora

nestoria

nettomorpha

neurias

neurocentra

neurocoma

neurogramma

neurogramma

neurographa

neurograpta

neurometra

neurophaea

neurophanes

neurophora

neuroplaca

neuroplecta

neuroscia

neurosema

neurota

neurota

neurotona

neurotypa

nicaea

nicomacha

nidicola

nidifica

nigrata

nigrescens

nigricincta

nigricola

nigricoma
TELPHUSA

GLYPHIPTERYX

MELEONOMA

EUCOSMA

GRACILARIA

CARPOSINA

CEROMITIA

PHYCIODYTA*

ANACAMPSIS

COMMATICA

ANTAEOTRICHA

TINEOLA

BRACHMIA

TINEA

THYRSOSTOMA

COPROMORPHA

CRYPTOPHASA

CARPOSINA

PYRODERCES

TINEA

SCIEROPEPLA

NEMOTOIS

STENOMA

MACROBATHRA

LIMNOECIA

SYLEPTA

ANTAEOTRICHA

BRACHMIA

HYPEURYNTIS

TORTRIX

TRICHEMBOLA

COMPSOLECHIA

IDIOPTERYX

BRACHMIA

MACHIMIA

CLYSIA

IMMA

OCYSTOLA

ATHLETA

PHASMATOGRAPHA*

EULECHRIA

LASPEYRESIA

HAPSIFERA

AUGASMA (?)

NEPTICULA

MELASINA

CORMOTYPA

HYPONOMEUTA

NARYCIA
1932, Exotic Microlepidoptera, 4: I94. (BM)

I888, Trans. Proc. New Zealand Inst. 20:87.

I930, Exotic Microlepidopiera, 3: 623. (BM)

I927, Exotic Microlepidoptera, 3: 333. (BM)

I88I, Proc. Linnean Soc. N.S. Wales, 5: I63.

I9I3, Exotic Microlepidoptera, I: 75. (BM)

I9I2, Exotic Microlepidoptera, I: 28 . (T)

I9I8, Ann. Transvaal Mus. 6: 58. (T)

I906, Journ. Bombay Nat. Hist. Soc. I7: I39. (BM)

I9I4, Trans. Ent. Soc. London, I9I4: 239. (BM)

I9I5, Exotic Microlepidoptera, I: 395. (BM)

I9I I, Ann. Transvaal Mus. 2: 239. (T)

I9I I, Trans. Linnean Soc. London, I4:273. (BM)

I928, Exotic Microlepidoptera, 3: 427.

I929, Exotic Microlepidoptera, 3: 499. (BM)

I926, Exotic Microlepidoptera, 3: 244.

I925, Exotic Microlepidoptera, 3: I 50.

I9Io, Proc. Limnean Soc. N.S. Wales, 35: I48.

I897, Proc. Linnean Soc. N.S. Wales, 22: 354.

I9I0, Rec. Indian Mus. 2: 23I. (K)

I930, Exotic Microlepidoptera, 4: I5.

I922, Exotic Microlepidoptera, 2: 536.

1925, Exotic Microlepidoptera, 3: I79. (BM)

I930, Exotic Microlepidoptera, 3: 6I4. (BM)

Igo9, Ann. Transvaal Mus. 2: 20, pl. 6, fig. 9. (T)

I939, Trans, R. Ent. Soc. London, 89: 48.

I923, Exotic Microlepidoptera, 2:6I4. (P)

I9I2, Ann. Transwaal Mus. 8: 9I. (T)

I926, Sarawak Mus. Journ. 3: I6I.

I932, Exotic Microlepidoptera, 4: 34I. (BM)

I926, Exotic Microlepidoptera, 3: 27I. (BM)

I922, Trans. Ent. Soc. London, I922: 98. (BM)

I933, Exotic Microlepidoptera, 4: 359. (BM)

1938, Institut des Parcs Nationaux du Congo Belge,

fasc. I4, p. I7, pl. 2, fig. 3 .

I930, Ann. Naturhist. Mus. Wien, 44: 23I. (v)

I938, Trans. R. Ent. Soc. London, 87: 504. (BM)

I906, Trans. Ent. Soc. London, I906: I84. (BM)

I885, Proc. Limnean Soc. N.S. Wales, 9: 1082.

I9I5, Exotic Microlepidoptera, I: 382 . (BM)

I928, Exotic Microlepidoptera, 3: 4I9. (BM)

I902, Trans. R. Soc. S. Australia, 26: I47.

I921, Ann. Transvaal Mus. 8: 61. (T)

I935, Rev. Française d'Entomol. $2: 56$. (P)

I9I2, Ann. Sonth African Mus. Io: 68. (SA)

I9I3, Ann. Transwaal Mus. 3: 326. (T)

I920, Ann. South African Mus. I7: 3IO. (SA)

I92I, Exotic Microlepidoptera, 2: 386 . (BM)

I9I2, Ann. South African Mus. IO: 66. (SA)

I927, Exotic Microlepidoptera, 3: 328. (BM) 


\begin{tabular}{|c|c|c|}
\hline nigridorsis & LEURONOMA & I92I, Ann. Transvaal Mus. 8: 68. (T) \\
\hline nigrispersa & SCYTHRIS & I9I8, Ann. Transvaal Mus. 6:37. (T) \\
\hline nigritula & DICELLITIS* & I9o8, Journ. Bombay Nat. Hist. Soc. I8: 6I6. (Bм) \\
\hline nimbata & ANTAEOTRICHA & I925, Exotic Microlepidoptera, 3: I75. (BM) \\
\hline nimbifera & ELACHISTA & I9I3, Ann. Transvaal Mus. 3: 322. (T) \\
\hline nimbigera & CHELARIA & I926, Exotic Microlepidoptera, 3: 283. \\
\hline imbosa & ANARSIA & I9I3, Ann. Transvaal Mus. 3: 300. (T) \\
\hline nimbosa & ARGYROPLOCE & $\begin{array}{l}\text { I920, Voyage de Ch. Alluaud et } R \text {. Jeannel en Afrique } \\
\text { Oriental, II, Microlepidoptera, p. } 64 . \text { (P) }\end{array}$ \\
\hline iphacma & STENOMA & I9I6, Exotic Microlepidoptera, I: 510. (Us) \\
\hline niphadarcha & PLATYPTILIA & I930, Exotic Microlepidoptera, 3: 566. (BM) \\
\hline niphadastra & ARGYROPLOCE & I92I, Ann. Transvaal Mus. 8: 57. (T) \\
\hline niphadia & CROSSOPHORA & I886, Proc. Linnean Soc. N.S. Wales, Io: 795. \\
\hline dobola & MACROBATHRA & I886, Proc. Linnean Soc. N.S. Wales, ro: 8 го. \\
\hline lopa & PTOCHAULA* & I920, Exotic Microlepidoptera, 2: 325. (BM) \\
\hline phadophanes & ELACHISTA & I937, Exotic Microlepidoptera, 5: Ioo. (ML) \\
\hline phadopla & ERECHTHIAS & I88 I, Proc. Linnean Soc. N.S. Wales, 5: 270. \\
\hline dosticha & OCTASPHALES & I930, Exotic Microlepidoptera, 3: 620. (BM) \\
\hline narcha & IMMA & I93I, Exotic Microlepidoptera, 4: I8o. (BM) \\
\hline niphaspis & EUCOSMA & I928, Exotic Microlepidoptera, 3: 44I. (T) \\
\hline stis & THIOTRICHA & I904, Proc. Limnean Soc. N.S. Wales, 29: 297. \\
\hline tra & EULIA & I93I, Exotic Microlepidoptera, 4: I5I. (V) \\
\hline niphatma & URODUS & I925, Exotic Microlepidoptera, 3: I30. (BM) \\
\hline niphaula & EPIPHTHORA & I904, Proc. Linnean Soc. N.S. Wales, 29: 263. \\
\hline as & PELTOPHORA & I884, Proc. Linnean Soc. N.S. Wales, 9: 730. \\
\hline es & NEMOTOIS & $\begin{array}{l}\text { I938, in Caradja and Meyrick, Deuts. Ent. Zeit. Iris, } 52 \text { : } \\
28 \text {. }\end{array}$ \\
\hline arpa & STATHMOPODA & 1937, Exotic Microlepidoptera, 5: I50. \\
\hline entra & COMPSOLECHIA & I922, Trans. Ent. Soc. London, I922: 90. (BM) \\
\hline phochalca & HOPLOPHANES & I897, Proc. Linnean Soc. N.S. Wales, 22: 4 I3. \\
\hline niphochlaena & PTILOGENES & I926, Exotic Microlepidoptera, 3: 233. (BM) \\
\hline niphochrysa & STAGMATOPHORA & I930, Exotic Microlepidoptera, $3: 546$. (вм) \\
\hline niphocosma & MELASINA & I934, Exotic Microlepidoptera, 4: 483 . (v) \\
\hline niphocremna & ACROCERCOPS & I932, Exotic Microlepidoptera, 4: 268. \\
\hline rossa & COLEOPHORA & I920, Ann. South African Mus. I7: 299. (SA) \\
\hline niphocrypta & SIMAETHIS & I930, Exotic Microlepidoptera, 4:6. (BM) \\
\hline niphocycla & HYPERCALLIA & I926, Exotic Microlepidoptera, 3: 3I4. (Bм) \\
\hline niphodelta & ARGYROPLOCE & I925, Exotic Microlepidoptera, 3: I43. (BM) \\
\hline niphodesma & OCYSTOLA & I885, Proc. Limnean Soc. N.S. Wales, 9: roSo. \\
\hline nipholecta & SCYTHRIS & I924, Exotic Microlepidoptera, 3:87. \\
\hline nipholoncha & HYPOSMOCOMA & 1935, Proc. Hawaiian Ent. Soc. 9:66. (SP) \\
\hline niphomesta & COLEOPHORA & I9I7, Exotic Microlepidoptera, 2: 73. \\
\hline niphomitra & LECITHOCERA & I93I, Exotic Microlepidoptera, 4: 78. (BM) \\
\hline niphopelta & IMMA & I930, Exotic Microlepidoptera, 4: 4. (BM) \\
\hline niphoptila & MERIDARCHIS & I930, Exotic Microlepidoptera, 3: 588. (BM) \\
\hline niphopyrrha & CALLITHRINCA & I927, Exotic Microlepidoptera, 3: 36I. (BuI) \\
\hline rrhabda & ARGYROCORYS* & $\begin{array}{l}\text { I938, Institut des Parcs Nationaux du Congo Belge, fasc. I4, } \\
\text { p. } 25 \text {. }\end{array}$ \\
\hline & TRICHEMBOLA & I926, Exotic Microlepidoptera, 3:272. (B.1) \\
\hline & EPICHORISTA & I9I7, Ann. South African Mus. I7: 2. (Si) \\
\hline
\end{tabular}




$\begin{array}{ll}\begin{array}{l}\text { niphosperma } \\ \text { niphospora }\end{array} & \text { ACROLEPIA } \\ \text { ARGYRESTHIA } \\ \text { niphosticta } & \text { STAGMATOPHORA } \\ \text { niphostoma } & \text { IMMA } \\ \text { niphostrota } & \text { ORNEODES } \\ \text { niphostrota } & \text { PROTHELYMNA } \\ \text { niphosyrphas } & \text { DEPRESSARIA } \\ \text { niphoxantha } & \text { LABDIA } \\ \text { niphozela } & \text { SCYTHRIS } \\ \text { niphozona } & \text { STATHMOPODA } \\ \text { nitescens } & \text { ANTAEOTRICHA } \\ \text { nitida } & \text { STATHMOPODA } \\ \text { nitrodes } & \text { A[R]CROLEPIA } \\ \text { nitrota } & \text { ANTAEOTRICHA } \\ \text { nivalis } & \text { PTEROPHORUS } \\ \text { niveipalpis } & \text { EUCOSMA } \\ & \\ \text { nivescens } & \text { EULIA } \\ \text { nivicolor } & \text { SCYTHRIS } \\ \text { nivifera } & \text { COLEOPHORA } \\ \text { nivosa } & \text { ZELLERIA }\end{array}$

nobilitata

nocticolor

noctivaga

noctivola

nocturna

nodata

nodicornis

nodifera

nodosa

nodulata

nomaditis

nomadopis

nomaea

nomaea

nomias

nomistis

nomodoxa

normalis

normifera

noserodes

noseropis

nota

notanthes

notaria

notaula

nothostigma
I93I, Ann.Mus.Nac.Hist.Nat.Buenos Aires, $36: 403$. (Bм) I938, in Caradja and Meyrick, Deuts. Ent. Zeit. Iris, 52: 23. (BM)

I936, Exotic Microlepidoptera, 5: 30.

I922, Exotic Microlepidoptera, 2: 483. (вм)

I9o8, Trans. Ent. Soc. London, I907: 507.

I907, Trans. Proc. New Zealand Inst. 39: i 6.

I93I, Exotic Microlepidoptera, 4: I20. (BM)

I930, Exotic Microlepidoptera, 3: 545 .

I93I, Rec. Cant. Mus. 3: 369.

1927, Exotic Microlepidoptera, 3: 379.

1925, Exotic Microlepidoptera, 3: I7I. (BM)

I9I3, Exotic Microlepidoptera, I: 93.

I9Io, Rec. Indian Mus. 2: 229. (K)

I9I6, Exotic Microlepidoptera, I : 497. (BM)

Igo8, Trans. Ent. Soc. London, I907: 499.

I938, Institut des Parcs Nationaux du Congo Belge,

fasc. I4, p. 8 .

I926, Exotic Microlepidoptera, 3:250. (BM)

I9I6, Exotic Microlepidoptera, 2: II.

I930, Exotic Microlepidoptera, 3: 625.

I938, in Caradja and Meyrick, Deuts. Ent. Zeit. Iris, 52:

I6. (BM)

I9I7, Exotic Microlepidoptera, 2: 6I.

I9I6, Exotic Microlepidoptera, I: 603.

I9II, Trans. Linnean Soc. London, I4:277. (BM)

I932, Exotic Microlepidoptera, 4: 342.

I9I4, Trans. Ent. Soc. London, I9I4: 252. (BM)

I897, Proc. Linnean Soc. N.S. Wales, 22: 373.

I9Io, Proc. Linnean Soc. N.S. Wales, 35: I80.

I931, in Joannis, Ann. Soc. Ent. France, 98 (Supp.): 724

[486]. (P)

I9I0, Trans. Ent. Soc. London, I9Io: 447. (BM)

I921, Exotic Microlepidoptera, 2: 440.

I9I6, Exotic Microlepidoptera, I: 594.

I935, Exotic Microlepidoptera, 4:578.

I9I7, Trans. Ent. Soc. London, I9I7: 27. (BM)

I9I4, Exotic Microlepidoptera, I: 2 I7. (BM)

I92I, Ann. Transvaal Mus. 8: 74. (T)

I889, Proc. Linnean Soc. N.S. Wales, (Ser. 2) 3: I579.

I897, Proc. Limnean Soc. N.S. Wales, 22: 395.

I9I8, Exotic Microlepidoptera, 2: I60. (Bм)

I927, Exotic Microlepidoptera, 3: 347. (BM)

1889, Proc. Limnean Soc. N.S. Wales, (Ser. 2) 3: 1658.

I928, Exotic Microlepidoptera, 3: 458. (BM)

I9I9, Exotic Microlepidoptera, 2: 265.

I936, Exotic Microlepidoptera, 4: 6Io. (BM)

I9I3, Exotic Microlepidoptera, I: II8.

I933, Exotic Microlepidoptera, 4: 355. (v)

I9I4, Trans. Ent. Soc. London, I9I4: 233. (BM) 


\begin{tabular}{|c|c|c|}
\hline nothrodes & EUCOSMA & I9I2, Ent. Mon. Mag. 48: 34. (Sub.) \\
\hline nothrodes & PROTOLECHIA & I92I, Exotic Microlepidoptera, 2: 429. (BM) \\
\hline nothropis & AUTOSTICHA & I921, Ann. Transvaal Mus. 8: 92. (T) \\
\hline notifera & STENOMA & I915, Exotic Microlepidoptera, I: $465 . \quad$ (BM) \\
\hline notifica & FRISILIA & I9Io, Journ. Bombay Nat. Hist. Soc. 20: 438. (вм) \\
\hline otocapna & ODITES & I925, in Caradja, Mem.Sect.Sti.Acad.Românā, 3:382. (BM) \\
\hline lotochlora & GELECHIA & I929, Exotic Microlepidoptera, 3: 489. (BM) \\
\hline otochorda & STAGMATOPHORA & I907, Journ. Bombay Nat. Hist. Soc. I7: 744 . \\
\hline otocosma & EULIA & I928, Proc. Hawaiian Ent. Soc. $7:$ 97. (SP) \\
\hline lotocrossa & THEMELIOTIS & I9I7, Exotic Microlepidoptera, 2: 89 . \\
\hline otogramma & STENOMA & I930, Ann. Naturhist. Mus. Wien, 44: 243. (v) \\
\hline otograpta & CAPUA & I9Io, Proc. Linnean Soc. N.S. Wales, 35: I98. \\
\hline otolopha & GLYPHIDOCERA & I929, Exotic Microlepidoptera, 3: 529. (вм) \\
\hline otolychna & HOMALOXESTIS & I936, Exotic Microlepidoptera, 5: 48. (BM) \\
\hline 1otometra & LYONETIA & I9I5, Exotic Microlepidoptera, I: 350. \\
\hline otomitra & MACROBATHRA & I924, Exotic Microlepidoptera, 3: 98. (BM) \\
\hline otomochla & STENOLECHIA & I935, Exotic Microlepidoptera, 4: 583. (BM) \\
\hline otophanes & MALACOGRAPTIS* & I922, Zool. Meded. 7: 89. (L) \\
\hline otophthalma & ACROCLITA & I933, Exotic Microlepidoptera, 4: 4I7. (BM) \\
\hline otoplecta & LEPTOCOPA* & I9I8, Exotic Microlepidoptera, 2: 22I. \\
\hline otopyrsa & COPOCENTRA & I935, Arb. morph. taxon. Ent. Berl. 3: I05. (D) \\
\hline otorrhoa & SCYTHRIS & I92I, Exotic Microlepidoptera, 2: 44I. (BM) \\
\hline tosaris & STENOMA & I925, Exotic Microlepidoptera, 3: I8I. (BM) \\
\hline otosema & CRYPHIOXENA & I922, Exotic Microlepidoptera, 2: 507. \\
\hline otospila & EPITHECTIS & I923, Exotic Microlepidoptera, 3: I5. \\
\hline otosticta & ODITES & I925, Exotic Microlepidoptera, 3: I57. \\
\hline gga & MACROBATHRA & I9I4, Exotic Microlepidoptera, I: 2I6. \\
\hline novalis & LIMNOECIA & I920, Exotic Microlepidoptera, 2: 32I. (BM) \\
\hline hoverca & AGRIOPHARA & I9I6, Exotic Microlepidoptera, I: 484. (вм) \\
\hline oxia & THEREUTIS & I921, Exotic Microlepidoptera, 2: 437. (BM) \\
\hline abeculosa & ODITES & I9I8, Ann. Transvaal Mus. 6: 54. (T) \\
\hline ubicincta & IMMA & I9I6, Exotic Microlepidoptera, I: 559. (вм) \\
\hline icincta & SIMAETHIS & $\begin{array}{l}\text { I938, in Caradja and Meyrick, Dents. Ent. Zeit. Iris, } 52 \text { : } \\
\text { 86. (Bм) }\end{array}$ \\
\hline era & OECOPHORA & I886, Proc. Linnean Soc. N.S. Wales, Io: 784 . \\
\hline ra & ORNEODES & I921, Exotic Microlepidoptera, 2: 408. \\
\hline gena & BRACHMIA & I9I I, Journ. Bombay Nat. Hist. Soc. $20: 720$. (BM) \\
\hline jigena & IMMA & I9Io, Trans. Ent. Soc. London, I9Io: 466. (Bм) \\
\hline losa & NARYCIA & $\begin{array}{l}\text { I920, Voyage de Ch. Alluaud et } R \text {. Jeannel en Afrique } \\
\text { Oriental, II, Microlepidoptera, p. Iog. (P) }\end{array}$ \\
\hline vaga & PACHNISTIS & I921, Zool. Meded. 6: I67. (L) \\
\hline ifraga & TIQUADRA & I9I9, Exotic Microlepidoptera, 2: 276. (BM) \\
\hline civora & STATHMOPODA & I932, Exotic Microlepidoptera, 4: 272. \\
\hline ivora & TINEA & I939, Trans. R. Ent. Soc. London, 89: 6I. \\
\hline learis & ANTAEOTRICHA & I9I3, Trans. Ent. Soc. London, I9I3: I8I. (BuI) \\
\hline leata & ARGYROPLOCE & I9I3, Ann. Transvaal Mus. 3:278. (т) \\
\hline nucleata & EUCROTALA* & I9I7, Exotic Microlepidoptera, $2: 96$. \\
\hline leolata & AMATHYNTIS & I9I I, Journ. Bombay Nat. Hist. Soc. 21: II3. \\
\hline & SARIDOSCELIS & I9I3, Exotic Microlepidoptera, I: I46. \\
\hline dicornis & CHOLOTIS & I9I3, Ann. Transvaal Mus. 3: 3os. (T) \\
\hline
\end{tabular}




nugax
numellata
numeraria
numerata
numeratrix
numerosa
nummifera
nummulata
nummulata
nuntia
nuptialis
nutrix
nyctalopis
nycteris
nycterodes
nycteropa
nycteropis
nyctias
nyctiphanes
nyctiphron
nyctiphronas
nyctopis
nyctora
nymphaea
nymphas
nymphidia
nymphocoma
nymphotima
nystalea

CHEZALA

EUCOSMA

MELASINA

RHYTHMOLOGA *

ATTEVA

HYPONOMEUTA

SPATALISTIS

CAPUA

EOMICHLA

STENOMA

MOMPHA

LABDIA

DEPRESSARIA

OECOPHORA

AMPHORITIS

STENOMA

ATYCHIA

PROTOLECHIA

COSMOPTERYX

LECITHOCERA

CRYPTOLECHIA

TRACHYPEPLA

BORKHAUSENIA

ELACHISTA

STENOMA

DEPRESSARIA

ARGYRESTHIA

STENOMA

CRYPTOLOGA*

$\begin{array}{ll}\begin{array}{l}\text { obarata } \\ \text { obducta }\end{array} & \text { SIMAETHIS } \\ \text { obelacma } & \text { PROTOCRYPTIS* } \\ \text { obeliscana } & \text { SEUCOPTERA } \\ \text { obeliscota } & \text { PROTOLECHIA } \\ \text { obelitis } & \text { EUCLEODORA } \\ \text { obelodes } & \text { STENOMA } \\ \text { obfuscata } & \text { BRACHMIA } \\ \text { obfuscatana } & \text { CAPUA } \\ \text { objecta } & \text { TELPHUSA } \\ \text { oblitescens } & \text { TINEOLA } \\ \text { obliviosa } & \text { PHILOBOTA } \\ \text { obmutescens } & \text { STENOMA } \\ \text { obnixa } & \text { LASPEYRESIA } \\ \text { obnoxia } & \text { TINEA } \\ \text { obnupta } & \text { AGRIOPHARA } \\ \text { obolaea } & \text { BORKHAUSENIA } \\ \text { obolarcha } & \text { HELIODINES } \\ \text { obolucha } & \text { DEPRESSARIA }\end{array}$

I920, Exotic Microlepidoptera, 2: 382.

I9I2, Journ. Bombay Nat. Hist. Soc. 2I: 868. (Bм)

IgI4, Ann. Transvaal Mus. 4: 204. (T)

I926, Exotic Microlepidoptera, 3: 249. (BM)

I930, Ann. Naturhist. Mus. Wien, 44: 262. (v)

I92I, Exotic Microlepidoptera, $2: 438$. (BM)

IgIo, Proc. Linnean Soc. N.S. Wales, 35: 287.

IgIo, Proc. Linnean Soc. N.S. Wales, 35: I87.

IgI6, Exotic Microlepidoptera, I: 546. (BM)

I925, Exotic Microlepidoptera, 3: I79. (BM)

I922, Exotic Microlepidoptera, 2: 577. (BM)

I928, Exotic Microlepidoptera, 3: 387.

I930, Exotic Microlepidoptera, 3: 62I.

I8go, Trans. Proc. New Zealand Inst. 22: 2 I9.

Ig05, Journ. Bombay Nat. Hist. Soc. I6: 602.

I9I5, Exotic Microlepidoptera, I: 422. (BM)

I920, Ann. South African Mus. I7: 294. (SA)

Igo4, Proc. Linnean Soc. N.S. Wales, 29: 353.

I9I5, Trans. Ent. Soc. London, I9I5: 208. (BM)

I93I, Exotic Microlepidoptera, 4:8I. (BM)

I93I, Exotic Microlepidoptera, 4: I25. (BM)

I883, New Zealand Journ. Sci. I: 522.

I9I5, Exotic Microlepidoptera, I: 297.

IgI I, Ann. Transvaal Mus. 2: 233. (T)

I9I6, Exotic Microlepidoptera, I: 534. (BM)

I9I8, Exotic Microlepidoptera, 2: 223. (BM)

I9I9, Exotic Microlepidoptera, 2: 234. (BM)

I93I, Exotic Microlepidoptera, 4: 4I. (BM)

I932, Exotic Microlepidoptera, 4:2 I9.

I92I, Zool. Meded. 6: I84. (L)

I93I, Exotic Microlepidoptera, 4: I6I.

I9I8, Ann. Transvaal Mus. 6: 4I. (T)

I88I, Proc. Linnean Soc. N.S. Wales, 6: 690.

I904, Proc. Linnean Soc. N.S. Wales, 29: $35^{8}$.

I9I4, Exotic Microlepidoptera, I: 259. (BM)

I9I5, Exotic Microlepidoptera, I: 456. (BM)

I92I, Exotic Microlepidoptera, 2: 436. (BM)

I88I, Proc. Linnean Soc. N.S. Wales, 6: 455.

I92I, Ann. Transvaal Mus. 8: 7o. (T)

I924, Exotic Microlepidoptera, 3: 7I. (BM)

I9I3, Exotic Microlepidoptera, I: I24.

I9I6, Exotic Microlepidoptera, I: 525. (BM)

I921, Zool. Meded. 6: I59. (L)

I9I7, Ann. South African Mus. I7: I3. (SA)

I9I6, Exotic Microlepidoptera, I: 483. (BM)

I9Io, Journ. Bombay Nat. Hist. Soc. 20: I43. (BM)

I909, Trans. Ent. Soc. London, I909: 35 .

1936, Exotic Microlepidoptera, 5: 5I. 


obovata
obruta
obscurata
obscurata
obsepta
obsepta

obserata
obsignata
obsordescens
obstans
obstinata
obstinata
obstricta
obstructa
obtecta
obtemperata
obtorta
obtrectans
obtrectata
obtruncata
obtusa
obumbrata
obusta

obversa

obvia obvoluta occaecata occidua oceanis oceanitis ocellaris ocellifera ochanota ochetaula ochlerodes ochlica ochlopa ochnias ochnodelta ochnosema ochnotoma ochracea ochracma ochrantha ochranthes ochraspis
STENOMA

GELECHIA

FUMEA

STROBISIA

DRAGMATUCHA

ORSODYTIS

NARYCIA

LECITHOCERA

POMPHOCRITA*

SCYTHRIS

AGDISTIS

EBODA

AUXIMOBASIS

PROCHOLA

LASPEYRESIA

PHTHORIMAEA

XYROSARIS

MELASINA

BRACHMIA

CHELARIA

STENOMA

ODITES

MOTHONICA

[MOTHONODES*]

ACROCERCOPS

ODITES

THIOTRICHA

DEPRESSARIA

PHILOBOTA

DICHOMERIS

STENOMA

COESYRA

EURYPLACA*

MACROBATHRA

MIMOSCOPA*

TELPHUSA

LANGASTIS*

SYNTOMACTIS

GELECHIA

MONOPIS

CNEPHASIA

TORTRIX

CHRYSORYCTIS

CRYPHIOTECIINA*

SCYTHRIS

CHRYSORYCTIS

ORNEODES
I93I, Exotic Microlepidoptera, 4: 40. (v)

I92I, Ann. Transvaal Mus. 8: 72. (T)

I9I7, Ann. Natal Mus. 3: 6I5. (T)

IgI I, Journ. Bombay Nat. Hist. Soc. 20: 728. (вм)

I9I8, Ann. Transvaal Mus. 6:24. (T)

I935, in Caradja and Meyrick, Materialien zu einer

Microlepidopteren Fauna der Chinesischen Provinzen

Kiangsu, Chekiang und Hunan, p. 70. (вм)

I9I9, Exotic Microlepidoptera, 2: 263.

I9I4, Exotic Microlepidoptera, I: 277.

I930, Ann. Naturhist. Mus. Wien, 44:238. (v)

I928, Exotic Microlepidoptera, 3: 4I3. (BM)

I920, Voyage de Ch. Alluaud et R. Jeannel en Afrique

Oriental, II, Microlepidoptera, p. 44. (P)

Igo8, Journ. Bombay Nat. Hist. Soc. I8: 624. (вм)

I9I8, Exotic Microlepidoptera, 2: I60.

I9I 5, Exotic Microlepidoptera, I: 332. (вм)

I922, Exotic Microlepidoptera, 2: 534. (вм)

I925, Bull. Soc. R. Ent. Egypte, 9: 209.

I924, Trans. Ent. Soc. London, I923: 554.

I930, Exotic Microlepidoptera, 3: 55I.

I922, Exotic Microlepidoptera, 2: 506. (P)

I923, Exotic Microlepidoptera, 3: 3०. (BM)

I9I6, Exotic Microlepidoptera, I : 5 I3.

I925, Exotic Microlepidoptera, 3: I56. (T)

I92I, Exotic Microlepidoptera, 2: 456 .

I9I5, Trans. Ent. Soc. London, I9I5: 230.

I9I4, Ann. South African Mus. I0: 250. (SA)

I9I8, Exotic Microlepidoptera, 2: I26. (BM)

I92I, Exotic Microlepidoptera, 2: 39I. (BM)

I884, Proc. Linnean Soc. N.S. Wales, 8: 507.

1920, Exotic Microlepidoptera, 2: 306. (BM)

I9I6, Exotic Microlepidotpera, I: 5I9. (BM)

I884, Proc. Linnean Soc. N.S. Wales, 9: 773.

I883, Proc. Linnean Soc. N.S. Wales, 7: 488.

I9I5, Exotic Microlepidoptera, I: 295. (BM)

I893, Proc. Linnean Soc. N.S. Wales, 7: 526 .

I926, Ann. South African Mus. 23: 329. (SA)

I9I4, Exotic Microlepidoptera, I : 267. (BM)

I897, Proc. Linnean Soc. N.S. Wales, 22: $38 \mathrm{I}$.

I9I3, Ann. Transwaal Mus. 3: 29I. (T)

I925, Treubia, 6: 432 .

I936, Trans. roy. Soc. N.Z. 66:28I.

I930, Trans. Ent. Soc. London, 78: 310. (1)

I893, Proc. Limnean Soc. N.S. Wales, $7: 553$.

I932, Exotic Microlepidoptera, 4: 207. (BM)

Igog, Amn. Transvaal Mus. 2: 22, pl. 7, fig. 4. (x)

I893, Proc. Limnean Soc. N.S. Wales, $7: 552$.

I929, Exotic Microlepidoptera, 3: 535. (B.1) 


\begin{tabular}{|c|c|c|}
\hline ochrastis & MELASINA & I937, Exotic Microlepidoptera, 5: II4. (T) \\
\hline ochraula & PYCNOSTOLA & I9I8, Ann. Transvaal Mus. 6: I4. (T) \\
\hline ochrescens & ARISTOTELIA & $\begin{array}{l}\text { I938, in Caradja and Meyrick, Deuts. Ent. Zeit. Iris, 52: } \\
\text { 3. (вм) }\end{array}$ \\
\hline ochrias & TETRASCHALIS & I908, Trans. Ent. Soc. London, I907: 475 . \\
\hline chricilia & PROCOMETIS & I92I, Ann. Transvaal Mus. 8: Io6. (т) \\
\hline ochriclivis & EULIA & I93I, in Caradja, Bull. Sect. sci. Acad. roum. I4: 63. \\
\hline ochridorsellum & CORISCIUM & I88I, Proc. Linnean Soc. N.S. Wales, 5: I66. \\
\hline ochrilactea & IMMA & I934, Pacific Ent. Surv. Publ. 7, art. 28, p. 353. \\
\hline ochripalpis & BRENTHIA & I920, Exotic Microlepidoptera, 2: 335. (BM) \\
\hline ochrobathra & BLASTOBASIS & I92I, Exotic Microlepidoptera, $2: 463$. (BM) \\
\hline ochrobyrsa & BRACHMIA & I92I, Ann. Transvaal Mus. 8: 92. (T) \\
\hline ochrocapna & LECITHOCERA & I923, Exotic Microlepidoptera, 3: 40. (BM) \\
\hline ochrocausta & LEISTOMORPHA (?) & I884, Proc. Linnean Soc. N.S. Wales, 8: 5II. \\
\hline ochrocephala & GRACILARIA & I88I, Proc. Linnean Soc. N.S. Wales, 5: I62. \\
\hline chrochalca & PROTOMACHA & I889, Proc. Linnean Soc. N.S. Wales, 3: I646. \\
\hline chrochorda & NOSYMNA & I924, Exotic Microlepidoptera, 3: II8. (BM) \\
\hline ochrocoma & MELASINA & I894, Trans. Ent. Soc. London, I894: 26. \\
\hline chrocorys & GELECHIA & I936, Exotic Microlepidoptera, 5: 43. (c) \\
\hline ochrocosma & EPITHECTIS & I9II, Ann. Transvaal Mus. 2: 230. \\
\hline ochrocrena & EREUNETIS & I92I, Zool. Meded. 6: I94. (L) \\
\hline ochrocrossa & DINOTRGPA* & I9I6, Exotic Microlepidoptera, I: 506. (BM) \\
\hline chrodelta & STATHMOPODA & I9I3, Exotic Microlepidoptera, I: 89 . \\
\hline ochrodesma & EPERMENIA & I9I3, Ann. Transvaal Mus. 3: 317. (T) \\
\hline ochrodeta & PHTHORIMAEA & I923, Exotic Microlepidoptera, 3: 23. (BM) \\
\hline ochrodryas & ODITES & I933, Exotic Microlepidoptera, 4: 434. (v) \\
\hline ochrodyta & CEROMITIA & I92I, Exotic Microlepidoptera, 2: 405. \\
\hline chrogastra & CHERSADAULA* & I923, Trans. Proc. New Zealand Inst. 54: I65. \\
\hline ochrogramma & HETEROGYMNA & I9I3, Exotic Microlepidoptera, I: 74. (BM) \\
\hline chrogramma & SCYTHRIS & I9I6, Exotic Microlepidoptera, 2: II. \\
\hline ochrograpta & PTOCHORYCTIS & I923, Exotic Microlepidoptera, 2: 6I2. (Bм) \\
\hline ochrogypsa & AUXOTRICHA* & I93I, Exotic Microlepidoptera, 4: I9o. (вм) \\
\hline chroleuca & ELACHISTA & I923, Trans. Proc. New Zealand Inst. 54: I67. \\
\hline chrolina & METRERNIS* & I9o6, Journ. Bombay Nat. Hist. Soc. I7: 4I4. \\
\hline chrolychna & PSEUDURGIS & I9I4, Ann. South African Mus. Io: 254. (SA) \\
\hline & $\begin{array}{l}\text { OECOPHORA } \\
\text { [HOPLOSTEGA*] }\end{array}$ & I886, Proc. Linnean Soc. N.S. Wales, Io: 78 I. \\
\hline omalla & PROBATOSTOLA* & I926, Ann. South African Mus. 23:344. (SA) \\
\hline hromeris & BUCCULATRIX & I928, Exotic Microlepidoptera, 3:398. (T) \\
\hline chrometra & LECITHOCERA & I933, Exotic Microlepidoptera, 4: 356. (BM) \\
\hline chromicta & PROCHOLA & I922, Exotic Microlepidoptera, 2: 580 . (Bм) \\
\hline chromorpha & BLASTOBASIS & I925, Exotic Microlepidoptera, 3: I 45. \\
\hline chronephela & ACROCERCOPS & I9o8, Journ. Bombay Nat. Hist. Soc. I8: 821. \\
\hline ochronota & PROTEROCOSMA & I886, Trans. Ent. Soc. London, I886: 294. \\
\hline ropa & LIPOPTYCHA & I905, Journ. Bombay Nat. Hist. Soc. I6: $5^{87}$. \\
\hline chrophaea & SPHYRELATA (?) & I883, Proc. Linnean Soc. N.S. Wales, 8: 36I. \\
\hline ochrophanes & YPSOLOPHUS & I907, Journ. Bombay Nat. Hist. Soc. I7: 98I. (BM) \\
\hline rophragma & ENCELIDOTIS* & I920, Ann. South African Mus. I7: 308. (sA) \\
\hline chrophthalma & TELPHUSA & I927, Exotic Microlepidoptera, 3:344. (T) \\
\hline chropis & PARASTEGA & I9I4, Trans. Ent. Soc. London, I9I4: 236. \\
\hline
\end{tabular}




$\begin{array}{ll}\text { ochropsamma } & \text { TINEOLA } \\ \text { ochroptera } & \text { COESYRA } \\ \text { ochroptila } & \text { CNEPHASIA } \\ \text { ochroptila } & \text { HAPSIFERA } \\ \text { ochrosaris } & \text { STENOMA } \\ \text { ochrosceles } & \text { HOMALOXESTIS } \\ \text { ochroschista } & \text { PHTHORIMAEA } \\ \text { ochrosema } & \text { STOMOPTERYX } \\ \text { ochrosidera } & \text { SISYRODONTA* } \\ \text { ochrospila } & \text { RECURVARIA } \\ \text { ochrospora } & \text { ATELOSTICHA } \\ \text { ochrostoma } & \text { BRACHMIA } \\ \text { ochrostoma } & \text { CACOECIA } \\ \text { ochrothicta } & \text { STENOMA } \\ \text { ochrotoma } & \text { TELPHUSA } \\ \text { ochrotricha } & \text { CEROMITIA } \\ \text { ochroxesta } & \text { TRICHOTAPHE } \\ \text { ochroxyla } & \text { CRYPTOLECHIA } \\ \text { ochroxysta } & \text { ARISTOTELIA } \\ \text { ochrozona } & \text { LIMNOECIA } \\ \text { ochrozona } & \text { ORNEODES } \\ \text { ochrura } & \text { PARALOGISTIS* } \\ \text { ochthophora } & \text { DICHOMERIS } \\ \text { ochthopsamma } & \text { MELASINA } \\ \text { ochthotoma } & \text { ALLOTALANTA } \\ \text { ochyrota } & \text { BRACHMIA } \\ \text { ocladias } & \text { EPIBLEMA } \\ \text { ocneropis } & \text { TRACHYPEPLA } \\ \text { ocnogramma } & \text { LASPEYRESIA } \\ \text { ocreata } & \text { ONEBALA } \\ & \text { ODEITOPISTS }\end{array}$

$\begin{array}{ll}\text { octacentra } & \text { STENOMA } \\ \text { octanoma } & \text { ETHMIA } \\ \text { octavana } & \text { BRACHMIA } \\ \text { octavia } & \text { HEMIMENE } \\ \text { octobola } & \text { MERIDARCHIS } \\ \text { octocentra } & \text { HYPONOMEUTA } \\ \text { octonias } & \text { LECITHOCERA } \\ \text { octophora } & \text { BRACHMIA } \\ \text { octosticta } & \text { SCHOENOTENES } \\ \text { oculata } & \text { IDIOSTYLA* } \\ \text { oculisignis } & \text { ASYMPHORODES } \\ \text { ocydroma } & \text { PERONEA }\end{array}$

ocymorpha

ocyphanes

ocypora

ocytpera

ocystola
I9o8, Proc. Zool. Soc. London, p. 739.

1884, Proc. Linnean Soc. N.S. Wales, 9:78r.

I9ro, Proc. Linnean Soc. N.S. Wales, 35: 279.

I9o8, Proc. Zool. Soc. London, p. 749.

I925, Exotic Microlepidoptera, 3: I99. (BM)

I9Io, Journ. Bombay Nat. Hist. Soc. 20: 44I. (BM)

I929, Exotic Microlepidoptera, 3: 494. (BM)

I932, Trans. Ent. Soc. London, 80: II3. (BM)

I922, Arkiv för Zool. vol. I4, part I5, p. 6.

I934, Exotic Microlepidoptera, 4: 510. (BM)

I928, Exotic Microlepidoptera, 3: 472. (BM)

I9I3, Ann. Transvaal Mus. 3:296. (T)

I9I8, Exotic Microlepidoptera, 2: I66. (BM)

I925, Exotic Microlepidoptera, 3: I8o. (BM)

I923, Exotic Microlepidoptera, 3: I7. (BM)

I9I2, Exotic Microlepidoptera, I: 29. (T)

I921, Ann. Transvaal Mus. 8: 86. (T)

1937, Exotic Microlepidoptera, 5:87. (T)

I929, Exotic Microlepidoptera, 3: 483. (BM)

I897, Proc. Linnean Soc. N.S. Wales, 22: 364 .

I907, Journ. Bombay Nat. Hist. Soc. I7: 730.

I9I3, Ann. Transvaal Mus. 3: 3I2. (T)

I936, Exotic Microlepidoptera, 5: 46. (BM)

I934, Exotic Microlepidoptera, 4: 5I9. (C)

I930, Exotic Microlepidoptera, 3: 6I6. (Bм)

I920, Ann. South African Mus. I7:285. (SA)

I906, Journ. Bombay Nat. Hist. Soc. I7: I37. (BM)

I937, Exotic Microlepidoptera, 5: I 20.

I9I0, Ann. South African Mus. 5: 4I2. (SA)

I9Io, Journ. Bombay Nat. Hist. Soc. 20: 45I. (BM)

I9I5, Exotic Microlepidoptera, I : 459. (BM)

I9I4, Supplementa Entomologica, No. 3, p. 55.

I9I I, Journ. Bombay Nat. Hist. Soc. 20: 7 I4. (BM)

I922, Exotic Microlepidoptera, 2: 528. (BM)

I925, Exotic Microlepidoptera, 3: I37.

I921, Ann. Transvaal Mus. 8: II7. (T)

I9Io, Trans. Ent. Soc. London, I9Io: 447. (BM)

I9I8, Ann. Transvaal Mus. 6: 25. (T)

I930, Exotic Microlepidoptera, 3: 6Iо. (BM)

I92I, Exotic Microlepidoptera, 2: 4I2. (BM)

1934, Pacific Ent. Surv. Publ. 7, art. 28, p. 349. (Bв)

I935, in Caradja and Meyrick, Materialien zu einer Micro-

lepidopteren Fauna der Chinesischen Provinzen Kiangsu,

Chekiang und Hunan, p. 5I.

I893, Proc. Linnean Soc. N.S. Wales, $7: 557$.

I937, Exotic Microlepidoptera, 5: 95. (T)

I932, Trans. Ent. Soc. London, 8o: I I5. (BM)

I9Io, Proc. Limnean Soc. N.S. Wales, 35: 23I. (MA)

I932, Exotic Microlepidoptera, 4: 259. (v) 


\begin{tabular}{|c|c|c|}
\hline ocytoma & MERIDARCHIS & $\begin{array}{l}\text { I938, in Caradja and Meyrick, Deuts. Ent. Zeit. Iris, } 52 \text { : } \\
\text { I4. (BM) }\end{array}$ \\
\hline odiosa & PLATYPTILIA & I924, Exotic Microlepidoptera, 3:93. (T) \\
\hline odorifera & PROTOLECHIA & I904, Proc. Linnean Soc. N.S. Wales, 29:37I. (MA) \\
\hline oecodoma & CRYPTOPHASA & I930, Exotic Microlepidoptera, 4: I4. \\
\hline oeconoma & ATOMOTRICHA & I9I4, Trans. Proc. New Zealand Inst. 46: iाo. \\
\hline oenombra & TRICHOTAPHE & I9I4, Exotic Microlepidoptera, I: 20I. (BM) \\
\hline oeconomica & PYCNOSTOLA & I920, Ann. South African Mus. I7: 280. (SA) \\
\hline oedalea & ARGYROPLOCE & I909, Journ. Bombay Nat. Hist. Soc. I9: 593. (BM) \\
\hline oemias & BATRACHEDRA & I909, Ann. South African Mus. 5: 359. (SA) \\
\hline oenochares & ANCHINIA & I924, Exotic Microlepidoptera, 3: Ioo. (T) \\
\hline oenochares & PLEMYRISTIS & I920, Exotic Microlepidoptera, 2: 364. (BM) \\
\hline oenochroa & ENARMONIA & I9I2, Ent. Mon. Mag. 48: 34. (Sub.) \\
\hline oenochyta & ACOMPSIA & I92I, Ann. Transvaal Mus. 8: 78. (T) \\
\hline oenodes & PHELOTROPA* & I9I5, Exotic Microlepidoptera, I: 4II. (BM) \\
\hline oenombra & TRICHOTAPHE & I9I4, Exotic Microlepidoptera, I: $20 \mathrm{I}$. \\
\hline oenopa & CROSSOPHORA & I886, Proc. Linnean Soc. N.S. Wales, Io: 796. \\
\hline oenopa & TORTRIX & I9Io, Proc. Linnean Soc. N.S. Wales, 35:23o. (MA) \\
\hline oenopella & GRACILARIA & I88I, Proc. Linnean Soc. N.S. Wales, 5: I4I. \\
\hline oenophanes & XYROPTILA* & I9o8, Trans. Ent. Soc. London, I907: 480. \\
\hline oenopis & TINEA & I9o8, Proc. Zool. Soc. London, p. 740. \\
\hline oenosema & EUSTALODES* & I927, Insects of Samoa, 3, Lepidoptera, fasc. 2, p. 82. (BM) \\
\hline officialis & LECITHOCERA & I9II, Ann. Transvaal Mus. 3:67. (вм) \\
\hline officiosa & BRACHMIA & I9I8, Exotic Microlepidoptera, 2: II3. \\
\hline officiosa & PHALONIA & I9I2, Ent. Mon. Mag. 48:35. (Sub.). \\
\hline ogmodes & CLINOGRAPTIS* & I932, Exotic Microlepidoptera, 4: 208. (вм) \\
\hline ogmolopha & STENOMA & I930, Exotic Microlepidoptera, 3: 557. (BM) \\
\hline ogmosaris & STENOMA & I9I5, Exotic Microlepidoptera, I: 4I5. (вм) \\
\hline ogmotona & COLEOPHORA & I9I7, Exotic Microlepidoptera, $2: 72$. \\
\hline ogygia & NEPTICULA & I889, Trans. Proc. New Zealand Inst. 2 I : I87. \\
\hline olearis & CNEPHASIA & I9I2, Exotic Microlepidoptera, I: Iо. (T) \\
\hline oleata & DICHOMERIS & I9I3, Ann. Transvaal Mus. 3: 305. (T) \\
\hline olenarcha & ARGYROPLOCE & I93I, Exotic Microlepidoptera, 4: I36. (вм) \\
\hline olenitis & MELASINA & I921, Bull. Mus. Nat. Hist. Nat. Paris, 20: 122. \\
\hline olga & ZELOSYNE & I9I5, Trans. Ent. Soc. London, I9I5: 201. \\
\hline oligachthes & EULIA & I932, Exotic Microlepidoptera, 4:257. (v) \\
\hline oligarcha & COPTOTELIA & I9I3, Trans. Ent. Soc. London, I9I3: I78. (BM) \\
\hline oligarchica & COMPSOTORNA* & I89o, Trans. R. Soc. S. Australia, I3: 4I. \\
\hline oligastra & GLYPHIPTERYX & I926, Exotic Microlepidoptera, 3: 308. (BM) \\
\hline oligectis & ODITES & I9I7, Exotic Microlepidoptera, 2: 56. (BM) \\
\hline oligosperma & NEPTICULA & I934, Exotic Microlepidoptera, 4: 468. \\
\hline olivescens & DICHOMERIS & I9I3, Journ. Bombay Nat. Hist. Soc. 22: I75. (Bм) \\
\hline olympia & COMOCRITIS* & I894, Trans. Ent. Soc. London, I894: 24. (BM) \\
\hline olympias & PHILOBOTA & I889, Proc. Linnean Soc. N.S. Wales, (Ser. 2) 3: I6ro. \\
\hline olyranta & USSARA & I93I, Exotic Microlepidoptera, 4: I84. (v) \\
\hline olyritis & NEPTICULA & I9I5, Trans. Ent. Soc. London, I9I5: 256. \\
\hline ombrodoca & ELACHISTA & I889, Trans. Proc. New Zealand Inst. 2 I: I79. \\
\hline ombromorpha & ANCHARCHA* & I920, Exotic Microlepidoptera, 2: 369. \\
\hline ombrophora & EULECHRIA & I883, Proc. Linnean Soc. N.S. Wales, 8: 322. \\
\hline omiastis & OPOGONA & I937, Exotic Microlepidoptera, 5: 88. (T \\
\hline
\end{tabular}


omichleuta omichleutis omichlodes omichlopis omichlota ommatias ommatopa omogramma omophaea omoptila omoscopa omosema omphacias omphacina omphacitis omphacopa omphalias omphalopa omphalopis omphalota oncodes oncota oncota oneropis onias onthostola onychias onychosema onychota onychotis opaca opalina opaula operaria operosa operosa operosa

operta ophiaula ophidias ophiocephala ophiocypha ophiodes ophiodesma ophiodora ophiodryas ophioglossa ophiogramma ophiograpta M.T.M. I-I 6
GYMNOBATHRA

MISCERA

STRUTHISCA

TINEA

COESYRA

ATOMOTRICHA*

PTILOGENES

ENDOPHTHORA*

HARMOLOGA

ANARSIA

HIEROXESTIS*

EULECHRIA

LECITHOCERA

OTOPTRIS

PHALONIA

STENOMA

MICROCOLONA

THOLEROSTOLA*

GELECHIA

GYMNOBATHRA

ANACAMPSIS

BUCCULATRIX

PROCALYPTIS

TRACHYPEPLA

PLATYPTILIA

MELASINA

LINDERA

ARGYROPLOCE

MACAROSTOLA

CROBYLOPHORA

BRACHMIA

NEMOTOIS

GELECHIA

THIOTRICHA

ARGYROPLOCE

CACOECIA

PALTODORA

[PYCNOSTOLA*]

EUCOSMA

GELECHIA

MACAROSTOLA

NARYCIA

DECADARCHIS

PHILOBOTA

SIMAETHIS

PICROTECHNA*

BORKHAUSENIA

LACTURA

XYLORYCTA

TORTRIX
I929, Trans. Proc. New Zealand Inst. 60: 489. 1907, Proc. Linnean Soc. N.S. Wales, 32: I05. I908, Proc. Zool. Soc. London, p. 742. I928, Exotic Microlepidoptera, 3: 427. (BM) I884, Proc. Linnean Soc. N.S. Wales, 9: 785 . I883, New Zealand Journ. Sci. I: 522. I926, Exotic Microlepidoptera, 3: 230. (BM) I888, Trans. Proc. New Zealand Inst. 20: 94. I926, Sarawak Mus. Journ. 3: I49.

I9I8, Exotic Microlepidoptera, 2: I47. (BM)

I893, Proc. Linnean Soc. N.S. Wales, $7: 567$.

1920, Exotic Microlepidoptera, 2: 372.

I9Io, Journ. Bombay Nat. Hist. Soc. 20: 445. (Bм)

I9I 5, Trans. Ent. Soc. London, I9I 5: 245.

I9I2, Ent. Mon. Mag. 48: 35. (Sub.)

I93I, Exotic Microlepidoptera, 4: 39. (BM)

I9I3, Ann. Transvaal Mus. 3: 309. (T)

I9I7, Trans. Ent. Soc. London, I9I7: 40. (BM)

I926, Ann. South African Mus. 23: 330. (SA)

I888, Trans. Proc. New Zealand Inst. 20: 8I.

I9I3, Ann. Transvaal Mus. 3: 285. (T)

I9I9, Exotic Microlepidoptera, 2: 287.

I9Io, Proc. Linnean Soc. N.S. Wales, 35: 204.

1936, Trans. roy. Soc. N.Z. 66: 282.

I9I6, Exotic Microlepidoptera, I: 559.

I937, Exotic Microlepidoptera, 5: II6.

I93I, Exotic Microlepidoptera, 4:96. (v)

I93I, Exotic Microlepidoptera, 4: I34.

I908," Proc. Zool. Soc. London, p. 735.

I9I5, Exotic Microlepidoptera, I: 345.

1927, in Caradja, Mem. Sect. Sti. Acad. Românā, 4: 42I.

I9I2, in Wytsman, Genera Insectorum, fasc. I33, p. 6.

I9I 2, Ann. Transvaal Mus. 3:65. (T)

I9I8, Exotic Microlepidoptera, 2: I25. (BM)

I9I I, Proc. Linnean Soc. N.S. Wales, 36: 272.

I908, Journ. Bombay Nat. Hist. Soc. I8: 6I6. (вм)

I909, Ann. Transvaal Mus. 2: Io, pl. 4, fig. I. (T)

I9I7, Trans. Ent. Soc. London, I9I7: 20. (BM)

I93I, Exotic Microlepidoptera, 4: 60. (BM)

I907, Proc. Linnean Soc. N.S. Wales, 32: 62.

1933, Exotic Microlepidoptera, 4: 4I5.

I934, Exotic Microlepidoptera, 4: 464.

I889, Proc. Limnean Soc. N.S. Wales, (Ser. 2) 3: I62 I.

I9I 5, Trans. Ent. Soc. London, I9I5: 2I6. (B.I)

I9I4, Exotic Microlepidoptera, I: 260. (BM)

I936, Trans. roy. Soc. N.Z. 66: 282 .

I924, Exotic Microlepidoptera, 3: I22.

I89o, Trans. R. Soc. S. Australia, I3: 58.

I932, Trans. Ent. Soc. London, So: IIO. (BM) 


\begin{tabular}{|c|c|c|}
\hline ophiomorpha & GELECHIA & I935, Exotic Microlepidoptera, 4: 560. (BM) \\
\hline ophionota & PYLAETIS* & I907, Journ. Bombay Nat. Hist. Soc. I7: 752. (Bм) \\
\hline ophiopa & AGRIOPHARA & I9I6, Exotic Microlepidoptera, I: 484 . (BM) \\
\hline ophiosema & THUDACA & I893, Proc. Linnean Soc. N.S. Wales, 7: 576 . \\
\hline ophitis & GELECHIA & I9I3, Ann. Transvaal Mus. 3: 29I. (T) \\
\hline ophiucha & LACTURA & I924, Exotic Microlepidoptera, 3: I23. \\
\hline ophiura & METATHRINCA* & I9o8, Journ. Bombay Nat. Hist. Soc. I8: 626. (вм) \\
\hline ophryodes & CYPHOTHYRIS* & I9I4, Exotic Microlepidoptera, I: 255. (BM) \\
\hline ophryodes & PHTHEOCHROA & I927, Exotic Microlepidoptera, 3: 368: (BM) \\
\hline ophryota & CTENOCOMPA & I9I7, Exotic Microlepidoptera, z: 93. \\
\hline phrysta & ANTAEOTRICHA & I9I2, Trans. Ent. Soc. London, I9I I : 708. (BM) \\
\hline ophthalmias & CAPUA & I9Io, Proc. Linnean Soc. N.S. Wales, 35: I99. (MA) \\
\hline ophthalmias & EULECHRIA & I888, Proc. Linnean Soc. N.S. Wales, 2: $95^{\circ}$. \\
\hline phthalmica & COESYRA & I884, Proc. Linnean Soc. N.S. Wales, 9: 775 . \\
\hline opifica & AMYDRIA & I9o8, Proc. Zool. Soc. London, p. 752. \\
\hline opisthopa & TRICHEMBOLA & I9I8, Exotic Microlepidoptera, 2: II5. (BM) \\
\hline oporaea & OECOPHORA & I883, New Zealand Journ. Sci. I: 524 . \\
\hline oporina & AMATHYNTIS & I9I I, Journ. Bombay Nat. Hist. Soc. 2I: II3. \\
\hline oporista. & ALLOTALANTA & I926, Exotic Microlepidoptera, 3: 3Іо. \\
\hline oppidana & PROCHOLA* & I9I5, Exotic Microlepidoptera, I: 33I. (вм) \\
\hline opportuna & LECITHOCERA & I923, Exotic Microlepidoptera, 3: 38 . (BM) \\
\hline opposita & MACROBATHRA & I920, Exotic Microlepidoptera, 2: 364 . \\
\hline oppressa & EULIA & I926, Exotic Microlepidoptera, 3: 258. (BM) \\
\hline opsarias & CNEPHASIA & I9II, Ann. Transvaal Mus. 2: 224. (T) \\
\hline opsia & EUCOSMA & I9II, Proc. Linnean Soc. N.S. Wales, 36: 247. \\
\hline opsias & EPERMINIA & I897, Proc. Linnean Soc. N.S. Wales, 22: 430. \\
\hline opsigona & TINEA & I9I I, Journ. Bombay Nat. Hist. Soc. 21 : I 23. \\
\hline opsonoma & DICHOMERIS & I9I4, Trans. Ent. Soc. London, I9I4: 28I. (BM) \\
\hline opsonoma & EUCOSMA & I9I8, Ann. Transvaal Mus. 6: 48 . (T) \\
\hline opsorrhoa & TRICHOTAPHE & I929, Exotic Microlepidoptera, 3: 5I2. (T) \\
\hline optabilis & TINEA & I9I6, Exotic Microlepidoptera, I: 604 . \\
\hline optalea & EULECHRIA & I902, Trans. R. Soc.S. Australia, 26: I5I. \\
\hline optania & AMYDRIA & I9o8, Proc. Zool. Soc. London, p. 752 . \\
\hline optanias & BACTRA & I9I I, Proc. Linnean Soc. N.S. Wales, 36: 253. \\
\hline optica & LASPEYRESIA & I9I 2, Journ. Bombay Nat. Hist. Soc. 2I : 875. (BM) \\
\hline optica & SIMAETHIS & I921, Zool. Meded. 6: I83. (L) \\
\hline opticaspis & STATHMOPODA & I93I, Exotic Microlepidoptera, 4: I75. \\
\hline opticodes & ZACORISCA & I927, Exotic Microlepidoptera, 3:370. \\
\hline opticosema & URODUS & I930, Ann. Naturhist. Mus. Wien, 44:262, pl. 2, fig. 24. (v) \\
\hline optima & TRYMALTIS & I9I I, Proc. Linnean Soc. N.S. Wales, 36: 294. \\
\hline optodes & MYRMECOZELA & I922, Exotic Microlepidoptera, 2: 59I. (BM) \\
\hline oratrix & TINEOLA & I9I3, Ann. Transvaal Mus. 3: 332. (T) \\
\hline orba & ELACHISTA & I921, Zool. Meded. 6: I86. (L) \\
\hline orbata & BRACHMIA & I9Io, Trans. Ent. Soc. London, I9IO: 450. (BM) \\
\hline orbiculata & STATHMOPODA & I9I3, Exotic Microlepidoptera, I: 9I. \\
\hline orbifera & ACROCERCOPS & Igo8, Journ. Bombay Nat. Hist. Soc. I8: 8I8. \\
\hline orbigera & SPATALISTIS & I9I2, Exotic Microlepidoptera, I: I4. (BM) \\
\hline orbitalis & EULECHRIA & I922, Exotic Microlepidoptera, 2: 5II. \\
\hline orbitosa & EULECHRIA & I920, Exotic Microlepidoptera, 2: 373. \\
\hline orchas & CRYPSITHYRIS & I907, Journ. Bombay Nat. Hist. Soc. I7: 753. \\
\hline
\end{tabular}


orchatias

orchestris

ordinalis

ordinaria

ordinata

ordinatella

orectis

oreographa

orescoa

orestias

orgadopa

organica

orgias

orgiastis

orgilopis

oriana

orianassa

oriarcha

oriarcha

oriastra

oriaula

oribatis

oricasis

orichàlcias

orichalcias

orichlora

origenes

originalis

orinarcha

orinephela

orinoma

orinympha

oriphaea

oriphanes

oriphanta

oriphylax

orites

oritis

orneopis

ornithias

ornithotypa

oroglypta

orophias

orophota

orophoxantha

orosema

orphania

orphania

orphanopa

orphica
PTEROPHORUS

OPOGONA

CEROSTOMA

COLEOPHORA

MYRMECOZELA

GRACILARIA

XYLORYCTA

TORTRIX

NEPHOGENES

OPOSTEGA

STENOMA

POLYCHROSIS

PSEUDATTERIA

PHILOBOTA

TELPHUSA

MALLOBATHRA

ACROCERCOPS

GRACILARIA

TORTRIX

NEPTICULA

PERSICOPTILA

ARISTOTELIA

CNEPHASIA

NEMOTOIS

OXYPTILUS

ARGYROPLOCE

GYMNOBATHRA

PROTONYCTIA*

BATRACHEDRA

CATHALISTIS*

PHILOBOTA

SIMAETHIS

PHILOBOTA

TORTRIX

HYPERCALLIA

MONOPIS

MIMAESEOPTILUS

NEPTICULA

STENOMA

BLABOPHANES

ARGYROPLOCE

LACTURA

EUCOSMA

SYMBOLISTIS*

OPOSTEGA

PLUTELLA

CARPOSINA

MACROSACES

PROMALACTIS

POLYCHROSIS
I908, Trans. Ent. Soc. London, I907: 5II.

I9I I, Journ. Bombay Nat. Hist. Soc. 2I : IIo.

I9I4, Exotic Microlepidoptera, I : 227.

I9I3, Ann. Transvaal Mus. 3: 324.

I921, Ann. Transvaal Mus. 8: I26. (T)

I88I, Proc. Linnean Soc. N.S. Wales, 5: I45.

I89o, Trans. R. Soc. S. Australia, I3: 60.

I909, Trans. Ent. Soc. London, I909: I6. (BM)

I883, Proc. Linnean Soc. N.S. Wales, 8: 376.

I88I, Proc. Linnean Soc. N.S. Wales, 5: I75.

I 925, Exotic Microlepidoptera, 3: I82. (BM)

I920, Exotic Microlepidoptera, 2: 348. (BM)

I930, Exotic Microlepidoptera, 3: 607. (BM)

I889, Proc. Linnean Soc. N.S. Wales, (Ser. 2) 3: I6zo.

I923, Exotic Microlepidoptera, 3: I6. (BM)

I9I7, Exotic Microlepidoptera, 2: 9I.

I932, Trans. Ent. Soc. London, 80: II6.

I9I5, Trans. Ent. Soc. London, I9I5: 239.

I9Io, Proc. Linnean Soc. N.S. Wales, 35: 240.

I9I7, Trans. Proc. New Zealand Inst. 49: 247.

I9I5, Exotic Microlepidoptera, I: 321 .

I9I7, Trans. Ent. Soc. London, p. 35. (BM)

I932, Exotic Microlepidoptera, 4: 342. (BM)

1893, Proc. Linnean Soc. N.S. Wales, 7: 484.

I9I6, Exotic Microlepidoptera, I: 557.

I920, Ann. South African Mus. I7: 279. (SA)

I936, Trans. roy. Soc. N.Z. 66: 282 .

I932, Exotic Microlepidoptera, 4: 220.

I9I7, Exotic Microlepidoptera, 2: 33.

I9I7, Ann. South African Mus. I7: I4. (SA)

I884, Proc. Linnean Soc. N.S. Wales, 8: 486.

I926, Exotic Microlepidoptera, 3: 304. (BM)

I889, Proc. Linnean Soc. N.S. Wales, (Ser. 2) 3: I6r3.

I930, Exotic Microlepidoptera, 3: 608. (BM)

I928, Exotic Microlepidoptera, 3: 473. (BM)

I924, Arkiv för Zool. vol. I6, part I4, p. 3.

I885, Trans. Proc. New Zealand Inst. I7: I26.

I9Io, Rec. Indian Mus. 2: 229. (K)

I925, Exotic Microlepidoptera, 3:221. (BM)

I888, Trans. Proc. New Zealand Inst. 20: 97.

I938, Trans. R. Ent. Soc. London, 87: 5II. (BM)

I930, Exotic Microlepidoptera, 3: 595. (BM)

I9o7, Journ. Bombay Nat. Hist. Soc. I7: 732. (BM)

I904, Proc. Linnean Soc. N.S. Wales, 29: 4I4.

I92I, Ann. Transvaal Mus. 8: I24. (T)

1932, Exotic Microlepidoptera, 4:228.

I9Io, Proc. Linnean Soc. N.S. Wales, 35: I5I.

I9Io, Journ. Bombay Nat. Hist. Soc. 20: I49. (BM)

I9I5, Exotic Microlepidoptera, I: 294. (BM)

I920, Exotic Microlepidoptera, 2: 347. (BM) 
orphnaea CRYPTOLECHIA

orphnoceros orphnocycla

orphnodes orphnogenes orphnographa orphnopa orphnophanes orphnopis orphnospila orsicoma orsobela orthacma orthacta orthadia orthaula orthias orthiasta orthiastis orthidias orthiodes orthiostyla orthobasis orthobathra orthocampta orthocapna orthocentra orthochaeta orthochlora orthochorda orthochrysa orthoclina orthocopa orthocosma orthoctenis orthodelta orthodeta orthodoxa orthodroma orthogona orthogramma orthographa ortholampra ortholeuca ortholocha orthomacha orthomastix orthomeris
COLEOPHORA

PERONEA

TINEA

EUCOSMA

PYRODERCES

PROCHOLA

TORTYRA

DOLIOTECHNA*

TINEA

RECURVARIA

AREOCOSMA*

DICHOMERIS

ARGYROPLOCE

TIMYRA

MISCERA

CNEPHASIA

TINEA

THIOTRICHA

COPROMORPHA

PERIACMA

DICHELOPA

PROCHOLA

DEPRESSARIA

BATTARISTIS

STENOMA

THALEROSTOMA*

COPTOTELIA

HOMALOXESTIS

SAGALASSA

HILAROGRAPHA

ALLOCLITA

TORTRIX

ARGYROPLOCE

GLYPHIDOCERA

ANTISPILA

GLYPHIPTERYX

MACHIMIA

THUDACA

SIMAETHIS

PELTOPHORA

STENOMA

STENOMA

GLYPHIPTERYX

ACROCERCOPS

GLYPHIPTERYX

BRACHMIA

COLPOMORPHA*
I931, Ann. Mus. Nac. Hist. Nat. Buenos Aires, 36: 397. (BM)

I937, Exotic Microlepidoptera, 5: 130.

I937, in Caradja and Meyrick, Deuts. Ent. Zeit. Iris, 5I: I76. (BM)

I9I I, Journ. Bombay Nat. Hist. Soc. 2I : I22.

I939, Trans. R. Ent. Soc. London, 89: 49. (c)

I936, Exotic Microlepidoptera, 5: 29. (C)

I922, Exotic Microlepidoptera, 2: 582. (BM)

I932, Exotic Microlepidoptera, 4: 275. (v)

I9I4, Exotic Microlepidoptera, I: I88. (BM)

I934, Exotic Microlepidoptera, 4: 5I6. (C)

I9I8, Exotic Microlepidoptera, 2: I30. (BM)

I9I7, Ann. South African Mus. I7: 7. (SA)

I926, Ann. South African Mus. 23: 332. (SA)

I908, Proc. Zool. Soc. London, p. 718.

I906, Journ. Bombay Nat. Hist. Soc. I7: I45. (Bм)

I907, Proc. Linnean Soc. N.S. Wales, 32: I02.

I9Io, Proc. Linnean Soc. N.S. Wales, 35: 273. (MA)

I928, Exotic Microlepidoptera, 3: 427.

I905, Journ. Bombay Nat. Hist. Soc. I6: 59I. (BM)

I927, Insects of Samoa, 3, Lepidoptera, fasc. 2, p. 96. (BM) I894, Trans. Ent. Soc. London, I894: 22. (BM)

1934, Pacific Ent. Surv. Publ. 7, art. 28, p. 343. (BB)

I922, Exotic Microlepidoptera, 2: 582. (BM)

I9I8, Ann. Transvaal Mus. 6: 3I. (T)

I9I4, Trans. Ent. Soc. London, I9I4:246. (BM)

I9I 2, Trans. Ent. Soc. London, I9I I: 7I7. (BM)

I9I7, Exotic Microlepidoptera, 2: 42.

I9I3, Trans. Ent. Soc. London, I9I3: I78. (BM)

I926, Sarawak Mus. Journ. 3: I56.

I922, Exotic Microlepidoptera, 2: 484. (BM)

I932, Exotic Microlepidoptera, 4: 274. (v)

I922, Exotic Microlepidoptera, 2: 547. (P)

I924, Trans. Proc. New Zealand Inst. 55: 66I.

I93I, Exotic Microlepidoptera, 4: I4I.

I923, Exotic Microlepidoptera, 3: 49. (BM)

I93I, Exotic Microlepidoptera, 4: I86.

I922, Exotic Microlepidoptera, 2: 490. (BM)

I9I2, Trans. Ent. Soc. London, I9I : 697. (BM)

I893, Proc. Linnean Soc. N.S. Wales, $7: 577$.

I886, Trans. Ent. Soc. London, I886: 287.

I884, Proc. Linnean Soc. N.S. Wales, 9: 725.

1925, Exotic Microlepidoptera, 3: 205. (BM)

I930, Exotic Microlepidoptera, 4: 29. (v)

I92I, Ann. Transvaal Mus. 8: II3. (T)

I908, Journ. Bombay Nat. Hist. Soc. I8: 8I7.

I920, Exotic Microlepidoptera, 2: 33I. (BM)

I93I, Exotic Microlepidoptera, 4:84. (BM)

I929, Exotic Microlepidoptera, 3: 528. (BM) 
orthometalla orthometra orthomochla orthomorpha orthopa orthopeda orthophaea orthophanes orthophracta orthophragma orthophrontis orthopyrga orthopyrrha orthoscia

orthostacta orthostathma orthota orthotenes orthotis orthotoma orthotona orthotricha orthozona orthrarcha orthridia orthriopa orthritis orthropis orthroptila ortygia orymagdis oschophora osmotris osphrantica osteacma osteitis osteopa osteosema ostiaria ostracitis ostracitis ostracodes ostracophanes

ostracopis ostrina othello othrarcha otiosa
CANCANODES* ODITES

CHELARIA

POLYCHROSIS

STENOMA

EUCOSMA

ANTAEOTRICHA COMPSISTIS

ANACAMPSIS

ATELOSTICHA

HOLCOCERA

LASPEYRESIA

HIEROCROBYLA * EULIA

ACROCERCOPS

CHELARIA

ZYGOLOPIIA

GLYPHIDOCERA

LOZOSTOMA

PHAEOSACES

ANTAEOTRICHA

NEMATOBOLA

BAEONOMA

CRYPTOLECHIA

STENOMA

ANTAEOTRICHA

LABDIA

CACOECIA

STENOMA

EUCOSMA

GLYPHIPTERYX EPITHECTIS

AMORBIA

COPHOMANTIS

ORTHENCHES

STATHMOPODA

ACROCERCOPS

EPITHECTIS

TINEA

ANATHAMNA*

MELASINA

DICHOMERIS

NARYCIA

ANISOCHORISTA

TINEA

TINEA

CRYPTOLECHIA

PIILOBOTA
I922, Exotic Microlepidoptera, 2: 499. (BM)

Igo8, Journ. Bombay Nat. Hist. Soc. I8: 634. (Bм)

I932, Exotic Microlepidoptera, 4:99. (BM)

I928, Exotic Microlepidoptera, 3: 443. (BM)

1932, Exotic Microlepidoptera, 4: 297. (v)

I934, Exotic Microlepidoptera, 4:484. (BM)

I930, Exotic Microlepidoptera, 4: 20. (v)

I905, Trans. Ent. Soc. London, I905: 243.

I9I4, Trans. Ent. Soc. London, I9I4: 257. (BM)

I9I6, Exotic Microlepidoptera, I: 548. (BM)

1932, Exotic Microlepidoptera, 4: 317. (v)

I922, Exotic Microlepidoptera, 2: 532. (BM)

I9I5, Exotic Microlepidoptera, I: 353.

I936, Veröff. Deutschen Kol.-Mus. I :330, pl. I3, figs. 23-24.

(H)

I9I8, Exotic Microlepidoptera, 2: I74.

I92I, Exotic Microlepidoptera, 2: 429. (BM)

I9I4, Exotic Microlepidoptera, I: 243. (BM)

I929, Trans. Ent. Soc. London, 76: 5I0. (BM)

I897, Proc. Linnean Soc. N.S. Wales, 22: 4I8.

I905, Journ. Bombay Nat. Hist. Soc. I6: 605. (вм)

I9I6, Exotic Microlepidoptera, I: 495. (BM)

I893, Proc. Linnean Soc. N.S. Wales, 7: 592.

I9I6, Exotic Microlepidoptera, I: 508. (BM)

I930, Exotic Microlepidoptera, 3: 578. (Bм)

I9I6, Exotic Microlepidoptera, I: 520. (BM)

I925, Exotic Microlepidoptera, 3: I66. (Bм)

I930, Exotic Microlepidoptera, 3: 545. (BM)

I902, Trans. Ent. Soc. London, I902: 573.

1935, Arb. morph. taxon. Ent. Berl. 3: I04. (D)

I9I7, Trans. Ent. Soc. London, I9I7: 22. (BM)

I909, Journ. Bombay Nat. Hist. Soc. I9: 435. (BM)

I9Io, Rec. Indian Mus. 2: 219. (K)

I932, Exotic Microlepidoptera, 4:263. (v)

I929, Exotic Microlepidoptera, 3: 525. (BM)

I93I, Ann. Mus. Nac. Hist. Nat. Buenos Aires, 36: 406.

I9I7, Exotic Microlepidoptera, 2: 6I. (BM)

I920, Exotic Microlepidoptera, 2: 292. (BM)

I929, Exotic Microlepidoptera, 3:486. (BM)

I927, Exotic Microlepidoptera, 3: 322.

I9I I, Proc. Linnean Soc. N.S. Wales, 36: 262.

I9I3, Ann. Transvaal Mus. 3: 334. (T)

I9I6, Exotic Microlepidoptera, I: 583. (BM)

I938, Institut des Parcs Nationaux du Congo Belge, fasc. I4,

p. 28 , pl. 3, fig. 7 .

I938, Trans. R. Ent. Soc. London, 87: 505. (BM)

I9I6, Exotic Microlepidoptera, I: 604.

I907, Journ. Bombay Nat. Hist. Soc. I7: 9S8. (BM)

I930, Exotic Microlepidoptera, 3: 578. (BM)

I92I, Exotic Microlepidoptera, 2: 385 . 


\begin{tabular}{|c|c|c|}
\hline toptera & IMMA & I906, Trans. Ent. Soc. London, I9o6: I86. (вм) \\
\hline ovigera & STATHMOPODA & I9I3, Exotic Microlepidoptera, I: 93. \\
\hline ovulata & THRIOPHORA* & I9II, Ann. Transvaal Mus. 2: 23I. (T) \\
\hline ovulifera & STENOMA & I925, Exotic Microlepidoptera, 3: I82. (вм) \\
\hline xalea & LECITHOCERA & I9Io, Journ. Bombay Nat. Hist. Soc. $20: 449$. (BM) \\
\hline xinopa & EUPRAGIA & I929, Trans. Ent. Soc. London, 76: 509. (BM) \\
\hline oxyacma & METACHANDA & I926, Ann. South African Mus. 23: 334. (SA) \\
\hline xyactis & STENOPTILIA & I922, Exotic Microlepidoptera, 2: 550. (вм) \\
\hline xybela & MACHIMIA & I93I, Exotic Microlepidoptera, 4: II9. (v) \\
\hline xycarpa & MUSURGA & I935, Exotic Microlepidoptera, 4: 562. (BM) \\
\hline xycentra & ANTAEOTRICHA & I9I6, Exotic Microlepidoptera, I: 497 . (BM) \\
\hline xychalca & GELECHIA & I937, Exotic Microlepidoptera, 5: I22. \\
\hline oxycharis & LABDIA & I921, Zool. Meded. 6: I68. (L) \\
\hline oxychasta & TELPHUSA & I929, Exotic Microlepidoptera, 3:487. (T) \\
\hline oxychlora & LABDIA & I932, Exotic Microlepidoptera, 4: 3I4. \\
\hline xycona & LECITHOCERA & I9Io, Journ. Bombay Nat. Hist. Soc. $20: 444$. (вм) \\
\hline oxycopis & GLYPHIPTERYX & I9I8, Exotic Microlepidoptera, 2: I95. (BM) \\
\hline oxycrates & ELACHISTA & I932, Exotic Microlepidoptera, 4:2I8. \\
\hline oxycrena & PARAPLEURIS* & I937, Exotic Microlepidoptera, 5: 82. (T) \\
\hline oxycryptis & SYMMOCA & I929, Exotic Microlepidoptera, 3: 5I6. (BM) \\
\hline oxydecta & STENOMA & I9I5, Exotic Microlepidoptera, I: 426. (BM) \\
\hline oxydelta & CALANTICA & I9I3, Exotic Microlepidoptera, I: I47. \\
\hline oxydelta & GRACILARIA & I908, Journ. Bombay Nat. Hist. Soc. I8: 831. \\
\hline oxydesma & OPOGONA & I9I8, Exotic Microlepidoptera, 2: I85. \\
\hline oxyglossa & COSMOPTERYX & I9o9, Ann. Transvaal Mus, 2: I8, pl. 6, fig. 3. (T) \\
\hline oxyglypta & ACROLEPIA & I929, Trans. Ent. Soc. London, 76: 5 I9. \\
\hline oxygramma & THIOTRICHA & I9I8, Exotic Microlepidoptera, 2: I23. (вм) \\
\hline oxygrammana & ACROCEUTHES & I88I, Proc. Linnean Soc. N.S. Wales, 6: 460. \\
\hline xygrapha & TRICHOTAPHE & I9I3, Ann. Transvaal Mus. 3: 3о2. (т) \\
\hline oxygrapta & LITHOCOLLETIS & I9I5, Trans. Ent. Soc. London, I9I5: 222. \\
\hline oxyina & CREMNOGENES* & I883, New Zealand Journ. Sci. I: $5^{25}$. \\
\hline oxyleuca & PYRODERCES & I9I5, Exotic Microlepidoptera, I: 3I5. \\
\hline oxyleuca & THIODIA & I9I2, Ent. Mon. Mag. 48: 34. (Sub.) \\
\hline oxylitha & HETERODERCES* & I929, Exotic Microlepidoptera, 3: 521. (BM) \\
\hline oxyloba & GNORIMOSCHEMA & I929, Exotic Microlepidoptera, 3: 492. (Bм) \\
\hline oxyloga & ACROCERCOPS & I928, Exotic Microlepidoptera, 3: 408. (T) \\
\hline oxylopha & PLUTELLA & I932, Trans. Ent. Soc. London, 80: II 8. \\
\hline oxymacha & EREUNETIS & I893, Proc. Limnean Soc. N.S. Wales, 7: 564 . \\
\hline oxymachaera & PHRYGANOSTOLA & I88I, Proc. Linnean Soc. N.S. Wales, 5: $25 \mathrm{I}$. \\
\hline oxymeris & EPITHECTIS & I929, Exotic Microlepidoptera, 3: 486. (вм) \\
\hline oxymochla & POLYCHROSIS & I9I7, Trans. Ent. Soc. London, I9I7: 24. (BM) \\
\hline oxymora & TINEA & I9I9, Exotic Microlepidoptera, 2:274. (BM) \\
\hline oxymoris & OINOPHILA & I9I6, Exotic Microlepidoptera, I: 6r9. \\
\hline oxymorpha & NASAMONICA* & I922, Exotic Microlepidoptera, 2: 555. (P) \\
\hline oxyntes & PTEROPHORUS & I9o8, Trans. Ent. Soc. London, I907: 498. \\
\hline oxyopa & PHYLLOCNISTIS & I9I8, Exotic Microlepidoptera, 2: I82. \\
\hline oxyopis & THIOTRICHA & I927, Insects of Samoa, 3, Lepidoptera, fasc. 2, p. 80. \\
\hline oxypercna & POLYCHROSIS & I930, Trans. Ent. Soc. London, 78: 3II. (P) \\
\hline oxypetra & HYPOSMOCOMA & I935, Proc. Hawaiian Ent. Soc. 9:65. (sP) \\
\hline oxyphaea & COLEOPHORA & I9I3, Ann. Transwaal Mus: $3: 324$. (T) \\
\hline
\end{tabular}


oxyphanes

oxyphanta

oxyphrontis

oxyphylla

oxyplaca

oxyplecta

oxypora

oxyprora

oxypselia

oxyptera

oxyptila

oxyschista

oxyscia

oxysema

oxysphena

oxyspila

oxystaura

oxystola

oxystoma

oxystyla

oxyteles

oxytheces

oxythectis

oxytoma

oxytona

oxytora

oxytricha

oxytypa

oxyxantha

oxyzona

oxyzona

ozodes

pabulicola

pacabilis

pacalis

pachnias

pachnitis

pachnocoma

pachnodes

pachnodes

pachnopis

pachnoscia

pachnostola

pachnota
MEGACRASPEDUS CITRINARCHA*

METACHANDA OCYPHRON* PTILOGENES

SCYTHRIS

PROCOMETIS

THYRSOSTOMA

IMMA

SCIEROPEPLA

PYRODERCES

STENOMA

STENOMA

PYRODERCES

PARECTOPA

ANACAMPSIS

PAMMENE

POLYHYMNO

NOTHOGENES

GEPHYRISTIS

GLAUCOSTOLA*

THIOTRICHA

ARISTOTELIA

PYRODERCES

ENDOPHTHORA

OCYSTOLA

GLYPHIPTERYX

SCLEROGRAPTIS*

COESYRA

ONEBALA

TRACHYDORA

EPERMENIA

EUTORNA

BATRACHEDRA

BLASTOBASIS

ACOLASTA

HERMENIAS

PSEUDODOXIA

ARGYROPLOCE

CHOLOTIS

ARISTOTELIA

EPORYCTA

PSEGMATICA*

CALLIBRYASTIS*
I904, Proc. Linnean Soc. N.S. Wales, 29: 276.

I938, in Caradja and Meyrick, Deuts. Ent. Zeit. Iris, 52: 88.

I930, Trans. Ent. Soc. London, 78: 3I5. (P)

I92I, Ann. Transvaal Mus. 8: I03. (T)

I929, Trans. Ent. Soc. London, 76: 5I8. (Bм)

I9I6, Exotic Microlepidoptera, 2: I4. (BM)

I908, Proc. Zool. Soc. London, p. 730.

I922, Exotic Microlepidoptera, 2: 50I. (P)

I929, Trans. Ent. Soc. London, 76: 503. (BM)

I89o, Trans. R. Soc. S. Australia, I3: 70.

I928, Exotic Microlepidoptera, 3: 389 .

I925, Exotic Microlepidoptera, 3: I87. (BM)

I923, Exotic Microlepidoptera, 2: 6I5. (P)

I897, Proc. Linnean Soc. N.S. Wales, 22: 35 I.

I934, Exotic Microlepidoptera, 4: 473.

I909, Ann. South African Mus. 5: 35I. (SA)

I935, in Caradja and Meyrick, Materialien zu einer Micro-

lepidopteren Fauna der Chinesischen Provinzen Kiangsu,

Chekiang und Hunan, p. 62. (BM)

I9I3, Ann. Transvaal Mus. 3:284. (T)

I938, in Caradja and Meyrick, Deuts. Ent. Zeit. Iris, 52: 28.

I9II, Ann. Transvaal Mus. 3: 79 .

I926, Ann. South African Mus. 23: 344. (SA)

I904, Proc. Linnean Soc. N.S. Wales, 29: 293.

I929, Exotic Microlepidoptera, 3: 48I. (BM)

I897, Proc. Linnean Soc. N.S. Wales, 22: 352.

I893, Proc. Linnean Soc. N.S. Wales, 7: $55^{8}$.

I885, Proc. Linnean Soc. N.S. Wales, 9: I064.

I928, Exotic Microlepidoptera, 3: 424. (BM)

I923, Exotic Microlepidoptera, 3: 3I. (BM)

I9I4, Exotic Microlepidoptera, I : 245.

I9Io, Journ. Bombay Nat. Hist. Soc. 20: 455. (BM)

I897, Proc. Linnean Soc. N.S. Wales, 22: 396.

I9I7, Exotic Microlepidoptera, 2: 68.

I906, Trans. R. Soc. S. Australia, 30: 43.

I922, Exotic Microlepidoptera, 2: 583 .

I922, Exotic Microlepidoptera, 2: 539. (BM)

Igo2, Trans. R. Soc. S. Australia, 26: I55.

I9I2, Journ. Bombay Nat. Hist. Soc. 2I: 852. (BM)

I922, Exotic Microlepidoptera, 2: 547. (BM)

I9I I, Proc. Linnean Soc. N. S. Wales, 36: 266.

I9I7, Exotic Microlepidoptera, 2: 44.

I939, Trans. R. Ent. Soc. London, 89: 53.

I9I5, Exotic Microlepidoptera, I : 376.

I930, Exotic Microlepidoptera, 3: 598. (BM)

I9I2, Exotic Microlepidoptera, I: I4. (BM) 


\section{pachyacma pachybathra pachybela pachyceros pachydesma pachydmeta pachygramma pachynta pachyntis pachypleura pachyscia pachyspila pachysticta pachystoma pachystoma}

$\begin{array}{ll}\text { pachyzona } & \text { PHTHINOSTOMA } \\ \text { pacifera } & \text { EULECHRIA } \\ \text { pacifica } & \text { PROTOLECHIA } \\ \text { pactolia } & \text { DRYADAULA } \\ \text { pactolias } & \text { PHILOBOTA } \\ \text { pactota } & \text { ANTAEOTRICHA } \\ \text { paenitens } & \text { PACHYSARIS } \\ \text { paeta } & \text { HETERODERCES } \\ \text { pagana } & \text { MACHIMIA } \\ \text { pagetodes } & \text { PROGONARMA } \\ \text { pagicola } & \text { GELECHIA } \\ \text { pagidotis } & \text { MACHIMIA } \\ \text { pagiducha } & \text { TINEA } \\ \text { pagiopa } & \text { ETHMIA } \\ \text { palacta } & \text { PARADORIS } \\ \text { palaearcha } & \text { GRACILARIA } \\ \text { palaechrysis } & \text { TINEA } \\ \text { palaeocosma } & \text { TORTYRA } \\ \text { palaeodes } & \text { IMMA } \\ \text { palaeographa } & \text { AEONOXENA* } \\ \text { palaeomorpha } & \text { GLYPHIPTERYX } \\ \text { palaeosema } & \text { COMMATARCHA* } \\ \text { palaeota } & \text { TARPHYSCELI } \\ \text { palaestrias } & \text { ANTAEOTRICHA } \\ \text { palamedes } & \text { LASPEYRESIA } \\ \text { palamota } & \text { ARISTOTELIA } \\ \text { palathodes } & \text { TIMYRA } \\ \text { palearis } & \text { EPITHECTIS } \\ \text { paliacma } & \text { ACROCERCOPS } \\ \text { palimpsesta } & \text { PSEUDODOXIA } \\ \text { palinorsa } & \text { POLYHYMNO } \\ \text { palirrhoa } & \text { COMMATICA } \\ \text { pallacopa } & \text { EPIBRONTIS } \\ & \end{array}$

I926, Ann. South African Mus. 23: 328. (SA) I92I, Exotic Microlepidoptera, 2: 394. (BM) I934, Exotic Microlepidoptera, 4: 509 . I921, Ann. Transvaal Mus. 8: 5o. (T) I9I8, Ann. Transvaal Mus. 6: 9. (T) 1929, Trans. Ent. Soc. London, 76: 493. (BM) I92I, Exotic Microlepidoptera, 2: 459. I9I3, Trans. Ent. Soc. London, I9I3: I97. (BM) I894, Trans. Ent. Soc. London, I894: I7. (BM) I92I, Exotic Microlepidoptera, 2: 448. (BM) I93I, Exotic Microlepidoptera, 4: I67. I905, Joum. Bombay Nat. Hist. Soc. I6: 6I9. I936, Exotic Microlepidoptera, 5: 49. (BM) I92I, Ann. Transvaal Mus. 8: IOI. (T) I920, Voyage de Ch. Alluaud et R. Jeannel en Afrique Oriental, II, Microlepidoptera, p. 98. (P) I92I, Ann. Transvaal Mus. 8: II4. (T) I914, Exotic Microlepidoptera, I: I65. I904, Proc. Linnean Soc. N.S. Wales, 29: 342. I902, Trans. Ent. Soc. London, I902: 577 I9I3, Exotic Microlepidoptera, I: I24. (MA) I9I5, Exotic Microlepidoptera, I: 39I. (MA) I923, Exotic Microlepidoptera, 2: 626. (BM) I929, Exotic Microlepidoptera, 3: 522. (BM) I9I6, Exotic Microlepidoptera, I : 549. (вм) I9I I, Trans. Linnean Soc. London, I4: 302. I936, Exotic Microlepidoptera, 5: 44. (BM) I9I8, Exotic Microlepidoptera, 2: 2I6. (BM) I932, Exotic Microlepidoptera, 4: 325 I9I8, Exotic Microlepidoptera, 2: I89. (BM) IgI I, Journ. Bombay Nat. Hist. Soc. 20: 736. (BM) I930, Exotic Microlepidoptera, 3: 582. (BM) I929, Journ. Fed. Malay States Mus. I4: 374. I926, Exotic Microlepidoptera, 3: 303. (BM) I9I4, Supplementa Entomologica, No. 3, p. 57. (D) I928, Exotic Microlepidoptera, 3: 432. (BM) I88I, Proc. Linnean Soc. N.S. Wales, 5: 242. I935, Exotic Microlepidoptera, 4: 594. (BM) I9I3, Exotic Microlepidoptera, I: I45. (BM) I9I6, Exotic Microlepidoptera, I: 502. (BM) I9I6, Exotic Microlepidoptera, I: 564. (BM) I926, Exotic Microlepidoptera, 3: 274. (BM) I9o6, Journ. Bombay Nat. Hist. Soc. I7: I44. (BM) I9I3, Exotic Microlepidoptera, I: 65. (BM) I93I, in Joannis, Ann. Soc. Ent. France, 98 (Supp.): 739 [50I]. (P) I9I5, Exotic Microlepidoptera, I: 308. (BM) I9o9, Ann. Transvaal Mus. 2: I5. I922, Trans. Ent. Soc. London, I922: 72. (BM) I922, Exotic Microlepidoptera, 2: 502 . 


\begin{tabular}{|c|c|c|}
\hline allacopis & ENDOPHTHORA & 918, Trans. Proc. New Zealand Inst. 50: I34. \\
\hline & LEPIDOSCIA & I893, Proc. Linnean Soc. N.S. Wales, 7: 5I3. \\
\hline al & ARGYROPLOCE & I909, Journ. Bombay Nat. Hist. Soc. I9: 595. (BN \\
\hline ella & EULECHRIA & I883, Proc. Linnean Soc. N.S. Wales, 7: 5 I 9. \\
\hline & HERYPSTIS & I9I2, Journ. Bombay Nat. Hist. Soc. 21: 862. \\
\hline tus & PTEROPHORUS & I908, Trans. Ent. Soc. London, I907: 495. \\
\hline ola & HERMENIAS & I912, Journ. Bombay Nat. Hist. Soc. 21: 853 . (в \\
\hline cola & MACROSARISTIS* & 1937, Exotic Microlepidoptera, 5: 78 . \\
\hline era & NEELYSIA & 1935, Proc. Hawaiian Ent. Soc. 9:63. (SP) \\
\hline ora & HYPOSMOCOMA & I928, Proc. Hawaiian Ent.Soc. 7: I04. (sP) \\
\hline les & EUCOSMA & I920, Exotic Microlepidoptera, 2: 344. (BM) \\
\hline & TINISSA & I9I7, Exotic Microlepidoptera, 2: 89. \\
\hline la & THIOTRICHA & I92I, Ann. Transvaal Mus. 8: 75. (T) \\
\hline 1anes & COSMOPTERYX & I909, Journ. Bombay Nat. Hist. Soc. I9: 4I7. \\
\hline & MESOPHERNA* & I893, Proc. Linnean Soc. N.S. Wales, 7: 5I5. \\
\hline & CEROMITIA & I9o8, Proc. Zool. Soc. London, p. 755. \\
\hline cha & PALTODORA & I9I3, Ann. Transvaal Mus. 3:28I. (T) \\
\hline & ARISTOTELIA & I904, Proc. Linnean Soc. N.S. Wales, 29: 290. \\
\hline phyra & URODUS & I935, Arb. morph. taxon. Ent. Berl. 3: 1o8. (D) \\
\hline ana & ISOCHORISTA & I88I, Proc. Limnean Soc. N.S. Wales, 6: 425. \\
\hline & ELINOSTOLA & I921, Exotic Microlepidoptera, 2: 473. (BM) \\
\hline & LYTROPHILA* & I9I3, Ann. Transvaal Mus. 3: 319. (т) \\
\hline & GELECHIA & I909, Ann. South African Mus. 5: 370. (SA) \\
\hline lca & HOPLOPHANES & I897, Proc. Linnean Soc. N.S. Wales, $22: 414$. \\
\hline & BRACHMIA & aal Mus. 2: 232. (T) \\
\hline omatica & ARISTOTELIA & I926, Exotic Microlepidoptera, 3: 274. (BM) \\
\hline & COESYRA & I884, Proc. Linnean Soc. N.S. Wales, 9: 766. \\
\hline & NEPTICULA & I920, Ann. South African Mus. I7: 312. (SA) \\
\hline iastis & THIOTRICHA & I921, Exotic Microlepidoptera, 2: 426 . (вм) \\
\hline copa & HOMALOXESTIS & I937, Exotic Microlepidoptera, 5:96. (c) \\
\hline & CHOLOTIS & I9I5, Exotic Microlepidoptera, I: 330. (Bм) \\
\hline lia & ODITES & I928, Exotic Microlepidoptera, 3: 434. (вм) \\
\hline & OPOGONA & I9II, Journ. Bombay Nat. Hist. Soc. 2I: I I I. \\
\hline & PLOCAMOSARIS* & I9I2, Trans. Ent. Soc. London, I9I I: 706. (Bм) \\
\hline & LEUCOPTERA & I9I5, Exotic Microlepidoptera, I: 346 \\
\hline & ORNEODES & I9I I, Journ. Bombay Nat. Hist. Soc. 21 : Io6. \\
\hline actis & SCYTHRIS & I933, Exotic Microlepidoptera, 4: 369. \\
\hline hela & ACROLOPHUS & I93I, Exotic Microlepidoptera, 4: ro3. (v) \\
\hline & COSMOPTERYX & I9o9, Journ. Bombay Nat. Hist. Soc. I9: 4I2. \\
\hline ana & DICHELIA & I88I, Proc. Linnean Soc. N.S. Wales, 6: 480. \\
\hline & IMMA & I9o6, Trans. Ent. Soc. London, I906: I9o. (вм) \\
\hline ista & TRICHOBOSCIS* & I929, Exotic Microlepidoptera, 3: 526. (BM) \\
\hline & CHOROPLECA & I9I7, Exotic Microlepidoptera, 2:8r. \\
\hline ches & STATHMOPODA & I897, Proc. Linnean Soc. N.S. Wales, 22: 321. \\
\hline & EULECHRIA & I883, Proc. Linnean Soc. N.S. Wales, 7: 520. \\
\hline erina & EPAGOGE & I9o8, Journ. Bombay Nat. Hist. Soc. I8: 6I8. (в \\
\hline eropa & BRACHMIA & I9I3, Amn. Transvaal Mus. 3: 296. (т) \\
\hline genes & STENOMA & I930, Exotic Microlepidoptera, 4: 30. (v) \\
\hline mima & MICROCOLONA & I9I7, Exotic Microlepidoptera, 2: 51. \\
\hline & DEPRESSARIA & Ann. South African Mus. I7: 289 . \\
\hline
\end{tabular}




$\begin{array}{ll}\text { panxantha } & \text { COESYRA } \\ \text { paphia } & \text { ARISTOTELIA } \\ \text { paphlactis } & \text { GELECHIA } \\ \text { parabola } & \text { PTOCHORYCTIS } \\ \text { paracapna } & \text { STENOMA } \\ \text { parachalca } & \text { SCYTHRIS } \\ \text { parachlora } & \text { METATHEORA* } \\ \text { paraclasta } & \text { MELASINA } \\ \text { paraclina } & \text { PORTHMOLOGA* } \\ \text { paraclista } & \text { OCYSTOLA } \\ \text { paracma } & \text { POLYHYMNO } \\ \text { paraconta } & \text { THIOTRICHA } \\ \text { paracosma } & \text { MNESARCHAEA* } \\ \text { paracosma } & \text { SCARDIA } \\ \text { paracremna } & \text { EUCOSMA } \\ \text { paracrypta } & \text { ANTAEOTRICHA } \\ \text { paracta } & \text { STENOMA } \\ \text { paracycla } & \text { COESYRA } \\ \text { paracyrta } & \text { XYLORYCTA } \\ \text { paradelpha } & \text { TORTYRA } \\ \text { paradelta } & \text { EUCOSMA } \\ \text { paradema } & \text { PSEUDODOXIA } \\ \text { paraderces } & \text { COESYRA } \\ \text { paradesma } & \text { ISOCHASTA* } \\ \text { paradisia } & \text { MACAROSTOLA } \\ \text { paradromis } & \text { AGRIOPHARA } \\ \text { paraglypta } & \text { ARCHYALA* } \\ \text { paraglypta } & \text { EUCOSMA } \\ \text { paraglyptis } & \text { PLATYPTILIA } \\ \text { Paragrama } & \text { XENOTHICTIS* } \\ \text { ARGYROPLOCE } \\ \text { COESYRA }\end{array}$

[HAPAloteucha*] I884, Proc. Linnean Soc. N.S. Wales, 9: 766. paragraphopis

$\begin{array}{ll}\text { paragrapta } & \text { PHRIXOSCELES } \\ \text { paraleuca } & \text { ARISTOTELIA } \\ \text { paralia } & \text { PROTOMACHA } \\ \text { paralia } & \text { TORTRIX } \\ \text { parallactis } & \text { TORODORA } \\ \text { paralonoma } & \text { TINEA } \\ \text { paraloxa } & \text { CAPUA } \\ \text { paraloxa } & \text { EURYTHECTA } \\ \text { paralyrgis } & \text { HELIOCAUSTA } \\ \text { paramima } & \text { TINEOLA } \\ \text { paramochla } & \text { STENOMA } \\ \text { paraneura } & \text { SEMIOCOSMA } \\ \text { paranthes } & \text { DICHOMERIS } \\ \text { paranympha } & \text { BRENTHIA } \\ \text { paraphanes } & \text { HAPLOPHYLAX* }\end{array}$

I884, Proc. Linnean Soc. N.S. Wales, 9: 784. I9I7, Trans. Ent. Soc. London, I9I7: 34. (BM) I9I2, Trans. Ent. Soc. London, I9I I: 693. (BM) I9I4, Journ. Bombay Nat. Hist. Soc. 22: 778 . (BM) I9I5, Exotic Microlepidoptera, I: 444. (BM) I9I6, Exotic Microlepidoptera, 2: I4. I9I9, Exotic Microlepidoptera, 2: 229. (T) I922, Exotic Microlepidoptera, 2: 605. (P) I9I4, Exotic Microlepidoptera, I:26I. (BM) I9I3, Exotic Microlepidoptera, I: II6. Igo9, Ann. Transvaal Mus. 2: I5, pl. 5, fig. 5. (T) I904, Proc. Linnean Soc. N.S. Wales, 29: 295. I886, Trans. Proc. New Zealand Inst. I8: I8o. Igo8, Proc. Zool. Soc. London, p. 753.

I9I3, Ann. Transvaal Mus. 3:275. (T)

I9I5, Exotic Microlepidoptera, I: 405. (BM) I9I5, Exotic Microlepidoptera, I: 425. (BM) I884, Proc. Linnean Soc. N.S. Wales, 9: 777. I905, Journ. Bombay Nat. Hist. Soc. I6: 602. (BM) I907, Proc. Linnean Soc. N.S. Wales, 32: 98. I925, Exotic Microlepidoptera, 3: I4I. (T) I9I4, Exotic Microlepidoptera, I: 264. (BM) I889, Proc. Linnean Soc. N.S. Wales, (Ser. 2) 3: I659. I886, Trans. Proc. New Zealand Inst. I8: I63. Igo8, Journ. Bombay Nat. Hist. Soc. I8: 826 . I9I5, Exotic Microlepidoptera, I : 408. (BM) I889, Trans. Proc. New Zealand Inst. 21: I59. I9I2, Ent. Mon. Mag. 48: 34. (Sub.) Igo8, Trans. Ent. Soc. London, Igo7: 484. I9Io, Proc. Linnean Soc. N.S. Wales, 35:280. Igog, Journ. Bombay Nat. Hist. Soc. I9: 598. (Bм) I937, in Caradja and Meyrick, Deuts. Ent. Zeit. Iris, 51: I 8 I.

I9I5, Trans. Ent. Soc. London, I9I5: 223. I929, Exotic Microlepidoptera, 3:482. (BM) I9I3, Exotic Microlepidoptera, I: I22. I9Io, Proc. Linnean Soc. N.S. Wales, 35: 243. I894, Trans. Ent. Soc. London, I894: I7. (BM) I928, Exotic Microlepidoptera, 3: 427. (BM) I9Io, Proc. Linnean Soc. N.S. Wales, 35: 200. (MA) I907, Trans. New Zealand Inst. 39: II6. I883, Proc. Linnean Soc. N.S. Wales, 7: 479. I9I7, Exotic Microlepidoptera, 2: 96. I9I8, Exotic Microlepidoptera, 2: 207. (BM) 1892, Trans. Proc. New Zealand Inst. 24: 2 I9. I936, Exotic Microlepidoptera, 5: 47. (BM) I9I2, Exotic Microlepidoptera, I: 53. (BM) I932, Exotic Microlepidoptera, 4: 3I5. 


paraphracta
paraphragma
paraphrictis
paraplecta
parapleura
paraptera
parapteryx
paraptila
paraschista
parascia

parasema

parasema

parasira

parasitica

parastacta

parastactis

parasticta

parasticta

parastrepta

parastropha

paratarsa

parathicta

paratma

paratrimma

paratrimma

paraula

paraxantha

paraxena

parazela

parazeucta

parazona

parca

parcens

pardalea

pardalias

pardalodes

pardalota

paredra

paredraea

parichniota

parichnota

parilis

pariodelta

parmata

parmifera

parmiferana

parmulata

parochra
PROCELEUSTIS*

POLYCHROSIS

MELASINA

STENOMA

GELECHIA

SCHOENOTENES

ADENONEURA

IDIOPHANTIS

LIOCROBYLA*

SYNTOMACTIS

[LEPTOZESTIS*]

ARGYROPLOCE

BRACHMIA

ANAPTILORA

EPERMENIA

OPSIGENES*

CAPUA

GRACILARIA

SPHALERACTIS

LASPEYRESIA

ADOXOPHYES

ANTICRATES

DIADOXASTIS*

IMMA

BORKHAUSENIA

BORKHAUSENIA

TELPHUSA

ANTICRATES

TINEA

BATTARISTIS

PROMALACTIS

GLYPHIPTERYX

ALUCITA

BASANASCA *

HAPSIFERA

MELEONOMA

STENOMA

HETEROGYMNA

SCYTHRIS

CACOECIA

CHELARIA

LASPEYRESIA

AGRIOPHARA

ANTISPILA

COMPSOLECHIA

COMOTECHNA

CAPUA

COMMATICA

TIMYRA
I9I4, Exotic Microlepidoptera, I: 268.

1922, Exotic Microlepidoptera, 2: 522. (P)

I908, Proc. Zool. Soc. London, p. 744.

I925, Exotic Microlepidoptera, 3: 2I4. (BM)

I886, Trans. Proc. New Zealand Inst. I8: I68.

I9Io, Trans. Ent. Soc. London, I9I0: 433. (BM)

I932, Exotic Microlepidoptera, 4:222. (BM)

I9I6, Exotic Microlepidoptera, I: 566. (вм)

I9I6, Exotic Microlepidoptera, 2: 5 .

I897, Proc. Linnean Soc. N.S. Wales, 22: 385.

I9I I, Proc. Linnean Soc. N.S. Wales, 36: 267.

I9I3, Exotic Microlepidoptera, I: 66. (BM)

I9I6, Exotic Microlepidoptera, I: 59I.

I930, Exotic Microlepidoptera, 3: 626. (BM)

I918, Ann. Transwaal Mus. 6: 30. (T)

I9Io, Proc. Linnean Soc. N.S. Wales, 35: I86.

I908, Journ. Bombay Nat. Hist. Soc. I8: 828.

I904, Proc. Linnean Soc. N.S. Wales, 29: 328.

I907, Journ. Bombay Nat. Hist. Soc. I8: I47. (BM)

I9I2, Exotic Microlepidoptera, I: 3. (BM)

I9I3, Exotic Microlepidoptera, I: I4I. (BM)

I9I3, Exotic Micralepidoptera, I: 79 .

I9I2, Exotic Microlepidoptera, I: 40. (BM)

I9I0, Trans. New Zealand Inst. 42: 65.

I9II, Trans. New Zealand Inst. 43: 63.

I9I6, Exotic Microlepidoptera, I: 568. (BM)

I907, Proc. Linnean Soc. N.S. Wales, $32: 85$.

I908, Proc. Zool. Soc. London, p. 740.

I929, Exotic Microlepidoptera, 3: 505. (BM)

I908, Journ. Bombay Nat. Hist. Soc. I8: 807. (BM)

1907, Proc. Linnean Soc. N.S. Wales, 32: 125.

I92 I, Exotic Microlepidoptera, 2: 42I. (BM)

I922, Exotic Microlepidoptera, 2: 594.

I9o8, Proc. Zool. Soc. London, p. 749.

I93I, Exotic Microlepidoptera, 4: I9I. (BM)

I9I8, Exotic Microlepidoptera, 2: 207. (BM)

I922, Exotic Microlepidoptera, 2: 55I. (BM)

1897, Proc. Linnean Soc. N.S. Wales, 22: 425.

I93I, Exotic Microlepidoptera, 4: I49. (BM)

I938, in Caradja and Meyrick, Deuts. Ent. Zeit. Iris, 52: 4.

(BM)

I921, Zool. Meded. 6: I60. (L)

I9I8, Exotic Microlepidoptera, 2: I97. (BM)

I929, Exotic Microlepidoptera, 3: 54I.

I9I8, Exotic Microlepidoptera, 2: I39. (BM)

I92I, Exotic Microlepidoptera, 2: 40I. (BM)

I88I, Proc. Limnean Soc. N.S. Wales, 6: 534. (MA)

I9I4, Trans. Ent. Soc. London, I9I4: 24I. (BM)

I906, Journ. Bombay Nat. Hist. Soc. I7: I46. (BM) 


\begin{tabular}{|c|c|}
\hline paroctas & CHELARIA \\
\hline parodaula & CEROSTOMA \\
\hline paroditis & PYRODERCES \\
\hline paroena & MACROTONA \\
\hline parolca & MACAROSTOLA \\
\hline paromias & OPOSTEGA \\
\hline paronyma & PHALONIA \\
\hline paropis & PYRODERCES \\
\hline paropta & STENOMA \\
\hline paroristis & BRACHMIA \\
\hline parorma & THERAPNIS* \\
\hline parorthota & CAPUA \\
\hline paroxynta & GELECHIA \\
\hline parthenia & STREPSICRATES \\
\hline parthenica & COLEOPHORA \\
\hline parthenica & THIOTRICHA \\
\hline parthenistis & MACROBATHRA \\
\hline parthenopa & HELIOCAUSTA \\
\hline parthenopis & LECITHOCERA \\
\hline particeps & ACROLOPHUS \\
\hline particulata & CHELARIA \\
\hline partiseca & STENOPTILIA \\
\hline parvisignana & STIGMONOTA \\
\hline parvisignis & EULIA \\
\hline parvula & COESYRA \\
\hline paryphaea & SPATALISTIS \\
\hline passaleuta & EPICHORISTA \\
\hline passalias & PHILOBOTA \\
\hline passalota & SABATINCA \\
\hline passalotana & ACROPOLITIS \\
\hline passiva & EUCOSMA \\
\hline pastas & TIMYRA \\
\hline pastor & BATRACHEDRA \\
\hline pastoralis & TINEA \\
\hline patarea & CHRESMARCHA \\
\hline patellata & ACROCERCOPS \\
\hline patellatus & TITANOPTILUS \\
\hline patellifera & STENOMA \\
\hline patens & STENOMA \\
\hline paterata & ARISTOTELIA \\
\hline paterata & CACOECIA \\
\hline pateropa & CRYPTOLECHIA \\
\hline patiens & SCYTHRIS \\
\hline patria & ORNEODES \\
\hline patriarcha & PLATYPTILIA \\
\hline patricia & ACROCERCOPS \\
\hline patriocosma & HEMIMENE \\
\hline & ELACHISTA \\
\hline
\end{tabular}

I9I3, Journ. Bombay Nat. Hist. Soc. 22: I66. (Вм) I938, in Caradja and Meyrick, Dents. Ent. Zeit. Iris, 52: 23.

1928, Exotic Microlepidoptera, 3: 390. (BM) Igo6, Journ. Bombay Nat. Hist. Soc. I7: I48. (вм)

I9II, Trans. Linnean Soc. London, I4: 292.

I9I5, Trans. Ent. Soc. London, I9I5: 240.

I932, Exotic Microlepidoptera, 4: 266.

1915, Exotic Microlepidoptera, I: 310. I9I6, Exotic Microlepidoptera, I: 536. (Bм) I9II, Journ. Bombay Nat. Hist. Soc. 20: 718. (ВM) I9Io, Journ. Bombay Nat. Hist. Soc. 20: I45. (BM) 1928, Exotic Microlepidoptera, 3: 453.

I931, Exotic Microlepidoptera, 4: 59. (BM) 1888, Trans. Proc. New Zealand Inst. 20: 73. I89I, Ent. Mon. Mag. 27: 59.

I904, Proc. Linnean Soc. N.S. Wales, 29: 297. 1889, Proc. Linnean Soc. N.S. Wales, (Ser. 2) 3: 1678. 1883, Proc. Linnean Soc. N.S. Wales, 7: 48I. 1932, Exotic Microlepidoptera, 4: 204. (BM) I913, Trans. Ent. Soc. London, I913: I97. (BM) I9I3, Journ. Bombay Nat. Hist. Soc. 22: г67. (вм) 1931, Ann. Mus. Nac. Hist. Nat. Buenos Aires, 36: 380. I88I, Proc. Linnean Soc. N.S. Wales, 6: 654. 1931, Exotic Microlepidoptera, 4: 152. (v) I884, Proc. Linnean Soc. N.S. Wales, 9: 783 . 1907, Journ. Bombay Nat. Hist. Soc. I7: 978. (ВM) I920, Voyage de Ch. Alluaud et R. Jeannel en Afrique

Oriental, II, Microlepidoptera, p. 53. (P)

I9I3, Exotic Microlepidoptera, I : I22.

I923, Trans. Proc. New Zealand Inst. 54: I69. I88I, Proc. Linnean Soc. N.S. Wales, 6: 436. I913, Ann. Transvaal Mus. 3: 273. (T) I908, Journ. Bombay Nat. Hist. Soc. I8: 444. (BM) 1936, Exotic Microlepidoptera, 5: 31.

I93I, Exotic Microlepidoptera, 4: 106. I924, Exotic Microlepidoptera, 3: IIo. I92I, Exotic Microlepidoptera, 2: 4I5. (BM) I9I3, Exotic Microlepidoptera, I: Iog. I93I, Exotic Microlepidoptera, 4: 34. (v) I9I3, Trans. Ent. Soc. London, I9I3: I89. (ви) I9I4, Trans. Ent. Soc. London, I9I4: 229. I9I4, Supplementa Entomologica, No. 3, p. 47. (D) I931, Exotic Microlepidoptera, 4: I25. (v) I92I, Ann. Transvaal Mus. 8: II6. (T) I92I, Exotic Microlepidoptera, 2: 408. I9I2, Ann. South African Mus. Io: 54. (SA) I908, Joutr. Bombay Nat. Hist. Soc. I8: 825. I927, Exotic Microlepidoptera, 3: 34I. (BM) I932, Exotic Microlepidoptera, 4: 216. (BM) 


\begin{tabular}{|c|c|c|}
\hline patrona & CASMARA & $\begin{array}{l}\text { I925, in Caradja, Mem. Sect. Sti. Acad. Românā, 3: 38I. } \\
\text { (BM) }\end{array}$ \\
\hline patula & STENOMA & I9I6, Exotic Microlepidoptera, I: 526. (вм) \\
\hline paulina & ACROCLITA & I925, Exotic Microlepidoptera, 3: I40. (BM) \\
\hline paurocentra & STENOMA & I9I2, Trans. Ent. Soc. London, I9II: 7I3. (BM) \\
\hline pauroconis & STENOMA & I932, Exotic Microlepidoptera, 4: 303. (v) \\
\hline paurodes & YPONOMEUTA & I907, Proc. Limnean Soc. N.S. Wales, 32: I50. \\
\hline aurogramma & EULECHRIA & I883, Proc. Linnean Soc. N.S. Wales, 7: 542 . \\
\hline pauroleuca & TRACHYRRHOPALA* & I926, Sarawak Mus. Journ. 3: I68. \\
\hline paurosperma & MYRMECOZELA & I926, Ann. South African Mus. 23: 342. (SA) \\
\hline pausimacha & POLYHYMNO & Igog, Ann. Transvaal Mus. 2: I4, pl. 5, fig. 3. (T) \\
\hline pavida & DEPRESSARIA & I9I3, Exotic Microlepidoptera, I: II4. (BM) \\
\hline pavidus & PTEROPHORUS & I9o8, Trans. Ent. Soc. London, I907: 493. \\
\hline pavimentata & ATTERIA & I9I3, Trans. Ent. Soc. London, I9I3: I7I. (BM) \\
\hline eectinata & ARDIOSTERES & I9I7, Exotic Microlepidoptera, 2: 9o. \\
\hline losa & NEMOTOIS & I92I, Zool. Meded. 6: 202. (L) \\
\hline pedaliota & CAPUA & 1936, Exotic Microlepidoptera, 5: 58. (вм) \\
\hline pedetis & PHILOBOTA & I884, Proc. Linnean Soc. N.S. Wales, 8: 488. \\
\hline is & POLYCHROSIS & I920, Exotic Microlepidoptera, 2: 347. (BM) \\
\hline ata & ODITES & I9I4, Ann. Transvaal Mus. 4: I98. (T) \\
\hline pedicata & SCHIFFERMUELLERIA & A I9I8, Ann. Transvaal Mus. 6:29. (T) \\
\hline pegaea & HYBROMA & I9I9, Exotic Microlepidoptera, 2: 24I. (BM) \\
\hline nitis & PHALONIA & I912, Ent. Mon. Mag. 48: 35. (Sub.) \\
\hline pelaea & AUTOSTICHA & Igo8, Journ. Bombay Nat. Hist. Soc. I8: 456. (Bм) \\
\hline pelanodes & NEPTICULA & $\begin{array}{l}\text { I920, Voyage de Ch. Alluaud et } R \text {. Jeannel en Afrique } \\
\text { Oriental, II, Microlepidoptera, p. II6. (P) }\end{array}$ \\
\hline pelargodes & SIMAETHIS & I921, Zool. Meded. 6: I83. (L) \\
\hline pelasta & CHOLOTIS & I9I5, Exotic Microlepidoptera, I: 329. (BM) \\
\hline pelecyntes & OXYPTILUS & I908, Trans. Ent. Soc. London, I907: 477. \\
\hline pelias & TRICHOPTILUS & I908, Trans. Ent. Soc. London, 1907: 472. \\
\hline pelina & EUCOSMA & I9I2, Ent. Mon. Mag. 48: 35. (Sub.) \\
\hline pelinactis & IMMA & I925, Exotic Microlepidoptera, 3: I33. \\
\hline aula & SCYTHRIS & I9I6, Exotic Microlepidoptera, 2: I4. \\
\hline pelinitis & STENOMA & I9I2, Trans. Ent. Soc. London, I9II: 7I4. (BM) \\
\hline pelinoma & OPOGONA & I93I, Exotic Microlepidoptera, 4: I64. \\
\hline pelinopa & MACHAERITIS & I902, Trans. R. Soc. S. Australia, 26: I33. \\
\hline pelinopis & COLEOPHORA & 1933, Exotic Microlepidoptera, 4:364. (v) \\
\hline pelitis & TRICHOTAPHE & I9I3, Journ. Bombay Nat. Hist. Soc. 22: I79. (BM) \\
\hline pellax & BRACHMIA & I9I I, Journ. Bombay Nat. Hist. Soc. 20: 7І 3. (вм) \\
\hline pellocoma & APHANOXENA* & I9I5, Exotic Microlepidoptera, I: 387 . (BM) \\
\hline peloceros & LECITHOCERA & I938, in Caradja and Meyrick, Deuts. Ent.Zeit.Iris, 52:86. \\
\hline pelochares & CRYPTOLECHIA & I934, Exotic Microlepidoptera, 4: 479. (вм) \\
\hline pelochlora & TINEOLA & $\begin{array}{l}\text { I920, Voyage de Ch. Alluand et R. Jeannel en Afrique } \\
\text { Oriental, II, Microlepidoptera, p. IOI. (P) }\end{array}$ \\
\hline pelochroa & XYSMATODOMA & 1893, Proc. Limnean Soc. N.S. Wales, 7: 501. \\
\hline pelochrosta & ODITES & I933, Exotic Microlepidoptera, 4: 434. (BM) \\
\hline pelochyta & SCYTHRIS & I9o9, Ann. Transvaal Mus. 2: 22, pl. 7, fig. 5. (T) \\
\hline pelochytana & ATELODORA* & I881, Proc. Linnean Soc. N.S. Wales, 6: 427 . \\
\hline pelocnista & GAESA & I939, Trans. R. Ent. Soc. London, 89: 55. (BM) \\
\hline pelocrossa & OPOSTEGA & I928, Exotic Microlepidoptera, 3:396. (T) \\
\hline
\end{tabular}


pelodelta

pelodes

pelodora

pelodoxa

pelogenes

pelogramma

pelograpta

pelomacta

pelomicta

pelomorpha

pelophaea

peloplaca

peloptera

peloptila

pelorrhoa

pelospila

pelospora

pelosticta

pelostrota

pelota

pelotricha

peloxantha

peloxythana

peltastes

peltastica

peltastis

peltata

peltias

peltophanes

peltophora

pelurga

pemphigodes

pendens

pendula

pendulata

penelope

penessa

penetralis

penetralis

penetrans

penetrata

penetratrix

penographa

pensilis

pensilis

pentacarpa

pentacentra
MACHAEROPTERIS

AUTOMOLA*

[AUTOSTICHA*]

EULECHRIA

MELASINA

EUTORNA

PROTOLECHIA

MERIDARCHIS

IMMA

STOMOPTERYX

LECITHOCERA

CRYPTOLECHIA

AMYDRIA

CLADARODES*

AGRIASTIS*

OPOSTEGA

TORTRIX

SYMMOCA

HELIOCAUSTA

MELASINA

PANCOENIA

DECADARCHIS

PLEUROTA

TORTRIX

OXYPTILUS

ARGYROPLOCE

TYMBOPHORA*

AEGERIA

PERSICOPTILA

GRACILARIA

STERIPHOTIS*

IMMA

GRAPHICOPTILA*

PHTHORIMAEA

MACROSACES

ARGYROPLOCE

ERIOCHRYSIS*

EPISTOMOTIS*

HEMIMENE

OTOPTRIS

RECURVARIA

TINEA

TELPHUSA

ACROCERCOPS

ANARSIA

CACOECIA

OINOPHILA

PALTODORA
I93I, in Joannis, Ann. Soc. Ent. France, 98 (Supp.): $744[506]$. (P)

I883, Ent. Mon. Mag. 20: 34. (BM)

I888, Proc. Linnean Soc. N.S. Wales, (Ser, 2) 2: 944.

I928, Exotic Microlepidoptera, 3: 43I.

I9o6, Trans. R. Soc. S. Australia, 30: 45 .

I904, Proc. Linnean Soc. N.S. Wales, 29:367.

I929, Trans. Ent. Soc. London, 76:502. (BM)

1922, Exotic Microlepidoptera, 2: 484. (BM)

I928, Bull. Hill Mus. 2: 234. (BM)

I93I, in Caradja, Bull. Sect. sci. Acad. roum. I4: 69. (BM)

I93I, Exotic Microlepidoptera, 4: I92. (BM)

I9I7, Ann. South African Mus. I7: I6. (SA)

I9Io, Rec. Indian Mus. 2: 230. (K)

I9I4, Trans. Ent. Soc. London, I9I4: 25I. (BM)

I9I5, Exotic Microlepidoptera, I: 352.

I932, Exotic Microlepidoptera, 4:255. (BM)

I927, Exotic Microlepidoptera, 3: 356.

I883, Proc. Linnean Soc. N.S. Wales, 7: 473.

1927, Exotic Microlepidoptera, 3: 328. (T)

I904, Proc. Linnean Soc. N.S. Wales, 29: 390.

I926, Trans. Ent. Soc. London, 74: 275.

I884, Proc. Linnean Soc. N.S. Wales, 9: 753.

I88I, Proc. Linnean Soc. N.S. Wales, 6: 5I4.

I908, Trans. Ent. Soc. London, I907: 479.

I92I, Ann. Transvaal Mus. 8: 57. (T)

I89o, Trans. R. Soc. S. Australia, I3: 56.

I932, Exotic Microlepidoptera, 4: 230.

I897, Proc. Linnean Soc. N.S. Wales, 22: 330.

I907, Proc. Linnean Soc. N.S. Wales, 32: 67.

I9I , Proc. Linnean Soc. N.S. Wales, 36: 259.

I921, Zool. Meded. 6: I8o. (L)

I93I, Exotic Microlepidoptera, 4: 97.

I9I8, Ann. Transvaal Mus. 6: I8. (T)

I9Io, Journ. Bombay Nat. Hist. Soc. 20: I 47. (Bм)

I9I I, Proc. Linnean Soc. N.S. Wales, 36: 279.

I937, Exotic Microlepidoptera, 5: I 55.

I906, Journ. Bombay Nat. Hist. Soc. I7: 4I6.

I9I6, Exotic Microlepidoptera, 2: 24. (BM)

I9I5, Trans. Ent. Soc. London, I9I5: 245 .

I923, Exotic Microlepidoptera, 3: I3. (BM)

I9I2, Ann. Transvaal Mus. 3: 8o. (T)

I93I, Journ. Linnean Soc. London, 37: 279. (BM)

I920, Exotic Microlepidoptera, 2: 294. (BM)

I9I3, Journ. Bombay Nat. Hist. Soc. 22: I68. (BM)

I920, Exotic Microlepidoptera, 2: 339. (BM)

I9I0, Trans. Ent. Soc. London, I9I0: 376.

I9I2, Ann. South African Mus. Io: 59. (SA) 


pentachora
pentachorda
pentachrysis
pentaclosta
pentacyba
pentacycla
pentadesma
pentadisca
pentadora
pentagama
pentagonaspis
pentagramma
pentalitha
pentalocha
pentalychna
pentameris

pentametra pentanoma pentaphylactis pentaplaca pentapyrga pentarcha pentargyra pentasema pentaspila pentasticta pentasticta pentastra pentathlopa pentatma pentatypa

pentaula pentaxantha pentazona pentazyga penthalea penthesilea penthicodes pentulias pepantica

peperistis peperitis peplophanes peplotoma peracuta
PERIACMA COSMOPTERYX LIMNOECIA PROMALACTIS CHOREUTIS ACROCERCOPS LITHOCOLLETIS MONOPIS GELECHIA STAGMATOPHORA EUCOSMA CALLIPRORA* ANTISPILA ACROCERCOPS LASPEYRESIA ARISTOTELIA

TINEA ARGYRESTHIA GRACILARIA ACROCERCOPS MOMPHA CRYPHIOMYSTIS* MICTOPSICHIA STATHMOPODA TINEA AULOTROPHA* LASPEYRESIA COMPSOLECHIA CRYPTOLECHIA MYRMECOZELA DECADARCHIS

PLUTELLA ERETMOCERA SESIA ARCHYALA BORKHAUSENIA [OECOPHORA] LYONETIA PROTOLECHIA IPHIERGA LECITHOCERA

GELECHIA PRAYS CALLIATHLA*

LOBESIA MEGACRASPEDUS
I9Io, Journ. Bombay Nat. Hist. Soc. 20: 158. (BM)

I9I5, Trans. Ent. Soc. London, I9I5: 203. (BM)

I93I, Exotic Microlepidoptera, 4:54.

I922, Exotic Microlepidoptera, 2: 542. (BM)

I927, Bull. Soc. R. Ent. Egypte, I926, p. I Iо.

I934, Exotic Microlepidoptera, 4:472.

I9I9, Exotic Microlepidoptera, 2: 230. (BM)

I924, Exotic Microlepidoptera, 3: 7I. (BM)

I9I7, Trans. Ent. Soc. London, I9I7: 5I. (BM)

I928, Exotic Microlepidoptera, 3: 388. (T)

I93I, Exotic Microlepidoptera, 4: I44. (BM)

I9I4, Trans. Ent. Soc. London, I9I4: 243. (BM)

1916, Exotic Microlepidoptera, 2: 9.

I9I2, in Wytsman, Genera Insectorum, fasc. I28, p. I5. I938, in Caradja and Meyrick, Deuts. Ent. Zeit. Iris, 52: 2. I93I, in Caradja, Bull. Sect. sci. Acad. roum. I4: 66. (BM)

I92I, Ann. Transvaal Mus. 8: I28. (T)

I9I3, Ann. Transvaal Mus. 3: 3I8. (T)

I938, Trans. R. Ent. Soc. London, 87:525.

I9I I, Trans. Linnean Soc. London, I4: $29 \mathrm{I}$.

1922, Exotic Microlepidoptera, 2: 577. (BM)

I922, Exotic Microlepidoptera, 2: 563. (BM)

I92I, Exotic Microlepidoptera, 2: 478. (BM)

I9I3, Exotic Microlepidoptera, I: 84.

I9I6, Exotic Microlepidoptera, I: 603. (MA)

I9I8, Ann. Transvaal Mus. 6:32. (T)

I9I6, Exotic Microlepidoptera, 2: 22. (BM)

I922, Trans. Ent. Soc. London, I922: 8I. (BM)

I933, Exotic Microlepidoptera, 4: 369. (v)

I936, Exotic Microlepidoptera, 5: 53 .

I920, Voyage de Ch. Alluand et R. Jeannel en Afrique

Oriental, II, Microlepidoptera, p. 96. (P)

I9I3, Exotic Microlepidoptera, I: I52.

1929, Exotic Microlepidoptera, 3: 543. (BM)

I9I8, Exotic Microlepidoptera, 2: I80.

I9I5, Trans. Droc. New Zealand Inst. 47: 204.

I905, Trans. Ent. Soc. London, I905: 239.

I92I, Exotic Microlepidoptera, $2: 457$.

I92I, Exotic Microlepidoptera, 2: 427. (BM)

I893, Proc. Linnean Soc. N.S. Wales, 7: 5IS.

I934, in Caradja and Meyrick, Deuts. Ent. Zeit. Iris, 4S: 36.

I926, Exotic Microlepidoptera, 3: 277. (BM)

I907, Journ. Bombay Nat. Hist. Soc. I7: 984. (вм)

I93I, Ann. Mus. Nac. Hist. Nat. Buenos Aires, 36: 405. (BM)

I928, Exotic Microlepidoptera, 3: 442. (BM)

I920, Ann. S. African Mus. I7:28I. 


$\begin{array}{ll}\text { peraea } & \text { EUCOSMA } \\ \text { peraeota } & \text { MACROBATHRA } \\ \text { peragrata } & \text { CRASIMORPHA } \\ \text { percastis } & \text { AMYDRIA } \\ \text { percnacma } & \text { VAZUGADA } \\ \text { percnaula } & \text { SCAEOSOPHA } \\ \text { percnitis } & \text { IZATHA } \\ \text { percnobathra } & \text { ANCYLIS } \\ \text { percnobela } & \text { BRACHMIA } \\ \text { percnocarpa } & \text { STENOMA } \\ \text { percnoceros } & \text { COLEOPHORA } \\ \text { percnochlaena } & \text { ARGYROPLOCE } \\ & \\ \text { percnocoma } & \text { CRYPTOLECHIA } \\ \text { percnodes } & \text { OPOGONA } \\ \text { percnogona } & \text { ANTAEOTRICHA } \\ \text { percnoleuca } & \text { GLYPHIDOCERA } \\ \text { percnombra } & \text { LIOPYCNAS* } \\ \text { percnomicta } & \text { DECADARCHIS } \\ \text { percnopa } & \text { HELIOSTIBES } \\ \text { percnophanes } & \text { ERETMOCERA } \\ \text { percnophylla } & \text { COLLOGENES* } \\ \text { percnoptila } & \text { PHTHEOCHROA } \\ \text { percnorma } & \text { EPIMORYCTIS* } \\ \text { percnoscia } & \text { HOLCOCERA } \\ \text { percnospila } & \text { ANACAMPSIS } \\ \text { percnotoxa } & \text { ATELOSTICHA } \\ \text { perdita } & \text { EULECHRIA } \\ \text { perducta } & \text { AGRIOPHARA } \\ \text { peregrina } & \text { DIPLODOMA } \\ \text { perfida } & \text { LECITHOCERA } \\ \text { perfidiosa } & \text { PHTHORIMAEA } \\ \text { perfixa } & \text { BUCCULATRIX } \\ \text { perflavescens } & \text { PHALONIA } \\ & \end{array}$

perflua perforata perfossa perfracta perfrenis perfricta perfuga perfumata perfusa periacta perianthes periarga periastra periastra periaula
I9I I, Proc. Linnean Soc. N.S. Wales, 36: 247.

I92I, Ann. Transvaal Mus. 8: 98. (T) I923, Exotic Microlepidoptera, 3: 33. (BM) Igo8, Proc. Zool. Soc. London, p. 753. I923, Exotic Microlepidoptera, 2: 623. (Bм) I9I4, Exotic Microlepidoptera, I: 254. (BM) I9o9, Trans. New Zealand Inst. 4I: I4. I933, Exotic Microlepidoptera, 4: 4I7. I9I I, Journ. Bombay Nat. Hist. Soc. 20: 723. (BM) 1925, Exotic Microlepidoptera, 3: I8I. (BM) I933, Exotic Microlepidoptera, 4:365. I938, Institut des Parcs Nationaux du Congo Belge, fasc. I4, p. IO. (c)

I930, Exotic Microlepidoptera, 3: 578. I9Io, Rec. Indian Mus. 2: 230. (K) I925, Exotic Microlepidoptera, 3: I70. (вм) I923, Exotic Microlepidoptera, 3: 48. (BM) I937, Exotic Microlepidoptera, 5: II3. I934, Pacific Ent. Surv. Publ. 7, art. 28, p. 355. (BB) I922, Ent. Mitteil. II: 47. (D) I929, Exotic Microlepidoptera, 3: 543. I93I, Exotic Microlepidoptera, 4: I42. I933, Exotic Microlepidoptera, 4: 446. (c) I930, Exotic Microlepidoptera, 3: 6I8. (BM) I932, Exotic Microlepidoptera, 4: 3I7: (v) I9I4, Trans. Ent. Soc. London, I9I4: 266. (BM) I920, Exotic Microlepidoptera, 2: 379. (BM) I883, Proc. Linnean Soc. N.S. Wales, 7: 547. I9I6, Exotic Microlepidoptera, I: 484. (BM) I9I9, Exotic Microlepidoptera, 2: 262. I9I8, Exotic Microlepidoptera, 2: I05. (BM) I9I7, Trans. Ent. Soc. London, I9I7: 4I. (BM) I9I5, Exotic Microlepidoptera, I: 354. I934, in Caradja and Meyrick, Deuts. Ent. Zeit. Iris, 48: 29.

I9I4, Exotic Microlepidoptera, I: 205. (MA) I9I I, Ann. Transvaal Mus. 3: 80. I9I7, Trans. Ent. Soc. London, I9I7: 33. (BM) I922, Exotic Microlepidoptera, 2: 493. (BM) 1920, Exotic Microlepidoptera, 2: $36 \mathbf{r}$. I920, Exotic Microlepidoptera, 2: 352. (BM) 1926, Trans. Ent. Soc. London, 74: 274. I927, Insects of Samoa, 3, Lepidoptera, fasc. 2, p. II2. I9I6, Exotic Microlepidoptera, I : 504. (BM) I9IO, Ann. South African Mus. 5: 4II. (SA) I922, Exotic Microlepidoptera, 2: 545. (P) I913, Ann. Transvaal Mus. 3:267. (T) I907, Journ. Bombay Nat. Hist. Soc. I7: 978. (BM) I9I o, Journ. Bombay Nat. Hist. Soc. 20: 454. I9I6, Exotic Microlepidoptera, I: 5Io. (BM) 
pericentra pericentrota perichalca pericharacta perichlora perichlora periclina periclyta pericrossa periculosa periculosa pericyclota pericyphana peridesma peridoxa perigramma perigrapta perigypsa perilithas perinyctis periochra periodica perioditis periopa periopta periorma periphanes periphora periphracta periphrictis periploca perirrhoa periscelta perischias periscia periscias perisepta perisphena perissarcha perisseuta perissopa perissopis perissosema peristera peristicta peristictis peristrepta peristylis peritoma peritura
HESPERARCHA* ETHMIA

HELIODINES HOMALOPSYCHA BORKHAUSENIA HOMALOXESTIS STATHMOPODA PETALANTHES MELASINA COESYRA LASPEYRESIA MACHIMIA STREPSICEROS STENOMA PROTANYSTIS PTOCHORYCTIS CROCANTHES LECITHOCERA POLYMNESTRA* HAPLODYTA PACHNISTIS BAREA MACHIMIA CAPUA MICTOPSICHIA CNEPHASIA ARISTAEA * PANCOENIA* LEUCOPTERA STENOMA IMMA STENOMA STENOMA URODUS LOCHEUTIS ODITES

TINEA GRACILARIA ACROLOPHUS MALLOBATHRA NEPTICULA ODITES

GELECHIA HOPLOPHANES EUCOSMA

PAMMENE PYRODERCES YPSOLOPHUS ADOXOPHYES CALIIPRORA
I9I8, Ann. Transvaal Mis. 6:38. (T)

I926, Ann. South African Mus. 23: 339. (SA)

I9I2, Exotic Microlepidoptera, I: 58.

I924, Exotic Microlepidoptera, 3: 70. (T)

I907, Trans. Proc. New Zealand Inst. 39: II8.

I9Io, Trans. Ent. Soc. London, I9Io: 446. (BM)

I938, Trans. R. Ent. Soc. London, 89: 520.

I883, Proc. Linnean Soc. N.S. Wales, 8: 337.

I907, Journ. Bombay Nat. Hist. Soc. I8: I58.

I9I3, Exotic Microlepidoptera, I: I20. (MA)

I9I3, Ann. Transvaal Mus. 3: 28o.

I920, Exotic Microlepidoptera, 2: 376. (BM)

I88I, Proc. Limnean Soc. N.S. Wales, 6: 685 .

I925, Exotic Microlepidoptera, 3:220. (BM)

I924, Exotic Microlepidoptera, 3:97. (BM)

I926, Sarawak Mus. Journ. 3: I6o.

I904, Proc. Linnean Soc. N.S. Wales, 29: 402.

I922, Zool. Meded. 7:85. (L)

I927, Exotic Microlepidoptera, 3: 33I. (T)

I889, Proc. Linnean Soc. N.S. Wales, (Ser. 2) 3: 1666.

I9I6, Exotic Microlepidoptera, I: 577.

I920, Exotic Microlepidoptera, 2: 308.

I928, Exotic Microlepidoptera, 3: 47I. (BM)

I9Io, Proc. Linnean Soc. N.S. Wales, 35: I84.

I9I3, Exotic Microlepidoptera, I: 99. (BM)

I9Io, Proc. Linnean Soc. N.S. Wales, 35:27I.

I907, Proc. Linnean Soc. N.S. Wales, 32: 52.

I904, Proc. Linnean Soc. N.S. Wales, 29: 380 .

I9I5, Exotic Microlepidoptera, I : 347.

I9I5, Exotic Microlepidoptera, I: 45I. (BM)

I9Io, Trans. Ent. Soc. London, I9Io: 466. (BM)

I930, Exotic Microlepidoptera, 4:28. (BM)

I9I5, Exotic Microlepidoptera, I: 457. (BM)

I925, Exotic Microlepidoptera, 3: I29. (BM)

I889, Proc. Linnean Soc. N.S. Wales, (Ser. 2) 3: I589.

I928, Exotic Microlepidoptera, 3: 434. (BM)

I922, Exotic Microlepidoptera, 2: 595. (P)

I905, Journ. Bombay Nat. Hist. Soc. I6: 613.

I9I9, Exotic Microlepidoptera, 2: 278. (BM)

I920, Trans. Proc. New Zealand Inst. 52: 32.

I9I9, Trans. Proc. New Zealand Inst. 5I: 354.

I936, Exotic Microlepidoptera, 5: 27. (BM)

I932, Exotic Microlepidoptera, 4:35I. (BM)

I897, Proc. Linnean Soc. N.S. Wales, 22: 4 I4.

I9I2, Ent. Mon. Mag. 48:34. (Sub.)

I9I2, Journ. Bombay Nat. Hist. Soc. 21:874. (BM).

I9I7, Exotic Microlepidoptera, 2: 39.

I904, Proc. Linnean Soc. N.S. Wales, 29: 43I.

I9I8, Exotic Microlepidoptera, 2: I67. (BM)

I922, Trans. Ent. Soc. London, I922: 70. (BM) 
perixantha perizeucta perjecta

perjura

perlucescens perlustrata

permagna

permeata

permissa

permota

permutata

perniciosa

pernocoma

perobtusa

peronacma

peronectis

peroneta

peronetris

peronodes

peropaca

perophora

perpensa

perpetua

perpetua

perquisita

perrupta

persecta

persephone

perseverans

persicopa

persistis

persita

personata

perspersa

perspicua

perspicua

perstricta

perterrita

pertexta

pertinax

pertinax

pertinens

perturbata

perumbrata

pervallata

perversa

perversa

pervicax

pesephena
AUTOSTICHA

HOMALOXESTIS

STENOMA

STENOMA

CTENOCOMPA

PYCNOSTOLA

COMPSOLECHIA

COESYRA

ANARSIA

MOMPHA

CACOECIA

HYLADAULA*

CRYPTOLECHIA

BATRACHEDRA

OTOCHARES

GELECHIA

TRACHYDORA

TIMYRA

LIMNOECIA

DICHELOPA

STENOMA

LECITHOCERA

ACROLOPHUS

EULECHRIA

ANACAMPSIS

LASPEYRESIA

EPICHORISTA

SCYTHRIS

TINEA

LACTURA

HYPERCALLIA

STENOMA

COESYRA

RHATHAMICTIS*

CRIMNOLOGA*

CYCLOPONYMPHA

ADOXOPHYES

ARISTOTELIA

ARGYROPLOCE

AROTROPHORA

STENOMA

GELECHIA

ACROCERCOPS

BRACHMIA

PROCHOLA

CRYPTOLECHIA

EPICHORISTA

EUCOSMA

PLEUROTA
I9I4, Exotic Microlepidoptera, I: 202. (BM)

I9Io, Journ. Bombay Nat. Hist. Soc. 20: 44I. (BM)

I93I, Exotic Microlepidoptera, 4:36. (v)

I925, Exotic Microlepidoptera, 3: 207. (BM)

I927, Exotic Microlepidoptera, 3: 328. (T)

I920, Ann. South African Mus. I7: 280. (SA)

1920, Ann. South African Mus. I7: 284. (SA)

I9I3, Exotic Microlepidoptera, I: II7.

I926, Ann. South African Mus. 23: 33I. (SA)

I9I7, Exotic Microlepidoptera, 2: 52. (BM)

I928, Exotic Microlepidoptera, 3: 457. (BM)

I920, Exotic Microlepidoptera, 2: 350́.

1930, Exotic Microlepidoptera, 3: 578.

I922, Exotic Microlepidoptera, 2: 583. (BM)

I9I9, Exotic Microlepidoptera, 2: 245. (BM)

I909, Ann. Transvaal Mus. 2: I2, pl. 4, fig. 9. (T)

1897, Proc. Linnean Soc. N.S. Wales, 22: 394.

I906, Journ. Bombay Nat. Hist. Soc. I7: I45. (Bм)

I9I5, Exotic Microlepidoptera, I: 3 I8.

1929, Trans. Ent. Soc. London, 76: 493. (BM)

I9I5, Exotic Microlepidoptera, I: 469. (BM)

I9I8, Exotic Microlepidoptera, 2: I53. (BM)

I9I3, Trans. Ent. Soc. London, I9I3: I96. (BM)

I9I3, Exotic Microlepidoptera, I: I6o. (MA)

I922, Trans. Ent. Soc. London, I922: 78. (Bм)

I922, Exotic Microlepidoptera, 2: 53I. (BM)

I9I4, Trans. Proc. New Zealand Inst. 46: I04.

I936, Exotic Microlepidoptera, 5: 63.

I926, Sarawak Mus. Journ. 3: I67.

I928, Exotic Microlepidoptera, 3: 4I6.

I9I4, Exotic Microlepidoptera, I: I9I. (BM)

I9I 5, Exotic Microlepidoptera, I: 463. (BM)

I884, Proc. Limnean Soc. N.S. Wales, 9: 774.

I924, Trans. Proc. New Zealand Inst. 55: 662.

I920, Voyage de Ch. Alluaud et R. Jeannel en Afrique

Oriental, II, Microlepidoptera, p. 62. (P)

I9I3, Ann. Transvaal Mus. 3: 328. (T)

I928, Exotic Microlepidoptera, 3: 453. (BM)

I923, Exotic Microlepidoptera, 3: II. (BM)

I920, Exotic Microlepidoptera, 2: 35I. (BM)

I9Io, Proc. Limnean Soc. N.S. Wales, 35: 265.

I9I5, Exotic Microlepidoptera, I: 459. (BM)

I93I, Journ. Linnean Soc. London, 37: 279. (BM)

I921, Exotic Microlepidoptera, 2: 469.

I9I8, Exotic Microlepidoptera, 2: II3. (BM)

I922, Exotic Microlepidoptera, 2: 58I. (BM)

I9I8, Exotic Microlepidoptera, 2: 222. (BM)

I9I2, Exotic Microlepidoptera, I: 9. (Bм)

I9I I, Proc. Limnean Soc. N.S. Wales, 36: 250.

I884, Proc. Linnean Soc. N.S. Wales, 9:75I. 


\begin{tabular}{|c|c|c|}
\hline pesseuta & COSMOCLOSTIS & I9o6, Journ. Bombay Nat. Hist. Soc. I7: I34. \\
\hline pessocrossa & ARISTOTELIA & I926, Exotic Microlepidoptera, 3: 273. (BM) \\
\hline pessota & LASPEYRESIA & I9II, Proc. Linnean Soc. N.S. Wales, 36: 290. \\
\hline petalacma & MACHAEROPTERIS & I932, Exotic Microlepidoptera, 4: 2 II. \\
\hline petalistis & ELACHISTA & I932, Exotic Microlepidoptera, 4: 2I7. \\
\hline petalitis & MACROBATHRA & I9I5, Exotic Microlepidoptera, I: 295. (Bм) \\
\hline petalocrossa & EULIA & I926, Exotic Microlepidoptera, 3: 250. (BM) \\
\hline petalodes & DICHOMERIS & I934, Exotic Microlepidoptera, 4: 5I2. (BM) \\
\hline petalonota & EUCOSMA & I937, Exotic Microlepidoptera, 5: I57. (o) \\
\hline petalopa & ACROCERCOPS & I934, Exotic Microlepidoptera, 4:470. \\
\hline petaloxantha & HAPSIFERA & I93I, Exotic Microlepidoptera, 4: 98. \\
\hline petasodes & ORSIMACHA* & I9I4, Exotic Microlepidoptera, I: I86. (BM) \\
\hline petraea & CARPOSINA & I9ro, Proc. Linnean Soc. N.S. Wales, 35: I5I. \\
\hline petraea & STENOPTILIA & I908, Trans. Ent. Soc. London, I907: 504. \\
\hline petraea & SYLLOCHITIS* & I9Io, Journ. Bombay Nat. Hist. Soc. 20: 462 . (вм) \\
\hline petrias & HARMOLOGA & I902, Trans. Ent. Soc. London, I902: 572. \\
\hline petrias & ZELLERIA & I907, Journ. Bombay Nat. Hist. Soc. I7: 746. (вм) \\
\hline petrinodes & GNORIMOSCHEMA & I904, Proc. Linnean Soc. N.S. Wales, 29: 3 I8. \\
\hline petrinopa & PERSICOPTILA & I9I5, Exotic Microlepidoptera, I: 320. \\
\hline petrinopis & CHELARIA & I934, Exotic Microlepidoptera, 4: 45I. (BM) \\
\hline petrodes & MELASINA & I9I4, Ann. South African Mus. Io: 252. (SA) \\
\hline petrodoxa & NARYCIA & I923, Trans. Proc. New Zealand Inst. 54: I69. \\
\hline petroeca & TALAEPORIA & I937, Exotic Microlepidoptera, 5: II3. \\
\hline petroglypta & PATROMASIA* & I926, Ann. South African Mus. 23: 347. (SA) \\
\hline petrographa & ANACAMPSIS & I922, Trans. Ent. Soc. London, I922: 79. (BM) \\
\hline petrograpta & SYMPETALISTIS* & I935, Exotic Microlepidoptera, 4:557. \\
\hline petrologa & DEMOBROTIS & I9I9, Exotic Microlepidoptera, 2: 254 . \\
\hline petromacha & ARGYROPLOCE & I93I, Exotic Microlepidoptera, 4: I28. (BM) \\
\hline petromorpha & COMPSOLECHIA & I922, Trans. Ent. Soc. London, I922: 84. (BM) \\
\hline petronoma & DEPRESSARIA & I934, Exotic Microlepidoptera, 4: 475. (BM) \\
\hline petrophanes & EULECHRIA & I9I4, Exotic Microlepidoptera, I: I62. \\
\hline petrophora & CAPUA & $\begin{array}{l}\text { I938, Institut des Parcs Nationaux du Congo Belge, fasc. I4, } \\
\text { p. 5, pl. I, fig. } 3 \text {. }\end{array}$ \\
\hline petrophracta & TORTRIX & I938, Trans. Ent. Soc. London, 87: 506. (BM) \\
\hline petroscia & HYPOSMOCOMA & I9I5, Exotic Microlepidoptera, I: 340. (BM) \\
\hline petrosema & PROTOTHEORA* & I9I7, Ann. South African Mus. I7: I9. \\
\hline petrosperma & CUPHOMANTIS* & I935, Exotic Microlepidoptera, 4:576. (c) \\
\hline petrota & MELEONOMA & I9I4, Exotic Microlepidoptera, I: 255. (BM) \\
\hline petrotoma & AUTOSTICHA & I9I6, Exotic Microlepidoptera, I: 587 . (Bм) \\
\hline petroxesta & LABDIA & I92I, Exotic Microlepidoptera, 2: 45I. (BM) \\
\hline petulans & HAPALOSARIS* & I9I7, Trans. Ent. Soc. London, I9I7: 37. (BM) \\
\hline petulans & PERONEA & I9I2, Exotic Microlepidoptera, I: I9. (BM) \\
\hline pexa & OPOSTEGA & I920, Exotic Microlepidoptera, 2: 358. \\
\hline phacelota & CHELARIA & I913, Journ. Bombay Nat. Hist. Soc. 22: I66. (Bм) \\
\hline phaeacma & ARGYROPLOCE & I93I, Exotic Microlepidoptera, 4: I29. \\
\hline phaeadelpha & OPOGONA & I934, Exotic Microlepidoptera, $4: 463$. \\
\hline phaedra & ANTICTENISTIS & I93I, Exotic Microlepidoptera, 4: I42. (v) \\
\hline edrella & ATELOSTICHA* & I883, Proc. Linnean Soc. N.S. Wales, 7: 49I. \\
\hline aedropis & TINEA & I926, Sarawak Mus. Journ. 3: I66. \\
\hline haedrospora & MNESIPATRIS* & I935, Exotic Microlepidoptera, 4: 559. \\
\hline
\end{tabular}




$\begin{array}{ll}\text { phaedrostoma } & \text { TRILOCHANA } \\ \text { phaedryntis } & \text { ENCHOCRATES } \\ \text { phaeobathra } & \text { USSARA } \\ \text { phaeocasis } & \text { MELASINA } \\ \text { phaeocausta } & \text { CRYPTOLECHIA } \\ \text { phaeocentra } & \text { COLEOPHORA } \\ \text { phaeocephala } & \text { TINEOLA } \\ \text { phaeoceros } & \text { CEROMITIA } \\ \text { phaeochalca } & \text { HOPLOPHANES } \\ \text { phaeochalca } & \text { OPOGONA } \\ \text { phaeochlora } & \text { ERIDACHTHA }\end{array}$

phaeochorda

phaeochtha

phaeocoma

phaeocoma

phaeocosma

phaeocrana

phaeocremna

phaeocremna

phaeocrossa

phaeocrossis

phaeodelta

phaeodes

phaeodesma

phaeodeta

phaeodora

phaeodryas

phaeogastra

phaeogenes

phaeograpta

phaeoleuca

phaeomalla

phaeometalla

phaeomicta

phaeomorpha

phaeomystis

phaeonephela

phaeonephes

phaeoneura

phaeopa

phaeopelta

phaeophanes

phaeopis

phaeopleura

phaeoplintha

phaeopsamma

phaeoptila

phaeosarca
ASYMPHORODES

CRYPTOPHASA

CEROMITIA

EPICHORISTA

COESYRA

OPOGONA

CILICITIS*

EUCOSMA

MACHIMIA

ERIDACHTHA

MERIDARCHIS

EUCOSMA

OCYSTOLA

ACROCERCOPS

HIEROMANTIS

LECITHOCERA

COSMOPTERYX

MELASINA

CROCANTHES

ATHRYPSIASTIS*

ACROLOPHUS

CHRYSOCENTRIS

EPITHECTIS

ACROCERCOPS

STENOMA

TINEA

MIMESEOPTILUS

STENOMA

STOMOPTERYX

ARGYROPLOCE

STENOMA

BACTRA

LIMNOECIA

STENOMA

EULECHRIA

TRACHYPEPLA

CYMOTRICHA
I934, Exotic Microlepidoptera, 4: 455.

I888, Proc. Linnean Soc. N.S. Wales, (Ser. 2) 2: 929.

I932, Exotic Microlepidoptera, 4:276. (v)

I934, Exotic Microlepidoptera, 4: 520. (C)

I934, Exotic Microlepidoptera, 4: 478. (BM)

I9I4, Exotic Microlepidoptera, I: 206.

I9I8, Ann. Transvaal Mus. 6: 44. (T)

I92I, Exotic Microlepidoftera, 2: 406.

I897, Proc. Linnean Soc. N.S. Wales, 22: 4 I4.

I9o8, Proc. Zool. Soc. London, p. 737.

I920, Voyage de Ch. Alluaud et $R$. Jeannel en Afrique

Oriental, II, Microlepidoptera, p. 76. (P)

I929, Trans. Ent. Soc. London, 76: 499.

I925, Exotic Microlepidoptera, 3: I47.

I9I2, Exotic Microlepidoptera, I: 28. (T)

I9I4, Exotic Microlepidoptera, I: I95. (BM)

I889, Proc. Linnean Soc. N.S. Wales, (Ser. 2) 3: I655.

I9I4, Exotic Microlepidoptera, I: 287.

I938, Trans. R. Ent. Soc. London, 87: 5I5.

I937, in Caradja and Meyrick, Deuts. Ent. Zeit. Iris, 5I:

I79. (BM)

I9I2, Trans. Ent. Soc. London, I9I I: 699. (Bм)

I937, Exotic Microlepidoptera, 5:96. (T)

I906, Journ. Bombay Nat. Hist. Soc. I7: I38.

I920, Exotic Microlepidoptera, 2: 344. (Sub.)

I9I3, Exotic Microlepidoptera, I: II5.

I927, Insects of Samoa, 3, Lepidoptera, fasc. 2, p. IoS.

I929, Exotic Microlepidoptera, 3: 54I.

I93I, in Caradja, Bull. Sect. sci. Acad. roum. I4: 68.

I9I7, Ent. Mon. Mag. 53: 257.

I9I9, Exotic Microlepidoptera, 2: 266.

I93I, Exotic Microlepidoptera, 4: 75. (BM)

I9Io, Trans. Ent. Soc. London, I9I0: $45^{8}$.

I9I3, Trans. Ent. Soc. London, I9I3: I98. (вм)

I937, Exotic Microlepidoptera, 5: I25. (c)

I936, Exotic Microlepidoptera, 5: 42. (c)

I9I9, Exotic Microlepidoptera, 2: 23I.

I925, Exotic Microlepidoptera, 3: I97. (BM)

I927, Exotic Microlepidoptera, 3: 322. (BM)

I886, Trans. Ent. Soc. London, I886: I9.

I9I3, Trans. Ent. Soc. London, I9I3: I87. (BM)

I9I8, Exotic Microlepidoptera, 2: I36. (BM)

I921, Zool. Meded. 6: I55. (L)

I9I2, Trans. Ent. Soc. London, I9I I: 7I4. (BM)

I9II, Proc. Linnean Soc. N.S. Wales, 36: 254.

I924, Exotic Microlepidoptera, 3: 90. (BM)

I9I5, Exotic Microlepidoptera, I: 424. (BM)

I9I3, Ann. Transvaal Mus. 3: 316. (T)

I905, Trans. Ent. Soc. London, I905: 236.

I93I, Exotic Microlepidoptera, 4: 68. (BM) 
phaeosaris phaeosceptra phaeoschista phaeosoma phaeospila phaeospora phaeostephes phaeostrota phaeothicta phaeotoxa phaeoxesta phaeoxyla phaeozona phaethontia phalacra phalacris phalacrodes phalacropa phalacropis phalaraea phalaranthes phalaraspis phalarista phalaritis phalarocosma phalaropa phalaropis phalarota phalerata phaloniodes phanaea phanarcha phaneracma phanerarcha phaneraula phanerodoxa phaneropa phaneropis phanerozona phanocrossa phanoptila phantasta phantastis pharangitis pharangodes

pharetrata pharetrata pharetria
ANTAEOTRICHA EULECHRIA ALUCITA OPOSTEGA SYNCRATERNIS ACROCERCOPS EULECHRIA RHYNCHOTONA* PSYCHROMNESTRA COMPSOLECHIA ZACORISCA EURYTHECTA COESYRA CRYPTOPHAGA STAGMATOPHORA LASPEYRESIA GNORIMOSCHEMA STENOMA ETNODONA* EPICHORISTA DICHELOPA CHOREUTIS ALOMENARCHA* TIPHA

CNEPHASIA GRACILARIA MOMPHA MICROCOLONA IMMA

EULIA . HOMONA AGANOPTILA* ASCALENIA ORNEODES OCHETOXENA* OEGOCONIA BATRACHEDRA PARECTOPA EMPEDAULA COMMATICA STAGMATOPHORA EPIPHTHORA PARACHANDA* HOMONA EUCOSMA

TIPHA [MNESTERIA*] TORTRIX GELECHIA
I9I5, Exotic Microlepidoptera, I: 394. (BM) I888, Proc. Linnean Soc. N.S. Wales, (Ser. 2) 2: 964. 1923, The Entomologist, 56: 277. I928, Exotic Microlepidoptera, 3: 396. (T) I922, Exotic Microlepidoptera, 2: 59I. (BM) I9I6, Exotic Microlepidoptera, I: 627. I888, Proc. Linnean Soc. N.S. Wales, (Ser. 2) 2: 956. I923, Exotic Microlepidoptera, 3: 35. (BM) I924, Exotic Microlepidoptera, 3: 89. I922, Trans. Ent. Soc. London, I922: 85. (BM) I924, Exotic Microlepidoptera, 3: III. I938, Trans. roy. Soc. N.Z. $67: 427$. I889, Proc. Linnean Soc. N.S. Wales, (Ser. 2) 3: I656. (A) I89o, Trans. R. Soc. S. Australia, I3: 36.

I9o9, Ann. Transvaal Mus. 2: 20, pl. 6, fig. 8. (T)

I9I2, Exotic Microlepidoptera, I: 64 .

I9I3, Ann. Transvaal Mus. 3: 293. (T)

I932, Exotic Microlepidoptera, 4: 303. (Us)

I9I5, Exotic Microlepidoptera, I: 289.

I920, Ann. South African Mus. I7: 276. (SA)

I934, Pacific Ent. Surv. Publ. 7, art. 28, p. 344. (вв)

1923, Exotic Microlepidoptera, 2: 6I7. (BM)

I930, Exotic Microlepidoptera, 3: 6I5. (BM)

I905, Journ. Bombay Nat. Hist. Soc. I6: 594. (BM)

1937, Exotic Microlepidoptera, 5: I28. (T)

I9I2, in Wytsman, Genera Insectorum, fasc. I28, p. 28.

I922, Exotic Microlepidoptera, 2: 578. (BM)

I9I5, Exotic Microlepidoptera, I: 335.

I906, Trans. Ent. Soc. London, I906: I95. (BM)

1932, Exotic Microlepidoptera, 4: 257. (v)

I9Io, Proc. Linnean Soc. N.S. Wales, 35: 2I5.

I9I5, Exotic Microlepidoptera, I: 334.

I92I, Ann. Transvaal Mus. 8: 96. (T)

I924, Exotic Microlepidoptera, 3:96. (T)

I920, Ann. South African Mus. I7: 305. (SA)

I926, Exotic Microlepidoptera, 3: 29I. (BM)

I9I4, Exotic Microlepidoptera, I: 286.

I9I5, Trans. Ent. Soc. London, I9I5: 233.

I922, Trans. Ent. Soc. London, I922: 65. (BM)

I922, Trans. Ent. Soc. London, I922: 72. (BM)

I9II, Ann. Transvaal Mus. 3: 70.

1904, Proc. Linnean Soc. N.S. Wales, 29: 266.

I927, Exotic Microlepidoptera, 3: 338. (BM)

I9Io, Proc. Linnean Soc. N.S. Wales, 35:2I4.

I920, Voyage de Ch. Alluaud et $R$. Jeamel en Afrique

Oriental, II, Microlepidoptera, p. $5^{8}$. (P)

I905, Journ. Bombay Nat. Hist. Soc. I6: 593. (Lost) I909, Ann. Transvaal Mus. 2: 6, pl. 2, fig. 7. (T) I886, Trans. Proc. New Zealand Inst. I8: I69. 
246

pharetrodes

SCARDIA

\section{pharetropa \\ pharetropis \\ pharetrucha \\ pharmacista \\ pharmactis \\ pharopeda \\ pharotoma \\ pharsotoma \\ phaselodes \\ phasganopa \\ phasianis \\ phasmatica}

phasmatopa

phaulocentra

phaulocosma

phaulodes

phaulodes

phaulomorpha

phaulopa

phaulopa

phauloptera

phauloscopa

phaulosema

phegophylla

phellodes

phellopis

phelotris

phenacistis

phenax

phepsalias

phepsalitis

pheretropa

phiaropis

philadelpha

philantha

philaromia

philerasta

phileris

phileris

philippa

philobrya

philocala

philocarpa

philocharis

philochersa
CATALECTIS

GLYPHIPTERYX

PHYLLOCNISTIS

OGMOCOMA*

BORKHAUSENIA

ACROCERCOPS

ENDOPHTHORA

HYPOSMOCOMA

STENOMA

ISOZYGA*

PROTOLECHIA

MERITASTIS

STENOMA

LATYPICA

EUTORNA

AUTOSTICHA

XYSMATODOMA

LASPEYRESIA

BACTRA

IYTROPHILA

TINEA

PHILOBOTA

TELPHUSA

OECOPHORA

CAPUA

PSALTICA

GELECHIA

BACTRA

MACHAEROPTERIS

ENDOPHTHORA

[OXYLYCHNA*]

COMPSOLECHIA

CRYPSITHYRIS

GRACILARIA

GYMNOBATHRA

TRICLONELLA

STATHMOPODA

LITHOCOLLETIS

EREUNETIS

LAMYRODES*

CACOECIA

ACROCLITA

SAROPLA

PYRODERCES

HYPERDASYS

BRACHMIA
I934, in Caradja and Meyrick, Deuts. Ent. Zeit. Iris, 48: 42.

I920, Exotic Microlepidoptera, 2: 362. (BM)

I907, Proc. Linnean Soc. N.S. Wales, $32:$ I25.

I92I, Ann. Transvaal Mus. 8: I24. (T)

I924, Trans. Ent. Soc. London, I923: 557.

I905, Trans. Ent. Soc. London, I905: 24I.

I9I6, Exotic Microlepidoptera, I: 626.

1888, Trans. Proc. New Zealand Inst. 20: 94.

I9I5, Exotic Microlepidoptera, I: 34I. (BM)

I93I, Exotic Microlepidobtera, 4: 39. (v)

I92I, Ann. Transvaal Mus. 8: гзо. (T)

I904, Proc. Linnean Soc. N.S. Wales, 29:382.

I937, in Caradja and Meyrick, Deuts. Ent. Zeit. Iris, 5I:

I75.

I9Io, Trans, Ent. Soc. London, I9Io: 460.

I92I, Ann. Transvaal Mus. 8: I3I. (T)

I9o6, Trans. $R$. Soc. S. Australia, 30: 45 .

I908, Journ. Bombay Nat. Hist. Soc. I 8: 459. (вм)

I893, Proc. Linnean Soc. N.S. Wales, 7: 499.

I927, Bull. Soc. R. Ent. Egypte, I926, p. I Io.

I9II, Proc. Linnean Soc. N.S. Wales, 36: 253.

I921, Ann. Transvaal Mus. 8: I33. (T)

I893, Proc. Linnean Soc. N.S. Wales, $7: 544$.

1884, Proc. Linnean Soc. N.S. Wales, 8: 484.

I920, Voyage de Ch. Alluaud et R. Jeannel en Afrique

Oriental, II, Microlepidoptera, p. 7o. (P)

I883, New Zealand Journ. Sci. I: 524 .

I9I0, Proc. Limnean Soc. N.S. Wales, 35: I83. (MA)

I9I4, Exotic Microlepidoptera, I: 262. (BM)

I909, Ann. South African Mus. 5: 37I. (SA)

I9o9, Journ. Bombay Nat. Hist. Soc. I9: 585. (BM)

I9I I, Journ. Bombay Nat. Hist. Soc. 2I: I29.

I907, Journ. Bombay Nat. Hist. Soc. I7: 750.

I922, Trans. Ent. Soc. London, I922: 94. (BM)

I93I, Exotic Microlepidoptera, 4: I05.

I92I, Exotic Microlepidoptera, 2: 470 .

I883, New Zealand Journ. Sci. I : 524.

I920, Exotic Microlepidoptera, 2: 365. (BM)

I936, Exotic Microlepidoptera, 4: 6I8.

I922, Exotic Microlepidoptera, 2: 560.

I893, Proc. Linnean Soc. N.S. Wales, 7: 563 .

I9Io, Proc. Linnean Soc. N.S. Wales, 35: I82.

I9I8, Exotic Microlepidoptera, 2: I65. (BM)

I920, Exotic Microlepidoptera, 2: 344. (BM)

I884, Proc. Linnean Soc. N.S. Wales, 9: 746.

I92I, Exotic Microlepidoptera, 2: 45I.

I9I5, Exotic Microlepidoptera, I: 344. (BM)

I938, Trans. R. Ent. Soc. London, 87: 5I4. (BM) 


\begin{tabular}{|c|c|}
\hline philochora & LOCHEUTIS* \\
\hline philochrysa & MACAROCOSMA* \\
\hline philocoma & EPIMARPTIS* \\
\hline philocompsa & ARGYROPLOCE \\
\hline philocremna & STENOPTILIA \\
\hline philodema & BRACHMIA \\
\hline philodoxa & TINGENTERA \\
\hline philogeorga & PYRODERCES \\
\hline philomacha & HOPLOPHANES \\
\hline philomela & ORNEODES \\
\hline philomela & STENOMA \\
\hline philomima & ARCHIMAGA \\
\hline philomusa & BRACHMIA \\
\hline philonoma & IMMA \\
\hline philonyma & CHOREUTIS \\
\hline philopatris & ELACHISTA \\
\hline philopoana & TORTRIX \\
\hline philopsamma & NEPHOGENES* \\
\hline philoptica & MYRMECOZELA \\
\hline philorectis & PLATYPTILIA \\
\hline philoscia & PARATHETA \\
\hline philosopha & BRACHMIA \\
\hline philostaura & EULECHRIA \\
\hline philotherma & EULECHRIA \\
\hline philoxena & COESYRA \\
\hline phlegyra & STATHMOPODA \\
\hline phlegyropa & ENCAMINA* \\
\hline phloeodes & PROTOLECHIA \\
\hline phloeotis & PTERNIDORA* \\
\hline phlogopa & GEN. ? [LACTURA] \\
\hline phlyctaenias & MARASMARCHA \\
\hline phlyctaenopa & RHOPALOSETIA* \\
\hline phlyctidopa & ISOCRITA \\
\hline phlyctidota & ZELOMORA \\
\hline phoberopis & LECITHOCERA \\
\hline phocina & TINEOLA \\
\hline phocodes & GONIONOTA \\
\hline phoebas & EULECHRIA \\
\hline phoenaula & GELECHIA \\
\hline phoenicaula & PARECTOPA \\
\hline phoenicis & EPIPHRACTIS* \\
\hline phoenicites & NEMOTOIS \\
\hline phoenicopa & RHADINASTIS \\
\hline phoenicopa & TINEA \\
\hline phoenicura & ETHMIA \\
\hline phoeniodes & ARGYROPLOCE \\
\hline
\end{tabular}

I883, Proc. Linnean Soc. N.S. Wales, 8: 342. 1931, Ann. Mus. Nac. Hist. Nat. Buenos Aires, 36: 393. (BM)

I9I4, Journ. Bombay Nat. Hist. Soc. 22: 776.

I92I, Zool. Meded. 6: I58. (Lost)

I930, Exotic Microlepidoptera, 3: 570 .

I938, in Caradja and Meyrick, Deuts. Ent. Zeit. Iris, $52: 7$. (BM)

I908, Journ. Bombay Nat. Hist. Soc. I 8: 452. (BM)

I933, Exotic Microlepidoptera, 4: 428.

1897, Proc. Linnean Soc. N.S. Wales, 22: 4I4.

I937, in Caradja and Meyrick, Deuts. Ent. Zeit. Iris, 5I:

I 70 .

1925, Exotic Microlepidoptera, 3: 224. (BM)

I9I 8, Exotic Microlepidoptera, 2: I7I. (BM)

I9I 8, Exotic Microlepidoptera, 2: II4. (BM)

1925, Exotic Microlepidoptera, 3: 132. (BM)

I9I2, Exotic Microlepidoptera, I: 55. (BM)

1932, Exotic Microlepidoptera, 4: 216.

I88I, Proc. Linnean Soc. N.S. Wales, 6: 5 I5.

I883, Proc. Linnean Soc. N.S. Wales, 8: 379.

I93I, Exotic Microlepidoptera, 4: 93.

I926, Exotic Microlepidoptera, 3: 298.

I902, Trans. R. Soc. S. Australia, 26: I74.

I9II, Journ. Bombay Nat. Hist. Soc. 20: 708. (Bм)

1883, Proc. Linnean Soc. N.S. Wales, 7: 537.

I883, Proc. Linnean Soc. N.S. Wales, 7: 534.

I884, Proc. Linnean Soc. N.S. Wales, 9: 779.

I889, Trans. Proc. New Zealand Inst. I889: I68.

I9I 5, Trans. Ent. Soc. London, I9I5: 2I4.

I904, Proc. Linnean Soc. N.S. Wales, 29: 355.

I9I I, Proc. Linnean Soc. N.S. Wales, 36: 286.

I886, Proc. Linnean Soc. N.S. Wales, I: 258. (A)

I9I I, Journ. Bombay Nat. Hist. Soc. 21 : 106.

I926, Exotic Microlepidoptera, 3: 24I. (BM)

I921, Ann. Transvaal Mus. 8: I04. (T)

I920, Ann. South African Mus. I7: 306. (SA)

I938, in Caradja and Meyrick, Deuts. Ent. Zeit. Iris, 52:

5. (BM)

I908, Proc. Zool. Soc. London, p. 740.

I909, Trans. Ent. Soc. London, I909: 27. (BM)

I907, Journ. Bombay Nat. Hist. Soc. I7: 742. (BM)

I913, Ann. Transvaal Mus. 3: 289. (T)

I934, Exotic Microlepidoptera, 4: 473.

I9o8, Proc. Zool. Soc. London, p. 732. (BMI)

I907, Journ. Bombay Nat. Hist. Soc. I 7 993.

I907, Journ. Bombay Nat. Hist. Soc. I 7: 982.

I893, Proc. Linnean Soc. N.S. Wales, 7: 542.

I932, Exotic Microlepidoptera, 4: 346. (BM)

I921, Ann. Transvaal Mus. 8: 59. (T) 
phoenissa

EULECHRIA

phoenocrossa

phoenogramma VAZUGADA

phoenoteles

phoenoxantha

pholas

pholidota

phollicodes

phorcis

phorcydia

phormictis

phormophora

phortax

phortegella

phortica

phorticopa

phoryntis

phosphora

phosphora

phosphora

phosphorodes

phosphoropa

phosphoropis

photaula

photinella

photinopa

photodotis

photodoxa

photophanes

phractopa

phratriastis

phricodes

phricotarsa

phrictodes

phrixopa

phronimopis

phryactis

phryctora

phryganitis

phthonera

phthoneropa

phthorodoxa

phthoromima

phthorosema

phylacopis

phylactis

phylarcha

phyllanthana

phyllanthina
CROCANTHES

PERSICOPTILA

EUCOSMA

GORGOPIS

STENOMA

TIMYRA

BATRACHEDRA

PLEUROTA

CYRICTODES*

GONIOTERMA

SAGEPHORA*

ANARSIA

EPERMENIA

EULECHRIA

CNEPHASIA

EROTIS*

GLYPHIPTERYX

GONADA

ANTERETHISTA

AMBONOSTOLA*

ORNEODES

EULECHRIA

MEGACRASPEDUS

PLEUROTA

NEMOTOIS

CALLICERASTIS

ACROCERCOPS

LECITHOCERA

ALUCITA

PHRIXOSCELES

TINISSA

PHYLLOCNISTIS

PERSICOPTILA

ANTAEOTRICHA

CAPUA

BRACHMIA

CTENOCOMPA

HOMALOXESTIS

[CARTERICA*]

CROSSOPHORA

PILOPREPES

IMMA

HELIOCAUSTA

SYNTOMACTIS

HELIOCAUSTA

ANTITHESIA

NEPTICULA
I902, Trans. R. Soc. S. Australia, 26: I47.

I925, Exotic Microlepidoptera, 3: I4I. (BM)

I930, Ann. Naturhist. Mus. Wien, 44: 227. (v)

I929, Exotic Microlepidoptera, 3: 5I9. (BM)

I923, Exotic Microlepidoptera, 3: 60. (BM)

I9I 2, Ent. Mon. Mag. 48: 34. (Sub.)

I92I, Ann. Transvaal Mus. 8: I4I. (T)

I9I6, Exotic Microlepidoptera, I: 533. (BM)

I908, Journ. Bombay Nat. Hist. Soc. I8: 444. (BM)

I897, Proc. Linnean Soc. N.S. Wales, 22: 307.

I9I3, Exotic Microlepidoptera, I: I2I. (MA)

I926, Exotic Microlepidoptera, 3: 284. (BM)

I9I5, Exotic Microlepidoptera, I: 383. (Bм)

I888, Trans. Proc. New Zealand Inst. 20: 96.

1913, Journ. Bombay Nat. Hist. Soc. 22: I67. (BM)

I92I, Exotic Microlepidoptera, 2: 464.

I902, Trans. R. Soc. S. Australia, 26: I50.

I9Io, Proc. Linnean Soc. N.S. Wales, 35: 274.

I9Io, Journ. Bombay Nat. Hist. Soc. 20: I46. (BM)

I907, Proc. Linnean Soc. N.S. Wales, 32: I26.

I922, Exotic Microlepidoptera, 2: 543. (P)

I922, Trans. Ent. Soc. London, I922: 66. (BM)

I935, Exotic Microlepidoptera, 4: 606.

I9I8, Ann. Transvaal Mus. 6: 55. (T)

I883, Proc. Linnean Soc. N.S. Wales, 7: 54I.

I920, Ann. South African Mus. I7: 28I. (SA)

I889, Proc. Linnean Soc. N.S. Wales, (Ser. 2) 3: 1650.

I930, Exotic Microlepidoptera, 3: 57I. (BM)

I9I7, Exotic Microlepidoptera, 2: 82.

I9I8, Exotic Microlepidoptera, 2: I77.

I929, Exotic Microlepidoptera, 3: 523. (BM)

I886, Trans. Ent. Soc. London, I886: 20.

I9I6, Exotic Microlepidoptera, I: 623.

I9Io, Trans. Ent. Soc. London, I9Io: 477.

I926, Exotic Microlepidoptera, 3: 264.

I928, Exotic Microlepidoptera, 3: 390.

I925, Exotic Microlepidoptera, 3: I67. (BM)

I9Io, Proc. Linnean Soc. N.S. Wales, 35: I9I.

I9II, Journ. Bombay Nat. Hist. Soc. 20: 722. (BM)

I9I9, Exotic Microlepidoptera, 2: 263.

I922, Exotic Microlepidoptera, 2: 505. (P)

I886, Proc. Linnean Soc. N.S. Wales, Io: 794.

I930, Exotic Microlepidoptera, 3: 6I7.

I9I2, Exotic Microlepidoptera, I: 40. (BM)

I888, Proc. Limnean Soc. N.S. Wales, 2: 935.

I897, Proc. Limnean Soc. N.S. Wales, 22: 387.

I883, Proc. Linnean Soc. N.S. Wales, $7: 476$.

I88I, Proc. Linnean Soc. N.S. Wales, 6: 64I.

I906, Trans. R. Soc. S. Australia, 30: 60. 


\begin{tabular}{|c|c|c|}
\hline ochlora & PLATYPEPLUS & I905, Journ. Bombay Nat. Hist. Soc. I6: 585. (BM) \\
\hline phyllocosma & STENOMA & I9I6, Exotic Microlepidoptera, I : 520. (BM) \\
\hline phyllodes & OSIDRYAS & I9I6, Exotic Microlepidoptera, $2: 7$. \\
\hline phyllodoxa & ARGYROPLOCE & I932, Exotic Microlepidoptera, 4: 3IO. (вм) \\
\hline phyllograpta & CALLIPHRACTIS* & I928, Exotic Microlepidoptera, 3: 476. (вм) \\
\hline hylloscia & EUCOSMA & I937, Exotic Microlepidoptera, 5: I58. (Bм) \\
\hline phylloxantha & STENOMA & I933, Exotic Microlepidoptera, 4: 435. (BM) \\
\hline phylotypa & ANCYLOMETIS & I930, Trans. Ent. Soc. London, 78: 3I7. (P) \\
\hline phyrsima & CEROMITIA & IgII, Ann. Transvaal Mus. $2: 239$. (T) \\
\hline salodes & RHOPOBOTA & I9Io, Trans. Ent. Soc. London, I9Io: 368. (Bм) \\
\hline atma & AMATHYNTIS* & I907, Journ. Bombay Nat. Hist. Soc. I7: 987. (Bм) \\
\hline physaula & PHILOBOTA & I9I4, Exotic Microlepidoptera, I: 273 . \\
\hline physeta & TRICHOTAPHE & I9I3, Ann. Transvaal Mus. 3: 302. (T) \\
\hline etopa & TORTRIX & I932, Trans. Ent. Soc. London, 80: III. (Bм) \\
\hline sica & EULECHRIA & I920, Exotic Microlepidoptera, 2: 37I. \\
\hline ocapna & DECADARCHIS & I929, Trans. Ent. Soc. London, 76:506. \\
\hline ocoma & DICHOMERIS & I926, Exotic Microlepidoptera, 3: 286. (Bм) \\
\hline otricha & STENOMA & I9I5, Exotic Microlepidoptera, I: 455 . (BM) \\
\hline ialea & HYPERCALLIA & I92I, Exotic Microlepidoptera, 2: 389 . (BM) \\
\hline bic & MELASINA & I9I7, Ann. Natal Mus. 3:6I6. (T) \\
\hline icoma & PLATYEDRA & I93I, Exotic Microlepidoptera, 4:6I. \\
\hline actis & EUCALYPTRA* & I92I, Zool. Meded. 6: I75. (L) \\
\hline antis & STENOMA & I930, Ann. Naturhist. Mus. Wien, 44: 253. (v) \\
\hline carpa & CARBATINA* & I9I3, Journ. Bombay Nat. Hist. Soc. 22: I82. (BM) \\
\hline ocentra & CRYPTOLECHIA & I92I, Exotic Microlepidoptera, 2: 395. (Bм) \\
\hline chalca & LABDIA & I937, Exotic Microlepidoptera, 5: I47. \\
\hline cosma & LEUCOPTERA & I9I5, Exotic Microlepidoptera, I: 346. \\
\hline odelta & EUCOSMA & I932, Trans. Ent. Soc. London, 80: III. (BM) \\
\hline odes & PERSICOPTILA & I9I5, Exotic Microlepidoptera, I: 322. \\
\hline dora & LECITHOCERA & I9I3, Ann. Transvaal Mus. 3: 294. (T) \\
\hline oglossa & PARECTOPA & I9I2, in Wytsman, Genera Insectorum, fasc. I28, p. 20. \\
\hline picrogramma & GELECHIA & I929, Exotic Microlepidoptera, 3: 489. (BM) \\
\hline oleuca & ILLANTIS* & I92I, Zool. Meded. 6: I87. (L) \\
\hline omorpha & TRACHYPEPLA & I928, Exotic Microlepidoptera, 3: 469. (BM) \\
\hline opa & PLATYBATHRA & I92I, Ann. Transvaal Mus. 8: 98. (т) \\
\hline ophaea & PSEUDODOXIA & I9Io, Journ. Bombay Nat. Hist. Soc. 20: I52. (BM) \\
\hline phanes & DICHOMERIS & I913, Ann. Transvaal Mus. 3: 304. (T) \\
\hline ophylla & ENCHOCRATES & I886, Proc. Linnean Soc. N.S. Wales, I0: 827 . \\
\hline picroscopa & MERIDARCHIS & I930, Exotic Microlepidoptera, 3: 588. (вм) \\
\hline stacta & HOMONA & I921, Zool. Meded. 6: I46. (L) \\
\hline oxesta & MYRMECOZELA & I926, Ann. South African Mus. 23: 342. (SA) \\
\hline picryntis & ENTHETICA* & I9I6, Exotic Microlepidoptera, I: 574 . (BMI) \\
\hline & PLATYPTILIA & I9I3, Exotic Microlepidoptera, I: Iog. \\
\hline oria & TRICLONELLA & I9I6, Exotic Microlepidoptera, I: 544. (BAI) \\
\hline pictrix & PSITTACASTIS & I92I, Exotic Microlepidoptera, 2: 399. (BM) \\
\hline picturata & MICTOPSICHIA & I9I2, Exotic Microlepidoptera, I: 35. (BN) \\
\hline & COMOCRITIS & I906, Journ. Bombay Nat. Hist. Soc. I7: 4I5. (вм) \\
\hline gnerata & ASCALENIA & I922, Exotic Microlepidoptera, 2: 5 So. (Bir) \\
\hline & CAPUA & I921, Zool. Meded. 6: I46. (L) \\
\hline ina & STAGMATOPHORA & I9I3, Ann. Transvaal Mus. 3: 307. (T) \\
\hline
\end{tabular}


pilaria

pileata

pileigera

pilifera

piligera

pilularis

pilulata

pinalea

pinarodes

pinarodes

pindarica

pinguicula

pinguis

pinguivora

pinicola

pinnata

pinnifera

piodes

piperata

pirastica

pirastis

piratica

piratodes

pissantha

pistarcha

pistopis

pithanodes

pithanopis

pityritis

placata

placata

placens

placida

placida

placida

placomorpha

placophanes

placoterma

placoxantha

placozona

pladarota

plaesiodes

plagiaula

plagifera

plagiocentra

plagiopa
SIMAETHIS

PYRODERCES

HIEROXESTIS

AMBLYXENA*

ACROCERCOPS

CEROMITIA

STEREOSTICHA*

ORNEODES

CARPOSINA

PSEUDODOXIA

COMOCRITIS

GELECHIA

MELASINA

PHYSOPTILA

FUMEA

ANARSIA

ACROLOPHUS

EULECHRIA

[ELAEONOMA*]

TORTRIX

ASCALENIA

AROTROPHORA

SCYTHRIS

ARTICOLLA

OTOPTRIS

AEGERIA

ZELLERIA

EULECHRIA

EPERMENIA

MEGACRASPEDUS

PERONEA

PSEUDODOXIA

HECTACMA

ARGYROPLOCE

PSEUDODOXIA

STATHMOPODA

MOERARCHIS

TRACHYDORA

COMMATICA

OCYSTOLA

OCHYROTICA

DICHOMERIS

PACHNISTIS

[PARALLACTIS*]

THECTOPHILA

TISCHERIA

BATRACHEDRA

ENSCEPASTRA*
I9I2, Exotic Microlepidoptera, I: 50. (BM)

I897, Proc. Linnean Soc. N.S. Wales, 22: 355.

I9I3, Ann. Transvaal Mus. 3: 330. (T)

I92I, Ann. Transvaal Mus. 8: I2I. (T)

I9I5, Trans. Ent. Soc. London, I9I5: 227.

I92I, Ann. Transvaal Mus. 8: I39. (T)

I9I3, Exotic Microlepidoptera, I: 83.

I908, Trans. Ent. Soc. London, I907: 506.

I9Io, Proc. Linnean Soc. N.S. Wales, 35: I52.

I9Io, Journ. Bombay Nat. Hist. Soc. 20: I52. (Bм)

I924, Exotic Microlepidoptera, 3: II8. (BM)

I929, Exotic Microlepidoptera, 3: 489. (BM)

I9I4, Supplementa Entomologica, No. 3, p. 6o. (D)

1934, Exotic Microlepidoptera, 4:466. (BM)

I937, Exotic Microlepidoptera, 5: II7.

I93I, Exotic Microlepidoptera, 4: 69. (BM)

I93I, Exotic Microlepidoptera, 4: Ioo. (v)

I902, Trans. R. Soc. S. Australia, 26: I48.

I9Io, Proc. Linnean Soc. N.S. Wales, 35: 239.

I937, Exotic Microlepidoptera, 5: 80.

I9Io, Proc. Linnean Soc. N.S. Wales, 35: 263.

I928, Exotic Microlepidoptera, 3: 4I4. (BM)

I930, Exotic Microlepidoptera, 3: 6ог. (вм)

I9I5, Trans. Ent. Soc. London, I9I5: 246.

I93I, Exotic Microlepidoptera, 4: 50.

I93I, Ann. Mus. Nac. Hist. Nat. Buenos Aires, 36: 398.

(BM)

I920, Exotic Microlepidoptera, 2: 309.

I92I, Exotic Microlepidoptera, 2: 463.

I904, Proc. Linnean Soc. N.S. Wales, 29: 278.

I9I2, Exotic Microlepidoptera, I: I7. (BM)

I9I4, Exotic Microlepidoptera, I : 265. (BM)

1920, Exotic Microlepidoptera, 2: 363. (BM)

I9II, Proc. Linnean Soc. N.S. Wales, 36: 268.

I9Io, Journ. Bombay Nat. Hist. Soc. 20: I5I. (Bм)

I9o8, Rec. Indian Mus. 2: 396. (K)

I922, Arkiv för Zool. vol. I4, part I5, p. I2.

I897, Proc. Linnean Soc. N.S. Wales, 22: 400.

I9I8, Exotic Microlepidoptera, 2: I46. (BM)

I885, Proc. Linnean Soc. N.S. Wales, 9: 1072.

I92I, Exotic Microlepidoptera, 2: 423.

I92I, Ann. Transvaal Mus. 8: 84. (T)

I920, Voyage de Ch. Alluaud et R. Jeannel en Afrique

Oriental, II, Microlepidoptera, p. 78. (P)

I938, Trans. roy. Soc. N.Z. 67: 427.

I9I5, Trans. Ent. Soc. London, I9I5: 246.

I897, Proc. Linnean Soc. N.S. Wales, 22: 309.

I920, Ann. South African Mus. I7: 30I. (SA) 


\begin{tabular}{|c|c|c|}
\hline plagiula & GYMNOGRAMMA & I923, Bull. Mus. Nat. Hist. Nat. Paris, 29: 565. \\
\hline plana & ANATHAMNA & Igr I, Proc. Linnean Soc. N.S. Wales, 36: 26I. \\
\hline plana & HYPOPHRICTIS & I920, Exotic Microlepidoptera, 2: 354 . \\
\hline plana & NARYCIA & I9I9, Exotic Microlepidoptera, 2: 262. \\
\hline planata & TRICHOTAPHE & I9I0, Rec. Indian Mus. 2: 222. (K) \\
\hline planeta & DEUTEROCOPUS & Igo8, Trans. Ent. Soc. London, I9o8: 473 . \\
\hline planetis & NEPTICULA & i9o6, Trans. R. Soc. S. Australia, 30: $5^{8}$. \\
\hline planetopa & PYRODERCES & I9I5, Exotic Microlepidoptera, I: 309. \\
\hline planicola & BRACHMIA & I932, Exotic Microlepidoptera, 4: 206. (BM) \\
\hline planicola & LIOPSEUSTIS* & I928, Exotic Microlepidoptera, 3: 429. (BM) \\
\hline planicoma & STENOMA & I925, Exotic Microlepidoptera, 3: I89. (BM) \\
\hline planiloqua & LIPOPTYCHA & I912, Ent. Mon. Mag. 48: 36. (Sub.) \\
\hline planodes & GELECHIA & I9I8, Exotic Microlepidoptera, 2: I34. (BM) \\
\hline plasmatica & TINEA & I9I I, Journ. Bombay Nat. Hist. Soc. 2I: I 22. \\
\hline plastica & TORTRIX & I9Io, Proc. Linnean Soc. N.S. Wales, 35: 234. \\
\hline plasticus & YPSOLOPHUS & I904, Proc. Linnean Soc. N.S. Wales, 29: 433. \\
\hline plasturga & OPOGONA & I9I I, Journ. Bombay Nat. Hist. Soc. 2I : II2. \\
\hline platamodes & DELTOBATHRA* & I923, Exotic Microlepidoptera, 3: 55. (BM) \\
\hline platanaspis & EUCOSMA & I 934, in Caradja and Meyrick, Deuts. Ent.Zeit. Iris, $48: 32$ \\
\hline platanodes & STENOPTILIA & I9I4, Supplementa Entomologica, No. 3, p. 47. (D) \\
\hline plathanana & CAPUA & I88I, Proc. Linnean Soc. N.S. Wales, 6: 457. \\
\hline platiastis & COMPSOLECHIA & I922, Trans. Ent. Soc. London, I922: 84 . (BM) \\
\hline platyacma & HELENODES & I930, Trans. Ent. Soc. London, 78: 3I9. (P) \\
\hline platyantha & ZACORISCA & I924, Exotic Microlepidoptera, 3: II3. \\
\hline platyaula & CRAMBODOXA* & I9I3, Trans. Ent. Soc. London, I9I 3: I74. (BM) \\
\hline platybyrsa & THYRSOTARSA* & I92I, Zool. Meded. 6: I88. (L) \\
\hline platycapna & TIMYRA & I9I6, Exotic Microlepidoptera, I: 569 . (BM) \\
\hline platychlora & LIMNOECIA & I9I5, Exotic Microlepidoptera, I: 318. \\
\hline platycitra & CROCANTHES & I93I, Exotic Microlepidoptera, 4:73. (BM) \\
\hline platycolpa & STENOMA & I9I5, Exotic Microlepidoptera, I: 465. (BM) \\
\hline platyconta & $\begin{array}{l}\text { MACROTONA } \\
\text { [NARTHECOCERO }\end{array}$ & Journ. Bombay Nat. Hist. Soc. I6: 597 . (Bм) \\
\hline osma & GRACILARIA & I9I2, in Wytsman, Genera Insectorum, fasc. I28, p. 30. \\
\hline remna & ARGYROPLOCE & $\begin{array}{l}\text { I935, in Caradja and Meyrick, Materialien zu einer Micio- } \\
\text { lepidopteren Fauna der Chinesischen Provinzen Kiangsu, } \\
\text { Chekiang und Hunan, p. 6r. (BM) }\end{array}$ \\
\hline platycycla & PROTHAMNODES* & I923, Exotic Microlepidoptera, 2:6I3. (BM) \\
\hline platycypha & AGRIOCEROS* & I928, Exotic Microlepidoptera, 3: 4I8. (BM) \\
\hline platydelta & DECADARCHIS & I907, Journ. Bombay Nat. Hist. Soc. I7: 75I. \\
\hline tydesma & ANTAEOTRICHA & I9I5, Exotic Microlepidoptera, I: 405. (BM) \\
\hline platydoxa & GELECHIA & I923, Exotic Microlepidoptera, 3:20. (BM) \\
\hline platydryas & LASPEYRESIA & I932, Exotic Microlepidoptera, 4: 226. (BM) \\
\hline platyleuca & MEGACRASPEDUS & I904, Proc. Linnean Soc. N.S. Wales, 29: 274. \\
\hline platyleucota & CAENORYCTA & I938, Trans. R. Ent. Soc. London, 87: 5I7. \\
\hline platyloxa & HAPSIFERA & I930, Exotic Microlepidoptera, 3: 553 . \\
\hline platymolybdis & ARGYROPLOCE & I930, Exotic Microlepidoptera, 3: 603. (BM) \\
\hline platyntis & TINEA & I894, Trans. Ent. Soc. London, I894: 28. \\
\hline latyochra & GLYPHIPTERYX & I920, Exotic Microlepidoptera, 2: 333. (ВM) \\
\hline yorma & LACTURA & I924, Exotic Microlepidopte"a, 3: I22. \\
\hline typhaea & TINEA & I926, Sarawak Mus. Journ. 3: 167. \\
\hline
\end{tabular}




\section{2}

platyphracta
platyphylla
platyptera
platyrrhoa
platyrrhyncha
platysaris

platysema

platyspora

platystega

platyterma

platyxantha

platyxipha

platyzona

platyzona

plausibilis

plebicola

plebigena

plectanodes

plectanopa

plectanota

plectica

plectocosma

plectodes

plectographa

plectospila

plectrantha

plectrodes

plectrophora

pleiadopa

plemochoa

pleonastes

pleonectis

plerota

plerotis

plesiasta

plesistia

pleuracma

pleuropa

pleurophanes

pleuroptila

pleurosaris

pleurotricha

plexigramma

plexigrapha

pleximorpha

plinthactis
TELPHUSA

STENOMA

SEMIOCOSMA

PLEUROTA

DECADARCHIS

TINEA

SAGALASSA

PTILOGENES

TORTRIX

STENOMA

PROMALACTIS

[ANORCOTA *]

CALLIPRORA

ARGYROPLOCE

MELASINA

HELIOCAUSTA

STENOMA

GYMNELEMA

HILAROGRAPHA

COLONANTHES*

MACHIMIA

STOMPHASTIS*

LASPEYRESIA

SIMAETHIS

ASYMPHORODES

ACROCERCOPS

COESYRA

ARGYRESTHIA

CENARCHIS

BRENTHIA

PHTHORIMAEA

STENOMA

SCYTHRIS

LASPEYRESIA

ANTAEOTRICHA

COSMOPTERYX

STENOMA

POLYHYMNO

TRICHOTAPHE

COPROMORPHA

TORTRIX

RECURVARIA

STENOMA

DICHOMERIS

[BROCHOMETIS*]

PHRIXOSCELES

STENOMA

PROTOLECHIA
1935, Exotic Microlepidoptera, 4:584. (вм)

I9I6, Exotic Microlepidoptera, I: 540. (вм)

I888, Trans. Proc. New Zealand Inst. 20: 80.

1923, Exotic Microlepidoptera, 3: 6I. (BM)

I928, Exotic Microlepidoptera, 3: 402. (BM)

I93I, Ann. Mus. Nac. Hist. Nat. Buenos Aires, 36:4II.

(BM)

I92I, Zool. Meded. 6: I8o. (L)

I932, Exotic Microlepidoptera, 4: 305. (v)

I920, Voyage de Ch. Alluaud et $R$. Jeannel en Afrique

Oriental, II, Microlepidoptera, p. 48 . (P)

I9I5, Exotic Microlepidoptera, I: 436. (BM)

I909, Trans. Ent. Soc. London, I9o9: I8. (BM)

I922, Trans. Ent. Soc. London, I922: 69. (BM)

I9I7, Trans. Ent. Soc. London, I9I7: 28 . (BM)

I905, Journ. Bombay Nat. Hist. Soc. I6: 6I6.

I9I3, Exotic Microlepidoptera, I : I28. (MA)

I9I8, Exotic Microlepidoptera, 2: 205. (BM)

1924, Exotic Microlepidoptera, 3: 78 . (T)

I92I, Exotic Microlepidoptera, 2: 480. (BM)

I923, Exotic Microlepidoptera, 3: I2. (BM)

I9I8, Exotic Microlepidoptera, 2: 2I6. (BM)

I9I 2, in Wytsman, Genera Insectorum, fasc. I28, p. I9.

I921, Ann. Transvaal Mus. 8: 64. (T)

I921, Ann. Transvaal Mus. 8: I II. (T)

I929, Trans. Ent. Soc. London, 76: 498.

I92I, Exotic Microlepidoptera, 2: 469 .

I9I3, Exotic Microlepidoptera, I: I20.

I9I3, Exotic Microlepidoptera, I : I35. (BM)

I 924, Trans. Ent. Soc. London, I923: 55I. (BM)

I92I, Ann. Transvaal Mus. 8: II2. (T)

I9I6, Trans. Proc. New Zealand Inst. 48: 4 I5.

I9I 5, Exotic Microlepidoptera, I : 475. (BM)

I897, Proc. Linnean Soc. N.S. Wales, 22: 423.

I92I, Ann. Transvaal Mus. 8: 63. (T)

1925, Exotic Microlepidoptera, 3: I65. (BM)

I9I9, Exotic Microlepidoptera, 2: 284.

I930, Exotic Microlepidoptera, 4:24. (BM)

I926, Exotic Microlepidoptera, 3: 280. (BM)

I921, Ann. Transvaal Mus. 8: 86. (T)

I905, Journ. Bombay Nat. Hist. Soc. I6: 606. (BM)

I937, Exotic Microlepidoptera, 5: I27. (T)

I923, Exotic Microlepidoptera, 3: I4. (BM)

I925, Exotic Microlepidoptera, I3: 206. (BM)

I922, Trans. Ent. Soc. London, I922: IIO. (BM)

I9I6, Exotic Microlepidoptera, I: 623.

I930, Exotic Microlepidoptera, 4: 32. (BM)

I904, Proc. Linnean Soc. N.S. Wales, 29: 363. 


plinthinana
plinthinopa
plinthiota
plinthoglypta
plinthograpta
plinthomicta
plinthotripta
plocamandra
plocamis
plocamopa

plocanota plocogastra plocogramma plumata plumbaginea plumbaria plumbata plumbea plumbifera plumbiflua plumbigera plumbosa plumigera pluvialis pneumatica podarga poecilantha poecilaspis poecilella poecilopa poetica pogonias

pogonopis polemarcha polemica polemistis poliantha poliarcha polias poliastis poliochra poliocoma poliocrana poliodes poliodes poliombra
STREPSICEROS LEPTOCROCA STATHMOPODA PYRGOTIS ARGYROPLOCE EOCHROIS MACHAEROPTERIS LECITHOCERA ACROCERCOPS ODITES

SCYTHRIS

SCYTHRIS

AGRIOPHARA

ARISTOTELIA

EUCOSMA

PROCELEUSTIS

CHOLOTIS

PERIACMA

HIEROXESTIS

STATHMOPODA

ENCRATORA*

TRICHOTAPHE

ANATARACTIS*

ORNEODES

GRACILARIA

HOMOSACES

ZACORISCA

IRIANASSA

EULECHRIA

SEMNOSTOMA

ARGYROPLOCE

ONEBALA

[CYNICOSTOLA*]

MYRMECOZELA

TISIS

CHELARIA

OCYSTOLA

SCYTHRIS

EUCHAETIS

CAPUA

MELASINA

TORTRIX

LECITHOCERA

OECOPHORA

AMIYDRIA

HOMADAULA

ANACAMPSIS
I88I, Proc. Linnean Soc. N.S. Wales, 6: 689. I920, Exotic Microlepidoptera, 2: 368.

I9Io, Trans. Ent. Soc. London, I9IO: 454.

I892, Trans. Proc. New Zealand Inst. 24: 218.

I93I, Exotic Microlepidoptera, 4: I35. (BM)

I9I4, Exotic Microlepidoptera, I : I93.

I9I9, Exotic Microlepidoptera, 2: 257.

1907, Journ. Bombay Nat. Hist. Soc. I7: 737. (Вм)

I908, Journ. Bombay Nat. Hist. Soc. I 8: 823.

I935, in Caradja and Meyrick, Materialien zu einer Micro-

lepidopteren Fauna der Chinesischen Provinzen Kiangsu,

Chekiang und Hunan, p. 84. (вм)

I897, Proc. Linnean Soc. N.S. Wales, 22: 425.

I93I, Journ. Linnean Soc. London, 37:282. (BM)

I9I5, Exotic Microlepidoptera, I: 407. (BM)

I9I7, Trans. Ent. Soc. London, I9I7: 30. (BM)

I93I, Exotic Microlepidoptera, 4: I27. (BM)

I921, Ann. Transvaal Mus. 8: I05. (T)

I9I5, Exotic Microlepidoptera, I: 330. (BM)

I9I9, Exotic Microlepidoptera, 2: 238. (BM)

I9I4, Exotic Microlepidoptera, I : 287.

I9I I, Trans. New Zealand Inst. 43: 75 .

1923, Exotic Microlepidoptera, 2:6I8. (BM)

I9I3, Ann. Transvaal Mus. 3: 302. (T)

I9I6, Exotic Microlepidoptera, I : 565 .

I908, Trans. Ent. Soc. London, I907: 505.

I920, Exotic Microlepidoptera, 2: 297.

I9I4, Exotic Microlepidoptera, I : 256. (BM)

I924, Exotic Microlepidoptera, 3: II3.

I923, Exotic Microlepidoptera, 2: 6I6. (BM)

I883, Proc. Linnean Soc. N.S. Wales, 7: 53I.

I9I8, Exotic Microlepidoptera, 2: I28. (BM)

I909, Journ. Bombay Nai. Hist. Soc. I9: 437. (BM)

I923, Exotic Microlepidoptera, 3: 43. (BM)

1926, Ann. South African Mus. 23: 342. (S.A)

I926, Sarawak Mus. Journ. 3: I55.

I935, Exotic Microlepidoptera, 4:589. (BM)

I902, Trans. R. Soc. S. Australia, 26: I34.

I92I, Exotic Microlepidoptera, 2: 44I.

I888, Proc. Linnean Soc. N.S. Wales, (Ser. 2) 2: 939.

I9I3, Trans. Proc. New Zealand Inst. 45: 26.

I937, Exotic Microlepidoptera, 5: 78. (T)

1920, Voyage de Ch. Alluaud et R. Jeannel en Afrique

Oriental, II, Microlepidoptera, p. 5I. (P)

I9I6, Exotic Microlepidoptera, I: 593.

I886, Proc. Limean Soc. N.S. Wales, Io: 787.

I909, Ann. South African Mus. 5: 362. (SA)

I907, Proc. Linnean Soc. N.S. Wales, 32: 74.

I922, Trans. Ent. Soc. London, I922: 77. (BMI) 


$\begin{array}{ll}\text { poliopa } & \text { AGRIOPHARA } \\ \text { poliopis } & \text { ACROLEPIA } \\ \text { polismatica } & \text { EUTORNA } \\ \text { politica } & \text { AROTROMIMA* } \\ \text { politicopa } & \text { GLAPHYRISTIS } \\ \text { politis } & \text { OECOPHORA } \\ \text { pollinaris } & \text { NEMOTOIS } \\ \text { polluta } & \text { ASYMPHORODES } \\ \text { polyactis } & \text { LIMNOECIA } \\ \text { polyaema } & \text { MUSURGA } \\ \text { polyantha } & \text { DINOCHORA } \\ \text { polyarcha } & \text { HOMONA } \\ \text { polyastra } & \text { USSARA } \\ \text { polyaula } & \text { THIOTRICHA } \\ \text { polycapnias } & \text { MELASINA } \\ \text { polycentra } & \text { TRETOSCOPA* } \\ \text { polychlora } & \text { TISIS } \\ \text { polychorda } & \text { NEMOTOIS } \\ \text { polychorda } & \text { PSEUDURGIS } \\ \text { polychroma } & \text { ERITHYMA } \\ \text { polychrysa } & \text { METAPODISTIS } \\ \text { polyclasta } & \text { ACROCERCOPS } \\ \text { polycosma } & \text { PARACHANDA } \\ \text { polydora } & \text { PHANEROZELA* } \\ \text { polydoxa } & \text { NEPTICULA } \\ \text { polyglypta } & \text { STENOMA } \\ \text { polygnampta } & \text { TRICHOTAPHE } \\ & \end{array}$

$\begin{array}{ll}\text { polygramma } & \text { GELECHIA } \\ \text { polygrapta } & \text { COMODICA } \\ \text { polyleuca } & \text { TRACHYPEPLA } \\ \text { polymorpha } & \text { ARGYROPLOCE } \\ \text { polyplecta } & \text { HEMIMENE } \\ \text { polyscia } & \text { HYPOPHRICTIS } \\ \text { polyspila } & \text { HECTOMANES } \\ \text { polystacta } & \text { SCARDIA } \\ \text { polysticha } & \text { NEUROZESTIS } \\ & \\ \text { polytechna } & \text { TORTRIX } \\ \text { polyxena } & \text { ACMOSARA } \\ \text { polyxena } & \text { EUCOSMA }\end{array}$

polyxesta polyzela pomifera pomivora pompeiana pompholycias
I9I6, Exotic Microlepidoptera, I: 485. (BM) I9I9, Exotic Microlepidoptera, 2: 227. (BM) I93I, Exotic Microlepidoptera, 4: I9I. (BM) I929, Exotic Microlepidoptera, 3: 532. (BM) I934, Exotic Microlepidoptera, 4: 509 . I888, Trans. Proc. New Zealand Inst. 20: 8I. I907, Journ. Bombay Nat. Hist. Soc. I7: 993. I929, Trans. Ent. Soc. London, 76:500. I921, Exotic Microlepidoptera, 2: 455. I923, Exotic Microlepidoptera, 3: 4. (BM) I924, Exotic Microlepidoptera, 3: 69. I924, Exotic Microlepidoptera, 3: I06. (T) I937, Exotic Microlepidoptera, 5: I26. (T) I9I8, Exotic Microlepidoptera, 2: I25. (BM) I922, Exotic Microlepidoptera, 2: 607. (P) I9I6, Exotic Microlepidoptera, I : 607. I926, Sarawak Mus. Journ. 3: I55. I9I4, Supplementa Entomologica, No. 3, p. 6I. (D) I9I3, Ann. Transvaal Mus. 3: 333. (T) I9I4, Exotic Microlepidoptera, I: 224. (BM) I934, Exotic Microlepidoptera, 4:523. (BM) I9I9, Exotic Microlepidoptera, 2: 232. I930, Ann. Naturhist. Mus. Wien, 44: 225. (v) I92I, Exotic Microlepidoptera, 2: 404. I9II, Journ. Bombay Nat. Hist. Soc. 2I: IO7. (BM) I9I5, Exotic Microlepidoptera, I: 427. (BM) I938, Institut des Parcs Nationaux du Congo Belge. fasc. I4, p. I3, pl. 2, fig. I.

I9I4, Ann. Transvaal Mus. 4: I92. (T) I924, Exotic Microlepidoptera, 3:82.

I93I, Trans. Proc. New Zealand Inst. 62: 95.

I932, Trans. Ent. Soc. London, 80: II2.

I922, Exotic Microlepidoptera, 2: 529. (BM) I9I7, Exotic Microlepidoptera, 2: 85.

I89o, Proc. Linnean Soc. N.S. Wales, (Ser. 2) 4: II27. I9I8, Ann. Transvaal Mus. 6: 47. (T) I938, Institut des Parcs Nationaux du Congo Belge, fasc. I4, p. 25, pl. 3, fig. I. I924, Exotic Microlepidoptera, 3: II4. (T) I887, Proc. Linnean Soc. N.S. Wales, (Ser. 2) I: I043. I937, in Caradja and Meyrick, Deuts. Ent. Zeit. Iris, 5I: I 79 .

I89o, Trans. R. Soc. S. Australia, I3: 67. I920, Exotic Microlepidoptera, 2: 33I. (BM) I9I3, Ann. Transvaal Mus. 3:3I0. (T) I920, Exotic Microlepidoptera, 2: 340. (BM) I925, Exotic Microlepidoptera, 3: I76. (BM) I935, in Caradja and Meyrick, Materialien zu einer Microlepidopteren Fauna der Chinesischen Provinzen Kiangsu, Chekiang und Hunan, p. 58. (BM) 
pompholyctis ponderata ponophora pontifera pontifex pontifica pontifica pontificalis pontifraga pontigera pontiseca popularis porinodes porophora porota porpacias porpanthes porphyracma porphyractis porphyrantha porphyrantha porphyranthes porphyrarcha porphyrarga

porphyraspis porphyraspis porphyraspis porphyrastis porphyratma porphyraula porphyrea porphyreana porphyreuta porphyrias

\section{porphyrina} porphyris porphyritica porphyritis porphyrocentra

porphyrogona TYRIOZELA* porphyrogramma TRICHOTAPHE porphyropa TINEA porphyrophanes DICHELOPA porphyropis COPOCENTRA porphyropla HOPLOPHANES porphyroscia PITYOCONA porphyrospila MACHLOTICA
I889, Proc. Linnean Soc. N.S. Wales, (Ser. 2) 3: I677. I9I6, Exotic Microlepidoptera, I: 488. (BM)

1897, Proc. Linnean Soc. N.S. Wales, 22: 375.

I932, Exotic Microlepidoptera, 4: I96. (BM)

I9I5, Trans. Ent. Soc. London, I9I5: 240.

I9I I, Trans. New Zealand Inst. 43: 74.

I9I9, Exotic Microlepidoptera, 2: 276. (BM)

1929, Trans. Ent. Soc. London, 76: 505.

I928, Exotic Microlepidoptera, 3: 444. (BM)

I927, Insects of Samoa, 3, Lepidoptera, fasc. 2, p. Iog.

I936, Exotic Microlepidoptera, 5: 52. (BM)

I904, Proc. Linnean Soc. N.S. Wales, 29: 282.

I9I5, Exotic Microlepidoptera, I : 454. (вM)

I929, Trans. Proc. New Zealand Inst. 60: 488.

I9I7, Exotic Microlepidoptera, 2: 50.

I9o8, Journ. Bombay Nat. Hist. Soc. I8: 625.

I906, Trans. Ent. Soc. London, I906: I80. (Bм)

I922, Exotic Microlepidoptera, 2: 564 .

I937, Exotic Microlepidoptera, 5: II9.

I9I3, Ann. Transvaal Mus. 3: 309. (T)

I9I3, Exotic Microlepidoptera, I: 90.

I921, Ann. Transvaal Mus. 8: I22. (T)

1929, Trans. Ent. Soc. London, 76: 501.

1934, in Caradja and Meyrick, Deuts. Ent. Zeit. Iris, 48:

37.

I933, Exotic Microlepidoptera, 4: 440. (C)

I930, Exotic Microlepidoptera, 3: 625. (BM)

I909, Trans. Ent. Soc. London, I909: 23. (вм)

I9I5, Exotic Microlepidoptera, I: 449. (BM)

I930, Exotic Microlepidoptera, 4: 5. (BM)

1927, Trans. Proc. New Zealand Inst. 58: 3 I4.

I886, Proc. Linnean Soc. N.S. Wales, Io: 820 .

I88I, Proc. Linnean Soc. N.S. Wales, 6: 443.

I9I7, Ann. South African Mus. Io: I3. (SA)

I920, Voyage de Ch. Alluaud et $R$. Jeannel en Afrique

Oriental, II, Microlepidoptera, p. 87.

I932, Exotic Microlepidoptera, 4: 284.

I907, Proc. Linnean Soc. N.S. Wales, 32: 79.

I9I4, Exotic Microlepidoptera, I: 237. (BM)

I886, Trans. Proc. New Zealand Inst. I8: I76.

I937, in Caradja and Meyrick, Deuts. Ent. Zeit. Iris, 5I: I 76 .

I93I, Exotic Microlepidoptera, 4: I85.

I9I4, Trans. Ent. Soc. London, I9I4:278. (BM)

1927, Exotic Microlepidoptera, 3: 332.

I934, Pacific Ent. Surv. Publ. 7, art. 28, p. 344. (вв)

1922, Exotic Microlepidop:era, 2: 587.

I897, Proc. Linnean Soc. N.S. Wales, 22: 413.

I927, Insects of Samoa, 3, Lepidoptera, fasc. 2, p. 76. (вм)

I926, Exotic Microlepidoptera, 3: 306. (v) 
$\begin{array}{ll}\text { porphyrota } & \text { TINEA } \\ \text { porpocosma } & \text { ARGYROPLOCE }\end{array}$

porpota

porpotis

porthmis

portuosa

postbarbata

postscripta

potamias

practica

practicodes

practicopa

practoria

praealbata

praealbescens

praecana

praecauta

praecentrix

praeceps

praeceps

praecepta

praeceptrix

praecincta

praecincta

praecipitalis

praecipitata

praecipua

praecipua

praecisa

praecisa

praeclara

praeclinata

praeclivis

praeclusa

praecolor

praeconia

praedator

praedatrix

praediata

praedicata

praedonia

praeducta

praefasciata

praefinita

praefixa

praeflava

praefracta
CYDIA

MACHIMIA

BUCCULATRIX

BATODES

PLATYPTILIA

ANTISPILA

EURYTHECTA

ACROLOPHUS

BORKHAUSENIA

MELASINA

ODITES

STATHMOPODA

ZOMEUTIS

PYRGOPTILA

STENOMA

SARISOPHORA

ASAPHISTIS*

STENOMA

MELASINA

TIMYRA

ANTISPILA

OPOGONA

GELOPHAULA

STOMOPTERYX

BUCCULATRIX

CNEPHASIA

ANTAEOTRICHA

HEMIMENE

ETHMIA

TORTRIX

HYPERCALLIA

ACROCERCOPS

COMOCRITIS

ARGYROTOXA

OXYPTILUS

CARYOLESTIS*

ASCALENIA

GELECHIA

MENEPTILA*

GAESA

PHTHEOCHROA

MOMPHA

ODITES

SCYTHRIS

APHTHONETUS
I893, Proc. Linnean Soc. N.S. Wales, 7: 538.

I935, in Caradja and Meyrick, Materialien zu einer Microlepidopteren Fauna der Chinesischen Provinzen Kiangsu,

Chekiang und Hunan, p. 59.

I907, Journ. Bombay Nat. Hist. Soc. I7: 733.

I9I4, Exotic Microlepidoptera, I : I79. (BM)

I908, Proc. Zool. Soc. London, p. 737.

I922, Exotic Microlepidoptera, 2: 495. (BM)

I938, Institut des Parcs Nationaux du Congo Belge, fasc. I4, P. 4, pl. I, fig. I.

I92I, Exotic Microlepidoptera, 2: 403.

Igog, Trans. New Zealand Inst. 4I: II.

I9I3, Trans. Ent. Soc. London, I9I3: I93. (BM)

I9I8, Exotic Microlepidoptera, 2: 2I3.

I934, Exotic Microlepidoptera, 4: 5I9. (c)

I908, Journ. Bombay Nat. Hist. Scc. I8: 63I. (BM)

I9I5, Exotic Microlepidoptera, I: 337.

I922, Exotic Microlepidoptera, 2: 505. (P)

I9I3, Exotic Microlepidoptera, I: I28. (MA)

I9I6, Exotic Microlepidoptera, I: 527. (Bм)

I93I, Exotic Microlepidoptera, 4: 78 .

I909, Journ. Bombay Nat. Hist. Soc. I9: 590. (BM)

I9I5, Exotic Microlepidoptera, I: 453. (BM)

I9I6, Exotic Microlepidoptera, I: 6I3.

I9Io, Journ. Bombay Nat. Hist. Soc. 20: 46I. (BM)

I92I, Exotic Microlepidoptera, 2: 403.

I9I6, Exotic Microlepidoptera, I : 620.

I934, Trans. roy. Soc. N.Z. 64: I52.

I9I8, Exotic Microlepidoptera, 2: I37. (BM)

I9I8, Ann. Transvaal Mus. 6: 42. (T)

I9I7, Trans. Ent. Soc. London, I9I7: II. (BM)

I9I2, Trans. Ent. Soc. London, I9I I: 709. (BM)

I9I6, Exotic Microlepidoptera, 2: 28. (BM)

I9Io, Trans. Ent. Soc. London, I9Io: 460. (BM)

I92I, Ann. Transvaal Mus. 8: 52. (T)

I92I, Exotic Microlepidoptera, 2: 389. (BM)

I9I4, Journ. Bombay Nat. Hist. Soc. 23: I20.

I9I4, Journ. Bombay Nat. Hist. Soc. 23: I29. (BM)

I937, Exotic Microlepidoptera, 5: I29. (C)

I9I0, Rec. Indian Mus. 2: 217. (K)

I934, Pacific Ent. Surv. Publ. 6, art. 22, p. IIo. (BB)

I922, Exotic Microlepidoptera, 2: 579. (BM)

1923, Exotic Microlepidoptera, 3: I9. (Sub.)

I9I5, Exotic Microlepidoptera, I: 333.

I929, Exotic Microlepidoptera, 3: 5II.

I932, Exotic Microlepidoptera, 4: 266. (v)

I9I7, Exotic Microlepidoptera, 2: 53. (BM)

I92I, Zool. Meded. 6: I74. (L)

I9r3, Ent. Mitteil. 2: 299. (D)

I935, Proc. Hawaiian Ent. Soc. 9:63. (SP) 


$\begin{array}{ll}\text { praefulgens } & \text { LAMPROZELA* } \\ \text { praefulva } & \text { LYONETIA } \\ \text { praefumata } & \text { EPERMENIA } \\ \text { praefusca } & \text { OPOSTEGA } \\ \text { praefuscata } & \text { PHALONIA } \\ \text { praegemina } & \text { ACROCERCOPS } \\ \text { praemaculata } & \text { CHELARIA }\end{array}$

praematura praematura praemiata praenigrata praenigratus praenivalis praenivea praenubila praepedita praeposita praeramis praerupta praescripta praesecta praeses praesignis praesiliens praestabilis praestricta praestrigata praestructa praesul

\section{praetexta} praetextata praetincta praeumbrata praeusta praevacua praevecta pragmatias pragmatica prasina prasinantha prasinaula prasinias prasinissa prasinochroa prasinodes prasinoleuca prasinopis prasinoptila

\section{HIEROXESTIS} SCYTHRIS HIEROMANTIS ZYGOLOPHA * PETROPHORUS [sic!] CAPNOLOCHA * ANACAMPSIS PTILOGENES MACHIMIA PHTHEOCHROA OEGOCONIA ANTAEOTRICHA PROTEOPTERYX ACROCERCOPS LECITHOCERA ANISOPLACA ARGYROPLOCE TINEA PARECTOPA DICHELOPA SCYTHRIS BORKHAUSENIA

CEROMITIA* TRICHOSTIBAS HYPONOMEUTA TINEA CALICOTIS DICHOMERIS CRYPTOLECHIA ODITES CRASPEDOTIS* AGRIASTIS PORPODRYAS* ACROLEPIA ARGYROPLOCE ACROCLITA COPROMORPHA ORTHENCHES GELECHIA CROCANTHES* ALSODRYAS
I9I6, Exotic Microlepidoptera, 2: 9 . I9I I, Journ. Bombay Nat. Hist. Soc. 2I: Iog. I9II, Ann. Transvaal Mus, 2: 236. (T) I9I3, Ann. Transvaal Mus. 3: 327. (T) I925, Bull. Soc. R. Ent. Egypte, 9: 207. I9I7, Ann. South African Mus. I7: I2. (SA) I931, in Caradja, Bull. Sect. sci. Acad. roum. I4: 67. (BM) I9o9, Ann. Transvaal Mus. 2: 26, pl. 8, fig. 5. (T) 1937, Exotic Microlepidoptera, 5: I45. I92I, Exotic Microlepidoptera, 2: 460. (Bм) I9I4, Exotic Microlepidoptera, I: 242. (BM) I92I, Exotic Microlepidoptera, 2: $42 \mathrm{I}$. I925, Exotic Microlepidoptera, 3: I52. I9I4, Trans. Ent. Soc. London, I9I4: 260. (BM) I926, Exotic Microlepidoptera, 3:23I. (BM) I920, Exotic Microlepidoptera, 2: 375 . I9I7, Trans. Ent. Soc. London, I9I7: 5. (BM) I9I8, Exotic Microlepidoptera, 2: I55. (BM) I9I5, Exotic Microlepidoptera, I: 394. (BM) I9I2, Ent. Mon. Mag. 48: 34. (Sub.) I922, Exotic Microlepidoptera, 2: 562. (BM) I9I9, Exotic Microlepidoptera, 2: 236. (BM) I9I3, Trans. Ent. Soc. London, I9I3: I75. (BM) I924, Exotic Microlepidoptera, 3:68. (BM) I927, Exotic Microlepidoptera, 3: 323. (BM) I9I8, Ann. Transvaal Mus. 6: 39. (T) I929, Trans. Ent. Soc. London, 76: 492. (BM) I922, Exotic Microlepidoptera, 2: 554 . I93I, Ann. Mus. Nac. Hist. Nat. Buenos Aires, 36: 39 I. (BM) I924, Exotic Microlepidoptera, 3: 79. (T) I9I3, Exotic Microlepidoptera, I: I40. I9I4, Journ. Bombay Nat. Hist. Soc. 23: I26. (BM) I9I9, Exotic Microlepidoptera, 2: 274. (BM) I922, Exotic Microlepidoptera, 2: 584. (BM) I922, Exotic Microlepidoptera, 2: 504. (P) 1929, Trans. Ent. Soc. London, 76:513. (BM) I9I4, Journ. Bombay Nat. Hist. Soc. 22: 780. (BM) I904, Proc. Limnean Soc. N.S. Wales, 29: 327. I9I4, Trans. Ent. Soc. London, I9I4: 25I. (BM) I920, Exotic Microlepidoptera, 2: 305. (BM) I927, Exotic Microlepidoptera, 3: 359. (BM) I9I6, Exotic Microlepidoptera, I: 562. (BM) I921, Zool. Meded. 6: I52. (L) I906, Trans. R. Soc. S. Australia, 30: 52. I886, Trans. Proc. New Zealand Inst. I8: I76. I921, Zool. Meded. 6: I6I. (L) I886, Trans. Ent. Soc. London, I886: 277. I922, Trans. Ent. Soc. London, I922: 75. (BM) 
prasinospila prasinospora prasochalca prasoleuca prasophanes prasophyta pratifera premnias prensans prensoria

presbytica primaeva primaria primaria primigena primigena primigenia primipilana primulacea princeps principalis prioleuca prionistis prionota priozona prisca priscata prisciformis

priscilla prismatica prismatica prismatopa pristica pristina privata proaula probata probatus probolactis probolaea probolaspis probolias probolias probolopis procax procax procellaris procellosa
PROTEOTERAS IMMA

TORTYRA AGRIOPHARA PROCHOLA SEMIOCOSMA STENOMA MICROSCHISMUS DICHOMERIS TINEA

COLEOPHORA SCARDIA

NEPTICULA POLYCHROSIS LASPEYRESIA NEPTICULA ANACAMPSIS ARISTOTELIA ADAINA HELIODINES STATHMOPODA GELECHIA TORTRIX ACROCERCOPS IMMA PROTOLECHIA CENARCHIS THAMNOSCELIS* [ISOTHAMNIS *] ELEGISTIS

GRACILARIA LASPEYRESIA BATTARISTIS TIMYRA CLADOPHANTIS GYMNOGRAMMA DRAGMATUCHA* TELPHUSA PTEROPHORUS LYONETIA CHELARIA ONEBALA EPAGOGE PTEROPHORUS ARISTOTELIA ALLOCOTA SPARGANOTHIS ACROCERCOPS EUCOSMA
I9I2, Ent. Mon. Mag. 48: 34. (Sub.) I9I5, Trans. Ent. Soc. London, I9I5: 215. I907, Proc. Linnean Soc. N.S. Wales, 32: 98. I9I6, Exotic Microlepidoptera, I: 483. (BM) I922, Exotic Microlepidoptera, 2: 581. (BM) I883, New Zealand Journ. Sci. I: 523. I925, Exotic Microlepidoptera, 3: 222. (BM) I9I3, Ann. Transvaal Mus. 3: 269. (T) I922, Trans. Ent. Soc. London, I922: III. (BM) I931, Ann. Mus. Nac. Hist. Nat. Buenos Aires, 36: 4I 2. (BM)

I921, Ann. Transvaal Mus. 8: I20. (T) I893, Proc. Linnean Soc. N.S. Wales, 7: 524. I9I3, Ann. Transvaal Mus. 3: 326. (T) I9o9, Ann. Transvaal Mus. 2: 9, pl. 3, fig. 8. (T) I9I2, Journ. Bombay Nat. Hist. Soc. 21:877. (BM) Igo6, Trans. R. Soc. S. Australia, 30: $5^{8}$. I9I8, Exotic Microlepidoptera, 2: I4I. (BM) I923. Exotic Microlepidoptera, 3: 8. (BM) I929, Trans. Ent. Soc. London, 76: 507. I906, Trans. R. Soc. S. Australia, 30: 54 . I9I3, Exotic Microlepidoptera, I: I54. I9II, Ann. Transvaal Mus. 3: 66. (BM) I928, Exotic Microlepidoptera, 3: 457 . I928, Exotic Microlepidoptera, 3: 407. (T) I906, Trans. Ent. Soc. London, I906: I78. (BM) I904, Proc. Linnean Soc. N.S. Wales, 29: 343. I924, Trans. Ent. Soc. London, I923: 552. (BM)

I935, Exotic Microlepidoptera, 4:558. I922, Exotic Microlepidoptera, 2: 603. I907, Journ. Bombay Nat. Hist. Soc. I7: 983. I9I I, Proc. Linnean Soc. N.S. Wales, 36: 289. I9I4, Trans. Ent. Soc. London, I9I4: 246. (BM) I9I6, Exotic Microlepidoptera, I: 57I. (BM) I925, Exotic Microlepidoptera, 3: I6o. (T) I924, Exotic Microlepidoptera, 3: I2I. (T) Igo8, Proc. Zool. Soc. London, p. 726. Igo9, Ann. Transvaal Mus. 2 : II, pl. 4, fig. 4. (T) I938, Trans. R. Ent. Soc. London, 87: 504. I9II, Trans. Linnean Soc. London, I4: 293. I9I3, Ann. Transvaal Mus. 3: 298. (T) I929, Exotic Microlepidoptera, 3: 508. (T) I907, Journ. Bombay Nat. Hist. Soc. I7: 977. I89I, Ent. Mon. Mag. 27: I2.

I923, Exotic Microlepidoptera, 3: 8. (BM) I9II, Trans. Linnean Soc. London, I4: 274. (BM) I9I2, Ent. Mon. Mag. 48: 34. (Sub.) I9I4, Ann. Transwaal Mus. 4: 20I. (T) I9I7, Trans. Ent. Soc. London, I9I7: I9. (BM) 


procellosa
procentra
proceros
prochalina
prochlora
prochyta
proclina
proclivis
proconsularis
procontias
procremna
procridias
procritica
procrossa
procrossa
procyphodes
prodela
prodelta
prodigella
prodigiosa

prodotis

prodroma profana profluens profuga profunda profusa progama progonopis prographa prograpta projecta projecta projectrix prolapsa prolectans proletaria proleuca

prolixa prolocha prolongata prolopha promacha promalacta prometopa prominens prominula prominula
ODITES

FRISILIA

IMMA

PHOTODOTIS*

ASCERODES*

EPAGOGE

LIMNOECIA

LECITHOCERA

CARPOSINA

PTEROPHORUS

THEMELIOTIS

URODUS

STENOMA

IMMA

TRICHOTAPHE

DICHOMERIS

HELIOZELA

METACHANDA

TORTYRA

EPICHORISTA

SAROPLA

ARGYROPLOCE

THIODIA

PHRIXOSCELES

NEPHOGENES

PROTEODES

EPITHECTIS

NEPTICULA

NEPTICULA

PANICOTRICHA*

PILOCRATES*

EUCOSMA

STENOMA

THEMELIOTIS

STOMOPTERYX

HYPERCALLIA

TINEA

CRYPTOPHAGA

[XEROCRATES*]

AUXIMOBASIS

ERIDACHTHA*

SPILONOTA

LASPEYRESIA

PYRODERCES

OPOGONA

UTUCA

EUCOSMA

ARISTOTELIA

COPTOTELIA
I908, Proc. Zool. Soc. London, p. 729. (BM)

I9I6, Exotic Microlepidoptera, I: 573. (BM)

I928, Exotic Microlepidoptera, 3: 422. (BM)

I9I I, Ann. Transvaal Mus. 2: 229. (T)

1905, Trans. Ent. Soc. London, I905: 234.

igo8, Journ. Bombay Nat. Hist. Soc. I8: 618. (Bм)

1907, Journ. Bombay Nat. Hist. Soc. I7: 745.

I9Io, Journ. Bombay Nat. Hist. Soc. 20: 446. (BM)

I921, Ann. Transvaal Mus. 8: 5I. (T)

I908, Trans. Ent. Soc. London, I907: 496.

1931, Exotic Microlepidoptera, 4: 99.

1935, Arb. morph. taxon. Ent. Berl. 3: 108. (D)

I925, Exotic Microlepidoptera, 3: 217. (BM)

I906, Trans. Ent. Soc. London, I906: 184. (BM)

I913, Journ. Bombay Nat. Hist. Soc. 22: I77. (BM)

I922, Trans. Ent. Soc. London, I922: I I5. (BM)

1897, Proc. Linnean Soc. N.S. Wales, 22: 404.

I9I I, Trans. Linnean Soc. London, I4:277. (BM)

1907, Proc. Linnean Soc. N.S. Wales, 32: 10o.

I920, Voyage de Ch. Alluaud et R. Jeannel en Afrique

Oriental, II, Microlepidoptera, p. 52. (P)

I9I4, Exotic Microlepidoptera, I : 246.

I9I3, Ann. Transvaal Mus. 3: 277. (T)

I9I2, Ent. Mon. Mag. 48: 34. (Sub.)

I9I6, Exotic Microlepidoptera, I: 623 .

I913, Exotic Microlepidoptera, I: 132. (MA)

I905, Trans. Ent. Soc. London, I905: 236.

I921, Ann. Transvaal Mus. 8: 67. (T)

I924, Trans. Proc. New Zealand Inst. 55: 662.

I92I, Trans. Proc. New Zealand Inst. 53: 336.

19I3, Ann. Transvaal Mus. 3: 296. (T)

1920, Exotic Microlepidoptera, 2: 299. (BM)

I921, Ann. Transvaal Mus. 8: 53. (T)

I9I5, Exotic Microlepidoptera, I : 466. (BM)

I930, Exotic Microlepidoptera, 3: 549 .

I9I8, Exotic Microlepidoptera, 2: I37. (BM)

I926, Exotic Microlepidoptera, 3: 314. (BM)

I921, Zool. Meded. 6: I99. (L)

I89o, Trans. R. Soc. S. Australia, I3: 3I.

I922, Exotic Microlepidoptera, 2: 540. (BM)

I9Io, Journ. Bombay Nat. Hist. Soc. 20: 440. (BM)

I932, Exotic Microlepidoptera, 4: 306. (v)

I912, Journ. Bombay Nat. Hist. Soc. 21:875. (BM)

I897, Proc. Linnean Soc. N.S. Wales, 22: 35 I.

I9I5, Exotic Microlepidoptera, I: 361.

I909, Trans. Ent. Soc. London, I909: I3.

I922, Exotic Microlepidoptera, 2: 5I7. (BM)

I9I3, Ann. Transvaal Mus. 3:282. (T)

I9I3, Trans. Ent. Soc. London, I9I3: I 8o. (B.I) 


\begin{tabular}{ll} 
promiscua & CAPUA \\
promonitrix & GELECHIA \\
prompta & ACROCERCOPS \\
promylaea & BATRACHEDRA \\
prona & TORTRIX \\
pronephela & BORKHAUSENIA \\
propalaea & NEPTICULA \\
propensa & ONEBALA \\
properans & LABDIA \\
prophantis & HYPOSMOCOMA \\
prophetica & LATHICROSSA \\
prophracta & CALAMOTIS* \\
propitia & ARGYROPLOCE \\
propitia & LECITHOCERA \\
propria & ADAINA \\
propycnota & ORYGOCERA \\
prorepta & GELECHIA \\
prosacta & ACROCERCOPS \\
prosarista & ZARATHA \\
prosarithma & MONOPIS \\
proscripta & PROTOLECHIA \\
prosecta & EULIA \\
prosectrix & ETHMIOPSIS* \\
& \\
\hline &
\end{tabular}

$\begin{array}{ll}\text { prosedra } & \text { ODITES } \\ \text { proserga } & \text { EPERMENIA } \\ \text { prosiliens } & \text { ADOXOPHYES } \\ \text { prospera } & \text { ACROCERCOPS } \\ \text { prospera } & \text { ARTICOLLA } \\ \text { prospiciens } & \text { ATOMOTRICHA } \\ \text { prospicua } & \text { DEPRESSARIA } \\ \text { prospicua } & \text { XYLORYCTA } \\ \text { prosticta } & \text { GRACILARIA } \\ \text { prostylias } & \text { CRANAODES } \\ \text { protadelpha } & \text { OECOPHORA } \\ \text { protaxia } & \text { TINEA } \\ \text { protecta } & \text { PHILOBOTA } \\ \text { proteis } & \text { LEPIDOTARSA } \\ \text { protelesta } & \text { HIEROXESTIS } \\ \text { proterandra } & \text { LOBESIA } \\ \text { proterischna } & \text { PLATYPTILIA } \\ \text { proteroclina } & \text { OEGOCONIA } \\ \text { proterospila } & \text { ZELLERIA } \\ \text { proterozona } & \text { STATHMOPODA } \\ \text { proterva } & \text { DIATHRYPTICA } \\ \text { prothyrodes } & \text { NARYCIA } \\ \text { prothyropa } & \text { CRYPTOLECHIA }\end{array}$

protocentra PICROSPORA
I922, Arkiv för Zool. vol. I4, part I5, p. 2.

I927, Exotic Microlepidoptera, 3: 345. (BM)

I9I6, Exotic Microlepidoptera, 2: 3.

I9I7, Exotic Microlepidoptera, 2: 35 .

I9II, Ann. Transvaal Mus. 2: 223. (BM)

I907, Trans. Proc. New Zealand Inst. 39: II9.

I889, Trans. Proc. New Zealand Inst. 2I: I87.

I9Io, Journ. Bombay Nat. Hist. Soc. 20: 456. (BM)

I927, Insects of Samoa, 3, Lepidoptera, fasc. 2, p. 90.

I9I5, Exotic Microlepidoptera, I: 342. (BM)

I927, Trans. Proc. New Zealand Inst. 57: 70 I.

I9I8, Exotic Microlepidoptera, 2: I89. (BM)

I9I8, Ann. Transvaal Mus. 6: I2. (T)

I9I3, Ann. Transvaal Mus. 3: 293. (T)

I92I, Ann. Transvaal Mus. 8: 50. (T)

I930, Exotic Microlepidoptera, 3: 577. (BM)

I923, Exotic Microlepidoptera, 3: I9. (Sub.)

I9I8, Exotic Microlepidoptera, 2: I75.

I909, Journ. Bombay Nat. Hist. Soc. I9: 4IO.

I928, Exotic Microlepidoptera, 3: 425.

I92I, Exotic Microlepidoptera, 2: 429. (BM)

I932, Exotic Microlepidoptera, 4: 259. (v)

I935, in Caradja and Meyrick, Materialien zu einer Micro. lepidopteren Fauna der Chinesischen Provinzen Kiangsu

Chekiang und Hunan, p. 69. (BM)

I9I5, Exotic Microlepidoptera, I: 378 . (BM)

I9I3, Ann. Transvaal Mus. 3: 3I8. (T)

I928, Exotic Microlepidoptera, 3: 454. (BM)

I920, Exotic Microlepidoptera, 2: 293. (BM)

I909, Journ. Bombay Nat. Hist. Soc. I9: 59I. (Bм)

I924, Trans. Proc. New Zealand Inst. 55: 662.

I9I4, Ann. South African Mus. Io: 249. (SA)

I9I4, Exotic Microlepidoptera, I : 205. (MA)

Igo9, Ann. South African Mus. 5: 374. (SA)

I927, Exotic Microlepidoptera, 3: 327. (BM)

I889, Proc. Linnean Soc. N.S. Wales, (Ser. 2) 3: I672.

I924, Exotic Microlepidoptera, 3: 72.

I920, Exotic Microlepidoptera, 2: 383.

I888, Proc. Linnean Soc. N.S. Wales, (Ser. 2) 2: 93 I.

I9I5, Exotic Microlepidoptera, I: 364 .

I92I, Zool. Meded. 6: I55. (L)

I935, Exotic Microlepidoptera, 4: 555. (c)

I938, Rev. Franç. Lépidopterologie, 9: 70.

I893, Proc. Linnean Soc. N.S. Wales, $7: 584$.

I938, Trans. R. Ent. Soc. London, 87:521.

1907, Proc. Linnean Soc. N.S. Wales, 32: I39.

I92I, Ann. Transvaal Mus. 8: I34. (T)

I938, in Caradja and Meyrick, Dents. Ent. Zeit. Iris, 52 :

II.

I92I, Ann. Transvaal Mus. 8: I32. 
protochlora protocrossa protocyma protodoxa protogramma protographa protograpta protolyca

protoma protomacra protomima protomochla protona protonistis

protophaes protophanes protorna protorthra protosaris protosema protosticha protothrinca protoxantha protozona protracta protrocha protypa protypica provocata proxena proxena prozona prudens prudens prudentula pruinosa pruneticola prymnaea prymnaea psacadias psacasta psacasta psacastis psalactis psalidota psaliodes psalmographa psalmophanes
TRACHYPEPLA IMMA ARGYROPLOCE LOZOSTOMA PLEUROTA OPOGONA TINEA LECITHOCERA

LECITHOCERA GLYPHIPTERYX HIEROXESTIS OPOSTEGA ANAPHANTIS EPISYRTA

EOCHROA ISOCRITA XYSMATODOMA NEPHOGENES ANTAEOTRICHA NEPTICULA OCYSTOLA TINEA HELIOCAUSTA GELECHIA PSEUDURGIS BRACHMIA AUTOSTICHA ASAPHOCRITA * CATAMACTA MACROBATHRA OCYSTOLA (?) DISSOPTILA EUCOSMA LECITHOCERA PTILOGENES PHILOBOTA CACOECIA SYSCALMA* SYSCALMA TINEA MESOLECTA* PALTODORA HEMIMENE ISOCRITA LIMNOECIA ACROCERCOPS STENOMA EUXANTHIS
I883, New Zealand Journ. Sci. I : 522. I909, Trans. Ent. Soc. London, I909: 36. (BM) I936, Exotic Microlepidoptera, 4:6I3. (BM) 1897, Proc. Linnean Soc. N.S. Wales, 22: 4I8. I884, Proc. Linnean Soc. N.S. Wales, 9: 75I. I9I I, Journ. Bombay Nat. Hist. Soc. 2I: II2. I935, Exotic Microlepidoptera, 4: 578 .

I938, in Caradja and Meyrick, Deuts. Ent. Zeit. Iris, 52 : 5. (BM) I9I4, Exotic Microlepidoptera, I: I98. (BM) I907, Proc. Linnean Soc. N.S. Wales, 32 : II7. I9I8, Exotic Microlepidoptera, 2: I85.

I935, Exotic Microlepidoptera, 4:567. I9Io, Trans. Ent. Soc. London, I9Io: 472. I930, Ann. Naturhist. Mus. Wien, 44: 266, pl. I, fig. 35 .

I883, Proc. Linnean Soc. N.S. Wales, $7: 457$. 1927, Exotic Microlepidoptera, 3: 382. (T) I893, Proc. Linnean Soc. N.S. Wales, $7: 495$. I883, Proc. Linnean Soc. N.S. Wales, 8: 378. I9I5, Exotic Microlepidoptera, I: 406. (BM) I92I, Ann. Transvaal Mus. 8: I40. (T) I885, Proc. Linnean Soc. N.S. Wales, 9: Io7I. I932, Exotic Microlepidoptera, 4:327.

I883, Proc. Linnean Soc. N.S. Wales, $7: 480$. I926, Trans. Ent. Soc. London, 74: 277. (Bм) I924, Exotic Microlepidoptera, 3: 74. (T) I9I6, Exotic Microlepidoptera, I: 576. (BM) I908, Journ. Bombay Nat. Hist. Soc. I8: 457. (BM) I93I, Exotic Microlepidoptera, 4: I78. (BM) I9I2, Exotic Microlepidoptera, I: I. (BM) I9I4, Exotic Microlepidoptera, I: 2 I6. I9I4, Ann. South African Mus. Io: 247. (SA) I9I4, Trans. Ent. Soc. London, I9I4: 235. (BM) I9I7, Trans. Ent. Soc. London, I9I7: 23. (BM) I9I8, Exotic Microlepidoptera, 2: 106. I926, Exotic Microlepidoptera, 3: 232. (BM) I884, Proc. Linnean Soc. N.S. Wales, 8: 495. I935, Exotic Microlepidoptera, 4: 569 . I920, Exotic Microlepidoptera, 2: 38I. I930, Exotic Microlepidoptera, 3: 6I9. I9I2, Ann. Transvaal Mus. 3:80. (T) I883, Proc. Linnean Soc. N.S. Wales, 8: 37 I. Igo8, Proc. Zool. Soc. London, p. 723. (T) I922, Exotic Microlepidoptera, 2: 527. (BM) I9I2, Ann. South African Mus. Io: 65. (SA) I917, Exotic Microlepidoftera, 2: 43. I926, Exotic Microlepidoptera, 3: 294. I93I, Exotic Microlepidoptera, 4: 35. (v) I925, Bull. Soc. R. Ent. Egypte, 9: 208. 


\begin{tabular}{|c|c|}
\hline psaltria & ORTHIOSTOLA \\
\hline psamathaula & BATTARISTIS \\
\hline psamathias & APODIA \\
\hline psamathina & OCYSTOLA \\
\hline psamatholeuca & ACRIA \\
\hline psamathota & SCYTHRIS \\
\hline psammacta & CYDIA \\
\hline psammaula & DECADARCHIS \\
\hline psamminella & BEDELLIA \\
\hline psamminitis & LASPEYRESIA \\
\hline psamminopa & ARGYRESTHIA \\
\hline psammochroa & PLUTELLA \\
\hline psammochtha & CRYPTOPHASA \\
\hline psammocryptis & HYPERSYMMOCA \\
\hline psammocyma & EPAGOGE \\
\hline psammodes & TRACHYDORA \\
\hline psammodina & AEGIDOMORPHA* \\
\hline psammodora & ENDROSIS \\
\hline psammodoxa & PACHYPSALTIS \\
\hline psammogona & NARYCIA \\
\hline psammolitha & GLYPHIDOCERA \\
\hline psammophila & ACROLOPHUS \\
\hline psammophricta & ACALYPTRIS* \\
\hline psammosticha & MELASINA \\
\hline psammota & MELEONOMA \\
\hline psammoxantha & PLEUROTA \\
\hline psaphara & DEMIOPHILA* \\
\hline psapharana & CACOECIA \\
\hline psapharogma & HAPSIFERA \\
\hline sapharota & EPALEURA \\
\hline
\end{tabular}

[TYPHOGENES*]

psarodes psarotricha psathyra pselaphistis pselaphotis pseliacma pseliota pselioxantha psephias psephonoma

psephophora PHLOEOPOLA psephophragma ATHRINACIA pseudacma STENOMA pseudagyrta MONOPIS pseudarcha CAPUA pseudochyta ANTAEOTRICHA pseudogramma CRYPTOPHASA pseudomantis MERIDARCHIS
I928, Exotic Microlepidoptera, 3: 479. (BM) I934, Exotic Microlepidoptera, 4: 450. (BM) I891, Ent. Mon. Mag. 27: 56. (BM) I885, Proc. Linnean Soc. N.S. Wales, 9: Io7o. I930, Exotic Microlepidoptera, 4: I2.

I9I3, Ann. Transvaal Mus. 3: 3I2. (T) Igo8, Proc. Zool. Soc. London, p. 721 . (BM) I92I, Exotic Microlepidoptera, 2: 459. I889, Trans. Proc. New Zealand Inst. 2I: I65. I9I3, Ann. Transvaal Mus. 3: 279. (T) I932, Exotic Microlepidoptera, 4: 339. (BM) I886, Trans. Proc. New Zealand Inst. I8: I79. I925, Exotic Microlepidoptera, 3: I47. I923, Exotic Microlepidoptera, 3:63. (BM) I908, Journ. Bombay Nat. Hist. Soc. I8: 6I7. (Bм) I897, Proc. Linnean Soc. N.S. Wales, 22: 394. I932, Exotic Microlepidoptera, 4:229. (BM) I92I, Ann. Transvaal Mus. 8: 99. (T) I925, Bull. Soc. R. Ent. Egypte, 9:217. I93I, Exotic Microlepidoptera, 4: 99. I923, Exotic Microlepidoptera, 3: 48. (BM) I93I, Exotic Microlepidoptera, 4: Ioo. (v) I92I, Exotic Microlepidoptera, 2: 4 IO. I92I, Ann. Transvaal Mus. 8: I35. (T) I9I5, Exotic Microlepidoptera, I: 304. I884, Proc. Linnean Soc. N.S. Wales, 9: 755. I 906, Journ. Bombay Nat. Hist. Soc. I7: I52. I883, Proc. Linnean Soc. N.S. Wales, 7: I74. I936, Exotic Microlepidoptera, 5: 55. (c)

I9I7, Exotic Microlepidoptera, 2: 79.

I9Io, Proc. Linnean Soc. N.S. Wales, 35: 238. I897, Proc. Linnean Soc. N.S. Wales, $22: 382$. I886, Proc. Linnean Soc. N.S. Wales, Io: 77 I. I9Io, Journ. Bombay Nat. Hist. Soc. 20: 456. (BM) I9I5, Trans. Ent. Soc. London, I9I5: 237. I92I, Ann. Transvaal Mus. 8: I44. (T) I920, Ann. South African Mus. I7: 294. (SA) I929, Trans. Ent. Soc. London, 76: 502. 1904, Proc. Linnean Soc. N.S. Wales, 29: $35^{2}$. I887, Proc. Linnean Soc. N.S. Wales, (Ser. 2) I: ro46. (A) I883, Proc. Linnean Soc. N.S. Wales, 8: 352. I929, Exotic Microlepidoptera, 3: 5I8. (BM) I9I8, Exotic Microlepidoptera, 2: 203. (BM) I9I9, Exotic Microlepidoptera, 2: 240. I9I0, Proc. Linnean Soc. N.S. Wales, 35: I88. I9I5, Exotic Microlepidoptera, I: 393. (BM) I930, Exotic Microlepidoptera, 4: I4. I920, Exotic Microlepidoptera, 2: 338. (BM) 


$\begin{array}{ll}\text { pseudometra } & \text { TRICHOTAPHE } \\ \text { pseudomorpha } & \text { EUCOSMA } \\ \text { pseudonectis } & \text { LASPEYRESIA } \\ \text { pseudonoma } & \text { ACROLOPHUS } \\ \text { pseudopis } & \text { LEPTOCROCA } \\ \text { pseudostoma } & \text { GLYPHIPTERYX } \\ \text { pseustis } & \text { ACROCLITA } \\ \text { psichiodes } & \text { EULECHRIA } \\ \text { psilachyra } & \text { SIMAETHIS } \\ \text { psilodoxa } & \text { STACHYOSTOMA* } \\ \text { psilomorpha } & \text { STENOMA } \\ \text { psilopa } & \text { BATRACHEDRA } \\ \text { psilopis } & \text { ATHEROPLA } \\ \text { psilopla } & \text { PELTOPHORA } \\ \text { psilostola } & \text { COESYRA } \\ \text { psilotis } & \text { XYLORYCTA }\end{array}$

psimythota psithyra psithyristis psittacopa psolocoma psoloessa psoralea psoricodes psoricopa psorodes psoropis

psychina psychopa psychra psychrasema

psychrodes psychrodora psychropa

psyllodecta ptarmica ptarmicopa pteridograpta pterocola pterocosma pterocosmana pterolopha pteroneura pteropis pterostoma pterotarsa

[MYRIOPLEURA*] GELECHIA BATRACHEDRA IMMA COPTOTELIA CRYPSITHYRIS ACROLOPHUS SYNTOMACTIS TORTRIX

IMMA TRACH YCENTRA EPICHORISTA

PHYLLOCNISTIS GLYPHIPTERYX AOCHLETA* PHALONIA

EPIPHTHORA PHTHEOCHROA COLEOPHORA

GYMNOGRAMMA TISCHERIA ARG YROPLOCE PHRIXOSCELES CYPHOSTICHA TINEA OISTOPIORA* LIMNOECIA DIACTENIS* THIOTRICHA GRACILARIA URANOTHYRIS*
I9I3, Journ. Bombay Nat. Hist. Soc. 22: I78. (вм) I9I6, Exotic Microlepidoptera, 2: I9. (BM) I907, Journ. Bombay Nat. Hist. Soc. I8: I46. (BM) I922, Exotic Microlepidoptera, 2: 608. (BM) I920, Exotic Microlepidoptera, 2: 368 . I922, Exotic Microlepidoptera, 2: 492. (BM) I9II, Proc. Linnean Soc. N.S. Wales, 36: 239. I9I4, Exotic Microlepidoptera, I: 2I9. I9I2, Exotic Microlepidoptera, I: 47. (BM) 1923, Exotic Microlepidoptera, 3: 28. (P) I9I5, Exotic Microlepidoptera, I: 470. (BM) I907, Journ. Bombay Nat. Hist. Soc. I7: 982. I889, Proc. Linnean Soc. N.S. Wales, (Ser. 2) 3: I652. I884, Proc. Linnean Soc. N.S. Wales, 9: 735. I889, Proc. Linnean Soc. N.S. Wales, (Ser. 2) 3: I66ı.
I905, Journ. Bombay Nat. Hist. Soc. I6: 602. (BM) I9I3, Ann. Transvaal Mus. 3: 293. (T) I889, Trans. Proc. Nerw Zealand Inst. 2I: I8I. I906, Trans. Ent. Soc. London, I906: I76. (BM) I9I2, Trans. Ent. Soc. London, I9II: 7OI. (BM) I931, Exotic Microlepidoptera, 4: I05. I932, Exotic Microlepidoptera, 4: 332. (v) I897, Proc. Linnean Soc. N.S. Wales, 22: 386. I9II, Ann. Transvaal Mus. 2: 223. (BM) I906, Trans. Ent. Soc. London, I906: I76. (вм) I907, Proc. Linnean Soc. N.S. Wales, 32 : I43. I920, Voyage de Ch. Alluaud et $R$. Jeannel en Afrique Oriental, II, Microlepidoptera, p. 5I. (P) I906, Trans. R. Soc. S. Australia, 30: 62. I 9o9, Journ. Bombay Nat. Hist. Soc. I9: 433. (BM) I883, New Zealand Journ. Sci. I : 523.

I937, in Caradja and Meyrick, Deuts. Ent. Zeit. Iris, 5 I: I7I. (BM)

I904, Proc. Linnean Soc. N.S. Wales, 29: 263. I936, Exotic Microlepidoptera, 5: 23. (BM) I920, Voyage de Ch. Alluaud et R. Jeannel en Afrique

Oriental, II, Microlepidoptera, p. 9I. (P) I924, Exotic Microlepidoptera, 3: I2I. (T) I908, Rec. Indian Mus. 2: 399. (K) I936, Exotic Microlepidoptera, 4:6I2. (BM) I935, Exotic Microlepidoptera, 4: 596. I9I4, Journ. Bombay Nat. Hist. Soc. 23: I22. I9I6, Exotic Microlepidoptera, I: 603. I88I, Proc. Linnean Soc. N.S. Wales, 6: 699. 1920, Exotic Microlepidoptera, 2: 320. I907, Journ. Bombay Nat. Hist. Soc. I7: 98o. (Bм) 1908, Journ. Bombay Nat. Hist. Soc. I8: 443. (вм) I922, Exotic Microlepidoptera, 2: 564 . I933, Exotic Microlepidoptera, 4: 4I7. 


\begin{tabular}{|c|c|c|}
\hline pterygota & COMMATICA & I929, Exotic Microlepidoptera, 3: 503. (BM) \\
\hline ptiladelpha & CROCIDOSEMA & I9I7, Trans. Ent. Soc. London, I9I7: I8. (BM) \\
\hline ptilallactis & STENOMA & I930, Exotic Microlepidoptera, 4: 24. (v) \\
\hline ptilastis & ARISTOTELIA & I909, Ann. South African Mus. 5: 369. (sa) \\
\hline ptilocentra & HYPEROPTICA* & $\begin{array}{l}\text { I934, in Caradja and Meyrick, Deuts. Ent. Zeit. Iris, } 48 \text { : } \\
\text { 40. }\end{array}$ \\
\hline ptilocharis & COLEOPHORA & $\begin{array}{l}\text { I938, Institut des Parcs Nationaux du Congo Belge, fasc. I4, } \\
\text { p. } 22 \text {, pl. 2, fig. } 9 .\end{array}$ \\
\hline ptilocnemis & TORTRIX & $\begin{array}{l}\text { I920, Voyage de Ch. Alluaud et } R \text {. Jeannel en Afrique } \\
\text { Oriental, II, Microlepidoptera, p. } 47 . \text { ( } \mathrm{P})\end{array}$ \\
\hline ptilocompa & DICHOMERIS & I922, Trans. Ent. Soc. London, I922: II3. (BM) \\
\hline ptilocrates & ANTAEOTRICHA & I932, Exotic Microlepidoptera, 4: 289. (v) \\
\hline ptilocrossa & CAPUA & I9I4, Exotic Microlepidoptera, I : 274. (BM) \\
\hline ptilodelta & PYRODERCES & I922, Exotic Microlepidoptera, 2: 570. (P) \\
\hline ptilodoxa & METACHANDA & I930, Trans. Ent. Soc. London, 78: 3I7. (P) \\
\hline ptilonota & ARGYROPLOCE & I92I, Ann. Transvaal Mus. 8: 57. (T) \\
\hline ptilopa & AGRIOPHARA & I9I3, Trans. Ent. Soc. London, I9I3: I83. (BM) \\
\hline ptiloscelis & HEPIALUS & I9I9, Exotic Microlepidoptera, 2: 230. (T) \\
\hline ptilosema & CERCONOTA & I9I8, Exotic Microlepidoptera, 2: 20I. (BM) \\
\hline ptilosoma & ARGYROPLOCE & I9I6, Exotic Microlepidoptera, I: 563. (BM) \\
\hline ptilosticta & SCIEROPEPLA & I9I5, Exotic Microlepidoptera, I: 377. \\
\hline ptilostoma & GLYPHIDOCERA & I935, Exotic Microlepidoptera, 4:565. (BM) \\
\hline ptilozona & CATALECTIS & I923, Exotic Microlepidoptera, 3: 64. (BM) \\
\hline ptisanopa & SYNCERASTIS* & I93I, Ann. Mus. Nac. Hist. Nat. Buenos Aires, 36: 4ro. \\
\hline ptochas & LECITHOCERA & I9I8, Exotic Microlepidoptera, 2: I04. (BM) \\
\hline ptochastis & BUCCULATRIX & I893, Proc. Linnean Soc. N.S. Wales, 7:602. \\
\hline ptochodora & MELASINA & I9I7, Exotic Microlepidoptera, 2: 94. \\
\hline ptochodoxa & CTENOCOMPA & I934, in Caradja and Meyrick, Deuts. Ent. Zeit. Iris, $48: 43$. \\
\hline ptochodryas & BRACHMIA & I923, Exotic Microlepidoptera, 3: 46. (BM) \\
\hline ptochogramma & COMPSOLECHIA & I922, Trans. Ent. Soc. London, I922: 93. (BM) \\
\hline ptochomorpha & SARISOPHORA & I923, Exotic Microlepidoptera, 3: 36 . (BM) \\
\hline ptochopa & ARTIASTIS & I889, Proc. Linnean Soc. N.S. Wales, (Ser. 2) 3: 1675. \\
\hline ptyalistis & MELASINA & I937, Exotic Microlepidoptera, 5: II4. \\
\hline ptychobathra & STENOMA & I930, Ann. Naturhist. Mus. Wien, 44: 245. (v) \\
\hline ptychocentra & STENOMA & I9I6, Exotic Microlepidoptera, I: 5Io. (BM) \\
\hline ptychocryptis & GLYPHIDOCERA & I929, Trans. Ent. Soc. London, 76: 5I I. (BM) \\
\hline ptychophora & EPITHECTIS & I9I4, Ann. South African Mus. Io: 244. (SA) \\
\hline ptychophthalma & a STENOMA & I930, Exotic Microlepidoptera, 4:3I. (BM) \\
\hline ptychoptila & ATOPOTORNA* & I932, Exotic Microlepidoptera, 4: 28I. (v) \\
\hline ptychora & LASPEYRESIA & I9o7, Journ. Bombay Nat. Hist. Soc. I8: I47. (BM) \\
\hline ptychosema & DICHOMERIS & I9I3, Journ. Bombay Nat. Hist. Soc. 22: I75. (BM) \\
\hline ptychospila & SYMPHORISTIS* & I9I8, Ann. Transvaal Mus. 6:56. (T) \\
\hline ptychospora & GRACILARIA & $\begin{array}{l}\text { I938, Institut des Parcs Nationaux du Congo Belge, } \\
\text { fasc. I4, p. 23. }\end{array}$ \\
\hline ptyoptera & ANISOPLACA & I886, Trans. Proc. New Zealand Inst. I8: I7I. \\
\hline ublicana & EULECHRIA & I9I4, Exotic Microlepidoptera, I: I68. (MA) \\
\hline puellaris & EULECHRIA & I883, Proc. Linnean Soc. N.S. Wales, 7: 522. \\
\hline ionaria & HOLCOCERA & I9I8, Exotic Microlepidoptera, 2: I6I. (BM) \\
\hline $\mathrm{x}$ & TROCHILIUM & I926, Exotic Microlepidoptera, 3: 266. \\
\hline ata & ETHMIA & IgIo, Trans. Ent. Soc. London, I9Io: 46I. \\
\hline
\end{tabular}




$\begin{array}{ll}\text { pulverata } & \text { CHOLOTIS } \\ \text { pulverea } & \text { BLASTOBASIS } \\ \text { pulverea } & \text { PHILOBOTA } \\ \text { pulverescens } & \text { TISCHERIA } \\ \text { pulverula } & \text { LASPEYRESIA } \\ \text { pulverulenta } & \text { EOCHROA }\end{array}$

pulvinata

pumicata

pumicea

pumicosa

pumicosa

pungens

punica

punicea

pura

purata

purgata

purifica

purificata

purpurascens

purpurata

purus

puteolata

putris

pycnitis

pycnochra

pycnodes

pycnograpta

pycnomias

pycnosaris

pycnostoma

pycnostrota

pycnota

pygmaea

pygmaea

pylartis

pylonias

pylonitis

pylonota

pylora

pyracma

pyractis

pyramidias

pyramidota

pyramis

pyrangela

pyranthis
[EOCHROIS*]

URODUS

COPHOMANTIS

ACROLOPHUS

ISOCHORISCA

PSEUDODOXIA

COMPSOLECHIA

PHILAGRAULA*

STENOMA

PHILONYMPHA

TORTRIX

PICROSPORA

COLEOPHORA

APETHISTIS

PERONEA

LAMPRYSTICA*

PTEROPHORUS

BRACHMIA

ELACHISTA

MICROCOLONA

LASPEYRESIA

NOTHRIS (?)

LASPEYRESIA

EULIA

LIMNOECIA

SPERMANTHRAX*

AGRIOPHANES*

LASPEYRESIA

ALUCITA

XYSMATODOMA

THIOTRICHA

ACROCERCOPS

EUCOSMA

DIASTATICA*

CAPUA

MACARANGELA

ARCHIMAGA*

PYRGOTIS

ACROCERCOPS

OCYSTOLA

OPOGONA

HILAROGRAPHA
1913, Ann. Transvaal Mus. 3: 308. (T)

I907, Journ. Bombay Nat. Hist. Soc. I8: I51.

I884, Proc. Linnean Soc. N.S. Wales, 8: 509.

I936, Exotic Microlepidoptera, 5: 57.

I9I2, Journ. Bombay Nat. Hist. Soc. 21 : 876.

(BM)

I883, Proc. Linnean Soc. N.S. Wales, $7: 454$.

I924, Exotic Microlepidoptera, 3: I28. (BM)

I929, Exotic Microlepidoptera, 3: 525. (BM)

I9r3, Trans. Ent. Soc. London, I913: I93. (BM)

I910, Proc. Linnean Soc. N.S. Wales, 35: I67. (MA)

I9I4, Exotic Microlepidoptera, I : 266. (BM)

1922, Trans. Ent. Soc. London, I922: 95. (BM)

1930, Exotic Microlepidoptera, 3:554. (BM)

I9I6, Exotic Microlepidoptera, I: 5I8. (BM)

I884, Proc. Linnean Soc. N.S. Wales, 9: 723.

I932, Exotic Microlepidoptera, 4: 254. (v)

I9I3, Ann. Transvaal Mus. 3: 332. (T)

I921, Ann. Transvaal Mus. 8: I20. (T)

I93I, Exotic Microlepidoptera, 4: 85. (BM)

I9I2, Trans. Ent. Soc. London, I9I I : 686. (BM)

I9I4, Supplementa Entomologica, No. 3, p. 58. (D)

I9I3, Ann. Transvaal Mus. 3: 268. (T)

I9I I, Journ. Bombay Nat. Hist. Soc. 20: 7I6. (BM)

I923, Exotic Microlepidoptera, 3: 60.

I9I5, Exotic Microlepidoptera, I: 334.

I920, Exotic Microlepidoptera, 2: 353. (BM)

I909, Ann. Transvaal Mus. 2: I7, pl. 5, fig. 9. (T)

I936, Exotic Microlepidoptera, 4:6I6. (BM)

I928, Proc. Hawaiian Ent. Soc. 7: 97. (SP)

I938, Trans. R. Ent. Soc. London, 87: 520.

I936, Exotic Microlepidoptera, 4: 625 .

I930, Exotic Microlepidoptera, 3: 600. (вм)

I9I4, Journ. Bombay Nat. Hist. Soc. 22: 772. (BM)

I890, Proc. Linnean Soc. N.S. Wales, (Ser. 2) 4: III2.

I893, Proc. Linnean Soc. N.S. Wales, 7: 500.

I908, Journ. Bombay Nat. Hist. Soc. I8: 44I. (ВM)

I92I, Exotic Microlepidoptera, 2: 465.

I932, Exotic Microlepidoptera, 4: 307. (BM)

I938, Institut des Parcs Nationaux du Congo Belge,

fasc. I4, p. 24.

1938, Institut des Parcs Nationaux du Congo Belge,

fasc. I4, p. 5, pl. I, fig. 4.

1893, Proc. Linnean Soc. N.S. Wales, 7: 589.

I905, Journ. Bombay Nat. Hist. Soc. 16: 609. (вм)

I902, Trans. Ent. Soc. London, I902: 57I.

1918, Ann. Transwaal Mus. 6:39. (T)

I885, Proc. Linnean Soc. N.S. Wales, 9: Io73.

I928, Exotic Microlepidoptera, 3: 400.

I907, Proc. Linnean Soc. N.S. Wales, 32: 91. 


$\begin{array}{ll}\begin{array}{l}\text { pyrarcha } \\ \text { pyraspis }\end{array} & \text { HYPERCALLIA } \\ \text { pyrelictis } & \text { LASPEYRESIA } \\ \text { pyrenodes } & \text { PARECTOPA } \\ \text { pyretodes } & \text { STENOMA } \\ & \text { NOEZA } \\ \text { [SEMIOMERIS*] } \\ \text { pyrgonota } & \text { PHLOEOPOLA } \\ \text { pyriata } & \text { STENOMA } \\ \text { pyrilampis } & \text { TINEA } \\ \text { pyrites } & \text { ENAEMA } \\ \text { pyritis } & \text { NEMOTOIS } \\ \text { pyrobathra } & \text { HEDNOPHORA* } \\ \text { pyrobola } & \text { STENOMA } \\ & \text { GONIONOTA }\end{array}$

pyrocalyx pyrocanthara pyrocausta pyrocephala pyrochalca pyrochorda pyrochroa pyrochrysa pyrocoma pyrocosma pyrodoxa pyrogastra pyrogramma pyrograpta pyroleuca pyromantis pyrometalla pyromyia pyronota pyronympha pyrophanes pyrophracta pyrophthalma pyropis pyroptera pyroptis pyrora pyrosema pyrosemana pyrosoma pyrosoma pyrostoma pyrota pyrotechnica pyrothorax
[CERYCOSTOLA*] MACHIMIA

ZACORISCA

HYPERCALLIA

HECATOMPEDA*

PROMALACTIS GONAEPA

SCARDIA

OCYSTOLA ERIOCOTTIS ATASTHALISTIS* MACROBATHRA GLYPHIPTERYX AGONOXENA MACHIMIA ZELLERIA TINAEGERIA OINOPHILA OEDEMATOPODA GONADA

LACTURA TIPHA ATHLOSTOLA* IMMA

NOEZA SYSCALMA TORTRICOPSIS EULECHRIA

PLEUROTA CACOECIA SESIA TINEA AEGERIA NEPHOGENES SATRAPIA ATTEVA
I9Io, Journ. Bombay Nat. Hist. Soc. 20: I44. (BM) I928, The Entomologist, 6I: 23I. (Lost) I927, Insects of Samoa, 3, Lepidoptera, fasc. 2, p. Io8. I9I5, Exotic Microlepidoptera, I: 448. (BM)

I9I4, Trans. Ent. Soc. London, I9I4: 277. (BM) I889, Proc. Linnean Soc. N.S. Wales, (Ser. 2) 3: I594. I930, Exotic Microlepidoptera, 4:25. (v) I9I7, Exotic Microlepidoptera, 2: 78 . I886, Proc. Linnean Soc. N.S. Wales, (Ser. 2) I: 257. (A) I907, Journ. Bombay Nat. Hist. Soc. I7: 992.

I9II, Ann. Transvaal Mus. 2: 233. I93I, Exotic Microlepidoptera, 4: 33. (BM)

I887, Proc. Linnean Soc. N.S. Wales, (Ser. 2) I: IołI. (A) 1922, Exotic Microlepidoptera, 2: 545. (P) I924, Exotic Microlepidoptera, 3: II2. I93I, Exotic Microlepidoptera, 4: I2I. (Us) I929, Exotic Microlepidoptera, 3: 543. (BM) I93I, Exotic Microlepidoptera, 4: I86. (BM) I9Io, Trans. Ent. Soc. London, I9Io: 442. I893, Proc. Linnean Soc. N.S. Wales, 7: 523 . I889, Proc. Linnean Soc. N.S. Wales, (Ser. 2) 3: 1663. I89I, Ent. Mon. Mag. 27: 58.

I886, Trans. Ent. Soc. London, I886: 280. I926, Exotic Microlepidoptera, 3: 310. I909, Journ. Bombay Nat. Hist. Soc. I9: 429. (BM) I924, Exotic Microlepidoptera, 3: 84. (BM) I932, Exotic Microlepidoptera, 4:278. (v) I893, Proc. Linnean Soc. N.S. Wales, 7: 585. I92I, Exotic Microlepidoptera, 2: 442. I928, Exotic Microlepidoptera, 3: 40I. (T) I929, Exotic Microlepidoptera, 3: 542. I924, Exotic Microlepidoptera, 3: 98. (BM) I924, Exotic Microlepidoptera, 3: I27. I905, Journ. Bombay Nat. Hist. Soc. I6: 594. (BM) I924, Exotic Microlepidoptera, 3: 97. I937, Exotic Microlepidoptera, 5: I53. (BM) I9I8, Exotic Microlepidoptera, 2: I49. (BM) I933, Exotic Microlepidoptera, 4:367. (cu) I902, Trans. R. Soc. S. Australia, 26: I43. I9I4, Exotic Microlepidoptera, I: I66. (MA) I884, Proc. Linnean Soc. N.S. Wales, 9: 754. I88I, Proc. Linnean Soc. N.S. Wales, 6: 496. I9I8, Exotic Microlepidoptera, 2: I80. I924, Rec. Indian Mus. 26: II4. (K) I927, Exotic Microlepidoptera, 3: 372. (BM) I889, Proc. Linnean Soc. N.S. Wales, (Ser. 2) 3: I604. I935, Exotic Microlepidoptera, 4: 566. (BM) I928, Exotic Microlepidoptera, 3: 415. 


\begin{tabular}{|c|c|c|}
\hline pyrothyris & MACHIMIA & I9I6, Exotic Microlepidoptera, I: 550. (вм) \\
\hline pyrotricha & TINEA & I893, Proc. Linnean Soc. N.S. Wales, 7: 536. \\
\hline pyroxantha & TINEA & I9I4, Exotic Microlepidoptera, I: 210. (BM) \\
\hline pyrozancla & GYMNOGRAMMA & I9I2, Ann. Transvaal Mus. 3:77. (T) \\
\hline pyrozela & COSMOPTERYX & I922, Exotic Microlepidoptera, 2: 566 . (Bм) \\
\hline pyrozona & LASPEYRESIA & I9I6, Exotic Microlepidoptera, 2: 24. (вм) \\
\hline pyrphora & THIOTRICHA & I9I8, Exotic Microlepidoptera, 2: I22. (вм) \\
\hline pyrrhanthes & GNORIMOSCHEMA & I904, Proc. Linnean Soc. N.S. Wales, 29: 318. \\
\hline pyrrhaspis & GRACILARIA & I93I, in Caradja, Bull. Sect. sci. Acad. roum. I4: 75. \\
\hline pyrrhias & STENOMA & I9I5, Exotic Microlepidoptera, I: 455. (вм) \\
\hline pyrrhitis & TRICHOTAPHE & I9I2, Ann. Transvaal Mus. 3:68. (т) \\
\hline pyrrhochyta & TISCHERIA & I936, Exotic Microlepidoptera, 5: 57. \\
\hline pyrrhoclista & SIMAETHIS & I922, Exotic Microlepidoptera, 2: 486. \\
\hline pyrrhocolona & EULIA & I926, Exotic Microlepidoptera, 3: 257. (вм) \\
\hline pyrrhocoma & TRICHOTHYRSA & I9I2, Exotic Microlepidoptera, I: 62. \\
\hline pyrrhocremna & PYRGOTIS & 1922, Exotic Microlepidoptera, 2: 494. \\
\hline pyrrhocrossa & ARGYROPLOCE & I9I2, Journ. Bombay Nat. Hist. Soc. 2 I : 874. (Bм) \\
\hline pyrrhodelta & EUXANTHIS & I93I, Exotic Microlepidoptera, 4: I59. (BM) \\
\hline pyrrhodes & PYRODERCES & I897, Proc. Linnean Soc. N.S. Wales, 22: 349. \\
\hline pyrrhogastra & EPICEPHALA & Igo8, Proc. Zool. Soc. London, p. 734 . \\
\hline pyrrhogramma & STATHMOPODA & I930, Exotic Microlepidoptera, $3: 585$. (вM) \\
\hline pyrrhonota & STENOMA & I9I5, Exotic Microlepidoptera, I: 446. (вм) \\
\hline pyrrhophrys & CYPHOTHYRIS & I932, Exotic Microlepidoptera, 4: 332. (cu) \\
\hline pyrrhophthalma & PHYTOMIMIA & I932, Exotic Microlepidoptera, 4:278. (вм) \\
\hline pyrrhopis & PROPHORAULA* & 1922, Trans. Ent. Soc. London, I922: 106. (вм) \\
\hline pyrrhoplaca & LYONETIA & I936, Exotic Microlepidoptera, 5: 57 \\
\hline pyrrhoplecta & DELONOMA & I9I8, Exotic Microlepidoptera, 2: 2I9. \\
\hline pyrrhopsamma & BRIAROSTOMA* & I920, Ann. South African Mus. I7: 290. (SA) \\
\hline pyrrhoptera & COESYRA & I884, Proc. Linnean Soc. N.S. Wales, 9: 780. \\
\hline pyrrhoptila & ENDOGRAPTIS* & 1927, Insects of Samoa, 3, Lepidoptera, fasc. 2, p. 92. \\
\hline pyrrhoschista & BRACHMIA & I934, Exotic Microlepidoptera, 4: 5I5. (BM) \\
\hline pyrrhoscia & COPROMORPHA & I935, Exotic Microlepidoptera, 4: 574. (вм) \\
\hline pyrrhota & PYRODERCES & I9I5, Exotic Microlepidoptera, I: 3I4. \\
\hline pyrrhotrota & HYPERCALLIA & I932, Exotic Microlepidoptera, 4: 280. (v) \\
\hline pyrrhoxantha & MACHIMIA & I93I, Exotic Microlepidoptera, 4: II9. \\
\hline pyrrl & TORTRIX & I9Io, Proc. Linnean Soc. N.S. Wales, 35: 226. \\
\hline pyrsocoma & TINEA & I937, Exotic Microlepidoptera, 5: 77. (T) \\
\hline pyrsogramma & DICHELOPA & I934, Pacific Ent. Surv. Publ. 7, art. 28, p. 343. (в \\
\hline pyrsopa & PHILOBOTA & I921, Exotic Microlepidoptera, 2: 385. \\
\hline pyrsophanes & PSITTACASTIS & I935, Arb. morph. taxon. Ent. Berl. 3: I04. (D) \\
\hline pythia & CHRESMARCHA & I920, Exotic Microlepidoptera, 2: 339. \\
\hline pythonaea & ANTAEOTRICHA & I9I6, Exotic Microlepidoptera, I: 49I. (Bм) \\
\hline pythonia & BACTRA & I909, Ann. Transvaal Mus. 2: 9, pl. 3, fig. 7. (T) \\
\hline pythonias & RHOPOBOTA & I9Io, Trans. Ent. Soc. London, I9Io: 434. (вм) \\
\hline pytinaea & LEPTOSACES & I902, Trans. R. Soc. S. Australia, 26: I57. \\
\hline pyxinodes & LECITHOCERA & I918, Exotic Microlepidoptera, 2: Iog. \\
\hline & $\begin{array}{l}\text { ELACHISTA } \\
\text { BACTRA }\end{array}$ & I9 \\
\hline & BACTRA & \\
\hline
\end{tabular}




$\begin{array}{ll}\text { quadricornis } & \text { PROTOTHEORA } \\ \text { quadrifrenis } & \text { LACTURA } \\ \text { quadrijuga } & \text { SABATINCA } \\ \text { quadrisecta } & \text { ACROCERCOPS } \\ \text { quaerenda } & \text { EULECHRIA } \\ \text { quaestoria } & \text { IMMA } \\ \text { quaestrix } & \text { BONDIA }\end{array}$

quaestuosa

quassa

quassata

quatiens

quercicola

quercivora

queribunda

querula

querula

quiescens

quieta

quietana

quincuncialis
PROTOSYNAEMA

MELASINA

BRACHMIA

ANTAEOTRICHA

DICHOMERIS

PAMMENE

HOMALOXESTIS

EUCOSMA

LECITHOCERA

STENOMA

BUCCULATRIX

HOLOCOLA

HETEROTACTIS*
I920, Ann. South African Mus. I7: 315. (SA)

1935, Arb. morph. taxon. Ent. Berl. 3: 108. (D)

I9I2, Trans. Proc. New Zealand Inst. 44: I26.

1932, Exotic Microlepidoptera, 4:267.

I920, Exotic Microlepidoptera, 2: 309.

I9II, Trans. Linnean Soc. London, I4:289. (BM)

I935, in Caradja and Meyrick, Materialien zu einer Micro-

lepidopteren Fauna der Chinesischen Provinzen Kiangsu,

Chekiang und Hunan, p. 85. (BM)

I924, Trans. Proc. New Zealand Inst. 55: 205.

I92I, Ann. Transvaal Mus. 8: I36. (T)

I93I, in Caradja, Bull. Sect. sci. A cad. roum. I4: 70. (BM)

I930, Ann. Naturhist. Mus. Wien, 44: 235. (v)

I92I, Exotic Microlepidoptera, 2: 433. (BM)

I920, Exotic Microlepidoptera, 2: 35I. (BM)

I922, Zool. Meded. 7: 83. (L)

I9I2, Trans. Proc. New Zealand Inst. 44: I25.

I9Io, Trans. Ent. Soc. London, I9Io: 449. (BM)

I9I6, Exotic Microlepidoptera, I: 5I4. (BM)

I9I3, Ann. Transvaal Mus. 3: 329. (T)

I88I, Proc. Linnean Soc. N.S. Wales, 6:673.

I928, Exotic Microlepidoptera, 3: 392.

$\begin{array}{ll}\text { rabida } & \text { THIOTRICHA } \\ \text { racemosa } & \text { GYMNOGRAMMA } \\ \text { radicata } & \text { ARISTOTELIA } \\ \text { radicicola } & \text { ANTAEOTRICHA } \\ \text { radiosa } & \text { OPOSTEGA } \\ \text { ramifera } & \text { MELASINA } \\ \text { ramigera } & \text { ACROCERCOPS } \\ \text { ramulata } & \text { GELECHIA } \\ \text { ramulosa } & \text { PERSICOPTILA } \\ \text { ranulana } & \text { ISOCHORISTA* } \\ \text { ranularis } & \text { NARYCIA } \\ \text { ranunculata } & \text { CNEPHASIA } \\ \text { rapax } & \text { OXYGRAPHA } \\ \text { rapida } & \text { AMYDRIA } \\ \text { raptans } & \text { ORSOTRICHA } \\ \text { raptor } & \text { OXYPTILUS } \\ \text { raricilia } & \text { ANTAEOTRICHA } \\ \text { raricoma } & \text { LEPIDOSCIA } \\ \text { rastrata } & \text { CNEPHASIA } \\ \text { rastricornis } & \text { ACROLOPHUS } \\ \text { rastrifera } & \text { STOMOPTERYX } \\ \text { ratifera } & \text { ENARMONIA } \\ \text { rationalis } & \text { LABDIA } \\ \text { rebellis } & \text { ARGYROPLOCE } \\ \text { rebellis } & \text { LEISTOGENES* } \\ \text { recidiva } & \text { LIMNOECIA } \\ & \end{array}$

I929, Exotic Microlepidoptera, 3: 500. (BM) I9I8, Ann. Transvaal Mus. 6: 38. (T) I9I7, Trans. Ent. Soc. London, I9I7: 35. (BM) I932, Exotic Microlepidoptera, 4: 292. (v) I9I3, Ann. Transvaal Mus. 3: 327. (T) I9I6, Exotic Microlepidoptera, I: 6 Iо. I920, Exotic Microlepidoptera, 2: 292. I926, Exotic Microlepidoptera, 3:278. (BM) I92I, Exotic Microlepidoptera, 2: 4I3. (BM) I88I, Proc. Linnean Soc. N.S. Wales, 6: 424. I922, Exotic Microlepidoptera, 2: 605. (P) I9I2, Trans. Ent. Soc. London, I9I I: 684. (BM) Igo8, Journ. Bombay Nat. Hist. Soc. I 8: 624. (BM) I909, Ann. South African Mus. 5:363. (SA) I920, Exotic Microlepidoptera, 2: 366. (BM) I908, Trans. Ent. Soc. London, I907: 478. I930, Exotic Microlepidoptera, 4:20. (v) I893, Proc. Limnean Soc. N.S. Wales, 7: 5 I3. I9Io, Ent. Mon. Mag. 2I: 2II. (BM) I932, Exotic Microlepidoptera, 4:330. (v) I9I8, Exotic Microlepidoptera, 2: I37. (BM) I9I2, Ent. Mon. Mag. 48: 34. (Sub.) I92I, Exotic Microlepidoptera, 2: 4II. (BM) I93I, Exotic Microlepidoptera, 4: I40. I927, Exotic Microlepidoptera, 3: 363. (BM) I9I2, Ann. Transvaal Mus. 3: 7I. (T) 


\begin{tabular}{|c|c|}
\hline reciproca & ANARSIA \\
\hline reciprocans & AGDISTIS \\
\hline recitata & GRACILARIA \\
\hline reclusa & PHALONIA \\
\hline econdita & STENOMA \\
\hline cordata & ORYGOCERA \\
\hline ecreata & SCYTHRIS \\
\hline recrepans & MACROBATHRA \\
\hline recta & COMPSOLECHIA \\
\hline rectangularis & AMORBIA \\
\hline tificata & STENOMA \\
\hline recurrens & STENOMA \\
\hline recurva & PROMALACTIS \\
\hline recurvata & LECITHOCERA \\
\hline redimita & PYRODERCES \\
\hline redintegrata & STENOMA \\
\hline referenda & EUCOSMA \\
\hline reflectrix & LASPEYRESIA \\
\hline reflua & ALLOCLITA \\
\hline luana & DIPTERINA \\
\hline & ANACAMPSIS \\
\hline refractrix & ANTAEOTRICHA \\
\hline refraga & HEMIMENE \\
\hline gens & ELAEODINA* \\
\hline rigerata & HYPONOMEUTA \\
\hline rigescens & LASPEYRESIA \\
\hline & HYSTEROSIA \\
\hline & MACHIMIA \\
\hline & PARECTOPA \\
\hline & STENOMA \\
\hline & STROBISIA \\
\hline
\end{tabular}

[SATRAPODOXA*]

regifica regionalis regressa regulifera regulus rejecta relapsa relata relaxata relecta relegata relevata relicta religata religiosa religiosa reliquatrix
I920, Exotic Microlepidoptera, 2: 300. (BM) 1924, Exotic Microlepidoptera, 3: 95. (T) I918, Exotic Microlepidoptera, 2: I78. I923, Exotic Microlepidoptera, 3: 53. (BM) I9I5, Exotic Microlepidoptera, I: 439. (BM) I921, Ann. Transvaal Mus. 8: 1oo. (T) I925, Bull. Soc. R. Ent. Egypte, 9: 2I4. I926, Exotic Microlepidoptera, 3: 310. (T) I922, Trans. Ent. Soc. London, I922: 86. (BM) I93I, Exotic Microlepidoptera, 4: I55. (v) I925, Exotic Microlepidoptera, 3: I8o. (BM) I925, Exotic Microlepidoptera, 3: 210. (BM) I9I4, Ann. Transvaal Mus. 4: I98. (T) I923, Exotic Microlepidoptera, 3: 39. (BM) I9I7, Exotic Microlepidoptera, 2: 38. I925, Exotic Microlepidoptera, 3: 218. (BM) I92I, Zool. Meded. 6: I54. (I) I928, Exotic Microlepidoptera, 3: 448. (BM) I9I4, Exotic Microlepidoptera, I: 268. (BM) I881, Proc. Linnean Soc. N.S. Wales, 6: 525 . I9I4, Trans. Ent. Soc. London, I9I4: 258. (BM) I930, Exotic Microlepidoptera, 4:2I. (v) I931, in Joannis, Ann. Soc. Ent. France, 98 (Supp.): $720[482] .(\mathrm{P})$

1926, Sarawak Mus. Journ. 3: I49.

I93I, Exotic Microlepidoptera, 4: I72. (BM)

1924, Exotic Microlepidoptera, 3: 68. (BM)

I9I2, Ent. Mon. Mag. 48: 35. (Sub.)

I916, Exotic Microlepidoptera, I : 549. (BM)

I9I5, Trans. Ent. Soc. London, I9 5: 234.

I926, Exotic Microlepidoptera, 3: 225. (BM)

I9I4, Trans. Ent. Soc. London, I9I4: 267.

I921, Exotic Microlepidoptera, 2: 390. (BM)

I934, Exotic Microlepidoptera, 4: 527. (c)

I9I6, Exotic Microlepidoptera, I: 620.

1933, Exotic Microlepidoptera, 4:362.

I906, Journ. Bombay Nat. Hist. Soc. I7: I35.

1922, Exotic Microlepidoptera, 2: 565 .

I928, Exotic Microlepidoptera, 3: 444. (BM)

1925, Exotic Microlepidoptera, 3: 207. (BM)

rgr6, Exotic Microlepidoptera, r: 586. (Bм)

I9I3, Ann. Transvaal Miss. 3: 335. (T)

1922, Nat. Hist. Juan Fernandez and Easter Islands, $3: 368$.

I920, Exotic Microlepidoptera, 2: 373.

I9I6, Exotic Microlepidoptera, I: 55I.

I922, Trans. Ent. Soc. London, I922: 85. (BM)

I9I7, Trans. Ent. Soc. London, I9I7: 21. (BM)

I923, Exotic Microlepidoptera, 3:28. (BM)

I928, Exotic Microlepidoptera, 3: 436. 
relucens

reluctans

remigera

remivola

remorsa

remota

renitens

renovata

repetita

repetitrix

replicata

reprehensa

reprobata

reprobata

repudiata

rescissa

resecta

residua

resignata

resiliens

resinosa

resoluta

resoluta

resoluta

resona

resonans

respersa

responsa

restincta

restricta

retecta

retiaria

reticens

reticulata

retifera

retorta

retracta

retroflexa

retrogressa

retrolapsa

revecta

revelata

reversa

revincta

revoluta

revoluta

revoluta
OCYSTOLA

CRATEROMBRIS*

STEGANOSTICHA*

TRIEROSTOLA*

STENOMA

DEPRESSARIA

MYRMECOZELA

NARYCIA

ODITES

GELECHIA

PHARMACIS

ANTAEOTRICHA

BORKHAUSENIA

MERIDARCHIS

ARISTOTELIA

PAMMENE

GELECHIA

LECITHOCERA

DICHOMERIS

ANTAEOTRICHA

ARISTOTELIA

BRACHMIA

PLATYPTILIA

PROTEOPTERYX

EXONCOTIS

CEROMITIA

MYRMECOZELA

LECITHOCERA

ISONOMEUTIS

MACHIMIA

TELPHUSA

LATYPICA

STENOMA

XYSMATODOMA

COLEOPHORA

DECADARCHIS

TRICHOTAPHE

LYONETIA

ACROCERCOPS

EONYMPHA

PROCHOLA

ASCALENIA

ENCRASIMA*

STATHMOPODA

EPAGOGE

GELECHIA

HAPSIFERA
I9I3, Exotic Microlepidoptera, I: II6.

I92I, Ann. Transvaal Mus. 8: I28. (T)

I92I, Ann. Transvaal Mus. 8: II8. (T)

I932, Exotic Microlepidoptera, 4: 23I.

1925, Exotic Microlepidoptera, 3: I95. (BM)

I92I, Exotic Microlepidoptera, 2:392. (BM)

1922, Exotic Microlepidoptera, 2: 59I. (BM)

I922, Exotic Microlepidoptera, 2: 605.

I932, Exotic Microlepidoptera, 4: 286. (BM)

I93I, Exotic Microlepidoptera, 4: 60. (BM)

I9I3, Ann. Transvaal Mus. 3: 270. (вм)

I926, Exotic Microlepidoptera, 3: 238. (BM)

1920, Exotic Microlepidoptera, 2: 307.

I920, Exotic Microlepidoptera, 2: 338. (BM)

1923, Exotic Microlepidoptera, 3:6. (BM)

I92I, Zool. Meded. 6: I59. (L)

I9I3, Ann. Transvaal Mus. 3: 288.

I923, Exotic Microlepidoptera, 3: 4I. (BM)

I929, Exotic Microlepidoptera, 3: 5Iо. (BM)

I925, Exotic Microlepidoptera, 3: I73. (BM)

I9I8, Exotic Microlepidoptera, 2: II8. (BM)

I9I8, Exotic Microlepidoptera, 2: II3. (BM)

I936, in Caradja and Meyrick, Deuts. Ent. Zeit. Iris, 50:

I54.

I9I2, Ent. Mon. Mag. 48: 34. (Sub.)

I929, Trans. Ent. Soc. London, 76: 5I9. (BM)

I9I8, Ann. Transvaal Mus. 6: 47. (T)

I9I9, Exotic Microlepidoptera, 2: 270. (BM)

I9I8, Exotic Microlepidoptera, 2: I08. (BM)

I923, Trans. Proc. New Zealand Inst. 54: I66.

I920, Exotic Microlepidoptera, 2: 310.

I92I, Ann. Transvaal Mus. 8: 70. (T)

I9I9, Exotic Microlepidoptera, 2: 258.

I9I7, Exotic Microlepidoptera, 2: 58. (T)

I893, Proc. Linnean Soc. N.S. Wales, 7: 499.

I922, Exotic Microlepidoptera, 2: 556. (BM)

I927, Insects of Samoa, 3, Lepidoptera, fasc. 2, p. II3.

I922, Trans. Ent. Soc. London, I922: Io9. (вм)

I93I, Exotic Microlepidoptera, 4: I63.

I92I, Exotic Microlepidoptera, 2: 467 .

I935, in Caradja and Meyrick, Materialien zu einer Micro-

lepidopteren Fauna der Chinesischen Provinzen Kiangsu.

Chekiang und Hunan, p. 79. (BM)

I922, Exotic Microlepidoptera, 2: 582. (BM)

1922, Exotic Microlepidoptera, 2: 579. (BM)

I9I6, Exotic Microlepidoptera, I: 594. (BM)

I92I, Ann. Transvaal Mus. 8: Iog. (T)

I908, Journ. Bombay Nat. Hist. Soc. I8: 6I8. (BM)

I918, Ann. Transvaal Mus. 6: I7. (T)

I9I4, Bull. Mus. Nat. Hist. Nat. Paris, 20: I22. (P) 


$\begin{array}{ll}\text { rhabdomaga } & \text { CAPUA } \\ \text { rhabdophanes } & \text { COSMOPTERYX } \\ \text { rhabducha } & \text { SCYTHRIS } \\ \text { rhabducha } & \text { STROBISIA } \\ \text { rhachias } & \text { PARAMORPHA } \\ \text { rhachitis } & \text { TRACHYDORA } \\ \text { rhacodyta } & \text { ANCYLIS } \\ \text { rhacota } & \text { ZELOMORA* } \\ \text { rhaptica } & \text { ORNEODES } \\ & \\ \text { rhaptocrossa } & \text { GRACILARIA } \\ \text { rhectaula } & \text { LABDIA } \\ \text { rhedaria } & \text { GELECHIA } \\ \text { rhetorica } & \text { ARGYROPLOCE } \\ \text { rhicnopa } & \text { LATYPICA } \\ \text { rhicnota } & \text { CHELARIA } \\ \text { rhinoceropa } & \text { GLYPHIPTERYX }\end{array}$

rhipidaspis rhipidaula rhipidoma rhipidura rhizobola rhizogramma rhizograpta

rhizomorpha rhizonoma rhizonympha rhizophaga rhizophora rhizosema rhodacris rhodanis rhodanopa rhodantha rhodantha rhodanthes rhodaspis rhodaula rhoditis rhodites rhodobapta rhodocentra rhodocharis rhodochlaena rhodochlora rhodochra rhodochranta
PERSICOPTILA STENOMA POLYCHROSIS NEPHOGENES EUCHAETIS AGELIARCHIS* SYNCHALARA

CALICOTIS IDIOMICTIS* PYRODERCES TRACHYTYLA* TIMYRA EULIA HESTIAULA * GLYPHIPTERYX SERICOSTOLA* CROCANTHES EUCOSMA LOXOTOMA LASPEYRESIA DALACA MIMOZELA* PHALONIA CRYPTOLECHIA MIEZA PECHYPTILA* TORTYRA DEPRESSARIA GELECHIA ARGYROPLOCE
I938, Trans. R. Ent. Soc. London, 87:505. (BM) I928, Exotic Microlepidoptera, 3: 396. (BM) I897, Proc. Linnean Soc. N.S. Wales, $22: 423$. I9I I, Journ. Bombay Nat. Hist. Soc. 20: 730. (BM) I9Io, Proc. Linnean Soc. N.S. Wales, 35: I55. I9I3, Ann. Transvaal Mus. 3: 310. (T) I938, Trans. R. Ent. Soc. London, 87: 510. (BM) I9I5, Exotic Microlepidoptera, I: 293. I920, Voyage de Ch. Alluaud et R. Jeannel en Afrique

Oriental, II, Microlepidoptera, p. 82. (P) I932, Exotic Microlepidoptera, 4:27I. 1927. Insects of Samoa, 3, Lepidoptera, fasc. 2, p. 89 . 1923, Exotic Microlepidoptera, 3: 20. (BM) 1920, Exotic Microlepidoptera, 2: 350. (BM) I9I9, Exotic Microlepidoptera, 2: 258. I9I6, Exotic Microlepidoptera, I: 580. (BM) I935, in Caradja and Meyrick, Materialien zu einer Microlepidopteren Fauna der Chinesischen Provinzen Kiangsu,

Chekiang und Hunan, p. 86. (BM) I928, Exotic Microlepidoptera, 3:390. I9I5, Exotic Microlepidoptera, I : 43I. (BM) I925, Exotic Microlepidoptera, 3: I42. (BM) I9I3, Exotic Microlepidoptera, I : I33. (MA) I888, Proc. Linnean Soc. N.S. Wales, (Ser. 2) 2: 937. I923, Exotic Microlepidoptera, 2: 623. (BM) I934, in Caradja and Meyrick, Deuts. Ent. Zeit. Iris, 48: $4 \mathrm{I}$.

I927, Insects of Samoa, 3, Lepidoptera, fasc. 2, p. 99.

I935, Exotic Microlepidoptera, 4: 57I.

I924, Exotic Microlepidoptera, 3: 90.

I93I, Exotic Microlepidoptera, 4: I07. I9I9, Exotic Microlepidoptera, 2: 236. (BM) I93I, Exotic Microlepidoptera, 4: I52. (v) I893, Proc. Linnean Soc. N.S. Wales, 7: 590. I9o9, Journ. Bombay Nat. Hist. Soc. I9: 434. (Bм) I927, Exotic Microlepidoptera, 3: 358. (BM) I9I8, Exotic Microlepidoptera, 2: 97. I907, Journ. Bombay Nat. Hist. Soc. I8: I38. (Bм) I9I5, Exotic Microlepidoptera, I: 382. (BM) I928, The Entomologist, 6I: 23I. (BM) I926, Ann. South African Mus. 23: 350. (Bм) I9I4, Exotic Microlepidoptera, I: 226. I9I2, Ent. Mon. Mag. 48: 34. (Sub.) I923, Trans. Proc. New Zealand Inst. 54: I66. I9I3, Exotic Microlepidoptera, I: I42. I921, Exotic Microlepidoptera, 2: $45^{2}$. I930, Exotic Microlepidoftera, 4: 5 . 1923, The Entomologist, 56:278. (BM) I913, Ann. Transvaal Mus. 3: 290. (T) I933, Exotic Microlepidoptera, 4: 4I9. (BM) 


$\begin{array}{ll}\begin{array}{l}\text { rhodochropa } \\ \text { rhodochrysa }\end{array} & \text { TORTRIX } \\ \text { rhodoclea } & \text { NICANTHES* } \\ \text { rhodoclista } & \text { ATELOSTICHA } \\ \text { rhodocnemis } & \text { PERSICOPTILA } \\ \text { rhodocolpa } & \text { STENOMA } \\ \text { rhodocosma } & \text { ARISTOTELIA } \\ \text { rhodocrossa } & \text { PACHNISTIS } \\ \text { rhododrosa } & \text { DEPRESSARIA } \\ \text { rhodogastra } & \text { DEPRESSARIA }\end{array}$

$\begin{array}{ll}\text { rhodoglauca } & \text { SCORPIOPSIS } \\ \text { rhodogramma } & \text { CALLIPRORA } \\ \text { rhodographa } & \text { LACTURA } \\ \text { rhodograpta } & \text { FILINOTA } \\ \text { rhodomicta } & \text { THIOTRICHA } \\ \text { rhodoneura } & \text { GYMNOGRAMMA } \\ \text { rhodopa } & \text { THIOTRICHA } \\ \text { rhodopetala } & \text { ZALITHIA } \\ & \text { [CHARISTICA } * \\ \text { rhodophaea } & \text { DICHOMERIS }\end{array}$

rhodophaea

rhodophanes

rhodopis

rhodopnoana

rhodopola

rhodoptila

rhodoscelis

rhodosema

rhodosoma

rhodospila

rhodospira

rhodota

rhodothicta

rhodothictis

rhodoxantha

rhomaeopa

rhombica

rhombifera

rhombocosma

rhombophora

rhombota

rhopalitis

rhopica
EUCOSMA

PERITORNEUTA

EOMYSTIS*

HELIOCOSMA

DIOCOSMA

HAPSIFERA

DEPRESSARIA

BORKHAUSENIA

PERCNARCHA

MACROBATHRA

ACROCERCOPS

PARADORIS

HIE ROXESTIS

SESIA

HELIOCAUSTA

PTILOGENES

COMPSOLECHIA

CHOROPLECA

ACROCERCOPS

BUCCULATRIX

AGRIOPHARA

[SYNCHALARA*]

ARGYROPLOCE

SPATALISTIS*
1927, Exotic Microlepidoptera, 3: 369.

I907, Journ. Bombay Nat. Hist. Soc. I7: 739. (Bм)

1928, Exotic Microlepidoptera, 3: 395. (BM)

I9I6, Exotic Microlepidoptera, I: 547. (BM)

I9I5, Exotic Microlepidoptera, I : $32 \mathrm{I}$.

I9I6, Exotic Microlepidoptera, I: 5I7. (BM)

I9I4, Trans. Ent. Soc. London, I9I4: 229. (BM)

I9I4, Trans. Ent. Soc. London, I9I4: 273. (BM)

I934, Exotic Microlepidoptera, 4:476. (BM)

I935, in Caradja and Meyrick, Materialien zu einer Micro-

lepidopteren Fauna der Chinesischen Provinzen Kiangsu.

Chekiang und Hunan, p. 8o. (Bм)

I930, Exotic Microlepidoptera, 3: 620.

I922, Trans. Ent. Soc. London, I922: 67. (BM)

I928, Exotic Microlepidoptera, 3:4I7.

I9I5, Trans. Ent. Soc. London, I9I5: 209. (BM)

I9I8, Exotic Microlepidoptera, 2: I26. (BM)

I909, Ann. South African Mus. 5: 374. (SA)

I908, Journ. Bombay Nat. Hist. Soc. I8: 442. (Bм)

1922, Trans. Ent. Soc. London, I922: I02. (BM)

I920, Voyage de Ch. Alluaud et R. Jeannel en Afrique

Oriental, II, Microlepidoptera, p. 73 .

I9I2, Ent. Mon. Mag. 48: 35. (Sub.)

I902, Trans. R. Soc. S. Australia, 26: I62.

I888, Proc. Linnean Soc. N.S. Wales, (Ser. 2) 2: 932.

I881, Proc. Linnean Soc. N.S. Wales, 6: 694.

I930, Exotic Microlepidoptera, 3: 621. (BM)

I920, Voyage de Ch. Alluaud et R. Jeannel en Afrique

Oriental, II, Microlepidoptera, p. I05. (P)

I920, Ann. South African Mus. I7: 288. (SA)

I931, Ann. Mus. Nac. Hist. Nat. Buenos Aires, 36: 390.

(BM)

I9I5, Trans. Ent. Soc. Lonton, I9I5: 212.

I886, Proc. Limnean Soc. N.S. Wales, Io: 8I4.

I939, Trans. R. Ent. Soc. London, 89:59.

I9I I, Journ. Bombay Nat. Hist. Soc. 20:735. (BM)

I9II, Trans. Linnean Soc. London, I4: 296.

I9I8, Exotic Microlepidoptera, 2: I79.

I888, Proc. Linnean Soc. N.S. Wales, (Ser. 2) 2: 933.

I93I, Exotic Microlepidoptera, 4:43. (v)

I922, Trans. Ent. Soc. London, I922: 95. (BM)

I9I7, Exotic Microlepidoptera, 2:82.

I9I I, Trans. Linnean Soc. London, I4: 292.

I926, Exotic Microlepidoptera, 3: 265. (BM)

I907, Journ. Bombay Nat. Hist. Soc. I7: 98I. (BM)

I920, Voyage de Ch. Alluaud et R. Jeannel en Afrique

Oriental, II, Microlepidoptera, p. 64. (P)

Igo7, Joum. Bombay Nat. Hist. Soc. I7: 979. (вм) 


\begin{tabular}{|c|c|}
\hline ne & SPILONOTA \\
\hline rhothias & HYPERTROPHA \\
\hline rhothias & PROCORONIS* \\
\hline thiastis & ACROCERCOPS \\
\hline rhothiaula & HIEROXESTIS \\
\hline rhothiodes & STENOMA \\
\hline rhothodoxa & CYNOMASTIX* \\
\hline rhymodes & EULECHRIA \\
\hline rhymogramma & EUCOSMA \\
\hline rhymotoma & ORNEODES \\
\hline rhynchacma & OPOGONA \\
\hline rhynchias & PLATYPEPLUS \\
\hline rhynchitis & TRACHYCENTRA \\
\hline rhynchograpta. & ACROCERCOPS \\
\hline rhyncholoba & PLATYPTILIA \\
\hline rhynchophora & PLATYPTILIA \\
\hline rhyodes & NOTHRIS \\
\hline rhyparias & ALUCITA \\
\hline rhysodes & BATRACHEDRA \\
\hline rhythmica & ELAPHRERGA* \\
\hline rhythmodes & BATTARISTIS \\
\hline thera & CACOECIA \\
\hline hythmologa & TORTRIX \\
\hline
\end{tabular}

$\begin{array}{ll}\text { rhythmopis } & \text { MELASINA } \\ \text { rhythmota } & \text { STATHMOPODA } \\ \text { rhytidota } & \text { CEROSTOMA }\end{array}$

$\begin{array}{ll}\begin{array}{l}\text { riciniata } \\ \text { ridicula } \\ \text { rigens } \\ \text { rigens }\end{array} & \begin{array}{l}\text { EUCOSMA } \\ \text { ACROLOPHUS } \\ \text { EPICNAPTIS* }\end{array} \\ \text { rigescens } & \text { EUCOSMA } \\ \text { rigida } & \text { IZATHA } \\ \text { rigida } & \text { CNEPHASIA } \\ \text { rimata } & \text { TINISSA } \\ \text { rimosa } & \text { SCIEROPEPLA } \\ \text { rimosa } & \text { ANCYLIS } \\ \text { rimulata } & \text { SCALMATICA } \\ & \text { STATHMOPODA }\end{array}$

$\begin{array}{ll}\text { riparia } & \text { GYPSONOMA } \\ \text { rivalis } & \text { ANACAMPSIS } \\ \text { rivifera } & \text { PHILONOME }\end{array}$

$\begin{array}{ll} & \text { [PHILOMONE, sic }] \\ \text { rivigera } & \text { SCYTHRIS } \\ \text { rivulata } & \text { GELECHIA } \\ \text { rixata } & \text { CEROMITIA } \\ \text { robiginosa } & \text { STENOMA } \\ \text { roborata } & \text { EULECHRIA }\end{array}$

I9Io, Trans. Ent. Soc. London, I9Io: 368. (BM)

I906, Trans. R. Soc. S. Australia, 30: 5I.

I9I I, Proc. Linnean Soc. N.S. Wales, 36:25I.

I921, Exotic Microlepidoptera, 2: 468. (BM)

I9II, Trans. Linnean Soc. London, I4: 295.

I9I5, Exotic Microlepidoptera, I: 437. (BM)

I930, Exotic Microlepidoptera, 3: 548.

I9I4, Exotic Microlepidoptera, I: I65.

I9I6, Exotic Microlepidoptera, 2: I7. (BM)

I92I, Exotic Microlepidoptera, 2: 408.

I920, Exotic Microlepidoptera, 2: 360.

I905, Journ. Bombay Nat. Hist. Soc. I6: 586. (BM)

I938, Trans. R. Ent. Soc. London, 87: 528.

I920, Exotic Microlepidoptera, 2: 292.

I924, Arkiv för Zool. vol. I6, part I4, p. I.

I888, Trans. Ent. Soc. London, I888: 239.

1909, Ann. Transvaal Mus. 2: 16, pl. 5, fig. 8. (т)

I9o8, Trans. Ent. Soc. London, I9o7: 489.

I9I7, Exotic Microlepidoptera, 2: 34 .

I922, Exotic Microlepidoptera, 2: 547. (BM)

I929, Exotic Microlepidoptera, 3: 506. (BM)

I924, Exotic Microlepidoptera, 3: Io6. (BM)

I937, in Caradja and Meyrick, Deuts. Ent. Zeit. Iris. 5 I:

I74.

I928, Exotic Microlepidoptera, 3: 43I.

I920, Exotic Microlepidoptera, 2: 324. (BM)

I938, in Caradja and Meyrick, Deuts. Ent. Zeit. Iris, 52 : 23.

I9II, Ann. Transvaal Mus. 2: 226. (BM)

I913, Trans. Ent. Soc. London, I9I3: I92. (BM)

I9I6, Exotic Microlepidoptera, I: 606.

1938, Institut des Parcs Nationaux du Congo Belge,

fasc. I4, p. 9 .

I929, Trans. Proc. New Zealand Inst. 6o: 490.

I910, Proc. Linnean Soc. N.S. Wales, 35: 275. (MA)

I9Io, Trans. Ent. Soc. London, I9Io: 477.

I89o, Trans. R. Soc. S. Australia, I3: 69.

I92I, Ann. Transvaal Mus. 8: 53. (т)

I9I I, Trans. Linnean Soc. London, I4: 306.

I920, Voyage de Ch. Alluaud et $R$. Jeannel en Afrique

Oriental, II, Microlepidoptera, p. 84. (P)

I933, Exotic Microlepidoptera, 4: 4I8. (BM)

I9I8, Exotic Microlepidoptera, 2: I4I. (BM)

I9I5, Trans. Ent. Soc. London, I9I5: $25 \mathrm{I}$.

I9I2, Ann. Transvaal Mus. 3: 7I. (T)

I927, Exotic Microlepidoptera, 3: 348. (BM)

I924, Exotic Microlepidoptera, 3: 8o.

I925, Exotic Microlepidoptera, 3: 2 I5. (BM)

I9I4, Exotic Microlepidoptera, I: I63. 


\begin{tabular}{|c|c|c|}
\hline \multicolumn{2}{|l|}{274} & RODOCHALCA \\
\hline rodochalca & !] CASMARA & $\begin{array}{l}\text { I934, in Caradja and Meyrick, Deuts. Ent. Zeit. Iris, } 48 \text { : } \\
\text { 37. }\end{array}$ \\
\hline rogifera & MACHIMIA & I9I4, Exotic Microlepidoptera, I: I84. (вM) \\
\hline roraria & CROCIDOSEMA & I9I7, Trans. Ent. Soc. London, I9I7: I6. (вм) \\
\hline rorida & OINOPHILA & I9II, Trans. Linnean Soc. London, I4: 298. \\
\hline rosaria & PTOCHORYCTIS & I907, Journ. Bombay Nat. Hist. Soc. I7: 740. (вм) \\
\hline roscida & IMMA & I922, Exotic Microlepidoptera, 2: 482. (BM) \\
\hline rosea & MACHIMIA & I920, Exotic Microlepidoptera, 2: 377 . (BM) \\
\hline roseata & ENDOPHTHORA & I9I3, Trans. Proc. New Zealand Inst. $45: 28$. \\
\hline roseola & SCYTHRIS & I9I2, Ann. South African Mus. I0: 63. (SA) \\
\hline rosicoma & SABATINCA & I9I4, Trans. Proc. New Zealand Inst. 46: II8. \\
\hline rosiflora & ATHRYPSIASTIS & I930, Exotic Microlepidoptera, 4: II. \\
\hline rostrata & MACRERNIS & I9o6, Journ. Bombay Nat. Hist. Soc. I7: I47. (вм) \\
\hline rostrifera & ANCYLIS & I9I2, Journ. Bombay Nat. Hist. Soc. 2I: 862. (Bм) \\
\hline rostrifera & ELASIPRORA* & I9I4, Trans. Ent. Soc. London, I9I4: 23I. (BM) \\
\hline rostriformis & STENOMA & I9I6, Exotic Microlepidoptera, I: 532. (вм) \\
\hline rostrigera & SCHIFFERMUELIERI & IA I9I9, Exotic Microlepidoptera, $2: 237$. (BM) \\
\hline rotalis & STAGMATOPHORA & IgIo, Trans. Ent. Soc. London, I9Io: 453. \\
\hline rotans & PHYLIOCNISTIS & I9I5, Trans. Ent. Soc. London, I9I5: 242. \\
\hline rotata & HIEROXESTIS & I9I5, Exotic Microlepidoptera, I: 364 \\
\hline rotigera & PARECTOPA & I93I, Ann. Mus. Nac. Hist. Nat. Buenos Aires, 36: 400. \\
\hline rubens & GONADA & I9I6, Exotic Microlepidoptera, I: 543. (вм) \\
\hline rubentula & $\begin{array}{l}\text { PACHNISTIS } \\
\text { [ERYTHRIASTIS*] }\end{array}$ & I9I4, Trans. Ent. Soc. London, I9I4: 273. (вм) \\
\hline rubidula & TRICHOTAPHE & I9I3, Ann. Transvaal Mus. 3: 303. (т) \\
\hline rubra & CRYPTOPHAGA & I89o, Trans. R. Soc. S. Australia, I3: 37. \\
\hline rubricata & EPIPHRACTIS & I9I3, Ann. Transvaal Mus. 3: 320. (T) \\
\hline rubriflora & LACTURA & I930, Exotic Microlepidoptera, 3: 596. (BM) \\
\hline rubripalpis & AEGERIA & I932, Exotic Microlepidoptera, 4: 337. \\
\hline rubripennis & ERETMOCERA & I9I5, Exotic Microlepidoptera, I: 337. \\
\hline rubripicta & STATHMOPODA & I921, Exotic Microlepidoptera, 2: 460 . \\
\hline rubritexta & MIEZA & I9I3, Exotic Microlepidoptera, I: I4I. \\
\hline rudis & MELASINA & I92I, Ann. Transvaal Mus. 8: I35. (T) \\
\hline ruens & ORNEODES & I929, Exotic Microlepidoptera, 3: 538. (Bм) \\
\hline rufa & HOPLITICA & I883, Proc. Linnean Soc. N.S. Wales, 7: 504. \\
\hline rufescens & EUCOSMA & I9I3, Ann. Transvaal Mus. 3:273. (T) \\
\hline ruficapilla & CAPUA & I932, Exotic Microlepidoptera, 4:252. (v) \\
\hline ruficolor & PROMALACTIS & I9I9, Exotic Microlepidoptera, 2: 237 . (BM) \\
\hline ruficoma & BUCCULATRIY & I93I, Exotic Microlepidoptera, 4: I63. \\
\hline ruficomana & BATHROTOMA & I881, Proc. Linnean Soc. N.S. Wales, 6: 676. \\
\hline rufimacula & DECADARCHIS & I934, Pacific Ent. Surv. Publ. 7, art. 28, p. 355. (вB) \\
\hline rufispinis & PTILOGENES & I932, Exotic Microlepidoptera, 4: 305. (v) \\
\hline rufitecta & MOLOPOSTOLA* & I920, Exotic Microlepidoptera, 2: 299. (BM) \\
\hline grisea & HELIOCAUSTA & I883, Proc. Linnean Soc. N.S. Wales, $7: 483$. \\
\hline ata & GLYPHIPTERYX & I9I5, Trans. Proc. New Zealand Inst. 47: 203. \\
\hline losa & EULECHRIA & I9I3, Exotic Microlepidoptera, I: I57. \\
\hline colana & DIPTERINA & I88I, Proc. Linnean Soc. N.S. Wales, 6: 526. \\
\hline Eera & ARGYROPLOCE & I9o9, Journ. Bombay Nat. Hist. Soc. I9: 602. (вм) \\
\hline cola & MACHIMIA & I9I4, Exotic Microlepidoptera, I: I8I. (BM) \\
\hline igena & PACHYSARIS* & I9I4, Trans. Ent. Soc. London. I9I4: 277. (BM) \\
\hline
\end{tabular}




\section{rusticana rusticata rusticula rusticula rutila}

\begin{tabular}{|c|c|}
\hline sabulosa & DICHELOPA \\
\hline saccharata & BUCCULATRIX \\
\hline saccharata & SCARDIA \\
\hline sacculata & OPOSTEGA \\
\hline sacharissa & SCYTHRIS \\
\hline sacra & STENOMA \\
\hline sacra & ZARCINIA \\
\hline sacrata & BATRACHEDRA \\
\hline sacricola & $\begin{array}{l}\text { ZALITHIA } \\
\text { [CERYCANGELA*] }\end{array}$ \\
\hline sacrifica & LITANEUTIS* \\
\hline sacrificus & PTEROPHORUS \\
\hline saeva & PLATYPTILIA \\
\hline sagaritis & ACROLOPHUS \\
\hline sagata & ARGYROPLOCE \\
\hline sagifera & GLYPHIDOCERA \\
\hline sagittans & SOPHRONIA \\
\hline sagittaria & ANARSIA \\
\hline sagittifera & DASCIA* \\
\hline sagmatias & OXYGRAPHA \\
\hline sagmatias & TRACHYCENTRA \\
\hline sagmatica & ANARSIA \\
\hline sagmatica & EULIA \\
\hline sagulata & AEOLANTHES \\
\hline salaconia & BRENTHIA \\
\hline salaconis & CACOECIA \\
\hline salaria & EPALEURA* \\
\hline salebrata & AROTROPHORA \\
\hline salebrosa & COMPSOLECHIA \\
\hline saleuta & ORTHENCHES \\
\hline saliaris & SCHOENOTENES \\
\hline salicoma & MELASINA \\
\hline saliens & LABDIA \\
\hline salina & BATRACHEDRA \\
\hline salinaria & AGRIOPHARA \\
\hline salinata & BRENTHIA \\
\hline salinatrix & ARISTOTELIA \\
\hline salsa & MESOPHERNA \\
\hline salsicola & EULECHRIA \\
\hline saltatoria & COPOCENTRA \\
\hline saltatrix & TINEA \\
\hline salticola & EUCOSMA \\
\hline salticola & PTEROPHORUS \\
\hline
\end{tabular}

I9I8, Exotic Microlepidoptera, 2: II3. (BM) I922, Exotic Microlepidoptera, 2: 494. I9II, Trans. Linnean Soc. London, I4: 268. (BM) I9I6, Exotic Microlepidoptera, 2: 7 . I9I2, Ann. South African Mus. Io: 64. (SA)

I9Io, Proc. Linnean Soc. N.S. Wales, 35: 284. I915, Trans. Ent. Soc. London, I9I5: 248. I9I4, Ann. Transvaal Mus. 4: 205. (T) I9I5, Trans. Ent. Soc. London, I9I5: 240. I9I3, Ann. Transvaal Mus. 3: 313. I9I8, Exotic Microlepidoptera, 2: 209. (BM) I925, Bull Soc. R. Ent. Egypte, 9: 2I4. I92I, Exotic Microlepidoptera, 2: 4I5.

I922, Trans. Ent. Soc. London, I922: I02. (BM) I9I3, Exotic Microlepidoptera, I: I43. (BM) 1926, Exotic Microlepidoptera, 3: 299. I930, Exotic Microlepidoptera, 3: 566. (BM) I932, Exotic Microlepidoptera, 4: 33I. (BM) I9I3, Ann. Transvaal Mus. 3:277. (T) 1929, Trans. Ent. Soc. London, 76: 510. 1923, Exotic Microlepidoptera, 3: 33. (Bм) I9I4, Journ. Bombay Nat. Hist. Soc. 22: 775. (вM) 1893, Proc. Linnean Soc. N.S. Wales, $7: 579$. I905, Journ. Bombay Nat. Hist. Soc. I6: 588. (BM) I907, Proc. Linnean Soc. N.S. Wales, 32: I44. I9I6, Exotic Microlepidoptera, I: 582. (BM) I9I2, Trans. Ent. Soc. London, I9I I: 680. (BM) I9I7, Exotic Microlepidoptera, 2: 53. (BM) I9Io, Trans. Ent. Soc. London, I9Io: 468. I9I2, Exotic Microlepidoptera, I: 5. (BM) I9I7, Ann. South African Mus. I7: I4. (SA) I9I0, Proc. Linnean Soc. N.S. Wales, 35:265. (MA) I9I8, Exotic Microlepidoptera, 2: I40. (BM) I913, Trans. Proc. New Zealand Inst. 45: 28. I928, Exotic Microlepidoptera, 3: 459. I9I8, Ann. Transvaal Mus. 6: 45. (T) I928, Exotic Microlepidoptera, 3:385. (BM) I921, Exotic Microlepidoptera, 2: 457. I93I, Exotic Microlepidoptera, 4: 43. I9I8, Exotic Microlepidoptera, 2: I93. (BM) I926, Exotic Microlepidoptera, 3: 273. (BM) I920, Exotic Microlepidoptera, 2: 353. (BM) I9I4, Exotic Microlepidoptera, I: I62. (MA) I922, Exotic Microlepidoptera, 2: 587. I930, Trans. Ent. Soc. London, 78: 322. (P) I9I3, Ann. Transvaal Mus. 3:27I. (T) I9I3, Trans. Ent. Soc. London, I913: I7o. 


saltuaria
saltuosa
salubricola
salubris
salutans
salva
samphoras
sana
sancta
sancticola
sanctifica
sanctificata
sandaracina
sandaracota
sandycitis

sandycitis sandycopa sandycota sanguiflua sanguinea sanguinolenta sanguipennis sanidopa sanidota sanifica saponacea sapphiritis sapphiropa sapphiropis sarcanthes sarcanthes sarcinata sarcinodes sarcinopa sarcinota sarcochlora sarcochroa sarcocrossa sarcodryas sarcographa sarcogypsa sarcoleuca sarcopa sarcophaea sarcophaga sarcophanes sarcopis sarcopis sarcostega
CAPUA BORKHAUSENIA

EULIA

STENOMA

ANTISPILA

ATHRYPSIASTIS

MACHAERITIS

ANTAEOTRICHA

CROBYLOPHORA

PROCHOLA

TINEA

CRYPTOLECHIA

SIMAETHIS

ZALITHIA

ANORTHOSIA

[MUSURGA*]

EUCOSMA

EPIMACTIS

ARGYROPLOCE

LACTURA

METZNERIA

LEPTOCROCA*

PARANTHRENE

CATHELOTIS*

TORTRIX

PROTEOPTERYX

LABDIA

STROBISIA

IRIANASSA*

OPOGONA

CARPOSINA

LIMNOECIA

ANTAEOTRICHA

CRYPTOLECHIA

PHYLOMICTIS

CRYPTOPHAGA

GELECHIA

MARASMARCHA

ACROCERCOPS

LABDIA

GELECHIA

LABDIA

MACROBATHRA

PROSAROTRA

HELIOCAUSTA

BLASTOBASIS

HELIOCAUSTA PRONOMEUTA* PRONOMEUTA CACOECIA
I922, Zocl. Meded. 7:8I. (L)

I9I4, Exotic Microlepidoptera, I: I72. (MA)

I93I, Exotic Microlepidoptera, 4: I53. (v)

1925, Exotic Microlepidoptera, 3: 208. (BM)

I92I, Ann. Transvaal Mus. 8: I08. (T)

I932, in Caradja, Bull. Sect. sci. Acad. roum. I5: 24.

I886, Proc. Linnean Soc. N.S. Wales, Io: 770.

1926, Exotic Microlepidoptera, 3: 235. (вM)

I9I5, Exotic Microlepidoptera, I: 345 .

I932, Exotic Microlepidoptera, 4: 3I5. (v)

I92I, Ann. Transvaal Mus. 8: I29. (T)

I936, Exotic Microlepidoptera, 5: 52. (вм)

I907, Journ. Bombay Nat. Hist. Soc. I7: 748. (Bм)

I9I4, Trans. Ent. Soc. London, I9I4: 269. (BM)

I907, Journ. Bombay Nat. Hist. Soc. I8: I50. (Bм)

I9I6, Exotic Microlepidoptera, I: 56I. (BM)

I9I7, Ann. South African Mus. I7: 9. (SA)

I9I2, Journ. Bombay Nat. Hist. Soc. 21:872. (вм)

I924, Exotic Microlepidoptera; 3: 123.

I934, Exotic Microlepidoptera, 4: 5I2. (c)

I886, Proc. Linnean Soc. N.S. Wales, I0: 775 .

1926, Exotic Microlepidoptera, 3:267. (BM)

1926, Exotic Microlepidoptera, 3: 242. (BM)

I9I2, Exotic Microlepidoptera, I: 7. (BM)

I9I 2, Ent. Mon. Mag. 48: 35. (Sub.)

I922, Exotic Microlepidoptera, 2: 569. (Bм)

I9I4, Trans. Ent. Soc. London, I9I4: 267. (BM)

I905, Journ. Bombay Nat. Hist. Soc. I6: 609. (вм)

I927, Insects of Samoa, 3, Lepidoptera, fasc. 2, p. Iog. I9I8, Trans. Proc. New Zealand Inst. 50: I33.

I921, Ann. Transvaal Mus. 8: 95. (T)

I9I 8, Exotic Microlepidoptera, 2: 200. (BM)

I921, Exotic Microlepidoptera, 2: 395.

I920, Exotic Microlepidoptera, 2: 322. (BM)

I89o, Trans. R. Soc. S. Australia, I3: 37 .

I929, Exotic Microlepidoptera, 3: 49I. (BM)

I932, Exotic Microlepidoptera, 4:25I.

I924, Exotic Microlepidoptera, 3: 86. (Вм)

I922, Exotic Microlepidoptera, 2: 570.

I9I7, Ann. South African Mus. I7: 3. (SA)

I932, Exotic Microlepidoptera, 4: 2I3.

I9I5, Exotic Microlepidoptera, I: 296.

I909, Ann. Transvaal Mus. 2: 23, pl. 7, fig. 8. (T)

I92 I, Exotic Microlepidoptera, 2: 386.

I902, Trans. $R$. Soc. S. Australia, 26: I69.

I888, Proc. Linnean Soc. N.S. Wales, (Ser. 2) 2: 936. I905, Journ. Bombay Nat. Hist. Soc. I6: 608. (BM)

I930, Exotic Microlepidoptera, 3: 562 .

I924, Exotic Microlepidoptera, 3: I07. (BM) 
sarcoxantha sarcoxantha sardania sardias sardiodes sardiopa sardiophanes sardonia sardonias sarisias sarista sarophila sartoria satelles satirica satrapella satrapis satrapodes satura saturata saturnina satyrisca satyrodes

satyropa saucropis saulopis saulotis saurates sauroderma saurodonta saurodoxa sauropa sauropis saurota saxea saxigera saxosa scabrata scabricoma scaeaspis

scaeocentra scaeocosma scaeodesma scaeodoxa scaeopa scaeosema scaeozona
CRYPTOPHASA GYMNOBATHRA ANTAEOTRICHA CERACE ARGYROPLOCE EUCOSMA SCHIFFERMUELLERIA I937, Exotic Microlepidoptera, 5: II9.

PAMPLUSIA PROCHARISTA* PROTOLECHIA ACROLOPHUS OPOGONA

TINEA STENOMA BATRACHEDRA EUPSELIA* HYPERCALLIA ADELA MACHIMIA DICHOMERIS ARISTOTELIA ACROLOPHUS SCARDIA [HOLACARTA*] STENOMA

TINEA GONIONOTA THIOTRICHA CARPOSINA PROTAPHREUTIS GLYPHIPTERYX HYPSIDRACON* MELASINA ACROCERCOPS BATRACHEDRA RECURVARIA CHELARIA XYSMATODOMA HAPSIFERA OPOGONA ARGYROPLOCE

HOLCOPOGON ANCYLOMETIS GRACILARIA TORTRIX PARAPHYLLIS* PYRODERCES OPOGONA
1937, Arb. morph. taxon. Ent. Berl. 4: 204. (D) I883, New Zealand Journ. Sci. I: 523.

1925, Exotic Microlepidoptera, 3: I68. (BM) I907, Journ. Bombay Nat. Hist. Soc. I7: 748. (вм) I922, Exotic Microlepidoptera, 2: 523. (BM) I9I2, Ent. Mon. Mag. 48: 34. (Sub.) I9o8, Proc. Zool. Soc. London, p. 7 I8. I922, Zool. Meded. 7:83. (L) I904, Proc. Linnean Soc. N.S. Wales, 29: 356. I9I3, Trans. Ent. Soc. London, I9I3: I99. (BM) I9I5, Exotic Microlepidoptera, I: 360. I9I I, Journ. Bombay Nat. Hist. Soc. 21 : 20. 1925, Exotic Microlepidoptera, 3: 202. (BM) I9I7, Exotic Microlepidoptera, 2: 33. I88I, Proc. Linnean Soc. N.S. Wales, 5: 220. I9I4, Exotic Microlepidoptera, I: I9I. (BM) I894, Trans. Ent. Soc. London, I894: 26. I9I4, Exotic Microlepidoptera, I: I82. (Вм) I923, Exotic Microlepidoptera, 2: 62I. (BM) I9I7, Trans. Ent. Soc. London, I9I7: 33. (BM) 1927, Exotic Microlepidoptera, 3: 329. (BM)

I9Io, Trans. Ent. Soc. London, I9Io: 477. I9I5, Exotic Microlepidoptera, I: 478. (BM) I9II, Trans. Linnean Soc. London, I4: 305. I909, Trans. Ent. Soc. London, I909: 28. (вм) I906, Journ. Bombay Nat. Hist. Soc. I7: г38. (вм) I9I3, Exotic Microlepidoptera, I: 75. (BM) I930, Trans. Ent. Soc. London, 78: 323. (P) I913, Exotic Microlepidoptera, I: 68. (BM) I934, Exotic Microlepidoptera, 4:526. (c) Igo8, Proc. Zool. Soc. London, p. 745.

I908, Journ. Bombay Nat. Hist. Soc. I8: 82I. I9II, Ann. Transvaal Mus. 2: 236.

I923, Exotic Microlepidoptera, 3: I4. (BM) I93I, in Caradja, Bull Sect. sci. Acad. roum. I4: 67. (BM) I893, Proc. Linnean Soc. N.S. Wales, 7: 496.

I9I6, Exotic Microlepidoptera, г : 6I6. I934, Exotic Microlepidoptera, 4: 463.

I937, in Caradja and Meyrick, Deuts. Ent. Zeit. Iris, 5I: I82. (BM)

I92I, Ann. Transvaal Mus. 8: 93. (T) I887, Trans. Ent. Soc. London, I887: 277. I928, Exotic Microlepidoptera, 3: 409. (BM) I935, Exotic Microlepidoptera, 4: 570. (C) I907, Proc. Linnean Soc. N.S. Wales, 32: I40. I905, Journ. Bombay Nat. Hist. Soc. I6: 607. I920, Voyage de Ch. Alluaud et R. Jeannel en Afrique Oriental, II, Microlepidoptera, P. 94. (P) 
scalata

scalena

scaleuta

scalifera

scaligera

scaiigera

scalmotoma

scalopias

scambodes

scambota

scammatias

scandalitis

scandaiota

scandalota

scandalotis

scansilis

scansoria

scaphopis

scaphula

scapularis

scapulata

scarabitis

scardamyctis

scarificata

scaripheuta

scariphista

scariphota

scaristis

scatebrosa

scatebrosa

scelerata

sceletias

sceletodes

sceletopa

sceletopa

sceletopis

sceliphrodes

sceliphrota

scenatica

scenias

scenica

scenica

scenites

scenodoxa

scenophora

sceptica

scepticopis

sceptrarcha
AGRIASTIS

LOZOSTOMA

COLEOPHORA

TRYMALITIS

COSMOPTERYX

EREUNETIS

PROMALACTIS

BACTRA

ARGYROPLOCE

XYROSARIS

LEUCOPTERA

HOMOSETIA

ACROCERCOPS

TORTRIX

TRACHYDORA

ARGYROPLOCE

GRACILARIA

OPOGONA

PICRORRHYNCHA*

STENOMA

BATRACHEDRA

SESIA

LEPYROTICA*

PHALONIA

PARODAEA*

CRYPTOLECHIA

COLEOPHORA

ARGYROPLOCE

ANCYLIS

SEMNOSTOMA

NARYCIA

MICROSCHISMUS

PROSELOTIS*

CROCANTHES

EUCOSMA

HETEROZYGA

CRYPTOPHASA

PSEUDURGIS

TINEA

ACROCERCOPS

EUCOSMA

PHYSOPTILA*

YPSOLOPHUS

LABDIA

SIMAETHIS

BARBAROSCARDIA

CYMOTRICHA

LECITHOCERA
I9I4, Trans. Ent. Soc. London, I9I4: 252. (BM)

I897, Proc. Linnean Soc. N.S. Wales, 22: 4 I6.

I9I I, Ann. Transvaal Mus. 2: 235. (T)

I9I2, Ann. South African Mus. Io: 58. (sA)

I909, Ann. Transvaal Mus. 2: 18, pl. 6, fig. 4. (T)

I9I I, Trans. Linnean Soc. London, I4: 300.

I9I8, Ann. Transvaal Mus. 6: 29. (T)

I9I I, Proc. Linnean Soc. N.S. Wales, 36: 255.

I9I I, Proc. Linnean Soc. N.S. Wales, 36: 277.

I928, Bull. Hill Mus. 2: 237.

r9o9, Ann. Transvaal Mus. 2: 26, pl. 8, fig. 4. (T)

I9I9, Exotic Microlepidoptera, 2: 252. (BM)

I9I4, Journ. Bombay Nat. Hist. Soc. 23: I20. (BM)

I9Io, Proc. Linnean Soc. N.S. Wales, 35: 254.

I92I, Ann. Transvaal Mus. 8: 97. (T)

I921, Zool. Meded. 6: I56. (L)

I9Io, Rec. Indian Mus. 5: 227. (K)

I909, Ann. South African Mus. 5: 376. (SA)

I922, Exotic Microlepidoptera, 2: 550. (BM)

I9I8, Exotic Microlepidoptera, 2: 209. (BM)

I9I7, Exotic Microlepidoptera, 2: 34.

I92I, Exotic Microlepidoptera, 2: 444.

I921, Zool. Meded. 6: I99. (L)

I9I7, Trans. Ent. Soc. London, I9I7:3. (BM)

I9I4, Exotic Microlepidoptera, I: 272.

I931, Ann. Mus. Nac. Hist. Nat. Buenos Aires, 36: 396.

(BM)

I9I2, Ann. Transvaal Mus. 3: 78. (T)

I9I I, Proc. Linnean Soc. N.S. Wales, 36: 270.

I9I2, Journ. Bombay Nat. Hist. Soc. 2I: 86I. (BM)

I9I8, Exotic Microlepidoptera, 2: I28. (BM)

I9I9, Exotic Microlepidoptera, 2: 262.

I9I I, Ann. Transvaal Mus. 2: 222. (T)

I9I4, Exotic Microlepidoptera, I: 276. (BM)

I9Io, Trans. Ent. Soc. London, I9Io: 445.

I912, Ent. Mon. Mag. 48: 35. (Sub.)

I9I5, Exotic Microlepidoptera, I: 30I. (BM)

I925, Exotic Microlepidoptera, 3: I50.

I923, Bull. Mus. Nat. Hist. Nat. Paris, 29: 566.

I9I I, Journ. Bombay Nat. Hist. Soc. 2I : II7.

I9I4, Journ. Bombay Nat. Hist. Soc. 23: I22.

I9I I, Ann. Transvaal Mus. 2: 227. (T)

I9I4, Journ. Bombay Nat. Hist. Soc. 22: 777. (BM)

I9o9, Ann. South African Mus. 5:37I. (SA)

I923, Exotic Microlepidoptera, 3: 57. (BM)

I922, Exotic Microlepidoptera, 2: 486. (BM)

I9Io, Ann. South African Mus. 5: 4I6. (SA)

I939, Trans. R. Ent. Soc. London, 89: 55 .

I920, Voyage de Ch. Alluaud et $R$. Jeannel en Afrique

Oriental, II, Microlepidoptera, p. 77. (P) 
sceptrifera

sceptrophora

schalidota

schematias

schematica

schismatias

schismatica

schistodes

schistopa

schoenoploca

schoenota

scholaea

scholarcha

scholasticus

scholias

sciacta

sciactis

sciadocoma

sciadopa

sciallactis

sciastis

sciaula

scierana

scieropa

scieropis

scierotoxa

sciochalca

sciocnesta

sciocolona

sciocosma

sciocrates

sciocrossa

sciocrypta

sciodelta

sciodes

sciodesma

sciodeta

sciodora

sciodoxa

sciodryas

sciogama

sciogramma

sciographa

sciograpta

scioleuca

sciombra

sciomima

sciomochla

scioneura
STENOMA

GELECHIA

EULECHRIA

ARISTOTELIA

GELECHIA

PYRODERCES

THEREUTIS*

ACROLOPHUS

LEPTOSACES

TINEA

TIMYRA

OECOPHORA

PERSICOPTILA

PTEROPHORUS

COMPSOLECHIA

TALAEPORIA

STEREMNIODES*

HIEROXESTIS

DEPRESSARIA

PARECTOPA

XYLORYCTA

MENECRATISTIS*

HYPERXENA*

PHILOBOTA

STREPSIMANES*

CARPOSINA

SCYTHRIS

STENOMA

PSEUDURGIS

SCHOENOTENES

MACHIMIA

COESYRA

GELECHIA

ACROCLITA

PROTOLECHIA

LEPIDOSCIA*

CRYPTOLECHIA

DICHOMERIS

ATTEVA

EULIA

STENOMA

ACRIA

CEROMITIA

CHELARIA

COLEOPHORA

NARYCIA

COMPSOLECHIA

NARYCIA

HOLCOPOGON
I9I6, Exotic Microlepidoptera, I: 524. (Bм)

I926, Exotic Microlepidoptera, 3: 276. (BM)

I888, Proc. Linnean Soc. N.S. Wales, (Ser. 2) $2: 955$.

I9II, Trans. Linnean Soc. London, I4: 27 I.

I886, Trans. Proc. New Zealand Inst. I8: I68.

1897, Proc. Linnean Soc. N.S. Wales, 22: 344.

I893, Proc. Linnean Soc. N.S. Wales, $7: 596$.

I9I3, Trans. Ent. Soc. London, I9I3: I92.

I902, Trans. R. Soc. S. Australia, 26: I56.

I9I I, Journ. Bombay Nat. Hist. Soc. 2I : II5.

I908, Journ. Bombay Nat. Hist. Soc. I8: 448. (вм)

I883, New Zealand Journ. Sci. I : 524 .

1915, Exotic Microlepidoptera, I: 32 I.

I924, Exotic Microlepidoptera, 3: 94. (BM)

I922, Trans. Ent. Soc. London, I922: 88. (BM)

I92I, Ann. Transvaal Mus. 8: I33. (T)

I923, Exotic Microlepidoptera, 3: 38. (BM)

I924, Trans. Ent. Soc. London, I923: 556.

I920, Exotic Microlepidoptera, 2: 3I5. (BM)

I928, Proc. Hawaiian Ent. Soc. 7 : I04. (SP)

I9I5, Exotic Microlepidoptera, I: 375 .

I933, Exotic Microlepidoptera, 4: 358. (cU)

I883, Proc. Linnean Soc. N.S. Wales, 7 : I77.

I889, Proc. Linnean Soc. N.S. Wales, (Ser. 2) 3: I6II.

I930, Exotic Microlepidoptera, 4: Io. (BM)

I924, Arkiv för Zool. vol. I6, part I4, p. 2.

I928, Exotic Microlepidoptera, 3:4I3. (BM)

I925, Exotic Microlepidoptera, 3: 213. (BM)

I9I4, Ann. South African Mus. Io: 254. (SA)

I938, Trans. R. Ent. Soc. London, 87:507. (BM)

I929, Trans. Ent. Soc. London, 76: 5I2. (BM)

I9I3, Exotic Microlepidoptera, I : II8.

I936, Exotic Microlepidoptera, 5: 44. (BM)

I92I, Exotic Microlepidoptera, 2: 446.

I904, Proc. Linnean Soc. N.S. Wales, 29: 347.

I893, Proc. Linnean Soc. N.S. Wales, 7: 5 I2.

1930, Exotic Microlepidoptera, 3: 578 .

I922, Exotic Microlepidoptera, 2: 504. (BM)

I908, Rec. Indian Mus. 2: 398. (K)

I926, Exotic Microlepidoptera, 3: 255. (BM)

I930, Exotic Microlepidoptera, 3: 558. (BM)

I9I5, Exotic Microlepidoptera, I: 38I.

I92I, Exotic Microlepidoptera, 2: 406. (BM)

I92I, Ann. Transvaal Mus. 8: 8o. (T)

I938, in Caradja and Meyrick, Deuts. Ent. Zeit. Iris, 52:

20.

I933, Exotic Microlepidoptera, 4: 4I4. (c)

I922, Trans. Ent. Soc. London, I922: 84. (BMI)

I928, Exotic Microlepidoptera, 3: 430. (B.I)

I929, Exotic Microlepidop'era, 3: 5 I5. 


$\begin{array}{ll}\begin{array}{l}\text { scionota } \\ \text { sciophaea }\end{array} & \begin{array}{l}\text { PTOCHORYCTIS } \\ \text { PLATYPTILIA }\end{array} \\ \text { sciophanes } & \text { EULECIIIA } \\ \text { sciophanta } & \text { PHYLLOCNISTIS } \\ \text { sciophracta } & \text { CRYPSITHYRIS } \\ \text { sciophthalma } & \text { STENOMA } \\ \text { scioplasta } & \text { ENCOLPOTIS } \\ & \\ \text { scioplecta } & \text { THIOTRICHA } \\ \text { scioplintha } & \text { PHRIXOSCELES } \\ \text { sciopola } & \text { PALTODORA } \\ \text { sciospila } & \text { STENOMA } \\ \text { sciospora } & \text { AUTOCNAPTIS* } \\ \text { scioterma } & \text { OPOSTEGA } \\ \text { sciotona } & \text { ANARSIA } \\ \text { sciotoxa } & \text { MACHIMIA } \\ \text { scioxantha } & \text { CROCANTHES } \\ \text { sciritis } & \text { BRACHYACMA } \\ & \text { [OXYSACTIS*] } \\ \text { sclerodes } & \text { GLYPHIPTERYX } \\ \text { sclerodes } & \text { STAGMATOPHORA } \\ \text { scleropa } & \text { ACROCLITA } \\ \text { scleropa } & \text { TORTRIX } \\ \text { sclerophracta } & \text { EULIA }\end{array}$

$\begin{array}{ll}\text { scleropis } & \text { AMYDRIA } \\ \text { sclerotricha } & \text { MEGACRASPEDUS } \\ \text { scolecias } & \text { ATTEVA } \\ \text { scolecitis } & \text { ARGYROPLOCE } \\ \text { scolia } & \text { ACOLASTA* } \\ \text { scoliandra } & \text { STENOMA } \\ \text { scolias } & \text { GLYPHIPTERYX } \\ \text { scoliastis } & \text { TRACHYBATHRA* } \\ \text { scoliograpta } & \text { ACROCERCOPS } \\ \text { scoliosema } & \text { LIMNOECIA } \\ \text { scoliozona } & \text { OPOSTEGA } \\ \text { scolopendrias } & \text { ARGYROPLOCE } \\ \text { scolopistis } & \text { HARPELLA } \\ \text { scoparcha } & \text { PSALTICA } \\ \text { scopodes } & \text { ACROLOPHUS } \\ \text { scopulata } & \text { ANACAMPSIS } \\ \text { scopulosa } & \text { CHELARIA } \\ \text { scopulosa } & \text { ONEBALA } \\ \text { scopulosana } & \text { BATHROTOMA } \\ \text { scoriodes } & \text { ORPHNOLECHIA } \\ \text { scoriopa } & \text { APHROZESTIS* } \\ \text { scoriopis } & \text { METASINA [Sic!] }\end{array}$

scoriota
I906, Journ. Bombay Nat. Hist. Soc. I7: 403. (BM) I920, Voyage de Ch. Alluaud et R. Jeannel en Afrique

Oriental, II, Microlepidoptera, p. 40. (P)

I883, Proc. Linnean Soc. N.S. Wales, 8: 323.

I9I5, Trans. Ent. Soc. London, I9I5: 24I.

I927, Insects of Samoa, 3, Lepidoptera, fasc. 2, p. II6.

I93I, Exotic Microlepidoptera, 4: 34. (v)

1920, Voyage de Ch. Alluaud et R. Jeannel en Afrique

Oriental, II, Microlepidoptera, p. 79. (P)

I9I8, Exotic Microlepidoptera, 2: I23. (BM)

I934, Exotic Microlepidoptera, 4: 470 .

I904, Proc. Linnean Soc. N.S. Wales, 29: 272.

I930, Exotic Microlepidoptera, 4:27. (v)

I925, Exotic Microlepidoptera, 4: 577. (BM)

I920, Exotic Microlepidoptera, 2: 358 .

I927, Exotic Microlepidoptera, 3: 353. (T)

I9I4, Exotic Microlepidoptera, I: I8o. (BM)

I9Io, Trans. Ent. Soc. London, I9Io: 443.

I9I8, Exotic Microlepidoptera, 2: I49. (BM)

I909, Journ. Bombay Nat. Hist. Soc. I9: 432.

I909, Ann. Transvaal Mus. 2: I9, pl. 6, fig. 7. (T)

I9I2, Journ. Bombay Nat. Hist. Soc. 2I: 857. (BM)

I9Io, Proc. Linnean Soc. N.S. Wales, 35: 236. (MA)

I936, Veröff. Deutschen Kol.-Mus. I : 330, pl. I3, fig. I9.

(H)

I9I4, Exotic Microlepidoptera, I : 2 I4.

I904, Proc. Linnean Soc. N.S. Wales, 29: 279.

I928, Exotic Microlepidoptera, 3: 4I9. (BM)

I9I6, Exotic Microlepidoptera, 2: 20. (BM)

I902, Trans. R. Soc. S. Australia, 26: I55.

I9I5, Exotic Microlepidoptera, I: 44I. (BM)

I9Io, Trans. New Zealand Inst. 42: 73 .

1907, Trans. Proc. New Zealand Inst. 39: II3.

I922, Exotic Microlepidoptera, 2: 562.

I897, Proc. Linnean Soc. N.S. Wales, 22: 366.

I9I5, Exotic Microlepidoptera, I : 35I.

I9I2, Journ. Bombay Nat. Hist. Soc. 2I:87I. (BM)

I909, Ann. S. African Mus. 5: 354. (SA)

I9I4, Exotic Microlepidoptera, I: 263. (BM)

I9I3, Trans. Ent. Soc. London, I9I3: I96. (BM)

I9I4, Trans. Ent. Soc. London, I9I4: 260.

I9I3, Journ. Bombay Nat. Hist. Soc. 22: I65. (BM)

I9Io, Journ. Bombay Nat. Hist. Soc. 20: 450.

I881, Proc. Linnean Soc. N.S. Wales, 6: 677 .

I9I5, Exotic Microlepidoptera, I: 382. (BM)

I93I, Exotic Microlepidoptera, 4: I56. (BM)

I934, in Caradja and Meyrick, Deuts. Ent. Zeit. Iris,

$48: 43$.

I9o9, Trans. New Zealand Inst. 4I: I6. 
scorpiastis scorpiodes scorpiura scorpiura scortea scotaea scotangela scotarcha scoteropis scotias scotinopa

scotinopis scotiodes scotochalca scotocleptes scriba scribaria scribarius scribula scriniata scriptifera scriptulata scrupulata scrupulosa scrupulosa scrutaria scrutatrix sculpta scutata scutellata scutifer scutifera scutiferana

scutiformis

scutigera scutulata scyrodes scytalias scythopa

scythrochalca scythrodes scythropa scythropa scythropis scytina
ARGYROPLOCE POLYCHROSIS DECADARCHIS PICROXENA* STENOMA THIOTRICHA TINEOLA PHTHONERODES* STEGASTA PSEUDODOXIA SCYTHRIS

NARYCIA EULECHRIA ASCALENIA TINEA MELASINA ODITES PTEROPHORUS OGMOGRAPTIS* CHELARIA LYONETIA ACROCERCOPS OROTHYNTIS* BACTRA PERIACMA MELASINA CATAMACTA ANCYLIS ANACAMPSIS AGRIOPHARA OXYPTILUS PSEUDURGIS CAPUA

[AEOLOSTOMA*] EUCOSMA

GRACILARIA COMOTECHNA MERIDARCHIS PARELLIPTIS* HYPERARCTIS*

URODUS TRICHOPTILUS BACTRA LINOSTICHA* EPICEPHALA PROTOLECHIA
I9I2, Journ. Bombay Nat. Hist. Soc. 21: 87I. (BM) I908, Proc. Zool. Soc. London, p. 7 I7.

I93I, Exotic Microlepidoptera, 4: I65.

I921, Zool. Meded. 6: I6I. (L)

I9I5, Exotic Microlepidoptera, I: 459. (BM)

I908, Journ. Bombay Nat. Hist. Soc. I8: 442. (BM)

I9I9, Exotic Microlepidoptera, 2: 245.

I890, Trans. R. Soc. S. Australia, I3: 45. (BM)

I93 I, Journ. Linnean Soc. London, 37: 280. (BM)

I921, Zool. Meded. 6: I7I. (L)

I935, in Caradja and Meyrick, Materialien zu einer Microlepidopteren Fauna der Chinesischen Provinzen Kiangsu,

Chekiang und Hunan, p. 87.

I897, Trans. Ent. Soc. London, I897: 383.

I902, Trans. R. Soc. S. Australia, 26: I5I.

I934, Exotic Microlepidoptera, 4: 509.

I934, Exotic Microlepidoptera, 4: 480. (v)

I921, Ann. Transvaal Mus. 8: г36. (т)

I9I5, Exotic Microlepidoptera, I: 380.

I926, Exotic Microlepidoptera, 3: 300.

I935, Exotic Microlepidoptera, 4: 600.

I9I3, Journ. Bombay Nat. Hist. Soc. 22: I63. (Вм)

I92I, Exotic Microlepidoptera, 2: 457.

I9I6, Exotic Microlepidoptera, 2: 2.

I9I3, Trans. Ent. Soc. London, I9I3: I9I. (BM)

I9I I, Ann. Transvaal Mus. 2: 227. (T)

I9Io, Journ. Bombay Nat. Hist. Soc. 20: I57. (BM)

I922, Exotic Microlepidoptera, 2: 606.

I9I2, Exotic Microlepidoptera, I: I. (BM)

I9I2, Exotic Microlepidoptera, I: 33. (BM)

I894, Trans. Ent. Soc. London, I894: I4.

I9I6, Exotic Microlepidoptera, I: 487. (BM)

I930, Exotic Microlepidoptera, 3: 564 .

I9I2, Ann. South African Mus. I0: 70. (SA)

I88I, Proc. Linnean Soc. N.S. Wales, 6: 456.

I93I, in Caradja, Bull. Sect. sci. Acad. roum. I4: 64. (BM)

I92I, Exotic Microlepidoptera, 2: 47 I.

I921, Exotic Microlepidoptera, 2: 400. (BM)

I9I6, Exotic Microlepidoptera, 2: 30. (BM)

I9Io, Journ. Bombay Nat. Hist. Soc. 20: 439. (BM)

I931, in Joannis, Ann. Soc. Ent. France, 98 (Supp.):

744 [506]. (P)

1932, Exotic Microlepidoptera, 4:285. (v)

I886, Trans. Ent. Soc. London, I886: I3.

I9II, Proc. Limnean Soc. N.S. Wales, 36: 254.

I883, Proc. Linnean Soc. N.S. Wales, 8: 339.

I930, Exotic Microlepidoptera, 3: $5^{\$ 3}$.

I904, Proc. Limnean Soc. N.S. Wales, 29: 374 . 
seclusa

secreta

secretifera

secta

sectifera

sectilis

sectionalis

secularis

secundata.

secura

secura

secutor

secutrix

sedata

sedifera

sediliata

sedilis

seditiosa

seditiosana

seducta

sedula

segetana

segmentata

segnis

segnis

sejuncta

selacta

selastis

selecta

selenaspis

selenia

seleniaca

selenias

selenitis

selenocycla

selenopa

selenophanes

selenopis

selenota

selenura

sellifera

selmatarcha

selmatias

selmatica

semalea

semantris

semaphora

sematacma

sematias

sematica
TORTRIX

XYROSARIS

ASCALENIA

PROTOLECHIA

MOMPHA

CONIASTIS*

EULIA

EUCRYPTOGONA

STENOMA

CACOECIA

CHLOROPHYTIS*

OXYPTILUS

MENDESIA

PLATYPTILIA

POLYCHROSIS

ARGYROPLOCE

THISIZIMA

CACOECIA

STREPSICEROS

STENOMA

SYNTOMACTIS

PALAEOBIA

STENOMA

TORTRIX

TRICHEMBOLA*

FRISILIA

HIEROXESTIS

ANTISPILA

EUXANTHIS

IMMA

PROTOLECHIA

COESYRA

COMPSOTROPHA*

GRACILARIA

LEUCOPTERA

PHYLLOCNISTIS

EREUNETIS

PYRODERCES

PERIACMA

SYNTOMACTIS

ANTAEOTRICHA

THROMBOGENES*

TIMYRA

ACROCERCOPS

EUCOSMA

EPICALLIMA

HOMALERNIS*

MEGACRASPEDUS

PTEROPHORUS

TELPHUSA
1926, Exotic Microlepidoptera, 3: 247. (BM)

I9I2, Ann. South African Mus, Io: 67. (SA)

I932, Trans. Ent. Soc. London, 80: II4.

I92I, Exotic Microlepidoptera, 2: 428. (BM)

1922, Exotic Microlepidoptera, 2: 579. (BM)

I9I6, Exotic Microlepidoptera, I: 601.

I932, Exotic Microlepidoptera, 4: 26o. (v)

I9I8, Ann. Transvaal Mus. 6: 45. (T)

I925, Exotic Microlepidoptera, 3:2 I4. (BM)

I9I0, Trans. Ent. Soc. London, I9Io: 432. (BM)

I9I2, Ann. South African Mus. IO: 72. (SA)

I9II, Ann. Transvaal Mus. 2: 218. (T)

I9I4, Ann. Transvaal Mus. 4: I96. (T)

I932, Exotic Microlepidoptera, 4:334.

I9I I, Proc. Limnean Soc. N.S. Wales, 36: 256.

I9I2, Trans. Ent. Soc. London, I9I I: 689. (Bм)

I907, Journ. Bombay Nat. Hist. Soc. I7: 989.

I92I, Zool. Meded. 6: I47. (L)

I881, Proc. Linnean Soc. N.S. Wales, 6: 684.

I9I8, Exotic Microlepidoptera, 2: 206. (BM)

I897, Proc. Linnean Soc. N.S. Wales, 22: 381.

I882, Proc. Linnean Soc. N.S. Wales, 6: 669.

I9I5, Exotic Microlepidoptera, I: 423. (BM)

I9I8, Exotic Microlepidoptera, 2: I68. (BM)

I9I8, Exotic Microlepidoptera, 2: I16.

I929, Exotic Microlepidoptera, 3:5I7. (BM)

I9II, Trans. Linnean Soc. London, I4: 294.

I926, Exotic Microlepidoptera, 3:26I.

I93I, Exotic Microlepidoptera, 4: I58. (v)

I925, Exotic Microlepidoptera, 3: I33. (BM)

I904, Proc. Linnean Soc. N.S. Wales, 29: 378.

1884, Proc. Linnean Soc. N.S. Wales, 9: 778.

I884, Proc. Linnean Soc. N.S. Wales, 8: 512.

I909, Trans. New Zelaand Inst. 4I: I5.

I930, Exotic Microlepidoptera, 4: 7 .

I915, Exotic Microlepidoptera, I: 348.

I88I, Proc. Linnean Soc. N.S. Wales, 5: 259.

I905, Journ. Bombay Nat. Hist. Soc. I6: 606.

I9I4, Exotic Microlepidoptera, I: 258. (BM)

I897, Proc. Linnean Soc. N.S. Wales, 22: 389 .

1925, Exotic Microlepidoptera, 3: I63. (BM)

I921, Ann. Transvaal Mus. 8: I37. (T)

I9o8, Journ. Bombay Nat. Hist. Soc. I8: 450. (вм)

I9I8, Exotic Microlepidoptera, 2: I74.

I9I2, Ent. Mon. Mag. 48: 35. (Sub.)

I906, Journ. Bombay Nat. Hist. Soc. I7: 409. (BM)

I908, Journ. Bombay Nat. Hist. Soc. I8: 620. (BM)

I921, Exotic Microlepidoptera, 2: 424. (BM)

igo8, Trans. Ent. Soc. London, I907: 496.

I9I3, Ann. Transvaal Mus. 3: 286. (T) 


$\begin{array}{ll}\text { sematopa } & \text { STENOMA } \\ \text { semiacma } & \text { ETHIROSTOMA* } \\ \text { semialba } & \text { HYPONOMEUTA } \\ \text { semialbata } & \text { PROCHOLA } \\ \text { semiarcha } & \text { HEMIMENE } \\ \text { semiberbis } & \text { COMOTECHNA } \\ \text { semicincta } & \text { SIMAETHIS } \\ \text { semicitra } & \text { IMMA } \\ \text { semiclara } & \text { IMMA } \\ \text { semiclausa } & \text { GRACILARIA } \\ \text { semicocta } & \text { EPICHORISTA } \\ \text { semicoronis } & \text { USSARA } \\ \text { semicretata } & \text { ORTHENCHES }\end{array}$

semiculta semicuprata semicuprea semicurva semidivisa semifervens semifixa semifulva semifusca semiglobata semigrisea semilutea seminalis seminata seminigera seminigrescens seminubila

semioceros semiophanes semiostrina

semiota semiota semiotarsa semiovata semipicta semiramis semirosa semirubra semirupta semisecta semisepta semisparsa semistricta
ARGYROPLOCE TRICHOTAPHE HOPLOPHANES EUCOSMA TINEA ENCAMINA BAREA SCHOENOTENES IULACTIS* TIQUADRA LYONETIA BLASTOBASIS COLEOPHORA TRICHOTAPHE ACROLOPHUS LOXOTOMA CACOECIA

STAGMATOPHORA ARISTOTELIA AEOLANTHES

CROSSOPHORA* XENOTHICTIS STRUTHOSCELIS ANTAEOTRICHA ARDEUTICA LABDIA GNORIMOSCHEMA OEDEMATOPODA LECITHOCERA LIMNOECIA ODITES GLYPHIPTERYX TANYZANCLA
I9I5, Exotic Microlepidoptera, I : 450. (BM)

I9I4, Trans. Ent. Soc. London, I9I4: 245. (BM)

I9I3, Exotic Microlepidoptera, I: I39.

I922, Exotic Microlepidoptera, 2: 58I. (Bм)

I922, Exotic Microlepidoptera, 2: 528. (BM)

I92I, Exotic Microlepidoptera, 2: 402. (BM)

I921, Zool. Meded. 6: I82. (L)

1937, Exotic Microlepidoptera, 5: I24. (ВM)

I929, Trans. Ent. Soc. London, 76: 503. (BM)

I92I, Exotic Microlepidoptera, 2: 47I.

I9I4, Trans. Proc. New Zealand Inst. 46: I04.

I932, Trans. Ent. Soc. London, 80: I I5.

I93I, Ann. Mus. Nac. Hist. Nat. Buenos Aires, 36: 406.

I9o9, Journ. Bombay Nat. Hist. Soc. I9: 604. (вм)

I922, Trans. Ent. Soc. London, I922: I07. (BM)

I897, Proc. Linnean Soc. N.S. Wales, 22: 412.

I9I 2, Journ. Bombay Nat. Hist. Soc. 21: 864. (вм)

I934, Exotic Microlepidoptera, 4:480.

I927, Exotic Microlepidoptera, 3: 379.

I9I5, Exotic Microlepidoptera, I : 298.

I908, Journ. Bombay Nat. Hist. Soc. I8: 620. (BM)

I9I8, Exotic Microlepidoptera, 2: I45.

I922, Exotic Microlepidoptera, 2: 599. (вм)

I932, Exotic Microlepidoptera, 4: 230.

I9I6, Exotic Microlepidoptera, I : 596.

I921, Zool. Meded. 6: I8g. (L)

I9I I, Trans. Linnean Soc. London, I4: 275. (BM)

I9I3, Trans. Ent. Soc. London, I9I3: I95. (вм)

I932, Exotic Microlepidoptera, 4:288. (v)

I931, in Joannis, Ann. Soc. Ent. France, 98 (Supp.): 7 II [473]. (P)

I935, Exotic Microlepidoptera, 4:572.

I9I8, Exotic Microlepidoptera, 2: II9. (BM)

I935, in Caradja and Meyrick, Materialien zu einer Micro-

lepidopteren Fauna der Chinesischen Provinzen Kiangsu,

Chekiang und Hunan, p. 82. (Вм)

1886, Proc. Linnean Soc. N.S. Wales, Io: 797.

I9ıo, Proc. Linnean Soc. N.S. Wales, 35: 280.

I9I6, Exotic Microlepidoptera, I : 545. (BM)

I926, Exotic Microlepidoptera, 3: 234. (BM)

I9I3, Trans. Ent. Soc. London, I9I3: I73. (вм)

I930, Exotic Microlepidoptera, 3: 545. (BM)

I929, Exotic Microlepidoptera, 3: 492. (BM)

I936, Exotic Microlepidoptera, 4: 6I9.

I9I0, Journ. Bombay Nat. Hist. Soc. 20: 445. (BM)

I928, Exotic Microlepidoptera, 3: 393. (T)

I930, Exotic Microlepidoptera, 4: IS.

I9I8, Exotic Microlepidoptera, 2: I95. (BM)

I920, Ann. South African Mus. I7: 286. (S.A) 


$\begin{array}{ll}\text { semistructa } & \text { CACOECIA } \\ \text { semisulphurea } & \text { OPOGONA } \\ \text { semitexta } & \text { PERONEA } \\ \text { semitincta } & \text { ZELLERIA } \\ \text { semitrunca } & \text { ARGYRESTHIA } \\ \text { semiumbrata } & \text { TINEA } \\ \text { semiusta } & \text { TELPHUSA } \\ \text { semivittata } & \text { MONOPIS } \\ \text { semias } & \text { GAESA } \\ \text { semnitis } & \text { EUCOSMA } \\ \text { semnocharis } & \text { PLATYPTILIA } \\ \text { semnochroa } & \text { PALTODORA } \\ \text { semınocrana } & \text { CRYPTOPHASA } \\ \text { semnodes } & \text { CRYPTOLECHIA } \\ \text { sennodora } & \text { LECITHOCERA } \\ \text { semnodoxa } & \text { HARPELLA }\end{array}$

semnodryas semnodryas semnolitha semnopa semnophanes semnopis semnospora semnostola

semnota
semocausta
semochlora
semocrossa
semophanes
semophanta
semophantis
semota
semotheta
senescens
senicula
senilis
sensilis
sepias
sepiogramma
septica
septicodes
septicola
septifera
sepulcralis
sepulcrana
sequens
sequestra

ARGYROPLOCE FUMEA LABDIA ANARSIA GRACILARIA PLATYPTILIA CRYPSICHARIS (?) PYRODERCES [CHOLOTIS*] COSMOPTERYX PHLOEOPOLA CHLOROCLYSTIS PELEOPODA PLEUROTA ELACHISTA ORNEODES MELASINA PARAMORPHA TONICA PALTODORA FRISILIA BORKHAUSENIA LOXOTROCHIS* CRYPTOPHASA HAPSIFERA EPIMARPTIS ENARMONIA ARGYROTOXA BRACHYSYMBOLA* EUCOSMA TINEA STENOMA
I937, Exotic Microlepidoptera, 5: I26.

I9I I, Journ. Bombay Nat. Hist. Soc. 2I: IIo.

I9I2, Exotic Microlepidoptera, I: I7. (BM)

1930, Exotic Microlepidoptera, 3: 562. (BM)

I9I4, Journ. Bombay Nat. Hist. Soc. 23: I23. (BM)

I920, Voyage de Ch. Alluaud et R. Jeannel en Afrique

Oriental, II, Microlepidoptera, p. I02. (P)

I922, Exotic Microlepidoptera, 2: 500. (P)

I927, Insects of Samoa, 3, Lepidoptera, fasc. 2, p. II5.

I926, Exotic Microlepidoptera, 3: 286. (BM)

I9I2, Ent. Mon. Mag. 48: 35. (Sub.)

I932, Exotic Microlepidoptera, 4: 335 .

I9I3, Ann. Transvaal Mus. 3: 28I. (T)

I928, Exotic Microlepidoptera, 3: 433 .

I9I I, Trans. New Zealand Inst. 43: 75 .

I933, Exotic Microlepidoptera, 4:357. (c)

I93I, Ann. Mus. Nac. Hist. Nat. Buenos Aires, 36: 392. (BM)

I936, Exotic Microlepidoptera, 4: 6I3. (BM)

I922, Exotic Microlepidoptera, 2: 604. (P)

I928, Exotic Microlepidoptera, 3: 386. (ВM)

I92I, Ann. Transvaal Mus. 8: 79. (T)

I9I8, Ann. Transvaal Mus. 6: 40. (T)

I93I, Exotic Microlepidoptera, 4: I76. (v)

I92I, Exotic Microlepidoptera, 2: 445.

I897, Proc. Linnean Soc. N.S. Wales, 22: 356.

I9I4, Journ. Bombay Nat. Hist. Soc. 22: 775 .

I883, Proc. Linnean Soc. N.S. Wales, 8: 350.

I9I9, Trans. Proc. New Zealand Inst. 5I: 349.

I930, Exotic Microlepidoptera, 4: I3. (BM)

I889, Proc. Linnean Soc. N.S. Wales, (Ser. 2) 3: I649.

I9I4, Exotic Microlepidoptera, I: 28I.

1929, Exotic Microlepidoptera, 3: 537. (BM)

I937, Exotic Microlepidoptera, 5: II5.

I9Io, Proc. Linnean Soc. N.S. Wales, 35: I54.

I9Io, Trans. Ent. Soc. London, I9Io: 456.

I9I3, Ent. Mitteil. 2: 298. (D)

I9Io, Journ. Bombay Nat. Hist. Soc. 20: 436.

I928, Exotic Microlepidoptera, 3: 467. (BM)

I906, Trans. Ent. Soc. London, r906: 205. (BM)

I938, Trans. R. Ent. Soc. London, 87: 5 I6.

Igo8, Proc. Zool. Soc. London, p. 750.

I9I7, Exotic Microlepidoptera, 2: 69.

I9I2, Ent. Mon. Mag. 48: 34. (Sub.)

I921, Zool. Meded. 6: I49. (Lost)

I9I2, Trans. Ent. Soc. London, I9II: 7 I8. (BM)

I927, Exotic Microlepidoptera, 3: 334. (BM)

I9I9, Exotic Microlepidoptera, 2: 248. (BM)

I9I8, Exotic Microlepidoptera, 2: 205. (BM) 
sequestrata

sera

serangias

serangodes

serangodes

serangota

seraphias

seraphica

serarcha

serena

serena

serenata

serenisca

serenus

seria

seriaiis

seriata

serica

sericanassa

sericata

sericodes

sericophaea

sericophthalma

sericopis

serina

serinopa

serpens

serpentina

serpula

serrata

serrata

serricornis

serriformis

serrigera

serrulatus

serruligera

sertifera

sertifera

sertigera

serva

servata

servicula

servilis

servula

sesamitis

sesamivora
CRANEODES [Sic!] PLUTELLA

EUCOSMA

ANTAEOTRICHA

POLYCHROSIS

PTILOTHYRIS

NEMOTOIS

ACROLEPIA

ANTAEOTRICHA

EPICHORISTA

TRICHOTAPHE

AGRIOSCELIS

IRENIDORA*

PTEROPHORUS

BACTRA

BRACHMIA

GRACILARIA

MEGACRASPEDUS

AEOLANTHES

HOPLITICA

MAROGA

CROBYLOPHANES*

DIPTYCHOPHORA

DICHELOPA

SCIEROPEPLA

NEPHANTIS*

PTEROPHORUS

PYRGOPTILA*

RHADINASTIS

OINOPHILA

TORTRIX

MICROSCHISMUS

ACROCERCOPS

ACROCERCOPS

TITANOPTILUS

PROTOTHEORA

MONOPIS

ORNEODES

HELCYSTOGRAMMA

MACHIMIA

NEMOTOIS

FUMEA

TINEA

COMMATICA

ALUCITA

PYRODERCES
1926, Sarawak Mus. Journ. 3: I64.

I886, Trans. Proc. New Zealand Inst. I8: I78.

I9I2, Ent. Mon. Mag. 48: 35. (Sub.)

I9I5, Exotic Microlepidoptera, I: 400.

1920, Exotic Microlepidoptera, 2: 346. (BM)

1932, Exotic Microlepidoptera, 4: 205. (BM)

1907, Journ. Bombay Nat. Hist. Soc. I7: 991.

I931, Ann. Mus. Nac. Hisi. Nat. Buenos Aires, 36: 402.

(BM)

I930, Exotic Microlepidoptera, 3: 556. (BM)

I9Io, Proc. Linnean Soc. N.S. Wales, 35: 256.

I909, Trans. Ent. Soc. London, I909: 23. (BM)

I9I3, Exotic Microlepidoptera, I: 96.

I938, in Caradja and Meyrick, Deuts. Ent. Zeit. Iris, 52:

7. (BM)

I9I3, Exotic Microlepidoptera, I: II3.

I9I7, Trans. Ent. Soc. London, I9I7: 24. (BM)

I908, Proc. Zool. Soc. London, p. 727.

I9I2, Exotic Microlepidoptera, I: 27. (T)

I9o9, Ann. South African Mus. 5: 369. (SA)

1934, in Caradja and Meyrick, Deuts. Ent. Zeit. Iris, 48:

39.

1883, Proc. Linnean Soc. N.S. Wales, 7: 497.

I9I5, Exotic Microlepidoptera, I: 372.

1938, Institut des Parcs Nationaux du Congo Belge, fasc. I4, p. I9.

I933, Exotic Microlepidoptera, 4:382.

I926, Trans. Ent. Soc. London, 74: 272. (BM)

I89o, Trans. R. Soc. S. Ausiralia, I3: 70.

1905, Journ. Bombay Nat. Hist. Soc. I6: 603. (BM)

I909, Ann. South African Mus. 5: 366. (SA)

I889, Proc. Linnean Soc. N.S. Wales, (Ser. 2) 3: I600.

1932, Exotic Microlepidoptera, 4: 2I6.

I9I4, Exotic Microlepidoptera, I: 209.

I9Io, Proc. Linnean Soc. N.S. Wales, 35:246. (MA)

I9I4, Ann. Transvaal Mus. 4: I87. (T)

1930, Exotic Microlepidoptera, 3: 580. (вм)

I9I5, Trans. Ent. Soc. London, I9I5: 232.

1935, Exotic Microlepidoptera, 4: 554 .

I920, Ann. South African Mus. I7: 3I4. (SA)

I9Io, Rec. Indian Mus. 2: 230. (K)

I92I, Exotic Microlepidoptera, 2: 406.

1923, Exotic Microlepidoptera, 3: 27. (BM)

1920, Exotic Microlepidoptera, 2: 375.

r925, in Caradja, Mem. Sect. Sti. Acal. Romanā, 3: 383.

I9I7, Exotic Microlepidoptera, 2: 92.

I9I4, Exotic Microlepidoptera, I: 2 I I.

I922, Trans. Ent. Soc. London, I922: 72. (BM)

1905, Journ. Bombay Nat. Hist. Soc. 16: $5 \mathrm{Sz}$.

I933, Exotic Microlepidoptera, 4: 427. 


\begin{tabular}{|c|c|c|}
\hline sesamodes & COMPSOLECHIA & I922, Trans. Ent. Soc. London, I922: 90. (BM) \\
\hline sesquitertia & TINEA & I909, Ann. South African Mus. 5: 361. (SA) \\
\hline setiacma & ACROLOPHUS & I923, Exotic Microlepidoptera, 2:6Io. (BM) \\
\hline setiotricha & MAROGA & I89o, Trans. R. Soc. S. Australia, I3: 40. \\
\hline etosa & CNEPHASIA & I9I7, Trans. Ent. Soc. London, I'9I7: Io. (BM) \\
\hline severa & HELIOCAUSTA & I883, Proc. Linnean Soc. N.S. Wales, 7: 470. \\
\hline sevocata & HEMIMENE & I9I6, Exotic Microlepidoptera, 2: 29. (BM) \\
\hline sexangula & PHYLLOCNISTIS & I9I5, Trans. Ent. Soc. London, I9I 5: 242. \\
\hline sextaria & TINEA & I9I 7, Exotic Microlepidoptera, $2: 76$. \\
\hline sibila & PHTHORIMAEA & I9I2, Ann. Transvaal Mus. 8:73. (T) \\
\hline sibyllina & CHRESMARCHA* & I9го, Proc. Linnean Soc. N.S. Wales, 35: 220. \\
\hline sicaria & SPORADARTHRA* & I9I I, Trans. Linnean Soc. London, I4: 303. \\
\hline sicariana & STREPSICEROS & I88I, Proc. Linnean Soc. N.S. Wales, 6: 69I. \\
\hline sicca & EPICHORISTA & I9I2, Exotic Microlepidoptera, I: 9. (BM) \\
\hline siccata & OINOPHILA & I9Io, Trans. Ent. Soc. London, I9Io: 375 . \\
\hline siccescens & EUCOSMA & I9I2, Ann. South African Mus. Io: 57 . (SA) \\
\hline siccinervis & ODITES & I930, Exotic Microlepidoptera, 4: I7. (BM) \\
\hline siccivora & AUTOSTICHA & I935, Exotic Microlepidoptera, 4: 592. (BM) \\
\hline sideracma & STATHMOPODA & I9I5, Exotic Microlepidoptera, I: 336. \\
\hline siderantha & EPAGOGE & I905, Journ. Bombay Nat. Hist. Soc. I6: 588. (вм) \\
\hline siderarcha & STRUTHISCA* & I905, Journ. Bombay Nat. Hist. Soc. I6: 6I5. \\
\hline sideraula & MNESTERIA & I9I6, Exotic Microlepidoptera, I: 573 . (BM) \\
\hline sideraula & PYRODERCES & \\
\hline & [ETHIRASTIS $*$ ] & xotic Microlepidoptera, I: 3I5. \\
\hline rias & HELIOZELA & I897, Proc. Linnean Soc. N.S. Wales, 22: 403. \\
\hline ina & LASIOSTEGA* & I932, Exotic Microlepidoptera, 4: 27I. \\
\hline deritis & NOTERAULA & I905, Trans. Ent. Soc. London, I905: 232. \\
\hline erodeta & OECOPHORA & I883, New Zealand Journ. Sci. I: 525 . \\
\hline eropa & RHADINASTIS & I997, Proc. Linnean Soc. N.S. Wales, 22: 312. \\
\hline deropetra & ARGYROPLOCE & I932, Exotic Microlepidoptera, 4: 309. (v) \\
\hline siderophanes & HEMIMENE & I922, Exotic Microlepidoptera, 2: 529. (BM) \\
\hline rota & CREMNOGENES & I888, Trans. Proc. New Zealand Inst. 20: 82. \\
\hline derota & PERONEA & I9I8, Exotic Microlepidoptera, 2: I7I. (BM) \\
\hline sideroxyla & ARGYROPLOCE & I93I, Exotic Microlepidoptera, 4: I33. (BM) \\
\hline ta & ISORRHOA & I9I7, Exotic Microlepidoptera, 2: 6I. \\
\hline sidonia & COESYRA & I9I3, Exotic Microlepidoptera, I: II9. (MA) \\
\hline sidonia & CROCANTHES & IgIo, Trans. Ent. Soc. London, I9Io: 442. \\
\hline ota & ISCHNODORIS* & I9I I, Journ. Bombay Nat. Hist. Soc. 20: 726. (BM) \\
\hline gillata & ZELLERIA & I893, Proc. Linnean Soc. N.S. Wales, $7: 587$. \\
\hline gillatrix & BRACHMIA & I9IO, Rec. Indian Mus. 2: 222. (K) \\
\hline & CROBYLOPHORA & I9I I, Journ. Bombay Nat. Hist. Soc. 21 : Io8. \\
\hline 1ophora & ERIODYTA & I 884, Proc. Linnean Soc. N.S. Wales, 8: 5I6. \\
\hline gnata & PHYLLOCNISTIS & I9I5, Exotic Microlepidoptera, I : 349. \\
\hline fera & MACHIMIA & I9I4, Exotic Microlepidoptera, I: I77. (BM) \\
\hline gnifera & POLYCHROSIS & I9I 2, Ent. Mon. Mag. 48: 34. (Sub.) \\
\hline gnifica & DEPRESSARIA & I9I 5, Trans. Ent. Soc. London, I9I5: 210. (BM) \\
\hline igera & PHILAUSTERA* & I927, Exotic Microlepidoptera, 3: 358. (BM) \\
\hline lens & PACHNISTIS & I935, Exotic Microlepidoptera, 4: 565. \\
\hline & BRYONYMPHA* & I930, Exotic Microlepidoptera, 3: 560. (BM) \\
\hline & BATRACHEDRA & I9I7, Exotic Microlepidoptera, 2: 35. \\
\hline
\end{tabular}




\begin{tabular}{|c|c|c|}
\hline silvestris & CHELARIA & I9I3, Journ. Bombay Nat. Hist. Soc. 22: I64. (BM) \\
\hline silvicola & SCIEROPEPLA & I89o, Trans. R. Soc. S. Australia, I3: 69. \\
\hline silvicolor & PHYTOMIMIA & I932, Exotic Microlepidoptera, 4:279. (v) \\
\hline simana & ANISOGONA & I88I, Proc. Linnean Soc. N.S. Wales, 6: 465. (вм) \\
\hline simbleuta & PTOCHORYCTIS & I907, Journ. Bombay Nat. Hist. Soc. I8: I50. (Bм) \\
\hline milana & ANISOGONA* & I88I, Proc. Linnean Soc. N.S. Wales, 6: 466. \\
\hline mulacrella & ALLOCOTA* & I904, Proc. Linnean Soc. N.S. Wales, 29: 420. \\
\hline mulatrix & STENOMA & I9I4, Ann. Transvaal Mus. 4: I99. (T) \\
\hline acera & HYPERCALLIA & I9o9, Ann. Transvaal Mus. 2: 22, pl. 7, fig. 6. (T) \\
\hline ndonia & CHOLOTIS & I9II, Trans. Linnean Soc. London, I4: 284 . \\
\hline ndonia & TINEA & I9I I, Journ. Bombay Nat. Hist. Soc. 2I: I I8. \\
\hline nistra & ARISTOTELIA & I904, Proc. Linnean Soc. N.S. Wales, 29: 287. \\
\hline nuosa & LECITHOCERA & I9Io, Journ. Bombay Nat. Hist. Soc. 20: 444. (BM) \\
\hline uosa & SPILONOTA & I9I7, Ann. South African Mus. I7:2. (SA) \\
\hline honaula & ACROCERCOPS & I93I, Exotic Microlepidoptera, $4: 47$. \\
\hline honias & AEOLANTHES & I9o8, Journ. Bombay Nat. Hist. Soc. I8: 637. (вм) \\
\hline phonistis & PHILOBOTA & I921, Exotic Microlepidoptera, $2: 385$. \\
\hline raea & HARMOLOGA & I885, Trans. Proc. New Zealand Inst. I7: I45. \\
\hline anta & TRICHOTAPHE & I9I3, Journ. Bombay Nat. Hist. Soc. 22: I 79. (BM) \\
\hline hora & STENOMA & I9I5, Exotic Microlepidoptera, I: 475. (вм) \\
\hline nia & PYRODERCES & I9I7, Exotic Microlepidoptera, 2: 37. \\
\hline renica & BASCANTIS* & I9I4, Trans. Proc. New Zealand Inst. 46: II5. \\
\hline iaca & BASSARODES* & I9Io, Trans. Ent. Soc. London, I9Io: 459. \\
\hline iana & TORTRIX & I88I, Proc. Linnean Soc. N.S. Wales, 6: 521. \\
\hline ina & PETASOBATHRA* & I9I5, Exotic Microlepidoptera, I: 355. \\
\hline iopa & CRYPTOLECHIA & I933, Exotic Microlepidoptera, 4: 368 . (BM) \\
\hline ota & ARISTOTELIA & I9o8, Proc. Zool. Soc. London, p. 724 . \\
\hline trata & ARGYROPLOCE & I9I I, Ann. Transvaal Mus. 2: 228. (Bм) \\
\hline strata & SCARDIA & I9I6, Exotic Microlepidoptera, I: 61 8. \\
\hline strophora & YPONOMEUTA & I909, Ann. South African Mus. 5: 358. (SA) \\
\hline syraea & LASIOCTENA* & I887, Trans. Ent. Soc. London, I887: 279. \\
\hline syraea & PROTOLECHIA & I904, Proc. Linnean Soc. N.S. Wales, 29: 359. \\
\hline yrana & HARMOLOGA & I883, Trans. Proc. New Zealand Inst. I5: 44. \\
\hline yranthes & DECADARCHIS & I930, Exotic Microlepidoptera, 4: 9. \\
\hline syrodes & MARASMARCHA & I92I, Ann. Transvaal Mus. 8: 50. (T) \\
\hline syrota & TITANOMIS* & I888, Trans. Proc. New Zealand Inst. 20: Io4. \\
\hline cha & SCYTHRIS & I9I8, Exotic Microlepidoptera, 2: I57. \\
\hline losa & MELASINA & $\begin{array}{l}\text { I920, Voyage de Ch. Alluand et R. Jeannel en Afrique } \\
\text { Oriental, II, Microlepidoptera, p. II3. (P) }\end{array}$ \\
\hline iens & BRACHMIA & I9I8, Exotic Microlepidoptera, 2: II4. (BM) \\
\hline ophaga & LOBESIA & I922, Exotic Microlepidoptera, 2: 534. (BM) \\
\hline urga & CARPOSINA & I9I2, Ann. South African Mus. Io: 55 . (SA) \\
\hline laragdaspis & NEMOTOIS & I924, Exotic Microlepidoptera, 3: 79. \\
\hline aragditis & EULIA & I9I2, Exotic Microlepidoptera, I: II. (BM) \\
\hline laragditis & SAPTHA & I905, Journ. Bombay Nat. Hist. Soc. I6: 6ro. (Bм) \\
\hline laragdopa & AMPHITHERA & I92I, Zool. Meded. 6: I92. (L) \\
\hline laragdophaea & EULIA & I932, Exotic Microlepidoptera, $4: 25 \mathrm{~S}$. (v) \\
\hline aragdophanes & S ARISTOPTILA* & I932, Exotic Microlepidoptera, 4: 219. \\
\hline aragdopis & TELPIIUSA & I926, Exotic Microlepidoptera, 3:275. (BM) \\
\hline enodes & EPICHORISTA & I9Io, Proc. Linncan Soc, N.S. Wales, 35: 256. \\
\hline
\end{tabular}


smileuta

smodicopa

sobria

socia

socialis

sociata

socors

socratica

solaris

solaris

solida

solidata

solita

sollennis

sollennis

sollers

sollicita

sollicitana

soloeca

soluta

solutrix

somphodes

somphota

sophista

sophistica

sophronia

sophronica

sophronistes

sophronistis

sophronopa

soporata

sordescens

sordidatana

sordidatus

sordidula

soreuta

soreutis

soritica

soritis

sorograpta

sortifera

sortilega

sortita

sosigona

sosisperma
ANTAEOTRICHA

AGRIOPHARA

MACROTONA*

PANCLINTIS*

HOMONA

BACTRA

CARPOSINA

ARGYROPLOCE

ARGYROPLOCE

PHILOBOTA

CACOECIA

EUCOSMA

PACHNISTIS

EUCOSMA

MICROCOLONA

PROCHOLA

HYPOPHRICTIS

STREPSICEROS

CRASPEDOTIS

SCYTHRIS

CHELARIA

CEROMITIA

TINEOLA

PYRODERCES

TELPHUSA

SCYTHRIS

OTONOMA

PTEROPHORUS

ONEBALA

[LARCOPHORA*] PHRICOGENES* CRYPSITHYRIS

LIPOPTYCHA

CAPUA

PTEROPHORUS

EPITHECTIS

IDIOPHANTIS

ENCHOCRATES

PSEUDOCRATES

ACROCERCOPS

CHELARIA

ANTAEOTRICHA

BRACHMIA

LEUCOPTERA

PROTOMACHA

COLEOPHORA
I915, Exotic Microlepidoptera, I: 397. (BM)

I9I5, Exotic Microlepidoptera, I: 409. (BM)

r904, Proc. Linnean Soc. N.S. Wales, 29: 407.

I929, Trans. Ent. Soc. London, 76: 5I2.

I912, Exotic Microlepidoptera, I: 3 .

1909, Journ. Bombay Nat. Hist. Soc. 19: 583. (BM)

1928, Exotic Microlepidoptera, 3: 403. (T)

I930, Trans. Ent. Soc. London, 78: 312. (P)

r9o9, Journ. Bombay Nat. Hist. Soc. 19: 605. (BM)

I913, Exotic Microlepidoptera, I: I26. (MA)

I908, Journ. Bombay Nat. Hist. Soc. 18:6I4. (BM)

I912, Journ. Bombay Nat. Hist. Soc. 21: 865. (BM)

I923, Exotic Microlepidoptera, 3: 45.

I913, Ann. Transvaal Mus. 3: 272. (T)

1897, Proc. Linnean Soc. N.S. Wales, 22: 377.

rgr7, Exotic Microlepidoptera, 2: 46 . (BM)

I9I7, Exotic Microlepidoptera, 2: 85.

I88I, Proc. Linnean Soc. N.S. Wales, 6: 687.

I904, Proc. Linnean Soc. N.S. Wales, 29: 326.

1916, Exotic Microlepidoptera, 2: 10.

I9I I, Ann. Transvaal Mus. 3:69. (BM)

I912, Ann. South African Mus. ro: 73. (SA)

I920, Voyage de Ch. Alluaud et R. Jeannel en Afrique

Oriental, II, Microlepidoptera, p. Ioo. (P)

I917, Exotic Microlepidoptera, 2: 36 .

1935, in Caradja and Meyrick, Materialien zu einer Micro-

lepidopteren Fauna der Chinesischen Provinzen Kiangsu,

Chekiang und Hunan, p. 67. (BM)

1935, Exotic Microlepidoptera, 4:555. (c)

I920, Exotic Microlepidoptera, 2: 32r. (BM)

1937, in Caradja and Meyrick, Deuts. Ent. Zeit. Iris, 51: I70.

I9I8, Exotic Microlepidoptera, 2: II2.

I93I, Exotic Microlepidoptera, 4: 72.

I9II, Journ. Bombay Nat. Hist. Soc. 21: II5.

I912, Ent. Mon. Mag. 48: 36. (Sub.)

1881, Proc. Linnean Soc. N.S. Wales, 6: 454.

I9I2, Ann. South African Mus. Io: 54. (SA)

1913, Ann. Transvaal Mus. 3: 285. (T)

r9o6, Journ. Bombay Nat. Hist. Soc. I7: 139. (BM)

I888, Proc. Linnean Soc. N.S. Wales, (Ser. 2) 2: 929.

I9I8, Exotic Microlepidoptera, 2: roo. (BM)

I915, Trans. Ent. Soc. London, I9I5: 224.

I931, Exotic Microlepidoptera, 4: 70. (BM)

I930, Exotic Microlepidoptera, 3: 557. (BM)

I9II, Journ. Bombay Nat. Hist. Soc. 20: 7I4. (BM)

I915, Exotic Microlepidoptera, x: 346.

1920, Ann. South African Mus. I7:286. (SA)

r936, Exotic Microlepidoptera, 4: 621. 


\begin{tabular}{|c|c|c|}
\hline tra & TINISSA & I932, Trans. Ent. Soc. London, 80: i 8. \\
\hline ctenis & MELASINA & I930, Exotic Microlepidoptera, 3: 552. (BM) \\
\hline spanista & IMMA & I930, Exotic Microlepidoptera, 4: 2. (BM) \\
\hline spanistis & SPILONOTA & I9I , Proc. Linnean Soc. N.S. Wales, 36: 232. \\
\hline aractis & EUCOSMA & I928, Exotic Microlepidoptera, $3: 440$. (BM) \\
\hline parganota & ANTAEOTRICHA & I9I5, Exotic Microlepidoptera, I: 389 . (вм) \\
\hline pargotis & HOMONA & I9ro, Proc. Linnean Soc. N.S. Wales, $35: 215$. \\
\hline arsa & NARYCIA & I921, Exotic Microlepidoptera, 2: 477. (BM) \\
\hline arsipalpis & APHANOSTOLA & I931, Exotic Microlepidoptera, 4: 57. (BM) \\
\hline ula & ELACHISTA & I92I, Ann. Transvaal Mus. 8: II4. (T) \\
\hline inodes & HECTACMA & I9I6, Exotic Microlepidoptera, I: 620. \\
\hline deta & TRACHYPEPLA & I883, New Zealand Journ. Sci. I: 522 . \\
\hline odes & EPIMACTIS & I9I4, Journ. Bombay Nat. Hist. Soc. 22: 779. (Bм) \\
\hline lodes & CEROMITIA & I920, Ann. South African Mus. I7: 3I2. (SA) \\
\hline oathias & PHARANGITIS* & I905, Journ. Bombay Nat. Hist. Soc. I6: 597. (Bм) \\
\hline ta & ACROLOPHUS & I9I9, Exotic Microlepidoptera, 2: 279. (BM) \\
\hline stis & OINOPHILA & I930, Trans. Ent. Soc. London, 78: 321. (P) \\
\hline 10 ta & CHELARIA & I9I3, Journ. Bombay Nat. Hist. Soc. 22: I65. (BM) \\
\hline sa & HELIOCAUSTA & I921, Exotic Microlepidoptera, 2: 387. (MA) \\
\hline lata & PHYLLOCNISTIS & I928, Exotic Microlepidoptera, 3: 405. \\
\hline osa & OPOGONA & I9I4, Exotic Microlepidoptera, I: 287. \\
\hline ata & PHILONOME & I920, Exotic Microlepidoptera, 2: 359. \\
\hline rix & CRYPSITHYRIS & I9I I, Journ. Bombay Nat. Hist. Soc. 2I: II5. \\
\hline ralis & SCHOENOTENES & I9I2, Exotic Microlepidoptera, I: I3. \\
\hline pectralis & TIPHA & I905, Journ. Bombay Nat. Hist. Soc. I6: 593. (B1 \\
\hline ta & IDIOPHANTIS & I9I I, Trans. Linnean Soc. London, I4:272. (BM) \\
\hline ella & EPIPHTHORA & I904, Proc. Linnean Soc. N.S. Wales, 29: 266. \\
\hline ocentra & DEPRESSARIA & I935, Exotic Microlepidoptera, 4: 593. (BM) \\
\hline rodes & BEDELLIA & I93I, Exotic Microlepidoptera, 4: I63. \\
\hline opa & PSELIASTIS & I897, Proc. Linnean Soc. N.S. Wales, $22: 407$. \\
\hline rophthalma & STENOMA & I932, Exotic Microlepidoptera, 4: 302. (v) \\
\hline ectropis & IMMA & I926, Sarawak Mus. Journ. 3: I63. \\
\hline ns & GLYPHIPTERYX & I922, Exotic Microlepidoptera, 2: 490. (Bм) \\
\hline laris & BATTARISTIS & I9I8, Exotic Microlepidoptera, 2: I42. (вм) \\
\hline latrix & CYDIA & I907, Journ. Bombay Nat. Hist. Soc. I8: I43. (B \\
\hline trix & MACHIMIA & I9I4, Exotic Microlepidoptera, I: I83. (Bм) \\
\hline fera & GELECHIA & I93I, Exotic Microlepidoptera, 4: 59. (BM) \\
\hline ligera & THIODIA & I9I2, Ent. Mon. Mag. 48: 35. (Sub.) \\
\hline & CRYPSITHYRIS & I908, Rec. Indian Mus. 2: 399. (K) \\
\hline eodes & ARGYROPLOCE & I93I, Exotic Microlepidoptera, 4: I3I. \\
\hline ns & CRYPTOLECHIA & I926, Sarawak Mus. Journ. 3: I59. \\
\hline atias & PTEROPHORUS & I908, Trans. Ent. Soc. London, I907: 499. \\
\hline natias & SYRMOLOGA & I9I9, Exotic Microlepidoptera, 2: 273. (BM) \\
\hline matica & CHOLOTIS & I9I5, Exotic Microlepidoptera, I: 328. \\
\hline atopis & ZEMIOCRITA & I933, Exotic Microlepidoptera, 4: 366. (BM) \\
\hline nidias & STENOMA & I932, Exotic Microlepidoptera, 4: 295. (v) \\
\hline nolitha & STENOMA & I9I5, Exotic Microlepidoptera, I: 432. (BM) \\
\hline nologa & BLASTOBASIS & I9I6, Exotic Microlepidoptera, I: 597. \\
\hline notoca & HELCANTHICA* & I932, Exotic Microlepidoptera, 4: 3I5. (v) \\
\hline s & ORYGOCERA & I927, Exotic Microlepidoptera, 3: 383 . (BM) \\
\hline
\end{tabular}




\begin{tabular}{|c|c|c|}
\hline dias & PHLOEOPOLA & I9I4, Exotic Microlepidoptera, I: I69. \\
\hline shae & TIMYRA & I9o8, Journ. Bombay Nat. Hist. Soc. I8: 450. (BM) \\
\hline phaerobola & GYMNOGRAMMA & I924, Exotic Microlepidoptera, 3: I20. \\
\hline phaerocopa & ARGYROPLOCE & $\begin{array}{l}\text { I93I, in Joannis, Ann. Soc. Ent. France, } 98 \text { (Supp.): } \\
\text { 7I9 [48I]. (P) }\end{array}$ \\
\hline phaerocosma & TERTHREUTIS* & I9I8, Exotic Microlepidoptera, 2: I70. (BM) \\
\hline osmana & a ANTITHESIA & I88I, Proc. Linnean Soc. N.S. Wales, 6: 642. \\
\hline delta & ACROCERCOPS & I935, Exotic Microlepidoptera, 4: 598. \\
\hline odoxa & ATTEVA & I9I8, Exotic Microlepidoptera, 2: I89. \\
\hline haerograpta & PROMALACTIS & I937, Exotic Microlepidoptera, 5: 82. (T) \\
\hline phora & PETALANTHES & I883, Proc. Linnean Soc. N.S. Wales, 8: 335. \\
\hline sticha & CERATOPHYSETIS* & I887, Proc. Linnean Soc. N.S. Wales, (Ser. 2) I: I045. \\
\hline toma & OPOGONA & I9I I, Journ. Bombay Nat. Hist. Soc. 2I: II I. \\
\hline rocha & ATTEVA & I936, Exotic Microlepidoptera, 5: 4I. (v) \\
\hline odes & EUCOSMA & I934, Ann. Mag. Nat. Hist. I4: 404. (BM) \\
\hline pa & TORTRIX & I9o9, Trans. Ent. Soc. London, I 909: I5. (BM) \\
\hline opis & BORKHAUSENIA & I902, Trans. R. Soc. S. Australia, 26: I68. \\
\hline $\mathrm{ca}$ & ASTHENOPTYCHA & I9io, Proc. Linnean Soc. N.S. Wales, 35: I80. \\
\hline ristis & TINEA & I9II, Journ. Bombay Nat. Hist. Soc. 2I: I2I. \\
\hline pa & HARMATITIS* & I9Io, Journ. Bombay Nat. Hist. Soc. $20: 460$. \\
\hline hila & EPITHECTIS & I936, Exotic Microlepidoptera, 4: 624. (BM) \\
\hline $\mathrm{pa}$ & CROCANTHES & $\begin{array}{l}\text { I933, Bull. Mus. R. Hist. Nat. Belgique, vol. 9, fasc. } 34 \text {, } \\
\text { p. I. }\end{array}$ \\
\hline ma & DECADARCHIS & I926, Trans. Ent. Soc. London, 74: 275. \\
\hline ista & CALIITHRINCA & I927, Insects of Samoa, 3, Lepidoptera, fasc. 2, p. I05. (вВ) \\
\hline lonistis & ODITES & I9o8, Journ. Bombay Nat. Hist. Soc. I8: 634. (BM) \\
\hline onita & STATHMOPODA & I92I, Exotic Microlepidoptera, 2: 46I. \\
\hline & CACOECIA & I9o9, Trans. New Zealand Inst. 4I: II. \\
\hline ias & SARIDOSCELIS* & I894, Trans. Ent. Soc. London, I894: 28. \\
\hline as & TIMYRA & I 905, Journ. Bombay Nat. Hist. Soc. I6: 596. \\
\hline iias & ODITES & I9I4, Journ. Bombay Nat. Hist. Soc. 22: 78I. (BM) \\
\hline es & PTEROPHORUS & I9I3, Exotic Microlepidoptera, I: I I2. \\
\hline is & EPICEPHALA & I93I, Exotic Microlepidoptera, 4: I68. \\
\hline pathra & PERONEA & I9I7, Trans. Ent. Soc. London, I9I7: I3. (BM) \\
\hline clina & LABDIA & I922, Exotic Microlepidoptera, 2: 570. \\
\hline copa & ASYMPHORODES & I929, Trans, Ent. Soc. London, 76: 499. \\
\hline osma & TINEA & I9I9, Trans. New Zealand Inst. 5I: 353. \\
\hline rossa & GRACILARIA & I934, Exotic Microlepidoptera, 4: 474. \\
\hline delta & BATTARISTIS & I922, Trans. Ent. Soc. London, I922: 75. (BM) \\
\hline oxa & STOMOPTERYX & I93I, Exotic Microlepidoptera, 4:63. (BM) \\
\hline rapta & LEUCOPTERA & I9I I, Journ. Bombay Nat. Hist. Soc. 2I: Io8. \\
\hline orpha & ARISTOTELIA & $\begin{array}{l}\text { I931, in Joannis, Ann. Soc. Ent. France, } 98 \text { (Supp.): } \\
722 \text { [484]. (P) }\end{array}$ \\
\hline ophora & DEUTEROPTILA* & I904, Proc. Linnean Soc. N.S. Wales, 29:4I9. \\
\hline & ACOMPSIA & I921, Ann. Transvaal Mus. 8: 79. (T) \\
\hline plecta & FILINOTA & I921, Exotic Microlepidoptera, 2: 390. (BM) \\
\hline oschista & DECADARCHIS & I93I, Exotic Microlepidoptera, 4: I66. \\
\hline osema & PYRODERCES & I897, Proc. Linnean Soc. N.S. Wales, 22: 350. \\
\hline pila & PARAXENISTIS & I9I9, Exotic Microlepidoptera, 2: 2 \\
\hline enota & HOFMANNIA & 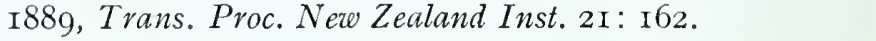 \\
\hline
\end{tabular}


sphenotoma sphenotona sphragidias sphragidopis sphragidota sphyrocopa sphyrota spicata spicea spicifera spiculata spiculifera spiculifera spiculifera

spiladias spiladorma spilanthes spilobathra spilocausta spilochorda spilocoma spilocrossa spilocryptis spilodes spilodesma spilodesma spilodoma spiloglypta spilographa spilonoma spilonoma spilophanes spilophora spilopis spilothorax spilothyris spilotoma spilozeucta spilozeucta spilozona spinicola spinifera spinigera spinitarsis spinosa spintherias spintheritis spintheritis spintheropa
AEOLARCHIS DECADARCHIS PHALONIA STENOMA AEOLOSCELIS TRICHOTAPHE MALLOBATHRA

ANARSIA

EUCOSMA

ORNEODES

COSMOPTERYX

ELACHISTA

EUCOSMA

MNESTERIA

[TEUCRODOXA*]

LECITHOCERA

ACROCLITA

PERITORNEUTA

THYROCOPA

ACROCLITA

AUTOSTICHA

TINEA

DOLIOTECHNA

AMORBIA

OPOSTEGA

CEROMITIA

ORNEODES

PHOTODOTIS

HYPOPHRICTIS

TORTRIX

CAPUA

SCHOENOTENES

MELASINA

EUCOSMA

BRACHMIA

STATHMOPODA

ALLOTALANTA

CACOECIA

CLADOPHANTIS

TAENIARCHIS*

STATHMOPODA

ANCYLIS

ACROLOPHUS

ANOMOXENA*

LYONETIA

MARASMARCHA

EUTORNA

BRENTHIA

PROMALACTIS

STAGMATOPHORA
I935, Exotic Microlepidoptera, 4:568.

I927, Insects of Samoa, 3, Lepidoptera, fasc. 2, p. III.

I932, Exotic Microlepidoptera, 4:265. (v)

I9I5, Exotic Microlepidoptera, I: 43I. (вм)

1897, Proc. Linnean Soc. N.S. Wales, 22: 329.

I9I8, Exotic Microlepidoptera, 2: I5o. (BM)

I9I7, Exotic Microlepidoptera, 2: 9I.

I9I8, Ann. Transvaal Mus. 6:2I. (T)

I9I2, Journ. Bombay Nat. Hist. Soc. 2I:867. (BM)

I9II, Ann. Transvaal Mus. 2: 22I. (T)

I9o9, Journ. Bombay Nat. Hist. Soc. I9: 420.

I922, Exotic Microlepidoptera, 2: 509.

I9I3, Ann. Transwaal Mus. 3:27I. (T)

I9I8, Exotic Microlepidoptera, 2: I52. (BM)

192I, Ann. Transvaal Mus. 8: 88. (T)

I932, Exotic Microlepidoptera, 4: 22I. (BM)

I934, Exotic Microlepidoptera, 4: 475. (BM)

I9I5, Exotic Microlepidoptera, I: 372. (вм)

I934, Exotic Microlepidoptera, 4: 484. (вм)

I9I6, Exotic Microlepidoptera, I: 588. (Bм)

I920, Ann. South African Mus. I7: 302. (SA)

I920, Exotic Microlepidoptera, 2: 380. (BM)

1932, Exotic Microlepidoptera, 4: 262. (v)

I9I5, Exotic Microlepidoptera, I: 35I.

I908, Proc. Zool. Soc. London, p. 754.

I908, Trans. Ent. Soc. London, I907: 508.

I9I8, Ann. Transvaal Mus. 6: I5. (T)

I930, Exotic Microlepidoptera, 3: 548 .

I937, Exotic Microlepidoptera, 5: I26. (BM)

I932, Trans. Ent. Soc. London, 80: I IO. (BM)

I938, Trans. R. Ent. Soc. London, 87: 507. (вм)

I92I, Ann. Transvaal Mus. 8: 137. (T)

I9I2, Ent. Mon. Mag. 48: 35. (Sub.)

I927, Exotic Microlepidoptera, 3:356. (T)

I927, Insects of Samoa, 3, Lepidoptera, fasc. 2, p. Ioo.

I922, Exotic Microlepidoptera, 2: 543. (BM)

I921, Zool. Meded. 6: I48. (L)

1927, Exotic Microlepidoptera, 3: 366. (T)

I93I, Exotic Microlepidoptera, 4: I54.

I9I3, Exotic Microlepidoptera, I: 90.

I927, Boll. Soc. Ent. Ital. 59: Io7.

I9I3, Trans. Ent. Soc. London, I9I3: I94. (BM)

I9I7, Trans. Ent. Soc. London, I9I7: 29. (BM)

1922, Exotic Microlepidoptera, 2: 557 . (BM)

I925, in Caradja, Bull. Sect. sci. Acad. roum. 3: 378.

I906, Trans. R. Soc. S. Australia, 30: 44.

I9Io, Trans. Ent. Soc. London, I9Io: 469.

I908, Journ. Bombay Nat. Hist. Soc. IS: SIо. (вм)

I934, Pacific Ent. Surv. Publ. 7, art. 28, p. 348. (BB) 


\begin{tabular}{|c|c|}
\hline spintheropis & STROBISIA \\
\hline spinulosa & TORTRIX \\
\hline spirocosma & LABDIA \\
\hline spirodoxa & GELECHIA \\
\hline spissa & CRYPSITHYRIS \\
\hline spissata & SCYTHRIS \\
\hline spodiaea & MERMERISTIS* \\
\hline podias & EUCOSMA \\
\hline spodina & TINEA \\
\hline spodinopis & STENOMA \\
\hline spodochtha & PYRODERCES \\
\hline spodocrossa & HYPONOMEUTA \\
\hline spodomicta & DECADARCHIS \\
\hline spodostrota & PARATHETA \\
\hline spodota & TORTRIX \\
\hline spoliatrix & ODITES \\
\hline sponditis & ARGYROPLOCE \\
\hline sponsalis & PROMALACTIS \\
\hline sporadarcha & HETEROZYGA \\
\hline sporadias & TORTRIX \\
\hline sporadica & SCYTHRIS \\
\hline sporadopa & NEPTICULA \\
\hline sporaea & CEROMITIA \\
\hline sporarcha & LYTROPHILA \\
\hline sporeuta & MYRRHINITIS* \\
\hline orimaea & LYGRONOMA* \\
\hline sporocentra & LYTROPHILA \\
\hline rochlora & LECITHOCERA \\
\hline rodectis & HIEROCROBYLA \\
\hline rodelta & TORTYRA \\
\hline na & NEMOTOIS \\
\hline mma & NOTHRIS \\
\hline
\end{tabular}

[EMPALACTIS*]

sporograpta sporomantis sporomochla sporosema sporozona spreta spumans spumescens spumifera spumosa spumosa

spyrathodes squalens squalida squalidella
I922, Trans. Ent. Soc. London, I922: IOI. (BM) I924, Exotic Microlepidoptera, 3: II4. (T) I92I, Exotic Microlepidoptera, 2: 4II. (BM) I93I, Journ. Linnean Soc. London, 37: 279. (BM) IgI8, Ann. Transvaal Mus. 6: 43. (T) I9I6, Exotic Microlepidoptera, 2: I2. I9I5, Exotic Microlepidoptera, I: 298. I9I2, Ent. Mon. Mag. 48: 35. (Sub.) 1893, Proc. Linnean Soc. N.S. Wales, $7: 543$. I93I, Exotic Microlepidoptera, 4: 35. (v) I905, Journ. Bombay Nat. Hist. Soc. I6: 607. I935, Exotic Microlepidoptera, 4: 602. (BM) I9I5, Exotic Microlepidoptera, I: 367. Igo2, Trans. R. Soc. S. Australia, 26: I73. I9Io, Proc. Linnean Soc. N.S. Wales, 35:235. (MA) I9I6, Exotic Microlepidoptera, I: 509. (BM) I9I8, Ann. Transwaal Mus. 6:50. (T) I920, Exotic Microlepidoptera, 2: 365. (BM) I92I, Zool. Meded. 6: I7I. (L) I920, Ann. South African Mus. I7: 275. (SA) I897, Proc. Linnean Soc. N.S. Wales, $22: 425$. IgI I, Journ. Bombay Nat. Hist. Soc. 21 : 108. Igo8, Proc. Zool. Soc. London, p. 756. I92I, Ann. Transvaal Mus. 8: I32. (T) I9I3, Ann. Transvaal Mus. 3: 32I. (T) I9I3, Exotic Microlepidoptera, I: Ioo. (BM) I923, Bull. Mus. Nat. Hist. Nat. Paris, 29: 565. I929, Exotic Microlepidoptera, 3: 523. (BM) I9I5, Exotic Microlepidoptera, I: 353 . I922, Exotic Microlepidoptera, 2: 485. (BM) I928, Exotic Microlepidoptera, 3: 464.

I921, Exotic Microlepidoptera, 2: 433. I932, Exotic Microlepidoptera, 4: 268. I93I, Exotic Microlepidoptera, 4: I84. (BM) I929, Exotic Microlepidoptera, 3: 49I. (BM) I922, Exotic Microlepidoptera, 2: 53I. (BM) I9I4, Trans. Ent. Soc. London, I9I4: 259. (BM) I920, Exotic Microlepidoptera, 2: 382 . I930, Exotic Microlepidoptera, 3: 605. (BM) I925, Exotic Microlepidoptera, 3: I3I. (BM) I9I8, Exotic Microlepidoptera, 2: I56. I9I3, Trans. Ent. Soc. London, I9I3: I73. (Bм) I920, Voyage de Ch. Alluaud et $R$. Jeannel en Afrique

Oriental, II, Microlepidoptera, p. III. (P) I922, Exotic Microlepidoptera, 2: 552. (BM) IgI4, Trans. Ent. Soc. London, IgI4: 282. (BM) 1926, Exotic Microlepidoptera, 3:272. (BM)

I884, Proc. Linnean Soc. N.S. Wales, 8: 496. 


\begin{tabular}{|c|c|}
\hline squamifera & LECITHOCERA \\
\hline stabilita & LIMNOECIA \\
\hline stabularia & MELASINA \\
\hline tabulata & HEMIMENE \\
\hline tachyophora & TIMYRA \\
\hline tactogramma & AXIAGASTA* \\
\hline stactopis & BRACHMIA \\
\hline stadaea & CRYPTOLECHIA \\
\hline stadias & PTEROPHORUS \\
\hline tadiaula & THUDACA \\
\hline tadiota & LINOSTICHA \\
\hline agmatias & CALLICERASTIS* \\
\hline tagmatopis & AUTOSTICHA \\
\hline tagnans & ASCALENIA \\
\hline tagnicola & MESOPHERNA \\
\hline tagnicolor & PTILOGENES \\
\hline tagnigera & SCAEOSOPHA \\
\hline nosa & SCYTHRIS \\
\hline actis & PALTODORA \\
\hline lactitis & EULIA \\
\hline alagmitis & ACROCERCOPS \\
\hline annifera & EUCLEODORA \\
\hline hiditis & LASPEYRESIA \\
\hline hylina & URODUS \\
\hline hylitis & MACHIMIA \\
\hline archa & GELECHIA \\
\hline stica & PLEUROTA \\
\hline asichlora & GLYPHIPTERYX \\
\hline asigastra & COMPSOLECHIA \\
\hline simodes & CHELARIA \\
\hline nopa & DICHOMERIS \\
\hline des & IPHIERGA* \\
\hline & TIMYRA \\
\hline rias & CROBYLOPHORA \\
\hline herot & COLEOPHORA \\
\hline hmodes & CEROMITIA \\
\hline thmota & OPOGONA \\
\hline & BUCCULATRIX \\
\hline ationaria & CARPOSINA \\
\hline & ATELOSTICHA \\
\hline ra & CHOLOTIS \\
\hline romacha & ACARTOPHILA \\
\hline ropa & EPICEPHALA \\
\hline rophora & LECITHOCERA \\
\hline & ANTAEOTRICHA \\
\hline & IMMA \\
\hline & TELPHUSA \\
\hline
\end{tabular}

1929, Exotic Microlepidoptera, 3: 525.

I9I5, Exotic Microlepidoptera, I: 3I9.

I908, Proc. Zool. Soc. London, p. 745.

I916, Exotic Microlepidoptera, 2: 25. (вм)

Igo8, Journ. Bombay Nat. Hist. Soc. I8: 445. (вм)

I930, Ann. Naturhist. Mus. Wien, 44: 266. pl. I, fig. 36.

(v)

1931, Exotic Microlepidoptera, 4:84. (вм)

I934, in Caradja and Meyrick, Deuts. Ent. Zeit. Iris, 48: 39.

I908, Trans. Ent. Soc. London, I907: 498.

I893, Proc. Linnean Soc. N.S. Wales, 7: 578 .

I889, Proc. Linnean Soc. N.S. Wales, (Ser. 2) 3: I580.

I9I6, Exotic Microlepidoptera, I: 600.

1923, Exotic Microlepidoptera, 3: 50. (BM)

I921, Ann. Transvaal Mus. 8: 96. (T)

I922, Exotic Microlepidoptera, 2: 596. (MA)

I926, Exotic Microlepidoptera, 3: 229. (BM)

1932, Exotic Microlepidoptera, 4: 277. (BM)

I9I3, Ann. Transvaal Mus. 3: 3I3. (T)

I904, Proc. Linnean Soc. N.S. Wales, 29: 27 I.

I93 I, Ann. Mus. Nac. Hist. Nat. Buenos Aires, 36: 382.

(BM)

I9I5, Trans. Ent. Soc. London, I9I5: 224.

I9I4, Exotic Microlepidoptera, I: 259. (BM)

1930, Exotic Microlepidoptera, 3: 604. (BM)

1932, Exotic Microlepidoptera, 4: 284.

I9I6, Exotic Microlepidoptera, I: 550. (вM)

I9I3, Ann. Transvaal Mus. 3: 290. (T)

I884, Proc. Linnean Soc. N.S. Wales, 9: 756.

I93I, Exotic Microlepidoptera, 4: I84. (BM)

I922, Trans. Ent. Soc. London, I922: 97. (вм)

I931, Exotic Microlepidoptera, 4: 70. (BM)

I937, Exotic Microlepidoptera, 5: 94. (T)

I893, Proc. Linnean Soc. N.S. Wales, 7: 5 I9.

I908, Journ. Bombay Nat. Hist. Soc. I8: 447. (BM)

1905, Journ. Bombay Nat. Hist. Soc. I6: 6r3.

I9I7, Exotic Microlepidoptera, 2: 72.

I908, Proc. Zool. Soc. London, p. 754.

I9II, Journ. Bombay Nat. Hist. Soc. 2I: IIo.

I92I, Zool. Meded. 6: I92. (L)

I928, Exotic Microlepidoptera, 3: 402. (BM)

1920, Exotic Microlepidoptera, 2: 379. (вM)

I9I5, Exotic Microlepidoptera, I: 327.

1932, Exotic Microlepidoptera, 4:283. (v)

I908, Journ. Bombay Nat. Hist. Soc. I8: SI3.

I93I, Exotic Microlepidcptera, 4: 8I. (вм)

I9I6, Exotic Microlepidoptera, I: 493. (BM)

I9I4, Exotic Microlepidoptera, I: 283. (BM)

I935, Exotic Microlepidoptera, 4:585. (BM) 


stelidias
stelitis
stella
stellans
stellaris
stellifera
stellulata
stelocosma
steloglypta
stelophanes
stelosema
stelota
stelucha
stemmatias
stemonias
stemonodes
stenacma
stenaspis
stenobathra
stenochlora
stenochorda
stenocraspeda
stenodoxa
stenomorpha
stenoptera
stenorma
stenota
stenothyris
stephanitis

stephanocoma

stephanodes stephanopa stephanopis stephanota stereocrossa stereodes stereodes stereodesma stereodoxa stereodyta stereogramma stereograpta stereoma stereomorpha stereopa stereopis stereosema stereota
PERIORYCTA MELASINA CORIDOMORPHA* ARGYROPLOCE XYSMATODOMA OCTASPHALES ANTIOCHTHA LASPEY RESIA PTILOGENES LECITHOCERA LASPEYRESIA HERMOGENES GLYPHIPTERYX MICTOPSICHIA THAUMATOLITA PARECTOPA GELECHIA POLYCHROSIS ANTAEOTRICHA NEMOPHORA CACOECIA LOZOSTOMA HYPONOMEUTA GLYPHIDOCERA COESYRA BRENTHIA EULECHRIA AEGERIA ATTERIA [MEGALODORIS*] ASMENISTIS

STENOMA TRACHY DORA ORNEODES LITHOCOLLETIS SIMAETHIS CNEPHASIA THEMELIOTIS* MACHIMIA ASCALENIA LOZOSTOMA BATTARISTIS PHYLLOCNISTIS EUCOSMA CAPUA CRANAODES* EUXANTHIS COESYRA ENDOPHTHORA
I922, Exotic Microlepidoptera, 2: 5II. I9o8, Proc. Zool. Soc. London, p. 743. I9I4, Trans. Proc. New Zealand Inst. 46: III. I922, Exotic Microlepidoptera, 2: 525. (BM) 1893, Proc. Linnean Soc. N.S. Wales, 7: 493. I9I4, Exotic Microlepidoptera, I: 225. I906, Journ. Bombay Nat. Hist. Soc. I7: I49. (BM) 1925, Exotic Microlepidoptera, 3: I45. (T) I93I, Exotic Microlepidoptera, 4: 44. (BM) I938, Trans. R. Ent. Soc. London, 87: 5I4. (BM) I93I, Exotic Microlepidobtera, 4: I43. (BM) 1908, Journ. Bombay Nat. Hist. Soc. I8: 628. (Bм) I909, Ann. South African Mus. 5: 375. (SA) I921, Zool. Meded. 6: I78. (L) I920, Exotic Microlepidoptera, 2: 374. (BM) I9I 5, Trans. Ent. Soc. London, I9I 5: 234. I935, Exotic Microlepidoptera, 4:585. (BM) I921, Ann. Transuaal Mus. 8: 55. (T) I932, Exotic Microlepidoptera, 4:289. (US) I9r2, in Wytsman, Genera Insectorum, fasc. I33, p. 3. I928, Exotic Microlepidoptera, 3: 456. (BM) 1897, Proc. Linnean Soc. N.S. Wales, 22: 4I9. I93I, Exotic Microlepidoptera, 4:87. (BM) 1923, Exotic Microlepidoptera, 3: 49. (BM) I884, Proc. Linnean Soc. N.S. Wales, 9: 780 . I9I5, Trans. Ent. Soc. London, I9I5: 2I9. (BM) I889, Proc. Linnean Soc. N.S. Wales, (Ser. 2) 3: 1567. I933, Exotic Microlepidoptera, 4: 4I6.

I9Io, Trans. Ent. Soc. London, I9Io: 433. (BM) I938, Institut des Parcs Nationaux du Congo Belge, fasc. I4, p. I3. I93I, Exotic Microlepidoptera, 4: 42. (BM) I897, Proc. Linnean Soc. N.S. Wales, 22: 399. I921, Exotic Microlepidoptera, 2: 407. I907, Proc. Limnean Soc. N.S. Wales, 32: $5 \mathrm{I}$. I92I, Ann. Transvaal Mus. 8: Iro. (T) I9Io, Proc. Linnean Soc. N.S. Wales, 35: 277. I9I0, Trans. Ent. Soc. London, I9Io: 476. I9I6, Exotic Microlepidoptera, I: 550. (BM) I925, Bull. Soc. R. Ent. Egypte, 9:212. 1897, Proc. Limnean Soc. N.S. Wales, 22: 4 I7. I9I4, Trans. Ent. Soc. London, I9I4: 249. (BM) I934, Exotic Microlepidoptera, 4: 469 . I9I2, Exotic Microlepidoptera, I: 33. (BM) I93I, in Caradja, Bull. Sect. sci. Acad. roum. I4: 62. I9I9, Exotic Microlepidoptera, 2: 239. (BM) I93I, Exotic Microlepidoptera, 4: I58. (v) I889, Proc. Linnean Soc. N.S. Wales, (Ser. 2) 3: I655. I9I4, Trans. Proc. New Zealand Inst. 46: II4. 
stericta

sterictis

sterilis

steriphota

sternitis

steropastis

steropodes

steropucha

sterrhomitra

stesichora

sthenarota

stibaropa

stibarodes

stibarodes

stibogramma

stibomorpha

stichocentra

stichoceros

stichograpta

stichospora

stictobathra

stictocosma

stictocrossa

stictoneura

stictonoma

stigmatias

stigmatias

stigmatica

stilata

stillata

stilpnota

stimulans

stimulata

stimulata

stimulatrix

stimuligera

stiphropa

stipularis

stipulata

stiriella

stirodes

stirpicola

stirpicola

stochastis

stoica

stolarcha

stolida
POLYCHROSIS

BRACHMIA

BATRACHEDRA

EULECHRIA

PLASMATICA *

SAGEPHORA

PACHYRHABDA *

PROTOSYNAEMA

STENOMA

PARADORIS

ANARSIA

ARGYROPLOCE

MALACYNTIS*

MELASINA

LABDIA

RECURVARIA

PLUTELLA

SPILONOTA

CRONODOXA

ELACHISTA

LASPEYRESIA

CHELARIA

PANDURISTA*

TORTRIX

GONIORRHOSTIS*

PILOSTIBES

TORTRIX

PHARMACIS

GLYPHIPTERYX

COMPSOLECHIA

ARGYRESTHIA

BRENTHIA

NEPTICULA

STATHMOPODA

TINEA

COLEOPHORA

DRIMYLASTIS

TORTRIX

COESYRA

OPOSTEGA

PHTHORIMAEA

LASPEYRESIA

LASPEYRESIA

CRYPTOPHAGA

IDIOPHANTIS

ISOCRITA*

STENOMA
I9I2, Ann. Transvaal Mus. 3:63. (T)

I9o8, Proc. Zool. Soc. London, p. 727.

1897, Proc. Linnean Soc. N.S. Wales, 22: 304.

I9I4, Exotic Microlepidoptera, I: I63. (MA)

I9I4, Exotic Microlepidoptera, I: 270. (BM)

I89i, Trans. Proc. New Zealand Inst. 23: Ioo.

1897, Proc. Linnean Soc. N.S. Wales, 22: 312.

I886, Trans. Proc. New Zealand Inst. I8: I74.

1925, Exotic Microlepidoptera, 3: I78. (BM)

I9I I, Journ. Bombay Nat. Hist. Soc. 20: 735.

$(\mathrm{BM})$

I926, Sarawak Mus. Journ. 3: I53.

I933, Exotic Microlepidoptera, 4: 420. (BM)

I9o8, Proc. Zool. Soc. London, p. 738.

I9o9, Ann. South African Mus. 5: 378. (SA)

1924, Exotic Microlepidoptera, 3: 90. (BM)

I929, Exotic Microlepidoptera, 3: 484. (BM)

I932, Trans. Ent. Soc. London, 80: II8.

I938, Trans. R. Ent. Soc. London, 87: 5IO. (BM)

I936, Exotic Microlepidoptera, 5: 54.

I932, Exotic Microlepidoptera, 4:217.

I9I6, Exotic Microlepidoptera, 2:22. (BM)

I920, Exotic Microlepidoptera, 2: 303. (BM)

I9I8, Exotic Microlepidoptera, 2: I67.

I930, Ann. Naturhist. Mus. Wien, 44:224. (v)

I93I, in Joannis, Ann. Soc. Ent. France, 98 (Supp.):

73I [493]. (P)

I89o, Trans. R. Soc. S. Australia, I3: 27. (A)

I9Io, Proc. Linnean Soc. N.S. Wales, 35: 237. (MA)

I909, Ann. South African Mus. 5: 369. (SA)

I9I2, Exotic Microlepidoptera, I : 57. (BM)

I922, Trans. Ent. Soc. London, I922: 93. (BM)

I9I3, Ann. Transvaal Mus. 3: 318. (T)

I920, Exotic Microlepidoptera, 2:336. (BM)

I9I3, Ann. Transvaal Mus. 3:326. (T)

I9I3, Exotic Microlepidoptera, I: 84 .

I93I, Ann. Mus. Nac. Hist. Nat. Buenos Aires, 36: 4 I3.

(BM)

1936, Exotic Microlepidoptera, 4: 622.

1924, Exotic Microlepidoptera, 3: 70.

I9Io, Proc. Linnean Soc. N.S. Wales, 35:226. (MA)

I9I8, Exotic Microlepidoptera, I: II9.

I88I, Proc. Linnean Soc. N.S. Wales, 5: I75.

I93I, Ann. Mus. Nac. Hist. Nat. Buenos Aires, 36: 385 .

(BM)

I926, Mem. Dept. Agric. India, 9: 259.

I927, Exotic Microlepidoptera, 3: 342. (BM)

I89o, Trans. R. Soc. S. Australia, I3: 30.

I907, Journ. Bombay Nat. Hist. Soc. I8: I49. (13M)

I909, Ann. South African Mus. 5: 372. (SA)

I9II, Ann. Transvaal Mus. 3: 75. (BM) 


\section{stolidota stomatocosma stomobapta stomota}

storestis strabo strabonia straminea

straminicornis strangalistis strangalota strangulata strategica

stratifera stratifica stratigera streblopa streblopis strepens strepsaula strepsibathra strepsicentra strepsicrena strepsidesma strepsidoma strepsigramma strepsineura strepsiploca strepsiptila strepsizona streptatma streptosema stricta strictiformis strictula strictulata strigifera strigosa strigulata strigulata stringens striolata stromatias strombodes strophaea strophala strophalodes
ACANTHEDRA*

STENOMA

LECITHOCERA CRYPTOLECHIA [MELEONOMA*] BRACHMIA MACROCIRCA* PTILOGENES CHILOIDES [NOTERAULA*] ONEBALA BRACHMIA PYRODERCES NESOXENA* PERCNARCHA

PROTOLECHIA MELASINA DICHOMERIS ARGYROPLOCE ISCHNOPHENAX* ELACHISTA TYRIOGRAPTIS* ARGYROPLOCE SYNCROTAULA* LECITHOCERA SIMAETHIS NARYCIA BRACHMIA

TINEA EPICEPHALA FRISILIA IMMA CHOREUTIS ULOCHORA* ACROCERCOPS PLATYPTILIA METZNERIA ORTHOSARIS* ASAPHARCHA* BRACHMIA DRACHMOBOLA LEPIDOSCIA ANTAEOTRICHA STENOMA DICHOMERIS EPIMACTIS ZELIERIA ACROCERCOPS STENOMA
I9I7, Exotic Microlepidoptera, 2: 66. (BM) I932, Exotic Microlepidoptera, 4: 304. (Us) I929, Exotic Microlepidoptera, 3: 524. (BM)

I9I0, Rec. Indian Mus. 5: 224. (K)

I9II, Journ. Bombay Nat. Hist. Soc. 20: 7II. (BM)

I931, Mitteil. Münch. Ent. Gesells. 21 : 38. 1930, Ann. Naturhist. Mus. Wien, 44:256.

I884, Trans. Proc. New Zealand Inst. I7: I42. I9Io, Journ. Bombay Nat. Hist. Soc. 20: 453. (BM) I9I I, Journ. Bombay Nat. Hist. Soc. 20: 722. (BM) I922, Exotic Microlepidoptera, 2: 572.

I929, Trans. Ent. Soc. London, 76: 507.

I930, Ann. Naturhist. Mus. Wien, 44: 26o, pl. I, fig. 38. (v)

I904, Proc. Linnean Soc. N.S. Wales, 29:366. I907, Journ. Bombay Nat. Hist. Soc. I8: I.59. I922, Trans. Ent. Soc. London, I922: III. (BM) I936, Exotic Microlepidoptera, 4: 6I4. (BM) I93I, Exotic Microlepidoptera, 4: I26. (BM) I922, Exotic Microlepidoptera, 2: 508. I934, Exotic Microlepidoptera, 4: 476. (v) I928, Exotic Microlepidoptera, 3: 446. I937, Exotic Microlepidoptera, 5: I43. I93I, Exotic Microlepidoptera, 4: 8I. (BM) I9I2, Exotic Microlepidoptera, I : 45. (BM) I920, Exotic Microlepidoptera, 2: 356. 1937, Exotic Microlepidoptera, 5: 97. (T) I926, Sarawak Mus. Journ. 3: I66. I9I8, Exotic Microlepidoptera, 2: I73. I9Io, Journ. Bombay Nat. Hist. Soc. 20: 436. (BM) I906, Trans. Ent. Soc. London, I906: 197. (BM) I938, Trans. $R$. Ent. Soc. London, 87: 523. (BM) I920, Exotic Microlepidoptera, 2: 3I9. (BM) I9o8, Journ. Bombay Nat. Hist. Soc. I8: 8I5. I932, Exotic Microlepidoptera, 4: 25I. I937, Exotic Microlepidoptera, 5: 90. (T) I9I4, Joum. Bombay Nat. Hist. Soc. 23: I26. (BM) I920, Ann. South African Mus. I7: 292. (SA) I9Io, Trans. Ent. Soc. London, I9Io: 450. (BM) I9Io, Proc. Linnean Soc. N.S. Wales, 35:285. I893, Proc. Linnean Soc. N.S. Wales, 7: 5I0. 1925, Exotic Microlepidoptera, 3: I64. (BM) I932, Exotic Microlepidoptera, 4: 302. (v) I9I8, Ann. Transvaal Mus. 6:23. (T) I9I4, Journ. Bombay Nat. Hist. Soc. 22: 779. (BM) I9I4, Journ. Bombay Nat. Hist. Soc. 23: I24. (BM) I9o8, Journ. Bombay Nat. Hist. Soc. I 8: 824. I9I5, Exotic Microlepidoptera, I : 454. 
strophalora

strophiacma

strophiaula

strophicodes

strophiella

strophiota

strophodina

strophopa

strophota

studiosa

stupefacta

stygeropa

stygia

stygiaula

stygnopa

\begin{tabular}{ll} 
stylograpta & ZELLERIA \\
stylonota & STENOMA \\
stylophora & BRENTHIA \\
stylota & ANARSIA \\
styphelana & PALAEOTOMA* \\
styracista & EULECHRIA \\
styracodes & PAROCHMASTIS \\
styracopa & STRYPHNODES* \\
suasoria & PHTHORIMAEA \\
suavis & BAEONOMA \\
subacida & SPARGANOTHIS \\
subacta & MELASINA \\
subacuta & PSEUDODOXIA \\
subaenea & NARYCIA \\
subagrestis & STASIXENA* \\
subalbata & BRACHYPSALTIS* \\
subcaerulea & PHTHORIMAEA \\
subcarinata & SAGEPHORA \\
subcarnea & PYRODERCES \\
subcretosa & ALUCITA \\
subcrocea & ASCALENIA \\
subcroceata & SCHOENOTENES \\
subcuprea & TINEA \\
subdecora & EUCOSMA \\
subdentata & DICHOMERIS \\
subdulcis & STENOMA \\
subflavescens & ADAINA \\
subfulvescens & ANARSIA \\
subfurcata & EXODITIS* \\
subfusca & ACROLOPHUS \\
subita & STENOMA \\
sublevata & METACHANDA \\
sublimicola & EPERMENIA \\
& \\
\hline
\end{tabular}

I9I2, Exotic Microlepidoptera, I: 5I. (BM)

1927, Insects of Samoa, 3, Lepidoptera, fasc. 2, p. 80. (BM)

I935, Exotic Microlepidoptera, 4:598.

I9I7, Exotic Microlepidoptera, 2: 5I.

I884, Proc. Linnean Soc. N.S. Wales, 8: 5 I3.

I9I I, Journ. Bombay Nat. Hist. Soc. 2I: II 8.

1932, Exotic Microlepidoptera, 4: 3II. (v)

I923, Exotic Microlepidoptera, 3: 46. (BM)

1926, Exotic Microlepidoptera, 3: 252. (BM)

I905, Journ. Bombay Nat. Hist. Soc. I6: 59I. (BM)

I9I6, Exotic Microlepidoptera, I: 523. (BM)

I925, Exotic Microlepidoptera, 3: І 87. (вм)

1922, Trans. Ent. Soc. London, I922: 71. (BM)

I933, Exotic Microlepidoptera, 4: 4I9.

1935, in Caradja and Meyrick, Materialien zu einer Micro-

lepidopteren Fauna der Chinesischen Provinzen Kiangsu,

Chekiang und Hunan, p. 57. (BM)

I907, Proc. Linnean Soc. N.S. Wales, 32: 7 I.

I9I5, Exotic Microlepidoptera, I : 447. (BM)

I920, Exotic Microlepidoptera, 2: 335. (BM)

I913, Journ. Bombay Nat. Hist. Soc. 22: I68. (BM)

I88I, Proc. Linnean Soc. N.S. Wales, 6: 423. (Bм)

I920, Exotic Microlepidoptera, 2: 370.

I9I7, Exotic Microlepidoptera, 2: 86.

I9I9, Exotic Microlepidoptera, 2: 259.

I9I8, Exotic Microlepidoptera, 2: I35. (BM)

I9I6, Exotic Microlepidoptera, I : 54I. (вм)

I9I7, Trans. Ent. Soc. London, I9I7: I3. (BM)

I919, Exotic Microlepidoptera, 2: 266.

I922, Exotic Microlepidoptera, 2: 5I4.

I921, Zool. Meded. 6: 201. (L)

I930, Exotic Microlepidoptera, 3: 573.

I93I, Exotic Microlepidoptera, 4:58. (v)

I9I8, Exotic Microlepidoptera, 2: I36. (BM)

I93I, Trans. Proc. New Zealand Inst. 62: 96.

1924, Trans. Ent. Soc. London, I923: 553.

I922, Exotic Microlepidoptera, 2: 549. (BM)

1923, Exotic Microlepidoptera, 3: 60.

I938, Trans. R. Ent. Soc. London, 87: 508. (BM)

I932, Exotic Microlepidoptera, 4: 209.

I922, Exotic Microlepidoptera, 2: 5I8. (BM)

I922, Trans. Ent. Soc. London, I922: I I3. (BM)

I925, Exotic Microlepidoptera, 3: I95. (BM)

I930, Exotic Microlepidoptera, 3: 568 .

I9I8, Ann. Transvaal Mus. 6:2I. (T)

I933, Exotic Microlepidoptera, 4: 433. (v)

I9I3, Trans. Ent. Soc. London, I9I3: I98. (вм)

I925, Exotic Microlepidoptcra, 3: 224. (B.1)

I924, Trans. Ent. Soc. London, I923: 548. (B.1)

I930, Exotic Microlepidoptera, 3:626. 


$\begin{array}{ll}\text { sublimis } & \text { CHOROPLECA } \\ \text { sublimis } & \text { PHALONIA } \\ \text { sublucens } & \text { CHARITOPSYCHA* } \\ \text { sublucida } & \text { OPOGONA } \\ \text { sublunaris } & \text { STENOMA } \\ \text { sublustris } & \text { COLONOPHORA } \\ \text { submersa } & \text { STENOMA } \\ \text { submetallica } & \text { COPOCENTRA } \\ \text { subnigrata } & \text { THEATRISTA* } \\ \text { subovalis } & \text { STENOMA } \\ \text { subpallida } & \text { HOMALOXESTIS } \\ \text { subpurpurea } & \text { HELIOZELA } \\ \text { subridens } & \text { DECADARCHIS } \\ \text { subrimosa } & \text { ARGYRESTHIA } \\ \text { subrosea } & \text { ARISTOTELIA } \\ \text { subroseata } & \text { PHTHORIMAEA } \\ \text { subrutila } & \text { CYMOTRICHA } \\ \text { subselliata } & \text { CARPOSINA } \\ \text { subsidiaria } & \text { CACOECIA } \\ \text { subsidiaris } & \text { ETHMIA }\end{array}$

\begin{tabular}{|c|c|}
\hline subsignata & SCYTHRIS \\
\hline substrata & BATRACHEDRA \\
\hline substricta & ANTAEOTRICHA \\
\hline substrigata & LEUCOPTERA \\
\hline substructa & PHTHEOCHROA \\
\hline subtigrina & CREPIDOCHARES* \\
\hline subtilis & EPICEPHALA \\
\hline subtincta & SYNTETRERNIS \\
\hline subusta & ASCALENIA \\
\hline subviolacea & OPOSTEGA \\
\hline subvirescens & NEPTICULA \\
\hline succensa & TANYZANCLA \\
\hline succulenta & OPOGONA \\
\hline sucinea & ODITES \\
\hline sucosa & THYROCOPA \\
\hline sulcata & FRISILIA \\
\hline sulfurata & ANTICRATES \\
\hline sulfuratella & STEGOMMATA \\
\hline sulfurea & OECOPHORA \\
\hline sulfurosa & CNEPHASIA \\
\hline sultana & OPOGONA \\
\hline summata & DICHOMERIS \\
\hline summivola & COLEOPHORA \\
\hline superans & APETHISTIS \\
\hline superciliaris & EPIPHRACTIS \\
\hline superciliosa & ANTAEOTRICHA \\
\hline superciliosa & EUCOSMA \\
\hline
\end{tabular}

I9I7, Exotic Microlepidoptera, 2: 8I.

I9I7, Trans. Ent. Soc. London, I9I7: 2. (BM)

I93I, Exotic Microlepidoptera, 4: I60.

1932, Exotic Microlepidoptera, 4: 23I.

1930, Ann. Naturhist. Mus. Wien, 44:248. (v)

I922, Exotic Microlepidoptera, 2: 576. (BM)

I9I5, Exotic Microlepidoptera, I: 429. (BM)

1922, Exotic Microlepidoptera, 2: 587.

I9I7, Exotic Microlepidoptera, 2: 95.

I932, Exotic Microlepidoptera, 4: 304. (Us)

I931, Exotic Microlepidoptera, 4: 76. (BM)

I934, Exotic Microlepidoptera, 4: 466.

1923, Exotic Microlepidoptera, 3: 64. (BM)

I932, Exotic Microlepidoptera, 4: 227. (BM)

I9I4, Trans. Ent. Soc. London, I9I4: 230. (BM)

I932, Exotic Microlepidoptera, 4: I96. (BM)

I923, Exotic Microlepidoptera, 3: I.

I92I, Ann. Transvaal Mus. 8: 5I. (T)

1924, Exotic Microlepidoptera, 3: I07. (BM)

I935, in Caradja and Meyrick, Materialien zu einer Micro-

lepidopteren Fauna der Chinesischen Provinzen Kiangsu,

Chekiang und Hunan, p. 9o. (BM)

I9I6, Exotic Microlepidoptera, 2: Io.

I9I6, Exotic Microlepidoptera, 2: 32.

I9I8, Exotic Microlepidoptera, 2: 200. (BM)

I928, Exotic Microlepidoptera, 3: 397.

1927, Exotic Microlepidoptera, 3: 367. (BM)

I922, Exotic Microlepidoptera, 2: 6оI. (BM)

I922, Exotic Microlepidoptera, 2: $56 \mathrm{I}$.

I922, Exotic Microlepidoptera, $2: 574$. (BM)

I921, Zool. Meded. 6: I7o. (L)

I920, Exotic Microlepidoptera, 2: 357.

I934, Exotic Microlepidoptera, 4:523.

I920, Exotic Microlepidoptera, 2: 383 .

I931, Exotic Microlepidoptera, 4: I64.

I9I5, Exotic Microlepidoptera, I: 378. (BM)

I9I5, Exotic Microlepidoptera, I: 37I. (BM)

I9Io, Journ. Bombay Nat. Hist. Soc. 20: 437. (BM)

I907, Proc. Linnean Soc. N.S. Wales, 32:84.

I88I, Proc. Linnean Soc. N.S. Wales, 5: I72.

1886, Proc. Limnean Soc. N.S. Wales, Io: 786 .

I9Io, Proc. Linnean Soc. N.S. Wales, 35: 278 .

I9II, Trans. Linnean Soc. London, I4: 293.

I9I3, Journ. Bombay Nat. Hist. Soc. 22: I72. (BM)

1930, Exotic Microlepidoptera, 3: 625.

I926, Exotic Microlepidoptera, 3:29I. (BM)

I930, Exotic Microlepidoptera, 3: 577. (BM)

I9I8, Exotic Microlepidoptera, 2: I98. (BM)

I920, Voyage de Ch. Alluaud et $R$. Jeannel en Afrique

Oriental, II, Microlepidoptera, p. 6o. (P) 


\begin{tabular}{|c|c|c|}
\hline superscripta & ODITES & I926, Ann. South African Mus. 23:337. (SA) \\
\hline supplex & ACROCERCOPS & I9I8, Exotic Microlepidoptera, 2: I75. \\
\hline suprema & STENOPTILIA & I926, Exotic Microlepidoptera, 3: 301. \\
\hline surda & ARISTOTELIA & I923, Exotic Microlepidoptera, 3:6. (вM) \\
\hline suspecta & PACHYRHABDA & I92I, Zool. Meded. 6: I76. (L) \\
\hline suspensa & GELECHIA & I923, Exotic Microlepidoptera, 3: I9. (BM) \\
\hline suspensilis & ACROLOPHUS & I93I, Exotic Microlepidoptera, 4: IOI. (v) \\
\hline suspiciosa & TINEA & IgI2, Ann. South African Mus. Io: 69. (SA) \\
\hline suspiciosus & PTEROPHORUS & I92I, Exotic Microlepidoptera, 2: 422. \\
\hline sustentata & STENOMA & I926, Exotic Microlepidoptera, 3: 225. (BM) \\
\hline susurrans & MELASINA & IgII, Ann. Transvaal Mus. 3:82. (BM) \\
\hline sutrix & SINDONOPHORA & I926, Ann. South African Mus. 23: 349. (SA) \\
\hline sybarita & MONOPIS & IgIo, Trans. Ent. Soc. London, I9Io: 474. \\
\hline sybaritis & TORTYRA & Igı2, Exotic Microlepidoptera, I: 37 . \\
\hline sycastis & STATHMOPODA & IgI7, Exotic Microlepidoptera, z: 62. \\
\hline sycophaga & STATHMOPODA & I9I3, Exotic Microlepidoptera, I: 87 . \\
\hline sycophanta & ORNEODES & Igo6, Journ. Bombay Nat. Hist. Soc. I7: I33. \\
\hline sycopola & SIMAETHIS & I88I, Proc. Linnean Soc. N.S. Wales, 5: 2II. \\
\hline syleuta & ERETMOCERA & I926, Ann. South African Mus. 23: 338. (SA) \\
\hline symbola & EUCOSMA & Igog, Ann. Transvaal Mus. 2: 7, pl. 3, fig. 3. (т) \\
\hline symbolaea & SIMAETHIS & I888, Trans. Proc. New Zealand Inst. $20: 85$. \\
\hline symbolaspis & EUCOSMA & I927, Exotic Microlepidoptera, 3: 334. (BM) \\
\hline symbolias & ACROCLITA & IgI2, Journ. Bombay Nat. Hist. Soc. 21: 857. (вм) \\
\hline symbolias & PYRODERCES & I9I5, Exotic Microlepidoptera, I: 3I3. \\
\hline symbolias & STAGMATOPHORA & Igo6, Trans. R. Soc. S. Australia, 30: 53 . \\
\hline symbolica & HELCYSTOGRAMMA & IgI4, Trans. Ent. Soc. London, I9I4: 270. (BM) \\
\hline symbolica & OPOSTEGA & I9I4, Ann. Transvaal Mus. 4:203. (T) \\
\hline symbolistis & ADOXOTRICHA* & $\begin{array}{l}\text { I938, Institut des Parcs Nationaux du Congo Belge, } \\
\text { fasc. I4, p. I5. }\end{array}$ \\
\hline symbolopa & PROMALACTIS & I935, Exotic Microlepidoptera, 4: 593. (BM) \\
\hline symbolopis & ACROCERCOPS & I936, Exotic Microlepidoptera, 5: 35 \\
\hline symmacha & EREUNETIS & I893, Proc. Linnean Soc. N.S. Wales, $7: 564$. \\
\hline symmathetica & DYSELPISTIS* & I938, Rev. Franç. Lépidopterologie, 9: 7 I. \\
\hline symmeles & LAMPRONIA & I9I9, Exotic Microlepidoptera, 2: 28I. (BM) \\
\hline symmerista & EUXANTHIS & $\begin{array}{l}\text { I935, in Caradja and Meyrick, Materialien zu einer Micro- } \\
\text { lepidopteren Fauna der Chinesischen Provinzen Kiangsu, } \\
\text { Chekiang und Hunan, p. } 47 .\end{array}$ \\
\hline symmetra & ATHRYPSIASTIS & I9I5, Exotic Microlepidoptera, I: 377. \\
\hline symmetra & CACOECIA & I9I8, Exotic Microlepidoptera, 2: I66. (вм) \\
\hline symmetropa & ACROCERCOPS & I939, Trans. R. Ent. Soc. London, 87: 59. \\
\hline symmictopa & ARGYROPLOCE & I930, Trans. Ent. Soc. London, $78: 3$ I2. (P) \\
\hline symmochlopa & CROCANTHES & I929, Exotic Microlepidoptera, 3: 5I9. \\
\hline symmochlota & DEPRESSARIA & I9I8, Exotic Microlepidoptera, 2: 223. (BMI) \\
\hline symmora & NEPTICULA & Igo6, Trans. R. Soc. S. Australia, 30: 59 . \\
\hline symmorias & EPERMENIA & I923, Exotic Microlepidoptera, 3: 52. (BM) \\
\hline symmorpha & EUTORNA & I889, Trans. Proc. New Zealand Inst. 21: I58. \\
\hline sympasta & HOLCOCERA & I9I8, Exotic Microlepidoptera, 2: I62. (вм) \\
\hline sympathetica & ACHANODES* & I922, Exotic Microlepidaptera, 2: 593. (в.1) \\
\hline symphanes & PHYLLOCNISTIS & I926, Exotic Microlepidoptera, 3: 264 . \\
\hline symphonica & STENOMA & I9I6, Exotic Microlepidoptera, I: 522. (BM) \\
\hline
\end{tabular}




$\begin{array}{ll}\text { symphoracma } & \text { THIOTRICHA } \\ \text { symphracta } & \text { STILBOSIS } \\ \text { symphyas } & \text { DECADARCHIS } \\ \text { symphyrta } & \text { CRYPSITHYRIS } \\ \text { sympiestis } & \text { URODUS } \\ \text { symplacota } & \text { PSEUDATTERIA } \\ \text { symplecta } & \text { TORTRIX } \\ \text { symplegadopa } & \text { STOMOPTERYX } \\ & \\ \text { symposias } & \text { GONIOTERMA } \\ \text { sympotica } & \text { MOMPHA }\end{array}$

symptomatica

synacma

synacta

synaema

synaphria

synaphrista

synarma

synarthra

synastra

synastra

synauges

synaula

syncapna

syncentra

syncentritis

synchorda

synchorda

synchorista

synchrysa

synchyta

synclepta

synclepta

synclera

synclina

synclinias

synclista

syncolleta

synconista

syncopaula

syncosma

syncrata

\section{LECITHOCERA \\ THIOTRICHA \\ MACROBATHRA \\ CHRYSORYCTIS \\ MACHAEROPTERIS \\ APOTHETOECA* \\ GLYPHIPTERYX \\ ZONOPETALA \\ MACROBATHRA \\ TORTRIX \\ PHILOBOTA}

XYLORYCTA

EUPSELIA

SISYROXENA*

THIOTRICHA

OENOE

SCHOENOTENES*

PTILOPSALTIS*

STATHMOPODA

PHLOEOPOLA

CHTHONOGENES*

METAPLATYNTIS*

CRYPTOLECHIA

PROMALACTIS

ACROCERCOPS

STILBOSIS

MERIDARCHIS

IMMA

GELECHIA

SPHENOGRYPA*
I927, Insects of Samoa, 3, Lepidoptera, fasc. 2, p. 81. (BM)

I9I7, Exotic Microlepidoptera, 2: 47. (Вм)

I927, Insects of Samoa, 3, Lepidoptera, fasc. 2,

p. II3.

I921, Zool. Meded. 6: I98. (L)

1925, Exotic Microlepidoptera, 3: I29. (BM)

I930, Exotic Microlepidoptera, 3: 606. (Bм)

I9I0, Ann. South African Mus. 5: 4I2. (SA)

1936, in Caradja and Meyrick, Deuts. Ent. Zeit. Iris, 50: I58. (BM)

I9I5, Exotic Microlepidoptera, I: 385. (Вм)

1931, Ann. Mus. Nac. Hist. Nat. Buenos Aires, 36: 387.

I93I, Exotic Microlepidoptera, 4: 79. (BM)

I9I8, Exotic Microlepidoptera, 2: 124. (BM)

1920, Exotic Microlepidoptera, 2: 364 .

I905, Journ. Bombay Nat. Hist. Soc. I6: 617.

(?) I922, Exotic Microlepidoptera, 2: 600.

1922, Nat. Hist. Juan Fernandez and Easter Islands, 3: 269.

I909, Journ. Bombay Nat. Hist. Soc. r9: 43I. (BM)

1886, Proc. Linnean Soc. N.S. Wales, 10: 829 .

I886, Proc. Linnean Soc. N.S. Wales, - Io: 8I5.

I9I8, Exotic Microlepidoptera, 2: 167. (Bм)

I889, Proc. Linnean Soc. N.S. Wales, (Ser. 2) 3: I622.

I89o, Trans. R. Soc. S. Australia, I3: 59.

1920, Exotic Microlepidoptera, 2: 317. (BM)

I9I6, Exotic Microlepidoptera, 2: 8. (T)

1935, Exotic Microlepidoptera, 4: 586. (BM)

I9I9, Exotic Microlepidoptera, 2: 249.

I 908, Journ. Bombay Nat. Hist. Soc. I8: 620. (BM)

I935, Exotic Microlepidoptera, 4: 577. (BM)

1924, Exotic Microlepidoptera, 3: 66. (Вм)

I883, Proc. Linnean Soc. N.S. Wales, 8: 355.

1938, in Caradja and Meyrick, Deuts. Ent. Zeit. Iris, 52:

4.

1938, Institut des Parcs Nationaux du Congo Belge, fasc. I4, p. I6. (BM)

I92I, Exotic Microlepidoptera, 2: 394. (вм)

I908, Journ. Bombay Nat. Hist. Soc. I8: 807. (BM)

I93I, Exotic Microlepidoptera, 4: 47.

I9I 7, Exotic Microlepidoptera, 2: 49.

I928, Exotic Microlepidoptera, 3: 404. (BM)

I9I8, Exotic Microlepidoptera, 2: I9I. (BM)

I937, Exotic Microlepidoptera, 5: 93. (T)

1920, Voyage de Ch. Alluaud et R. Jeannel en Afrique

Oriental, II, Microlepidoptera, p. $7 \mathrm{I}$. (P)

1921, Exotic Microlepidoptera, 2: 465 . (BM) 
syncratopa

TELPHUSA

syncrita

syncrotaula

syncrypta

syndecta

syndelta

syndeltias

syndesma

syndicastis

syndyas

synecta

synedra

synercta

synestia

synethes

syngalactis

synglypta

syngonarcha

syngramma

syngraphopa

syngraphopis

syngrapta

synneura

synneurota

synocha

synodias

synodonta

synolca

synomotis

synophrys

synora

synorista

syntaracta

syntona

syntonopis

syntricha

syntripta

syntrocha

syntropa

syntropha

syrictis

syringias

syringota

syrista

syrmatica

syrmatodes

syrmeutis
STOMOPTERYX

CERANTHES

CHELARIA

GLYPHIPTERYX.

TELPHUSA

ARGYROPLOCE

OEGOCONIA

STENOMA

DICHOMERIS *

GNORIMOSCHEMA

AGRIOPHARA

ANTAEOTRICHA

RECURVARIA

ELACHISTA

PYRODERCES

PHYLLOCNISTIS

COPHOMANTIS

ACROCERCOPS

BATTARISTIS

STENOMA

TRICHOTAPHE

CEROMITIA

CROCIDOSEMA

BATTARISTIS

SARIDOSCELIS

THIOTRICHA

CRYPSITHYRIS

ACROCLITA

SPHENARCHES*

MACHAERITIS

GLYPHIPTERYX

LIMNOECIA

CACOECIA

BRACHMIA

OINOPHILA

TIQUADRA

BRACHYDOXA*

PHILOBOTA

LECITHOCERA

RECURVARIA

ANATHAMNA

TRICHOTAPHE

ACROCERCOPS

SCYTHRIS

BLASTOBASIS

BORKHAUSENIA
I935, in Caradja and Meyrick, Materialien zu einer Microlepidopteren Fauna der Chinesischen Provinzen Kiangsu, Chekiang und Hunan, p. 66. (вм)

1926, Exotic Microlepidoptera, 3: 28I. (BM)

1926, Exotic Microlepidoptera, 3: 317. (T)

I9I6, Exotic Microlepidoptera, I: 580 . (BM)

I9I5, Trans. Ent. Soc. London, I915: 22I. (BM)

I92I, Ann. Transvaal Mus. 8: 69. (T)

I938, Trans. R. Ent. Soc. London, 87: 512. (BM)

1926, Ann. South African Mus. 23: 333. (SA)

I929, Trans. Ent. Soc. London, 76: 5I6. (BM)

I926, Exotic Microlepidoptera, 3: 286.

I909, Ann. Transvaal Mus.2: I2, pl. 4, fig. Iо. (T)

I9I6, Exotic Microlepidoptera, I: 485. (BM)

I925, Exotic Microlepidoptera, 3: I73. (вм)

I939, Trans. R. Ent. Soc. London, 89: 53.

I897, Proc. Linnean Soc. N.S. Wales, 22: 333.

1928, Exotic Microlepidoptera, 3: 389. (вм)

1918, Exotic Microlepidoptera, 2: I83.

I926, Exotic Microlepidoptera, 3: 290. (BM)

I9I4, Journ. Bombay Nat. Hist. Soc. 23: I20.

1922, Trans. Ent. Soc. London, I922: 73. (ВM)

I930, Ann. Naturhist. Mus. Wien, 44: 240. (v)

I921, Ann. Transwaal Mus. 8: 85. (T)

I921, Ann. Transvaal Mus. 8: I38. (T)

1926, Trans. Ent. Soc. London, 74: 276. (BM)

I922, Trans. Ent. Soc. London, I922: 74. (BM)

1932, Exotic Microlepidoptera, 4:228.

I936, Exotic Microlepidoptera, 5: 45. (Bм)

I9I7, Exotic Microlepidoptera, 2: 74.

I9II, Proc. Linnean Soc. N.S. Wales, 36: 238.

I886, Trans. Ent. Soc. London, I886: I7.

I889, Proc. Linnean Soc. N.S. Wales, (Ser. 2) 3: I668.

1922, Exotic Microlepidoptera, 2: 492. (вм)

I897, Proc. Linnean Soc. N.S. Wales, 22: 360.

I909, Subantarctic Is. Nerw Zealand, I: 73.

I923, Exotic Microlepidoptera, 3: 48. (BM)

I9I0, Trans. Ent. Soc. London, I9I0: 377.

I922, Exotic Microlepidoptera, 2: 598. (BM)

I9I7, Exotic Microlepidoptera, 2: 83.

I93I, Exotic Microlepidoptera, 4: I89. (вм)

I9I8, Exotic Microlepidoptera, 2: I09. (13:)

I936, Exotic Microlepidoptera, 5: 43. (BM)

I9II, Proc. Linnean Soc. N.S. Wales, 36:262.

1926, Exotic Microlepidoptera, 3: 287. (BM)

I926, Exotic Microlepidoptera, 3: 293.

I9I6, Exotic Microlepidoptera, 2: II.

I922, Exotic Microlepidoptera, 2: 53 8.

I931, Ann. Mus. Nac. Hist. Nat. Buenos Aires, 36: 392.

(B.I) 


$\begin{array}{ll}\text { syrphacopis } & \text { ACROLEPIA } \\ \text { syrphetias } & \text { GRACILARIA } \\ \text { syrphetodes } & \text { BRACHMIA } \\ \text { syrphetopa } & \text { PHTHORIMAEA } \\ \text { syrtica } & \text { PARATHETA* } \\ \text { syrtodes } & \text { EUCOSMA } \\ \text { syrtopa } & \text { CHANYSTIS } \\ \text { systelitis } & \text { CEROMITIA } \\ \text { systematica } & \text { ETHMIA } \\ \text { systolaea } & \text { MELASINA }\end{array}$

$\begin{array}{ll}\text { tabellaria } & \text { COSMOPTERYX } \\
\text { tabellata } & \text { PARASPISTES } \\
\text { tabellifera } & \text { CRATEROBATHRA* } \\
\text { tabernalis } & \text { MELASINA } \\
\text { tabescens } & \text { CACOECIA } \\
\text { tabifica } & \text { EPACTRIS } \\
\text { tabularia } & \text { TORTYRA } \\
\text { tabulata } & \text { PROTOLECHIA } \\
\text { tabulatrix } & \text { GYMNOGRAMMA } \\
\text { tachygrapha } & \text { HIEROXESTIS } \\
\text { tachytoma } & \text { CYNICOCRATES* } \\
\text { tacita } & \text { AGRIOSCELIS* } \\
\text { taciturna } & \text { DEPRESSARIA } \\
\text { taciturna } & \text { MACHAEROPTERIS } \\
\text { tactica } & \text { DICHOMERIS } \\
\text { taedifera } & \text { TRICHOTHYRSA } \\
\text { taeniarcha } & \text { ACROCERCOPS } \\
\text { taenias } & \text { EUMACHAERISTIS* }\end{array}$

taeniata taeniodesma taganista

talantias talantias talantodes talaria talarodes talaroscia

tamiodes tantalota tanyacta tanydora

tanyleuca tanylopha tanymorpha

CAPUA

CNEPHASIA

CHRYSORYCTIS

EPIMACTIS

MNESISTEGA*

MELASINA

MELASINA

LEPTOZANCLA*

SARISOPHORA

DYSOPTUS

STREPTOTHYRIS*

OPOGONA

ENSCEPASTRA

EUPLOCAMUS

BUCCULATRIX
I9I9, Exotic Microlepidoptera, 2: 228. (BM) I907, Journ. Bombay Nat. Hist. Soc. I7: 984. I906, Journ. Bombay Nat. Hist. Soc. I7: I52. (BM) I926, Exotic Microlepidoptera, 3: 278. (BM) I902, Trans. R. Soc. S. Australia, 26: I74. I9I2, Ent. Mon. Mag. 48: 35. (Sub.) I9II, Trans. Linnean Soc. London, I4:28I. (BM) I92I, Ann. Transvaal Mus. 8: I38. (T) I922, Exotic Microlepidoptera, 2: 552. (BM) I908, Proc. Zool. Soc. London, p. 747.

I908, Proc. Zool. Soc. London, p. 733.

19I3, Journ. Bombay Nat. Hist. Soc. 22: I7o. (Bм)

I927, Exotic Microlepidoptera, 3: 379.

I9II, Trans. Linnean Soc. London, I4: 305.

I92I, Zool. Meded. 6: I47. (L)

I921, Zool. Meded. 6: I98. (L)

I9I2, Exotic Microlepidoptera, I: 37.

I904, Proc. Linnean Soc. N.S. Wales, 29:367.

1930, Exotic Microlepidoptera, 3: 597. (BM)

I9I5, Exotic Microlepidoptera, I: 365.

1935, Exotic Microlepidoptera, 4:566. (BM)

I9I3, Exotic Microlepidoptera, I : 96.

I9I0, Journ. Bombay Nat. Hist. Soc. 20: I66. (BM)

I9I I, Journ. Bombay Nat. Hist. Soc. 21: I28.

I9I8, Exotic Microlepidoptera, 2: I52. (BM)

I9I2, Exotic Microlepidoptera, I: 62.

1932, Exotic Microlepidoptera, 4: 268.

1938, in Caradja and Meyrick, Deuts. Ent. Zeit. Iris, 52:

24.

homilostola* I9I7, Exotic Microlepidoptera, 2: 92. (BM)
I934, Exotic Microlepidoptera, 4:525. (BM)

I920, Voyage de Ch. Alluaud et $R$. Jeannel en Afrique

Oriental, II, Microlepidoptera, p. 54. (P)

I893, Proc. Linnean Soc. N.S. Wales, 7: $55^{2}$.

I908, Joum. Bombay Nat. Hist. Soc. I8: 631. (BM)

I9I8, Exotic Microlepidoptera, 2: I0I. (BM)

I924, Exotic Microlepidoptera, 3: 77. (T)

I927, Exotic Microlepidoptera, 3: 329. (T)

I920, Voyage de Ch. Alluaud et R. Jeannel en Afrique

Oriental, II, Microlepidoptera, p. I08. (P)

I9Io, Trans. Ent. Soc. London, I9Io: 445.

I9I9, Exotic Microlepidoptera, 2: 267. (BM)

I9I8, Ann. Transvaal Mus. 6:32. (T)

I920, Voyage de Ch. Alluaud et R. Jeannel en Afrique

Oriental, II, Microlepidoptera, p. 93. (P)

I936, Exotic Microlepidoptera, 4: 621.

1932, Exotic Microlepidoptera, 4: 329.

I9I5, Trans. Ent. Soc. London, I9I5: 250. 


\begin{tabular}{|c|c|}
\hline anyopa & PYCNAGORASTIS* \\
\hline anyopis & ELACHISTA \\
\hline tanyphaea & MELASINA \\
\hline tanyptera & TORTRIX \\
\hline rrhina & THYRSOSTOMA \\
\hline scia & EULECHRIA \\
\hline ysphena & ACROCERCOPS \\
\hline ysta & ANTAEOTRICHA \\
\hline ystis & TINEA \\
\hline zancla & LACHARISSA* \\
\hline chroa & OPOGONA \\
\hline cosma & LASPEYRESIA \\
\hline nopa & PHYCODES \\
\hline phanes & EUCOSMA \\
\hline spila & CHERSOMORPHA * \\
\hline iopis & RECURVARIA \\
\hline rites & MEGACRASPEDUS \\
\hline arocopa & CRYPTOLECHIA \\
\hline ronoma & CHELARIA \\
\hline rospila & METACHANDA \\
\hline chodes & SPILONOTA \\
\hline chota & CAPUA \\
\hline ctis & BORKHAUSENIA \\
\hline tis & CARPOSINA \\
\hline xias & PERISTACTIS* \\
\hline alea & EPORYCTA* \\
\hline & BLASTOBASIS \\
\hline cheuta & BLASTOBASIS \\
\hline coma & COLEOPHORA \\
\hline des & DICHELOPA \\
\hline ta & HIEROXESTIS \\
\hline & AMPHIGENES* \\
\hline & CRYPTOLECHIA \\
\hline pis & LACISTODES* \\
\hline acta & ETHMIA \\
\hline cha & PACHYPSALTIS \\
\hline & $\begin{array}{l}\text { EREUNETIS } \\
\text { [PETASACTIS*] }\end{array}$ \\
\hline nica & SCIOPETRIS* \\
\hline anicopa & OCTASPHALES \\
\hline nitis & TORTRIX \\
\hline nographa & MICROCOLONA \\
\hline ifera & PHILOBOTA \\
\hline igera & ARG YROPLOCE \\
\hline tonica & PSEUDURGIS* \\
\hline tonica & XYROPTILA \\
\hline nivora & PHYLLOCNISTIS \\
\hline & STENOMA \\
\hline 12 & CALLIPHRACTIS \\
\hline
\end{tabular}

1937, Exotic Microlepidoptera, 5: 8I. (т)

I932, Exotic Microlepidoptera, 4: 2I8.

I924, Exotic Microlepidoptera, 3: 77. (T)

I9ro, Proc. Linnean Soc. N.S. Wales, 35: 228.

I921, Zool. Meded. 6: I62. (L)

1883, Proc. Linnean Soc. N.S. Wales, 8: 322.

1928, Exotic Microlepidoptera, 3: 408. (T)

I9I5, Exotic Microlepidoptera, I: 402. (BM)

I908, Proc. Linnean Soc. London, p. 74I.

1937, Exotic Microlepidoptera, 5: 94. (T)

I934, Exotic Microlepidoptera, 4:463.

I9I4, Exotic Microlepidoptera, I: I97. (BM)

I909, Journ. Bombay Nat. Hist. Soc. I9: 423. (Bм)

I921, Exotic Microlepidoptera, 2: 447.

1926, Exotic Microlepidoptera, 3: 243. (BM)

I929, Exotic Microlepidoptera, 3: 485. (BM)

I937, Exotic Microlepidoptera, 5: 9I. (T)

I926, Exotic Microlepidoptera, 3: 3I7. (BM)

I932, Exotic Microlepidoptera, 4: I99. (BM)

I930, Trans. Ent. Soc. London, 78: 3I5. (P)

I9I I, Proc. Linnean Soc. N.S. Wales, 36: 23 I.

I9ıo, Proc. Linnean Soc. N.S. Wales, 35: 192.

I9I5, Exotic Microlepidoptera, I: 297.

I9ro, Proc. Linnean Soc. N.S. Wales, 35: 147.

I9I6, Exotic Microlepidoptera, I: 602.

I908, Proc. Zool. Soc. London, p. 729.

I902, Trans. R. Soc. S. Australia, 26: I7o.

I909, Ann. South African Mus. 5:372. (SA)

I9I7, Exotic Microlepidoptera, 2: 7I.

I9Io, Proc. Linnean Soc. N.S. Wales, 35: 284.

I9II, Trans. Linnean Soc. London, I4: 295.

I92I, Exotic Microlepidoptera, 2: 437.

I928, Exotic Microlepidoptera, 3: 477. (BM)

I921, Ann. Transvaal Mus. 8: 92. (T)

I920, Voyage de Ch. Alluand et R. Jeannel en Afrique

Oriental, $I I$, Microlepidoptera, p. 9o. (P)

I9I6, Exotic Microlepidoptera, I: 6I5.

I888, Trans. Proc. New Zealand Inst. 20: 92.

I89I, Ent. Mon. Mag. 27: 58 .

I920, Exotic Microlepidoptera, 2: 3І6. (вм)

I9Io, Proc. Linnean Soc. N.S. Wales, 35:245. (MA)

I928, Exotic Microlepidoptera, 3: 39I.

I909, Ann. South African Mus. 5: 373. (SA)

I9Io, Trans. Ent. Soc. London, I9Io: 436. (BM)

Igo8, Proc. Zool. Soc. London, p. 741.

I9I4, Supplementa Entomologica, No. 3, p. 46 . (D)

I936, Exotic Microlepidoptera, 5: 3I.

I9I5, Exotic Microlepidoptera, I: 429. (B.I)

I937, Exotic Microlepidoptera, 5: 121. 


\begin{tabular}{|c|c|}
\hline teganitis & GONIONOTA \\
\hline tegminata & EPIMIMASTIS \\
\hline tegulata & MACAROSTOLA \\
\hline egulifera & DACTYLETHRA \\
\hline elamonia & DRIMYLASTIS* \\
\hline eleacma & PLATYPTILIA \\
\hline elearcha & ACROCERCOPS \\
\hline telemacha & MNESISTEGA \\
\hline teleochra & TINEA \\
\hline teleodelta & GRACILARIA \\
\hline teleogramma & LACTURA \\
\hline eleopa & EVETRIA \\
\hline teleosema & ANTAEOTRICHA \\
\hline teleozona & STATHMOPODA \\
\hline telephanta & TORTRIX \\
\hline telesia & CARPOSINA \\
\hline telestica & ADOXOPHYES \\
\hline telestis & ACROCERCOPS \\
\hline teleturga & PANSEPTA* \\
\hline teleutopa & PROMALACTIS \\
\hline ffera & EPITHECTIS \\
\hline telifera & PHALONIA \\
\hline teligera & ABACISTIS \\
\hline teligera & COSMOPTERYX \\
\hline telopis & PROTOLECHIA \\
\hline temenitis & EUCOSMA \\
\hline penitis & PROTOLECHIA \\
\hline enodes & TIMYRA \\
\hline temenopis & ARGYROPLOCE \\
\hline temeraria & CROCANTHES \\
\hline temperata & CRYPTOLECHIA \\
\hline temperatior & PHYLLOCNISTIS \\
\hline tempestiva & CENOPIS \\
\hline tempestiva & STENOMA \\
\hline temulenta & CNEPHASIA \\
\hline iulenta & YPONOMEUTA \\
\hline tenax & COSMOPTERYX \\
\hline tenax & EUCOSMA \\
\hline tenax & MARASMARCHA \\
\hline tenebrosa & CHELARIA \\
\hline tenella & PARASELENA \\
\hline tenera & ACROCERCOPS \\
\hline tenontias & CNEPHASIA \\
\hline tentatrix & PASSALACTIS \\
\hline tentoria & STAGMATOPHORA \\
\hline tepens & CYMOTRICHA \\
\hline tephraea & ANTIOPALA* \\
\hline tephraea & LASPEYRESIA \\
\hline tephrantha & PTYCHOXENA* \\
\hline & SYNTOMACTIS \\
\hline
\end{tabular}

I909, Trans. Ent. Soc. London, I909: 26. I9I6, Exotic Microlepidoptera, I: 590. I908, Journ. Bombay Nat. Hist. Soc. I8: 827. I932, Exotic Microlepidoptera, 4: 20I. (BM) I907, Journ. Bombay Nat. Hist. Soc. I7: 988. I932, Exotic Microlepidoptera, 4: 250. I908, Journ. Bombay Nat. Hist. Soc. I 8: 82I. I927, Exotic Microlepidoptera, 3:356. (BM) I893, Proc. Linnean Soc. N.S. Wales, 7: 546. I926, Exotic Microlepidoptera, 3: 295. I924, Exotic Microlepidoptera, 3: I28. I927, Exotic Microlepidoptera, 3: 333. I925, Exotic Microlepidoptera, 3: I65. (BM) I92I, Ann. Transvaal Mus. 8: Iog. (T) I9Io, Proc. Linnean Soc. N.S. Wales, 35: 250. I9Io, Proc. Linnean Soc. N.S. Wales, 35: I50. I930, Trans. Ent. Soc. London, 78: 310. (P) I9II, Ent. Mon. Mag. 47:2I3. I9I5, Exotic Microlepidoptera, I: 377. I934, Exotic Microlepidoptera, 4: 52I. (BM) I9IO, Rec. Indian Mus. 5: 220. (K) I9I2, Ent. Mon. Mag. 48: 35. (Sub.) I9I4, Ann. Transvaal Mus. 4: 200. (T) I9I5, Trans. Ent. Soc. London, I9I5: 207. (BM) I904, Proc. Linnean Soc. N.S. Wales, 29: 338. I9II, Trans. Linnean Soc. London, I4: 269. I904, Proc. Linnean Soc. N.S. Wales, 29: 34 I. I922, Ent. Mitteil. II : 44. (D) I936, Exotic Microlepidoptera, 4: 6I4. (BM) I9Io, Trans. Ent. Soc. London, I9Io: 443. I9I0, Journ. Bombay Nat. Hist. Soc. 20: I65. (BM) I936, Exotic Microlepidoptera, 5: 3I. I9I2, Ent. Mon. Mag. 48: 34. (Sub.) I9I6, Exotic Microlepidoptera, I: 529. (BM) I9I2, Exotic Microlepidoptera, I: II. (T) I9Io, Rec. Indian Mus. 5: 228. (K) I9I5, Trans. Ent. Soc. London, I9I5: 207. (BM) I920, Ann. South African Mus. I7: 278. (SA) I9I3, Ann. Transvaal Mus. 3:268. (T) I920, Exotic Microlepidoptera, 2: 301 . I9Io, Proc. Linnean Soc. N.S. Wales, 35: I64. (BM) I9I4, Exotic Microlepidoptera, I: 284. I9I2, Trans. Ent. Soc. London, I9II: 685. (BM) 1935, Proc. Ent. Soc. London, Io: 49. I9II, Trans. Linnean Soc. London, I4: 283. I923, Exotic Microlepidoptera, 3: 2. I889, Proc. Linnean Soc. N.S. Wales, (Ser. 2) 3: I647. I9II, Proc. Linnean Soc. N.S. Wales, 36: 287. I9I6, Exotic Microlepidoptera, I: 6I6. (т) I897, Proc. Linnean Soc. N.S. Wales, 22: 385 . 


\begin{tabular}{|c|c|}
\hline tephrastis & NOTHRIS \\
\hline tephrina & PLEUROTA \\
\hline tephrinitis & TINEA \\
\hline tephrinopa & NOTHRIS \\
\hline tephroclysta & XERANTICA* \\
\hline tephroclystis & ALLOTALANTA \\
\hline tephrodelta & EULIA \\
\hline tephrodes & YPSOLOPHUS \\
\hline tephrodesma & STENOMA \\
\hline tephroleuca & PILOTOCOMA* \\
\hline tephromorpha & PERONEA \\
\hline tephrophanes & BORKHAUSENIA \\
\hline tephropis & HOMOSETIA \\
\hline tephroplintha & CHELARIA \\
\hline tephroptila & CHELARIA \\
\hline tephrota & SYNTOMAULA* \\
\hline tephroxesta & CYMOTRICHA \\
\hline tepida & ARTIASTIS* \\
\hline tepidata & BRACHMIA \\
\hline tepidus & PTEROPHORUS \\
\hline teratias & ATTEVA \\
\hline teratopa & MACHIMIA \\
\hline teraturga & PERIACMA \\
\hline terebrata & PTEROLONCHE \\
\hline terenaula & COLEOPHORA \\
\hline terenodes & BEDELLIA \\
\hline terenopa & PYRODERCES \\
\hline terenopis & XYLORYCTA \\
\hline teretracma & SOPHRONIA \\
\hline tergemina & OPOGONA \\
\hline termanthes & THIOTRICHA \\
\hline termatias & MALACOGNOSTIS* \\
\hline termias & CACOECIA \\
\hline terminata & XYLORYCTA \\
\hline terpnodes & PHYLOPATRIS* \\
\hline terrena & PROCOMETIS \\
\hline terrenus & PTEROPHORUS \\
\hline terrestris & MELASINA \\
\hline terricola & SAPHENEUTIS \\
\hline terrigena & MACROTONA \\
\hline territa & MYRMECOZELA \\
\hline tesseraria & IMMA \\
\hline tesserata & ORNEODES \\
\hline tessulata & CHELARIA \\
\hline tessulatana & CACOECIA \\
\hline testacea & COMPSOSARIS* \\
\hline testata & TROPHIMAEA \\
\hline testifica & DEPRESSARIA \\
\hline testudinata & DICHOMERIS \\
\hline
\end{tabular}

I904, Proc. Linnean Soc. N.S. Wales, 29: 427.

I884, Proc. Linnean Soc. N.S. Wales, 9: $75^{\circ}$.

I9I2, Ann. Transvaal Mus. 3: 81. (T)

I929, Exotic Microlepidoptera, 3: 496. (BM)

1930, Exotic Microlepidoptera, 3: 553. (BM)

I930, Exotic Microlepidoptera, 3: 6I6. (BM)

1932, Exotic Microlepidoptera, 4: 260. (v)

I909, Ann. Transvaal Mus. 2: I8, pl. 6, fig. 2. (T)

I9I6, Exotic Microlepidoptera, I: 5II. (BM)

I9I3, Ann. Transvaal Mus. 3: 33I. (T)

I930, Exotic Microlepidoptera, 3: 6I3. (BM)

I929, Trans. Proc. New Zealand Inst. 60: 488.

I9I9, Exotic Microlepidoptera, 2: 25I. (BM)

1923, Exotic Microlepidoptera, 3: 30. (BM)

1931, Exotic Microlepidoptera, 4: 70. (Bм)

I9I4, Exotic Microlepidoptera, I: 236. (BM)

I93I, Exotic Microlepidoptera, 4: 68. (BM)

I889, Proc. Linnean Soc. N.S. Wales, (Ser. 2) 3: I674.

1922, Exotic Microlepidoptera, 2: 505. (P)

1926, Exotic Microlepidoptera, 3: 299.

I907, Proc. Linnean Soc. N.S. Wales, 32: 79.

I920, Exotic Microlepidoptera, 2: 3Io.

I9I4, Exotic Microlepidoptera, I: 257. (BM)

I924, Exotic Microlepidoptera, 3:87. (T)

I927, Exotic Microlepidoptera, 3: 374. (T)

I9I5, Exotic Microlepidoptera, I: 353.

I9I7, Exotic Microlepidoptera, 2: 39.

I9I5, Exotic Microlepidoptera, I: 374.

I927, Exotic Microlepidoptera, 3: 353. (BM)

I915, Exotic Microlepidoptera, I: 36r.

1929, Exotic Microlepidoptera, 3: 500. (Bм)

I926, Sarawak Mus. Journ. 3: I6I.

I9I8, Exotic Microlepidoptera, 2: I64. (BM)

I92I, Exotic Microlepidoptera, 2: 444.

I923, Exotic Microlepidoptera, 3: I5. (BM)

I908, Proc. Zool. Soc. London, p. 73I.

1936, Exotic Microlepidoptera, 5: 64.

I9I4, Ann. Transvaal Mus. 4:204. (T)

I9I5, Exotic Microlepidoptera, I: 289.

1904, Proc. Limnean Soc. N.S. Wales, 29: 406.

I920, Ann. South African Mus. I7: 303. (SA)

I906, Trans. Ent. Soc. London, I906: I 87. (BM)

I9I8, Ann. Transvaal Mus. 6: 34. (T)

I92I, Exotic Microlepidoptera, 2: 43I.

I88I, Proc. Limnean Soc. N.S. Wales, 6: 506.

I9I4, Trans. Ent. Soc. London, I9I4: 234. (BM)

I9I I, Journ. Bombay Nat. Hist. Soc. 2I: I 24.

I920, Exotic Microlepidoptera, 2: 316. (Sub.)

I934, in Caradja and Meyrick, Deuts. Ent. Zeit. Iris, 4S: 34. 


testudinea
tetanota
tetanota
tetrabola
tetracentra
tetracercella
tetrachorda
tetrachrysa
tetraclina

tetracola

tetracona

tetraconcha

tetracosma

tetracrena

tetractis

tetradelta

tetradesma

tetradeta

tetradyas

tetragama

tetraglossa

tetragona

tetragonopa

tetragramma

tetragrapha

tetragrapta

tetraleuca

tetralina

tetrameris

tetrametra

tetramitra

tetrancyla

tetranoma

tetrapeda

tetrapetra

tetrapetra

tetraphala

tetraphracta

tetraphragma

tetraphyta

tetraplanetis

tetraplecta

tetraploa

tetraploca

tetraptila

tetrarcha

tetrarcha
PSEUDOCENTRIS*

ARGYROPLOCE

BUCCULATRIX

STENOMA

HETEROBATHRA

COMODICA*

EUMENODORA

GLYPHIPTERYX

TIMYRA

[HETERALCIS*]

EUCROTALA

PLATYPEPLUS

XESTOCASIS

ARISTOTELIA

ACROCERCOPS

SCHIFFERMUELlERIA I936, Exotic Microlepidoptera, 4:623.

ANTIOCHTHA

PYRODERCES

ACROCERCOPS

ZONOCHARES*

CHELARIA

DIACHALASTIS*

PHILOBOTA

BRACHMIA

COSMOPTERYX

POLYHYMNO

TELPHUSA

GELECHIA

HILAROGRAPHA

GELECHIA

NOTHRIS

LIMNOECIA

ACROLOPHUS

AEGERIA

AUTOSTICHA

ERISTHENODES*

STENOMA

THIOTRICHA

ADOXOPHYES

LEPIDOSCIA

PYRAMIDOBELA

LIMNOECIA

CALLIPRORA

PROTOLECHIA

ARGYROPLOCE

SEMODICTIS*

ARGYROPLOCE

COPROMORPHA
I92I, Exotic Microlepidoptera, 2: 395. (BM)

I9o9, Journ. Bombay Nat. Hist. Soc. I9: 602. (вм)

I9I8, Exotic Microlepidoptera, 2: I83.

I9I3, Trans. Ent. Soc. London, I9I3: I89. (BM)

I906, Trans. R. Soc. S. Australia, 30: 47.

I88I, Proc. Linnean Soc. N.S. Wales, 5: 255.

I924, Exotic Microlepidoptera, 3: 9I.

I907, Journ. Bombay Nat. Hist. Soc. I7: 749.

I906, Journ. Bombay Nat. Hist. Soc. I7: I43.

I9I9, Exotic Microlepidoptera, 2: 257.

I907, Journ. Bombay Nat. Hist. Soc. I7: 73I. (вм)

I9I7, Exotic Microlepidoptera, 2: 62.

I904, Proc. Limnean Soc. N.S. Wales, 29: 289.

I908, Journ. Bombay Nat. Hist. Soc. I8: 8I9.

I906, Journ. Bombay Nat. Hist. Soc. I7: I50. (Bм)

I897, Proc. Linnean Soc. N.S. Wales, 22: 352.

I926, Exotic Microlepidoptera, 3: 292.

I922, Exotic Microlepidoptera, 2: 596.

1935, Exotic Microlepidoptera, 4: 589. (BM)

I920, Exotic Microlepidoptera, 2: 363. (вм)

I889, Proc. Linnean Soc. N.S. Wales, (Ser. 2) 3: I623. I935, in Caradja and Meyrick, Materialien zu einer Micro,

lepidopteren Fauna der Chinesischen Provinzen Kiangsu-

Chekiang und Hunan, p. 75. (BM)

I9I5, Trans. Ent. Soc. London, I9I5: 203. (BM)

I9I3, Ann. Transvaal Mus. 3: 283. (P)

I937, Exotic Microlepidoptera, 5: I2I.

I9I8, Ann. Transvaal Mus. 6: I8. (T)

I930, Exotic Microlepidoptera, 4: I.

I926, Exotic Microlepidoptera, 3: 277. (Bм)

I9I3, Ann. Transvaal Mus. 3: 300. (T)

I93I, Exotic Microlepidoptera, 4: 54.

I9I3, Trans. Ent. Soc. London, I9I3: 195. (BM)

I932, Exotic Microlepidoptera, 4: 337.

I908, Journ. Bombay Nat. Hist. Soc. I8: 458. (вм)

1935, Exotic Microlepidoptera, 4:560.

I925, Exotic Microlepidoptera, 3: I94. (BM)

I886, Trans. Proc. New Zealand Inst. I8: I64.

1938, Trans. R. Ent. Soc. London, 87: 505. (BM)

I92I, Exotic Microlepidoptera, 2: 476.

I93I, Exotic Microlepidoptera, 4: I74. (Us)

I897, Proc. Linnean Soc. N.S. Wales, 22: 367 .

I922, Trans. Ent. Soc. London, I922: 68. (BM)

I904, Proc. Linnean Soc. N.S. Wales, 29: 338.

I928, Exotic Microlepidoptera, 3: 446.

I9o9, Ann. Transvaal Mus. 2: I6, pl. 5, fig. 7. (T)

I920, Exotic Microlepidoptera, 2: 349. (BM)

I9I6, Exotic Microlepidoptera, I: 555. 


\begin{tabular}{|c|c|}
\hline tetrargyra & PLEUROTA \\
\hline tetrarma & STATHMOPODA \\
\hline tetrarrhyncha & METAGRYPA* \\
\hline traschema & CYMOTRICHA \\
\hline tetrascia & IMMA \\
\hline trasema & GLYPHIPTERYX \\
\hline tetraspila & MELASINA \\
\hline tetrasticta & CRYPTOLECHIA \\
\hline trasticta & LASPEYRESIA \\
\hline tratoma & GLYPHIPTERYX \\
\hline etratypa & GRACILARIA \\
\hline traula & OCNOPHILA \\
\hline traxoa & ANOMOXENA \\
\hline etrazona & ARDIOSTERES \\
\hline trazyga & STATHMOPODA \\
\hline troa & OPOSTEGA \\
\hline tetroctas & DACTYLETHRA* \\
\hline etropa & TINEA \\
\hline trophthalma & COSMOPTERYX \\
\hline rortha & COMPSOLECHIA \\
\hline ucra & GRACILARIA \\
\hline xta & TINEA \\
\hline extifera & GELECHIA \\
\hline textilis & EULECHRIA \\
\hline textoria & COLEOPHORA \\
\hline extrina & MACHIMIA \\
\hline halamaula & PYRODERCES \\
\hline lalamectis & CROCANTHES \\
\hline alamepola & OCYSTOLA \\
\hline lameuta & EUCOSMA \\
\hline lamia & PALPARIA \\
\hline alamias & PESSOGRAPTIS* \\
\hline lamitis & ARISTOTELIA \\
\hline lamobathra & ANTAEOTRICHA \\
\hline lamopa & DICHOMERIS \\
\hline lamota & HETEROCROSSA \\
\hline assias & GRACILARIA \\
\hline lassinana & HOLOCOLA* \\
\hline lassitis & SPILONOTA \\
\hline era & ANCYLIS \\
\hline lerodes & BORKHAUSENIA \\
\hline leropa & STENOMA \\
\hline leropis & METACHANDA* \\
\hline iarcha & HILAROGRAPHA \\
\hline liastis & OLETHREUTES \\
\hline lophora & ELACHISTA \\
\hline podes & DICHOMERIS \\
\hline ycra & HAPLOCHROIS \\
\hline inodes & ACROLOPHUS \\
\hline nח & PARASELENA* \\
\hline
\end{tabular}

I928, Exotic Microlepidoptera, 3: 473. (вм)

I9I3, Exotic Microlepidoptera, I: 86.

1933, Exotic Microlepidoptera, 4: 446.

I93I, Exotic Microlepidoptera, 4: 67. (BM)

I9I2, Exotic Microlepidoptera, I: 38. (BM)

I883, Proc. Linnean Soc. N.S. Wales, 7 : I9I.

1905, Journ. Bombay Nat. Hist. Soc. I6: 615.

I9I7, Ann. South African Mus. I7: 6. (SA)

I9o9, Trans. Ent. Soc. London, I909: I4. (BM)

I9I3, Exotic Microlepidoptera, I: 68. (BM)

1928, Exotic Microlepidoptera, 3: 4IO.

1926, Ann. South African Mus. 23: 345. (SA)

I9I7, Trans. Ent. Soc. London, I9I7: 30. (Вм)

I920, Exotic Microlepidoptera, 2: 356.

I936, Exotic Microlepidoptera, 4:618.

I907, Journ. Bombay Nat. Hist. Soc. I7: 986.

I906, Journ. Bombay Nat. Hist. Soc. I7: I53.

I893, Proc. Linnean Soc. N.S. Wales, 7: 54I.

I921, Ann. Transvaal Mus. 8: 94. (T)

I922, Trans. Ent. Soc. London, I922: 92. (Вм)

I933, Exotic Microlepidoptera, 4: 363 .

I931, Trans. Proc. New Zealand Inst. 62: 97.

I9I3, Ann. Transvaal Mus. 3:288. (T)

I906, Trans. R. Soc. S. Australia, 30: 36 .

I920, Ann. Transvaal Mus. 8: I20. (T)

I9I4, Exotic Microlepidoptera, I: I77. (BM)

I9I5, Exotic Microlepidoptera, I: 3I4.

1929, Exotic Microlepidoptera, 3: 52 I. (BM)

I885, Proc. Linnean Soc. N.S. Wales, 9: Io8I.

I9I8, Ann. Transvaal Mus. 6: 49. (T)

I883, Proc. Linnean Soc. N.S. Wales, 7: 432.

1923, Exotic Microlepidoptera, 3: 30. (BM)

I9o8, Journ. Bombay Nat. Hist. Soc. I8: 437. (Bм)

I932, Exotic Microlepidoptera, 4: 290. (v)

I922, Trans. Ent. Soc. London, I922: II2. (BM)

I909, Trans. New Zealand Inst. 4I: I2.

I88I, Proc. Linnean Soc. N.S. Wales, 5: I58.

I881, Proc. Linnean Soc. N.S. Wales, 6: 672.

I9Io, Trans. Ent. Soc. London, I9ro: 434. (вм)

I907, Journ. Bombay Nat. Hist. Soc. I8: I42. (BM)

I9I6, Trans. Proc. New Zealand Inst. 48: 4 I6.

I9I6, Exotic Microlepidoptera, I: 529. (BM)

I9I I, Trans. Linnean Soc. London, I4: 278. (BM)

I920, Exotic Microlepidoptera, 2: 328. (BM)

I9I2, Ent. Mon. Mag. 48: 34. (Sub.)

I889, Trans. Proc. New Zealand Inst. 2 I: I78.

I922, Trans. Ent. Soc. London, I922: III. (BM)

I897, Proc. Linnean Soc. N.S. Wales, 22: 3 II.

I9I9, Exotic Microlepidoptera, 2: 2So. (BM)

I9I0, Proc. Linnean Soc. N.S. WVales, 35: I65. (вм). 


thamnolopha
thapsina
thapsinopa
thapsonota
tharsalea
tharsaleopa

ASAPHARCHA

ORNEODES

ANTAEOTRICHA

HIEROXESTIS

STATHMOPODA

CACOECIA

$\begin{array}{ll}\text { tharsyntis } & \text { PHILOBOTA } \\ \text { theatrica } & \text { AEOLERNIS } \\ \text { theconoma } & \text { LECITHOCERA } \\ \text { thematica } & \text { EUCOSMA } \\ \text { themelia } & \text { TRICHOTAPHE } \\ \text { themelias } & \text { MEROCRATES* } \\ \text { themeliota } & \text { APAPHRISTIS* } \\ \text { themerodes } & \text { LINOSTICHA } \\ \text { themeropis } & \text { PLEUROTA } \\ \text { theodora } & \text { DOLEROTHERA } \\ \text { theophila } & \text { PALPARIA } \\ \text { theorella } & \text { EUPSELIA } \\ \text { theoretica } & \text { ANTAEOTRICHA } \\ \text { theorica } & \text { PELTOPHORA } \\ \text { theoris } & \text { AEOLOSCELIS } \\ \text { therina } & \text { EPICHORISTA } \\ \text { theriosema } & \text { MERIDARCHIS } \\ \text { theristis } & \text { PAMMENE } \\ \text { thermastris } & \text { ANTICRATES } \\ \text { thermistis } & \text { COESYRA } \\ \text { thermochalca } & \text { NEMOTOIS } \\ \text { thermocharis } & \text { CROCANTHES } \\ \text { thermochroa } & \text { PELTOPHORA } \\ \text { thermoconis } & \text { PHALONIA } \\ \text { thermodes } & \text { HEPIALUS } \\ \text { thermodryas } & \text { DICHOMERIS } \\ \text { thermogramma CALLIPRORA } & \text { COLEOPHORA } \\ \text { thermoleuca } & \text { CONTAEOTRICHA } \\ \text { thermopa } & \text { MACROSACES* } \\ \text { thermopetra } & \text { ARGYROPLOCE } \\ \text { thermophaea } & \text { SIROGENES* } \\ \text { thermopis } & \text { AUTOSTICHA } \\ \text { thermopsamma } & \text { GNATHOTONA* } \\ \text { thermoxantha } & \text { LYSIGRAPHA } \\ \text { thermoxyla } & \text { HYPOSMOCOMA } \\ \text { thermurga } & \text { CARPOSINA } \\ \text { thesaurella } & \text { HYPERTROPHA* } \\ \text { thesaurina } & \text { SATRAPIA* } \\ \text { thesmia } & \text { ODITES } \\ \text { thesmiopa } & \text { DICHOMERIS } \\ \text { thesmophila } & \text { CRYPTOLECHIA } \\ \text { thesmophora } & \text { ANTA }\end{array}$

1932, Exotic Microlepidoptera, 4: 286. (v)

I905, Journ. Bombay Nat. Hist. Soc. I6: 583.

I9I6, Exotic Microlepidoptera, I: 498. (BM)

I9I5, Exotic Microlepidoptera, I: 362.

I9I4, Ann. Transwaal Mus. 4: I99. (T)

I935, in Caradja and Meyrick, Materialien zu einer Microlepidopteren Fauna der Chinesischen Provinzen Kiangsu.

Chekiang und Hunan, p. 5o. (BM)

I922, Arkiv för Zool. vol. I4, part I5, p. 8 .

I9I4, Exotic Microlepidoptera, I : 270. (BM)

I926, Sarawak Mus. Journ. 3: 156. (BM)

I9I8, Ann. Transvaal Mus. 6: 48. (T)

I9I3, Trans. Ent. Soc. London; I9I3: I75. (BM)

I93I, Exotic Microlepidoptera, 4:62. (BM)

I9I5, Exotic Microlepidoptera, I: 292.

I902, Trans. R. Soc. S. Australia, 26: I52.

I884, Proc. Linnean Soc. N.S. Wales, 9: 749.

I926, Exotic Microlepidoptera, 3: 265.

I886, Proc. Linnean Soc. N.S. Wales, Io: 825 .

I88I, Proc. Linnean Soc. N.S. Wales, 5: 222.

I932, Exotic Microlepidoptera, 4: 292. (v)

I884, Proc. Linnean Soc. N.S. Wales, 9: 729.

I9o6, Journ. Bombay Nat. Hist. Soc. I7: 4Io.

I9Io, Proc. Linnean Soc. N.S. Wales, 35: 259.

I928, Exotic Microlepidoptera, 3: 404. (BM)

I9I2, Journ. Bombay Nat. Hist. Soc. 2I: 874. (BM)

I9I4, Journ. Bombay Nat. Hist. Soc. 23: I29. (BM)

I889, Proc. Linnean Soc. N.S. Wales (Ser. 2) 3: I654.

I933, Exotic Microlepidoptera, 4:363.

I93I, Exotic Microlepidoptera, 4: 75.

I884, Proc. Linnean Soc. N.S. Wales, 9: 730.

I925, Exotic Microlepidoptera, 3: I39. (BM)

I921, Ann. Transvaal Mus. 8: I43. (T)

I923, Exotic Microlepidoptera, 2: 621. (BM)

I929, Exotic Microlepidoptera, 3:502. (BM)

I922, Exotic Microlepidoptera, 2: 556. (BM)

I905, Journ. Bombay Nat. Hist. Soc. I6: 604. (BM)

I930, Exotic Microlepidoptera, 3: 602. (BM)

1923, Exotic Microlepidoptera, 3: 3. (BM)

I923, Exotic Microlepidoptera, 3: 50. (вм)

I93I, Exotic Microlepidoptera, 4: I24. (v)

I9I4, Exotic Microlepidoptera, I: I85. (BM)

I9I5, Exotic Microlepidoptera, I: 343. (BM)

I929, Exotic Microlepidoptera, 3: 540. (T) (Sub.)

I88I, Proc. Linnean Soc. N.S. Wales, 5: 209.

I886, Proc. Linnean Soc. N.S. Wrales, Io: 823 .

I9I7, Exotic Microlepidoptera, 2: 57.

I922, Trans. Ent. Soc. London, I922: II4. (BM)

I930, Trans. Ent. Soc. London, 78: 3I8. (P)

I9I5, Exotic Microlepidoptera, I: 392. (вм) 


\begin{tabular}{|c|c|c|}
\hline thespia & STENOMA & I9I5, Exotic Microlepidoptera, I: 474. (BM) \\
\hline thespias & COMOCRITIS & I9og, Journ. Bombay Nat. Hist. Soc. I9: 423. (BM) \\
\hline thetias & CROSSOPHORA & I886, Proc. Linnean Soc. N.S. Wales, Io: 796. \\
\hline thetica & ARISTOTELIA & I904, Proc. Linnean Soc. N.S. Wales, 29: 289. \\
\hline thetodes & GYMNOBATHRA & I902, Trans. Ent. Soc. London, I902: 574 . \\
\hline thetopa & NEPHOGENES & IgIo, Trans. Ent. Soc. London, I9Io: 455 . (BM) \\
\hline thiacma & LYONETIA & I9I5, Exotic Microlepidoptera, I: 350 . \\
\hline hiadelpha & OPOGONA & I934, Exotic Microlepidoptera, 4: 463. \\
\hline thiantha & MONOPIS & I9Io, Trans. Ent. Soc. London, I9Io: 473. \\
\hline thianthes & IMMA & I927, Exotic Microlepidoptera, 3: 375. (BM) \\
\hline hiasarcha & EPICROESA & I907, Proc. Linnean Soc. N.S. Wales, 32: 95. \\
\hline itis & PROMALACTIS & I908, Journ. Bombay Nat. Hist. Soc. I8: 8o7. (вм) \\
\hline odes & PARECTOPA & IgI2, in Wytsman, Genera Insectorum, fasc. I28, p. $2 \mathrm{I}$. \\
\hline odes & ATTERIA & IgIo, Proc. Linnean Soc. N.S. Wales, 35:221. \\
\hline atis & $\begin{array}{l}\text { OCYSTOLA } \\
\text { [TRACHYSTOLA*] }\end{array}$ & 1885, Proc. Linnean Soc. N.S. Wales, 9: Io62. \\
\hline liatma & HYPOSMOCOMA & I935, Proc. Hawaiian Ent. Soc. 9:67. (SP) \\
\hline clasta & POLYHYMNO & I926, Exotic Microlepidoptera, 3: 280. (T) \\
\hline des & CRASPEDOTIS & I904, Proc. Linnean Soc. N.S. Wales, 29: 327. \\
\hline iobapta & CAENORYCTA & I930, Exotic Microlepidoptera, 4: I5. \\
\hline lioclora & LECITHOCERA & I93I, Exotic Microlepidoptera, 4: 79. (BM) \\
\hline des & RECURVARIA & I9I7, Trans. Ent. Soc. London, I9I7: 38. (BM) \\
\hline dora & LECITHOCERA & I9I4, Supplementa Entomologica, No. 3, p. 5I. \\
\hline thiodyta & CAPUA & I93I, Exotic Microlepidoptera, 4: I48. (v) \\
\hline thiogramma & PHILOBOTA & I889, Proc. Linnean Soc. N.S. Wales, (Ser. 2) 3: I607. \\
\hline ychna & ELECTROGRAPHA* & I9I2, Exotic Microlepidoptera, I: 63. (вм) \\
\hline thiopeda & IDIOPHANTIS & I93I, Exotic Microlepidoptera, 4:63. (Bм) \\
\hline phaea & COLEOPHORA & I9I7, Exotic Microlepidoptera, 2: 70. \\
\hline oma & PLATYPTILIA & $\begin{array}{l}\text { I920, Voyage de Ch. Alluand et } R \text {. Jeannel en Afrique } \\
\text { Oriental, II, Microlepidoptera, p. } 39 . \text { ( })\end{array}$ \\
\hline toma & ONEBALA & I929, Exotic Microlepidoptera, 3: 509. (BM) \\
\hline 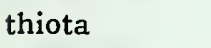 & CERANTHES* & I9I8, Ann. Transvaal Mus. 6: 33. (T) \\
\hline topa & LOBESIA & $\begin{array}{l}\text { I937, in Caradja and Meyrick, Dents. Ent. Zeit. Iris, 5I: } \\
\text { I8I. }\end{array}$ \\
\hline sias & SAPHENEUTIS & I9I5, Exotic Microlepidoptera, I: $28 \mathrm{~g}$. \\
\hline oenarcha & LASPEYRESIA & IgI I, Proc. Linnean Soc. N.S. Wales, 36:29I. \\
\hline loenatica & COESYRA & I920, Exotic Microlepidoptera, 2: 3I2. \\
\hline 1olaea & PARAPSECTRIS* & IgII, Ann. Transvaal Mus. 2: 23I. (T) \\
\hline tholeraula & EULIA & IgI 2, Trans. Ent. Soc. London, I9I I: 680. (BM) \\
\hline tholerodes & SCARDIA & I894, Trans. Ent. Soc. London, I 894: 27. \\
\hline 1oleromicta & BRACHMIA & I93I, Exotic Microlepidoptera, 4:84. (BM) \\
\hline tholeropa & BRIARAULA* & I922, Exotic Microlepidoptera, 2: 590. \\
\hline tholeropis & EUCOSMA & $\begin{array}{l}\text { I934, in Caradja and Meyrick, Deuts. Ent. Zeit. Iris, } 48 \text { : } \\
\text { 32. }\end{array}$ \\
\hline ias & DOLICHOTORNA* & I9Io, Journ. Bombay Nat. Hist. Soc. 20: 439. (BM) \\
\hline tholochorda & TORNODOXA* & I921, Exotic Microlepidoptera, 2: 432. (BM) \\
\hline tholodella & GYMNOBATHRA & I883, New Zealand Journ. Sci. I: 523 . \\
\hline lodes & STENOMA & I9I5, Exotic Microlepidoptera, I: 457 . (B.I) \\
\hline thologramma & STENOMA & I932, Exotic Microlepidoptera, 4: 300. (v) \\
\hline tholomicta & ACROLOPHUS & I929, Trans. Ent. Soc. London, 76:521. (в.1) \\
\hline
\end{tabular}




\begin{tabular}{|c|c|}
\hline tholoneura & COLEOPHORA \\
\hline thoracias & TINGENTERA \\
\hline thoracica & ANACAMPSIS \\
\hline thoracista & CHOLOTIS \\
\hline thoracta & HAPLODYTA* \\
\hline thoraea & ETHMIA \\
\hline thorybodes & THIOTRICHA ${ }^{*}$ \\
\hline thraneuta & BRACHMIA \\
\hline thranias & BORKHAUSENIA \\
\hline thranitis & STRUTHISCA \\
\hline thrasycosma & XYLORYCTA \\
\hline thrasydora & CROCANTHES \\
\hline thrasydoxa & PLATYPTILIA \\
\hline thrasymacha & DECADARCHIS \\
\hline thrasymedes & CATOLBISTIS* \\
\hline thrasynta & ILINGIOTIS \\
\hline thrasyzela & COSMOPTERYX \\
\hline thraumatias & DECADARCHIS \\
\hline thraustica & ACROCERCOPS \\
\hline threnodes & PLATYPEPLUS \\
\hline thriambica & GRACILARIA \\
\hline thriambis & CHLIDANOTA* \\
\hline thriambis & PSALTRIODES* \\
\hline thrincotis & EULECHRIA \\
\hline thriophora & SYRMOLOGA \\
\hline thrombodes & $\begin{array}{l}\text { PTOCHEUUSA } \\
\left.\text { [MERIDORMA }{ }^{*}\right]\end{array}$ \\
\hline thrombota & COPROMORPHA \\
\hline thrylodes & ACROCERCOPS \\
\hline thrypsandra & TRICHOTAPHE \\
\hline thrypsiphila & CHRYSOCLISTA \\
\hline thryptica & HYLOGRAPTIS* \\
\hline thrypticopa & TRACHYNTIS \\
\hline thurifica & ARISTOTELIA \\
\hline thyellia & PERITORNEUTA \\
\hline thyellias & EPIPHTHORA \\
\hline thyellopa & PLATYPTILIA \\
\hline thyellopis & SPILONOTA \\
\hline thylacandra & STENOMA \\
\hline thylacaula & ACROCERCOPS \\
\hline thylacitis & PERONEA \\
\hline thylacosaris & STENOMA \\
\hline thylacura & HEMIMENE \\
\hline thymelopa & ARGYROPLOCE \\
\hline thymiata & NOTHRIS \\
\hline thymiota & STENOMA \\
\hline thymodes & OCYSTOLA \\
\hline thymophanes & GRACILARIA \\
\hline
\end{tabular}

I936, Exotic Microlepidoptera, 4: 622.

1908, Journ. Bombay Nat. Hist. Soc. I8: 452. (вм)

I9I2, Ann. Transvaal Mus. 3: 67. (T)

I9I5, Exotic Microlepidoptera, I: 327.

I886, Proc. Linnean Soc. N.S. Wales, Io: 764 .

I910, Trans. Ent. Soc. London, I910: 46r.

I886, Trans. Proc. New Zealand Inst. 18: I64.

I9II, Journ. Bombay Nat. Hist. Soc. 20: 712.

I905, Trans. Ent. Soc. London, I905: 240.

I905, Journ. Bombay Nat. Hist. Soc. I6: 6I5.

I9I5, Exotic Microlepidoptera, I: 374.

IgIo, Trans. Ent. Soc. London, I9Io: 444.

I926, Exotic Microlepidoptera, 3: 297.

I9I8, Exotic Microlepidoptera, 2: I86.

I930, Exotic Microlepidoptera, 3: 624. (вм)

I9I4, Trans. Ent. Soc. London, I9I4: 275. (вм)

I9I5, Trans. Ent. Soc. London, I9I5: 206. (BM)

I9I6, Exotic Microlepidoptera, p. 620.

I908, Rec. Indian Mus. 2: 398. (K)

I905, Journ. Bombay Nat. Hist. Soc. I6: 585. (Lost)

I907, Journ. Bombay Nat. Hist. Soc. I7: 744.

I906, Journ. Bombay Nat. Hist. Soc. I7: 4I3. (Вм)

I902, Trans. R. Soc. S. Australia, 26: I38.

I888, Proc. Limnean Soc. N.S. Wales, (Ser. 2) 2: 965.

I9I9, Exotic Microlepidoptera, 2: 273. (BM)

I9I4, Trans. Ent. Soc. London, I9I4: 232. (BM)

I9I6, Exotic Microlepidoptera, I: 556.

I930, Exotic Microlepidoptera, 3: 58o.

1923, Exotic Microlepidoptera, 3: 5. (вм)

I9I7, Exotic Microlepidoptera, 2: 52.

I9I0, Trans. Ent. Soc. London, 1910: 45I.

I902, Trans. R. Soc. S. Australia, 26: I53.

I928, Proc. Hawaiian Ent. Soc. 7: 102. (SP)

I902, Trans. R. Soc. S. Australia, 26: I62.

I904, Proc. Linnean Soc. N.S. Wales, 29: 262. (мA)

I926, Exotic Microlepidoptera, 3: 298.

I926, Trans. Ent. Soc. London, 74:273. (Вм)

1915, Exotic Microlepidoptera, I: 468. (Bм)

1932, Exotic Microlepidoptera, 4: 269.

I920, Voyage de Ch. Alluaud et R. Jeannel en Afrique

Oriental, II, Microlepidoptera, p. $57 .(\mathrm{P})$

19I5, Exotic Microlepidoptera, I: 4I9. (BM)

I922, Exotic Microlepidoptera, 2: 527. (вм)

I938, in Caradja and Meyrick, Deuts. Ent. Zeit. Iris, 52:

I. (BM)

1929, Exotic Microlepidoptera, 3: 497. (BM)

I9I5, Exotic Microlepidoptera, I: 45I. (BM)

1885, Proc. Linnean Soc. N.S. Wales, 9: го6г.

I928, Exotic Microlepidoptera, 3: 4II. 


\begin{tabular}{|c|c|}
\hline thymopis & MICROCOLONA \\
\hline thymora & IMMA \\
\hline thyraula & GELECHIA \\
\hline thyreota & EPACTRIS \\
\hline thyriditis & IMMA \\
\hline thyridopa & COPTOTELIA \\
\hline thyridota & PROTOLECHIA \\
\hline thyrota & SYMMOCA \\
\hline thyrsicola & $\begin{array}{l}\text { HYPELICTIS } \\
{[\text { [DEIMNESTRA*] }}\end{array}$ \\
\hline thyrsitis & HIMOTICA* \\
\hline thyrsodoxa & GYMNOSOPHISTIS* \\
\hline thyrsogastra & GLYPHIDOCERA \\
\hline thyrsophora & TRACHYDORA \\
\hline thyrsoptera & PROTOLECHIA \\
\hline thyrsota & ZATRICHODES* \\
\hline thysanarcha & EPIPHRACTIS \\
\hline thysanodes & STENOMA \\
\hline thysanoma & CACOECIA \\
\hline thysanora & ANACAMPSIS \\
\hline thysanota & ACROCLITA \\
\hline thysanota & CUPHODES* \\
\hline thysiarcha & EOMICHLA \\
\hline thystas & ARGYROPLOCE \\
\hline thyteria & HOPLITICA \\
\hline tibicina & SCYTHRIS \\
\hline tignaria & XYLORYCTA \\
\hline tigroides & SIMAETHIS \\
\hline timaea & PARECTOPA \\
\hline timida & EULECHRIA \\
\hline timidus & PTEROPHORUS \\
\hline timioceros & LECITHOCERA \\
\hline tinactis & STENOMA \\
\hline tincta & OPOSTEGA \\
\hline tinctoria & HERPYSTIS \\
\hline tinthalea & CHEREUTA* \\
\hline titania & PHYLLOCNISTIS \\
\hline titanitis & GRACILARIA \\
\hline titanota & SPYRIDARCHA* \\
\hline tofina & CNEPHASIA \\
\hline tolerans & EUCOSMA \\
\hline tomaea & PROTERODESMA \\
\hline tomicodes & SIMAETHIS \\
\hline tonaea & DEMOPRACTIS* \\
\hline tonans & IMMA \\
\hline tonica & ARGYROPLOCE \\
\hline tonitrualis & EUCOSMA \\
\hline nosticha & LASPEYRESIA \\
\hline insa & CHELARIA \\
\hline
\end{tabular}

1897, Proc. Linnean Soc. N.S. Wales, 22: 375. I906, Trans. Ent. Soc. London, I906: 203. (BM) I886, Trans. Proc. New Zealand Inst. 18: I67. I9II, Journ. Bombay Nat. Hist. Soc. 21 : II4. I906, Trans. Ent. Soc. London, I906: I80. (Bм) I9I2, Trans. Ent. Soc. London, I9I I: 701. (BM) 1904, Proc. Linnean Soc. N.S. Wales, 29: 355. 1929, Exotic Microlepidoptera, 3: 516.

1913, Journ. Bombay Nat. Hist. Soc. 22: I7I. (BM) I9I 2, Trans. Ent. Soc. London, I9I I: 705. (BM)

1934, Exotic Microlepidoptera, 4: 454. (o) I929, Exotic Microlepidoptera, 3: 529. (BM) 1897, Proc. Linnean Soc. N.S. Wales, 22: 392. 1904, Proc. Linnean Soc. N.S. Wales, 29: 38r. I9I4, Exotic Microlepidoptera, I: 247. (BM) I9I 8, Ann. Transvaal Mus. 6: 53. (T) I9I5, Exotic Microlepidoptera, I: 434. (BM) I9Io, Proc. Linnean Soc. N.S. Wales, 35: 217. I9I4, Trans. Ent. Soc. London, I9I4: 26I. (BM) I9I2, Journ. Bombay Nat. Hist. Soc. 2I : 860. (BM) I897, Proc. Linnean Soc. N.S. Wales, 22: 314. 1928, Exotic Microlepidoptera, 3: 469. (вм) I9I I, Proc. Linnean Soc. N.S. Wales, 36: 276. 1889, Proc. Linnean Soc. N.S. Wales, (Ser. 2) 3: 1596. 1916, Exotic Microlepidoptera, 2: I2. (BM) I921, Exotic Microlepidoptera, 2: 445. I92I, Zool. Meded. 6: I8I. (L) I9I4, Exotic Microlepidoptera, I: 285. I9I4, Exotic Microlepidoptera, I : I65. 1908, Trans. Ent. Soc. London, I907: 494. I938, in Caradja and Meyrick, Deuts. Ent. Zeit. Iris, 52: 86.

I9I5, Exotic Microlepidoptera, I: 4I4. (BM)

I9I8, Ann. Transvaal Mus. 6: 4I. (T) 1916, Exotic Microlepidoptera, 2: 16. (Bм) I906, Trans. R. Soc. S. Australia, $30: 33$. 1928, Exotic Microlepidoptera, 3: 405. I92I, Ann. Transvaal Mus. 8: 122. (T) I9I3, Exotic Microlepidoptera, I: I47. I922, Exotic Microlepidoptera, 2: 498. (Bм) I930, Exotic Microlepidoptera, 3: 599. (BM) I9II, Trans. Linnean Soc. London, I4: 306. I930, Exotic Microlepidoptera, 4:5. (BM) I9I8, Exotic Microlepidoptera, 2: I54. 1925, Exotic Microlepidoptera, 3: I35. (BM) I909, Journ. Bombay Nat. Hist. Soc. 19: 606. (вм1) I934, Ann. Mag. Nat. Hist. (Io) I4: 405. (BM) I922, Exotic Microlepidoptera, 2: 53 I. (B.M) I9I3, Journ. Bombay Nat. Hist. Soc. 22: I64. (вм) 


$\begin{array}{ll}\text { tonsoria } & \text { ARGYROPLOCE } \\ \text { toparcha } & \text { PHYLLOCNISTIS } \\ \text { topazias } & \text { NEMOTOIS } \\ \text { topica } & \text { PHILOBOTA } \\ \text { toraula } & \text { COSMOPTERYX } \\ \text { toreutica } & \text { SYNTOMACTIS } \\ \text { toreutis } & \text { TIMYRA } \\ \text { tormentata } & \text { IMMA } \\ \text { tornastis } & \text { BACTRA } \\ \text { tornocarpa } & \text { PHTHEOCHROA } \\ \text { tornogramma } & \text { ANTAEOTRICHA } \\ \text { tornograpta } & \text { HEMIMENE } \\ \text { tornophanes } & \text { ARGYROPLOCE } \\ \text { tornoptila } & \text { COMPSOLECHIA } \\ \text { tornota } & \text { PYRGOTIS } \\ \text { torodetta } & \text { LASPEYRESIA } \\ \text { torodoxa } & \text { LABDIA } \\ \text { torogramma } & \text { CACOECIA } \\ \text { torophanes } & \text { CRYPTOLECHIA }\end{array}$

torophracta IMMA

$\begin{array}{ll}\text { torophragma } & \text { STENOMA } \\ \text { toropis } & \text { ELACHISTA } \\ \text { torosema } & \text { HAPLODYTA } \\ \text { torpens } & \text { PAROCYSTOLA } \\ \text { torquata } & \text { ARGYROPLOCE } \\ \text { torquens } & \text { LYONETIA } \\ \text { torrefacta } & \text { BRACHMIA } \\ & \text { [CATELAPHRIS*] } \\ \text { torrens } & \text { EUCOSMA } \\ \text { torrescens } & \text { EPORGASTIS } \\ \text { torrescens } & \text { PETELIACMA } \\ \text { torrida } & \text { PHAEOSACES } \\ \text { torridula } & \text { SIMAETHIS } \\ \text { torridus } & \text { DEUTEROCOPUS } \\ \text { torta } & \text { OROTHYNTIS } \\ \text { torticornis } & \text { ARGYROPLOCE } \\ \text { tortuosa } & \text { CHELARIA } \\ \text { tortuosa } & \text { EPICHORISTA } \\ \text { torva } & \text { BRACHMIA } \\ \text { tothastis } & \text { ACROCLITA } \\ \text { toxastis } & \text { TIMYRA } \\ \text { toxochorda } & \text { PLATYPTILIA } \\ \text { toxocosma } & \text { AGATHACTIS* } \\ \text { toxogramma } & \text { ARGYROPLOCE } \\ \text { toxolyca } & \text { CYMOTRICHA } \\ & \end{array}$

I9o9, Journ. Bombay Nat. Hist. Soc. I9: 592. (BM)

I9I8, Exotic Microlepidoptera, 2: I82.

I893, Proc. Linnean Soc. N.S. Wales, 7: 485 .

I9I4, Exotic Microlepidoptera, I: 250.

I9Io, Trans. Ent. Soc. London, IgIo: $45^{2}$.

I897, Proc. Linnean Soc. N.S. Wales, 22: 387.

Igo8, Journ. Bombay Nat. Hist. Soc. I 8: 449.

I921, Ann. Transvaal Miss. 8: IIO. (T)

I909, Journ. Bombay Nat. Hist. Soc. I9: 586. (BM)

1932, Exotic Microlepidoptera, 4:267. (v)

I925, Exotic Microlepidoptera, 3: I7I. (BM)

I927, Exotic Microlepidoptera, 3: 34I. (BM)

I930, Exotic Microlepidoptera, 3: 603. (BM)

I922, Trans. Ent. Soc. London, I922: 96. (BM)

I907, Trans. Proc. New Zealand Inst. 39: II4.

I9I4, Journ. Bombay Nat. Hist. Soc. 22: 772. (BM)

I928, Exotic Microlepidoptera, 3: 386. (Bм)

I897, Trans. Ent. Soc. London, I897: 388.

I935, in Caradja and Meyrick, Materialien zu einer Micro-

lepidopteren Fauna der Chinesischen Provinzen Kiangsu,

Chekiang und Hunan, p. 8I. (BM)

I935, in Caradja and Meyrick, Materialien zu einer Micro-

lepidopteren Fauna der Chinesischen Provinzen Kiangsu,

Chekiang und Hunan, p. 86. (BM)

I9I5, Exotic Microlepidoptera, I: 439. (BM)

I897, Proc. Linnean Soc. N.S. Wales, 22: 336.

I889, Proc. Linnean Soc. N.S. Wales, (Ser. 2) 3: 1665.

I920, Exotic Microlepidoptera, 2: 382. (MA)

I927, Exotic Microlepidoptera, 3: 339. (BM)

I922, Exotic Microlepidoptera, 2: $55^{8}$.

I9I4, Ann. South African Mus. Io: 245. (SA)

I927, Exotic Microlepidoptera, 3: 334. (BM)

I921, Ann. Transvaal Mus. 8: 82. (T)

I9I2, Exotic Microlepidoptera, I: I2. (BM)

I894, Trans. Ent. Soc. London, I894: 20. (Вм)

I926, Exotic Microlepidoptera, 3: 304. (BM)

I913, Exotic Microlepidoptera, I: Io6.

I922, Exotic Microlepidoptera, 2: 597. (BM)

I931, Exotic Microlepidoptera, 4: I37. (BM)

I9I3, Journ. Bombay Nat. Hist. Soc. 22: I65. (Вм)

I9I2, Exotic Microlepidoptera, I: 8. (T)

I9I4, Exotic Microlepidoptera, I: 278. (BM)

I9I I, Proc. Linnean Soc. N.S. Wales, 36: 243.

Igo8, Journ. Bombay Nat. Hist. Soc. I8: 449. (BM)

I934, Ann. Mag. Nat. Hist. (Io) I4: 402.

I929, Exotic Microlepidoptera, 3: 50I. (BM)

I925, Exotic Microlepidoptera, 3: I43.

I934, in Caradja and Meyrick, Deuts. Ent. Zeit. Iris, 48: 35. 


toxomacha
toxophanes
toxophila
toxophragma
trabeata
trabeata
trachelista
trachyacma
trachyclina

trachycnemis trachycosma trachydelta trachydesma trachydyta trachymorpha

trachynota
trachyphaea
trachyphanes
trachyphloeus
trachypsamma
trachyptera
trachyptila
trachyspila
trachyxyla
tractrix
tragoditis

tragoptila
tranquilla
tranquilla
transcissa
transcripta
transcutata
transecta
transennata
transfilata
transfixa
transformata
transformis
transfossa
transfuga
transfumata
transfusa
transjecta
translineata

GRACILARIA PSALTICA ORNEODES NARYCIA THUDACA TINAEGERIA [PERCNARCHA*] BLASTOBASIS GLYPHIDOCERA OPOGONA

COMPSOLECHIA

GELECHIA

HY PONOMEUTA ORNEODES CHELARIA CHELARIA

ACROCLITA XYLESTHIA PROTOLECHIA PTEROPHORUS SCARDIA ORNEODES IMMA CHELARIA TRICLONELLA ANTAEOTRICHA CAPUA

TINEA CRYPTOLECHIA SPILONOTA COSMOPTERYX BLASTOBASIS CACOECIA ACROCERCOPS MICROCOLONA ARISTOTELIA CATAMACTA METALLOCRATES* ARGYROPLOCE CRYPTOLECHIA CRYPTOLECHIA EREUNETIS EREUNETIS IMMA SPATALISTIS
I883, Proc. Linnean Soc. N.S. Wales, 7: 197. I9Io, Journ. Bombay Nat. Hist. Soc. 20: I 54. (Bм) I 9o6, Journ. Bombay Nat. Hist. Soc. I7: I33. 1937, Exotic Microlepidoptera, 5: I55.

I893, Proc. Linnean Soc. N.S. Wales, $7: 578$.

I9o9, Trans. Ent. Soc. London, Igog: I8. I921, Ann. Transvaal Mus. 8: II7. (T) I93I, Journ. Linnean Soc. London, 37:28I. (BM) I935, in Caradja and Meyrick, Materialien zu einer Microlepidopteren Fauna der Chinesischen Provinzen Kiangsu,

Chekiang und Hunan, p. 92.

I922, Trans. Ent. Soc. London, I922: 89. (вм)

I923, Exotic Microlepidoptera, 3:2I. (BM)

I931, Exotic Microlepidoptera, 4: I72. (BM)

I929, Exotic Microlepidoptera, 3: 534 .

I920, Exotic Microlepidoptera, 2: 304. (BM)

I927, Insects of Samoa, 3, Lepidoptera, fasc. 2, p. 83. (BM)

I926, Sarawak Mus. Journ. 3: I5o. (BM)

I921, Ann. Transvaal Mus. 8: I3I. (T)

I904, Proc. Linnean Soc. N.S. Wales, 29: 343.

I926, Exotic Microlepidoptera, 3: 300.

I907, Journ. Bombay Nat. Hist. Soc. I7: 989.

I906, Journ. Bombay Nat. Hist. Soc. I7: 134.

I92I, Exotic Microlepidoptera, 2: 480. (BM)

I933, Exotic Microlepidoptera, 4: 354. (BM)

I920, Exotic Microlepidoptera, 2: 366 . (Bм)

I925, Exotic Microlepidoptera, 3: I72. (BM)

I935, in Caradja and Meyrick, Materialien zu einer Microlepidopteren Fauna der Chinesischen Provinen Kiangsu, Chekiang und Hunan, p. 47.

I917, Exotic Microlepidoptera, 2: 78 .

I927, Exotic Microlepidoptera, 3: 383. (T)

IgII, Proc. Linnean Soc. N.S. Wales, 36: 233.

I9I4, Exotic Microlepidoptera, I: 203.

I9I8, Exotic Microlepidoptera, 2: I58.

I935, Exotic Microlepidoptera, 4: 569.

I931, Exotic Microlepidoptera, 4: I69.

I922, Exotic Microlepidoptera, 2: 575. (BM)

I927, Insects of Samoa, 3, Lepidoptera, fasc. 2, p. 77.

I924, Trans. Proc. New Zealand Inst. 55: 203.

I930, Exotic Microlepidoptera, 3: 579. (BMI)

I921, Ann. Transvaal Mus. 8: 6o. (T)

I926, Exotic Microlepidoptera, 3: 3IS. (B.I)

I9I I, Ann. Transwaal Mus. 3: 74. (BM)

I9I5, Exotic Microlepidoptera, I: 366.

I921, Zool. Meded. 6: I94. (L)

I936, Exotic Microlepidoptera., 4: 616. (BMI)

I921, Zool. Meded. 6: I5I. (L) 


translucens
translucida
transmutata
transtrifera
transtrifera
transvecta
transvola
trapezantha
trapezias
trapeziodes
trapezitis
trapezoides
trapezopa
trasias

traumatias

trematias

tremefacta

tremula

trepida

trepida

trepidans

triacma

triacmias

triacmopa

triacris

triactis

triadelpha

triadias

triaenota

triallactis

triancycla

triangulana

triannulata

triannulatus

triarcha

triardis

triargyra

triaria

triastis

triatma

triaula

tribalanota

tribapta

tribolana

tribolopa
TINEA

CACOECIA

EUCOSMA

CEROMITIA

POLYCHROSIS

STATHMOPODA

BACTRA

MONOPIS

COMPSOLECHIA

EULIA

EUCOSMA

TINEA

ACROLEPIA

LASPEYRESIA

MYROPHILA

[XENORRHYTHMA*] I923, Exotic Microlepidoptera, 2:625. (BM)

ARISTOTELIA

COLEOPHORA

EUCOSMA

EUCOSMA

NEPTICULA

MELASINA

IDIOGLOSSA

SIMAETHIS

STENOMA

ACROCERCOPS

ACROCERCOPS

CAPUA

PTEROPHORUS

ANARSIA

DEPRESSARIA

URODUS

HOLOCOLA

CHELARIA

DEUTEROCOPUS

LITHOCOLLETIS

IMMA

TRICLONELLA

PHALANGITIS

PSEUDODOXIA

SCYTHRIS

APHTHONETUS

GELECHIA

HOMONA

DIPTERINA

HIEROMANTIS

1926, Exotic Microlepidoptera, 3:253. (BM)
I9I7, Exotic Microlepidoptera, 2: 78.

Ig08, Journ. Bombay Nat. Hist. Soc. I8: 6r6. (Вм)

I93I, Exotic Microlepidoptera, 4: I46.

I9I2, Exotic Microlepidoptera, I: 29. (T)

I920, Exotic Microlepidoptera, 2: 346. (BM)

1927, Exotic Microlepidoptera, 3: 377.

I924, Trans. Ent. Soc. London, I923: 546.

1927, Exotic Microlepidoptera, 3:321.

I922, Trans. Ent. Soc. London, I922: 87. (вм)

I9I2, Ent. Mon. Mag. 48: 34. (Sub.)

1935, Exotic Microlepidoptera, 4: 579.

I9I4, Exotic Microlepidoptera, I: 208.

I928, Exotic Microlepidoptera, 3: 448. (BM)

I9I3, Ann. Transvaal Mus. 3:282. (P)

I92I, Exotic Microlepidoptera, 2: 472.

I909, Ann. Transvaal Mus. 2: 6, pl. 3, fig. I. (T)

I9II, Proc. Linnean Soc. N.S. Wales, 36:249.

I9o6, Trans. R. Soc. S. Australia, 30:6I.

I920, Voyage de Ch. Alluaud et $R$. Jeannel en Afrique

Oriental, II, Microlepidoptera, p. II2. (P)

I9I3, Exotic Microlepidoptera, I: 77.

I926, Exotic Microlepidoptera, 3: 304. (BM)

I93I, Exotic Microlepidoptera, 4: 37. (v)

I908, Journ. Bombay Nat. Hist. Soc. I8: 8I8.

I930, Trans. Ent. Soc. London, 78: 319. (P)

I920, Voyage de Ch. Alluaud et R. Jeannel en Afrique

Oriental, II, Microlepidoptera, p. 46. (P)

I908, Trans. Ent. Soc. London, I908: 494.

I9I3, Journ. Bombay Nat. Hist. Soc. 22: I69. (BM)

I935, Exotic Microlepidoptera, 4: 594. (BM)

I931, Exotic Microlepidoptera, 4:89. (v)

I88I, Proc. Linnean Soc. N.S. Wales, 6: 670.

IgI I, Ann. Transvaal Mus. 3: 69. (вм)

I9I3, Exotic Microlepidoptera, I: I07.

I908, Journ. Bombay Nat. Hist. Soc. I8: 8I I.

I906, Trans. Ent. Soc. London, I906: I74. (BM)

I920, Exotic Microlepidoptera, 2:366. (BM)

I907, Proc. Linnean Soc. N.S. Wales, 32 : 137.

I9Io, Journ. Bombay Nat. Hist. Soc. 20: I5I. (BM)

1935, Trans. roy. Soc. N.Z. 65: 304.

I935, Proc. Hawaiian Ent. Soc. 9: 63. (SP)

I935, in Caradja and Meyrick, Materialien zu einer Micro-

lepidopteren Fawna der Chinesischen Provinzen Kiangsu,

Chekiang und Hunan, p. 67.

I928, Exotic Microlepidoptera, 3: 455.

I88I, Proc. Linnean Soc. N.S. Wales, 6: 525.

I924, Exotic Microlepidoptera, 3: 66. (BM) 


\begin{tabular}{|c|c|}
\hline tribolopis & TELPHUSA \\
\hline bomias & STENOMA \\
\hline ribonia & STENOPTILIA \\
\hline achia & ENTHETICA \\
\hline achynta & LABDIA \\
\hline icalyx & ACROCERCOPS \\
\hline ricapna & PARACLADA* \\
\hline ricapsis & STENOMA \\
\hline arpa & DEMOBROTIS \\
\hline isis & EUTORNA \\
\hline iusta & ACROLOPHUS \\
\hline usta & ORNEODES \\
\hline ensa & EUPHILTRA \\
\hline ensa & TORTRIX \\
\hline entra & LASPEYRESIA \\
\hline entra & NEPTICULA \\
\hline icentrota & BATTARISTIS \\
\hline eros & ACROCERCOPS \\
\hline richaeola & LABDIA \\
\hline halca & DECADARCHIS \\
\hline ralina & PROTOLECHIA \\
\hline haracta & STENOMA \\
\hline haspis & RECURVARIA \\
\hline ichinaspis & PHTHORIMAEA \\
\hline ichinota & IMMA \\
\hline hoceros & OPOGONA \\
\hline locnemis & ACROCLITA \\
\hline hocolpa & STENOMA \\
\hline hocrossa & EUCOSMA \\
\hline hocyma & BRACHMIA \\
\hline lodeta & ANARSIA \\
\hline lodora & DECADARCHIS \\
\hline lodora & STATHMOPODA \\
\hline lodyta & MELASINA \\
\hline hogramma & EXAULISTIS* \\
\hline lograpta & ARGYROPLOCE \\
\hline Ioneura & STENOMA \\
\hline Lonota & ANTAEOTRICHA \\
\hline hophysa & PARECTOPA \\
\hline hopla & HOMALOXESTIS \\
\hline horda & STENOMA \\
\hline hosema & PROTOLECHIA \\
\hline rosoma & ARGY ROPLOCE \\
\hline hostola & GELECHIA \\
\hline hostrota & MNESIPYRGA* \\
\hline aroa & ADOXOPHYES \\
\hline aroa & IYYERCALLIA \\
\hline & TIPHA \\
\hline
\end{tabular}

[HETERODELTIS*]

trichrysa
1927. Exotic Microlepidoptera, 3: 344. I9I5, Exotic Microlepidoptera, I: 4I7. (BM) I92I, Exotic Microlepidoptera, 2: 423. I923, Exotic Microlepidoptera, 3: 38. (BM) 1928, Exotic Microlepidoptera, 3: 388. I921, Exotic Microlepidoptera, 2: 465. I9II, Trans. Linnean Soc. London, I4: 288. I930, Exotic Microlepidoptera, 4:26. (v) I9I3, Ann. Transvaal Mus. 3: 33I. (T) I9o6, Trans. R. Soc. S. Australia, 30: 42. I9I3, Trans. Ent. Soc. London, I9I3: I94. (BM) I9o8, Trans. Ent. Soc. London, I907: 506. I9I8, Exotic Microlepidoptera, 2: 219. I9I2, Exotic Microlepidoptera, I: 6. (BM) I907, Journ. Bombay Nat. Hist. Soc. I7: 734. (вм) I889, Trans. Proc. New Zealand Inst. 2I: I87. I93I, Exotic Microlepidoptera, 4: 64. (v) 1926, Ann. South African Mus. 23: 340. (SA) 1933, Exotic Microlepidoptera, 4: 427. I936, Exotic Microlepidoptera, 5: 58. I904, Proc. Linnean Soc. N.S. Wales, 29: 377. 1925, Exotic Microlepidoptera, 3: I99. (BM) I9I8, Exotic Microlepidoptera, 2: I3I. (BM) I9I7, Trans. Ent. Soc. London, I9I7: 4I. (BM) I9o6, Trans. Ent. Soc. London, I906: I85. (BM) I930, Exotic Microlepidoptera, 4:8. I9I4, Exotic Microlepidoptera, I: 275. I9I5, Exotic Microlepidoptera, I: 445. (Bм) I9I6, Exotic Microlepidoptera, I: 563. (BM) I923, Exotic Microlepidoptera, 3: 47. (BM) I904, Proc. Linnean Soc. N.S. Wales, 29: 4I5. I9I I, Trans. Linnean Soc. London, I4: 299. Igo9, Ann. Transvaal Mus. 2: 21, pl. 7, fig. I. (T) I924, Exotic Microlepidoptera, 3: 77. (BM) I9I I, Ann. Transvaal Mus. 3: 77. (T) IgII, Proc. Linnean Soc. N.S. Wales, 36: 275. I9I3, Trans. Ent. Soc. London, I9I3: I 89. (BM) I926, Exotic Microlepidoptera, 3: 235. (BM) I9I5, Trans. Ent. Soc. London, I9I5: 235. I929, Exotic Microlepidoptera, 3:5I8. I9I2, Trans. Ent. Soc. London, I9II: 7I3. (BM) I904, Proc. Linnean Soc. N.S. Wales, 29: 347. I9I4, Supplementa Entomologica, No. 3, p. 48. (D) I923, Exotic Microlepidoptera 3:22. (BM) I9I3, Trans. Ent. Soc. London, I9I3: I7I. (B.I) I902, Trans. Ent. Soc. London, I902: 57 S. I902, Trans. R. Soc. S. Australia, 26: I4I.

I906, Journ. Bombay Nat. Hist. Soc. I7: I42. (BM) I920, Exotic Microlepidoptera, 2:319. (BM) 


tricirrha
tricolona
tricolorana
tricornis
tricoronata
tricosma
tricrates
tricrena
tricrocota
tricuneatella
tricyanitis
tricycla
tricyma
tridectis
tridelta
tridentifera
tridesma
tridesma
tridora
tridryas
trierica
trierodes
triexoda
trifera

ACROCERCOPS

VANICELA

HELICTOPHANES

ANARSIA

COESYRA

CHELARIA

NEMOTOIS

CATORYCTIS

IMMA

GRACILARIA

SIMAETHIS

DIOCOSMA

ACROCERCOPS

TINEA

PYROZELA

MELASINA

ADELA

CERCONOTA*

CUPHODES

STATHMOPODA

PSITTACASTIS*

TINEA

LACTURA

LECITHOCERA

trifida

trifilata

triflua

triformis

trifurcata

trigama

triglenopa

triglossa

triglypta

trigonaspis

trigonias

trigonodes

trigonometra

trigonomis

trigonopis

trigonoptila

trigonospila

trigramma

trigrapha

trijuncta

trilampas

trileucota

trilicella

trilinguis

trilitha
1935, Exotic Microlepidoptera, 4: 598.

I913, Exotic Microlepidoptera, I: 8I.

I88I, Proc. Linnean Soc. N.S. Wales, 6: 638.

I9I3, Journ. Bombay Nat. Hist. Soc. 22: I68. (BM)

I920, Exotic Microlepidoptera, 2: 312.

1933, Exotic Microlepidoptera, 4: 355. (BM)

I922, Exotic Microlepidoptera, 2: 537.

I89o, Trans. R. Soc. S. Australia, I3: 44.

I935, Exotic Microlepidoptera, 4:574. (BM)

I88I, Proc. Linnean Soc. N.S. Wales, 5: I46.

I925, Treubia, E: 43I.

Igo9, Ann. Transvaal Mus. 2: 22, pl. 7, fig. 7. (T)

I go8, Journ. Bombay Nat. Hist. Soc. I8: 8I9.

I893, Proc. Linnean Soc. N.S. Wales, 7: 538.

1907, Journ. Bombay Nat. Hist. Soc. I7: 747. (BM)

1927, Exotic Microlepidoptera, 3: 328. (T)

I9I2, in Wytsman, Genera Insectorum, fasc. I33, p. I0.

I9I5, Exotic Microlepidoptera, I: 386. (BM)

IgI I, Trans. Linnean Soc. London, I4: 285 .

I934, Exotic Microlepidoptera, 4: 52I.

I909, Trans. Ent. Soc. London, I909: 2I. (BM)

I9I I, Journ. Bombay Nat. Hist. Soc. 2I: II7.

I924, Exotic Microlepidoptera, 3: I25.

I938, Institut des Parcs Nationaux du Congo Belge,

fasc. I4, p. I4, pl. 2, fig. 2.

I921, Exotic Microlepidoptera, 2: 462 .

1932, Exotic Microlepidoptera, 4: 272.

I9II, Ann. Transvaal Mus. 2: 235. (T)

I927, Insects of Samoa, 3, Lepidoptera, fasc. 2, p. 89 .

I9I8, Exotic Microlepidoptera, 2: 215. (BM)

I928, Exotic Microlepidoptera, 3:476. (BM)

I929, Exotic Microlepidoptera, 3: 520.

I904, Proc. Linnean Soc. N.S. Wales, 29: 284.

I933, Exotic Microlepidoptera, 4: 354. (BM)

I907, Proc. Linnean Soc. N.S. Wales, 32: I3I.

I904, Proc. Linnean Soc. N.S. Wales, 29: 408.

I887, Trans. Ent. Soc. London, I887: 277. (BM)

I93I, Journ. Linnean Soc. London, 37: 277.

I907, Journ. Bombay Nat. Hist. Soc. I7: 986.
I907, Journ. Bombay Nat. Hist. Soc. I7: 737 .

I921, Ann. Transvaal Mus. 8:63. (T)

I897, Proc. Limnean Soc. N.S. Wales, $22: 376$.

I9I4, Trans. Ent. Soc. London, I9I4: 243.

I907, Journ. Bombay Nat. Hist. Soc. I7: 736. (BM)

I934, Exotic Microlepidoptera, 4: 5I3. (BM)

I9I8, Exotic Microlepidoptera, 2: I93. (BM)

I921, Exotic Microlepidoptera, 2: 464.

I885, Proc. Linnean Soc. N.S. Wales, 9: Io8I.

1920, Ann. South African Mus. I7: 302. (SA)

I908, Proc. Linnean Soc. N.S. Wales, 32: Io8.

(BM)

ORSODYTIS

EPERMENIA

OCYSTOLA

TINEA

BRENTHIA 


trilithopa
triloba
trilobopa
trilopha
triloxias
trilychna
trimachaera
trimelaena
trimelanota
trimeris
trimetalla
trimetra
trimetropa
trimetropis
trimitra
trimolybda
trimolybdias
trimorpha
trinephela
trinervis
trinotata
trinummulata
triochrias
triophthalma
triortha
triorthias
triorthota
tripedila
tripetala
triphona
triphracta
triphracta
triplaca
triplaca
triplacodes
triplacomis
triplacopa
triplacopis
triplaneta
triplectectiplectra
triplea
triplatynta

triple

EUCOSMA

STATHMOPODA

EULIA

COSMOPTERYX

AEOLOSCELIS

CHRYSOCLISTA

BRENTHIA

ACROCLITA

HYPOSMOCOMA

BATRACHEDRA

ACROCERCOPS

CHIONOGENES*

METACHANDA

PROTOLECHIA

STAGMATOPHORA

ANACAMPSIS

STATHMOPODA

MACROBATHRA

TELPHUSA

AULACOMIMA*

STRYPHNOCOPA*

SCYTHRIS

SCYTHRIS

LECITHOCERA

PHYLLOCNISTIS

CHELARIA

EPICHORISTA

GLYPHIPTERYX

MONOPIS

THYRSOSTOMA

ECHINOPHRICTIS*

PLATYPTILA

ACROCERCOPS

PELTOSARIS*

DOLIOTECHNA

LITHOCOLLETIS

MONOPIS

GELECHIA

LIMNOECIA

PROTEROCOSMA*

EUPLOCAMUS

PACHYRHABDA

PHILOBOTA

STENOMA

XYSTOCEROS*

LITHOCOLLETIS

ARGYRESTHIA

APHANOXENA
I937, in Caradja and Meyrick, Deuts. Ent. Zeit. Iris, 5I: I78. (BM)

I913, Exotic Microlepidoptera, I: 85.

I926, Exotic Microlepidoptera, 3: 249. (BM)

1922, Exotic Microlepidoptera, 2: 566. (BM)

I907, Journ. Bombay Nat. Hist. Soc. I7: 983.

I928, Exotic Microlepidoptera, 3: 394.

I927, Insects of Samoa, 3, Lepidoptera, fasc. 2, p. I03.

1922, Exotic Microlepidoptera, 2: 52I. (BM)

I935, Proc. Hawaiian Ent. Soc. 9: 66. (SP)

I897, Proc. Linnean Soc. N.S. Wales, 22: 309.

I9I5, Trans. Ent. Soc. London, I9I5: 226.

I9I3, Exotic Microlepidoptera, I: I44.

I937, Exotic Microlepidoptera, 5: I45. (T)

I922, Arkiv för Zool. vol. I4, part I5, p. 5.

I9I3, Ann. Transvaal Mus. 3: 308. (T)

I9I4, Trans. Ent. Soc. London, I9I4: 258. (BM)

I926, Trans. New Zealand Inst. 56: 4I6.

I889, Proc. Linnean Soc. N.S. Wales, (Ser. 2) 3: 1675.

I929, Exotic Microlepidoptera, 3:487. (T)

Ig04, Proc. Linnean Soc. N.S. Wales, 29: 395.

I920, Exotic Microlepidoptera, 2: 307. (BM)

I922, Exotic Microlepidoptera, 2: 554. (BM)

I9I6, Exotic Microlepidoptera, 2: I5. (BM)

I9Io, Rec. Indian Mus. 5: 220. (K)

I906, Trans. R. Soc. S. Australia, 30:63.

I935, Exotic Microlepidoptera, 4: 589. (BM)

1927, Trans. Proc. New Zealand Inst. 57: 698.

I9I2, Exotic Microlepidoptera, I: 58. (BM)

I905, Journ. Bombay Nat. Hist. Soc. 16: 6r8.

I927, Insects of Samoa, 3, Lepidoptera, fasc. 2, p. 79. (Вм)

I922, Exotic Microlepidoptera, 2: 588.

I932, Exotic Microlepidoptera, 4: 334.

I908, Journ. Bombay Nat. Hist. Soc. I8: 8I 7.

I902, Trans. R. Soc. S. Australia, 26: I4I.

I920, Exotic Microlepidoptera, 2: 38I. (BM)

I936, Exotic Microlepidoptera, 5: 32.

I932, Trans. Ent. Soc. London, 80: I20.

I9I2, Ann. South African Mus. Io: 6I. (SA)

I92I, Exotic Microlepidoptera, 2: 453.

I886, Trans. Ent. Soc. London, I886: 293.

I934, in Caradja and Meyrick, Deuts. Ent. Zeit. Iris, t5: $^{5}$ 42.

I9I3, Ann. Transvaal Mus. 3: 3IO. (T)

I913, Exotic Microlepidoptera, I: I23.

I9I5, Exotic Microlepidoptera, I: 423. (BM)

I9I4, Exotic Microlepidoptera, I: 253. (BM)

I9I4, Journ. Bombay Nat. Hist. Soc. 23: IIS.

I9I4, Lepidopterorum Catalogus, pars I9, p. 4. (Sub.)

I9I6, Exotic Microlepidoptera, I: 490. (B.1) 
triploca

tripoda

tripolis

tripselia

triptila

triptycha

tripudians

tripunctella

trirecta

triscalma

triscelis

triscia

trisecta

triselena

triselena

trisema

trisemanta

trisigillata

trisignis

trisinuata

trisphaera

trissobathra

trissochroa

trissodelta

trissodesma

trissophricta

trissoptila

trissorrhiza

trissostacta

trissoxantha

tristephana

tristicta

tristicta

tristriata

tristrigata

trisulca

trita

trithalama

trithrona

trithyra

trithyra

trithyris

tritochlora

tritocosma

tritogramma

tritorrhecta
PHYLLOCNISTIS

AEOLOSCELIS

ACROCERCOPS

GLYPHIPTERYX

HYPOSMOCOMA

COESYRA

CHOROPLECA

DOLEROMIMA

STENOMA

ACROCERCOPS

GELECHIA

MELASINA

ZARATHA

GLYPHIPTERYX

STATHMOPODA

LIMNOECIA

METACHANDA

ACROCERCOPS

GELECHIA

ANTAEOTRICHA

HELIOZELA

DOLIOTECHNA

GRACILARIA

LIMNOECIA

PTILOCHARES*

EULIA

ACROCERCOPS

STATHMOPODA

OPOGONA

STROBISIA

[CATOPTRISTIS*]

EUPSELIA

BATRACHEDRA

LOZOSTOMA

EULIA

PHTHORIMAEA

HARMOLOGA

[GELOPHAULA*]

TINEA

MOMPHA

EUPSELIA

ARGYROPLOCE

MACROBATHRA

AEGERIA

HARMOLOGA

HOPLOPHANES*

ANTAEOTRICHA

LITHOCOLLETIS
1928, Exotic Microlepidoptera, 3: 406.

I9I3, Ann. Transvaal Mus. 3: 3II. (T)

I921, Zool. Meded. 6: 190. (L)

I907, Proc. Linnean Soc. N.S. Wales, 32: I29.

19I5, Exotic Microlepidoptera, I: 340. (вM)

1884, Proc. Linnean Soc. N.S. Wales, 9: 771.

1924, Exotic Microlepidoptera, 3: 65.

I902, [Meyr. nec Walk.] Trans. R. Soc. S. Australia, 26: I59.

I93I, Exotic Microlepidoptera, 4: 38. (BM)

I9I6, Exotic Microlepidoptera, 2: I.

I9I7, Ann. South African Mus. I7: 3. (SA)

I9I4, Exotic Microlepidoptera, I: 212.

I9I5, Exotic Microlepidoptera, I: 335.

188I, Proc. Linnean Soc. N.S. Wales, 5: 234.

1897, Proc. Linnean Soc. N.S. Wales, 22: 318.

1897, Proc. Linnean Soc. N.S. Wales, 22: 361.

1930, Trans. Ent. Soc. London, 78: 316. (P)

I92I, Exotic Microlepidoptera, 2: 470.

1908, Proc. Zool. Soc. London, p. 725.

1930, Exotic Microlepidoptera, 4: 21. (BM)

1897, Proc. Linnean Soc. N.S. Wales, 22: 405.

I920, Exotic Microlepidoptera, 2: 380. (BM)

I931, Exotic Microlepidoptera, 4: 48.

I922, Exotic Microlepidoptera, 2: 573.

I887, Proc. Linnean Soc. N.S. Wales, (Ser. 2) I: 1047.

1932, Exotic Microlepidoptera, 4: 259. (v)

I921, Exotic Microlepidoptera, 2: 4I7.

I939, Trans. R. Ent. Soc. London, 89: 58.

1934, Pacific Ent. Surv. Publ. 7, art. 28, p. 354. (вB)

1922, Trans. Ent. Soc. London, I922: Ioo. (BM)

I9I5, Exotic Microlepidoptera, I: 306.

I902, Trans. Ent. Soc. London, I902: 579.

I897, Proc. Linnean Soc. N.S. Wales, 22: 4I9.

I93I, Exotic Microlepidoptera, 4: I5I. (v)

I938, Institut des Parcs Nationaux du Congo Belge, fasc. I4, p. I2.

I9I6, Trans. Proc. New Zealand Inst. 48: 4I4.

I925, Treubia, 6: 433 .

I922, Exotic Microlepidoptera, 2: 578 . (BM)

I906, Trans. R. Soc. S. Australia, 30: 39.

I92I, Ann. Transvaal Mus. 8: 6I. (T)

I886, Proc. Limnean Soc. N.S. Wales, 10: 808 .

I926, Exotic Microlepidoptera, 3: 266. (BM)

I9I2, Trans. Proc. New Zealand Inst. 44: I20.

I897, Proc. Linnean Soc. N.S. Wales, 22: 4II.

I925, Exotic Microlepidoptera, 3: I76. (BM)

I935, Exotic Microlepidoptera, 4: 596. 


\begin{tabular}{|c|c|c|}
\hline tha & EOCHROIS & I886, Proc. Linnean Soc. N.S. Wales, Io: 828. \\
\hline triturata & FRISILIA & I9I4, Exotic Microlepidoptera, I: 277. (BM) \\
\hline tritypa & STENOMA & I909, Trans. Ent. Soc. London, I909: 32. (BM) \\
\hline triumphalis & IDIOGLOSSA & I9I8, Ann. Transvaal Mus. 6: 56. (T) \\
\hline ivallata & ANTAEOTRICHA & I934, Exotic Microlepidoptera, 4: 465. (v) \\
\hline trivia & PHILOBOTA & I9I5, Exotic Microlepidoptera, I: 303. \\
\hline trivia & TORTRIX & I9I3, Ent. Mitteil. 2: 297. (D) \\
\hline trivialis & BORKHAUSENIA & I9I4, Exotic Microlepidoptera, I: I72. \\
\hline trivialis & PTISANORA* & I9I3, Ann. Transvaal Mus. 3: 334. (т) \\
\hline ncta & PYRODERCES & I897, Proc. Linnean Soc. N.S. Wales, 22: 353. \\
\hline antha & METACHANDA & I9I I, Trans. Linnean Soc. London, I4: 276. (BM) \\
\hline phias & ARGYROPLOCE & I939, Trans. R. Ent. Soc. London, 89: 5I. (c) \\
\hline sta & OINOPHILA & I9I0, Trans. Ent. Soc. London, I9I0: 376. \\
\hline eucta & PTILOGENES & I930, Ann. Naturhist. Mus. Wien, 44: 258. (v) \\
\hline zona & PSELIASTIS* & I897, Proc. Linnean Soc. N.S. Wales, 22: 407. \\
\hline iga & PHLOEOPOLA & I9I4, Exotic Microlepidoptera, I: I69. \\
\hline haea & TINEA & I9I I, Trans. Linnean Soc. London, I4: 304. \\
\hline hala & PROCOMETIS & I9o8, Journ. Bombay Nat. Hist. Soc. I8: 635. (BM) \\
\hline chaula & ARGYRESTHIA & $\begin{array}{l}\text { I938, in Caradja and Meyrick, Deuts. Ent. Zeit. Iris, } 52 \\
\text { 22. (BM) }\end{array}$ \\
\hline hias & PROTOLECHIA & I92I, Exotic Microlepidoptera, 2: 428. \\
\hline hilodes & PERONEA & I9I2, Trans. Ent. Soc. London, I9II: 687. (BM) \\
\hline histis & STENOMA & I9I6, Exotic Microlepidoptera, I: 5I8. (BM) \\
\hline hoscia & ANTAEOTRICHA & I9I5, Exotic Microlepidoptera, I: 396. (вм) \\
\hline ochosticha & PHRIXOSCELES* & I9o8, Journ. Bombay Nat. Hist. Soc. I8: 8I5. \\
\hline octis & PIGRITIA & I922, Exotic Microlepidoptera, 2: 538. (вM) \\
\hline trogalia & SIMAETHIS & I9I2, Exotic Microlepidoptera, I: 43. (BM) \\
\hline merodes & CRYPSITHYRIS & I921, Zool. Meded.6: I98. (L) \\
\hline aea & POLYHYMNO & I9o8, Proc. Zool. Soc. London, p. 728. \\
\hline aea & SYNTOMACTIS & I897, Proc. Linnean Soc. N.S. Wales, $22: 389$. \\
\hline trophias & AMYDRIA & Igo8, Proc. Zool. Soc. London, p. 753. \\
\hline trophiodes & EUCosma & I9o8, Journ. Bombay Nat. Hist. Soc. I8: 61 3. (BM) \\
\hline his & OPOGONA & I9I3, Ann. Transvaal Mus. 3: 33o. (T) \\
\hline tropica & EULECHRIA & I888, Proc. Linnean Soc. N.S. Wales, (Ser. 2) 2: 955. \\
\hline dculenta & BACTRA & I9o9, Journ Bombay Nat. Hist. Soc. I9: 586 . (BM) \\
\hline culenta & MACHAEROPTERIS & I9I6, Exotic Microlepidoptera, I: 6I4. \\
\hline ca & MACHIMIA & I930, Ann. Naturhist. Mus. Wien, 44: 232. (v) \\
\hline catula & AGRIOPHARA & I9I3, Trans. Ent. Soc. London, 46: I85. (BM) \\
\hline hota & BRACHYACMA & I929, Exotic Microlepidoptera, 3: 5IO. (BM) \\
\hline gaula & MACHIMIA & I9I2, Trans. Ent. Soc. London, I9I : 699. (вм) \\
\hline godana & TORTRIX & I88I, Proc. Linnean Soc. N.S. Wales, 6: 5I5. \\
\hline nalopa & STENOMA & I925, Exotic Microlepidoptera, 3: 2II. (BM) \\
\hline ohera & TINEA & I893, Proc. Limnean Soc. N.S. Wales, 7: 54I. \\
\hline pherantis & ANTISPILA & I9I6, Exotic Microlepidoptera, 2: 8. \\
\hline pheropa & PISISTRATA* & I924, Exotic Microlepidoptera, 3: 8I. (13) \\
\hline phoxantha & EPIPHRACTIS & I930, Trans. Ent. Soc. London, $78: 3$ I8. (P) \\
\hline erculata & LICHENAULA & I89o, Trans. R. Soc. S. Australia, I3: 50 . \\
\hline icen & PHANERODOXA* & I92I, Exotic Microlepidoptera, 2: 393. (BM) \\
\hline gera & CRATINITIS* & I935, Exotic Microlepidoptera, 4: 56r. (13:) \\
\hline yurialis & TINEA & I932, Exotic Microlepidoptera, 4: 326. \\
\hline
\end{tabular}


tumens

tumescens

STENOMA

CTENOCOMPA

tumida

tumida

tumulata

tumulata

tumulifera

tumultuosa

tunicata

turbata

turbida

turbinalis

turbinaris

turbinata

turbinea

turbinea

turbulenta

turgida

turifera

turilega

turmaria

turrigera

turrita

tylephora

tylobathra

tylodes

tylogramma

tylonota

tylota

tympanias

tympanista

typhicola

typhlodes

typhlopa

typhlopis

typhodes

typhonica

typicantha

typicodes

typota

tyranna

tyrannica

tyranthes

tyrastis

tyreuta
PACHYRHABDA

ANCYLIS

CYDIA

STENOMA

MICROCOLONA

PHALANGITIS

MACHAEROCRATES*

MACHAEROPTERIS

EPIMACTIS

TRICLONELLA

CLYSIA

HOMALOXESTIS

PERIACMA

PERIACMA

PERIACMA

TRICHOTAPHE

LASPEYRESIA

SIMAETHIS

ARGYROPLOCE

STILBOSIS

TRICHOTAPHE

THIOTRICHA

LECITHOCERA

TINEA

ENDOPHTHORA

CAPUA

MELASINA

HERMOGENES

ORTHIOSTOLA

SCIEROPEPLA*

HEMIMENE

MONOPIS

BRACHMIA

MYRMECOZELA

ERETMOCERA

EULECHRIA

EUCOSMA

ODITES

OCYSTOLA

CHRYSORYCTIS

ETHMIA

PRAYS

SCLEROPHRICTA*
I9I6, Exotic Microlepidoptera, I: 5II. (BM)

I935, in Caradja and Meyrick, Materialien zu einer Micro-

lepidopteren Fauna der Chinesischen Provinzen Kiangsu,

Chekiang und Hunan, p. 93. (BM)

I9I3, Exotic Microlepidoptera, I: 94.

I9I2, Exotic Microlepidoptera, I: 30. (Вм)

rgo8, Proc. Zool. Soc. London, p. 720. (BM)

I9I6, Exotic Microlepidoptera, I : 538. (Bм)

I92I, Zool. Meded. 6: I69. (L)

I907, Proc. Linnean Soc. N.S. Wales, 32: I37.

I931, Exotic Microlepidoptera, 4: I76. (v)

I922, Exotic Microlepidoptera, 2: 600.

I9I4, Journ. Bombay Nat. Hist. Soc. 22: 779. (BM)

1933, Exotic Microlepidoptera, 4: 366. (BM)

I928, Exotic Microlepidoptera, 3: 435. (BM)

I9Io, Journ. Bombay Nat. Hist. Soc. 20: 442. (BM)

IgI6, Exotic Microlepidoptera, x: 554. (BM)

I9I8, Exotic Microlepidoptera, 2: 22I.

I9Io, Journ. Bombay Nat. Hist. Soc. 20: x58. (BM)

r9I 8, Ann. Transvaal Mus. 6:24. (T)

I9I2, Journ. Bombay Nat. Hist. Soc. 2I: 876. (вм)

1924, Trans. Ent. Soc. London, I923: 554. (BM)

I93I, Exotic Microlepidoptera, 4: I3I.

I92I, Exotic Microlepidoptera, 2: 4I4.

I9I4, Trans. Ent. Soc. London, I9I4: 279. (BM)

1935, in Caradja and Meyrick, Materialien zu einer Micro-

lepidopteren Fauna der Chinesischen Provinzen Kiangsu,

Chekiang und Hunan, p. 68. (Bм)

I93I, in Caradja, Bull. Sect. sci. Acad. roum. I4: 69. (BM)

I9I9, Exotic Microlepidoptera, 2: 247. (BM)

I924, Trans. Proc. New Zealand Inst. 55: 206.

I926, Exotic Microlepidoptera, 3:247. (BM)

I9I6, Exotic Microlepidoptera, I: 6I3.

I9o8, Journ. Bombay Nat. Hist. Soc. I8: 628. (вм)

I 928, Exotic Microlepidoptera, 3: 479. (вм)

I886, Trans. Proc. New Zealand Inst. I8: I65.

I93I, Exotic Microlepidoptera, 4: I42.

1925, Rec. Cant. Mus. 2: 274.

IgII, Journ. Bombay Nat. Hist. Soc. 20: 7I5. (BM)

I9I7, Exotic Microlepidoptera, 2: 88.

I9I7, Exotic Microlepidoptera, 2: 64 .

I9I8, Exotic Microlepidoptera, 2: 2I4.

I9I2, Ent. Mon. Mag. 48: 35. (Sub.)

I9I5, Exotic Microlepidoptera, I: 379.

I885, Proc. Linnean Soc. N.S. Wales, 9: ro68.

I893, Proc. Linnean Soc. N.S. Wales, 7: 549.

I934, Exotic Microlepidoptera, 4: 459. (c)

I907, Proc. Linnean Soc. N.S. Wales, 32: 75.

IgI8, Ann. Transvaal Mus. 6: 46 . (T) 


\begin{tabular}{|c|c|}
\hline tyriancha & PARECTOPA \\
\hline tyriarcha & LIMNOECIA \\
\hline tyriochalca & AMPHITHERA \\
\hline tyriochrysa & NEMOTOIS \\
\hline tyriocoma & ORPHANOCLERA * \\
\hline tyriopla & HIEROXESTIS \\
\hline tyrobathra & LEPIDOSCIA \\
\hline tyrochlora & CEROMITIA \\
\hline tyrochyta & CRYPTOLECHIA \\
\hline tyrocnista & IMMA \\
\hline tyrocnistis & TISCHERIA \\
\hline tyrocopa & THRASYDOXA* \\
\hline tyrocrossa & STENOMA \\
\hline tyrodes & HYPONOMEUTA \\
\hline tyrophanes & MELASINA \\
\hline tyrophthora & SPATALISTIS \\
\hline tyropiesta & STENOPTILIA \\
\hline tyropis & BORKHIAUSENIA \\
\hline tyroplaca & IMMA \\
\hline tyrota & TRICHOCIRCA* \\
\hline tyrotoma & LIMNOECIA \\
\hline tyroxantha & PHILOBOTA \\
\hline tyroxesta & STENOMA \\
\hline
\end{tabular}

$\begin{array}{ll}\text { uberana } & \text { HELICTOPHANES* } \\ \text { uberrima } & \text { HYPERCALLIA } \\ \text { ulmicola } & \text { PERONEA } \\ \text { ulocoma } & \text { SCALIDEUTIS } \\ \text { ulosema } & \text { STENOMA } \\ \text { ultrix } & \text { PROTEOPTERYX } \\ \text { umbellifera } & \text { EULIA } \\ \text { umbraculata } & \text { EUXANTHIS } \\ \text { umbratilis } & \text { TORTRIX } \\ \text { umbrigera } & \text { TRICLONELLA } \\ \text { umbrinervis } & \text { STENOMA } \\ \text { umbrosa } & \text { EULECHRIA } \\ \text { umbrosa } & \text { MERITASTIS* } \\ \text { uncta } & \text { GLYPHIPTERYX } \\ \text { uncticoma } & \text { STENOMA } \\ \text { unctoria } & \text { PACHYRHABDA } \\ \text { undifraga } & \text { ACROCERCOPS } \\ \text { undina } & \text { APATETRIS } \\ \text { undulata } & \text { MELASINA } \\ \text { unguentaria } & \text { HELIOCAUSTA } \\ \text { unguentata } & \text { STENOMA } \\ \text { unguifera } & \text { GLYPIIPTERYX }\end{array}$

I920, Exotic Microlepidoptera, 2: 296. (BM)

I9I9, Exotic Microlepidoptera, 2: 234.

I930, Exotic Microlepidoptera, 3: 58\%.

I935, in Caradja and Meyrick, Materialien zu einer Micro-

lepidopteren Fauna der Chinesischen Provinzen Kiangsu,

Chekiang und Hunan, p. 94.

1925, Treubia, 6: 430.

I927, Insects of Samoa, 3, Lepidoptera, fasc. 2, p. Iro.

1893, Proc. Limnean Soc. N.S. Wales, 7: 5 II.

Igo8, Proc. Zool. Soc. London, p. 756.

I9Io, Journ. Bombay Nat. Hist. Soc. 20: I64. (BM)

1906, Trans. Ent. Soc. London, I906: I99. (BM)

1934, Exotic Microlepidoptera, 4: 461.

I9I2, Exotic Microlepidoptera, I: 60.

1925, Exotic Microlepidoptera, 3: 183. (Bм)

I9I3, Exotic Microlepidoptera, I: I38. (BM)

I917, Ann. Natal Mus. 3:616. (T)

I9I2, Exotic Microlepidoptera, I: I5. (BM)

I932, Trans. Ent. Soc. London, 80: Iog.

1935, Exotic Microlepidoptera, 4: 566.

1925, Exotic Microlepidoptera, 3: I34. (BM)

I920, Voyage de Ch. Alluaud et R. Jeannel en Afrique

Oriental, II, Microlepidoptera, p. 86. (P)

1938, Trans. R. Ent. Soc. London, 87: 520.

I884, Proc. Linnean Soc. N.S. Wales, 8: 497.

1925, Exotic Microlepidoptera, 3: 213. (BM)

I88I, Proc. Linnean Soc. N.S. Wales, 6: 639.

I9I4, Exotic Microlepidoptera, I: I90. (BM)

1930, Exotic Microlepidoptera, 3: 612. (BM)

I9I8, Exotic Microlepidoptera, 2: 220. (BM)

I930, Exotic Microlepidoptera, 3: 559.

IgI2, Ent. Mon. Mag. 48: 34. (Sub.)

I926, Exotic Microlepidoptera, 3: 254. (BM)

I9I8, Ann. Transuaal Mus. 6: 8. (Bм)

I922, Exotic Microlepidoptera, 2: 496.

1929, Trans. Ent. Soc. London, 76: 512.

1930, Ann. Naturhist Mus. Wien, +4: 241. (v)

I9I4, Exotic Microlepidoptera, I: I67. (MA)

I9Io, Proc. Limnean Soc. N.S. Wales, 35: 255. (M.А)

I9I3, Exotic Microlepidoptera, I: IOI. (BM)

1916, Exotic Microlepidoptera, I: 528. (вм)

I9I2, Ann. Transvaal Mus. 3: 7I. (T)

I931, Exotic Microlepidoptera, 4: 47.

r921, Ann. Transwaal Mus. 8: 64. (T)

I9Ir, Amn. Transvaal Mus. 2: 239.

I921, Exotic Microlepidoptera, 2: 380.

I930, Ann. Naturhist. Mus. Wien, 4t: 250. (v)

I922, Exotic Microlepidoptern, $2:+89$. (BM) 


\begin{tabular}{|c|c|}
\hline ungulata & ORTHENCHES \\
\hline ungulifera & PTILOGENES \\
\hline uniformis & $\begin{array}{c}\text { OECOPHORA } \\
\text { [GUESTIA*] }\end{array}$ \\
\hline unilorata & HYPERCALLIA \\
\hline unisecta & STENOMA \\
\hline unisignis & STENOMA \\
\hline univoca & BUCCULATRIX \\
\hline uranarcha & MACARANGELA \\
\hline uranatma & LASPEYRESIA \\
\hline uranauges & AEGERIA \\
\hline uranaula & MAGOSTOLIS* \\
\hline urania & CHRYSOCENTRIS \\
\hline uraninas & AETHERASTIS* \\
\hline uranitis & IMMA \\
\hline uranopa & IRIANASSA \\
\hline uranophanes & STENOMA \\
\hline uranopis & ZALITHIA * \\
\hline uranopla & IDIOPOGON* \\
\hline urantha & STAGMATOPHORA \\
\hline urbana & ACROCERCOPS \\
\hline urbica & NEPTICULA \\
\hline urnigera & EUCOSMA \\
\hline urosema & PHTHORIMAEA \\
\hline ursina & CACOECIA \\
\hline ursinella & ARCTOCOMA* \\
\hline ursula & METACHORISTA* \\
\hline usitata & DEPRESSARIA \\
\hline uvida. & OPOSTEGA \\
\hline
\end{tabular}

vacans

vacata

vacuefacta

vadata

vadosa

vafra

vagata

vagula

valida

vallaris

vallata

vallicola

vallifera

vallifica

valligera
I9I3, Exotic Microlepidoptera, I: I49.

I929, Trans. Ent. Soc. London, 76:517. (BM)

I886, Proc. Linnean Soc. N.S. Wales, Io: 781.

I933, Exotic Microlepidoptera, 4: 368. (BM)

I930, Exotic Microlepidoptera, 4: 26. (v)

I932, Exotic Microlepidoptera, 4: 296. (v)

I9I8, Exotic Microlepidoptera, 2: I85.

I893, Proc. Linnean Soc. N.S. Wales, 7: 588.

1936, Exotic Microlepidoptera, 4: 615. (BM)

I926, Exotic Microlepidoptera, 3: 266.

I887, Proc. Linnean Soc. N.S. Wales, (Ser. 2) I: I040.

1920, Ann. South African Mus. I7: 297. (SA)

I909, Journ. Bombay Nat. Hist. Soc. I9: 422. (BM)

I9Io, Trans. Ent. Soc. London, I9I0: 465 . (вм)

I927, Insects of Samoa, 3, Lepidoptera, fasc. 2, p. IOI.

I93I, Exotic Microlepidoptera, 4: 4I. (BM)

1894, Trans. Ent. Soc. London, p. I8. (BM)

1934, Exotic Microlepidoptera, 4: 456.

I9I4, Supplementa Entomologica, No. 3, p. 53. (D)

I908, Journ. Bombay Nat. Hist. Soc. I8: 816.

1913, Ann. Transwaal Mus. 3: 326. (T)

1937, Exotic Microlepidoptera, 5: 158.

I9I7, Trans. Ent. Soc. London, p. 43. (BM)

I910, Proc. Linnean Soc. N.S. Wales, 35: 217.

I88I, Proc. Linnean Soc. N.S. Wales, 5: I7I.

I938, Trans. R. Ent. Soc. London, 87: 510. (BM)

I883, [Meyr. nec Butl.] Ent. Mon. Mag. 20: 33. (Sub.)

1915, Exotic Microlepidoptera, I: 352.

I9I6, Exotic Microlepidoptera, I: 5I9. (BM)

I925, Exotic Microlepidoptera, 3: I70. (BM)

1925, Exotic Microlepidoptera, 3: I48.

1922, Exotic Microlepidoptera, 2: 580. (BM)

1925, Exotic Microlepidoptera, 3: I53.

1921, Ann. Transvaal Mus. 8: I34. (T)

Igr6, Trans. Proc. Nero Zealand Inst. 48: 416.

1919, Exotic Microlepidoptera, 2: 262.

19I9, Exotic Microlepidoptera, 2: 286. (BM)

I9I5, Trans. Ent. Soc. London, I9I5: 243.

1897, Proc. Limnean Soc. N.S. W'ales, 22: 428.

1925, in Wytsman, Genere Insectornm, fasc. 184, p. 44.

(Sub.)

I9I4, Exotic Microlepidoptera, I: 226. (BMI)

1935, in Caradja and Meyrick, Materialien zu einer Micro-

lepidopteren Fauna der Chinesischen Provinzen Kiangsu,

Chekiang und Hunan, p. 49.

1929, Trans. Ent. Soc. London, 76:498. 


\begin{tabular}{|c|c|}
\hline vanescens & HAPLODYTA \\
\hline vannifera & NEPTICULA \\
\hline vannifera & STENOMA \\
\hline vanula & ACROCERCOPS \\
\hline vaporalis & DALACA \\
\hline vaporata & EULECHRIA \\
\hline variana & STEGASTA* \\
\hline variata & GLYPHIPTERYX \\
\hline varicosa & MELASINA \\
\hline variegata & EULECHRIA \\
\hline variegatus & OXYPTILUS \\
\hline varius & TRICHOPTILUS \\
\hline vasculigera & ENDOTHENIA \\
\hline vasifera & STENOMA \\
\hline vastata & ELACHISTA \\
\hline vates & THAMBOTRICHA * \\
\hline vaticina & IMMA \\
\hline vecors & BRACHMIA \\
\hline vectaria & ANARSIA \\
\hline vectigalis & CYANARMOSTIS* \\
\hline vegrandis & COESYRA \\
\hline velata & EULECHRIA \\
\hline velifera & OPOSTEGA \\
\hline veligera & DIACTENIS \\
\hline velipotens & ODITES \\
\hline velitans & TORTRIX \\
\hline velitaris & BRACHMIA \\
\hline velox & BATRACHEDRA \\
\hline venatrix & ANTICRATES \\
\hline venatrix & ARGYROTOXA \\
\hline venefica & COSMOPTERYX \\
\hline venenata & EPICEPHALA \\
\hline veneranda & EPICEPHALA \\
\hline veneratrix & TORTRIX \\
\hline venerea & PHYCODES \\
\hline veniflua & CRYPTOLECHIA \\
\hline ventilatrix & STENOMA \\
\hline ventosa & DICHOMERIS \\
\hline venusta & OEDEMATOPODA \\
\hline vera & PHILOBOTA \\
\hline verax & BATRACHEDRA \\
\hline verax & BUCCULATRIX \\
\hline verax & PTEROPHORUS \\
\hline verberata & BRACHMIA \\
\hline verberata & PLEXIPPICA* \\
\hline verecunda & AMYDRIA \\
\hline verecunda & CRUSIMETRA * \\
\hline veridica & PROMALACTIS \\
\hline
\end{tabular}

I9I5, Exotic Microlepidoptera, I: 301.

I9I4, Ann. Transvaal Mus. 4:203.

I9I5, Exotic Microlepidoptera, I: 4I6. (BM)

I9I2, in Wytsman, Genera Insectorum, fasc. I28, p. I7.

I92I, Ann. Transvaal Mus. 8: I42. (T)

I9I4, Exotic Microlepidoptera, I: 240. (BM)

I904, Proc. Linnean Soc. N.S. Wales, 29: 3I4.

I9I3, Exotic Microlepidoptera, I: I04. (BM)

I920, Voyage de Ch. Alluaud et R. Jeannel en Afrique

Oriental, II, Microlepidoptera, p. III. (P)

I883, Proc. Linnean Soc. N.S. Wales, 7: 528.

I920, Ann. South African Mus. I7: 274. (SA)

I909, Ann. Transvaal Mus. 2: 2, pl. I, fig. 2. (T)

I938, Institut des Parcs Nationaux du Congo Belge,

fasc. I4, p. 9, pl. I, fig. 7 .

1925, Exotic Microlepidoptera, 3: 204. (BM)

I932, Exotic Microlepidoptera, 4: 217.

I924, Trans. Proc. New Zealand Inst. 55: 205.

I9I2, Exotic Microlepidoptera, I: 39. (BM)

I9I8, Exotic Microlepidoptera, 2: II2. (BM)

I9I8, Ann. Transvaal Mus. 6:2I. (T)

1927, Exotic Microlepidoptera, 3: 380. (Bм)

I884, Proc. Linnean Soc. N.S. Wales, 9: 790.

I9I4, Exotic Microlepidoptera, I: 239. (BM)

1920, Exotic Microlepidoptera, 2: 357.

I928, Exotic Microlepidoptera, 3: 458. (Bм)

I935, Exotic Microlepidoptera, 4: 572. (BM)

I923, Exotic Microlepidoptera, 3: 54. (P)

I913, Ann. Transvaal Mus. 3: 295.

1897, Proc. Linnean Soc. N.S. Wales, 22: 302.

I9I4, Journ. Bombay Nat. Hist. Soc. 23: I28. (вм)

I930, Exotic Microlepidoptera, 3:6I2. (BM)

I9I5, Trans. Ent. Soc. London, I9I5: 204. (BM)

I935, Exotic Microlepidoptera, 4: 596.

I9o9, Ann. Transvaal Mus. 2: 24, pl. 8, fig. I. (T)

I9I2, Ent. Mon. Mag. 48: 35. (Sub.)

I921, Zool. Meded. 6: I8I. (L)

I9I4, Exotic Microlepidoptera, I: 227. (BM)

I9I6, Exotic Microlepidoptera, I: 527. (BM)

I9I3, Ann. Transvaal Mus. 3: 304. (T)

I9I3, Exotic Microlepidoptera, I: 97.

I9I4, Exotic Microlepidoptera, I: 250.

I9I7, Exotic Microlepidoptera, 2: 33.

I9I8, Exotic Microlepidoptera, 2: I84.

Igog, Ann. Transvaal Mus. 2: 3, pl. I, fig. 5. (T)

I912, Ann. Transvaal Mus. 3: 68. (BM)

I9I2, Ann. South African Mus. Iо (3):67. (вм)

I9II, Ann. Transuaal Mus. 3: 83 .

I9I2, Journ. Bombay Nat. Hist. Soc. 21: 855. (B.M)

I9I3, Ann. Transwaal Mus. 3:3I5. 
vermicularis PHILEDONE

yermiculata

vermiformis

verna

vernacula

vernalis

versabilis

versatilis

versatrix

versicolor

versuta

vertebrata

verticosa

verticosa

veruta

vesana

vesperascens

vespertilio

vespertina

vestigata

vestigialis

veterana

veterascens

veterata

vetula

vetusta

vexata

vexillaris

vexillata

vexillifera

viatica

viatica

viatrix

vibrans

vibrans

vibrata

vibratrix

vicana

vicaria

victrix

victrix

vicularis

viduata

viduus

vigescens

vigilans

vigilans

vigilax
ARGYROPLOCE

EPICEPHALA

TINEA

MACHAEROPTERIS

ERIODYTA

EULIA

PHANERODOXA

MACHETIS

GLYPHIPTERYX

ATOMOTRICHA

STATHMOPODA

CHELARIA

FRISILIA

ANARSIA

CENARCHIS*

ENCHOCRATES

ACROLOPHUS

CRYPTOLECHIA

RECURVARIA

EPICHORISTA

PHALANGITIS*

LEURONOMA

CENARCHIS

TINEA

SYMMOCA

STENOMA

COSMOPTERYX

COPTOTELIA

ZACORISCA

ACROCERCOPS

MONOPIS

ANISOPLACA

GRACILARIA

OXYPTILUS

PHTHOROPOEA

HELIOSTIBES

ARISTOTELIA

PHTHORIMAEA

LASPEYRESIA

STROBISIA

AUTOSTICHA

EULECHRIA

TRICHOPTILUS

ACROCLITA

HYSTEROSIA

ILINGIOTIS

ONEBALA
I935, in Caradja and Meyrick, Materialien zu einer Microlepidopteren Fauna der Chinesischen Provinzen Kiangsu. Chekiang und Hunan, p. 48. (BM)

I9I2, Trans. Ent. Soc. London, I9II: 69o. (Вм)

1936, Exotic Microlepidoptera, 5: 33.

1924, Exotic Microlepidoptera, 3: 72. (BM)

I9I I, Journ. Bombay Nat. Hist. Soc. 21: 130.

I883, Proc. Linnean Soc. N.S. Wales, 8: 5I9.

I926, Exotic Microlepidoptera, 3: 25I. (BM)

I92I, Exotic Microlepidoptera, 2: 394. (BM)

I9r4, Exotic Microlepidoptera, I: I7I. (MA)

I9r3, Exotic Microlepidoptera, I: ro3. (Lost)

I9I4, Trans. Proc. New Zealand Inst. 46: rog.

I9I4, Supplementa Entomologica, No. 3, p. 55. (D)

I913, Journ. Bombay Nat. Hist. Soc. 22: I66. (BM)

I9I4, Journ. Bombay Nat. Hist. Soc. 22: 772. (Bм)

I9I8, Exotic Microlepidoptera, 2: I48. (BM)

I924, Trans. Ent. Soc. London, I923: 550. (BM)

I921, Exotic Microlepidoptera, 2: 390.

I93I, Exotic Microlepidoptera, 4: I02. (v)

I9Io, Journ. Bombay Nat. Hist. Soc. 20: I62. (BM)

I929, Exotic Microlepidoptera, 3: 485. (BM)

I9I4, Ann. South African Mus. Io: 243. (SA)

I907, Proc. Linnean Soc. N.S. Wales, 32 : I38.

I9I8, Ann. Transvaal Mus. 6: I6. (T)

I924, Trans. Ent. Soc. London, I923: 552. (BM)

1893, Proc. Linnean Soc. N.S. Wales, 7: 544.

I93I, Exotic Microlepidoptera, 4: 72. (v)

I9I5, Exotic Microlepidoptera, I: 444. (BM)

I909, Journ. Bombay Nat. Hist. Soc. I9: 4I8.

I9I3, Trans. Ent. Soc. London, I9I3: I79. (BM)

I924, Exotic Microlepidoptera, 3: II2.

I916, Exotic Microlepidoptera, I: 625.

I9II, Journ. Bombay Nat. Hist. Soc. 2I: II4.

I921, Zool. Meded. 6: I65. (L)

I9I8, Ann. Transvaal Mus. 6: 40. (T)

I92I, Ann. Transvaal Mus. 8: 49. (T)

I9I3, Ann. Transvaal Mus. 3: 335. (T)

I927, Trans. Proc. New Zealand Inst. 57: 702.

I9I7, Trans. Ent. Soc. London, I9I7: 36. (BM)

I92I, Ann. Transvaal Mus. 8: 74. (T)

r9I8, Ann. Transvaal Mus. 6: I2. (T)

I9II, Journ. Bombay Nat. Hist. Soc. 20: 727. (BM)

I9I I, Journ. Bombay Nat. Hist. Soc. 20: 725.

I920, Exotic Microlepidoptera, 2: 37 I.

I9I7, Ann. South African Mus. I7: I. (SA)

I920, Exotic Microlepidoptera, 2: 343. (BM)

I9I2, Ent. Mon. Mag. 48: 35. (Sub.)

I9I4, Trans. Ent. Soc. London, I9I4: 275. (BM)

I9Io, Journ. Bombay Nat. Hist. Soc. 20: 455. (BM) 


$\begin{array}{ll}\text { vigilis } & \text { COLEOPHORA } \\ \text { vilis } & \text { TIQUADRA } \\ \text { viminea } & \text { PARECTOPA } \\ \text { vinacea } & \text { HOMALEDRA } \\ \text { vinaria } & \text { TRACHYPEPLA } \\ \text { vincta } & \text { HYSTEROSIA } \\ \text { vinculata } & \text { ARGYROPLOCE } \\ \text { vinculigera } & \text { ARGYROPLOCE } \\ \text { vindemians } & \text { ARGYROPLOCE } \\ \text { vindex } & \text { GORGOPIS } \\ \text { vindex } & \text { PHALARITICA* } \\ \text { vindicata } & \text { ADOXOPHYES } \\ \text { vindicatrix } & \text { HAEMANGELA } \\ \text { vineata } & \text { ANTITHYRA* } \\ \text { vinifera } & \text { STENOMA } \\ \text { vinolenta } & \text { ACOMPSIA } \\ \text { vinosa } & \text { PERSICOPTILA } \\ \text { violaria } & \text { TRICHOTAPHE } \\ \text { violascens } & \text { GNORISMONEURA } \\ \text { violenta } & \text { BATRACHEDRA } \\ \text { violescens } & \text { LASPEYRESIA } \\ \text { viperina } & \text { ECHIOMIMA } \\ \text { virens } & \text { PRASOLITHITES* } \\ \text { virginalis } & \text { BRENTHIA } \\ \text { virginea } & \text { DOLIOTECHNA } \\ \text { virgularia } & \text { STOCHASTICA* } \\ \end{array}$

$\begin{array}{ll}\text { virgulata } & \text { LITHOCOLLETIS } \\ \text { viridans } & \text { SOROTACTA* } \\ \text { viridans } & \text { SPILONOTA } \\ \text { viridescens } & \text { PERONEA } \\ \text { viridescens } & \text { ZALITHIA } \\ \text { virilis } & \text { PLATYPTILIA } \\ \text { viscata } & \text { ZONOPETALA } \\ \text { viscida } & \text { CEROMITIA } \\ \text { viscosa } & \text { PACHYRHABIA } \\ \text { vitiata } & \text { MERIDARCHIS } \\ \text { viticola } & \text { DEUTEROCOPUS } \\ \text { vitiosa } & \text { SPILONOTA } \\ \text { vitrea } & \text { HYALOPSEUSTIS* } \\ \text { vitreola } & \text { STENOMA } \\ \text { vittigera } & \text { EUCOSMA } \\ \text { vivax } & \text { TRICHOPTILUS } \\ \text { vivida } & \text { HYPERCALLIA } \\ \text { vociferans } & \text { FILINOTA } \\ \text { volitans } & \text { STENOMA } \\ \text { volubilis } & \text { COMPSOLECHIA } \\ \text { volucris } & \text { BATRACHEDRA } \\ \text { voluntaria } & \text { PHALONIA } \\ \text { voluptaria } & \text { MACHIMIA }\end{array}$

I925, Bull. Soc. R. Ent. Egypte, 9:2I5. I922, Exotic Microlepidoptera, 2: 598. (BM)

I9I5, Trans. Ent. Soc. London, I9I5: 237. I922, Exotic Microlepidoptera, 2: 574. (BM)

I9I4, Trans. Proc. New Zealand Inst. 46: 108.

I9I2, Ent. Mon. Mag. 48: 35. (Sub.)

I9I6, Exotic Microlepidoptera, 2: 21 .

I939, Trans. R. Ent. Soc. London, 89: 50. (BM)

I92I, Exotic Microlepidoptera, 2: 449.

I939, Trans. R. Ent. Soc. London, 89: 62.

I9I3, Exotic Microlepidoptera, I: 83.

I9I0, Proc. Linnean Soc. N.S. Wales, 35: 207.

I936, Exotic Microlepidoptera, 4: 6I9.

I906, Journ. Bombay Nat. Hist. Soc. I7: 404. (BM)

I9I6, Exotic Microlepidoptera, I: 52I. (BM)

I9I9, Exotic Microlepidoptera, 2: 235. (BM)

I92I, Exotic Microlepidoptera, 2: 452.

I9I4, Trans. Ent. Soc. London, I9I4: 279.

I934, Exotic Microlepidoptera, 4:525. (BM)

I9I6, Exotic Microlepidoptera, 2: 3I. (BM)

I9I8, Ann. Transvaal Mus. 6:5I. (T)

I9I5, Exotic Microlepidoptera, I: 373 .

I9I2, Trans. Ent. Soc. London, I9II: 707. (BM)

I9I2, Exotic Microlepidoptera, I: 52.

I9I4, Exotic Microlepidoptera, I: I88. (BM)

I938, in Caradja and Meyrick, Deuts. Ent. Zeit. Iris, 52:

6. (BM)

I9I4, Journ. Bombay Nat. Hist. Soc. 23: I I8.

I9I4, Trans. Ent. Soc. London, I9I4: 254. (BM)

I9I7, Trans. Ent. Soc. London, I9I7: I5. (BM)

I9I2, Trans. Ent. Soc. London, I9I I: 686. (BM)

I9I8, Exotic Microlepidoptera, 2: I43. (BM)

I9I6, Exotic Microlepidoptera, I: 558.

I9I4, Exotic Microlepidoptera, I: I94. (MA)

I92I, Exotic Microlepidoptera, 2: 405.

I9I3, Exotic Microlepidoptera, I: 94.

I9I3, Exotic Microlepidoptera, I: 72. (BM)

I9II, Journ. Bombay Nat. Hist. Soc. 21: I04.

I9II, Proc. Linnean Soc. N.S. Wales, 36: 230.

I925, Exotic Microlepidoptera, 3: I58. (BM)

I925, Exotic Microlepidoptera, 3: 209. (BM)

1932, Exotic Microlepidoptera, 4: 224.

I909, Ann. Transvaal Mus. 2: I, pl. I, fig. I. (T)

I924, Exotic Microlepidoptera, 3: I03. (BM)

I930, Exotic Microlepiàoptera, 3: 574. (P)

I925, Exotic Microlepidoptera, 3: 220. (BM)

I922, Trans. Ent. Soc. London, I922: 9I. (BM)

I897, Proc. Linnean Soc. N.S. Wales, 22: 306.

I9I2, Ent. Mon. Mag. 48: 35. (Sub.)

I9I4, Exotic Microlepidoptera, I: I76. (B.I) 


voluta
voluta
volutana
vomerata
vorax
vorticata
vorticosa
votaria
votiva
vulcanicola
vulgaris
vulgata
vulpecularis
vulpicoma
vulturina

xanthacma

xantharcha

xanthargyra

xanthaspis

xanthastis

xanthastis

xanthaula

xanthistia

xanthobyrsa

xanthocapna

xanthocarpa

xanthocausta

xanthocephala

xanthoceros

xanthochalca

xanthochares

xanthocharis

xanthochorda

xanthochra

xanthochyta

xanthoclina

xanthoclista

xanthocoma

xanthocosma

xanthocrana

xanthocrena

xanthocrita

xanthocrossa

xanthocycla

xanthodelta
MERITASTIS

PROTOLECHIA

PALAEOBIA

CEROSTOMA

PHTHORIMAEA

PHALONIA

MELASINA

SESIA

HETEROBATHRA

LOTISMA

CAPUA

SCYTHRIS

EUCOSMA

SPATALISTIS

ACROClITA

ALLOTALANTA

IMMA

NEMOTOIS

THIOTRICHA

MESOLECTA

TINEOLA

TIMYRA

CROCANTHES

STENOMA

PSEUDATTERIA

COMMATICA

CAMINEUTIS*

PROTOLECHIA

ADELA

LECITHOCERA

PROTANYSTIS

HOMALOXESTIS

HAPALOTHYMA*

PSALTICA

CROBYLOPHORA

AEMYLURGIS*

ENCRASIMA

CEROMITIA

BRACHMIA

MELASINA

BATRACHEDRA

OPOGONA

EULECHRIA

AMNIODES*

COMPSOTROPHA

[EUTHICTIS*]
I9I2, Trans. Ent. Soc. London, I9II: 677. (вм)

I904, Proc. Linnean Soc. N.S. Wales, 29: 34I.

I881, Proc. Linnean Soc. N.S. Wales, 6: 663.

I924, Exotic Microlepidoptera, 3: 88.

I939, Trans. R. Ent. Soc. London, 89: 53. (вм)

I9I2, Trans. Ent. Soc. London, I9II: 673. (Bм)

I930, Exotic Microlepudoptera, 3: 55I.

I92I, Exotic Microlepidoptera, $2: 444$.

I922, Exotic Microlepidoptera, 2: 548. (Bм)

1932, Exotic Microlepidoptera, 4:285. (v)

I921, Zool. Meded. 6: I45. (L)

I9I4, Ann. Transwaal Mus. 4: I97. (T)

I932, Trans. Ent. Soc. London, 80: III. (BM)

I932, Exotic Microlepidoptera, 4:26I. (v)

I936, Exotic Microlepidoptera, 4: 6Iо. (Bм)

I938, in Caradja and Meyrick, Deuts. Ent. Zeit. Iris, 52:

8. (BM)

I906, Trans. Ent. Soc. London, I906: I85. (BM)

I922, Exotic Microlepidoptera, 2: 536.

I9I8, Exotic Microlepidoptera, 2: I22. (BM)

I889, Proc. Linnean Soc. N.S. Wales, (Ser. 2) 3: I602. I9o8, Proc. Zool. Soc. London, p. 738.

I908, Journ. Bombay Nat. Hist. Soc. I8: 448. (Bм)

I93I, Exotic Microlepidoptera, 4: 73. (вм)

I9I5, Exotic Microlepidoptera, I: 447. (BM)

I930, Exotic Microlepidoptera, 3: 607. (BM)

I922, Trans. Ent. Soc. London, I922: 73. (BM)

I929, Exotic Microlepidoptera, 3: 544. (BM)

I904, Proc. Linnean Soc. N.S. Wales, 29: 376.

1937, Exotic Microlepidoptera, 5: 98. (C)

I9I4, Exotic Microlepidoptera, I: I99. (BM)

I938, Trans. R. Ent. Soc. London, 87: 52I.

I929, Exotic Microlepidoptera, 3: 518.

I9I9, Exotic Microlepidoptera, 2: 288.

I9Io, Journ. Bombay Nat. Hist. Soc. 20: I54. (Bм)

I9I8, Ann. Transvaal Mus. 6: 4I.

1936, Arb. morph. taxon. Ent. Berl. 3: I08. (D)

I923, Exotic Microlepidoptera, 3: 50. (BM)

I9I7, Ann. Natal Mus. 3: 6I7. (T)

I923, Exotic Microlepidoptera, 3: 46. (BM)

I93I, Exotic Microlepidoptera, 4: I04.

I9I7, Exotic Microlepidoptera, 2: 34 .

I9II, Journ. Bombay Nat. Hist. Soc. 2I: III.

I888, Proc. Linnean Soc. N.S. Wales, (Ser. 2) 2: 959.

I938, in Caradja and Meyrick, Deuts. Ent. Zeit. Iris, 52 :

I3. (BM)

I889, Proc. Limean Soc. N.S. Wales, (Ser. 2) 3: I636. 


$\begin{array}{ll}\text { xanthodes } & \text { ALUCITA } \\ \text { xanthodesma } & \text { STATHMOPODA } \\ \text { xanthodeta } & \text { DICHOMERIS } \\ \text { xanthodisca } & \text { PSELIASTIS } \\ \text { xanthodora } & \text { THIOTRICHA } \\ \text { xanthodoxa } & \text { MELOTELES* } \\ \text { xanthodryas } & \text { PHALONIA }\end{array}$

xanthogastra xanthoglypta xanthogramma xanthograpta xantholeuca xantholitha xanthoma xanthomela xanthomias xanthomicta xanthomima xanthomochla xanthomorpha xanthonota xanthonympha xanthopa xanthopeda xanthopetala xanthophaea xanthophanes xanthopharella xanthophracta xanthophylla xanthopis xanthoplaca xanthoplecta xanthopleura xanthoplitis xanthoprocta xanthoptila xanthoria xanthorrhabda xanthosarca xanthosaris xanthoschema xanthosema

xanthospora xanthostephana EULECHRIA xanthostola xanthoteles xanthotoxa ENARMONIA ASYMPHORODES LECITHOCERA

ARGYROPLOCE IMMA

SIMAETHIS AUTOSTICHA CYMONYMPHA* ETHMIA STATHMOPODA IMMA AUGANGELA* BORKHAUSENIA PYROZELA* STATHMOPODA PLACANTHES* ERETMOCERA SESIA ANTOLAEA* OXYCRATES* STENOMA LECITHOCERA ANTHINORA* GRACILARIA NEMOTOIS BUCCULATRIX LIMNOECIA MACROBATHRA GLYPHIPTERYX ETHMIA STATHMOPODA PHYCODES ANTAEOTRICHA ENCOLPOTIS* OINOPHILA CRYPTOLECHIA ACRIA

NESOPHYLAX* BORKHAUSENIA IIILAROGRAPHA
I890, Proc. Linnean Soc. N.S. Wales, 6: III2. I93I, Exotic Microlepidoptera, 4: I75. I9I3, Ann. Transvaal Mus. 3: 305. (T) I897, Proc. Linnean Soc. N.S. Wales, 22: 407. I923, Exotic Microlepidoptera, 3: 24. (BM) I920, Ann. South African Mus. I7: 289. (SA) I936, in Caradja and Meyrick, Deuts. Ent. Zeit. Iris, 50: I55. (BM) I92I, Zool. Meded. 6: I56. (L) I928, Exotic Microlepidoptera, 3: 423. (BM) I912, Exotic Microlepidoptera, I: 42. (BM) I916. Exotic Microlepidoptera, I: 588. (BM) I927, Insects of Samoa, 3, Lepidoptera, fasc. 2, p. I05. I929, Trans. Ent. Soc. London, 76:5I9. (BM) 1897, Proc. Linnean Soc. N.S. Wales, 22: 323. I930, Exotic Microlepidoptera, 4: 3. (BM) I932, Exotic Microlepidoptera, 4: 338. I9I6, Trans. Proc. New Zealand Inst. 48: 4I5. I906, Journ. Bombay Nat. Hist. Soc. I7: 4I5. (BM) I9I3, Exotic Microlepidoptera, I: 90. I923, Exotic Microlepidoptera, 3: 42. (BM) IgIo, Trans. Ent. Soc. London, I9IO: 455. I92I, Exotic Microlepidoptera, 2: 443. I9I4, Journ. Bombay Nat. Hist. Soc. 22: 780. (B.1) I930, Trans. Ent. Soc. London, 78: 3I4. (P) I93I, Exotic Microlepidoptera, 4: 4I. I926, Sarawak Mus. Journ. 3: I58. (BM) I9I4, Trans. Ent. Soc. London, I9I4: 256. (BM) I88I, Proc. Linnean Soc. N.S. Wales, 5: I4I. I930, Exotic Microlepidoptera, 3: 624. I931, Exotic Microlepidoptera, 4: I64. I920, Exotic Microlepidoptera, 2: 3 I9. I902, Trans. R. Soc. S. Australia, 26: I67. I922, Exotic Microlepidoptera, 2: 49I. (BM) I93I, Exotic Microlepidoptera, 4: I74. (BM) I908, Proc. Zool. Soc. London, p. 733. I9I4, Exotic Microlepidoptera, I: 283. I9I2, Trans. Ent. Soc. London, I9II: 709. (Bu) I909, Ann. South African Mus. 5: 352. (SA) I9I5, Exotic Microlepidoptera, I: 356. I9I7, Ann. South African Mus. I7: 7. (SA) Igo8, Journ. Bombay Nat. Hist. Soc. I8: 636. (BM) I926, Exotic Microlepidoptera, 3: 320.

I931, Ann. Mus. Nac. Hist. Nat. Buenos Aires, 36: 390. (BM)

I9I2, Ent. Mon. Mag. 48: 34. (Sub.) I888, Proc. Limean Soc. N.S. Wales, 2: 952. I934, Pacific Ent.Serv. Publ. 7, art. 28, p. 350. (вв) I923, Exotic Microlepidoptera, 3: 38. (BM) I920, Exotic Microlepidoptera, 2: 329. (BM) 


$\begin{array}{ll}\begin{array}{l}\text { xanthotricha } \\ \text { xanthotyla } \\ \text { xanthuris } \\ \text { xenadelpha } \\ \text { xenarcha }\end{array} & \begin{array}{l}\text { RECURVARIA } \\ \text { GELECHIA }\end{array} \\ \text { VANICELA } \\ \text { EUCOSMA } \\ \text { xenaula } & \text { BUCCULATRIX } \\ \text { xenias } & \text { OECOPHORA } \\ \text { xenica } & \text { EREUNETIS } \\ \text { xenica } & \text { IOPTERA } \\ \text { xenicopa } & \text { NOCHELODES* } \\ \text { xenicopis } & \text { CEROSTOMA } \\ & \\ \text { xenicosma } & \text { STATHMOPODA } \\ \text { xenoctenis } & \text { METAHEPIALUS } \\ \text { xenodes } & \text { TINEA } \\ \text { xenodoxa } & \text { OPOSTEGA } \\ \text { xenomima } & \text { PHILOBOTA } \\ \text { xenomorpha } & \text { HETEROCIYYTA } \\ \text { xenophaea } & \text { ODITES }\end{array}$

xenophila xenopis xenosema xeranta xerastis xeraula xerochroa xerodes xerodoxa xeroloxa xeronoma xeropa xerophaga

xerophanes xerophanta

xerophila xerophloea xerophylla xeropis xerospila xestobela xestobyrsa xestochalca xestolitha xestophanes xiphias

TEMELUCHA*

BRACHMIA

TORTRIX

CHELARIA

LASPEYRESIA

EUCOSMA

PITYOCONA*

DASMOPHORA*

CIRCICA

DICHOMERIS

TORTRIX

PROTOLECHIA

ASYMPHORODES

ACROLEPIA
I9I7, Trans. Ent. Soc. London, I9I7: 38. (BM)

I930, Exotic Microlepidoptera, 3: 546. (BM)

I927, Exotic Microlepidoptera, 3: 346. (BM)

I889, Trans. Proc. New Zealand Inst. 2I: I66.

I920, Voyage de Ch. Alluaud et $R$. Jeannel en Afrique

Oriental, II, Microlepidoptera, p. 6o. (P)

I893, Proc. Linnean Soc. N.S. Wales, 7: 60I.

I891, Ent. Mon. Mag. 27: 57.

I9I I, Trans. Linnean Soc. London, I4: 301.

I9I3, Exotic Microlepidoptera, I : 157 .

I920, Exotic Microlepidoptera, 2: 367 . (BM)

I938, in Caradja and Meyrick, Deuts. Ent. Zeit. Iris, 52:

23.

1922, Exotic Microlepidoptera, 2: 585 .

I926, Ann. South African Mus. 23: 35I. (SA)

I9o9, Trans. Ent. Soc. London, Igog: 42. (BM)

I893, Proc. Linnean Soc. N.S. Wales, 7: 608.

I9I3, Exotic Microlepidoptera, I: I22.

I906, Trans. R. Soc. S. Australia, 30: 47.

I93I, in Caradja, Bull. Sect. sci. Acad. roum. I4: 74. (BM)

I920, Exotic Microlepidoptera, 2: 3I3.

I889, Proc. Linnean Soc. N.S. Wales, (Ser. 2) 3: I679.

I920, Exotic Microlepidoptera, 2: 369. (BM)

I9I7, Exotic Microlepidoptera, 2: 68.

I905, Journ. Bombay Nat. Hist. Soc. I6: 599. (BM)

I9I0, Journ. Bombay Nat. Hist. Soc. 20: I44. (BM)

I92I, Ann. Transvaal Mus. 8: 66. (T)

I886, Trans. Ent. Soc. London, I886: I4.

I925, Bull. Soc. R. Ent. Egypte, 9: 2I6.

I935, Exotic Microlepidoptera, 4:602. (BM)

I935, Exotic Microlepidoptera, 4: 59I.

I909, Ann. Transvaal Mus. 2: 25, pl. 8, fig. 3. (T)

[PHILARACHNIS*]

GNORIMOSCHEMA
I9I4, Ent. Mon. Mag. 50: 2I9. (BM)

I909, Trans. Ent. Soc. London, I909: I5. (BM)

I93I, in Joannis, Ann. Soc. Ent. France, 98 (Supp.): 724 [486].

I939, Trans. R. Ent. Soc. London, 89: 5I. (BM)

I933, Exotic Microlepidoptera, 4: 4I8. (BM)

I904, Proc. Linnean Soc. N.S. Wales, 29: 320.

I9I8, Exotic Microlepidoptera, 2: II7. (BM)

I9I9, Exotic Microlepidoptera, 2: 268.

I888, Trans. Proc. New Zealand Inst. 20: 89.

I92I, Ann. Transvaal Mus. 8: 82. (T)

I926, Exotic Microlepidoptera, 3:248. (BM)

1904, Proc. Linnean Soc. N.S. Wales, 29: 354.

I934, Pacific Ent.Surv. Publ. 7, art. 28, p. 35I. (BB)

I93I, Ann. Mus. Nac. Hist. Nat. Buenos Aires, 36: $40 x$.

(BM) 
xiphodes

xiphostola

xiphura

xuthias

xuthocoma

xuthogastra

xuthomitra

xuthosaris

xuthoterma

xyleuta

xylinarcha

xylinaspis

xylinaula

xylinochra

xylinopa

xylinopis

xylobathra

xylochroa

xylocosma

xylocrossa

xylodelta

xylodes

xylodes

xylodesma

xylodeta

xylodoma

xylodryas

xyloglypta

xylogramma

xylograpta

xylomorpha

xylonitis

xylophaea

xylophaea

xylophracta

xylophragma

xylophthora

xylopsamma

xyloptera

xylopterella

xylospila

xylotechna

xylotoma

xylotripta

xylozona

xylurga

xyrias

xyridota
SYNTETRERNIS*

PHILOBOTA

URODUS

PROTOLECHIA

MACROBATHRA

COSMOPTERYX

NEPTICULA

ANTAEOTRICHA

COESYRA

DEPRESSARIA

BONDIA

AGRIOPHARA

LABDIA

SCYTHRIS

STENOMA

PSOROSTICHA

GELECHIA

EPITHECTIS

ANTAEOTRICHA

LASPEYRESIA

ARGYROPLOCE

NARTHECOCEROS*

TORTRIX

ALLOCLITA

STEREODMETA*

CYPHORYCTIS*

PERONEA

GELECHIA

LECITHOCERA

STENOMA

TRACHYEDRA*

NARYCIA

CAPUA

GELECHIA

CLADOPHANTIS*

ANTISPASTIS*

EPICNAPTIS

PLATYPTILIA

TELPHUSA

EULECHRIA

ARISTOTELIA

CHELARIA

TORTRIX

HOMALOXESTIS

PELOCNISTIS*

STENOMA

LEPTOCROCA

GLYPHIPTERYX
1922, Exotic Microlepidoptera, 2: 574. (BM)

I884, Proc. Linnean Soc. N.S. Wales, 8: 482.

I93I, Exotic Microlepidoptera, 4: 9o. (v)

1904, Proc. Linnean Soc. N.S. Wales, 29:36r.

I886, Proc. Linnean Soc. N.S. Wales, I0: 8I3.

I9Io, Trans. Ent. Soc. London, I9Io: 452.

I921, Ann. Transvaal Mus. 8: I40. (T)

I925, Exotic Microlepidoptera, 3: I75. (BM)

I920, Exotic Microlepidoptera, 2: 3 II.

I9I3, Exotic Microlepidoptera, I: II5. (BM)

I930, Exotic Microlepidoptera, 3: 589. (BM)

I9I5, Exotic Microlepidoptera, I: 407.

I935, Exotic Microlepidoptera, 4:605.

I931, Exatic Microlepidoptera, 4: I79.

I925, Exotic Microlepidoptera, 3:203. (BM)

I93I, in Caradja, Bull. Sect. sci. Acad. roum. I4: 72. (Вм)

I936, Veröff. Deutschen Kol.-Mus. I : 33I, pl. I3, figs. 28-30.

(H)

1926, Exotic Microlepidoptera, 3: 275. (BM)

I9I6, Exotic Microlepidoptera, I: 49I. (BM)

I939, Trans. R. Ent. Soc. London, 89: 52. (BM)

I928, Exotic Microlepidoptera, 3: 444. (BM)

Igo6, Journ. Bombay Nat. Hist. Soc. I7: I48. (BM)

IgIo, Proc. Linnean Soc. N.S. Wales, 35: 224.

IgI2, Ann. Transvaal Mus. 3: 72. (T)

I93I, Exotic Microlepidoptera, 4:65. (v)

I934, Exotic Microlepidoptera, 4:465. (v)

I927, Insects of Samoa, 3, Lepidoptera, fasc. 2, p. 69. (BM)

I923, Exotic Microlepidoptera, 3: 22. (BM)

I922, Zool. Meded. 7:86. (L)

I931, Exotic Microlepidoptera, 4: 40. (V)

I929, Exotic Microlepidoptera, 3: 498. (BM)

I935, Exotic Microlepidoptera, 4: 578. (c)

IgI2, Trans. Ent. Soc. London, I9I I: 675. (BM)

I92I, Ann. Transvaal Mus. 8: 7I. (T)

I9I8, Ann. Transvaal Mus. 6: 34. (T)

I926, Exotic Microlepidoptera, 3: 307. (BM)

I922, Exotic Microlepidoptera, 2: 596.

I908, Trans. Ent. Soc. London, I907: 488.

1932, Exotic Microlepidoptera, 4: 350. (BM)

I883, [Meyr. nec Walk.] Proc. Linnean Soc. N.S. Wales,

7:543.

1928, Proc. Hawaiian Ent. Soc. 7: 100. (SP)

I932, Exotic Microlepidoptera, 4: I99. (BM)

I89I, Ent. Mon. Mag. 27: I3. (BM)

I9I8, Exotic Microlepidoptera, 2: I02. (BM)

I932, Exotic Microlepidoptera, 4: I93. (BM)

I9I3, Trans. Ent. Soc. London, I9I3: ISS. (в.)

I93I, Rec. Cant. Mus. 3: 368.

I9I8, Exotic Microlepidoptera, 2: I94. (BM) 
xystidophora

xystidota

xystodes

xystopala

xystrota

xystrota

xythopterana

$\begin{array}{ll}\text { zacentra } & \text { HETEROGYMNA } \\ \text { zacharis } & \text { HYBROMA } \\ \text { zachroa } & \text { NOEZA } \\ & \text { [EUNEBRISTIS*] } \\ \text { zachrysa } & \text { GRACILARIA } \\ \text { zadocaea } & \text { ACROCERCOPS } \\ \text { zaleuca } & \text { NYMPHONIA } \\ \text { zaleuta } & \text { PROSCHISTI* } \\ \text { zalias } & \text { APATETRIS } \\ \text { zalocoma } & \text { PLEUROTA } \\ \text { zalocoma } & \text { TINEA } \\ \text { zalocrossa } & \text { STENOPTILIA } \\ \text { zalodes } & \text { STATHMOPODA } \\ \text { zalodisca } & \text { GLYPHIPTERYX } \\ \text { zalomorpha } & \text { MELASINA } \\ \text { zamenopa } & \text { ACROCERCOPS } \\ \text { zanclistes } & \text { OXYPTILU } \\ \text { zanclogramma } & \text { STENOMA } \\ \text { zanclophora } & \text { ACROLOPHUS } \\ \text { zanclotoma } & \text { COESYRA } \\ \text { zaplaca } & \text { CONOPOMORPHA } \\ \text { zapyra } & \text { ANTICRATES } \\ \text { zapyra } & \text { HILAROGRAPHA } \\ \text { zapyrana } & \text { STIGMONOTA } \\ \text { zapyropis } & \text { LYONETIA } \\ \text { zapyrrha } & \text { CAPUA } \\ \text { zarifa } & \text { DIOCOSMA } \\ \text { zascia } & \text { CTENOCOMPA } \\ \text { zathea } & \text { COSMOPTERYX } \\ \text { zatrophana } & \text { HARMOLOGA } \\ \text { zelaea } & \text { ETHMIA } \\ \text { zelaea } & \text { EURYTHECTA } \\ \text { zelantha } & \text { ARGYROPLOCE } \\ \text { zelopa } & \text { OPOSTEGA } \\ \text { zelosaris } & \text { STENOLECHIA } \\ \text { zelota } & \text { EUCOSMA } \\ \text { zelota } & \text { GLYPHIPTERYX } \\ \text { zelotica } & \text { PHILAGRIAS } \\ \text { zelotis } & \text { PYRGOPTILA } \\ \text { zelotypa } & \text { EULIA } \\ & \end{array}$

NEPHANTIS

ACROCERCOPS

BACTRA

AROTROPHORA
I893, Proc. Linnean Soc. N.S. Wales, 7: 548. I9I8, Exotic Microlepidoptera, 2: 2I5. (BM)

I9I6, Exotic Microlepidoptera, 2: 6.

[EUPETOCHIRA*]

I908, Proc. Zool. Soc. London, p. 729.

I9I5, Trans. Ent. Soc. London, I9I5: 224.

IgII, Trans. New Zealand Inst. 43: 62.

I88I, Proc. Linnean Soc. N.S. Wales, 6: 529.

I9I3, Exotic Microlepidoptera, I: 73. (BM)

I9I9, Exotic Microlepidoptera, 2:24I. (BM)

I9I4, Trans. Ent. Soc. London, I9I4: 278. (BM)

1907, Journ. Bombay Nat. Hist. Soc. I7: 983.

I9I2, in Wytsman, Genera Insectorum, fasc. I28, p. I7.

I9I3, Exotic Microlepidoptera, I: I36.

I907, Journ. Bombay Nat. Hist. Soc. I7: 73I. (вм)

I922, Exotic Microlepidoptera, 2: 500.

1884, Proc. Linnean Soc. N.S. Wales, 9: 749.

I9I I, Journ. Bombay Nat. Hist. Soc. 2I : I2I. (BM)

I907, Ent. Mon. Mag. 43: I46.

I9I3, Exotic Microlepidoptera, I: 93.

I920, Exotic Microlepidoptera, 2: 332. (BM)

I930, Exotic Microlepidoptera, 3: 550.

I934, Exotic Microlepidoptera, 4: 524.

I905, Journ. Bombay Nat. Hist. Soc. I6: 58I.

I9I5, Exotic Microlepidoptera, I: 4I7. (BM)

I922, Exotic Microlepidoptera, 2: 608. (BM)

I884, Proc. Linnean Soc. N.S. Wales, 9: 773.

1907, Proc. Linnean Soc. N.S. Wales, 32: 54.

I907, Proc. Linnean Soc. N.S. Wales, $32: 85$.

I886, Trans. Ent. Soc. London, I886: 286.

I88I, Proc. Linnean Soc. N.S. Wales, 6: 653.

I9I5, Trans. Ent. Soc. London, I9I5: 243.

I936, Exotic Microlepidoptera, 5: 59.

I921, Ann. Transvaal Mus. 8: I03. (T)

I920, Ann. South African Mus. I7: 309. (SA)

I9I7, Exotic Microlepidoptera, 2: 36.

1883, Trans. Proc. New Zealand Inst. I5: 46.

I906, Journ. Bombay Nat. Hist. Soc. I 7: 409. (BM)

I905, Trans. Ent. Soc. London, I905: 233.

IgI I, Proc. Linnean Soc. N.S. Wales, 36: 265.

I905, Journ. Bombay Nat. Hist. Soc. I6: 613.

I923, Exotic Microlepidoptera, 3: I2. (BM)

I9I6, Exotic Microlepidoptera, 2: I8. (BM)

I888, Trans. Proc. New Zealand Inst. 20:86.

I932, Trans. Ent. Soc. London, 80: II9.

I902, Trans. R. Soc. S. Australia, 26: I42.

I9I2, Trans. Ent. Soc. London, I9II: 679. (BM) 


$\begin{array}{ll}\text { zelotypa } & \text { PROCELEUSTIS } \\ \text { zeloxantha } & \text { CRYPTOLECHIA } \\ \text { zemiodes } & \text { EULECHRIA } \\ \text { zenobia } & \text { COSMOPTERYX } \\ \text { zephyritis } & \text { STENOMA } \\ \text { zestica } & \text { TISCHERIA } \\ \text { zesticopa } & \text { CHELARIA } \\ \text { zestodes } & \text { TORTRIX } \\ \text { zetes } & \text { PTEROPHORUS } \\ \text { zetetica } & \text { ARISTOTELIA } \\ \text { zeugmatica } & \text { SCYTHRIS } \\ \text { zobeida } & \text { STENOMA } \\ \text { zomeuta } & \text { SIMAETHIS } \\ \text { zomias } & \text { DICHOMERIS } \\ \text { zonaea } & \text { APHNOGENES* } \\ \text { zonarcha } & \text { XYSMATODOMA } \\ \text { zonastra } & \text { ARGYROPLOCE } \\ \text { zonias } & \text { CROCANTHES } \\ \text { zonites } & \text { OXYPTILUS } \\ \text { zonoceros } & \text { METANOMEUTA }\end{array}$

zonochares zonocopa zonodecta zonodeta zonodoxa zoncphanes zonoreas zonosphaera

$\begin{array}{ll}\text { zonostola } & \text { COESYRA } \\ \text { zonostoma } & \text { DICHOMERIS } \\ \text { zonotarsa } & \text { GRACILARIA } \\ \text { zonoteles } & \text { OXYTIECTA } \\ \text { zonotorna } & \text { MELODRYAS } \\ \text { zophaula } & \text { MALLOBATHRA } \\ \text { zopherana } & \text { STREPSICEROS } \\ \text { zopherandra } & \text { ACROCERCOPS } \\ \text { zopheropa } & \text { PSELDOIOXIA } \\ \text { zopheropis } & \text { LECITIOCERA } \\ \text { zopherota } & \text { PHIOEOGRAPTIS } \\ \text { zopherotarsa } & \text { GRACILARIA } \\ \text { zophochalca } & \text { EPITIICTIS } \\ \text { zophocosina } & \text { HYSTIROSIA } \\ \text { zophocrana } & \text { OPOGONA } \\ \text { zophocrossa } & \text { GLYPIIIDOCERA } \\ \text { zophodes } & \text { IMMA } \\ \text { zophodes } & \text { OECOPHORA }\end{array}$

LITHOCOLLETIS PSALTICA COLEOPHOLAS* LIMENARCHIS* PALAEOMICRA METACHARISTIS* NEMOTOIS OLBOTHREPTA
I9I8, Ann. Transvaal Mus. 6: 54. (T) 1934, Exotic Microlepidoptera, 4: 478. (BM) I902, Trans. R. Soc. S. Australia, 26: I49. I92I, Zool. Meded. 6: I68. (L) 1925, Exotic Microlepidoptera, 3:2I8. (BM) I9II, Ann. Transvaal Mus. 2: 238. (T) I929, Exotic Microlepidoptera, 3: 5I4. (BM) I924, Trans. Proc. New Zealand Inst. 55: 203. 1930, Exotic Microlepidoptera, 3: 569. (BM) I934, Exotic Microlepidoptera, 4: 5IO. (BM) I93I, Exotic Microlepidoptera, 4: I79. (BM) I93I, Exotic Microlepidoptera, 4: 33. (BM) I9I2, Trans. Proc. New Zealand Inst. 44: I2I. I9I4, Trans. Ent. Soc. London, I9I4: 283. (BM) I92I, Ann. Transvaal Mus. 8: 88. (T) I893, Proc. Linnean Soc. N.S. Wales, 7: 494. I93I, in Caradja, Bull. Sect. sci. Acad. roum. I4: 65. I904, Proc. Linnean Soc. N.S. Wales, 29: 403. I9I3, Exotic Microlepidoptera, I: I05. I935, in Caradja and Meyrick, Materialien zu einer Microlepidopteren Fauna der Chinesischen Provinzen Kiangsu,

Chekiang und Hunan, p. 88. (BM)

I933, Exotic Microlepidoptera, 4:36r. I9I4, Exotic Microlepidoptera, I: 26I. (BM) I939, Trans. R. Ent. Soc. London, 89: 59. I926, Exotic Microlepidoptera, 3: 288. I888, Trans. Proc. New Zealand Inst. 20: 9I. I922, Exotic Microlepidoptera, 2: 602. (P) 1924, Exotic Microlepidoptera, 3: 79.

I935. in Caradja and Meyrick. Materialien zu einer Microlepidopteren Fanna der Chinesischen Provinzen Kiangsu, Chekiang und Hunan, p. 73. (BM) I884, Proc. Linnean Soc. N.S. Wales, 9: 772. I9I4, Trans. Ent. Soc. London, I9I4: 28 I. I936, Exotic Microlepidoptera, 5: 39 . I885, Proc. Linnean Soc. N.S. Wales, 9: 1053. I925, Exotic Microlepidoptera, 3: I3I. I920, Ann. South African Mus. I7: 307. (SA) I881, Proc. Limnean Soc. N.S. Wales, 6: 68.5. I931, Exotic Microlepidoptera, 4: I69. Igo6, Journ. Bombay Nat. Hist. Soc. I7: 408. (Вм) 1931, Exotic Microlcpidoptera, 4: 78. (BM) I 904, Proc. Linnean Soc. N.S. Wales, 29: 394. I936, Exotic Microlepidoptera, 5:39. I9I8, Trans. Proc. New Zealand Inst. 50: I33. I93I, Exotic Microlepidoptera, +: 159. (В.I) 1915, Exotic Microlepidoptere, I: 360. 1929, Exotic Microlcpidoptera, 3: 530. (B.I) I909, Trans. Ent. Soc. London, Igo9: 35. (B.1) ISS6, Proc. Linnean Soc. N.S. Wales, ro: $7 s_{4}$. 


$\begin{array}{ll}\text { zophodesma } & \text { HYPERTROPHA } \\ \text { zophoessa } & \text { EULECHRIA } \\ \text { zotica } & \text { LIMNOECIA } \\ \text { zygaenura } & \text { EPISANNINA } \\ \text { zygiana } & \text { PYRGOTIS } \\ \text { zygitis } & \text { STRUTHISCA } \\ \text { zygodelta } & \text { HERMENIAS } \\ \text { zygodes } & \text { TINEA } \\ \text { zygodonta } & \text { OPOGONA } \\ \text { zygogramma } & \text { LASPEYRESIA }\end{array}$

$\begin{array}{ll}\text { zygonoma } & \text { ACROCERCOPS } \\ \text { zygophora } & \text { PHLOEOPOLA } \\ \text { zygophorus } & \text { YPSOLOPHUS } \\ \text { zygospila } & \text { ETHMIA } \\ \text { zygoterma } & \text { CHOROPLECA } \\ \text { zygotoma } & \text { STEGASTA } \\ \text { zymogramma } & \text { AMORBIA } \\ \text { zymota } & \text { CRYPSITHYRIS } \\ \text { zymota } & \text { MERIDARCHIS } \\ \text { zymotica } & \text { COLEOPHORA } \\ \text { zymotis } & \text { TELPHUSA }\end{array}$

I906, Trans. R. Soc. S. Australia, 30: 5 I. I883, Proc. Linnean Soc. N.S. Wales, 7: 515. I92I, Exotic Microlepidoptera, 2: 455 . I933, Exotic Microlepidoptera, 4: 4I6. I883, Trans. P oc. New Zealand Inst. I5: 39. 1907, Journ. Bombay Nat. Hist. Soc. I8: I55. I938, Trans. R. Ent. Soc. London, 87: 509. (Bм) I9I8, Ann. Transvaal Mus. 6: 44. I93I, Exotic Microlepidoptera, 4: I64. I935, in Caradja and Meyrick, Materialien zu einer Microlepidopteren Fauna der Chinesischen Provinzen Kiangsu, Chekiang und Hunan, p. 63.

I921, Exotic Microlepidoptera, 2: 4I6.

I889, Proc. Linnean Soc. N.S. Wales, (Ser. 2) 3: I592.

I904, Proc. Limnean Soc. N.S. Wales, 29: 430.

1934, Exotic Microlepidoptera, 4: 459 .

I9I7, Exotic Microlepidoptera, 2: 82.

I9I7, Trans. Ent. Soc. London, I9I7: 48. (BM)

I926, Exotic Microlepidoptera, 3: 260. (вм)

I9I7, Exotic Microlepidoptera, 2: 74.

I9Io, Proc. Linnean Soc. N.S. Wales, 35: 146.

I931, Journ. Linnean Soc. London, 37: 283.

I909, Ann. Transvaal Mus. 2: II, pl. 4, fig. 7. (T) 
. 
$\left[\begin{array}{ll}2 \\ 1\end{array}\right.$

- 1 vinitr 1955

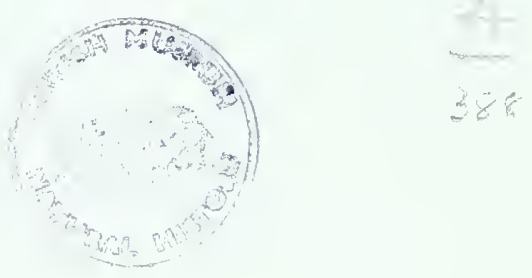



